Final Report on the Background Soil Characterization

Project at the Oak Ridge Reservation,

Oak Ridge, Tennessee

\title{
Volume 2-Data
}

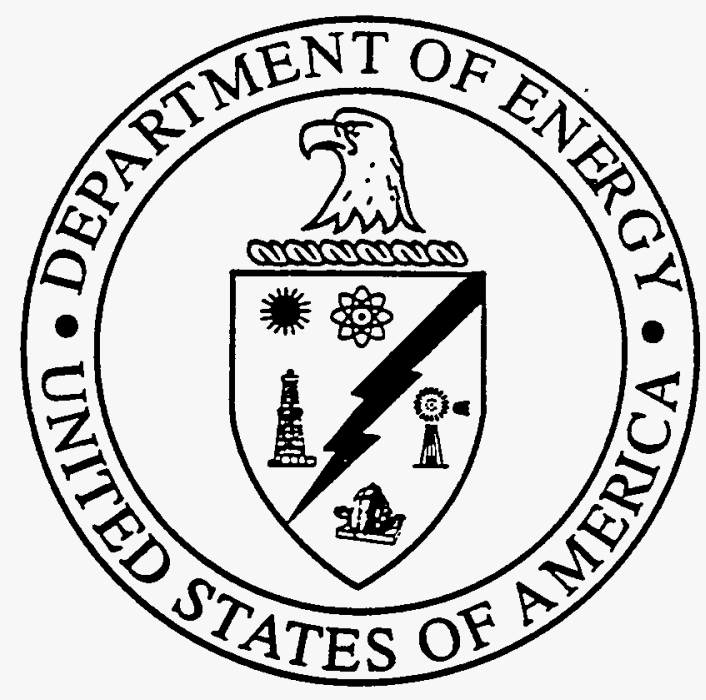




\section{DISCLAIMER}

This report was prepared as an account of work sponsored by an agency of the United States Government. Neither the United States Government nor any agency thereof, nor any of their employees, makes any warranty, express or implied, or assumes any legal liability or responsibility for the accuracy, completeness, or usefulness of any information, apparatus, product, or process disclosed, or represents that its use would not infringe privately owned rights. Reference herein to any specific commercial product, process, or service by trade name, trademark, manufacturer, or otherwise, does not necessarily constitute or imply its endorsement, recommendation, or favoring by the United States Government or any agency thereof. The views and opinions of authors expressed herein do not necessarily state or reflect those of the United States Government or any agency thereof.

This report has been reproduced directly from the best available copy.

Available to DOE and DOE contractors from the Office of Scientific and Technical Information, P.O. Box 62, Oak Ridge, TN 37831; prices available from 615-576-8401.

Available to the public from the National Technical Information Service, U.S. Department of Commerce, 5285 Port Royal Rd., Springfield, VA 22161. 


\section{DISCLAIMER}

Portions of this document may be illegible in electronic image products. Images are produced from the best available original document. 
DOE/OR/01-1175/V2

ES/ER/TM-84/V2

Final Report on the Background Soil Characterization Project at the Oak Ridge Reservation, Oak Ridge, Tennessee

\section{Volume 2-Data}

Environmental Restoration Division

P.O. Box 2003

Oak Ridge, Tennessee 37831-7298

Date Issued-October 1993

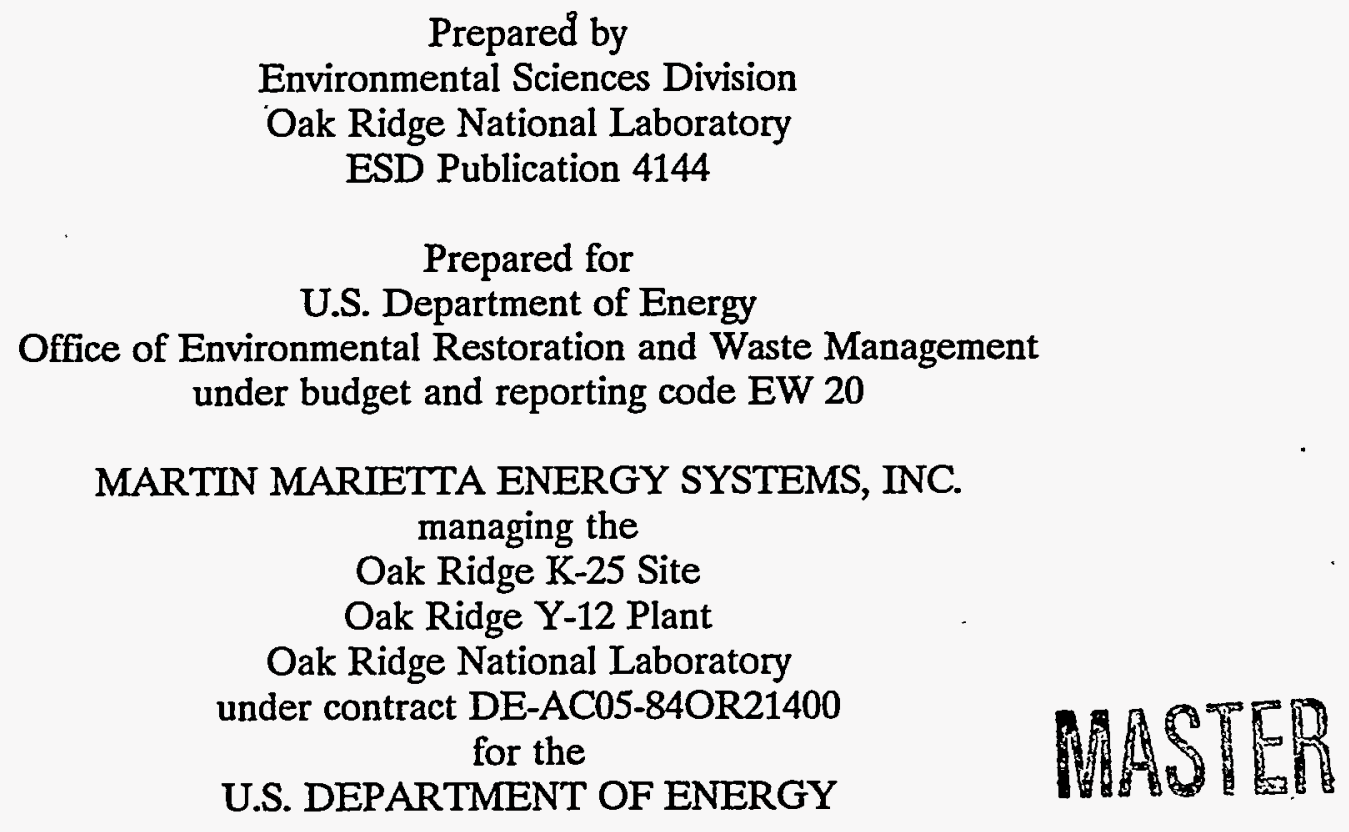




\section{CONTENTS}

VOLUME 2

TABLES $\ldots \ldots \ldots \ldots \ldots \ldots \ldots \ldots \ldots \ldots \ldots \ldots \ldots \ldots \ldots \ldots \ldots \ldots \ldots \ldots \ldots$

PREFACE TO VOLUME $2 \ldots \ldots \ldots \ldots \ldots \ldots \ldots \ldots \ldots \ldots$ vii

Appendix A. SITE DESCRIPTIONS, SOIL PROFILE DESCRIPTIONS, AND GENERAL ANALYSIS OF SITES $\ldots \ldots \ldots \ldots \ldots \ldots \ldots \ldots$ A-1

A.1 OAK RIDGE RESERVATION SITE DESCRIPTIONS ......... A-3

A.2 ROANE COUNTY SITE DESCRIPTIONS ... . . . . . . . . . A-17

A.3 ANDERSON COUNTY SITE DESCRIPTIONS ............. A.21

A.4 OAK RIDGE RESERVATION SOIL PROFILES ............ A A-25

A.5 ROANE COUNTY SOIL PROFILES . . . . . . . . . . . . . A A 97

A.6 ANDERSON COUNTY SOIL PROFILES ............... A-121

A.7 SITE LOCATIONS . . . . . . . . . . . . . . . . . . . A

A.7.1 Oak Ridge Reservation Site Locations $\ldots \ldots \ldots \ldots \ldots \ldots \ldots$ A . . . . .

A.7.2 Latitudes and Longitudes for Roane and

Anderson Counties ......................... A-146

Appendix B. SCREENING ANALYSIS DATA $\ldots \ldots \ldots \ldots \ldots \ldots \ldots \ldots$ B-1

Appendix C. ORGANIC ANALYSIS DATA $\ldots \ldots \ldots \ldots \ldots \ldots \ldots \ldots \ldots$

Appendix D. INORGANIC ANALYSIS DATA $\ldots \ldots \ldots \ldots \ldots \ldots \ldots \ldots \ldots$

Appendix E. RADIONUCLIDE ANALYSIS DATA $\ldots \ldots \ldots \ldots \ldots \ldots \ldots$

Appendix F. RELATION OF SAMPLE NUMBERS TO LABORATORY

SAMPLE DELIVERY GROUPS (SDGs) ............. F-1

Appendix G. SUMMARY OF STATISTICALLY TREATED DATA AND THE SIGNIFICANCE LEVEL OF DIFFERENCES

IN THE DATA . . . . . . . . . . . . . . . . . . . G

Appendix H. NEUTRON ACTIVATION ANALYSIS (NAA) DATA . . . . . . H-1

Appendix I. ICP/MS ANALYSIS DATA ................. I-1

Appendix J. OCCURRENCES OF REJECTED DATA $\ldots \ldots \ldots \ldots \ldots \ldots \ldots$ J-1 


\section{TABLES}

B.1 Volatile organic analysis results for soil samples $\ldots \ldots \ldots \ldots \ldots \ldots$ B-3

B.2 Weighted gamma screening results for soil samples $\ldots \ldots \ldots \ldots \ldots$ B-71

B.3 Unweighted gamma screening results for soil samples $\ldots \ldots \ldots \ldots \ldots$ B-91

C.1 Organic analysis results for soil samples $\ldots \ldots \ldots \ldots \ldots \ldots \ldots \ldots$ C-3

D.1 Inorganic analysis results for composite soil samples $\ldots \ldots \ldots \ldots \ldots$ D-3

E.1 Radionuclide analysis results for composite soil samples $\ldots \ldots \ldots \ldots$ E-3

E.2 Tritium analysis results for noncomposited soil samples $\ldots \ldots \ldots \ldots$ E-53

F.1 Relation of sample numbers to laboratory sample delivery groups (SDGs) $\ldots \ldots \ldots \ldots \ldots \ldots \ldots \ldots \ldots, \quad F-3$

G.1 Summary statistics for NAA data $\ldots \ldots \ldots \ldots \ldots \ldots \ldots \ldots \ldots$ G-5

G.2 Summary statistics for ICP/MS data $\ldots \ldots \ldots \ldots \ldots \ldots \ldots \ldots$ G-20

G.3 Significance levels for comparing inorganics $\ldots \ldots \ldots \ldots \ldots \ldots \ldots$ G-28

G.4 Significance levels for comparing PAHs $\ldots \ldots \ldots \ldots \ldots \ldots \ldots \ldots$ G-30

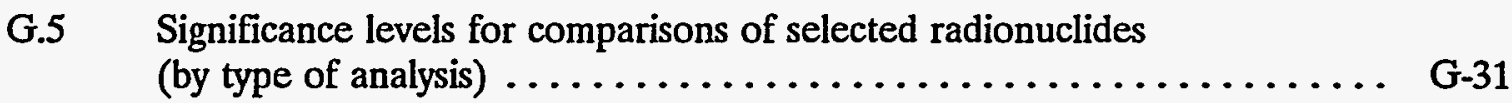

G.6 Comparisons of horizons for inorganics $\ldots \ldots \ldots \ldots \ldots \ldots \ldots \ldots$ G-32

G.7 Comparisons of horizons for selected radionuclides $\ldots \ldots \ldots \ldots \ldots$ G-47

G.8 Summary statistics for ORR inorganics-overall $\ldots \ldots \ldots \ldots \ldots \ldots$ G-54

G.9 Summary statistics for ORR radionuclides-overall $\ldots \ldots \ldots \ldots \ldots$ G-57

G.10 Summary statistics for ORR PAHs-overall $\ldots \ldots \ldots \ldots \ldots \ldots \ldots$ G-59

H.1 NAA analysis results for composite soil samples $\ldots \ldots \ldots \ldots \ldots \ldots \ldots$ H-3

I.1 ICP/MS metals results for composite soil samples $\ldots \ldots \ldots \ldots \ldots \ldots$ I-3

J.1 Occurrences of rejected data $\ldots \ldots \ldots \ldots \ldots \ldots \ldots \ldots \ldots \ldots, \quad$ J-3 


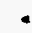




\section{PREFACE TO VOLUME 2}

This volume contains the data from the Background Soil Characterization Project. When available, the following validation qualifiers are used in the appendixes. When validation qualifiers are not available, the corresponding contract laboratory data qualifiers appearing on the next page are used.

Definitions of data validation qualifiers

\begin{tabular}{ll}
\hline Qualifier & Definition \\
\hline $\mathrm{U}$ & $\begin{array}{l}\text { The analyte was analyzed for but was not detected above the } \\
\text { reported sample quantitation limit. }\end{array}$ \\
The analyte was positively identified; the associated numerical \\
value is the approximate concentration of the analyte in the \\
sample. \\
The analysis indicates the presence of an analyte for which there is \\
presumptive evidence to make a tentative identification. \\
JN \\
$\begin{array}{l}\text { The analysis indicates the presence of an analyte that has been } \\
\text { tentatively identified, and the associated numerical value represents } \\
\text { its approximate concentration. } \\
\text { The analyte was not detected above the reported sample } \\
\text { quantitation limit. However, the reported quantitation limit is } \\
\text { approximate and may or may not represent the actual limit of } \\
\text { quantitation necessary to accurately and precisely measure the } \\
\text { analyte in the sample. } \\
\text { The sample results are rejected because of serious deficiencies in } \\
\text { the ability to analyze the sample and met quality control criteria. } \\
\text { The presence or absence of the analyte cannot be verified. } \\
\text { The laboratory did not register this compound, but there was } \\
\text { presumptive evidence of a compound that was within the retention } \\
\text { time window but was not reported. No other qualification of the } \\
\text { data was made. } \\
\text { The laboratory did not report the compound, but there was } \\
\text { presumptive evidence of a compound that was within the retention } \\
\text { time window but was not reported. The data were qualified as } \\
\text { estimated, J, because of other discrepancies with the data. }\end{array}$ \\
$\begin{array}{l}\text { The laboratory did not report the compound, but there was } \\
\text { evidence of a compound that was within the retention time window } \\
\text { but was not reported. The data were qualified as unusable, } \mathrm{R}, \\
\text { because of other discrepancies with the data. }\end{array}$ \\
\end{tabular}


Definitions of contract laboratory data qualifiers

Organic

\begin{tabular}{|c|l||}
\hline Qualifier & Explanation \\
\hline U & Indicates compound was analyzed for but not detected. \\
J & Indicates an estimated value. \\
N & $\begin{array}{l}\text { Indicates presumptive evidence of a compound [used only for tentatively } \\
\text { identified compounds (TICs)]. }\end{array}$ \\
P & $\begin{array}{l}\text { Used for pesticide/aroclor target analytes when there is greater than 25\% } \\
\text { difference for detected concentrations between the two gas chromatograph (GC) } \\
\text { columns. The lower of the two is reported and flagged. }\end{array}$ \\
C & $\begin{array}{l}\text { Used for pesticide results where the identification has been confirmed by } \\
\text { GC/mass spectrograph (MS). }\end{array}$ \\
B & $\begin{array}{l}\text { Used when the analyte is found in the associated blank as well as in the sample. } \\
\text { Identifies all compounds in an analyte at a secondary dilution factor. } \\
\text { D }\end{array}$ \\
A & $\begin{array}{l}\text { Indicates that a TIC is a suspected aldol-condensation product. } \\
\text { Xther specific flags may be used to properly define the results. If they are used } \\
\text { they must be fully described and attached to the Sample Data Package. }\end{array}$ \\
\hline
\end{tabular}

Inorganic

\begin{tabular}{|c|c|}
\hline Qualifier & Explanation \\
\hline $\mathrm{U}$ & Indicates compound was analyzed for but not detected. \\
\hline $\mathbf{J}$ & Indicates an estimated value. \\
\hline $\mathbf{N}$ & Spiked sample recovery not within control limits. \\
\hline E & Reported value estimated because of the presence of interference. \\
\hline $\mathbf{M}$ & Duplicate injection precision not met. \\
\hline B & $\begin{array}{l}\text { Reported value was obtained from a reading that was less than the CRDL, but } \\
\text { greater than or equal to the instrument detection limit (IDL). }\end{array}$ \\
\hline $\mathbf{S}$ & Reported value was determined by the method of standard additions (MSA). \\
\hline W & $\begin{array}{l}\text { Post-digestion spike for furnace atomic absorption is out of control limits, while } \\
\text { sample absorbance is less that } 50 \% \text { of spike absorbance. }\end{array}$ \\
\hline * & Duplicate analysis not within control limits. \\
\hline+ & Correlation coefficient for the MSA is less than 0.995 . \\
\hline
\end{tabular}


Abbreviations used for formation-locations

\begin{tabular}{||c|l||}
\hline Abbreviation & Definition \\
\hline DG-AND & Dismal Gap-Anderson County \\
DG-ROA & Dismal Gap-Roane County \\
DG-ORR & Dismal Gap-Oak Ridge Reservation \\
NL-ORR & Nolichucky-Oak Ridge Reservation \\
CHI-BV & Chickamauga-Bethel Valley \\
CHI-K25 & Chickamauga-K-25 Area \\
CHE-ORR & Chepultepec-Oak Ridge Reservation \\
CR-ORR & Copper Ridge-Oak Ridge Reservation \\
CR-AND & Copper Ridge-Anderson County \\
CR-ROA & Copper Ridge-Roane County \\
\hline
\end{tabular}




\section{Appendix A}

\section{SITE DESCRIPTIONS, SOIL PROFILE DESCRIPTIONS, AND}

GENERAL ANALYSIS OF SITES 



\section{A.1 OAK RIDGE RESERVATION SITE DESCRIPTIONS}

ORR Site 2. This site is located off Bear Creek Road close to the Clinch River, grid location N29300, E10300. The actual site is located northeast of the K-25 water treatment plant. Follow the road to where the power line right-of-way (ROW) crosses it. Then turn right, go up hill for about $200 \mathrm{ft}$, then left into the woods about $50 \mathrm{ft}$. The site is located in a stand of large oak, red maple, sweet gum and white pine that was probably cleared and pastured at one time but evidently never plowed. The underlying geology is the Dismal Gap Formation. The soils are typical of the soils of largest extent that were mapped on this formation. The soils are very heterogeneous. The geologic dip in the $C$ horizon varies from about $30^{\circ}$ to nearly vertical. Differential weathering of thin strata gives rise to very abrupt and irregular degrees of weathering with some strata being very highly weathered and other strata still hard and dense.

ORR Site 3. This site is located across Bear Creek Road from the Scientific Ecology Group plant, Grid N 29300, E11200. The actual site is located about $50 \mathrm{ft}$ north of the cut bank on the north side of the road. The vegetation on this site consists of old-field successional forest that has not been disturbed since the field was abandoned. Most of the old pines have died and fallen over. The present regrowth consists of young pine,oak, hickory, sassafras, and cedar with a sparse ground cover of honeysuckle and poison ivy. The underlying geology is the Nolichucky Shale. Because this site is on a stable landform, the soils were not badly eroded before abandonment.

ORR Site 5. This site is located on the south side of Bear Creek Road about $300 \mathrm{ft}$ west of the main entrance to the Central Training Facility and about $25 \mathrm{ft}$ south of the security fence, grid N29300, E15300. The site is located in an old field that was severely eroded before or after abandonment. Shallow gullies are on either side of this site. Vegetation is old-field successional forest. Most of the old pines have died and fallen over. Present vegetation consists of a dense stand of young pines along with cedar, oak, dogwood, and sweet gum along with a generous stand of honeysuckle and greenbriar. This site is the only ORR site that has a thick organic layer consisting primarily of mosses. The soils on this site are very similar to those of ORR Site 3, except that they are more eroded with the plow layer resting directly on the clayey subsoil beneath. The underlying geology is the Nolichucky Shale.

ORR Site 10. This site is located about $300 \mathrm{ft}$ north of the Tennessee Valley Authority (TVA) power line ROW that parallels Bear Creek Road on the north side of the road, Grid N30200, E21460. This site is located in an old field that had been severely eroded some time before abandonment. Vegetation is old-field successional forest that is now mostly hardwoods consisting of white oak, hickory cedar, and ash. The pine have disappeared. This site, being fairly open, has a dense ground cover of honeysuckle, Virginia creeper and poison ivy. The soils on this site are more typical of soils on steeper slopes. In fact, this site is the steepest of the ORR Phase 1 sites. The underlying geology is the lower Dismal Gap Formation, with strata in the $\mathrm{C}$ and $\mathrm{Cr}$ horizons nearly vertical.

ORR Site 11. This site is located about $50 \mathrm{ft}$ north of the TVA power line ROW that parallels Bear Creek Road on the north side of the road, ORR Site 10 is located about $250 \mathrm{ft}$ upslope. This site is located in an old field that had been severely eroded prior to or after abandonment. Vegetation is old-field successional forest. Many pines have died and fallen over. There is considerable young brush and sprouts of dogwood, sassafras, cherry, red maple, 
and sweet gum along with an abundant ground cover of poison ivy and honeysuckle. The soils are typical of stable uplands underlain by the upper Dismal Gap Formation.

ORR Site 13. This site is located south of the TVA power line ROW that parallels Bear Creek Road on the north side. The actual site, Grid N29480, E23220, is located about $100 \mathrm{ft}$ south of the ROW in forest. The site is located in an old farmyard. The remains of the chimney are located about $200 \mathrm{ft}$ east. Present vegetation consists of sugar maple and tulip poplar. The surface soil should have a higher $\mathrm{pH}$ and higher fertility. There is much evidence of worm activity on this site The only ground cover is a thick stand of poison ivy. The actual soil pit is situated within a brecciated zone of the Nolichucky Shale. Because of the past tectonic brecciation the soil is deeply weathered.

ORR Site 15. This site is located close to the intersection of the natural gas line ROW and the TVA power line ROW, Grid N29700, E25720. The route into this site and ORR Site 16 is through the first gate west of the Bear Creek and Route 95 intersection on the north side of the road. This field road is followed until the intersection of the TVA power line and the gas line ROW is crossed, then about $25 \mathrm{ft}$ north of the gas line ROW. The site is located in a severely eroded old field which was planted to loblolly pines. The trees are about 40 to $50 \mathrm{y}$ old and nearly ready for harvesting for poles. There is a hardwood understory consisting of oak, red maple, dogwood and a sparse ground cover of honeysuckle and grasses. The underlying geology is the lower Nolichucky Shale, which in this area, is fairly permeable as the saprolite fragments are mostly coated with red iron oxides.

ORR Site 16. This site is located close to the intersection of the gas line ROW and the TVA power line ROW, Grid N29700, E25928. The route into this site and ORR Site 16 is through the first gate west of the Bear Creek and Route 95 intersection on the north side of the road. This field road is followed until the intersection of the TVA power line and the gas line ROW is crossed, then along the power line ROW. The site is located on a sideslope of a severely eroded old field which was planted to loblolly pines. The trees are about 40 to $50 \mathrm{y}$ old and nearly ready for harvesting for poles. There is a hardwood understory consisting of oak, red maple, dogwood and a sparse ground cover of honeysuckle and grasses. The soils are typical that are underlain by the middle to lower Nolichucky Shale, which in this area, is less permeable than at ORR Site 15 , as the saprolite fragments are mostly coated with black manganese oxides and clay plugged.

ORR Site 19. This site is located east of the Bear Creek and Route 95 intersection within the area known as the Bear Creek Low-level Waste Disposal Development and Demonstration (LLWDDD) Site. The route to this site starts at the intersection of Bear Creek Road and Gum Hollow Road. Follow Gum Hollow Road to the intersection with the TVA power line ROW. Turn left and follow this road to the intersection with the telegraph ROW. Turn right onto the telegraph ROW road for about $400 \mathrm{ft}$. The site is about $60 \mathrm{ft}$ east into the woods. The site is located in an old field. Vegetation is old-field successional forest. Most of the old pines have died and fallen over. Present vegetation is mostly young hardwoods of cherry, sweet gum, cedar, poplar, dogwood, and Virginia pine. The ground surface is leaf covered with only scattered honeysuckle and poison ivy. The soils are typical of stable land surfaces that are underlain by the steeply dipping lower Dismal Gap Formation. This site has thin very fine grained sandstone strata in the $C$ horizon. The original location of this site was in a stand of young recently replanted pines and was moved about $300 \mathrm{ft}$ to the present location. 
ORR Site 21. This site is located in the area known as the Bear Creek LLWDDD. The route to this site starts at the intersection of Bear Creek Road and Gum Hollow Road. Follow Gum Hollow Road to the intersection with the TVA power line ROW. Turn left and follow this roâd for about $1,600 \mathrm{ft}$. The site is in the woods on the south side of the ROW, Grid N29700, E30940. The site is located in an old field that had been severely eroded before abandonment. Vegetation is old-field successional forest and some planted loblolly pines. Some pines have been harvested or have died and fallen over. Present vegetation consists of cedar, poplar, dense sourwood, oak, and red maple. Some grape vines and some prairie flowering plants are on the site. In addition to leaves, mosses and lichens cover part of the ground surface. The soils are typical for moderate slopes that are underlain by the Nolichucky Shale. Note that this site was moved a considerable distance from its original location. The original location was underlain by a deeply weathered section of the lower Maynardville Limestone which interbeds with the uppermost Nolichucky. When soil mapping was done in the Bear Creek LLWDDD site, this particular area was thought to be part of the Nolichucky, since the Maynardville was supposed to be high-grade limestone which gives rise to much different soils. Subsequent mapping and study finally put this shale-limestone interbedded area into the Maynardville which necessitated moving the site onto about the middle of the Nolichucky.

ORR Site 22. This site is located within the area known as the Bear Creek LLWDDD. The route to this site starts at the intersection of Bear Creek Road and Gum Hollow Road. Follow Gum Hollow Road to the intersection with the TVA power line ROW. Turn left and follow this road for about $800 \mathrm{ft}$. There is a cluster of wells on the north side of the ROW. Turn right onto a rough track and go to the top of the ridge then turn left and go down to where a large windthrow blocks the track. The site is about $100 \mathrm{ft}$ beyond the windthrow then about $50 \mathrm{ft}$ south into the woods, Grid N30520, E31250. Vegetation at this site is old-field successional forest of Virginia pine, shortleaf pine, dogwood, and cedar. The surface is leaf covered and there is a sparse stand of honeysuckle. This site is located in the transition zone between the Dismal Gap Formation and the Rogersville Shale. The saprolite in the sampling pit was mostly Rogersville with a very steep dip.

ORR Site 23. This site is located within the area known as the Bear Creek LLWDDD. The route to this site starts at the intersection of Bear Creek Road and Gum Hollow Road. Follow Gum Hollow Road to the intersection with the TVA power line ROW. Turn left and follow this road for about $800 \mathrm{ft}$. There is a cluster of wells on the north side of the ROW. Turn right onto a rough track for about $50 \mathrm{ft}$. The site is in the woods about $20 \mathrm{ft}$ to the east, Grid N29800, E31830. Vegetation at this site is old-field successional forest. Most of the pines have died and fallen over. Present vegetation is red maple, sourwood and dogwoods, plus a ground cover of honeysuckle and poison ivy. The soils are typical examples of the Dismal Gap Formation. The $\mathrm{C}$ horizon at this site contained glauconitic strata that were sampled.

ORR Site 24. This site is located within the area known as the Bear Creek LLWDDD. The route to this site starts at the intersection of Bear Creek Road and Gum Hollow Road. Follow Gum Hollow Road to the intersection with the TVA power line ROW. Turn left and follow this road for about $600 \mathrm{ft}$. The site is on the south side of the ROW then into the woods about $100 \mathrm{ft}$, Grid, N29900, E31980. The vegetation at this site is old-field successional forest with some planted loblolly pine about 30 y old. Present vegetation is a dense stand of poplar, dogwood, cedar, pine, and red maple with a sparse ground cover of poison ivy and honeysuckle. The soils are typical examples of the Nolichucky Shale. Of interest were the 
thick manganese oxide coatings on fragment surfaces in the $\mathrm{C} \& \mathrm{Cr}$ horizons that were covered by translocated clay.

ORR Site 25. This site is located within the area known as the Bear Creek LLWDDD. The route to this site starts at the intersection of Bear Creek Road and Gum Hollow Road. Follow Gum Hollow Road to the intersection with the TVA power line ROW. Turn left and follow this road for about $400 \mathrm{ft}$. The site is on the south side of the ROW then into the woods about $100 \mathrm{ft}$, Grid, N29800, E32290. Present vegetation on this site is planted loblolly pines that are about 40 to 50 y old. There is an understory of red maple, dogwood, and sweet gum. The ground surface is covered by a thick layer of pine needles and there is a moderate ground cover of poison ivy and honeysuckle. The soils are typical examples of the Nolichucky Shale.

ORR Site 26. This site is located east of the ORR area known as the Bear Creek LLWDDD. The route to this site starts at the intersection of Bear Creek Road and Gum Hollow Branch Road. Turn off Bear Creek onto Gum Hollow. Go to where the TVA power line ROW crosses. Turn right down the ROW track for about $1400 \mathrm{ft}$. The site is about 75 to $80 \mathrm{ft}$ north of the ROW in the woods, Grid N30000, E34370. The site is in a severely eroded old field that was abandoned well before 1942. Vegetation is old-field successional forest where hardwoods have supplanted the early pine stage. Present forest vegetation consists of red maple, oak, hickory, and a few cedar. The forest canopy is sufficiently dense that there is very little vegetative ground cover. The ground surface is covered with leaves. The soils are typical examples of the Dismal Gap Formation.

ORR Site 27. This site is located east of the ORR area known as the Bear Creek LLWDDD. The route to this site starts at the intersection of Bear Creek Road and Gum Hollow Branch Road. Turn off Bear Creek onto Gum Hollow. Go to where the TVA power line ROW crosses. Turn right down the ROW track for about $1800 \mathrm{ft}$. The site is about $100 \mathrm{ft}$ north of the ROW in the woods, Grid N30100, E34990. ORR Site 27 is separated from ORR Site 26 by a drainageway. The site is in an eroded old field that was abandoned well before 1942. Vegetation is old-field successional forest where hardwoods have supplanted the early pine stage. Present forest vegetation consists of red and black oak, white oak, a few Virginia pine and some dead cedars. The understory consists of beech, red maple, dogwood. Scattered ferns occur under the dense forest canopy, otherwise the ground surface is covered by leaves. This site and ORR Site 2 have the oldest forest vegetation for any of the ORR Phase 1 sites. The soils on this site are typical examples of the Dismal Gap Formation.

ORR Site 28. This site occurs along the new haul road that was constructed a few years ago to transport cover materials from the Chestnut Ridge borrow area to the Y-12 burial grounds. The route to this site starts at the intersection of Bear Creek Road and the new haul road. Turn onto the new haul road. The site is about $300 \mathrm{ft}$ beyond the gate on Bear Creek into the woods about $20 \mathrm{ft}$ from the edge of the TVA north-south power line ROW, Grid N29500, E34580. The site is in a very severely eroded old field when it was abandoned. Vegetation is old-field successional forest. Most of the older pines have either been harvested or died and fallen over. Present vegetation consists of a dense stand of young Virginia pine along with cedar and poplar. There is a sparse ground cover of honeysuckle, mosses, and ferns. The soils are typical examples of the Nolichucky Shale.

$O R R$ Site 31. This site occurs along the new haul road that was constructed a few years ago to transport cover materials from the Chestnut Ridge borrow area to the Y-12 burial 
grounds. The route to this site starts at the intersection of Bear Creek Road and the new haul road. Turn onto the new haul road. The site is about $3400 \mathrm{ft}$ from the intersection. The site is on the south side of the haul road and approximately $200 \mathrm{ft}$ into cutover woods, Grid N29900, E35820: The site is in a very severely eroded old field when it was abandoned: Vegetation is old-field successional forest. The pines were harvested 10 to $15 \mathrm{y}$ ago. Present vegetation consists of a dense stand of sweet gum, dogwood, cedar, hickory, poplar, and ash. There is a sparse ground cover of honeysuckle and poison ivy. Even though there had been some tree harvesting, the actual site showed little, if any, disturbance from the harvest. This site occurs low on a long sideslope. Initial soil investigations indicated that there might be some alluvial or colluvial capping. However, when the soil pit was opened the soil morphology indicated that the upper $61 \mathrm{~cm}$ of the soil formed in late Pleistocene Age or early Holocene Age slope wash that was derived from the Nolichucky Shale. The clay enriched subsoil has a clayey texture, but the substratum beneath did not contain any relic rock structure.

ORR Site 32. This site occurs along the new haul road that was constructed a few years ago to transport cover materials from the Chestnut Ridge borrow area to the Y-12 burial grounds. The route to this site starts at the intersection of Bear Creek Road and the new haul road. Turn onto the new haul road. The site is about $5400 \mathrm{ft}$ from the intersection and about $100 \mathrm{ft}$ Grid north into the woods from the edge of the TVA power line ROW, Grid N30200, E36760. Vegetation is old-field successional forest. Most of the pine trees were harvested or have died and fallen over. Present vegetation consists of a dense stand of hardwood underbrush plus abundant honeysuckle and some greenbriar. The soils on this site are typical examples of the upper Dismal Gap Formation.

ORR Site 33. This site occurs along the new haul road that was constructed a few years ago to transport cover materials from the Chestnut Ridge borrow area to the $\mathrm{Y}-12$ burial grounds. The route to this site starts at the intersection of Bear Creek Road and the new haul road. Turn onto the new haul road. The site is about $5700 \mathrm{ft}$ from the intersection and about 75 to $100 \mathrm{ft}$ Grid north into the woods from the edge of the TVA power line ROW, Grid N30000, E36970. This site is about 250-300 ft east of site 32. Vegetation is old-field successional forest. Most of the pine trees were harvested or have died and fallen over. Present vegetation consists of a dense stand of hardwood underbrush plus abundant honeysuckle and some greenbriar. The soils on this site are typical examples of the middle Dismal Gap Formation.

ORR Site 35. This site occurs along the new haul road that was constructed a few years ago to transport cover materials from the Chestnut Ridge borrow area to the $\mathrm{Y}-12$ burial grounds. The route to this site starts at the intersection of Bear Creek Road and the new haul road. Turn onto the new haul road. The site is about $6000 \mathrm{ft}$ from the intersection and about $150 \mathrm{ft}$ Grid north into the woods from the edge of the TVA power line ROW, Grid N30000, E37280. This site is about $250-300 \mathrm{ft}$ east of site 33 . Vegetation is old-field successional forest. Most of the pine trees were harvested or have died and fallen over. Present vegetation consists of a dense stand of sweet gum, dogwood, haw, sourwood and some poplar. About $90 \%$ of the ground surface is leaf covered with the remainder covered by honeysuckle, mosses and other forbes (weeds). The soils on this site are typical examples of the lower Dismal Gap Formation.

ORR Site 41. This site occurs along the new haul road that was constructed a few years ago to transport cover materials from the Chestnut Ridge borrow area to the Y-12 burial grounds. The route to this site starts at the intersection of Bear Creek Road and the new 
haul road. Turn onto the new haul road. The site is about $7400 \mathrm{ft}$ from the intersection and about $400 \mathrm{ft}$ Grid north into the woods from the edge of the TVA power line ROW, Grid N30200, E39570. This site occurs in an old field that was severely eroded prior to or after abandonment. Vegetation is old-field successional forest. Most older pines have died and fallen over. The present vegetation is a fairly dense regrowth of pine, red maple, beech, and poplar. The ground surface is covered with leaves. The soils are typical examples of the Middle Dismal Gap Formation.

ORR Site 42. This site occurs on the new haul road that was constructed a few years ago to transport cover materials from the Chestnut Ridge borrow area to the Y-12 burial grounds. The route to this site starts at the intersection of Bear Creek Road and the new haul road. Turn onto the new haul road. The site is about $8100 \mathrm{ft}$ from the intersection and about $30 \mathrm{ft}$ Grid north into the woods from the edge of the TVA power line ROW, Grid N30000, $\mathrm{E} 40500$. This site is in an old field that was severely eroded prior to or after abandonment. Vegetation is old-field successional forest. Most of the old pine trees have died and fallen over or were harvested. Present vegetation is a dense regrowth of poplar, sweet gum, cedar, oak, dogwood, and beech. There is a sparse ground cover of honeysuckle and poison ivy. The soils on this site are fairly typical examples of the middle to upper Nolichucky Shale.

ORR Site 43. This site is the easternmost site on the ORR. It is fairly close to west end of the Y-12 burial grounds. This site occurs on the new haul road that was constructed a few years ago to transport cover materials from the Chestnut Ridge borrow area to the Y-12 burial grounds. The route to this site starts at the intersection of Bear Creek Road and the new haul road. Turn onto the new haul road. The site is about $9100 \mathrm{ft}$ from the intersection and about $200 \mathrm{ft}$ Grid north into the woods from the edge of the TVA power line ROW, Grid N30400, E41460. This site occurs in an old field that was severely eroded prior to or after abandonment. Vegetation is old-field successional forest. Present vegetation is red maple, poplar, abundant dogwood along with a dense ground cover of honeysuckle and poison ivy. The soils on this site are typical of stable upland positions that are underlain by the lower Dismal Gap Formation. This site is very close to the Rogersville Shale boundary.

ORR Site 45. This site is situated on Chestnut Ridge and is underlain by the Copper Ridge Formation. The landform is convex with a slope gradient of about $25 \%$. The forest consists of older trees, but trees have been removed periodically. From the stumps present and their state of decay, it appears that the last logging was done 15 to 20 years ago. A recent woods road was cut to within $100-150 \mathrm{ft}$ of this site to allow access for drilling wells. The soil on this site is a typical residual soil, and there is no evidence of any accelerated erosion.

ORR Site 50. This site on Chestnut Ridge is underlain by residuum of the Chepultepec Formation. The landform is a long, gently sloping spur ridge with a $8 \%$ slope gradient. Present forest is old-field successional that is presently dominated by oaks, but there are still some red cedars along with white pine seedlings and red maple sprouts. The ground surface is leaf covered and there is no evidence of recent disturbance. The soil is representative of Chepultepec soils except for a thicker surface organic horizon.

ORR Site 51. This site is located on Chestnut Ridge and underlain by residuum of the Copper Ridge Formation. The landform is a convex sideslope with a slope gradient of about $15 \%$. Present forest is old-field successional but most of the original pines have died and fallen over. Present trees are mostly tulip poplar, red maple and sugar maple. This site is within 75 to $80 \mathrm{ft}$ of a recent clear cut that was replanted to loblolly pine is the past 15 to 
20 years. This site is also within $80 \mathrm{ft}$ of a recent woods road that provided access to well drilling sites. The soil is typical of Copper Ridge soils.

ORR Site 52 . This site is located on Chestnut Ridge and is underlain by residuum of the Chepultepec Formation. The landform is a doubly convex shoulder of a sideslope. The slope gradient is about $12 \%$. Present forest is old-field successional but most of the original pines have died and fallen over releasing the present hardwoods that are dominated by red maple, along with a few cedars and tulip poplar. The mostly leaf covered ground surface also has ferns and honeysuckle. The soil is typical of Chepultepec soils.

ORR Site 53. This site is situated on Chestnut Ridge and is underlain by residuum of the Chepultepec Formation, but the upper $36 \mathrm{~cm}$ of the soil consists of colluvium. The landform is a convex sideslope about a third of the way below a doubly convex shoulder with a slope gradient of about $20 \%$. Present forest is old-field successional, but the old field had been abandoned well before 1940. Present trees consist of white oak with an understory of dogwood, sourwood, and black gum. The actual soil pit revealed a complicated situation. The upper $36 \mathrm{~cm}$ of the sampled face had colluvium to a depth of $36 \mathrm{~cm}$, but colluvium extended to a depth of $70 \mathrm{~cm}$ on the left half of the pit face while the right half of the pit had clayey residuum at a depth of $36 \mathrm{~cm}$.

ORR Site 54. This site is situated on Chestnut Ridge and is underlain by residuum of the Copper Ridge Formation. The landform is a convex sideslope with a slope gradient of about $30 \%$. Present forest vegetation consists of older cutover trees that are presently dominated by chestnut oak with an understory of red maple. The forest at this site is very open. The soil is typical of Copper Ridge Soils.

ORR Site 55. This site is situated on Chestnut Ridge and is underlain by residuum of the Copper Ridge Formation. This site is located on a middle part of a convex sideslope between old alluvium above and colluvium below. Slope gradient is about $26 \%$ with a northeast aspect. Present vegetation is cutover forest dominated by chestnut oak, and with sugar maple and tulip poplar understory and a lower level of dogwood and black gum and oak seedlings. This site does not ever appear to have been plowed in the past due to the very high surficial chert content.The upper $27 \mathrm{~cm}$ of the soil consisted of colluvium. The shaded aspect has resulted in a darker than typical surface layer and there is considerable evidence of worm activity.

ORR Site 58. This site is situated on Chestnut Ridge and is underlain by residuum of the Copper Ridge Formation. The site is on the edge of a large, slightly convex ridgetop with a $6 \%$ slope gradient. Present forest is dominated by older hardwoods of red oak, sugar maple and some tulip poplar and an understory of sprouts and seedlings. The soil is a very typical Copper Ridge soil.

ORR Site 59. This site is situated on Chestnut Ridge and close to Route 95. It is underlain by residuum of the Copper Ridge Formation. The landform is a broad, convex, lower middle sideslope with a slope gradient of about $20 \%$. This site was a pasture field before abandonment and does not appear to have been plowed nor badly eroded. Present forest is old-field successional with some mature shortleaf pine, mature white oak and an understory of dogwood, beech, red maple and sassafras. The soil is a typical example of a Copper Ridge soil. 
ORR Site 60. This site is situated on Chestnut Ridge and is underlain by residuum of the Copper Ridge Formation. The landform is a doubly convex shoulder that steepens off into a drainageway. Slope gradient at the pit site is about $25 \%$. Forest on this site is old-field successional. The original pines have either died and fallen over or were harvested. Present trees are red maple and oak, along with a few white pine and.Virginia pine. There is an old field road about $20 \mathrm{ft}$ uphill. The soil at this site was eroded in the past but otherwise is a typical example of a Copper Ridge soil.

ORR Site 62. This site is situated on Chestnut Ridge and is underlain by residuum of the Copper Ridge Formation. The landform is a broad, slightly convex, ridgetop with a slope gradient of about $4 \%$. Forest is old-field successional. The original pines have all died and fallen over. Present canopy trees are oak and hickory with a red maple and dogwood understory and seedlings of red maple and sassafras. This site is about $80 \mathrm{ft}$ east of a recent clearcut that was replanted to loblolly pine. The soil is a typical example of Copper Ridge soils.

ORR Site 64. This site is situated on Chestnut Ridge and is underlain by residuum of the Copper Ridge Formation. The landform is the top of a slightly convex ridge with a slope gradient of about 3 to 5\%. The forest is old-field successional. The early pines haver all died and fallen over or else were harvested. Present trees consist of a few large oaks and tulip poplar, and a thick sapling stand of red maple. There was no evidence of any recent disturbance.The soil is a typical example of a Copper Ridge soil.

ORR Site 66. This site is situated on Chestnut Ridge and is underlain by residuum of the Chepultepec Formation. This landform is a doubly convex spur below a higher bench landform and there is a slope gradient of about $15 \%$. The forest at this site is a severely eroded old-field successional type that was dominated by Virginia pine. Most pines are still alive and standing. Some poplar, red maple trees are growing into the canopy and there is a thick understory of red maple-saplings and dogwood. The pit site is in a thick stand of ferns. The soil at this site is a typical example of a Chepultepec soil.

ORR Site 68. This site is situated on Chestnut Ridge and is underlain by residuum of the Chepultepec residuum. This site has a surficial layer of colluvium 40 to $50 \mathrm{~cm}$ thick. The landform is a convex sideslope below a colluvial bench and close to a recent tree throw mound. The actual pit has a colluvial layer of variable thickness that may be an older tree throw site. Present forest consists of older trees. There was a partial cutting about 15 to 20. years ago. Present tall trees are oaks, hickories and subcanopy trees of red maple and tulip poplar with a good stand of sprouts and saplings.

ORR Site 73. This site is situated on Chestnut Ridge and is underlain by residuum of the Chepultepec Formation. The landform is a doubly convex spur just below a topographic bench landform. Slope gradient is about $15 \%$. The forest consists of older trees and there has been harvesting from time to time. Stumps are near the pit. Present canopy trees are tulip poplar, oak and red maple. There is a thick sapling stand of oak, cherry, dogwood, and red maple. The soil is a typical example of a Chepultepec soil. The pit sampling face cut through an old stump hole, but this section was not sampled.

ORR Site 74. This site is situated on Chestnut Ridge and is underlain by residuum of the Chepultepec Formation. The landform is a convex shoulder on the lower part of a long spur ridge. Slope gradient is about $14 \%$. The forest is old-field successional, but the site is located 
close to an old fence row where old trees have barbed wire in them. The site was probably a pasture field at one time but it does not appear to have been plowed. The soil has a slightly higher sand content in the surface layer, but otherwise is a typical example of a Chepultepec söil:

ORR Site 75. This site is situated on Chestnut Ridge and is underlain by residuum of the Copper Ridge Formation. The landform is a broad, doubly convex, ridgetop with a slope gradient of about $13 \%$ and a shady northeast aspect. Forest is old-field successional. The original pines are being replaced by a thick stand of young pines, tulip poplar, black gum, sourwood and dogwood. The soil at this site has a thicker and darker surface than is otherwise typical of Chepultepec soils, but this soil is typical on this shaded aspect for Chepultepec soils.

ORR Site 77. This site is situated on Chestnut Ridge and is underlain by residuum of the Chepultepec Formation. The landform is a broad, slightly convex ridgetop with a slope gradient of 2 to $3 \%$. Forest is old-field successional. A few of the original pines are still standing along with taller red cedars. Most of the present canopy trees are now oaks, hickories, red maple and cherry. The ground surface is leaf covered. The soil is a typical example of a Chepultepec soil.

ORR Site 78. This site is situated on Chestnut Ridge and is underlain by residuum of the Chepultepec residuum. The landform is a broad, slightly convex ridgetop with a slope gradient of 4 to $5 \%$. The forest here is old-field successional. Most of the original pines have died and fallen. Present canopy tree are oaks and tulip poplar and a lower story of red maple, sassafras and cherry. The ground surface is leaf covered. This soil at this site has a surficial capping of ancient colluvium that is about $36 \mathrm{~cm}$ thick.

ORR Site 83. This site is situated on Chestnut Ridge and is underlain by residuum of the Copper Ridge Formation. The landform here is an elongated, doubly convex ridgetop, with a slope gradient of about 5\%. The forest is old-field successional. Most of the original pines have died and fallen over. The present trees are red maple, a few pines, poplars, dogwoods, cherry and sourwood along with some cedars. The ground surface is leaf covered. The soil is a typical example of a Copper Ridge soil.

ORR Site 85. This site is situated on Chestnut Ridge and is underlain by residuum of the uppermost Chepultepec Formation. The landform is a lower third of a long convex sideslope. The slope gradient here is about $48 \%$ and the site is on a shaded north-northeast aspect. Forest here is older growth that has been cutover from time to time. Present canopy trees are red oak, sugar maple, white pine and magnolia. There is abundant poison ivy and ferns. The ground surface is leaf covered. The upper $42 \mathrm{~cm}$ of the soil consists of creep materials.

ORR Site 86. This site is situated on Chestnut Ridge and is underlain by residuum of the uppermost Chepultepec Formation and perhaps some of the Longview Formation. The landform is a doubly convex, steep sideslope about halfway between the road and the creek. Slope gradient is about $45 \%$. Forest here is old-growth, but which has been cutover from time to time. Canopy trees are Chestnut oak and a few hickories along with some smaller red maples and sassafras. The entire soil has a very high chert content and that makes this soil different from a typical Chepultepec soil. 
ORR Site 90. This site is on Chestnut Ridge and close to Chestnut Ridge road. It is underlain by residuum of the Chepultepec Formation. The landform is a small, doubly convex spur that is surrounded by old alluvium. Slope gradient is about $6 \%$.

Present vegetation is a 30 to 40 year old stand of planted loblolly pine. There is a thick understory of red maple, roses, honeysuckle and blackberry briars. The soil is typical of lower Chepultepec soils that have sandstone fragments and a slightly higher sand content in the surface layer. This site is about $60 \mathrm{ft}$ north of Chestnut Ridge Road and has received road dust additions and perhaps other contaminants.

ORR Site 91. This site is situated on Chestnut Ridge and is underlain by residuum of the Copper Ridge Formation. The landform is a long convex sideslope spur. Slope gradient is about 7\% and on a shaded north aspect. The forest is an older one as there were several chestnut stumps near the pit. Present trees are tulip poplar, cherry, post oak,white oak plus one chestnut sprout about $15 \mathrm{ft}$ tall with a 1 inch $\mathrm{dbh}$. The ground is leaf covered. Except for a darker and thicker surface layer this soil is a typical example of a Copper Ridge soil.

ORR Site 93. This site is located in Bethel Valley east of Building 1505, very close to SWSA-1 and about $200 \mathrm{ft}$ into the woods south of the intersection of the blacktop road and the dirt road. This site is underlain by residuum of the Chickamauga Group Unit G. The landform is a broad convex spur that extends to the north from Haw Ridge. The slope gradient is about $7 \%$. Present forest is dominated by hardwoods but there are pines, a few large cedars, oaks and ash. The ground is leaf covered and there is also abundant poison ivy. The initial rad scan gave a higher than normal ground level reading of $70 \mathrm{CPM}$. The soil at this site was fairly typical of deeper Chickamauga soils. Depth to rock was highly variable below $85 \mathrm{~cm}$.

ORR Site 99. This site is located east of Building 1505 along the blacktop road that goes west to SWSA-1. This site is on the north side of the road and about $50 \mathrm{ft}$ into the woods. The site is underlain by residuum of the Chickamauga Group-Unit $G$. The landform is a lower convex sideslope with numerous gullies. The pit was dug between gullies. Slope gradient was about 5\%. Forest consists of old-field successional Virginia pine and shortleaf pine. There is a thick sapling stand of red maple and dogwood along with honeysuckle and poison ivy. The ground surface is about $70 \%$ covered with mosses and lichens and the remainder covered with pine needles and leaves. The soil at this site was deeply weathered and no rock was encountered to a depth of about $8 \mathrm{ft}$.

ORR Site 100. This site is located just west of Building 1505 , about $300 \mathrm{ft}$ south of the blacktop road that goes to SWSA-1. This site is underlain by residuum of the Chickamauga Group Unit $G$. The landform is on a fairly broad convex spur that drops off of Haw Ridge. Slope gradient is about $12 \%$. This site is in a severely eroded woods pasture. The open forest is dominated by large oaks, some white pine and sugar maple along with beech and maple saplings. There were blueberry bushes on the site. The ground surface was leaf covered. This site was unusual for Chickamauga soils in that there were blueberries and a surface organic horizon, an indication of deep weathering and acid soils. No hard rock was encountered in the soil pit but a $\mathrm{Cr}$ (paralithic) horizon was encountered at a depth of $84 \mathrm{~cm}$.

ORR Site 101. This site is located in the edge of the woods immediately east of the new water treatment plant and about $50 \mathrm{ft}$ into the woods. The landform is a convex spur coming off of Haw Ridge. The soils had been severely eroded before abandonment. Slope gradient was about $25 \%$. Present forest is dominated by mature cedar, oak and beech along with 
saplings of sugar maple, beech and dogwood. The ground surface was leaf covered and there was no evidence of any recent disturbance. The soil at this site was typical of Unit $G$ Chickamauga soils where rock is commonly encountered below a depth of about $50 \mathrm{~cm}$.

ORR Site 102. This site is located east of Building 4500 and about $300 \mathrm{ft}$ south of the road. The site is located in an old farm house yard or a barnyard. It is underlain by residuum of the Chickamauga Group. The landform is a broad, slightly convex sideslope with a slope gradient of about $3 \%$. The present vegetation consists of an open stand of mature oaks and Virginia pine. The ground surface has considerable grass, honeysuckle and poison ivy. There was no evidence of any recent disturbance. The soils were typical Chickamauga soils with rock encountered at a depth of $101 \mathrm{~cm}$.

ORR Site 103. This site is located about $100 \mathrm{ft}$ east of the paved road that goes south to the old Health Physics Reactor and across the road from a group of trailers. The site is underlain by residuum of the Chickamauga Group. The landform is the mid part of a doubly convex sideslope with a slope gradient of about $24 \%$. The forest is old-field successional. Some of the original pines remain along with a few older oaks. Small trees are beech, dogwood, gum and cedar. This site had been severely eroded prior to abandonment. The soils were deeply weathered at this site with no rock encountered within $105 \mathrm{~cm}$.

ORR Site 104. This site is located east of the X-10 complex, and about $300 \mathrm{ft}$ south of the powerline ROW. The site is underlain by deeply weathered residuum of the Chickamauga Group. The landform is a doubly convex sideslope with a slope gradient of about $13 \%$. Present vegetation is old-field successional forest. Most of the original pines and cedars have disappeared. Present trees consist of oaks, red maple and hickories. There are some lowbush blueberries and the ground surface is leaf covered.

ORR Site 108. This site is located in Bethel Valley east of the $\mathrm{X}-10$ complex. It is underlain by shallow residuum of the Chickamauga Group with depth to rock varying from 53 to $95 \mathrm{~cm}$ in the soil pit. The landform is a doubly convex sideslope with a slope gradient of about $9 \%$. Present vegetation is old-field successional forest with some large pines, more smaller pines and an understory of hardwoods. There is also fairly thick brush along with honeysuckle and poison ivy on the forest floor. The forest floor is mostly leaf covered but there are small patches of mosses.

ORR Site 110. This site is located east of ORR Site 108 and farther east of the X-10 complex. This site is underlain by residuum of the Chickamauga Group. The landform is a convex, elongated ridgetop with a slope gradient at the soil pit of about $2 \%$. This site is in an area where experiments had been located in the past. Present vegetation is old-field successional forest with pines, cedars and oaks as canopy trees. There are also smaller beech and dogwood along with weeds and honeysuckle. This site had been severely eroded prior to abandonment. The soil had thin rock ledges at several depths in the pit face that was sampled.

ORR Site 115. This site is located in Bethel Valley, east of ORR Site 110 but in the same vicinity, and close to the Walker Branch embayment of Melton Hill Lake. This site is underlain by shallow residuum of the Chickamauga Group Unit E. Depth to rock on the site ranged from above the ground surface to a maximum depth of $75 \mathrm{~cm}$. The landform is a convex ridgetop with a slope gradient of about $3 \%$. Present vegetation is old-field successional forest of Virginia pine, red cedar, oaks, hickories, ash and dogwood. Poison ivy was abundant. The site does not appear to have been plowed in the past, but was probably a woods pasture. 
Depth to rock in the soil pit was between 25 and $45 \mathrm{~cm}$ except for one corner that was $75 \mathrm{~cm}$ deep.

ORR Site 116. This site is located on a peninsula of the Walker Branch Embayment and about $500 \mathrm{ft}$ south of Bethel Valley road. This site is underlain by deeply weathered residuum of the Chickamauga Group Unit F. There were some rounded gravels on the surface, an indication that this site was covered with alluvium sometime in the past. A flint chip was found in the old Ap horizon, an indication that this area has been used for quite some time in the past. The landform is a slightly elongated, slightly convex ridgetop with a slope gradient of about $3 \%$. Present vegetation is a planted stand of loblolly pine with an estimated age of about 40 years. There are a few hardwoods in the understory and there is abundant honeysuckle. This site is located fairly close to an old house or barn.

ORR Site 117. This site is located on a peninsula located in the Walker Branch embayment and about $300 \mathrm{ft}$ south of ORR Site 116. This site is underlain by residuum of the Chickamauga Group Unit G. Rock was encountered in the soil pit at a depth of $105 \mathrm{~cm}$. The landform is a slightly convex spur that was once covered with old alluvium. Slope gradient on the site was about $2 \%$. Present vegetation is a planted loblolly pine plantation with approximately 40 year old trees. Landform is a slightly convex spur that extends out into the lake and the slope gradient at the site is about $2 \%$. This site was farmed in the past.

ORR Site 118. This site is located in the K-25 section of the ORR. This site along with sites ORR Site 119 and ORR Site 120 occur in the only, almost undisturbed, stand of native hardwoods left in the East Fork area of Poplar Creek. The site is underlain by deeply weathered residuum of the East Fork section of the Chickamauga Group. The landform is a doubly convex spur between two small drainageways. Slope gradient is about $6 \%$, This site is underlain by deeply weathered residuum of the East Fork Chickamauga Group. Present vegetation is old growth hardwoods of white oak, American beech, cherry and sugar maple. This part of the forest does not appear to have been plowed but the site has been eroded, probably the results of cattle pasturing or burning off the ground cover. The soil is typical of most deep Chickamauga soils.

ORR Site 119. This site is located in the K-25 section of the ORR, and within the only area of old growth hardwoods in the area. The site is underlain by residuum of the East Fork section of the Chickamauga Group. The landform is a narrow convex ridge with a slope gradient of about $3 \%$. Vegetation at this site is cutover woods. Present trees are cedars along with oaks, ash and hardwood saplings. Depth to rock varied from $30 \mathrm{~cm}$ to more than $100 \mathrm{~cm}$ in the soil pit.

ORR Site 120. This site is located in the K-25 section of the ORR. It is underlain by deeply weathered residuum of the East Fork section of the Chickamauga Group. The landform is a sideslope with slight convexity and a slope gradient of about $4 \%$. Present vegetation is old growth hardwoods dominated by beech. This does not appear to have aver been plowed but was probably a woods pasture. The soil on this site had a surficial layer of old alluvium that was about $27 \mathrm{~cm}$ thick.

ORR Site 121. This site is located in the $\mathrm{K}-25$ section of the ORR and about $200 \mathrm{ft}$ south of the old county road. This site is also about $200 \mathrm{ft}$ north of an old cemetery. The landform is a convex shoulder of a broad, slightly convex ridgetop. Slope gradient is about $5 \%$. Present vegetation is a mature stand of old-field successional forest. Dominant canopy trees are 
Virginia pine with an understory of hardwoods. The forest floor is open but covered with pine needles. This site had been plowed and was severely eroded at abandonment.

orr ORR Site T22. This site is located in the $\mathrm{K}=25$ section of the ORR. This site is underlain by deeply weathered residuum of the East Fork section of the Chickamauga Group. The landform at this site is an elongated but narrow ridgetop with a slope gradient of about $6 \%$. Present vegetation is a planted loblolly pine plantation with trees about 40 years old. There are abundant briars and honeysuckle. This site is located close to an old farm building site. This site has been subjected to some erosion.

ORR Site 123. This site is located in the K-25 section of the ORR. This site is underlain by deeply weathered residuum of the East Fork section of the Chickamauga Group. The landform is a convex sideslope below a prominent cherty ridge. Present vegetation is a loblolly pine plantation with trees about 40 years old. This site had been severely eroded prior to abandonment.

ORR Site 124. This site is located in the K-25 section of the ORR. And just west of a rock bluff overlooking the East Fork of Poplar Creek. This site is underlain at shallow depth by rocks of the East Fork section of the Chickamauga Group. Landform is a broad but slightly convex ridgetop with a slope gradient of about $2 \%$ at the pit site. The site is located near the edge of a loblolly pine plantation. The soil surface is leaf covered and there has been no recent disturbance, but there had been disturbance when the pines were planted.

ORR Site 125. This site is located in the K-25 section of the ORR, and close to ORR Site 123 and ORR Site 124. The site is underlain by deeply weathered residuum of the East Fork section of the Chickamauga Group. The landform is a very gently sloping, slightly convex ridge with a slope gradient of about $3 \%$ at the site. Present vegetation is loblolly pine about 40 years old. This site had been severely eroded before abandonment.

ORR Site 126. This site is located in the K-25 section of the ORR. and on the south side of the Oak Ridge Turnpike about $500 \mathrm{ft}$. This site is underlain by deeply weathered residuum of the East Fork section of the Chickamauga Group. The actual soil pit was in a brecciated zone. Several sandstone fragments from the Rockwood sandstone of East Fork Ridge were in the subsoil. The landform is the convex top of a narrow spur ridge with a slope gradient of about $5 \%$. Present vegetation is old-field successional forest, presently a mix of pines and hardwoods. There is abundant poison ivy ground cover and considerable mosses. The soils were severely eroded prior to abandonment.

ORR Site 127. This site is located in the K-25 section of the ORR, and on the south side of the Oak Ridge Turnpike about $300 \mathrm{ft}$. The site is underlain by deeply weathered residuum of the East Fork Chickamauga Group. The landform is a broad convex ridgetop with a slope gradient of about $2 \%$. Present vegetation is old-field successional forest of Virginia pine, red cedar, ash, some tulip poplar álong with abundant ground cover of poison ivy and honeysuckle. The soil was severely eroded prior to abandonment.

ORR Site 128. This site is located in the K-25 section of the ORR, and on the south side of the Oak Ridge Turnpike about $400 \mathrm{ft}$. The site is underlain by deeply weathered residuum of the East Fork section of the Chickamauga Group. The landform is an interfluve between the heads of two gullies and with a slope gradient of about $4 \%$. Present vegetation is old-field successional forest that is dominated by Virginia pine, red cedar along with dogwood and red 


\section{A-16}

maple. There is abundant poison ivy and honeysuckle. The soil at this site was severely eroded prior to abandonment.

ORR Site 129. This site is located in the K-25 section of the ORR. IT is located on the old county road as shown on the site location map. The actual site is about $250 \mathrm{ft}$ east of the gravel road. This site is underlain by deeply weathered residuum of the East Fork section of the Chickamauga Group, and also very close to the Whiteoak Mountain Fault. The landform is the lower convex sideslope with a slope gradient of about $11 \%$. Present vegetation is old-field successional forest. This site was probably a pasture and was not severely eroded before abandonment. Present trees are white pine, and a few large oaks. There are also some holly trees, plus red maple and oak sprouts. 


\section{A.2 ROANE COUNTY SITE DESCRIPTIONS}

ROANE Site 3. On the Pattie Gap quadrangle, go south on Dry Fork Valley Road. This property lies west from Salem Baptist Church. The site is in the hardwood forest $550 \mathrm{ft}$ west of the church, $350 \mathrm{ft}$ southwest of Stamp Creek, and $200 \mathrm{ft}$ southeast of the power lines. The present vegetation is oak, sweetgum, red maple, dogwood, and poison ivy. There was no evidence of a plow layer. The underlying geology is the Dismal Gap Formation. The site lies between several rills and gullies in a convex toeslope position.

ROANE Site 7. On the Pattie Gap quadrangle, go south on Dry Fork Valley Road. After passing Blue Springs Road, the power lines will cross the road. The site is in the hardwood thicket $1500 \mathrm{ft}$ southwest of the intersection of Blue Springs Road and Dry Fork Valley Road. It is $750 \mathrm{ft}$ west of the intersection of the power lines and Dry Fork Valley Road. It is $600 \mathrm{ft}$ west of Dry Fork Creek. This site has been cleared at one time. The present vegetation is tulip poplar, dogwood, red maple, and some Virginia pine. Age of the deciduous trees indicated they had been established at least 40 years. There was evidence of a plow layer. There are many spots where rills are beginning to develop. The underlying geology is the Dismal Gap Formation. There is evidence of colluvium on this site. Oddly, in several places sandstone fragments can be seen laying on the surface. Presently, this area is occupied by cattle for pasture.

ROANE Site 8. On the Pattie Gap quadrangle, go south on Dry Fork Valley Road. After passing Blue Spring Road, the power lines will cross the road. The site is in the hardwood thicket $1800 \mathrm{ft}$ southwest of the intersection of Blue Spring Road and Dry Fork Valley. It is $500 \mathrm{ft}$ west of the intersection of the power lines and Dry Fork Valley Road. It is $300 \mathrm{ft}$ east of Roane Site 7. This site has been cleared at one time. The present vegetation is tulip poplar, dogwood, red maple, and some Virginia pine. Age of the deciduous trees indicated they had been established at least 40 years. There was evidence of a plow layer. There are many spots where rills are beginning to develop. The underlying geology is the Dismal Gap Formation. There is evidence of colluvium on this site. Oddly, in several places sandstone fragments can be seen laying on the surface. Presently, this area is occupied by cattle for pasture.

ROANE Site 9. On the Pattie Gap quadrangle, go south on Dry Fork Valley Road. This property lies west from Salem Baptist Church. The site is in the hardwood forest $500 \mathrm{ft}$ west of the church, $500 \mathrm{ft}$ southwest of Stamp Creek, and $200 \mathrm{ft}$ southeast of the power lines. The present vegetation is oak hardwoods mixed with Virginia pine. There was no evidence of a plow layer. The underlying geology is the Dismal Gap Formation. The site lies between several rills and gullies in a convex toeslope position.

ROANE Site 10. On the Pattie Gap quadrangle, go south on Dry Fork Valley Road. Turn west on to Gage Road. After crossing Stamp Creek, the site is on a small knob $300 \mathrm{ft}$ northeast from Gage Road and $350 \mathrm{ft}$ northwest of Dry Fork Valley Road. It lies $100 \mathrm{ft}$ west from the creek. This site has been cleared at one time. The present vegetation is shortleaf pine, red maple, and oak. Age of the deciduous trees indicated they had been established at least 40 years. There was evidence of a plow layer. The underlying geology is the Dismal Gap Formation. There is evidence of alluvium on this site. Several large rounded rocks were on site and in the profile. The area north of this site was a sagegrass field with multiple gullies and rills. Presently, this area is occupied by cattle for pasture. 
ROANE Site 13. On the Pattie Gap quadrangle, go south on Dry Fork Valley Road. Go past Gage Road. The site is in the forest $3000 \mathrm{ft}$ south from Gage Road and $500 \mathrm{ft}$ west from Dry Fork Valley Road. It is $250 \mathrm{ft}$ west from the creek. The present vegetation is Virginia pine, red maple, dogwood, poison ivy, and chestnut oak. Age of the deciduous trees indicated they had been established at least 40 years. There was evidence of a plow layer. The underlying geology is the Dismal Gap Formation. The site is at the bottom of a long slope in a bench position. There is evidence of alluvium in the area but none was seen in this profile.

ROANE Site 14. On the Pattie Gap quadrangle, go south on Dry Fork Valley Road. Go past Gage Road. The site is in the forest $3200 \mathrm{ft}$ south from Gage Road and $600 \mathrm{ft}$ west from Dry Fork Valley Road. It is $250 \mathrm{ft}$ west from. the creek. This site is on a rise $200 \mathrm{ft}$ south of Roane 13. The present vegetation is tulip poplar, red maple, dogwood, poison ivy, and chestnut oak. The understory vegetation is thick. Age of the deciduous trees indicated they had been established at least 40 years. There was evidence of a plow layer. The underlying geology is the Dismal Gap Formation. However, this formation is very thin and in digging the soil pit the Rogersville Formation was also exposed. The site is in a midslope position. The slope rapidly drops into a drain below the site.

ROANE Site 17. On the Pattie Gap quadrangle, go south on Dry Fork Valley Road. The site is in the pine thicket $1000 \mathrm{ft}$ west of Dry Fork Valley Road and $3000 \mathrm{ft}$ north of Waterford Lane. The present vegetation is Virginia pine, eastern red cedar, and sweetgum. This land has not been cleared in the last 40 years. There was no evidence of a plow layer. The underlying geology is the Dismal Gap Formation. The site lies between the convergence of two drains on a convex slope.

ROANE Site 19. On the Pattie Gap quadrangle, go south on Dry Fork Valley Road. This property lies west of Salem Baptist Church. The site is in the hardwood forest $750 \mathrm{ft}$ west of the church, $750 \mathrm{ft}$ southwest of the creek, and $200 \mathrm{ft}$ southeast of the power lines. The present vegetation is oak, red maple, hickory, eastern red cedar, and dogwood. There was evidence of a plow layer. The underlying geology is the Dismal Gap Formation. The site lies between several rills and gullies on a convex toeslope.

ROANE Site 20. On the Pattie Gap quadrangle, go south on Dry Fork Valley Road. This property lies west of Salem Baptist Church. The site is in the hardwood forest $950 \mathrm{ft}$ northwest of the church, $1000 \mathrm{ft}$ southwest of Stamp Creek, and $200 \mathrm{ft}$ southeast of the power lines. The present vegetation is chestnut oak, sweetgum, and dogwood. There was evidence of a plow layer. The underlying geology is the Dismal Gap Formation. The site lies between several rills and gullies in a toeslope position. Highly eroded.

ROANE Site 21. On the Pattie Gap quadrangle, go south on Dry Fork Valley Road. This property lies west of Salem Baptist Church. The site is in the hardwood forest $1100 \mathrm{ft}$ west of the church, $1200 \mathrm{ft}$ southwest of Stamp Creek, and $200 \mathrm{ft}$ southeast of the power lines. The present vegetation is oak, red maple, tulip poplar, sweetgum, and ground pine. There was evidence of a plow layer. The underlying geology is the Dismal Gap Formation. The site lies between several rills and gullies in a midslope position.

ROANE Site 22. On the Pattie Gap quadrangle, go south on Dry Fork Valley Road. This property lies west of Salem Baptist Church. The site is in the hardwood forest $1400 \mathrm{ft}$ west of the church, $1500 \mathrm{ft}$ southwest of Stamp Creek, and $200 \mathrm{ft}$ southeast of the power lines. The 
present vegetation is oak, Virginia pine, tulip poplar, eastern red cedar, and ground pine. There was evidence of a plow layer. The underlying geology is the Dismal Gap Formation. The site lies between several rills and gullies in a midslope position on a bench.

ROANE Site 33. This site is on the Pattie Gap Quadrangle. The site is $500 \mathrm{ft}$ east from Dry Fork Valley Road, $500 \mathrm{ft}$ north from Gage Road, and $400 \mathrm{ft}$ northeast from an old limestone quarry that is marked on the topo map. This area has been under hardwood forest for the last 40 years. The primary constituents are redbuds, scarlet oak, hickory, dogwood, and blackjack oak. The area has been burned over at some time in the past. Scars on the trunks of the older trees were very evident. The underlying geology is the Copper Ridge Formation.

ROANE Site 34. This site is on the Pattie Gap Quadrangle. The site is $1000 \mathrm{ft}$ southeast from Dry Fork Valley Road, $1200 \mathrm{ft}$ northeast from Gage Road, and $1000 \mathrm{ft}$ northeast from an old limestone quarry that is marked on the topo map. This area has been under hardwood forest for the last 40 years. The primary constituents are scarlet oak, black oak, Virginia pine, and blackjack oak. The area has been burned over at some time in the past. Scars on the trunks of the older trees were very evident. The underlying geology is the Copper Ridge Formation.

ROANE Site 35. This site is on the Pattie Gap Quadrangle. The site is $1000 \mathrm{ft}$ southeast from Dry Fork Valley Road, $1000 \mathrm{ft}$ northeast from Gage Road, and $100 \mathrm{ft}$ west from an old logging road. This area has been under hardwood forest for the last 40 years. The primary constituents are scarlet oak, chestnut oak, hickory, dogwood, sassafras and blackjack oak. The area has been burned over at some time in the past. Scars on the trunks of the older trees were very evident. The underlying geology is the Copper Ridge Formation.

ROANE Site 39. Located on the Pattie Gap Quadrangle, this site lies $500 \mathrm{ft}$ north from Williams Road and $1000 \mathrm{ft}$ southeast from the intersection of Williams Road and Dry Fork Valley Road. This area been forested for the last 50 years. The primary forest species were chestnut oak, scarlet oak, blackjack oak, and dogwood. The underlying geology is the Copper Ridge Formation.

ROANE Site 40. Located on the Pattie Gap Quadrangle, Roane 40 lies $900 \mathrm{ft}$ north from Williams Road and $1500 \mathrm{ft}$ southeast from Dry Fork Valley Road. This area been forested for the last 50 years. The primary forest species were chestnut oak, white oak, hickory, and Virginia pine. The underlying geology is the Copper Ridge Formation.

ROANE Site 41. On the Pattie Gap quadrangle, this site is $1200 \mathrm{ft}$ northeast from Williams Road $1800 \mathrm{ft}$ southeast from Dry Fork Valley Road. This area been forested for the last 50 years. The primary forest species were chestnut oak, scarlet oak, and white oak. The underlying geology is the Copper Ridge Formation.

ROANE Site 42. This site is located on the Pattie Gap quadrangle $1500 \mathrm{ft}$ southeast from Dry Fork Valley Road and $50 \mathrm{ft}$ northeast of an old logging road. It is $1000 \mathrm{ft}$ south of the powerlines. This area was under deciduous forest, primarily red maple, blackjack oak, chestnut oak, and red sumac. The area has not been cleared in the last 40 years. The underlying geology is the Copper Ridge Formation.

ROANE Site 43. The site is on the Pattie Gap Quadrangle. Located $1300 \mathrm{ft}$ southeast from Dry Fork Valley Road, this site is $100 \mathrm{ft}$ northeast of an old logging road and $1200 \mathrm{ft}$ 
south from the power lines. This area was under deciduous forest, primarily sassafras, dogwood, chestnut oak, and red sumac. The area has not been cleared in the last 40 years. The underlying geology is the Copper Ridge Formation.

ROANE Site 44. The site is on the Pattie Gap Quadrangle and located $1000 \mathrm{ft}$ southeast from Dry Fork Valley Road and $1400 \mathrm{ft}$ south from the power lines. This area was under deciduous forest, primarily dogwood, maple, blackjack oak, chestnut oak, and tulip poplar. The area has not been cleared in the last 40 years. The underlying geology is the Copper Ridge Formation.

ROANE Site 45. The site is on the Pattie Gap Quadrangle. Roane 45 is located $1500 \mathrm{ft}$ southeast from Dry Fork Valley Road, $100 \mathrm{ft}$ west of an old logging road, and $1600 \mathrm{ft}$ south from the power lines. This area was under deciduous forest, primarily sassafras, dogwood, chestnut oak, and hickory. The area has not been cleared in the last 40 years. The underlying geology is the Copper Ridge Formation.

ROANE Site 46. The site is on the Pattie Gap Quadrangle. Located $1500 \mathrm{ft}$ southeast from Dry Fork Valley Road, this site is $100 \mathrm{ft}$ west of an old logging road and $1800 \mathrm{ft}$ south from the power lines. This area was under deciduous forest, primarily sassafras, dogwood, chestnut oak, and red maple. The area has not been cleared in the last 40 years. The underlying geology is the Copper Ridge Formation.

ROANE Site 47. The site is on the Pattie Gap Quadrangle. This site is located $1400 \mathrm{ft}$ southeast from Dry Fork Valley Road. It is $200 \mathrm{ft}$ west of an old logging road and $2100 \mathrm{ft}$ south from the power lines. This area was under deciduous forest, primarily tulip poplar, dogwood, oak, and red maple. The area has not been cleared in the last 40 years. The underlying geology is the Copper Ridge Formation. 


\section{A.3 ANDERSON COUNTY SITE DESCRIPTIONS}

ANDERSON Site 1. The site is in a hardwood thicket adjacent to the Old Andersonville Pike between Hinds Creek Valley Road and US 441. Located in the Norris Quadrangle, this site is approximately $1400 \mathrm{ft}$ northeast of Old Anderson Pike and $1000 \mathrm{ft}$ west from Hinds Creek Road. This site is $200 \mathrm{ft}$ northeast of Anderson Site 10. The site had been cleared at one time. The present vegetation is tulip poplar, dogwood, red maple, and some Virginia pine. Age of the deciduous trees indicated they had been established at least 50 years. The underlying geology is the Dismal Gap Formation. There was a water seep at approximately 40 centimeters. The slope dips strongly (30\%) approximately $50 \mathrm{ft}$ from the site. Presently, this area is occupied by cattle for pasture.

ANDERSON Site 3. This site is located on the Big Ridge Park quadrangle. Take Highway 441 north. Turn right on to Hinds Creek Road. After several miles, the road forks. Take the road on the left, which is Judson Road. The site is in a hardwood forest $100 \mathrm{ft}$ northwest of Judson Road and 0.4 miles south from the intersection of Judson Road and Moore Valley Road (Cooper's Gap). The present vegetation is moss, poison ivy, dogwood, red maple, and Virginia pine. Age of the deciduous trees indicated they had been established at least 50 years. The underlying geology is the Dismal Gap Formation. The site is on a wide slope. The surface drops to a drainage way approximately $150 \mathrm{ft}$ to the northwest. There are no houses near this site, although there are indications of an old home site approximately $300 \mathrm{ft}$ southwest of the site near Judson Road.

ANDERSON Site 4. Located on the Big Ridge Park quadrangle, this site is in a hardwood forest $300 \mathrm{ft}$ northwest of Judson Road and 0.6 miles south from the intersection of Judson Road and Moore Valley Road (Cooper's Gap). The present vegetation is poison ivy, dogwood, elm, cherry, sourwood, red maple, and Virginia pine. Age of the deciduous trees indicated they had been established at least 50 years. The underlying geology is the Dismal Gap Formation. The site is in the middle of a steep slope. It was difficult to find a good level position in which to dig.

ANDERSON Site 5. Located on the Big Ridge Park quadrangle, this site is in a hardwood forest $300 \mathrm{ft}$ northwest of Judson Road and 0.6 miles south from the intersection of Judson Road and Moore Valley Road (Cooper's Gap). This site is $300 \mathrm{ft}$ south from Anderson Site 4, but slightly higher on the slope. The present vegetation is poison ivy, dogwood, oak, and Virginia pine. Age of the deciduous trees indicated they had been established at least 50 years. The underlying geology is the Dismal Gap Formation. The site is in the middle of a steep slope. It was difficult to find a good level position in which to dig.

ANDERSON Site 9. This site is on the Powell quadrangle. Take Highway 441 north. Turn left onto East Wolf Valley Road. Go past Nolan Lane. The site is approximately $1300 \mathrm{ft}$ north of East Wolf Valley Road and 0.25 miles west from Nolan Lane: It lies in a hardwood forest about $100 \mathrm{ft}$ south of an old logging road downslope toward East Wolf Valley Road. The surrounding forest shows evidence of having been logged in the last 40 years. The site in a midslope position on a convex slope. The trees on this site are older and probably not logged as recently as those closer to the logging road. The present vegetation is tulip poplar, dogwood, red maple, and some Virginia pine. The underlying geology is the Dismal Gap Formation. This site is $300 \mathrm{ft}$ north of Anderson Site 19. 
ANDERSON Site 10. This site is in a hardwood thicket adjacent to the Old Andersonville Pike between Hinds Creek Valley Road and US 441. Located in the Norris Quadrangle, this site is approximately $1200 \mathrm{ft}$ northeast of Old Anderson Pike and $1000 \mathrm{ft}$ west from Hinds Creek Road. This site had been cleared at one time. The present vegetation is tulip poplar, dogwood, red maple, and some Virginia pine. Age of the deciduous trees indicated they had been established at least 50 years. The underlying geology is the Dismal Gap Formation. The site is in a level upland position. Presently, this area is occupied by cattle for pasture.

ANDERSON Site 11. Site is located on the Norris Quadrangle. Take Highway 441 north. Turn right on to Hinds Creek Road. Drive several miles until two signs saying Hidden Drive are passed. This site is located $600 \mathrm{ft}$ northwest from Hinds Creek Road and $1000 \mathrm{ft}$ northwest from the Hidden Drive sign. It is $700 \mathrm{ft}$ south of a small creek. The present forest vegetation is tulip poplar, may apple, beech, red maple, and hickory. The understory vegetation is very thick. This site has probably been cleared at some time. Age of the deciduous trees indicated they had been established at least 50 years. The underlying geology is the Dismal Gap Formation.

ANDERSON Site 12. This site is on the Big Ridge Park Quadrangle. Take Highway 441 north. Turn right on to Hinds Creek Road. Drive several miles until Hill Road enters on the right. This site is located $500 \mathrm{ft}$ northwest from the intersection of Hill Road and Hinds Creek Road and $100 \mathrm{ft}$ west of the power lines. It is $200 \mathrm{ft}$ west from Anderson Site 22 on a wide slope. A drain lies between sites 12 and 22. The present forest vegetation is dogwood, sweetgum, hickory, and Virginia pine. A thick carpet of grass underlies the trees. This site has probably been cleared at some time. Age of the deciduous trees indicated they had been established at least 50 years. The underlying geology is the Dismal Gap Formation. The site presently is used as pasture for cattle.

ANDERSON Site 19. This site is on the Powell quadrangle. Take Highway 441 north. Turn left onto East Wolf Valley Road. Pass Nolan Lane. The site is in a hardwood forest on the $300 \mathrm{ft}$ south of an old logging road downslope toward East Wolf Valley Road. It is approximately $1000 \mathrm{ft}$ north of East Wolf Valley Road and 0.25 miles west from Nolan Lane. The surrounding forest shows evidence of having been logged in the last 40 years. The site in a midslope position on a convex slope. The trees on this site are older and probably not logged as recently as those closer to the logging road. The present vegetation is tulip poplar, sassafras, red maple, moss, and some Virginia pine. The underlying geology is the Dismal Gap Formation. This site is $300 \mathrm{ft}$ south of Anderson Site 9.

ANDERSON Site 20. Located on the Big Ridge Park quadrangle, the site is in a hardwood forest $100 \mathrm{ft}$ northwest of Judson Road and 0.3 miles south from the intersection of Judson Road and Moore Valley Road (Cooper's Gap). The present vegetation is moss, poison ivy, dogwood, red maple, and Virginia pine. Age of the deciduous trees indicated they had been established at least 50 years. The underlying geology is the Dismal Gap Formation. The site is on a wide slope. There are several areas in which rills have developed and erosion is evident. There are no houses near this site, although there are indications of an old home site approximately $500 \mathrm{ft}$ southwest of the site near Judson Road.

ANDERSON Site 21. Located on the Big Ridge Park Quadrangle, this site is located $700 \mathrm{ft}$ northwest from the intersection of Hill Road and Hinds Creek Road and $150 \mathrm{ft}$ west of the power lines. It is $200 \mathrm{ft}$ northeast from Anderson Site 12. The site is located in a saddle between two slopes at the head of a drain. The present forest vegetation is dogwood, 
sweetgum, hickory, and Virginia pine. A thick carpet of grass underlies the trees. This site has probably been cleared at some time. Age of the deciduous trees indicated they had been established at least 50 years. The underlying geology is the Dismal Gap Formation. The site présently is used for pasture fór cattle.

ANDERSON Site 22. This site is located on the Big Ridge Park Quadrangle. It lies $500 \mathrm{ft}$ northwest from the intersection of Hinds Creek Road and Hill Road and $300 \mathrm{ft}$ west of the power lines. It is $200 \mathrm{ft}$ east from Anderson Site 12 on a convex slope. A drain lies between sites 12 and 22 . The present forest vegetation is dogwood, tulip poplar, eastern red cedar, and Virginia pine. A thick carpet of grass underlies the trees. This site has probably been cleared at some time. Age of the deciduous trees indicated they had been established at least 50 years. The underlying geology is the Dismal Gap Formation. The site presently is used as pasture for cattle.

ANDERSON Site 31. Anderson 31 is located on the Powell Quadrangle. Situated on top of a large knob, this site is $1000 \mathrm{ft}$ west of the Powell/Knox water tank, $300 \mathrm{ft}$ south of the dirt road leading to the water tank, and $800 \mathrm{ft}$ northeast from Heiskell Road. This area has been undisturbed for the last 50 years. Vegetation is primarily hardwoods-sassafras, red oak, chestnut oak, and some Virginia pines. The underlying geology is the Copper Ridge Formation.

ANDERSON Site 32. This site is on the Powell Quadrangle. Located $200 \mathrm{ft}$ north of Anderson 31, the site is $1200 \mathrm{ft}$ southwest of the water tank, $100 \mathrm{ft}$ south of the dirt road, $800 \mathrm{ft}$ northeast from Heiskell Road. This area has been undisturbed for the last 50 years. Vegetation is primarily hardwoods-sassafras, hickory, red oak, and chestnut oak. The underlying geology is the Copper Ridge Formation.

ANDERSON Site 33. This site is located on the Powell Quadrangle. Located $500 \mathrm{ft}$ southwest from Anderson 32, this site is $700 \mathrm{ft}$ southwest of the water tank, $600 \mathrm{ft}$ south of the dirt road, $900 \mathrm{ft}$ northeast from Heiskell Road. This area has been undisturbed for the last 50 years. Vegetation is primarily hardwoods-sassafras, dogwood, hickory, red oak, and chestnut oak. The underlying geology is the Copper Ridge Formation.

ANDERSON Site 34. On the Powell Quadrangle, Anderson 34 is located across the dirt access road $500 \mathrm{ft}$ north from Anderson 33 . It is $500 \mathrm{ft}$ northwest of the water tank, $100 \mathrm{ft}$ north of the dirt road, $1400 \mathrm{ft}$ northeast from Heiskell Road. This area has been undisturbed for the last 50 years. Vegetation is primarily hardwoods-sassafras, dogwood, red oak, and chestnut oak. The underlying geology is the Copper Ridge Formation.

ANDERSON Site 35. Located on the Powell Quadrangle, Anderson 35 is $200 \mathrm{ft}$ northeast from Anderson 34. This site is $700 \mathrm{ft}$ northwest of the water tank, $150 \mathrm{ft}$ north of the dirt road, $1500 \mathrm{ft}$ northeast from Heiskell Road. This area has been undisturbed for the last 50 years. Vegetation is primarily hardwoods-sassafras, dogwood, red oak, and chestnut oak. The underlying geology is the Copper Ridge Formation.

ANDERSON Site 36. Located on the Powell Quadrangle, this site is $700 \mathrm{ft}$ northiest of the water tank, $200 \mathrm{ft}$ north of the dirt road, $1600 \mathrm{ft}$ northeast from Heiskell Road. This area has been undisturbed for the last 50 years. Vegetation is primarily hardwoods-red oak, yellow poplar, hickory, and white oak. The underlying geology is the Copper Ridge Formation. 
ANDERSON Site 37. Located on the Big Ridge Park Quadrangle, the site is $800 \mathrm{ft}$ west of Mt. Hebron church and $700 \mathrm{ft}$ southeast from Hinds Creek Road. The area has been under forest for the last 40 years with vegetation primarily red cedar, red maple, and oaks. The underlying geology is the Copper Ridge Formation.

ANDERSON Site 38. The site is located on the Big Ridge Park Quadrangle. The site is $550 \mathrm{ft}$ west of Mt. Hebron church and $650 \mathrm{ft}$ southeast from Hinds Creek Road. The area has been under forest for the last 40 years with vegetation primarily red cedar, red maple, and red/black oaks. The underlying geology is the Copper Ridge Formation.

ANDERSON Site 39. Located on the Big Ridge Park Quadrangle, this site is $1000 \mathrm{ft}$ south from Mount Zion Church and $1000 \mathrm{ft}$ southeast from Hinds Creek Valley Road. Site has been under forest for the last 40 years with vegetation primarily red oak, hickory, and red maple. The underlying geology is the Copper Ridge Formation.

ANDERSON Site 40. Located on the Big Ridge Park Quadrangle, this site is $1200 \mathrm{ft}$ south from Mount Zion Church and $1500 \mathrm{ft}$ southeast from Hinds Creek Valley Road. It is $500 \mathrm{ft}$ west from Anderson Site 42. Site has been under forest for the last 40 years with vegetation primarily Virginia pine, ferns, and red maple.

ANDERSON Site 41. This site is located on the Big Ridge Park Quadrangle. It is $1500 \mathrm{ft}$ south from Mount Zion Church and $1700 \mathrm{ft}$ southeast from Hinds Creek Valley Road. It is $200 \mathrm{ft}$ west from Anderson Site 42. Site has been under forest for the last 40 years with vegetation primarily Virginia pine, ferns, dogwood, and red maple.

ANDERSON Site 42. Located on the Big Ridge Park Quadrangle. This site is located $1200 \mathrm{ft}$ south from Mount Zion Church and $1800 \mathrm{ft}$ southeast from Hinds Creek Valley Road. It is $800 \mathrm{ft}$ southeast from Anderson Site 39. Site has been under forest for the last 40 years with vegetation primarily Virginia pine, ferns, and red maple. 


\title{
A.4 OAK RIDGE RESERVATION SOIL PROFILES
}

\author{
ORR Site 2
}

(See pp. 20, 66, 67, 70, 71, 91 in Phase I Field Book 1)

Location:

Classification:

Geomorphic Position:

Slope and Aspect:

Parent Material(s):

Vegetation:

\section{Described By:}

Sampling Crew:

Rad Scan:

Weather Yesterday:

Present Weather:

Date:
Oak Ridge Reservation, Grid N29300, E10300.

Ruptic Ultic Dystrochrepts; clayey Bt, loamy-skeletal Bw, C, and $\mathrm{Cr}$, mixed, thermic

Middle of convex sideslope

$16 \%$ west-northwest

Middle Dismal Gap Formation

Oaks, few red maple, sweet gum, and white pine. This site was probably cleared and probably pastured but never plowed

D. A. Lietzke

Lietzke, Wilson

Air 25; ground level, 45

Partly cloudy, cool, scattered showers

Partly cloudy, cool

May 6, 1992

\section{Soil Description:}

Oi $\quad 4$ to $0 \mathrm{~cm}$; leaf litter, $100 \%$ coverage, few tree sprouts

A 0 to $5 \mathrm{~cm}$; dark brown (10YR 3/3) silt loam; strong fine granular structure; very friable; abrupt wavy boundary

E $\quad 5$ to $14 \mathrm{~cm}$; yellowish-brown (10YR 5/4) silt loam; weak fine subangular blocky structure; very friable; clear wavy boundary

BE 14 to $20 \mathrm{~cm}$; yellowish-brown (10YR 5/6) shaly silty clay loam; weak fine subangular blocky structure; friable; clear wavy boundary

$\mathrm{Bt} 20$ to $32 \mathrm{~cm}$; yellowish-red (5YR 5/6) shaly clay; strong medium subangular blocky structure; firm; all peds coated with yellowish-brown (10YR 5/6) materials; abrupt irregular boundary

$\mathrm{BC} \& \mathrm{Cr} 32$ to $74 \mathrm{~cm}$; strong brown (7.5YR 5/6) fragment interiors in $\mathrm{BC}$ materials and light yellowish-brown (2.5Y 6/4) fragment interiors in $\mathrm{C}$ and $\mathrm{Cr}$ materials. Fine earth and clay flows are strong brown (7.5YR 4/6); nearly all rock controlled structure; firm; much glauconite in $\mathrm{BC}$ materials; abrupt irregular boundary

Cr $\quad 74$ to $80 \mathrm{~cm}$; Not described

Notes: The ESD composite samples were collected from a pit face oriented with strike. There was less variability than the field duplicate samples that were collected from a pit face oriented across the strike. See pp. 66 and 67 in the field log book for sketches. The field duplicate samples were collected from the appropriate inclined soil horizon as shown in the sketch on p. 67. Depths of sampling are: A, 0 to $14 \mathrm{~cm} ; \mathrm{B}, 20$ to $32 \mathrm{~cm}$;, 32 to $50 \mathrm{~cm}$. 


\section{ORR Site 3}

(See pp. 94, 109 and 128 in Phase I Field Book 1 and pp. 4 and 5 in Phase I Field Book 2)

Location:

Classification:

Geomorphic Position:

Slope and Aspect:

Parent Material(s):

Vegetation:

Described By:

Sampling Crew:

Rad. Scan:

Weather Yesterday:

Present Weather:

Date:
Oak Ridge Reservation, Grid N29300, E11200. This site is within $50 \mathrm{ft}$ of the top edge of Bear Creek road embankment.

Typic hapludults; clayey, mixed, thermic

Broad slightly convex sideslope

$5 \%$ south

Middle Nolichucky Shale

Old field successional forest. Most old pines have died and fallen over. Present regrowth is V. pine, W. pine, oak, hickory, sassafras. cedar, with a sparse poison ivy ground cover.

D. A. Lietzke

Lietzke, Burgoa

Air 50 ; ground level, 40

Clear, very warm, with an evening shower

Partly cloudy, very warm, and humid

July 22, 1992

\section{Soil Description:}

Oi $\quad 3$ to $0 \mathrm{~cm}$; leaf litter and pine needles

A $\quad 0$ to $5 \mathrm{~cm}$; dark brown (10YR 3/3) silt loam; strong fine granular structure; very friable; abrupt wavy boundary

Ap $\quad 5$ to $15 \mathrm{~cm}$; yellowish-brown (10YR 5/4) silt loam; moderate medium granular structure; friable; abrupt wavy boundary

B\&E 15 to $18 \mathrm{~cm}$; dark yellowish-brown (10YR 4/6) B part, and yellowish-brown (10YR 5/6) E part; silty clay loam; weak medium subangular blocky structure; friable; abrupt wavy boundary

Bt 18 to $40 \mathrm{~cm}$; strong brown (7.5YR 4/6) clay; moderate medium subangular blocky structure; firm; strong brown (7.5YR 4/4) ped coatings; abrupt irregular boundary

C $\quad 40$ to $51 \mathrm{~cm}$; light olive brown (2.5Y 5/4) fragment interiors; plugged with strong brown (7.5YR 5/4) clay; all rock structure; abrupt irregular boundary

Cr $\quad 51$ to $60 \mathrm{~cm}$; brown (7.5YR 5/2) fragment interiors with most surfaces coated with dark red (2.5YR 3/6) iron-clay complexes

Notes: Sampling depths for ESD composites are: A, 0 to $15 \mathrm{~cm} ; \mathrm{B}, 18$ to $40 \mathrm{~cm}$;, 40 to $51 \mathrm{~cm}$. 


\section{ORR Site 5}

(See pp. 95, 109, and 133 in Phase I Field Book 1 and pp. 1 and 2 in Phase I Field Book 2)

Location:

Classification:

Geomorphic Position:

Slope and Aspect:

Parent Material(s):

Vegetation:

\section{Described By:}

Sampling Crew:

Rad. Scan:

Weather Yesterday:

Present Weather:

Date:
Oak Ridge Reservation, Grid N29300, E15300, about $300 \mathrm{ft}$ west of Central Training facility main entrance and $25 \mathrm{ft}$ south of the boundary fence

Typic hapludults; clayey, mixed, thermic

Broad bench landform on a convex spur

$1 \%$

Middle Nolichucky Shale with several carbonate strata

Old field successional forest. Most old pines have died and fallen over. Present regrowth is a dense stand of young pines, cedar, oak, dogwood, and sweet gum along with a generous stand of honeysuckle and greenbriar.

D. A. Lietzke

Lietzke, Burgoa

Air 40, ground level, 50

Clear and very warm with showers during the past evening

Cloudy and cooler

July 22, 1992

\section{Soil Description:}

Oi $\quad 5$ to $3 \mathrm{~cm}$; mosses and pine needles

Oe $\quad 3$ to $0 \mathrm{~cm}$; hemic materials, sapric materials and a dense root mat

Ap 0 to $10 \mathrm{~cm}$; yellowish-brown (10YR 5/4) silt loam; moderate fine granular structure; very friable; abrupt wavy boundary

Bt 10 to $40 \mathrm{~cm}$; strong brown (7.5YR $4 / 6)$ clay or silty clay; moderate medium subangular blocky structure; firm; strong brown (7.5YR 4/4) ped coatings; abrupt irregular boundary

$\mathrm{C} \& \mathrm{Cr} \quad 40$ to $60 \mathrm{~cm}$; olive brown $(2.5 \mathrm{Y} 4 / 3)$ fragment interiors with red and black surface coatings; strong brown (7.5YR 5/4) clay coatings on some surfaces and also as plugged zones

Notes: This is the only ORR Phase I site that has a thick moss layer. Sampling depths are: A, 0 to $10 \mathrm{~cm} ; \mathrm{B}, 10$ to $40 \mathrm{~cm}$;, 40 to $55 \mathrm{~cm}$. 


\section{ORR Site 10}

(See pp. 21, 39, 54, 55, and 91 in Phase I Field Book 1)

Location:

Classification:

Geomorphic Position:

Slope and Aspect:

Parent Material(s):

Vegetation:

Described By:

Sampling Crew:

Rad. Scan:

Weather Yesterday:

Present Weather:

Date:
Oak Ridge Reservation, Grid N30200, E21460

Typic dystrochrepts; loamy-skeletal, mixed, thermic

Upper convex sideslope just below ridge crest

$20 \%$ south southwest

Lower Dismal Gap Formation

Hardwood forest that regenerated from older severely eroded old-field forest succession. Present trees are white oak, hickory, cedar, ash. Ground cover of leaves and thick stand of poison ivy, five-leaf ivy and honeysuckle.

D. A. Lietzke

Lietzke, Wilson, Farmer

Air, 20; ground level, 20

Cool, widely scattered showers in the morning with clearing and warmer in the PM as the front went through

Clear and warm

May 1, 1992

\section{Soil Description:}

Oi $\quad 4$ to $0 \mathrm{~cm}$; leaf litter

Oa $\quad 0$ to $1 \mathrm{~cm}$; sapric and hemic materials with a root mat

A 1 to $12 \mathrm{~cm}$; brown (10YR 4/3) shaly silt loam; strong fine granular structure; very friable; clear wavy boundary

$\mathrm{Bw} \quad 12$ to $24 \mathrm{~cm}$; yellowish-brown (10YR $5 / 6$ ) shaly or very shaly silt loam or silty clay loam; moderate medium subangular blocky structure; very friable; some peds coated with darker materials; abrupt irregular boundary. (An intermittent $\mathrm{Bt}$ horizon, formed in a carbonate stratum was dug out before sampling for ESD composites started.)

C 24 to $46 \mathrm{~cm}$; light olive brown $(2.5 \mathrm{Y} 5 / 6)$ fragment interiors; most fragment surfaces thickly coated with yellowish-brown (10YR 5/4) silty materials. There are thin seams of red (2.5YR 4/8) clay originally derived from carbonate seams in the rock; 60 to $90 \%$ geologic structure; interbedded with harder $\mathrm{Cr}$ materials at lower depths

$\mathrm{Cr} \quad 46$ to $80 \mathrm{~cm}$; light olive brown (2.5Y 5/6) fragment interiors; surface coatings of dark red (2.5YR 3/6) and yellowish-brown (10YR 5/4)

Notes: Three sides of the pit were Typic Dystrochrepts. The fourth side had a Bt horizon but it was confined to a thin carbonate stratum and it had disappeared when the pit was enlarged to sample for ESD composites. The Oa horizon was not sampled. Sample depths are: A, 1 to $12 \mathrm{~cm}: \mathrm{B}, 12$ to $24 \mathrm{~cm} ; \mathrm{C}, 24$ to $46 \mathrm{~cm}$. 


\section{ORR Site 11}

(See pp. 16, 17, 32, and 91 in Phase I Field Book 1)

Location:

Classification:

Geomorphic Position:

Slope and Aspect:

Parent Material(s):

Vegetation:

Described By:

Sampling Crew:

Rad. Scan:

Weather Yesterday:

Present Weather.

Date:
Oak Ridge Reservation, about $50 \mathrm{ft}$ grid north of TVA power line ROW; Grid N29800, E21460

Typic Hapludults; clayey, mixed, thermic

Lower slightly convex sideslope; severely eroded before or after abandonment

$13 \%$ southwest

Upper Dismal Gap Formation and transition zone to lower Nolichucky

Old field regrowth. Most pines have died and have fallen over. Young brush and sprouts of dogwood, sassafras, cherry, red maple and sweet gum, with an abundant ground cover of poison ivy and honeysuckle

D. A. Lietzke

Lietzke, Wilson, Phillips, Farmer

Air, 15; ground level, 70

Partly cloudy and cool

Clear and warmer

March 17, 1992

\section{Soil Description:}

Oi $\quad 4$ to $0 \mathrm{~cm}$; leaves and pine needles

Oa $\quad 0$ to $1 \mathrm{~cm}$; black (10YR 2/1) organic matter and root mat

Ap $\quad 1$ to $18 \mathrm{~cm}$; brown (10YR 4/3) silty clay loam with fresh shale fragments; moderate fine granular structure; friable; common fine roots; abrupt wavy boundary

Bt 18 to $36 \mathrm{~cm}$; yellowish-red (5YR 5/6) silty clay or clay; moderate medium subangular blocky structure; friable; few fine roots and few medium roots; strong brown (7.5YR 4/6) clay coats on all ped faces; gradual wavy boundary

BC $\quad 36$ to $48 \mathrm{~cm}$; strong brown $(7.5 \mathrm{YR} 4 / 6)$ shaly silty clay or shaly clay; moderate coarse prismatic parting to moderate medium to coarse subangular blocky structure; firm; many fragments oriented with respect to the geologic strike and dip; yellowish and reddish parent materials saprolitic colors also present

C $\quad 48$ to $85 \mathrm{~cm}$; red (2.5YR 4/8) and yellowish-red (5YR 5/8 fragment interiors; material easily crushes to shaly or very shaly silty clay loam; 70 to $90 \%$ rock structure; yellowish-red (5YR 4/6) coatings on many fragment faces

$\mathrm{Cr} \quad 85$ to $132 \mathrm{~cm}$; fragment interiors range from red to olive; some fragment faces are coated with dark red (10R 3/6) while other fragment surfaces coated with yellowish-brown (10YR 5/4) clay; some fragments with light olive gray (5Y 6/2) fringes indicating wetness conditions; light brownish-gray (2.5Y 6/2) flow zones also occur in this horizon; fine roots in flow zones

Notes: Sampling depths are: A, 1 to $18 \mathrm{~cm} ; \mathrm{B}, 23$ to $36 \mathrm{~cm}$;, 51 to $62 \mathrm{~cm}$. 


\section{ORR Site 13}

(See pp. 95, 109, 135 in Phase I Field Book 1 and pp. 12 and 13 of Phase I Field Book 2)

Location:

Classification:

Geomorphic Position:

Slope and Aspect:

Parent Material(s):

Vegetation:

Described By:

Sampling Crew:

Rad. Scan:

Weather Yesterday:

Present Weather:

Date:
Oak Ridge Reservation, Grid N29480, E23220

Typic hapludults; clayey, mixed, thermic

Broad convex ridgetop

$3 \%$ northwest

Middle Nolichucky Shale

Old house site, probably a yard at one time. Present vegetation is maple and poplar with a dense ground cover of poison ivy.

D. A. Lietzke

Lietzke, Burgoa

Air, 50; ground level, 60

Partly cloudy, very warm

Cloudy, very humid

July 22, 1992

\section{Soil Description:}

Oi 2 to $0 \mathrm{~cm}$; leaf litter

Ap $\quad 0$ to $23 \mathrm{~cm}$; brown (10YR 4/3) silt loam or shaly silt loam; strong fine granular structure; very friable; abrupt wavy boundary

$\mathrm{Bt} 23$ to $46 \mathrm{~cm}$; strong brown (7.5YR 4/4) clay or shaly clay; moderate medium subangular blocky structure; friable; brown (10YR 4/4) ped coatings; clear wavy boundary

C $\quad 46$ to $69 \mathrm{~cm}$; brecciated zone with many disoriented small fragments with olive yellow (2.5Y 6/6) interiors; rock controlled structure; friable; crushes easily to a shaly or very shaly silty clay loam or silty clay

Notes: This site is in a brecciated zone that is deeply weathered. Sampling depths for ESD composites are: A, 0 to $23 \mathrm{~cm}$; B, 23 to $46 \mathrm{~cm}$; C, 46 to $56 \mathrm{~cm}$. 


\section{ORR Site 15}

(See pp. 95, 111, 116, and 117 in Phase I Field Book 1)

Location:

Classification:

Geomorphic Position:

Slope and Aspect:

Parent Material(s):

Vegetation:

\section{Described By: \\ Sampling Crew: \\ Rad. Scan: \\ Weather Yesterday: \\ Present Weather: \\ Date:}

Oak Ridge Reservation, Grid N29700, E25720, about $25 \mathrm{ft}$ north of gas line ROW

Typic hapludults; clayey, mixed, thermic

Broad, slightly convex, ridgetop

2-3\% south southwest

Lower Nolichucky Shale

Stand of 40 to 50 -year-old loblolly pines, with an understory of oaks, red maple, dogwood, and a ground cover of honeysuckle and grasses

D. A. Lietzke

Lietzke, Wilson

Air, 30; ground level, 40

Cloudy with scattered showers, cool

Partly cloudy and cool

May 27, 1992

\section{Soil Description:}

Oi $\quad 2$ to $0 \mathrm{~cm}$; pine needles and a few leaves

A 0 to $2 \mathrm{~cm}$; brown (10YR 4/3) silt loam; moderate fine granular structure; very friable; abrupt wavy boundary

Ap 2 to $16 \mathrm{~cm}$; yellowish-brown (10YR 5/6) silt loam or silty clay loam; weak medium granular structure; friable; abrupt wavy boundary

$\mathrm{Bt} \quad 16$ to $36 \mathrm{~cm}$; yellowish-red (5YR 5/6) clay; moderate medium subangular blocky structure; firm; strong brown (7.5YR 4/6) clay films on all ped surfaces; few parent material reds and yellows in some ped interiors in the lower part; abrupt irregular boundary

$\mathrm{C} / \mathrm{Cr} \quad 36$ to $60 \mathrm{~cm}$; olive yellow (2.5Y 6/8) fragment interiors and thin strong brown (7.5YR 4/6) clay seams; yellowish-red (5YR 5/6) clay flows coating C horizon material; most $\mathrm{Cr}$ horizon fragments coated with red iron oxides; all rock controlled structure; $\mathrm{C}$ and $\mathrm{Cr}$ materials occur in thin strata that had weathered differentially with respect to each other

$\mathrm{Cr} \quad 60$ to $70 \mathrm{~cm}$; brown (7.5YR 4/4) fragment interiors of less weathered strata and yellowish-red (5YR 4/6) fragment interiors of more highly weathered strata; dark red $(2.5 \mathrm{YR} 3 / 6-3 / 4)$ iron oxides on many fragment surfaces

Notes: Sampling depths are: A, 0 to $16 \mathrm{~cm} ; \mathrm{B}, 16$ to $36 \mathrm{~cm}$, C, 36 to $60 \mathrm{~cm}$. 


\section{ORR Site 16}

(See pp. 98, 11, and 113 in Phase I Field Book 1)

Location:

Classification:

Geomorphic Position:

Slope and Aspect:

Parent Material(s):

Vegetation:

Described By:

Sampling Crew:

Rad. Scan:

Weather Yesterday:

Present Weather:

Date:
Oak Ridge Reservation, Grid N29700; E25928

Typic hapludults; clayey, mixed, thermic

Sideslope, slight convexity

$10 \%$ southeast; severely eroded before abandonment

Middle Nolichucky Shale

Loblolly Pine plantation with approximately 40-year-old trees; understory of dogwoods, oaks, sweetgum. Sparse ground cover of honeysuckle and poison ivy

D. A. Lietzke

Lietzke, Burgoa

Air, 45; ground level, 60

Clear and warm

Clear and warm

July 20, 1992

\section{Soil Description:}

$\mathrm{Oi} \quad 3$ to $0 \mathrm{~cm}$; pine needles and some leaves

A $\quad 0$ to $7 \mathrm{~cm}$; dark yellowish-brown (10YR 4/4) shaly silt loam; moderate fine granular structure; very friable; abrupt wavy boundary

Ap $\quad 7$ to $20 \mathrm{~cm}$; yellowish-brown (10YR 5/4) shaly silty clay loam; moderate medium granular structure; very friable; clear wavy boundary

$\mathrm{Bt} 20$ to $33 \mathrm{~cm}$; strong brown (7.5YR 4/6) clay or shaly clay; moderate medium subangular blocky structure; friable; all peds coated; clear wavy boundary

$\mathrm{BC} \quad 33$ to $43 \mathrm{~cm}$; strong brown (7.5YR 5/4) very shaly silty clay loam; weak medium subangular blocky structure and areas of rock controlled structure; firm; strong brown (7.5YR 5/4) clay coatings on most fragment surfaces, with the clay covering a thick layer of black manganese oxides; abrupt irregular boundary

C 43 to $88 \mathrm{~cm}$; yellowish-brown (10YR 5/6) fragment interiors; crushes to shaly silty clay or very shaly silty clay loam; $90 \%$ rock controlled structure; clay plugged; some black coatings; firm; abrupt irregular boundary

Notes: Sampling depths for ESD composites are: A, 0 to $20 \mathrm{~cm} ; \mathrm{B}, 20$ to $33 \mathrm{~cm} ; \mathrm{C}, 43$ to $63 \mathrm{~cm}$. 


\section{ÓRR Site 19}

(See pp. 21, 33, 35, 37, 42, 43, and 91 in Phase I Field Book 1)

Location:

Classification:

Geomorphic Position:

Slope and Aspect:

Parent Material(s):

Vegetation:

Described By:

Sampling Crew:

Rad. Scan:

Weather Yesterday:

Present Weather:

Date:
Oak Ridge Reservation, $60 \mathrm{ft}$ east of telegraph ROW within the Bear Creek LLWDDD; Grid N33000, E28530

Typic hapludults; clayey, mixed, thermic

Top of broad spur ridge

$4 \%$ west

Lower Dismal Gap Formation

Old-field forest. Most pines have fallen. Young hardwoods of cherry, sweet gum, cedar, poplar, dogwood and virginia pine. Sparse ground cover of honeysuckle. Ground surface, $100 \%$ leaf covered.

D. A. Lietzke

Lietzke, Wilson, Farmer

Air, 30; ground level, 40

Cool, cloudy, light showers

Cool, cloudy with cold front moving through

April 27, 1992

\section{Soil Description:}

$\mathrm{Oi} \quad 5$ to $0 \mathrm{~cm}$; leaf litter

oa $\quad 0$ to $1 \mathrm{~cm}$; sapric and hemic materials and dense root mat

A 1 to $6 \mathrm{~cm}$; dark brown (10YR 3/3) silt loam; moderate fine granular structure; very friable; clear wavy boundary

Ap 6 to $16 \mathrm{~cm}$; yellowish-brown (10YR 5/4) shaly silt loam; moderate fine granular structure; very friable; abrupt wavy boundary

BE 16 to $25 \mathrm{~cm}$; strong brown $(7.5 \mathrm{YR} 5 / 6)$ shaly silty clay loam; weak medium subangular blocky structure; friable; clear wavy boundary

Bt 25 to $51 \mathrm{~cm}$; yellowish-red (5YR $4 / 6)$ silty clay or clay; moderate medium subangular blocky structure; friable; strong brown (7.5YR 5/6) clay on all ped faces; clear wavy boundary

BC 51 to $54 \mathrm{~cm}$; transition to $\mathrm{C}$ horizon with loss of soil structure and start of geologic strike and dip controlled rock structure

C 54 to $84 \mathrm{~cm}$; alternating strata of brown (7.5YR 4/3) very fine grained sandstone saprolite and shale-siltstone saprolite. Saprolite is plugged with yellowish-red (5YR $4 / 6)$ clay

Cr 84 to $90 \mathrm{~cm}$; Harder shale strata. Fragment interiors are olive brown (2.5Y 4/4). Most surface are coated with black manganese oxides and some yellowish-red (5YR 4/6) clay

Notes: The $\mathrm{Bt}$ horizon is continuous around the pit. The $\mathrm{Cr}$ horizon in shale starts at $60 \mathrm{~cm}$ depth while the $\mathrm{Cr}$ in the sandstone strata occurs at a depth of 80 to $84 \mathrm{~cm}$ below the surface. Sampling depths are: A, 0 to $16 \mathrm{~cm} ; \mathrm{B}, 25$ to $51 \mathrm{~cm}$;, 54 to $74 \mathrm{~cm}$. This site was moved from its original location, which was in a young stand of planted pines, to a location in an old field successional forest. 


\section{ORR Site 21}

(See pp. 99, 107, 139, 158, and 159 in Phase I Field Book 1)

Location:

Classification:

Geomorphic Position:

Slope and Aspect:

Parent Material(s):

Vegetation:

Described By:

Sampling Crew:

Rad. Scan:

Weather Yesterday:

Present Weather:

Date:
Oak Ridge Reservation, Grid N29700, E30940. Site in old field, grown up to pines

Ruptic Ultic Dystrochrepts; clayey $\mathrm{Bt}$, loamy-skeletal $\mathrm{Bw}, \mathrm{C}$ and $\mathrm{Cr}$, mixed, thermic

Nearly level bench-like area on a convex sideslope

$9 \%$ south, severely eroded

Middle Nolichucky Shale

Old field successional forest with a few tall pines remaining along with cedar, poplar, dense stands of sourwood, oak and red maple. Some grape vines and some mosses and lichen groundcover

D. A. Lietzke

Lietzke, Burgoa

Air, 35; ground level, 80

Clear and warm

Partly cloudy and very warm

July 21, 1992

\section{Soil Description:}

Oi $\quad 2$ to $0 \mathrm{~cm}$; leaf litter

Ap 0 to $10 \mathrm{~cm}$; dark yellowish-brown (10YR 4/6) shaly silty clay loam; moderate medium granular structure; friable, abrupt wavy boundary

Bt $\quad 10$ to $26 \mathrm{~cm}$; yellowish-red (5YR 5/6) shaly clay; strong medium subangular blocky structure; firm; strong brown (7.5YR 4/4) ped coatings; abrupt irregular boundary

$\mathrm{Cr} 26$ to $48 \mathrm{~cm}$; inclined shaly strata with brown (7.5YR 4/2) fragment interiors; red (10YR 4/6) surface coatings of iron-clay complex; abrupt irregular boundary

2C 48 to $63 \mathrm{~cm}$; yellowish-brown and yellowish-red saprolitic materials that crushes to silty clay loam; this stratum impregnated with red iron compounds and black manganese oxides

$3 \mathrm{Cr} \quad 63$ to $65 \mathrm{~cm}$; harder shale saprolite

Notes: This site was moved from its original location. The original location was on the Maynardville Formation. See the Phase 1 map for the original and moved locations. Sampling depths are: A, 0 to $10 \mathrm{~cm} ; \mathrm{B}, 10$ to $26 \mathrm{~cm} ; \mathrm{C}, 26$ to $48 \mathrm{~cm}$. 


\section{ORR Site 22}

(See pp. 24, 35, 37, 46, 47, and 91 in Phase I Field Book 1)

Location:

Classification:

Geomorphic Position: Slope and Aspect: Parent Material(s):

Vegetation:

Described By: Sampling Crew: -

Rad. Scan:

Weather Yesterday: Present Weather:

Date:
Oak Ridge Reservation, within Bear Creek LLWDDD site; Grid N30520, E31250

Ruptic Ultic Dystrochrepts; clayey Bt, loamy-skeletal C and $\mathrm{Cr}$ horizons; mixed, thermic

Broad convex ridge crest

$3 \%$ southwest

Lower Dismal Gap Formation and transition to Rogersville shale. The pit was in mostly Rogersville saprolite.

Old-field woods of virginia pine, shortleaf pine, dogwoods, cedars. Surface was $100 \%$ leaf covered and with a sparse groundcover of honeysuckle.

D. A. Lietzke

Lietzke, Wilson, Farmer

Air, 40; ground level, 40

Cloudy with scattered showers

Cloudy, cool, with scattered showers

April 28, 1992

Soil Description:

Oi\&Oa 2 to $0 \mathrm{~cm}$; leaf litter, litter fragments and root mat

A $\quad 0$ to $5 \mathrm{~cm}$; dark grayish-brown (10YR 4/2) silt loam; moderate fine granular structure; very friable; clear wavy boundary

Ap $\quad 5$ to $15 \mathrm{~cm}$; brown (10YR 5/3) shaly silt loam; weak medium granular structure; friable; abrupt wavy' boundary

Bt 15 to $27 \mathrm{~cm}$; yellowish-red (5YR 4/6) clay; moderate medium subangular blocky structure; firm; brown (7.5YR 4/4) clay on all ped faces; abrupt irregular boundary

$\mathrm{C} \& \mathrm{Cr} 1 \quad 27$ to $46 \mathrm{~cm}$; Alternating thin strata of soft and hard shale-siltstone saprolite with light olive brown (2.5Y 5/4) fragment interiors; plugged with brown (7.5YR 4/4) clay

Cr2 46 to $54 \mathrm{~cm}$; harder shale saprolite with most fragment surfaces coated with dark red $(2.5 Y R 3 / 4-3 / 6)$ iron oxides

Notes: Sampling depths are: A, 0 to $15 \mathrm{~cm} ; \mathrm{B}, 15$ to $27 \mathrm{~cm} ; \mathrm{C}, 27$ to $46 \mathrm{~cm}$. 
ORR Site 23

(See pp. 101, 107, 120, and 121 in Phase I Field Book 1)

Location:

Classification:

Geomorphic Position:

Slope and Aspect:

Parent Material(s):

Vegetation:

Described By:

Sampling Crew:

Rad. Scan:

Weather Yesterday:

Present Weather:

Date:
Oak Ridge Reservation, Grid N 29800, E 31830; about $50 \mathrm{ft}$ north of TVA power line ROW and about $20 \mathrm{ft}$ east of road that goes to ORR-22

Ruptic Ultic Dystrochrepts; clayey Bt, loamy-skeletal Bw, C, and $\mathrm{Cr}$, mixed, thermic

Interfluve between two drainageways

$2 \%$ to $3 \%$ south

Lower Nolichucky Shale

Old-field pines; most have died and fallen over. Present canopy vegetation is red maple, sourwood and dogwoods. Groundcover of honeysuckle and poison ivy.

D. A. Lietzke

Lietzke, Wilson, Burgoa

Air, 30; ground level, 70

Partly cloudy and cool

Cloudy with showers

May 28, 1992

\section{Soil Description:}

$\mathrm{Oi} \quad 4$ to $2 \mathrm{~cm}$; pine needles and leaves

Oa 2 to $0 \mathrm{~cm}$; hemic materials, sapric organic materials, many fine roots, and a root mat at the mineral soil surface. (The Oa has variable thickness ranging from $0 \mathrm{~cm}$ to about $2 \mathrm{~cm}$ in the area that was cleared of organic materials for sampling the mineral soil.)

A $\quad 0$ to $1 \mathrm{~cm}$; dark yellowish-brown (10YR 4/4) shaly silt loam; moderate fine granular structure; very friable; abrupt wavy boundary

Ap 1 to $12 \mathrm{~cm}$; dark yellowish-brown (10YR 4/6) shaly silty clay loam; weak medium granular structure; friable; abrupt wavy boundary

Bt 12 to $30 \mathrm{~cm}$; yellowish-red (5YR 4/6) shaly clay; moderate fine subangular blocky structure; firm; strong brown (7.5YR 4/4) coatings on all ped surfaces; abrupt irregular boundary

$\mathrm{C} \& \mathrm{Cr} \quad 30$ to $50 \mathrm{~cm}$; strong brown (7.5YR 5/8) glauconitic fragment interiors and reddishbrown (5YR 4/4) clay flows; rock-oriented strike and dip structure

$\mathrm{Cr} \& \mathrm{C} \quad 50$ to $60 \mathrm{~cm}$; Thin strata of both $\mathrm{C}$ and $\mathrm{Cr}$ siltstone saprolite materials. In addition to the siltstone stratum, there is a red (10R 4/6) glauconitic stratum and a confined flow zone stratum dominated by light brownish-gray (10YR 6/2) wetness mottles; some fragments in the $C$ horizon are coated with reddish-brown (5YR 4/4) clay. Siltstone fragment interiors are yellowish-brown (10YR 5/6).

Notes: This pit was dug and sampled for ESD composites and gamma scan samples in a light rain. The profile was not described until June 1, 1992. Sampling depths are: A, 0 to $12 \mathrm{~cm} ; \mathrm{B}, 12$ to $30 \mathrm{~cm} ; \mathrm{C}, 30$ to $50 \mathrm{~cm}$. 


\section{ORR Site 24}

(See pp. 101, 107 and 108 in Phase I Field Book 1 and pp. 8 and 9 in Phase I Field Book 2)

Location:

Classification:

Geomorphic Position:

Slope and Aspect:

Parent Material(s):

Vegetation:

Described By:

Sampling Crew:

Rad. Scan:

Weather Yesterday:

Present Weather:

Date:
Oak Ridge Reservation, Grid N29900, E31980

Ruptic Ultic Dystrochrepts; clayey $\mathrm{Bt}$, loamy-skeletal, $\mathrm{Bw}, \mathrm{C}$ and $\mathrm{Cr}$, mixed, thermic

Convex sideslope

$8 \%$ North

Middle Nolichucky Shale

Old field successional forest with some planted loblolly pine about 30 y old; dense undergrowth of poplar, dogwood, cedar, pine, red maple; sparse ground cover of poison ivy and honeysuckle

D. A. Lietzke

Lietzke, Burgoa

Air, 50; ground level, 70

Clear to partly cloudy, very warm with a shower in the evening Partly cloudy, very warm, very humid

July 22, 1992

\section{Soil Description:}

Oi $\quad 4$ to $1 \mathrm{~cm}$; pine needles and some leaves

Oa\&Oe 1 to $0 \mathrm{~cm}$; hemic materials, sapric materials and a root mat

A $\quad 0$ to $5 \mathrm{~cm}$; dark brown (10YR 3/3) silt loam; strong fine granular structure; very friable; abrupt wavy boundary

Ap 5 to $19 \mathrm{~cm}$; dark yellowish-brown (10YR 4/4) shaly silt loam; moderate fine granular structure; very friable; abrupt wavy boundary

Bw\&Bt 19 to $26 \mathrm{~cm}$; dark yellowish-brown (10YR 4/4) shaly or very shaly silty clay loam; weak medium subangular blocky structure; friable; abrupt wavy boundary

$\mathrm{C} \& \mathrm{Cr} 26$ to $49 \mathrm{~cm}$; light olive brown $(2.5 \mathrm{Y} 5 / 3)$ fragment interiors; rock controlled structure; yellowish-brown (10YR 5/4) coatings on most fragment surfaces. These coatings cover thick black manganese oxide coatings

Cr\&C 49 to $60 \mathrm{~cm}$; highly weathered shale and siltstone saprolite

Notes: Sampling depths for ESD composites are: A, 0 to $19 \mathrm{~cm} ; \mathrm{B}, 19$ to $26 \mathrm{~cm}$ C, 26 to $49 \mathrm{~cm}$. 


\section{ORR Site 25}

(See pp. 95, 107, 124, 125 in Phase I Field Book 1)

Location:

Classification:

Geomorphic Position:

Slope and Aspect:

Parent Material(s):

Vegetation:

Described By:

Sampling Crew:

Rad. Scan:

Weather Yesterday:

Present Weather:

Date:
Oak Ridge Reservation, Grid N 29800, E 32290; site about $50 \mathrm{ft}$ south of TVA electric power line ROW

Ruptic Ultic Dystrochrepts; clayey Bt, loamy-skeletal Bw, C and $\mathrm{Cr}$, mixed, thermic

Upland summit, slightly convex

2 to $3 \%$ southeast

Middle Nolichucky Shale

Loblolly pine plantation, 40 to $50 \mathrm{y}$ old. Lower story of red maple, dogwood, and sweet gum; thick layer of pine needles on the ground surface along with moderate poison ivy and honeysuckle

D. A. Lietzke

Lietzke, Wilson, Burgoa

Air, 45; ground level, 60

Partly cloudy and cool

Overcast with showers

May 28, 1992

\section{Soil Description:}

Oi $\quad 4$ to $2 \mathrm{~cm}$; pine needles and leaves

Oe\&Oa 2 to $0 \mathrm{~cm}$; sapric materials, hemic materials and root mat

A 0 to $1 \mathrm{~cm}$; yellowish-brown (10YR 5/4) shaly silt loam; moderate fine granular structure; very friable; abrupt wavy boundary

Ap $\quad 1$ to $17 \mathrm{~cm}$; yellowish-brown (10YR 5/4) shaly silty clay loam; weak medium granular structure; friable; abrupt wavy boundary

Bt\&Bw 17 to $34 \mathrm{~cm}$; brown (7.5YR 4/4) shaly clay; about 50\% moderate medium subangular blocky structure in Bt strata and rock controlled strike and dip structure in Bw; Fragment interiors are light yellowish-brown (2.5Y 6/4); firm; abrupt irregular boundary.

$\mathrm{C} \& \mathrm{Cr} \quad 34$ to $50 \mathrm{~cm}$; strong brown (7.5YR 5/4) very shaly clay; strike and dip controlled orientation of shale fragments; light olive brown shale fragment interiors; abrupt irregular boundary

$\mathrm{Cr} \quad 50$ to $60 \mathrm{~cm}$; light olive brown (2.5Y 5/4) fragment interiors; red and black coatings on fragment surfaces; thin carbonate seams are red (2.5YR 4/8) clay; larger cracks contain strong brown (7.5YR 5/4) translocated clay

Notes: ESD composite samples were collected in a light rain. The ESD gamma scan samples were collected and the profile described on the 1st of June, 1992 when the weather was partly cloudy. Sampling depths are: A, 0 to $17 \mathrm{~cm} ; \mathrm{B}, 17$ to $34 \mathrm{~cm}$; , 34 to $50 \mathrm{~cm}$. 


\section{ORR Site 26}

(See pp. 24, 74, 75, 78, 79, 91 in Phase I Field Book 1)

Location:

Classification:

Geomorphic Position:

Slope and Aspect:

Parent Material(s):

Vegetation:

Described By:

Sampling Crew:

Rad. Scan:

Weather Yesterday:

Present Weather:
Oak Ridge Reservation, Grid N 30000, E34370. The site is 75 to $80 \mathrm{ft}$ north of the TVA power line ROW

Ruptic Ultic Dystrochrepts; clayey Bt, loamy-skeletal Bw, C, Cr, mixed, thermic

Lower convex sideslope

$14 \%$ south southeast

Middle Dismal Gap Formation

This site has been plowed but was abandoned before the Atomic Energy Commission (AEC) takeover in 1942. Present forest is hardwoods of red maple, oaks, hickories, and a few cedars.

D. A. Lietzke

Lietzke, Wilson

Air, 15; ground level, 25

Partly cloudy in the morning with afternoon scattered showers

Cloudy, scattered showers, cool. Got rained out before completing sampling and soil description.

May 11, 1992

Date:

Soil Description:

Oi $\quad 4$ to $0 \mathrm{~cm}$; leaf litter and root mat

A $\quad 0$ to $6 \mathrm{~cm}$; very dark grayish-brown (10YR 3/2) shaly silt loam; strong fine granular structure; very friable; abrupt wavy boundary

Ap 6 to $15 \mathrm{~cm}$; dark yellowish-brown (10YR $4 / 4)$ shaly silt loam; moderate fine granular structure; very friable; abrupt wavy boundary

Bt 15 to $30 \mathrm{~cm}$; red $(2.5 \mathrm{YR} 4 / 6)$ shaly clay or shaly silty clay; moderate medium subangular blocky structure; friable; strong brown (7.5YR) coatings on all ped faces; abrupt irregular boundary

BC\&C 30 to $50 \mathrm{~cm}$; light olive brown (2.5Y 5/4) fragment interiors; soil matrix is yellowish-red (5YR 4/6) very shaly clay. BC has some weak soil structure. the Chas all rock structure; abrupt irregular boundary.

$\mathrm{C} \& \mathrm{Cr} \quad 50$ to $80 \mathrm{~cm}$; olive brown (2.5Y 4/4) fragment interiors; Cr fragments coated with black manganese oxides; $C$ horizon fragments coated with yellowish-red (5YR 4/6) and strong brown (7.5YR 5/6) materials

Notes: The $\mathrm{BC} \& \mathrm{C}$ and $\mathrm{C} \& \mathrm{Cr}$ horizons occur in thin strata that have geologic strike and dip. It was not feasible to describe each separately, nor could they be individually sampled. Sampling depths: A, 0 to $15 \mathrm{~cm} ; \mathrm{B}, 15$ to $30 \mathrm{~cm} ; \mathrm{C}, 30$ to $60 \mathrm{~cm}$. The field duplicate samples, because they were sampled from another side of the pit have different depths as follows: A, 0 to $15 \mathrm{~cm} ; \mathrm{B}, 18$ to $36 \mathrm{~cm}$; and C, 20 to $40 \mathrm{~cm}$. 


\section{ORR Site 27}

(See pp. 8, 9, 25, 32, 91 in Phase I Field Book 1)

Location:

Classification:

Geomorphic Position:

Slope and Aspect:

Parent Material(s):

Vegetation:

Described By:

Sampling Crew:

Rad Scan:

Previous Weather:

Weather Today:

Date:
Oak Ridge Reservation, Bear Creek area; $100 \mathrm{ft}$ north of TVA power line right-of-way; Grid N30100, E34990

Ruptic Ultic Dystrochrepts; clayey Bt and loamy-skeletal Bw, C, and $\mathrm{Cr}$ horizons; mixed, thermic

Mid side slope with slight convexity

7\% southwest

Upper Dismal Gap Formation

Old-field regrowth of red and black oak, chestnut oak, white oak, a few virginia pine, some dead cedars; understory of beech, oak, red maple, dogwood, and a few green herbs; ground covered $100 \%$ by leaves

D. A. Lietzke

Lietzke, Wilson, Phillips, Farmer

Air, 10; ground level, 15

Rain and snow showers on March 14th and 15th

Clear, $18^{\circ} \mathrm{F}$ to $20^{\circ} \mathrm{F}$ in morning

March 16, 1992

Soil Description:

$\mathrm{Oi} \quad 4$ to $0 \mathrm{~cm}$; leaf litter

A1 0 to $5 \mathrm{~cm}$; very dark grayish-brown (10YR 3/2) silt loam; strong fine granular structure; very friable; many fine roots; abrupt wavy boundary

Ap 5 to $10 \mathrm{~cm}$; dark brown (10YR 3/3) silt loam; moderate fine granular structure; very friable; common fine roots; abrupt wavy boundary

$\mathrm{AB} \quad 10$ to $23 \mathrm{~cm}$; yellowish-brown (10YR 5/4) silt loam; weak fine subangular blocky structure; few medium roots; clear wavy boundary

Bt 23 to $34 \mathrm{~cm}$; strong brown (7.5YR 4/6) silty clay loam; moderate medium subangular blocky structure; few roots; abrupt irregular boundary

C $\quad 34$ to $74 \mathrm{~cm}$; olive brown (2.5Y 4/4-5/4) fragment interiors; material crushes to a shaly or very shaly silty clay loam; yellowish-red (5YR 4/6) clay coatings on some fragment faces while other fragment faces coated with black manganese oxides in the lower part of the horizon; more than $70 \%$ rock controlled structure; abrupt irregular boundary

$\mathrm{Cr} \quad 74$ to $80 \mathrm{~cm}$; olive brown (2.5Y 4/4) fragment interiors; dark red (2.5YR 3/6) and black coatings on most fragment faces

Notes: Depth to the $\mathrm{Cr}$ varied from 45 to $74 \mathrm{~cm}$ across the face that was sampled. Only the Argillic part of the soil was described and sampled. One side of the pit had $\mathrm{Cr}$ materials coming to within $20 \mathrm{~cm}$ of the surface. This old-field site was abandoned some time before the AEC takeover and had already reverted to hardwoods. Depths of sampling for ESD composites are: A, 0 to $10 \mathrm{~cm} ; \mathrm{B}, 23$ to $34 \mathrm{~cm} ; \mathrm{C}, 34$ to $53 \mathrm{~cm}$. 


\section{ORR Site 28}

(See pp. 103, 107, 129 in Phase I Field Book 1 and pp. 152 and. 153 in Phase I Field Book 2)

Location:

Classification:

Geomorphic Position:

Slope and Aspect:

Parent Material(s):

Vegetation:

Described By:

Sampling Crew:

Rad. Scan:

Weather Yesterday:

Present Weather:

Date:
Oak Ridge Reservation, Grid N29500, E34580, about $20 \mathrm{ft}$ grid west of edge of power line ROW

Ruptic Ultic Dystrochrepts; clayey Bt, loamy-skeletal Bw, C and Cr horizons; mixed, thermic

Mid sideslope with convexity. Severely eroded before abandonment

$5 \%$ southeast

Middle Nolichucky Shale

Old field successional forest where older pines have been harvested; present vegetation of young pines, cedars and poplar; sparse ground cover of honeysuckle, mosses and ferns

D. A. Lietzke

Lietzke, Burgoa

Air, 45; ground level, 60

Clear and warm

Clear and warm

July 21, 1992

Soil Description:

Oi $\quad 2$ to $0 \mathrm{~cm}$; mosses, pine needles, leaves and root mat

A $\quad 0$ to $2 \mathrm{~cm}$; brown (10YR 4/3) silt loam or shaly silt loam; strong fine granular structure; very friable; abrupt wavy boundary

Ap 2 to $16 \mathrm{~cm}$; yellowish-brown (10YR 5/4) silt loam or shaly silt loam; moderate fine granular structure; very friable; abrupt wavy boundary

Bt 16 to $25 \mathrm{~cm}$; yellowish-brown (10YR 5/8) shaly clay; moderate medium subangular blocky structure; friable; strong brown (7.5YR 5/6) ped coatings; abrupt irregular boundary

C 25 to $35 \mathrm{~cm}$; light olive brown (2.5Y 5/4) fragment interiors; red (2.5YR 4/6) iron-clay coatings on some fragment surfaces; other areas plugged with yellowishred (5YR 5/4) clay; abrupt irregular boundary

Cr $\quad 35$ to $52 \mathrm{~cm}$; brown (10YR 4/3) fragment interiors; most surface coated with red and yellowish-red clay and iron oxides

Notes: If the Bt horizon is continuous around all four sides of the pit the soil is classified as a Typic Hapludult. If the $\mathrm{Bt}$ horizon is interrupted by a $\mathrm{Bw}, \mathrm{C}$ or $\mathrm{Cr}$ horizon then the soil is classified as a Ruptic Ultic Dystrochrept. Sampling depths are: A, 0 to $16 \mathrm{~cm}$; $B, 16$ to $25 \mathrm{~cm} ; \mathrm{C}, 26$ to $35 \mathrm{~cm}$. Depths of sampling for ESD composites are: A, 0 to $10 \mathrm{~cm} ; \mathrm{B}, 23$ to $34 \mathrm{~cm}$;, 34 to $53 \mathrm{~cm}$. 


\section{ORR Site 31}

(See pp. 103, 113, 114, 156, 157 in Phase I Field Book 1)

Location:

Classification:

Geomorphic Position:

Slope and Aspect:

Parent Material(s):

Vegetation:

\section{Described By: \\ Sampling Crew: \\ Rad. Scan: \\ Weather Yesterday: \\ Present Weather: \\ Date:}

Oak Ridge Reservation, Grid N29900, E35820

Typic Hapludults; clayey, mixed, thermic

Lower part of long slightly convex sideslope

$3 \%$ southeast

Upper Nolichucky Shale

Old field successional forest. The older pines have been harvested in the past 10 to $15 \mathrm{y}$. Dense regrowth of sweet gum, dogwood, cedar, hickory, poplar, and ash. Sparse ground cover of honeysuckle and poison ivy.

D. A. Lietzke

Lietzke, Burgoa

Air, 35 ; ground level, 60

Clear and warm

Partly cloudy, very warm and humid

July 21, 1992

\section{Soil Description:}

Oi $\quad 2$ to $0 \mathrm{~cm}$; leaf litter

A $\quad 0$ to $8 \mathrm{~cm}$; brown (10YR 4/3) silt loam; strong fine granular structure; very friable; abrupt wavy boundary

Ap 8 to $19 \mathrm{~cm}$; yellowish-brown (10YR 5/4) silt loam; moderate fine granular structure; friable; abrupt wavy boundary

Bt 19 to $40 \mathrm{~cm}$; dark yellowish-brown (10YR 4/6) silty clay; moderate medium subangular blocky structure; friable; dark yellowish-brown (10YR 4/4) ped coatings; clear wavy boundary

C 40 to $61 \mathrm{~cm}$; yellowish-brown (10YR 5/4) silt loam or silty clay loam; weak coarse subangular blocky structure; firm; light brownish-gray (10YR 6/2) wetness mottles; strong brown (7.5YR 4/4) ped zones, but most peds are not coated; abrupt irregular boundary

$2 \mathrm{Cr} \quad 61$ to $70 \mathrm{~cm}$; dark reddish-gray (5YR 4/2) fragment interiors, with surfaces coated with black manganese oxides

Notes: The upper $61 \mathrm{~cm}$ appears to be colluvium of entirely Nolichucky origins and of early Holocene age. Sampling depths are: A, 0 to $19 \mathrm{~cm}$; B, 19 to $40 \mathrm{~cm}$;, 40 to $55 \mathrm{~cm}$. 


\section{ORR Site 32}

(See pp. 25, 33, 35, 37, 50, 51, 91 in Phase I Field Book 1)

Location:

Classification:

Geomorphic Position: Slope and Aspect:

Parent Material(s):

Vegetation:

Described By:

Sampling Crew:

Rad. Scan:

Weather Yesterday:

Present Weather:

Date:
Oak Ridge Reservation, about $100 \mathrm{ft}$ grid north of TVA power line ROW; Grid N30200, E36760

Ruptic Ultic Dystrochrepts; clayey Bt and loamy-skeletal, Bw, C and $\mathrm{Cr}$ horizons; mixed, thermic

Mid-point of long convex sideslope

$12 \%$ south

Upper Dismal Gap Formation

Old-field. Pines have almost all died and fallen over. Present vegetation is thick hardwood underbrush and abundant honeysuckle.

D. A. Lietzke

Lietzke, Wilson, Farmer

Air, 15; ground level, 40

Cloudy, cool, widely scattered showers

Cloudy, cooler with clearing in the afternoon

April 28, 1992

\section{Soil Description:}

Oi $\quad 4$ to $0 \mathrm{~cm}$; leaf litter and root mat

A $\quad 0$ to $5 \mathrm{~cm}$; dark brown (10YR 3/3) silt loam; strong fine granular structure; very friable; abrupt wavy boundary

Ap 5 to $20 \mathrm{~cm}$; dark yellowish-brown (10YR 4/4) silt loam or shaly silt loam; moderate fine granular structure; very friable; abrupt wavy boundary

Bt 20 to $32 \mathrm{~cm}$; yellowish-red (5YR $4 / 6)$ silty clay or clay; moderate medium subangular blocky structure; strong brown (7.5YR 5/4) clay coatings on all ped surfaces; clear irregular boundary

C $\quad 32$ to $53 \mathrm{~cm}$; light olive brown (2.5Y 5/6) fragment interiors coated with brown (7.5YR 4/4) clay; 60 to $80 \%$ rock controlled structure; clay plugged; abrupt irregular boundary

$\mathrm{Cr} \quad 53$ to $64 \mathrm{~cm}$; light olive brown (2.5Y 5/6) fragment interiors; many fragment faces coated with black manganese oxides; some fragment faces coated with red (2.5YR $4 / 6$ ) iron-clay materials; some strong brown ( $7.5 \mathrm{YR} 4 / 6)$ clay in thin flow zone seams

Notes: Sampling depths: A, 0 to $20 \mathrm{~cm} ; \mathrm{B}, 20$ to $32 \mathrm{~cm}$;, 32 to $53 \mathrm{~cm}$. 


\section{ORR Site 33}

(See pp. 25, 35, 37, 62, 63, 91 in Phase I Field Book 1)

Location:

Classification:

Geomorphic Position:

Slope and Aspect:

Parent Material(s):

Vegetation:

Described By:

Sampling Crew:

Rad. Scan:

Weather Yesterday:

Present Weather:

Date:
Oak Ridge Reservation, Grid N30000, E36970

Ruptic Ultic Dystrochrepts; Clayey Bt, loamy-skeletal Bw, C, and $\mathrm{Cr}$ horizons, mixed, thermic

Middle of slightly convex lower sideslope

$13 \%$ south-southwest

Middle Dismal Gap Formation

Original old-field successional forest of pines has died and fallen over. Present vegetation consists of dogwood, beech, sweet gum, poplar, red maple, pine, with a thick ground cover of honeysuckle.

D. A. Lietzke

Lietzke, Wilson

Air, 25; ground level, 40

Early morning showers, then clearing and very windy

Clear, sunny afternoon with a light breeze

May 4, 1992

\section{Soil Description:}

Oi $\quad 7$ to $0 \mathrm{~cm}$; leaf litter, twigs and small branches, and a dense root mat

A 0 to $5 \mathrm{~cm}$; dark yellowish-brown (10YR 4/4) silt loam; strong fine granular structure; very friable; abrupt wavy boundary

Ap 5 to $12 \mathrm{~cm}$; yellowish-brown (10YR 5/6) shaly silt loam; moderate fine granular structure; friable; abrupt wavy boundary

Bt 12 to $27 \mathrm{~cm}$; yellowish-red (5YR 5/6) clay; moderate medium subangular blocky structure; firm; strong brown (7.5YR 5/6) clay films on all ped faces; clear wavy boundary

BC 27 to $53 \mathrm{~cm}$; strong brown (7.5YR 5/6) shaly or very shaly silty clay loam; weak coarse subangular blocky structure; firm; 10 to $50 \%$ rock structure in thin harder strata; abrupt irregular boundary

C $\quad 53$ to $63 \mathrm{~cm}$; olive brown (2.5Y 4/4) fragment interiors; thick strong brown (7.5YR $5 / 6)$ coatings on most fragment surfaces and plugging cracks and fractures; abrupt irregular boundary

$\mathrm{Cr} \quad 63$ to $80 \mathrm{~cm}$; light olive brown (2.5Y 5/6) fragment interiors; surfaces coated with black manganese oxides or yellowish-brown (10YR 5/8) iron hydroxides

Notes: Sampling depths are: A, 0 to $12 \mathrm{~cm} ; \mathrm{B}, 12$ to $27 \mathrm{~cm} ; \mathrm{C}, 53$ to $63 \mathrm{~cm}$. 


\section{ORR Site 35}

(See pp. 28, 37, 39, 58, 59, 91 in Phase I Field Book 1)

Location:

Classification:

Geomorphic Position:

Slope and Aspect:

Parent Material(s):

Vegetation:

Described By:

Sampling Crew:

Rad. Scan:

Weather Yesterday:

Present Weather:

Date:
Oak Ridge Reservation, Grid N30000, E37280. Site is located about $150 \mathrm{ft}$ north of cleared edge of ROW. Typic Hapludults; clayey, mixed, thermic Convex area just below rest of ridge $13 \%$ south southwest Lower Dismal Gap Formation

Old field successional forest where most pines have died and fallen or were harvested. Present vegetation is sweet gum, dogwood, haw, sourwood with some poplar. About $90 \%$ of ground surface is leaf covered the remainder is covered by honeysuckle, mosses and other forbes.

D. A. Lietzke Lietzke, Wilson, Farmer

Air, 15; ground level, 35

Cloudy with early morning showers, then clearing and windy in the afternoon

Clear and windy

May 4, 1992

\section{Soil Description:}

Oi $\quad 2$ to $0 \mathrm{~cm}$; leaf litter

A $\quad 0$ to $8 \mathrm{~cm}$; dark brown (10YR 3/3) silt loam; strong fine granular structure; very friable; abrupt wavy boundary

Ap 8 to $19 \mathrm{~cm}$; brown (10YR $5 / 3$ ) silt loam; weak fine granular structure; very friable; abrupt wavy boundary

E\&B 19 to $31 \mathrm{~cm}$; yellowish-brown (10YR 5/4) silt loam, E-part, and strong brown (7.5YR 5/6) silty clay loam, B-part; weak medium subangular blocky structure; very friable; abrupt irregular boundary

Bt $\quad 31$ to $52 \mathrm{~cm}$; strong brown (7.5YR 4/6) clay; moderate medium subangular blocky structure; friable; peds coated with yellowish-red (5YR 4/6) clay; abrupt irregular boundary

BC $\quad 52$ to $63 \mathrm{~cm}$; strong brown (7.5YR 4/6) shaly clay; weak medium subangular blocky structure; with about 10 to $25 \%$ rock structure remaining; highly clay plugged. Abrupt irregular boundary

C\&Cr 63 to $80 \mathrm{~cm}$; dark yellowish-brown (10YR 4/6) fragment interiors; some fragment surface coated with yellowish-red (5YR 4/6) clay and others coated with red (2.5YR 4/6) iron-clay materials

Notes: Sampling depths are: A, 0 to $19 \mathrm{~cm} ; \mathrm{B}, 31$ to $52 \mathrm{~cm}$;, 63 to $80 \mathrm{~cm}$. 


\section{ORR Site 41}

(See pp. 12, 13, 28, 29, 91 in Phase I Field Book 1)

Location: $\quad$ Oak Ridge Reservation, about $400 \mathrm{ft}$ north of the TVA ROW; Grid N30200, E39570

Classification: Typic Hapludults; clayey, mixed, thermic

Geomorphic Position:

Slope and Aspect:

Parent Material(s):

Vegetation:

Described By:

Sampling Crew:

Rad. Scan:

Weather Yesterday:

Present Weather:

Date:
Convex summit of small ridge. Soils were severely eroded prior to or after abandonment.

2-3\%

Middle Dismal Gap Formation

Old-field successional forest. Most older pines have died and fallen over. Present regrowth of pines red maple, beech and poplar. Ground surface covered with leaves.

D. A. Lietzke

Lietzke, Wilson, Phillips, Farmer

Air, 10; ground level, 50

Partly cloudy

Clear and warmer

March 17, 1992

\section{Soil Description:}

$\mathrm{Oi} \quad 4$ to $0 \mathrm{~cm}$; leaf litter

A $\quad 0$ to $5 \mathrm{~cm}$; very dark grayish-brown (10YR 3/2) silt loam; moderate fine granular structure; very friable; many fine roots; abrupt wavy boundary

Ap 5 to $12 \mathrm{~cm}$; dark yellowish-brown (10YR 4/4) heavy silt loam; weak fine granular structure; very friable; common fine roots; abrupt wavy boundary

Bt 12 to $28 \mathrm{~cm}$; strong brown $(7.5 \mathrm{YR} 4 / 6)$ silty clay loam or silty clay; moderate medium subangular blocky structure; friable; common medium roots and common fine roots; strong brown (7.5YR 5/6) clay flows on ped faces; clear wavy boundary

BC 28 to $40 \mathrm{~cm}$; strong brown (7.5YR 5/6) shaly silty clay or shaly clay; weak coarse subangular blocky structure; some areas without soil structure; firm; few fine roots; clay plugged with abrupt irregular boundary

C 40 to $64 \mathrm{~cm}$; strong brown (7.5YR 4/6) and yellowish-brown (10YR 5/8) fragment interiors; material crushes easily to a shaly or very shaly silty clay loam; 60 to $80 \%$ rock controlled structure; friable; few fine roots; considerable strong brown (7.5YR $4 / 6$ ) clay on fragment surfaces; abrupt irregular boundary

Cr1 64 to $80 \mathrm{~cm}$; harder fragment interiors of strong brown (7.5YR 4/6) and yellowishbrown (10YR 5/8); most fragments coated with dark red (10R 3/6) iron oxides

$\mathrm{Cr} 2$. 80 to $90 \mathrm{~cm}$; harder fragment interiors of light olive gray $(5 \mathrm{Y} 6 / 2)$ and yellowishbrown (10YR 5/8) with a few red coatings; most fragments are wet and this horizon is at the top of a perched water zone.

Notes: Sampling depths for ESD composites are: A, 0 to $12 \mathrm{~cm}$;, 14 to $28 \mathrm{~cm} ; \mathrm{C}, 50$ to $60 \mathrm{~cm}$. 
ORR Site 42

(See pp. 105, 111, 143, 154, 155 in Phase I Field Book 1)

Location:

Classification:

Geomorphic Position:

Slope and Aspect:

Parent Material(s):

Vegetation:

Described By:

Sampling Crew:

Rad. Scan:

Weather Yesterday:

Present Weather:

Date:
Oak Ridge Reservation, Grid N30000, E40500. The site is in woods about $30 \mathrm{ft}$ from edge of power line ROW.

Ruptic Ultic Dystrochrepts; clayey Bt, loamy-skeletal Bw, C, Cr horizon; mixed, thermic

Long, gentle, convex side slope

$3 \%$ south

Middle to upper Nolichucky Shale

Old field successional where most older pines have died and fallen over or were harvested; dense regrowth of poplar, sweet gum, cedar, oak, dogwood, and beech. Sparse honeysuckle and poison ivy groundcover

D. A. Lietzke

Lietzke, Burgoa

Air, 30; ground level, 90

Clear and warm

Clear and warm

July 21,1992 .

Soil Description:

Oi $\quad 2$ to $0 \mathrm{~cm}$; leaves

Oa $\quad 0$ to $1 \mathrm{~cm}$; hemic materials, sapric materials and root mat

Ap 1 to $15 \mathrm{~cm}$; yellowish-brown (10YR 5/4) silt loam or shaly silt loam; moderate fine granular structure; very friable; abrupt wavy boundary

Bt 15 to $33 \mathrm{~cm}$; dark yellowish-brown (10YR 4/4) shaly clay; moderate medium subangular blocky structure; firm; all peds coated with brown (10YR 5/3) material; abrupt irregular boundary

C 33 to $47 \mathrm{~cm}$; mottled dark yellowish-brown (10YR 4/4), brown (10YR 5/3) and grayish-brown (10YR 5/2) wetness streaks; extremely shaly clay; all rock controlled structure; abrupt irregular boundary

$\mathrm{Cr} \quad 47$ to $56 \mathrm{~cm}$; brown (10YR 4/3) fragment interiors; red (10R 4/6) and black coatings on most fragment surfaces; some grayish-brown (10YR 5/2) in flow zones

Notes: The Bw and Bt were sampled together. The Bw had similar morphology but higher fragment content. This site has limestone seams 2 - to 5 -cm-thick that have weathered out to a yellowish-brown clay. Some of this clayey material was included in the very shaly $C$ horizon sample. Sampling depths are: A, 1 to $15 \mathrm{~cm}$; VB, 15 to $33 \mathrm{~cm} ; \mathrm{C}, 33$ to $47 \mathrm{~cm}$. 


\section{ORR Site 43}

(See pp. 28, 82, 83, 86, 87, 91 in Phase I Field Book 1)

Location:

Classification:

Geomorphic Position:

Slope and Aspect:

Parent Material(s):

Vegetation:

Described By:

Sampling Crew:

Rad Scan:

Weather Yesterday:

Present Weather:

Date:
Oak Ridge Reservation, Grid N30400, E41460, about $200 \mathrm{ft}$ grid north of edge of ROW

Typic Hapludults; clayey, mixed, thermic

Broad, slightly convex sideslope

2-3\% south-southwest

Lower Dismal Gap Formation within transition zone to Rogersville Shale

Old field hardwoods of red maple, poplar, abundant dogwood, beech, with thick honeysuckle, and poison ivy groundcover

D. A. Lietzke

Lietzke, Wilson

Air, 20; ground level, 55

Clear, warmer, last rain on May 9th

Clear and warm

May 11, 1992

\section{Soil Description:}

Oi $\quad 4$ to $0 \mathrm{~cm}$; leaf litter

A $\quad 0$ to $6 \mathrm{~cm}$; dark brown (10YR 3/3) silt loam; strong fine granular structure; very friable; abrupt wavy boundary

Ap $\quad 6$ to $15 \mathrm{~cm}$; brown (10YR 5/3) silt loam or shaly silt loam; moderate fine granular structure; very friable; abrupt wavy boundary

Bt1 15 to $37 \mathrm{~cm}$; strong brown $(7.5 \mathrm{YR} 4 / 6)$ clay or silty clay; moderate medium subangular blocky structure; firm; clear wavy boundary

$\mathrm{Bt} 237$ to $51 \mathrm{~cm}$; strong brown ( 7.5 YR 4/6) shaly silty clay; moderate medium and coarse subangular blocky structure; firm; Manganese oxide plugged at base of horizon; abrupt irregular boundary

C 51 to $70 \mathrm{~cm}$; light olive, brown fragment interiors; very soft saprolite with yellowishred (2.5 YR 4/6) clay streaks and yellowish-red (5YR 4/6) soil material coating some fragment surfaces and filling spaces

Notes: Sampling depths: A, 0 to $15 \mathrm{~cm} ; \mathrm{B}, 15$ to $37 \mathrm{~cm}$;, 51 to $70 \mathrm{~cm}$. Sampling depths for ORR-43 field duplicates are: A, 0 to $15 \mathrm{~cm} ; \mathrm{B}, 15$ to $40 \mathrm{~cm} ; \mathrm{C}, 53$ to $70 \mathrm{~cm}$. The reason for the different depths is that strike and dip oriented subsoil and substratum horizons vary around the sides of a pit. 
ORR Site 45

(See pp. 28, 29, and 42 in Phase II Field Book 2)

Location:

Classification:

Geomorphic position:

Slope and aspect:

Parent material(s):

Vegetation:

Described by:

Sampling crew:

Rad scan:

Weather yesterday:

Present weather:

Date:
Oak Ridge Reservation, Chestnut Ridge, Grid N27600, E18400

Typic Hapludults; clayey, mixed, thermic

Convex sideslope just above a slope break into a deeply incised, but stable, head of a drainageway

$25 \%$ northwest

Copper Ridge Residuum

Older woods that have been cut over several times. Oak and chestnut stumps are still present. Some logging was done 15 to 20 years ago. Present vegetation consists of red oak, black oak, post oak, with a lower story of gum, sourwood, sugar maple and some sassafras sprouts. The ground surface is leaf covered. A woods road about $100-150 \mathrm{ft}$ away was bulldozed a few years ago to allow access for the drilling of wells.

D. A. Lietzke

Lietzke and Wilson

Air, 30; ground level, 60

Clear, breezy and cooler

Clear, brisk, breezy

September 30, 1992

\section{Soil Description:}

$\mathrm{Oi} \quad 3$ to $0 \mathrm{~cm}$; leaf litter

A 0 to $5 \mathrm{~cm}$; dark grayish-brown (10YR $4 / 2$ ) cherty silt loam; strong fine granular structure; very friable; clear wavy boundary

E1 5 to $17 \mathrm{~cm}$; pale brown $(2.5 \mathrm{Y} 6 / 3)$ cherty silt loam; moderate fine granular structure; very friable; gradual wavy boundary

E2 17 to $30 \mathrm{~cm}$; light yellowish-brown (10YR 6/4) cherty silt loam; moderate fine granular structure; very friable; gradual irregular boundary

B\&E $\quad 30$ to $48 \mathrm{~cm}$; strong brown (7.5YR 5/6) cherty silty clay loam B part, and yellowishbrown (10YR 5/6) cherty silt loam E part; moderate fine subangular blocky structure; very friable; abrupt irregular boundary

Bt1 48 to $70 \mathrm{~cm}$; red (2.5YR 4/6) cherty clay; $40 \%$ clay content) moderate medium subangular blocky structure; friable; gradual wavy boundary

$\mathrm{Bt} 270$ to $120 \mathrm{~cm}$; red clay (60-70\% clay content); augered

BC $\quad 120$ to $170 \mathrm{~cm}$; red clayey saprolitic materials with high chroma yellowish saprolite colors

Notes: Sampling depths: A horizon, 0 to $17 \mathrm{~cm}$; B horizon, 48 to $60 \mathrm{~cm}$; $\mathrm{C}$ horizon, 150 to $170 \mathrm{~cm}$. 


\section{ORR Site 50}

(See pp. 31, 81, 82, and 87 in Phase II Field Book 2)

Location:

Classification:

Geomorphic position:

Slope and aspect:

Parent material(s):

Vegetation:

Described by:

Sampling crew:

Rad scan:

Weather yesterday:

Present weather:

Date:
Oak Ridge Reservation, Chestnut Ridge, Grid N26600, E19800

Typic Paleudults; clayey, kaolinitic, thermic

Long, gently sloping, spur ridge

$8 \%$ southeast

Chepultepec Residuum

Old-field successional forest. Present vegetation consists of an overstory of oaks along with a few red cedars, white pine seedlings and red maple sprouts. The ground surface is leaf covered.

D. A. Lietzke

Lietzke, Farmer, Wilson, Massengale, Burgoa

Air, 40; ground level, 55

Rain, heavy at times

Clear and getting warmer

November 3, 1992

\section{Soil Description:}

$\mathrm{Oi} \quad 3$ to $0 \mathrm{~cm}$; leaf litter

$\mathrm{Oa} \quad 0$ to $2 \mathrm{~cm}$; sapric materials and a root mat

A $\quad 0$ to $12 \mathrm{~cm}$; dark grayish-brown (10YR 4/2) cherty loam; strong fine granular structure; very friable; clear wavy boundary

E 12 to $25 \mathrm{~cm}$; pale brown (10YR 6/3) cherty loam; weak fine granular structure; very friable; abrupt irregular boundary

B\&E $\quad 25$ to $34 \mathrm{~cm}$; yellowis-red (5YR 5/6) B part and strong brown (7.5YR 5/6) E part; cherty or non-cherty clay loam; weak fine subangular blocky structure; friable; abrupt irregular boundary

Bt1 34 to $50 \mathrm{~cm}$; red $(2.5 \mathrm{YR} 4 / 6)$ clay or cherty clay; moderate fine and medium subangular blocky structure; firm; gradual wavy boundary

Bt2 50 to $80 \mathrm{~cm}$; red very cherty clay with yellowish-brown mottles (augered)

Bt3 80 to $160 \mathrm{~cm}$; mottled red, yellowish-red and yellowish-brown cherty clay (augered)

Notes: Sampling depths for primary samples: A horizon, 0 to $16 \mathrm{~cm}$; B horizon, 40 to $50 \mathrm{~cm}$; C horizon, 140 to $160 \mathrm{~cm}$. Sampling depths for field duplicates: A horizon, 0 to $10 \mathrm{~cm}$; B horizon, 34 to $50 \mathrm{~cm}$; C horizon, 140 to $160 \mathrm{~cm}$. 


\section{ORR Site 51}

(See pp. 25, 26, 30, and 36 in Phase II Field Book 2)

Location:

Classification:

Geomorphic position:

Slope and aspect:

Parent material(s):

Vegetation:

Described by:

Sampling crew:

Rad scan:

Weather yesterday:

Present weather.

Date:
Oak Ridge Reservation, Chestnut Ridge, Grid N28400, E21600

Typic Paleudults; clayey, kaolinitic, thermic

Convex sideslope

$15 \%$ northwest

Copper Ridge Residuum

Old field successional forest with most of the original pines having died and fallen over releasing poplar, red maple and sugar maple. This site is within 75 to $80 \mathrm{ft}$ of a previously cleared area that has been planted to loblolly pine in the past 15 to 20 years. This site is also within $80 \mathrm{ft}$ of a recently bulldozed woods road to get access to drill wells.

D. A. Lietzke

Lietzke and Wilson

Air, 30; ground level, 45

Cloudy and rainy

Clearing, partly cloudy

September 28, 1992

\section{Soil Description:}

$\mathrm{Oi} \quad 3$ to $0 \mathrm{~cm}$; leaf litter

A $\quad 0$ to $10 \mathrm{~cm}$; brown (10YR $4 / 3)$ cherty silt loam;strong fine granular structure; very friable; clear wavy boundary

Ap 10 to $20 \mathrm{~cm}$; brown (10YR 4/3) cherty silt loam; moderate fine granular structure; very friable; clear wavy boundary

B\&E 20 to $35 \mathrm{~cm}$; yellowish-red (5YR 5/6) B part, and pale brown (10YR 6/3) E part; cherty silt loam; moderate fine subangular blocky structure; very friable; abrupt irregular boundary

Bt1 35 to $75 \mathrm{~cm}$; red $(2.5 \mathrm{YR} 4 / 6)$ cherty clay; moderate medium subangular blocky structure; firm; gradual wavy boundary, base of pit

Bt2 75 to $165 \mathrm{~cm}$; red cherty clay with yellowish-brown mottles

Notes: Sampling depths: A horizon, 0 to $20 \mathrm{~cm}$; B horizon, 40 to $60 \mathrm{~cm}$; $C$ horizon, 140 to $170 \mathrm{~cm}$. 


\section{ORR Site 52}

(See pp. 32, 57, and 58 in Phase II Field Book 2)

Location:

Classification:

Geomorphic position:

Slope and aspect:

Parent material(s):

Vegetation:

Described by:

Sampling crew:

Rad scan:

Weather yesterday:

Present weather:

Date:
Oak Ridge Reservation, Chestnut Ridge, Grid N26600, E20200

Typic Hapludults; clayey, mixed, thermic

Doubly convex sideslope shoulder

$12 \%$ south

Chepultepec residuum

Old field successional forest. Most pines have fallen. Present vegetation is red maple, few red cedars and poplar. There is a fairly thick understory of red maple and oak seedlings. There are scattered ferns and honeysuckle of the leaf covered ground surface.

D. A. Lietzke

Lietzke, Farmer, and Massengale

Air, 20; ground level, 55

Clear

Clear and warmer

October 14, 1992

\section{Soil Description:}

Oi $\quad 4$ to $0 \mathrm{~cm}$; leaf litter

A $\quad 0$ to $7 \mathrm{~cm}$; dark grayish-brown (10YR 4/2) cherty or very cherty silt loam; strong fine granular structure; very friable; clear wavy boundary

Ap 7 to $18 \mathrm{~cm}$; brown (10YR 4/3) cherty silt loam; moderate fine granular structure; very friable; abrupt wavy boundary

E\&B 18 to $27 \mathrm{~cm}$; brown (10YR 5/3) E part and strong brown (7.5YR 5/6) B part; silt loam or silty clay loam; weak fine subangular blocky structure; very friable; abrupt irregular boundary

B\&E 27 to $45 \mathrm{~cm}$; yellowish-red (5YR 5/6) B part and strong brown (7.5YR 5/6) E part; silty clay loam; moderate fine and medium subangular blocky structure; friable; gradual irregular boundary

Bt1 45 to $63 \mathrm{~cm}$; yellowish-red (5YR 5/6) clay; moderate to strong fine and medium subangular blocky structure; friable; gradual wavy boundary

Bt2 63 to $100 \mathrm{~cm}$; mottled, red, yellowish-red with yellowish-brown drainage mottles; augered

$\mathrm{BC} \quad 100$ to $170 \mathrm{~cm}$; mottled clay plugged saprolitic materials

Notes: Sampling depths: A horizon, 0 to $18 \mathrm{~cm}$; $B$ horizon, 45 to $60 \mathrm{~cm}$; $C$ horizon, 140 to $170 \mathrm{~cm}$. 


\section{ORR Site 53}

(See pp. 33, 55, and 56 in Phase II Field Book 2)

Location:

Classification:

Geomorphic position:

Slope and aspect:

Parent material(s):

Vegetation:

Described by:

Sampling crew:

Rad scan:

Weather yesterday:

Present weather:

Date:
Oak Ridge Reservation, Chestnut Ridge, N26200, E22400

Typic Hapludults; clayey, mixed, thermic

About a third of the way below a doubly convex shoulder $20 \%$ south-southeast

Chepultepec Residuum with a $36 \mathrm{~cm}$ thick capping of colluvium Old field abandoned well before 1940 . Present vegetation is white oak, with and understory of dogwood, sourwood, black gum and a sparse ground cover of oak and maple sprouts and considerable chert cobbles.

D. A. Lietzke

Lietzke, Farmer, and Massengale

Air, 30; ground level, 60

Clear, cool

Clear and warmer

October 13, 1992

Soil Description:

Oi $\quad 3$ to $0 \mathrm{~cm}$; leaf litter

A 0 to $9 \mathrm{~cm}$; dark grayish-brown (10YR 4/2) cherty or very cherty silt loam; strong fine granular structure; very friable; clear wavy boundary

Ap 9 to $16 \mathrm{~cm}$; brown (10YR 4/3) cherty silt loam; moderate fine granular structure; very friable; clear wavy boundary

Bt1 16 to $36 \mathrm{~cm}$; yellowish-brown (10YR 5/6) cherty silt loam or cherty loam; weak fine subangular blocky structure; very friable; abrupt irregular boundary (base of colluvium)

2Bt2 36 to $59 \mathrm{~cm}$; yellowish-red (5YR 5/6) cherty or very cherty clay; moderate fine and medium subangular blocky structure; firm; gradual wavy boundary

2Bt3 59 to $101 \mathrm{~cm}$; mottled reds and yellows clay (augered)

2BC 101 to $160 \mathrm{~cm}$; clay plugged saprolitic materials

Notes: The upper $36 \mathrm{~cm}$ of the soil is colluvium. The left half of the pit had colluvium to a depth of $70 \mathrm{~cm}$. The ESD composite sampled the B horizon of the residuum while the gamma samples extended almost through the colluvium. Sampling depths: A horizon, 0 to $16 \mathrm{~cm}$; B horizon, 36 to $59 \mathrm{~cm}$; C horizon, 140 to $160 \mathrm{~cm}$. 


\section{A-54}

\section{ORR Site 54}

(See pp. 9, 47, 48, 53 in Phase II Field Book 2)

Location:

Classification:

Geomorphic position:

Slope and aspect:

Parent material(s):

Vegetation:

Described by:

Sampling crew:

Rad scan:

Weather yesterday:

Present weather:

Date:
Oak Ridge Reservation, Chestnut Ridge, Grid N28200, E22500

Typic Hapludults; clayey, mixed, thermic

Middle of convex sideslope

$30 \%$ northwest

Copper Ridge Residuum

Older, cutover, forest. Present canopy trees of chestnut oak with smaller red maples. The forest floor is covered with leaves along with a few dogwood sprouts. The forest is very open here.

D. A. Lietzke

Lietzke, Farmer, and Harris

Air, 40; ground level, 60

Clear

Clear and cool

October 12, 1992

Soil Description:

$\mathrm{Oi} \quad 3$ to $0 \mathrm{~cm}$; leaf litter

A $\quad 0$ to $6 \mathrm{~cm}$; brown (10YR 5/3) cherty silt loam; strong fine granular structure; very friable; clear wavy boundary

E 6 to $19 \mathrm{~cm}$; light yellowish-brown (10YR 6/4) cherty silt loam; moderate fine granular structure; friable; gradual irregular boundary (includes E\&B horizon)

B\&E 19 to $29 \mathrm{~cm}$; strong brown (7.5YR 5/6) B part and light yellowish-brown (10YR 6/4) E part, cherty silty clay loam; moderate fine subangular blocky structure; friable; gradual irregular boundary

Bt1 29 to $51 \mathrm{~cm}$; red $(2.5 \mathrm{YR} 4 / 6)$ cherty clay; strong fine and medium subangular blocky structure; firm; gradual wavy boundary

Bt2 51 to $130 \mathrm{~cm}$; red $(2.5 \mathrm{YR} 4 / 6)$ cherty clay with some high chroma yellowish saprolite mottles; moderate medium and coarse subangular blocky structure; firm; gradual wavy boundary

BC $\quad 130$ to $165 \mathrm{~cm}$; red and yellow clay saprolitic materials that are clay plugged

Notes: Sampling depths: A horizon, 0 to $12 \mathrm{~cm}$; B horizon, 29 to $51 \mathrm{~cm} \mathrm{C}$ horizon, 155 to $165 \mathrm{~cm}$. 


\section{ORR Site 55}

(See pp. 8, 23, 24, and 35 in Phase II Field Book 2)

Location:

Classification:

Geomorphic position:

Slope and aspect:

Parent material(s):

Vegetation:
Described by:

Sampling crew:

Rad scan:

Weather yesterday:

Present weather.

Date:
Oak Ridge Reservation, Chestnut Ridge, Grid N28400, E23400 Typic Paleudults; clayey, kaolinitic, thermic

Mid, convex, sideslope between old alluvium above and colluvium below. This pit has about $27 \mathrm{~cm}$ of colluvium over residuum.

$26 \%$ northeast

Copper Ridge Residuum

Cut over forest. Soil does not appear to have been plowed due to the very high surficial chert content, but was probably a woods pasture with some past șoil disturbance. Present vegetation consists of chestnut oaks in the canopy along with a few poplar, a few midlevel sugar maples and lower level dogwood and black gum and sprouts of sugar maple and oak. The ground surface is leaf covered.

D. A. Lietzke

Lietzke and Wilson

Air, 40; ground level, 60

Cloudy with showers

Clearing

September 28,1992

Soil Description:

$\mathrm{Oi} \quad 4$ to $0 \mathrm{~cm}$; leaf litter

A 0 to $9 \mathrm{~cm}$; dark brown (10YR 3/3) very cherty silt loam; strong fine granular structure; very friable; clear irregular boundary

E\&A 9 to $13 \mathrm{~cm}$; Yellowish-brown (10YR 5/4) E part and dark brown (10YR 3/3) A Part (worm activity); moderate fine granular structure; very friable; clear wavy boundary

Bt 13 to $27 \mathrm{~cm}$; yellowish-brown (10YR $5 / 6)$ cherty silty clay loam; weak fine subangular blocky structure; very friable; clear wavy boundary, base of colluvium

2E\&B 27 to $45 \mathrm{~cm}$; yellowish-brown (10YR 5/6) and strong brown (7.5YR 5/6) cherty silty clay loam; weak fine subangular blocky structure; very friable; gradual irregular boundary

2Bt1 45 to $71 \mathrm{~cm}$; yellowish-red (5YR $5 / 6$ ) cherty clay; moderate medium subangular blocky structure; friable; gradual wavy boundary

2Bt2 71 to $165 \mathrm{~cm}$; red $(2.5 \mathrm{YR} 4 / 8)$ cherty clay; moderate coarse and medium subangular blocky structure; firm

Notes: Sampling depths: A horizon, 0 to $13 \mathrm{~cm}$; B horizon, 45 to $60 \mathrm{~cm}$; $\mathrm{C}$ horizon, 140 to $165 \mathrm{~cm}$. 
ORR Site 58

(See pp. 16, 17, 19, and 27 in Phase II Field Book 2)

Location:

Classification:

Geomorphic position:

Slope and aspect:

Parent material(s):

Vegetation:

Described by:

Sampling crew:

Rad scan:

Weather yesterday:

Present weather:

Date:
Oak Ridge Reservation, Chestnut Ridge, Grid N28000, E25000

Typic Paleudults; clayey, kaolinitic, thermic

Edge of large, slightly convex, hilltop

$6 \%$ southeast

Copper Ridge

Old field successional. Present vegetation consists of older growth hardwoods of red oak, sugar maple and some poplar, dogwood and hickory understory, and red maple,oak, sassafras, black gum, and dogwood sprouts. Ground surface is leaf covered and there are some boulder-sized chert blocks nearby.

D. A. Lietzke

Lietzke and Burgoa

Air, 25; ground level, 50

Partly cloudy with an evening shower

Partly cloudy, warm, very humid with a shower during the pit digging

September 21, 1992

\section{Soil Description:}

Oi $\quad 4$ to $0 \mathrm{~cm}$; leaf litter

A $\quad 0$ to $5 \mathrm{~cm}$; brown (10YR $4 / 3$ ) very cherty silt loam; moderate fine granular structure; very friable; clear wavy boundary

Ap 5 to $17 \mathrm{~cm}$; dark yellowish-brown (10YR 4/4) very cherty silt loam or silty clay loam; moderate fine granular structure; very friable; abrupt wavy boundary

B\&E 17 to $30 \mathrm{~cm}$; yellowish-red (5/YR 5/8) B part and strong brown (7.5YR 5/6) E Part, cherty silty clay loam; moderate fine subangular blocky structure; friable; clear wavy boundary

Bt1 30 to $36 \mathrm{~cm}$; red (2.5YR 4/8) cherty silty clay loam; moderate fine subangular blocky structure; friable; clear wavy boundary

Bt2 36 to $120 \mathrm{~cm}$; red $(2.5 \mathrm{YR} 4 / 6)$ cherty or very cherty clay; moderate fine and medium subangular blocky structure; firm; gradual wavy boundary

Bt3 120 to $173 \mathrm{~cm}$; red cherty clay or clay with some yellowish mottles

Notes: Sampling depths: A horizon, 0 to $127 \mathrm{~cm}$; B horizon, 36 to $50 \mathrm{~cm}$; C horizon, 140 to $173 \mathrm{~cm}$. 


\section{ORR Site 59}

(See pp. 6, 14, 15, and 18 in Phase II Field Book 2)

Location:

Classification:

Geomorphic position:

Slope and aspect:

Parent material(s):

Vegetation:

Described by:

Sampling crew:

Rad scan:

Weather yesterday:

Present weather:

Date:
Oak Ridge Reservation, Chestnut Ridge, Grid N28000, E26600

Typic Paleudults; clayey, kaolinitic, thermic

Broad, convex, lower middle sideslope

$20 \%$ northwest

Copper Ridge residuum

This site appears to have been in an old field or pasture that was not badly eroded prior to abandonment. The soil does not appear to have been plowed. Present forest is old field-successional. with some mature shortleaf pine, mature white oak, and an understory of dogwood, beech, red maple and sassafras sprouts, some blueberry plants and a few hickory sprouts. The forest floor is covered with leaves.

Lietzke

Lietzke and Burgoa

Air, 45; ground level 50

Partly cloudy with a shower during the evening

Cloudy

September 21, 1992

\section{Soil Description:}

$\mathrm{Oi} \quad 4$ to $0 \mathrm{~cm}$; leaf litter

A $\quad 0$ to $5 \mathrm{~cm}$; dark brown (10YR 3/3) very cherty silt loam; moderate fine granular structure; very friable; clear wavy boundary

E 5 to $17 \mathrm{~cm}$; yellowish-brown (10YR 5/4) very cherty silt loam; weak fine granular structure; very friable; clear wavy boundary

E\&B 17 to $29 \mathrm{~cm}$; yellowish-brown (10YR 5/4) E part, and strong brown (7.5YR 5/6) B part, cherty silty clay loam; weak fine subangular blocky structure; very friable; clear irregular boundary

B\&E 29 to $43 \mathrm{~cm}$; red (2.5YR 4/6) B part and strong brown (7.5YR 5/8) E part, cherty silty clay loam or cherty clay; moderate fine subangular blocky structure; friable; gradual irregular boundary

Bt1 43 to $100 \mathrm{~cm}$; red (2.5YR 4/6) cherty clay; moderate medium subangular blocky structure; firm; gradual wavy boundary

Bt2 100 to $140 \mathrm{~cm}$; red (2.5YR 4.6 clay) augered

$\mathrm{Bt3} / \mathrm{BC} 140$ to $165 \mathrm{~cm}$; red cherty or very cherty clay with yellowish-brown, high chroma, parent material mottles

Notes: Sampling depths: A horizon, 0 to $17 \mathrm{~cm}$; B horizon, 43 to $68 \mathrm{~cm}$; C horizon, 140 to $165 \mathrm{~cm}$. 
ORR Site 60

(See pp. 7, 37, 38, and 39 in Phase II Field Book 2)

Location:

Classification:

Geomorphic position:

Slope and aspect:

Parent material(s):

Vegetation:

Described by:

Sampling crew:

Rad scan:

Weather yesterday:

Present weather:

Date:
Oak Ridge Reservation, Chestnut Ridge, Grid N27800, E27400

Typic hapludults; clayey, mixed, thermic

Doubly convex shoulder that breaks off into a drainageway

$25 \%$ southwest

Copper Ridge Residuum

Old field successional forest. Pines have either been harvested,or died and fallen over. Present canopy trees are oaks, red maple along with a few white pine and Virginia pine. There is an old field road about $20 \mathrm{ft}$ uphill of this site. The ground surface is leaf covered along with sprouts of blueberry, sassafras, red maple and oak along with greenbriar and a few young cedars.

D. A. Lietzke Lietzke, Farmer and Harris

Air, 45; ground level, 60

Rainy all day

Cloudy with a few sprinkles

October 5, 1992

\section{Soil Description:}

$\mathrm{Oi} \quad 3$ to $0 \mathrm{~cm}$; leaf litter

A $\quad 0$ to $4 \mathrm{~cm}$; dark yellowish-brown (10YR 4/4) very cherty silt loam; moderate fine granular structure; very friable; clear wavy boundary

Ap 4 to $11 \mathrm{~cm}$; brown (7.5YR 5/4) cherty or very cherty silty clay loam; moderate fine granular structure; very friable; abrupt wavy boundary

Bt1 11 to $30 \mathrm{~cm}$; red $(2.5 \mathrm{YR} 4 / 6)$ cherty clay; moderate fine subangular blocky structure; friable; gradual wavy boundary

Bt2 30 to $70 \mathrm{~cm}$; red $(2.5 \mathrm{YR} 4 / 6)$ cherty or very cherty clay; moderate medium subangular blocky structure; firm; a few vertical streaks of strong brown (7.5YR $5 / 6)$ silty clay loam; gradual wavy boundary

Bt3 70 to $140 \mathrm{~cm}$; red $(2.5 Y \mathrm{YR} 4 / 6)$ cherty clay; moderate coarse subangular blocky structure; very firm; gradual wavy boundary

BC $\quad 140$ to $175 \mathrm{~cm}$; red cherty clay with high chroma yellowish saprolite colors

Notes: Sampling depths: A horizon, 0 to $11 \mathrm{~cm}$; B horizon, 20 to $40 \mathrm{~cm}$; C horizon, 145 to $175 \mathrm{~cm}$. 


\section{ORR Site 62}

(See pp. 4, 21, 22, and 34 in Phase II Field Book 2)

Location:

Classification:

Geomorphic position:

Slope and aspect:

Parent material(s):

Vegetation:

Described by:

Sampling crew:

Rad scan:

Weather yesterday:

Present weather:

Date:
Oak Ridge Reservation, Chestnut Ridge, Grid 27900, E29700

Typic paleudults; clayey, kaolinitic, thermic

Broad, slightly convex, ridgetop

$3-4 \%$ south

Copper Ridge residuum

Old field successional forest where original pines have all died and fallen over. Present vegetation consists of oaks and hickories with red maple and dogwood understory and seedlings of red maple and sassafras. The forest floor is leaf covered. This site is. located about 75 to $80 \mathrm{ft}$ east of an area that was cut over and replanted to loblolly pine.

D. A. Lietzke

Lietzke and Wilson

Air, 20; ground level, 50; reading at a depth of $20 \mathrm{~cm}$ in the pit, 65

Cloudy with showers

Cloudy

September 28, 1992

Soil Description:

Oi $\quad 3$ to $0 \mathrm{~cm}$; leaf litter

A $\quad 0$ to $5 \mathrm{~cm}$; dark brown (10YR 3/3) cherty silt loam; strong fine granular structure; very friable; clear wavy boundary

Ap $\quad 5$ to $15 \mathrm{~cm}$; brown (10YR $4 / 3$ ) cherty silt loam; moderate fine granular structure; very friable; clear wavy boundary

E $\quad 15$ to $23 \mathrm{~cm}$; yellowish-brown (10YR 5/4) cherty silt loam; weak fine granular structure; very friable; clear irregular boundary (includes E\&B horizon in the lower part)

B\&E 23 to $36 \mathrm{~cm}$; yellowish-red (5YR 5/8) silty clay loam B part and light yellowishbrown (10YR 6/4) silt loam E part; weak fine subangular blocky structure; very friable; clear irregular boundary

Bt1 36 to $55 \mathrm{~cm}$; red (2.5YR $4 / 8$ ) cherty clay; moderate medium subangular blocky structure; firm; gradual wavy boundary

Bt2 55 to $140 \mathrm{~cm}$; red (2.5YR 4/6) cherty clay with yellowish-brown mottles

Bt3 140 to $163 \mathrm{~cm}$; highly mottled red and yellowish-brown clay with a 2-chroma wetness streak in the auger samples

Notes: Sampling depths: A horizon, 0 to $15 \mathrm{~cm}$; B horizon, 36 to $50 \mathrm{~cm}$; $\mathrm{C}$ horizon, 140 to $163 \mathrm{~cm}$. 
ORR Site 64

(See pp. 5, 51, and 52 in Phase II Field Book 2)

Location:

Classification:

Geomorphic position:

Slope and aspect:

Parent material(s):

Vegetation:

Described by:

Sampling crew:

Rad scan:

Weather yesterday:

Present weather:

Date:
Oak Ridge Reservation, Chestnut Ridge, Grid N27200, E30000

Typic Hapludults; clayey, mixed, thermic

Top of broad, slightly convex ridge

3-5\% north-northeast

Copper Ridge Residuum

Old field successional forest. The early pines have all disappeared. There are a few taller oaks and poplar. There is a thick sapling stand of red maple along with sprouts of red maple, beech, ash, along with a few ferns and a few white pine sprouts. D. A. Lietzke Lietzke, Farmer and Harris

Air, 30; ground level, 50

Clear

Clear

October 12, 1992

\section{Soil Description:}

$\mathrm{Oi} \quad 4$ to $0 \mathrm{~cm}$; leaf litter

A 0 to $8 \mathrm{~cm}$; dark brown (10YR 3/3) silt loam; strong fine and medium granular structure; very friable; clear wavy boundary

Ap $\quad 8$ to $19 \mathrm{~cm}$; dark yellowish-brown (10YR 4/4) silt loam; moderate fine granular structure; very friable; clear wavy boundary

$\mathrm{BE} \quad 19$ to $26 \mathrm{~cm}$; not described

Bt1 26 to $58 \mathrm{~cm}$; red (2.5YR 4/8) clay; strong fine and medium subangular blocky structure; firm; gradual wavy boundary

BC 58 to $64 \mathrm{~cm}$; yellow (10YR 7/8) and yellowish-red (5YR 5/8) saprolite that easily crushes to silt loam or silty clay loam. There are also vertical clay flows throughout.

CB 64 to $165 \mathrm{~cm}$; yellow (10YR 7/8) and yellowish-red (5YR 5/8) saprolite that crushes to silt loam or silty clay loam (augered)

Notes: Sampling depths are: A horizon, 0 to $19 \mathrm{~cm}$; $\mathrm{B}$ horizon, 26 to $50 \mathrm{~cm}$; $\mathrm{C}$ horizon, 150 to $160 \mathrm{~cm}$. 


\section{ORR Site 66}

(See pp. 69, 83, and 84 in Phase II Field Book 2)

Location:

Classification:

Geomorphic position:

Slope and aspect:

Parent material(s):

Vegetation:

Described by:

Sampling crew:

Rad scan:

Weather yesterday:

Present weather:

Date:
Oak Ridge Reservation, Chestnut Ridge, Grid N27000, E31000

Typic hapludults; clayey, mixed, thermic Doubly convex spur below a bench landform $15 \%$ southeast

Chepultepec Residuum

Severely eroded old-field successional forest. Many pines are still standing. There are a few poplar, some red maple and many red maple saplings and dogwood. Pit is in a dense stand of ferns.

D. A. Lietzke

Lietzke, Farmer, Harris, Massengale

Air, 35; ground level, 55

Rain ending

Clear with high cirrus moving in for the next storm.

November 3, 1992

\section{Soil Description:}

$\mathrm{Oi} \quad 2$ to $0 \mathrm{~cm}$; leaf litter

Ap 0 to $13 \mathrm{~cm}$; brown (10YR 4/3) cherty silty clay loam; moderate fine granular structure; very friable; abrupt wavy boundary

B\&E 13 to $21 \mathrm{~cm}$; yellowish-red (5YR 5/8) B part and yellowish-brown (10YR 5/4) E part; silty clay loam or cherty silty clay loam; moderate fine subangular blocky structure; friable; abrupt irregular boundary

Bt1 21 to $57 \mathrm{~cm}$; red $(2.5 \mathrm{YR} 4 / 6)$ cherty clay; moderate fine and medium subangular blocky structure; firm; gradual wavy boundary

Bt2 57 to $120 \mathrm{~cm}$; yellowish-red (5YR 5/8) and yellow (2.5Y 7/8) cherty clay. A 10YR $7 / 1$ ) flow zone was also observed in the auger cuttings

BC 120 to 160 ; highly mottled clay plugged saprolitic materials that are very cherty

Notes: Sampling depths for primary samples: A horizon, 0 to $12 \mathrm{~cm}$; B horizon, 16 to $30 \mathrm{~cm}$; C horizon, 140 to $165 \mathrm{~cm}$. Sampling depths for field duplicates: A horizon, 0 to $14 \mathrm{~cm}$; B horizon, 30 to $45 \mathrm{~cm}$; C horizon, 150 to $173 \mathrm{~cm}$. 
ORR Site 68

(See pp. 70, 78, and 79 in Phase II Field Book 2)

Location:

Classification:

Geomorphic position:

Slope and aspect:

Parent material(s):

Vegetation:

Described by:

Sampling crew:

Rad scan:

Weather yesterday:

Present weather.

Date:
Oak Ridge Reservation, Chestnut Ridge, Grid N26800, E31700

Typic paleudults; clayey, kaolinitic, thermic

Convex sideslope below a colluvial bench. The pit has a colluvial capping of variable depth. An old tree throw nearby may be the agent responsible for the surface mixing.

$25 \%$ northwest

Chepultepec Residuum with a surficial colluvial capping of 40 to $50 \mathrm{~cm}$

Older forest from which trees have been periodically harvested. The most recent cutting was done approximately 15 to 25 years ago. The taller trees are oaks and hickories with subcanopy trees of red maple and poplar and younger saplings and sprouts of cherry, hickory, sourwood, oak and sassafras. The forest floor is leaf covered.

D. A. Lietzke

Lietzke

Air, 20; ground level, 45

Cloudy with occasional drizzle

Rain earlier then clearing

October 28, 1992

\section{Soil Description:}

Oi $\quad 4$ to $0 \mathrm{~cm}$; leaf litter

A 0 to $10 \mathrm{~cm}$; dark grayish-brown (10YR 4/2) cherty silt loam; moderate fine granular structure; very friable; abrupt irregular boundary (ant burrow or termite burrow fillings into the $\mathrm{E}$ beneath)

E $\quad 10$ to $32 \mathrm{~cm}$; light yellowish-brown (10YR 6/4) cherty silt loam; weak fine granular structure; very friable; gradual wavy boundary

Bt $\quad 32$ to $50 \mathrm{~cm}$; yellowish-brown (10YR 5/6) very cherty silty clay loam; weak fine subangular blocky structure; friable to firm with some brittleness; abrupt irregular boundary

2E\&B $\quad 50$ to $68 \mathrm{~cm}$; light yellowish-brown (10YR 6/4) E part and strong brown (7.5YR $5 / 8$ ) B part; very cherty to extremely cherty silty clay loam; weak coarse subangular blocky structure; friable with some brittle areas; abrupt irregular boundary (includes 2B\&E horizon)

2Bt1 68 to $90 \mathrm{~cm}$; yellowish-red (5YR 5/8) cherty clay; moderate fine and medium subangular blocky structure; firm; gradual wavy boundary

2Bt2 90 to $170 \mathrm{~cm}$; highly mottled red, yellowish-red, and yellowish-brown clay or cherty clay. There are some 2 chroma wetness streaks.

Notes: Sampling depths; A horizon, 0 to $10 \mathrm{~cm}$; B horizon, 68 to $90 \mathrm{~cm}$; C horizon, 150 to $175 \mathrm{~cm}$ 
ORR Site 73

(See pp. 72, 85, and 86 in Phase II Field Book 2)

Location:

Classification:

Geomorphic position:

Slope and aspect:

Parent material(s):

Vegetation:

Described by:

Sampling crew:

Rad scan:

Weather yesterday:

Present weather:

Date:
Oak Ridge Reservation, Chestnut Ridge, Grid N26800, E34000

Typic hapludults; clayey, mixed, thermic

Doubly convex spur just below a topographic bench landform $15 \%$ northwest

Chepultepec Residuum

Old woods that has been logged from time to time. Stumps are near the pit. Present canopy trees are poplar, oak and red maple. There is a thick sapling stand of oak, cherry, dogwood and red maple. There are a few ferns and sprouts of oak, cedar and cherry. The ground surface is leaf covered.

D. A. Lietzke

Lietzke, Farmer, Harris, Massengale, Justice

Air, 15; ground level, 55

Clearing

Clear but clouds moving in

November 3, 1992

\section{Soil Description:}

Oi $\quad 3$ to $0 \mathrm{~cm}$; leaf litter

A 0 to $10 \mathrm{~cm}$; very dark grayish-brown (10YR $3 / 2$ ) cherty silt loam; strong fine granular structure; very friable, clear wavy boundary

E 10 to $27 \mathrm{~cm}$; pale brown (10YR 6/3) cherty silt loam; moderate fine granular structure; very friable; abrupt irregular boundary

B\&E 27 to $46 \mathrm{~cm}$; light yellowish-brown (10YR 6/4) E part and strong brown (7.5YR 5/6) B part; cherty silty clay loam; moderate fine subangular blocky structure; very friable; abrupt irregular boundary

Bt 46 to $60 \mathrm{~cm}$; yellowish-red (5YR 5/6) clay or cherty clay; strong fine and medium subangular blocky structure; friable; gradual wavy boundary

$\mathrm{BC} \quad 60$ to $80 \mathrm{~cm}$; yellowish-red and red clay plugged saprolitic materials with high chroma yellow mottles (augered)

CB 80 to $160 \mathrm{~cm}$; yellow (10YR 7/6-7/8) saprolitic materials with red zones and clay plugs; also black manganese oxide zones

Notes: This pit cuts through an old stump hole. Sampling depths for primary samples: A horizon, 0 to $14 \mathrm{~cm}$; B horizon, 33 to $55 \mathrm{~cm}$; C horizon, 145 to $160 \mathrm{~cm}$. Sampling depths for field duplicates: A horizon, 0 to $10 \mathrm{~cm}$; $B$ horizon, 30 to $50 \mathrm{~cm}$; C horizon, 140 to $160 \mathrm{~cm}$. 


\section{ORR Site 74}

(See pp. 71, 76, and 77 in Phase II Field Book 2)

Location:

Classification:

Geomorphic position:

Slope and aspect:

Parent material(s):

Vegetation:

Described by:

Sampling crew:

Rad scan:

Weather yesterday:

Present weather:

Date:
Oak Ridge Reservation, Chestnut Ridge, Grid N27200, E34000

Typic Hapludults; clayey, mixed, thermic

Convex shoulder on the lower end of a long spur ridge

$14 \%$ northwest

Chepultepec Residuum

Old-field successional forest The site is next to an old fence row. There is barbed wire sticking out of a nearby tree. Older trees are red and white oak. The younger trees are black gum, sweet gum, oaks, red maple. Only one old pine remains standing. There are sprouts of red maple, beech, cherry, sourwood and some ferns. The ground surface is leaf covered. This site was probably a pasture. It does not appear to have been plowed.

D. A. Lietzke

Lietzke, Farmer, Petrowski

Air, 15; ground level, 60

Clear and cool

Clear and cool

October 26, 1992

\section{Soil Description:}

Oi $\quad 4$ to $0 \mathrm{~cm}$; leaf litter

A $\quad 0$ to $6 \mathrm{~cm}$; dark grayish-brown (10YR 4/2) cherty loam; moderate fine granular structure; very friable; clear wavy boundary

E1 6 to $16 \mathrm{~cm}$; brown (10YR 5/3) cherty loam; weak fine granular structure; very friable; clear wavy boundary

E2 16 to $29 \mathrm{~cm}$; brownish-yellow (10YR 6/4) cherty loam or cherty fine sandy loam; weak fine granular structure; very friable; abrupt irregular boundary

B\&E 29 to $41 \mathrm{~cm}$; strong brown (7.5YR 5/8) and brownish-yellow (10YR 6/4) sandy clay loam or clay loam; weak fine subangular blocky structure; friable; abrupt irregular boundary

$\mathrm{Bt} \quad 69$ to $120 \mathrm{~cm}$; yellowish-red (5YR 5/8) clay; moderate fine and medium subangular blocky structure; firm; gradual wavy boundary

BC1 69 to $120 \mathrm{~cm}$; yellowish-red (5YR 5/8) with high chroma yellow mottles, highly clay plugged saprolitic materials along with chert blocks

$\mathrm{BC2} 120$ to $160 \mathrm{~cm}$; very cherty saprolitic materials. (It took more than $2 \mathrm{~h}$ to penetrate from 69 to $160 \mathrm{~cm}$.)

Notes: Sampling depths: A horizon, 0 to $12 \mathrm{~cm}$; B horizon, 41 to $61 \mathrm{~cm}$; C horizon, 140 to $160 \mathrm{~cm}$ 
ORR Site 75

(See pp. 3, 40, and 41 in Phase II Field Book 2)

Location:

Classification:

Geomorphic position:

Slope and aspect:

Parent material(s):

Vegetation:

Described by:

Sampling crew:

Rad scan:

Weather yesterday:

Present weather:

Date:
Oak Ridge Reservation, Chestnut Ridge, Grid N27200, E35400

Typic Paleudults; clayey, kaolinitic, thermic, (dark surface phase). Broad, doubly convex, ridgetop $13 \%$ northeast

Copper Ridge Residuum

Old field successional forest. Original pines are being replaced by a thick stand of young pines, poplar, black gum, sourwood and dogwood with some sprouts plus honeysuckle on the forest floor. The ground surface is covered with leaves and fallen pines.

D. A. Lietzke

Lietzke and Harris

Air, 20; ground level, 50

Cloudy

Clear, cool and breezy

October 6, 1992

Soil Description:

$\mathrm{Oi} \quad 2$ to $0 \mathrm{~cm}$; leaf litter

A $\quad 0$ to $4 \mathrm{~cm}$; very dark grayish-brown (10YR 3/2) cherty silt loam; strong medium granular structure; very friable; abrupt wavy boundary

Ap 4 to $11 \mathrm{~cm}$; dark brown (10YR 3/3) cherty silt loam; strong fine granular structure; very friable; clear wavy boundary

EB $\quad 11$ to $20 \mathrm{~cm}$; strong brown \&.5YR 4/6) cherty silt loam; strong fine subangular blocky structure; very friable; gradual wavy boundary

BE 20 to $30 \mathrm{~cm}$; yellowish-red (5YR $5 / 6)$ cherty or very cherty silty clay loam; moderate fine subangular blocky structure; very friable; clear wavy boundary

Bt1 30 to $49 \mathrm{~cm}$; red $(2.5 \mathrm{YR} 4 / 6)$ cherty clay; strong fine and medium subangular blocky structure; firm; gradual wavy boundary

Bt2 49 to $78 \mathrm{~cm}$; red $(2.5 \mathrm{YR} 4 / 6)$ cherty or very cherty clay; moderate medum subangular blocky structure; firm; gradual wavy boundary

Bt3 78 to $165 \mathrm{~cm}$; red cherty or very cherty clay (augered)

Notes: Sampling depths: A horizon, 0 to $11 \mathrm{~cm}$; B horizon, 30 to $49 \mathrm{~cm}, \mathrm{C}$ horizon, 150 to $165 \mathrm{~cm}$ 


\section{ORR Site 77}

(See pp. 43, 61, 62, and 63 in Phase II Field Book 2)

Location:

Classification:

Geomorphic position:

Slope and aspect:

Parent material(s):

Vegetation:

Described by:

Sampling crew:

Rad scan:

Weather yesterday:

Present weather:

Date:
Oak Ridge Reservation, Chestnut Ridge, Grid N26600, E35200

Typic Hapludults; clayey, kaolinitic, thermic

Broad, slightly convex, ridgetop

2-3\% south-southeast

Chepultepec Residuum

Old field successional forest with a few of the original pines still standing along with a few cedars. Present canopy trees are oaks, hickories, red maple and cherry. There are sprouts of oak, sassafras, maple, and poplar. The ground surface is leaf covered. D. A. Lietzke Lietzke, Harris

Air, 25; ground level, 45

Cloudy with showers then clearing in the afternoon

Clear morning with frost in the low places

October 19, 1992

\section{Soil Description:}

$\mathrm{Oi} \quad 4$ to $0 \mathrm{~cm}$; leaf litter and a root mat

A $\quad 0$ to $5 \mathrm{~cm}$; dark brown (10YR $3 / 3-3 / 2$ ) cherty loam; strong fine granular structure; very friable; clear wavy boundary

Ap $\quad 5$ to $14 \mathrm{~cm}$; dark brown (10YR 3/3) cherty loam; moderate fine and medium granular structure; very friable; abrupt wavy boundary

E 14 to $24 \mathrm{~cm}$; yellowish-brown (10YR 5/4) cherty loam or cherty silt loam; weak fine granular structure; very friable; abrupt irregular boundary. (The lower part of the $\mathrm{E}$ horizon contains thin $\mathrm{E} \& \mathrm{~B}$, and $\mathrm{B} \& \mathrm{E}$ horizons.)

Bt1 24 to $40 \mathrm{~cm}$; yellowish-red (5YR 5/8) cherty clay loam or cherty sandy clay loam; moderate medium subangular blocky structure; friable; gradual wavy boundary

Bt2 40 to $75 \mathrm{~cm}$; mottled yellowish-red and yellowish-brown clay (augered)

Bt3 75 to 130; yellowish-red clay

$\mathrm{BC} / \mathrm{CB} 130$ to $160 \mathrm{~cm}$; saprolitic materials in hues of reds and yellows; partially plugged with soft manganese oxides

Notes: Sampling depths: A horizon, 0 to $14 \mathrm{~cm}$; B horizon, 40 to $60 \mathrm{~cm}$; $C$ horizon, 140 to $160 \mathrm{~cm}$; field duplicate depths: A horizon, 0 to $14 \mathrm{~cm}$; B horizon, 45 to $60 \mathrm{~cm}$; C horizon, 140 to $160 \mathrm{~cm}$ 


\section{ORR Site 78}

(See pp. 44, 59, and 60 in Phase II Field Book 2)

Location:

Classification:

Geomorphic position:

Slope and aspect:

Parent material(s):

Vegetation:

Described by:

Sampling crew:

Rad scan:

Weather yesterday:

Present weather:

Date:
Oak Ridge Reservation, Chestnut Ridge, Grid N26700, E35600

Typic Hapludults; clayey, kaolinitic, thermic

Broad, slightly convex ridgetop

4-5\% southeast

Chepultepec Residuum that is covered by about $36 \mathrm{~cm}$ of ancient colluvium

Old field successional forest. Most of the original pines have died and fallen over. Present vegetation consists of oaks and some poplars in the canopy and a few lower story red maples. There are sprouts of red maple, sassafras, and cherry. There is some honeysuckle. The ground surface is leaf covered.

D. A. Lietzke

Lietzke, Farmer, G.Lee

Air, 30; ground level, 65

Clear and warm

Clear and warmer

October 15, 1992

Soil Description:

Oi $\quad 4$ to $0 \mathrm{~cm}$; leaf litter

A $\quad 0$ to $5 \mathrm{~cm}$; very dark grayish-brown (10YR $3 / 2$ ) cherty loam; strong medium granular structure; very friable; clear wavy boundary

Ap 5 to $15 \mathrm{~cm}$; dark grayish-brown (10YR 4/2) cherty loam or cherty silt loam; strong fine granular structure; very friable; abrupt wavy boundary

E 15 to $36 \mathrm{~cm}$; light yellowish-brown (10YR 6/4) very cherty loam; moderate fine granular structure; very friable; abrupt wavy boundary. (A thin Bt horizon (7.5YR $5 / 6)$ is included at the base.)

2B\&E 36 to $48 \mathrm{~cm}$; strong brown (7.5YR 5/6) B part and pale brown (10YR 6/3) E part cherty clay loam; moderate fine subangular blocky structure; friable; abrupt irregular boundary

2Bt1 48 to $60 \mathrm{~cm}$; yellowish-red (5YR 5/8) clay; moderate to strong fine and medium subangular blocky structure; firm; gradual wavy boundary

2Bt2 60 to $120 \mathrm{~cm}$; yellowish-red and red clay with yellowish-brown drainage mottles (augered)

2BC 120 to $160 \mathrm{~cm}$; highly clay plugged, very cherty, saprolitic materials of reds and yellows

Notes: Sampling depths: A horizon, 0 to $15 \mathrm{~cm}$; B horizon, 48 to $60 \mathrm{~cm}$; C horizon, 140 to $150 \mathrm{~cm}$ 


\section{ORR Site 83}

(See pp. 2, 49, 50, and 54 in Phase II Field Book 2)

Location:

Classification:

Geomorphic position:

Slope and aspect:

Parent material(s):

Vegetation:

Described by:

Sampling crew:

Rad scan:

Weather yesterday:

Present weather:

Date:
Oak Ridge Reservation, Chestnut Ridge, Grid N27800, E37600

Typic Hapludults; clayey, mixed, thermic

Elongated, doubly convex, ridgetop

$5 \%$ south-southwest

Copper Ridge Residuum

Old field successional forest. The early pines have nearly all died and fallen over. Present forest consists of red maple, a few pines, poplars. cherry, sourwood along with some cedars and dogwood. The ground surface is leaf covered.

D. A. Lietzke

Lietzke, Farmer and Harris

Air, 20; ground level, 50

Clear, breezy and cool

Clear and cool

October 12, 1992

\section{Soil Description:}

Oi $\quad 3$ to $0 \mathrm{~cm}$; leaf litter

A $\quad 0$ to $6 \mathrm{~cm}$; very dark grayish-brown (10YR 3/2) cherty silt loam; strong fine and medium granular structure; very friable; clear wavy boundary

Ap 6 to $16 \mathrm{~cm}$; dark brown (10YR 3/3) cherty silt loam; strong fine granular structure; very friable; clear wavy boundary

B\&E 16 to $24 \mathrm{~cm}$; yellowish-red (5YR 5/6) B part and strong brown (7.5YR 4/6) E part; silty clay loam or cherty silty clay loam; moderate fine subangular blocky structure; very friable; clear wavy boundary

Bt1 24 to $53 \mathrm{~cm}$; red $(2.5 Y R \quad 4 / 6)$ clay or cherty clay; strong fine and medium subangular blocky structure; friable; gradual wavy boundary

Bt2 53 to $90 \mathrm{~cm}$; red (2.5YR $4 / 8)$ clay or cherty clay; moderate medium subangular blocky structure; firm; gradual wavy boundary

BC 90 to $160 \mathrm{~cm}$; mottled red and yellow clay-plugged saprolitic materials (augered)

Notes: Sampling depths: A horizon, 0 to $15 \mathrm{~cm}$; B horizon, 24 to $53 \mathrm{~cm}$; C horizon, 100 to $170 \mathrm{~cm}$. (Because of the chert content, two auger holes were required to collect enough sample. The first auger hole penetrated from 150 to $170 \mathrm{~cm}$ with only sporadic recovery. The second hole was sampled from 100 to $140 \mathrm{~cm}$ depth. Penetration ceased in this hole at $140 \mathrm{~cm}$.) 


\section{ORR Site 85}

(See pp. 46, 66, and 67 in Phase II Field Book 2)

Location:

Classification:

Geomorphic position:

Slope and aspect:

Parent material(s):

Vegetation:

Described by:

Sampling crew:

Rad scan:

Weather yesterday:

Present weather:

Date:
Oak Ridge Reservation, Chestnut Ridge, Grid N26000, E37800

Typic or Humic Hapludults; clayey, kaolinitic, thermic

Lower third of long convex sideslope that goes all the way to the creek

48\% east-northeast

Chepultepec Residuum with a capping of creep materials $42 \mathrm{~cm}$ thick. This site is very close to the Longview Formation.

Cut over forest probably never plowed because of the steepness, but probably pastured. Present trees are red oak, sugar maple, white pine and umbrella magnolia. There is abundant poison ivy ground cover along with some ferns. The ground surface is leaf covered.

D. A. Lietzke

Lietzke, Harris

Air, 25; ground level, 55

Clear

Partly cloudy

October 20, 1992

\section{Soil Description:}

Oi $\quad 4$ to $0 \mathrm{~cm}$; leaf litter

A1 0 to $16 \mathrm{~cm}$; dark brown (10YR 3/3) cherty silt loam; strong medium granular structure; very friable; clear wavy boundary

A 16 to $23 \mathrm{~cm}$; dark yellowish-brown (10YR 3/4) cherty silt loam; strong fine granular structure; very friable; abrupt irregular boundary

E\&A 23 to $31 \mathrm{~cm}$; yellowish-brown (10YR 5/8) E part and dark yellowish-brown (10YR 4/4) A part; cherty silt loam; moderate fine granular structure; very friable; abrupt irregular boundary (worm activity?)

B\&E $\quad 31$ to $42 \mathrm{~cm}$; strong brown (7.5YR 4/4) B part, and dark yellowish-brown (10YR 4/4) E part; cherty silty clay loam; weak fine subangular blocky structure; friable; abrupt irregular boundary

Bt 42 to $80 \mathrm{~cm}$; red $(2.5 Y R \quad 4 / 6)$ clay or cherty clay with yellowish-brown clay flows on ped faces; strong medium subangular blocky structure; firm; gradual wavy boundary

BC 80 to $160 \mathrm{~cm}$; red saprolitic materials with high chroma yellowish mottles; clay; some manganese oxides in discrete zones

Notes: Sampling depths for primary samples: A horizon, 0 to $15 \mathrm{~cm}$; B horizon, 42 to $60 \mathrm{~cm}$; $C$ horizon, 140 to $160 \mathrm{~cm}$. Field duplicate sampling depths are the same. 


\section{ORR Site 86}

(See pp. 45, 64, and 65 in Phase II Field Book 2)

Location:

Classification:

Geomorphic position:

Slope and aspect:

Parent material(s):

Vegetation:

Described by:

Sampling crew:

Rad scan:

Weather yesterday:

Present weather:

Date:
Oak Ridge Reservation, Chestnut Ridge, Grid N26000, E38200

Typic Hapludults; clayey skeletal, kaolinitic, thermic

Steep doubly convex sideslope between Chestnut Ridge Road and the creek

$45 \%$ southwest

Uppermost Chepultepec Residuum, but may be in the Longview Old growth forest but which has been periodically logged. Present trees are chestnut oaks and some hickories along with some smaller red maple and small sassafras, oak and maple sprouts.

D. A. Lietzke

Lietzke, Harris

Air, 25; ground level, 50

Clear and frosty

Cloudy

October 20, 1992

\section{Soil Description:}

$\mathrm{Oi} \quad 3$ to $0 \mathrm{~cm}$; leaf litter and root mat

A $\quad 0$ to $3 \mathrm{~cm}$; very dark grayish-brown (10YR 3/2) extremely cherty silt loam; strong medium granular structure; very friable; abrupt wavy boundary

E $\quad 3$ to $18 \mathrm{~cm}$; pale brown $(2.5 \mathrm{Y} 6 / 3)$ extremely cherty silt loam; moderate fine granular structure; very friable; clear wavy boundary

EB 18 to $40 \mathrm{~cm}$; yellowish-brown (10YR $5 / 6)$ extremely cherty silty clay loam; weak fine subangular blocky structure; very friable; gradual wavy boundary

BE 40 to $56 \mathrm{~cm}$; strong brown (7.5YR $5 / 6)$ extremely cherty silty clay loam; moderate fine subangular blocky structure; very friable; gradual wavy boundary

Bt 56 to $95 \mathrm{~cm}$; strong brown (7.5YR 5/6-5/8) extremely cherty clay or very cherty clay; moderate medium subangular blocky structure; friable; gradual wavy boundary

BC 95 to $160 \mathrm{~cm}$; mottled red and yellow clayey saprolitic materials (augered)

Notes: Sampling depths for primary samples: A horizon, 0 to $15 \mathrm{~cm}$; B horizon, 32 to $50 \mathrm{~cm}$; C horizon, 140 to $155 \mathrm{~cm}$. Field duplicate sampling depths: A horizon, 0 to $18 \mathrm{~cm}$; B horizon, 56 to $70 \mathrm{~cm}$; C horizon, 140 to $155 \mathrm{~cm}$. 
ORR Site 90

(See pp. 73, 74, and 75 in Phase II Field Book 2)

Location:

Classification:

Geomorphic position:

Slope and aspect:

Parent material(s):

Vegetation:

Described by:

Sampling crew:

Rad scan:

Weather yesterday:

Present weather:

Date:
Oak Ridge Reservation, Chestnut Ridge, Grid N28000, E40800 Typic Hapludults; clayey, mixed, thermic

Small, doubly convex, spur ridge that is surrounded by old alluvium

$6 \%$ southeast

Chepultepec Residuum

Present vegetation consists of a 30- to 40-year-old stand of planted loblolly pine. The understory is red maple, poplar and seedling pines. Groundcover is honeysuckle, roses, and blackberry briars.

D. A. Lietzke

Lietzke, Farmer, Petrowski

Air, 15; ground level, 55

Clear and cool with some fog

Clear and cool but with a warming trend

October 26, 1992

\section{Soil Description:}

$\mathrm{Oi} \quad 4$ to $0 \mathrm{~cm}$; leaf litter, pine needles and honeysuckle roots and stems

Ap $\quad 0$ to $12 \mathrm{~cm}$; brown (10YR 4/3) cherty loam; moderate fine granular structure; friable; abrupt wavy boundary

E\&B 12 to $27 \mathrm{~cm}$; light yellowish-brown (10YR 6/4) E part, and brown (7.5YR 5/4) B part; cherty loam or cherty sandy clay loam; weak fine granular structure; friable; abrupt irregular boundary. (This horizon also includes a B\&E horizon in the lower part.)

Bt1 27 to $59 \mathrm{~cm}$; yellowish-red (5YR 5/8) clay or cherty clay; moderate medium and coarse subangular blocky structure; firm; gradual wavy boundary

Bt2 95 to $120 \mathrm{~cm}$; yellowish-red and red clay with yellowish-brown mottles. (augered

BC $\quad 120$ to $160 \mathrm{~cm}$; mottled reds and high chroma yellows; clay-plugged saprolitic materials

Notes: Many fragments are sandstone. This site is about $60 \mathrm{ft}$ north of Chestnut Ridge Road with a gravel surface. The soil surface probably has received dust additions and perhaps other contaminants. Sampling depths: A horizon, 0 to $12 \mathrm{~cm}$; B horizon, 27 to $40 \mathrm{~cm}$; C horizon, 140 to $160 \mathrm{~cm}$. 


\section{ORR Site 91}

(See pp. 1, 10, 11, 12, 13, and 20 in Phase II Field Book 2)

Location:

Classification:

Geomorphic position:

Slope and aspect:

Parent material(s):

Vegetation:

Described by:

Sampling crew:

Rad. Scan:

Weather yesterday:

Present weather.

Date:
Oak Ridge Reservation, Chestnut Ridge near Roane-Anderson County line, Grid N27000, E41000 Typic Paleudults; clayey, kaolinitic, thermic

Long convex sideslope spur with some shallow dolines nearby and some boulder-sized chert on the surface

$7 \%$ north

Copper Ridge Residuum

Old woodlot with remains of chestnut stumps and barbed wire imbedded in a tree about $3 \mathrm{ft}$ from the pit. Present trees are poplar, cherry, post and white oak, one chestnut sprout about 15 -ft-tall and 1-in. dbh plus red maple and dogwood. Leaf covered ground and sprouts of sassafras, red maple and hickory. D. A. Lietzke

Lietzke and Burgoa

Air, 50; ground level 65

Partly cloudy and warm with a shower during the night

Cloudy and damp

September 21, 1992

\section{Soil Description:}

$\mathrm{Oi} \quad 4$ to $0 \mathrm{~cm}$; leaf litter

A $\quad 0$ to $5 \mathrm{~cm}$; dark brown (10YR $3 / 3$ ) cherty or very cherty silt loam; strong fine granular structure; very friable; abrupt wavy boundary

E 5 to $18 \mathrm{~cm}$; yellowish-brown (10YR $5 / 4$ ) cherty or very cherty silt loam; moderate fine granular structure; very friable; clear wavy boundary

B\&E 18 to $30 \mathrm{~cm}$; yellowish-red (5YR 5/6) B part, and strong brown (7.5YR 5/6) E part; silt loam or silty clay loam; weak fine subangular blocky structure; very friable; clear wavy boundary

Bt1 30 to $52 \mathrm{~cm}$; red (2.5YR $4 / 8)$, cherty clay; moderate medium subangular blocky structure; firm; gradual wavy boundary

Bt2 52 to $79 \mathrm{~cm}$; red $(2.5 \mathrm{YR} 4 / 6)$ clay or cherty clay; moderate medium subangular blocky structure and angular blocky structure; firm; gradual wavy boundary

Bt3 79 to $155 \mathrm{~cm}$; red $(2.5 \mathrm{YR} 4 / 6)$ cherty clay with strong brown and yellowish-brown mottles

Notes: Sampling depths: A Horizon, 0 to $18 \mathrm{~cm}$; B horizon, 30 to $52 \mathrm{~cm}$; C horizon, 135 to $155 \mathrm{~cm}$ 


\section{ORR Site 93}

(See pp. 1, 5, and 6 in Phase II Field Book 3)

Location:

Classification:

Geomorphic position:

Slope and aspect:

Parent material(s):

Vegetation:

Described by:

Sampling crew:

Rad scan:

Weather yesterday:

Present weather:

Date:
Oak Ridge Reservation, Bethel Valley, Grid N21600, E26500

Typic Hapludalfs; fine, mixed, thermic

Broad, convex spur extending to the north from Haw Ridge

7\% northwest

Unit G Chickamauga-Bethel Valley Section

Old field successional forest in a severely eroded old field. Present vegetation is dominated by hardwoods but there are still pines and a few large cedars. a few large oaks and ash along with sprouts of maple, dogwood and ash. There is abundant poison ivy. The ground surface is leaf-covered.

D. A. Lietzke

Lietzke, Burgoa

Air, 50; ground level, 70

Clear and cool with frost at night

Clear and cool with frost at night

November 16, 1992

\section{Soil Description:}

Oi $\quad 4$ to $0 \mathrm{~cm}$; leaf litter

A $\quad 0$ to $2 \mathrm{~cm}$; very dark grayish-brown (10YR $3 / 2$ ), silty clay loam; strong medium granular structure; friable; abrupt wavy boundary

Ap 2 to $11 \mathrm{~cm}$; dark yellowish-brown (10YR 4/4), silty clay loam; weak fine granular structure;firm; abrupt wavy boundary

Bt1 11 to $22 \mathrm{~cm}$; yellowish-red (5YR 4/6) clay; strong fine and medium angular and subangular blocky structure; very firm; yellowish-brown (10YR 5/6) coatings on all ped surfaces; clear wavy boundary

$\mathrm{Bt} 222$ to $45 \mathrm{~cm}$; yellowish-red (5YR 5/6) and red (2.5YR 5/6) clay; weak coarse prismatic structure parting to strong fine and medium angular blocky structure; pale brown (2.5Y 6/3) ped coatings; very firm; clear wavy boundary

$\mathrm{BC} \quad 45$ to $85 \mathrm{~cm}$; yellowish-brown (10YR 5/4) clay; massive; abrupt irregular boundary

$\mathrm{R} \quad 85 \mathrm{~cm}$ limestone rock ledge. Depth to rock is highly variable below a depth of $85 \mathrm{~cm}$

Notes: Sampling depths; A horizon, 0 to $11 \mathrm{~cm}$; B horizon, 12 to $22 \mathrm{~cm}$; C horizon, 65 to $80 \mathrm{~cm}$ 


\section{ORR Site 99}

(See pp. 1, 7, and 8 in Phase II Field Book 3)

Location:

Classification:

Geomorphic position:

Slope and aspect:

Parent material(s):

Vegetation:

Described by:

Sampling crew:

Rad scan:

Weather yesterday:

Present weather:

Date:
Oak Ridge Reservation, Bethel Valley, Grid 22000, E27750

Typic Hapludults; clayey, mixed, thermic

Lower convex sideslope, very severely eroded landform. The pit is located on an narrow spur ridge between two gullies.

$5 \%$ northwest

Unit G Chickamauga Residuum. Bethel Valley section

Old-field successional pines with most Virginia and shortleaf pines still in place. There is a thick sapling stand of red maple and dogwood along with honey suckle and poison ivy. The ground surface is about $70 \%$ covered with mosses and lichens with the remainder covered by needles and leaves.

D. A. Lietzke

Lietzke, Burgoa

Air, 40; ground level, 60

Clear and cool with frost at night

Clear and cool with frost at night

November 16, 1992

\section{Soil Description:}

$\mathrm{Oi} \quad 4$ to $0 \mathrm{~cm}$; needles, leaves, mosses, and lichens

A $\quad 0$ to $2 \mathrm{~cm}$; dark brown (10YR 3/3) silt loam; moderate fine granular structure; very friable; abrupt wavy boundary

Ap 2 to $15 \mathrm{~cm}$; brown (7.5YR 4/4) silty clay loam; weak fine granular structure; friable; abrupt wavy boundary

Bt1 15 to $32 \mathrm{~cm}$; yellowish-red (5YR 4/6) clay or silty clay; moderate fine and medium subangular blocky structure; friable; gradual wavy boundary

Bt2 32 to $87 \mathrm{~cm}$; brown (7.5YR 4/4) clay or silty clay; moderate fine subangular blocky structure; friable; many soft manganese oxide bodies throughout; gradual wavy boundary

BC $\quad 87$ to $180 \mathrm{~cm}$; brown (7.5YR 4/4) clay (augered)

$\mathrm{CB} .180$ to $200 \mathrm{~cm}$; yellowish-brown (10YR 5/4) clay with many soft black manganese oxide bodies throughout

Notes: Sampling depths: A horizon, 0 to $15 \mathrm{~cm}$; B horizon, 18 to $32 \mathrm{~cm}$; $C$ horizon, 98 to $113 \mathrm{~cm}$ 


\section{ORR Site 100}

- (See pp. 1, 9, and 10 in Phase II Field Book 3)

Location:

Classification:

Geomorphic position:

Slope and aspect:

Parent material(s):

Vegetation:

Described by:

Sampling crew:

Rad scan:

Weather yesterday:

Present weather:

Date:
Oak Ridge Reservation, Bethel Valley, Grid N21600, E28000

Typic Hapludults clayey, mixed, thermic

Doubly convex spur off of Haw Ridge

$12 \%$ northwest

Unit G Chickamauga Residuum of Bethel Valley section

Severely eroded old woods pasture field vegetation. The open forest stand is dominated by large oaks, some white pine and sugar maple. There are beech and maple saplings and oak sprouts along with some lowbush blueberry. The ground surface is leaf covered.

D. A. Lietzke

Lietzke, Burgoa

Air, 50; ground level, 70

Clear and cool

Clear and cool

November 16, 1992

\section{Soil Description:}

Oi $\quad 4$ to $0 \mathrm{~cm}$; leaves

$\mathrm{Oa} \quad 0$ to $3 \mathrm{~cm}$; sapric materials, hemic materials and a root mat

Ap $\quad 3$ to $6 \mathrm{~cm}$; brown (10YR 4/3) silty clay loam; moderate fine granular structure; friable; abrupt wavy boundary

Bt1 6 to $25 \mathrm{~cm}$; strong brown (7.5YR 5/8) clay; moderate medium prismatic structure parting to moderate fine subangular blocky structure; firm; thick yellowish-brown (10YR 5/4) coatings on prism faces; clear wavy boundary

Bt2 25 to $48 \mathrm{~cm}$; strong brown (7.5YR 5/8) clay with some brownish-yellow (10YR 6/6) ped interiors; moderate coarse prismatic structure parting to moderate fine angular blocky structure; very firm; thick yellowish-brown (10YR 5/4) coatings on prism faces; clear wavy boundary

C $\quad 48$ to $84 \mathrm{~cm}$; brownish-yellow (10YR 6/6) saprolitic materials that easily crush to clay; some ped faces in the upper part that are coated with strong brown (7.5YR $5 / 8$ ) clay

$\mathrm{Cr} \quad 84$ to $100 \mathrm{~cm}$; olive yellow (2.5Y 6/6) saprolite with red and black coatings in larger cracks and on joint surfaces

Notes: Sampling depths: A horizon, 0 to $6 \mathrm{~cm}$; B horizon, 10 to $25 \mathrm{~cm}$; $C$ horizon, 55 to $70 \mathrm{~cm}$ 
ORR Site 101

(See pp. 2, 13, 14, and 15 in Phase II Field Book 3)

Location:

Classification:

Geomorphic position:

Slope and aspect:

Parent material(s):

Vegetation:

Described by:

Sampling crew:

Rad scan:

Weather yesterday:

Present weather:

Date:
Oak Ridge Reservation, Bethel Valley, Grid N21250, E31550

Typic Hapludalfs; fine, mixed, thermic

Doubly convex sideslope off of Haw Ridge

$25 \%$ northwest

Unit G Chickamauga Residuum of Bethel Valley Section

Mature cedars, oaks and beech along with sugar maple, beech and dogwood saplings and sprouts. This site was evidently never plowed but was probably a woods pasture

D. A. Lietzke

Lietzke, Farmer, Harris

Air, 60; ground level, 80

Clear

Clear

November 19, 1992

\section{Soil Description:}

Oi $\quad 5$ to $0 \mathrm{~cm}$; leaves

A $\quad 0$ to $13 \mathrm{~cm}$; very dark grayish-brown (10YR 3/2) clay or silty clay; strong coarse granular structure; firm or very firm; clear wavy boundary

$\mathrm{AB} \quad 13$ to $23 \mathrm{~cm}$; brown (10YR 4/3) clay; strong medium and coarse subangular and angular blocky structure; very firm; peds coated with dark grayish-brown (10YR 4/2) materials; gradual wavy boundary

Bt 23 to $50 \mathrm{~cm}$; yellowish-brown (10YR 5/4) clay; moderate medium and coarse prismatic parting to strong medium angular blocky structure; very firm; ped coatings of dark grayish-brown (10YR 4/2); gradual wavy boundary

C $\quad 50$ to $70 \mathrm{~cm}$; light yellowish-brown (2.5Y 6/4) clay; weak coarse prismatic structure in the upper part to massive in the lower part; abrupt irregular boundary. Some thin limestone ledges were in this horizon and were removed from the pit.

$\mathrm{R} \quad 70 \mathrm{~cm}$; limestone rock ledge at strike and dip

Notes: Sampling depths: A horizon, 0 to $12 \mathrm{~cm}$; B horizon, 23 to $50 \mathrm{~cm}$; $C$ horizon, 60 to $70 \mathrm{~cm}$ 


\section{ORR Site 102}

(See pp. 2, 13, 14, and 17 in Phase II Field Book 3)

Location:

Classification:

Geomorphic position:

Slope and aspect:

Parent material(s):

Vegetation:

Described by:

Sampling crew:

Rad scan:

Weather yesterday:

Present weather:

Date:
Oak Ridge Reservation, Bethel Valley, Grid N21300, E34900

Typic Hapludalss; fine, mixed, thermic

Broad, slightly convex ridge

$3 \%$ northwest

Chickamauga Residuum of the Bethel Valley Section

This site is probably in the front yard of an old house site. Present vegetation consists of scattered large oaks and large Virginia pines. Poison ivy ground cover is very abundant. This site was relatively uneroded before abandonment.

D. A. Lietzke

Lietzke, Farmer, and Harris

Air, 40; ground level, 60: In top of auger hole, 70

Clear and warm

Clear but clouding in

November 19, 1992

Soil Description:

Oi $\quad 5$ to $0 \mathrm{~cm}$; leaves, needles and leaf litter

A $\quad 0$ to $10 \mathrm{~cm}$; dark yellowish-brown (10YR 3/4) silty clay loam; strong coarse granular structure; friable; clear wavy boundary

BA 10 to $20 \mathrm{~cm}$; dark brown (7.5YR $3 / 4$ ) silty clay loam; moderate fine subangular blocky structure; firm; clear wavy boundary

Bt 20 to $34 \mathrm{~cm}$; yellowish-red (5YR 4/6) clay; weak coarse prismatic parting to strong medium and coarse angular and subangular blocky structure; very firm; strong brown (7.5YR 5/4) clay flows on most ped surfaces and especially heavy on prism surfaces; gradual wavy boundary

BC $\quad 34$ to $101 \mathrm{~cm}$; saprolitic materials with ped in the upper part decreasing with depth; colors are red (2.5YR 5/6), strong brown (7.5YR 5/6) and light yellowish-brown (2.5Y 6/4) clay; weak coarse prismatic structure; very firm; abrupt irregular boundary

$\mathrm{R} \quad 101 \mathrm{~cm}$; limestone ledge. Depth to rock in this pit ranges from 34 to $101 \mathrm{~cm}$ and perhaps deeper in a few places. Other small slabs of limestone were removed when digging the pit.

Notes: Sampling depths: A horizon, 0 to $10 \mathrm{~cm}$; B horizon, 20 to $34 \mathrm{~cm}$; $C$ horizon, 90 to $101 \mathrm{~cm}$ 
ORR Site 103

(See pp. 2, 12, 13, and 16 in Phase II Field Book 3)

Location:

Classification:

Geomorphic position:

Slope and aspect:

Parent material(s):

Vegetation:

Described by:

Sampling crew:

Rad scan:

Weather yesterday:

Present weather:

Date:
Oak Ridge Reservation, Bethel Valley, Grid N20800, E36400

Typic Hapludults; clayey, mixed, thermic

Middle section of a doubly convex sideslope

$24 \%$ northwest

Chickamauga Residuum from the Bethel Valley Section

Old field successional forest. Some of the original pines remain along with a few larger oaks. Small trees are beech, dogwood, gum and cedar. This site was severely eroded before abandonment.

D. A. Lietzke

Lietzke, Farmer and Harris

Air, 60; ground level, 80; top of auger hole, 90

Clear and dry

Clear and dry

November 19, 1992

\section{Soil Description:}

$\mathrm{Oi} \quad 5$ to $0 \mathrm{~cm}$; leaf litter

A $\quad 0$ to $4 \mathrm{~cm}$ (varies from 0 to $7 \mathrm{~cm}$ in an old stump hole); dark yellowish-brown (10YR 4/4) loam; moderate fine granular structure; very friable; abrupt wavy boundary. This A horizon consists largely of Rome materials that have moved down the slope.

2Bt 4 to $30 \mathrm{~cm}$; yellowish-red (5YR 4/6) clay; strong medium and coarse subangular blocky structure; very firm; strong brown (7.5YR 5/4) ped coatings; gradual wavy boundary. The dominant structure is probably large prismatic.

2BC $\quad 30$ to $77 \mathrm{~cm}$; saprolitic materials but having ped structure; ped interiors are brownish-yellow (10YR 6/6) and yellowish-red (5YR 4/6) clay; weak coarse prismatic structure; very firm. Peds are coated with both yellowish-red and red clay; gradual wavy boundary.

2CB $\quad 77$ to $105 \mathrm{~cm}$; red and yellow saprolitic materials that easily crush to clay. No rock was exposed in the bottom of the pit.

Notes: Sampling depths: A Horizon, 0 to $4 \mathrm{~cm}$; B Horizon, 4 to $15 \mathrm{~cm}$; $\mathrm{C}$ horizon, 90 to $100 \mathrm{~cm}$ 


\section{ORR Site 104}

(See pp. 3, 21, 22, 30, and 31 in Phase II Field Book 3)

Location:

Classification:

Geomorphic position:

Slope and aspect:

Parent material(s):

Vegetation:

Described by:

Sampling crew:

Rad scan:

Weather yesterday:

Present weather:

Date:
Oak Ridge Reservation, Bethel Valley, Grid N21800, E44000

Typic Hapludults; clayey, mixed, thermic

Doubly convex sideslope

$13 \%$ northwest

Chickamauga Residuum of the Bethel Valley Section

Old field successional forest. The original pines and cedars have mostly disappeared, but some remain. Present trees are all hardwoods consisting of oaks, red maple, and hickory. There are some lowbush blueberries on the forest floor. The ground surface is leaf covered.

D. A. Lietzke

Lietzke, Burgoa

Air, 40; ground level, 65; top of auger hole 85

Cloudy and cold

Partly cloudy to mostly cloudy

November 30, 1992

\section{Soil Description:}

Oi $\quad 4$ to $0 \mathrm{~cm}$; leaves and leaf litter

$\mathrm{Oa} \quad 0$ to $1 \mathrm{~cm}$; black organic matter and a root mat

$\mathrm{E} \quad 1$ to $4 \mathrm{~cm}$; pale brown $(2.5 \mathrm{Y} 5 / 3)$ silt loam; weak fine granular structure; very friable; clear wavy boundary. Contains some Rome pebbles.

Ap $\quad 4$ to $11 \mathrm{~cm}$; brown (10YR 4/3) silt loam; weak fine granular structure; very friable; abrupt wavy boundary. Contains some Rome pebbles.

Bt 11 to $47 \mathrm{~cm}$; yellowish-red (5YR 5/8) clay; weak coarse prismatic parting to strong medium subangular blocky structure; very firm; gradual wavy boundary.

$\mathrm{BC} \quad 47$ to $100 \mathrm{~cm}$; multi-colored saprolitic materials with clay texture; moderate medium and coarse angular blocky structure; peds have saprolitic interiors; peds are coated with reddish-brown (5YR 5/4) clay.

Sampling depths: A horizon, 0 to $11 \mathrm{~cm}$; B horizon, 17 to $40 \mathrm{~cm}$; C horizon, 75 to $95 \mathrm{~cm}$. 
ORR Site 108

(See pp. 3, 23, 24, 30, and 31 in Phase II Field Book 3)

Location:

Classification:

Geomorphic position:

Slope and aspect:

Parent material(s):

Vegetation:

Described by:

Sampling crew:

Rad scan:

Weather yesterday:

Present weather:

Date:
Oak Ridge Reservation, Bethel Valley, Grid N22250, E45000

Typic Hapludalfs; fine, mixed, thermic

Doubly convex sideslope

9\% northwest

Chickamauga Residuum of the Bethel Valley Section

Old field successional forest with some large pines and a more smaller pines and a lower story of hardwoods;. There is fairly thick brush along with honey suckle and poison ivy. The forest floor is mostly leaf covered but there are patches of moses.

\section{A. Lietzke}

Lietzke, Burgoa

$\mathrm{Air}, 50$; ground level, 60 ; top of auger hole, 70

Cloudy and cold

Mostly cloudy

November 30, 1992

Soil Description:

Oi $\quad 3$ to $0 \mathrm{~cm}$; leaf litter and pine needles

Oe $\quad 0$ to $1 \mathrm{~cm}$; partially decomposed litter

Ap $\quad 0$ to $7 \mathrm{~cm}$; dark yellowish-brown (10YR 4/4) silty clay loam; moderate fine granular structure; very friable; abrupt wavy boundary

$\mathrm{Bt} \quad 7$ to $38 \mathrm{~cm}$; red $(2.5 / \mathrm{YR} 4 / 6)$ clay; strong fine and medium angular blocky structure; firm; clear wavy boundary

BC $\quad 38$ to $53 \mathrm{~cm}$; collapsed saprolitic materials with clay texture; highly mottled yellowish-brown, yellowish-red along with 2 and 3 chroma wetness mottles; weak medium and coarse angular blocky structure; abrupt irregular boundary

$\mathrm{R} \quad 53 \mathrm{~cm}$; limestone rock with highly variable depth ranging from 53 to $95 \mathrm{~cm}$

Notes: Sampling depths: A horizon, 0 to $7 \mathrm{~cm}$; B horizon, 12 to $20 \mathrm{~cm}$; $C$ horizon, 80 to $90 \mathrm{~cm}$. The $\mathrm{BC}$ horizon that was sampled at a depth of 80 to $90 \mathrm{~cm}$ is not underlain directly by limestone as is the $\mathrm{BC}$ horizon described above. Where the $\mathrm{BC}$ horizon is thicker and deeper to rock, it has a yellowish-red (5YR 4/6) color along with Chroma 3 wetness mottles and higher chroma parent material colors. 
ORR Site 110

(See pp. 4, 25, 26, 30, and 38 in Phase II Field Book 3)

Location:

Classification:

Geomorphic position:

Slope and aspect:

Parent material(s):

Vegetation:

Described by:

Sampling crew:

Rad scan:

Weather yesterday:

Present weather:

Date:
Oak Ridge Reservation, Bethel Valley, Grid N22400, E45800

Typic Hapludalfs; fine, mixed, thermic

Elongated convex ridge

$2 \%$

Chickamauga Residuum of the Bethel Valley Section

Old field successional forest with pines, oaks and cedars. There are also beech and hickory sprouts along with weeds and honeysuckle. This site was severely eroded before abandonment. D. A. Lietzke Lietzke, Burgọa

Air, 40; ground level, 50; top of auger hole, 90

Cloudy and cold

Cloudy

November 30, 1992

\section{Soil Description:}

Oi $\quad 5$ to $0 \mathrm{~cm}$; leaves and leaf litter

A $\quad 0$ to $3 \mathrm{~cm}$; dark brown (10YR $3 / 3$ ) silty clay loam; strong medium granular structure; very friable; abrupt wavy boundary

Bt 3 to $40 \mathrm{~cm}$; yellowish-red (5YR 4/6) clay; strong fine, medium and coarse angular blocky structure; firm; dark yellowish-brown (10YR 4/4) ped coatings; gradual wavy boundary

BC $\quad 40$ to $75 \mathrm{~cm}$; brown (7.5YR 4/4) clay with yellowish-brown (10YR 5/4) drainage mottles; moderate coarse prismatic structure; gradual wavy boundary

CB $\quad 75$ to $85 \mathrm{~cm}$; light olive brown (2.5Y 5/6) and yellowish-brown (10YR 5/6) clay; strong fine angular blocky structure. There were rock ledges in several locations on all pit faces.

Notes: Sampling depths: A horizon, 0 to $3 \mathrm{~cm}$; B horizon, 3 to $20 \mathrm{~cm}$; $\mathrm{C}$ horizon, 75 to $85 \mathrm{~cm}$. 
ORR Site 115

(See pp. 11, 30, 36, and 37 in Phase II Field Book 3)

Location:

Classification:

Geomorphic position:

Slope and aspect:

Parent material(s):

Vegetation:

Described by:

Sampling crew:

Rad scan:

Weather yesterday:

Present weather:

Date:
Oak Ridge Reservation, Bethel Valley, Grid N22500, E46700

Typic Hapludalfs; fine, mixed, thermic

Convex ridgetop

$3 \%$ southeast

Unit E Chickamauga, Bethel Valley Section Residuum

Old field successional forest of Virginia pine, cedar, oaks, hickories, ash, dogwood, with a good stand of poison ivy and some honeysuckle. This site does not appear to have been plowed, but was probably a woods pasture.

D. A. Lietzke Lietzke, Farmer, Petrowski

Air, 50; ground level, 70

Cloudy to partly cloudy, less. windy but still cold

Clouding in again

December 3, 1992

\section{Soil Description:}

$\mathrm{Oi} \quad 4$ to $0 \mathrm{~cm}$; leaf litter

A $\quad 0$ to $4 \mathrm{~cm}$; dark brown (10YR 3/3) silt loam; strong fine and medium granular structure; very friable; abrupt wavy boundary

EB $\quad 4$ to $8 \mathrm{~cm}$; yellowish-brown (10YR 5/4) silt loam; moderate fine granular structure; very friable; clear wavy boundary

$\mathrm{Bt} \quad 8$ to $60 \mathrm{~cm}$; yellowish-red (5YR 4/6) clay; moderate fine subangular blocky structure; firm; brown (7.5YR 5/3) ped coatings; gradual wavy boundary

BC 60 to $75 \mathrm{~cm}$; dark yellowish-brown (10YR 4/4) clay; strong fine angular blocky structure; very firm; abrupt irregular boundary

R $75 \mathrm{~cm}$; limestone. Depth to rock in the pit ranged from 25 to $45 \mathrm{~cm}$ except on the west side where this soil was described and sampled.

Notes: Sampling depths: A horizon 0, to $8 \mathrm{~cm}$; B horizon, 10 to $30 \mathrm{~cm}$; $C$ horizon, 60 to $75 \mathrm{~cm}$. 


\section{ORR Site 116}

(See pp. 11, 31, 32, and 33 in Phase II Field Book 3)

Location:

Classification:

Geomorphic position:

Slope and aspect:

Parent material(s):

Vegetation:

Described by:

Sampling crew:

Rad scan:

Weather yesterday:

Present weather:

Date:

Soil Description:

Oi $\quad 4$ to $0 \mathrm{~cm}$; pine needles

A $\quad 0$ to $5 \mathrm{~cm}$; dark reddish-brown (5YR $3 / 2)$ silty clay loam; strong medium granular structure; friable; abrupt wavy boundary

Ap 5 to $12 \mathrm{~cm}$; dark reddish-brown (5YR 3/4) silty clay loam or silty clay; moderate fine subangular blocky structure; firm; abrupt wavy boundary

Bt 12 to $38 \mathrm{~cm}$; dark reddish-brown (2.5YR 3/4) clay; strong fine and medium prismatic parting to strong medium angular blocky structure; very firm; brown (7.5YR 4/3) ped coatings; gradual wavy boundary

BC $\quad 38$ to $70 \mathrm{~cm}$; clayey saprolitic materials in reds and yellows; moderate coarse prismatic parting to moderate coarse angular blocky structure; very firm;all ped surfaces coated with brown ( $7.5 Y R$ 4/3) clay; there are a few 2 chroma wetness mottles in old root channels; gradual irregular boundary

$\mathrm{CB} \quad 70$ to $85 \mathrm{~cm}$; saprolitic materials that easily crush to clay; colors are mixed yellows and reds. There are a few 2 chroma wetness mottled in flow pathways.

Notes: Sampling depths: A horizon, 0 to $12 \mathrm{~cm}$; B horizon, 16 to $30 \mathrm{~cm}$; $\mathrm{C}$ horizon, 70 to $85 \mathrm{~cm}$. 


\section{A-84}

\section{ORR Site 117}

(See pp. 11, 31, 34, and 35 in Phase II Field Book 3)

Location:

Classification:

Geomorphic position:

Slope and aspect:

Parent material(s):

Vegetation:

Described by:

Sampling crew:

Rad scan:

Weather yesterday:

Present weather:

Date:
Oak Ridge Reservation, Bethel Valley, Grid N22500, E49000 Typic Hapludalfs; fine, mixed, thermic Slightly convex spur, once covered by alluvium. Some rounded fragments remain in the surface layer.

$2 \%$

Unit G Chickamauga Residuum, Bethel Valley Section Loblolly Pine plantation

D. A. Lietzke Lietzke, Farmer, Petrowski

Air, 40; ground level, 70; top of auger hole, 80

Cloudy, cold, windy

Clear to partly cloudy, clouding in from the west for the next storm

December 3, 1992

\section{Soil Description:}

Oi $\quad 6$ to $0 \mathrm{~cm}$; pine needles

0a $\quad 0$ to $2 \mathrm{~cm}$; highly decomposed pine needles

A 2 to $7 \mathrm{~cm}$; dark yellowish-brown (10YR 3/4) silt loam; strong medium granular structure; very friable; clear wavy boundary

Ap $\quad 7$ to $15 \mathrm{~cm}$; brown (7.5YR 4/4) clay loam; strong medium and coarse granular structure; friable; abrupt wavy boundary

$\mathrm{Bt} \quad 15$ to $34 \mathrm{~cm}$; yellowish-red (5YR 5/8) clay; moderate coarse prismatic parting to weak medium and coarse angular blocky structure; very firm; strong brown (7.5YR $4 / 6$ ) coatings on prism faces; gradual wavy boundary

BC $\quad 34$ to $57 \mathrm{~cm}$; mottled red, yellowish-red, strong brown, and yellowish-brown clay; moderate medium prismatic parting to weak coarse angular blocky structure; very firm; most ped interiors are saprolitic; gradual wavy boundary

CB $\quad 57$ to $106 \mathrm{~cm}$; highly mottled red, yellowish-red, strong brown, yellowish-brown and olive brown clayey saprolitic materials; moderate fine and medium prismatic structure; very firm; abrupt irregular boundary

$\mathrm{R} \quad 106 \mathrm{~cm}$; limestone rock

Notes: Sampling depths: A horizon, 0 to $15 \mathrm{~cm}$; B horizon, 15 to $30 \mathrm{~cm}$; $C$ horizon, 85 to $95 \mathrm{~cm}$. 


\section{ORR Site 118}

(See pp. 19, 39, 46, 47, and 49 in Phase II Field Book 3)

Location:

Classification:

Geomorphic position:

Slope and aspect:

Parent material(s):

Vegetation:

Described by:

Sampling crew:

Rad scan:

Weather yesterday:

Present weather:

Date:
Oak Ridge Reservation, K-25 area, Grid N39800, E23000

Typic Hapludults; clayey, mixed, thermic

Doubly convex spur ridge between two drainageways

$6 \%$ west

Residuum of East Fork Chickamauga Section

Hardwood forest of large white oaks, beech, cherry, sugar maple. This forest does not appear to have been plowed or ever pastured.

D. A. Lietzke

Lietzke, Burgoa

Air, 50; ground level, 60; top of auger hole, 60

Cloudy and cold

Cloudy and cold

December 8, 1992

\section{Soil Description:}

Oi $\quad 4$ to $0 \mathrm{~cm}$; leaves

A $\quad 0$ to $5 \mathrm{~cm}$; very dark grayish-brown (10YR $3 / 2$ ) silty clay loam; strong medium and coarse granular structure; friable; abrupt wavy boundary

$\mathrm{Bt} \quad 5$ to $28 \mathrm{~cm}$; yellowish-red (5YR 5/6) clay; moderate medium and coarse prismatic parting to moderate medium subangular blocky and angular blocky structure; very firm; yellowish-red (5YR 4/6) clay films on prism surfaces; gradual wavy boundary

BC 28 to $69 \mathrm{~cm}$; yellowish-red with bright brownish-yellow saprolitic colors; clay; moderate coarse prismatic parting to moderate medium angular blocky structure; very firm; peds coated with yellowish-red (5YR 4/6) clay; gradual wavy boundary

CB 69 to $100 \mathrm{~cm}$; brownish-yellow (10YR 6/6) saprolitic materials with clay texture; streaks of yellowish-red materials throughout; weak fine angular blocky structure; very firm

Notes: Sampling depths: A horizon, 0 to $5 \mathrm{~cm}$; B horizon, 7 to $20 \mathrm{~cm}$; $\mathrm{C}$ horizon, 70 to $80 \mathrm{~cm}$. 


\section{ORR Site 119}

(See pp. 19, 39, 51, 56, and 57 in Phase II Field Book 3)

Location:

Classification:

Geomorphic position:

Slope and aspect:

Parent material(s):

Vegetation:

Described by:

Sampling crew:

Rad scan:

Weather yesterday:

Present weather:

Date:
Oak Ridge Reservation, K-25 area, Grid 40000, E23000

Typic Hapludalfs; fine, mixed, thermic

Narrow convex ridge

3\% west

Residuum of East Fork Chickamauga Section

Cutover woods. Present trees consist of cedars, some oak, ash and hardwood saplings. Site was probably never plowed but was probably pastured.

D. A. Lietzke

Lietzke, Burgoa and Farmer

Air, 50; ground level, 70; top of auger hole, 70

Mostly clear

Clear but with high cirrus overhead

January 4, 1993

\section{Soil Description:}

Oi $\quad 4$ to $0 \mathrm{~cm}$; leaf litter

A1 0 to $5 \mathrm{~cm}$; dark brown (10YR 3/3) silt loam; strong medium granular structure; very friable; clear wavy boundary

A2 5 to $16 \mathrm{~cm}$; brown (10YR 4/3) silt loam; moderate fine granular structure; very friable; clear wavy boundary

Bt $\quad 16$ to $59 \mathrm{~cm}$; yellowish-red (5YR 5/8) clay; strong fine to coarse angular blocky structure; very firm; gradual wavy boundary

BC $\quad 59$ to $90 \mathrm{~cm}$; mottled strong brown and yellowish-brown clay; strong fine angular blocky structure; very firm; several pressure faces

$\mathrm{R} \quad 90 \mathrm{~cm}$; limestone. Depth to rock varies from $30 \mathrm{~cm}$ to more than $100 \mathrm{~cm}$.

Notes: Sampling depths: A horizon, 0 to $15 \mathrm{~cm}$; B horizon, 20 to $30 \mathrm{~cm}$; $C$ horizon, 75 to $88 \mathrm{~cm}$. 
ORR Site 120

(See pp. 19, 39, 44, 45, and 48 in Phase II Field Book 3)

Location:

Classification:

Geomorphic position:

Slope and aspect:

Parent material(s):

Vegetation:

Described by:

Sampling crew:

Rad scan:

Weather yesterday:

Present weather:

Date:
Oak Ridge Reservation, K-25 area, Grid N39800, E23250

Typic Hapludults; clayey, mixed, thermic

Sideslope with slight convexity, about 15 to $20 \mathrm{ft}$ away from a recent subsidence

$4 \%$ west

Thin alluvium over residuum of East Fork Chickamauga Section Hardwoods, never pastured but cutover from time to time; large beech

D. A. Lietzke

Lietzke, Burgoa

Air, 45; ground level, 60; top of auger hole, 60

Cloudy, cold with drizzle

Cloudy

December 7, 1992

\section{Soil Description:}

$\mathrm{Oi} \quad 6$ to $0 \mathrm{~cm}$; leaves and other litter

A $\quad 0$ to $10 \mathrm{~cm}$; dark brown (7.5YR 3/4) loam; strong fine and medium granular structure; very friable; clear wavy boundary

$\mathrm{AB} \quad 10$ to $17 \mathrm{~cm}$; reddish-brown (5YR 4/4) loam; moderate fine subangular blocky structure; very friable; clear wavy boundary

Bt1 17 to $27 \mathrm{~cm}$; red.(2.5YR 4/6) clay loam; moderate fine subangular blocky structure; friable; few sandstone pebbles and gravels; abrupt wavy boundary

2Bt2 27 to $74 \mathrm{~cm}$; red (2.5YR 4/6) clay; strong fine and medium subangular blocky structure; friable to firm; few chert fragments; gradual wavy boundary

2BC $\quad 74$ to $80 \mathrm{~cm}$; red (2.5YR 4/8) clay; strong fine angular blocky structure; friable; has some yellow, high chroma saprolite colors; gradual wavy boundary

2CB 80 to $100 \mathrm{~cm}$; red, yellowish-red and brownish-yellow clayey saprolitic materials with dull yellowish-brown wetness mottles; moderate medium angular blocky structure; very firm

Notes: Sampling depths: A horizon, 0 to $10 \mathrm{~cm}$; B horizon, 26 to $40 \mathrm{~cm}$; $C$ horizon, 85 to $100 \mathrm{~cm}$. 
ORR Site 121

(See pp. 20, 39, 51, 58, and 59 in Phase II Field Book 3)

Location:

Classification:

Geomorphic position:

Slope and aspect:

Parent material(s):

Vegetation:

Described by:

Sampling crew:

Rad scan:

Weather yesterday:

Present weather:

Date:
Oak Ridge Reservation, K-25 area, Grid N42100, E26200

Typic Hapludults or Ultic Hapludalfs: clayey, mixed, thermic

Shoulder of broad, slightly convex ridgetop

5\% northwest

Residuum of East Fork Chickamauga Section

Old field successional forest of Virginia pine and an understory of gum, hickory, red maple and beech

D. A. Lietzke

Lietzke, Burgoa and Farmer

Air, 45; ground level, 55; top of auger hole, 55

Cloudy

Mostly clear, but with high thin cirrus overhead

January 4, 1993

\section{Soil Description:}

Oi $\quad 4$ to $0 \mathrm{~cm}$; leaves and needles along with some mosses

A $\quad 0$ to $3 \mathrm{~cm}$; very dark brown (10YR 2/2) silt loam; strong medium granular structure; very friable; abrupt wavy boundary

Ap $\quad 3$ to $20 \mathrm{~cm}$; brown (10YR 4/3) silty clay loam; moderate fine granular structure; friable; abrupt wavy boundary

Bt 20 to $30 \mathrm{~cm}$; brown (7.5YR 4/3-4/4) clay; moderate medium angular blocky structure; very firm; abrupt wavy boundary

$\mathrm{BC} 130$ to $51 \mathrm{~cm}$; dark reddish-gray (5YR 4/2) clay; strong fine angular blocky structure; has red iron oxide streaks and 2 chroma wetness mottles and coatings on some ped surfaces; abrupt wavy boundary

$\mathrm{BC} 251$ to $70 \mathrm{~cm}$; highly mottled yellowish-red, olive yellow and gray clay; strong fine angular blocky structure; very firm; abrupt wavy boundary

CB 70 to $100 \mathrm{~cm}$; reddish-gray (10R 5/1) saprolitic material; very soft and crushes easily to clay; There are red iron oxide streaks throughout that are on cracks and along flow zones; massive.

Notes: Sampling depths: A horizon, 0 to $20 \mathrm{~cm}$; B horizon, 23 to $30 \mathrm{~cm}$; $\mathrm{C}$ horizon, 70 to $90 \mathrm{~cm}$. 
ORR Site 122

(See pp. 27, 39, 49, 66, and 67 in Phase II Field Book 3)

Location:

Classification:

Geomorphic position:

Slope and aspect:

Parent material(s):

Vegetation:

Described by:

Sampling crew:

Rad scan:

Weather yesterday:

Present weather:

Date:
Oak Ridge Reservation, K-25 area, Grid N41300, E27250

Typic Hapludults or Ochreptic Hapludults; clayey, mixed, thermic Elongated, convex, narrow ridgetop

$6 \%$ northwest

Residuum of East Fork Chickamauga Section (Pond Springs Siltstone)

Loblolly pine plantation. There are abundant honeysuckle vines and briars.

D. A. Lietzke

Lietzke, Burgoa and Farmer

Air, 40; ground level, 50; top of auger hole, 50

Mostly clear

Was clear earlier but now completely cloudy

January 4, 1993

\section{Soil Description:}

Oi $\quad 3$ to $0 \mathrm{~cm}$; pine needles and debris

Ap $\quad 0$ to $10 \mathrm{~cm}$; brown (10YR 4/3) cherty silty clay loam; moderate fine granular structure; friable; abrupt wavy boundary

Bt 10 to $21 \mathrm{~cm}$; strong brown (7.5YR 5/6) clay; strong fine and medium subangular blocky structure; very firm; gradual wavy boundary. There is a layer of lag chert gravel about $2 \mathrm{~cm}$ thick at the top of this horizon.

BC 21 to $56 \mathrm{~cm}$; brownish-yellow (10YR 6/6) silty clay saprolitic materials; moderate coarse prismatic parting to strong medium angular blocky structure, but most ped interiors are saprolitic; very firm. There are 2 chroma wetness mottles at a depth of $30 \mathrm{~cm}$; prism faces are coated with brown (7.5YR 4/4) clay; gradual wavy boundary.

CB 56 to $80 \mathrm{~cm}$; olive yellow $(2.5 \mathrm{Y} 6 / 6)$ saprolite with silty clay texture; fracture faces coated with red iron oxides and yellowish-red clay

Notes: Sampling depths: A horizon, 0 to $12 \mathrm{~cm}$; B horizon, 15 to $30 \mathrm{~cm}$; $\mathrm{C}$ horizon, 70 to $80 \mathrm{~cm}$. 
ORR Site 123

(See pp. 27, 39, 51, 54, and 55 in Phase II Field Book 3)

Location:

Classification:

Geomorphic position:

Slope and aspect:

Parent material(s):

Vegetation:

Described by:

Sampling crew:

Rad scan:

Weather yesterday:

Present weather:

Date:
Oak Ridge Reservation, K-25 area, Grid 41200, E29750

Typic Hapludults; clayey, mixed, thermic

Convex sideslope below prominent cherty ridge

$7 \%$ west

Residuum of East Fork Chickamauga Section

Loblolly pine plantation. There is very little undergrowth except for young pines, honeysuckle and greenbriar.

D. A. Lietzke

Lietzke, Burgoa

Air, 45; ground level, 55; top of auger hole, 70

Partly cloudy

Cloudy

January 4, 1993

\section{Soil Description:}

$\mathrm{Oi} \quad 5$ to $0 \mathrm{~cm}$; pine needles

A $\quad 0$ to $3 \mathrm{~cm}$; dark brown (10YR 3/3) silty clay loam; moderate coarse granular structure; friable; clear wavy boundary

Ap 3 to $16 \mathrm{~cm}$; brown (7.5YR 4/4) clay; weak medium subangular blocky structure; firm; abrupt wavy boundary

Bt 16 to $42 \mathrm{~cm}$; red (2.5YR 4/6) clay; with a few fine yellowish-brown (10YR 5/6) mottles; moderate medium subangular blocky structure; very firm; gradual wavy boundary

$\mathrm{BC} \quad 42$ to $90 \mathrm{~cm}$; highly mottled red and yellowish-brown saprolitic materials; clay; strong fine angular blocky structure; very firm

Notes: Sampling depths: A horizon, 0 to $5 \mathrm{~cm}$; B horizon, 8 to $20 \mathrm{~cm}$; $\mathrm{C}$ horizon, 80 to $90 \mathrm{~cm}$. 


\section{ORR Site 124}

(See pp. 27, 39, 50, 64, and 65 in Phase II Field Book 3)

Location:

Classification:

Geomorphic position:

Slope and aspect:

Parent material(s):

Vegetation:

Described by:

Sampling crew:

Rad scan:

Weather yesterday:

Present weather:

Date:
Oak Ridge Reservation, K-25 area, Grid N40900, E30400

Typic Hapludalfs; fine, mixed, thermic

Broad, slightly convex landform. The site is located about $100 \mathrm{ft}$ west of a bedrock escarpment to the creek and at the edge of a loblolly pine plantation.

$2 \%$

Residuum of East Fork Chickamauga Section

Loblolly pine plantation. About $30 \mathrm{ft}$ in from the east edge in an old field.

D. A. Lietzke

Lietzke, Burgoa and Farmer

Air, 40; ground level, 45; top of auger hole, 50

Partly cloudy

Cloudy

January 4, 1993

\section{Soil Description:}

Oi $\quad 5$ to $0 \mathrm{~cm}$; needles, some leaves and many poison ivy and honeysuckle stems

A 0 to $7 \mathrm{~cm}$; dark brown (10YR 3/3) cherty silt loam; strong coarse granular structure; very friable; clear wavy boundary

Ap 7 to $18 \mathrm{~cm}$; brown (10YR 4/3) cherty silty clay loam; moderate medium subangular blocky structure; friable; clear wavy boundary

$\mathrm{Bt} 18$ to $64 \mathrm{~cm}$; yellowish-red (5YR 4/6) clay; moderate coarse prismatic parting to moderate medium angular blocky structure; very firm; strong brown (7.5YR 5/4) clay flows on prism faces; gradual wavy boundary

BC 64 to $90 \mathrm{~cm}$; red (2.5YR 4/6) clay; strong fine angular blocky structure; very firm; yellowish-brown flow streaks; abrupt irregular boundary

$\mathrm{R} 990 \mathrm{~cm}$; limestone. Depth to rock in this pit varied from near the surface on one end to more than $100 \mathrm{~cm}$ on the side that was described and sampled.

Notes: Considerable charcoal and partially burned wood fragments were on the surface and in the A horizon. Sampling depths: A horizon, 0 to $10 \mathrm{~cm}$; B horizon, 15 to $30 \mathrm{~cm}$; C horizon, 80 to $90 \mathrm{~cm}$. 
ORR Site 125

(See pp. 28, 39, 51, 60, and 61 in Phase II Field Book 3)

Location:

Classification:

Geomorphic position:

Slope and aspect:

Parent material(s):

Vegetation:

Described by:

Sampling crew:

Rad scan:

Weather yesterday:

Present weather:

Date:

\section{Soil Description:}

$\mathrm{Oi} \quad 4$ to $0 \mathrm{~cm}$; pine needles

Oe $\quad 0$ to $2 \mathrm{~cm}$; partially decomposed organic materials and a dense root mat

A $\quad 2$ to $4 \mathrm{~cm}$; dark brown (10YR $3 / 3$ ) cherty silty clay loam; moderate medium granular structure; friable; abrupt wavy boundary

Ap $\quad 4$ to $12 \mathrm{~cm}$; brown (7.5YR 4/4) cherty silty clay loam; weak fine subangular blocky structure; friable; abrupt wavy boundary

$\mathrm{Bt} \quad 12$ to $48 \mathrm{~cm}$; red (2.5YR 5/8) clay; moderate very coarse prismatic parting to strong medium and coarse subangular blocky structure; very firm; gradual wavy boundary

BC $\quad 48$ to $90 \mathrm{~cm}$; yellowish-red (5YR 5/6) and brownish-yellow (10YR 6/8) clayey saprolitic materials; strong fine angular blocky structure; very firm; clay plugged

Notes: Sampling depths: A horizon, 0 to $6 \mathrm{~cm}$; B horizon, 20 to $40 \mathrm{~cm}$; $C$ horizon, 70 to $90 \mathrm{~cm}$. 
ORR Site 126

(See pp. 28, 39, 42, 43, and 48 in Phase II Field Book 3)

Location:

Classification:

Geomorphic position:

Slope and aspect:

Parent material(s):

Vegetation:

Described by: .

Sampling crew:

Rad scan:

Weather yesterday:

Present weather:

Date:
Oak Ridge Reservation, K-25 area, Grid N38950, E28750

Typic Hapludults; clayey, mixed, thermic

Convex top of spur ridge

$5 \%$ north

Residuum of East Fork Chickamauga Section

Old field successional forest. Presently a mix of pines and hardwoods along with a poison ivy, honeysuckle and moss ground cover.

D. A. Lietzke

Lietzke, Burgoa

Air, 40; ground level, 50; top of auger hole, 50

Cloudy, cold, drizzle

Cloudy and becoming colder

December 7, 1992

\section{Soil Description:}

Oi $\quad 3$ to $0 \mathrm{~cm}$; leaves, pine needles and debris

A $\quad 0$ to $5 \mathrm{~cm}$; brown (7.5YR 4/4) silty clay loam; moderate fine granular structure; friable; clear wavy boundary

Ap 5 to $12 \mathrm{~cm}$; strong brown (7.5YR 4/6) silty clay loam; moderate medium and coarse granular structure; friable; clear wavy boundary

$\mathrm{Bt} \quad 12$ to $30 \mathrm{~cm}$; strong brown (7.5YR 5/6) clay; strong fine subangular blocky structure; firm; common Rockwood sandstone fragments; gradual wavy boundary

BC $\quad 30$ to $70 \mathrm{~cm}$; mottles red, yellowish-red, strong brown, and yellow saprolitic materials that easily crush to clay; strong fine angular blocky structure, but most ped interiors are saprolite; firm; contains Rockwood sandstone fragments; gradual wavy boundary.

$\mathrm{CB} \quad 70$ to $100 \mathrm{~cm}$; strike and dip yellowish-brown, yellow, strong brown, and yellowishred saprolitic materials

Notes: This pit is in a brecciated zone in the Chickamauga which may explain the Rockwood sandstone fragments. Sampling depths: A horizon, 0 to $11 \mathrm{~cm}$; B horizon, 20 to $30 \mathrm{~cm}$; C horizon, 80 to $90 \mathrm{~cm}$. 
ORR Site 127

(See pp. 28, 39, 51, 52, and 53 in Phase II Field Book 3)

Location:

Classification:

Geomorphic position:

Slope and aspect:

Parent material(s):

Vegetation:

Described by:

Sampling crew:

Rad scan:

Weather yesterday:

Present weather:

Date:
Oak Ridge Reservation, K-25 area, Grid N39500, E28650

Typic Hapludults; clayey, mixed, thermic

Convex ridgetop

$2 \%$, nearly level

Residuum of East Fork Chickamauga Section

Old field successional forest of Virginia pines, cedar, ash, poplar along with abundant poison ivy and honeysuckle

D. A. Lietzke

Lietzke, Burgoa

Air, 40; ground level, 65; top of auger hole, 65

Cloudy

Partly cloudy but there was a shower earlier.

January 7, 1993

\section{Soil Description:}

$\mathrm{Oi} \quad 5$ to $0 \mathrm{~cm}$; leaves, needles and many poison ivy and honeysuckle stems

A $\quad 0$ to $5 \mathrm{~cm}$; dark brown (10YR 3/3) silt loam; strong coarse granular structure; very friable; abrupt wavy boundary

Ap 5 to $14 \mathrm{~cm}$; dark yellowish-brown (10YR 4/6) clay; moderate fine subangular blocky structure; firm; abrupt wavy boundary

$\mathrm{Bt} 14$ to $28 \mathrm{~cm}$; yellowish-brown (10YR 5/6) clay; strong fine and medium angular blocky structure; very firm; gradual wavy boundary

BC 28 to $75 \mathrm{~cm}$; saprolitic materials with a clay texture, yellowish-brown (10YR 5/4-5/6) color; enriched black manganese oxide zones show the geologic strike and dip; red (2.5YR 4/8) iron oxide zones on some joint and fracture faces

Notes: Sampling depths: A horizon, 0 to $7 \mathrm{~cm}$; B horizon, 12 to $20 \mathrm{~cm}$; $\mathrm{C}$ horizon, 65 to $75 \mathrm{~cm}$. 
ORR Site 128

(See pp. 29, 39, 51, 62, and 63 in Phase II Field Book 3)

Location:

Classification:

Geomorphic position:

Slope and aspect:

Parent material(s):

Vegetation:

Described by:

Sampling crew:

Rad scan:

Weather yesterday:

Present weather:

Date:
Oak Ridge Reservation, K-25 area, Grid N38900, E29600

Typic Hapludults; clayey, mixed, thermic

Interfluve between the heads of two drainageways. The area is very eroded and gullied, but the site is only moderately eroded.

4\% northwest

Residuum of East Fork Chickamauga Section

Old Field successional forest. Present trees are Virginia pine, cedar, along with some dogwood and red maple. There is abundant poison ivy and honeysuckle.

D. A. Lietzke

Lietzke, Farmer

Air, 40; ground level, 60; top of auger hole, 65

Cloudy

Mostly cloudy

January 7, 1993

\section{Soil Description:}

Oi $\quad 2$ to $0 \mathrm{~cm}$; needles and leaves

A $\quad 0$ to $5 \mathrm{~cm}$; dark brown (10YR 3/3) silt loam; strong medium granular structure; very friable; abrupt wavy boundary

Ap 5 to $17 \mathrm{~cm}$; brown (7.5YR 4/4) silty clay loam with some chert fragments; moderate fine subangular blocky structure; friable; abrupt wavy boundary

$\mathrm{Bt} \quad 17$ to $43 \mathrm{~cm}$; yellowish-red (5YR 5/6) clay; moderate medium prismatic parting to strong medium angular blocky structure; Very firm; prism faces coated with dark yellowish-brown (10YR 4/4) clay; gradual wavy boundary

$\mathrm{BC} \quad 43$ to $90 \mathrm{~cm}$; mottled yellowish-red and yellowish-brown saprolitic materials with a clay texture; There are some 2 chroma wetness mottles and also some black manganese oxide enriched zones in the lower part. There are some ped faces in the upper part and there are some chert fragments throughout.

Notes: Sampling depths: A horizon, 0 to $21 \mathrm{~cm}$; B horizon, 23 to $35 \mathrm{~cm}$; $C$ horizon, 70 to $90 \mathrm{~cm}$. 
ORR Site 129

(See pp. 29, 39, 40, 41, and 48 in Phase II Field Book 3)

Location:

Classification:

Geomorphic position:

Slope and aspect:

Parent material(s):

Vegetation:

Described by:

Sampling crew:

Rad scan:

Weather yesterday:

Present weather:

Date:
Oak Ridge Reservation, K-25 area, Grid N36100, E26300

Typic Hapludults; clayey, mixed, thermic

Doubly convex sideslope

$11 \%$ northwest

Residuum of East Fork Chickamauga Section

Old field successional forest. This site was probably a pasture. Present vegetation consists of a few large hardwoods. White pine is growing and reproducing. There are a few holly, red maple, and oak sprouts.

D. A. Lietzke

Lietzke, Burgoa

Air, 30; ground level, 45; top of auger hole, 60

Cloudy and cold

Cloudy to partly cloudy and slightly warmer

December 7, 1992

\section{Soil Description:}

Oi $\quad 5$ to $0 \mathrm{~cm}$; leaf litter

A $\quad 0$ to $5 \mathrm{~cm}$; brown (10YR 4/3) loam; strong fine granular structure; very friable; has a few Rome pebbles and gravels and a higher sand content; clear wavy boundary

Ap $\quad 5$ to $13 \mathrm{~cm}$; dark yellowish-brown (10YR 4/4) loam; moderate fine granular structure; very friable; abrupt wavy boundary

Bt 13 to $47 \mathrm{~cm}$; yellowish-red (5YR 4/6) clay; strong fine and medium subangular blocky structure; friable; gradual wavy boundary

BC $\quad 47$ to $80 \mathrm{~cm}$; mottled yellowish-red, red, strong brown and yellowish-brown clay; strong fine and medium angular blocky structure; friable; has some saprolitic character; gradual wavy boundary

CB $\quad 80$ to $120 \mathrm{~cm}$; mottled strong brown, yellowish-brown and brownish-yellow saprolitic materials with a clayey texture; weak fine and medium angular blocky structure; very firm

Notes: Sampling depths: A horizon, 0 to $13 \mathrm{~cm}$; B horizon, 15 to $30 \mathrm{~cm}$; C horizon, 100 to $116 \mathrm{~cm}$. 


\section{AS ROANE COUNTY SOIL PROFLLES}

\section{ROANE Site 3}

Location:

Classification:

Control Section:

Geomorphic position:

Slope and aspect:

Parent material(s):

Vegetation:

Described by:

Sampling crew:

Time Since Last Rain:

Present weather:

Date:
Pattie Gap Quadrangle, $550 \mathrm{ft}$ west from Salem Baptist Church, $200 \mathrm{ft}$ southeast from power line, $350 \mathrm{ft}$ west of Stamp Creek Typic Hapludults; fine-loamy, mixed, thermic

31 to $81 \mathrm{~cm}$ of the argillic horizon

Convex toeslope position

$11 \%$ southeast

Thin colluvial mantle over Dismal Gap Formation

Oak, red maple, sweetgum, dogwood, poison ivy

J. L. Branson

Branson, Timpson, Morris

0 days

$75^{\circ} \mathrm{F}$ raining during sampling

May 7, 1992

\section{Soil Description:}

Oi $\quad 4$ to $0 \mathrm{~cm}$; leaves and pine needles

Ap $\quad 0$ to $15 \mathrm{~cm}$; dark yellowish-brown (10YR 4/4) loam; moderate medium granular structure; friable; clear wavy boundary

BE 15 to $31 \mathrm{~cm}$; strong brown (7.5YR 5/6) and dark yellowish-brown (10YR 4/4) silt loam; moderate medium subangular blocky structure; friable; clear wavy boundary

Bt $\quad 131$ to $53 \mathrm{~cm}$; yellowish-brown (10YR 5/6) clay loam; weak coarse subangular blocky structure; firm; gradual wavy boundary

$\mathrm{Bt} 253$ to $85 \mathrm{~cm}$; mottled yellowish-brown (10YR 5/6), red (2.5YR 4/6), and light yellowish-brown (2.5Y 6/4) clay loam; moderate medium subangular blocky structure; firm; gradual smooth boundary

C $85+\mathrm{cm}$; mottled yellowish-brown (10YR 5/6), dark red (2.5YR 3/6), and light brownish-gray (2.5Y 6/2) loam; rock controlled structure; firm

Notes: This site is on a footslope in an area that is highly dissected. The area around the site has numerous rills and gullies. This area shows no evidence of having been cleared or farmed within the last $50 \mathrm{y}$. The area $300 \mathrm{ft}$ east of this site has been clearcut within the last 60 days. 


\section{ROANE Site 7}

Location:

Classification:

Control Section:

Geomorphic position:

Slope and aspect:

Parent material(s):

Vegetation:

Described by:

Sampling crew:

Time Since Last Rain:

Present weather:

Date:
Pattie Gap Quadrangle, Hurricane Ridge; $1550 \mathrm{ft}$ southwest from the intersection of Blue Springs Road and Dry Fork Valley Road, $750 \mathrm{ft}$ west of the intersection of the power lines and Dry Fork Valley Road.

Typic Hapludults; fine-loamy, mixed, thermic

16 to $56 \mathrm{~cm}$ of the argillic horizon

Upland

$4 \%$ south

Colluvium over Dismal Gap Formation

Virginia pine, red maple, dogwood, tulip poplar

J. L. Branson

Branson, Livingston

1 day

$65^{\circ} \mathrm{F}$ cloudy, sprinkled during sampling

May 6, 1992

\section{Soil Description:}

A 0 to $6 \mathrm{~cm}$; very dark grayish-brown (10YR 3/2) silt loam; moderate fine granular structure; friable; abrupt smooth boundary

Ap 6 to $16 \mathrm{~cm}$; yellowish-brown (10YR 5/4) silt loam; weak fine subangular blocky structure; friable; clear smooth boundary

$\mathrm{Bt} \quad 116$ to $44 \mathrm{~cm}$; yellowish-brown (10YR 5/6) silty clay loam; moderate medium subangular blocky structure; friable; clear smooth boundary

2Bt2 44 to $56 \mathrm{~cm}$; mottled red (2.5YR 4/6), strong brown (7.5YR 4/6), and light brownish-gray (2.5Y 6/2) clay; weak medium subangular blocky structure; firm; gradual smooth boundary

2C 56 to $93 \mathrm{~cm}$; mottled: red (2.5YR 4/6), strong brown (7.5YR 5/6), and light brownish-gray ( $2.5 \mathrm{Y} 6 / 2$ ) clay; rock controlled structure; firm; clear wavy boundary

$2 \mathrm{Cr} \quad 93+\mathrm{cm}$; soft red shale fragments with some olive and yellow shale

Notes: This site is in a group of deciduous trees surrounded by cattle pasture. Indications are that area that had been cleared at one time and plowed. This area has not been cleared or cropped in the last $50 \mathrm{y}$. There is evidence of erosion with small rills beginning to form and shallow surface horizons. Presently the land is open to cattle. 


\section{ROANE Site 8}

Location:

Classification:

Control Section:

Geomorphic position:

Slope and aspect:

Parent material(s):

Vegetation:

Described by:

Sampling crew:

Time Since Last Rain:

Present weather.

Date:
Pattie Gap Quadrangle, Hurricane Ridge; $1800 \mathrm{ft}$ southwest from the intersection of Blue Springs Road and Dry Fork Valley Road, $550 \mathrm{ft}$ west of the intersection of the power lines and Dry Fork Valley Road, $300 \mathrm{ft}$ east of Roane Site 7

Typic Hapludults; fine-loamy, mixed, thermic

17 to $67 \mathrm{~cm}$ of the argillic horizon

Upland

$6 \%$ south

Colluvium over Dismal Gap Formation

Virginia pine, red maple, dogwood, sweetgum

J. L. Branson

Branson, Livingston

1 day

$50^{\circ} \mathrm{F}$ cloudy, overcast

May 6, 1992

\section{Soil Description:}

A 0 to $5 \mathrm{~cm}$; very dark grayish-brown (10YR 3/2) silt loam; strong fine granular structure; very friable; abrupt smooth boundary

Ap 5 to $17 \mathrm{~cm}$; yellowish-brown (10YR 5/6) silt loam; moderate fine granular structure; very friable; clear smooth boundary

Bt1 17 to $44 \mathrm{~cm}$; strong brown (7.5YR 5/6) silty clay loam; moderate medium subangular blocky structure; friable; gradual smooth boundary

2Bt2 44 to $80 \mathrm{~cm}$; strong brown (7.5YR 5/6) clay; moderate medium angular blocky structure; firm; gradual smooth boundary

2C 80 to $95 \mathrm{~cm}$; mottled red (2.5YR 4/6), yellowish-brown (5YR 4/6), and light reddish-brown (2.5YR 6/4) clay; rock controlled structure; very firm consistency

Notes: This site is in a group of deciduous trees surrounded by cattle pasture. Indications are that area that had been cleared at one time and plowed. This area has not been cleared or cropped in the last $50 \mathrm{y}$. There is evidence of erosion with small rills beginning to form and shallow surface horizons. Presently the land is open to cattle. 


\section{ROANE Site 9}

Location:

Classification:

Control Section:

Geomorphic position:

Slope and aspect:

Parent material(s):

Vegetation:

Described by:

Sampling crew:

Time Since Last Rain:

Present weather:

Date:
Pattie Gap Quadrangle, Hurricane Ridge; $500 \mathrm{ft}$ west from Salem Baptist Church, $200 \mathrm{ft}$ southeast from power line, $500 \mathrm{ft}$ southwest of Stamp Creek

Ochreptic Hapludults; fine-loamy, mixed, thermic, shallow.

30 to $52 \mathrm{~cm}$ of the argillic horizon

Colluvial footslope position

$12 \%$ southeast

Dismal Gap Formation

Oak hardwoods mixed with Virginia pine

J. L. Branson

Branson, Timpson

3 days

$76^{\circ} \mathrm{F}$ sunny

April 14, 1992

Soil Description:

Oi $\quad 2$ to $0 \mathrm{~cm}$; leaves and twigs

A $\quad 0$ to $9 \mathrm{~cm}$; dark grayish-brown (10YR 4/2) silt loam or loam; moderate medium subangular blocky structure; very friable; clear wavy boundary

E 9 to $16 \mathrm{~cm}$; brown (10YR 5/3) silt loam or loam; moderate medium subangular blocky structure; friable; clear wavy boundary

$\mathrm{BE} \quad 16$ to $30 \mathrm{~cm}$; yellowish-brown (10YR 5/6) silt loam; weak medium subangular blocky structure; friable; clear wavy boundary

Bt $\quad 30$ to $52 \mathrm{~cm}$; yellowish-red (5YR 5/6) silty clay loam; weak medium subangular blocky structure; friable consistency; clear wavy boundary

$\mathrm{Cr} \quad 52+\mathrm{cm}$; soft red shale with some olive and yellowish-brown strata

Notes: This site is on a footslope in an area that is highly dissected. The area around the site has numerous rills and gullies. This area shows no evidence of having been cleared or farmed within the last 50 years. 


\section{ROANE Site 10}

Location:

Classification:

Control Section:

Geomorphic position:

Slope and aspect:

Parent material(s):

Vegetation:

Described by:

Sampling crew:

Time Since Last Rain:

Present weather:

Date:
Pattie Gap Quadrangle, Hurricane Ridge; $300 \mathrm{ft}$ northeast from Gage Road, $350 \mathrm{ft}$ northwest from Dry Fork Valley Road, $100 \mathrm{ft}$ west of Stamp Creek

Ochreptic Hapludults; fine-loamy, mixed, thermic, shallow

18 to $28 \mathrm{~cm}$ of the argillic horizon

Upland bench

$3 \%$ southeast

Thin alluvial capping over Dismal Gap Formation shortleaf pine, red maple, oak

J. L. Branson

Branson, Gallagher, Morris

2 days

$85^{\circ} \mathrm{F}$ sunny

May 15, 1992

\section{Soil Description:}

O $\quad$ i7 to $5 \mathrm{~cm}$; leaf litter

Oa $\quad 5$ to $0 \mathrm{~cm}$; decomposed plant material

Ap $\quad 0$ to $18 \mathrm{~cm}$; dark yellowish-brown (10YR 4/4) silt loam; weak medium granular structure; friable; clear irregular boundary

2Bt 18 to $28 \mathrm{~cm}$; yellowish-brown (10YR 5/4) shaly clay loam; moderate fine subangular blocky structure; firm; clear smooth boundary

2BC 28 to $40 \mathrm{~cm}$; yellowish-brown (10YR 5/4) shaly clay; moderate medium angular blocky structure; firm; clear smooth boundary

2CB 40 to $62 \mathrm{~cm}$; strong brown (7.5YR 4/6) very shaly clay; moderate medium angular blocky structure; firm; clear smooth boundary

2Cr1 62 to $83 \mathrm{~cm}$; soft red shale with yellow and olive shale fragments

$2 \mathrm{Cr} 283$ to $108+\mathrm{cm}$; soft green shale with a thick band of manganese stained material and concretions

Notes: This site is in a group of deciduous trees surrounded by cattle pasture. Indications were the area had been cleared at one time and plowed, but not within the last 50 years. The site area is at the bottom of a long slope. This looks to be a terrace remanent. There were rounded rock fragments in the profile and a large rock approximately $20 \mathrm{~cm}$ in diameter. There is evidence of erosion with small rills and gullies forming in the sagegrass field to the north of the site. Presently the land is open to cattle. 


\section{ROANE Site 13}

Location:

Classification:

Control Section:

Geomorphic position:

Slope and aspect:

Parent material(s):

Vegetation:

Described by:

Sampling crew:

Time Since Last Rain:

Present weather.

Date:
Pattie Gap Quadrangle, Hurricane Ridge, $500 \mathrm{ft}$ west from Dry Fork Valley Road, $3000 \mathrm{ft}$ south from Gage Road, $250 \mathrm{ft}$ west of Stamp Creek

Typic Hapludults; fine-loamy, mixed, thermic

13 to $63 \mathrm{~cm}$ of the argillic horizon

Upland bench

$3 \%$ east

Dismal Gap Formation

Virginia pine, red maple, chestnut oak, dogwood, poison ivy

J. L. Branson

Branson, Gallagher, Timpson

1 day

$75^{\circ} \mathrm{F}$ and sunny

May 14, 1992

\section{Soil Description:}

A $\quad 0$ to $2.5 \mathrm{~cm}$; very dark brown (10YR 2/2) loam; strong fine granular structure; friable; abrupt smooth boundary

Ap 2.5 to $13 \mathrm{~cm}$; yellowish-brown (10YR 5/4) and brown (10YR 5/3) silt loam; weak fine and medium granular structure; friable; clear wavy boundary

Bt1 13 to $50 \mathrm{~cm}$; strong brown (7.5YR 4/6) on clay skins and strong brown ped interiors (7.5YR 5/6); clay; moderate fine and medium subangular blocky structure; firm; clear wavy boundary

Bt2 50 to $78 \mathrm{~cm}$; strong brown (7.5YR 4/6) clay; weak medium and fine subangular blocky structure; firm; clear wavy boundary

C 78 to $93+\mathrm{cm}$; strong brown (7.5YR 4/6) clay; rock controlled structure; firm

Notes: This site is in a wooded area. The site area is at the bottom of a long slope. Because it is in a low position, the possibility of this being an area of terrace smears is likely. However, there were no indications in this profile to substantiate this point. 


\section{ROANE Site 14}

Location:

Classification:

Control Section:

Geomorphic position:

Slope and aspect:

Parent material(s):

Vegetation:

Described by:

Sampling crew:

Time Since Last Rain:

Present weather:

Date:
Pattie Gap Quadrangle, Hurricane Ridge, $600 \mathrm{ft}$ west from Dry Fork Valley Road, $3200 \mathrm{ft}$ south of Gage Road, $250 \mathrm{ft}$ west of Stamp Creek

Typic Hapludults; fine-loamy, mixed, thermic 19 to $58 \mathrm{~cm}$ of the argillic horizon

Upland on a convex sideslope

$13 \%$ east

Dismal Gap Formation and Rogersville Formation transition zone red maple, oak, tulip poplar, dogwood, poison ivy

J. L. Branson

Branson, Gallagher, Timpson

1 day

$80^{\circ} \mathrm{F}$ and sunny

May 14, 1992

\section{Soil Description:}

A $\quad 0$ to $8 \mathrm{~cm}$; very dark brown (10YR 2/2) silt; moderate, medium granular structure; very friable; clear smooth boundary

Ap 8 to $19 \mathrm{~cm}$; brown (10YR 4/3) loam; moderate medium granular structure; friable; clear wavy boundary

Bt 19 to $41 \mathrm{~cm}$; strong brown (7.5YR 4/6) silty clay loam; weak medium subangular blocky structure; firm; clear wavy boundary

Bt\&C 41 to $58 \mathrm{~cm}$; mottled red (2.5YR 4/6), grayish-brown (10YR 5/2), and olive brown (2.5Y 4/4); channery silty clay; moderate medium subangular blocky structure; firm; gradual wavy boundary

$\mathrm{Cr} \quad 58$ to $104+\mathrm{cm}$; primarily dark red and olive shale

Notes: This site is in a wooded area. The site area is in the middle of a short slope. The Dismal Gap is very thin and fluctuates in this area. The site showed some indications of having Dismal Gap residuum, but when the pit was opened there was more Rogersville shale than Dismal Gap. This area has a great deal of groundcover, primarily vines and poison ivy. The area from the site drops immediately into a drainageway. 


\section{ROANE Site 17}

Location:

Classification:

Control Section:

Geomorphic position:

Slope and aspect:

Parent material(s):

Vegetation:

Described by:

Sampling crew:

Time Since Last Rain:

Present weather:

Date:
Pattie Gap Quadrangle, Hurricane Ridge, $1000 \mathrm{ft}$ west from Dry Fork Valley Road, $3000 \mathrm{ft}$ north from Waterford Lane Typic Hapludults; clayey, kaolinitic, thermic 28 to $78 \mathrm{~cm}$ of the argillic horizon

Upland on the crown of a convex slope

$2 \%$ east

Dismal Gap Formation

Virginia pine, eastern red cedar, sweetgum

J. L. Branson

Branson, Timpson

1 day

$65^{\circ} \mathrm{F}$ cloudy, light rain

April 20, 1992

\section{Soil Description:}

Oi $\quad 2$ to $0 \mathrm{~cm}$; pine needles, leaves, and twigs

A $\quad 0$ to $15 \mathrm{~cm}$; yellowish-brown (10YR 5/4) silt loam; weak fine granular structure; friable; clear smooth boundary

$\mathrm{AB} \quad 15$ to $28 \mathrm{~cm}$; dark yellowish-brown (10YR 4/6) silt loam; moderate fine subangular blocky structure; friable; clear smooth boundary

Bt1 28 to $60 \mathrm{~cm}$; yellowish-red (5YR 4/6) clay; moderate medium subangular blocky structure; friable; gradual smooth boundary

Bt2 60 to $80 \mathrm{~cm}$; yellowish-red (5YR 4/6) clay; moderate medium subangular blocky structure; friable; clear smooth boundary

C 80 to $104+\mathrm{cm}$; mottled yellowish-red (5YR 4/6), strong brown (7.5YR 4/6), and light olive brown (2.5Y 5/4) clay loam; rock controlled structure; friable

Notes: This site lies between the convergence of two drains, both of which continually carry at least some flowing water. The site is on a convex slope in an area that had been cleared at one time. Presently the land is open to cattle. 


\section{ROANE Site 19}

Location:

Classification:

Control Section:

Geomorphic position:

Slope and aspect:

Parent material(s):

Vegetation:

Described by:

Sampling crew:

Time Since Last Rain:

Present weather:

Date:
Pattie Gap Quadrangle, Hurricane Ridge; $750 \mathrm{ft}$ west from Salem Baptist Church, $200 \mathrm{ft}$ southeast from power lines, $750 \mathrm{ft}$ southwest of Stamp Creek

Typic Hapludults; fine-loamy, mixed, thermic

18 to $47 \mathrm{~cm}$ of the argillic horizon

Convex toeslope position

$15 \%$ southeast

Dismal Gap Formation

Oak, red maple, hickory, dogwood, red cedar

J. L. Branson

Branson, Gallagher

2 days

$80^{\circ} \mathrm{F}$ and sunny

April 23, 1992

\section{Soil Description:}

Oe 2 to $0 \mathrm{~cm}$; decomposing leaves

A 0 to $6 \mathrm{~cm}$; very dark grayish-brown (10YR 3/2) silt loam; strong fine granular structure; very friable; abrupt smooth boundary

Ap 6 to $18 \mathrm{~cm}$; dark yellowish-brown (10YR 4/4) silt loam; weak medium granular structure; very friable; clear smooth boundary

$\mathrm{Bt} \quad 18$ to $47 \mathrm{~cm}$; strong brown (7.5YR 4/6) clay loam; moderate medium subangular blocky structure; friable; clear wavy boundary

$\mathrm{Cr} \quad 47$ to $98+\mathrm{cm}$; soft red shale with yellow and green shale fragments

Notes: This site is on a toeslope in an area that is highly dissected. The area around the site has numerous rills and gullies. This area shows no evidence of having been cleared or farmed within the last 50 years. 


\section{A-106}

\section{ROANE Site 20}

Location:

Classification:

Geomorphic position:

Slope and aspect:

Parent material(s):

Vegetation:

Described by:

Sampling crew:

Time Since Last Rain:

Present weather:

Date:
Pattie Gap Quadrangle, Hurricane Ridge; $950 \mathrm{ft}$ northwest from Salem Baptist Church, $200 \mathrm{ft}$ southeast from power lines, $1000 \mathrm{ft}$ southwest of Stamp Creek

Ochreptic Hapludults; fine-loamy, mixed, thermic, shallow

Convex toeslope position

$12 \%$ southeast

Dismal Gap Formation

Oak, sweetgum, dogwood, poison ivy

J. L. Branson

Branson, Gallagher

1 day

$60^{\circ} \mathrm{F}$ and partly cloudy

May 5, 1992

\section{Soil Description:}

$\mathrm{Oi} \quad 2$ to $0 \mathrm{~cm}$; leaves and pine needles

Ap $\quad 0$ to $12 \mathrm{~cm}$; yellowish-brown (10YR 5/4) silt loam; weak medium granular structure; very friable; clear smooth boundary

Bt 12 to $35 \mathrm{~cm}$; yellowish-red (5YR 4/6) clay; strong medium subangular blocky structure; firm; gradual smooth boundary

C $\quad 35$ to $52 \mathrm{~cm}$; dark red $(2.5 \mathrm{YR} 3 / 6)$ saprolite that crushes easily to clay; rock controlled structure; abrupt irregular boundary

$\mathrm{Cr} \quad 52$ to $72 \mathrm{~cm}$; weathered reddish and olive shale 


\section{ROANE Site 21}

Location:

Classification:

Control Section:

Geomorphic position:

Slope and aspect:

Parent material(s):

Vegetation:

Described by:

Sampling crew:

Time Since Last Rain:

Present weather:

Date:
Pattie Gap Quadrangle, Hurricane Ridge; $1100 \mathrm{ft}$. west from Salem Baptist Church, $200 \mathrm{ft}$ southeast from power line, $1200 \mathrm{ft}$ southwest of creek

Typic Hapludults; fine-loamy, mixed, thermic

18 to $64 \mathrm{~cm}$ of the argillic horizon

Midslope

$13 \%$ southeast

Dismal Gap Formation

Oak, tulip poplar, Virginia pine, sweetgum, red maple, club mosses

J. L. Branson

Branson, Morris

2 days

$80^{\circ} \mathrm{F}$ and sunny

May 11, 1992

Soil Description:

Oi $\quad 3$ to $0 \mathrm{~cm}$; leaves, moss, and pine needles

A 0 to $5 \mathrm{~cm}$; brown (10YR $4 / 3$ ) shaly silty clay loam; moderate, medium granular structure; very friable; clear smooth boundary

Ap 5 to $18 \mathrm{~cm}$; dark grayish-brown (10YR 4/2) silty clay loam; weak medium subangular blocky structure; friable; clear wavy boundary.

Bt1 18 to $36 \mathrm{~cm}$; strong brown (7.5YR $4 / 6$ ) clay loam; moderate medium subangular blocky structure; firm; clear smooth boundary

Bt2 36 to $64 \mathrm{~cm}$; strong brown (7.5YR 5/6) clay loam; moderate coarse subangular blocky structure; firm; clear smooth boundary

BC $\quad 64$ to $74 \mathrm{~cm}$; brown (7.5YR 5/4) silty clay loam; weak medium subangular blocky structure; friable; gradual smooth boundary

$\mathrm{Cr} \quad 74+\mathrm{cm}$; soft red shale with some olive and yellowish-brown thin strata

Notes: This site is on a convex slope in an area that is highly dissected. The area around the site has numerous rills and gullies. This area shows no evidence of having been cleared or farmed within the last $50 \mathrm{y}$. Surfaces nearby show evidence of extensive erosion and shallow depths. 


\section{ROANE Site 22}

Location:

Classification:

Control Section:

Geomorphic position:

Slope and aspect:

Parent material(s):

Vegetation:

Described by:

Sampling crew:

Time Since Last Rain:

Present weather:

Date:
Pattie Gap Quadrangle, Hurricane Ridge, $1400 \mathrm{ft}$ west from Salem Baptist Church, $200 \mathrm{ft}$ southeast from power line, $1500 \mathrm{ft}$ west of Stamp Creek

Typic Hapludults; fine-loamy, mixed, thermic

19 to $56 \mathrm{~cm}$ of the argillic horizon

Midslope bench

$13 \%$ southeast

Dismal Gap Formation

Oak, tulip poplar, Virginia pine, red cedar, club mosses

J. L. Branson

Branson, Gallagher

1 day

$60^{\circ} \mathrm{F}$ and overcast; sprinkled lightly during sampling

May 5, 1992

\section{Soil Description:}

Oi $\quad 3$ to $0 \mathrm{~cm}$; leaves and pine needles

Ap $\quad 0$ to $19 \mathrm{~cm}$; yellowish-brown (10YR 5/6) silt loam; moderate medium subangular blocky structure; friable; clear, smooth boundary

Bt1 19 to $29 \mathrm{~cm}$; strong brown (7.5YR 5/8) clay loam; moderate medium subangular blocky structure; firm; clear smooth boundary

Bt2 29 to $45 \mathrm{~cm}$; strong brown (7.5YR 4/6) clay loam; moderate medium subangular blocky structure; firm; clear smooth boundary

Bt\&C 45 to $56 \mathrm{~cm}$; red (2.5YR 4/6) and light olive brown (2.5Y 5/4) clay; weak coarse subangular blocky structure; firm; clear smooth boundary

C 56 to $100+\mathrm{cm}$; mottled red (2.5YR 4/6), strong brown (7.5YR 5/8), and light yellowish-brown (2.5Y 6/4) clay loam; rock controlled structure

Notes: This site is on a slight bench in an area that is highly dissected. The area around the site has numerous rills and gullies. This area shows no evidence of having been cleared or farmed within the last 50 years. Surfaces nearby show evidence of extensive erosion and shallow depths. 


\section{ROANE Site 33}

Location:

Classification:

Geomorphic position:

Slope and aspect:

Parent material(s):

Vegetation:

Described by:

Sampling crew:

Time since last rain:

Present weather:

Date:
Pattie Gap Quadrangle, Gage Hill; $500 \mathrm{ft}$ east from Dry Fork Valley Roád, $500 \mathrm{ft}$ northeast from Gage Road; $400 \mathrm{ft}$ northèast from the old limestone quarry

- Typic Paleudults; clayey, mixed, thermic

A double convex side slope approximately midway between the top of the ridge and the valley floor

$39 \%, 265 \mathrm{~W}$

Copper Ridge (lower)

Redbuds, scarlet oak, hickory, dogwood, black jack oak

J.L. Branson

Janice Branson, Mike Timpson

2 days

$85^{\circ} \mathrm{F}$ and sunny and humid

September 1, 1992

Soil Description:

Surface covered with 2-3 in.-diam (some, 20 in.-diam) limestone cobbles

$\mathrm{Oi} / \mathrm{Oa} \quad 5-0 \mathrm{~cm}$; intermittent leaf litter, twigs, roots

Ap $\quad 0-10 \mathrm{~cm}$; strong brown (7.5 YR 5/6) silt loam; weak coarse subangular blocky structure; firm; $15 \%$ chert fragments; clear smooth boundary

Bt1 10-20 cm; red (2.5 YR 4/8) clay; strong medium subangular blocky structure; firm to very firm; clear smooth boundary

Bt2 20-110 cm; red (2.5 YR 4/6) clay; moderate medium subangular blocky structure; firm; gradual smooth boundary

Bt3 110-147 cm; red (2.5 YR 4/6) clay; moderate medium subangular blocky structure; friable 


\section{ROANE Site 34}

Location:

Classification:

Geomorphic position:

Slope and aspect:

Parent material(s):

Vegetation:

Described by:

Sampling crew:

Time since last rain:

Present weather:

Date:
Pattie Gap Quadrangle, Gage Hill; $1000 \mathrm{ft}$ southeast from Dry Fork Valley Road, $1200 \mathrm{ft}$ northeast from Gage Road, $1000 \mathrm{ft}$ northeast of the limestone quarry

Typic Hapludults; clayey, mixed, thermic

Upland just below the break of the ridge crest

$10 \%, 380^{\circ} \mathrm{NW}$

Copper Ridge Formation

Chestnut oak, blackjack oak, black oak, scarlet oak, Virginia pine

J.L. Branson

Sonja Entrekin, Mike Timpson, and Janice Branson

5 days

$60^{\circ} \mathrm{F}$ and cloudy and overcast

November 11, 1992

\section{Soil Description:}

Oi $\quad 5-0 \mathrm{~cm}$; leaf litter, roots

A $\quad 0-4 \mathrm{~cm}$; very. dark grayish-brown (10YR 3/2) and very dark brown (10YR 2/2) very fine sandy loam; moderate fine granular structure; very friable; clear smooth boundary

Ap 4-16 cm; dark yellowish-brown (10YR 4/6) silt loam; fine medium subangular blocky structure; friable; $5 \%$ chert fragments; clear smooth boundary

Bt1 16-27 cm; yellowish-red (5YR 5/8) silty clay loam; fine medium subangular blocky structure; friable; clear smooth boundary

Bt2 27-48 cm; yellowish-red (5YR 5/8) clay; moderate medium subangular blocky structure; firm; clear smooth boundary

BC $\quad 48-75 \mathrm{~cm}$; red (2.5YR 4/8) clay; moderate medium angular blocky structure; firm; gradual smooth boundary

C 75-105 cm; mottled red (2.5YR 5/8 and 10R 4/8) and yellow (2.5Y 7/6) saprolitic materials; clay; massive; firm 


\section{ROANE Site 35}

Location:

Classification:

Geomorphic position:

Slope and aspect:

Parent material(s):

Vegetation:

Described by:

Sampling crew:

Time since last rain:

Present weather:

Date:
Pattie Gap Quadrangle, Gage Hill; $100 \mathrm{ft}$ west from an old logging road, $1000 \mathrm{ft}$ southeast from Dry Fork Valley Road; $1800 \mathrm{ft}$ northeast from Gage Road

Typic Paleudults; clayey, mixed, thermic

Upper side slope just below crest of the ridge $19 \%, 340^{\circ} \mathrm{N}$

Surficial colluvium and residuum of the Copper Ridge Formation Dogwood, chestnut oak, scarlet oak, blackjack oak, hickory, sassafras

J.L. Branson

Rick Livingston, and Janice Branson

1 day

$85^{\circ} \mathrm{F}$ and sunny

August 31, 1992

\section{Soil Description:}

Oi $\quad 5-0 \mathrm{~cm}$; leaf litter, roots, twigs; $5 \%$ chert fragments

A $\quad 0-10 \mathrm{~cm}$; brown (10YR 4/3) silt loam; moderate fine granular structure; very friable; $5 \%$ chert fragments; clear smooth boundary

Ap 10-16 cm; brown (10YR 4/3) silt loam; weak fine subangular blocky structure; friable; $5 \%$ chert fragments; clear smooth boundary

Bt1 16-23 cm; strong brown (7.5YR 5/6) silty clay loam; weak fine subangular blocky structure; friable; $5 \%$ chert fragments; clear smooth boundary

Bt2 23-46 cm; yellowish-red (5YR 5/6) clay loam; moderate medium subangular blocky structure; friable; $10 \%$ chert fragments; gradual wavy boundary

2Bt3 46-73 cm; red (2.5YR 4/6) clay; moderate medium angular blocky structure; firm; $20 \%$ chert fragments; gradual smooth boundary

Bt4 73-110 cm; red (2.5YR 4/6) clay; moderate medium angular blocky structure; firm; $10 \%$ chert fragments; gradual smooth boundary

Bt5 110-135 cm; red (2.5 YR 5/8) clay; moderate medium angular blocky structure; firm; gradual smooth boundary

Bt5 135-180 cm; red (2.5 YR 5/8) clay; moderate medium subangular blocky structure; friable; $10 \%$ chert fragments; gradual smooth boundary

BC 180-195 cm; red (2.5 YR 5/8) clay; weak medium subangular blocky structure; friable 


\section{ROANE Site 39}

Location:

Classification:

Geomorphic position:

Slope and aspect:

Parent material(s):

Vegetation:

Described by:

Sampling crew:

Time since last rain:

Present weather:

Date:
Pattie Gap Quadrangle, Walker Ridge; $1000 \mathrm{ft}$ southeast from Dry Fork Valley Road, $500 \mathrm{ft}$ north from Williams Road Typic Paleudults; fine-loamy, siliceous, thermic

Foot slope near a depression $14 \%, 220^{\circ} \mathrm{SW}$

Surficial colluvium and residuum of the Copper Ridge Formation Chestnut oak, scarlet oak, blackjack oak, dogwood

J.L. Branson

Mike Timpson, Dr. J.T. Ammons, and Janice Branson

1 day

$50^{\circ} \mathrm{F}$ and sunny and windy

November 13, 1992

\section{Soil Description:}

$\mathrm{Oi} \quad 5-0 \mathrm{~cm}$; roots, leaf litter

A $\quad 0-7 \mathrm{~cm}$; brown (10YR 5/3) cherty silt loam; weak medium granular structure; very friable; $20 \%$ chert fragments; abrupt smooth boundary

E $\quad 7-34 \mathrm{~cm}$; brownish-yellow (10YR 6/6) silt loam or loam; weak medium subangular blocky structure; very friable; clear smooth boundary

Bt1 34-61 cm; strong brown (7.5YR 5/6) loam; moderate medium subangular blocky structure; friable; clear wavy boundary

Bt2 61-95 cm; yellowish-red (5YR 5/8) cherty clay loam; moderate medium angular blocky structure; firm; $20 \%$ chert fragments; gradual smooth boundary; A large pocket of Btx material present in the Bt2 horizon

2BC 95-146 cm; red (2.5YR 4/8) cherty clay; massive; $20 \%$ chert fragments; firm 


\section{ROANE Site 40}

Location:

Classification:

Geomorphic position:

Slope and aspect:

Parent material(s):

Vegetation:

Described by:

Sampling crew:

Time since last rain:

Present weather:

Date:
Pattie Gap Quadrangle, Walker Ridge; $900 \mathrm{ft}$ north from Williams Road, $1500 \mathrm{ft}$ southeast from the intersection of Williams Road and Dry Fork Valley Road

Typic Paleudults; clayey, mixed, thermic

Upland on a doubly convex slope at the top of the knob $7 \%, 320^{\circ} \mathrm{NW}$

Surficial colluvium and residuum of the Copper Ridge Formation Chestnut oak, white oak, hickory, Virginia pine

J.L. Branson

Dr. J.T. Ammons, and Janice Branson

3 days

$80^{\circ} \mathrm{F}$ and partly cloudy; heavy rain during sampling

September 8, 1992

\section{Soil Description:}

Oi $\quad 4-0 \mathrm{~cm}$; leaf litter, roots

E 0-18 cm; pale brown (10YR 6/3) silt loam; weak fine granular structure; friable; $10 \%$ chert fragments; clear smooth boundary

Bt1 18-29 cm; reddish-yellow (7.5YR 7/6) silty clay loam; weak fine subangular blocky structure with some weak medium subangular blocky structure; friable; $2 \%$ chert fragments; gradual smooth boundary

Bt2 29-52 cm; yellowish-red (5YR 5/8) clay loam or clay; moderate medium subangular blocky structure; firm; $10 \%$ chert fragments; clear smooth boundary

2Bt3 52-75 cm; red (2.5YR 4/8) cherty clay; moderate medium subangular blocky structure; friable; $20 \%$ chert fragments; clear smooth boundary

2BC 75-102 cm; red (2.5YR 4/8) cherty clay; weak fine and medium subangular structure; $25 \%$ chert fragments; friable 


\section{ROANE Site 41}

Location: $\quad$ Pattie Gap Quadrangle, Walker Ridge; $1800 \mathrm{ft}$ southeast from

Classification:

Geomorphic position: Dry Fork Valley Road, $1200 \mathrm{ft}$ northeast from Williams Road

Slope and aspect: Typic Hapludults; clayey, mixed, thermic

Parent material(s): Midslope $18 \%, 160^{\circ} \mathrm{S}$

Vegetation:

Described by:

Sampling crew:

Copper Ridge Formation

White oak, scarlet oak, chestnut oak

J.L. Branson

Time since last rain:

Mike Timpson, Dr. J.T. Ammons, and Janice Branson

1 day

Present weather:

$45^{\circ} \mathrm{F}$ and sunny

Date:

November 13, 1992

\section{Soil Description:}

Oi $\quad 5-0 \mathrm{~cm}$; leaf litter

Oa\&A $\quad 0-2 \mathrm{~cm}$; a mix of mineral matter and leaf litter

E 2-17 cm; dark yellowish-brown (10YR 4/4) cherty loam or silt loam; fine medium granular structure; friable; $20 \%$ chert fragments; clear smooth boundary

$\mathrm{BE} \quad 17-33 \mathrm{~cm}$; light brown (7.5YR 6/4) cherty loam; fine medium subangular blocky structure; friable; $20 \%$ chert fragments; clear smooth boundary

Bt1 33-58 cm; red (2.5YR 5/8) cherty clay or clay loam; moderate medium subangular blocky structure; friable; $25 \%$ chert fragments; clear smooth boundary

Bt2 58-74 cm; red (2.5YR 4/8) cherty clay or clay loam; weak coarse subangular blocky structure; firm; $25 \%$ chert fragments; clear smooth boundary

C 74-102 cm; red (2.5YR 4/8) cherty clay loam; massive; $25 \%$ chert fragments; friable 


\section{ROANE Site 42}

Location:

Classification:

Geomorphic position:

Slope and aspect:

Parent material(s):

Vegetation:

Described by:

Sampling crew:

Time since last rain:

Present weather:

Date:
Pattie Gap Quadrangle, Walker Ridge; $50 \mathrm{ft}$ northeast of an old logging road, $1000 \mathrm{ft}$ south of the power lines, $1500 \mathrm{ft}$ southeast from Dry Fork Valley Road

Typic Hapludults; clayey, mixed, thermic

On a long slope $1 / 3$ of the way from the top

$19 \%, 100^{\circ}$ east

Copper Ridge Formation

Chestnut oak, blackjack oak, red maple, sumac

J.L. Branson

Mike Timpson, and Janice Branson

1 day

$90^{\circ} \mathrm{F}$ and sunny

September 9, 1992

\section{Soil Description:}

Oi $\quad 5-0 \mathrm{~cm}$; root mat, leaf litter

E1 0-15 cm; light brownish-gray (10YR 6/2) very cherty silt loam; weak fine granular structure; very friable; $40 \%$ chert fragments; clear smooth boundary

E2 $15-35 \mathrm{~cm}$; very pale brown (10YR $7 / 3$ ) cherty silt loam; weak medium subangular blocky structure, very friable; $30 \%$ chert fragments; clear smooth boundary

Bt1 35-55 cm; yellowish-red (5YR 5/8) cherty clay loam; weak coarse subangular blocky structure; friable; $30 \%$ chert fragments; clear smooth boundary

Bt2 55-80 cm; red (2.5YR 5/8) cherty clay; moderate medium subangular blocky structure; firm; $30 \%$ chert fragments; clear smooth boundary

Bt3 80-125 cm; red (2.5YR 4/8) cherty clay; moderate medium subangular blocky structure; firm; $25 \%$ chert fragments; gradual smooth boundary 


\section{ROANE Site 43}

Location:

Classification:

Geomorphic position:

Slope and aspect:

Parent material(s):

Vegetation:

Described by:

Sampling crew:

Time since last rain:

Present weather:

Date:
Pattie Gap Quadrangle, Walker Ridge; $1300 \mathrm{ft}$ northeast of an old logging road, $1200 \mathrm{ft}$ south of the power lines, $1300 \mathrm{ft}$ southeast of Dry Fork Valley Road

Typic Paleudults; fine-loamy, siliceous, thermic

Midslope on a convex slope just before a major slope break $23 \%, 120^{\circ}$ east

Surficial colluvium and residuum of the Copper Ridge Formation

Chestnut oak, dogwood, sumac, sassafras

J.L. Branson

Mike Timpson, and Janice Branson

1 day

$90^{\circ} \mathrm{F}$

September 9, 1992

\section{Soil Description:}

$\mathrm{Oi} \quad 5-0 \mathrm{~cm}$; thick root mat, leaf litter

E1 0-18 cm; light yellowish-brown (10YR 6/4) cherty silt loam; weak medium subangular blocky structure; friable; $30 \%$ chert fragments; clear smooth boundary

Bt1 18-48 cm; (7.5 YR 5/8) cherty silt loam; weak, medium subangular blocky structure; friable; clear, smooth boundary; $30 \%$ coarse fragments

Bt2 48-72 cm; strong brown (7.5YR 5/8) cherty clay loam; weak medium subangular blocky structure; friable; $20-25 \%$ chert fragments; clear smooth boundary

2Bt3 72-90 cm; yellowish-red (5YR 5/8) cherty clay; moderate medium subangular blocky structure; friable; 30-35\% chert fragments; clear smooth boundary

BC $\quad 90-110 \mathrm{~cm} ;$ red (2.5 YR 5/8) cherty clay; massive; friable 


\section{ROANE Site 44}

\section{Location:}

Classification:

Geomorphic position:

Slope and aspect:

Parent material(s):

Vegetation:

Described by:

Sampling crew:

Time since last rain:

Present weather:

Date:
Pattie Gap Quadrangle, Walker Ridge; $1400 \mathrm{ft}$ south of the power lines, $1000 \mathrm{ft}$ southeast from Dry Fork Valley Road Typic Paleudults; fine-loamy, siliceous, thermic Midslope just above a change to a steeper slope of $35 \%$. $23 \%, 140^{\circ}$ southeast

Surficial colluvium and residuum of the Copper Ridge Formation Dogwood, maple, blackjack oak, chestnut oak, tulip poplar

J.L. Branson

Sonja Entrekin, Janice Branson

3 days

$80^{\circ} \mathrm{F}$ and partly sunny to cloudy

September 2, 1992

Soil Description:

Oi $\quad 5-0 \mathrm{~cm}$; leaf litter, roots, twigs

E1 0-14 cm; brown (10YR 5/3) silt loam; weak fine granular structure; very friable; $10 \%$ chert fragments; clear smooth boundary

E2 14-34 cm; very pale brown (10YR 7/4) silt loam; weak medium and fine subangular blocky structure; very friable; $4 \%$ chert fragments; clear wavy boundary

E3 34-47 cm; very pale brown (10YR 7/4) cherty silt loam; moderate medium subangular blocky structure; friable; $20 \%$ chert fragments; clear wavy boundary

Bt1 47-88 cm; reddish-yellow (7.5YR 6/6) cherty clay loam; moderate medium subangular blocky structure; friable, $30 \%$ chert fragments; gradual wavy boundary

2Bt2 . 88-115 cm; strong brown (7.5YR 5/8) clay; moderate medium subangular blocky structure; friable; $10 \%$ chert fragments; clear irregular boundary

$\mathrm{R} \quad 115 \mathrm{~cm}$; ran into a solid carbonate rock. Unsure as to whether it is a large "floater" or a pinnacle 


\section{ROANE Site 45}

Location:

Classification:

Geomorphic position:

Slope and aspect:

Parent material(s):

Vegetation:

Described by:

Sampling crew:

Time since last rain:

Present weather:

Date:
Pattie Gap Quadrangle, Walker Ridge; $1600 \mathrm{ft}$ south of the power lines, $1500 \mathrm{ft}$ southeast from Dry Fork Valley Road

Typic Paleudults; clayey, mixed, thermic

Midslope

$23 \%, 20^{\circ}$ southwest

Surficial colluvium and residuum of the Copper Ridge Formation Sassafras, chestnut oak, hickory, dogwood

J.L. Branson

Rick Livingston, Sonja Entrekin, Dr. J.T. Ammons, and Janice Branson

2 days

$75^{\circ} \mathrm{F}$ and sunny

October 7, 1992

Soil Description:

Oi $\quad 5-0 \mathrm{~cm}$; leaf litter, twigs, roots

A 0-6 cm; brown (10YR 5/3) silt loam; weak medium granular structure; very friable; $5-10 \%$ chert fragments; abrupt smooth boundary

E 6-23 cm; light yellowish-brown (10YR 6/4) silt loam; weak fine subangular blocky structure; friable; $2 \%$ chert fragments; clear wavy boundary

Bt1 23-33 cm; strong brown (7.5YR 5/6) silt loam; weak fine subangular blocky structure; friable; $5 \%$ chert fragments; clear wavy boundary

2Bt2 33-61 cm; yellowish-red (5YR 5/8) clay; moderate medium angular and subangular blocky structure; friable; $10 \%$ chert fragments; clear wavy boundary

2Bt3 61-94 cm; yellowish-red (5YR 5/6) clay; weak medium angular and subangular blocky structure; friable; $10 \%$ chert fragments; clear wavy boundary

2BC 94-109 cm; red (2.5YR 4/8) clay; massive; $10 \%$ chert fragments; friable 


\section{ROANE Site 46}

Location:

Classification:

Geomorphic position:

Slope and aspect:

Parent material(s):

Vegetation:

Described by:

Sampling crew:

Time since last rain:

Present weather:

Date:
Pattie Gap Quadrangle, Walker Ridge; $1800 \mathrm{ft}$ south of the power lines, 1500 southeast from Dry Fork Valley Road

Typic Paleudults; fine-loamy, siliceous, thermic

Midslope on a doubly convex slope, $50 \mathrm{ft}$. above a major slope break

$23 \%, 240^{\circ}$ southwest

Ancient alluvial capping and residuum of the Copper Ridge Formation

Red maple, dogwood

J.L. Branson

Rick Livingston, Sonja Entrekin, Dr. J.T. Ammons, and Janice Branson

2 days

$80^{\circ} \mathrm{F}$ and sunny

October 7, 1992

\section{Soil Description:}

Oi $\quad 5-0 \mathrm{~cm}$; leaf litter, twigs, and roots

A 0-12 cm; dark yellowish-brown (10YR 4/4) silt loam; weak fine and medium granular structure; friable; 7\% chert fragments; clear smooth boundary

AB 12-19 cm; strong brown (7.5YR 4/6) silt loam; weak medium subangular blocky structure; friable; clear smooth boundary .

BE 19-28 cm; red (2.5YR 4/8) loam; weak medium subangular blocky; friable; clear smooth boundary

Bt1 28-43 cm; red (2.5YR 4/8) clay loam; moderate medium subangular blocky structure; friable; $5 \%$ chert fragments; clear smooth boundary

Bt2 43-64 cm; red (2.5YR 4/6) clay; moderate medium subangular blocky structure; friable; $5 \%$ chert fragments; gradual smooth boundary

$2 \mathrm{Bt3} \quad 64-104 \mathrm{~cm}$; red (2.5YR 4/8) clay; massive; $13 \%$ chert fragments; friable 


\section{A-120}

\section{ROANE Site 47}

Location:

\section{Classification:}

Geomorphic position:

Slope and aspect:

Parent material(s):

Vegetation:

Described by:

Sampling crew:

Time since last rain:

Present weather:

Date:
Pattie Gap Quadrangle, Walker Ridge; $2100 \mathrm{ft}$ south of the power lines, $1400 \mathrm{ft}$ southeast from Dry Fork Valley Road

Typic Paleudults; fine-loamy, siliceous, thermic

Midslope just above where the slope breaks to $50 \%$

$25 \%, 20^{\circ}$ north

Surficial colluvium and residuum of the Copper Ridge Formation Tulip poplar, red maple, dogwood

J.L. Branson

Rick Livingston, Sonja Entrekin, Dr. J.T. Ammons, and Janice Branson

2 days

$80^{\circ} \mathrm{F}$ and sunny

October 7, 1992

\section{Soil Description:}

Oi $\quad 5-0 \mathrm{~cm}$; leaf litter; twigs, roots

A $\quad 0-13 \mathrm{~cm}$; dark yellowish-brown (10YR 4/4) silt loam; moderate medium granular structure; very friable; $2 \%$ chert fragments; clear smooth boundary

Ap 13-22 cm; dark yellowish-brown (10YR 4/4) silt loam; weak medium subangular blocky structure; very friable; $2 \%$ chert fragments; clear smooth boundary

Bt1 22-43 cm; strong brown (7.5YR 5/6) loam; weak medium subangular blocky structure; friable; $2 \%$ chert fragments; clear smooth boundary

2Bt2 43-61 cm; yellowish-red (5YR 5/8) clay loam; weak medium and coarse subangular blocky structure; friable to firm; $5 \%$ chert fragments; clear smooth boundary

2BC 61-89 cm; red (2.5YR 4/8) clay; moderate medium subangular blocky structure; 5\% chert fragments; very firm 


\section{A.6 ANDERSON COUNTY SOIL PROFILES}

\section{ANDERSON Site 1}

Location:

Classification:

Control Section:

Geomorphic position:

Slope and aspect:

Parent material(s):

Vegetation:

Described by:

Sampling crew:

Time Since Last Rain:

Present weather:

Date:
Norris Quadrangle, Pine Ridge; $1400 \mathrm{ft}$ northeast of Old Anderson Pike, $1000 \mathrm{ft}$ west from Hinds Creek Road Ochreptic Hapludults; clayey, kaolinitic, thermic, shallow 28 to $44 \mathrm{~cm}$ argillic horizon

Midslope

$6 \%$ north

Dismal Gap Formation

Virginia pine, tulip poplar, red maple, dogwood

J. L. Branson

Branson, Livingston, Gallagher

0 day

$85^{\circ} \mathrm{F}$ and partly cloudy; heavy rain during sampling

July 17, 1992

\section{Soil Description:}

A 0 to $10 \mathrm{~cm}$; dark brown (10YR 3/3) silt loam; weak medium granular structure; very friable; clear smooth boundary

Ap $\quad 10$ to $28 \mathrm{~cm}$; yellowish-brown (10YR 5/6) loam; weak medium granular structure; friable ; clear wavy boundary

Bt 28 to $44 \mathrm{~cm}$; yellowish-red (5YR 5/6) channery clay; moderate medium subangular blocky structure; firm; clear wavy boundary

$\mathrm{Cr} \quad 44$ to $104 \mathrm{~cm}$; soft olive, red, and yellow shale

Notes: This site had been cleared at one time. Age of the deciduous trees indicated they had been established at least $50 \mathrm{y}$. No evidence of a plow layer. Slope dips strongly $(30 \%)$ approximately $50 \mathrm{ft}$ from the site. Presently, this area is occupied by cattle for pasture. 


\section{ANDERSON Site 3}

Location:

Classification:

Control Section:

Geomorphic position:

Slope and aspect:

Parent material(s):

Vegetation:

Described by:

Sampling crew:

Time Since Last Rain:

Present weather:

Date:
Big Ridge Park Quadrangle, Pine Ridge; $100 \mathrm{ft}$ northwest from Judson Road, 0.4 miles south from Moore Valley Road (also known as Cooper Gap)

Typic Hapludults; fine-loamy, mixed, thermic

16 to $53 \mathrm{~cm}$ entire argillic horizon

Upland, convex slope

$7 \%$ southeast

Dismal Gap Formation

Virginia Pine, dogwood, red maple, mosses, poison ivy, sumac, red cedar

J. L. Branson

Branson, Timpson, Livingston, Gallagher, Entrekin

0 days

$85^{\circ} \mathrm{F}$ and heavy rain during sampling

July 15,1992

\section{Soil Description:}

$\mathrm{Oi} \quad 2$ to $0 \mathrm{~cm}$; moss and pine needles

A $\quad 0$ to $4 \mathrm{~cm}$; dark brown (10YR 4/3) loam; moderate medium granular structure; friable; abrupt smooth boundary

Ap 4 to $16 \mathrm{~cm}$; yellowish-brown (10YR 5/4) loam; moderate fine granular structure; friable; clear smooth boundary

Bt1 16 to $40 \mathrm{~cm}$; yellowish-red (5YR 4/6) clay; moderate medium angular blocky structure; firm; clear wavy boundary

Bt2 40 to $53 \mathrm{~cm}$; yellowish-red (5YR 4/6) clay loam; moderate medium angular blocky structure; firm; clear wavy boundary

$\mathrm{Cr} \quad 53$ to $90 \mathrm{~cm}$; soft olive, red, and yellow shale 


\section{ANDERSON Site 4}

Location:

Classification:

Control Section:

Geomorphic position:

Slope and aspect:

Parent material(s):

Vegetation:

Described by:

Sampling crew:

Time Since Last Rain:

Present weather:

Date:
Big Ridge Park Quadrangle, Pine Ridge; $300 \mathrm{ft}$ northwest from Judson Road, 0.6 miles south from Moore Valley Road (also known as Cooper Gap)

Typic Hapludults; fine-loamy, mixed, thermic

24 to $70 \mathrm{~cm}$ argillic horizon

Midslope, convex shape

$24 \%$ southeast

Dismal Gap Formation

Virginia pine, dogwood, red maple, elm, poison ivy, cherry, sourwood

J. L. Branson

Branson, Timpson, Livingston, Gallagher, Entrekin

4 days

$85^{\circ} \mathrm{F}$ and cloudy

July 15,1992

\section{Soil Description:}

$\mathrm{Oi} \quad 3$ to $0 \mathrm{~cm}$; leaf litter, small roots, and pine needles

A $\quad 0$ to $5 \mathrm{~cm}$; yellowish-brown (10YR 5/4) loam; weak medium granular structure; very friable; abrupt smooth boundary

Ap 5 to $24 \mathrm{~cm}$; yellowish-brown (10YR 5/6) silt loam; weak fine granular structure; friable; abrupt smooth boundary

Bt1 24 to $37 \mathrm{~cm}$; strong brown (7.5YR 5/6) silty clay loam; moderate fine subangular blocky structure; friable; clear smooth boundary

Bt2 37 to $53 \mathrm{~cm}$; yellowish-red (5YR 4/6) clay loam; moderate medium subangular blocky structure; friable; clear wavy boundary

$\mathrm{Bt} \& \mathrm{Cr} \quad 53$ to $70 \mathrm{~cm}$; Bt: yellowish-red (5YR 5/6) clay loam; moderate medium subangular block structure; friable; $\mathrm{Cr}$ : soft olive, red, and yellow shale; abrupt wavy boundary

$\mathrm{Cr} \quad 70$ to $100 \mathrm{~cm}$; soft olive, red, and yellow shale 


\section{ANDERSON Site 5}

Location:

Classification:

Control Section:

Geomorphic position:

Slope and aspect:

Parent material(s):

Vegetation:

Described by:

Sampling crew:

Time Since Last Rain:

Present weather:

Date:
Big Ridge Park Quadrangle, Pine Ridge; $300 \mathrm{ft}$ northwest from Judson Road, 0.6 miles south from Moore Valley Road (also known as Cooper Gap), $300 \mathrm{ft}$ south of Anderson Site 4 Typic Hapludults; fine-loamy, mixed, thermic 6 to $56 \mathrm{~cm}$ of the argillic horizon

Midslope, convex shape

$25 \%$ southeast

Dismal Gap Formation

Virginia pine, dogwood, oak, poison ivy

J. L. Branson

Branson, Timpson, Livingston, Gallagher, Entrekin

4 days

$85^{\circ} \mathrm{F}$ and cloudy

July 15, 1992

\section{Soil Description:}

Oi 2 to $0 \mathrm{~cm}$; leaf litter, small roots, and pine needles

A $\quad 0$ to $6 \mathrm{~cm}$; dark yellowish-brown (10YR 4/4) loam; weak medium granular structure; very friable; clear smooth boundary

Ap 6 to $15 \mathrm{~cm}$; dark yellowish-brown (10YR 4/6) loam; weak fine granular structure; friable; clear smooth boundary

Bt1 15 to $45 \mathrm{~cm}$; yellowish-brown (10YR 5/6) clay loam; moderate medium subangular blocky structure; friable; clear smooth boundary

Bt2 45 to $70 \mathrm{~cm}$; strong brown (7.5YR 5/8) clay loam; moderate medium subangular blocky structure; friable; clear smooth boundary

$\mathrm{Cr} \quad 70$ to $122 \mathrm{~cm}$; soft olive, red, and yellow shale 


\section{ANDERSON Site 9}

Location:

Classification:

Control Section:

Geomorphic position:

Slope and aspect:

Parent material(s):

Vegetation:

Described by:

Sampling crew:

Time Since Last Rain:

Present weather:

Date:
Powell Quadrangle, Pine Ridge; $1300 \mathrm{ft}$ north from East Wolf Valley Road, 0.25 miles west from Nolan Road, $300 \mathrm{ft}$ north from Anderson Site 19

Ochreptic Hapludults; clayey, kaolinitic, thermic, shallow

12 to $35 \mathrm{~cm}$ of the argillic horizon

Middle portion of a convex slope

$15 \%$ southeast

Dismal Gap Formation

Virginia pine, red maple, tulip poplar, dogwood

J. L. Branson

Branson, Timpson, Livingston, Gallagher, Entrekin

2 days

$75^{\circ} \mathrm{F}$ and cloudy

July 17,1992

\section{Soil Description:}

$\mathrm{Oi} \quad 2$ to $0 \mathrm{~cm}$; leaves and pine needles

A $\quad 0$ to $5 \mathrm{~cm}$; dark brown (10YR 3/3) loam; moderate fine granular structure; very friable; clear, smooth boundary

Ap 5 to $12 \mathrm{~cm}$; yellowish-brown (10YR 5/4) loam; weak fine and medium granular structure; friable; clear smooth boundary

$\mathrm{Bt} \quad 12$ to $35 \mathrm{~cm}$; strong brown (7.5YR 5/6) clay; moderate medium and coarse subangular blocky structure; firm; clear wavy boundary

BC $\quad 35$ to $50 \mathrm{~cm}$; strong brown (7.5YR $4 / 6)$ clay; $60 \%$ of structure is rock controlled; firm; clear wavy boundary

CB 50 to $62 \mathrm{~cm}$; strong brown (7.5YR 5/6) clay loam; $80 \%$ structure is rock controlled; firm; clear, wavy boundary

$\mathrm{Cr} \quad 62$ to $105 \mathrm{~cm}$; soft olive, red, and yellow shale 


\section{ANDERSON Site 10}

Location:

Classification:

Control Section:

Geomorphic position:

Slope and aspect:

Parent material(s):

Vegetation:

Described by:

Sampling crew:

Time Since Last Rain:

Present weather:

Date:
Norris Quadrangle, Pine Ridge; $1200 \mathrm{ft}$ northeast of Old Anderson Pike, $1000 \mathrm{ft}$ west from Hinds Creek Road

Typic Hapludults; fine-loamy, mixed, thermic

40 to $77 \mathrm{~cm}$ argillic horizon

Upland slightly convex summit

$1 \%$ northeast

Dismal Gap Formation

Virginia pine, tulip poplar, red maple, dogwood

J. L. Branson

Branson, Livingston, Gallagher

0 day

$85^{\circ} \mathrm{F}$ and partly cloudy; heavy rain during sampling

July 17, 1992

\section{Soil Description:}

$\mathrm{Oi} \quad 2$ to $0 \mathrm{~cm}$; roots and leaf litter

A $\quad 0$ to $10 \mathrm{~cm}$; very dark grayish-brown (10YR 3/2) silt loam; weak medium granular structure; friable; clear wavy boundary

E 10 to $21 \mathrm{~cm}$; brown (10YR 5/3) silt loam; weak medium granular structure; friable; clear smooth boundary

$\mathrm{BE} 21$ to $40 \mathrm{~cm}$; yellowish-brown (10YR 5/4) and strong brown (7.5YR 5/6) clay loam; weak medium subangular blocky structure; friable; clear smooth boundary

Bt $\quad 40$ to $77 \mathrm{~cm}$; yellowish-red (5YR 5/6) clay loam; weak medium subangular blocky structure; friable; clear smooth boundary

$\mathrm{Cr} \quad 77$ to $136 \mathrm{~cm}$; soft olive, red, and yellow shale 


\section{ANDERSON Site 11}

Location:

Classification:

Control Section:

Geomorphic position:

Slope and aspect:

Parent material(s):

Vegetation:

Described by:

Sampling crew:

Time Since Last Rain:

Present weather:

Date:
Norris Quadrangle, Pine Ridge; $600 \mathrm{ft}$ northwest from Hinds Creek Road, $1000 \mathrm{ft}$ northwest from Hidden Drive

Typic Hapludults; fine-loamy, mixed, thermic

18 to $56 \mathrm{~cm}$ of the argillic horizon

Upland position

$12 \%$ southeast

Dismal Gap Formation

tulip poplar, may apple, beech, red maple, hickory

J. L. Branson

Branson, Timpson, Livingston, Gallagher, Entrekin

2 days

$65^{\circ} \mathrm{F}$ and partly cloudy

May 27, 1992 (sampled for gamma samples and description) July 16, 1992 (sampled for zero contamination samples)

Soil Description:

Oa 2 to $0 \mathrm{~cm}$; crumbly organic matter

A 0 to $6 \mathrm{~cm}$; brown (10YR $4 / 3$ ) silt loam; moderate fine granular structure; very friable; clear wavy boundary

Ap 6 to $12 \mathrm{~cm}$; yellowish-brown (10YR 5/6) silt loam or loam; weak fine granular structure; friable; clear wavy boundary

BE 12 to $18 \mathrm{~cm}$; strong brown (7.5YR 5/6) and brown (7.5YR 5/4) loam; moderate fine subangular blocky structure; friable; clear wavy.boundary

Bt1 18 to $30 \mathrm{~cm}$; brown (7.5YR 5/4) clay; weak medium subangular blocky parting to moderate fine subangular blocky structure; firm; clear wavy boundary; yellowish-red (5YR 5/6) on ped faces

Bt2 30 to $56 \mathrm{~cm}$; strong brown (7.5YR 5/6) clay loam; weak medium subangular blocky parting to moderate fine subangular blocky structure; friable; yellowish-red (5YR $5 / 6)$ on clay skins; clear irregular boundary

CB 56 to $92 \mathrm{~cm}$; brown (7.5YR 5/4); clay loam; about $50 \%$ fine medium subangular blocky structure, remainder is rock controlled; friable to firm; gradual wavy boundary

Cr $\quad 92$ to $140 \mathrm{~cm}$; soft olive, red, and yellowish-brown shale 
ANDERSON Site 12

Location:

Classification:

Control Section:

Geomorphic position:

Slope and aspect:

Parent material(s):

Vegetation:

Described by:

Sampling crew:

Time Since Last Rain:

Present weather:

Date:
Big Ridge Park Quadrangle, Pine Ridge; $500 \mathrm{ft}$ northwest of Hinds Creek Road, $100 \mathrm{ft}$ west from power lines, $200 \mathrm{ft}$ west from Anderson Site 22

Typic Hapludults; fine-loamy, mixed, thermic

16 to $56 \mathrm{~cm}$ argillic horizon

Midslope

$15 \%$ southeast

Dismal Gap Formation

Virginia pine, dogwood, sweetgum, hickory

J. L. Branson

Branson, Timpson, Livingston, Gallagher, Entrekin

1 day

$80^{\circ} \mathrm{F}$ and partly cloudy

July 16, 1992

\section{Soil Description:}

$\mathrm{Oi} \quad 2$ to $0 \mathrm{~cm}$; leaf litter and pine needles

A $\quad 0$ to $7 \mathrm{~cm}$; dark brown (10YR 3/3) silt loam; moderate fine granular structure; very friable; abrupt smooth boundary

Ap 7 to $16 \mathrm{~cm}$; yellowish-brown (10YR 5/4) loam; weak medium granular structure; friable; clear, wavy boundary

Bt1 16 to $41 \mathrm{~cm}$; strong brown (7.5YR 4/6) clay; moderate coarse subangular blocky structure; firm; clear, wavy boundary

Bt2 41 to $56 \mathrm{~cm}$; yellowish-red (5YR 5/6) clay; moderate coarse subangular blocky structure; firm; clear wavy boundary

$\mathrm{Cr} 156$ to $90 \mathrm{~cm}$; soft olive, red, and yellow shale

$\mathrm{Cr} 290$ to $122 \mathrm{~cm}$; soft shale primarily red with some olive and yellowish-brown colors

Notes: This site had been cleared at one time. Age of the deciduous trees indicated they had been established at least 50 y. Slope dips strongly (30\%) off to the side. Presently, this area is occupied by cattle for pasture. 


\section{ANDERSON Site 19}

Location:

Classification:

Control Section:

Geomorphic position:

Slope and aspect:

Parent material(s):

Vegetation:

Described by:

Sampling crew:

Time Since Last Rain:

Present weather.

Date:
Powell Quadrangle, Pine Ridge; $100 \mathrm{ft}$ north from East Wolf Valley Road, 0.25 miles west from Nolan Road, $300 \mathrm{ft}$ south from Anderson Site 9

Typic Hapludults; clayey, kaolinitic, thermic 23 to $72 \mathrm{~cm}$ of the argillic horizon

Lower portion of a convex slope

$6 \%$ southeast

Dismal Gap Formation

Virginia pine, red maple, tulip poplar, sassafras, mosses

J. L. Branson

Branson, Timpson, Livingston, Gallagher, Entrekin

2 days

$75^{\circ} \mathrm{F}$ and cloudy

July 17, 1992.

\section{Soil Description:}

Oi $\quad 4$ to $0 \mathrm{~cm}$; moss, small roots, and pine needles

A $\quad 0$ to $13 \mathrm{~cm}$; very dark grayish-brown (10YR 3/2) silt loam; moderate medium granular structure; very friable; abrupt smooth boundary

Ap 13 to $23 \mathrm{~cm}$; yellowish-brown (10YR 5/6) loam; weak fine and medium granular structure; friable; abrupt smooth boundary

Bt1 23 to $39 \mathrm{~cm}$; yellowish-red (5YR 5/6) clay; moderate medium subangular blocky structure; firm; clear smooth boundary

Bt2 39 to $72 \mathrm{~cm}$; yellowish-red (5YR 5/8) clay; moderate medium subangular blocky structure; firm; clear wavy boundary

BC $\quad 72$ to $95 \mathrm{~cm}$; yellowish-red (5YR 5/8) clay loam; weak medium subangular blocky structure; friable; gradual wavy boundary

Cr $\quad 95$ to $135 \mathrm{~cm}$; soft olive, red, and yellow shale 


\section{ANDERSON Site 20}

Location:

Classification:

Control Section:

Geomorphic position:

Slope and aspect:

Parent material(s):

Vegetation:

Described by:

Sampling crew:

Time Since Last Rain:

Present weather:

Date:
Big Ridge Park Quadrangle, Pine Ridge; $100 \mathrm{ft}$ northwest from Judson Road, 0.3 miles south from Moore Valley Road (also known as Cooper Gap), $300 \mathrm{ft}$ north of Anderson Site 3

Typic Hapludults; clayey, kaolinitic, thermic

13 to $63 \mathrm{~cm}$ of the argillic horizon

Upland, convex slope, area is highly eroded

$12 \%$ southwest

Dismal Gap Formation

Virginia pine, dogwood, tulip poplar, poison ivy, mosses

J. L. Branson

Branson, Timpson, Livingston, Gallagher, Entrekin

0 days

$85^{\circ} \mathrm{F}$ and heavy rain during sampling

July 15,1992

\section{Soil Description:}

$\mathrm{Oi} \quad 2$ to $0 \mathrm{~cm}$; moss and leaf litter

A $\quad 0$ to $5 \mathrm{~cm}$; dark brown (7.5YR 4/4) silt loam; moderate medium granular structure; friable; clear smooth boundary

Ap 5 to $13 \mathrm{~cm}$; yellowish-brown (10YR 5/6) silt loam; weak fine granular structure; friable; gradual smooth boundary

Bt1 13 to $21 \mathrm{~cm}$; strong brown (7.5YR 5/6) silty clay loam; moderate medium subangular blocky structure; friable; abrupt smooth boundary

Bt2 21 to $42 \mathrm{~cm}$; yellowish-red (5YR 5/6) clay; moderate medium subangular blocky structure; firm; clear wavy boundary

Bt3 42 to $84 \mathrm{~cm}$; red (2.5YR 4.8) clay; strong, medium subangular blocky structure; friable; clear, wavy boundary

$\mathrm{Cr} \quad 84$ to $90 \mathrm{~cm}$; soft light olive, red and yellowish-brown shale 


\section{ANDERSON Site 21}

Location:

Classification:

Control Section:

Geomorphic position:

Slope and aspect:

Parent material(s):

Vegetation:

Described by:

Sampling Crew:

Time Since Last Rain:

Present weather:

Date:
Big Ridge Park Quadrangle, Pine Ridge; $700 \mathrm{ft}$ northwest from Hinds Creek Road, $150 \mathrm{ft}$ west from power lines, $200 \mathrm{ft}$ northeast from Anderson Site 12

Ochreptic Hapludults; clayey, kaolinitic, thermic, shallow

12 to $28 \mathrm{~cm}$ argillic horizon

Upland in the curve connecting two parallel slopes at the top of a small ridge

$11 \%$ southeast

Dismal Gap Formation

Virginia pine, dogwood, sweetgum, hickory

J. L. Branson

Branson, Timpson, Livingston, Gallagher, Entrekin

1 day

$80^{\circ} \mathrm{F}$ and partly cloudy to sunny

July 16, 1992

\section{Soil Description:}

$\mathrm{Oi} \quad 2$ to $0 \mathrm{~cm}$; leaf litter and pine needles

A $\quad 0$ to $6 \mathrm{~cm}$; dark brown (10YR 3/3) silt loam; moderate medium granular structure; friable; abrupt smooth boundary

Ap 6 to $12 \mathrm{~cm}$; yellowish-brown (10YR 5/4) loam; moderate medium granular structure; friable; clear smooth boundary

Bt 12 to $28 \mathrm{~cm}$; yellowish-red (5YR 4/6) clay; strong medium subangular blocky structure; firm; clear wavy boundary

$\mathrm{Cr} \quad 28$ to $87 \mathrm{~cm}$; soft olive, red, and yellow shale

Notes: This site had been cleared at one time. Age of the deciduous trees indicated they had been established at least $50 \mathrm{y}$. Slope dips strongly (30\%) approximately $50 \mathrm{ft}$ from the site. Presently, this area is occupied by cattle for pasture. 


\section{ANDERSON Site 22}

Location:

Classification:

Control Section:

Geomorphic position:

Slope and aspect:

Parent material(s):

Vegetation:

Described by:

Sampling crew:

Time Since Last Rain:

Present weather:

Date:
Big Ridge Park Quadrangle, Pine Ridge; $500 \mathrm{ft}$ northwest from the intersection of Hill Road and Hind's Creek Road, $300 \mathrm{ft}$ west of the power lines. It is $200 \mathrm{ft}$ east from Anderson Site 12 on a convex slope.

Ochreptic Hapludults; fine-loamy, mixed, thermic, shallow

4 to $28 \mathrm{~cm}$ entire argillic horizon

Upland

$18 \%$ southeast

Dismal Gap Formation

Virginia Pine, dogwood, cedar, poison ivy, red cedar

J. L. Branson

Branson, Livingston

0 days

$85^{\circ} \mathrm{F}$ and heavy rain during sampling

July 15, 1992 (zero contamination sampling)

July 16, 1992 (gamma sampling)

\section{Soil Description:}

Oi $\quad 2$ to $0 \mathrm{~cm}$; roots, leaf litter, and pine needles

A $\quad 0$ to $4 \mathrm{~cm}$; dark brown (10YR 4/3) loam; weak fine granular structure; friable; abrupt smooth boundary

Ap $\quad 4$ to $14 \mathrm{~cm}$; yellowish-brown (10YR.5/4) silt loam; weak medium granular structure; friable; clear wavy boundary

$\mathrm{Bt} \quad 14$ to $28 \mathrm{~cm}$; yellowish-red (5YR 5/6) clay; moderate medium subangular blocky structure; firm; clear irregular boundary

$\mathrm{Cr} \quad 28$ to $98 \mathrm{~cm}$; soft olive, red, and yellow shale

Note: A water seep occurred at approximately $50 \mathrm{~cm}$. 


\section{ANDERSON Site 31}

Location:

Classification:

Geomorphic position:

Slope and aspect:

Parent material(s):

Vegetation:

Described by:

Sampling crew:

Time since last rain:

Present weather:

Date:
Powell Quadrangle, Chestnut Ridge; $1000 \mathrm{ft}$ west of the Knox/Powell water tank, $300 \mathrm{ft}$ south of dirt road, $800 \mathrm{ft}$ northeast from Heiskell Road

Typic Hapludults; clayey, mixed, thermic

Lower slope just above a colluvial bench

$18 \%, 240^{\circ}$ southwest

Surficial colluvium and residuum of the Copper Ridge Formation

Virginia pine, sassafras, red oak, chestnut oak

J.L. Branson

Mike Timpson, Mike Haarbauer, and Janice Branson

1 day

$40^{\circ} \mathrm{F}$ and sunny and clear

December 5, 1992

\section{Soil Description:}

Oi $\quad 5-0 \mathrm{~cm}$; leaf litter, twigs

A $\quad 0-11 \mathrm{~cm}$; very dark grayish-brown and dark grayish-brown (10YR 3/2 and 10YR 4/2) gravelly silt loam; moderate fine granular parting to weak medium subangular blocky structure; very friable; $15 \%$ coarse fragments; clear smooth boundary

E1 11-20 cm; grayish-brown (10YR 5/2) silt loam; weak medium subangular blocky structure; very friable; clear smooth boundary

E2 20-49 cm; light yellowish-brown (10YR 6/4) silt loam; weak medium subangular blocky structure; very friable; $10 \%$ coarse fragments; clear smooth boundary

$\mathrm{Bt} \quad 49-61 \mathrm{~cm}$; brown (7.5YR 5/4) loam or clay loam; moderate medium subangular blocky structure; friable; $10 \%$ coarse fragments; clear smooth boundary

2Bt 61-87 cm; yellowish-red (5YR 5/6) clay; moderate medium subangular blocky structure; firm; $5 \%$ coarse fragments; clear smooth boundary

2BC 87-100 cm; yellowish-red (5YR 5/8) cherty clay loam; moderate medium and fine subangular blocky structure; friable; $15 \%$ coarse fragments; gradual smooth boundary

2C 100-132 cm; strong brown (7.5YR 5/8) and light yellowish-brown (2.5Y 6/4) saprolitic materials that easily crush to cherty clay; massive; friable 


\section{ANDERSON Site 32}

Location:

Classification:

Geomorphic position:

Slope and aspect:

Parent material(s):

Vegetation:

Described by:

Sampling crew:

Time since last rain:

Present weather:

Date:
Powell Quadrangle, Chestnut Ridge; $1200 \mathrm{ft}$ southwest from water tank, and $100 \mathrm{ft}$ south of access road, $800 \mathrm{ft}$ northeast of Heiskell Road

Typic Paleudults; clayey, mixed, thermic

Midslope

$13 \%, 270^{\circ}$ west

Surficial colluvium and residuum of the Copper Ridge Formation

Chestnut oak, hickory, red oak, sassafras, hickory

J.L. Branson

Lara Buckner, Rick Livingston, and Janice Branson

1 day

$40^{\circ} \mathrm{F}$ and clear and sunny

December 5, 1992

\section{Soil Description:}

Oi $\quad 10-2 \mathrm{~cm}$; hardwood leaf litter .

$\mathrm{Oa} \quad 2-0 \mathrm{~cm}$; highly decomposed leaves, twigs, and roots

A $\quad 0-7 \mathrm{~cm}$; dark brown (10YR 3/3) cherty silt loam; weak fine granular structure; very friable; $50 \%$ chert fragments; clear smooth boundary

E 7-38 cm; light yellowish-brown (10YR 6/4) silt loam; weak fine subangular blocky structure; very friable; clear smooth boundary

$\mathrm{BE} \quad 38-45 \mathrm{~cm}$; strong brown (7.5YR 5/8) silty clay loam; moderate fine subangular blocky structure; friable; $10-15 \%$ chert; clear wavy boundary

2Bt1 45-91 cm; yellowish-red (5YR 5/8) clay; moderate medium subangular blocky structure; friable; $5-10 \%$ chert fragments; gradual smooth boundary

2Bt2 91-120 cm; yellowish-red (5YR 5/8) clay; moderate medium subangular blocky structure; firm; $5-10 \%$ chert fragments; clear wavy boundary

2BC 120-144 cm; yellowish-red (5YR 5/8) gravelly clay; moderate medium subangular blocky structure; $20-30 \%$ chert; friable 


\section{ANDERSON Site 33}

Location:

Classification:

Geomorphic position:

Slope and aspect:

Parent material(s):

Vegetation:

Described by:

Sampling crew:

Time since last rain:

Present weather:

Date:
Powell Quadrangle, Pine Ridge; $700 \mathrm{ft}$ southwest of the water tank, $100 \mathrm{ft}$ south of old access road, $900 \mathrm{ft}$ northeast of Heiskell Road

Typic Paleudults; clayey, mixed, thermic

Upper portion of long slope

$10 \%, 240^{\circ}$ southwest

Copper Ridge Formation

Red oak, chestnut oak, hickory, dogwood, sassafras

J.L. Branson

Mike Morris, Steve Travis, and Janice Branson

1 day

$40^{\circ} \mathrm{F}$

December 5, 1992

\section{Soil Description:}

$\mathrm{Oi} \quad 7-2 \mathrm{~cm}$; leaf litter

Oa 2-0 cm; decomposed leaves

A $\quad 0-3 \mathrm{~cm}$; very dark grayish-brown (10YR $3 / 2)$ extremely cherty silt loam; weak fine granular structure; very friable; $70 \%$ chert fragments; abrupt smooth boundary

E1 3-21 cm; light yellowish-brown (2.5Y 6/4) very cherty silt loam; weak medium subangular blocky structure; very friable; $50 \%$ chert fragments; clear smooth boundary

E2 21-36 cm; brownish-yellow (10YR 6/6) silt loam; weak medium subangular blocky structure; friable; $10 \%$ chert fragments; gradual smooth boundary

Bt1 36-46 cm; reddish-yellow (7.5 YR 6/8) cherty clay loam; moderate medium subangular blocky structure; friable; $25 \%$ chert fragments; clear smooth boundary

2Bt2 46-62 cm; yellowish-red (5YR 5/8) cherty clay loam; moderate medium subangular blocky structure; friable; $15 \%$ chert fragments; clear smooth boundary

2Bt3 62-75 cm; yellowish-red (5YR 5/8) cherty clay; moderate medium subangular blocky structure; firm; $25 \%$ chert fragments; gradual wavy boundary

2BC 75-117 cm; yellowish-red (5YR 5/8) clay; moderate medium subangular blocky structure; firm 


\section{ANDERSON Site 34}

Location: $\quad$ Powell Quadrangle, Chestnut Ridge; $500 \mathrm{ft}$ northwest of water

Classification:

Geomorphic position:

Slope and aspect:

Parent material(s):

Vegetation:

Described by:

Sampling crew:

Time since last rain:

Present weather:

Date: tank, $100 \mathrm{ft}$ north of access road, $1400 \mathrm{ft}$ northeast from Heiskell Road, $500 \mathrm{ft}$ north from Anderson Site 33

Typic Paleudults; fine-loamy, mixed, thermic

Upland, midslope

$14 \%, 290^{\circ}$ west

Surficial colluvium and residuum of the Copper Ridge Formation

Chestnut oak, sassafras, dogwood, red maple

J.L. Branson

Mike Timpson, Mike Haarbauer, Janice Branson

1 day

$35^{\circ} \mathrm{F}$ and clear

December 5, 1992

\section{Soil Description:}

Oi $\quad 5-0 \mathrm{~cm}$; leaf litter

A $\quad$ 0-7 cm; brown (10YR 4/3) silt loam; weak medium granular structure; very friable; abrupt smooth boundary

E1 7-27 cm; yellowish-brown (10YR 5/4) silt loam; weak medium subangular blocky structure; very friable; clear smooth boundary

E2 27-38 cm; brownish-yellow (10YR 6/8) silt loam; weak medium, subangular blocky structure; friable; clear wavy boundary

Bt1 38-52 cm; reddish-yellow (7.5YR 6/8) silty clay loam; moderate medium subangular blocky structure; friable; few chert fragments; clear smooth boundary

2Bt2 52-94 cm; reddish-yellow (5YR 6/8) clay loam; moderate medium subangular blocky; friable; $13 \%$ chert fragments; gradual smooth boundary

2BC 94-140 cm; yellowish-red (5YR 5/8) clay; weak medium subangular blocky structure; firm 


\section{ANDERSON Site 35}

Location:

Classification:

Geomorphic position:

Slope and aspect:

Parent material(s):

Vegetation:

Described by:

Sampling crew:

Time since last rain:

Present weather:

Date:
Powell Quadrangle, Chestnut Ridge; $700 \mathrm{ft}$ northwest of water tank, $150 \mathrm{ft}$ north of the access road, $1500 \mathrm{ft}$ northeast from Heiskell Road

Typic Paleudults; fine-loamy, mixed, thermic

Upland, upper sideslope

$8 \%, 20^{\circ}$ north

Colluvium and residuum from the Copper Ridge Formation

Chestnut oak, sassafras, scarlet oak, white oak, red maple

J.L. Branson

Mike Morris, Steve Travis, and Janice Branson

1 day

$35^{\circ} \mathrm{F}$ and clear

December 5, 1992

\section{Soil Description:}

$\mathrm{Oi} \quad 5-0 \mathrm{~cm}$; leaf litter

A $\quad 0-12 \mathrm{~cm}$; brown (10YR 4/3) silt loam; weak medium subangular blocky structure; very friable; clear smooth boundary

E $\quad 12-27 \mathrm{~cm}$; dark yellowish-brown (10YR 4/6) silt loam; weak medium subangular blocky structure; very friable; clear smooth boundary

Bt1 27-46 cm; strong brown (7.5YR 5/6) silty clay loam; moderate medium subangular blocky structure; friable; clear smooth boundary

Bt2 46-62 cm; strong brown (7.5YR 5/8) clay loam; moderate medium subangular blocky structure; friable; clear smooth boundary

2Bt3 62-85 cm; yellowish-red (5YR 5/8) cherty clay loam; moderate, medium subangular blocky structure; friable; $20 \%$ chert fragments; gradual smooth boundary

2C $\quad 85-125 \mathrm{~cm}$; red (2.5YR 4/8) clay; massive; firm 


\section{ANDERSON Site 36}

Location:

Classification:

Geomorphic position:

Slope and aspect:

Parent material(s):

Vegetation:

Described by:

Sampling crew:

Time since last rain:

Present weather:

Date:
Powell Quadrangle, Chestnut Ridge; $700 \mathrm{ft}$ northwest of water tank, $200 \mathrm{ft}$ north of access road, $1600 \mathrm{ft}$ northeast from Heiskell Road

Typic Paleudults; fine-loamy, mixed, thermic

Midslope just above a bench

$32 \%, 350^{\circ}$ north

Surficial colluvium and residuum of the Copper Ridge Formation Yellow poplar, white oak, hickory, red oak

J.L. Branson

Lara Buckner, Rick Livingston, and Janice Branson

1 day

$35^{\circ} \mathrm{F}$ and clear

December 5, 1992

\section{Soil Description:}

Oi $\quad 7-2 \mathrm{~cm}$; leaf litter

Oa $\quad 2-0 \mathrm{~cm}$; decomposed leaves, twigs

A $\quad 0-8 \mathrm{~cm}$; dark yellowish-brown (10YR 3/4) silt loam; weak medium granular structure; very friable; $5 \%$ chert fragments; clear smooth boundary

E $\quad 8-26 \mathrm{~cm}$; brown (10YR 5/3) silt loam; weak medium subangular blocky structure; very friable; $5 \%$ chert fragments; clear smooth boundary

BE 26-42 cm; yellowish-brown (10YR 5/4) silt loam; weak medium subangular blocky structure; friable; $5 \%$ chert fragments; clear wavy boundary

Bt $\quad 42-75 \mathrm{~cm}$; yellowish-red (5YR 5/8) clay loam; moderate medium subangular blocky structure; friable; $10 \%$ chert fragments; clear wavy boundary

BC 75-90 cm; yellowish-red (5YR 5/8) very cherty clay loam; moderate medium subangular blocky structure; $60 \%$ chert fragments; firm 


\section{ANDERSON Site 37}

Location:

Classification:

Geomorphic position:

Slope and aspect:

Parent material(s):

Vegetation:

Described by:

Sampling crew:

Time since last rain:

Present weather:

Date:
Big Ridge Park Quadrangle, Chestnut Ridge; $800 \mathrm{ft}$ west of Mt. Hebron Church, $700 \mathrm{ft}$ southeast from Hinds Creek Road, $250 \mathrm{ft}$ southwest from Anderson Site 38

Typic Paleudults; clayey, mixed, thermic

Upland, upper sideslope

$16 \%, 280^{\circ}$ west

Copper Ridge Formation

Cedar, red maple, oaks

J.L. Branson

Dr. J.T. Ammons, and Janice Branson

2 days

$35^{\circ} \mathrm{F}$ and overcast and cloudy

December 12, 1992

\section{Soil Description:}

Oi $\quad 5-0 \mathrm{~cm}$; leaf litter

Ap 0-19 cm; dark yellowish-brown (10YR 4/4) cherty silt loam; moderate medium subangular blocky structure; friable; $15 \%$ chert fragments; clear smooth boundary

Bt1 19-33 cm; yellowish-red (5YR 5/8) clay loam; moderate medium subangular blocky structure; friable; clear smooth boundary

Bt2 33-80 cm; yellowish-red (5YR 5/8) clay; strong medium subangular blocky structure; firm; $10 \%$ chert fragments; gradual smooth boundary

Bt3 80-100 cm; red (2.5YR 4/6) clay; strong medium subangular blocky structure; firm; clear smooth boundary

BC $\quad 100-123 \mathrm{~cm}$; red (2.5YR 4/6) clay; moderate medium subangular blocky structure; firm 


\section{ANDERSON Site 38}

Location:

Classification:

Geomorphic position:

Slope and aspect:

Parent material(s):

Vegetation:

Described by:

Sampling crew:

Time since last rain:

Present weather:

Date:
Big Ridge Park Quadrangle, Chestnut Ridge; $550 \mathrm{ft}$ west from Mt. Hebron Church, and $650 \mathrm{ft}$ southeast from Hinds Creek Road

Typic Paleudults; clayey, mixed, thermic

Upland, upper slope

$13 \%, 20^{\circ}$ north

Surficial colluvium and residuum of the Copper Ridge Formation Red cedar, privet, red maple, red and black oaks

J.L. Branson

Dr. J.T. Ammons, and Janice Branson

2 days

$35^{\circ} \mathrm{F}$ and overcast and cloudy

December 12, 1992

\section{Soil Description:}

Oi $\quad 5-0 \mathrm{~cm}$; leaf litter, roots

A $\quad 0-12 \mathrm{~cm}$; dark yellowish-brown (10YR 4/4) cherty silt loam; moderate medium granular structure; very friable; $20 \%$ chert fragments; clear smooth boundary

E 12-29 cm; yellowish-brown (10YR 5/4) silt loam; weak medium, subangular blocky structure; very friable; $10 \%$ chert fragments; gradual smooth boundary

Bt1 29-40 cm; yellowish-red (5YR 5/6) cherty silty clay loam; weak fine subangular blocky structure; friable; $15 \%$ coarse fragments; clear smooth boundary

$2 \mathrm{Bt} 240-80 \mathrm{~cm}$; red (2.5YR 4/6) clay; moderate medium, subangular blocky structure; firm; $5 \%$ chert fragments; gradual smooth boundary

2BC 80-120 cm; red (2.5YR 4/6) clay; moderate medium subangular blocky structure; firm 


\section{ANDERSON Site 39}

Location:

Classification:

Geomorphic position:

Slope and aspect:

Parent material(s):

Vegetation:

Described by:

Sampling crew:

Time since last rain:

Present weather:

Date:
Big Ridge Park Quadrangle, Chestnut Ridge; $1000 \mathrm{ft}$ south from Mount Zion Church, $1000 \mathrm{ft}$ southeast from Hinds Creek Valley Road

Typic Paleudults; clayey, mixed, thermic Midslope on a short bench

$5 \%, 310^{\circ}$ northwest

Surficial old alluvium and residuum of the Copper Ridge Formation

Red oak, hickory, red maple

J.L. Branson

Mike Timpson, Rick Livingston, Sonja Entrekin, and Janice Branson

1 day

$20^{\circ} \mathrm{F}$ and cold with wind chill and snow flurries

December 11, 1992

\section{Soil Description:}

$\mathrm{Oi} \quad 6-0 \mathrm{~cm}$; leaves

A $\quad 0-8 \mathrm{~cm}$; dark yellowish-brown (10YR 3/4) cherty silt loam; moderate medium granular structure; very friable; $20 \%$ coarse fragments; clear smooth boundary

BA 8-22 cm; strong brown (7.5YR 4/6) silt loam; weak medium subangular blocky structure; very friable; $5 \%$ chert fragments; clear smooth boundary

Bt1 22-34 cm; yellowish-red (5YR 5/6) clay loam; weak medium subangular blocky structure; friable; $5 \%$ chert fragments; clear smooth boundary

Bt2 34-60 cm; red (2.5YR 4/6) clay; moderate medium subangular blocky structure; firm; 5\% chert fragments; gradual smooth boundary

BC 74-114 cm; red (2.5YR 4/6) clay; weak moderate subangular blocky structure; firm 


\section{ANDERSON Site 40}

Location:

Classification:

Geomorphic position:

Slope and aspect:

Parent material(s):

Vegetation:

Described by:

Sampling crew:

Time since last rain:

Present weather:

Date:
Big Ridge Park Quadrangle, Chestnut Ridge; $1200 \mathrm{ft}$ south from Mt. Zion Church, $1500 \mathrm{ft}$ southeast from Hinds Creek Valley Road

Typic Paleudults; clayey, mixed, thermic

Midslope upland

$21 \%, 10^{\circ}$ north

Surficial old alluvium and residuum of the Copper Ridge Formation

Virginia Pine, ferns, red maple

J.L. Branson

Rick Livingston, Sonja Entrekin, Mike Timpson, and Janice Branson

1 day

Cold, wind chill $20^{\circ} \mathrm{F}$ and snow flurries

December 11, 1992

Soil Description:

Oi $\quad 5-0 \mathrm{~cm}$; leaves, pine needles

A 0-19 cm; dark yellowish-brown (10YR 4/4) cherty silt loam; weak medium subangular blocky structure; very friable; $15 \%$ chert fragments; clear smooth boundary

Bt1 19-36 cm; strong brown (7.5YR 4/6) clay loam; weak medium subangular blocky structure; friable; $5 \%$ chert fragments; clear smooth boundary

2Bt2 36-72 cm; yellowish-red (5YR 4/6) clay; moderate medium subangular blocky structure; firm; $10 \%$ chert fragments; gradual smooth boundary

2BC 72-104 cm; yellowish-red (5YR 5/6) clay; weak medium subangular blocky structure; firm 


\section{ANDERSON Site 41}

Location:

Classification:

Geomorphic position:

Slope and aspect:

Parent material(s):

Vegetation:

Described by:

Sampling crew:

Time since last rain:

Present weather:

Date:
Big Ridge Park Quadrangle, Chestnut Ridge; $1500 \mathrm{ft}$ south from Mt. Zion Church, $1700 \mathrm{ft}$ southeast from Hinds Creek Valley Road

Typic Paleudults; clayey, mixed, thermic

Midslope

$18 \%, 1 / 2^{\circ}$ north

Surficial colluvium and residuum of the Copper Ridge Formation

Red Maple, Ferns, Dogwood, Virginia Pine

J.L. Branson

Mike Timpson, Rick Livingston, Sonja Entrekin, and Janice Branson

1 day

$20^{\circ} \mathrm{F}$ wind chill, cold, and snow flurries

December 11, 1992

Soil Description:

$\mathrm{Oi} \quad 5-0 \mathrm{~cm}$; leaf litter, pine needles

A $\quad 0-11 \mathrm{~cm}$; dark yellowish-brown (10YR 3/4) cherty silt loam; moderate medium granular structure; very friable; $20 \%$ chert fragments; clear smooth boundary

AB $\quad 11-27 \mathrm{~cm}$; brown (7.5YR 4/4) silt loam; weak medium, subangular blocky structure; friable; clear smooth boundary

Bt1 27-38 cm; strong brown (7.5YR 4/6) clay loam; moderate medium, subangular blocky structure; friable; $3 \%$ chert fragments; clear smooth boundary

2Bt2 38-70 cm; yellowish-red (5YR 4/6) clay; moderate coarse subangular blocky structure; firm; $5 \%$ chert fragments; gradual smooth boundary

2BC 70-125 cm; yellowish-red (5YR 5/6) clay; weak medium subangular blocky structure; friable 


\section{ANDERSON Site 42}

Location:

Classification:

Geomorphic position:

Slope and aspect:

Parent material(s):

Vegetation:

Described by:

Sampling crew:

Time since last rain:

Present weather:

Date:
Big Ridge Park Quadrangle, Chesnut Ridge; $1200 \mathrm{ft}$ south of Mount Zion Church, $1800 \mathrm{ft}$ southeast from Hinds Creek Valley Road

Typic Paleudults; fine-loamy, siliceous, thermic

Upper portion of a long slope

$19 \%, 1 / 2^{\circ}$ north

Surficial colluvium and residuum of the Copper Ridge Formation

Virginia Pine, Red Maple, Ferns

J.L. Branson

Mike Timpson, Rick Livingston, Sonja Entrekin, and Janice Branson

1 day

$20^{\circ} \mathrm{F}$, cold, wind chill with snow flurries

December 11, 1992

\section{Soil Description:}

Oi $\quad 5-0 \mathrm{~cm}$; leaves, pine needles

A $\quad 0-24 \mathrm{~cm}$; dark brown (10YR 3/3) silt loam; weak medium subangular structure; very friable; $5 \%$ chert fragments; clear smooth boundary

Bt1 24-39 cm; strong brown (7.5YR 4/6) loam; weak medium subangular blocky structure; friable; clear smooth boundary

Bt2 39-53 cm; strong brown (7.5YR 5/6) cherty clay loam; moderate medium subangular blocky structure; friable; $15 \%$ chert fragments; clear smooth boundary

2Bt3 53-81 cm; yellowish-red (5YR 5/6) clay; moderate medium subangular blocky structure; firm; $5 \%$ chert fragments; gradual smooth boundary

2BC 81-93 + cm; yellowish-red (5YR 5/8) cherty clay; massive; 20\% coarse fragments; firm 


\section{A.7 SITE LOCATIONS}

\section{A.1.1 Oak Ridge Reservation Site Locations}

The following locations are approximate. Key: DG = Dismal Gap, NOL = Nolichucky, $\mathrm{CR}=$ Copper Ridge, $\mathrm{CH}=$ Chepultepec, $\mathrm{BV}=$ Chickamauga-Bethel Valley, and $\mathrm{EF}=$ Chickamauga, East Fork or K-25 section.

\begin{tabular}{|c|c|c|c|c|c|}
\hline Site & North & East & Site & North & East \\
\hline $2 \mathrm{DG}$ & 29300 & 10300 & $45 \mathrm{CR}$ & 27600 & 18400 \\
\hline $3 \mathrm{NOL}$ & 29300 & 11200 & $50 \mathrm{CH}$ & 26600 & 19800 \\
\hline 5 NOL & 29300 & 15300 & $51 \mathrm{CR}$ & 28400 & 21600 \\
\hline $10 \mathrm{DG}$ & 30200 & 21460 & $52 \mathrm{CH}$ & 26600 & 20200 \\
\hline $11 \mathrm{DG}$ & 29800 & 21460 & $53 \mathrm{CH}$ & 26200 & 22400 \\
\hline $13 \mathrm{NOL}$ & 29480 & 23220 & $54 \mathrm{CR}$ & 28200 & 22500 \\
\hline 15 NOL & 29700 & 25720 & $55 \mathrm{CR}$ & 28400 & 23400 \\
\hline $16 \mathrm{NOL}$ & 29700 & 25928 & $58 \mathrm{CR}$ & 28000 & 25000 \\
\hline $19 \mathrm{DG}$ & 33000 & 28530 & $59 \mathrm{CR}$ & 28000 & 26600 \\
\hline $21 \mathrm{NOL}$ & 29700 & 30940 & $60 \mathrm{CR}$ & 27800 & 27400 \\
\hline $22 \mathrm{DG}$ & 30520 & 31250 & $62 \mathrm{CR}$ & 27900 & 29700 \\
\hline $23 \mathrm{NOL}$ & 29800 & 31830 & $64 \mathrm{CR}$ & 27200 & 30000 \\
\hline 24 NOL & 29900 & 31980 & $66 \mathrm{CH}$ & 27000 & 31000 \\
\hline $25 \mathrm{NOL}^{\circ}$ & 29800 & 32290 & $68 \mathrm{CH}$ & 26800 & 31700 \\
\hline $26 \mathrm{DG}$ & 30000 & 34370 & $73 \mathrm{CH}$ & 26800 & 34000 \\
\hline 27 DG & 30100 & 34990 & $74 \mathrm{CH}$ & 27200 & 34000 \\
\hline 28 NOL & 29500 & 34580 & $75 \mathrm{CR}$ & 27200 & 35400 \\
\hline 31 NOL & 29900 & 35820 & $77 \mathrm{CH}$ & 26600 & 35200 \\
\hline $32 \mathrm{DG}$ & 30200 & 36760 & $78 \mathrm{CH}$ & 26700 & 35600 \\
\hline 33 DG & 30000 & 36970 & $83 \mathrm{CR}$ & 27800 & 37600 \\
\hline $35 \mathrm{DG}$ & 30000 & 37280 & $85 \mathrm{CH}$ & 26000 & 37800 \\
\hline $41 \mathrm{DG}$ & 30200 & 39570 & $86 \mathrm{CH}$ & 26000 & 38200 \\
\hline 42 NOL & 30000 & 40500 & $90 \mathrm{CH}$ & 28000 & 40800 \\
\hline $43 \mathrm{DG}$ & 30400 & 41460 & $91 \mathrm{CR}$ & 27000 & 41000 \\
\hline $93 \mathrm{BV}$ & 21600 & 26500 & $118 \mathrm{EF}$ & 39800 & 23000 \\
\hline 99 BV & 22000 & 27750 & $119 \mathrm{EF}$ & 40000 & 23000 \\
\hline $100 \mathrm{BV}$ & 21600 & 28000 & $120 \mathrm{EF}$ & 39800 & 23250 \\
\hline $101 \mathrm{BV}$ & 21250 & 31550 & $121 \mathrm{EF}$ & 42100 & 26200 \\
\hline $102 \mathrm{BV}$ & 21300 & 34900 & $122 \mathrm{EF}$ & 41300 & 27250 \\
\hline $103 \mathrm{BV}$ & 20800 & 36400 & $123 \mathrm{EF}$ & 41200 & 29750 \\
\hline 104 BV & 21800 & 44000 & $124 \mathrm{EF}$ & 40900 & 30400 \\
\hline $108 \mathrm{BV}$ & 22250 & 45000 & $125 \mathrm{EF}$ & 40600 & 29800 \\
\hline $110 \mathrm{BV}$ & 22400 & 45800 & $126 \mathrm{EF}$ & 38950 & 28750 \\
\hline $115 \mathrm{BV}$ & 22500 & 46700 & $127 \mathrm{EF}$ & 39500 & 28650 \\
\hline $116 \mathrm{BV}$ & 22100 & 49000 & $128 \mathrm{EF}$ & 38900 & 29600 \\
\hline $117 \mathrm{BV}$ & 22500 & 49000 & $129 \mathrm{EF}$ & 36100 & 26300 \\
\hline
\end{tabular}


A7.2 Latitudes and Longitudes for Roane and Anderson Counties

Site number $\quad$ Latitude

ANDERSON COUNTY

1

3

4

5

9

10

11

12

19

20

21

22

31

32

33

34

35

36

37

38

39

40

41

42

$$
\begin{aligned}
& 36^{\circ} 11^{\prime} 48^{\prime \prime} \\
& 36^{\circ} 11^{\prime} 40^{\prime \prime} \\
& 36^{\circ} 06^{\prime} 28^{\prime \prime} \\
& 36^{\circ} 11^{\prime} 29^{\prime \prime} \\
& 36^{\circ} 06^{\prime} 00^{\prime \prime} \\
& 36^{\circ} 08^{\prime} 47^{\prime \prime} \\
& 36^{\circ} 09^{\prime} 10^{\prime \prime} \\
& 36^{\circ} 10^{\prime} 32^{\prime \prime} \\
& 36^{\circ} 11^{\prime} 01^{\prime \prime} \\
& 36^{\circ} 11^{\prime} 32^{\prime \prime} \\
& 36^{\circ} 11^{\prime} 36^{\prime \prime} \\
& 36^{\circ} 10^{\prime} 34^{\prime \prime} \\
& 36^{\circ} 06^{\prime} 19^{\prime \prime} \\
& 36^{\circ} 06^{\prime} 21^{\prime \prime} \\
& 36^{\circ} 06^{\prime} 19^{\prime \prime} \\
& 36^{\circ} 06^{\prime} 20^{\prime \prime} \\
& 36^{\circ} 06^{\prime} 25^{\prime \prime} \\
& 36^{\circ} 06^{\prime} 24^{\prime \prime} \\
& 36^{\circ} 10^{\prime} 18^{\prime \prime} \\
& 36^{\circ} 10^{\prime} 20^{\prime \prime} \\
& 36^{\circ} 11^{\prime} 04^{\prime \prime} \\
& 36^{\circ} 11^{\prime} 01^{\prime} \\
& 36^{\circ} 10^{\prime} 58^{\prime \prime} \\
& 36^{\circ} 11^{\prime} 01^{\prime \prime}
\end{aligned}
$$

Longitude
$83^{\circ} 56^{\prime} 57^{\prime \prime}$
$83^{\circ} 57^{\prime} 05^{\prime \prime}$
$83^{\circ} 57^{\prime} 18^{\prime \prime}$
$83^{\circ} 57^{\prime} 22^{\prime \prime}$
$84^{\circ} 03^{\prime} 42^{\prime \prime}$
$84^{\circ} 00^{\prime} 58^{\prime \prime}$
$84^{\circ} 00^{\prime} 36^{\prime \prime}$
$83^{\circ} 58^{\prime} 45^{\prime \prime}$
$83^{\circ} 58^{\prime} 14^{\prime \prime}$
$83^{\circ} 57^{\prime} 14^{\prime \prime}$
$83^{\circ} 57^{\prime} 14^{\prime \prime}$
$83^{\circ} 58^{\prime} 14^{\prime \prime}$
$84^{\circ} 02^{\prime} 55^{\prime \prime}$
$84^{\circ} 02^{\prime} 54^{\prime \prime}$
$84^{\circ} 02^{\prime} 50^{\prime \prime}$
$84^{\circ} 02^{\prime} 47^{\prime \prime}$
$84^{\circ} 02^{\prime} 51^{\prime \prime}$
$84^{\circ} 02^{\prime} 15^{\prime \prime}$
$83^{\circ} 58^{\prime} 36^{\prime \prime}$
$83^{\circ} 58^{\prime} 34^{\prime \prime}$
$83^{\circ} 57^{\prime} 06^{\prime \prime}$
$83^{\circ} 57^{\prime} 04^{\prime \prime}$
$83^{\circ} 57^{\prime} 01^{\prime \prime}$
$83^{\circ} 56^{\prime} 57^{\prime \prime}$

\section{ROANE COUNTY}

$\begin{array}{ll}3 & 35^{\circ} 43^{\prime} 02^{\prime \prime} \\ 7 & 35^{\circ} 40^{\prime} 41^{\prime \prime} \\ 8 & 35^{\circ} 40^{\prime} 38^{\prime \prime} \\ 9 & 35^{\circ} 42^{\prime} 53^{\prime \prime} \\ 10 & 35^{\circ} 44^{\prime} 41^{\prime \prime} \\ 13 & 35^{\circ} 44^{\prime} 22^{\prime \prime} \\ 14 & 35^{\circ} 44^{\prime} 19^{\prime \prime} \\ 17 & 35^{\circ} 41^{\prime} 31^{\prime \prime} \\ 19 & 35^{\circ} 42^{\prime} 50^{\prime \prime} \\ 20 & 35^{\circ} 42^{\prime} 47^{\prime \prime} \\ 21 & 35^{\circ} 42^{\prime} 44^{\prime \prime} \\ 22 & 35^{\circ} 42^{\prime} 46^{\prime \prime} \\ 33 & 35^{\circ} 44^{\prime} 25^{\prime \prime} \\ 34 & 35^{\circ} 440^{\prime \prime} \\ 35 & 35^{\circ} 44^{\prime} 33^{\prime \prime} \\ 39 & 35^{\circ} 42^{\prime} 00^{\prime \prime} \\ 40 & 35^{\circ} 41^{\prime} 59^{\prime \prime}\end{array}$

$84^{\circ} 35^{\prime} 07^{\prime \prime}$

$84^{\circ} 36^{\prime} 55^{\prime \prime}$

$84^{\circ} 36^{\prime} 53^{\prime \prime}$

$84^{\circ} 35^{\prime} 14^{\prime \prime}$

$84^{\circ} 33^{\prime} 37^{\prime \prime}$

$84^{\circ} 33^{\prime} 57^{\prime \prime}$

$84^{\circ} 33^{\prime} 59^{\prime \prime}$

$84^{\circ} 36^{\prime} 21^{\prime \prime}$

$84^{\circ} 35^{\prime} 16^{\prime \prime}$

$84^{\circ} 35^{\prime} 19^{\prime \prime}$

$84^{\circ} 35^{\prime} 22^{\prime \prime}$

$84^{\circ} 35^{\prime} 18^{\prime \prime}$

$84^{\circ} 33^{\prime} 33^{\prime \prime}$

$84^{\circ} 33^{\prime} 30^{\prime \prime}$

$84^{\circ} 33^{\prime} 26^{\prime \prime}$

$84^{\circ} 35^{\prime} 30^{\prime \prime}$

$84^{\circ} 35^{\prime} 30^{\prime \prime}$ 


\section{A-147}

\section{Site number}

41

42

43

44

45

46

47 $\underline{\text { Latitude }}$

$35^{\circ} 42^{\prime} 00^{\prime \prime}$

$35^{\circ} 40^{\prime} 14^{\prime \prime}$

$35^{\circ} 40^{\prime} 16^{\prime \prime}$

$35^{\circ} 40^{\prime} 16^{\prime \prime}$

$35^{\circ} 40^{\prime} 12^{\prime \prime}$

$35^{\circ} 40^{\prime} 09^{\prime \prime}$

$35^{\circ} 40^{\prime} 06^{\prime \prime}$
Longitude

$84^{\circ} 35^{\prime} 28^{\prime \prime}$

$84^{\circ} 36^{\prime} 42^{\prime \prime}$

$84^{\circ} 36^{\prime} 44^{\prime \prime}$

$84^{\circ} 36^{\prime} 48^{\prime \prime}$

$84^{\circ} 36^{\prime} 50^{\prime \prime}$

$84^{\circ} 36^{\prime} 54^{\prime \prime}$

$84^{\circ} 36^{\prime} 58^{\prime \prime}$ 
Appendix B

SCREENING ANALYSIS DATA 



\section{B-3}

Table B.1. Volatile organic analysis results for soil samples

\begin{tabular}{lcc}
\hline & A & A horizon \\
Analysis & Units & horizon \\
\end{tabular}

Location=AND; Formation=COPPER RIDGE; Site=31; Samp id=2256; Dup id=' '; Phase=2

\begin{tabular}{|c|c|c|}
\hline 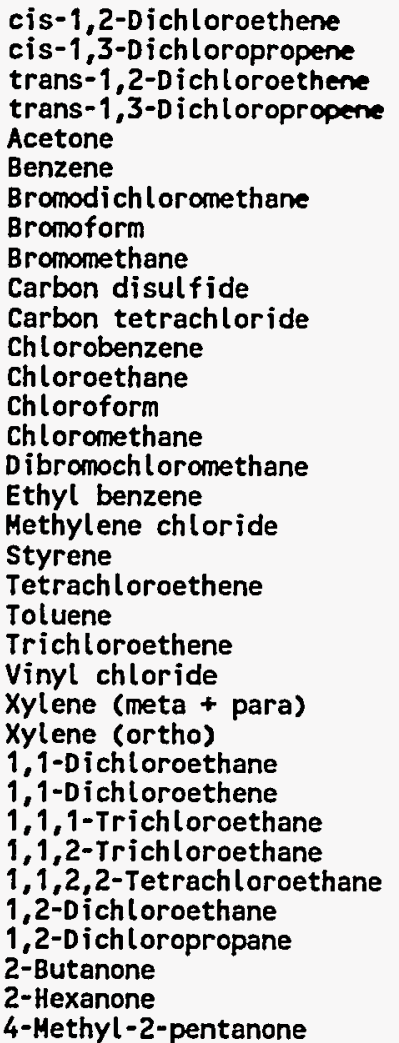 & $\begin{array}{l}\mu g / k g \\
\mu g / k g \\
\mu g / k g \\
\mu g / k g \\
\mu g / k g \\
\mu g / k g \\
\mu g / k g \\
\mu g / k g \\
\mu g / k g \\
\mu g / k g \\
\mu g / k g \\
\mu g / k g \\
\mu g / k g \\
\mu g / k g \\
\mu g / k g \\
\mu g / k g \\
\mu g / k g \\
\mu g / k g \\
\mu g / k g \\
\mu g / k g \\
\mu g / k g \\
\mu g / k g \\
\mu g / k g \\
\mu g / k g \\
\mu g / k g \\
\mu g / k g \\
\mu g / k g \\
\mu g / k g \\
\mu g / k g \\
\mu g / k g \\
\mu g / k g \\
\mu g / k g \\
\mu g / k g \\
\mu g / k g \\
\mu g / k g\end{array}$ & $\begin{array}{r}5 \\
5 \\
5 \\
5 \\
18 \\
5 \\
5 \\
5 \\
10 \\
5 \\
5 \\
5 \\
10 \\
5 \\
10 \\
5 \\
5 \\
5 \\
5 \\
5 \\
5 \\
5 \\
10 \\
5 \\
5 \\
5 \\
5 \\
5 \\
5 \\
5 \\
5 \\
5\end{array}$ \\
\hline
\end{tabular}

Location=AND; Formation=COPPER RIDGE; Site=32; Samp id=2258; Dup id=' '; Phase=2

\begin{tabular}{|c|c|c|}
\hline $\begin{array}{l}\text { cis-1,2-Dichloroethene } \\
\text { cis-1,3-Dichloropropene } \\
\text { trans-1,2-Dichloroethene } \\
\text { trans-1,3-Dichloropropene } \\
\text { Acetone } \\
\text { Benzene } \\
\text { Bromodichloromethane } \\
\text { Bromoform } \\
\text { Bromomethane } \\
\text { Carbon disulfide } \\
\text { Carbon tetrachloride } \\
\text { Chlorobenzene } \\
\text { Chloroethane } \\
\text { Chloroform } \\
\text { Chloromethane } \\
\text { Dibromochloromethane } \\
\text { Ethyl benzene } \\
\text { Methylene chloride } \\
\text { Styrene } \\
\text { Tetrachloroethene } \\
\text { Toluene } \\
\text { Trichloroethene } \\
\text { vinyl chloride } \\
\text { Xylene (meta + para) } \\
\text { Xylene (ortho) } \\
1,1-0 \text { ichloroethane } \\
1,1-D i c h l o r o e t h e n e \\
1,1,1-\text { Trichloroethane } \\
1,1,2-\text { Trichloroethane }\end{array}$ & $\begin{array}{l}\mu g / \mathbf{k g} \\
\mu g / \mathbf{k g} \\
\mu g / \mathbf{k g} \\
\mu g / \mathbf{k g} \\
\mu g / \mathbf{k g} \\
\mu g / \mathbf{k g} \\
\mu g / \mathbf{k g} \\
\mu g / \mathbf{k g} \\
\mu g / \mathbf{k g} \\
\mu g / \mathbf{k g} \\
\mu g / \mathbf{k g} \\
\mu g / \mathbf{k g} \\
\mu g / \mathbf{k g} \\
\mu g / \mathbf{k g} \\
\mu g / \mathbf{k g} \\
\mu g / \mathbf{k g} \\
\mu g / \mathbf{k g} \\
\mu g / \mathbf{k g} \\
\mu g / \mathbf{k g} \\
\mu g / \mathbf{k g} \\
\mu g / \mathbf{k g} \\
\mu g / \mathbf{k g} \\
\mu g / \mathbf{k g} \\
\mu g / \mathbf{k g} \\
\mu g / \mathbf{k g} \\
\mu g / \mathbf{k g} \\
\mu g / \mathbf{k g} \\
\mu g / \mathbf{k g} \\
\mu g / \mathbf{k g}\end{array}$ & $\begin{array}{r}5 \\
5 \\
5 \\
5 \\
9 \\
5 \\
5 \\
5 \\
10 \\
5 \\
5 \\
5 \\
10 \\
5 \\
10 \\
5 \\
5 \\
5 \\
5 \\
5 \\
5 \\
5 \\
10 \\
5\end{array}$ \\
\hline
\end{tabular}


B-4

Table B.1 (continued)

\begin{tabular}{|c|c|c|c|c|c|}
\hline Analysis & Units & $\begin{array}{c}\text { A } \\
\text { horizon }\end{array}$ & Qualifier & $\begin{array}{l}\text { A horizon } \\
\text { field dup }\end{array}$ & Qualifier \\
\hline $\begin{array}{l}\text { 1,1,2,2-Tetrachloroethane } \\
1,2-D \text { ichloroethane } \\
1,2-\text { Dichloropropane } \\
\text { 2-Butanone } \\
\text { 2-Hexanone } \\
\text { 4-Methyl-2-pentanone }\end{array}$ & $\begin{array}{l}\mu g / \mathrm{kg} \\
\mu \mathrm{g} / \mathrm{kg} \\
\mu \mathrm{g} / \mathrm{kg} \\
\mu \mathrm{g} / \mathrm{kg} \\
\mu \mathrm{g} / \mathrm{kg} \\
\mu \mathrm{g} / \mathrm{kg}\end{array}$ & $\begin{array}{r}5 \\
5 \\
5 \\
7 \\
50 \\
50\end{array}$ & $\begin{array}{l}\mathbf{U} \\
\mathbf{U} \\
\mathbf{U} \\
\mathbf{J} \\
\mathbf{U} \\
\mathbf{U}\end{array}$ & & \\
\hline
\end{tabular}

Location $=A N D ;$ Formation $=$ COPPER RIDGE; Site=33; Samp id=2261; Dup id=' '; Phase=2

\begin{tabular}{|c|c|c|}
\hline $\begin{array}{l}\text { cis-1,2-Dichloroethene } \\
\text { cis-1,3-Dichloropropene } \\
\text { trans-1,2-Dichloroethene } \\
\text { trans-1,3-Dichloropropene } \\
\text { Acetone } \\
\text { Benzene } \\
\text { Bromodichloromethane } \\
\text { Bromoform } \\
\text { Bromomethane } \\
\text { Carbon disulfide } \\
\text { Carbon tetrachloride } \\
\text { Chlorobenzene } \\
\text { Chloroethane } \\
\text { Chloroform } \\
\text { Chloromethane } \\
\text { Dibromochloromethane } \\
\text { Ethyl benzene } \\
\text { Methylene chloride } \\
\text { styrene } \\
\text { Tetrachloroethene } \\
\text { Toluene } \\
\text { Trichloroethene } \\
\text { Vinyl chloride } \\
\text { Xylene (meta + para) } \\
\text { Xylene (ortho) } \\
1,1 \text {-D ichloroethane } \\
1,1-D i c h l o r o e t h e n e \\
1,1,1-\text {-Trichloroethane } \\
1,1,2-\text {-Trichloroethane } \\
1,1,2,2-T \text { - Tetrachloroethane } \\
1,2-D \text { ichloroethane } \\
1,2-D \text { ichloropropane } \\
2-\text { Butanone } \\
2-\text { Hexanone } \\
4-\text { Methyl-2-pentanone }\end{array}$ & 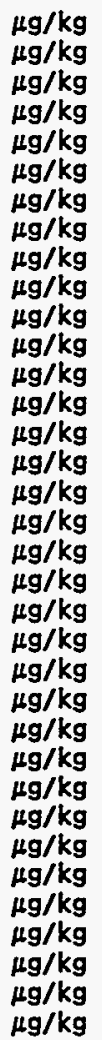 & $\begin{array}{r}5 \\
5 \\
5 \\
5 \\
100 \\
5 \\
5 \\
5 \\
10 \\
5 \\
5 \\
5 \\
10 \\
5 \\
10 \\
5 \\
5 \\
5 \\
5 \\
5 \\
5 \\
5 \\
10 \\
5 \\
5 \\
5 \\
5 \\
5 \\
5 \\
5 \\
5 \\
5 \\
100 \\
50 \\
50\end{array}$ \\
\hline
\end{tabular}

Location=AND; Formation=COPPER RIDGE; Site=34; Samp id=2264; Dup id=' '; Phase=2

\begin{tabular}{|c|c|c|}
\hline $\begin{array}{l}\text { cis-1,2-Dich loroethene } \\
\text { cis-1,3-D ichloropropene } \\
\text { trans-1,2-D ichloroethene } \\
\text { trans-1,3-D ichloropropene } \\
\text { Acetone } \\
\text { Benzene } \\
\text { Bromodichloromethane } \\
\text { Bromoform } \\
\text { Bromomethane } \\
\text { Carbon disul fide } \\
\text { Carbon tetrachloride } \\
\text { Chlorobenzene } \\
\text { Chloroethane } \\
\text { Chloroform } \\
\text { Chloromethane } \\
\text { Dibromochloromethane } \\
\text { Ethyl benzene } \\
\text { Methylene chloride } \\
\text { Styrene } \\
\text { Tetrachloroethene } \\
\text { Toluene } \\
\text { Trichloroethene } \\
\text { Vinyl chloride }\end{array}$ & 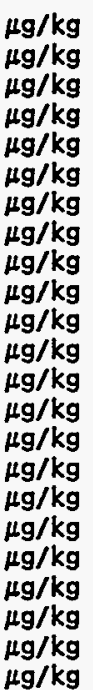 & $\begin{array}{r}5 \\
5 \\
5 \\
5 \\
100 \\
5 \\
5 \\
5 \\
10 \\
5 \\
5 \\
5 \\
10 \\
5 \\
10 \\
5 \\
5 \\
5 \\
5 \\
5 \\
5 \\
5 \\
10\end{array}$ \\
\hline
\end{tabular}


Table B.1 (continued)

\begin{tabular}{|c|c|c|c|c|c|c|c|}
\hline Analysis & Units & $\begin{array}{c}\text { A } \\
\text { horizon }\end{array}$ & Qualifie & $\begin{array}{l}\text { A horizon } \\
\text { field dup }\end{array}$ & Qualifier & & \\
\hline $\begin{array}{l}\text { Xylene (meta + para) } \\
\text { Xylene (ortho) } \\
1,1-D \text { ichloroethane } \\
1,1-\text { Dichloroethene } \\
1,1,1-\text { Trichloroethane } \\
1,1,2-\text { Trichloroethane } \\
1,1,2,2-\text { Tetrachloroethane } \\
1,2-D \text { ichloroethane } \\
1,2-\text { ichloropropane } \\
\text { 2-Butanone } \\
\text { 2-Hexanone } \\
\text { 4-Hethyl-2-pentanone }\end{array}$ & $\begin{array}{l}\mu g / \mathrm{kg} \\
\mu g / \mathrm{kg} \\
\mu g / \mathrm{kg} \\
\mu g / \mathrm{kg} \\
\mu g / \mathrm{kg} \\
\mu g / \mathrm{kg} \\
\mu g / \mathrm{kg} \\
\mu g / \mathrm{kg} \\
\mu g / \mathrm{kg} \\
\mu g / \mathrm{kg} \\
\mu g / \mathrm{kg} \\
\mu \mathrm{g} / \mathrm{kg}\end{array}$ & $\begin{array}{r}5 \\
5 \\
5 \\
5 \\
5 \\
5 \\
5 \\
5 \\
5 \\
100 \\
50 \\
50\end{array}$ & $\begin{array}{l}\mathbf{U} \\
\mathbf{U} \\
\mathbf{U} \\
\mathbf{U} \\
\mathbf{U} \\
\mathbf{U} \\
\mathbf{U} \\
\mathbf{U} \\
\mathbf{U} \\
\mathbf{U} \\
\mathbf{U}\end{array}$ & $z$ & & . & $\therefore 7$ \\
\hline
\end{tabular}

Location=AND; Formation=COPPER RIDGE; Site=35; Samp id=2267; Dup id=' '; Phase=2

\begin{tabular}{|c|c|c|}
\hline $\begin{array}{l}\text { cis-1,2-Dichloroethene } \\
\text { cis-1,3-Dichloropropene } \\
\text { trans-1,2-Dichloroethene } \\
\text { trans-1,3-Dichloropropene } \\
\text { Acetone } \\
\text { Benzene } \\
\text { Bromodichloromethane } \\
\text { Bromoform } \\
\text { Bromomethane } \\
\text { Carbon disulfide } \\
\text { Carbon tetrachloride } \\
\text { Chlorobenzene } \\
\text { Chloroethane } \\
\text { Chloroform } \\
\text { Chloromethane } \\
\text { Dibromochloromethane } \\
\text { Ethyl benzene } \\
\text { Methylene chloride } \\
\text { Styrene } \\
\text { Tetrachloroethene } \\
\text { Toluene } \\
\text { Trichloroethene } \\
\text { Vinyl chloride } \\
\text { xylene (meta + para) } \\
\text { xylene (ortho) } \\
1,1-D i c h l o r o e t h a n e \\
1,1-D i c h l o r o e t h e n e \\
1,1,1-T r i c h l o r o e t h a n e \\
1,1,2-\text { Trichloroethane } \\
1,1,2,2-T e t r a c h l o r o e t h a n e \\
1,2-D \text { ichloroethane } \\
1,2-D \text { ichloropropane } \\
2-\text { Butanone } \\
2-\text { Hexanone } \\
4-\text { Methyl-2-pentanone }\end{array}$ & $\begin{array}{l}\mu g / k g \\
\mu g / k g \\
\mu g / k g \\
\mu g / k g \\
\mu g / k g \\
\mu g / k g \\
\mu g / k g \\
\mu g / k g \\
\mu g / k g \\
\mu g / k g \\
\mu g / k g \\
\mu g / k g \\
\mu g / k g \\
\mu g / k g \\
\mu g / k g \\
\mu g / k g \\
\mu g / k g \\
\mu g / k g \\
\mu g / k g \\
\mu g / k g \\
\mu g / k g \\
\mu g / k g \\
\mu g / k g \\
\mu g / k g \\
\mu g / k g \\
\mu g / k g \\
\mu g / k g \\
\mu g / k g \\
\mu g / k g \\
\mu g / k g \\
\mu g / k g \\
\mu g / k g \\
\mu g / k g \\
\mu g / k g \\
\mu g / k g\end{array}$ & $\begin{array}{r}5 \\
5 \\
5 \\
5 \\
18 \\
5 \\
5 \\
5 \\
10 \\
5 \\
5 \\
5 \\
10 \\
5 \\
10 \\
5 \\
5 \\
5 \\
5 \\
5 \\
5 \\
5 \\
10 \\
5 \\
5 \\
5 \\
5 \\
5 \\
5 \\
5 \\
5 \\
5 \\
5 \\
51 \\
51\end{array}$ \\
\hline
\end{tabular}

Location=AND; Formation=COPPER RIDGE; Site=36; Samp id=2270; Dup id=' '; Phase=2

\begin{tabular}{|c|c|c|}
\hline $\begin{array}{l}\text { cis-1,2-D ichloroethene } \\
\text { cis-1,3-Dichloropropene } \\
\text { trans-1,2-D ichloroethene } \\
\text { trans }-1,3-0 \text { ichloropropene } \\
\text { Acetone } \\
\text { Benzene } \\
\text { Bromodichloromethane } \\
\text { Bromoform } \\
\text { Bromomethane } \\
\text { Carbon disulfide } \\
\text { Carbon tetrachloride } \\
\text { Chlorobenzene } \\
\text { Chloroethane } \\
\text { Chloroform } \\
\text { Chloromethane } \\
\text { Dibromochloromethane } \\
\text { Ethyl benzene }\end{array}$ & $\begin{array}{l}\mu g / k g \\
\mu g / k g \\
\mu g / k g \\
\mu g / k g \\
\mu g / k g \\
\mu g / k g \\
\mu g / k g \\
\mu g / k g \\
\mu g / k g \\
\mu g / k g \\
\mu g / k g \\
\mu g / k g \\
\mu g / k g \\
\mu g / k g \\
\mu g / k g \\
\mu g / k g \\
\mu g / k g\end{array}$ & $\begin{array}{r}5 \\
5 \\
5 \\
5 \\
10 \\
5 \\
5 \\
5 \\
10 \\
5 \\
5 \\
5 \\
10 \\
5 \\
10\end{array}$ \\
\hline
\end{tabular}


B-6

Table B.1 (continued)

\begin{tabular}{|c|c|c|c|c|c|}
\hline Analysis & Units & $\begin{array}{c}\text { A } \\
\text { horizon }\end{array}$ & Qualifier & $\begin{array}{l}\text { A horizon } \\
\text { field dup }\end{array}$ & Qualifier \\
\hline $\begin{array}{l}\text { Freon- } 113 \\
\text { Methylene chloride } \\
\text { Styrene } \\
\text { Tetrachloroethene } \\
\text { Toluene } \\
\text { Trichloroethene } \\
\text { Trichlorofluoromethane } \\
\text { Vinyl acetate } \\
\text { vinyl chloride } \\
\text { xylene (meta + para) } \\
\text { xylene (ortho) } \\
1,1-D \text { ichloroethane } \\
1,1-D \text { ichloroethene } \\
1,1,1-\text { Trichloroethane } \\
1,1,2-\text { Trichloroethane } \\
1,1,2,2-\text { Tetrachloroethane } \\
1,2-D \text { ichlorobenzene } \\
1,2-D \text { ichloroethane } \\
1,2-D \text { ichloropropane } \\
1,3-D \text { ichlorobenzene } \\
1,4-D \text { ichlorobenzene } \\
\text { 2-Butanone } \\
2-\text { Chloroethylvinyl ether } \\
\text { 2-Hexanone } \\
\text { 4-Methyl-2-pentanone }\end{array}$ & 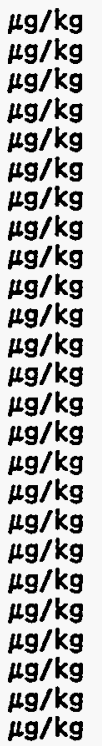 & $\begin{array}{r}5 \\
5 \\
5 \\
5 \\
5 \\
5 \\
5 \\
5 \\
10 \\
5 \\
5 \\
5 \\
5 \\
5 \\
5 \\
5 \\
5 \\
5 \\
5 \\
5 \\
5 \\
10 \\
5 \\
10 \\
10\end{array}$ & $\begin{array}{l}\mathbf{U} \\
\mathbf{U} \\
\mathbf{U} \\
\mathbf{U} \\
\mathbf{U} \\
\mathbf{U} \\
\mathbf{U} \\
\mathbf{U} \\
\mathbf{U} \\
\mathbf{U} \\
\mathbf{U} \\
\mathbf{U} \\
\mathbf{U} \\
\mathbf{U} \\
\mathbf{U} \\
\mathbf{U} \\
\mathbf{U} \\
\mathbf{U} \\
\mathbf{U} \\
\mathbf{U} \\
\mathbf{U} \\
\mathbf{U} \\
\mathbf{U} \\
\mathbf{U}\end{array}$ & & \\
\hline
\end{tabular}

Location=AND; Formation=COPPER RIDGE; Site=37; Samp id=2272; Dup id=' '; Phase=2

\begin{tabular}{|c|c|c|c|}
\hline $\begin{array}{l}\text { cis-1,2-Dichloroethene } \\
\text { cis-1,3-D ichloropropene } \\
\text { trans-1,2-Dichloroethene } \\
\text { trans-1,3-Dichloropropene } \\
\text { Acetone } \\
\text { Benzene } \\
\text { Bromodichloromethane } \\
\text { Bromoform } \\
\text { Bromomethane } \\
\text { Carbon disulfide } \\
\text { Carbon tetrachloride } \\
\text { Chlorobenzene } \\
\text { Chloroethane } \\
\text { Chloroform } \\
\text { Chloromethane } \\
\text { Dibromochloromethane } \\
\text { Ethyl benzene } \\
\text { Methylene chloride } \\
\text { Styrene } \\
\text { Tetrachloroethene } \\
\text { Toluene } \\
\text { Trichloroethene } \\
\text { Vinyl chloride } \\
\text { Xylene (meta + para) } \\
\text { xylene (ortho) } \\
1,1-D i c h l o r o e t h a n e \\
1,1-D i c h l o r o e t h e n e \\
1,1,1-\text { Trichloroethane } \\
1,1,2-\text { Trichloroethane } \\
1,1,2,2-T e t r a c h l o r o e t h a n e \\
1,2-D \text { ichloroethane } \\
1,2-D \text { ichloropropane } \\
2-\text { - Butanone } \\
2-\text { Hexanone } \\
\text { 4-Methyl-2-pentanone }\end{array}$ & 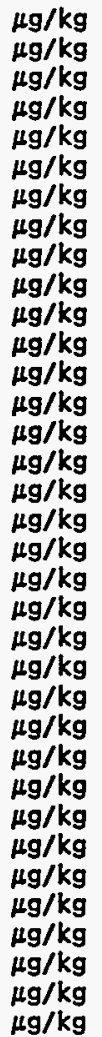 & $\begin{array}{r}5 \\
5 \\
5 \\
5 \\
100 \\
5 \\
5 \\
5 \\
10 \\
5 \\
5 \\
5 \\
10 \\
5 \\
10 \\
5 \\
5 \\
5 \\
5 \\
5 \\
5 \\
5 \\
10 \\
5 \\
5 \\
5 \\
5 \\
5 \\
5 \\
5 \\
5 \\
5 \\
100 \\
50 \\
50\end{array}$ & . \\
\hline
\end{tabular}

Location=AND; Formation=COPPER RIDGE; Site=38; Samp id=2274; Dup id=' '; Phase=2

cis-1,2-Dichloroethene cis-1,3-Dichloropropene trans-1, 2-D ichloroethene trans-1,3-D ichloropropene

$\begin{array}{lll}\mu \mathrm{g} / \mathrm{kg} & 5 & U \\ \mu \mathrm{g} / \mathrm{kg} & 5 & U \\ \mu \mathrm{g} / \mathrm{kg} & 5 & U \\ \mu \mathrm{g} / \mathrm{kg} & 5 & U\end{array}$


Table B.1 (continued)

\begin{tabular}{|c|c|c|c|c|c|c|}
\hline Analysis & Units & $\begin{array}{c}A \\
\text { horizon }\end{array}$ & Qualifier & $\begin{array}{l}\text { A horizon } \\
\text { field dup }\end{array}$ & Qualifier & \\
\hline $\begin{array}{l}\text { Acetone } \\
\text { Benzene } \\
\text { Bromodichloromethane } \\
\text { Bromoform } \\
\text { Bromomethane } \\
\text { Carbon disulfide } \\
\text { Carbon tetrachloride } \\
\text { Chlorobenzene } \\
\text { Chloroethane } \\
\text { Chloroform } \\
\text { Chloromethane } \\
\text { Dibromochloromethane } \\
\text { Ethyl benzene } \\
\text { Methylene chloride } \\
\text { Styrene } \\
\text { Tetrachloroethene } \\
\text { Toluene } \\
\text { Trichloroethene } \\
\text { vinyl chloride } \\
\text { Xylene (meta + para) } \\
\text { Xylene (ortho) } \\
1,1-D \text { ichloroethane } \\
1,1-D \text { ichloroethene } \\
1,1,1-\text { Trichloroethane } \\
1,1,2-\text { Trichloroethane } \\
1,1,2,2-\text { Tetrachloroethane } \\
1,2-0 \text { ichloroethane } \\
1,2-0 \text { ichloropropane } \\
2-\text { Butanone } \\
2-\text { Hexanone } \\
4-\text { Methyl-2-pentanone }\end{array}$ & 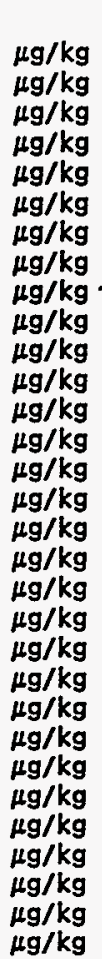 & $\begin{array}{r}100 \\
5 \\
5 \\
5 \\
10 \\
5 \\
5 \\
5 \\
10 \\
5 \\
10 \\
5 \\
5 \\
5 \\
5 \\
5 \\
5 \\
5 \\
10 \\
5 \\
5 \\
5 \\
5 \\
5 \\
5 \\
5 \\
5 \\
5 \\
8 \\
50 \\
50\end{array}$ & $\begin{array}{l}\mathbf{U} \\
\mathbf{U} \\
\mathbf{U} \\
\mathbf{U} \\
\mathbf{U} \\
\mathbf{U} \\
\mathbf{U} \\
\mathbf{U} \\
\mathbf{U} \\
\mathbf{U} \\
\mathbf{U} \\
\mathbf{U} \\
\mathbf{U} \\
\mathbf{U} \\
\mathbf{U} \\
\mathbf{U} \\
\mathbf{U} \\
\mathbf{U} \\
\mathbf{U} \\
\mathbf{U} \\
\mathbf{U} \\
\mathbf{U} \\
\mathbf{U} \\
\mathbf{U} \\
\mathbf{U} \\
\mathbf{U} \\
\mathbf{U} \\
\mathbf{U} \\
\mathbf{U} \\
\mathbf{U} \\
\mathbf{U}\end{array}$ & ' & & $\cdots$ \\
\hline
\end{tabular}

Location=AND; Formation=COPPER RIDGE; Site=39; Samp id=2276; Dup id=' '; Phase=2

\begin{tabular}{|c|c|c|}
\hline $\begin{array}{l}\text {,2-Dichloroethene } \\
\text {,3-Dichloropropene } \\
;-1,2-D i c h l o r o e t h e n e \\
\text {-1,3-Dich loropropene } \\
\text { one } \\
\text { ne } \\
\text { dichloromethane } \\
\text { form } \\
\text { methane } \\
\text { on disulfide } \\
\text { on tetrachloride } \\
\text { obenzene } \\
\text { oethane } \\
\text { oform } \\
\text { omethane } \\
\text { mochloromethane } \\
\text { benzene } \\
\text { lene chloride } \\
\text { ne } \\
\text { chloroethene } \\
\text { ne } \\
\text { loroethene } \\
\text { chloride } \\
\text { le (meta + para) } \\
\text { e (ortho) } \\
\text { ichloroethane } \\
\text { ichloroethene } \\
\text { - Trichloroethane } \\
\text { - Trichloroethane } \\
2-\text { Tetrachloroethane } \\
\text { ichloroethane } \\
\text { ichloropropane } \\
\text { anone } \\
\text { anone } \\
\text { hyl-2-pentanone }\end{array}$ & 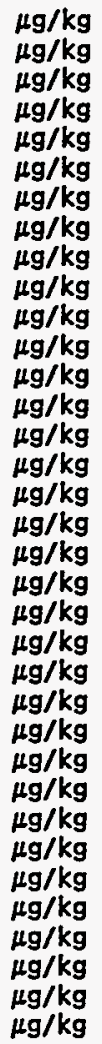 & $\begin{array}{r}5 \\
5 \\
5 \\
5 \\
100 \\
5 \\
5 \\
5 \\
10 \\
5 \\
5 \\
5 \\
10 \\
5 \\
10 \\
5 \\
5 \\
5 \\
5 \\
5 \\
5 \\
5 \\
10 \\
5 \\
5 \\
5 \\
5 \\
5 \\
5 \\
5 \\
5 \\
5 \\
100 \\
50 \\
50\end{array}$ \\
\hline
\end{tabular}


B-8

Table B.1 (continued)

\begin{tabular}{lcc} 
& A & A horizon \\
Analysis & Units horizon & Qualifier field dup Qualifier \\
\hline
\end{tabular}

Location=AND; Formation=COPPER RIDGE; Site=40; Samp id=2278; Dup id=' '; Phase=2

\begin{tabular}{|c|c|c|}
\hline 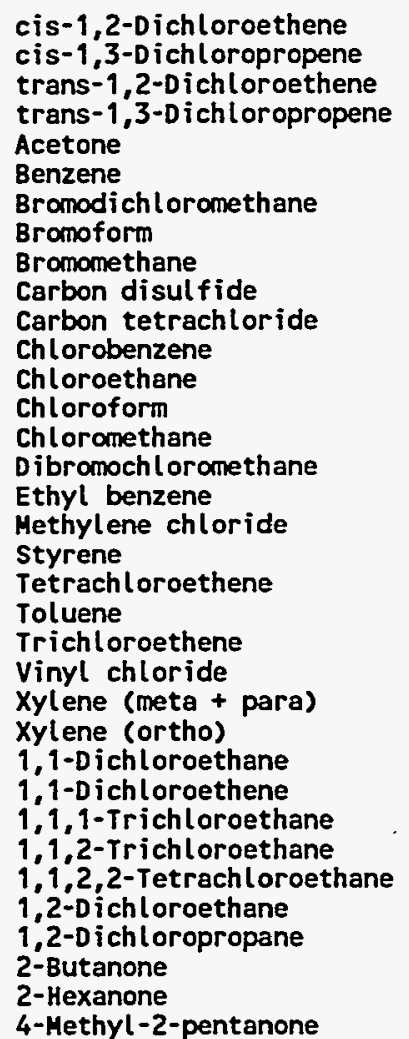 & 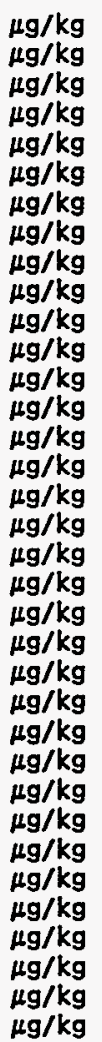 & $\begin{array}{r}5 \\
5 \\
5 \\
5 \\
6 \\
5 \\
5 \\
5 \\
10 \\
5 \\
5 \\
5 \\
10 \\
5 \\
10 \\
5 \\
5 \\
5 \\
5 \\
5 \\
5 \\
5 \\
10 \\
5 \\
5 \\
5 \\
5 \\
5 \\
5 \\
5 \\
5 \\
5 \\
100 \\
50 \\
50\end{array}$ \\
\hline
\end{tabular}

Location=AND; Formation=COPPER RIDGE; Site=41; Samp id =2280; Dup id=' '; Phase=2

\begin{tabular}{|c|c|c|}
\hline $\begin{array}{l}\text { cis-1,2-Dichloroethene } \\
\text { cis-1,3-Dichloropropene } \\
\text { trans-1,2-Dichloroethene } \\
\text { trans-1,3-Dichloropropene } \\
\text { Acetone } \\
\text { Benzene } \\
\text { Bromodichloromethane } \\
\text { Bromoform } \\
\text { Bromomethane } \\
\text { Carbon disulfide } \\
\text { Carbon tetrachloride } \\
\text { Chlorobenzene } \\
\text { Chloroethane } \\
\text { Chloroform } \\
\text { Chloromethane } \\
\text { Dibromochloromethane } \\
\text { Ethyl benzene } \\
\text { Methylene chloride } \\
\text { Styrene } \\
\text { Tetrachloroethene } \\
\text { Toluene } \\
\text { Trichloroethene } \\
\text { Vinyl chloride } \\
\text { Xylene (meta + para) } \\
\text { Xylene (ortho) } \\
1,1-D \text { ichloroethane } \\
1,1-D \text { ichloroethene } \\
1,1,1-\text { Trichloroethane } \\
1,1,2-\text { Trichloroethane } \\
1,1,2,2-T e t r a c h l o r o e t h a n e \\
1,2-D \text { ichloroethane }\end{array}$ & 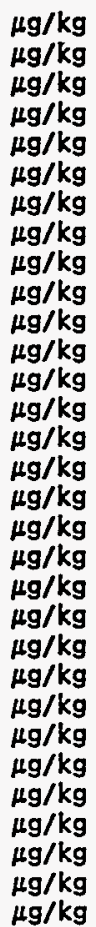 & $\begin{array}{r}5 \\
5 \\
5 \\
5 \\
100 \\
5 \\
5 \\
5 \\
10 \\
5 \\
5 \\
5 \\
10 \\
5 \\
10 \\
5 \\
5\end{array}$ \\
\hline
\end{tabular}


Table B.1 (continued)

\begin{tabular}{|c|c|c|c|c|c|}
\hline Analysis & Units & $\begin{array}{c}\text { A } \\
\text { horizon }\end{array}$ & Qualifier & $\begin{array}{l}\text { A horizon } \\
\text { field dup }\end{array}$ & \\
\hline $\begin{array}{l}\text { 1,2-Dichloropropane } \\
\text { 2-Butanone } \\
\text { 2-Hexanone } \\
\text { 4-Methyl-2-pentanone }\end{array}$ & $\begin{array}{l}\mu \mathrm{g} / \mathrm{kg} \\
\mu \mathrm{g} / \mathrm{kg} \\
\mu \mathrm{g} / \mathrm{kg} \\
\mu \mathrm{g} / \mathrm{kg}\end{array}$ & $\begin{array}{r}5 \\
100 \\
50 \\
50\end{array}$ & $\begin{array}{l}\mathbf{U} \\
\mathbf{U} \\
\mathbf{U} \\
\mathbf{U}\end{array}$ & $\because \quad \because$ & $\eta^{\prime \prime}$ \\
\hline
\end{tabular}

Location $=A N D$; Formation=COPPER RIDGE; Site $=42$; Samp id=2282; Dup id=' '; Phase=2

\begin{tabular}{|c|c|c|}
\hline $\begin{array}{l}\text { cis-1,2-Dichloroethene } \\
\text { cis-1,3-Dichloropropene } \\
\text { trans-1,2-D ichloroethene } \\
\text { trans-1,3-Dichloropropene } \\
\text { Acetone } \\
\text { Benzene } \\
\text { Bromodichloromethane } \\
\text { Bromoform } \\
\text { Bromomethane } \\
\text { Carbon disulfide } \\
\text { Carbon tetrach loride } \\
\text { Chlorobenzene } \\
\text { Chloroethane } \\
\text { Chloroform } \\
\text { Chloromethane } \\
\text { Dibromochloromethane } \\
\text { Ethyl benzene } \\
\text { Methylene chloride } \\
\text { Styrene } \\
\text { Tetrachloroethene } \\
\text { Toluene } \\
\text { Trichloroethene } \\
\text { Vinyl chloride } \\
\text { Xylene (meta + para) } \\
\text { xylene (ortho) } \\
1,1-D i c h l o r o e t h a n e \\
1,1-D i c h l o r o e t h e n e \\
1,1,1-\text {-Trichloroethane } \\
1,1,2-\text { Trichloroethane } \\
1,1,2,2-T \text { Tetrachloroethane } \\
1,2-0 \text { ichloroethane } \\
1,2-D \text { ichloropropane } \\
2-\text { Butanone } \\
2-\text { Hexanone } \\
4-\text { Hethyl-2-pentanone }\end{array}$ & $\begin{array}{l}\mu g / \mathbf{k g} \\
\mu g / \mathbf{k g} \\
\mu g / \mathbf{k g} \\
\mu g / k g \\
\mu g / k g \\
\mu g / k g \\
\mu g / k g \\
\mu g / k g \\
\mu g / k g \\
\mu g / k g \\
\mu g / k g \\
\mu g / k g \\
\mu g / k g \\
\mu g / k g \\
\mu g / k g \\
\mu g / k g \\
\mu g / k g \\
\mu g / k g \\
\mu g / k g\end{array}$ & $\begin{array}{r}5 \\
5 \\
5 \\
11 \\
5 \\
5 \\
5 \\
10 \\
5 \\
5 \\
5 \\
10 \\
5 \\
10 \\
5 \\
5 \\
5 \\
5 \\
5 \\
5 \\
5 \\
10 \\
5 \\
5 \\
5 \\
5 \\
5 \\
5 \\
5 \\
5 \\
5 \\
100 \\
50 \\
50\end{array}$ \\
\hline
\end{tabular}

Location=AND; Formation=DISMAL GAP; Site=1; Samp id=2029; Dup id =' '; Phase=1

cis-1,2-Dichloroethene

cis-1,3-Dichloropropene

trans-1,2-Dichloroethene

trans-1,3-Dichloropropene

Acetone

Benzene

Bromodichloromethane . $\quad \mu \mathrm{g} / \mathrm{kg}$

Bromoform

Bromomethane

Carbon disulfide

Carbon tetrachloride

Chlorobenzene

Chloroethane

Chloroform

Chloromethane

Dibromoch loromethane

Ethyl benzene

Methylene chloride

Styrene

Tetrachloroethene

Toluene

Trichloroethene

Vinyl chloride

Xylene (meta + para)

Xylene (ortho)

$\begin{array}{lrl}\mu g / k g & 5 & U \\ \mu g / k g & 5 & U \\ \mu g / k g & 5 & U \\ \mu g / k g & 5 & U \\ \mu g / k g & 100 & U \\ \mu g / k g & 5 & U \\ \mu g / k g & 5 & U \\ \mu g / k g & 5 & U \\ \mu g / k g & 10 & U \\ \mu g / k g & 5 & U \\ \mu g / k g & 5 & U \\ \mu g / k g & 5 & U \\ \mu g / k g & 10 & U \\ \mu g / k g & 5 & U \\ \mu g / k g & 10 & U \\ \mu g / k g & 5 & U \\ \mu g / k g & 5 & U \\ \mu g / k g & 5 & U \\ \mu g / k g & 5 & U \\ \mu g / k g & 5 & U \\ \mu g / k g & 5 & U \\ \mu g / k g & 5 & U \\ \mu g / k g & 10 & U \\ \mu g / k g & 5 & U \\ \mu g / k g & 5 & U\end{array}$


B-10

Table B.1 (continued)

\begin{tabular}{|c|c|c|c|c|c|}
\hline Analysis & Units & $\begin{array}{c}\text { A } \\
\text { horizon }\end{array}$ & Qualifier & $\begin{array}{l}\text { A horizon } \\
\text { field dup }\end{array}$ & Qualifier \\
\hline $\begin{array}{l}\text { 1,1-Dichloroethane } \\
\text { 1,1-Dichloroethene } \\
\text { 1,1,1-Trichloroethane } \\
\text { 1,1,2-Trichloroethane } \\
\text { 1,1,2,2-Tetrachloroethane } \\
\text { 1,2-Dichloroethane } \\
\text { 1,2-Dichloropropane } \\
\text { 2--Butanone } \\
\text { 2-Hexanone } \\
\text { 4-Methyl-2-pentanone }\end{array}$ & $\begin{array}{l}\mu g / k g \\
\mu g / k g \\
\mu g / k g \\
\mu g / k g \\
\mu g / k g \\
\mu g / k g \\
\mu g / k g \\
\mu g / k g \\
\mu g / k g \\
\mu g / k g\end{array}$ & $\begin{array}{r}5 \\
5 \\
5 \\
5 \\
5 \\
5 \\
5 \\
100 \\
50 \\
50\end{array}$ & $\begin{array}{l}U \\
U \\
U \\
U \\
U \\
U \\
U \\
U \\
U \\
U\end{array}$ & & \\
\hline
\end{tabular}

Location=AND; Formation=DISMAL GAP; Site=10; Samp id=2028; Dup id=' '; Phase=1

\begin{tabular}{|c|c|c|}
\hline 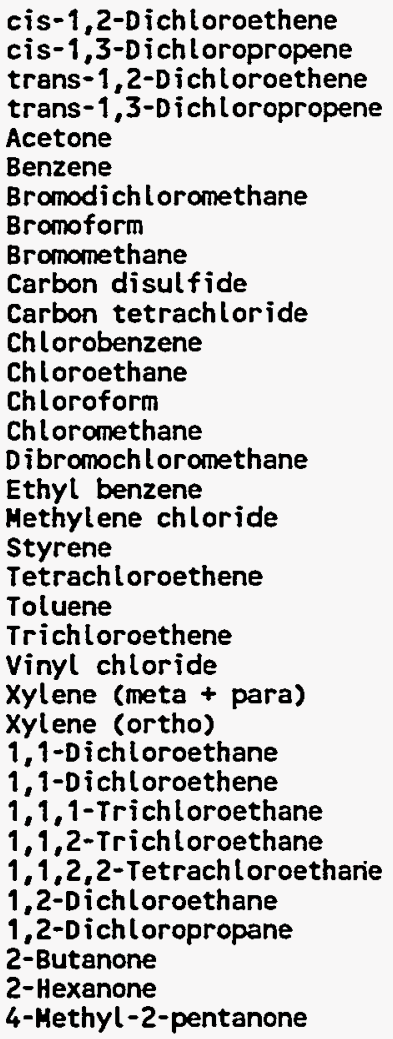 & $\begin{array}{l}\mu g / k g \\
\mu g / k g \\
\mu g / k g \\
\mu g / k g \\
\mu g / k g \\
\mu g / k g \\
\mu g / k g \\
\mu g / k g \\
\mu g / k g \\
\mu g / k g \\
\mu g / k g \\
\mu g / k g \\
\mu g / k g \\
\mu g / k g \\
\mu g / k g \\
\mu g / k g \\
\mu g / k g \\
\mu g / k g \\
\mu g / k g \\
\mu g / k g \\
\mu g / k g \\
\mu g / k g \\
\mu g / k g \\
\mu g / k g \\
\mu g / k g \\
\mu g / k g \\
\mu g / k g \\
\mu g / k g \\
\mu g / k g \\
\mu g / k g \\
\mu g / k g \\
\mu g / k g \\
\mu g / k g \\
\mu g / k g \\
\mu g / k g\end{array}$ & $\begin{array}{r}5 \\
5 \\
5 \\
5 \\
6 \\
5 \\
5 \\
5 \\
10 \\
5 \\
5 \\
5 \\
10 \\
5 \\
10 \\
5 \\
5 \\
5 \\
5 \\
5 \\
5 \\
5 \\
10 \\
5 \\
5 \\
5 \\
5 \\
5 \\
5 \\
5 \\
5 \\
5 \\
100 \\
50 \\
50\end{array}$ \\
\hline
\end{tabular}

Location=AND; Formation=DISMAL GAP; Site=11; Samp id=2111; Dup id=' '; Phase=1

cis-1,2-Dichloroethene
cis-1,3-Dichloropropene
trans-1,2-Dichloroethene
trans-1,3-Dichloropropene
Acetone
Benzene
Bromodichloromethane
Bromoform
Bromomethane
Carbon disulfide
Carbon tetrachloride
Chlorobenzene
Chloroethane
Chloroform
Chloromethane
Dibromochloromethane
Ethyl benzene
Methylene chloride
Styrene

\begin{tabular}{|c|c|}
\hline $\begin{array}{l}\mu \mathrm{g} / \mathrm{kg} \\
\mu \mathrm{g} / \mathrm{kg} \\
\mu \mathrm{g} / \mathrm{kg} \\
\mu \mathrm{g} / \mathrm{kg} \\
\mu \mathrm{g} / \mathrm{kg} \\
\mu \mathrm{g} / \mathrm{kg} \\
\mu \mathrm{g} / \mathrm{kg} \\
\mu \mathrm{g} / \mathrm{kg} \\
\mu \mathrm{g} / \mathrm{kg} \\
\mu \mathrm{g} / \mathrm{kg} \\
\mu \mathrm{g} / \mathrm{kg} \\
\mu \mathrm{g} / \mathrm{kg} \\
\mu \mathrm{g} / \mathrm{kg} \\
\mu \mathrm{g} / \mathrm{kg} \\
\mu \mathrm{g} / \mathrm{kg} \\
\mu \mathrm{g} / \mathrm{kg} \\
\mu \mathrm{g} / \mathrm{kg} \\
\mu \mathrm{g} / \mathrm{kg} \\
\mu \mathrm{g} / \mathrm{kg}\end{array}$ & $\begin{array}{r}5 \\
5 \\
5 \\
5 \\
5 \\
5 \\
5 \\
5 \\
10 \\
5 \\
5 \\
5 \\
10 \\
5 \\
10 \\
5 \\
5\end{array}$ \\
\hline
\end{tabular}


B-11

Table B.1 (continued)

\begin{tabular}{|c|c|c|c|c|c|c|}
\hline Analysis & Units & $\stackrel{A}{\text { horizon }}$ & Qualifier & $\begin{array}{l}\text { A horizon } \\
\text { field dup }\end{array}$ & Qualifier & \\
\hline $\begin{array}{l}\text { Tetrachloroethene } \\
\text { Toluene } \\
\text { Trichloroethene } \\
\text { Vinyl chloride } \\
\text { xylene (meta + para) } \\
\text { xylene (ortho) } \\
\text { 1,1-Dichloroethane } \\
\text { 1,1-Dichloroethene } \\
1,1,1-\text {-Trichloroethane } \\
1,1,2-\text { Trichloroethane } \\
1,1,2,2-\text { Tetrachloroethane } \\
1,2-0 \text { ichloroethane } \\
1,2-D \text { ichloropropane } \\
\text { 2-Butanone } \\
\text { 2-Hexanone } \\
\text { 4-Methyl-2-pentanone }\end{array}$ & 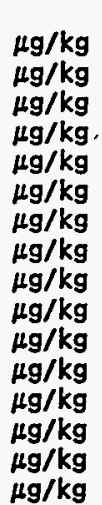 & $\begin{array}{r}5 \\
5 \\
5 \\
10 \\
5 \\
5 \\
5 \\
5 \\
5 \\
5 \\
5 \\
5 \\
5 \\
99 \\
50 \\
50\end{array}$ & $\begin{array}{l}U \\
U \\
U \\
U \\
U \\
U \\
U \\
U \\
U \\
U \\
U \\
U \\
U \\
U \\
U \\
U\end{array}$ & & . & : \\
\hline
\end{tabular}

Location=AND; Formation=DISMAL GAP; Site=12; Samp id=2031; Dup id=' '; Phase=1

\begin{tabular}{|c|c|c|}
\hline 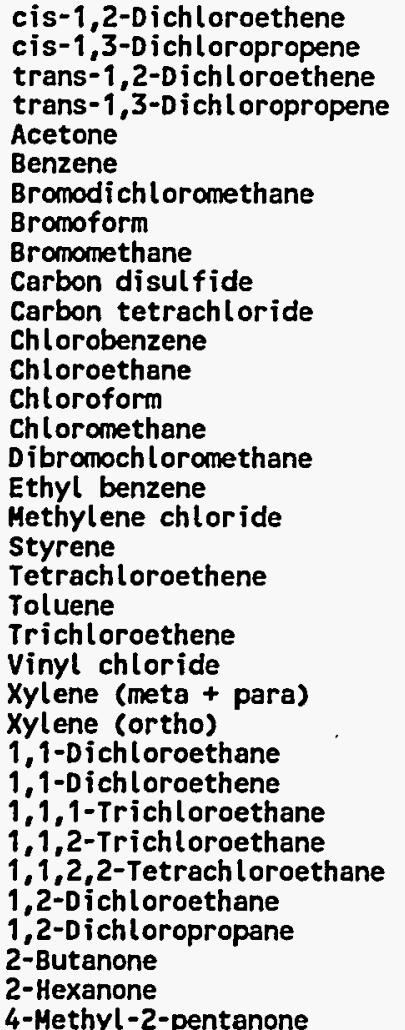 & 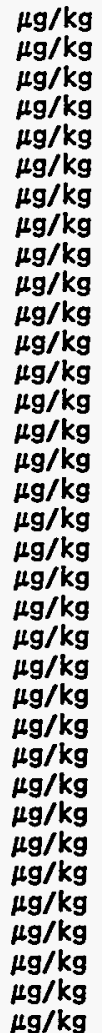 & $\begin{array}{r}5 \\
5 \\
5 \\
5 \\
7 \\
5 \\
5 \\
5 \\
10 \\
5 \\
5 \\
5 \\
10 \\
5 \\
10 \\
5 \\
5 \\
5 \\
5 \\
5 \\
5 \\
5 \\
10 \\
5 \\
5 \\
5 \\
5 \\
5 \\
5 \\
5 \\
5 \\
5 \\
100 \\
50 \\
50\end{array}$ \\
\hline
\end{tabular}

Location=AND; Formation=DISMAL GAP; Site=19; Samp id=2027; Dup id=' '; Phase=1

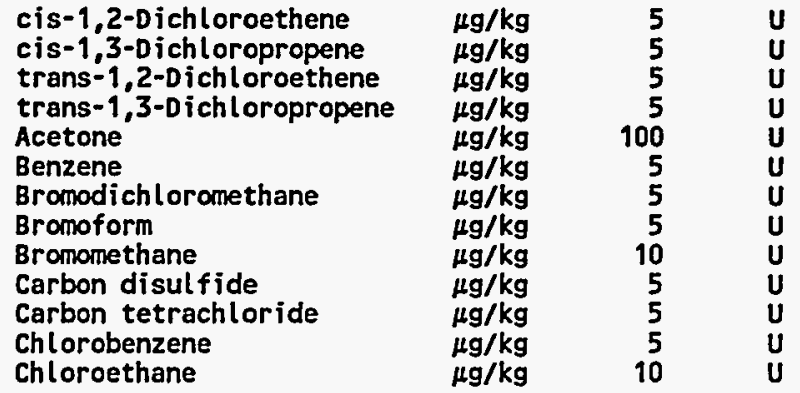


B-12

Table B.1 (continued)

\begin{tabular}{|c|c|c|c|c|c|}
\hline Analysis & Units & $\underset{\text { horizon }}{\mathbf{A}}$ & Qualifier & $\begin{array}{l}\text { A horizon } \\
\text { field dup }\end{array}$ & Qualifier \\
\hline $\begin{array}{l}\text { Chloroform } \\
\text { Chloromethane } \\
\text { Dibromochloromethane } \\
\text { Ethyl benzene } \\
\text { Hethylene chloride } \\
\text { Styrene } \\
\text { Tetrachloroethene } \\
\text { Toluene } \\
\text { Trichloroethene } \\
\text { Vinyl chloride } \\
\text { xylene (meta + para) } \\
\text { xylene (ortho) } \\
1,1-D \text { ichloroethane } \\
1,1-\text { Dichloroethene } \\
1,1,1-\text { Trichloroethane } \\
1,1,2-\text { Trichloroethane } \\
1,1,2,2-\text { Tetrachloroethane } \\
1,2-D \text { ichloroethane } \\
1,2-D \text { ichloropropane } \\
\text { 2--Butanone } \\
\text { 2-Hexanone } \\
\text { 4-Methyl-2-pentanone }\end{array}$ & 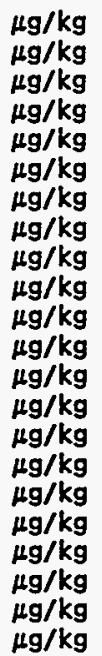 & $\begin{array}{r}5 \\
10 \\
5 \\
5 \\
5 \\
5 \\
5 \\
5 \\
5 \\
10 \\
5 \\
5 \\
5 \\
5 \\
5 \\
5 \\
5 \\
5 \\
5 \\
100 \\
50 \\
50\end{array}$ & $\begin{array}{l}U \\
U \\
U \\
U \\
U \\
U \\
U \\
U \\
U \\
U \\
U \\
U \\
U \\
U \\
U \\
U \\
U \\
U \\
U \\
U \\
U \\
U\end{array}$ & & \\
\hline
\end{tabular}

Location=AND; Formation=DISMAL GAP; Site=20; Samp id=2069; Dup id=' '; Phase=1

\begin{tabular}{|c|c|c|}
\hline 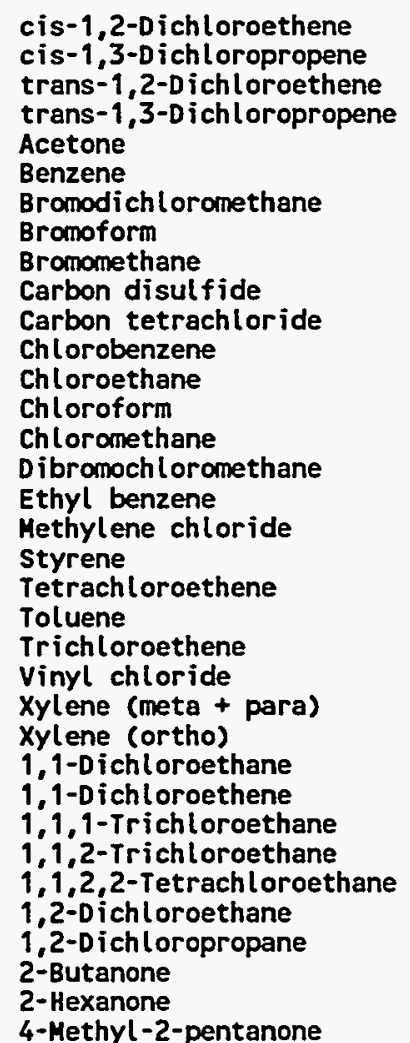 & 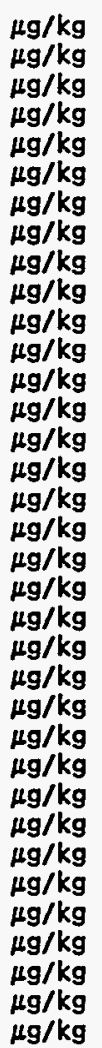 & $\begin{array}{r}5 \\
5 \\
5 \\
5 \\
5 \\
5 \\
5 \\
5 \\
10 \\
5 \\
5 \\
5 \\
10 \\
5 \\
10 \\
5 \\
5 \\
5 \\
5 \\
5 \\
5 \\
5 \\
10 \\
5 \\
5 \\
5 \\
5 \\
5 \\
5 \\
5 \\
5 \\
5 \\
100 \\
50 \\
50\end{array}$ \\
\hline
\end{tabular}

Location=AND; Formation=DISMAL GAP; Site=21; Samp id=2036; Dup id=' '; Phase=1

$\begin{array}{llll}\text { cis-1,2-Dichloroethene } & \mu g / \mathrm{kg} & 5 & \mathrm{U} \\ \text { cis-1,3-Dichloropropene } & \mu \mathrm{g} / \mathrm{kg} & 5 & \mathrm{U} \\ \text { trans-1,2-Dichloroethene } & \mu \mathrm{g} / \mathrm{kg} & 5 & \mathrm{U} \\ \text { trans-1,3-Dichloropropene } & \mu \mathrm{g} / \mathrm{kg} & 5 & \mathrm{U} \\ \text { Acetone } & \mu \mathrm{g} / \mathrm{kg} & 6 & \mathrm{~J} \\ \text { Benzene } & \mu \mathrm{gg} & 5 & U \\ \text { Bromodichloromethane } & \mu \mathrm{g} / \mathrm{kg} & 5 & \mathrm{U}\end{array}$


B-13

Table B.1 (continued)

\begin{tabular}{|c|c|c|c|c|c|}
\hline Analysis & Units & $\begin{array}{c}\text { A } \\
\text { horizon }\end{array}$ & Qualifier & $\begin{array}{l}\text { A horizon } \\
\text { field dup }\end{array}$ & Qualifier \\
\hline $\begin{array}{l}\text { Bromoform } \\
\text { Bromomethane } \\
\text { Carbon disulfide } \\
\text { Carbon tetrachloride } \\
\text { Chlorobenzene } \\
\text { Chloroethane } \\
\text { Chloroform } \\
\text { Chloromethane } \\
\text { Dibromochloromethane } \\
\text { Ethyl benzene } \\
\text { Methylene chloride } \\
\text { Styrene } \\
\text { Tetrachloroethene } \\
\text { Toluene } \\
\text { Trichloroethene } \\
\text { Vinyl chloride } \\
\text { Xylene (meta + para) } \\
\text { xylene (ortho) } \\
\text { 1,1-Dichloroethane } \\
\text { 1,1-Dichloroethene } \\
\text { 1,1,1-Trichloroethane } \\
\text { 1,1,2-Trichloroethane } \\
1,1,2,2-\text { Tetrachloroethane } \\
1,2-D \text { ichloroethane } \\
1,2-D \text { ichloropropane } \\
\text { 2-Butanone } \\
\text { 2-Hexanone } \\
\text { 4-Hethyl-2-pentanone }\end{array}$ & $\begin{array}{l}\mu g / \mathrm{kg} \\
\mu g / \mathrm{kg} \\
\mu g / \mathrm{kg} \\
\mu g / \mathrm{kg} \\
\mu g / \mathrm{kg} \\
\mu g / \mathrm{kg} \\
\mathrm{\mu g} / \mathrm{kg} \\
\mu g / \mathrm{kg} \\
\mu g / \mathrm{kg} \\
\mu g / \mathrm{kg} \\
\mu g / \mathrm{kg} \\
\mathrm{\mu g} / \mathrm{kg} \\
\mu g / \mathrm{kg} \\
\mu g / \mathrm{kg} \\
\mu g / \mathrm{kg} \\
\mu g / \mathrm{kg} \\
\mu g / \mathrm{kg} \\
\mu g / \mathrm{kg} \\
\mu g / \mathrm{kg} \\
\mu g / \mathrm{kg} \\
\mu g / \mathrm{kg} \\
\mu g / \mathrm{kg} \\
\mu g / \mathrm{kg} \\
\mu g / \mathrm{kg} \\
\mu g / \mathrm{kg} \\
\mu g / \mathrm{kg} \\
\mu g / \mathrm{kg} \\
\mu g / \mathrm{kg}\end{array}$ & $\begin{array}{r}5 \\
10 \\
5 \\
5 \\
5 \\
10 \\
5 \\
10 \\
5 \\
5 \\
5 \\
5 \\
5 \\
5 \\
5 \\
10 \\
5 \\
5 \\
5 \\
5 \\
5 \\
5 \\
5 \\
5 \\
5 \\
100 \\
50 \\
50\end{array}$ & $\begin{array}{l}U \\
U \\
U \\
U \\
U \\
U \\
U \\
U \\
U \\
U \\
U \\
U \\
U \\
U \\
U \\
U \\
U \\
U \\
U \\
U \\
U \\
U \\
U \\
U \\
U \\
U \\
U \\
U\end{array}$ & & \\
\hline
\end{tabular}

Location=AND; Formation=DISMAL GAP; Site=22; Samp id=2030; Dup id=' '; Phase=1

\begin{tabular}{|c|c|c|}
\hline $\begin{array}{l}\text { cis-1,2-Dichloroethene } \\
\text { cis-1,3-Dichloropropene } \\
\text { trans-1,2-Dichloroethene } \\
\text { trans-1,3-Dichloropropene } \\
\text { Acetone } \\
\text { Benzene } \\
\text { Bromodichloromethane } \\
\text { Bromoform } \\
\text { Bromomethane } \\
\text { Carbon disulfide } \\
\text { Carbon tetrachloride } \\
\text { Chlorobenzene } \\
\text { Chloroethane } \\
\text { Chloroform } \\
\text { Chloromethane } \\
\text { Dibromochloromethane } \\
\text { Ethyl benzene } \\
\text { Methylene chloride } \\
\text { Styrene } \\
\text { Tetrachloroethene } \\
\text { Toluene } \\
\text { Trichloroethene } \\
\text { vinyl chloride } \\
\text { Xylene (meta + para) } \\
\text { xylene (ortho) } \\
1,1-D i c h l o r o e t h a n e \\
1,1-D i c h l o r o e t h e n e \\
1,1,1-T r i c h l o r o e t h a n e \\
1,1,2-T r i c h l o r o e t h a n e \\
1,1,2,2-T e t r a c h l o r o e t h a n e \\
1,2-D i c h l o r o e t h a n e \\
1,2-D i c h l o r o p r o p a n e \\
2-\text { Butanone } \\
2-\text { Hexanone } \\
4-\text { Methyl-2-pentanone }\end{array}$ & 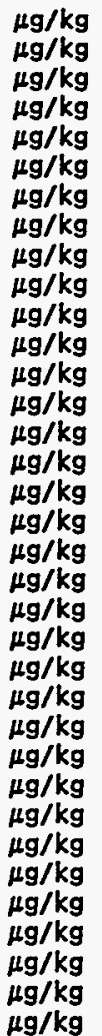 & $\begin{array}{r}5 \\
5 \\
5 \\
5 \\
10 \\
5 \\
5 \\
5 \\
10 \\
5 \\
5 \\
5 \\
10 \\
5 \\
10 \\
5 \\
5 \\
5 \\
5 \\
5 \\
5 \\
5 \\
10 \\
5 \\
5 \\
5 \\
5 \\
5 \\
5 \\
5 \\
5 \\
5 \\
100 \\
50 \\
50\end{array}$ \\
\hline
\end{tabular}




\section{B-14}

Table B.1 (continued)

\begin{tabular}{|c|c|c|c|c|c|}
\hline Analysis & Units & $\underset{\text { horizon }}{\text { A }}$ & Qualifier & $\begin{array}{l}\text { A horizon } \\
\text { field dup }\end{array}$ & Qualifier \\
\hline
\end{tabular}

Location=AND; Formation=DISMAL GAP; Site=3; Samp id=2032; Dup id=' '; Phase=1

\begin{tabular}{|c|c|c|}
\hline $\begin{array}{l}\text { cis-1,2-D ichloroethene } \\
\text { cis-1,3-D ichloropropene } \\
\text { trans-1,2-Dichloroethene } \\
\text { trans-1,3-D ichloropropene } \\
\text { Acetone } \\
\text { Benzene } \\
\text { Bromodichloromethane } \\
\text { Bromoform } \\
\text { Bromomethane } \\
\text { Carbon disulfide } \\
\text { Carbon tetrachloride } \\
\text { Chlorobenzene } \\
\text { Chloroethane } \\
\text { Chloroform } \\
\text { Chloromethane } \\
\text { Dibromochloromethane } \\
\text { Ethyl benzene } \\
\text { Methylene chloride } \\
\text { Styrene } \\
\text { Tetrachloroethene } \\
\text { Toluene } \\
\text { Trichloroethene } \\
\text { Vinyl chloride } \\
\text { Xylene (meta + para) } \\
\text { Xylene (ortho) } \\
1,1-D \text { ichloroethane } \\
1,1-D i c h l o r o e t h e n e \\
1,1,1-T r i c h l o r o e t h a n e \\
1,1,2-\text { Trichloroethane } \\
1,1,2,2-T e t r a c h l o r o e t h a n e \\
1,2-D \text { ichloroethane } \\
1,2-D \text { ichloropropane } \\
2-\text { - Butanone }\end{array}$ & 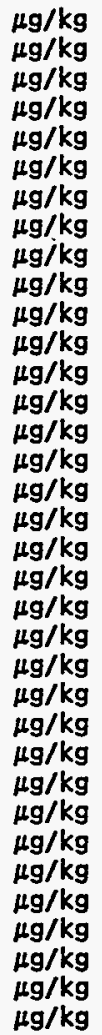 & $\begin{array}{r}5 \\
5 \\
5 \\
5 \\
5 \\
5 \\
5 \\
5 \\
10 \\
5 \\
5 \\
5 \\
10 \\
5 \\
10 \\
5 \\
5 \\
5 \\
5 \\
5 \\
5 \\
5 \\
10 \\
5 \\
5 \\
5 \\
5 \\
5 \\
5 \\
5 \\
5 \\
5 \\
100 \\
50 \\
50\end{array}$ \\
\hline
\end{tabular}

Location=AND; Formation=DISMAL GAP; Site=4; Samp id=2033; Dup id=' '; Phase=1

\begin{tabular}{|c|c|c|}
\hline 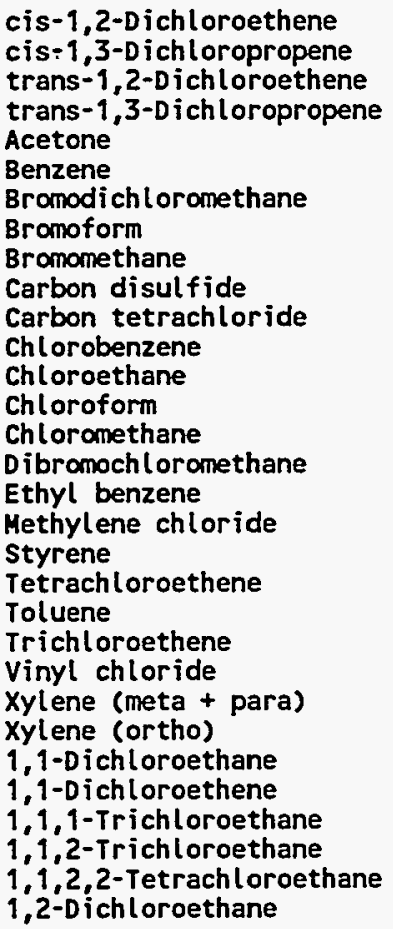 & 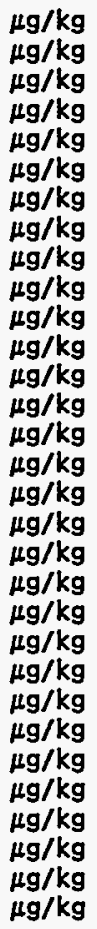 & $\begin{array}{r}5 \\
5 \\
5 \\
5 \\
13 \\
5 \\
5 \\
5 \\
10 \\
5 \\
5 \\
5 \\
10 \\
5 \\
10 \\
5 \\
5 \\
5 \\
5 \\
5 \\
5 \\
5 \\
10 \\
5\end{array}$ \\
\hline
\end{tabular}




\section{B-15}

Table B.1 (continued)

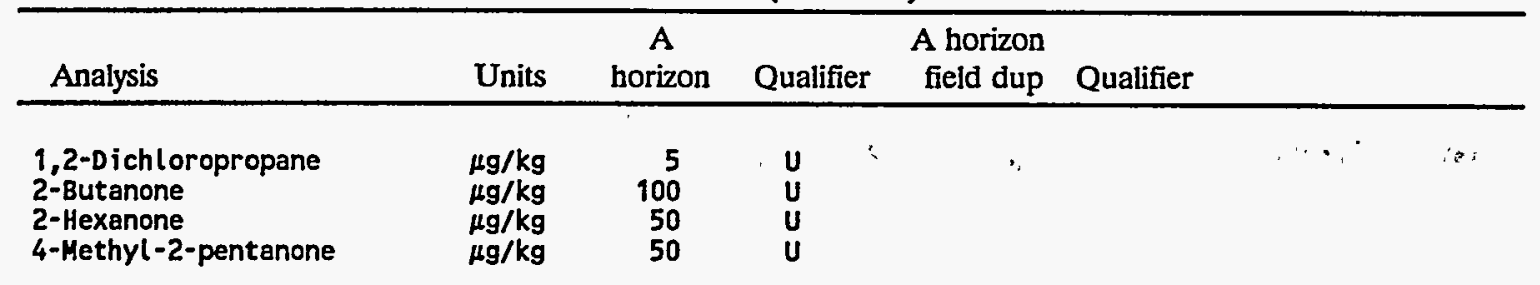

Location=AND; Formation=DISMAL GAP; Site=5; Samp id=2034; Dup id=' '; Phase=1

\begin{tabular}{|c|c|c|}
\hline 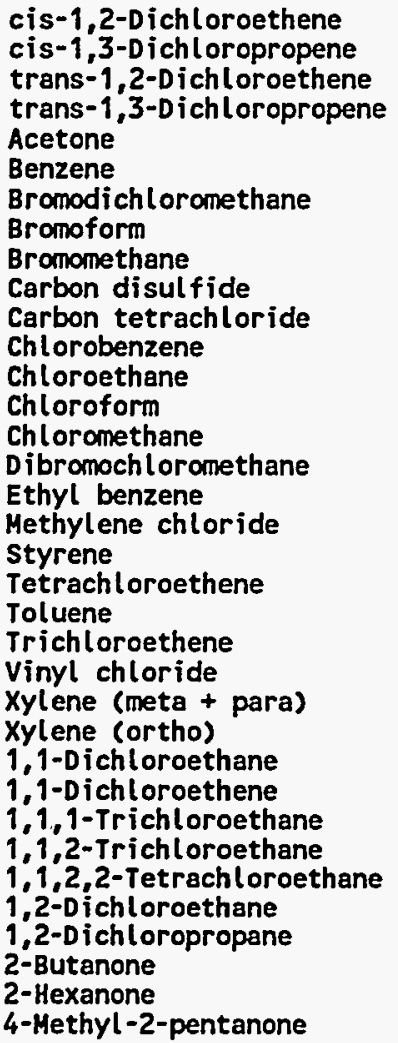 & 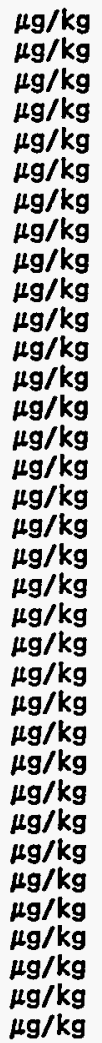 & $\begin{array}{r}5 \\
5 \\
8 \\
5 \\
5 \\
5 \\
10 \\
5 \\
5 \\
5 \\
10 \\
5 \\
10 \\
5 \\
5 \\
5 \\
5 \\
5 \\
5 \\
5 \\
10 \\
5 \\
5 \\
5 \\
5 \\
5 \\
5 \\
5 \\
5 \\
5 \\
100 \\
50 \\
50\end{array}$ \\
\hline
\end{tabular}

Location=AND; Formation=DISMAL GAP; Site=9; Samp id=2026; Dup id=' '; Phase=1

$\begin{array}{ll}\text { cis-1,2-Dichloroethene } & \mu \mathrm{g} / \mathrm{kg} \\ \text { cis-1,3-Dichloropropene } & \mu \mathrm{g} / \mathrm{kg} \\ \text { trans-1,2-Dichloroethene } & \mu \mathrm{g} / \mathrm{kg} \\ \text { trans-1,3-Dichloropropene } & \mu \mathrm{g} / \mathrm{kg} \\ \text { Acetone } & \mu \mathrm{g} / \mathrm{kg} \\ \text { Benzene } & \mu \mathrm{g} / \mathrm{kg} \\ \text { Bromodichloromethane } & \mu \mathrm{g} / \mathrm{kg} \\ \text { Bromoform } & \mu \mathrm{g} / \mathrm{kg} \\ \text { Bromomethane } & \mu \mathrm{g} / \mathrm{kg} \\ \text { Carbon disulfide } & \mu \mathrm{g} / \mathrm{kg} \\ \text { Carbon tetrachloride } & \mu \mathrm{g} / \mathrm{kg} \\ \text { Chlorobenzene } & \mu \mathrm{g} / \mathrm{kg} \\ \text { Chloroethane } & \mu \mathrm{g} / \mathrm{kg} \\ \text { Chloroform } & \mu \mathrm{g} / \mathrm{kg} \\ \text { Chloromethane } & \mu \mathrm{g} / \mathrm{kg} \\ \text { Dibromochloromethane } & \mu \mathrm{g} / \mathrm{kg} \\ \text { Ethyl benzene } & \mu \mathrm{g} / \mathrm{kg} \\ \text { Methylene chloride } & \mu \mathrm{g} / \mathrm{kg} \\ \text { Styrene } & \mu \mathrm{g} / \mathrm{kg} \\ \text { Tetrachloroethene } & \mu \mathrm{g} / \mathrm{kg} \\ \text { Toluene } & \mu \mathrm{g} / \mathrm{kg} \\ \text { Trichloroethene } & \mu \mathrm{g} / \mathrm{kg} \\ \text { Vinyl chloride } & \mu \mathrm{g} / \mathrm{kg} \\ \text { Xylene (meta }+ \text { para) } & \mu \mathrm{g} / \mathrm{kg} \\ \text { Xylene (ortho) } & \mu \mathrm{g} / \mathrm{kg}\end{array}$

$\begin{array}{rl}5 & U \\ 5 & U \\ 5 & U \\ 5 & U \\ 8 & J \\ 5 & U \\ 5 & U \\ 5 & U \\ 10 & U \\ 5 & U \\ 5 & U \\ 5 & U \\ 10 & U \\ 5 & U \\ 10 & U \\ 5 & U \\ 5 & U \\ 5 & U \\ 5 & U \\ 5 & U \\ 5 & U \\ 5 & U \\ 10 & U \\ 5 & U \\ 5 & U\end{array}$


Table B.1 (continued)

\begin{tabular}{|c|c|c|c|c|c|}
\hline Analysis & Units & $\begin{array}{c}\mathrm{A} \\
\text { horizon }\end{array}$ & Qualifier & $\begin{array}{l}\text { A horizon } \\
\text { field dup }\end{array}$ & Qualifier \\
\hline $\begin{array}{l}\text { 1,1-Dichloroethane } \\
1,1-D \text { ichloroethene } \\
1,1,1-\text { Trijchloroethane } \\
1,1,2-\text { Trichloroethane } \\
1,1,2,2-\text { Tetrachloroethane } \\
\text { 1,2-Dichloroethane } \\
1,2-0 \text { ichloropropane } \\
\text { 2-Butanone } \\
\text { 2-Hexanone } \\
\text { 4-Methyl-2-pentanone }\end{array}$ & $\begin{array}{l}\mu \mathrm{g} / \mathrm{kg} \\
\mu \mathrm{g} / \mathrm{kg} \\
\mu \mathrm{g} / \mathrm{kg} \\
\mu \mathrm{g} / \mathrm{kg} \\
\mu \mathrm{g} / \mathrm{kg} \\
\mu \mathrm{g} / \mathrm{kg} \\
\mu \mathrm{g} / \mathrm{kg} \\
\mu \mathrm{g} / \mathrm{kg} \\
\mu \mathrm{g} / \mathrm{kg} \\
\mu \mathrm{g} / \mathrm{kg}\end{array}$ & $\begin{array}{r}5 \\
5 \\
5 \\
5 \\
5 \\
5 \\
5 \\
100 \\
50 \\
50\end{array}$ & $\begin{array}{l}U \\
U \\
U \\
U \\
U \\
U \\
U \\
U \\
U \\
U\end{array}$ & & \\
\hline
\end{tabular}

Location=ORR; Formation=CHEPULTEPEC; Site=50; Samp id=1547; Dup id =' '; Phase =2

\begin{tabular}{|c|c|c|}
\hline 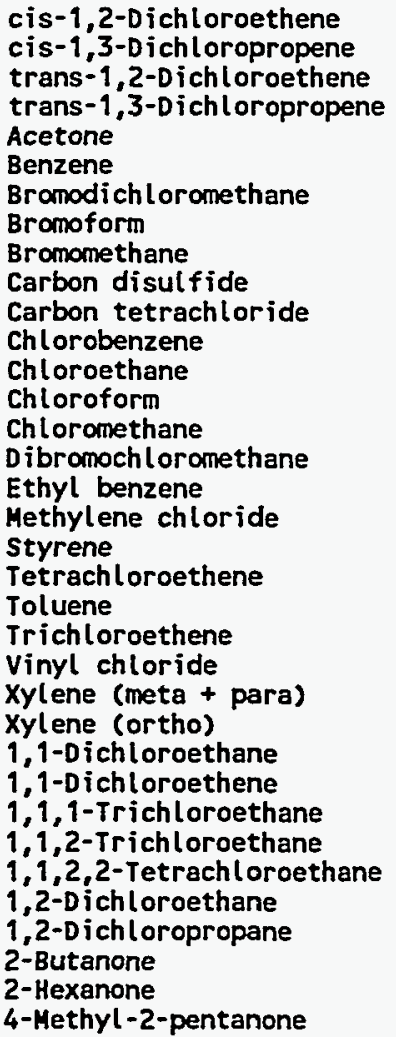 & 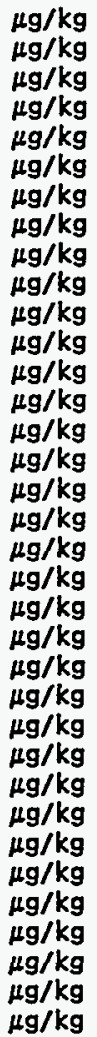 & $\begin{array}{r}5 \\
5 \\
5 \\
100 \\
5 \\
5 \\
5 \\
10 \\
5 \\
5 \\
5 \\
10 \\
5 \\
10 \\
5 \\
5 \\
5 \\
5 \\
5\end{array}$ \\
\hline
\end{tabular}

Location=ORR; Formation=CHEPULTEPEC; Site=52; Samp id=1550; Dup id=' '; Phase=2

$\begin{array}{llrl}\text { cis-1,2-Dichloroethene } & \mu \mathrm{g} / \mathrm{kg} & 5 & \mathrm{U} \\ \text { cis-1,3-Dichloropropene } & \mu \mathrm{g} / \mathrm{kg} & 5 & \mathrm{U} \\ \text { trans-1,2-Dichloroethene } & \mu \mathrm{g} / \mathrm{kg} & 5 & \mathrm{U} \\ \text { trans-1,3-Dichloropropene } & \mu \mathrm{g} / \mathrm{kg} & 5 & \mathrm{U} \\ \text { Acetone } & \mu \mathrm{g} / \mathrm{kg} & 7 & \mathrm{~J} \\ \text { Benzene } & \mu \mathrm{g} / \mathrm{kg} & 5 & \mathrm{U} \\ \text { Bromodichloromethane } & \mu \mathrm{gg} & 5 & \mathrm{U} \\ \text { Bromoform } & \mu \mathrm{g} / \mathrm{kg} & 5 & \mathrm{U} \\ \text { Bromomethane } & \mu \mathrm{g} / \mathrm{kg} & 10 & \mathrm{U} \\ \text { Carbon disulfide } & \mu \mathrm{g} / \mathrm{kg} & 5 & \mathrm{U} \\ \text { Carbon tetrachloride } & \mu \mathrm{g} / \mathrm{kg} & 5 & \mathrm{U} \\ \text { Chlorobenzene } & \mu \mathrm{g} / \mathrm{kg} & 5 & \mathrm{U} \\ \text { Chloroethane } & \mu \mathrm{gg} & 10 & \mathrm{U} \\ \text { Chloroform } & \mu \mathrm{g} / \mathrm{kg} & 5 & \mathrm{U} \\ \text { Chloromethane } & \mu \mathrm{g} / \mathrm{kg} & 10 & \mathrm{U} \\ \text { Dibromochloromethane } & \mu \mathrm{g} / \mathrm{kg} & 5 & \mathrm{U} \\ \text { Ethyl benzene } & \mu \mathrm{kg} & 5 & \mathrm{U} \\ \text { Methylene chloride } & \mu \mathrm{g} / \mathrm{kg} & 5 & \mathrm{U} \\ \text { Styrene } & \mu \mathrm{g} / \mathrm{kg} & 5 & \mathrm{U}\end{array}$


Table B.1 (continued)

\begin{tabular}{|c|c|c|c|c|c|c|}
\hline Analysis & Units & $\begin{array}{c}\mathrm{A} \\
\text { horizon }\end{array}$ & Qualifier & $\begin{array}{l}\text { A horizon } \\
\text { field dup }\end{array}$ & Qualifier & \\
\hline $\begin{array}{l}\text { Tetrachloroethene } \\
\text { Toluene } \\
\text { Trichloroethene } \\
\text { Vinyl chloride } \\
\text { Xylene (meta + para) } \\
\text { Xylene (ortho) } \\
\text { 1,1-D ichloroethane } \\
\text { 1,1-Dichloroethene } \\
1,1,1-\text { Trichloroethane } \\
1,1,2-\text { Trichloroethane } \\
1,1,2,2-\text { Tetrachloroethane } \\
1,2-0 \text { ichloroethane } \\
1,2-\text { ichloropropane } \\
\text { 2-Butanone } \\
\text { 2-Hexanone } \\
\text { 4-Methyl-2-pentanone }\end{array}$ & $\begin{array}{l}\mu \mathrm{g} / \mathrm{kg} \\
\mu \mathrm{g} / \mathrm{kg} \\
\mu \mathrm{g} / \mathrm{kg} \\
\mu \mathrm{g} / \mathrm{kg} \\
\mu \mathrm{g} / \mathrm{kg} \\
\mu \mathrm{g} / \mathrm{kg} \\
\mu \mathrm{g} / \mathrm{kg} \\
\mu \mathrm{g} / \mathrm{kg} \\
\mu \mathrm{g} / \mathrm{kg} \\
\mu \mathrm{g} / \mathrm{kg} \\
\mu \mathrm{g} / \mathrm{kg} \\
\mu \mathrm{g} / \mathrm{kg} \\
\mu \mathrm{g} / \mathrm{kg} \\
\mu \mathrm{g} / \mathrm{kg} \\
\mu \mathrm{g} / \mathrm{kg} \\
\mu \mathrm{g} / \mathrm{kg}\end{array}$ & $\begin{array}{r}5 \\
5 \\
5 \\
10 \\
5 \\
5 \\
5 \\
5 \\
5 \\
5 \\
5 \\
5 \\
5 \\
5 \\
50 \\
50\end{array}$ & $\begin{array}{l}\mathbf{U} \\
\mathbf{U} \\
\mathbf{U} \\
\mathbf{U} \\
\mathbf{U} \\
\mathbf{U} \\
\mathbf{U} \\
\mathbf{U} \\
\mathbf{U} \\
\mathbf{U} \\
\mathbf{U} \\
\mathbf{U} \\
\mathbf{J} \\
\mathbf{U} \\
\mathbf{U}\end{array}$ & "r & & \\
\hline
\end{tabular}

Location=ORR; Formation=CHEPULTEPEC; Site=53; Samp id=1552; Dup id=' '; Phase =2

\begin{tabular}{|c|c|c|}
\hline 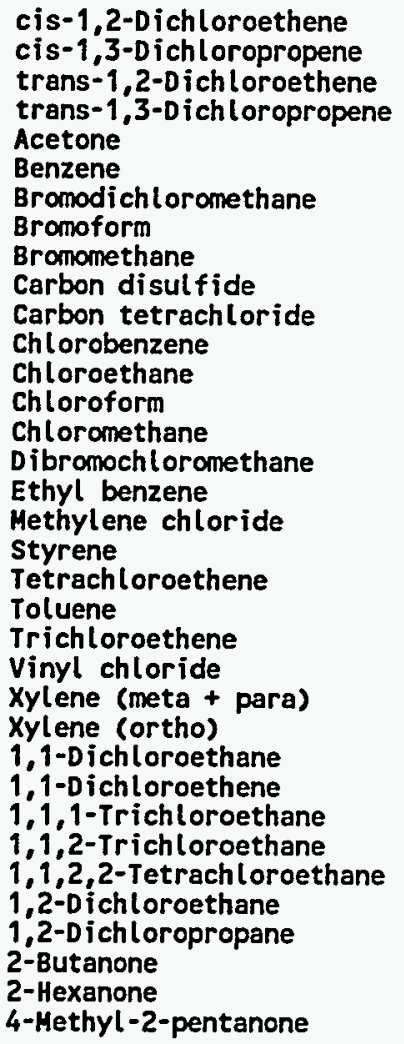 & $\begin{array}{l}\mu g / k g \\
\mu g / k g \\
\mu g / k g \\
\mu g / k g \\
\mu g / k g \\
\mu g / k g \\
\mu g / k g \\
\mu g / k g \\
\mu g / k g \\
\mu g / k g \\
\mu g / k g \\
\mu g / k g \\
\mu g / k g \\
\mu g / k g \\
\mu g / k g \\
\mu g / k g \\
\mu g / k g \\
\mu g / k g \\
\mu g / k g \\
\mu g / k g \\
\mu g / k g \\
\mu g / k g \\
\mu g / k g \\
\mu g / k g \\
\mu g / k g \\
\mu g / k g \\
\mu g / k g \\
\mu g / k g \\
\mu g / k g \\
\mu g / k g \\
\mu g / k g \\
\mu g / k g \\
\mu g / k g \\
\mu g / k g \\
\mu g / k g\end{array}$ & $\begin{array}{r}5 \\
5 \\
5 \\
5 \\
100 \\
5 \\
5 \\
5 \\
10 \\
5 \\
5 \\
5 \\
10 \\
5 \\
10 \\
5 \\
5 \\
5 \\
5 \\
5 \\
5 \\
5 \\
10 \\
5 \\
5 \\
5 \\
5 \\
5 \\
5 \\
5 \\
5 \\
5 \\
100 \\
50 \\
50\end{array}$ \\
\hline
\end{tabular}

Location=ORR; Formation=CHEPULTEPEC; Site=66; Samp id=1737; Dup id=' '; Phase=2

cis-1,2-Dichloroethene cis-1,3-Dichloropropene trans-1,2-Dichloroethene trans-1,3-Dichloropropene Acetone

Benzene

Bromodichloromethane

Bromoform

Bromomethane

Carbon disulfide

Carbon tetrachloride

Chlorobenzene

Chloroethane

$\begin{array}{lrl}\mu \mathrm{g} / \mathrm{kg} & 5 & \mathrm{U} \\ \mu \mathrm{g} / \mathrm{kg} & 5 & U \\ \mu \mathrm{g} / \mathrm{kg} & 5 & U \\ \mu \mathrm{g} / \mathrm{kg} & 5 & U \\ \mu \mathrm{g} / \mathrm{kg} & 100 & U \\ \mu \mathrm{g} / \mathrm{kg} & 5 & U \\ \mu \mathrm{g} / \mathrm{kg} & 5 & U \\ \mu \mathrm{g} / \mathrm{kg} & 5 & U \\ \mu \mathrm{g} / \mathrm{kg} & 10 & U \\ \mu \mathrm{g} / \mathrm{kg} & 5 & U \\ \mu \mathrm{g} / \mathrm{kg} & 5 & U \\ \mu \mathrm{g} / \mathrm{kg} & 5 & U \\ \mu \mathrm{g} / \mathrm{kg} & 10 & U\end{array}$




\section{B-18}

Table B.1 (continued)

\begin{tabular}{|c|c|c|c|c|c|}
\hline Analysis & Units & $\underset{\text { horizon }}{A}$ & Qualifier & $\begin{array}{l}\text { A horizon } \\
\text { field dup }\end{array}$ & Qualifier \\
\hline $\begin{array}{l}\text { Chloroform } \\
\text { Chloromethane } \\
\text { Dibromochloromethane } \\
\text { Ethyl benzene } \\
\text { Methylene chloride } \\
\text { styrene } \\
\text { Tetrachloroethene } \\
\text { Toluene } \\
\text { Trichloroethene } \\
\text { Vinyl chloride } \\
\text { Xylene (meta + para) } \\
\text { xylene (ortho) } \\
\text { 1,1-Dichloroethane } \\
\text { 1,1-Dichloroethene } \\
1,1,1-\text { Trichloroethane } \\
1,1,2-\text { Trichloroethane } \\
1,1,2,2-T e t r a c h l o r o e t h a n e \\
1,2-\text { ichloroethane } \\
1,2-0 \text { ichloropropane } \\
\text { 2--Butanone } \\
\text { 2-Hexanone } \\
\text { 4-Methyl-2-pentanone }\end{array}$ & $\begin{array}{l}\mu \mathrm{g} / \mathrm{kg} \\
\mu \mathrm{g} / \mathrm{kg} \\
\mu \mathrm{g} / \mathrm{kg} \\
\mu \mathrm{g} / \mathrm{kg} \\
\mu \mathrm{g} / \mathrm{kg} \\
\mu \mathrm{g} / \mathrm{kg} \\
\mu \mathrm{g} / \mathrm{kg} \\
\mu \mathrm{g} / \mathrm{kg} \\
\mu \mathrm{g} / \mathrm{kg} \\
\mu \mathrm{g} / \mathrm{kg} \\
\mu \mathrm{g} / \mathrm{kg} \\
\mu \mathrm{g} / \mathrm{kg} \\
\mu \mathrm{g} / \mathrm{kg} \\
\mu \mathrm{g} / \mathrm{kg} \\
\mu \mathrm{g} / \mathrm{kg} \\
\mu \mathrm{g} / \mathrm{kg} \\
\mu \mathrm{g} / \mathrm{kg} \\
\mu \mathrm{g} / \mathrm{kg} \\
\mu \mathrm{g} / \mathrm{kg} \\
\mu \mathrm{g} / \mathrm{kg} \\
\mu \mathrm{g} / \mathrm{kg} \\
\mu \mathrm{g} / \mathrm{kg}\end{array}$ & $\begin{array}{r}5 \\
10 \\
5 \\
5 \\
5 \\
5 \\
5 \\
5 \\
5 \\
10 \\
5 \\
5 \\
5 \\
5 \\
5 \\
5 \\
5 \\
5 \\
5 \\
100 \\
50 \\
50\end{array}$ & $\begin{array}{l}U \\
U \\
U \\
U \\
U \\
U \\
U \\
U \\
U \\
U \\
U \\
U \\
U \\
U \\
U \\
U \\
U \\
U \\
U \\
U \\
U \\
U\end{array}$ & & \\
\hline
\end{tabular}

Location=ORR; Formation=CHEPULTEPEC; Site=68; Samp id=1740; Dup id=' '; Phase=2

\begin{tabular}{|c|c|c|}
\hline 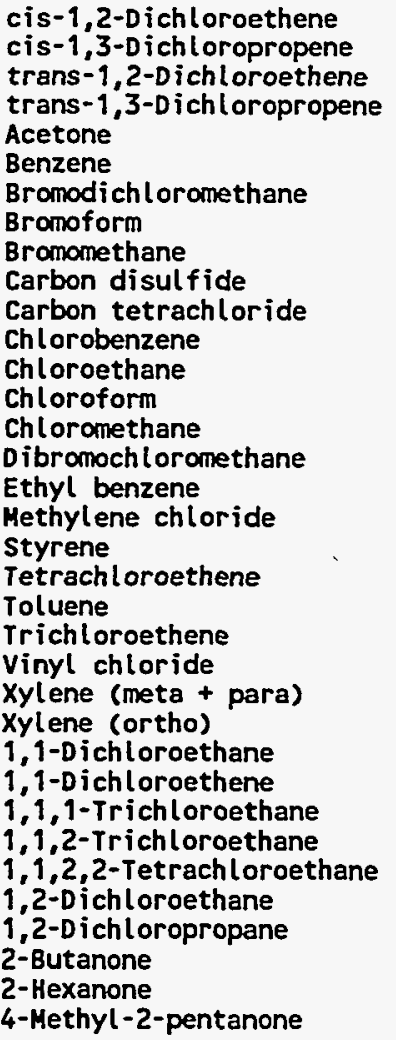 & 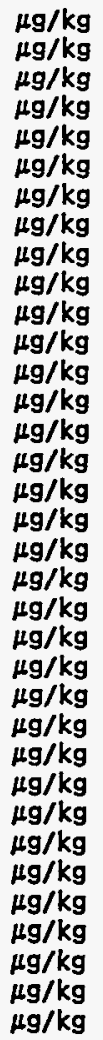 & $\begin{array}{r}5 \\
5 \\
5 \\
5 \\
22 \\
5 \\
5 \\
5 \\
10 \\
5 \\
5 \\
5 \\
10 \\
5 \\
10 \\
5 \\
5 \\
5 \\
5 \\
5 \\
5 \\
5 \\
10 \\
5 \\
5 \\
5 \\
5 \\
5 \\
5 \\
5 \\
5 \\
5 \\
100 \\
50 \\
50\end{array}$ \\
\hline
\end{tabular}

Location=ORR; Formation=CHEPULTEPEC; Site=73; Samp id=1743; Dup id =' '; Phase=2

$\begin{array}{llrl}\text { cis-1,2-Dichloroethene } & \mu g / k g & 5 & U \\ \text { cis-1,3-Dichloropropene } & \mu g / k g & 5 & U \\ \text { trans-1,2-Dichloroethene } & \mu g / k g & 5 & U \\ \text { trans-1,3-Dichloropropene } & \mu g / k g & 5 & U \\ \text { Acetone } & \mu g / k g & 13 & J \\ \text { Benzene } & \mu g / k g & 5 & U \\ \text { Bromodichloromethane } & \mu g / k g & 5 & U\end{array}$


Table B.1 (continued)

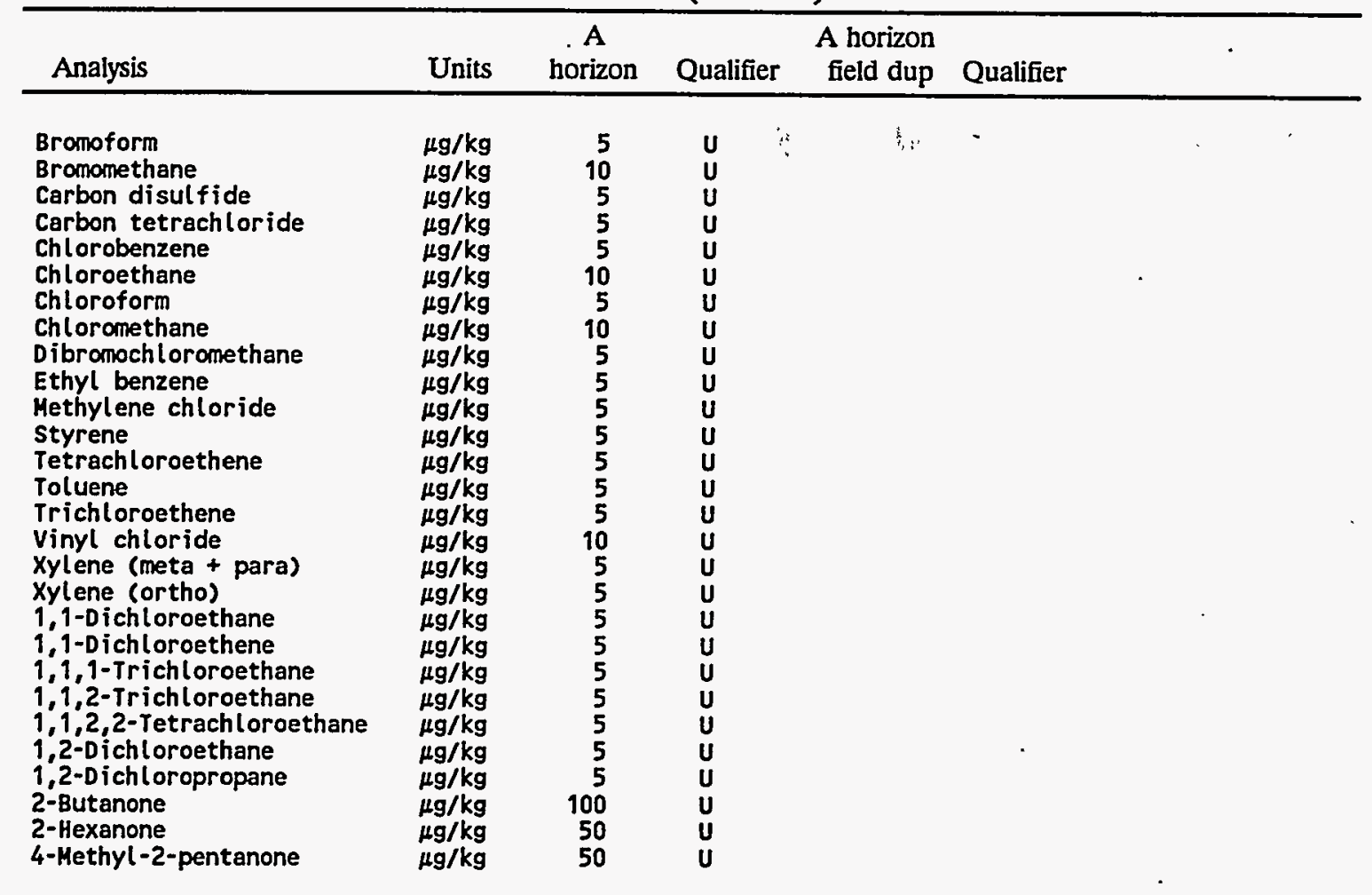

Location=ORR; Formation=CHEPULTEPEC; Site=74; Samp id =1745; Dup id=' '; Phase $=2$

\begin{tabular}{|c|c|c|}
\hline 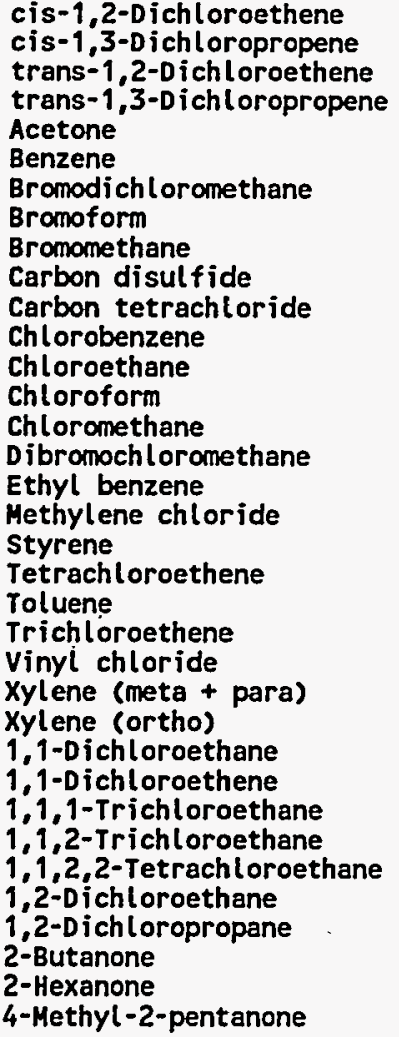 & 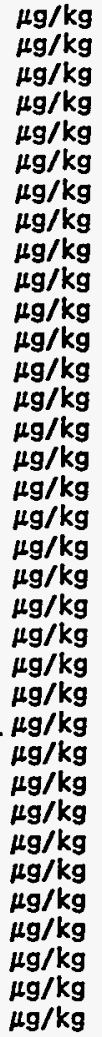 & $\begin{array}{r}5 \\
5 \\
5 \\
5 \\
5 \\
5 \\
10 \\
5 \\
5 \\
5 \\
10 \\
5 \\
10 \\
5 \\
5 \\
5 \\
5 \\
5 \\
5 \\
5 \\
10 \\
5 \\
5 \\
5 \\
5 \\
5 \\
5 \\
5 \\
5 \\
5 \\
100 \\
50 \\
50\end{array}$ \\
\hline
\end{tabular}


Table B.1 (continued)

\begin{tabular}{lccc}
\hline & A & A horizon \\
Analysis & Units & horizon & Qualifier field dup Qualifier \\
\hline
\end{tabular}

Location=ORR; Formation=CHEPULTEPEC; Site=77; Samp id=1599; Dup id=' '; Phase=2

\begin{tabular}{|c|c|c|}
\hline 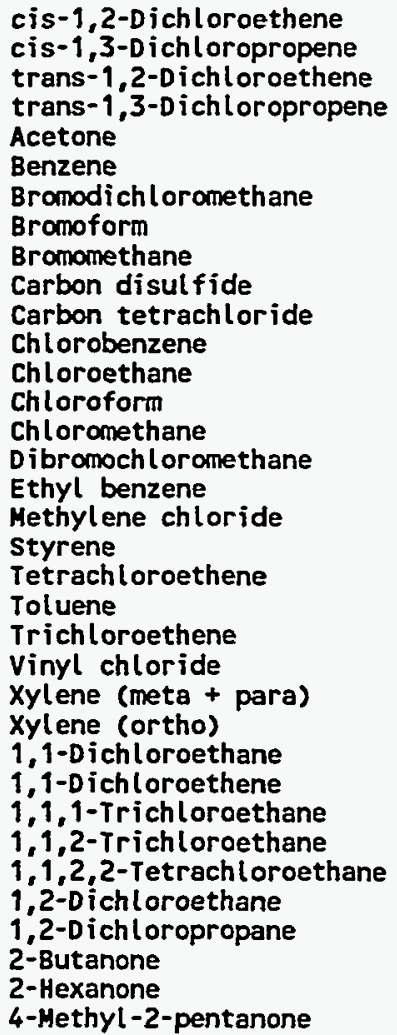 & 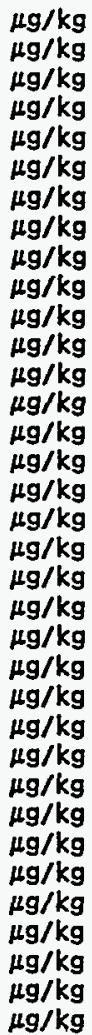 & $\begin{array}{r}5 \\
5 \\
5 \\
5 \\
100 \\
5 \\
5 \\
5 \\
10 \\
5 \\
5 \\
5 \\
10 \\
5 \\
10 \\
5 \\
5 \\
5 \\
5 \\
5 \\
5 \\
5 \\
10 \\
5 \\
5 \\
5 \\
5 \\
5 \\
5 \\
5 \\
5 \\
100 \\
50 \\
50\end{array}$ \\
\hline
\end{tabular}

Location=ORR; Formation=CHEPULTEPEC; Site=78; Samp id=1601; Dup id=' '; Phase=2

\begin{tabular}{|c|c|c|}
\hline $\begin{array}{l}\text { cis-1,2-Dichloroethene } \\
\text { cis-1,3-Dichloropropene } \\
\text { trans-1,2-Dichloroethene } \\
\text { trans-1,3-Dichloropropene } \\
\text { Acetone } \\
\text { Benzene } \\
\text { Bromodichloromethane } \\
\text { Bromoform } \\
\text { Bromomethane } \\
\text { Carbon disulfide } \\
\text { Carbon tetrachloride } \\
\text { Chlorobenzene } \\
\text { Chloroethane } \\
\text { Chloroform } \\
\text { Chloromethane } \\
\text { Dibromochloromethane } \\
\text { Ethyl benzene } \\
\text { Methylene chloride } \\
\text { Styrene } \\
\text { Tetrachloroethene } \\
\text { Toluene } \\
\text { Trichloroethene } \\
\text { vinyl chloride } \\
\text { Xylene (meta + para) } \\
\text { Xylene (ortho) } \\
1,1-D \text { ichloroethane } \\
1,1-D i c h l o r o e t h e n e \\
1,1,1-\text { Trichloroethane } \\
1,1,2-\text { Trichloroethane } \\
1,1,2,2-\text { Tetrachloroethane } \\
1,2-D \text { ichloroethane }\end{array}$ & 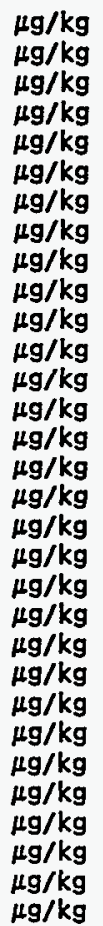 & $\begin{array}{r}5 \\
5 \\
5 \\
5 \\
100 \\
5 \\
5 \\
5 \\
10 \\
5 \\
5 \\
5 \\
10 \\
5 \\
10 \\
5\end{array}$ \\
\hline
\end{tabular}


B-21.

Table B.1 (continued)

\begin{tabular}{|c|c|c|c|c|c|c|}
\hline Analysis & Units & $\begin{array}{c}A \\
\text { horizon }\end{array}$ & Qualifier & $\begin{array}{l}\text { A horizon } \\
\text { field dup }\end{array}$ & Qualifier. & \\
\hline $\begin{array}{l}\text { 1,2-Dichloropropane } \\
\text { 2-Butanone } \\
\text { 2-Hexanone } \\
\text { 4-Methyl-2-pentanone }\end{array}$ & $\begin{array}{l}\mu \mathrm{g} / \mathrm{kg} \\
\mu \mathrm{g} / \mathrm{kg} \\
\mu \mathrm{g} / \mathrm{kg} \\
\mu \mathrm{g} / \mathrm{kg}\end{array}$ & $\begin{array}{r}5 \\
100 \\
50 \\
50\end{array}$ & $\begin{array}{l}\mathbf{u} \\
\mathbf{U} \\
\mathbf{U} \\
\mathbf{U}\end{array}$ & ' & . & \\
\hline
\end{tabular}

Location=ORR; Formation=CHEPULTEPEC; Site=85; Samp id=1605; Dup id =' '; Phase=2

\begin{tabular}{|c|c|c|}
\hline 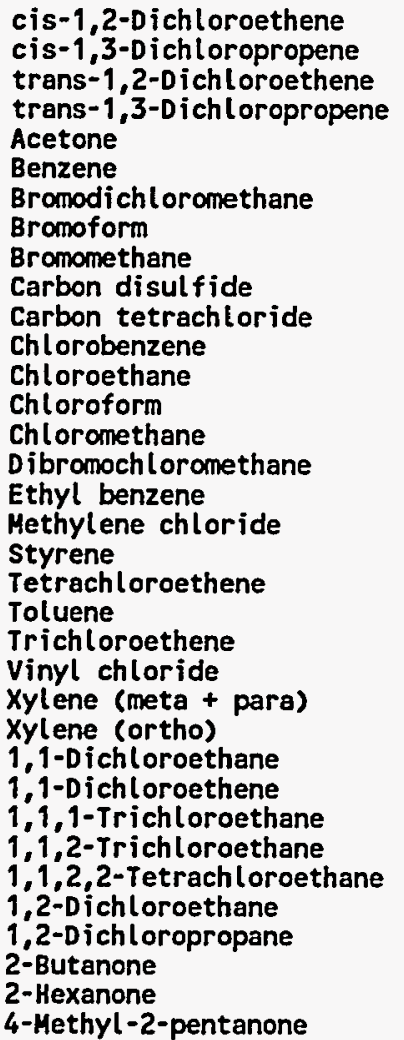 & 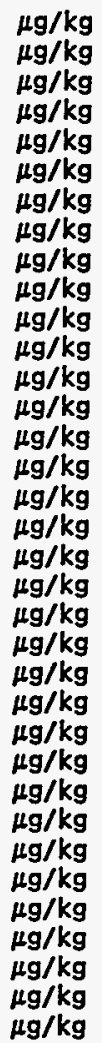 & $\begin{array}{r}5 \\
5 \\
5 \\
5 \\
8 \\
5 \\
5 \\
5 \\
10 \\
5 \\
5 \\
5 \\
10 \\
5 \\
10 \\
5 \\
5 \\
5 \\
5 \\
5 \\
5 \\
5 \\
10 \\
5 \\
5 \\
5 \\
5 \\
5 \\
5 \\
5 \\
5 \\
5 \\
100 \\
50\end{array}$ \\
\hline
\end{tabular}

Location $=$ ORR; Formation=CHEPULTEPEC; Site=86; Samp id=1603; Dup id=' '; Phase $=2$

\begin{tabular}{|c|c|c|}
\hline $\begin{array}{l}\text { cis-1,2-D ichloroethene } \\
\text { cis-1,3-Dichloropropene } \\
\text { trans-1,2-Dichloroethene } \\
\text { trans-1,3-D ichloropropene } \\
\text { Acetone } \\
\text { Benzene } \\
\text { Bromodichlorome thane } \\
\text { Bromoform } \\
\text { Bromomethane } \\
\text { Carbon disulfide } \\
\text { Carbon tetrachloride } \\
\text { Chlorobenzene } \\
\text { Chloroethane } \\
\text { Chloroform } \\
\text { Chloromethane } \\
\text { Dibromochloromethane } \\
\text { Ethyl benzene } \\
\text { Methylene chloride } \\
\text { Styrene } \\
\text { Tetrachloroethene } \\
\text { Toluene } \\
\text { Trichloroethene } \\
\text { Vinyl chloride } \\
\text { Xylene (meta + para) } \\
\text { Xylene (ortho) }\end{array}$ & $\begin{array}{l}\mu g / k g \\
\mu g / k g \\
\mu g / k g \\
\mu g / k g \\
\mu g / k g \\
\mu g / k g \\
\mu g / k g \\
\mu g / k g \\
\mu g / k g \\
\mu g / k g \\
\mu g / k g \\
\mu g / k g \\
\mu g / k g \\
\mu g / k g \\
\mu g / k g \\
\mu g / k g \\
\mu g / k g \\
\mu g / k g \\
\mu g / k g \\
\mu g / k g \\
\mu g / k g \\
\mu g / k g \\
\mu g / k g \\
\mu g / k g \\
\mu g / k g\end{array}$ & $\begin{array}{r}100 \\
5 \\
10 \\
5\end{array}$ \\
\hline
\end{tabular}


Tabłe B.1 (continued)

\begin{tabular}{|c|c|c|c|c|c|}
\hline Analysis & Units & $\begin{array}{c}\text { A } \\
\text { horizon }\end{array}$ & Qualifier & $\begin{array}{l}\text { A horizon } \\
\text { field dup }\end{array}$ & Qualifier \\
\hline $\begin{array}{l}\text { 1,1-Dichloroethane } \\
\text { 1,1-Dichloroethene } \\
1,1,1-T r \text { ichloroethane } \\
1,1,2-\text { Trichloroethane } \\
1,1,2,2-\text { Tetrachloroethane } \\
\text { 1,2-Dichloroethane } \\
\text { 1,2-Dichloropropane } \\
\text { 2-Butanone } \\
\text { 2-Hexanone } \\
\text { 4-Methyl-2-pentanone }\end{array}$ & $\begin{array}{l}\mu \mathrm{g} / \mathrm{kg} \\
\mu \mathrm{g} / \mathrm{kg} \\
\mu \mathrm{g} / \mathrm{kg} \\
\mu \mathrm{g} / \mathrm{kg} \\
\mu \mathrm{g} / \mathrm{kg} \\
\mu \mathrm{g} / \mathrm{kg} \\
\mu \mathrm{g} / \mathrm{kg} \\
\mu \mathrm{g} / \mathrm{kg} \\
\mu \mathrm{g} / \mathrm{kg} \\
\mu \mathrm{g} / \mathrm{kg}\end{array}$ & $\begin{array}{r}5 \\
5 \\
5 \\
5 \\
5 \\
5 \\
5 \\
100 \\
50 \\
50\end{array}$ & $\begin{array}{l}\mathbf{U} \\
\mathbf{U} \\
\mathbf{U} \\
\mathbf{U} \\
\mathbf{U} \\
\mathbf{U} \\
\mathbf{U} \\
\mathbf{U} \\
\mathbf{U} \\
\mathbf{U}\end{array}$ & & \\
\hline \multicolumn{6}{|c|}{ Location $=$ ORR; Formation $=$ CHEPULTEPEC; Site $=90 ;$ Samp id $=1748 ;$ Dup id =' '; Phase $=2$} \\
\hline $\begin{array}{l}\text { cis-1,2-Dichloroethene } \\
\text { cis-1,3-Dichloropropene } \\
\text { trans-1,2-Dichloroethene } \\
\text { trans-1,3-Dichloropropene } \\
\text { Acetone } \\
\text { Benzene } \\
\text { Bromodichloromethane } \\
\text { Bromoform } \\
\text { Bromomethane } \\
\text { Carbon disulfide } \\
\text { Carbon tetrachloride } \\
\text { Chlorobenzene } \\
\text { Chloroethane } \\
\text { Chloroform } \\
\text { Chloromethane } \\
\text { Dibromochloromethane } \\
\text { Ethyl benzene } \\
\text { Methylene chloride } \\
\text { Styrene } \\
\text { Tetrachloroethene } \\
\text { Toluene } \\
\text { Trichloroethene } \\
\text { Vinyl chloride } \\
\text { Xylene (meta + para) } \\
\text { Xylene (ortho) } \\
1,1-D \text { ichloroethane } \\
1,1-D i c h l o r o e t h e n e \\
1,1,1-\text { Trichloroethane } \\
1,1,2-\text { Trichloroethane } \\
1,1,2 \text {, -Tetrachloroethane } \\
1,2-D \text { ichloroethane } \\
1,2-D \text { ichloropropane } \\
2-\text { Butanone } \\
2-\text { Hexanone } \\
\text { 4-Methyl-2-pentanone }\end{array}$ & 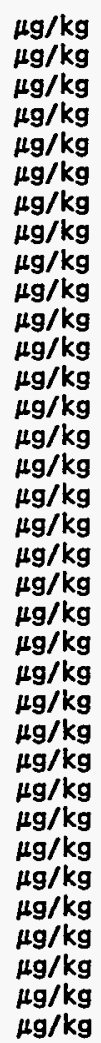 & $\begin{array}{r}5 \\
5 \\
5 \\
5 \\
6 \\
5 \\
5 \\
5 \\
10 \\
5 \\
5 \\
5 \\
10 \\
5 \\
10 \\
5 \\
5 \\
5 \\
5 \\
5 \\
5 \\
5 \\
10 \\
5 \\
5 \\
5 \\
5 \\
5 \\
5 \\
5 \\
5 \\
5 \\
100 \\
50 \\
50\end{array}$ & $\begin{array}{l}U \\
U \\
U \\
U \\
J \\
U \\
U \\
U \\
U \\
U \\
U \\
U \\
U \\
U \\
U \\
U \\
U \\
U \\
U \\
U \\
U \\
U \\
U \\
U \\
U \\
U \\
U \\
U \\
U \\
U \\
U \\
U \\
U \\
U \\
U\end{array}$ & & \\
\hline
\end{tabular}

Location=ORR; Formation=CHICKAMAUGA Bethel V; Site=100; Samp id=1905; Dup id=' '; Phase=2

$\begin{array}{llrl}\text { cis-1,2-Dichloroethene } & \mu \mathrm{g} / \mathrm{kg} & 5 & \mathrm{U} \\ \text { cis-1,3-Dichloropropene } & \mu \mathrm{g} / \mathrm{kg} & 5 & \mathrm{U} \\ \text { trans-1,2-Dichloroethene } & \mu \mathrm{g} / \mathrm{kg} & 5 & \mathrm{U} \\ \text { trans-1,3-Dichloropropene } & \mu \mathrm{g} / \mathrm{kg} & 5 & \mathrm{U} \\ \text { Acetone } & \mu \mathrm{g} / \mathrm{kg} & 7 & \mathrm{~J} \\ \text { Benzene } & \mu \mathrm{g} / \mathrm{kg} & 5 & \mathrm{U} \\ \text { Bromodichloromethane } & \mu \mathrm{g} / \mathrm{kg} & 5 & \mathrm{U} \\ \text { Bromoform } & \mu \mathrm{g} / \mathrm{kg} & 5 & \mathbf{U} \\ \text { Bromomethane } & \mu \mathrm{g} / \mathrm{kg} & 10 & \mathbf{U} \\ \text { Carbon disulfide } & \mu \mathrm{g} / \mathrm{kg} & 5 & \mathbf{U} \\ \text { Carbon tetrachloride } & \mu \mathrm{g} / \mathrm{kg} & 5 & \mathbf{U} \\ \text { Chlorobenzene } & \mu \mathrm{g} / \mathrm{kg} & 5 & \mathbf{U} \\ \text { Chloroethane } & \mu \mathrm{g} / \mathrm{kg} & 10 & \mathbf{U} \\ \text { Chloroform } & \mu \mathrm{g} / \mathrm{kg} & 5 & \mathbf{U} \\ \text { Chloromethane } & \mu \mathrm{gg} / \mathrm{kg} & 10 & \mathbf{U} \\ \text { Dibromochloromethane } & \mu \mathrm{g} / \mathrm{kg} & 5 & \mathbf{U} \\ \text { Ethyl benzene } & \mu \mathrm{g} / \mathrm{kg} & 5 & \mathbf{U}\end{array}$




\section{B-23}

Table B.1 (continued)

\begin{tabular}{|c|c|c|c|c|c|}
\hline Analysis & Units & $\begin{array}{c}\text { A } \\
\text { horizon }\end{array}$ & Qualifier & $\begin{array}{l}\text { A horizon } \\
\text { field dup }\end{array}$ & Qualifier \\
\hline $\begin{array}{l}\text { Methylene chloride } \\
\text { Styrene } \\
\text { Tetrachloroethene } \\
\text { Toluene. } \\
\text { Trichloroethene } \\
\text { Vinyl chloride } \\
\text { xylene (meta + para) } \\
\text { xylene (ortho) } \\
1,1-D \text { ichloroethane } \\
1,1-D i c h l o r o e t h e n e \\
1,1,1-\text { Trichloroethane } \\
1,1,2-\text { Trichloroethane } \\
1,1,2,2-T e t r a c h l o r o e t h a n e \\
1,2-\text { ichloroethane } \\
1,2-D i c h l o r o p r o p a n e \\
\text { 2-Butanone } \\
\text { 2-Hexanone } \\
\text { 4-Methyl-2-pentanone. }\end{array}$ & 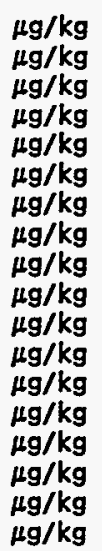 & $\begin{array}{r}5 \\
5 \\
5 \\
5 \\
5 \\
10 \\
5 \\
5 \\
5 \\
5 \\
5 \\
5 \\
5 \\
5 \\
5 \\
100 \\
50 \\
50\end{array}$ & $\begin{array}{l}U \\
U \\
U \\
U \\
U \\
U \\
U \\
U \\
U \\
U \\
U \\
U \\
U \\
U \\
U \\
U \\
U \\
U \\
U\end{array}$ & . & \\
\hline
\end{tabular}

Location=ORR; Formation=CHICKAMAUGA Bethel V; Site=101; Samp id=1896; Dup id=' '; Phase=2

\begin{tabular}{|c|c|c|}
\hline 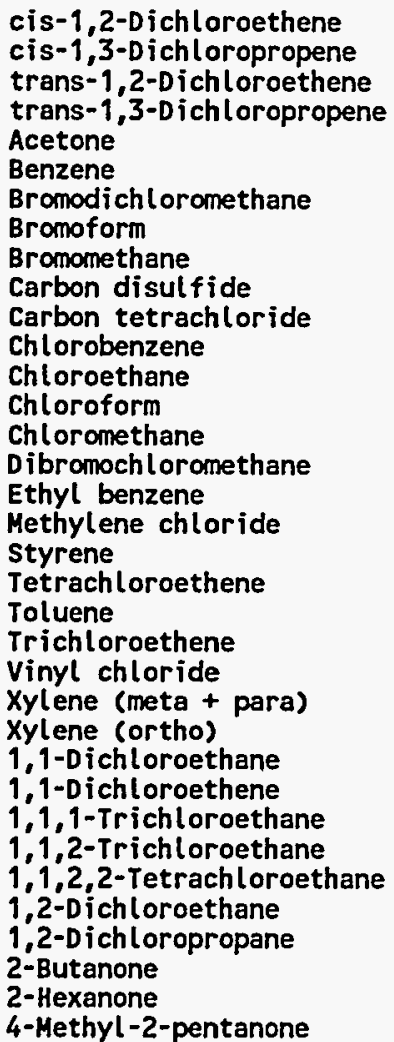 & 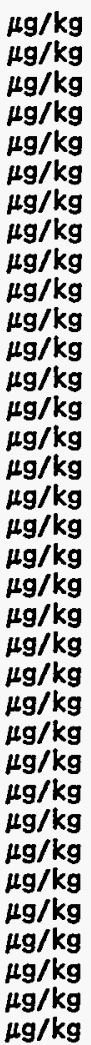 & $\begin{array}{r}5 \\
5 \\
5 \\
5 \\
11 \\
5 \\
5 \\
5 \\
10 \\
5 \\
5 \\
5 \\
10 \\
5 \\
10 \\
5 \\
5 \\
5 \\
5 \\
5 \\
5 \\
5 \\
10 \\
5 \\
5 \\
5 \\
5 \\
5 \\
5 \\
5 \\
5 \\
5 \\
100 \\
50 \\
50\end{array}$ \\
\hline
\end{tabular}

Location=ORR; Formation=CHICKAMAUGA Bethel V; Site=102; Samp id=1893; Dup id=' '; Phase=2

cis-1,2-Dichloroethene

cis-1,3-Dichloropropene trans-1,2-Dich loroethene trans-1,3-Dich loropropene Acetone Benzene Bromodichloromethane Bromoform $\mu \mathrm{g} / \mathrm{kg}$ $\mu \mathrm{g} / \mathrm{kg}$ $\mu \mathrm{g} / \mathrm{kg}$ $\mu \mathrm{g} / \mathrm{kg}$ $\mu \mathrm{g} / \mathrm{kg}$ $\mu \mathrm{g} / \mathrm{kg}$ $\mu \mathrm{g} / \mathrm{kg}$ $\mu \mathrm{g} / \mathrm{kg}$

$\begin{array}{ll}5 & U \\ 5 & U \\ 5 & U \\ 5 & U \\ 9 & J \\ 5 & U \\ 5 & U \\ 5 & U\end{array}$


Table B.1 (continued)

\begin{tabular}{|c|c|c|c|c|c|}
\hline Analysis & Units & $\begin{array}{c}\text { A } \\
\text { horizon }\end{array}$ & Qualifier & $\begin{array}{l}\text { A horizon } \\
\text { field dup }\end{array}$ & Qualifier \\
\hline 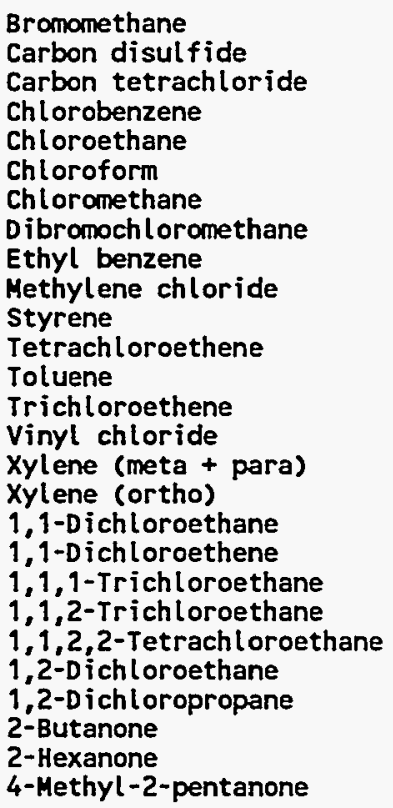 & 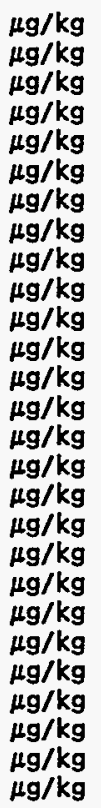 & $\begin{array}{r}10 \\
5 \\
5 \\
5 \\
10 \\
5 \\
10 \\
5 \\
5 \\
5 \\
5 \\
5 \\
5 \\
5 \\
10 \\
5 \\
5 \\
5 \\
5 \\
5 \\
5 \\
5 \\
5 \\
5 \\
100 \\
50 \\
50\end{array}$ & $\begin{array}{l}U \\
U \\
U \\
U \\
U \\
U \\
U \\
U \\
U \\
U \\
U \\
U \\
U \\
U \\
U \\
U \\
U \\
U \\
U \\
U \\
U \\
U \\
U \\
U \\
U \\
U \\
U\end{array}$ & & \\
\hline
\end{tabular}

Location=ORR; Formation=CHICKAMAUGA Bethel V; Site=103; Samp id=1890; Dup id=' '; Phase=2

\begin{tabular}{|c|c|c|}
\hline $\begin{array}{l}\text { Benzene } \\
\text { Bromodichloromethane } \\
\text { Bromoform } \\
\text { Bromomethane } \\
\text { Carbon disulfide } \\
\text { Carbon tetrachloride } \\
\text { Chlorobenzene } \\
\text { Chloroethane } \\
\text { Chloroform } \\
\text { Chloromethane } \\
\text { Dibromochloromethane } \\
\text { Ethyl benzene } \\
\text { Methylene chloride } \\
\text { Styrene } \\
\text { Tetrachloroethene } \\
\text { Toluene } \\
\text { Trichloroethene } \\
\text { Vinyl chloride } \\
\text { xylene (meta + para) } \\
\text { xylene (ortho) } \\
1,1-D \text { ichloroethane } \\
1,1-D \text { ichloroethene } \\
1,1,1-\text { Trichloroethane } \\
1,1,2-T r i c h l o r o e t h a n e \\
1,1,2,2-\text { Tetrachloroethane } \\
1,2-D \text { ichloroethane } \\
1,2-D \text { ichloropropane } \\
2-8 \text { tanone } \\
\text { 2-Hexanone } \\
4-\text { Methyl-2-pentanone }\end{array}$ & $\begin{array}{l}\mu g / \mathbf{k g} \\
\mu g / \mathbf{k g} \\
\mu g / \mathbf{k g} \\
\mu \mathrm{g} / \mathrm{kg} \\
\mu \mathrm{g} / \mathrm{kg} \\
\mu \mathrm{g} / \mathrm{kg} \\
\mu \mathrm{g} / \mathrm{kg} \\
\mu \mathrm{g} / \mathrm{kg} \\
\mu \mathrm{g} / \mathrm{kg} \\
\mu \mathrm{g} / \mathrm{kg} \\
\mu \mathrm{g} / \mathrm{kg} \\
\mu \mathrm{g} / \mathrm{kg} \\
\mu \mathrm{g} / \mathrm{kg} \\
\mu \mathrm{g} / \mathrm{kg} \\
\mu \mathrm{g} / \mathrm{kg} \\
\mu \mathrm{g} / \mathrm{kg} \\
\mu \mathrm{g} / \mathrm{kg} \\
\mu \mathrm{g} / \mathrm{kg} \\
\mu \mathrm{g} / \mathrm{kg} \\
\mu \mathrm{g} / \mathrm{kg} \\
\mu \mathrm{g} / \mathrm{kg} \\
\mu \mathrm{g} / \mathrm{kg} \\
\mu \mathrm{g} / \mathrm{kg} \\
\mu \mathrm{g} / \mathrm{kg} \\
\mu \mathrm{g} / \mathrm{kg} \\
\mu \mathrm{g} / \mathrm{kg} \\
\mu \mathrm{g} / \mathrm{kg} \\
\mu \mathrm{g} / \mathrm{kg} \\
\mu \mathrm{g} / \mathrm{kg} \\
\mu \mathrm{g} / \mathrm{kg} \\
\mu \mathrm{g} / \mathrm{kg} \\
\mu \mathrm{g} / \mathrm{kg} \\
\mu \mathrm{g} / \mathrm{kg} \\
\mu \mathrm{g} / \mathrm{kg} \\
\mu \mathrm{g} / \mathrm{kg}\end{array}$ & $\begin{array}{r}5 \\
5 \\
5 \\
5 \\
11 \\
5 \\
5 \\
5 \\
10 \\
5 \\
5 \\
5 \\
10 \\
5 \\
10 \\
5 \\
5 \\
5 \\
5 \\
5 \\
5 \\
5 \\
10 \\
5 \\
5 \\
5 \\
5 \\
5 \\
5 \\
5 \\
5 \\
5 \\
100 \\
50 \\
50\end{array}$ \\
\hline
\end{tabular}


Table B.1 (continued)

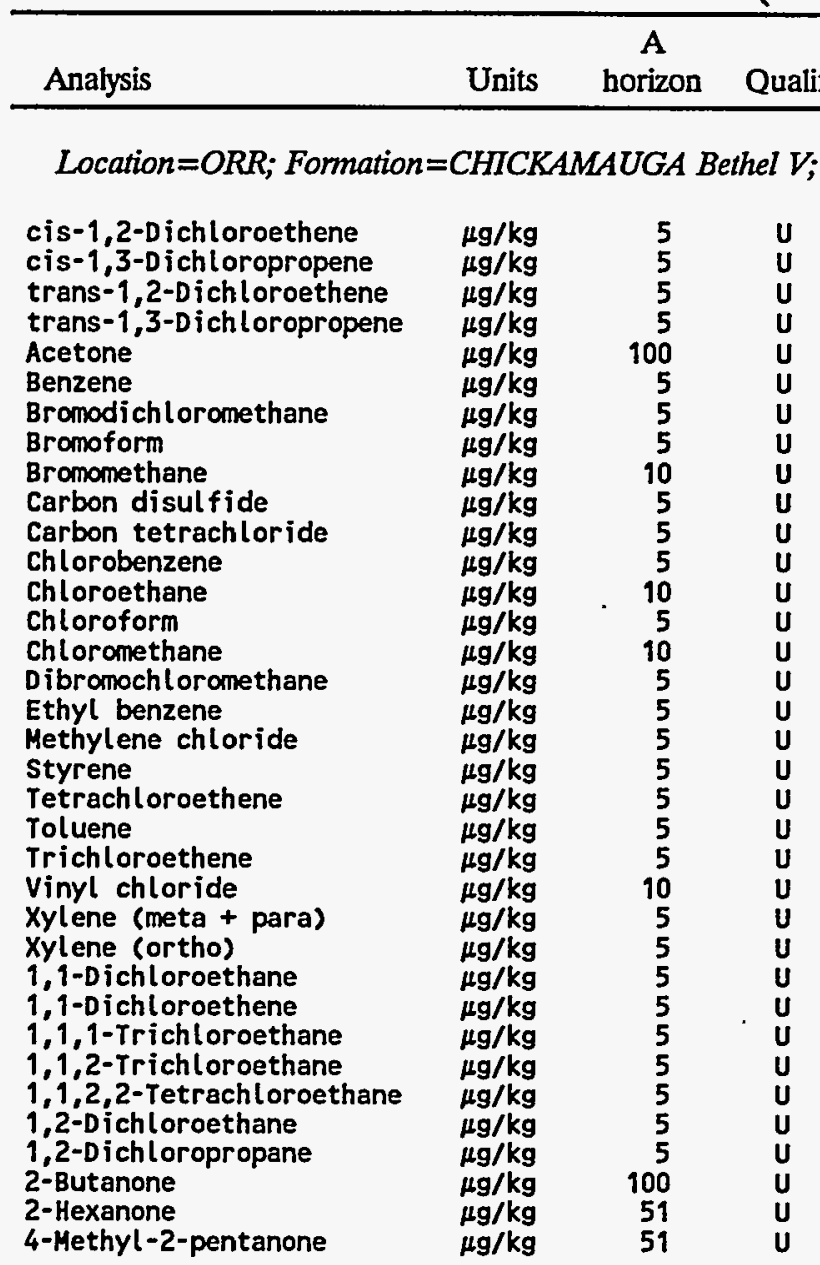

Location=ORR; Formation=CHICKAMAUGA Bethel V; Site=108; Samp id=1966; Dup id=' '; Phase=2

$\begin{array}{llrl}\text { cis-1,2-Dichloroethene } & \mu g / k g & 5 & U \\ \text { cis-1,3-Dichloropropene } & \mu g / k g & 5 & U \\ \text { trans-1,2-Dichloroethene } & \mu g / k g & 5 & U \\ \text { trans-1,3-Dichloropropene } & \mu g / k g & 5 & U \\ \text { Acetone } & \mu g / k g & 100 & U \\ \text { Benzene } & \mu g / k g & 5 & U \\ \text { Bromodichloromethane } & \mu g / k g & 5 & U \\ \text { Bromoform } & \mu g / k g & 5 & U \\ \text { Bromomethane } & \mu g / k g & 10 & U \\ \text { Carbon disulfide } & \mu g / k g & 5 & U \\ \text { Carbon tetrachloride } & \mu g / k g & 5 & U \\ \text { Chlorobenzene } & \mu g / k g & 5 & U \\ \text { Chloroethane } & \mu g / k g & 10 & U \\ \text { Chloroform } & \mu g / k g & 5 & U \\ \text { Chloromethane } & \mu g / k g & 10 & U \\ \text { Dibromochloromethane } & \mu g / k g & 5 & U \\ \text { Ethyl benzene } & \mu g / k g & 5 & U \\ \text { Methylene chloride } & \mu g / k g & 5 & U \\ \text { Styrene } & \mu g / k g & 5 & U \\ \text { Tetrachloroethene } & \mu g / k g & 5 & U \\ \text { Toluene } & \mu g / k g & 5 & U \\ \text { Trichloroethene } & \mu g / k g & 5 & U \\ \text { Vinyl chloride } & \mu g / k g & 10 & U \\ \text { Xylene (meta tora) } & \mu g / k g & 5 & U \\ \text { Xylene (ortho) } & \mu g / k g & 5 & U \\ \text { 1, 1-Dichloroethane } & \mu g / k g & 5 & U \\ \text { 1,1-Dichloroethene } & \mu g / k g & 5 & U \\ \text { 1,1,1-Trichloroethane } & \mu g / k g & 5 & U\end{array}$


Table B.1 (continued)

\begin{tabular}{|c|c|c|c|c|c|}
\hline Analysis & Units & $\begin{array}{c}\mathbf{A} \\
\text { horizon }\end{array}$ & Qualifier & $\begin{array}{l}\text { A horizon } \\
\text { field dup }\end{array}$ & Qualifier \\
\hline $\begin{array}{l}\text { 1,1,2-Trichloroethane } \\
\text { 1,1,2,2-Tetrachloroethane } \\
\text { 1,2-Dichloroethane } \\
\text { 1,2-Dichloropropane } \\
\text { 2-Butanone } \\
\text { 2-Hexanone } \\
\text { 4-Methyl-2-pentanone }\end{array}$ & $\begin{array}{l}\mu \mathrm{g} / \mathrm{kg} \\
\mu \mathrm{g} / \mathrm{kg} \\
\mu \mathrm{g} / \mathrm{kg} \\
\mu \mathrm{g} / \mathrm{kg} \\
\mu \mathrm{g} / \mathrm{kg} \\
\mu \mathrm{g} / \mathrm{kg} \\
\mu \mathrm{g} / \mathrm{kg}\end{array}$ & $\begin{array}{r}5 \\
5 \\
5 \\
5 \\
9 \\
50 \\
-\quad 50\end{array}$ & $\begin{array}{l}\mathrm{U} \\
\mathrm{U} \\
\mathrm{U} \\
\mathrm{U} \\
\mathrm{J} \\
\mathrm{U} \\
\mathrm{U}\end{array}$ & & . \\
\hline \multicolumn{6}{|c|}{ Location=ORR; Formation=CHICKAMAUGA Bethel V; Site=110; Samp id=1969; Dup id=' '; Phase=2 } \\
\hline $\begin{array}{l}\text { cis-1,2-Dichloroethene } \\
\text { cis-1,3-Dichloropropene } \\
\text { trans-1,2-Dichloroethene } \\
\text { trans-1,3-Dichloropropene } \\
\text { Acetone } \\
\text { Benzene } \\
\text { Bromodichloromethane } \\
\text { Bromoform } \\
\text { Bromomethane } \\
\text { Carbon disulfide } \\
\text { Carbon tetrachloride } \\
\text { Chlorobenzene } \\
\text { Chloroethane } \\
\text { Chloroform } \\
\text { Chloromethane } \\
\text { Dibromochloromethane } \\
\text { Ethyl benzene } \\
\text { Methylene chloride } \\
\text { Styrene } \\
\text { Tetrachloroethene } \\
\text { Toluene } \\
\text { Trichloroethene } \\
\text { Vinyl chloride } \\
\text { Xylene (meta + para) } \\
\text { Xylene (ortho) } \\
1,1-D \text { ichloroethane } \\
\text { 1,1-Dichloroethene } \\
1,1,1-\text { Trichloroethane } \\
1,1,2-\text { Trichloroethane } \\
1,1,2,2-\text { Tetrachloroethane } \\
1,2-D \text { ichloroethane } \\
1,2-D \text { ichloropropane } \\
2-\text { Butanone } \\
2-\text { Hexanone } \\
\text { 4-Methyl-2-pentanone }\end{array}$ & $\begin{array}{l}\mu g / \mathbf{k g} \\
\mu g / \mathbf{k g} \\
\mu g / \mathbf{k g} \\
\mu g / \mathbf{k g} \\
\mu g / \mathbf{k g} \\
\mu g / \mathbf{k g} \\
\mu g / \mathbf{k g} \\
\mu g / \mathbf{k g} \\
\mu g / \mathbf{k g} \\
\mu g / \mathbf{k g} \\
\mu g / \mathbf{k g} \\
\mu g / \mathbf{k g} \\
\mu g / \mathbf{k g} \\
\mu g / \mathbf{k g} \\
\mu g / \mathbf{k g} \\
\mu g / \mathbf{k g} \\
\mu g / \mathbf{k g} \\
\mu g / \mathbf{k g} \\
\mu g / \mathbf{k g} \\
\mu g / \mathbf{k g} \\
\mu g / \mathbf{k g} \\
\mu g / \mathbf{k g} \\
\mu g / \mathbf{k g} \\
\mu g / \mathbf{k g} \\
\mu g / \mathbf{k g} \\
\mu g / \mathbf{k g} \\
\mu g / \mathbf{k g} \\
\mu g / \mathbf{k g} \\
\mu g / \mathbf{k g} \\
\mu g / \mathbf{k g} \\
\mu g / \mathbf{k g} \\
\mu g / \mathbf{k g}\end{array}$ & $\begin{array}{r}5 \\
5 \\
5 \\
5 \\
5 \\
5 \\
5 \\
5 \\
10 \\
5 \\
5 \\
5 \\
10 \\
5 \\
10 \\
5 \\
5 \\
5 \\
5 \\
5 \\
5 \\
5 \\
10 \\
5 \\
5 \\
5 \\
5 \\
5 \\
5 \\
5 \\
5 \\
5 \\
100 \\
50 \\
50\end{array}$ & $\begin{array}{l}U \\
U \\
U \\
U \\
J \\
U \\
U \\
U \\
U \\
U \\
U \\
U \\
U \\
U \\
U \\
U \\
U \\
U \\
U \\
U \\
U \\
U \\
U \\
U \\
U \\
U \\
U \\
U \\
U \\
U \\
U \\
U \\
U \\
U \\
U\end{array}$ & & \\
\hline
\end{tabular}

Location=ORR; Formation=CHICKAMAUGA Bethel V; Site=115; Samp id=1972; Dup id=' '; Phase=2

\begin{tabular}{|c|c|c|}
\hline $\begin{array}{l}\text { cis-1,2-Dichloroethene } \\
\text { cis-1,3-Dichloropropene } \\
\text { trans-1,2-Dichloroethene } \\
\text { trans-1,3-Dichloropropene } \\
\text { Acetone } \\
\text { Benzene } \\
\text { Bromodichloromethane } \\
\text { Bromoform } \\
\text { Bromomethane } \\
\text { Carbon disulfide } \\
\text { Carbon tetrachloride } \\
\text { Chlorobenzene } \\
\text { Chloroethane } \\
\text { Chloroform } \\
\text { Chloromethane } \\
\text { Dibromochloromethane } \\
\text { Ethyl benzene } \\
\text { Methylene chloride } \\
\text { Styrene }\end{array}$ & $\begin{array}{l}\mu \mathrm{g} / \mathrm{kg} \\
\mu \mathrm{g} / \mathrm{kg} \\
\mu \mathrm{g} / \mathrm{kg} \\
\mu \mathrm{g} / \mathrm{kg} \\
\mu \mathrm{g} / \mathrm{kg} \\
\mu \mathrm{g} / \mathrm{kg} \\
\mu \mathrm{g} / \mathrm{kg} \\
\mu \mathrm{g} / \mathrm{kg} \\
\mu \mathrm{g} / \mathrm{kg} \\
\mu \mathrm{g} / \mathrm{kg} \\
\mu \mathrm{g} / \mathrm{kg} \\
\mu \mathrm{g} / \mathrm{kg} \\
\mu \mathrm{g} / \mathrm{kg} \\
\mu \mathrm{g} / \mathrm{kg} \\
\mu \mathrm{g} / \mathrm{kg} \\
\mu \mathrm{g} / \mathrm{kg} \\
\mu \mathrm{g} / \mathrm{kg} \\
\mu \mathrm{g} / \mathrm{kg} \\
\mu \mathrm{g} / \mathrm{kg}\end{array}$ & $\begin{array}{r}5 \\
5 \\
5 \\
5 \\
5 \\
5 \\
5 \\
5 \\
10 \\
5 \\
5 \\
5 \\
10 \\
5 \\
10 \\
5\end{array}$ \\
\hline
\end{tabular}


Table B.1 (continued)

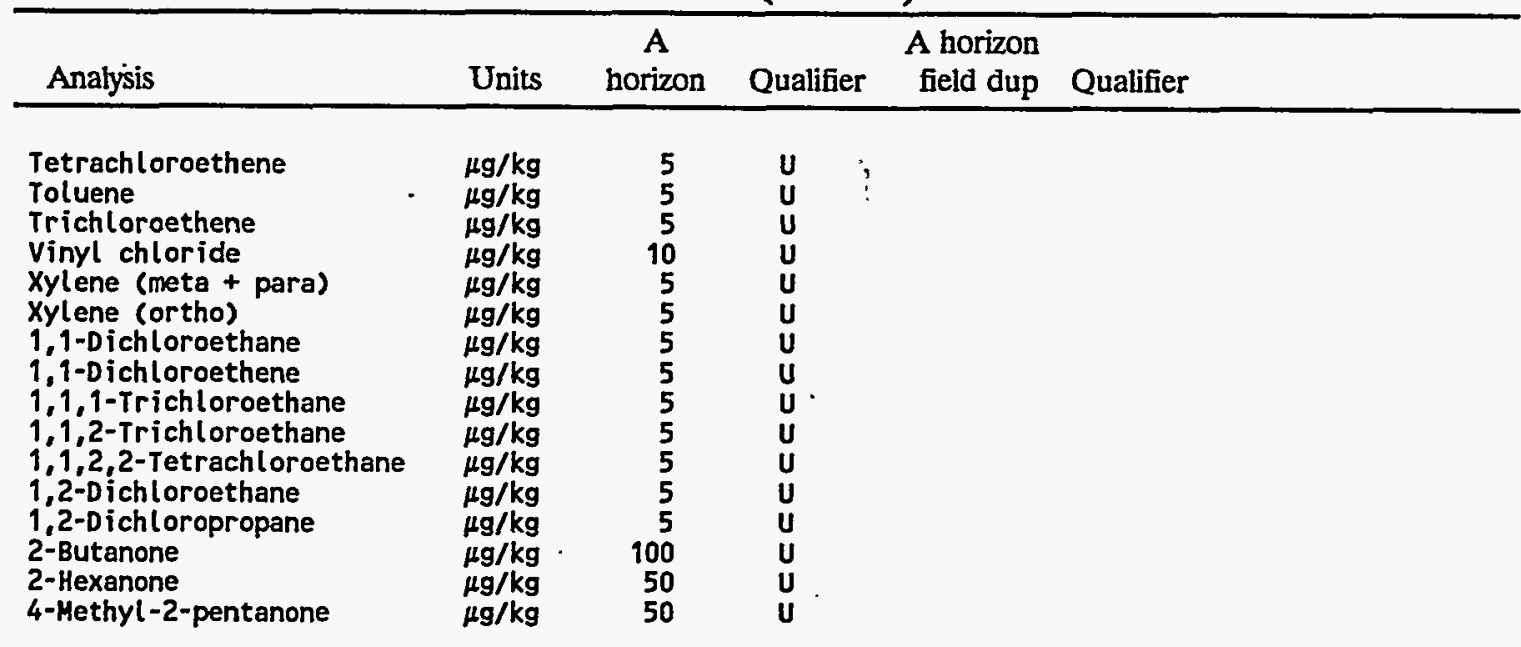

Location=ORR; Formation =CHICKAMAUGA Bethel V; Site=116; Samp id=1975; Dup id=' '; Phase=2

\begin{tabular}{|c|c|c|}
\hline 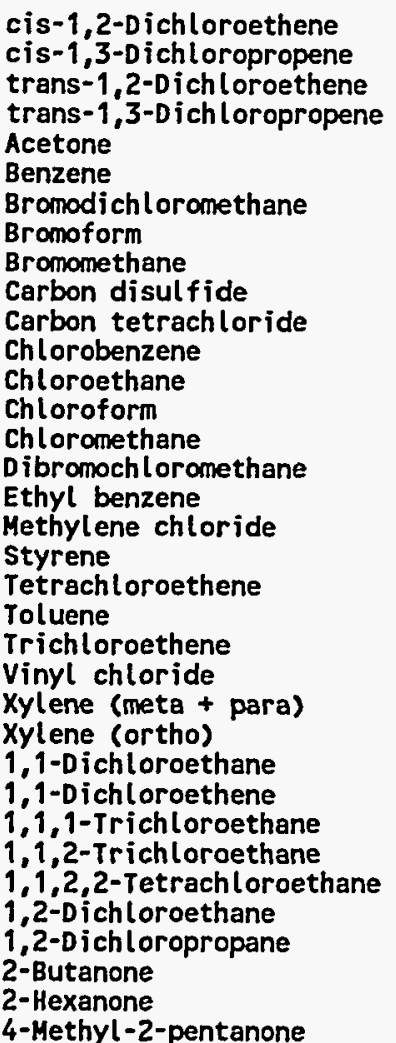 & $\begin{array}{l}\mu g / k g \\
\mu g / k g \\
\mu g / k g \\
\mu g / k g \\
\mu g / k g \\
\mu g / k g \\
\mu g / k g \\
\mu g / k g \\
\mu g / k g \\
\mu g / k g \\
\mu g / k g \\
\mu g / k g \\
\mu g / k g \\
\mu g / k g \\
\mu g / k g \\
\mu g / k g \\
\mu g / k g \\
\mu g / k g \\
\mu g / k g \\
\mu g / k g \\
\mu g / k g \\
\mu g / k g \\
\mu g / k g \\
\mu g / k g \\
\mu g / k g \\
\mu g / k g \\
\mu g / k g \\
\mu g / k g \\
\mu g / k g \\
\mu g / k g \\
\mu g / k g \\
\mu g / k g \\
\mu g / k g \\
\mu g / k g \\
\mu g / k g\end{array}$ & $\begin{array}{r}5 \\
5 \\
5 \\
5 \\
7 \\
5 \\
5 \\
5 \\
10 \\
5 \\
5 \\
5 \\
10 \\
5 \\
10 \\
5 \\
5 \\
5 \\
5 \\
5 \\
5 \\
5 \\
10 \\
5 \\
5 \\
5 \\
5 \\
5 \\
5 \\
5 \\
5 \\
5 \\
6 \\
49 \\
49\end{array}$ \\
\hline
\end{tabular}

Location=ORR; Formation=CHICKAMAUGA Bethel V; Site=117; Samp id=1978; Dup id=' '; Phase=2

$\begin{array}{llrl}\text { cis-1,2-Dichloroethene } & \mu \mathrm{g} / \mathrm{kg} & 5 & \mathrm{U} \\ \text { cis-1,3-Dichloropropene } & \mu \mathrm{g} / \mathrm{kg} & 5 & \mathrm{U} \\ \text { trans-1,2-Dichloroethene } & \mu \mathrm{g} / \mathrm{kg} & 5 & \mathrm{U} \\ \text { trans-1,3-Dichloropropene } & \mu \mathrm{g} / \mathrm{kg} & 5 & \mathrm{U} \\ \text { Acetone } & \mu \mathrm{g} / \mathrm{kg} & 98 & \mathrm{U} \\ \text { Benzene } & \mu \mathrm{g} / \mathrm{kg} & 5 & \mathrm{U} \\ \text { Bromodichloromethane } & \mu \mathrm{g} / \mathrm{kg} & 5 & \mathrm{U} \\ \text { Bromoform } & \mu \mathrm{g} / \mathrm{kg} & 5 & \mathrm{U} \\ \text { Bromomethane } & \mu \mathrm{g} / \mathrm{kg} & 10 & \mathrm{U} \\ \text { Carbon disulfide } & \mu \mathrm{g} / \mathrm{kg} & 5 & \mathrm{U}\end{array}$


Table B.1 (continued)

\begin{tabular}{|c|c|c|c|c|c|}
\hline Analysis & Units & $\underset{\text { horizon }}{A}$ & Qualifier & $\begin{array}{l}\text { A horizon } \\
\text { field dup }\end{array}$ & Qualifier \\
\hline $\begin{array}{l}\text { Carbon tetrachloride } \\
\text { Chlorobenzene } \\
\text { Chloroethane } \\
\text { Chloroform } \\
\text { Chloromethane } \\
\text { Dibromochloromethane } \\
\text { Ethyl benzene } \\
\text { Methylene chloride } \\
\text { styrene } \\
\text { Tetrachloroethene } \\
\text { Toluene } \\
\text { Trichloroethene } \\
\text { vinyl chloride } \\
\text { Xylene (meta + para) } \\
\text { xylene (ortho) } \\
1,1-D \text { ichloroethane } \\
1,1-\text { Dichloroethene } \\
1,1,1-\text { Trichloroethane } \\
1,1,2-\text { Trichloroethane } \\
1,1,2,2-\text { Tetrachloroethane } \\
1,2-0 \text { ichloroethane } \\
1,2-D \text { ichloropropane } \\
\text { 2-Butanone } \\
\text { 2-Hexanone } \\
\text { 4-Methyl-2-pentanone }\end{array}$ & $\begin{array}{l}\mu g / k g \\
\mu g / k g \\
\mu g / k g \\
\mu g / k g \\
\mu g / k g \\
\mu g / k g \\
\mu g / k g \\
\mu g / k g \\
\mu g / k g \\
\mu g / k g \\
\mu g / k g \\
\mu g / k g \\
\mu g / k g \\
\mu g / k g \\
\mu g / k g \\
\mu g / k g \\
\mu g / k g \\
\mu g / k g \\
\mu g / k g \\
\mu g / k g \\
\mu g / k g \\
\mu g / k g \\
\mu g / k g \\
\mu g / k g \\
\mu g / k g\end{array}$ & $\begin{array}{r}5 \\
5 \\
10 \\
5 \\
10 \\
5 \\
5 \\
5 \\
5 \\
5 \\
5 \\
5 \\
10 \\
5 \\
5 \\
5 \\
5 \\
5 \\
5 \\
5 \\
5 \\
5 \\
7 \\
49 \\
49\end{array}$ & $\begin{array}{l}U \\
U \\
U \\
U \\
U \\
U \\
U \\
U \\
U \\
U \\
U \\
U \\
U \\
U \\
U \\
U \\
U \\
U \\
U \\
U \\
U \\
U \\
J \\
U \\
U\end{array}$ & & \\
\hline
\end{tabular}

Location=ORR; Formation=CHICKAMAUGA Bethel V; Site=93; Samp id=1899; Dup id=' '; Phase=2

\begin{tabular}{|c|c|c|}
\hline 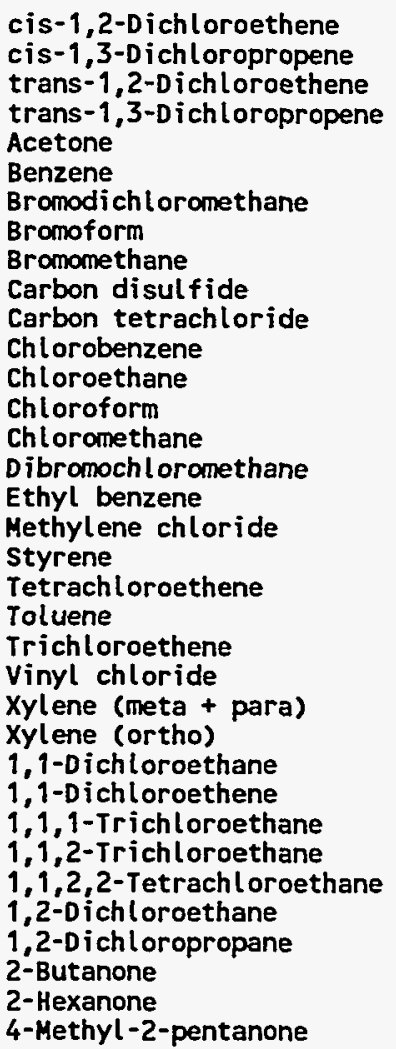 & $\begin{array}{l}\mu g / \mathbf{k g} \\
\mu g / \mathbf{k g} \\
\mu g / \mathbf{k g} \\
\mu g / \mathbf{k g} \\
\mu g / \mathbf{k g} \\
\mu g / \mathbf{k g} \\
\mu g / \mathbf{k g} \\
\mu g / \mathbf{k g} \\
\mu g / \mathbf{k g} \\
\mu g / \mathbf{k g} \\
\mu g / \mathbf{k g} \\
\mu g / \mathbf{k g} \\
\mu g / \mathbf{k g} \\
\mu g / \mathbf{k g} \\
\mu g / \mathbf{k g} \\
\mu g / \mathbf{k g} \\
\mu g / \mathbf{k g} \\
\mu g / \mathbf{k g} \\
\mu \mathrm{g} / \mathbf{k g} \\
\mu g / \mathbf{k g} \\
\mu g / \mathbf{k g} \\
\mu g / \mathbf{k g} \\
\mu g / \mathbf{k g} \\
\mu g / \mathbf{k g} \\
\mu g / \mathbf{k g} \\
\mu g / \mathbf{k g} \\
\mu g / \mathbf{k g} \\
\mu g / \mathbf{k g} \\
\mu g / \mathbf{k g} \\
\mu g / \mathbf{k g} \\
\mu g / \mathbf{k g} \\
\mu g / \mathbf{k g} \\
\mu g / \mathbf{k g} \\
\mu g / \mathbf{k g} \\
\mu g / \mathbf{k g}\end{array}$ & $\begin{array}{r}5 \\
5 \\
5 \\
5 \\
10 \\
5 \\
5 \\
5 \\
10 \\
5 \\
5 \\
5 \\
10 \\
5 \\
10 \\
5 \\
5 \\
5 \\
5 \\
5 \\
5 \\
5 \\
10 \\
5 \\
5 \\
5 \\
5 \\
5 \\
5 \\
5 \\
5 \\
5 \\
100 \\
50 \\
50\end{array}$ \\
\hline
\end{tabular}


Tabłe B.1 (continued)

\begin{tabular}{|c|c|c|c|}
\hline Analysis & Units & $\begin{array}{c}\text { A } \\
\text { horizon }\end{array}$ & Qual \\
\hline \multicolumn{4}{|c|}{ Location=ORR; Formation =CHICKAMAUGA Bethel V } \\
\hline 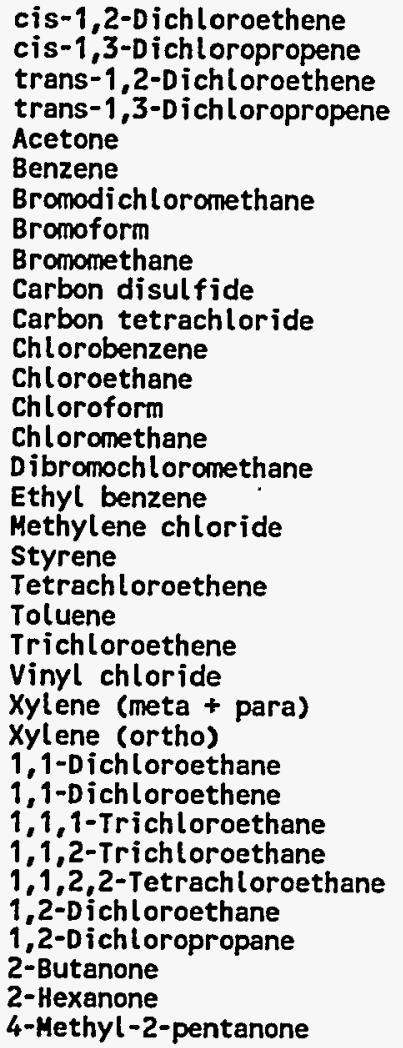 & $\begin{array}{l}\mu g / \mathbf{k g} \\
\mu g / k g \\
\mu g / k g \\
\mu g / k g \\
\mu g / k g \\
\mu g / k g \\
\mu g / k g \\
\mu g / k g \\
\mu g / k g \\
\mu g / k g \\
\mu g / k g \\
\mu g / k g \\
\mu g / k g \\
\mu g / k g \\
\mu g / k g \\
\mu g / k g \\
\mu g / k g \\
\mu g / k g \\
\mu g / k g \\
\mu g / k g \\
\mu g / k g \\
\mu g / k g \\
\mu g / k g \\
\mu g / k g \\
\mu g / k g \\
\mu g / k g \\
\mu g / k g \\
\mu g / k g \\
\mu g / k g \\
\mu g / k g \\
\mu g / k g \\
\mu g / k g \\
\mu g / k g \\
\mu g / k g \\
\mu g / k g\end{array}$ & $\begin{array}{r}5 \\
5 \\
5 \\
5 \\
5 \\
5 \\
5 \\
5 \\
10 \\
5 \\
5 \\
5 \\
10 \\
5 \\
10 \\
5 \\
5 \\
5 \\
5 \\
5 \\
5 \\
5 \\
10 \\
5 \\
5 \\
5 \\
5 \\
5 \\
5 \\
5 \\
5 \\
5 \\
5 \\
50 \\
50\end{array}$ & $\begin{array}{l}U \\
U \\
U \\
U \\
J \\
U \\
U \\
U \\
U \\
U \\
U \\
U \\
U \\
U \\
U \\
U \\
U \\
U \\
U \\
U \\
U \\
U \\
U \\
U \\
U \\
U \\
U \\
U \\
U \\
U \\
U \\
U \\
J \\
U \\
U\end{array}$ \\
\hline
\end{tabular}

Location=ORR; Formation=CHICKAMAUGA K-25; Site=118; Samp id=4094; Dup id=' '; Phase=2

cis-1,2-Dichloroethene cis-1,3-Dichloropropene trans-1,2-Dichloroethene trans-1,3-Dichloropropene Acetone

Benzene

Bromodichloromethane

Bromoform

Bromomethane

Carbon disulfide

Carbon tetrachloride

Chlorobenzene

Chloroethane

Chloroform

Chloromethane

Dibromochloromethane

Ethyl benzene

Methylene chloride

styrene

Tetrachloroethene

Toluene

Trichloroethene

Vinyl chloride

xylene (meta + para)

xylene (ortho)

1,1-Dichloroethane

1,1-Dichloroethene

$1,1,1$-Trichloroethane

$1,1,2-$ Trichloroethane
A horizon

field dup Qualifier 
B-30

Table B.1 (continued)

\begin{tabular}{|c|c|c|c|c|c|}
\hline Analysis & Units & $\underset{\text { horizon }}{A}$ & Qualifier & $\begin{array}{l}\text { A horizon } \\
\text { field dup }\end{array}$ & Qualifier \\
\hline $\begin{array}{l}\text { 1,1,2,2-Tetrachloroethane } \\
1,2-D \text { ichloroethane } \\
\text { 1,2-Dichloropropane } \\
\text { 2-Butanone } \\
\text { 2-Hexanone } \\
\text { 4-Methyl-2-pentanone }\end{array}$ & $\begin{array}{l}\mu g / \mathrm{kg} \\
\mu \mathrm{g} / \mathrm{kg} \\
\mu \mathrm{g} / \mathrm{kg} \\
\mu \mathrm{g} / \mathrm{kg} \\
\mu \mathrm{g} / \mathrm{kg} \\
\mu \mathrm{g} / \mathrm{kg}\end{array}$ & $\begin{array}{r}5 \\
5 \\
5 \\
100 \\
50 \\
50\end{array}$ & $\begin{array}{l}\mathbf{U} \\
\mathbf{U} \\
\mathbf{U} \\
\mathbf{U} \\
\mathbf{U} \\
\mathbf{U}\end{array}$ & & \\
\hline
\end{tabular}

Location=ORR; Formation=CHICKAMAUGA K-25; Site=119; Samp id=4126; Dup id=' '; Phase=2

\begin{tabular}{|c|c|c|}
\hline 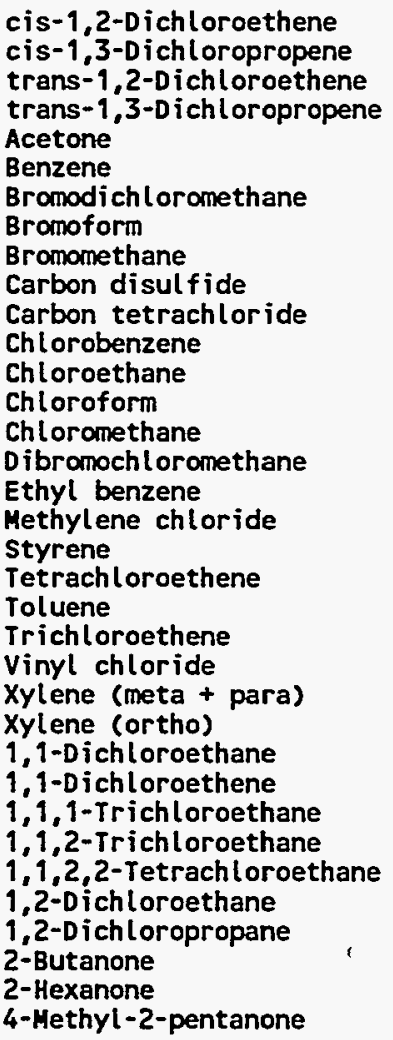 & 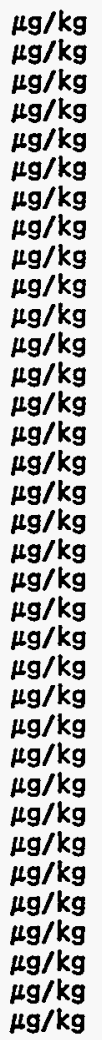 & $\begin{array}{r}5 \\
5 \\
5 \\
5 \\
100 \\
5 \\
5 \\
5 \\
10 \\
5 \\
5 \\
5 \\
10 \\
5 \\
10 \\
5 \\
5 \\
5 \\
5 \\
5 \\
5 \\
5 \\
10 \\
5 \\
5 \\
5 \\
5 \\
5 \\
5 \\
5 \\
5 \\
5 \\
100 \\
50 \\
50\end{array}$ \\
\hline
\end{tabular}

Location=ORR; Formation=CHICKAMAUGA K-25; Site=120; Samp id=4091; Dup id =' '; Phase=2

\begin{tabular}{|c|c|c|}
\hline $\begin{array}{l}\text { cis-1,2-D ichloroethene } \\
\text { cis-1,3-Dichloropropene } \\
\text { trans-1,2-Dichloroethene } \\
\text { trans-1,3-Dichloropropene } \\
\text { Acetone } \\
\text { Benzene } \\
\text { Bromodichloromethane } \\
\text { Bromoform } \\
\text { Bromomethane } \\
\text { Carbon disulfide } \\
\text { Carbon tetrachloride } \\
\text { Chlorobenzene } \\
\text { Chloroethane } \\
\text { Chloroform } \\
\text { Chloromethane } \\
\text { Dibromochloromethane } \\
\text { Ethyl benzene } \\
\text { Methylene chloride } \\
\text { styrene } \\
\text { Tetrachloroethene } \\
\text { Toluene } \\
\text { Trichloroethene } \\
\text { Vinyl chloride }\end{array}$ & 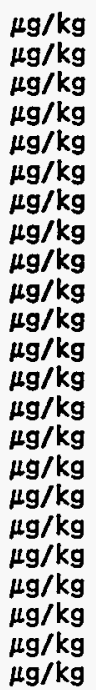 & $\begin{array}{r}5 \\
5 \\
5 \\
5 \\
100 \\
5 \\
5 \\
5 \\
10 \\
5 \\
5 \\
5 \\
10 \\
5 \\
10 \\
5 \\
5 \\
5 \\
5 \\
5 \\
5 \\
5 \\
10\end{array}$ \\
\hline
\end{tabular}




\section{B-31}

Table B.1 (continued)

\begin{tabular}{|c|c|c|c|c|c|c|}
\hline Analysis ${ }^{\circ}$ & Units & $\begin{array}{c}A \\
\text { horizon }\end{array}$ & Qualifier & $\begin{array}{l}\text { A horizon } \\
\text { field dup }\end{array}$ & Qualifier & \\
\hline $\begin{array}{l}\text { Xylene (meta + para) } \\
\text { Xylene (ortho) } \\
1,1-D \text { ichloroethane } \\
1,1-\text { Dichloroethene } \\
1,1,1-\text { Trichloroethane } \\
1,1,2-\text { Trichloroethane } \\
1,1,2,2-\text { Tetrachloroethene } \\
\text { 1,2-Dichloroethane } \\
\text { 1,2-Dichloropropane } \\
\text { 2-Butanone } \\
\text { 2-Hexanone } \\
\text { 4-Methyl-2-pentanone }\end{array}$ & $\begin{array}{l}\mu g / \mathrm{kg} \\
\mu g / \mathrm{kg} \\
\mu g / \mathrm{kg} \\
\mu g / \mathrm{kg} \\
\mu g / \mathrm{kg} \\
\mu g / \mathrm{kg} \\
\mu g / \mathrm{kg} \\
\alpha g / \mathrm{kg} \\
\mu g / \mathrm{kg} \\
\alpha g / \mathrm{kg} \\
\mu g / \mathrm{kg} \\
\mu g / \mathrm{kg}\end{array}$ & $\begin{array}{r}5 \\
5 \\
5 \\
5 \\
5 \\
5 \\
5 \\
5 \\
5 \\
100 \\
50 \\
50\end{array}$ & $\begin{array}{l}\mathbf{U} \\
\mathbf{U} \\
\mathbf{U} \\
\mathbf{U} \\
\mathbf{U} \\
\mathbf{U} \\
\mathbf{U} \\
\mathbf{U} \\
\mathbf{U} \\
\mathbf{U} \\
\mathbf{U} \\
\mathbf{U}\end{array}$ & 8 & & $4=$ \\
\hline
\end{tabular}

Location=ORR; Formation=CHICKAMAUGA K-25; Site=121; Samp id=4128; Dup id=' '; Phase=2

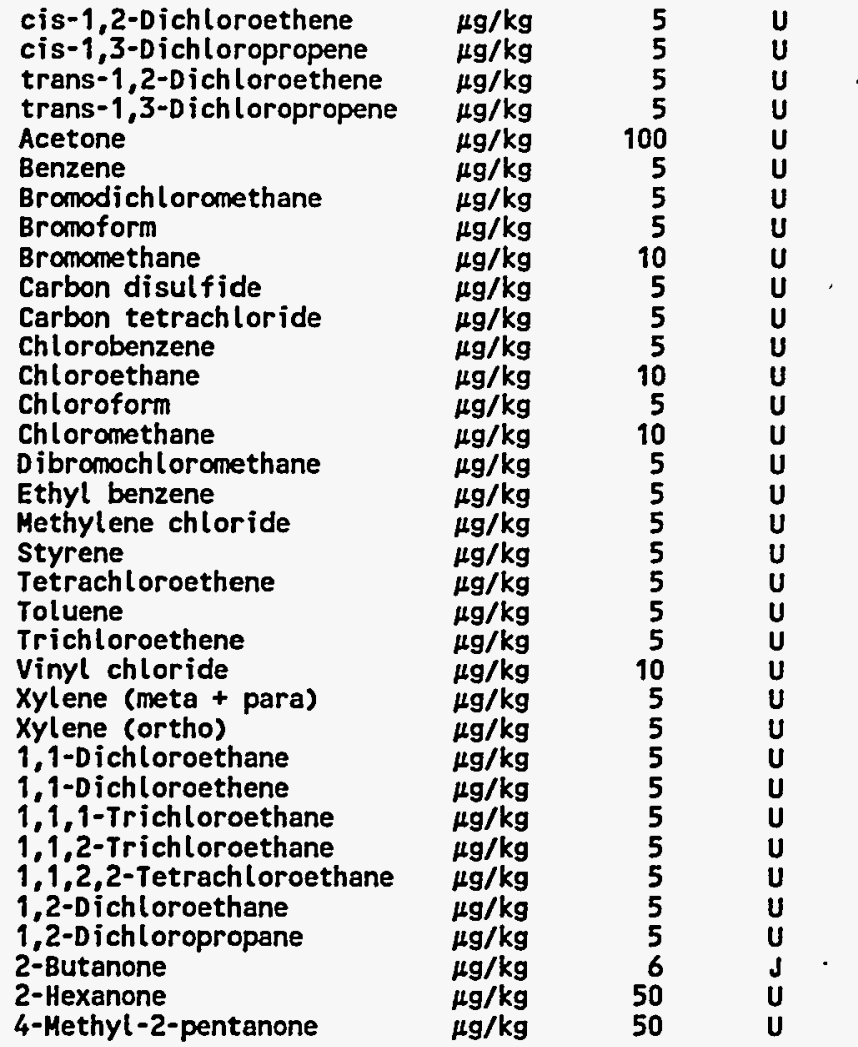

Location=ORR; Formation=CHICKAMAUGA K-25; Site=122; Samp id=4104; Dup id=' '; Phase=2

cis-1,2-Dichloroethene cis-1,3-Dichloropropene trans-1,2-Dichloroethene trans-1,3-Dichloropropene Acetone

Benzene

Bromodichloromethane

Bromoform

Bromomethane

Carbon disulfide

Carbon tetrachloride

Chlorobenzene

Chloroethane

Chloroform

Chloromethane

Dibromochloromethane

Ethyl benzene

$\begin{array}{lrl}\mu \mathrm{g} / \mathrm{kg} & 5 & U \\ \mu \mathrm{g} / \mathrm{kg} & 5 & U \\ \mu \mathrm{g} / \mathrm{kg} & 5 & U \\ \mu \mathrm{g} / \mathrm{kg} & 5 & U \\ \mu \mathrm{g} / \mathrm{kg} & 98 & U \\ \mu \mathrm{g} / \mathrm{kg} & 5 & U \\ \mu \mathrm{g} / \mathrm{kg} & 5 & U \\ \mu \mathrm{g} / \mathrm{kg} & 5 & U \\ \mu \mathrm{g} / \mathrm{kg} & 10 & U \\ \mu \mathrm{g} / \mathrm{kg} & 5 & U \\ \mu \mathrm{g} / \mathrm{kg} & 5 & U \\ \mu \mathrm{g} / \mathrm{kg} & 5 & U \\ \mu \mathrm{g} / \mathrm{kg} & 10 & U \\ \mu \mathrm{g} / \mathrm{kg} & 5 & U \\ \mu \mathrm{g} / \mathrm{kg} & 10 & U \\ \mu \mathrm{g} / \mathrm{kg} & 5 & U \\ \mu \mathrm{g} / \mathrm{kg} & 5 & U\end{array}$




\section{B-32}

Table B.1 (continued)

\begin{tabular}{|c|c|c|c|c|c|}
\hline Anatysis & Units & $\underset{\text { horizon }}{A}$ & Qualifier & $\begin{array}{l}\text { A horizon } \\
\text { field dup }\end{array}$ & Qualifier \\
\hline $\begin{array}{l}\text { Methylene chloride } \\
\text { Styrene } \\
\text { Tetrachloroethene } \\
\text { Toluene } \\
\text { Trichloroethene } \\
\text { Vinyl chloride } \\
\text { Xylene (meta + para) } \\
\text { Xylene (ortho) } \\
\text { 1,1-Dichloroethane } \\
\text { 1,1-Dichloroethene } \\
\text { 1,1,1-Trichloroethane } \\
\text { 1,1,2-Trichloroethane } \\
1,9,2,2-\text { Tetrachloroethane } \\
\text { 1,2-Dichloroethane } \\
1,2-D \text { ichloropropane } \\
\text { 2-Butanone } \\
\text { 2-Hexanone } \\
\text { 4-Hethyl-2-pentanone }\end{array}$ & $\begin{array}{l}\mu g / \mathrm{kg} \\
\mu g / \mathrm{kg} \\
\mu \mathrm{g} / \mathrm{kg} \\
\mu g / \mathrm{kg} \\
\mu g / \mathrm{kg} \\
\mu g / \mathrm{kg} \\
\mu \mathrm{g} / \mathrm{kg} \\
\mu g / \mathrm{kg} \\
\mu \mathrm{g} / \mathrm{kg} \\
\mu \mathrm{g} / \mathrm{kg} \\
\mu g / \mathrm{kg} \\
\mu \mathrm{g} / \mathrm{kg} \\
\mu \mathrm{g} / \mathrm{kg} \\
\mu g / \mathrm{kg} \\
\mu \mathrm{g} / \mathrm{kg} \\
\mu \mathrm{g} / \mathrm{kg} \\
\mu \mathrm{g} / \mathrm{kg} \\
\mu \mathrm{g} / \mathrm{kg}\end{array}$ & $\begin{array}{r}5 \\
5 \\
5 \\
5 \\
5 \\
10 \\
5 \\
5 \\
5 \\
5 \\
5 \\
5 \\
5 \\
5 \\
5 \\
98 \\
49 \\
49\end{array}$ & $\begin{array}{l}U \\
U \\
U \\
U \\
U \\
U \\
U \\
U \\
U \\
U \\
U \\
U \\
U \\
U \\
U \\
U \\
U \\
U\end{array}$ & & \\
\hline
\end{tabular}

Location=ORR; Formation=CHICKAMAUGA K-25; Site=123; Samp id=4130; Dup id=' '; Phase=2

\begin{tabular}{|c|c|c|}
\hline 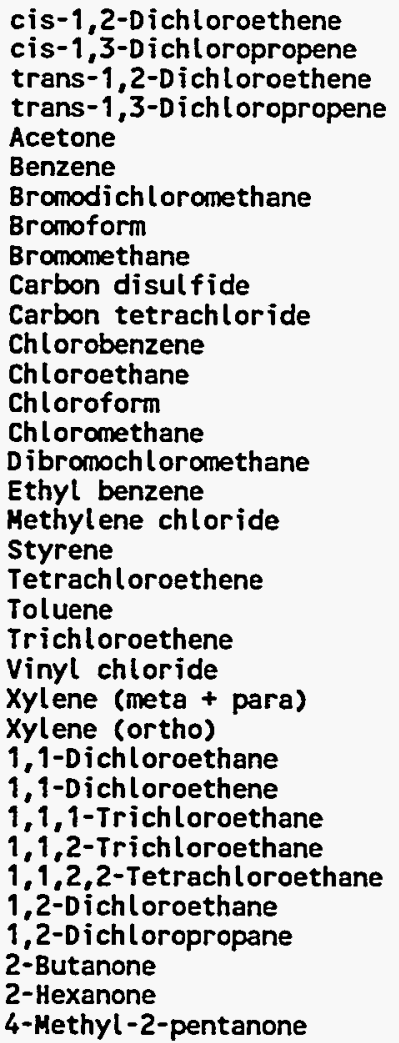 & $\begin{array}{l}\mu g / \mathrm{kg} \\
\mu \mathrm{g} / \mathrm{kg} \\
\mu \mathrm{g} / \mathrm{kg} \\
\mu \mathrm{g} / \mathrm{kg} \\
\mu \mathrm{g} / \mathrm{kg} \\
\mu \mathrm{g} / \mathrm{kg} \\
\mu \mathrm{g} / \mathrm{kg} \\
\mu \mathrm{g} / \mathrm{kg} \\
\mu \mathrm{g} / \mathrm{kg} \\
\mu \mathrm{g} / \mathrm{kg} \\
\mu \mathrm{g} / \mathrm{kg} \\
\mu \mathrm{g} / \mathrm{kg} \\
\mu \mathrm{g} / \mathrm{kg} \\
\mu \mathrm{g} / \mathrm{kg} \\
\mu \mathrm{g} / \mathrm{kg} \\
\mu \mathrm{g} / \mathrm{kg} \\
\mu \mathrm{g} / \mathrm{kg} \\
\mu \mathrm{g} / \mathrm{kg} \\
\mu \mathrm{g} / \mathrm{kg} \\
\mu \mathrm{g} / \mathrm{kg} \\
\mu \mathrm{kg} \\
\mu \mathrm{kg} / \mathrm{kg} \\
\mu \mathrm{k} / \mathrm{kg} \\
\mu \mathrm{g} / \mathrm{kg} \\
\mu \mathrm{g} / \mathrm{kg} \\
\mu \mathrm{g} / \mathrm{kg} \\
\mu \mathrm{g} / \mathrm{kg} \\
\mu \mathrm{g} / \mathrm{kg} \\
\mu \mathrm{g} / \mathrm{kg} \\
\mu \mathrm{g} / \mathrm{kg} \\
\mu \mathrm{g} / \mathrm{kg} \\
\mu \mathrm{g} / \mathrm{kg} \\
\mu \mathrm{g} / \mathrm{kg} \\
\mu \mathrm{g} / \mathrm{kg} \\
\mu \mathrm{g} / \mathrm{kg}\end{array}$ & $\begin{array}{r}5 \\
5 \\
5 \\
5 \\
19 \\
5 \\
5 \\
5 \\
10 \\
5 \\
5 \\
5 \\
10 \\
5 \\
10 \\
5 \\
5 \\
5 \\
5 \\
5 \\
5 \\
5 \\
10 \\
5 \\
5 \\
5 \\
5 \\
5 \\
5 \\
5 \\
5 \\
5 \\
100 \\
50 \\
50\end{array}$ \\
\hline
\end{tabular}

Location=ORR; Formation=CHICKAMAUGA K-25; Site=124; Samp id=4114; Dup id=' '; Phase=2

$\begin{array}{llrl}\text { cis-1,2-Dichloroethene } & \mu \mathrm{g} / \mathrm{kg} & 5 & \mathrm{U} \\ \text { cis-1,3-Dichloropropene } & \mu \mathrm{g} / \mathrm{kg} & 5 & \mathrm{U} \\ \text { trans-1,2-Dichloroethene } & \mu \mathrm{g} / \mathrm{kg} & 5 & \mathrm{U} \\ \text { trans-1,3-Dichloropropene } & \mu \mathrm{g} / \mathrm{kg} & 5 & \mathrm{U} \\ \text { Acetone } & \mu \mathrm{g} / \mathrm{kg} & 98 & \mathrm{U} \\ \text { Benzene } & \mu \mathrm{g} / \mathrm{kg} & 5 & U \\ \text { Bromodichloromethane } & \mu \mathrm{g} / \mathrm{kg} & 5 & U \\ \text { Bromoform } & \mu \mathrm{g} / \mathrm{kg} & 5 & U \\ \text { Bromomethane } & \mu \mathrm{g} / \mathrm{kg} & 10 & \mathrm{U} \\ \text { Carbon disulfide } & \mu \mathrm{g} / \mathrm{kg} & 5 & U \\ \text { Carbon tetrachloride } & \mu \mathrm{g} / \mathrm{kg} & 5 & U\end{array}$


Table B.1 (continued)

\begin{tabular}{|c|c|c|c|c|c|c|}
\hline Analysis & Units & $\begin{array}{c}A \\
\text { horizon }\end{array}$ & Qualifier & $\begin{array}{l}\text { A horizon } \\
\text { field dup }\end{array}$ & Qualifier & \\
\hline 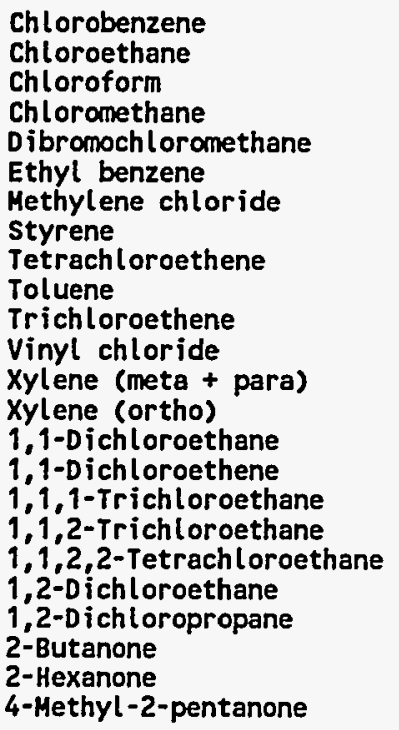 & $\begin{array}{l}\mu g / k g \\
\mu g / k g \\
\mu g / k g \\
\mu g / k g \\
\mu g / k g \\
\mu g / k g \\
\mu g / k g \\
\mu g / k g \\
\mu g / k g \\
\mu g / k g \\
\mu g / k g \\
\mu g / k g \\
\mu g / k g \\
\mu g / k g \\
\mu g / k g \\
\mu g / k g \\
\mu g / k g \\
\mu g / k g \\
\mu g / k g \\
\mu g / k g \\
\mu g / k g \\
\mu g / k g \\
\mu g / k g \\
\mu g / k g\end{array}$ & $\begin{array}{r}5 \\
10 \\
5 \\
10 \\
5 \\
5 \\
5 \\
5 \\
5 \\
5 \\
5 \\
10 \\
5 \\
5 \\
5 \\
5 \\
5 \\
5 \\
5 \\
5 \\
5 \\
98 \\
49 \\
49\end{array}$ & $\begin{array}{l}U \\
U \\
U \\
U \\
U \\
U \\
U \\
U \\
U \\
U \\
U \\
U \\
U \\
U \\
U \\
U \\
U \\
U \\
U \\
U \\
U \\
U \\
U \\
U\end{array}$ & 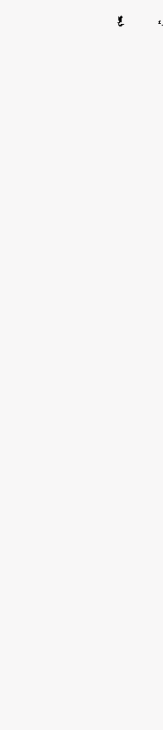 & 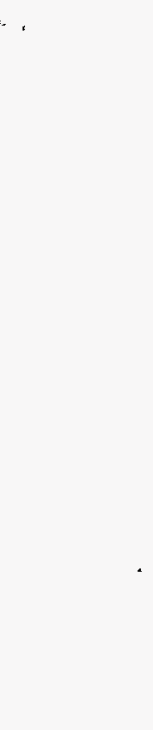 & $98 \%$ \\
\hline
\end{tabular}

Location=ORR; Formation=CHICKAMAUGA K-25; Site=125; Samp id=4132; Dup id=' '; Phase=2

\begin{tabular}{|c|c|c|}
\hline 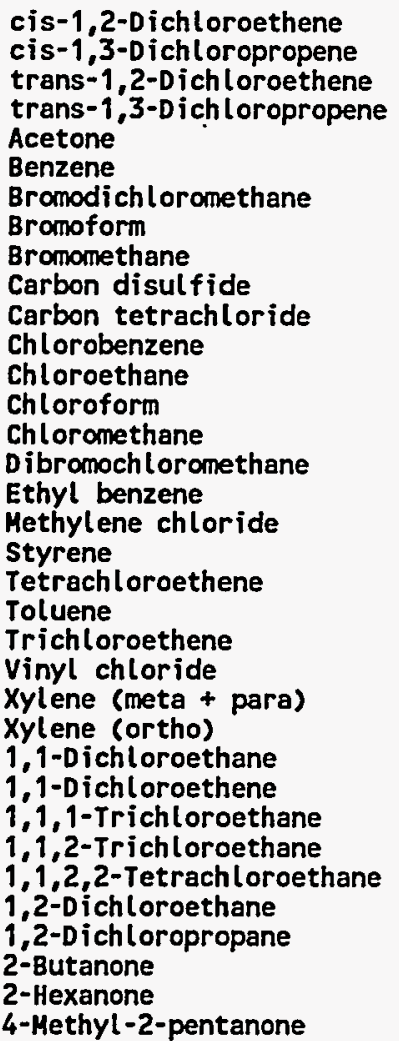 & $\begin{array}{l}\mu g / \mathbf{k g} \\
\mu g / k g \\
\mu g / k g \\
\mu g / k g \\
\mu g / k g \\
\mu g / k g \\
\mu g / k g \\
\mu g / k g \\
\mu g / k g \\
\mu g / k g \\
\mu g / k g \\
\mu g / k g \\
\mu g / k g \\
\mu g / k g \\
\mu g / k g \\
\mu g / k g \\
\mu g / k g \\
\mu g / k g \\
\mu g / k g \\
\mu g / k g \\
\mu g / k g \\
\mu g / k g \\
\mu g / k g \\
\mu g / k g \\
\mu g / k g \\
\mu g / k g \\
\mu g / k g \\
\mu g / k g \\
\mu g / k g \\
\mu g / k g \\
\mu g / k g \\
\mu g / k g \\
\mu g / k g \\
\mu g / k g \\
\mu g / k g\end{array}$ & $\begin{array}{r}5 \\
5 \\
12 \\
5 \\
5 \\
5 \\
10 \\
5 \\
5 \\
5 \\
10 \\
5 \\
10 \\
5 \\
5 \\
5 \\
5 \\
5 \\
5 \\
5 \\
10 \\
5 \\
5 \\
5 \\
5 \\
5 \\
5 \\
5 \\
5 \\
5 \\
100 \\
50 \\
50\end{array}$ \\
\hline
\end{tabular}

Location=ORR; Formation=CHICKAMAUGA K-25; Site=126; Samp id=4088; Dup id=' '; Phase=2

cis-1,2-Dichloroethene cis-1,3-Dichloropropene trans-1,2-Dichloroethene trans-1,3-Dichloropropene Acetone

$\begin{array}{lll}\mu g / \mathrm{kg} & 5 & U \\ \mu \mathrm{g} / \mathrm{kg} & 5 & \mathrm{U} \\ \mu \mathrm{g} / \mathrm{kg} & 5 & \mathrm{U} \\ \mu \mathrm{g} / \mathrm{kg} & 5 & \mathrm{U} \\ \mu \mathrm{g} / \mathrm{kg} & 5 & \mathrm{~J}\end{array}$


B-34

Table B.1 (continued)

\begin{tabular}{|c|c|c|c|c|c|}
\hline Analysis & Units & $\begin{array}{c}A \\
\text { horizon }\end{array}$ & Qualifier & $\begin{array}{l}\text { A horizon } \\
\text { field dup }\end{array}$ & Qualifier \\
\hline $\begin{array}{l}\text { Benzene } \\
\text { Bromodichloromethane } \\
\text { Bromoform } \\
\text { Bromomethane } \\
\text { Carbon disulfide } \\
\text { Carbon tetrachloride } \\
\text { Chlorobenzene } \\
\text { Chloroethane } \\
\text { Chloroform } \\
\text { Chloromethane } \\
\text { Dibromochloromethane } \\
\text { Ethyl benzene } \\
\text { Methylene chloride } \\
\text { Styrene } \\
\text { Tetrachloroethene } \\
\text { Toluene } \\
\text { Trichloroethene } \\
\text { Vinyl chloride } \\
\text { Xylene (meta + para) } \\
\text { Xylene (ortho) } \\
1,1-D \text { ichloroethane } \\
1,1-D \text { ichloroethene } \\
1,1,1 \text {-Trichloroethane } \\
1,1,2-\text { Trichloroethane } \\
1,1,2,2-\text { Tetrachloroethane } \\
1,2-D \text { ichloroethane } \\
1,2-D \text { ichloropropane } \\
2-\text { Butanone } \\
2-\text { Hexanone } \\
\text { 4-Methyl-2-pentanone }\end{array}$ & 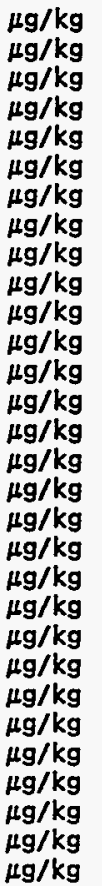 & $\begin{array}{r}5 \\
5 \\
5 \\
10 \\
5 \\
5 \\
5 \\
10 \\
5 \\
10 \\
5 \\
5 \\
5 \\
5 \\
5 \\
5 \\
5 \\
10 \\
5 \\
5 \\
5 \\
5 \\
5 \\
5 \\
5 \\
5 \\
5 \\
100 \\
50 \\
50\end{array}$ & $\begin{array}{l}U \\
U \\
U \\
U \\
U \\
U \\
U \\
U \\
U \\
U \\
U \\
U \\
U \\
U \\
U \\
U \\
U \\
U \\
U \\
U \\
U \\
U \\
U \\
U \\
U \\
U \\
U \\
U \\
U \\
U \\
U \\
U \\
U \\
U\end{array}$ & & \\
\hline
\end{tabular}

Location=ORR; Formation=CHICKAMAUGA K-25; Site=127; Samp id=4134; Dup id=' '; Phase=2

cis-1,2-Dichloroethene cis-1,3-Dichloropropene trans-1,2-D ichloroethene trans-1,3-Dichloropropene Acetone

Benzene

Bromodichloromethane

Bromoform

Bromomethane

Carbon disulfide

Carbon tetrachloride

Chlorobenzene

Chloroethane

Chloroform

Chloromethane

Dibromoch loromethane

Ethyl benzene

Hethylene chloride

Styrene

Tetrachloroethene

Toluene

Trichloroethene

Vinyl chloride

Xylene (meta + para)

Xylene (ortho)

1,1-Dichloroethane

1, 1-Dichloroethene

1,1,1-Trichloroethane

1,1,2-Trichloroethane

$1,1,2,2-$ Tetrachloroethane

1,2-Dichloroethane

1,2-Dichloropropane

2-Butanone

2-Hexanone

4-Hethyl-2-pentanone

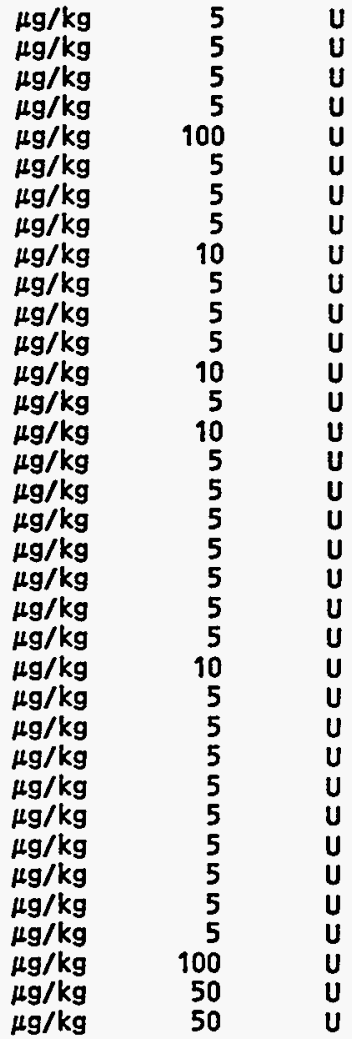


Table B.1 (continued)

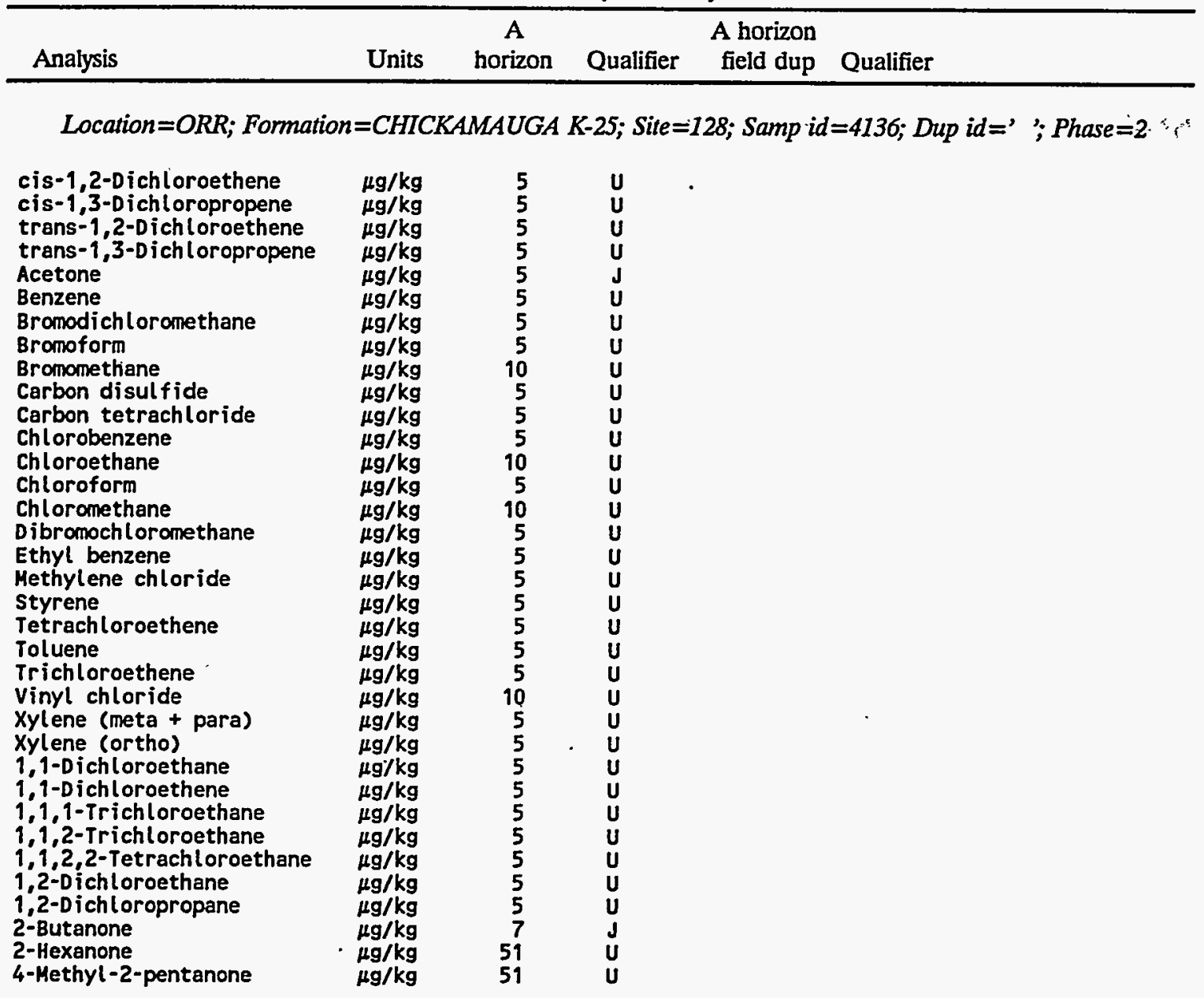

Location=ORR; Formation=CHICKAMAUGA K-25; Site=129; Samp id=4085; Dup id=' '; Phase=2

\begin{tabular}{|c|c|c|}
\hline $\begin{array}{l}\text { cis-1,2-Dichloroethene } \\
\text { cis-1,3-Dichloropropene } \\
\text { trans-1,2-Dichloroethene } \\
\text { trans-1,3-Dichloropropene } \\
\text { Acetone } \\
\text { Benzene } \\
\text { Bromodichloromethane } \\
\text { Bromoform } \\
\text { Bromomethane } \\
\text { Carbon disulfide } \\
\text { Carbon tetrachloride } \\
\text { Chlorobenzene } \\
\text { Chloroethane } \\
\text { Chloroform } \\
\text { Chloromethane } \\
\text { Dibromochloromethane } \\
\text { Ethyl benzene } \\
\text { Methylene chloride } \\
\text { Styrene } \\
\text { Tetrachloroethene } \\
\text { Toluene } \\
\text { Trichloroethene } \\
\text { Vinyl chloride } \\
\text { Xylene (meta + para) } \\
\text { xylene (ortho) } \\
1,1-0 \text { ichloroethane } \\
1,1-D \text { ichloroethene } \\
1,1,1-T r i c h l o r o e t h a n e \\
1,1,2-T r i c h l o r o e t h a n e \\
1,1,2,2-T e t r a c h l o r o e t h a n e \\
1,2-D i c h l o r o e t h a n e\end{array}$ & 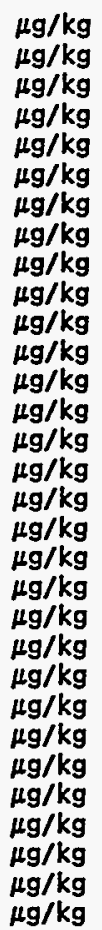 & $\begin{array}{r}5 \\
5 \\
5 \\
5 \\
100 \\
5 \\
5 \\
5 \\
10 \\
5 \\
5 \\
5 \\
10 \\
5 \\
10 \\
5 \\
5 \\
5 \\
5 \\
5 \\
5 \\
5 \\
10 \\
5\end{array}$ \\
\hline
\end{tabular}


Table B.1 (continued)

\begin{tabular}{lcccc}
\hline \multicolumn{1}{c}{ Analysis } & Units & $\begin{array}{c}\text { A } \\
\text { horizon }\end{array}$ & Qualifier & $\begin{array}{c}\text { A horizon } \\
\text { field dup }\end{array}$ \\
\hline & & & & \\
1,2-Dichloropropane & $\mu \mathrm{g} / \mathrm{kg}$ & 5 & $\mathrm{U}$ & \\
2-Butanone & $\mu \mathrm{g} / \mathrm{kg}$ & 5 & $\mathrm{BJ}$ & \\
2-Hexanone & $\mu \mathrm{g} / \mathrm{kg}$ & 50 & $\mathrm{U}$ & \\
4-Hethyl-2-pentanone & $\mu \mathrm{g} / \mathrm{kg}$ & 50 & $\mathrm{U}$
\end{tabular}

Location=ORR; Formation=COPPER RIDGE; Site=45; Samp id=1543; Dup id=' '; Phase=2

\begin{tabular}{|c|c|c|}
\hline $\begin{array}{l}\text { cis-1,2-Dichloroethene } \\
\text { cis-1,3-Dichloropropene } \\
\text { trans-1,2-Dichloroethene } \\
\text { trans-1,3-Dichloropropene } \\
\text { Acetone } \\
\text { Benzene } \\
\text { Bromodichloromethane } \\
\text { Bromoform } \\
\text { Bromomethane } \\
\text { Carbon disulfide } \\
\text { Carbon tetrachloride } \\
\text { Chlorobenzene } \\
\text { Chloroethane } \\
\text { Chloroform } \\
\text { Chloromethane } \\
\text { Dibromochloromethane } \\
\text { Ethyl benzene } \\
\text { Methylene chloride } \\
\text { Styrene } \\
\text { Tetrachloroethene } \\
\text { Toluene } \\
\text { Trichloroethene } \\
\text { Vinyl chloride } \\
\text { xylene (meta + para) } \\
\text { xylene (ortho) } \\
1,1 \text {-Dichloroethane } \\
1,1-D i c h l o r o e t h e n e \\
1,1,1-T r i c h l o r o e t h a n e \\
1,1,2-T r i c h l o r o e t h a n e \\
1,1,2,2-T \text {-Trachloroethane } \\
1,2-D \text { ichloroethane } \\
1,2-D \text { ichloropropane } \\
2-\text { Butanone } \\
2-\text { Hexanone } \\
4-\text { Methyl-2-pentanone }\end{array}$ & $\begin{array}{l}\mu g / k g \\
\mu g / k g \\
\mu g / k g \\
\mu g / k g \\
\mu g / k g \\
\mu g / k g \\
\mu g / k g \\
\mu g / k g \\
\mu g / k g \\
\mu g / k g \\
\mu g / k g \\
\mu g / k g \\
\mu g / k g \\
\mu g / k g \\
\mu g / k g \\
\mu g / k g \\
\mu g / k g \\
\mu g / k g \\
\mu g / k g \\
\mu g / k g \\
\mu g / k g \\
\mu g / k g \\
\mu g / k g \\
\mu g / k g \\
\mu g / k g \\
\mu g / k g \\
\mu g / k g \\
\mu g / k g \\
\mu g / k g \\
\mu g / k g \\
\mu g / k g \\
\mu g / k g \\
\mu g / k g \\
\mu g / k g \\
\mu g / k g\end{array}$ & $\begin{array}{r}5 \\
5 \\
5 \\
5 \\
100 \\
5 \\
5 \\
5 \\
10 \\
5 \\
5 \\
5 \\
10 \\
5 \\
10 \\
5 \\
5 \\
5 \\
5 \\
5 \\
5 \\
5 \\
10 \\
5 \\
5 \\
5 \\
5 \\
5 \\
5 \\
5 \\
5 \\
5 \\
100 \\
50 \\
50\end{array}$ \\
\hline
\end{tabular}

Location=ORR; Formation=COPPER RIDGE; Site=51; Samp id=1545; Dup id =' '; Phase $=2$

\begin{tabular}{|c|c|c|}
\hline $\begin{array}{l}\text { cis-1,2-Dichloroethene } \\
\text { cis-1,3-Dichloropropene } \\
\text { trans-1,2-Dichloroethene } \\
\text { trans-1,3-Dichloropropene } \\
\text { Acetone } \\
\text { Benzene } \\
\text { Bromodichloromethane } \\
\text { Bromoform } \\
\text { Bromomethane } \\
\text { Carbon disul fide } \\
\text { Carbon tetrachloride } \\
\text { Chlorobenzene } \\
\text { Chloroethane } \\
\text { Chloroform } \\
\text { Chloromethane } \\
\text { Dibromochloromethane } \\
\text { Ethyl benzene } \\
\text { Hethylene chloride } \\
\text { Styrene } \\
\text { Tetrachloroethene } \\
\text { Toluene } \\
\text { Trichloroethene } \\
\text { Vinyl chloride } \\
\text { Xylene (meta + para) } \\
\text { Xylene (ortho) }\end{array}$ & $\begin{array}{l}\mu g / \mathbf{k g} \\
\mu g / k g \\
\mu g / k g \\
\mu g / k g \\
\mu g / k g \\
\mu g / k g \\
\mu g / k g \\
\mu g / k g \\
\mu g / k g \\
\mu g / k g \\
\mu g / k g \\
\mu g / k g \\
\mu g / k g \\
\mu g / k g \\
\mu g / k g \\
\mu g / k g \\
\mu g / k g \\
\mu g / k g \\
\mu g / k g \\
\mu g / k g \\
\mu g / k g \\
\mu g / k g \\
\mu g / k g \\
\mu g / k g \\
\mu g / k g\end{array}$ & $\begin{array}{r}5 \\
5 \\
5 \\
5 \\
7 \\
5 \\
5 \\
5 \\
10 \\
5 \\
5 \\
5 \\
10 \\
5 \\
10 \\
5 \\
5 \\
5 \\
5 \\
5 \\
5 \\
5 \\
10\end{array}$ \\
\hline
\end{tabular}


Table B.1 (continued)

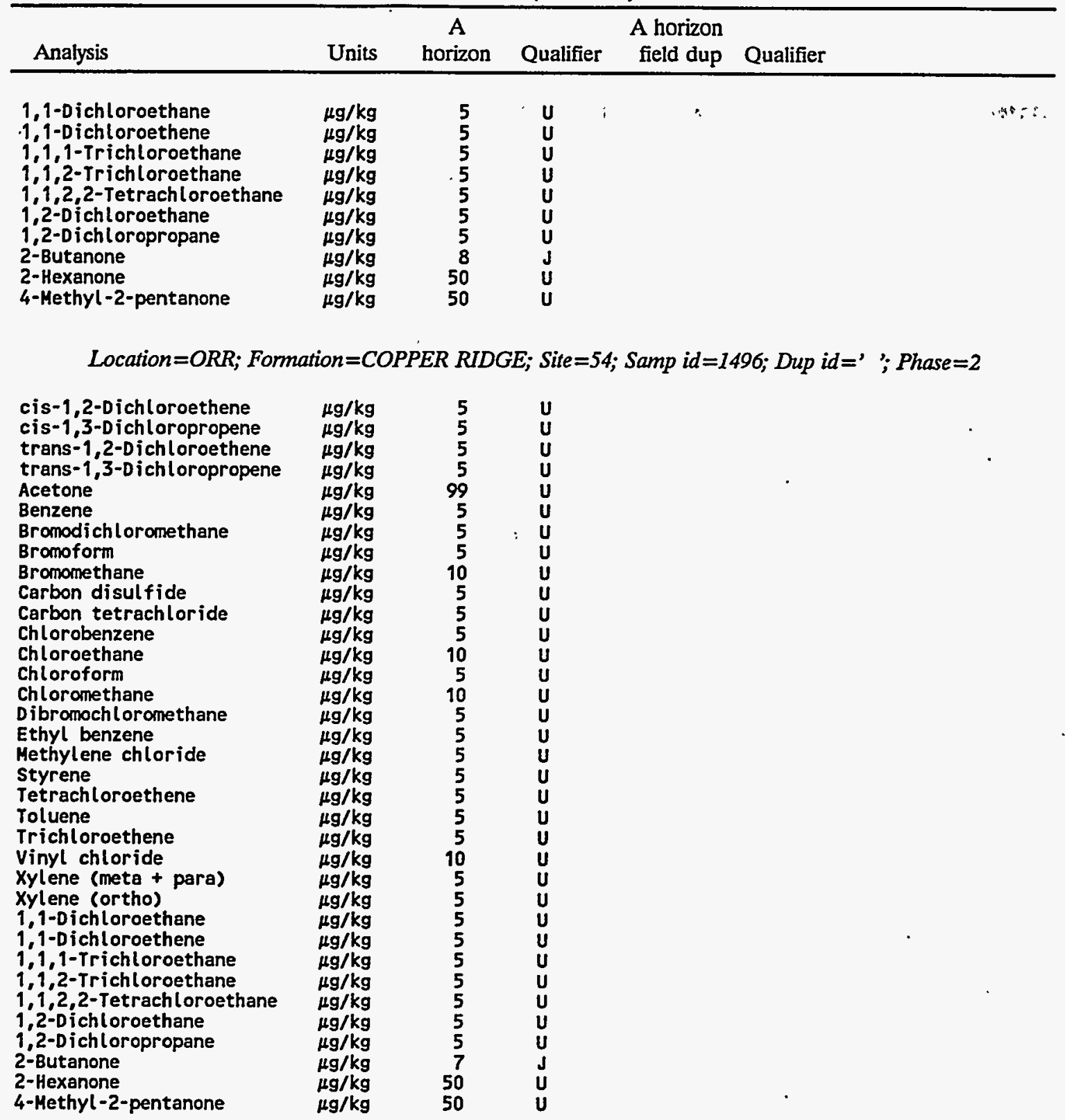

Location=ORR; Formation=COPPER RIDGE; Site=55; Samp id=1494; Dup id=' '; Phase=2

$\begin{array}{llrl}\text { cis-1,2-Dichloroethene } & \mu \mathrm{g} / \mathrm{kg} & 5 & \mathrm{U} \\ \text { cis-1,3-Dichloropropene } & \mu \mathrm{g} / \mathrm{kg} & 5 & \mathrm{U} \\ \text { trans-1,2-Dichloroethene } & \mu \mathrm{g} / \mathrm{kg} & 5 & \mathrm{U} \\ \text { trans-1,3-Dichloropropene } & \mu \mathrm{g} / \mathrm{kg} & 5 & \mathrm{U} \\ \text { Acetone } & \mu \mathrm{g} / \mathrm{kg} & 6 & \mathrm{~J} \\ \text { Benzene } & \mu \mathrm{g} / \mathrm{kg} & 5 & \mathrm{U} \\ \text { Bromodichloromethane } & \mu \mathrm{g} / \mathrm{kg} & 5 & \mathrm{U} \\ \text { Bromoform } & \mu \mathrm{g} / \mathrm{kg} & 5 & U \\ \text { Bromomethane } & \mu \mathrm{g} / \mathrm{kg} & 10 & \mathrm{U} \\ \text { Carbon disulfide } & \mu \mathrm{g} / \mathrm{kg} & 5 & \mathrm{U} \\ \text { Carbon tetrachloride } & \mu \mathrm{g} / \mathrm{kg} & 5 & \mathrm{U} \\ \text { Chlorobenzene } & \mu \mathrm{g} / \mathrm{kg} & 5 & U \\ \text { Chloroethane } & \mu \mathrm{g} / \mathrm{kg} & 10 & U \\ \text { Chloroform } & \mu \mathrm{g} / \mathrm{kg} & 5 & U \\ \text { Chloromethane } & \mu \mathrm{g} / \mathrm{kg} & 10 & U \\ \text { Dibromochloromethane } & \mu \mathrm{g} / \mathrm{kg} & 5 & \mathrm{U} \\ \text { Ethyl benzene } & \mu \mathrm{g} / \mathrm{kg} & 5 & U \\ \text { Methylene chloride } & \mu \mathrm{g} / \mathrm{kg} & 5 & U \\ \text { Styrene } & \mu \mathrm{g} / \mathrm{kg} & 5 & U\end{array}$




\section{B-38}

Table B.1 (continued)

\begin{tabular}{|c|c|c|c|c|c|}
\hline Analysis & Units & $\begin{array}{c}\text { A } \\
\text { horizon }\end{array}$ & Qualifier & $\begin{array}{l}\text { A horizon } \\
\text { field dup }\end{array}$ & Qualifier \\
\hline $\begin{array}{l}\text { Tetrachloroethene } \\
\text { Toluene } \\
\text { Trichloroethene } \\
\text { Vinyl chloride } \\
\text { Xylene (meta + para) } \\
\text { Xylene (ortho) } \\
\text { 1,1-Dichloroethane } \\
1,1-\text { Dichloroethene } \\
1,1,1-\text { Trichloroethane } \\
1,1,2-\text { Trichloroethane } \\
1,1,2,2-\text { Tetrachloroethane } \\
1,2-\text { ichloroethane } \\
\text { 1,2-Dichloropropane } \\
\text { 2-Butanone } \\
\text { 2-Hexanone } \\
\text { 4- - Methyl-2-pentanone }\end{array}$ & $\begin{array}{l}\mu g / k g \\
\mu g / k g \\
\mu g / k g \\
\mu g / k g \\
\mu g / k g \\
\mu g / k g \\
\mu g / k g \\
\mu g / k g \\
\mu g / k g \\
\mu g / k g \\
\mu g / k g \\
\mu g / k g \\
\mu g / k g \\
\mu g / k g \\
\mu g / k g \\
\mu g / k g\end{array}$ & $\begin{array}{r}5 \\
5 \\
5 \\
10 \\
5 \\
5 \\
5 \\
5 \\
5 \\
5 \\
5 \\
5 \\
5 \\
98 \\
49 \\
49\end{array}$ & $\begin{array}{l}U \\
U \\
U \\
U \\
U \\
U \\
U \\
U \\
U \\
U \\
U \\
U \\
U \\
U \\
U \\
U \\
U\end{array}$ & & \\
\hline
\end{tabular}

Location=ORR; Formation=COPPER RIDGE; Site $=58$; Samp id =1541; Dup id=' '; Phase $=2$

\begin{tabular}{|c|c|c|}
\hline 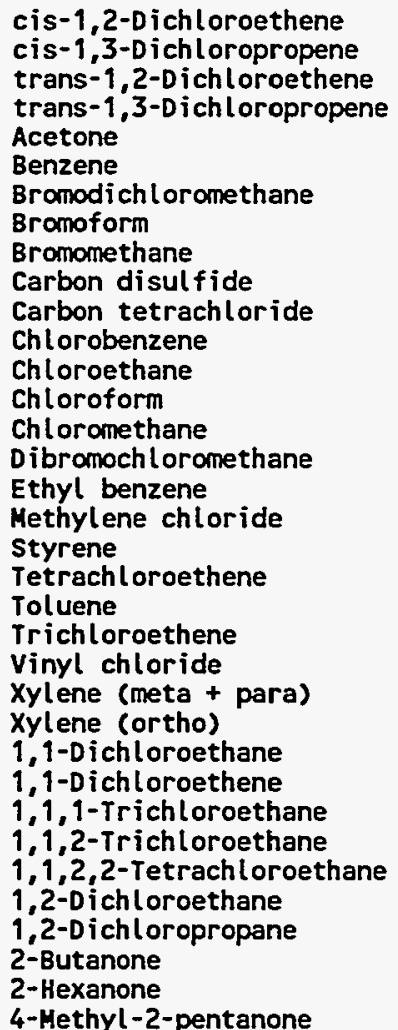 & 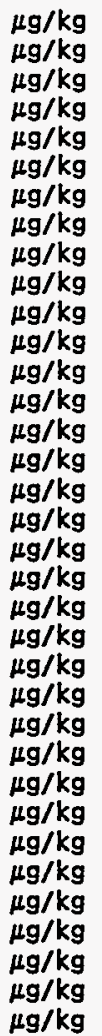 & $\begin{array}{r}5 \\
5 \\
5 \\
5 \\
7 \\
5 \\
5 \\
5 \\
10 \\
5 \\
5 \\
5 \\
10 \\
5 \\
10 \\
5 \\
5 \\
5 \\
5 \\
5 \\
5 \\
5 \\
10 \\
5 \\
5 \\
5 \\
5 \\
5 \\
5 \\
5 \\
5\end{array}$ \\
\hline
\end{tabular}

Location=ORR; Formation=COPPER RIDGE; Site=59; Samp id=1490; Dup id=' '; Phase =2

$\begin{array}{llrl}\text { cis-1,2-Dichloroethene } & \mu \mathrm{g} / \mathrm{kg} & 5 & \mathrm{U} \\ \text { cis-1,3-Dichloropropene } & \mu \mathrm{g} / \mathrm{kg} & 5 & \mathrm{U} \\ \text { trans-1,2-Dichloroethene } & \mu \mathrm{g} / \mathrm{kg} & 5 & \mathrm{U} \\ \text { trans-1,3-Dichloropropene } & \mu \mathrm{g} / \mathrm{kg} & 5 & \mathrm{U} \\ \text { Acetone } & \mu \mathrm{g} / \mathrm{kg} & 100 & \mathrm{U} \\ \text { Benzene } & \mu \mathrm{g} / \mathrm{kg} & 5 & \mathrm{U} \\ \text { Bromodichloromethane } & \mu \mathrm{g} / \mathrm{kg} & 5 & \mathrm{U} \\ \text { Bromoform } & \mu \mathrm{g} / \mathrm{kg} & 5 & \mathrm{U} \\ \text { Bromomethane } & \mu \mathrm{g} / \mathrm{kg} & 10 & U \\ \text { Carbon disulfide } & \mu \mathrm{g} / \mathrm{kg} & 5 & \mathrm{U} \\ \text { Carbon tetrachloride } & \mu \mathrm{g} / \mathrm{kg} & 5 & U \\ \text { Chlorobenzene } & \mu \mathrm{g} / \mathrm{kg} & 5 & U \\ \text { Chloroethane } & \mu \mathrm{g} / \mathrm{kg} & 10 & U\end{array}$


Table B.1 (continued)

\begin{tabular}{|c|c|c|c|c|c|}
\hline Analysis & Units & $\begin{array}{c}A \\
\text { horizon }\end{array}$ & Qualifier & $\begin{array}{l}\text { A horizon } \\
\text { field dup }\end{array}$ & Qualifier \\
\hline $\begin{array}{l}\text { Chloroform } \\
\text { Chloromethane } \\
\text { Dibromochloromethane } \\
\text { Ethyl benzene } \\
\text { Methylene chloride } \\
\text { styrene } \\
\text { Tetrachloroethene } \\
\text { Toluene } \\
\text { Trichloroethene } \\
\text { vinyl chloride } \\
\text { xylene (meta + para) } \\
\text { xylene (ortho) } \\
1,1-\text { Dichloroethane } \\
\text { 1,1-Dichloroethene } \\
1,1,1-\text { Trichloroethane } \\
1,1,2-\text { Trichloroethane } \\
1,1,2,2-T \text {-Trachloroethane } \\
\text { 1,2--Dichloroethane } \\
1,2-\text { Dichloropropane } \\
\text { 2--Butanone } \\
\text { 2-Hexanone } \\
\text { 4-Methyl-2-pentanone }\end{array}$ & 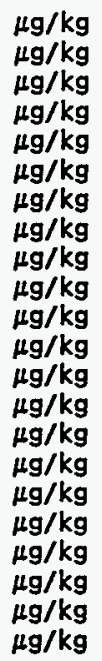 & $\begin{array}{r}5 \\
10 \\
5 \\
5 \\
5 \\
5 \\
5 \\
5 \\
5 \\
10 \\
5 \\
5 \\
5 \\
5 \\
5 \\
5 \\
5 \\
5 \\
5 \\
100 \\
50 \\
50\end{array}$ & $\begin{array}{l}U \\
U \\
U \\
U \\
U \\
U \\
U \\
U \\
U \\
U \\
U \\
U \\
U \\
U \\
U \\
U \\
U \\
U \\
U \\
U \\
U \\
U\end{array}$ & & \\
\hline
\end{tabular}

Location=ORR; Formation=COPPER RIDGE; Site=60; Samp id=1492; Dup id=' '; Phase=2

\begin{tabular}{|c|c|c|}
\hline $\begin{array}{l}\text { cis-1,2-Dichloroethene } \\
\text { cis-1,3-Dichloropropene } \\
\text { trans-1,2-Dichloroethene } \\
\text { trans-1,3-Dichloropropene } \\
\text { Acetone } \\
\text { Benzene } \\
\text { Bromodichloromethane } \\
\text { Bromoform } \\
\text { Bromomethane } \\
\text { Carbon disulfide } \\
\text { Carbon tetrachloride } \\
\text { Chlorobenzene } \\
\text { Chloroethane } \\
\text { Chloroform } \\
\text { Chloromethane } \\
\text { Dibromochloromethane } \\
\text { Ethyl benzene } \\
\text { Methylene chloride } \\
\text { Styrene } \\
\text { Tetrachloroethene } \\
\text { Toluene } \\
\text { Trichloroethene } \\
\text { vinyl chloride } \\
\text { Xylene (meta + para) } \\
\text { Xylene (ortho) } \\
1,1-0 \text { ichloroethane } \\
1,1-0 \text { ichloroethene } \\
1,1,1-\text { Trichloroethane } \\
1,9,2-T r i c h l o r o e t h a n e \\
1,9,2,2-\text { Tetrachloroethane } \\
1,2-0 \text { ichloroethane } \\
1,2-0 \text { ichloropropane } \\
2-\text {-Butanone } \\
2-\text { Hexanone } \\
4-\text { Methyl-2-pentanone }\end{array}$ & 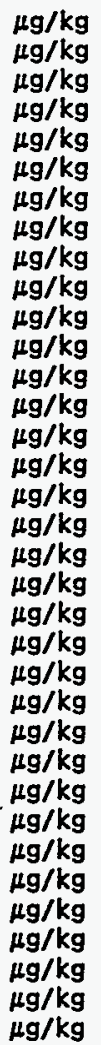 & $\begin{array}{r}5 \\
5 \\
5 \\
5 \\
5 \\
5 \\
5 \\
5 \\
10 \\
5 \\
5 \\
5 \\
10 \\
5 \\
10 \\
5 \\
5 \\
5 \\
5 \\
5 \\
5 \\
5 \\
10 \\
5 \\
5 \\
5 \\
5 \\
5 \\
5 \\
5 \\
5 \\
5 \\
100 \\
50 \\
50\end{array}$ \\
\hline
\end{tabular}

Location=ORR; Formation=COPPER RIDGE; Site=62; Samp id=1479; Dup id =' '; Phase=2

$\begin{array}{lrrr}\text { cis-1,2-Dichloroethene } & \mu g / \mathrm{kg} & 5 & U \\ \text { cis-1,3-Dichloropropene } & \mu g / \mathrm{kg} & 5 & U \\ \text { trans-1,2-Dichloroethene } & \mu \mathrm{g} / \mathrm{kg} & 5 & U \\ \text { trans-1,3-Dichloropropene } & \mu g / \mathrm{kg} & 5 & U \\ \text { Acetone } & \mu \mathrm{g} / \mathrm{kg} & 100 & U \\ \text { Benzene } & \mu \mathrm{gg} & 5 & U \\ \text { Bromodichloromethane } & \mu \mathrm{g} / \mathrm{kg} & 5 & U\end{array}$


Table B.1 (continued)

\begin{tabular}{|c|c|c|c|c|c|c|c|}
\hline Analysis & Units & $\begin{array}{c}\text { A } \\
\text { horizon }\end{array}$ & Qualifier & $\begin{array}{l}\text { A horizon } \\
\text { field dup }\end{array}$ & Qualifier & & \\
\hline $\begin{array}{l}\text { Bromoform } \\
\text { Bromomethane } \\
\text { Carbon disulfide } \\
\text { Carbon tetrachloride } \\
\text { Chlorobenzene } \\
\text { Chloroethane } \\
\text { Chloroform } \\
\text { Chloromethane } \\
\text { Dibromochloromethane } \\
\text { Ethyl benzene } \\
\text { Methylene chloride } \\
\text { Styrene } \\
\text { Tetrachloroethene } \\
\text { Toluene } \\
\text { Trichloroethene } \\
\text { Vinyl chloride } \\
\text { xylene (meta + para) } \\
\text { xylene (ortho) } \\
1,1-D i c h l o r o e t h a n e \\
1,1-D i c h l o r o e t h e n e \\
1,1,1-\text { Trichloroethane } \\
1,1,2-\text { Trichloroethane } \\
1,1,2,2-T e t r a c h l o r o e t h a n e \\
1,2-D \text { ichloroethane } \\
1,2-D \text { ichloropropane } \\
\text { 2-Butanone } \\
\text { 2-Hexanone } \\
\text { 4-Methyl-2-pentanone }\end{array}$ & 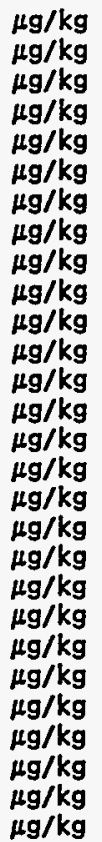 & $\begin{array}{r}5 \\
10 \\
5 \\
5 \\
5 \\
10 \\
5 \\
10 \\
5 \\
5 \\
5 \\
5 \\
5 \\
5 \\
5 \\
10 \\
5 \\
5 \\
5 \\
5 \\
5 \\
5 \\
5 \\
5 \\
5 \\
100 \\
50 \\
50\end{array}$ & $\begin{array}{l}U \\
U \\
U \\
U \\
U \\
U \\
U \\
U \\
U \\
U \\
U \\
U \\
U \\
U \\
U \\
U \\
U \\
U \\
U \\
U \\
U \\
U \\
U \\
U \\
U \\
U \\
U \\
U\end{array}$ & $:$ & & $?$ & F斻: \\
\hline
\end{tabular}

Location=ORR; Formation=COPPER RIDGE; Site=64; Samp id=1482; Dup id=' '; Phase=2

\begin{tabular}{|c|c|c|}
\hline $\begin{array}{l}\text { cis-1,2-Dichloroethene } \\
\text { cis-1,3-Dichloropropene } \\
\text { trans-1,2-Dichloroethene } \\
\text { trans-1,3-Dichloropropene } \\
\text { Acetone } \\
\text { Benzene } \\
\text { Bromodichloromethane } \\
\text { Bromoform } \\
\text { Bromomethane } \\
\text { Carbon disulfide } \\
\text { Carbon tetrachloride } \\
\text { Chlorobenzene } \\
\text { Chloroethane } \\
\text { Chloroform } \\
\text { Chloromethane } \\
\text { Dibromochloromethane } \\
\text { Ethyl benzene } \\
\text { Methylene chloride } \\
\text { Styrene } \\
\text { Tetrachloroethene } \\
\text { Toluene } \\
\text { Trichloroethene } \\
\text { vinyl chloride } \\
\text { Xylene (meta + para) } \\
\text { Xylene (ortho) } \\
1,1-D \text { ichloroethane } \\
1,1-D i c h l o r o e t h e n e \\
1,1,1-T r i c h l o r o e t h a n e \\
1,1,2-T r i c h l o r o e t h a n e \\
1,1,2,2-T e t r a c h l o r o e t h a n e \\
1,2-0 \text { ichloroethane } \\
1,2-0 \text { ichloropropane } \\
2-\text { Butanone } \\
2-H e x a n o n e \\
4-\text { Methyl-2-pentanone }\end{array}$ & $\begin{array}{l}\mu g / \mathbf{k g} \\
\mu g / \mathbf{k g} \\
\mu g / \mathbf{k g} \\
\mu g / \mathbf{k g} \\
\mu g / \mathbf{k g} \\
\mu g / \mathbf{k g} \\
\mu g / \mathbf{k g} \\
\mu g / \mathbf{k g} \\
\mu g / \mathbf{k g} \\
\mu g / \mathbf{k g} \\
\mu g / \mathbf{k g} \\
\mu g / \mathbf{k g} \\
\mu g / \mathbf{k g} \\
\mu g / \mathbf{k g} \\
\mu g / \mathbf{k g} \\
\mu g / \mathbf{k g} \\
\mu g / \mathbf{k g} \\
\mu g / \mathbf{k g} \\
\mu g / \mathbf{k g} \\
\mu g / \mathbf{k g} \\
\mu g / \mathbf{k g} \\
\mu g / \mathbf{k g} \\
\mu g / \mathbf{k g} \\
\mu g / \mathbf{k g} \\
\mu g / \mathbf{k g} \\
\mu g / \mathbf{k g} \\
\mu g / \mathbf{k g} \\
\mu g / \mathbf{k g} \\
\mu g / \mathbf{k g} \\
\mu g / \mathbf{k g} \\
\mu g / \mathbf{k g} \\
\mu g / \mathbf{k g} \\
\mu g / \mathbf{k g}\end{array}$ & $\begin{array}{r}5 \\
5 \\
5 \\
5 \\
6 \\
5 \\
5 \\
5 \\
10 \\
5 \\
5 \\
5 \\
10 \\
5 \\
10 \\
5 \\
5 \\
5 \\
5 \\
5 \\
5 \\
5 \\
10 \\
5 \\
5 \\
5 \\
5 \\
5 \\
5 \\
5 \\
5 \\
5\end{array}$ \\
\hline
\end{tabular}


Table B.1 (continued)

\begin{tabular}{lccc}
\hline & A & A horizon \\
Analysis & Units & horizon & Qualifier \\
field dup & Qualifier \\
\hline
\end{tabular}

Location=ORR; Formation=COPPER RIDGE; Site=75; Samp id=1476; Dup id=' '; Phase =2 it:

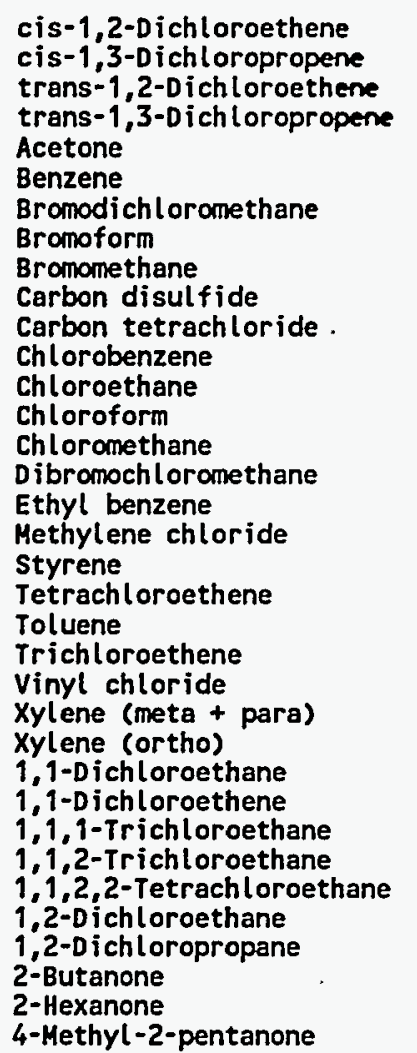

$\begin{array}{lrl}\mu g / k g & 5 & U \\ \mu g / k g & 5 & U \\ \mu g / k g & 5 & U \\ \mu g / k g & 5 & U \\ \mu g / k g & 100 & U \\ \mu g / k g & 5 & U \\ \mu g / k g & 5 & U \\ \mu g / k g & 5 & U \\ \mu g / k g & 10 & U \\ \mu g / k g & 5 & U \\ \mu g / k g & 5 & U \\ \mu g / k g & 5 & U \\ \mu g / k g & 10 & U \\ \mu g / k g & 5 & U \\ \mu g / k g & 10 & U \\ \mu g / k g & 5 & U \\ \mu g / k g & 5 & U \\ \mu g / k g & 5 & U \\ \mu g / k g & 5 & U \\ \mu g / k g & 5 & U \\ \mu g / k g & 5 & U \\ \mu g / k g & 5 & U \\ \mu g / k g & 10 & U \\ \mu g / k g & 5 & U \\ \mu g / k g & 5 & U \\ \mu g / k g & 5 & U \\ \mu g / k g & 5 & U \\ \mu g / k g & 5 & U \\ \mu g / k g & 5 & U \\ \mu g / k g & 5 & U \\ \mu g / k g & 5 & U \\ \mu g / k g & 5 & U \\ \mu g / k g & 100 & U \\ \mu g / k g & 50 & U \\ \mu g / k g & 50 & U \\ & & 5\end{array}$

Location=ORR; Formation=COPPER RIDGE; Site=83; Samp id=1473; Dup id =' '; Phase=2

cis-1,2-Dichloroethene

cis-1,3-Dichloropropene

trans-1,2-Dichloroethene

trans-1,3-D ich loropropene

Acetone

Benzene

Bromodichloromethane

Bromoform

Bromomethane

Carbon disulfide

Carbon tetrachloride

Chlorobenzene

Chloroethane

Chloroform

Chloromethane

Dibromochloromethane

Ethyl benzene

Methylene chloride

Styrene

Tetrachloroethene

Toluene

Trichloroethene

Vinyl chloride

Xylene (meta + para) $\mu \mathrm{g} / \mathrm{kg}$

Xylene (ortho)

1,1-Dichloroethane

1,1-Dichloroethene

$\mu \mathrm{g} / \mathrm{kg}$

$\mu \mathrm{g} / \mathrm{kg}$

$\mu \mathrm{g} / \mathrm{kg}$

$\mu \mathrm{g} / \mathrm{kg}$

$\mu \mathrm{g} / \mathrm{kg}$

$\mu \mathrm{g} / \mathrm{kg}$

$\mu \mathrm{g} / \mathrm{kg}$

$\mu \mathrm{g} / \mathrm{kg}$

$\mu \mathrm{g} / \mathrm{kg}$

$\mu \mathrm{g} / \mathrm{kg}$

$\mu \mathrm{g} / \mathrm{kg}$

$\mu \mathrm{g} / \mathrm{kg}$

$\mu \mathrm{g} / \mathrm{kg}$

$\mu \mathrm{g} / \mathrm{kg}$

$\mu \mathrm{g} / \mathrm{kg}$

$\mu \mathrm{g} / \mathrm{kg}$

$\mu \mathrm{g} / \mathrm{kg}$

$\mu g / \mathrm{kg}$

$\mu \mathrm{g} / \mathrm{kg}$

$\mu \mathrm{g} / \mathrm{kg}$

$\mu \mathrm{g} / \mathrm{kg}$

$\mu \mathrm{g} / \mathrm{kg}$

$\mu \mathrm{g} / \mathrm{kg}$

1,1,1-Trichloroethane

$\mu \mathrm{g} / \mathrm{kg}$

$\mu \mathrm{g} / \mathrm{kg}$

1,1,2-Trichloroethane

$\mu \mathrm{g} / \mathrm{kg}$

$1,1,2,2-T e t r a c h l o r o e t h a n e \quad \mu g / k g$

1,2-D ichloroethane

$\mu \mathrm{g} / \mathrm{kg}$

$\begin{array}{rr}5 & U \\ 5 & U \\ 5 & U \\ 5 & U \\ 100 & U \\ 5 & U \\ 5 & U \\ 5 & U \\ 10 & U \\ 5 & U \\ 5 & U \\ 5 & U \\ 10 & U \\ 5 & U \\ 10 & U \\ 5 & U \\ 5 & U \\ 5 & U \\ 5 & U \\ 5 & U \\ 5 & U \\ 5 & U \\ 10 & U \\ 5 & U \\ 5 & U \\ 5 & U \\ 5 & U \\ 5 & U \\ 5 & U \\ 5 & U \\ 5 & U\end{array}$


Table B.1 (continued)

\begin{tabular}{lcccc}
\hline \multicolumn{1}{c}{ Analysis } & \multicolumn{1}{c}{$\begin{array}{c}\text { A horizon } \\
\text { horizon }\end{array}$} & Qualifier & $\begin{array}{c}\text { field dup } \\
\text { Qualifier }\end{array}$ \\
\hline 1,2-Dichloropropane & $\mu \mathrm{g} / \mathrm{kg}$ & 5 & $\mathrm{U}$ & \\
2-Butanone & $\mu \mathrm{kg}$ & 100 & $\mathrm{U}$ & \\
2-Hexanone & $\mu \mathrm{kg} / \mathrm{kg}$ & 50 & $\mathrm{U}$ & \\
4-Methyl-2-pentanone & $\mu \mathrm{g} / \mathrm{kg}$ & 50 & $\mathrm{U}$
\end{tabular}

Location=ORR; Formation=COPPER RIDGE; Site=91; Samp id=1470; Dup id=' '; Phase=2

\begin{tabular}{|c|c|c|}
\hline 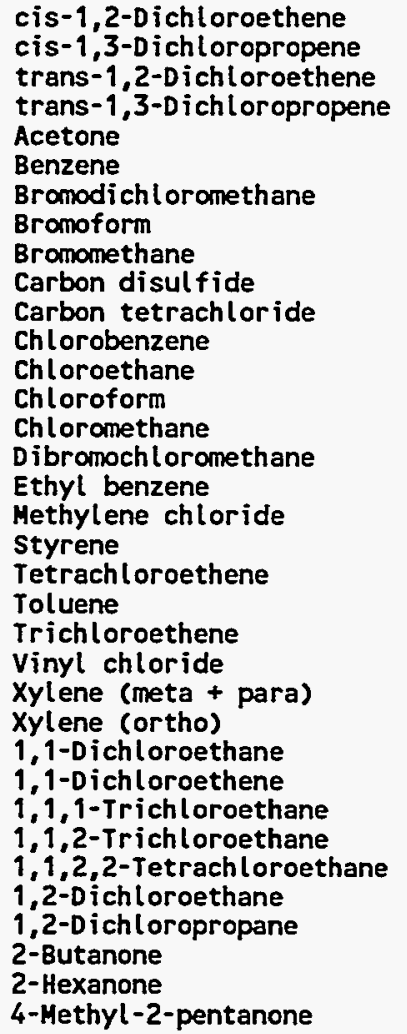 & $\begin{array}{l}\mu g / k g \\
\mu g / k g \\
\mu g / k g \\
\mu g / k g \\
\mu g / k g \\
\mu g / k g \\
\mu g / k g \\
\mu g / k g \\
\mu g / k g \\
\mu g / k g \\
\mu g / k g \\
\mu g / k g \\
\mu g / k g \\
\mu g / k g \\
\mu g / k g \\
\mu g / k g \\
\mu g / k g \\
\mu g / k g \\
\mu g / k g \\
\mu g / k g \\
\mu g / k g \\
\mu g / k g \\
\mu g / k g \\
\mu g / k g \\
\mu g / k g \\
\mu g / k g \\
\mu g / k g \\
\mu g / k g \\
\mu g / k g \\
\mu g / k g \\
\mu g / k g \\
\mu g / k g \\
\mu g / k g \\
\mu g / k g \\
\mu g / k g\end{array}$ & $\begin{array}{r}5 \\
5 \\
5 \\
5 \\
100 \\
5 \\
5 \\
5 \\
10 \\
5 \\
5 \\
5 \\
10 \\
5 \\
10 \\
5 \\
5 \\
5 \\
5 \\
5 \\
5 \\
5 \\
10 \\
5 \\
5 \\
5 \\
5 \\
5 \\
5 \\
5 \\
5 \\
100 \\
50 \\
50\end{array}$ \\
\hline
\end{tabular}

Location=ORR; Formation=DISMAL GAP; Site=10; Samp id=1258; Dup id=' '; Phase=1

$\begin{array}{llrl}\text { cis-1,2-Dichloroethene } & \mu \mathrm{g} / \mathrm{kg} & 5 & \mathrm{U} \\ \text { cis-1,3-Dichloropropene } & \mu \mathrm{g} / \mathrm{kg} & 5 & \mathrm{U} \\ \text { trans-1,2-Dichloroethene } & \mu \mathrm{g} / \mathrm{kg} & 5 & \mathrm{U} \\ \text { trans-1,3-Dichloropropene } & \mu \mathrm{g} / \mathrm{kg} & 5 & \mathrm{U} \\ \text { Acetone } & \mu \mathrm{g} / \mathrm{kg} & 14 & \mathrm{BJ} \\ \text { Benzene } & \mu \mathrm{g} / \mathrm{kg} & 5 & \mathrm{U} \\ \text { Bromodichloromethane } & \mu \mathrm{g} / \mathrm{kg} & 5 & \mathrm{U} \\ \text { Bromoform } & \mu \mathrm{g} / \mathrm{kg} & 5 & \mathrm{U} \\ \text { Bromomethane } & \mu \mathrm{g} / \mathrm{kg} & 10 & \mathrm{U} \\ \text { Carbon disulfide } & \mu \mathrm{g} / \mathrm{kg} & 5 & \mathrm{U} \\ \text { Carbon tetrachloride } & \mu \mathrm{g} / \mathrm{kg} & 5 & \mathrm{U} \\ \text { Chlorobenzene } & \mu \mathrm{g} / \mathrm{kg} & 5 & \mathrm{U} \\ \text { Chloroethane } & \mu \mathrm{g} / \mathrm{kg} & 10 & \mathrm{U} \\ \text { Chloroform } & \mu \mathrm{g} / \mathrm{kg} & 5 & \mathrm{U} \\ \text { Chloromethane } & \mu \mathrm{g} / \mathrm{kg} & 10 & \mathrm{U} \\ \text { Dibromochloromethane } & \mu \mathrm{g} / \mathrm{kg} & 5 & \mathrm{U} \\ \text { Ethyl benzene } & \mu \mathrm{g} / \mathrm{kg} & 5 & \mathrm{U} \\ \text { Freon-113 } & \mu \mathrm{g} / \mathrm{kg} & 10 & \mathrm{U} \\ \text { Methylene chloride } & \mu \mathrm{g} / \mathrm{kg} & 5 & \mathrm{U} \\ \text { Styrene } & \mu \mathrm{g} / \mathrm{kg} & 5 & \mathrm{U} \\ \text { Tetrachloroethene } & \mu \mathrm{g} / \mathrm{kg} & 5 & \mathrm{U} \\ \text { Toluene } & \mu \mathrm{g} / \mathrm{kg} & 5 & \mathrm{U} \\ \text { Trichloroethene } & \mu \mathrm{g} / \mathrm{kg} & 5 & \mathrm{U} \\ \text { Trichlorofluoromethane } & \mu \mathrm{g} / \mathrm{kg} & 10 & \mathrm{U} \\ \text { Vinyl acetate } & \mu \mathrm{g} / \mathrm{kg} & 10 & \mathrm{U}\end{array}$


Table B.1 (continued)

\begin{tabular}{|c|c|c|c|c|c|c|}
\hline Analysis & Units & $\begin{array}{c}\mathrm{A} \\
\text { horizon }\end{array}$ & Qualifier & $\begin{array}{l}\text { A horizon } \\
\text { field dup }\end{array}$ & Qualifier & \\
\hline $\begin{array}{l}\text { Vinyl chloride } \\
\text { xylene (meta + para) } \\
\text { xylene (ortho) } \\
\text { 1,1-Dichloroethane } \\
1,1-D \text { ichloroethene } \\
1,1,1 \text {-Trichloroethane } \\
1,1,2-\text { Trichloroethane } \\
1,1,2,2-\text { Tetrachloroethane } \\
1,2-D \text { ichlorobenzene } \\
\text { 1,2-Dichloroethane } \\
1,2-D \text { ichloropropane } \\
\text { 1,3-Dichlorobenzene } \\
\text { 1,4-Dichlorobenzene } \\
\text { 2-Butanone } \\
\text { 2-Chloroethylvinyl ether } \\
\text { 2-Hexanone } \\
\text { 4-Methyl-2-pentanone }\end{array}$ & $\begin{array}{l}\mu g / \mathbf{k g} \\
\mu g / \mathbf{k g} \\
\mu g / \mathbf{k g} \\
\mu g / \mathbf{k g} \\
\mu g / \mathbf{k g} \\
\mu g / \mathbf{k g} \\
\mu g / \mathbf{k g} \\
\mu g / \mathbf{k g} \\
\mu g / \mathbf{k g} \\
\mu g / \mathbf{k g} \\
\mu g / \mathbf{k g} \\
\mu g / \mathbf{k g} \\
\mu g / \mathbf{k g} \\
\mu g / \mathbf{k g} \\
\mu g / \mathbf{k g} \\
\mu g / \mathbf{k g} \\
\mu g / \mathbf{k g}\end{array}$ & $\begin{array}{r}10 \\
5 \\
5 \\
5 \\
5 \\
5 \\
5 \\
5 \\
5 \\
5 \\
5 \\
5 \\
5 \\
98 \\
5 \\
49 \\
49\end{array}$ & $\begin{array}{l}U \\
U \\
U \\
U \\
U \\
U \\
U \\
U \\
U \\
U \\
U \\
U \\
U \\
U \\
U \\
U \\
U\end{array}$ & $:$ & 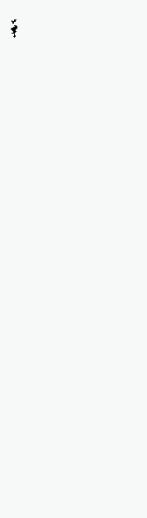 & $i^{2}=4$ \\
\hline
\end{tabular}

Location=ORR; Formation=DISMAL GAP; Site=11; Samp id=1259; Dup id=' '; Phase=1

\begin{tabular}{|c|c|c|}
\hline 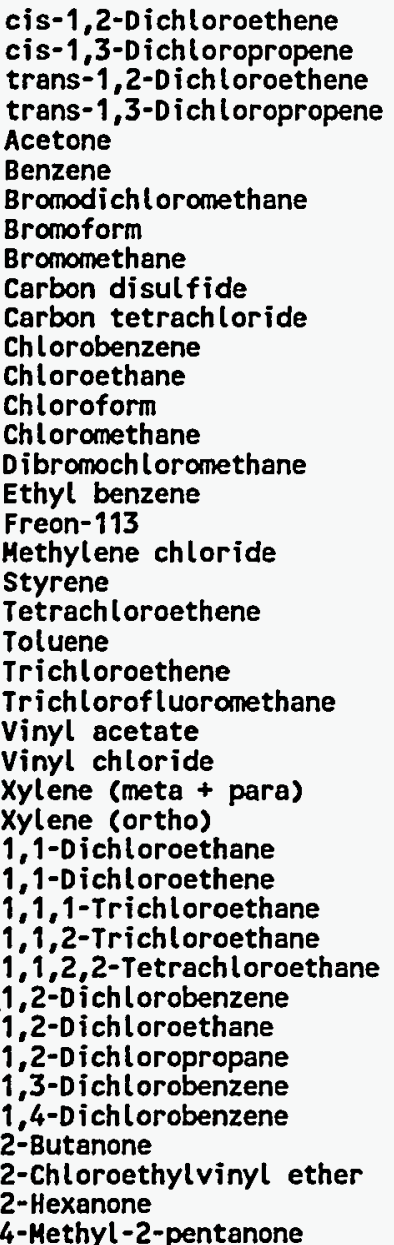 & 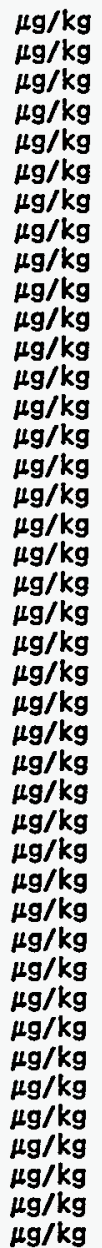 & $\begin{array}{r}5 \\
5 \\
5 \\
5 \\
52 \\
5 \\
5 \\
5 \\
10 \\
5 \\
5 \\
5 \\
10 \\
5 \\
10 \\
5 \\
5 \\
10 \\
5 \\
5 \\
5 \\
5 \\
5 \\
10 \\
10 \\
10 \\
5 \\
5 \\
5 \\
5 \\
5 \\
5 \\
5 \\
5 \\
5 \\
5 \\
5 \\
5 \\
100 \\
5 \\
50 \\
50\end{array}$ \\
\hline
\end{tabular}

Location=ORR; Formation=DISMAL GAP; Site=19; Samp id=1260; Dup id=' '; Phase=1

$\begin{array}{llrl}\text { cis-1,2-Dichloroethene } & \mu \mathrm{g} / \mathrm{kg} & 5 & \mathrm{U} \\ \text { cis-1,3-Dichloropropene } & \mu \mathrm{g} / \mathrm{kg} & 5 & \mathrm{U} \\ \text { trans-1,2-Dichloroethene } & \mu \mathrm{g} / \mathrm{kg} & 5 & \mathrm{U} \\ \text { trans-1,3-Dichloropropene } & \mu \mathrm{g} / \mathrm{kg} & 5 & \mathrm{U} \\ \text { Acetone } & \mu \mathrm{g} / \mathrm{kg} & 41 & \mathrm{BJ}\end{array}$


Table B.1 (continued)

\begin{tabular}{|c|c|c|c|c|c|}
\hline Analysis & Units & $\begin{array}{c}\text { A } \\
\text { horizon }\end{array}$ & Qualifier & $\begin{array}{l}\text { A horizon } \\
\text { field dup }\end{array}$ & Qualifier \\
\hline 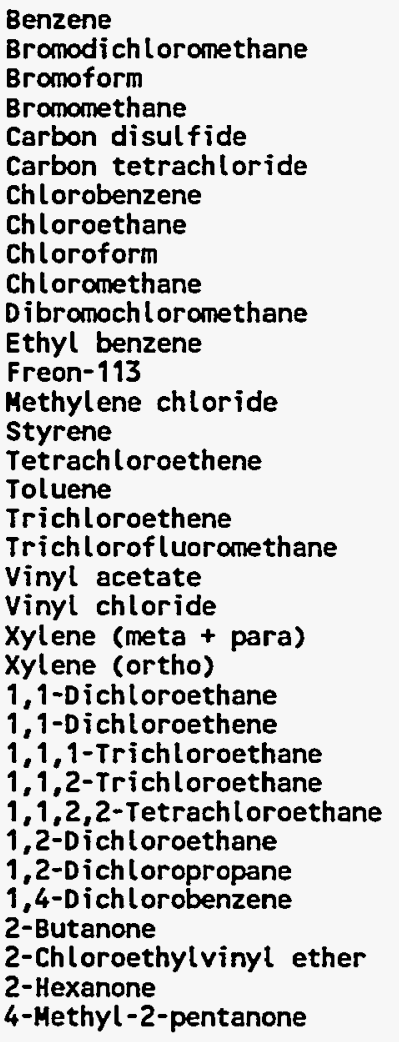 & $\begin{array}{l}\mu g / \mathbf{k g} \\
\mu g / \mathbf{k g} \\
\mu g / \mathbf{k g} \\
\mu g / \mathbf{k g} \\
\mu g / \mathbf{k g} \\
\mu g / \mathbf{k g} \\
\mu g / \mathbf{k g} \\
\mu g / \mathbf{k g} \\
\mu g / \mathbf{k g} \\
\mu g / \mathbf{k g} \\
\mu g / \mathbf{k g} \\
\mu g / \mathbf{k g} \\
\mu g / \mathbf{k g} \\
\mu g / \mathbf{k g} \\
\mu g / \mathbf{k g} \\
\mu g / \mathbf{k g} \\
\mu g / \mathbf{k g} \\
\mu g / \mathbf{k g} \\
\mu g / \mathbf{k g} \\
\mu g / \mathbf{k g} \\
\mu g / \mathbf{k g} \\
\mu g / \mathbf{k g} \\
\mu g / \mathbf{k g} \\
\mu g / \mathbf{k g} \\
\mu g / \mathbf{k g} \\
\mu g / \mathbf{k g} \\
\mu g / \mathbf{k g} \\
\mu g / \mathbf{k g} \\
\mu g / \mathbf{k g} \\
\mu g / \mathbf{k g} \\
\mu g / \mathbf{k g} \\
\mu g / \mathbf{k g} \\
\mu g / \mathbf{k g} \\
\mu g / \mathbf{k g}\end{array}$ & $\begin{array}{r}5 \\
5 \\
5 \\
10 \\
5 \\
5 \\
5 \\
10 \\
5 \\
10 \\
5 \\
5 \\
5 \\
5 \\
5 \\
5 \\
5 \\
5 \\
5 \\
5 \\
10 \\
5 \\
5 \\
5 \\
5 \\
5 \\
5 \\
5 \\
5 \\
5 \\
5 \\
100 \\
5 \\
50 \\
50\end{array}$ & $\begin{array}{l}\mathbf{U} \\
\mathbf{U} \\
\mathbf{U} \\
\mathbf{U} \\
\mathbf{U} \\
\mathbf{U} \\
\mathbf{U} \\
\mathbf{U} \\
\mathbf{U} \\
\mathbf{U} \\
\mathbf{U} \\
\mathbf{U} \\
\mathbf{U} \\
\mathbf{U} \\
\mathbf{U} \\
\mathbf{U} \\
\mathbf{U} \\
\mathbf{U} \\
\mathbf{U} \\
\mathbf{U} \\
\mathbf{U} \\
\mathbf{U} \\
\mathbf{U} \\
\mathbf{U} \\
\mathbf{U} \\
\mathbf{U} \\
\mathbf{U} \\
\mathbf{U} \\
\mathbf{U} \\
\mathbf{U} \\
\mathbf{U} \\
\mathbf{U} \\
\mathbf{U} \\
\mathbf{U} \\
\mathbf{U} \\
\mathbf{U}\end{array}$ & & \\
\hline \multicolumn{6}{|c|}{ Location $=$ ORR; Formation $=$ DISMAL GAP; Site $=2 ;$ Samp id $=1257 ;$ Dup id =' '; Phase $=1$} \\
\hline $\begin{array}{l}\text { cis-1,2-Dichloroethene } \\
\text { cis-1,3-Dichloropropene } \\
\text { trans-1,2-Dichloroethene } \\
\text { trans-1,3-Dichloropropene } \\
\text { Acetone } \\
\text { Benzene } \\
\text { Bromodichloromethane } \\
\text { Bromoform } \\
\text { Bromomethane } \\
\text { Carbon disulfide } \\
\text { Carbon tetrachloride } \\
\text { Chlorobenzene } \\
\text { Chloroethane } \\
\text { Chloroform } \\
\text { Chloromethane } \\
\text { Dibromochloromethane } \\
\text { Ethyl benzene } \\
\text { Freon-113 } \\
\text { Methylene chloride } \\
\text { Styrene } \\
\text { Tetrachloroethene } \\
\text { Toluene } \\
\text { Trichloroethene } \\
\text { Trichlorofluoromethane } \\
\text { Vinyl acetate } \\
\text { Vinyl chloride } \\
\text { Xylene (meta + para) } \\
\text { Xylene (ortho) } \\
1,1-D \text { ichloroethane } \\
1,1-D i c h l o r o e t h e n e \\
1,1,1-\text { Trichloroethane } \\
1,1,2-\text { Trichloroethane } \\
1,1,2,2-\text { Tetrachloroethane }\end{array}$ & $\begin{array}{l}\mu g / \mathbf{k g} \\
\mu g / \mathbf{k g} \\
\mu g / \mathbf{k g} \\
\mu g / \mathbf{k g} \\
\mu g / \mathbf{k g} \\
\mu g / \mathbf{k g} \\
\mu g / \mathbf{k g} \\
\mu g / \mathbf{k g} \\
\mu g / \mathbf{k g} \\
\mu g / \mathbf{k g} \\
\mu g / \mathbf{k g} \\
\mu g / \mathbf{k g} \\
\mu g / \mathbf{k g} \\
\mu g / \mathbf{k g} \\
\mu g / \mathbf{k g} \\
\mu g / \mathbf{k g} \\
\mu g / \mathbf{k g} \\
\mu g / \mathbf{k g} \\
\mu g / \mathbf{k g} \\
\mu g / \mathbf{k g} \\
\mu g / \mathbf{k g} \\
\mu g / \mathbf{k g} \\
\mu g / \mathbf{k g} \\
\mu g / \mathbf{k g} \\
\mu g / \mathbf{k g} \\
\mu g / \mathbf{k g} \\
\mu g / \mathbf{k g} \\
\mu g / \mathbf{k g} \\
\mu g / \mathbf{k g} \\
\mu g / \mathbf{k g} \\
\mu g / \mathbf{k g} \\
\mu g / \mathbf{k g}\end{array}$ & $\begin{array}{r}5 \\
5 \\
5 \\
5 \\
26 \\
5 \\
5 \\
5 \\
10 \\
5 \\
5 \\
5 \\
10 \\
8 \\
10 \\
5 \\
5 \\
5 \\
5 \\
5 \\
5 \\
5 \\
5 \\
5 \\
5 \\
10 \\
5 \\
5 \\
5 \\
5 \\
5 \\
5 \\
5\end{array}$ & $\begin{array}{l}U \\
U \\
U \\
U \\
B J \\
U \\
U \\
U \\
U \\
U \\
U \\
U \\
U \\
U \\
\\
U \\
U \\
U \\
U \\
U \\
U \\
U \\
U \\
U \\
U \\
U \\
U \\
U \\
U \\
U \\
U \\
U \\
U \\
U \\
U \\
U\end{array}$ & & \\
\hline
\end{tabular}


Table B.1 (continued)

\begin{tabular}{|c|c|c|c|c|c|c|}
\hline Analysis & Units & $\begin{array}{c}A \\
\text { horizon }\end{array}$ & Qualifier & $\begin{array}{l}\text { A horizon } \\
\text { field dup }\end{array}$ & Qualifier & \\
\hline $\begin{array}{l}\text { 1,2-Dichloroethane } \\
\text { 1,2-Dichloropropane } \\
\text { 1,4-Dichlorobenzene } \\
\text { 2-Butanone } \\
\text { 2-Chloroethylvinyl ether } \\
\text { 2-Hexanone } \\
\text { 4-Hethyl-2-pentanone }\end{array}$ & $\begin{array}{l}\mu \mathrm{g} / \mathrm{kg} \\
\mu \mathrm{g} / \mathrm{kg} \\
\mu \mathrm{g} / \mathrm{kg} \\
\mu \mathrm{g} / \mathrm{kg} \\
\mu \mathrm{g} / \mathrm{kg} \\
\mu \mathrm{g} / \mathrm{kg} \\
\mu \mathrm{g} / \mathrm{kg}\end{array}$ & $\begin{array}{r}5 \\
5 \\
5 \\
99 \\
5 \\
50 \\
50\end{array}$ & $\begin{array}{l}\mathbf{U} \\
\mathbf{U} \\
\mathbf{U} \\
\mathbf{U} \\
\mathbf{U} \\
\mathbf{U} \\
\mathbf{U}\end{array}$ & s & $\therefore$ & $\because \approx$ \\
\hline
\end{tabular}

Location=ORR; Formation=DISMAL GAP; Site=22; Samp id=1261; Dup id=' '; Phase=1

\begin{tabular}{|c|c|c|}
\hline 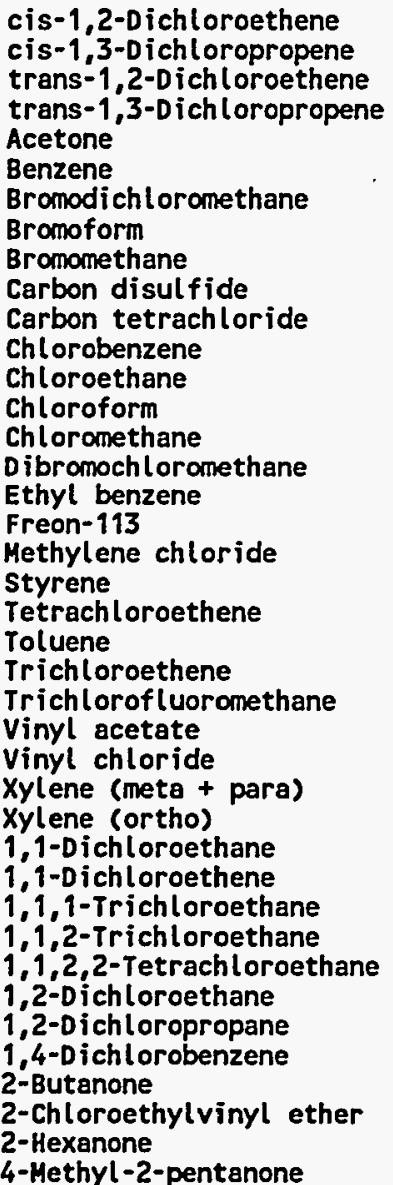 & $\begin{array}{l}\mu g / k g \\
\mu g / k g \\
\mu g / k g \\
\mu g / k g \\
\mu g / k g \\
\mu g / k g \\
\mu g / k g \\
\mu g / k g \\
\mu g / k g \\
\mu g / k g \\
\mu g / k g \\
\mu g / k g \\
\mu g / k g \\
\mu g / k g \\
\mu g / k g \\
\mu g / k g \\
\mu g / k g \\
\mu g / k g \\
\mu g / k g \\
\mu g / k g \\
\mu g / k g \\
\mu g / k g \\
\mu g / k g \\
\mu g / k g \\
\mu g / k g \\
\mu g / k g \\
\mu g / k g \\
\mu g / k g \\
\mu g / k g \\
\mu g / k g \\
\mu g / k g \\
\mu g / k g \\
\mu g / k g \\
\mu g / k g \\
\mu g / k g \\
\mu g / k g \\
\mu g / k g \\
\mu g / k g \\
\mu g / k g \\
\mu g / k g \\
k\end{array}$ & $\begin{array}{r}5 \\
5 \\
5 \\
5 \\
26 \\
5 \\
5 \\
5 \\
10 \\
5 \\
5 \\
5 \\
10 \\
5 \\
10 \\
5 \\
5 \\
5 \\
5 \\
5 \\
5 \\
5 \\
5 \\
5 \\
5 \\
10 \\
5 \\
5 \\
5 \\
5 \\
5 \\
5 \\
5 \\
5 \\
5 \\
5 \\
100 \\
5 \\
51 \\
51\end{array}$ \\
\hline
\end{tabular}

Location=ORR; Formation=DISMAL GAP; Site=26; Samp id=1262; Dup id=' '; Phase=1

cis-1,2-Dichloroethene

cis-1,3-Dichloropropene

trans-1,2-Dichloroethene

trans-1,3-Dichloropropene

Acetone

Benzene

Bromodichloromethane

Bromoform

Bromomethane

Carbon disulfide

Carbon tetrachloride

Chlorobenzene

Chloroethane

Chloroform

Chloromethane

Dibromochloromethane

Ethyl benzene

$\begin{array}{lrl}\mu g / \mathbf{k g} & 5 & U \\ \mu g / \mathbf{k g} & 5 & U \\ \mu g / \mathrm{kg} & 5 & U \\ \mu g / \mathrm{kg} & 5 & U \\ \mu \mathrm{g} / \mathrm{kg} & 64 & \mathrm{BJ} \\ \mu \mathrm{g} / \mathrm{kg} & 5 & U \\ \mu \mathrm{g} / \mathrm{kg} & 5 & U \\ \mu \mathrm{g} / \mathrm{kg} & 5 & U \\ \mu \mathrm{g} / \mathrm{kg} & 10 & U \\ \mu \mathrm{g} / \mathrm{kg} & 5 & U \\ \mu \mathrm{g} / \mathrm{kg} & 5 & U \\ \mu \mathrm{g} / \mathrm{kg} & 5 & U \\ \mu \mathrm{g} / \mathrm{kg} & 10 & U \\ \mu \mathrm{g} / \mathrm{kg} & 5 & U \\ \mu \mathrm{g} / \mathrm{kg} & 10 & U \\ \mu \mathrm{g} / \mathrm{kg} & 5 & U \\ \mu \mathrm{g} / \mathrm{kg} & 5 & U\end{array}$




\section{B-46}

Table B.1 (continued)

\begin{tabular}{|c|c|c|c|c|c|}
\hline Analysis & Units & $\begin{array}{c}\text { A } \\
\text { horizon }\end{array}$ & Qualifier & $\begin{array}{l}\text { A horizon } \\
\text { field dup }\end{array}$ & Qualifier \\
\hline 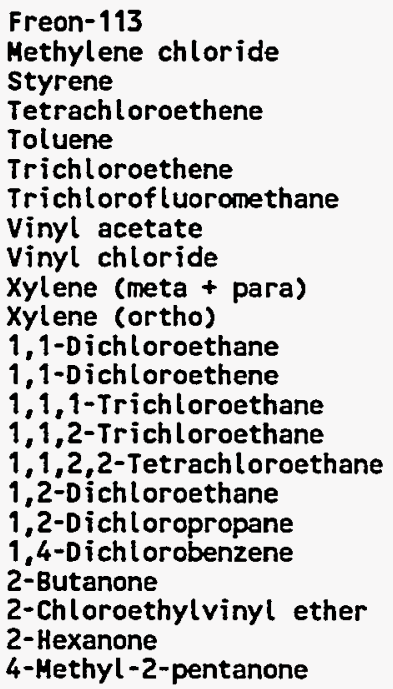 & 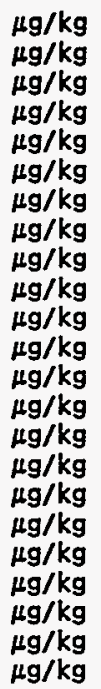 & $\begin{array}{r}5 \\
5 \\
5 \\
5 \\
5 \\
5 \\
5 \\
5 \\
10 \\
5 \\
5 \\
5 \\
5 \\
5 \\
5 \\
5 \\
5 \\
5 \\
5 \\
100 \\
5 \\
50 \\
50\end{array}$ & $\begin{array}{l}U \\
U \\
U \\
U \\
U \\
U \\
U \\
U \\
U \\
U \\
U \\
U \\
U \\
U \\
\mathbf{U} \\
\mathbf{U} \\
\mathbf{U} \\
\mathbf{U} \\
\mathbf{U} \\
\mathbf{U} \\
\mathbf{U} \\
\mathbf{U} \\
\mathbf{U}\end{array}$ & & \\
\hline
\end{tabular}

Location=ORR; Formation=DISMAL GAP; Site=27; Samp id=1263; Dup id=' '; Phase=1

\begin{tabular}{|c|c|c|}
\hline 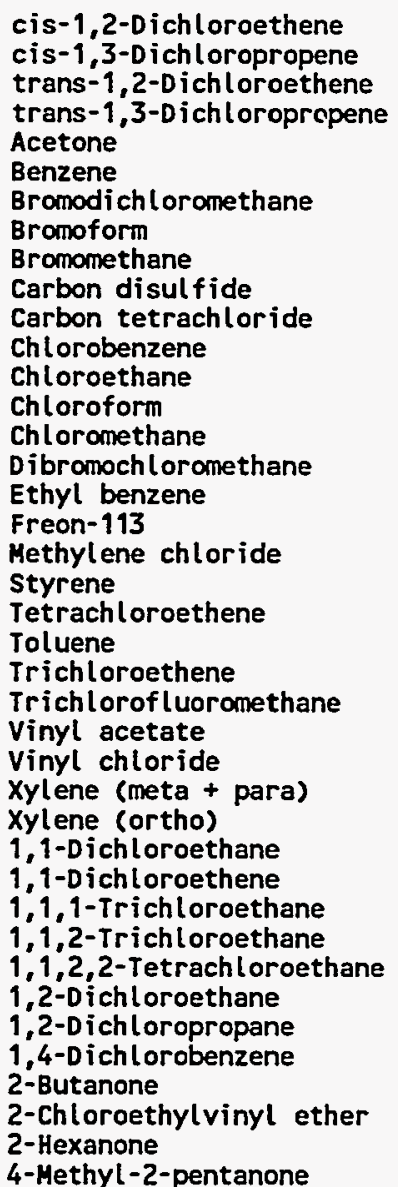 & 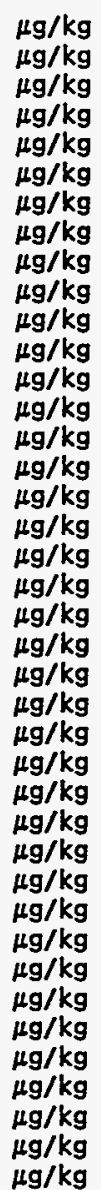 & $\begin{array}{r}5 \\
5 \\
5 \\
5 \\
53 \\
5 \\
5 \\
5 \\
10 \\
5 \\
5 \\
5 \\
10 \\
5 \\
10 \\
5 \\
5 \\
5 \\
5 \\
5 \\
5 \\
5 \\
5 \\
5 \\
5 \\
10 \\
5 \\
5 \\
5 \\
5 \\
5 \\
5 \\
5 \\
5 \\
5 \\
5 \\
8\end{array}$ \\
\hline
\end{tabular}


Table B.1 (continued)

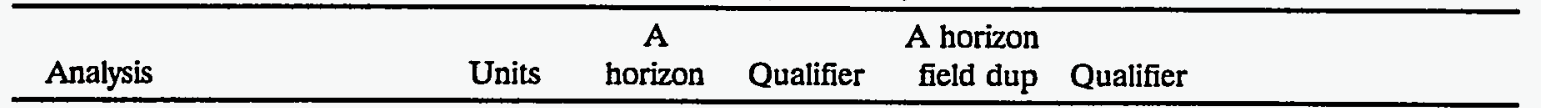

Location=ORR; Formation=DISMAL GAP; Site=32; Samp id=1268; Dup id=' '; Phase=1 ' t $x^{\prime}$

\begin{tabular}{|c|c|}
\hline $\begin{array}{l}\text { chloromethane } \\
\text { orm } \\
\text { thane } \\
\text { disulfide } \\
\text { tetrachloride } \\
\text { senzene } \\
\text { thane } \\
\text { orm } \\
\text { ethane } \\
\text { chloromethane } \\
\text { renzene } \\
13 \\
\text { ne chloride } \\
\text { loroethene } \\
\\
\text { roethene } \\
\text { rofluoromethane } \\
\text { ictate } \\
\text { hloride } \\
\text { (meta + para) } \\
\text { cortho) } \\
\text { hloroethane } \\
\text { hloroethene } \\
\text { richloroethane } \\
\text { richloroethane } \\
\text { - Tetrachloroethane } \\
\text { hloroethane } \\
\text { hloropropane } \\
\text { hlorobenzene } \\
\text { one } \\
\text { oethylvinyl ether } \\
\text { one } \\
\text { l-2-pentanone }\end{array}$ & \\
\hline
\end{tabular}

$\begin{array}{rr}5 & U \\ 5 & U \\ 5 & U \\ 5 & U \\ 12 & J \\ 5 & U \\ 5 & U \\ 5 & U \\ 10 & U \\ 5 & U \\ 5 & U \\ 5 & U \\ 10 & U \\ 5 & U \\ 10 & U \\ 5 & U \\ 5 & U \\ 5 & U \\ 5 & U \\ 5 & U \\ 5 & U \\ 5 & U \\ 5 & U \\ 5 & U \\ 5 & U \\ 10 & U \\ 5 & U \\ 5 & U \\ 5 & U \\ 5 & U \\ 5 & U \\ 5 & U \\ 5 & U \\ 5 & U \\ 5 & U \\ 5 & U \\ 98 & U \\ 5 & U \\ 49 & U \\ 49 & U\end{array}$

Location=ORR; Formation=DISMAL GAP; Site=33; Samp id=1264; Dup id=' '; Phase=1

\begin{tabular}{|c|c|c|}
\hline $\begin{array}{l}\text { cis-1,2-Dichloroethene } \\
\text { cis-1,3-Dichloropropene } \\
\text { trans-1,2-D ichloroethene } \\
\text { trans-1,3-D ichloropropene } \\
\text { Acetone } \\
\text { Benzene } \\
\text { Bromodichloromethane } \\
\text { Bromoform } \\
\text { Bromomethane } \\
\text { Carbon disulfide } \\
\text { Carbon tetrachloride } \\
\text { Chlorobenzene } \\
\text { Chloroethane } \\
\text { Chloroform } \\
\text { Chloromethane } \\
\text { Dibromochloromethane } \\
\text { Ethyl benzene } \\
\text { Freon-113 } \\
\text { Methylene chloride } \\
\text { Styrene } \\
\text { Tetrachloroethene } \\
\text { Toluene } \\
\text { Trichloroethene } \\
\text { Trichlorofluoromethane } \\
\text { Vinyl acetate } \\
\text { Vinyl chloride }\end{array}$ & $\begin{array}{l}\mu g / k g \\
\mu g / k g \\
\mu g / k g \\
\mu g / k g \\
\mu g / k g \\
\mu g / k g \\
\mu g / k g \\
\mu g / k g \\
\mu g / k g \\
\mu g / / k g \\
\mu g / k g \\
\mu g / k g \\
\mu g / k g \\
\mu g / k g \\
\mu g / k g \\
\mu g / k g \\
\mu g / k g \\
\mu g / k g \\
\mu g / k g \\
\mu g / k g \\
\mu g / k g \\
\mu g / k g \\
\mu g / k g \\
\mu g / k g \\
\mu g / k g \\
\mu g / k g\end{array}$ & $\begin{array}{r}5 \\
5 \\
5 \\
5 \\
55 \\
5 \\
5 \\
5 \\
10 \\
5 \\
5 \\
5 \\
10 \\
5 \\
10 \\
5 \\
5 \\
5 \\
5 \\
5 \\
5 \\
5 \\
5 \\
5\end{array}$ \\
\hline
\end{tabular}


Table B.1 (continued)

\begin{tabular}{|c|c|c|c|c|c|}
\hline Analysis & Units & $\begin{array}{c}A \\
\text { horizon }\end{array}$ & Qualifier & $\begin{array}{l}\text { A horizon } \\
\text { field dup }\end{array}$ & Qualifier \\
\hline $\begin{array}{l}\text { Xylene (meta + para) } \\
\text { Xylene (ortho) } \\
\text { 1,1-Dichloroethane } \\
1,1-D \text { ichloroethene } \\
1,1,1-\text { Trichloroethane } \\
1,1,2-\text { Trichloroethane } \\
1,1,2,2-\text { Tetrachloroethane } \\
\text { 1,2-Dichloroethane } \\
\text { 1,2-Dichloropropane } \\
\text { 1,4-Dichlorobenzene } \\
\text { 2-Butanone } \\
\text { 2-Chloroethylvinyl ether } \\
\text { 2-Hexanone } \\
\text { 4-Hethyl-2-pentanone }\end{array}$ & $\begin{array}{l}\mu \mathrm{g} / \mathrm{kg} \\
\mu \mathrm{g} / \mathrm{kg} \\
\mu \mathrm{g} / \mathrm{kg} \\
\mu \mathrm{g} / \mathrm{kg} \\
\mu \mathrm{g} / \mathrm{kg} \\
\mu \mathrm{g} / \mathrm{kg} \\
\mu \mathrm{g} / \mathrm{kg} \\
\mu \mathrm{g} / \mathrm{kg} \\
\mu \mathrm{g} / \mathrm{kg} \\
\mu \mathrm{g} / \mathrm{kg} \\
\mu \mathrm{g} / \mathrm{kg} \\
\mu \mathrm{g} / \mathrm{kg} \\
\mu \mathrm{g} / \mathrm{kg} \\
\mu \mathrm{g} / \mathrm{kg}\end{array}$ & $\begin{array}{r}5 \\
5 \\
5 \\
5 \\
5 \\
5 \\
5 \\
5 \\
5 \\
5 \\
100 \\
5 \\
50 \\
50\end{array}$ & $\begin{array}{l}\mathbf{U} \\
\mathbf{U} \\
\mathbf{U} \\
\mathbf{U} \\
\mathbf{U} \\
\mathbf{U} \\
\mathbf{U} \\
\mathbf{U} \\
\mathbf{U} \\
\mathbf{U} \\
\mathbf{U} \\
\mathbf{U} \\
\mathbf{U} \\
\mathbf{U}\end{array}$ & & \\
\hline
\end{tabular}

Location=ORR; Formation=DISMAL GAP; Site=35; Samp id=1265; Dup id=' '; Phase=1

\begin{tabular}{|c|c|c|}
\hline 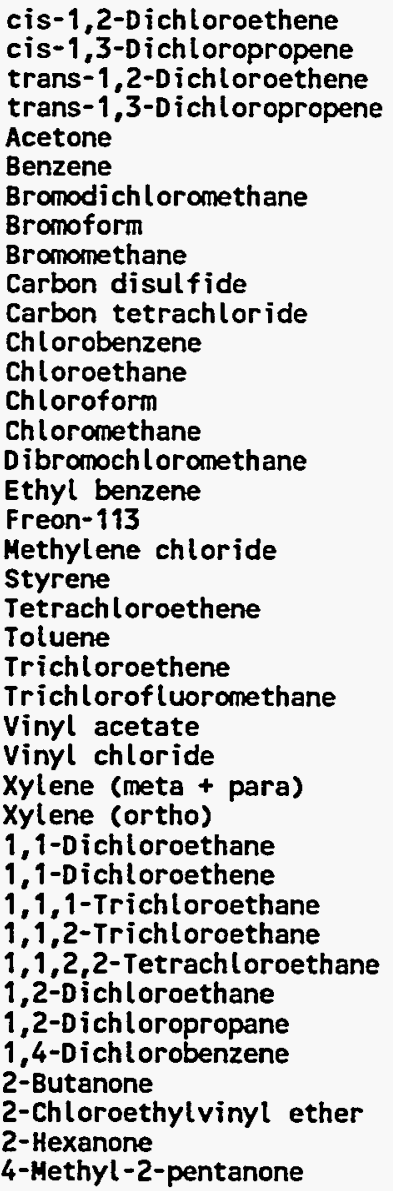 & 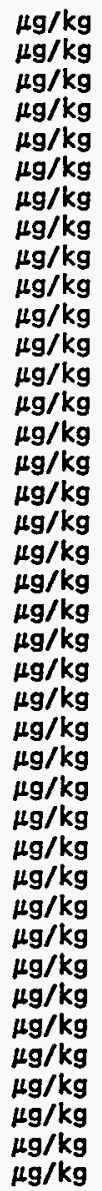 & $\begin{array}{r}5 \\
5 \\
5 \\
5 \\
7 \\
5 \\
5 \\
5 \\
10 \\
5 \\
5 \\
5 \\
10 \\
5 \\
10 \\
5 \\
5 \\
5 \\
5 \\
5 \\
5 \\
5 \\
5 \\
5 \\
5 \\
10 \\
5 \\
5 \\
5 \\
5 \\
5 \\
5 \\
5 \\
5 \\
5 \\
5 \\
6 \\
5 \\
50 \\
50\end{array}$ \\
\hline
\end{tabular}

Location=ORR; Formation=DISMAL GAP; Site=41; Samp id=1266; Dup id=' '; Phase=1

cis-1,2-Dichloroethene

cis-1,3-D ichloropropene

trans-1,2-D ichloroethene

trans-1,3-D ichloropropene

Acetone

Benzene

Bromodichloromethane

Bromoform

Bromomethane

Carbon disulfide $\mu \mathrm{g} / \mathrm{kg}$

$\mu \mathrm{g} / \mathrm{kg}$

$\mu \mathrm{g} / \mathrm{kg}$

$\mu \mathrm{g} / \mathrm{kg}$

$\mu \mathrm{g} / \mathrm{kg}$

$\mu \mathrm{g} / \mathrm{kg}$

$\mu \mathrm{g} / \mathrm{kg}$

$\mu \mathrm{g} / \mathrm{kg}$

$\mu \mathrm{g} / \mathrm{kg}$

$\mu \mathrm{g} / \mathrm{kg}$

$\begin{array}{rl}5 & U \\ 5 & U \\ 5 & U \\ 5 & U \\ 16 & J \\ 5 & U \\ 5 & U \\ 5 & U \\ 10 & U \\ 5 & U\end{array}$


Table B.1 (continued)

\begin{tabular}{|c|c|c|c|c|c|c|}
\hline Analysis & Units & $\begin{array}{c}A \\
\text { horizon }\end{array}$ & Qualifier & $\begin{array}{l}\text { A horizon } \\
\text { field dup }\end{array}$ & Qualifier & \\
\hline 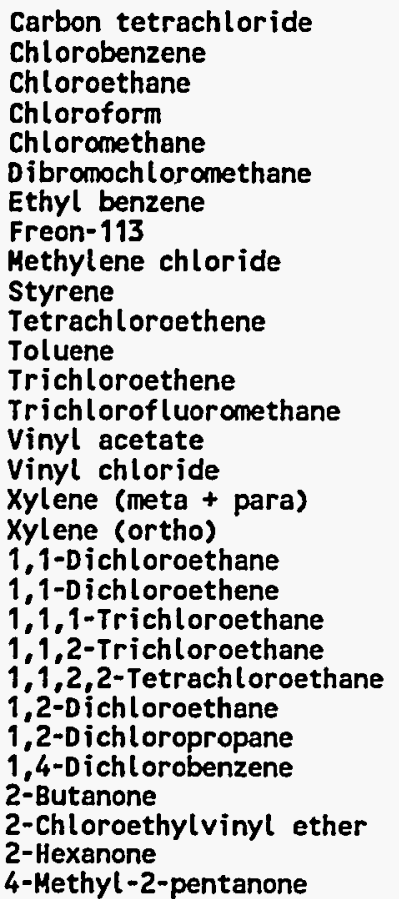 & $\begin{array}{l}\mu g / k g \\
\mu g / k g \\
\mu g / k g \\
\mu g / k g \\
\mu g / k g \\
\mu g / k g \\
\mu g / k g \\
\mu g / k g \\
\mu g / k g \\
\mu g / k g \\
\mu g / k g \\
\mu g / k g \\
\mu g / k g \\
\mu g / k g \\
\mu g / k g \\
\mu g / k g \\
\mu g / k g \\
\mu g / k g \\
\mu g / k g \\
\mu g / k g \\
\mu g / k g \\
\mu g / k g \\
\mu g / k g \\
\mu g / k g \\
\mu g / k g \\
\mu g / k g \\
\mu g / k g \\
\mu g / k g \\
\mu g / k g \\
\mu g / k g\end{array}$ & $\begin{array}{r}5 \\
5 \\
10 \\
5 \\
10 \\
5 \\
5 \\
5 \\
5 \\
5 \\
5 \\
5 \\
5 \\
5 \\
5 \\
10 \\
5 \\
5 \\
5 \\
5 \\
5 \\
5 \\
5 \\
5 \\
5 \\
5 \\
99 \\
5 \\
49 \\
49\end{array}$ & $\begin{array}{l}U \\
U \\
U \\
U \\
U \\
U \\
U \\
U \\
U \\
U \\
U \\
U \\
U \\
U \\
U \\
U \\
U \\
U \\
U \\
U \\
U \\
U \\
U \\
U \\
U \\
U \\
U \\
U \\
U \\
U \\
U \\
U \\
U\end{array}$ & & & $\because \therefore:-x^{2}$ \\
\hline
\end{tabular}

Location=ORR; Formation=DISMAL GAP; Site=43; Samp id=1267; Dup id=' '; Phase=1

cis-1,2-Dichloroethene

cis-1,3-D ich loropropene

trans-1,2-D ichloroethene

trans-1,3-Dichloropropene

Acetone

Benzene

Bromodich loromethane

Bromoform

Bromomethane

Carbon disulfide

Carbon tetrachloride

Chlorobenzene

Chloroethane

Chloroform

Chloromethane

Dibromochloromethane

Ethyl benzene

Freon-113

Methylene chloride

Styrene

Tetrachloroethene

Toluene

Trichloroethene

Trichlorof luoromethane

Vinyl acetate

Vinyl chloride

Xylene (meta + para)

Xylene (ortho)

1,1-Dichloroethane

1,1-Dichloroethene

1,1,1-Trichloroethane

$1,1,2$-Trichloroethane

$1,1,2,2-$ Tetrachloroethane

1,2-D ichloroethane

1,2-Dichloropropane

1,4-D ichlorobenzene

2-Butanone

2-Chloroethylvinyl ether

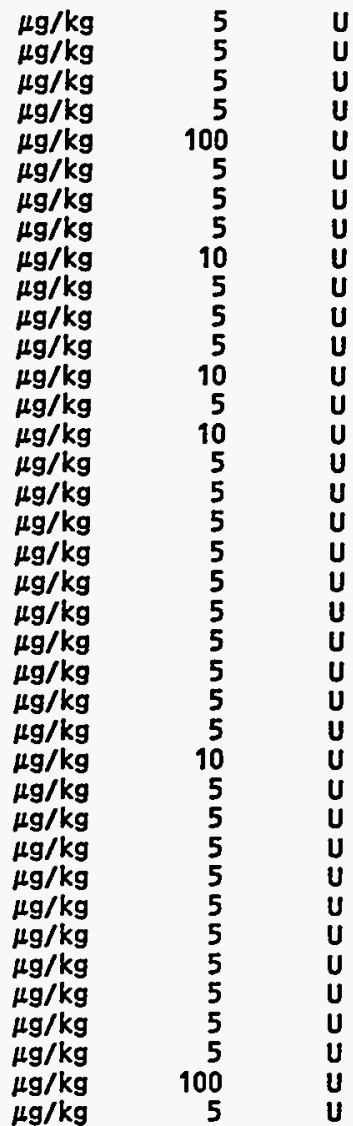




\section{B-50}

Table B.1 (continued)

\begin{tabular}{|c|c|c|c|c|c|}
\hline Analysis & Units & $\begin{array}{c}\text { A } \\
\text { horizon }\end{array}$ & Qualifier & $\begin{array}{l}\text { A horizon } \\
\text { field dup }\end{array}$ & Qualifier \\
\hline $\begin{array}{l}\text { 2-Hexanone } \\
\text { 4-Hethyl-2-pentanone }\end{array}$ & $\begin{array}{l}\mu \mathrm{g} / \mathrm{kg} \\
\mu \mathrm{g} / \mathrm{kg}\end{array}$ & $\begin{array}{l}50 \\
50\end{array}$ & $\begin{array}{l}\mathbf{U} \\
\mathbf{U}\end{array}$ & & \\
\hline \multicolumn{6}{|c|}{ Location=ORR; Formation=NOLICHUCKY; Site=13; Samp id=1273; Dup id=' '; Phase=1 } \\
\hline 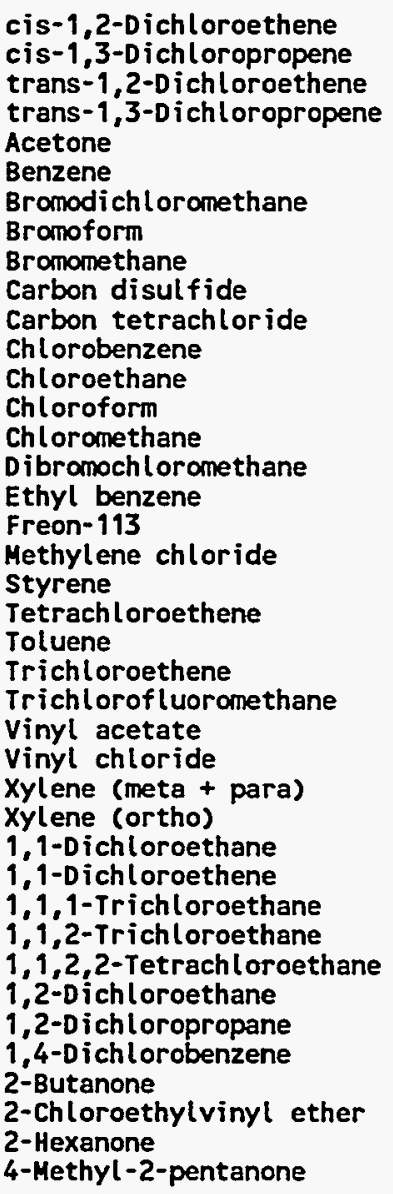 & 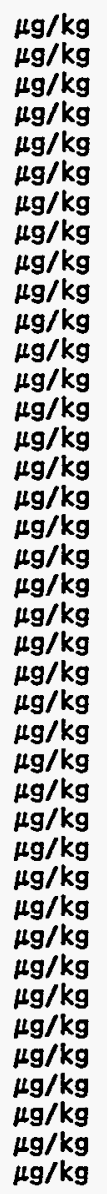 & $\begin{array}{r}5 \\
5 \\
5 \\
5 \\
10 \\
5 \\
5 \\
5 \\
10 \\
5 \\
5 \\
5 \\
10 \\
5 \\
10 \\
5 \\
5 \\
5 \\
5 \\
5 \\
5 \\
5 \\
5 \\
5 \\
5 \\
10 \\
5 \\
5 \\
5 \\
5 \\
5 \\
5 \\
5 \\
5 \\
5 \\
5 \\
99 \\
5 \\
50 \\
50\end{array}$ & 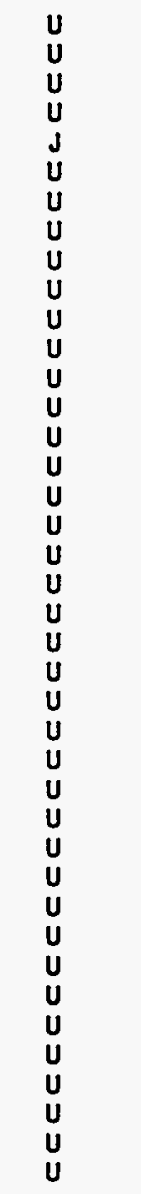 & & \\
\hline
\end{tabular}

Location=ORR; Formation=NOLICHUCKY; Site=15; Samp id=1275; Dup id=' '; Phase=1

cis-1,2-Dichloroethene

cis-1,3-D ich loropropene

trans-1,2-D ichloroethene

trans-1,3-Dichloropropene

Acetone

Benzene

Bromodich loromethane

Bromoform

Bromomethane

Carbon disulfide

Carbon tetrachloride

Chlorobenzene

Chloroethane

Chloroform

Chloromethane

Dibromochloromethane

Ethyl benzene

Freon-113

Methylene chloride

Styrene

Tetrachloroethene

Toluene

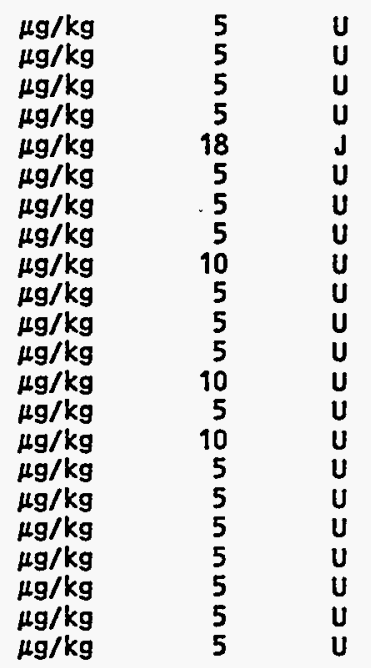




\section{B-51}

Table B.1 (continued)

\begin{tabular}{|c|c|c|c|c|c|c|}
\hline Analysis & Units & $\begin{array}{c}A \\
\text { horizon }\end{array}$ & Qualifier & $\begin{array}{l}\text { A horizon } \\
\text { field dup }\end{array}$ & Qualifier & \\
\hline 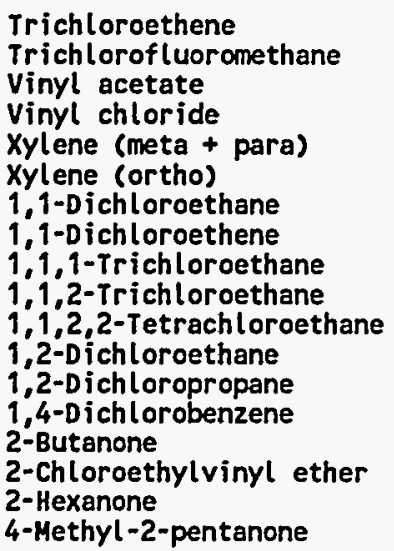 & $\begin{array}{l}\mu g / \mathrm{kg} \\
\mu g / \mathrm{kg} \\
\mu \mathrm{g} / \mathrm{kg} \\
\mu \mathrm{g} / \mathrm{kg} \\
\mu \mathrm{g} / \mathrm{kg} \\
\mu \mathrm{g} / \mathrm{kg} \\
\mu \mathrm{g} / \mathrm{kg} \\
\mu \mathrm{g} / \mathrm{kg} \\
\mu g / \mathrm{kg} \\
\mu \mathrm{g} / \mathrm{kg} \\
\mu \mathrm{g} / \mathrm{kg} \\
\mu \mathrm{g} / \mathrm{kg} \\
\mu \mathrm{g} / \mathrm{kg} \\
\mu \mathrm{g} / \mathrm{kg} \\
\mu \mathrm{g} / \mathrm{kg} \\
\mu g / \mathrm{kg} \\
\mu \mathrm{g} / \mathrm{kg} \\
\mu \mathrm{g} / \mathrm{kg}\end{array}$ & $\begin{array}{r}5 \\
5 \\
5 \\
10 \\
5 \\
5 \\
5 \\
5 \\
5 \\
5 \\
5 \\
5 \\
5 \\
5 \\
98 \\
5 \\
49 \\
49\end{array}$ & $\begin{array}{l}U \\
U \\
U \\
U \\
U \\
U \\
U \\
U \\
U \\
U \\
U \\
U \\
U \\
U \\
U \\
U \\
U \\
U\end{array}$ & $\therefore$ & $=$ & $x:-1$ \\
\hline
\end{tabular}

Location=ORR; Formation=NOLICHUCKY; Site=16; Samp id=1276; Dup id=' '; Phase=1

\begin{tabular}{|c|c|c|}
\hline 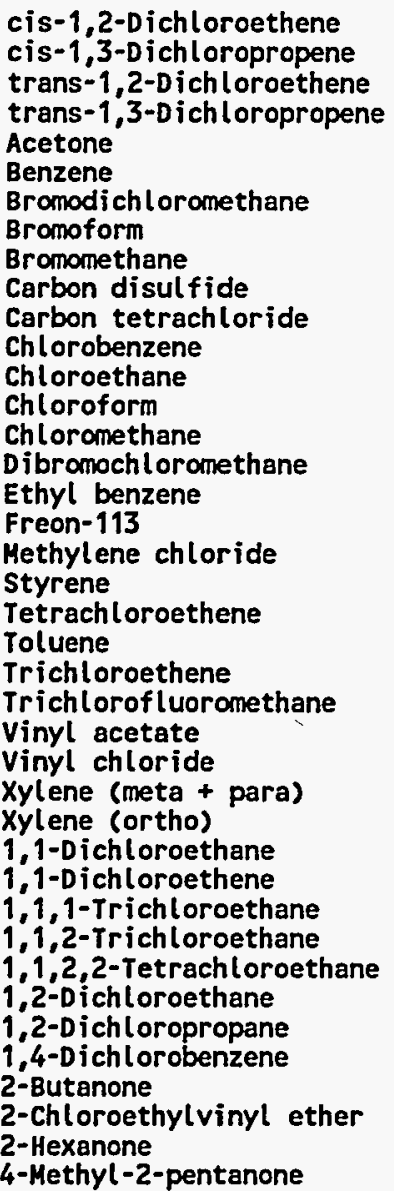 & 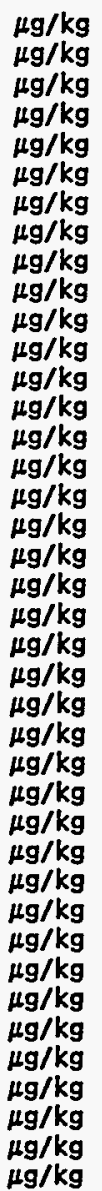 & $\begin{array}{r}5 \\
5 \\
5 \\
5 \\
13 \\
5 \\
5 \\
5 \\
10 \\
5 \\
5 \\
5 \\
10 \\
5 \\
10 \\
5 \\
5 \\
5 \\
5 \\
5 \\
5 \\
5 \\
5 \\
5 \\
5 \\
10 \\
5 \\
5\end{array}$ \\
\hline
\end{tabular}

Location=ORR; Formation=NOLICHUCKY; Site=21; Samp id =1279; Dup id =1280; Phase=1

cis-1,2-Dichloroethene $\quad \mu \mathrm{g} / \mathrm{kg}$

cis-1,3-Dichloropropene $\quad \mu \mathrm{g} / \mathrm{kg}$

trans $-1,2-D i c h l o r o e t h e n e \quad \mu g / k g$

trans-1,3-Dichloropropene $\quad \mu \mathrm{g} / \mathrm{kg}$

Acetone $\mu \mathrm{g} / \mathrm{kg}$

$\begin{array}{rr}5 & U \\ 5 & U \\ 5 & U \\ 5 & U \\ 11 & J\end{array}$


Table B.1 (continued)

\begin{tabular}{|c|c|c|c|c|c|}
\hline Analysis & Units & $\begin{array}{c}\text { A } \\
\text { horizon }\end{array}$ & Qualifier & $\begin{array}{l}\text { A horizon } \\
\text { field dup }\end{array}$ & Qualifier \\
\hline 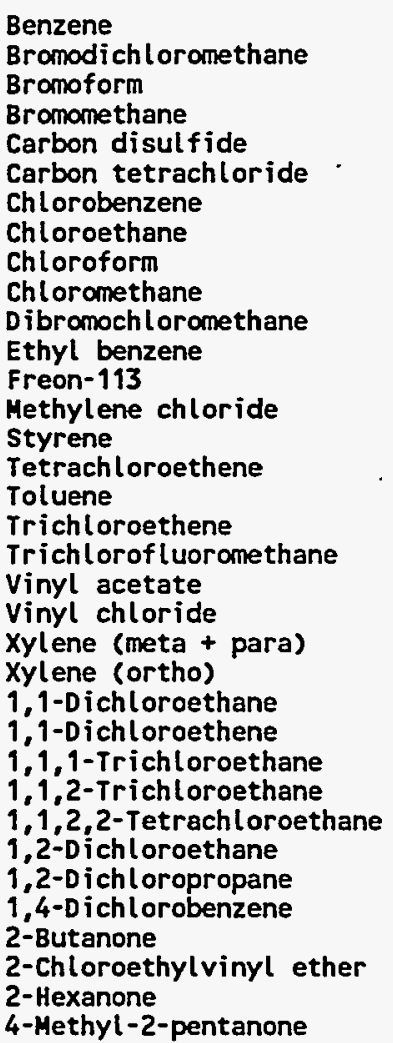 & 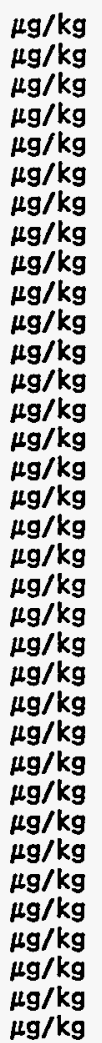 & $\begin{array}{r}5 \\
5 \\
5 \\
10 \\
5 \\
5 \\
5 \\
10 \\
5 \\
10 \\
5 \\
5 \\
5 \\
5 \\
5 \\
5 \\
5 \\
5 \\
5 \\
5 \\
10 \\
5 \\
5 \\
5 \\
5 \\
5 \\
5 \\
5 \\
5 \\
5 \\
5 \\
98 \\
5 \\
49 \\
49\end{array}$ & 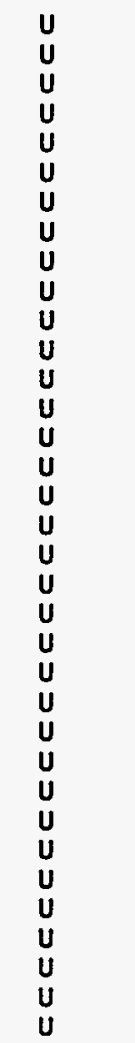 & $\begin{array}{r}5 \\
5 \\
5 \\
10 \\
5 \\
5 \\
5 \\
10 \\
5 \\
10 \\
5 \\
5 \\
5 \\
5 \\
5 \\
5 \\
5 \\
5 \\
5 \\
5 \\
10 \\
5 \\
5 \\
5 \\
5 \\
5 \\
5 \\
5 \\
5 \\
5 \\
5 \\
100 \\
5 \\
50 \\
50\end{array}$ & 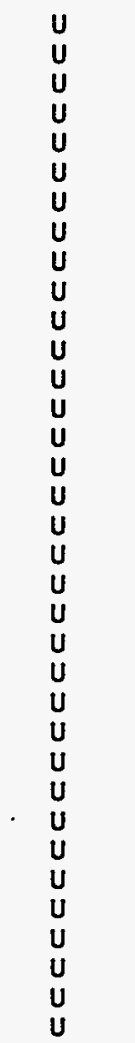 \\
\hline
\end{tabular}

Location=ORR; Formation=NOLICHUCKY; Site=23; Samp id =1281; Dup id =1282; Phase $=1$

$\begin{array}{ll}\text { cis-1,2-Dichloroethene } & \mu \mathrm{g} / \mathrm{kg} \\ \text { cis-1,3-Dichloropropene } & \mu \mathrm{g} / \mathrm{kg} \\ \text { trans-1,2-Dichloroethene } & \mu \mathrm{g} / \mathrm{kg} \\ \text { trans-1,3-Dichloropropene } & \mu \mathrm{g} / \mathrm{kg} \\ \text { Acetone } & \mu \mathrm{g} / \mathrm{kg} \\ \text { Benzene } & \mu \mathrm{g} / \mathrm{kg} \\ \text { Bromodichloromethane } & \mu \mathrm{g} / \mathrm{kg} \\ \text { Bromoform } & \mu \mathrm{g} / \mathrm{kg} \\ \text { Bromomethane } & \mu \mathrm{g} / \mathrm{kg} \\ \text { Carbon disulfide } & \mu \mathrm{g} / \mathrm{kg} \\ \text { Carbon tetrachloride } & \mu \mathrm{g} / \mathrm{kg} \\ \text { Chlorobenzene } & \mu \mathrm{g} / \mathrm{kg} \\ \text { Chloroethane } & \mu \mathrm{g} / \mathrm{kg} \\ \text { Chloroform } & \mu \mathrm{g} / \mathrm{kg} \\ \text { Chloromethane } & \mu \mathrm{g} / \mathrm{kg} \\ \text { Dibromochloromethane } & \mu \mathrm{g} / \mathrm{kg} \\ \text { Ethyl benzene } & \mu \mathrm{g} / \mathrm{kg} \\ \text { Freon-113 } & \mu \mathrm{g} / \mathrm{kg} \\ \text { Methylene chloride } & \mu \mathrm{g} / \mathrm{kg} \\ \text { Styrene } & \mu \mathrm{g} / \mathrm{kg} \\ \text { Tetrachloroethene } & \mu \mathrm{g} / \mathrm{kg} \\ \text { Toluene } & \mu \mathrm{g} / \mathrm{kg} \\ \text { Trichloroethene } & \mu \mathrm{g} / \mathrm{kg} \\ \text { Trichlorofluoromethane } & \mu \mathrm{g} / \mathrm{kg} \\ \text { Vinyl acetate } & \mu \mathrm{g} / \mathrm{kg} \\ \text { Vinyl chloride } & \mu \mathrm{g} / \mathrm{kg} \\ \text { Xylene (meta + para) } & \mu \mathrm{g} / \mathrm{kg} \\ \text { Xylene (ortho) } & \mu \mathrm{g} / \mathrm{kg} \\ \text { 1, 1-Dichloroethane } & \mu \mathrm{g} / \mathrm{kg} \\ \text { 1, 1-Dichloroethene } & \mu \mathrm{g} / \mathrm{kg} \\ \text { 1, 1, 1-Trichloroethane } & \mu \mathrm{g} / \mathrm{kg} \\ \text { 1, 1,2-Trichloroethane } & \mu \mathrm{g} / \mathrm{kg} \\ \text { 1, 1,2,2-Tetrachloroethane } & \mu \mathrm{g} / \mathrm{kg} \\ & \end{array}$

$\begin{array}{rrrr}5 & U & 5 & U \\ 5 & U & 5 & U \\ 5 & U & 5 & U \\ 5 & U & 5 & U \\ 14 & J & 18 & J \\ 5 & U & 5 & U \\ 5 & U & 5 & U \\ 5 & U & 5 & U \\ 10 & U & 10 & U \\ 5 & U & 5 & U \\ 5 & U & 5 & U \\ 5 & U & 5 & U \\ 10 & U & 10 & U \\ 5 & U & 5 & U \\ 10 & U & 10 & U \\ 5 & U & 5 & U \\ 5 & U & 5 & U \\ 5 & U & 5 & U \\ 5 & U & 5 & U \\ 5 & U & 5 & U \\ 5 & U & 5 & U \\ 5 & U & 5 & U \\ 5 & U & 5 & U \\ 5 & U & 5 & U \\ 5 & U & 5 & U \\ 10 & U & 10 & U \\ 5 & U & 5 & U \\ 5 & U & 5 & U \\ 5 & U & 5 & U \\ 5 & U & 5 & U \\ 5 & U & 5 & U \\ 5 & U & 5 & U \\ 5 & U & 5 & U\end{array}$


Table B.1 (continued)

\begin{tabular}{|c|c|c|c|c|c|c|}
\hline Analysis & Units & $\begin{array}{c}\text { A } \\
\text { horizon }\end{array}$ & Qualifier & $\begin{array}{l}\text { A horizon } \\
\text { field dup }\end{array}$ & Qualifier & \\
\hline $\begin{array}{l}\text { 1,2-Dichloroethane } \\
\text { 1,2-Dichloropropane } \\
\text { 1,4-Dichlorobenzene } \\
\text { 2-Butanone } \\
\text { 2-Chloroethylvinyl ether } \\
\text { 2-Hexanone } \\
\text { 4-Hethyl-2-pentanone }\end{array}$ & $\begin{array}{l}\mu \mathrm{g} / \mathrm{kg} \\
\mu \mathrm{g} / \mathrm{kg} \\
\mu \mathrm{g} / \mathrm{kg} \\
\mu \mathrm{g} / \mathrm{kg} \\
\mu \mathrm{g} / \mathrm{kg} \\
\mu \mathrm{g} / \mathrm{kg} \\
\mu \mathrm{g} / \mathrm{kg}\end{array}$ & $\begin{array}{r}5 \\
5 \\
5 \\
99 \\
5 \\
50 \\
50\end{array}$ & $\begin{array}{l}\mathbf{U} \\
\mathbf{U} \\
\mathbf{U} \\
\mathbf{U} \\
\mathbf{U} \\
\mathbf{U} \\
\mathbf{U}\end{array}$ & $\begin{array}{r}5 \\
5 \\
5 \\
100 \\
5 \\
50 \\
50\end{array}$ & $\begin{array}{l}\mathbf{U} \\
\mathbf{U} \\
\mathbf{U} \\
\mathbf{U} \\
\mathbf{U} \\
\mathbf{U} \\
\mathbf{U}\end{array}$ & 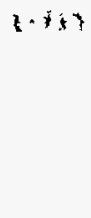 \\
\hline
\end{tabular}

Location=ORR; Formation=NOLICHUCKY; Site=24; Samp id=1284; Dup id=1285; Phase $=1$

\begin{tabular}{|c|c|c|c|c|}
\hline 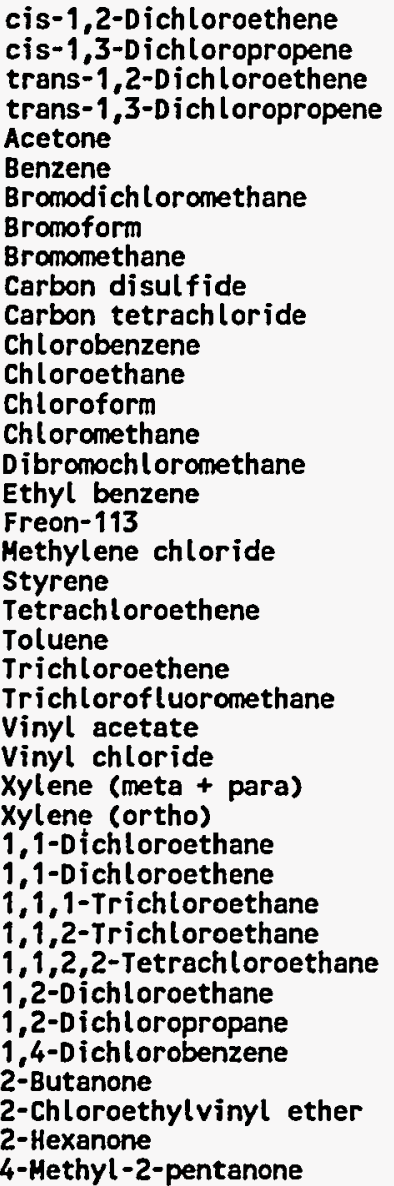 & 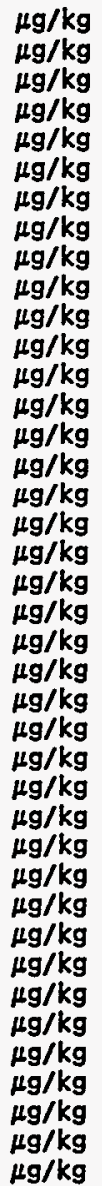 & $\begin{array}{r}5 \\
5 \\
5 \\
5 \\
100 \\
5 \\
5 \\
5 \\
10 \\
5 \\
5 \\
5 \\
10 \\
5 \\
10 \\
5 \\
5 \\
5 \\
5 \\
5 \\
5 \\
5 \\
5 \\
5 \\
5 \\
10 \\
5 \\
5 \\
5 \\
5 \\
5 \\
5 \\
5 \\
5 \\
5 \\
5 \\
100 \\
5 \\
50 \\
50\end{array}$ & 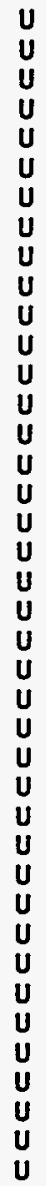 & $\begin{array}{r}5 \\
5 \\
5 \\
5 \\
10 \\
5 \\
5 \\
5 \\
10 \\
5 \\
5 \\
5 \\
10 \\
5 \\
10 \\
5 \\
5 \\
5 \\
5 \\
5 \\
5 \\
5 \\
5 \\
5 \\
5 \\
10 \\
5 \\
5 \\
5 \\
5 \\
5 \\
5 \\
5 \\
5 \\
5 \\
5 \\
100 \\
5 \\
50 \\
50\end{array}$ \\
\hline
\end{tabular}

Location=ORR; Formation=NOLICHUCKY; Site=25; Samp id=1274; Dup id=' '; Phase=1

$\begin{array}{llrl}\text { cis-1,2-Dichloroethene } & \mu \mathrm{g} / \mathrm{kg} & 5 & \mathrm{U} \\ \text { cis-1,3-Dichloropropene } & \mu \mathrm{g} / \mathrm{kg} & 5 & \mathrm{U} \\ \text { trans-1,2-Dichloroethene } & \mu \mathrm{g} / \mathrm{kg} & 5 & \mathrm{U} \\ \text { trans-1,3-Dichloropropene } & \mu \mathrm{g} / \mathrm{kg} & 5 & \mathrm{U} \\ \text { Acetone } & \mu \mathrm{g} / \mathrm{kg} & 24 & \mathrm{~J} \\ \text { Benzene } & \mu \mathrm{g} / \mathrm{kg} & 5 & \mathrm{U} \\ \text { Bromodichloromethane } & \mu \mathrm{g} / \mathrm{kg} & 5 & \mathrm{U} \\ \text { Bromoform } & \mu \mathrm{g} / \mathrm{kg} & 5 & \mathrm{U} \\ \text { Bromomethane } & \mu \mathrm{g} / \mathrm{kg} & 10 & U \\ \text { Carbon disulfide } & \mu \mathrm{gg} & 5 & U \\ \text { Carbon tetrachloride } & \mu \mathrm{g} / \mathrm{kg} & 5 & U \\ \text { Chlorobenzene } & \mu \mathrm{g} / \mathrm{kg} & 5 & U \\ \text { Chloroethane } & \mu \mathrm{g} / \mathrm{kg} & 10 & U \\ \text { Chloroform } & \mu g / \mathrm{kg} & 5 & U \\ \text { Chloromethane } & \mu \mathrm{gg} & 10 & U \\ \text { Dibromochloromethane } & \mu \mathrm{g} / \mathrm{kg} & 5 & U \\ \text { Ethyl benzene } & \mu \mathrm{g} / \mathrm{kg} & 5 & U\end{array}$


Table B.1 (continued)

\begin{tabular}{|c|c|c|c|c|c|}
\hline Analysis & Units & $\begin{array}{c}\text { A } \\
\text { horizon }\end{array}$ & Qualifier & $\begin{array}{l}\text { A horizon } \\
\text { field dup }\end{array}$ & Qualifier \\
\hline 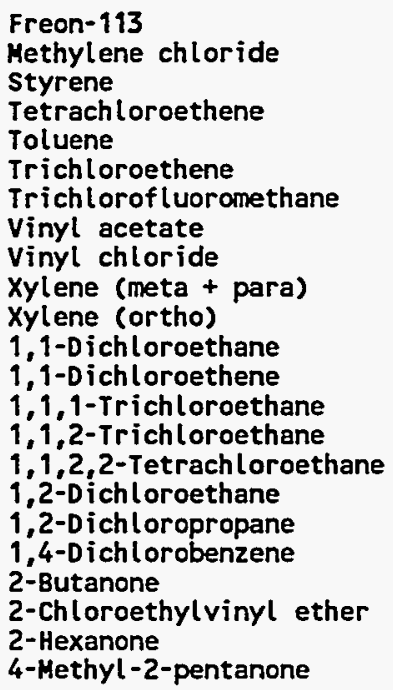 & 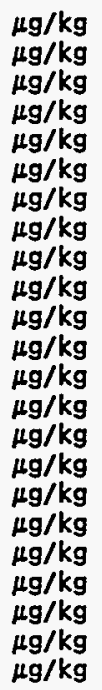 & $\begin{array}{r}5 \\
5 \\
5 \\
5 \\
5 \\
5 \\
5 \\
5 \\
10 \\
5 \\
5 \\
5 \\
5 \\
5 \\
5 \\
5 \\
5 \\
5 \\
5 \\
98 \\
5 \\
49 \\
49\end{array}$ & $\begin{array}{l}U \\
U \\
U \\
U \\
U \\
U \\
U \\
U \\
U \\
U \\
U \\
U \\
U \\
U \\
U \\
U \\
U \\
U \\
U \\
U \\
U \\
U \\
U\end{array}$ & & \\
\hline
\end{tabular}

Location=ORR; Formation=NOLICHUCKY; Site=28; Samp id =1286; Dup id=1287; Phase $=1$

\begin{tabular}{|c|c|c|c|c|}
\hline 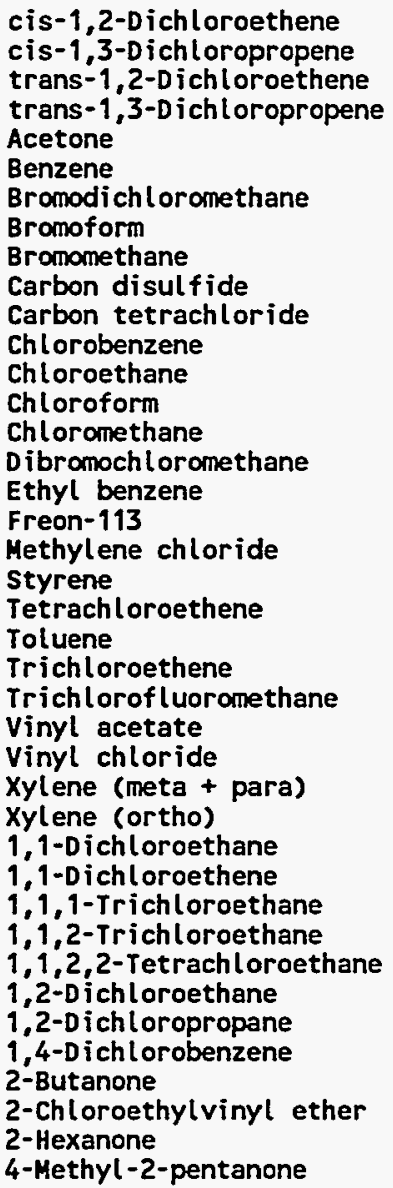 & 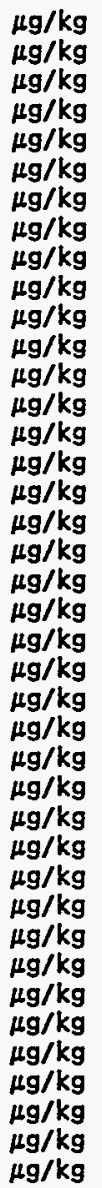 & $\begin{array}{r}5 \\
5 \\
5 \\
5 \\
22 \\
5 \\
5 \\
5 \\
10 \\
5 \\
5 \\
5 \\
10 \\
5 \\
10 \\
5 \\
5 \\
5 \\
5 \\
5 \\
5 \\
5 \\
5 \\
5 \\
5 \\
10 \\
5 \\
5 \\
5 \\
5 \\
5 \\
5 \\
5 \\
5 \\
5 \\
5 \\
98 \\
5 \\
49 \\
49\end{array}$ & 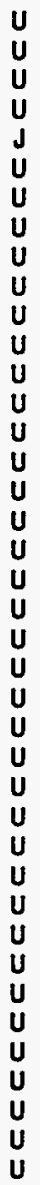 & $\begin{array}{r}5 \\
5 \\
5 \\
5 \\
14 \\
5 \\
5 \\
5 \\
10 \\
5 \\
5 \\
5 \\
10 \\
5 \\
10 \\
5 \\
5 \\
5 \\
5 \\
5 \\
5 \\
5 \\
5 \\
5 \\
5 \\
10 \\
5 \\
5 \\
5 \\
5 \\
5 \\
5 \\
5 \\
5 \\
5 \\
5 \\
100 \\
5 \\
50 \\
50\end{array}$ \\
\hline
\end{tabular}




\section{B-55}

Table B.1 (continued)

\begin{tabular}{|c|c|c|c|c|c|}
\hline Analysis & Units & $\begin{array}{c}A \\
\text { horizon }\end{array}$ & Qualifier & $\begin{array}{l}\text { A horizon } \\
\text { field dup }\end{array}$ & Qualifier \\
\hline \multicolumn{6}{|c|}{ Location=ORR; Formation=NOLICHUCKY; Site=3; Samp id =1271; Dup id=' '; Phase =1 } \\
\hline 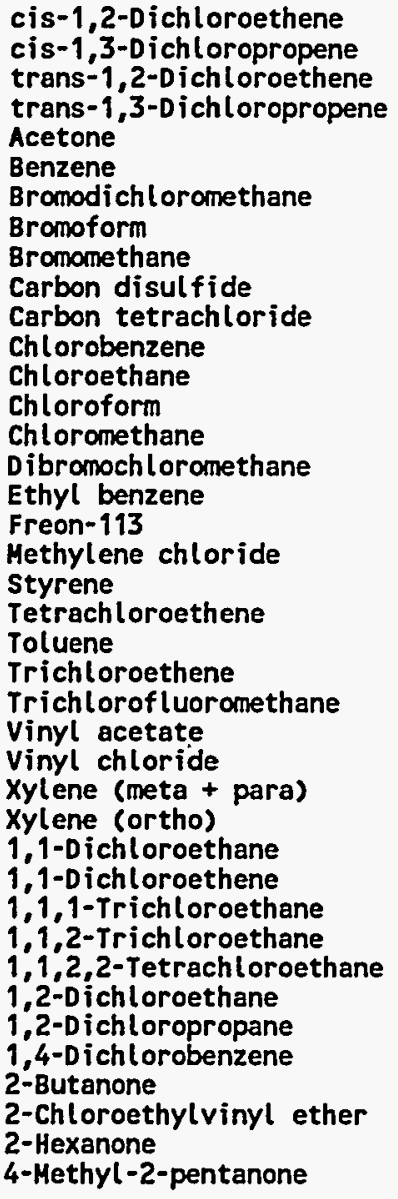 & 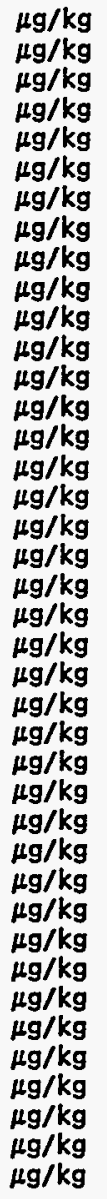 & $\begin{array}{r}5 \\
5 \\
5 \\
5 \\
12 \\
5 \\
5 \\
5 \\
10 \\
5 \\
5 \\
5 \\
10 \\
5 \\
10 \\
5 \\
5 \\
5 \\
5 \\
5 \\
5 \\
5 \\
5 \\
5 \\
5 \\
10 \\
5 \\
5 \\
5 \\
5 \\
5 \\
5 \\
5 \\
5 \\
5 \\
5 \\
98 \\
5 \\
49 \\
49\end{array}$ & 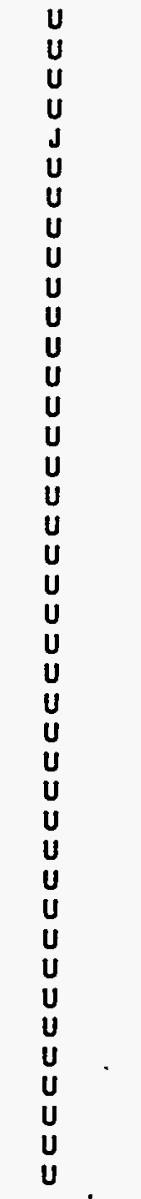 & $\cdot$ & \\
\hline
\end{tabular}

Location=ORR; Formation=NOLICHUCKY; Site=31; Samp id=1288; Dup id=1289; Phase=1

$\begin{array}{ll}\text { cis-1,2-Dichloroethene } & \mu \mathrm{g} / \mathrm{kg} \\ \text { cis-1,3-Dichloropropene } & \mu \mathrm{g} / \mathrm{kg} \\ \text { trans-1,2-Dichloroethene } & \mu \mathrm{g} / \mathrm{kg} \\ \text { trans-1,3-Dichloropropene } & \mu \mathrm{g} / \mathrm{kg} \\ \text { Acetone } & \mu \mathrm{g} / \mathrm{kg} \\ \text { Benzene } & \mu \mathrm{g} / \mathrm{kg} \\ \text { Bromodichloromethane } & \mu \mathrm{g} / \mathrm{kg} \\ \text { Bromoform } & \mu \mathrm{g} / \mathrm{kg} \\ \text { Bromomethane } & \mu \mathrm{g} / \mathrm{kg} \\ \text { Carbon disulfide } & \mu \mathrm{g} / \mathrm{kg} \\ \text { Carbon tetrachloride } & \mu \mathrm{g} / \mathrm{kg} \\ \text { Chlorobenzene } & \mu \mathrm{g} / \mathrm{kg} \\ \text { Chloroethane } & \mu \mathrm{g} / \mathrm{kg} \\ \text { Chloroform } & \mu \mathrm{g} / \mathrm{kg} \\ \text { Chloromethane } & \mu \mathrm{g} / \mathrm{kg} \\ \text { Dibromochloromethane } & \mu \mathrm{g} / \mathrm{kg} \\ \text { Ethyl benzene } & \mu \mathrm{g} / \mathrm{kg} \\ \text { Freon-113 } & \mu \mathrm{g} / \mathrm{kg} \\ \text { Methylene chloride } & \mu \mathrm{g} / \mathrm{kg} \\ \text { Styrene } & \mu \mathrm{g} / \mathrm{kg} \\ \text { Tetrachloroethene } & \mu \mathrm{g} / \mathrm{kg} \\ \text { Toluene } & \mu \mathrm{g} / \mathrm{kg} \\ \text { Trichloroethene } & \mu \mathrm{g} / \mathrm{kg} \\ \text { Trichlorofluoromethane } & \mu \mathrm{g} / \mathrm{kg} \\ \text { Vinyl acetate } & \mu \mathrm{g} / \mathrm{kg}\end{array}$

$\begin{array}{rrrr}5 & U & 5 & U \\ 5 & U & 5 & U \\ 5 & U & 5 & U \\ 5 & U & 5 & U \\ 27 & J & 78 & J \\ 5 & U & 5 & U \\ 5 & U & 5 & U \\ 5 & U & 5 & U \\ 10 & U & 10 & U \\ 5 & U & 5 & U \\ 5 & U & 5 & U \\ 5 & U & 5 & U \\ 10 & U & 10 & U \\ 5 & U & 5 & U \\ 10 & U & 10 & U \\ 5 & U & 5 & U \\ 5 & U & 5 & U \\ 5 & U & 5 & U \\ 5 & U & 5 & U \\ 5 & U & 5 & U \\ 5 & U & 5 & U \\ 5 & U & 5 & U \\ 5 & U & 5 & U \\ 24 & & 5 & U \\ 5 & U & 5 & U\end{array}$




\section{B-56}

Table B.1 (continued)

\begin{tabular}{|c|c|c|c|c|c|}
\hline Analysis & Units & $\begin{array}{c}\text { A } \\
\text { horizon }\end{array}$ & Qualifier & $\begin{array}{l}\text { A horizon } \\
\text { field dup }\end{array}$ & Qualifier \\
\hline 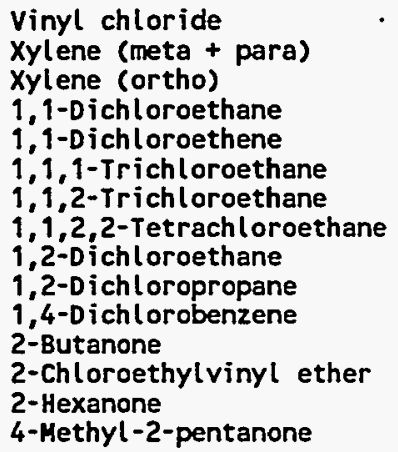 & $\begin{array}{l}\mu g / \mathrm{kg} \\
\mu g / \mathrm{kg} \\
\mu g / \mathrm{kg} \\
\mu g / \mathrm{kg} \\
\mu g / \mathrm{kg} \\
\mu g / \mathrm{kg} \\
\mu g / \mathrm{kg} \\
\mu g / \mathrm{kg} \\
\mu g / \mathrm{kg} \\
\mu g / \mathrm{kg} \\
\mu g / k g \\
\mu g / k g \\
\mu g / k g \\
\mu g / k g \\
\mu g / \mathrm{kg}\end{array}$ & $\begin{array}{r}10 \\
5 \\
5 \\
5 \\
5 \\
5 \\
5 \\
5 \\
5 \\
5 \\
5 \\
100 \\
5 \\
50 \\
50\end{array}$ & $\begin{array}{l}U \\
U \\
U \\
U \\
U \\
U \\
U \\
U \\
U \\
U \\
U \\
U \\
U \\
U \\
\mathbf{U}\end{array}$ & $\begin{array}{r}10 \\
5 \\
5 \\
5 \\
5 \\
5 \\
5 \\
5 \\
5 \\
5 \\
5 \\
7 \\
5 \\
50 \\
50\end{array}$ & $\begin{array}{l}\mathbf{U} \\
\mathbf{U} \\
\mathbf{U} \\
\mathbf{U} \\
\mathbf{U} \\
\mathbf{U} \\
\mathbf{U} \\
\mathbf{U} \\
\mathbf{U} \\
\mathbf{U} \\
\mathbf{U} \\
\mathbf{B J} \\
\mathbf{U} \\
\mathbf{U} \\
\mathbf{U}\end{array}$ \\
\hline
\end{tabular}

Location=ORR; Formation=NOLICHUCKY; Site=42; Samp id=1290; Dup id=1291; Phase=1

\begin{tabular}{|c|c|c|c|c|}
\hline 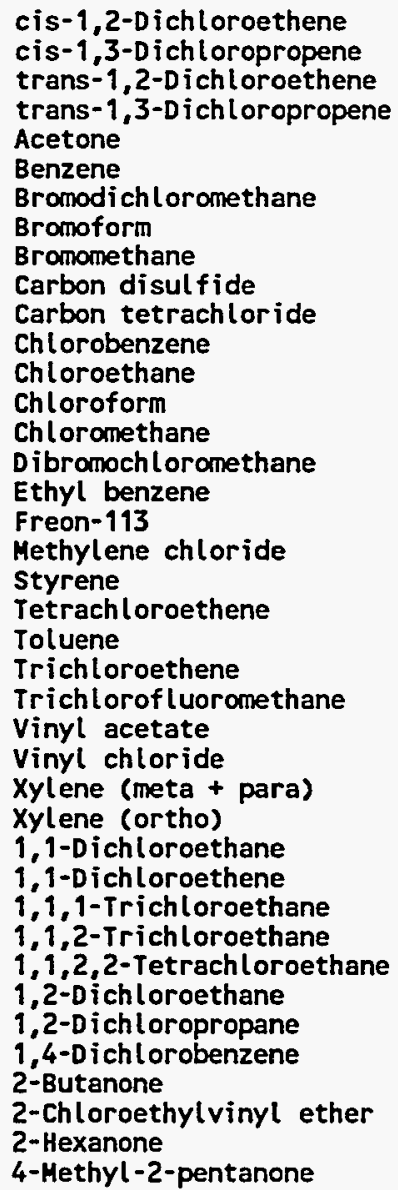 & 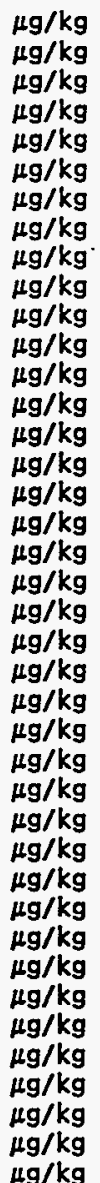 & $\begin{array}{r}5 \\
5 \\
5 \\
5 \\
18 \\
5 \\
5 \\
5 \\
10 \\
5 \\
5 \\
5 \\
10 \\
5 \\
10 \\
5 \\
5 \\
5 \\
5 \\
5 \\
5 \\
5 \\
5 \\
5 \\
5 \\
10 \\
5 \\
5 \\
5 \\
5 \\
5 \\
5 \\
5 \\
5 \\
5 \\
5 \\
5 \\
5 \\
5 \\
5 \\
5\end{array}$ & 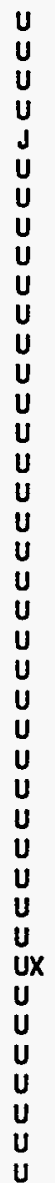 & 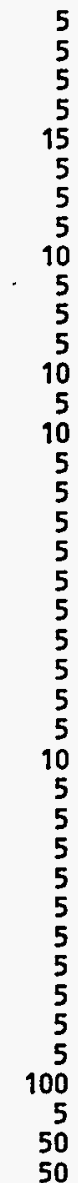 \\
\hline
\end{tabular}

Location=ORR; Formation=NOLICHUCKY; Site=5; Samp id=1272; Dup id=' '; Phase=1

cis-1,2-Dichloroethene
cis-1,3-D ichloropropene
trans-1,2-D ichloroethene
trans-1,3-Dichloropropene
Acetone
Benzene
Bromodichloromethane
8romoform
Bromomethane

$\begin{array}{lrl}\mu g / \mathrm{kg} & 5 & \mathrm{U} \\ \mu \mathrm{g} / \mathrm{kg} & 5 & \mathrm{U} \\ \mu \mathrm{g} / \mathrm{kg} & 5 & \mathrm{U} \\ \mu \mathrm{g} / \mathrm{kg} & 5 & \mathrm{U} \\ \mu \mathrm{g} / \mathrm{kg} & 19 & \mathrm{~J} \\ \mu \mathrm{g} / \mathrm{kg} & 5 & \mathrm{U} \\ \mu \mathrm{g} / \mathrm{kg} & 5 & \mathrm{U} \\ \mu \mathrm{g} / \mathrm{kg} & 5 & \mathrm{U} \\ \mu \mathrm{g} / \mathrm{kg} & 10 & \mathrm{U}\end{array}$




\section{B-57}

Table B.1 (continued)

\begin{tabular}{|c|c|c|c|c|c|c|c|}
\hline Analysis & Units & $\begin{array}{c}\text { A } \\
\text { horizon }\end{array}$ & Qualifier & $\begin{array}{l}\text { A horizon } \\
\text { field dup }\end{array}$ & Qualifier & & \\
\hline 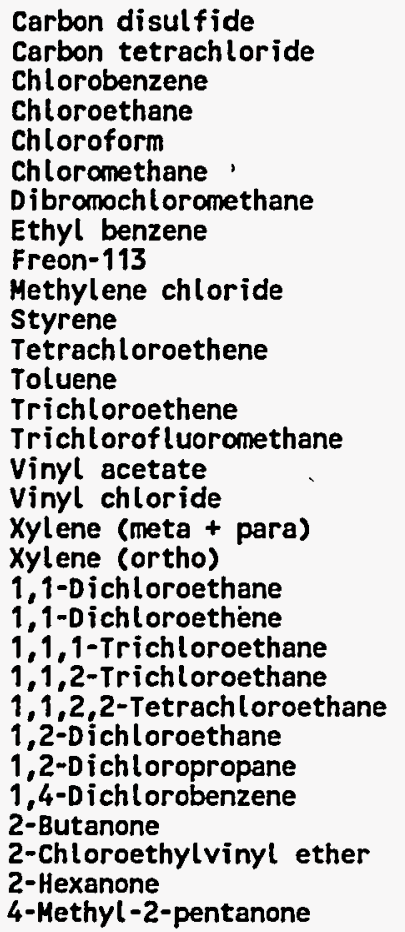 & 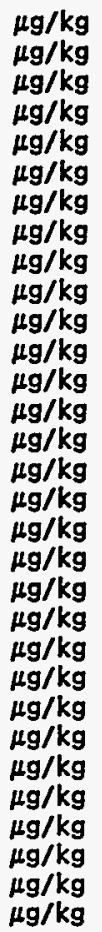 & $\begin{array}{r}5 \\
5 \\
5 \\
10 \\
5 \\
10 \\
5 \\
5 \\
5 \\
5 \\
5 \\
5 \\
5 \\
5 \\
5 \\
5 \\
10 \\
5 \\
5 \\
5 \\
5 \\
5 \\
5 \\
5 \\
5 \\
5 \\
5 \\
99 \\
5 \\
50 \\
50\end{array}$ & 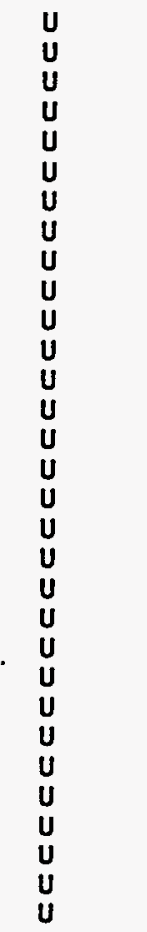 & . & . & $\xi$ & $1 t^{2}, x^{2}$ \\
\hline
\end{tabular}

Location=ROA; Formation=COPPER RIDGE; Site=33; Samp id=3202; Dup id=' '; Phase=2

\begin{tabular}{|c|c|c|}
\hline 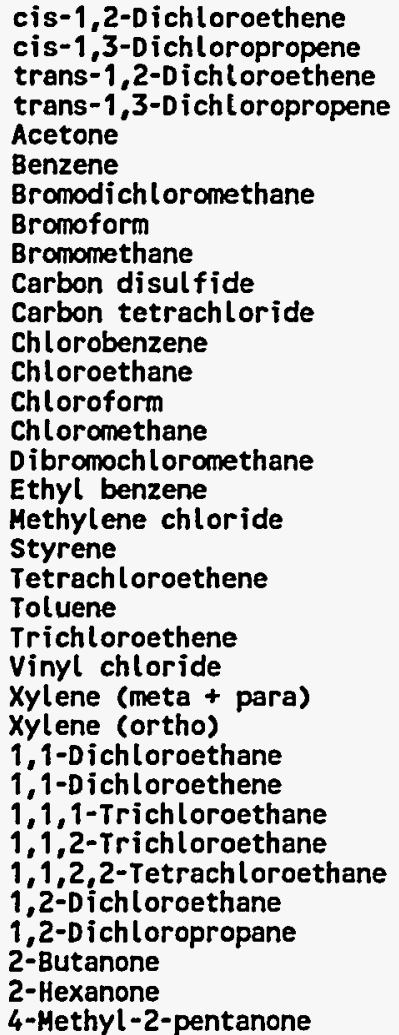 & 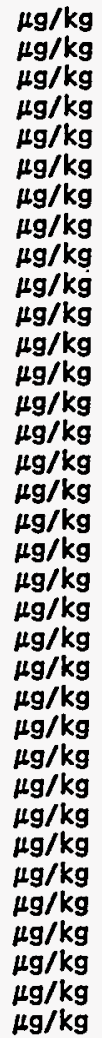 & $\begin{array}{r}5 \\
5 \\
5 \\
5 \\
8 \\
5 \\
5 \\
5 \\
10 \\
5 \\
5 \\
5 \\
10 \\
5 \\
10 \\
5 \\
5 \\
5 \\
5 \\
5 \\
5 \\
5 \\
10 \\
5 \\
5 \\
5 \\
5 \\
5 \\
5 \\
5 \\
5 \\
5 \\
100 \\
50 \\
50\end{array}$ \\
\hline
\end{tabular}




\section{B-58}

Table B.1 (continued)

\begin{tabular}{|c|c|c|c|c|c|}
\hline Analysis & Units & $\underset{\text { horizon }}{\mathrm{A}}$ & Qualifier & $\begin{array}{l}\text { A horizon } \\
\text { field dup }\end{array}$ & Qualifier \\
\hline
\end{tabular}

Location=ROA; Formation=COPPER RIDGE; Site=34; Samp id=3205; Dup id=' '; Phase=2

\begin{tabular}{|c|c|c|}
\hline 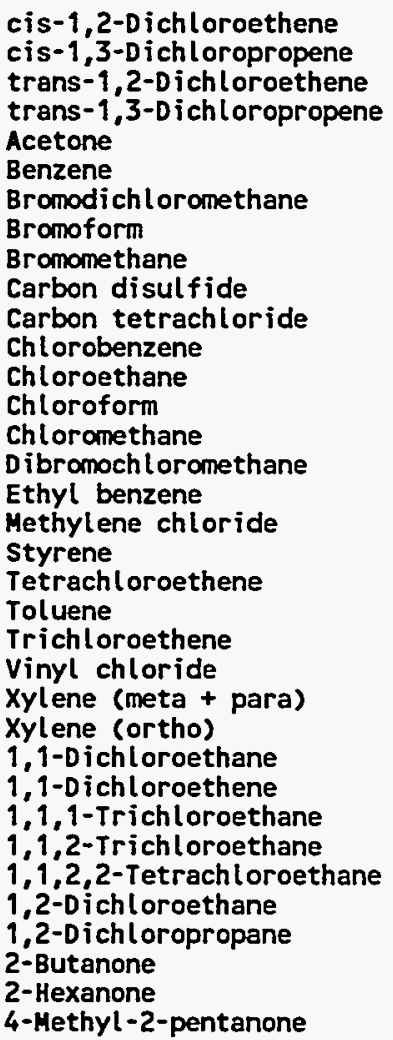 & $\begin{array}{l}\mu g / k g \\
\mu g / k g \\
\mu g / k g \\
\mu g / k g \\
\mu g / k g \\
\mu g / k g \\
\mu g / k g \\
\mu g / k g \\
\mu g / k g \\
\mu g / k g \\
\mu g / k g \\
\mu g / k g \\
\mu g / k g \\
\mu g / k g \\
\mu g / k g \\
\mu g / k g \\
\mu g / k g \\
\mu g / k g \\
\mu g / k g \\
\mu g / k g \\
\mu g / k g \\
\mu g / k g \\
\mu g / k g \\
\mu g / k g \\
\mu g / k g \\
\mu g / k g \\
\mu g / k g \\
\mu g / k g \\
\mu g / k g \\
\mu g / k g \\
\mu g / k g \\
\mu g / k g \\
\mu g / k g \\
\mu g / k g \\
\mu g / k g\end{array}$ & $\begin{array}{r}5 \\
5 \\
5 \\
5 \\
17 \\
5 \\
5 \\
5 \\
10 \\
5 \\
5 \\
5 \\
10 \\
5 \\
10 \\
5 \\
5 \\
5 \\
5 \\
5 \\
5 \\
5 \\
10 \\
5 \\
5 \\
5 \\
5 \\
5 \\
5 \\
5 \\
5 \\
5 \\
100 \\
50 \\
50\end{array}$ \\
\hline
\end{tabular}

Location=ROA; Formation=COPPER RIDGE; Site=35; Samp id=3208; Dup id=' '; Phase=2

\begin{tabular}{|c|c|c|}
\hline $\begin{array}{l}\text { cis-1,2-Dichloroethene } \\
\text { cis-1,3-Dichloropropene } \\
\text { trans-1,2-D ichloroethene } \\
\text { trans-1,3-Dichloropropene } \\
\text { Acetone } \\
\text { Benzene } \\
\text { Bromodichloromethane } \\
\text { Bromoform } \\
\text { Bromomethane } \\
\text { Carbon disulfide } \\
\text { Carbon tetrachloride } \\
\text { Chlorobenzene } \\
\text { Chloroethane } \\
\text { Chloroform } \\
\text { Chloromethane } \\
\text { Dibromochloromethane } \\
\text { Ethyl benzene } \\
\text { Methylene chloride } \\
\text { Styrene } \\
\text { Tetrachloroethene } \\
\text { Toluene } \\
\text { Trichloroethene } \\
\text { vinyl chloride } \\
\text { Xylene (meta + para) } \\
\text { Xylene (ortho) } \\
1,1-D i c h l o r o e t h a n e \\
1,1-D i c h l o r o e t h e n e \\
1,1,1-\text { Trichloroethane } \\
1,1,2-\text { Trichloroethane } \\
1,1,2,2-T e t r a c h l o r o e t h a n e \\
1,2-D i c h l o r o e t h a n e\end{array}$ & 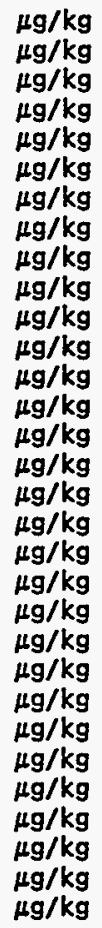 & $\begin{array}{r}5 \\
5 \\
5 \\
5 \\
100 \\
5 \\
5 \\
5 \\
10 \\
5 \\
5 \\
5 \\
10 \\
5 \\
10 \\
5 \\
5 \\
5 \\
5\end{array}$ \\
\hline
\end{tabular}


Table B.1 (continued)

\begin{tabular}{|c|c|c|c|c|c|c|}
\hline Analysis & Units & $\begin{array}{c}\text { A } \\
\text { horizon }\end{array}$ & Qualifier & $\begin{array}{l}\text { A horizon } \\
\text { field dup }\end{array}$ & Qualifier & \\
\hline $\begin{array}{l}\text { 1,2-Dich Loropropane } \\
\text { 2-Butanone } \\
\text { 2-Hexanone } \\
\text { 4-Hethyl-2-pentanone }\end{array}$ & $\begin{array}{l}\mu g / \mathrm{kg} \\
\mu g / \mathrm{kg} \\
\mu g / \mathrm{kg} \\
\mu g / \mathrm{kg}\end{array}$ & $\begin{array}{r}5 \\
100 \\
50 \\
50\end{array}$ & $\begin{array}{l}\mathbf{U} \\
\mathbf{U} \\
\mathbf{U} \\
\mathbf{U}\end{array}$ & $\therefore$ & & $z_{z}^{*} \cdot i$ \\
\hline
\end{tabular}

Location=ROA; Fomation =COPPER RIDGE; Site=39; Samp id=3213; Dup id =' '; Phase=2

\begin{tabular}{|c|c|c|}
\hline 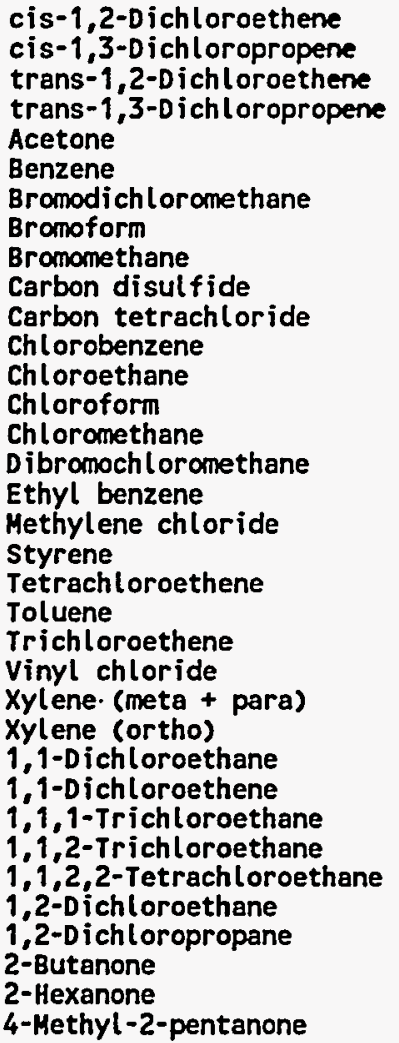 & $\begin{array}{l}\mu g / k g \\
\mu g / k g \\
\mu g / k g \\
\mu g / k g \\
\mu g / k g \\
\mu g / k g \\
\mu g / k g \\
\mu g / k g \\
\mu g / k g \\
\mu g / k g \\
\mu g / k g \\
\mu g / k g \\
\mu g / k g \\
\mu g / k g \\
\mu g / k g \\
\mu g / k g \\
\mu g / k g \\
\mu g / k g \\
\mu g / k g \\
\mu g / k g \\
\mu g / k g \\
\mu g / k g \\
\mu g / k g \\
\mu g / k g \\
\mu g / k g \\
\mu g / k g \\
\mu g / k g \\
\mu g / k g \\
\mu g / k g \\
\mu g / k g \\
\mu g / k g \\
\mu g / k g \\
\mu g / k g \\
\mu g / k g \\
\mu g / k g\end{array}$ & $\begin{array}{r}5 \\
5 \\
5 \\
5 \\
100 \\
5 \\
5 \\
5 \\
10 \\
5 \\
5 \\
5 \\
10 \\
5 \\
10 \\
5 \\
5 \\
5 \\
5 \\
5 \\
5 \\
5 \\
10 \\
5 \\
5 \\
5 \\
5 \\
5 \\
5 \\
5 \\
5 \\
5 \\
100 \\
50 \\
50\end{array}$ \\
\hline
\end{tabular}

Location=ROA; Formation=COPPER RIDGE; Site=40; Samp id=3210; Dup id=' '; Phase=2

\begin{tabular}{|c|c|c|}
\hline $\begin{array}{l}\text { cis-1,2-Dichloroethene } \\
\text { cis-1,3-Dichloropropene } \\
\text { trans-1,2-Dichloroethene } \\
\text { trans-1,3-Dichloropropene } \\
\text { Acetone } \\
\text { Benzene } \\
\text { Bromodichloromethane } \\
\text { Bromoform } \\
\text { Bromomethane } \\
\text { Carbon disulfide } \\
\text { Carbon tetrachloride } \\
\text { Chlorobenzene } \\
\text { Chloroethane } \\
\text { Chloroform } \\
\text { Chloromethane } \\
\text { Dibromochloromethane } \\
\text { Ethyl benzene } \\
\text { Methylene chloride } \\
\text { Styrene } \\
\text { Tetrachloroethene } \\
\text { Toluene } \\
\text { Trichloroethene } \\
\text { Vinyl chloride } \\
\text { Xylene (meta + para) } \\
\text { Xylene (ortho) }\end{array}$ & $\begin{array}{l}\mu g / \mathbf{k g} \\
\mu g / k g \\
\mu g / k g \\
\mu g / k g \\
\mu g / k g \\
\mu g / k g \\
\mu g / k g \\
\mu g / k g \\
\mu g / k g \\
\mu g / k g \\
\mu g / k g \\
\mu g / k g \\
\mu g / k g \\
\mu g / k g \\
\mu g / \mathbf{k g} \\
\mu g / \mathbf{k g} \\
\mu g / \mathbf{k g} \\
\mu g / \mathbf{k g} \\
\mu g / \mathbf{k g} \\
\mu g / \mathbf{k g} \\
\mu g / \mathbf{k g} \\
\mu g / \mathbf{k g} \\
\mu g / \mathbf{k g} \\
\mu g / \mathbf{k g} \\
\mu g / \mathbf{k g}\end{array}$ & $\begin{array}{r}5 \\
5 \\
5 \\
5 \\
14 \\
5 \\
5 \\
5 \\
10 \\
5 \\
5 \\
5 \\
10 \\
5 \\
10 \\
5 \\
5 \\
5 \\
5 \\
5 \\
5 \\
5 \\
10 \\
5\end{array}$ \\
\hline
\end{tabular}


Table B.1 (continued)

\begin{tabular}{|c|c|c|c|c|c|}
\hline Analysis & Units & $\begin{array}{c}\text { A } \\
\text { horizon }\end{array}$ & Qualifier & $\begin{array}{l}\text { A horizon } \\
\text { field dup }\end{array}$ & Qualifier \\
\hline $\begin{array}{l}\text { 1,1-Dichloroethane } \\
1,1-D i c h l o r o e t h e n e \\
1,1,1-T r i c h l o r o e t h a n e \\
1,1,2-T r i c h l o r o e t h a n e \\
1,1,2,2-T e t r a c h l o r o e t h a n e \\
1,2-D \text { ichloroethane } \\
1,2-D \text { ichloropropane } \\
\text { 2-Butanone } \\
\text { 2-Hexanone } \\
\text { 4-Methyl-2-pentanone }\end{array}$ & $\begin{array}{l}\mu g / \mathrm{kg} \\
\mu g / \mathrm{kg} \\
\mu g / \mathrm{kg} \\
\mu g / \mathrm{kg} \\
\mu g / \mathrm{kg} \\
\mu g / \mathrm{kg} \\
\mu g / \mathrm{kg} \\
\mu g / \mathrm{kg} \\
\mu g / \mathrm{kg} \\
\mu g / \mathrm{kg}\end{array}$ & $\begin{array}{r}5 \\
5 \\
5 \\
5 \\
5 \\
5 \\
5 \\
100 \\
50 \\
50\end{array}$ & $\begin{array}{l}U \\
U \\
U \\
U \\
U \\
U \\
U \\
U \\
U \\
U \\
U\end{array}$ & & \\
\hline
\end{tabular}

Location=ROA; Formation=COPPER RIDGE; Site $=41$; Samp id=3216; Dup id =' '; Phase $=2$

\begin{tabular}{|c|c|c|}
\hline 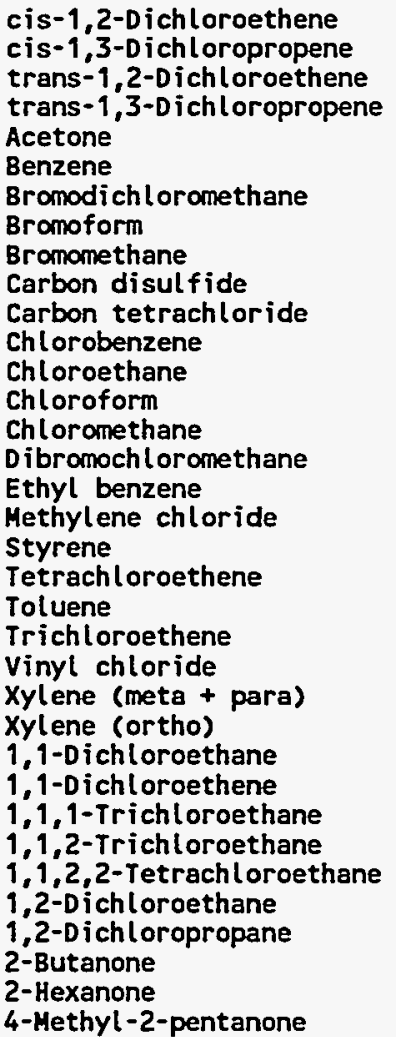 & 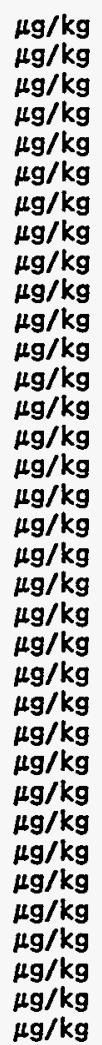 & $\begin{array}{r}5 \\
10 \\
5 \\
5 \\
5 \\
5 \\
5 \\
5 \\
5 \\
5 \\
5 \\
100 \\
50 \\
50\end{array}$ \\
\hline
\end{tabular}

\section{Location=ROA; Formation=COPPER RIDGE; Site =42; Samp id=3234; Dup id =' '; Phase $=2$}

\begin{tabular}{|c|c|c|}
\hline $\begin{array}{l}\text { cis-1,2-Dichloroethene } \\
\text { cis-1,3-Dichloropropene } \\
\text { trans-1,2-D ichloroethene } \\
\text { trans-1,3-Dichloropropene } \\
\text { Acetone } \\
\text { Benzene } \\
\text { Bromodichloromethane } \\
\text { Bromoform } \\
\text { Bromomethane } \\
\text { Carbon disulfide } \\
\text { Carbon tetrachloride } \\
\text { Chlorobenzene } \\
\text { Chloroethane } \\
\text { Chloroform } \\
\text { Chloromethane } \\
\text { Dibromochloromethane } \\
\text { Ethyl benzene } \\
\text { Methylene chloride } \\
\text { Styrene }\end{array}$ & $\begin{array}{l}\mu g / k g \\
\mu g / k g \\
\mu g / k g \\
\mu g / k g \\
\mu g / k g \\
\mu g / k g \\
\mu g / k g \\
\mu g / k g \\
\mu g / k g \\
\mu g / k g \\
\mu g / k g \\
\mu g / k g \\
\mu g / k g \\
\mu g / k g \\
\mu g / k g \\
\mu g / k g \\
\mu g / k g \\
\mu g / k g \\
\mu g / k g\end{array}$ & $\begin{array}{r}5 \\
5 \\
5 \\
5 \\
26 \\
5 \\
5 \\
5 \\
10 \\
5 \\
5 \\
5 \\
10 \\
5 \\
10\end{array}$ \\
\hline
\end{tabular}


Table B.1 (continucd)

\begin{tabular}{|c|c|c|c|c|c|c|}
\hline Analysis & Units & $\begin{array}{c}\text { A } \\
\text { horizon }\end{array}$ & Qualifier & $\begin{array}{l}\text { A horizon } \\
\text { field dup }\end{array}$ & Qualifier & \\
\hline $\begin{array}{l}\text { Tetrachloroethene } \\
\text { Toluene } \\
\text { Trichloroethene } \\
\text { Vinyl chloride } \\
\text { xylene (meta + para) } \\
\text { xylene (ortho) } \\
1,1-D \text { ichloroethane } \\
1,1-0 \text { ichloroethene } \\
1,1,1-\text { Trichloroethane } \\
1,1,2-\text { Trichloroethane } \\
1,1,2,2-\text { Tetrachloroethane } \\
1,2-D i c h \text { loroethane } \\
1,2-\text { Dichloropropane } \\
\text { 2--Butanone } \\
\text { 2-Hexanone } \\
\text { 4-Methyl-2-pentanone }\end{array}$ & $\begin{array}{l}\mu g / k g \\
\mu g / k g \\
\mu g \\
\mu g / k g \\
\mu g / k g \\
\mu g / k g \\
\mu g / k g \\
\mu g / k g \\
\mu g / k g \\
\mu g / k g \\
\mu g / k g \\
\mu g / k g \\
\mu g / k g \\
\mu g / k g \\
\mu g / k g \\
\mu g / k g \\
\mu g / k g\end{array}$ & $\begin{array}{r}5 \\
5 \\
5 \\
50 \\
5 \\
5 \\
5 \\
5 \\
5 \\
5 \\
5 \\
5 \\
5 \\
5 \\
100 \\
50 \\
50\end{array}$ & $\begin{array}{l}U \\
U \\
U \\
U \\
U \\
U \\
U \\
U \\
U \\
U \\
U \\
U \\
U \\
U \\
U \\
U \\
U\end{array}$ & $:$ & & $\therefore 3.4$ \\
\hline
\end{tabular}

Location=ROA; Formation=COPPER RIDGE; Site=43; Samp id=3230; Dup id=3232; Phase =2

\begin{tabular}{|c|c|c|c|c|}
\hline 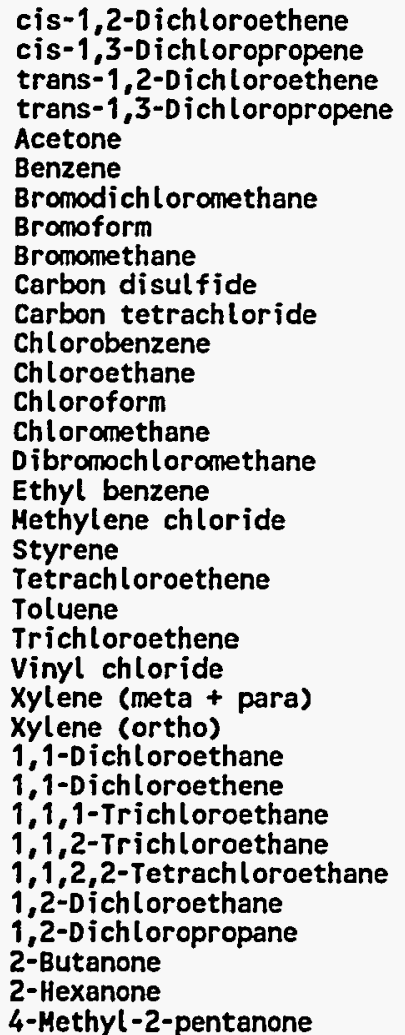 & $\begin{array}{l}\mu g / k g \\
\mu g / k g \\
\mu g / k g \\
\mu g / k g \\
\mu g / k g \\
\mu g / k g \\
\mu g / k g \\
\mu g / k g \\
\mu g / k g \\
\mu g / k g \\
\mu g / k g \\
\mu g / k g \\
\mu g / / k g \\
\mu g / k g \\
\mu g / k g \\
\mu g / k g \\
\mu g / k g \\
\mu g / k g \\
\mu g / k g \\
\mu g / k g \\
\mu g / k g \\
\mu g / k g \\
\mu g / k g \\
\mu g / k g \\
\mu g / k g \\
\mu g / k g \\
\mu g / k g \\
\mu g / k g \\
\mu g / k g \\
\mu g / k g \\
\mu g / k g \\
\mu g / k g \\
\mu g / k g \\
\mu g / k g \\
\mu g / k g\end{array}$ & $\begin{array}{r}5 \\
5 \\
5 \\
5 \\
9 \\
5 \\
5 \\
5 \\
5 \\
10 \\
5 \\
5 \\
5 \\
10 \\
5 \\
10 \\
5 \\
5 \\
5 \\
5 \\
5 \\
5 \\
5 \\
10 \\
5 \\
5 \\
5 \\
5 \\
5 \\
5 \\
5 \\
5 \\
5 \\
100 \\
50 \\
50\end{array}$ & $\begin{array}{l}U \\
U \\
U \\
U \\
J \\
U \\
U \\
U \\
U \\
U \\
U \\
U \\
\mathbf{U} \\
\mathbf{U} \\
\mathbf{U} \\
\mathbf{U} \\
\mathbf{U} \\
\mathbf{U} \\
\mathbf{U} \\
\mathbf{U} \\
\mathbf{U} \\
\mathbf{U} \\
\mathbf{U} \\
\mathbf{U} \\
\mathbf{U} \\
\mathbf{U} \\
\mathbf{U} \\
\mathbf{U} \\
\mathbf{U} \\
\mathbf{U} \\
\mathbf{U} \\
\mathbf{U} \\
\mathbf{U}\end{array}$ & $\begin{array}{r}5 \\
5 \\
5 \\
5 \\
5 \\
5 \\
5 \\
5 \\
5 \\
10 \\
5 \\
5 \\
5 \\
10 \\
5 \\
10 \\
5 \\
5 \\
5 \\
5 \\
5 \\
5 \\
5 \\
10 \\
5 \\
5 \\
5 \\
5 \\
5 \\
5 \\
5 \\
5 \\
5 \\
100 \\
50 \\
50\end{array}$ \\
\hline
\end{tabular}

Location=ROA; Formation=COPPER RIDGE; Site=44; Samp id=3228; Dup id=' '; Phase=2

\begin{tabular}{|c|c|}
\hline $\begin{array}{l}\text { cis-1,2-Dichloroethene } \\
\text { cis-1,3-Dichloropropene } \\
\text { trans-1,2-Dichloroethene } \\
\text { trans-1,3-Dichloropropene } \\
\text { Acetone } \\
\text { Benzene } \\
\text { Bromodichloromethane } \\
\text { Bromoform } \\
\text { Bromomethane } \\
\text { Carbon disulfide } \\
\text { Carbon tetrachloride } \\
\text { Chlorobenzene } \\
\text { Chloroethane }\end{array}$ & $\begin{array}{l}\mu g / \mathrm{kg} \\
\mu \mathrm{g} / \mathrm{kg} \\
\mu g / \mathrm{kg} \\
\mu g / \mathrm{kg} \\
\mu g / \mathrm{kg} \\
\mu g / \mathrm{kg} \\
\mu g / \mathrm{kg} \\
\mu g / \mathrm{kg} \\
\mu g / \mathrm{kg} \\
\mu g / \mathrm{kg} \\
\mu \mathrm{g} / \mathrm{kg} \\
\mu \mathrm{g} / \mathrm{kg} \\
\mu g / \mathrm{kg}\end{array}$ \\
\hline
\end{tabular}


Table B.1 (continued)

\begin{tabular}{|c|c|c|c|c|c|}
\hline Analysis & Units & $\begin{array}{c}\text { A } \\
\text { horizon }\end{array}$ & Qualifier & $\begin{array}{l}\text { A horizon } \\
\text { field dup }\end{array}$ & Qualifier \\
\hline $\begin{array}{l}\text { Chloroform } \\
\text { Chloromethane } \\
\text { Dibromochloromethane } \\
\text { Ethyl benzene } \\
\text { Methylene chloride } \\
\text { styrene } \\
\text { Tetrachloroethene } \\
\text { Toluene } \\
\text { Trichloroethene } \\
\text { vinyl chloride } \\
\text { xylene (meta + para) } \\
\text { Xylene (ortho) } \\
1,1-D \text { ichloroethane } \\
1,1-D i c h l o r o e t h e n e \\
1,1,1-T r i c h l o r o e t h a n e \\
1,1,2-T r i c h l o r o e t h a n e \\
1,1,2,2-T e t r a c h l o r o e t h a n e \\
1,2-D \text { ichloroethane } \\
1,2-D \text { ichloropropane } \\
2-8 \text { tanone } \\
\text { 2-Hexanone } \\
\text { 4-Methyl-2-pentanone }\end{array}$ & $\begin{array}{l}\mu g / k g \\
\mu g / k g \\
\mu g / k g \\
\mu g / k g \\
\mu g / k g \\
\mu g / k g \\
\mu g / k g \\
\mu g / k g \\
\mu g / k g \\
\mu g / k g \\
\mu g / k g \\
\mu g / k g \\
\mu g / k g \\
\mu g / k g \\
\mu g / k g \\
\mu g / k g \\
\mu g / k g \\
\mu g / k g \\
\mu g / k g \\
\mu g / k g \\
\mu g / k g \\
\mu g / k g\end{array}$ & $\begin{array}{r}5 \\
10 \\
5 \\
5 \\
5 \\
-\quad 5 \\
5 \\
5 \\
5 \\
10 \\
5 \\
5 \\
5 \\
5 \\
5 \\
5 \\
5 \\
5 \\
5 \\
100 \\
50 \\
50\end{array}$ & $\begin{array}{l}U \\
U \\
U \\
U \\
U \\
U \\
U \\
U \\
U \\
U \\
U \\
U \\
U \\
U \\
U \\
U \\
U \\
U \\
U \\
U \\
U \\
U\end{array}$ & & \\
\hline
\end{tabular}

Location=ROA; Formation=COPPER RIDGE; Site=45; Samp id=3226; Dup id=' '; Phase=2

\begin{tabular}{|c|c|c|}
\hline 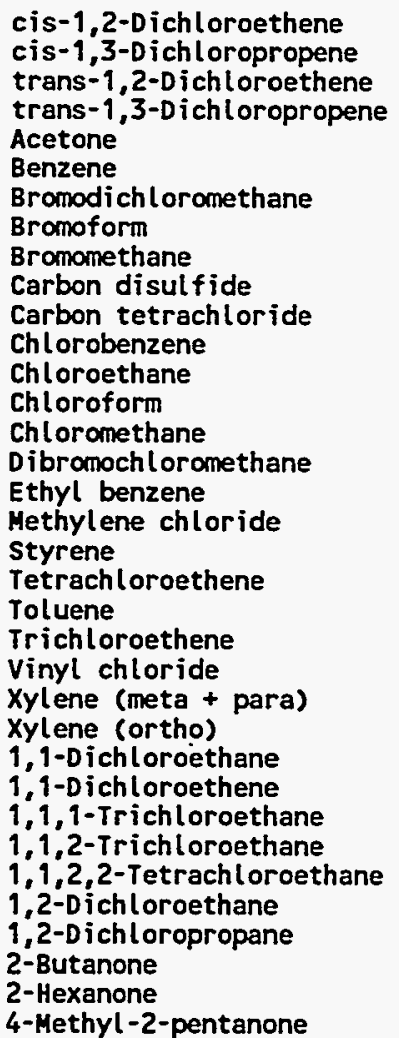 & 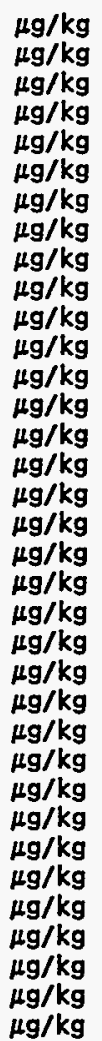 & $\begin{array}{r}5 \\
5 \\
5 \\
5 \\
6 \\
5 \\
5 \\
5 \\
10 \\
5 \\
5 \\
5 \\
10 \\
5 \\
10 \\
5 \\
5 \\
5 \\
5 \\
5 \\
5 \\
5 \\
10 \\
5 \\
5 \\
5 \\
5 \\
5 \\
5 \\
5 \\
5 \\
5 \\
100 \\
50 \\
50\end{array}$ \\
\hline
\end{tabular}

Location=ROA; Formation=COPPER RIDGE; Site=46; Samp id=3224; Dup id=' '; Phase=2

$\begin{array}{ll}\text { cis-1,2-Dichloroethene } & \mu \mathrm{g} / \mathrm{kg} \\ \text { cis-1,3-Dichloropropene } & \mu \mathrm{g} / \mathrm{kg} \\ \text { trans-1,2-Dichloroethene } & \mu \mathrm{g} / \mathrm{kg} \\ \text { trans-1,3-Dichloropropene } & \mu g / \mathrm{kg} \\ \text { Acetone } & \mu \mathrm{g} / \mathrm{kg} \\ \text { Benzene } & \mu g / \mathrm{kg} \\ \text { Bromodichloromethane } & \mu g / \mathrm{kg}\end{array}$

Bromodichloromethane $\quad \mu \mathrm{g} / \mathrm{kg}$ 
B-63

Table B.1 (continued)

\begin{tabular}{|c|c|c|c|c|c|c|}
\hline Analysis & Units & $\begin{array}{c}\text { A } \\
\text { horizon }\end{array}$ & Qualifier & $\begin{array}{l}\text { A horizon } \\
\text { field dup }\end{array}$ & Qualifier & \\
\hline $\begin{array}{l}\text { Bromoform } \\
\text { Bromomethane } \\
\text { Carbon disulfide } \\
\text { Carbon tetrachloride } \\
\text { Chlorobenzene } \\
\text { Chloroethane } \\
\text { Chloroform } \\
\text { Chloromethane } \\
\text { Dibromochloromethane } \\
\text { Ethyl benzene } \\
\text { Methylene chloride } \\
\text { Styrene } \\
\text { Tetrachloroethene } \\
\text { Toluene } \\
\text { Trichloroethene } \\
\text { Vinyl chloride } \\
\text { xylene (meta + para) } \\
\text { Xylene (ortho) } \\
\text { 1,1-Dichloroethane } \\
\text { 1,1-Dichloroethene } \\
\text { 1,1,1-Trichloroethane } \\
\text { 1,1,2-Trichloroethane } \\
\text { 1,1,2,2-Tetrachloroethane } \\
\text { 1,2-Dichloroethane } \\
\text { 1,2-Dichloropropane } \\
\text { 2-Butanone } \\
\text { 2-Hexanone } \\
\text { 4-Hethyl-2-pentanone }\end{array}$ & $\begin{array}{l}\mu g / k g \\
\mu g / k g \\
\mu g / k g \\
\mu g / k g \\
\mu g / k g \\
\mu g / k g \\
\mu g / k g \\
\mu g / k g \\
\mu g / k g \\
\mu g / k g \\
\mu g / k g \\
\mu g / k g \\
\mu g / k g \\
\mu g / k g \\
\mu g / k g \\
\mu g / k g \\
\mu g / k g \\
\mu g / k g \\
\mu g / k g \\
\mu g / k g \\
\mu g / k g \\
\mu g / k g \\
\mu g / k g \\
\mu g / k g \\
\mu g / k g \\
\mu g / k g \\
\mu g / k g \\
\mu g / k g\end{array}$ & $\begin{array}{r}5 \\
10 \\
5 \\
5 \\
5 \\
10 \\
5 \\
10 \\
5 \\
5 \\
5 \\
5 \\
5 \\
5 \\
5 \\
10 \\
5 \\
5 \\
5 \\
5 \\
5 \\
5 \\
5 \\
5 \\
5 \\
100 \\
50 \\
50\end{array}$ & $\begin{array}{l}U \\
U \\
U \\
U \\
U \\
U \\
U \\
U \\
U \\
U \\
U \\
U \\
U \\
U \\
U \\
U \\
U \\
U \\
U \\
U \\
U \\
U \\
U \\
U \\
U \\
U \\
U \\
U\end{array}$ & 1 & . & if $f_{0}$ is \\
\hline
\end{tabular}

Location=ROA; Formation=COPPER RIDGE; Site=47; Samp id=3222; Dup id=' '; Phase=2

\begin{tabular}{|c|c|c|}
\hline 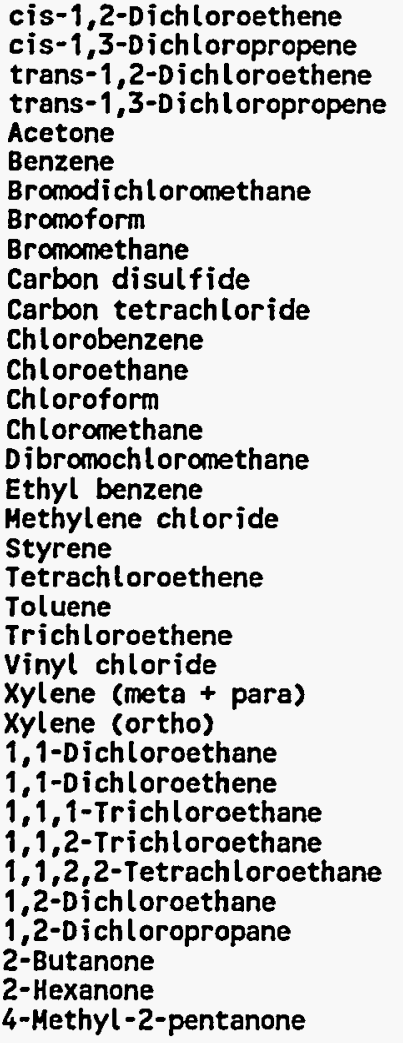 & $\begin{array}{l}\mu g / k g \\
\mu g / k g \\
\mu g / k g \\
\mu g / k g \\
\mu g / k g \\
\mu g / k g \\
\mu g / k g \\
\mu g / k g \\
\mu g / k g \\
\mu g / k g \\
\mu g / k g \\
\mu g / k g \\
\mu g / k g \\
\mu g / k g \\
\mu g / k g \\
\mu g / \mathbf{k g} \\
\mu g / k g \\
\mu g / k g \\
\mu g / k g \\
\mu g / k g \\
\mu g / k g \\
\mu g / k g \\
\mu g / k g \\
\mu g / k g \\
\mu g / k g \\
\mu g / k g \\
\mu g / k g \\
\mu g / k g \\
\mu g / k g \\
\mu g / k g \\
\mu g / k g \\
\mu g / k g \\
\mu g / k g \\
\mu g / k g \\
\mu g / k g\end{array}$ & $\begin{array}{r}5 \\
5 \\
5 \\
5 \\
5 \\
5 \\
5 \\
5 \\
10 \\
5 \\
5 \\
5 \\
10 \\
5 \\
10 \\
5 \\
5 \\
5 \\
5 \\
5 \\
5 \\
5 \\
10 \\
5 \\
5 \\
5 \\
5 \\
5 \\
5 \\
5 \\
5 \\
5 \\
100 \\
50 \\
50\end{array}$ \\
\hline
\end{tabular}


Table B.1 (continued)

\begin{tabular}{|c|c|c|c|c|c|}
\hline Analysis & Units & $\underset{\text { horizon }}{\text { A }}$ & Qualifier & $\begin{array}{l}\text { A horizon } \\
\text { field dup }\end{array}$ & Qualifier \\
\hline
\end{tabular}

Location=ROA; Formation=DISMAL GAP; Site=10; Samp id=3166; Dup id=' '; Phase=1

\begin{tabular}{|c|c|c|}
\hline 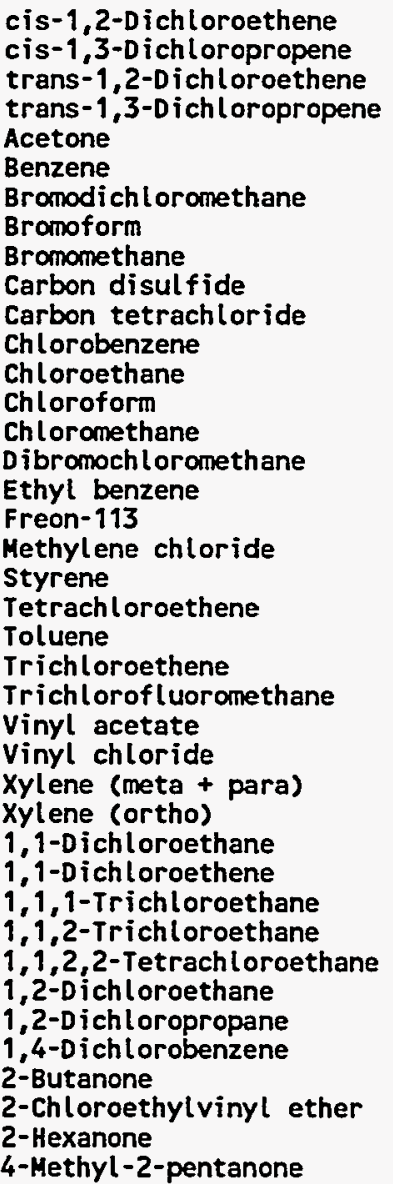 & 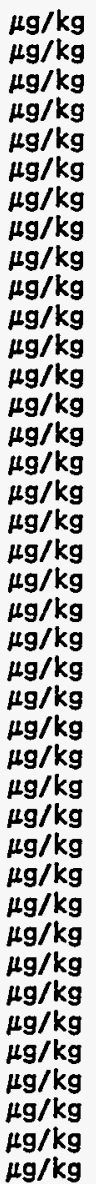 & $\begin{array}{r}5 \\
5 \\
5 \\
5 \\
8 \\
5 \\
5 \\
5 \\
10 \\
5 \\
5 \\
5 \\
10 \\
5 \\
10 \\
5 \\
5 \\
5 \\
5 \\
5 \\
5 \\
5 \\
5 \\
5 \\
5 \\
10 \\
5 \\
5 \\
5 \\
5 \\
5 \\
5 \\
5 \\
5 \\
5 \\
5 \\
100 \\
5 \\
50 \\
50\end{array}$ \\
\hline
\end{tabular}

Location=ROA; Formation=DISMAL GAP; Site=13; Samp id =3125; Dup id =3137; Phase $=1$

cis-1,2-Dichloroethene

cis-1,3-Dichloropropene

trans-1,2-Dichloroethene

trans-1,3-Dichloropropene

Acetone

Benzene

Bromodichloromethane

Bromoform

Bromomethane

Carbon disulfide

Carbon tetrachloride

Chlorobenzene

Chloroethane

Chloroform

Chloromethane

Dibromochloromethane

Ethyl benzene

Freon-113

Methylene chloride

Styrene

Tetrachloroethene

Toluene

Trichloroethene

Trichlorof luoromethane

Vinyl acetate

vinyl chloride

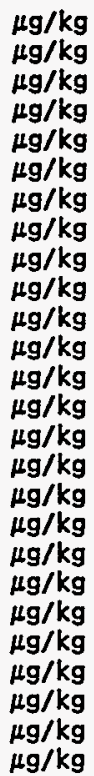

$\mu \mathrm{g} / \mathrm{kg}$

$\mu \mathrm{g} / \mathrm{kg}$

$\mu \mathrm{g} / \mathrm{kg}$

$\mu \mathrm{g} / \mathrm{kg}$

$\mu \mathrm{g} / \mathrm{kg}$

$\mu \mathrm{g} / \mathrm{kg}$

$\mu \mathrm{g} / \mathrm{kg}$

$\mu \mathrm{g} / \mathrm{kg}$

$\mu \mathrm{g} / \mathrm{kg}$

$\mu \mathrm{g} / \mathrm{kg}$

$\mu \mathrm{g} / \mathrm{kg}$

$\mathrm{g} / \mathrm{kg}$

$\mu \mathrm{g} / \mathrm{kg}$

$\mu \mathrm{g} / \mathrm{kg}$

$\mu \mathrm{g} / \mathrm{kg}$

$\mu \mathrm{g} / \mathrm{kg}$

$\mathrm{Hg} / \mathrm{kg}$

$\mu \mathrm{g} / \mathrm{kg}$

$\begin{array}{rr}5 & U \\ 5 & U \\ 5 & U \\ 5 & U \\ 8 & J \\ 5 & U \\ 5 & U \\ 5 & U \\ 10 & U \\ 5 & U \\ 5 & U \\ 5 & U \\ 10 & U \\ 5 & U \\ 10 & U \\ 5 & U \\ 5 & U \\ 5 & U \\ 5 & U \\ 5 & U \\ 5 & U \\ 5 & U \\ 5 & U \\ 5 & U \\ 5 & U \\ 10 & U\end{array}$




\section{B- 65}

Table B.1 (continued)

\begin{tabular}{|c|c|c|c|c|c|c|}
\hline Analysis & Units & $\begin{array}{c}A \\
\text { horizon }\end{array}$ & Qualifier & $\begin{array}{l}\text { A horizon } \\
\text { field dup }\end{array}$ & Qualifier & \\
\hline $\begin{array}{l}\text { Xylene (meta + para) } \\
\text { Xylene (ortho) } \\
\text { 1,1-Dichloroethane } \\
1,1-\text { Dichloroethene } \\
1,1,1-\text { Trichloroethane } \\
1,1,2-\text { Trichloroethane } \\
1,1,2,2-\text { Tetrachloroethane } \\
\text { 1,2-Dichloroethane } \\
\text { 1,2-Dichloropropane } \\
\text { 1,4-Dichlorobenzene } \\
\text { 2-Butanone } \\
\text { 2-Chloroethylvinyl ether } \\
\text { 2-Hexanone } \\
\text { 4- Methyl-2-pentanone }\end{array}$ & $\begin{array}{l}\mu g / k g \\
\mu g / k g \\
\mu g / k g \\
\mu g / k g \\
\mu g / k g \\
\mu g / k g \\
\mu g / k g \\
\mu g / k g \\
\mu g / k g \\
\mu g / k g \\
\mu g / k g \\
\mu g / k g \\
\mu g / k g \\
\mu g / k g\end{array}$ & $\begin{array}{r}5 \\
5 \\
5 \\
5 \\
5 \\
5 \\
5 \\
5 \\
5 \\
5 \\
79 \\
5 \\
50 \\
50\end{array}$ & $\begin{array}{l}U \\
U \\
U \\
U \\
U \\
U \\
U \\
U \\
U \\
U \\
B J \\
U \\
U \\
U\end{array}$ & $\begin{array}{l}5 \\
5 \\
5 \\
5 \\
5 \\
5 \\
5 \\
5 \\
5 \\
5 \\
9 \\
5 \\
49 \\
49\end{array}$ & $\begin{array}{l}U \\
U \\
U \\
U \\
U \\
U \\
U \\
U \\
U \\
\mathbf{U} \\
\mathbf{B J} \\
\mathbf{U} \\
\mathbf{U} \\
\mathbf{U}\end{array}$ & $\therefore \cdots$ \\
\hline
\end{tabular}

Location=ROA; Formation=DISMAL GAP; Site=14; Samp id=3146; Dup id=' ; Phase=1

\begin{tabular}{|c|c|c|}
\hline 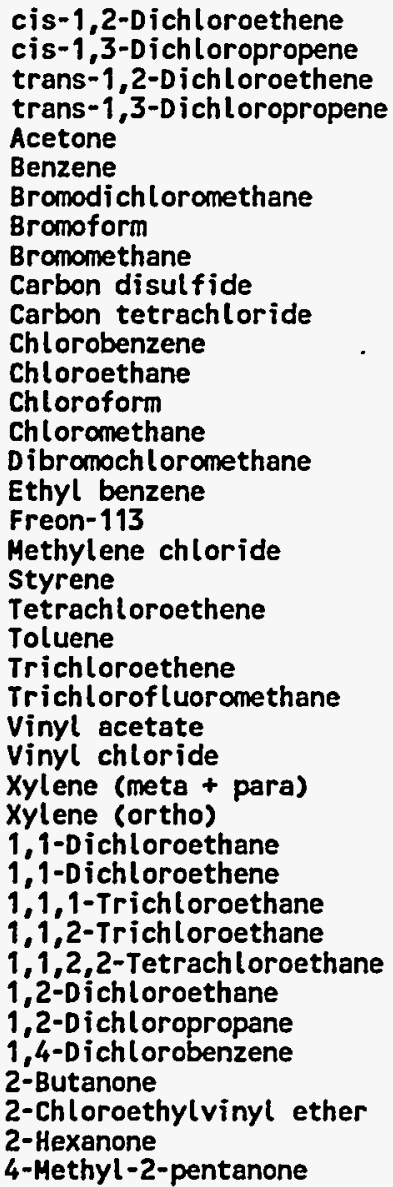 & $\begin{array}{l}\mu g / \mathrm{kg} \\
\mu \mathrm{g} / \mathrm{kg} \\
\mu \mathrm{g} / \mathrm{kg} \\
\mu \mathrm{g} / \mathrm{kg} \\
\mu \mathrm{g} / \mathrm{kg} \\
\mu \mathrm{g} / \mathrm{kg} \\
\mu \mathrm{g} / \mathrm{kg} \\
\mu \mathrm{g} / \mathrm{kg} \\
\mu g / \mathrm{kg} \\
\mu \mathrm{g} / \mathrm{kg} \\
\mu \mathrm{g} / \mathrm{kg} \\
\mu \mathrm{g} / \mathrm{kg} \\
\mu \mathrm{g} / \mathrm{kg} \\
\mu \mathrm{g} / \mathrm{kg} \\
\mu \mathrm{g} / \mathrm{kg} \\
\mu \mathrm{g} / \mathrm{kg} \\
\mu \mathrm{g} / \mathrm{kg} \\
\mu \mathrm{g} / \mathrm{kg} \\
\mu \mathrm{g} / \mathrm{kg} \\
\mu \mathrm{g} / \mathrm{kg} \\
\mu \mathrm{g} / \mathrm{kg} \\
\mu \mathrm{g} / \mathrm{kg} \\
\mu \mathrm{g} / \mathrm{kg} \\
\mu \mathrm{g} / \mathrm{kg} \\
\mu \mathrm{g} / \mathrm{kg} \\
\mu \mathrm{g} / \mathrm{kg} \\
\mu \mathrm{g} / \mathrm{kg} \\
\mu \mathrm{g} / \mathrm{kg} \\
\mu \mathrm{g} / \mathrm{kg} \\
\mu \mathrm{g} / \mathrm{kg} \\
\mu \mathrm{g} / \mathrm{kg} \\
\mu \mathrm{g} / \mathrm{kg} \\
\mu \mathrm{k} / \mathrm{kg} \\
\mu \mathrm{g} / \mathrm{kg} \\
\mu \mathrm{k} / \mathrm{kg} \\
\mu \mathrm{g} / \mathrm{kg} \\
\mu \mathrm{g} / \mathrm{kg} \\
\mu \mathrm{g} / \mathrm{kg} \\
\mu \mathrm{g} / \mathrm{kg} \\
\mu \mathrm{g} / \mathrm{kg}\end{array}$ & $\begin{array}{r}5 \\
5 \\
5 \\
5 \\
8 \\
5 \\
5 \\
5 \\
10 \\
5 \\
5 \\
5 \\
10 \\
5 \\
10 \\
5 \\
5 \\
5 \\
5 \\
5 \\
5 \\
5 \\
5 \\
5 \\
5 \\
10 \\
5 \\
5 \\
5 \\
5 \\
5 \\
5 \\
5 \\
5 \\
5 \\
5 \\
35 \\
5 \\
50 \\
50\end{array}$ \\
\hline
\end{tabular}

\section{Location=ROA; Formation=DISMAL GAP; Site=17; Samp id=3184; Dup id=' '; Phase=1}

$\begin{array}{llrl}\text { cis-1,2-Dichloroethene } & \mu \mathrm{g} / \mathrm{kg} & 5 & \mathrm{U} \\ \text { cis-1,3-Dichloropropene } & \mu \mathrm{g} / \mathrm{kg} & 5 & \mathrm{U} \\ \text { trans-1,2-Dichloroethene } & \mu \mathrm{g} / \mathrm{kg} & 5 & \mathrm{U} \\ \text { trans-1,3-Dichloropropene } & \mu \mathrm{g} / \mathrm{kg} & 5 & \mathrm{U} \\ \text { Acetone } & \mu \mathrm{g} / \mathrm{kg} & 10 & \mathrm{~J} \\ \text { Benzene } & \mu \mathrm{g} / \mathrm{kg} & 5 & \mathrm{U} \\ \text { Bromodichloromethane } & \mu \mathrm{g} / \mathrm{kg} & 5 & \mathrm{U} \\ \text { Bromoform } & \mu \mathrm{g} / \mathrm{kg} & 5 & \mathrm{U} \\ \text { Bromomethane } & \mu \mathrm{g} / \mathrm{kg} & 10 & U \\ \text { Carbon disulfide } & \mu \mathrm{g} / \mathrm{kg} & 5 & \mathrm{U}\end{array}$


Table B.1 (continued)

\begin{tabular}{|c|c|c|c|c|c|}
\hline Analysis & Units & $\begin{array}{c}\text { A } \\
\text { horizon }\end{array}$ & Qualifier & $\begin{array}{l}\text { A horizon } \\
\text { field dup }\end{array}$ & Qualifier \\
\hline $\begin{array}{l}\text { Carbon tetrachloride } \\
\text { Chlorobenzene } \\
\text { Chloroethane } \\
\text { Chloroform } \\
\text { Chloromethane } \\
\text { Dibromochloromethane } \\
\text { Ethyl benzene } \\
\text { Freon-113 } \\
\text { Methylene chloride } \\
\text { Styrene } \\
\text { Tetrachloroethene } \\
\text { Toluene } \\
\text { Trichloroethene } \\
\text { Trichlorofluoromethane } \\
\text { Vinyl acetate } \\
\text { Vinyl chloride } \\
\text { Xylene (meta + para) } \\
\text { Xylene (ortho) } \\
1,1-D \text { ichloroethane } \\
1,1-D \text { ichloroethene } \\
1,1,1-\text { Trichloroethane } \\
1,1 \text { 1, -Trichloroethane } \\
1,1,2,2-\text { Tetrachloroethane } \\
1,2-D \text { ichloroethane } \\
1,2-D \text { ichloropropane } \\
1,4-D \text { ichlorobenzene } \\
\text { 2-Butanone } \\
2-\text { Chloroethylvinyl ether } \\
\text { 2-Hexanone } \\
\text { 4- Methyl-2-pentanone }\end{array}$ & $\begin{array}{l}\mu g / k g \\
\mu g / k g \\
\mu g / k g \\
\mu g / k g \\
\mu g / k g \\
\mu g / k g \\
\mu g / k g \\
\mu g / k g \\
\mu g / k g \\
\mu g / k g \\
\mu g / k g \\
\mu g / k g \\
\mu g / k g \\
\mu g / k g \\
\mu g / k g \\
\mu g / k g \\
\mu g / k g \\
\mu g / k g \\
\mu g / k g \\
\mu g / k g \\
\mu g / k g \\
\mu g / k g \\
\mu g / k g \\
\mu g / k g \\
\mu g / k g \\
\mu g / k g \\
\mu g / k g \\
\mu g / k g \\
\mu g / k g \\
\mu g / k g\end{array}$ & $\begin{array}{r}5 \\
5 \\
10 \\
5 \\
10 \\
5 \\
5 \\
5 \\
5 \\
5 \\
5 \\
5 \\
5 \\
5 \\
5 \\
10 \\
5 \\
5 \\
5 \\
5 \\
5 \\
5 \\
5 \\
5 \\
5 \\
5 \\
8 \\
5 \\
50 \\
50\end{array}$ & $\begin{array}{l}U \\
U \\
U \\
U \\
U \\
U \\
U \\
U \\
U \\
U \\
U \\
U \\
U \\
U \\
U \\
U \\
U \\
U \\
U \\
U \\
U \\
U \\
U \\
U \\
U \\
U \\
B J \\
U \\
U \\
U\end{array}$ & & \\
\hline
\end{tabular}

Location=ROA; Formation=DISMAL GAP; Site=19; Samp id=3199; Dup id=' '; Phase=1

\begin{tabular}{|c|c|c|}
\hline 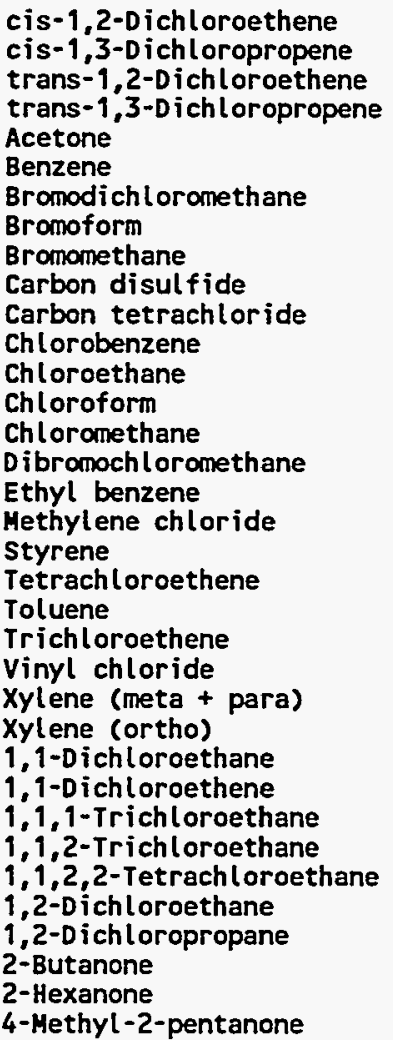 & $\begin{array}{l}\mu g / k g \\
\mu g / k g \\
\mu g / k g \\
\mu g / k g \\
\mu g / k g \\
\mu g / k g \\
\mu g / k g \\
\mu g / k g \\
\mu g / k g \\
\mu g / k g \\
\mu g / k g \\
\mu g / k g \\
\mu g / k g \\
\mu g / k g \\
\mu g / k g \\
\mu g / k g \\
\mu g / k g \\
\mu g / k g \\
\mu g / k g \\
\mu g / k g \\
\mu g / k g \\
\mu g / k g \\
\mu g / k g \\
\mu g / k g \\
\mu g / k g \\
\mu g / k g \\
\mu g / k g \\
\mu g / k g \\
\mu g / k g \\
\mu g / k g \\
\mu g / k g \\
\mu g / k g \\
\mu g / k g \\
\mu g / k g \\
\mu g / k g\end{array}$ & $\begin{array}{r}5 \\
5 \\
5 \\
5 \\
100 \\
5 \\
5 \\
5 \\
10 \\
5 \\
5 \\
5 \\
10 \\
5 \\
10 \\
5 \\
5 \\
5 \\
5 \\
5 \\
5 \\
5 \\
10 \\
5 \\
5 \\
5 \\
5 \\
5 \\
5 \\
5 \\
5 \\
5 \\
100 \\
50 \\
50\end{array}$ \\
\hline
\end{tabular}


Table B.1 (continued)

\begin{tabular}{lccc}
\hline & A & A horizon \\
Analysis & Units & horizon & Qualifier field dup Qualifier \\
\hline
\end{tabular}

Location=ROA; Formation=DISMAL GAP; Site=20; Samp id =3198; Dup id=' '; Phase=1 '. ',

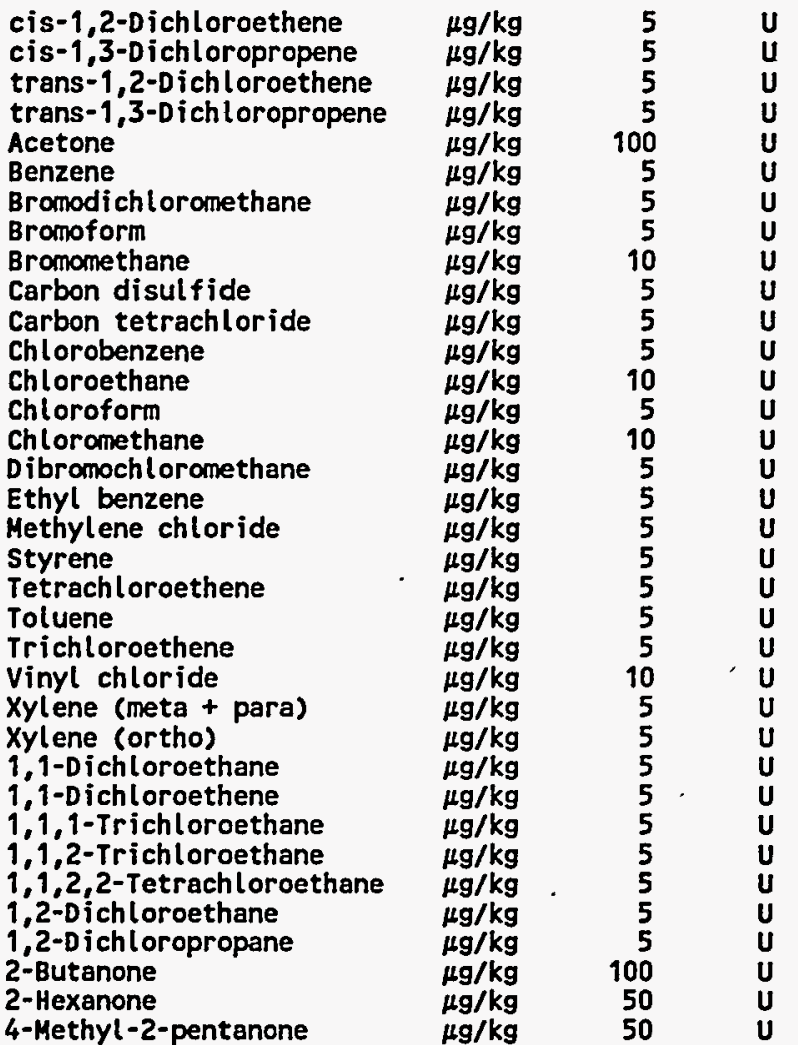

Location=ROA; Formation=DISMAL GAP; Site=21; Samp id=3196; Dup id=' '; Phase=1

cis-1,2-Dichloroethene cis-1,3-D ichloropropene trans-1,2-Dichloroethene trans-1,3-Dichloropropene Acetone

Benzene

Bromodichloromethane

Bromoform

Bromomethane

Carbon disulfide

Carbon tetrachloride

Chlorobenzene

Chloroethane

Chloroform

Chloromethane

Dibromochloromethane

Ethyl benzene

Methylene chloride

Styrene

Tetrachloroethene

Toluene

Trichloroethene

Vinyl chloride

Xylene (meta + para)

Xylene (ortho)

1,1-Dichloroethane

1,1-Dichloroethene

1,1,1-Trichloroethane

1,1,2-Trichloraethane

$1,1,2,2$-Tetrachloroethane

1,2-Dichloroethane

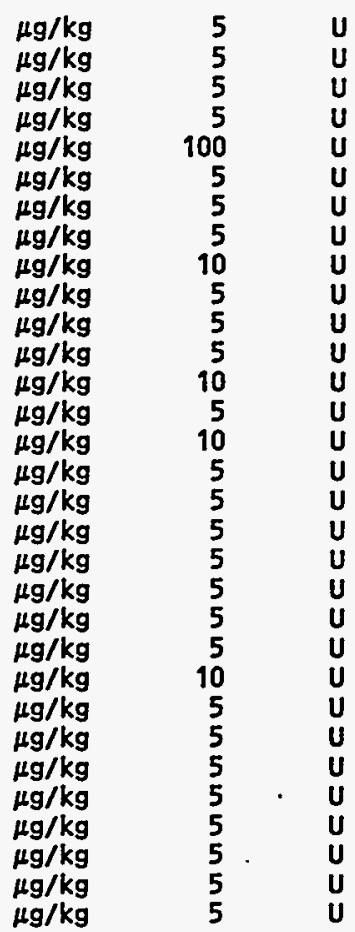


Table B.1 (continued)

\begin{tabular}{lcccc}
\hline \multicolumn{1}{c}{ Analysis } & Units & $\begin{array}{c}\text { A horizon } \\
\text { horizon }\end{array}$ & Qualifier & $\begin{array}{r}\text { field dup } \\
\text { Qualifier }\end{array}$ \\
\hline & & & & \\
1,2-Dichloropropane & $\mu \mathrm{g} / \mathrm{kg}$ & 5 & $\mathrm{U}$ & \\
2-Butanone & $\mu \mathrm{g} / \mathrm{kg}$ & 100 & $\mathrm{U}$ & \\
2-Hexanone & $\mu \mathrm{g} / \mathrm{kg}$ & 50 & $\mathrm{U}$
\end{tabular}

Location=ROA; Formation=DISMAL GAP; Site=22; Samp id=3197; Dup id=' '; Phase=1

\begin{tabular}{|c|c|c|}
\hline 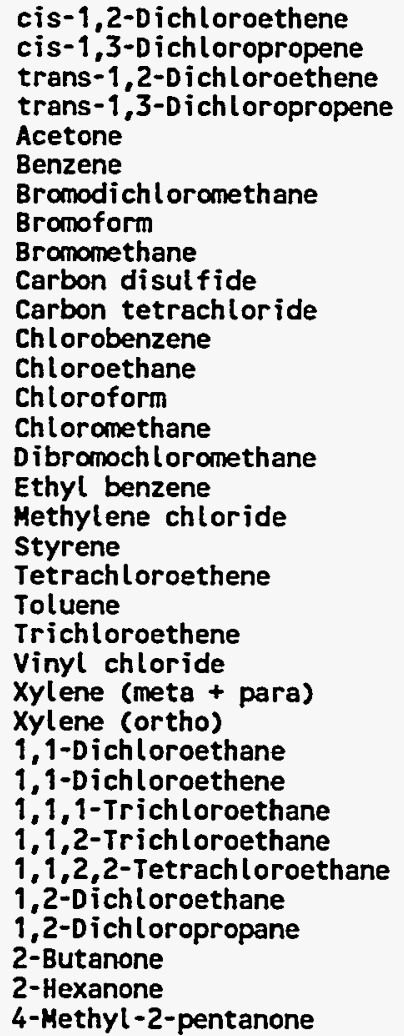 & 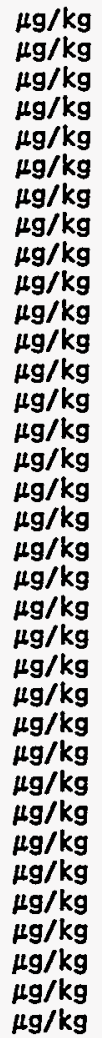 & $\begin{array}{r}5 \\
5 \\
5 \\
5 \\
98 \\
5 \\
5 \\
5 \\
10 \\
5 \\
5 \\
5 \\
10 \\
5 \\
10 \\
5 \\
5 \\
5 \\
5 \\
5 \\
5 \\
5 \\
10 \\
5 \\
5 \\
5 \\
5 \\
5 \\
5 \\
5 \\
5 \\
5 \\
98 \\
49 \\
49\end{array}$ \\
\hline
\end{tabular}

Location=ROA; Formation=DISMAL GAP; Site=3; Samp id=3201; Dup id=' '; Phase=1

\begin{tabular}{|c|c|c|}
\hline $\begin{array}{l}\text { cis-1,2-Dichloroethene } \\
\text { cis-1,3-Dichloropropene } \\
\text { trans-1,2-Dichloroethene } \\
\text { trans-1,3-Dichloropropene } \\
\text { Acetone } \\
\text { Benzene } \\
\text { Bromodichloromethane } \\
\text { Bromoform } \\
\text { Bromomethane } \\
\text { Carbon disulfide } \\
\text { Carbon tetrachloride } \\
\text { Chlorobenzene } \\
\text { Chloroethane } \\
\text { Chloroform } \\
\text { Chloromethane } \\
\text { Dibromochloromethane } \\
\text { Ethyl benzene } \\
\text { Methylene chloride } \\
\text { Styrene } \\
\text { Tetrachloroethene } \\
\text { Toluene } \\
\text { Trichloroethene } \\
\text { Vinyl chloride } \\
\text { Xylene (meta + para) } \\
\text { Xylene (ortho) }\end{array}$ & $\begin{array}{l}\mu g / k g \\
\mu g / k g \\
\mu g / k g \\
\mu g / k g \\
\mu g / k g \\
\mu g / k g \\
\mu g / k g \\
\mu g / k g \\
\mu g / k g \\
\mu g / k g \\
\mu g / k g \\
\mu g / k g \\
\mu g / k g \\
\mu g / k g \\
\mu g / k g \\
\mu g / k g \\
\mu g / k g \\
\mu g / k g \\
\mu g / k g \\
\mu g / k g \\
\mu g / k g \\
\mu g / k g \\
\mu g / k g \\
\mu g / k g \\
\mu g / k g\end{array}$ & $\begin{array}{r}5 \\
5 \\
5 \\
5 \\
5 \\
5 \\
5 \\
5 \\
10 \\
5 \\
5 \\
5 \\
10 \\
5 \\
10 \\
5 \\
5 \\
5 \\
5 \\
5 \\
5 \\
5 \\
10 \\
5 \\
5\end{array}$ \\
\hline
\end{tabular}


Table B.1 (continued)

\begin{tabular}{|c|c|c|c|c|c|c|}
\hline Analysis & Units & $\begin{array}{c}A \\
\text { horizon }\end{array}$ & Qualifier & $\begin{array}{l}\text { A horizon } \\
\text { field dup }\end{array}$ & Qualifier & \\
\hline $\begin{array}{l}\text { 1,1-Dichloroethane } \\
\text { 1,1-Dichloroethene } \\
1,1,1-\text { Trjchloroethane } \\
1,1,2-\text { Trichloroethane } \\
1,1,2,2-\text { Tetrochloroethane } \\
\text { 1,2-Dichloroethane } \\
\text { 1,2-Dichloropropane } \\
\text { 2--Butanone } \\
\text { 2-Hexanone } \\
\text { 4-Hethyl-2-pentanone }\end{array}$ & $\begin{array}{l}\mu g / \mathrm{kg} \\
\mu g / \mathrm{kg} \\
\mu g / \mathrm{kg} \\
\mu g / \mathrm{kg} \\
\mu g / \mathrm{kg} \\
\mu g / \mathrm{kg} \\
4 g / \mathrm{kg} \\
\mu g / \mathrm{kg} \\
\mu g / \mathrm{kg} \\
\mu g / \mathrm{kg}\end{array}$ & $\begin{array}{r}5 \\
5 \\
5 \\
5 \\
5 \\
5 \\
5 \\
5 \\
50 \\
50\end{array}$ & $\begin{array}{l}\mathbf{U} \\
\mathbf{U} \\
\mathbf{U} \\
\mathbf{U} \\
\mathbf{U} \\
\mathbf{U} \\
\mathbf{U} \\
\mathbf{B J} \\
\mathbf{U} \\
\mathbf{U}\end{array}$ & . & . & $\because A$ \\
\hline
\end{tabular}

Location=ROA; Fomation =DISMAL GAP; Site=7; Samp id=3185; Dup id=' '; Phase=1

\begin{tabular}{|c|c|c|}
\hline 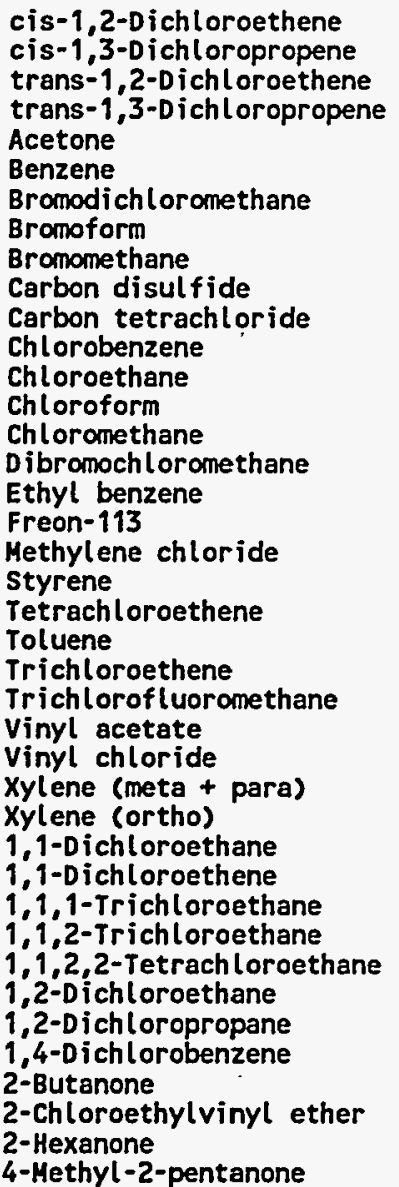 & 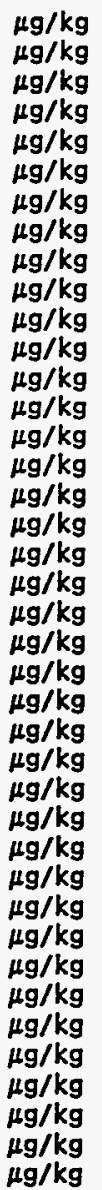 & $\begin{array}{r}5 \\
5 \\
5 \\
5 \\
7 \\
5 \\
5 \\
5 \\
10 \\
5 \\
5 \\
5 \\
10 \\
5 \\
10 \\
5 \\
5 \\
5 \\
5 \\
5 \\
5 \\
5 \\
5 \\
5 \\
5 \\
10 \\
5 \\
5 \\
5 \\
5 \\
5 \\
5 \\
5 \\
5 \\
5 \\
5 \\
100 \\
5 \\
50 \\
50\end{array}$ \\
\hline
\end{tabular}

Location=ROA; Formation=DISMAL GAP; Site=8; Samp id=3186; Dup id=' '; Phase=1

cis-1,2-Dichloroethene
cis-1,3-Dichloropropene
trans-1,2-Dichloroethene
trans-1,3-Dichloropropene
Acetone
Benzene
Bromodichloromethane
Bromoform
Bromomethane
Carbon disulfide
Carbon tetrachloride
Chlorobenzene
Chloroethane
Chloroform

$\begin{array}{lrl}\mu \mathrm{g} / \mathrm{kg} & 5 & U \\ \mu \mathrm{g} / \mathrm{kg} & 5 & U \\ \mu \mathrm{g} / \mathrm{kg} & 5 & U \\ \mu \mathrm{g} / \mathrm{kg} & 5 & U \\ \mu \mathrm{g} / \mathrm{kg} & 7 & J \\ \mu \mathrm{g} / \mathrm{kg} & 5 & U \\ \mu \mathrm{g} / \mathrm{kg} & 5 & U \\ \mu \mathrm{g} / \mathrm{kg} & 5 & U \\ \mu \mathrm{g} / \mathrm{kg} & 10 & U \\ \mu \mathrm{g} / \mathrm{kg} & 5 & U \\ \mu \mathrm{g} / \mathrm{kg} & 5 & U \\ \mu \mathrm{g} / \mathrm{kg} & 5 & U \\ \mu \mathrm{g} / \mathrm{kg} & 10 & U \\ \mu \mathrm{g} / \mathrm{kg} & 5 & U\end{array}$


B-70

Table B.1 (continued)

\begin{tabular}{|c|c|c|c|c|c|}
\hline Analysis & Units & $\begin{array}{c}\text { A } \\
\text { horizon }\end{array}$ & Qualifier & $\begin{array}{l}\text { A horizon } \\
\text { field dup }\end{array}$ & Qualifier \\
\hline $\begin{array}{l}\text { Chloromethane } \\
\text { Dibromochloromethane } \\
\text { Ethyl benzene } \\
\text { Freon-113 } \\
\text { Hethylene chloride } \\
\text { Styrene } \\
\text { Tetrachloroethene } \\
\text { Toluene } \\
\text { Trichloroethene } \\
\text { Trichlorofluoromethane } \\
\text { Vinyl acetate } \\
\text { Vinyl chloride } \\
\text { Xylene (meta + para) } \\
\text { xylene (ortho) } \\
\text { 1,1-Dichloroethane } \\
\text { 1,1-Dichloroethene } \\
\text { 1,1,1-Trichloroethane } \\
\text { 1,1,2-Trichloroethane } \\
\text { 1,1,2,2-Tetrachloroethane } \\
\text { 1,2-Dichloroethane } \\
\text { 1,2-Dichloropropane } \\
\text { 1,4-Dichlorobenzene } \\
\text { 2--Butanone } \\
\text { 2-Chloroethylvinyl ether } \\
\text { 2-Hexanone } \\
\text { 4- Methyl-2-pentanone }\end{array}$ & 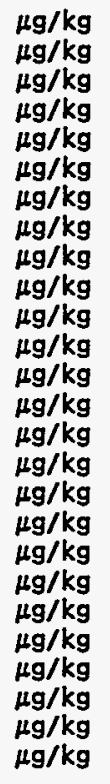 & $\begin{array}{r}10 \\
5 \\
5 \\
5 \\
5 \\
5 \\
5 \\
5 \\
5 \\
5 \\
5 \\
10 \\
5 \\
5 \\
5 \\
5 \\
8 \\
5 \\
5 \\
5 \\
5 \\
5 \\
100 \\
5 \\
50 \\
50\end{array}$ & $\begin{array}{l}U \\
U \\
U \\
U \\
U \\
U \\
U \\
U \\
U \\
U \\
U \\
U \\
U \\
U \\
U \\
U \\
\\
U \\
U \\
U \\
U \\
U \\
U \\
U \\
U \\
U\end{array}$ & & . \\
\hline \multicolumn{6}{|c|}{ Location $=$ ROA; Formation =DISMAL GAP; Site=9; Samp id=3200; Dup id=' '; Phase=1 } \\
\hline $\begin{array}{l}\text { cis-1,2-Dichloroethene } \\
\text { cis-1,3-D ichloropropene } \\
\text { trans-1,2-D ichloroethene } \\
\text { trans-1,3-D ichloropropene } \\
\text { Acetone } \\
\text { Benzene } \\
\text { Bromodichloromethane } \\
\text { Bromoform } \\
\text { Bromomethane } \\
\text { Carbon disulfide } \\
\text { Carbon tetrachloride } \\
\text { Chlorobenzene } \\
\text { Chloroethane } \\
\text { Chloroform } \\
\text { Chloromethane } \\
\text { Dibromochloromethane } \\
\text { Ethyl benzene } \\
\text { Methylene chloride } \\
\text { Styrene } \\
\text { Tetrachloroethene } \\
\text { Toluene } \\
\text { Trichloroethene } \\
\text { Vinyl chloride } \\
\text { Xylene (meta + para) } \\
\text { Xylene (ortho) } \\
1,1-D \text { ichloroethane } \\
1,1-D i c h l o r o e t h e n e \\
1,1,1-\text { Trichloroethane } \\
1,1,2-\text {-Trichloroethane } \\
1,1,2,2-T e t r a c h l o r o e t h a n e \\
1,2-D \text { ichloroethane } \\
1,2-D \text { ichloropropane } \\
2-\text {-Butanone } \\
2-\text { Hexanone } \\
\text { 4-Methyl-2-pentanone } \\
\end{array}$ & 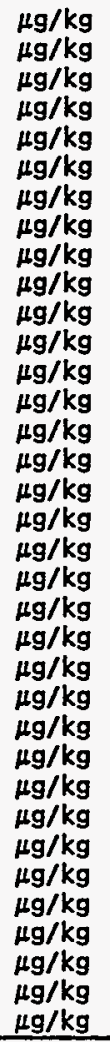 & $\begin{array}{r}5 \\
5 \\
5 \\
5 \\
100 \\
5 \\
5 \\
5 \\
10 \\
5 \\
5 \\
5 \\
10 \\
5 \\
10 \\
5 \\
5 \\
5 \\
5 \\
5 \\
5 \\
5 \\
10 \\
5 \\
5 \\
5 \\
5 \\
5 \\
5 \\
5 \\
5 \\
5 \\
100 \\
50 \\
50 \\
\end{array}$ & $\begin{array}{l}U \\
U \\
U \\
\mathbf{U} \\
\mathbf{U} \\
\mathbf{U} \\
\mathbf{U} \\
\mathbf{U} \\
\mathbf{U} \\
\mathbf{U} \\
\mathbf{U} \\
\mathbf{U} \\
\mathbf{U} \\
\mathbf{U} \\
\mathbf{U} \\
\mathbf{U} \\
\mathbf{U} \\
\mathbf{U} \\
\mathbf{U} \\
\mathbf{U} \\
\mathbf{U} \\
\mathbf{U} \\
\mathbf{U} \\
\mathbf{U} \\
\mathbf{U} \\
\mathbf{U} \\
\mathbf{U} \\
\mathbf{U} \\
\mathbf{U} \\
\mathbf{U} \\
\mathbf{U} \\
\mathbf{U} \\
\mathbf{U} \\
\mathbf{U} \\
\mathbf{U} \\
\mathbf{U}\end{array}$ & & \\
\hline
\end{tabular}


Table B.2 Weighted gamma screening results for soil samples

\begin{tabular}{|c|c|c|c|c|c|c|c|}
\hline \multirow[b]{2}{*}{ Analysis } & \multicolumn{6}{|c|}{ Depth $(\mathrm{cm})$} & \multirow[b]{2}{*}{$25-30$} \\
\hline & Units & $00-05$ & $05-10$ & $10-15$ & $15-20$ & $20-25$ & \\
\hline \multicolumn{8}{|c|}{$\begin{array}{c}\text { Location }=A N D ; \text { Formation }=C O P P E R \text { RIDGE; Site }=31 ; 00-05=2203 ; 05-10=2204 ; 10-15= \\
15-20=2206 ; 20-25=2207 ; 25-30=2208 ; \text { Phase }=2\end{array}$} \\
\hline $\begin{array}{l}\text { Cesium-137 } \\
\text { Potassium-40 } \\
\text { Radium-226 } \\
\text { Thor ium-232 } \\
\text { Uranium-235 } \\
\text { Uranium-238 }\end{array}$ & $\begin{array}{l}\mathrm{pCi} / \mathrm{g} \\
\mathrm{pCi} \mathrm{g} \\
\mathrm{pCi} / \mathrm{g} \\
\mathrm{pCi} / \mathrm{g} \\
\mathrm{pCi} / \mathrm{g} \\
\mathrm{pCi} \mathrm{g}\end{array}$ & $\begin{array}{l}0.73256 \\
1.73709 \\
0.50704 \\
0.35396 \\
0.22720 \\
1.87793\end{array}$ & $\begin{array}{l}0.37976 \\
1.91586 \\
0.55514 \\
0.44699 \\
0.05335 \\
1.02629\end{array}$ & $\begin{array}{l}0.10332 \\
2.10093 \\
0.60491 \\
0.51127\end{array}$ & $\begin{array}{l}0.06438 \\
2.45081 \\
0.59503 \\
0.44704 \\
0.07322 \\
2.46377\end{array}$ & $\begin{array}{l}0.03224 \\
2.01757 \\
0.61582 \\
0.48347 \\
0.08380 \\
1.80648\end{array}$ & $\begin{array}{l}0.00804 \\
2.12749 \\
0.60063 \\
0.52314 \\
0.05514 . \\
0.99122\end{array}$ \\
\hline
\end{tabular}

Location $=A N D ;$ Formation $=$ COPPER RIDGE; Site $=32 ; 00-05=2212 ; 05-10=2213 ; 10-15=2214 ;$ $15-20=2215 ; 20-25=2216 ; 25-30=2217 ;$ Phase $=2$

Cesium- 137 Potassium-40 Radium-226 Thorium-232 Uranium-235 Uranium-238

$\begin{array}{ll}\mathrm{pCi} / \mathrm{g} & 0.78489 \\ \mathrm{pCi} / \mathrm{g} & 1.51591 \\ \mathrm{pCi} / \mathrm{g} & 0.52326 \\ \mathrm{pCi} / \mathrm{g} & 0.63228 \\ \mathrm{pCi} / \mathrm{g} & 0.13848 \\ \mathrm{pCi} / \mathrm{g} & 2.21315\end{array}$

0.18635
2.47573
0.67759
0.56257
0.10924
2.03654

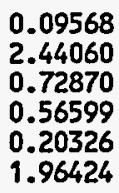

0.02333
2.82340
0.78198
0.58130
0.13177
1.97959

$\begin{array}{ll}0.00318 & 0.00304 \\ 3.28989 & 4.18094 \\ 0.72411 & 0.78168 \\ 0.57422 & 0.69264 \\ 0.13257 & 0.08669 \\ 2.23510 & 1.58676\end{array}$

Location $=A N D ;$ Formation $=$ COPPER RIDGE; Site $=33 ; 00-05=2221 ; 05-10=2222 ; 10-15=2223$; $15-20=2224 ; 20-25=2225 ; 25-30=2226 ;$ Phase $=2$

\begin{tabular}{|c|c|c|c|c|c|c|c|}
\hline $\begin{array}{l}\text { Cesium-137 } \\
\text { Potassium-40 } \\
\text { Radium-226 } \\
\text { Thorium-232 } \\
\text { Uranium-235 } \\
\text { Uranium-238 }\end{array}$ & $\begin{array}{l}\mathrm{pCi} / \mathrm{g} \\
\mathrm{pCi} / \mathrm{g} \\
\mathrm{pCi} / \mathrm{g} \\
\mathrm{pCi} / \mathrm{g} \\
\mathrm{pCi} / \mathrm{g} \\
\mathrm{pCi} / \mathrm{g}\end{array}$ & $\begin{array}{l}0.86805 \\
1.77329 \\
0.56678 \\
0.34199 \\
0.13487 \\
0.60911\end{array}$ & $\begin{array}{l}0.21344 \\
2.26005 \\
0.61646 \\
0.40075 \\
0.17374 \\
1.99912\end{array}$ & $\begin{array}{l}0.08081 \\
2.34045 \\
0.78564 \\
0.45246 \\
0.19540 \\
2.17822\end{array}$ & $\begin{array}{l}0.04379 \\
2.42785 \\
0.73838 \\
0.44757 \\
0.11977 \\
0.87519\end{array}$ & $\begin{array}{l}0.03354 \\
2.41589 \\
0.74191 \\
0.45138 \\
0.11891 \\
1.66225\end{array}$ & $\begin{array}{l}0.00490 \\
2.58916 \\
0.69533 \\
0.47055 \\
0.07893 \\
1.26452\end{array}$ \\
\hline
\end{tabular}

Location $=A N D ;$ Formation $=C O P P E R$ RIDGE; Site $=34 ; 00-05=2230 ; 05-10=2231 ; 10-15=2232$; $15-20=2233 ; 20-25=2234 ; 25-30=2235 ;$ Phase $=2$

$\begin{array}{llllllll}\text { Cesium-137 } & \text { pCi/g } & 0.72579 & 0.49465 & 0.36771 & 0.18624 & 0.12188 & 0.05804 \\ \text { Potassium-40 } & \text { pCi/g } & 3.47889 & 3.01606 & 3.26976 & 2.74865 & 3.08371 & 3.20147 \\ \text { Radium-226 } & \text { pCi/g } & 0.95364 & 0.94179 & 0.98998 & 0.91094 & 0.87060 & 0.88093 \\ \text { Thorium-232 } & \text { pCi/g } & 0.61858 & 0.64955 & 0.65605 & 0.60943 & 0.58014 & 0.58501 \\ \text { Uranium-235 } & \text { pCi/g } & 0.19392 & 0.16706 & 0.15656 & 0.14339 & 0.05529 & 0.06248 \\ \text { Uranium-238 } & \text { pCi/g } & 2.57243 & 1.69957 & 1.53984 & 2.36820 & 1.99701 & 1.24430\end{array}$

Location $=A N D ;$ Formation $=C O P P E R$ RIDGE; Site $=35 ; 00-05=2239 ; 05-10=2240 ; 10-15=2241$; $15-20=2242 ; 20-25=2243 ; 25-30=2244 ;$ Phase $=2$

$\begin{array}{llllllll}\text { Cesium-137 } & \text { pCi/g } & 0.53150 & 0.34390 & 0.07837 & 0.11647 & 0.00954 & 0.00458 \\ \text { Potassium-40 } & \text { pCi/g } & 2.74278 & 4.17352 & 3.08020 & 3.97701 & 4.17611 & 3.97210 \\ \text { Radium-226 } & \text { PCi/g } & 0.75353 & 0.88048 & 0.90777 & 0.83604 & 0.85575 & 0.75441 \\ \text { Thorium-232 } & \text { PCi/g } & 0.83221 & 0.77173 & 0.72306 & 0.69508 & 0.78523 & 0.75844 \\ \text { Uranium-235 } & \text { pCi/g } & 0.18258 & 0.19934 & 0.27231 & 0.18573 & 0.07653 & 0.05508 \\ \text { Uranium-238 } & \text { PCi/g } & 2.40473 & 3.34121 & 3.20313 & 3.02868 & 1.60243 & 1.42560\end{array}$

Location $=A N D ;$ Formation $=C O P P E R$ RIDGE; Site $=36 ; 00-05=2248 ; 05-10=2249 ; 10-15=2250$; $15-20=2251 ; 20-25=2252 ; 25-30=2253 ;$ Phase $=2$

$\begin{array}{llllllll}\text { Cesium-137 } & \mathrm{pCi} / \mathrm{g} & 0.47755 & 0.17720 & 0.15596 & 0.02985 & 0.02905 & 0.00658 \\ \text { Potassium-40 } & \mathrm{pCi} / \mathrm{g} & 3.38401 & 3.09831 & 3.38601 & 2.97470 & 2.85441 & 3.77005 \\ \text { Radium-226 } & \mathrm{pCi} / \mathrm{g} & 0.91612 & 0.87731 & 0.86417 & 0.83839 & \mathbf{0 . 7 8 1 5 5} & 0.78228 \\ \text { Thorium-232 } & \mathrm{pCi} / \mathrm{g} & 0.57038 & 0.57493 & 0.57045 & 0.58378 & 0.58734 & 0.65262 \\ \text { Uranium-235 } & \mathrm{pCi} / \mathrm{g} & 0.19948 & 0.18655 & 0.17641 & 0.17061 & 0.15859 & 0.07353 \\ \text { Uranium-238 } & \mathrm{pCi} / \mathrm{g} & \mathbf{0 . 8 8 4 6 4} & 2.72216 & 2.84852 & 3.19673 & 1.83871 & 1.51672\end{array}$


Table B.2 (continued)

\begin{tabular}{llllllll}
\hline & \multicolumn{8}{c}{ Depth $(\mathrm{cm})$} \\
\cline { 2 - 7 } Analysis & Units & $00-05$ & $05-10$ & $10-15$ & $15-20$ & $20-25$ & $25-30$ \\
\hline
\end{tabular}

Location $=A N D ;$ Formation $=C O P P E R$ RIDGE; Site $=37 ; 00-05=2287 ; 05-10=2288 ; 10-15=2289$; $15-20=2290 ; 20-25=2291 ; 25-30=2292 ;$ Phase $=2$

$\begin{array}{llllllll}\text { Cesium-137 } & \text { pCi/g } & 1.07299 & 0.69610 & 0.57877 & 0.37496 & 0.05467 & 0.00813 \\ \text { Potassium-40 } & \text { pCi/g } & 3.78546 & 2.68513 & 2.50862 & 2.38283 & 3.25296 & 4.80193 \\ \text { Radium-226 } & \text { pCi/g } & 1.05112 & 0.82304 & 0.91127 & 0.78462 & 0.82812 & 1.03727 \\ \text { Thorium-232 } & \text { pCi/g } & 0.70981 & 0.58473 & 0.54484 & 0.48605 & 0.47490 & 0.60464 \\ \text { Uranium-235 } & \text { pCi/g } & 0.22852 & 0.12887 & 0.15109 & 0.14342 & 0.05152 & 0.10662 \\ \text { Uranium-238 } & \text { pCi/g } & 2.86259 & 3.95884 & 0.28402 & 1.71699 & 0.83669 & 1.91426\end{array}$

Location $=A N D ;$ Formation $=C O P P E R$ RIDGE; Site $=38 ; 00-05=2296 ; 05-10=2297 ; 10-15=2298$; $15-20=2299 ; 20-25=2300 ; 25-30=2301 ;$ Phase $=2$

$\begin{array}{llllllll}\text { Cesium-137 } & \text { pCi/g } & 1.02183 & 0.61949 & 0.30099 & 0.08881 & 0.03800 & 0.00725 \\ \text { Potassium-40 } & \text { pCi/g } & 4.92117 & 3.82435 & 4.38945 & 3.54379 & 4.02833 & 3.84108 \\ \text { Radium-226 } & \text { pCi/g } & 0.77056 & 0.65535 & 0.75966 & 0.67264 & 0.69858 & 0.69676 \\ \text { Thorium-232 } & \text { pCi/g } & 0.55760 & 0.44787 & 0.48326 & 0.40735 & 0.43187 & 0.41008 \\ \text { Uranium-235 } & \text { pCi/g } & 0.22540 & 0.15650 & 0.13089 & 0.03143 & 0.03642 & 0.06034 \\ \text { Uranium-238 } & \text { pCi/g } & 4.28717 & 3.04269 & 2.38541 & 0.93944 & 1.08003 & 1.18812\end{array}$

Location $=A N D ;$ Formation $=C O P P E R$ RIDGE; Site $=39 ; 00-05=2331 ; 05-10=2332 ; 10-15=2333$; $15-20=2334 ; 20-25=2335 ; 25-30=2336 ;$ Phase $=2$

$\begin{array}{llllllll}\text { Cesium-137 } & \text { pCi/g } & 0.44619 & 0.34745 & 0.19369 & 0.11172 & 0.02742 & 0.01409 \\ \text { Potassium-40 } & \text { pCi/g } & 2.33674 & 2.91416 & 2.90200 & 2.67502 & 3.02655 & 2.53587 \\ \text { Radium-226 } & \text { pCi/g } & 0.87742 & 0.98273 & 0.99521 & 0.87104 & 0.81176 & 0.76207 \\ \text { Thorium-232 } & \text { pCi/g } & 0.62021 & 0.69604 & 0.72401 & 0.63007 & 0.61198 & 0.63045 \\ \text { Uranium-235 } & \text { pCi/g } & 0.18926 & 0.10230 & 0.15834 & 0.11007 & 0.07944 & 0.08620 \\ \text { Uranium-238 } & \text { pCi/g } & 4.09139 & 2.68236 & 2.76682 & 2.53132 & 3.05158 & 0.90203\end{array}$

Location $=A N D ;$ Formation $=C O P P E R$ RIDGE; Site $=40 ; 00-05=2322 ; 05-10=2323 ; 10-15=2324$; $15-20=2325 ; 20-25=2326 ; 25-30=2327 ;$ Phase $=2$

$\begin{array}{llllllll}\text { Cesium-137 } & \text { pCi/g } & 0.85211 & 0.39631 & 0.07536 & 0.03244 & 0.01784 & 0.00179 \\ \text { Potassium-40 } & \text { pCi/g } & 3.90597 & 4.28751 & 4.56551 & 4.96497 & 4.48585 & 3.86398 \\ \text { Radium-226 } & \text { pCi/g } & 0.94750 & 0.96505 & 0.92617 & 0.95940 & 0.97082 & 0.93871 \\ \text { Thorium-232 } & \text { pCi/g } & 0.83800 & 0.90142 & 0.98676 & 1.02443 & 0.98031 & 0.94777 \\ \text { Uranium-235 } & \text { pCi/g } & 0.19173 & 0.18276 & 0.07901 & 0.10528 & 0.11387 & 0.06734 \\ \text { Uranium-238 } & \text { pCi/g } & 5.72968 & 3.86449 & 3.26952 & 2.32441 & 1.17050 & 1.38489\end{array}$

Location $=A N D ;$ Formation $=C O P P E R$ RIDGE; Site $=41 ; 00-05=2314 ; 05-10=2315 ; 10-15=2316$; $15-20=2317 ; 20-25=2318 ; 25-30=2319 ;$ Phase $=2$

\begin{tabular}{|c|c|c|c|c|c|c|c|}
\hline $\begin{array}{l}\text { Cesium-137 } \\
\text { Potassium-40 } \\
\text { Radium-226 } \\
\text { Thorium-232 } \\
\text { Uranium-235 } \\
\text { Uranium-238 }\end{array}$ & $\begin{array}{l}\mathrm{pCi} / \mathrm{g} \\
\mathrm{pCi} / \mathrm{g} \\
\mathrm{pCi} / \mathrm{g} \\
\mathrm{pCi} / \mathrm{g} \\
\mathrm{pCi} / \mathrm{g} \\
\mathrm{pCi} / \mathrm{g}\end{array}$ & $\begin{array}{l}1.13664 \\
4.48473 \\
0.99084 \\
0.89809 \\
0.19794 \\
1.45992\end{array}$ & $\begin{array}{l}0.91528 \\
4.87910 \\
1.08735 \\
0.91022 \\
0.19653 \\
1.69972\end{array}$ & $\begin{array}{l}0.51366 \\
4.86342 \\
1.11169 \\
0.90059 \\
0.21888 \\
2.79876\end{array}$ & $\begin{array}{l}0.08159 \\
4.51613 \\
1.07832 \\
0.90530 \\
0.12762 \\
1.60756\end{array}$ & $\begin{array}{l}0.02022 \\
5.48717 \\
1.08703 \\
1.01890 \\
0.08010 \\
2.22816\end{array}$ & $\begin{array}{l}0.00519 \\
4.97048 \\
1.00715 \\
0.92744 \\
0.10685 \\
1.76352\end{array}$ \\
\hline
\end{tabular}

Location $=A N D ;$ Formation $=C O P P E R$ RIDGE; Site $=42 ; 00-05=2305 ; 05-10=2306 ; 10-15=2307$; $15-20=2308 ; 20-25=2309 ; 25-30=2310 ;$ Phase $=2$

\begin{tabular}{|c|c|c|c|c|c|c|c|}
\hline $\begin{array}{l}\text { Cesium- } 137 \\
\text { Potassium-40 } \\
\text { Radium-226 } \\
\text { Thorium-232 } \\
\text { Uranium-235 } \\
\text { Uranium-238 }\end{array}$ & $\begin{array}{l}p C i / g \\
p C i / g \\
p C i / g \\
p C i / g \\
p C i / g \\
p C i / g\end{array}$ & $\begin{array}{l}0.66215 \\
4.56549 \\
1.25763 \\
0.98103 \\
0.19771 \\
2.86540\end{array}$ & $\begin{array}{l}0.33570 \\
4.08042 \\
1.32136 \\
0.92221 \\
0.19160 \\
2.77620\end{array}$ & $\begin{array}{l}0.15513 \\
4.74424 \\
1.30281 \\
0.94853 \\
0.12521 \\
3.04342\end{array}$ & $\begin{array}{l}0.03418 \\
5.25863 \\
1.19369 \\
0.99471 \\
0.10293 \\
3.50521\end{array}$ & $\begin{array}{l}0.02752 \\
5.20465 \\
1.09153 \\
0.89235 \\
0.11995 \\
2.18517\end{array}$ & $\begin{array}{l}0.01134 \\
5.39969 \\
1.13332 \\
0.95286 \\
0.10290 \\
1.68820\end{array}$ \\
\hline
\end{tabular}


B-73

Table B.2 (continued)

\begin{tabular}{|c|c|c|c|c|c|c|c|}
\hline \multirow[b]{2}{*}{ Analysis } & \multicolumn{6}{|c|}{ Depth (cm) } & \multirow[b]{2}{*}{$25-30$} \\
\hline & Units & $00-05$ & $05-10$ & $10-15$ & $15-20$ & $20-25$ & \\
\hline \multicolumn{8}{|c|}{$\begin{array}{l}\text { Location=AND; Formation=DISMAL GAP; Site }=1 ; 00-05=2161 ; 05-10=2162 ; 10-15=2163 ; 15 \\
20-25=2165 ; 25-30=2166 ; \text { Phase }=1\end{array}$} \\
\hline $\begin{array}{l}\text { Cesium-137 } \\
\text { Potassium-40 } \\
\text { Redium-226 } \\
\text { Thorium-232 } \\
\text { Uranium-235 } \\
\text { Uranium-238 }\end{array}$ & $\begin{array}{l}\mathrm{pCi} / \mathrm{g} \\
\mathrm{pCi} / \mathrm{g} \\
\mathrm{pCi} \mathrm{g} \\
\mathrm{pCi} / \mathrm{g} \\
\mathrm{pCi} \mathrm{g} \\
\mathrm{pCi} / \mathrm{g}\end{array}$ & $\begin{array}{r}1.0405 \\
15.3184 \\
0.5906 \\
0.8824\end{array}$ & $\begin{array}{r}0.1665 \\
17.2832 \\
0.6374 \\
0.9491 \\
1.5935\end{array}$ & $\begin{array}{r}0.0405 \\
24.2441 \\
0.6665 \\
1.1162\end{array}$ & $\begin{array}{r}28.9573 \\
0.5316 \\
1.1710 \\
\\
3.2284\end{array}$ & $\begin{array}{r}27.5187 \\
0.5379 \\
1.1096\end{array}$ & $\begin{array}{r}0.0024 \\
26.7698 \\
0.5178 \\
1.1050 \\
0.0548 \\
1.0655\end{array}$ \\
\hline
\end{tabular}

Location $=A N D ;$ Formation $=D I S M A L$ GAP; Site $=10 ; 00-05=2151 ; 05-10=2152 ; 10-15=2153 ; 15-20=2154$; $20-25=2155 ; 25-30=2156 ;$ Phase $=1$

Cesium-137

Potassium-40

Radium-226

Thorium-232

Uranium-235

Uranium-238

$\begin{array}{lr}p C i / g & 1.735 \\ \text { pCi/g } & 11.801 \\ \text { pCi/g } & 0.758 \\ \text { pCi/g } & 0.970 \\ \text { pCi/g } & 0.223 \\ \text { pCi/g } & 4.079\end{array}$

$\begin{array}{rr}0.4507 & 0.0784 \\ 11.8693 & 12.2725 \\ 0.6983 & 0.7517 \\ 0.8848 & 0.9176 \\ 0.1365 & 0.1481 \\ 2.6709 & 1.0747\end{array}$

0.1257

12.5808

0.7760

0.9236

0.1374

0.6061

0.0380

16.4545

0.7012

1.0609

0.1045

1.2632

0.0010

18.3984

0.7227

1.0965

0.0549

1.0706

Location $=A N D$; Formation $=D I S M A L$ GAP; Sire $=11 ; 00-05=2008 ; 05-10=2009 ; 10-15=2010 ; 15-20=2011$; $20-25=2012 ; 25-30=2013 ;$ Phase $=1$

$\begin{array}{llrrrrrr}\text { Cesium-137 } & \text { PCi/g } & 1.3817 & 0.3935 & 0.1478 & 0.0640 & 0.0289 & 0.0109 \\ \text { Potassium-40 } & \text { PCi/g } & 27.4654 & 26.5695 & 27.4711 & 30.7996 & 34.7116 & 35.2470 \\ \text { Radium-226 } & \text { PCi/g } & 0.5614 & 0.5051 & 0.4774 & 0.5233 & 0.5704 & 0.6033 \\ \text { Thorium-232 } & \text { PCi/g } & 0.8008 & 0.8003 & 0.8681 & 0.9982 & 1.1924 & 1.2761 \\ \text { Uranium-235 } & \text { PCi/g } & 0.3359 & 0.1403 & 0.1355 & 0.0286 & 0.1508 & 0.0515 \\ \text { Uranium-238 } & \text { pCi/g } & 2.9494 & 2.7268 & 2.5674 & 1.3655 & 3.0768 & 1.9468\end{array}$

Location $=A N D$; Formation $=D I S M A L$ GAP; Site $=12 ; 00-05=2084 ; 05-10=2085 ; 10-15=2086 ; 15-20=2087$; $20-25=2088 ; 25-30=2089 ;$ Phase $=1$

$\begin{array}{llrrrrrr}\text { Cesium-137 } & \text { PCi/g } & 1.0709 & 0.1372 & 0.0255 & 0.0175 & 0.0314 & 0.0386 \\ \text { Potassium-40 } & \text { PCi/g } & 24.4291 & 26.2972 & 30.1365 & 32.0915 & 34.8006 & 33.3333 \\ \text { Radium-226 } & \text { PCi/g } & 0.5542 & 0.6450 & 0.6896 & 0.7044 & 0.7390 & 0.6715 \\ \text { Thorium-232 } & \text { PCi/g } & 0.7771 & 0.8903 & 0.9694 & 1.2797 & 1.3595 & 1.3283 \\ \text { Uranium-235 } & \text { pCi/g } & 0.1039 & & 0.1844 & 0.2166 & 0.1761 & 0.2364 \\ \text { Uranium-238 } & \text { pCi/g } & & & & 4.7411 & 3.5144 & 4.4903\end{array}$

Location $=A N D ;$ Formation $=D I S M A L$ GAP; Site $=19 ; 00-05=2124 ; 05-10=2125 ; 10-15=2126 ; 15-20=2127$; 20-25=2128; 25-30=2129; Phase $=1$

$\begin{array}{llrrrrrr}\text { Cesium-137 } & \text { PCj/g } & 2.1956 & 0.3944 & 0.0641 & 0.0341 & 0.0048 & 0.0092 \\ \text { Potassium-40 } & \text { pCj/g } & 10.8236 & 13.7935 & 14.9526 & 19.0090 & 21.5009 & 21.5915 \\ \text { Radium-226 } & \text { pCi/g } & 0.5843 & 0.7096 & 0.6770 & 0.6492 & 0.6780 & 0.6273 \\ \text { Thorium-232 } & \text { pCi/g } & 0.8043 & 1.0076 & 1.0909 & 1.2560 & 1.3327 & 1.2870 \\ \text { Uranium-235 } & \text { pCi/g } & 0.1024 & 0.1452 & 0.1432 & 0.1029 & 0.1650 & 0.0666 \\ \text { Uranium-238 } & \text { pCi/g } & 0.9936 & 0.8918 & 2.4189 & 2.7871 & 2.3963 & 1.3233\end{array}$

Location $=A N D$; Formation $=D I S M A L$ GAP; Site $=20 ; 00-05=2074 ; 05-10=2075 ; 10-15=2076 ; 15-20=2077$; $20-25=2078 ; 25-30=2079 ;$ Phase $=1$

$\begin{array}{llrrrrrr}\text { Cesium-137 } & \mathrm{pCi} / \mathrm{g} & 1.1134 & 0.0549 & 0.0363 & & 0.0403 & \\ \text { Potassium-40 } & \mathrm{pCi/g} & 17.3193 & 16.7671 & 18.7084 & 21.6877 & 20.6868 & 19.2249 \\ \text { Radium-226 } & \mathrm{pCi} / \mathrm{g} & 0.7066 & 0.6747 & 0.6899 & 0.7344 & 0.7655 & 0.5821 \\ \text { Thorium-232 } & \mathrm{pCi/g} & 1.0379 & 0.9686 & 1.1993 & 1.4205 & 1.4101 & 1.2856 \\ \text { Uranium-235 } & \mathrm{pCi/g} & & & & & 0.2252 & 0.0902 \\ \text { Uranium-238 } & \mathrm{pCi/g} & & & & & 4.0640 & \end{array}$


Table B.2 (continued)

\begin{tabular}{llllllll}
\hline & \multicolumn{8}{c}{ Depth (cm) } \\
\cline { 2 - 7 } Anatysis & Units & $00-05$ & $05-10$ & $10-15$ & $15-20$ & $20-25$ & $25-30$ \\
\hline
\end{tabular}

Location =AND; Formation $=$ DISMAL GAP; Site $=21 ; 00-05=2105 ; 05-10=2106 ; 10-15=2107 ; 15-20=2108$; $20-25=2109 ; 25-30=2110 ;$ Phase $=1$

$\begin{array}{llrrrrrr}\text { Cesium-137 } & \mathrm{pCi} / \mathrm{g} & 0.8445 & 0.1873 & 0.0844 & 0.0787 & 0.0172 & 0.0154 \\ \text { Potassium-40 } & \mathrm{pCi} / \mathrm{g} & 21.1842 & 21.1774 & 29.5182 & 28.1374 & 26.8110 & 28.8829 \\ \text { Radium-226 } & \mathrm{pCi} / \mathrm{g} & 0.4616 & 0.5328 & 0.5716 & 0.4946 & 0.5707 & 0.5243 \\ \text { Thorium-232 } & \mathrm{pCi} / \mathrm{g} & 0.8694 & 0.8480 & 1.1365 & 1.1462 & 1.1399 & 1.2445 \\ \text { Uranium-235 } & \mathrm{pCi/g} & & & & 0.1963 & 0.2800 & 0.2204 \\ \text { Uranium-238 } & \mathrm{pCi/g} & & & 5.6762 & 3.7402 & 4.0914 & 4.3602\end{array}$

Location =AND; Formation =DISMAL GAP; Site =22; 00-05=2094; 05-10=2095; 10-15=2096; 15-20=2097; 20-25=2098; $25-30=2099 ;$ Phase $=1$

$\begin{array}{llrrrrrr}\text { Cesium-137 } & \mathrm{pCi/g} & 0.5353 & 0.0534 & 0.0255 & 0.0269 & 0.0248 & 0.0075 \\ \text { Potassium-40 } & \mathrm{pCi/g} & 29.6099 & 26.6361 & 27.9045 & 25.3770 & 28.1517 & 29.2053 \\ \text { Radium-226 } & \mathrm{PCi} / \mathrm{g} & 0.6262 & 0.6201 & 0.6371 & 0.6038 & 0.6204 & 0.5593 \\ \text { Thorium-232 } & \mathrm{PCi} / \mathrm{g} & 1.0610 & 1.0666 & 1.2126 & 1.2661 & 1.1991 & 1.1141 \\ \text { Uranium-235 } & \mathrm{pCi/g} & 0.1576 & 0.1492 & 0.1560 & 0.1608 & 0.1619 & 0.0565 \\ \text { Uranium-238 } & \mathrm{pCi/g} & 3.1628 & 2.6898 & 3.2686 & 3.3346 & 2.9950 & 1.2268\end{array}$

Location $=A N D ;$ Formation =DISMAL GAP; Site =3; 00-05=2063; 05-10=2064; 10-15=2065; 15-20=2066; $20-25=2067 ; 25-30=2068 ;$ Phase $=1$

$\begin{array}{llrrrrrr}\text { Cesium-137 } & \mathrm{PCi} / \mathrm{g} & 0.5910 & 0.1505 & 0.0473 & 0.0247 & 0.0148 & \\ \text { Potassium-40 } & \mathrm{PCi} / \mathrm{g} & 22.0278 & 20.0736 & 20.3150 & 22.0944 & 23.9437 & 22.9413 \\ \text { Radium-226 } & \mathrm{PCi} / \mathrm{g} & 0.5822 & 0.6817 & 0.5955 & 0.5933 & 0.6653 & 0.5522 \\ \text { Thorium-232 } & \mathrm{PCi} / \mathrm{g} & 1.1588 & 1.1267 & 1.1139 & 1.2530 & 1.3566 & 1.3722 \\ \text { Uranium-235 } & \mathrm{PCi/g} & 0.1369 & & 0.1372 & 0.0526 & & \\ \text { Uranium-238 } & \mathrm{PCi} / \mathrm{g} & & 2.1177 & & 1.6575 & & 4.4991\end{array}$

Location=AND; Formation $=D I S M A L$ GAP; Site $=4 ; 00-05=2043 ; 05-10=2044 ; 10-15=2045 ; 15-20=2046$; $20-25=2047 ; 25-30=2048 ;$ Phase $=1$

\begin{tabular}{|c|c|c|c|c|c|c|c|}
\hline $\begin{array}{l}\text { Cesium- } 137 \\
\text { Potassium-40 } \\
\text { Radium-226 } \\
\text { Thorium-232 } \\
\text { Uranium-235 } \\
\text { Uranium- } 238\end{array}$ & $\begin{array}{l}\mathrm{pCi} / \mathrm{g} \\
\mathrm{pCi} / \mathrm{g} \\
\mathrm{pCi} / \mathrm{g} \\
\mathrm{pCi} \mathrm{g} \\
\mathrm{pCi} / \mathrm{g} \\
\mathrm{pCi} / \mathrm{g}\end{array}$ & $\begin{array}{r}1.4287 \\
38.6418 \\
0.6041 \\
1.0622\end{array}$ & $\begin{array}{r}0.1510 \\
35.0762 \\
0.5853 \\
0.9718\end{array}$ & $\begin{array}{r}0.0498 \\
34.7543 \\
0.5594 \\
0.9949\end{array}$ & $\begin{array}{r}0.0627 \\
38.6450 \\
0.5266 \\
1.1156\end{array}$ & $\begin{array}{r}0.0692 \\
39.4480 \\
0.5971 \\
1.2681\end{array}$ & $\begin{array}{r}0.0263 \\
36.5679 \\
0.5300 \\
1.1060\end{array}$ \\
\hline \multicolumn{8}{|c|}{$\begin{array}{l}\text { Location }=A N D ; \text { Formation }=D I S M A L G A P ; \text { Site }=5 ; 00-05=2053 ; 05-10=2054 ; 10-15=2055 ; 1 \\
\qquad 20-25=2057 ; 25-30=2058 ; \text { Phase }=1\end{array}$} \\
\hline $\begin{array}{l}\text { Cesium-137 } \\
\text { Potassium-40 } \\
\text { Radium-226 } \\
\text { Thorium-232 } \\
\text { Uranium-235 } \\
\text { Uranium-238 }\end{array}$ & $\begin{array}{l}\mathrm{pCi} / \mathrm{g} \\
\mathrm{pCi} / \mathrm{g} \\
\mathrm{pCi} / \mathrm{g} \\
\mathrm{pCi} / \mathrm{g} \\
\mathrm{pCi} / \mathrm{g} \\
\mathrm{pCi} / \mathrm{g}\end{array}$ & $\begin{array}{r}0.7101 \\
42.2530 \\
0.5661 \\
1.1098\end{array}$ & $\begin{array}{r}0.0924 \\
42.8831 \\
0.5771 \\
1.1491 \\
0.2555\end{array}$ & $\begin{array}{r}0.0095 \\
42.2073 \\
0.5308 \\
1.1711 \\
\\
3.4697\end{array}$ & $\begin{array}{r}42.2013 \\
0.5410 \\
1.1772\end{array}$ & $\begin{array}{r}39.4710 \\
0.5666 \\
1.1599 \\
0.2688\end{array}$ & $\begin{array}{r}36.9445 \\
0.6190 \\
1.1139 \\
0.0803 \\
1.1683\end{array}$ \\
\hline
\end{tabular}

Location=AND; Formation =DISMAL GAP; Site=9; $00-05=2137 ; 05-10=2138 ; 10-15=2139 ; 15-20=2140$; $20-25=2141 ; 25-30=2142 ;$ Phase $=1$

Cesium- 137

Potassium-40

Radium-226

Thorium-232

Uranium-235

Uranium-238

$\begin{array}{lrr}\text { pCi/g } & 1.2969 & 0.2461 \\ \text { pCi/g } & 17.1819 & 18.9354 \\ \text { pCi/g } & 0.5175 & 0.7025 \\ \text { pCi/g } & 0.8142 & 1.2333 \\ \text { pCi/g } & & 0.4942 \\ \text { pCi/g } & 1.1784 & \end{array}$

$\begin{array}{rr}0.0855 & 0.0120 \\ 20.4686 & 22.2786 \\ 0.7756 & 0.8913 \\ 1.4551 & 1.4779 \\ & 0.2734 \\ & 5.1406\end{array}$

$\begin{array}{rr}0.0403 & 0.0043 \\ 22.6142 & 20.6699 \\ 0.7526 & 0.7397 \\ 1.5131 & 1.3998 \\ 0.2443 & 0.0979 \\ 4.5186 & 2.2496\end{array}$


Table B.2 (continued)

\begin{tabular}{llllllll}
\hline & \multicolumn{1}{c}{ Depth $(\mathrm{cm})$} \\
\cline { 2 - 8 } Analysis & Units & $00-05$ & $05-10$ & $10-15$ & $15-20$ & $20-25$ & $25-30$ \\
\hline
\end{tabular}

Location =ORR; Formation $=$ CHEPULTEPEC; Site $=50 ; 00-05=1847 ; 05-10=1848 ; 10-15=1849$; $15-20=1850 ; 20-25=1851 ; 25-30=1852 ;$ Phase $=2$

$\begin{array}{llllllll}\text { Cesium-137 } & \text { PCi/g } & 2.10241 & 0.15775 & 0.15342 & 0.08184 & 0.02196 & 0.01190 \\ \text { Potassium-40 } & \text { PCi/g } & 1.86521 & 1.64010 & 1.62390 & 1.87998 & 1.96848 & 2.04277 \\ \text { Radium-226 } & \text { PCi/g } & 0.84902 & 0.60450 & 0.80096 & 0.82490 & 0.76813 & 0.75780 \\ \text { Thorium-232 } & \text { PCi/g } & 0.85090 & 0.89647 & 0.73944 & 0.71344 & 0.76171 & 0.77049 \\ \text { Uranium-235 } & \text { PCi/g } & 0.27659 & 0.15540 & 0.06980 & 0.11901 & & 0.06413 \\ \text { Uranium-238 } & \text { PCi/g } & 4.98833 & 2.55921 & 2.21355 & 2.00319 & 1.78579 & 1.61368\end{array}$

Location $=O R R$; Formation $=$ CHEPULTEPEC; Site $=52 ; 00-05=1654 ; 05-10=1655 ; 10-15=1656$; $15-20=1657 ; 20-25=1658 ; 25-30=1659 ;$ Phase $=2$

$\begin{array}{llllllll}\text { Cesium-137 } & \text { PCi/g } & 0.94567 & 0.75691 & 0.21236 & 0.08661 & 0.02230 & 0.00585 \\ \text { Potassium-40 } & \text { PCi/g } & 1.83657 & 2.36038 & 2.40621 & 2.51101 & 2.53563 & 2.75268 \\ \text { Radium-226 } & \text { PCi/g } & 0.66880 & 0.92030 & 0.85986 & 0.86925 & 0.83692 & 0.83155 \\ \text { Thorium-232 } & \text { PCi/g } & 0.77927 & 0.79053 & 0.78731 & 0.70907 & 0.69291 & 0.66490 \\ \text { Uranium-235 } & \text { PCi/g } & 0.20318 & 0.17013 & 0.15691 & 0.30185 & 0.10554 & 0.07704 \\ \text { Uranium-238 } & \text { pCi/g } & 2.70509 & 2.42274 & 2.12015 & 1.70132 & 1.85996 & 1.23402\end{array}$

Location $=$ ORR; Formation $=$ CHEPULTEPEC; Site $=53 ; 00-05=1642 ; 05-10=1643 ; 10-15=1644 ;$ $15-20=1645 ; 20-25=1646 ; 25-30=1647 ;$ Phase $=2$

$\begin{array}{llllllll}\text { Cesium-137 } & \mathrm{PCi} / \mathrm{g} & 0.55692 & 0.34886 & 0.10377 & 0.02640 & 0.02519 & 0.00505 \\ \text { Potassium-40 } & \mathrm{PCi} / \mathrm{g} & 1.45076 & 2.12170 & 2.43330 & 2.52317 & 2.43012 & 2.29705 \\ \text { Radium-226 } & \mathrm{PCi/g} & \mathbf{0 . 4 3 2 6 7} & \mathbf{0 . 7 1 1 8 1} & 0.63744 & \mathbf{0 . 6 5 9 4 0} & 0.63295 & 0.52831 \\ \text { Thorium-232 } & \mathrm{PCi} / \mathrm{g} & \mathbf{0 . 4 4 1 7 1} & \mathbf{0 . 5 2 6 4 1} & \mathbf{0 . 4 8 0 8 7} & \mathbf{0 . 5 0 5 9 3} & \mathbf{0 . 4 7 3 4 5} & \mathbf{0 . 3 9 7 8 5} \\ \text { Uranium-235 } & \mathrm{PCi} / \mathrm{g} & \mathbf{0 . 0 8 8 0 0} & \mathbf{0 . 0 6 0 9 9} & \mathbf{0 . 1 0 4 3 6} & \mathbf{0 . 0 4 9 8 5} & \mathbf{0 . 0 1 2 6 1} & \mathbf{0 . 0 4 1 3 5}\end{array}$

Uranium-235

$\mathrm{pCi} / \mathrm{g} \quad 0.08800$

0.060990 .10436

$0.04985 \quad 0.01261 \quad 0.04135$

Location $=O R R ;$ Formation $=C H E P U L T E P E C ;$ Site $=66 ; 00-05=1821 ; 05-10=1822 ; 10-15=1823$; $15-20=1824 ; 20-25=1825 ; 25-30=1826 ;$ Phase $=2$

$\begin{array}{llllllll}\text { Cesium-137 } & \text { PCi/g } & 0.60910 & 0.21391 & 0.13298 & 0.02826 & 0.01737 & 0.00263 \\ \text { Potassium-40 } & \text { PCi/g } & 2.40029 & 2.47074 & 3.49271 & 3.68101 & 4.55206 & 6.08771 \\ \text { Radium-226 } & \text { PCi/g } & 0.67443 & 0.66434 & 0.82011 & 0.74611 & 0.74032 & 0.75872 \\ \text { Thorium-232 } & \text { PCi/g } & 0.48728 & 0.52861 & 0.59798 & 0.63006 & 0.84123 & 0.96222 \\ \text { Uranium-235 } & \text { PCi/g } & 0.19274 & 0.10026 & 0.16056 & 0.10993 & 0.06597 & 0.04559\end{array}$

Location $=$ ORR; Formation $=$ CHEPULTEPEC; Site $=68 ; 00-05=1787 ; 05-10=1788 ; 10-15=1789$; $15-20=1790 ; 20-25=1791 ; 25-30=1792 ;$ Phase $=2$

$\begin{array}{llllllll}\text { Cesium-137 } & \text { PCi/g } & 0.85939 & 0.56481 & 0.29776 & 0.08572 & 0.01469 & 0.00865 \\ \text { Potassium-40 } & \text { PCi/g } & 2.25573 & 2.18213 & 2.00510 & 2.37480 & 2.49411 & 2.69501 \\ \text { Radium-226 } & \text { PCi/g } & 0.70901 & 0.66641 & 0.76377 & 0.71273 & 0.76090 & 0.72938 \\ \text { Thorium-232 } & \text { pCi/g } & 0.48110 & 0.50472 & 0.47951 & 0.51198 & 0.50780 & 0.52553 \\ \text { Uranium-235 } & \text { PCi/g } & 0.11856 & 0.14985 & 0.14525 & 0.10973 & 0.12596 & 0.02980 \\ \text { Uranium-238 } & \text { PCi/g } & 2.04822 & 1.90646 & 2.31187 & 1.88291 & 1.79329 & 1.05443\end{array}$

Location =ORR; Formation $=$ CHEPULTEPEC; Site $=73 ; 00-05=1841 ; 05-10=1842 ; 10-15=1843$; $15-20=1844 ; 20-25=1845 ; 25-30=1846 ;$ Phase $=2$

\begin{tabular}{|c|c|c|c|c|c|c|c|}
\hline $\begin{array}{l}\text { Cesium-137 } \\
\text { Potassium-40 } \\
\text { Radium-226 } \\
\text { Thorium-232 } \\
\text { Uranium-235 } \\
\text { Uranium-238 }\end{array}$ & $\begin{array}{l}\mathrm{pCi} / \mathrm{g} \\
\mathrm{pCi} / \mathrm{g} \\
\mathrm{pCi} / \mathrm{g} \\
\mathrm{pCi} / \mathrm{g} \\
\mathrm{pCi} / \mathrm{g} \\
\mathrm{pCi} / \mathrm{g}\end{array}$ & $\begin{array}{l}1.72656 \\
3.99339 \\
0.87470 \\
0.61118 \\
0.13422 \\
3.25721\end{array}$ & $\begin{array}{l}0.74266 \\
3.34947 \\
0.86587 \\
0.55512 \\
0.24984 \\
2.56090\end{array}$ & $\begin{array}{l}0.30316 \\
3.84441 \\
0.85662 \\
0.52520 \\
0.07828 \\
2.77254\end{array}$ & $\begin{array}{l}0.02544 \\
5.33054 \\
0.86176 \\
0.62364 \\
1.76096\end{array}$ & $\begin{array}{l}0.03622 \\
8.42374 \\
1.08019 \\
0.92944 \\
0.22091 \\
3.88758\end{array}$ & $\begin{array}{l}0.02451 \\
8.59054 \\
1.19483 \\
0.98424 \\
0.11329 \\
2.29655\end{array}$ \\
\hline
\end{tabular}


Table B.2 (continued)

\begin{tabular}{llllllll}
\hline & \multicolumn{8}{c}{ Depth (cm) } \\
\cline { 2 - 7 } Analysis & Units & $00-05$ & $05-10$ & $10-15$ & $15-20$ & $20-25$ & $25-30$ \\
\hline
\end{tabular}

Location =ORR; Formation=CHEPULTEPEC; Site $=74 ; 00-05=1773 ; 05-10=1774 ; 10-15=1775$; $15-20=1776 ; 20-25=1777 ; 25-30=1778 ;$ Phase $=2$

$\begin{array}{llllllll}\text { Cesium-137 } & \text { pci/g } & 0.96025 & 0.26977 & 0.04590 & 0.02125 & 0.01732 & 0.00500 \\ \text { Potassium-40 } & \text { pCi/g } & 2.25082 & 1.91660 & 2.34893 & 2.74624 & 2.80257 & 3.75242 \\ \text { Radium-226 } & \text { pCi/g } & 0.59209 & 0.54595 & 0.69166 & 0.68604 & 0.64524 & 0.77262 \\ \text { Thorium-232 } & \text { pCi/g } & 0.37507 & 0.37835 & 0.40800 & 0.43511 & 0.45368 & 0.56593 \\ \text { Uranium-235 } & \text { pCi/g } & 0.05496 & 0.11169 & 0.13967 & 0.08435 & 0.10323 & 0.02788 \\ \text { Uranium-238 } & \text { pci/g } & 2.81928 & 2.03545 & 2.34166 & 2.32901 & 1.49243 & 2.01357\end{array}$

Location $=$ ORR; Formation $=$ CHEPULTEPEC; Site $=77 ; 00-05=1690 ; 05-10=1691 ; 10-15=1692$; $15-20=1693 ; 20-25=1694 ; 25-30=1695 ;$ Phase $=2$

$\begin{array}{llllllll}\text { Cesium-137 } & \mathrm{pCi} / \mathrm{g} & 1.49008 & 0.67372 & 0.07884 & 0.01549 & 0.00605 & 0.00173 \\ \text { Potassium-40 } & \mathrm{pCi/g} & 2.05836 & 2.47857 & 2.65682 & 2.71133 & 3.23189 & 2.85856 \\ \text { Radium-226 } & \mathrm{pCi/g} & 0.67308 & 0.72096 & 0.76309 & 0.70380 & 0.72528 & 0.78096 \\ \text { Thorium-232 } & \mathrm{pCi/g} & 0.60401 & 0.67281 & 0.65027 & 0.64995 & 0.70600 & 0.62037 \\ \text { Uranium-235 } & \mathrm{pCi/g} & 0.17543 & & 0.13445 & 0.09349 & 0.06048 & 0.06344 \\ \text { Uranium-238 } & \mathrm{pCi/g} & 2.86800 & 2.67554 & 2.13459 & 2.10577 & 1.49298 & 1.08271\end{array}$

Location $=O R R ;$ Formation $=$ CHEPULTEPEC; Site $=78 ; 00-05=1672 ; 05-10=1673 ; 10-15=1674 ;$ $15-20=1675 ; 20-25=1676 ; 25-30=1677 ;$ Phase $=2$

$\begin{array}{llllllll}\text { Cesium-137 } & \text { PCi/g } & 0.84300 & 0.59938 & 0.15047 & 0.02233 & 0.00533 & 0.00178 \\ \text { Potassium-40 } & \text { PCi/g } & 2.78335 & 2.96564 & 2.77574 & 3.38102 & 2.37894 & 2.77230 \\ \text { Radium-226 } & \text { PCi/g } & 0.87625 & 0.86353 & 0.76337 & 0.84107 & 0.66695 & 0.72479 \\ \text { Thorium-232 } & \text { PCi/g } & 0.53698 & 0.57751 & 0.54178 & 0.73098 & 0.52900 & 0.58087 \\ \text { Uranium-235 } & \text { PCi/g } & 0.15346 & 0.17103 & 0.14345 & 0.14361 & 0.03349 & 0.05811 \\ \text { Uranium-238 } & \text { pCi/g } & 3.23746 & 2.13588 & 1.80648 & 2.18807 & 0.86810 & 1.31160\end{array}$

Location $=O R R ;$ Formation $=$ CHEPULTEPEC; Site $=85 ; 00-05=1726 ; 05-10=1727 ; 10-15=1728$; $15-20=1729 ; 20-25=1730 ; 25-30=1731 ;$ Phase $=2$

$\begin{array}{llllllll}\text { Cesium-137 } & \text { PCi/g } & 0.99675 & 0.47968 & 0.14051 & 0.04096 & 0.02292 & 0.00261 \\ \text { Potassium-40 } & \text { PCi/g } & 3.62987 & 3.96800 & 4.71797 & 4.42078 & 4.19563 & 4.24248 \\ \text { Radium-226 } & \text { pCi/g } & 0.82100 & 0.81967 & 0.87965 & 0.77504 & 0.70771 & 0.71151 \\ \text { Thorium-232 } & \text { pCi/g } & 0.61926 & 0.62927 & 0.66659 & 0.64630 & 0.59914 & 0.58887 \\ \text { Uranium-235 } & \text { pCi/g } & 0.19439 & 0.15737 & 0.18623 & 0.10504 & 0.11408 & 0.01763 \\ \text { Uranium-238 } & \text { pCi/g } & 1.82446 & 1.03794 & 3.13245 & 2.89798 & 1.41758 & 1.14031\end{array}$

Location=ORR; Formation $=$ CHEPULTEPEC; Site $=86 ; 00-05=1708 ; 05-10=1709 ; 10-15=1710$; $15-20=1711 ; 20-25=1712 ; 25-30=1713 ;$ Phase $=2$

$\begin{array}{llllllll}\text { Cesium-137 } & \text { PCi/g } & 1.02891 & 0.22752 & 0.16600 & 0.05582 & 0.01965 & 0.00924 \\ \text { Potassium-40 } & \text { pCi/g } & 0.76543 & 1.25940 & 2.69652 & 3.11371 & 2.67786 & 3.06805 \\ \text { Radium-226 } & \text { PCi/g } & 0.48283 & 0.50695 & 0.67018 & 0.65021 & 0.57922 & 0.55953 \\ \text { Thorium-232 } & \text { PCi/g } & 0.10907 & 0.18651 & 0.42869 & 0.44718 & 0.44317 & 0.41546 \\ \text { Uranium-235 } & \text { PCi/g } & \mathbf{0 . 1 3 1 5 8} & \mathbf{0 . 1 1 1 7 8} & 0.28019 & 0.12743 & 0.16563 & 0.05644 \\ \text { Uranium-238 } & \text { pCi/g } & 2.63913 & 1.62807 & 3.29619 & 2.92163 & 1.67386 & 1.26766\end{array}$

Location=ORR; Formation $=C H E P U L T E P E C ;$ Site $=90 ; 00-05=1757 ; 05-10=1758 ; 10-15=1759$; $15-20=1760 ; 20-25=1761 ; 25-30=1762 ;$ Phase $=2$

Cesium- 137 Potossium-40 Radium-226 Thorium-232 Uranium-235 Uranium-238

$\begin{array}{ll}\mathrm{PCi} / \mathrm{g} & \mathbf{0 . 9 9 0 9 6} \\ \mathrm{PCi} / \mathrm{g} & 1.82854 \\ \mathrm{PCi} / \mathrm{g} & 0.52324 \\ \mathrm{PCi} / \mathrm{g} & 0.55453 \\ \mathrm{PCi} / \mathrm{g} & 0.06764 \\ \mathrm{PCi} / \mathrm{g} & 0.87774\end{array}$

0.38078
1.52587
0.51375
0.51412
0.19008
1.60235

0.09616

2.02061

0.57124

0.56661

0.04453

1.98372
0.05189

2.09026

0.45103

0.45697

0.09235

0.99219
0.03466

3.45065

0.55692

0.57644

0.09054

2.10338
0.01477

5.31109

0.54744

0.77534

0.01592

0.70735 
Table B2 (continued)

\begin{tabular}{|c|c|c|c|c|c|c|c|}
\hline \multirow[b]{2}{*}{ Analysis } & \multicolumn{6}{|c|}{ Depth (cm) } & \multirow[b]{2}{*}{$25-30$} \\
\hline & Units & $00-05$ & $05-10$ & $10-15$ & $15-20$ & $20-25$ & \\
\hline \multicolumn{8}{|c|}{$\begin{aligned} \text { Location }=\text { ORR; Formation }=\text { CHICKAMAUGA Bethel V; Site }=100 ; 00-05=1883 ; 05-10=1884 ; 1 \\
$\[ 15-20=1886 ; 20-25=1887 ; 25-30=1888 ; \text { Phase }=2 \]$\end{aligned}$} \\
\hline $\begin{array}{l}\text { Cesium-137 } \\
\text { Potassium-40 } \\
\text { Radium-226 } \\
\text { Thorium-232 } \\
\text { Uranium-235 }\end{array}$ & $\begin{array}{l}\mathrm{pCi} / \mathrm{g} \\
\mathrm{pCi} / \mathrm{g} \\
\mathrm{pCi} / \mathrm{g} \\
\mathrm{pCi} / \mathrm{g} \\
\mathrm{pCi} / \mathrm{g}\end{array}$ & $\begin{array}{r}4.1484 \\
25.1193 \\
0.7243 \\
1.1726 \\
0.3127\end{array}$ & $\begin{array}{r}0.0887 \\
26.7140 \\
0.6227 \\
1.2362 \\
0.1906\end{array}$ & $\begin{array}{r}0.0276 \\
33.1802 \\
0.6057 \\
1.4337 \\
0.1705\end{array}$ & $\begin{array}{r}0.0266 \\
34.0221 \\
0.5096 \\
1.4361 \\
0.1704\end{array}$ & $\begin{array}{r}0.0265 \\
32.0928 \\
0.5329 \\
1.3364 \\
0.1602\end{array}$ & $\begin{array}{r}0.0328 \\
35.9458 \\
0.6249 \\
1.5226 \\
0.0234\end{array}$ \\
\hline \multicolumn{8}{|c|}{$\begin{aligned} \text { Location }=\text { ORR; Formation }= & \text { CHICKAMAUGA Bethel V; Site }=101 ; 00-05=1920 ; 05-10=1921 ; 1 \\
& 15-20=1923 ; 20-25=1924 ; 25-30=1925 ; \text { Phase }=2\end{aligned}$} \\
\hline $\begin{array}{l}\text { Cesium-137 } \\
\text { Potassium-40 } \\
\text { Radium-226 } \\
\text { Thorium-232 } \\
\text { Uranium-235 }\end{array}$ & $\begin{array}{l}\mathrm{pCi} / \mathrm{g} \\
\mathrm{pCi} / \mathrm{g} \\
\mathrm{pCi} / \mathrm{g} \\
\mathrm{pCi} / \mathrm{g} \\
\mathrm{pCi} / \mathrm{g}\end{array}$ & $\begin{array}{r}2.8195 \\
25.8557 \\
0.6397 \\
1.2295 \\
0.2592\end{array}$ & $\begin{array}{r}1.1620 \\
28.3762 \\
0.6137 \\
1.2602 \\
0.2144\end{array}$ & $\begin{array}{r}0.4971 \\
28.7446 \\
0.6723 \\
1.3272 \\
0.2153\end{array}$ & $\begin{array}{r}0.1861 \\
27.6445 \\
0.5617 \\
1.2671 \\
0.0694\end{array}$ & $\begin{array}{r}0.1093 \\
28.1725 \\
0.5790 \\
1.2781 \\
0.1559\end{array}$ & $\begin{array}{r}0.0669 \\
29.3659 \\
0.6154 \\
1.3224 \\
0.0400\end{array}$ \\
\hline
\end{tabular}

Location=ORR; Formation $=$ CHICKAMAUGA Bethel V; Site $=102 ; 00-05=1914 ; 05-10=1915 ; 10-15=1916$; $15-20=1917 ; 20-25=1918 ; 25-30=1919 ;$ Phase $=2$

$\begin{array}{llrrrrrr}\text { Cesium-137 } & \text { PCi/g } & 2.3368 & 1.2347 & 0.2961 & 0.0872 & 0.0399 & 0.0081 \\ \text { Potassium-40 } & \text { PCi/g } & 14.8798 & 13.0872 & 14.4717 & 15.4992 & 17.6216 & 18.9912 \\ \text { Radium-226 } & \text { PCi/g } & 0.8934 & 0.7905 & 0.8284 & 0.7152 & 0.8199 & 0.6996 \\ \text { Thorium-232 } & \text { pCi/g } & 1.5042 & 1.2724 & 1.3420 & 1.2938 & 1.3094 & 1.2783 \\ \text { Uranium-235 } & \text { pCi/g } & 0.1685 & 0.2083 & 0.1290 & 0.1575 & 0.1638 & 0.1154\end{array}$

Location=ORR; Formation=CHICKAMAUGA Bethel V; Site $=103 ; 00-05=1908 ; 05-10=1909 ; 10-15=1910$; $15-20=1911 ; 20-25=1912 ; 25-30=1913 ;$ Phase $=2$

$\begin{array}{llrrrrrr}\text { Cesium-137 } & \text { pCi/g } & 1.6265 & 0.1131 & 0.1092 & 0.0111 & 0.0431 & 0.0171 \\ \text { Potassium-40 } & \text { pCi/g } & 34.4526 & 28.9176 & 32.0552 & 32.0828 & 30.8301 & 31.4933 \\ \text { Radium-226 } & \text { pCi/g } & 0.7153 & 0.5756 & 0.5848 & 0.6318 & 0.5546 & 0.5441 \\ \text { Thorium-232 } & \text { pCi/g } & 1.1411 & 1.2310 & 1.4093 & 1.4282 & 1.4327 & 1.4606 \\ \text { Uranium-235 } & \text { pCi/g } & 0.2528 & 0.1986 & 0.2215 & 0.2268 & 0.0536 & 0.0964\end{array}$

Location=ORR; Formation=CHICKAMAUGA Bethel V; Site=104; 00-05=1981; 05-10=1982; 10-15=1983; $15-20=1984 ; 20-25=1985 ; 25-30=1986 ;$ Phase $=2$

$\begin{array}{llrrrrrr}\text { Cesium-137 } & \text { pCi/g } & 2.6889 & 0.3540 & 0.1038 & 0.0318 & 0.0055 & 0.0153 \\ \text { Potassium-40 } & \text { pCi/g } & 23.3107 & 20.5458 & 24.8031 & 24.6567 & 26.8630 & 29.6826 \\ \text { Radium-226 } & \text { PCi/g } & 0.8708 & 0.5928 & 0.6250 & 0.5898 & 0.5504 & 0.5771 \\ \text { Thorium-232 } & \text { PCi/g } & 0.8802 & 0.6620 & 0.8729 & 1.0192 & 1.2982 & 1.4975 \\ \text { Uranium-235 } & \text { PCi/g } & 0.2882 & 0.1099 & 0.1624 & 0.1116 & 0.1568 & 0.0328 \\ \text { Uranium-238 } & \text { pCi/g } & & & 1.1765 & 4.7232 & 2.5866 & \end{array}$

Location=ORR; Formation=CHICKAMAUGA Bethel V; Site=108; 00-05=1987; 05-10=1988; 10-15=1989; $15-20=1990 ; 20-25=1991 ; 25-30=1992 ;$ Phase $=2$

\begin{tabular}{|c|c|c|c|c|c|c|c|}
\hline $\begin{array}{l}\text { Cesium-137 } \\
\text { Potassium-40 } \\
\text { Radium-226 } \\
\text { Thorium-232 } \\
\text { Uranium-235 } \\
\text { Uranium-238 }\end{array}$ & $\begin{array}{l}\mathrm{pCi} / \mathrm{g} \\
\mathrm{pCi} / \mathrm{g} \\
\mathrm{pCi} / \mathrm{g} \\
\mathrm{pCi} / \mathrm{g} \\
\mathrm{pCi} / \mathrm{g} \\
\mathrm{pCi} i / g\end{array}$ & $\begin{array}{l}1.20756 \\
7.91772 \\
0.82065 \\
1.25172 \\
0.27053\end{array}$ & $\begin{array}{r}0.1196 \\
11.3295 \\
0.7505 \\
1.3449 \\
0.1960\end{array}$ & $\begin{array}{r}0.0615 \\
19.7268 \\
0.8196 \\
1.5114 \\
3.3103\end{array}$ & $\begin{array}{r}0.0138 \\
22.2148 \\
0.7283 \\
1.5877 \\
0.0121 \\
3.3971\end{array}$ & $\begin{array}{r}0.0263 \\
24.7961 \\
0.7129 \\
1.6662 \\
0.2420 \\
3.2116\end{array}$ & $\begin{array}{r}0.0032 \\
24.9512 \\
0.6790 \\
1.7229 \\
0.0587 \\
1.2403\end{array}$ \\
\hline
\end{tabular}

Location=ORR; Formation=CHICKAMAUGA Bethel V; Site=110; 00-05=4032; 05-10=4033; $10-15=4034$; $15-20=4035 ; 20-25=4036 ; 25-30=4037 ;$ Phase $=2$

$\begin{array}{lllllrrr}\text { Cesium-137 } & \text { pCi/g } & 0.98105 & 0.17764 & 0.0869 & 0.0644 & 0.0079 & 0.0303 \\ \text { Potassium-40 } & \text { pCi/g } & 8.69078 & 9.51336 & 13.1116 & 12.5245 & 15.5835 & 14.0385\end{array}$


Tabłe B.2 (continued)

\begin{tabular}{llllllll}
\hline & \multicolumn{8}{c}{ Depth (cm) } \\
\cline { 2 - 8 } Analysis & Units & $00-05$ & $05-10$ & $10-15$ & $15-20$ & $20-25$ & $25-30$ \\
\hline & & & & & & & \\
Radium-226 & PCi/g & 0.99604 & 0.76348 & 0.8960 & 0.7386 & 1.1463 & 0.7284 \\
Thorium-232 & PCi/g & 1.33402 & 1.19840 & 1.4199 & 1.2539 & 1.4847 & 1.3361 \\
Uranium-235 & PCi/g & 0.21108 & 0.24451 & 0.2253 & 0.2437 & 0.1642 & 0.0353 \\
Uranium-238 & PCi/g & & & & 1.9764 & 2.6670 & 0.8714
\end{tabular}

Location=ORR; Fomation =CHICKAMAUGA Bethel V; Site=115; 00-05=4026; 05-10=4027; 10-15=4028; $15-20=4029 ; 20-25=4030 ; 25-30=4031 ;$ Phase $=2$

$\begin{array}{llllllrr}\text { Cesium-137 } & \text { PCi/g } & 1.63932 & 0.25246 & 0.06675 & 0.03083 & 0.0328 & 0.0248 \\ \text { Potassium-40 } & \text { PCi/g } & \mathbf{6 . 9 4 5 7 0} & 7.89016 & 8.43249 & 9.54118 & 10.9773 & 12.2497 \\ \text { Radium-226 } & \text { PCi/g } & 0.93882 & 0.91230 & 0.82885 & 0.82391 & 0.9375 & 1.0977 \\ \text { Thorium-232 } & \text { PCi/g } & 1.30092 & 1.41934 & 1.31554 & 1.38067 & 1.4694 & 1.4943 \\ \text { Uranium-235 } & \text { pCi/g } & 0.23453 & 0.17123 & 0.13255 & 0.17080 & 0.1233 & 0.1020 \\ \text { Uranium-238 } & \text { pCi/g } & 4.88783 & 0.91213 & 2.53600 & 0.64080 & 2.9670 & 1.1241\end{array}$

Location $=O R R ;$ Formation $=C H I C K A M A U G A$ Bethel V; Site $=116 ; 00-05=4000 ; 05-10=4001 ; 10-15=4002$; $15-20=4003 ; 20-25=4004 ; 25-30=4005 ;$ Phase $=2$

$\begin{array}{llrrrrrr}\text { Cesium-137 } & \text { PCi/g } & 1.1171 & 0.3121 & 0.0817 & 0.0378 & 0.0271 & 0.0148 \\ \text { Potassium-40 } & \text { PCi/g } & 14.5564 & 15.3602 & 16.4251 & 19.7006 & 23.4032 & 23.7906 \\ \text { Radium-226 } & \text { PCi/g } & 0.9115 & 0.7440 & 0.7302 & 0.6853 & 0.6189 & 0.6274 \\ \text { Thorium-232 } & \text { PCi/g } & 1.2114 & 1.2710 & 1.2793 & 1.3579 & 1.4752 & 1.4623 \\ \text { Uranium-235 } & \text { PCi/g } & 0.0889 & 0.1848 & 0.2112 & 0.1527 & 0.0219 & 0.0520\end{array}$

Location=ORR; Formation $=$ CHICKAMAUGA Bethel V; Site $=117 ; 00-05=4013 ; 05-10=4014 ; 10-15=4015$; $15-20=4016 ; 20-25=4017 ; 25-30=4018 ;$ Phase $=2$

$\begin{array}{lllllrrr}\text { Cesium-137 } & \text { PCi/g } & 0.86595 & 0.48475 & 0.18540 & 0.0538 & 0.0156 & 0.0068 \\ \text { Potassium-40 } & \text { PCi/g } & 7.99921 & 8.07801 & 9.88111 & 21.2407 & 23.5741 & 24.3898 \\ \text { Radium-226 } & \text { PCi/g } & 0.79833 & 0.71445 & 0.70534 & 0.6280 & 0.6423 & 0.5496 \\ \text { Thorium-232 } & \text { PCi/g } & 0.99564 & 1.00883 & 1.06998 & 1.4101 & 1.4237 & 1.3773 \\ \text { Uranium-235 } & \text { PCi/g } & 0.19169 & 0.17305 & 0.16859 & 0.1576 & 0.0988 & 0.0413\end{array}$

Location=ORR; Formation=CHICKAMAUGA Bethel V; Site=93;00-05=1871;05-10=1872; 10-15=1873; $15-20=1874 ; 20-25=1875 ; 25-30=1876 ;$ Phase $=2$

$\begin{array}{llrrrrrr}\text { Cesium-137 } & \text { PCi/g } & 1.6874 & 0.1548 & 0.0816 & 0.0515 & 0.0442 & 0.0358 \\ \text { Potassium-40 } & \text { pCi/g } & 14.7053 & 15.5027 & 21.5573 & 22.5600 & 22.2651 & 27.2214 \\ \text { Radium-226 } & \text { pCi/g } & 0.7843 & 0.6856 & 0.6729 & 0.6466 & 0.6053 & 0.8620 \\ \text { Thorium-232 } & \text { pCi/g } & 1.3124 & 1.2895 & 1.4183 & 1.4014 & 1.3593 & 1.5816 \\ \text { Uranium-235 } & \text { pCi/g } & 0.2159 & 0.3361 & 0.1650 & 0.1771 & 0.1643 & 0.1450\end{array}$

Location=ORR; Formation=CHICKAMAUGA Bethel V; Site=99; 00-05=1877; 05-10=1878; 10-15=1879; $15-20=1880 ; 20-25=1881 ; 25-30=1882 ;$ Phase $=2$

$\begin{array}{llrrrrrr}\text { Cesium-137 } & \mathrm{pCi} / \mathrm{g} & 1.3424 & 0.3349 & 0.2164 & 0.0335 & \mathbf{0 . 0 1 3 5} & \mathbf{0 . 0 0 7 0} \\ \text { Potassium-40 } & \mathrm{PCi} / \mathrm{g} & 12.6348 & 12.2586 & 14.3657 & 14.7168 & 15.0907 & 16.1017 \\ \text { Radium-226 } & \mathrm{PCi} / \mathrm{g} & 0.9160 & 0.8829 & 0.9142 & 0.8761 & 0.8538 & 0.8647 \\ \text { Thorium-232 } & \mathrm{PCi} / \mathrm{g} & 1.3127 & 1.3233 & 1.3863 & 1.3169 & 1.2438 & 1.2936 \\ \text { Uranium-235 } & \mathrm{PCi} / \mathrm{g} & 0.2068 & 0.1909 & \mathbf{0 . 1 6 1 0} & 0.1638 & 0.1449 & \mathbf{0 . 0 4 6 1}\end{array}$

Location=ORR; Formation =CHICKAMAUGA K-25; Site =118; $00-05=4077 ; 05-10=4078 ; 10-15=4079$; $15-20=4080 ; 20-25=4081 ; 25-30=4082 ;$ Phase $=2$

$\begin{array}{llrrrrrr}\text { Cesium-137 } & \mathrm{pCi} / \mathrm{g} & 4.3547 & 0.2531 & 0.0345 & 0.0791 & 0.0535 & 0.0194 \\ \text { Potassium-40 } & \mathrm{pCi} / \mathrm{g} & 34.7095 & 25.7962 & 33.0683 & 30.6392 & 27.2936 & 30.2019 \\ \text { Radium-226 } & \mathrm{PCi} / \mathrm{g} & 1.4909 & 0.8481 & 0.8304 & 0.8499 & 0.7395 & 0.6780 \\ \text { Thorium-232 } & \mathrm{PCi} / \mathrm{g} & 2.8539 & 1.7956 & 2.1456 & 2.0113 & 1.8393 & 1.8581 \\ \text { Uranium-235 } & \mathrm{PCi} / \mathrm{g} & 0.8611 & 0.2830 & 0.3192 & 0.4587 & 0.2699 & 0.1299 \\ \text { Uranium-238 } & \mathrm{pCi} / \mathrm{g} & 10.7360 & 6.0008 & 5.7285 & 4.5065 & \mathbf{5 . 6 3 7 6} & 2.2427\end{array}$


Table B.2 (continued)

\begin{tabular}{|c|c|c|c|c|c|c|c|}
\hline \multirow[b]{2}{*}{ Analysis } & \multicolumn{6}{|c|}{ Depth $(\mathrm{cm})$} & \multirow[b]{2}{*}{$25-30$} \\
\hline & Units & $00-05$ & $05-10$ & $10-15$ & $15-20$ & $20-25$ & \\
\hline \multirow{2}{*}{\multicolumn{8}{|c|}{$\begin{array}{c}\text { Location=ORR; Formation }=\text { CHICKAMAUGA K-25; Site }=119 ; 00-05=4180 ; 05-10=4181 ; 10- \\
15-20=4183 ; 20-25=4184 ; 25-30=4185 ; \text { Phase }=2\end{array}$}} \\
\hline & & & & & & & \\
\hline $\begin{array}{l}\text { Cesium-137 } \\
\text { Potassium-40 } \\
\text { Redium-226 } \\
\text { Thorium-232 } \\
\text { Uranium-235 } \\
\text { Uranium-238 }\end{array}$ & $\begin{array}{l}\mathrm{pCi} / \mathrm{g} \\
\mathrm{pCi} / \mathrm{g} \\
\mathrm{pCi} / \mathrm{g} \\
\mathrm{pCi} / \mathrm{g} \\
\mathrm{pCi} / \mathrm{g} \\
\mathrm{pCi} / \mathrm{g}\end{array}$ & $\begin{array}{r}1.4584 \\
12.2804 \\
1.0550 \\
1.2823 \\
0.3896 \\
5.9931\end{array}$ & $\begin{array}{r}0.5068 \\
10.2521 \\
0.8003 \\
1.1452 \\
0.2970 \\
3.4204\end{array}$ & $\begin{array}{r}0.0501 \\
10.5291 \\
0.7489 \\
1.1784 \\
0.1517 \\
2.9711\end{array}$ & $\begin{array}{r}0.0382 \\
15.0735 \\
0.7660 \\
1.2036 \\
0.1877 \\
1.8796\end{array}$ & $\begin{array}{r}0.0369 \\
18.5972 \\
0.6768 \\
1.1759 \\
0.2034 \\
2.7881\end{array}$ & $\begin{array}{r}0.0148 \\
23.4081 \\
0.6063 \\
1.3051 \\
0.0602 \\
1.2742\end{array}$ \\
\hline
\end{tabular}

Location=ORR; Formation $=C H I C K A M A U G A$ K-25; Site $=120 ; 00-05=4071 ; 05-10=4072 ; 10-15=4073$; $15-20=4074 ; 20-25=4075 ; 25-30=4076 ;$ Phase $=2$

$\begin{array}{lllllllr}\text { Cesium-137 } & \text { pCi/g } & 1.33960 & 0.53536 & 0.17735 & 0.04010 & 0.03723 & 0.0070 \\ \text { Potassium-40 } & \text { pCi/g } & 5.20520 & 5.79742 & 5.51634 & 5.34278 & 8.32013 & 11.1744 \\ \text { Radium-226 } & \text { pCi/g } & 1.40231 & 1.10239 & 1.22453 & 0.87288 & 1.11442 & 1.0019 \\ \text { Thorium-232 } & \text { PCi/g } & 1.32052 & 1.14549 & 1.30369 & 1.05907 & 1.35182 & 1.4632 \\ \text { Uranium-235 } & \text { PCi/g } & 0.30442 & 0.16768 & 0.06091 & 0.20111 & 0.18003 & 0.0937 \\ \text { Uranium-238 } & \text { pCi/g } & 4.10116 & 4.11602 & 2.05312 & 2.03516 & 3.98922 & 2.2493\end{array}$

Location=ORR; Formation =CHICKAMAUGA K-25; Site $=121 ; 00-05=4186 ; 05-10=4187 ; 10-15=4188$; $15-20=4189 ; 20-25=4190 ; 25-30=4191 ;$ Phase $=2$

\begin{tabular}{|c|c|c|c|c|c|c|c|}
\hline $\begin{array}{l}\text { Cesium- } 137 \\
\text { Potassium-40 } \\
\text { Radium-226 } \\
\text { Thorium-232 } \\
\text { Uranium-235 } \\
\text { Uranium-238 }\end{array}$ & $\begin{array}{l}\mathrm{pCi} / \mathrm{g} \\
\mathrm{pCi} i / g \\
\mathrm{pCi} / \mathrm{g} \\
\mathrm{pCi} / \mathrm{g} \\
\mathrm{pCi} / \mathrm{g} \\
\mathrm{pCi} i / g\end{array}$ & $\begin{array}{l}1.10186 \\
6.80667 \\
0.76879 \\
0.90520 \\
0.16449 \\
3.46693\end{array}$ & $\begin{array}{l}0.07836 \\
6.49062 \\
0.65263 \\
0.83863 \\
0.06696 \\
2.61692\end{array}$ & $\begin{array}{l}0.01962 \\
7.92026 \\
0.63570 \\
0.83011 \\
0.04179 \\
2.38197\end{array}$ & $\begin{array}{r}0.0274 \\
11.9132 \\
0.6228 \\
0.9086 \\
0.1466 \\
2.5582\end{array}$ & $\begin{array}{r}0.0343 \\
26.9167 \\
0.5058 \\
1.2449 \\
0.1748 \\
3.9474\end{array}$ & $\begin{array}{r}0.0111 \\
31.8098 \\
0.5205 \\
1.3416 \\
0.0450 \\
1.8486\end{array}$ \\
\hline
\end{tabular}

Location=ORR; Formation=CHICKAMAUGA K-25; Site=122;00-05=4192; 05-10=4193; 10-15=4194; $15-20=4195 ; 20-25=4196 ; 25-30=4197 ;$ Phase $=2$

$\begin{array}{llrlrrrr}\text { Cesium-137 } & \mathrm{PCi} / \mathrm{g} & 1.1869 & 0.56579 & 0.1236 & 0.0663 & 0.0213 & 0.0142 \\ \text { Potassium-40 } & \mathrm{pCi} / \mathrm{g} & 13.6981 & 9.91624 & 15.5927 & 23.9834 & 26.2110 & 26.3081 \\ \text { Radium-226 } & \mathrm{PCi} / \mathrm{g} & 1.1588 & 0.89597 & 0.8200 & 0.9734 & 0.9900 & 0.7625 \\ \text { Thorium-232 } & \mathrm{PCi} / \mathrm{g} & 1.2927 & 0.98060 & 1.1210 & 1.5849 & 1.5897 & 1.5327 \\ \text { Uranium-235 } & \mathrm{PCi} / \mathrm{g} & \mathbf{0 . 2 8 6 4} & 0.03328 & 0.1158 & 0.2249 & \mathbf{0 . 2 2 2 0} & 0.0644 \\ \text { Uranium-238 } & \mathrm{PCi} / \mathrm{g} & \mathbf{8 . 0 2 0 4} & \mathbf{3 . 5 5 5 6 5} & \mathbf{2 . 1 7 2 2} & \mathbf{3 . 8 5 1 1} & \mathbf{1 . 7 4 3 5} & \mathbf{1 . 3 4 4 1}\end{array}$

Location=ORR; Formation $=$ CHICKAMAUGA K-25; Site $=123 ; 00-05=4210 ; 05-10=4211 ; 10-15=4212$; $15-20=4213 ; 20-25=4214 ; 25-30=4215 ;$ Phase $=2$

$\begin{array}{lllrrrrr}\text { Cesium-137 } & \text { pCi/g } & \mathbf{0 . 8 0 6 5 7} & 0.3396 & 0.1059 & 0.0482 & 0.1111 & 0.0144 \\ \text { Potassium-40 } & \text { pCi/g } & 8.40190 & 10.2694 & 14.3937 & 24.2186 & 22.5278 & 19.9658 \\ \text { Radium-226 } & \text { pCi/g } & 0.67741 & 0.6301 & 0.7143 & 0.8308 & 0.7935 & 0.7137 \\ \text { Thorium-232 } & \text { PCi/g } & 1.20688 & 1.1700 & 1.4841 & 2.0776 & 1.9009 & 1.7373 \\ \text { Uranium-235 } & \text { pCi/g } & 0.12577 & 0.1699 & 0.2119 & 0.2363 & 0.1229 & 0.0764 \\ \text { Uranium-238 } & \text { pCi/g } & 3.45669 & 3.2591 & 3.7070 & 5.0393 & 4.4112 & 1.7216\end{array}$

Location=ORR; Formation=CHICKAMAUGA K-25; Site $=124 ; 00-05=4204 ; 05-10=4205 ; 10-15=4206$; $15-20=4207 ; 20-25=4208 ; 25-30=4209 ;$ Phase $=2$

$\begin{array}{lllllrrr}\text { Cesium-137 } & \text { pCi/g } & 1.27278 & 0.59321 & 0.09693 & 0.0314 & 0.0353 & 0.0101 \\ \text { Potassium-40 } & \text { pCi/g } & 7.54343 & 6.55849 & 7.74315 & 21.3926 & 23.8636 & 24.8610 \\ \text { Radium-226 } & \text { pCi/g } & 0.90399 & 0.65415 & 0.60807 & 0.6839 & 0.6701 & 0.5720 \\ \text { Thorium-232 } & \text { pCi/g } & 1.24992 & 1.18075 & 1.10543 & 1.4016 & 1.3920 & 1.4174 \\ \text { Uranium-235 } & \text { pCi/g } & 0.33240 & 0.18125 & 0.14240 & 0.1807 & 0.1839 & 0.0437 \\ \text { Uranium-238 } & \text { pCi/g } & 5.16611 & 2.85472 & 2.44710 & 3.6553 & 4.1227 & 1.2276\end{array}$


B-80

Table B.2 (continued)

\begin{tabular}{llllllll}
\hline & \multicolumn{7}{c}{ Depth $(\mathrm{cm})$} \\
\cline { 2 - 7 } Analysis & Units & $00-05$ & $05-10$ & $10-15$ & $15-20$ & $20-25$ & $25-30$ \\
\hline
\end{tabular}

Location=ORR; Formation=CHICKAMAUGA K-25; Site=125; 00-05=4198; 05-10=4199; $10-15=4200$; $15-20=4201 ; 20-25=4202 ; 25-30=4203 ;$ Phase $=2$

$\begin{array}{lllllrrr}\text { Cesium-137 } & \mathrm{pCi} / \mathrm{g} & \mathbf{0 . 9 9 4 0 2} & 0.24710 & 0.07979 & 0.0291 & \mathbf{0 . 0 3 1 0} & \mathbf{0 . 0 1 2 5} \\ \text { Potassium-40 } & \mathrm{pCi/g} & 6.7583 & 6.71753 & 9.54873 & 13.8157 & 17.4617 & 18.2644 \\ \text { Radium-226 } & \mathrm{pCi/g} & 0.73304 & 0.63580 & 0.66263 & 0.6297 & 0.6617 & 0.6110 \\ \text { Thorium-232 } & \mathrm{pCi/g} & 0.88512 & 0.94483 & 0.95977 & 1.1324 & 1.2079 & 1.2172 \\ \text { Uranium-235 } & \mathrm{pci/g} & 0.2058 & 0.10260 & 0.15377 & 0.1574 & 0.1611 & 0.0546 \\ \text { Uranium-238 } & \mathrm{pCi/g} & 2.97745 & 2.33500 & 2.59780 & 3.3636 & 5.2730 & 1.0867\end{array}$

Location =ORR; Formation $=$ CHICKAMAUGA $K-25 ;$ Site $=126 ; 00-05=4065 ; 05-10=4066 ; 10-15=4067 ;$ $15-20=4068 ; 20-25=4069 ; 25-30=4070 ;$ Phase $=2$

$\begin{array}{llrrrrrr}\text { Cesium-137 } & \mathrm{pCi} / \mathrm{g} & 1.4562 & 0.2313 & 0.0681 & 0.0342 & 0.0119 & 0.0071 \\ \text { Potassium-40 } & \mathrm{pCi} / \mathrm{g} & 12.6823 & 12.7013 & 14.8319 & 14.7306 & 19.5074 & 21.7761 \\ \text { Radium-226 } & \mathrm{pCi} / \mathrm{g} & 1.1450 & 0.8826 & 0.7798 & 0.7959 & 0.7794 & 0.7892 \\ \text { Thorium-232 } & \mathrm{pCi} / \mathrm{g} & 1.4928 & 1.3104 & 1.2581 & 1.2906 & 1.4133 & 1.5751 \\ \text { Uranium-235 } & \mathrm{pCi} / \mathrm{g} & 0.3047 & 0.1723 & 0.1682 & 0.1784 & 0.1815 & 0.0628 \\ \text { Uranium-238 } & \mathrm{pCi} / \mathrm{g} & 1.9494 & 1.3067 & 3.1178 & 3.2683 & 3.1206 & 1.1513\end{array}$

Location=ORR; Formation $=$ CHICKAMAUGA $K-25 ;$ Site $=127 ; 00-05=4241 ; 05-10=4242 ; 10-15=4243$; $15-20=4244 ; 20-25=4245 ; 25-30=4246 ;$ Phase $=2$

$\begin{array}{llrrrrrr}\text { Cesium-137 } & \text { PCi/g } & 2.6830 & 0.2583 & 0.0805 & 0.0453 & 0.0707 & 0.0080 \\ \text { Potassium-40 } & \text { pCi/g } & 25.9252 & 25.8546 & 28.1656 & 29.7531 & 30.2571 & 16.8718 \\ \text { Radium-226 } & \text { PCi/g } & 0.9024 & 0.9525 & 0.9015 & 0.7875 & 0.7977 & 0.4991 \\ \text { Thorium-232 } & \text { PCi/g } & 1.8306 & 1.7610 & 1.7809 & 1.7608 & 1.9787 & 1.0384 \\ \text { Uranium-235 } & \text { PCi/g } & 0.3840 & 0.1811 & 0.2298 & 0.2540 & 0.3645 & 0.0757 \\ \text { Uranium-238 } & \text { PCi/g } & 7.5973 & 2.6635 & 5.2910 & 4.8094 & 5.5249 & 1.7233\end{array}$

Location=ORR; Formation $=$ CHICKAMAUGA K-25; Süte $=128 ; 00-05=4247 ; 05-10=4248 ; 10-15=4249$; $15-20=4250 ; 20-25=4251 ; 25-30=4252 ;$ Phase $=2$

$\begin{array}{llrrrrrr}\text { Cesium-137 } & \text { PCi/g } & 1.61619 & 0.2387 & 0.09971 & 0.0314 & 0.0310 & 0.0217 \\ \text { Potassium-40 } & \text { PCi/g } & 9.82509 & 10.3859 & 9.26337 & 15.4870 & 19.8834 & 20.2383 \\ \text { Radium-226 } & \text { PCi/g } & 0.96177 & 0.7177 & 0.74336 & 0.8062 & 0.7147 & 0.6801 \\ \text { Thorium-232 } & \text { PCi/g } & 1.17616 & 1.1405 & 1.06324 & 1.3554 & 1.5291 & 1.4257 \\ \text { Uranium-235 } & \text { PCi/g } & 0.30702 & 0.1698 & 0.17603 & 0.2328 & 0.1300 & 0.1301 \\ \text { Uranium-238 } & \text { PCi/g } & 4.62269 & 2.1094 & 3.21695 & 1.9292 & 3.5529 & 2.1577\end{array}$

Location=ORR; Formation $=$ CHICKAMAUGA K-25; Site $=129 ; 00-05=4059 ; 05-10=4060 ; 10-15=4061$; $15-20=4062 ; 20-25=4063 ; 25-30=4064 ;$ Phase $=2$

$\begin{array}{lllllrrr}\text { Cesium-137 } & \text { PCi/g } & 1.61429 & 0.29571 & 0.10771 & 0.0762 & 0.0481 & 0.0151 \\ \text { Potassium-40 } & \text { pCi/g } & 4.54902 & 4.97201 & 7.60954 & 12.3434 & 16.7873 & 19.0190 \\ \text { Radium-226 } & \text { pCi/g } & 0.86919 & 0.89614 & 0.93261 & 0.9577 & 0.8687 & 0.8283 \\ \text { Thorium-232 } & \text { PCi/g } & 0.88852 & 0.90314 & 1.08012 & 1.4345 & 1.6353 & 1.9284 \\ \text { Uranium-235 } & \text { pCi/g } & 0.19919 & 0.18315 & 0.18858 & 0.2403 & 0.4288 & 0.0976 \\ \text { Uranium-238 } & \text { pCi/g } & 5.28711 & 3.22948 & 4.15098 & 3.8462 & 4.1144 & 1.8656\end{array}$

Location $=O R R$; Formation $=$ COPPER RIDGE; Site $=45 ; 00-05=1593 ; 05-10=1594 ; 10-15=1595$; $15-20=1596 ; 20-25=1597 ; 25-30=1598 ;$ Phase $=2$

$\begin{array}{llllllll}\text { Cesium-137 } & \text { pCi/g } & 1.31459 & 0.21678 & 0.05398 & 0.01377 & 0.01854 & 0.00991 \\ \text { Potassium-40 } & \text { pCi/g } & 2.40802 & 2.43464 & 3.15164 & 2.80080 & 2.76635 & 2.72684 \\ \text { Radium-226 } & \text { pCi/g } & 0.82141 & 0.84477 & 0.96069 & 0.88417 & 0.89114 & 0.86004 \\ \text { Thorium-232 } & \text { pCi/g } & 0.55643 & 0.58987 & 0.62893 & 0.65335 & 0.72237 & 0.71819 \\ \text { Uranium-235 } & \text { pCi/g } & 0.12673 & 0.12308 & 0.12736 & 0.04333 & 0.09776 & 0.07162\end{array}$


B-81

Table B2 (continued)

\begin{tabular}{llllllll}
\hline & \multicolumn{7}{c}{ Depth $(\mathrm{cm})$} \\
\cline { 2 - 7 } Analysis & Units & $00-05$ & $05-10$ & $10-15$ & $15-20$ & $20-25$ & $25-30$ \\
\hline
\end{tabular}

Location=ORR; Formation $=$ COPPER RIDGE; Site $=51 ; 00-05=1566 ; 05-10=1567 ; 10-15=1568$; $15-20=1569 ; 20-25=1570 ; 25-30=1571 ;$ Phase $=2$

$\begin{array}{llllllll}\text { Cesium-137 } & \text { PCi/g } & 0.73767 & 0.46321 & 0.20490 & 0.07874 & 0.01963 & 0.00013 \\ \text { Potassium-40 } & \text { PCi/g } & 2.45618 & 2.77590 & 2.59210 & 2.97926 & 3.18076 & 2.77015 \\ \text { Radium-226 } & \text { PCi/g } & 0.72157 & 0.77017 & 0.74240 & 0.79845 & 0.78172 & 0.68494 \\ \text { Thorium-232 } & \text { PCi/g } & 0.66572 & 0.67044 & 0.60575 & 0.62838 & 0.60392 & 0.57781 \\ \text { Uranium-235 } & \text { PCi/g } & 0.07907 & 0.12186 & 0.12679 & 0.23184 & 0.12207 & 0.04404\end{array}$

Location=ORR; Formation $=$ COPPER RIDGE; Site $=54 ; 00-05=1630 ; 05-10=1631 ; 10-15=1632$; $15-20=1633 ; 20-25=1634 ; 25-30=1635 ;$ Phase $=2$

$\begin{array}{llllllll}\text { Cesium-137 } & \text { PCi/g } & 0.64576 & 0.37812 & 0.14020 & 0.06296 & 0.04645 & 0.05509 \\ \text { Potassium-40 } & \text { PCi/g } & 2.71302 & 3.43090 & 3.56716 & 4.12647 & 4.10096 & 4.28849 \\ \text { Radium-226 } & \text { PCi/g } & 0.82373 & 1.01040 & 1.01885 & 1.01716 & 0.99389 & 1.21019 \\ \text { Thorium-232 } & \text { PCi/g } & 0.53051 & 0.61884 & 0.62160 & 0.60884 & 0.68825 & 0.70076 \\ \text { Uranium-235 } & \text { PCi/g } & 0.01508 & 0.20106 & 0.12349 & 0.03315 & \mathbf{0 . 1 2 2 8 3} & \mathbf{0 . 0 8 2 0 8}\end{array}$

Location $=O R R ;$ Formation $=$ COPPER RIDGE; Site $=55 ; 00-05=1560 ; 05-10=1561 ; 10-15=1562 ;$ $15-20=1563 ; 20-25=1564 ; 25-30=1565 ;$ Phase $=2$

$\begin{array}{llllllll}\text { Cesium-137 } & \text { PCi/g } & 0.61139 & 0.55791 & 0.39879 & 0.12312 & 0.01598 & 0.01582 \\ \text { Potassium-40 } & \text { PCi/g } & 1.90430 & 2.45251 & 3.71398 & 3.40865 & 3.52387 & 3.40780 \\ \text { Radium-226 } & \text { PCi/g } & 0.67114 & 0.80242 & 0.97207 & 1.00194 & 0.97963 & 0.92247 \\ \text { Thorium-232 } & \text { pCi/g } & 0.41367 & 0.52104 & 0.61295 & 0.61924 & 0.63580 & 0.56552 \\ \text { Uranium-235 } & \text { pCi/g } & 0.14904 & 0.14581 & 0.07268 & 0.14143 & \mathbf{0 . 0 9 6 2 7} & \mathbf{0 . 0 5 6 5 9}\end{array}$

Location=ORR; Formation $=$ COPPER RIDGE; Site $=58 ; 00-05=1519 ; 05-10=1520 ; 10-15=1521$; $15-20=1522 ; 20-25=1523 ; 25-30=1524 ;$ Phase $=2$

$\begin{array}{llllllll}\text { Cesium-137 } & \text { PCi/g } & 0.66312 & 0.33993 & 0.17862 & 0.02516 & 0.01537 & 0.00034 \\ \text { Potassium-40 } & \text { PCi/g } & 2.94865 & 3.14324 & 3.82270 & 3.22667 & 3.00775 & 3.68430 \\ \text { Radium-226 } & \text { PCi/g } & 0.65135 & 0.76370 & 0.88086 & 0.80912 & 0.71954 & 0.92610 \\ \text { Thorium-232 } & \text { PCi/g } & 0.59720 & 0.67898 & 0.78012 & 0.66765 & 0.60329 & 0.83300 \\ \text { Uranium-235 } & \text { pCi/g } & 0.12708 & 0.12637 & 0.12423 & 0.07819 & 0.08214 & 0.08181\end{array}$

Location=ORR; Formation $=$ COPPER RIDGE; Site $=59 ; 00-05=1513 ; 05-10=1514 ; 10-15=1515$; $15-20=1516 ; 20-25=1517 ; 25-30=1518 ;$ Phase $=2$

$\begin{array}{llllllll}\text { Cesium-137 } & \text { PCi/g } & 1.27053 & 0.19831 & 0.09355 & 0.03261 & 0.01504 & 0.01884 \\ \text { Potassium-40 } & \text { PCi/g } & 2.84551 & 2.95404 & 2.67501 & 2.81630 & 3.16815 & 3.05796 \\ \text { Radium-226 } & \text { PCi/g } & 0.82470 & 0.91875 & 0.91501 & 0.98392 & 0.89643 & 0.89360 \\ \text { Thorium-232 } & \text { PCi/g } & 0.51482 & 0.68239 & 0.65350 & 0.68352 & 0.65714 & 0.60265 \\ \text { Uranium-235 } & \text { pCi/g } & 0.15886 & 0.07964 & 0.22271 & 0.06912 & 0.11979 & 0.05196\end{array}$

Location=ORR; Formation =COPPER RIDGE; Site $=60 ; 00-05=1572 ; 05-10=1573 ; 10-15=1574$; $15-20=1575 ; 20-25=1576 ; 25-30=1577 ;$ Phase $=2$

$\begin{array}{llllllll}\text { Cesium-137 } & \text { pCi/g } & 0.71665 & 0.19398 & 0.07772 & 0.03267 & 0.02987 & 0.03054 \\ \text { Potassium-40 } & \text { pCi/g } & 1.61427 & 1.93660 & 2.02797 & 2.22403 & 2.99855 & 3.12826 \\ \text { Radium-226 } & \text { pCi/g } & 0.70957 & 0.73995 & 0.74825 & 0.76166 & 0.88828 & 0.92520 \\ \text { Thorium-232 } & \text { pCi/g } & \mathbf{0 . 5 6 2 3 5} & \mathbf{0 . 6 2 5 7 7} & 0.67116 & 0.78586 & 0.98420 & 1.03009 \\ \text { Uranium-235 } & \text { pCi/g } & \mathbf{0 . 1 5 5 8 9} & \mathbf{0 . 2 0 9 9 0} & \mathbf{0 . 1 4 7 8 4} & \mathbf{0 . 1 1 9 0 1} & \mathbf{0 . 1 3 2 0 3} & \mathbf{0 . 0 5 1 3 4}\end{array}$

Location $=O R R ;$ Formation $=$ COPPER RIDGE; Site $=62 ; 00-05=1554 ; 05-10=1555 ; 10-15=1556$; $15-20=1557 ; 20-25=1558 ; 25-30=1559 ;$ Phase $=2$

$\begin{array}{llllllll}\text { Cesium-137 } & \text { PCi/g } & 1.46219 & 0.44444 & 0.12942 & 0.08320 & 0.03744 & 0.02214 \\ \text { Potassium-40 } & \text { PCi/g } & 2.74205 & 2.17922 & 2.39099 & 2.37025 & 2.51268 & 2.40552 \\ \text { Radium-226 } & \text { PCi/g } & 0.92132 & 0.82577 & 0.93225 & 0.75297 & 0.80691 & 0.77066\end{array}$


B-82

Table B.2 (continued)

\begin{tabular}{llllllll}
\hline & \multicolumn{10}{c}{ Depth (cm) } \\
\cline { 2 - 8 } Analysis & Units & $00-05$ & $05-10$ & $10-15$ & $15-20$ & $20-25$ & $25-30$ \\
\hline & & & & & & & \\
Thorium-232 & pCi/g & 0.76372 & 0.73340 & 0.76144 & 0.73456 & 0.74223 & 0.65953 \\
Uranium-235 & pCi/g & 0.17870 & 0.08602 & 0.13667 & 0.11608 & 0.04808 & 0.05601
\end{tabular}

Location $=O R R ;$ Formation $=C O P P E R$ RIDGE; Site $=64 ; 00-05=1624 ; 05-10=1625 ; 10-15=1626$; $15-20=1627 ; 20-25=1628 ; 25-30=1629 ;$ Phase $=2$

$\begin{array}{llllllll}\text { Cesium-137 } & \mathrm{pCi} / \mathrm{g} & 1.35825 & 0.55949 & 0.13025 & 0.05076 & 0.02776 & 0.00672 \\ \text { Potassium-40 } & \mathrm{pCi/g} & 4.57810 & 4.35225 & 3.88484 & 4.25317 & 4.20743 & 5.00062 \\ \text { Radium-226 } & \mathrm{pCi} / \mathrm{g} & 1.14307 & 1.26928 & 1.19442 & 1.05443 & 1.09406 & 1.12214 \\ \text { Thorium-232 } & \mathrm{pCi} / \mathrm{g} & 0.63299 & 0.57065 & 0.60510 & 0.59333 & 0.62899 & 0.63976 \\ \text { Uranium-235 } & \mathrm{pCi/g} & 0.18705 & 0.15061 & \mathbf{0 . 0 8 5 5 7} & \mathbf{0 . 1 0 7 5 1} & \mathbf{0 . 0 6 5 2 2} & \mathbf{0 . 0 4 0 5 0}\end{array}$

Location =ORR; Formation $=$ COPPER RIDGE; Site $=75 ; 00-05=1584 ; 05-10=1585 ; 10-15=1586$; $15-20=1587 ; 20-25=1588 ; 25-30=1589 ;$ Phase $=2$

\begin{tabular}{|c|c|c|c|c|c|c|c|}
\hline $\begin{array}{l}\text { Cesium-137 } \\
\text { Potassium-40 } \\
\text { Radium-226 } \\
\text { Thorium-232 } \\
\text { Uranium-235 }\end{array}$ & $\begin{array}{l}\mathrm{pCi} / \mathrm{g} \\
\mathrm{pCi} / \mathrm{g} \\
\mathrm{pCi} / \mathrm{g} \\
\mathrm{pCi} / \mathrm{g} \\
\mathrm{pCi} / \mathrm{g}\end{array}$ & $\begin{array}{l}1.20643 \\
3.12891 \\
0.96178 \\
0.85230 \\
0.16778\end{array}$ & $\begin{array}{l}0.70914 \\
3.66351 \\
1.01614 \\
0.82211 \\
0.02727\end{array}$ & $\begin{array}{l}0.09526 \\
3.27692 \\
1.00702 \\
0.76017 \\
0.04514\end{array}$ & $\begin{array}{l}0.00893 \\
3.49178 \\
0.84096 \\
0.61532 \\
0.17811\end{array}$ & $\begin{array}{l}0.00797 \\
3.33220 \\
0.96231 \\
0.69202 \\
0.11366\end{array}$ & $\begin{array}{l}0.00902 \\
3.78174 \\
1.09401 \\
0.81179 \\
0.05796\end{array}$ \\
\hline
\end{tabular}

Location =ORR; Formation $=C O P P E R$ RIDGE; Site $=83 ; 00-05=1636 ; 05-10=1637 ; 10-15=1638$; $15-20=1639 ; 20-25=1640 ; 25-30=1641 ;$ Phase $=2$

$\begin{array}{llllllll}\text { Cesium-137 } & \mathrm{pCi} / \mathrm{g} & 1.43142 & 0.45581 & 0.07816 & 0.01367 & 0.01237 & 0.00939 \\ \text { Potassium-40 } & \mathrm{pCi} / \mathrm{g} & 3.16801 & 3.04970 & 3.32586 & 3.63522 & 3.64562 & 3.91081 \\ \text { Radium-226 } & \mathrm{pCi/g} & 0.87328 & \mathbf{0 . 9 3 3 0 9} & 1.02638 & 1.01598 & 1.03243 & 1.06284 \\ \text { Thorium-232 } & \mathrm{pCi/g} & 0.74561 & \mathbf{0 . 7 7 3 7 4} & \mathbf{0 . 7 1 9 6 6} & 0.76888 & 0.75811 & 0.80789 \\ \text { Uranium-235 } & \mathrm{pCi} / \mathrm{g} & \mathbf{0 . 0 7 8 7 6} & \mathbf{0 . 1 0 2 1 1} & \mathbf{0 . 0 5 1 4 5} & \mathbf{0 . 0 6 3 7 7} & 0.09445 & \mathbf{0 . 1 3 1 0 8}\end{array}$

Location $=O R R ;$ Formation $=$ COPPER RIDGE; Site $=91 ; 00-05=1525 ; 05-10=1526 ; 10-15=1527 ;$ $15-20=1528 ; 20-25=1529 ; 25-30=1530 ;$ Phase $=2$

$\begin{array}{llllllll}\text { Cesium-137 } & \text { pCi/g } & 1.55850 & 0.79606 & 0.11417 & 0.01851 & 0.01913 & 0.00043 \\ \text { Potassium-40 } & \text { pCi/g } & 3.18634 & 3.35480 & 3.00799 & 3.32103 & 3.72947 & 3.91862 \\ \text { Radium-226 } & \text { pCi/g } & 1.01708 & 1.00546 & 1.06773 & 1.05082 & 1.06568 & 0.97347 \\ \text { Thorium-232 } & \text { pCi/g } & 0.72699 & 0.77791 & 0.82529 & 0.79722 & 0.83680 & 0.82024 \\ \text { Uranium-235 } & \text { pCi/g } & 0.19485 & 0.13070 & 0.11341 & 0.06298 & 0.20416 & 0.10720\end{array}$

Location=ORR; Formation=DISMAL GAP; Site=10; 00-05=1129; 05-10=1130; 10-15=1131; 15-20=1132; $20-25=1133 ; 25-30=1134 ;$ Phase $=1$

$\begin{array}{llrrrrrr}\text { Cesium-137 } & \text { PCi/g } & 1.3656 & 0.8561 & 0.2386 & 0.1730 & 0.0296 & 0.0148 \\ \text { Potassium-40 } & \text { PCi/g } & 22.0029 & 23.1574 & 16.1954 & 19.3380 & 17.1920 & 17.0671 \\ \text { Radium-226 } & \text { PCi/g } & 0.7174 & 0.6503 & 0.4937 & 0.5869 & 0.8397 & 0.5044 \\ \text { Thorium-232 } & \text { PCi/g } & 1.1676 & 1.2337 & 0.9389 & 1.1499 & 1.1452 & 1.0976 \\ \text { Uranium-235 } & \text { PCi/g } & 0.1695 & 0.1837 & 0.0671 & 0.1521 & 0.1485 & 0.0242 \\ \text { Uranium-238 } & \text { PCi/g } & 3.5354 & 3.6073 & 2.3943 & 2.8313 & 2.4951 & 0.4048\end{array}$

Location=ORR; Formation =DISMAL GAP; Site =11; 00-05=1040; 05-10=1041; 10-15=1042; 15-20=1043; $20-25=1044 ; 25-30=1045 ;$ Phase $=1$

$\begin{array}{llrrrrrr}\text { Cesium-137 } & \text { PCi/g } & 1.3536 & 0.4110 & 0.1274 & 0.0915 & 0.0520 & 0.0220 \\ \text { Potassium-40 } & \text { PCi/g } & 27.3335 & 26.2783 & 24.7049 & 22.4150 & 29.1504 & 30.8557 \\ \text { Radium-226 } & \text { PCi/g } & 0.8693 & 0.7244 & 0.6513 & 0.6776 & 0.7581 & 0.8685 \\ \text { Thorium-232 } & \text { PCi/g } & 1.4545 & 1.3252 & 1.3623 & 1.3867 & 1.5856 & 1.6720 \\ \text { Uranium-235 } & \text { PCi/g } & 0.1348 & 0.1714 & 0.1145 & 0.1162 & 0.1236 & 0.1354\end{array}$


Table B.2 (continued)

\begin{tabular}{|c|c|c|c|c|c|c|c|}
\hline \multirow[b]{2}{*}{ Analysis } & \multicolumn{7}{|c|}{ Depth (cm) } \\
\hline & Units & 00-05 & $05-10$ & $10-15$ & $15-20$ & $20-25$ & $25-30$ \\
\hline \multicolumn{8}{|c|}{$\begin{array}{l}\text { Location }=O R R ; \text { Formation }=\text { DISMAL GAP; Site }=19 ; 00-05=1093 ; 05-10=1094 ; 10-15=1095 ; 1 \\
\qquad 20-25=1097 ; 25-30=1098 ; \text { Phase }=1\end{array}$} \\
\hline $\begin{array}{l}\text { Cesium- } 137 \\
\text { Potassium-40 } \\
\text { Radium-226 } \\
\text { Thorium-232 } \\
\text { Uranium-235 } \\
\text { Uranium-238 }\end{array}$ & $\begin{array}{l}\mathrm{pCi} / \mathrm{g} \\
\mathrm{pCi} / \mathrm{g} \\
\mathrm{pCi} i / g \\
\mathrm{pCi} / \mathrm{g} \\
\mathrm{pCi} / \mathrm{g} \\
\mathrm{pCi} / \mathrm{g}\end{array}$ & $\begin{array}{l}1.15976 \\
9.78573 \\
0.55824 \\
0.69456 \\
0.13685 \\
2.35735\end{array}$ & $\begin{array}{r}0.0860 \\
12.2284 \\
0.6651 \\
0.9246 \\
0.1363 \\
2.1892\end{array}$ & $\begin{array}{r}0.0184 \\
13.9657 \\
0.6638 \\
0.9387 \\
0.0849 \\
0.6857\end{array}$ & $\begin{array}{r}0.0049 \\
15.6700 \\
0.7204 \\
1.0822 \\
0.0545 \\
1.4209\end{array}$ & $\begin{array}{r}0.0072 \\
17.5225 \\
0.7024 \\
1.1208 \\
0.0412 \\
0.5366\end{array}$ & $\begin{array}{r}0.0044 \\
19.3992 \\
0.7533 \\
1.2394 \\
0.1009 \\
1.7466\end{array}$ \\
\hline
\end{tabular}

Location=ORR; Formation =DISMAL GAP; Site =2; $00-05=1183 ; 05-10=1184 ; 10-15=1185 ; 15-20=1186$; 20-25=1187; 25-30=1188; Phase $=1$

$\begin{array}{llrrrrrr}\text { Cesium-137 } & \text { PCi/g } & 1.7189 & 0.2115 & 0.0747 & 0.0422 & 0.0284 & 0.0131 \\ \text { Potassium-40 } & \text { PCi/g } & 15.1972 & 16.3894 & 19.4542 & 21.4761 & 23.1861 & 22.8751 \\ \text { Radium-226 } & \text { PCi/g } & 0.9044 & 0.7510 & 0.6634 & 0.6080 & 0.7755 & 0.5888 \\ \text { Thorium-232 } & \text { PCi/g } & 1.0133 & 0.9939 & 1.0647 & 1.0588 & 1.1916 & 1.3749 \\ \text { Uranium-235 } & \text { PCi/g } & 0.0967 & 0.1449 & 0.1463 & 0.1388 & 0.1908 & 0.1606 \\ \text { Uranium-238 } & \text { PCi/g } & 3.2767 & 2.3829 & 2.3630 & 2.0251 & 2.4461 & 2.7837\end{array}$

Location=ORR; Formation =DISMAL GAP; Site =22;00-05=1100; 05-10=1101; 10-15=1102; 15-20=1103; 20-25=1104; $25-30=1105 ;$ Phase $=1$

$\begin{array}{llrrrrrr}\text { Cesium-137 } & \text { PCi/g } & 1.5205 & 0.3829 & 0.0907 & 0.0285 & 0.0265 & 0.0101 \\ \text { Potassium-40 } & \text { PCi/g } & 13.5478 & 15.9145 & 18.1063 & 26.8466 & 28.8003 & 27.9817 \\ \text { Radium-226 } & \text { PCi/g } & 0.6656 & 0.6936 & 0.7541 & 0.7897 & 0.6973 & 0.5653 \\ \text { Thorium-232 } & \text { PCi/g } & 0.9694 & 1.0370 & 1.1640 & 1.4235 & 1.4542 & 1.4183 \\ \text { Uranium-235 } & \text { PCi/g } & 0.1789 & 0.1117 & & 0.1689 & & 0.0530 \\ \text { Uranium-238 } & \text { pCi/g } & 3.2313 & 2.3585 & 2.4392 & 2.9163 & 2.8677 & 1.3261\end{array}$

Location=ORR; Formation=DISMAL GAP; Site=26;00-05=1207;05-10=1208; 10-15=1209; 15-20=1210; $20-25=1211 ; 25-30=1212 ;$ Phase $=1$

$\begin{array}{llrrrrrr}\text { Cesium-137 } & \text { PCi/g } & 1.3703 & 0.2945 & 0.0957 & 0.0251 & 0.0116 & 0.0129 \\ \text { Potassium-40 } & \text { PCi/g } & 18.3172 & 17.9569 & 20.0332 & 20.9431 & 21.5800 & 26.3039 \\ \text { Radium-226 } & \text { PCi/g } & 0.8390 & 0.5569 & 0.6158 & 0.6438 & 0.6505 & 0.6739 \\ \text { Thorium-232 } & \text { PCi/g } & 1.0653 & 0.9838 & 1.0935 & 1.1993 & 1.3079 & 1.4047 \\ \text { Uranium-235 } & \text { PCi/g } & 0.1761 & 0.1001 & 0.1578 & 0.1472 & 0.1718 & 0.0495 \\ \text { Uranium-238 } & \text { pCi/g } & 3.3415 & 2.8359 & 2.7844 & 2.9593 & 2.5301 & 1.6265\end{array}$

Location=ORR; Formation =DISMAL GAP; Site $=27 ; 00-05=1009 ; 05-10=1010 ; 10-15=1011 ; 15-20=1012 ;$ 20-25=1013; 25-30=1014; Phase $=1$

$\begin{array}{llrrrrrr}\text { Cesium-137 } & \text { PCi/g } & 1.2572 & 0.3332 & 0.1850 & 0.0111 & 0.0082 & 0.0013 \\ \text { Potassium-40 } & \text { PCi/g } & 12.6756 & 14.8813 & 14.8364 & 15.4542 & 16.6053 & 16.9683 \\ \text { Radium-226 } & \text { PCi/g } & 0.7552 & 0.8678 & 0.6976 & 0.7580 & 0.7483 & 0.6641 \\ \text { Thorium-232 } & \text { PCi/g } & 0.9894 & 1.1002 & 1.0336 & 1.0406 & 1.0213 & 0.9938 \\ \text { Uranium-235 } & \text { PCi/g } & 0.1628 & 0.1161 & 0.1443 & 0.1126 & 0.1126 & 0.0949\end{array}$

Location=ORR; Formation=DISMAL GAP; Site =32; 00-05=1087; 05-10=1088; 10-15=1089; 15-20=1090; $20-25=1091 ; 25-30=1092 ;$ Phase $=1$

Cesium-137 Potassium-40 Radium-226 Thorium-232 Uranium-235 Uranium-238

$\begin{array}{lrr}\mathrm{PCj} / \mathrm{g} & 1.0464 & 0.2435 \\ \mathrm{PCi} / \mathrm{g} & 15.4072 & 15.6139 \\ \mathrm{PCi} / \mathrm{g} & 0.6552 & 0.5872 \\ \mathrm{PCi} / \mathrm{g} & 1.0246 & 0.9444 \\ \mathrm{PCi} / \mathrm{g} & 0.0761 & 0.051 \\ \mathrm{PCi} / \mathrm{g} & 1.0328 & 1.2187\end{array}$

0.1281

15.8296

0.6975

0.9937

0.1186

2.0979
0.0150

16.9094

0.6931

1.0446

0.0571

1.2029
0.0001

20.7083

0.6028

1.1923

0.0617

0.8208
0.0005

21.1364

0.5331

1.0643

0.0455

0.7408 


\section{B-84}

Table B.2 (continued)

\begin{tabular}{llllllll}
\hline & \multicolumn{8}{c}{ Depth (cm) } \\
\cline { 2 - 7 } Analysis & Units & $00-05$ & $05-10$ & $10-15$ & $15-20$ & $20-25$ & $25-30$ \\
\hline
\end{tabular}

Location $=O R R ;$ Formation $=D I S M A L$ GAP; Site $=33 ; 00-05=1109 ; 05-10=1110 ; 10-15=1111 ; 15-20=1112$; 20-25=1113; 25-30=1114; Phase $=1$

$\begin{array}{llrrrrrr}\text { Cesium-137 } & \mathrm{pCi} / \mathrm{g} & 2.6559 & 0.3720 & 0.0489 & 0.0306 & 0.0283 & 0.0200 \\ \text { Potassium-40 } & \mathrm{pCi} / \mathrm{g} & 19.1068 & 17.4362 & 19.8696 & 18.0485 & 16.6901 & 19.5761 \\ \text { Radium-226 } & \mathrm{pCi} / \mathrm{g} & 1.2161 & 0.7522 & 0.6108 & 0.6012 & 0.6634 & 0.7477 \\ \text { Thorium-232 } & \mathrm{pCi} / \mathrm{g} & 1.3637 & 1.0643 & 1.1503 & 1.1354 & 1.1780 & 1.1920 \\ \text { Uranium-235 } & \mathrm{pCi} / \mathrm{g} & 0.1426 & 0.1776 & 0.1254 & 0.1659 & & 0.1333 \\ \text { Uranium-238 } & \mathrm{pCi} / \mathrm{g} & 7.8303 & 3.4469 & 3.7913 & 3.1028 & 2.1457 & 2.4243\end{array}$

Location $=O R R ;$ Formation $=$ DISMAL GAP; Site $=35 ; 00-05=1116 ; 05-10=1117 ; 10-15=1118 ; 15-20=1119$; 20-25=1120; 25-30=1121; Phase $=1$

$\begin{array}{lllllllr}\text { Cesium-137 } & \text { pCi/g } & 0.87748 & 0.09969 & 0.02041 & 0.01806 & 0.00300 & 0.0191 \\ \text { Potassium-40 } & \text { pCi/g } & 6.20873 & 5.68209 & 5.81069 & 6.62107 & 7.71612 & 10.6939 \\ \text { Radium-226 } & \text { pCi/g } & 0.72322 & 0.81582 & 0.88972 & 0.75208 & 0.71532 & 0.8264 \\ \text { Thorium-232 } & \text { pCi/g } & 0.80594 & 0.94075 & 0.90856 & 0.89607 & 0.89224 & 1.0554 \\ \text { Uranium-235 } & \text { pCi/g } & 0.11851 & 0.10982 & 0.13253 & \mathbf{0 . 1 2 6 1 2} & \mathbf{0 . 0 2 2 1 0} & 0.1065 \\ \text { Uranium-238 } & \text { pCi/g } & 3.08327 & 2.05896 & 0.75256 & 3.83585 & 1.26806 & 2.4462\end{array}$

Location=ORR; Formation =DISMAL GAP; Site=41;00-05=1028; 05-10=1029; 10-15=1030; 15-20=1031; 20-25=1032; 25-30=1033; Phase $=1$

$\begin{array}{llrlrrrr}\text { Cesium-137 } & \text { PCi/g } & 2.0686 & 0.60128 & 0.3249 & 0.0779 & 0.0207 & 0.0216 \\ \text { Potassium-40 } & \text { PCi/g } & 10.7427 & 9.92154 & 11.0152 & 18.5631 & 18.6508 & 20.0673 \\ \text { Radium-226 } & \text { PCi/g } & 0.7561 & 0.72228 & 0.6847 & 0.6112 & 0.5917 & 0.7496 \\ \text { Thorium-232 } & \text { PCi/g } & 1.0634 & 1.00867 & 1.0485 & 1.4132 & 1.5553 & 1.6006 \\ \text { Uranium-235 } & \text { PCi/g } & 0.1896 & 0.13170 & 0.1131 & 0.1402 & 0.1234 & 0.1315\end{array}$

Location=ORR; Formation=DISMAL GAP; Site =43; 00-05=1240;05-10=1241; 10-15=1242; 15-20=1243; $20-25=1244 ; 25-30=1245 ;$ Phase $=1$

$\begin{array}{llrrrrrr}\text { Cesium-137 } & \text { PCi/g } & 1.2980 & 0.3329 & 0.0690 & 0.0321 & 0.0300 & 0.0385 \\ \text { Potassium-40 } & \text { PCi/g } & 16.9967 & 16.2910 & 15.8280 & 17.6780 & 18.1977 & 22.3797 \\ \text { Radium-226 } & \text { PCi/g } & 0.8646 & 0.9468 & 0.8196 & 0.7193 & 0.6794 & 0.7219 \\ \text { Thorium-232 } & \text { PCi/g } & 1.2621 & 1.1425 & 1.1133 & 1.2199 & 1.1961 & 1.2745 \\ \text { Uranium-235 } & \text { PCi/g } & 0.2573 & 0.2029 & & 0.1681 & 0.1775 & 0.1858 \\ \text { Uranium-238 } & \text { PCi/g } & 4.9464 & 2.6392 & 1.7212 & 2.7298 & 5.6479 & 4.1528\end{array}$

Location $=O R R ;$ Formation $=$ NOLICHUCKY; Site $=13 ; 00-05=1355 ; 05-10=1356 ; 10-15=1357 ; 15-20=1358$; $20-25=1359 ; 25-30=1360 ;$ Phase $=1$

$\begin{array}{llrrrrrr}\text { Cesium-137 } & \text { PCi/g } & 1.2523 & 0.4773 & 0.0890 & 0.0208 & 0.0035 & 0.0064 \\ \text { Potassium-40 } & \text { PCi/g } & 15.2972 & 13.4125 & 13.4367 & 12.9746 & 12.8163 & 18.2887 \\ \text { Radium-226 } & \text { PCi/g } & 0.8298 & 0.7163 & 0.6500 & 0.6454 & 0.5691 & 0.6463 \\ \text { Thorium-232 } & \text { PCi/g } & 1.2134 & 1.1583 & 1.1003 & 1.0305 & 0.9680 & 1.2154 \\ \text { Uranium-235 } & \text { PCi/g } & 0.1304 & 0.1522 & 0.1367 & 0.1359 & 0.1241 & 0.0759 \\ \text { Uranium-238 } & \text { PCi/g } & 2.7410 & 2.1689 & 2.1121 & 1.9954 & 0.7563 & 1.1959\end{array}$

Location $=O R R ;$ Formation $=$ NOLICHUCKY; Site $=15 ; 00-05=1307 ; 05-10=1308 ; 10-15=1309 ; 15-20=1310$; $20-25=1311 ; 25-30=1312 ;$ Phase $=1$

$\begin{array}{llllllrr}\text { Cesium-137 } & \text { pCi/g } & 1.31838 & 0.37165 & 0.06814 & 0.03282 & 0.0439 & 0.0376 \\ \text { Potassium-40 } & \text { PCi/g } & 7.57668 & 7.42748 & 8.37181 & 9.91225 & 12.7679 & 15.2212 \\ \text { Radium-226 } & \text { PCi/g } & \mathbf{0 . 7 5 4 8 8} & 0.74257 & 0.81304 & 0.68292 & 0.7380 & 0.8035 \\ \text { Thorium-232 } & \text { PCi/g } & 1.10444 & 1.17495 & 1.21709 & 1.32450 & 1.5444 & 1.6724 \\ \text { Uranium-235 } & \text { PCi/g } & \mathbf{0 . 2 5 5 4 4} & 0.19953 & 0.19982 & 0.26038 & 0.2233 & 0.2312 \\ \text { Uranium-238 } & \text { pCi/g } & 3.92091 & 3.55493 & 3.02203 & 0.85926 & 3.2127 & 3.7710\end{array}$


Table B.2 (continued)

\begin{tabular}{|c|c|c|c|c|c|c|c|}
\hline \multirow[b]{2}{*}{ Analysis } & \multicolumn{7}{|c|}{ Depth (cm) } \\
\hline & Units & $00-05$ & $05-10$ & $10-15$ & $15-20$ & $20-25$ & $25-30$ \\
\hline \multicolumn{8}{|c|}{$\begin{array}{l}\text { Location }=\text { ORR; Formation }=\text { NOLICHUCKY; Site }=16 ; 00-05=1343 ; 05-10=1344 ; 10-15=1345 ; \\
\qquad 20-25=1347 ; 25-30=1348 ; \text { Phase }=1\end{array}$} \\
\hline $\begin{array}{l}\text { Cesium-137 } \\
\text { Potassium-40 } \\
\text { Radium-226 } \\
\text { Thorium-232 } \\
\text { Uranium-235 } \\
\text { Uranium-238 }\end{array}$ & $\begin{array}{l}\mathrm{PCi} / \mathrm{g} \\
\mathrm{pCi} / 9 \\
\mathrm{PCi} / 9 \\
\mathrm{pCi} / 9 \\
\mathrm{PCi} / \mathrm{g} \\
\mathrm{pCi} / 9\end{array}$ & $\begin{array}{r}1.4118 \\
18.6789 \\
0.8334 \\
1.4208\end{array}$ & $\begin{array}{r}0.3658 \\
17.0942 \\
0.7990 \\
1.4286 \\
0.1074 \\
0.7675\end{array}$ & $\begin{array}{r}0.0694 \\
16.7292 \\
0.7655 \\
1.3369 \\
3.3155\end{array}$ & $\begin{array}{r}0.0235 \\
16.9414 \\
0.7565 \\
1.3412\end{array}$ & $\begin{array}{r}12.1189 \\
0.7995 \\
1.3938\end{array}$ & $\begin{array}{r}0.0261 \\
13.0763 \\
0.7460 \\
1.3556 \\
0.0920 \\
1.0901\end{array}$ \\
\hline
\end{tabular}

Location=ORR; Formation $=$ NOLICHUCKY; Site $=21 ; 00-05=1367 ; 05-10=1368 ; 10-15=1369 ; 15-20=1370$; $20-25=1371 ; 25-30=1372 ;$ Phase $=1$

$\begin{array}{llrrrrrr}\text { Cesium-137 } & \mathrm{PCi} / \mathrm{g} & 3.0932 & 0.1176 & 0.0299 & 0.0294 & 0.0157 & 0.0081 \\ \text { Potassium-40 } & \mathrm{PCi} / \mathrm{g} & 34.9695 & 30.5231 & 24.6009 & 23.5949 & 27.9409 & 36.0979 \\ \text { Radium-226 } & \mathrm{PCi} / \mathrm{g} & 0.7174 & 0.6222 & 0.7803 & 0.5500 & 0.5476 & 0.5080 \\ \text { Thorium-232 } & \mathrm{PCi} / \mathrm{g} & 1.7714 & 1.6407 & 1.6096 & 1.7286 & 1.6812 & 1.4985 \\ \text { Uranium-235 } & \mathrm{PCi} / \mathrm{g} & 0.2569 & 0.0629 & 0.1863 & 0.1076 & 0.2444 & 0.0296 \\ \text { Uranium-238 } & \mathrm{PCi} / \mathrm{g} & 4.6271 & 3.0044 & 2.9325 & 3.2139 & 2.6644 & 1.2930\end{array}$

Location =ORR; Formation $=$ NOLICHUCKY; Sile $=23 ; 00-05=1316 ; 05-10=1317 ; 10-15=1318 ; 15-20=1319$; $20-25=1320 ; 25-30=1321 ;$ Phase $=1$

$\begin{array}{llrrrrrr}\text { Cesium-137 } & \text { pCi/g } & 1.0747 & 0.5820 & 0.0663 & 0.0405 & 0.0283 & 0.0380 \\ \text { Potassium-40 } & \text { pCi/g } & 10.2992 & 12.4700 & 21.3062 & 27.4506 & 28.2411 & 29.2868 \\ \text { Radium-226 } & \text { pCi/g } & 0.7428 & 0.7925 & 0.6529 & 0.5557 & 0.6003 & 0.5588 \\ \text { Thorium-232 } & \text { pCi/g } & 1.1539 & 1.2869 & 1.5108 & 1.6378 & 1.6599 & 1.6898 \\ \text { Uranium-235 } & \text { pCi/g } & 0.2182 & 0.2202 & 0.2275 & 0.2288 & 0.2327 & 0.2311 \\ \text { Uranium-238 } & \text { pCi/g } & 2.5799 & 2.7668 & 3.8144 & 4.3977 & 4.8436 & 2.5994\end{array}$

Location=ORR; Formation=NOLICHUCKY; Site =24; 00-05=1361; 05-10=1362; 10-15=1363; 15-20=1364; $20-25=1365 ; 25-30=1366 ;$ Phase $=1$

$\begin{array}{llrrrrrr}\text { Cesium-137 } & \mathrm{pCi} / \mathrm{g} & 1.8149 & 0.4331 & 0.1023 & 0.0399 & 0.0273 & 0.0016 \\ \text { Potassium-40 } & \mathrm{PCi} / \mathrm{g} & 29.1557 & 25.3314 & 27.3521 & 28.6304 & 30.9524 & 31.1790 \\ \text { Radium-226 } & \mathrm{pCi} / \mathrm{g} & 0.8650 & 0.6682 & 0.6839 & 0.6464 & 0.6724 & 0.6171 \\ \text { Thorium-232 } & \mathrm{PCi} / \mathrm{g} & 1.5330 & 1.3191 & 1.5141 & 1.5585 & 1.6765 & 1.6022 \\ \text { Uranium-235 } & \mathrm{pCi} / \mathrm{g} & 0.2297 & 0.1567 & 0.1659 & 0.1657 & 0.1667 & 0.0492 \\ \text { Uranium-238 } & \mathrm{pCi} / \mathrm{g} & 4.1051 & 4.5234 & 1.1107 & 2.5580 & 2.8852 & 0.9021\end{array}$

Location=ORR; Formation =NOLICHUCKY; Site $=25 ; 00-05=1325 ; 05-10=1326 ; 10-15=1327 ; 15-20=1328$; $20-25=1329 ; 25-30=1330 ;$ Phase $=1$

\begin{tabular}{|c|c|c|c|c|c|c|c|}
\hline $\begin{array}{l}\text { Cesium-137 } \\
\text { Potassium-40 } \\
\text { Radium-226 } \\
\text { Thorium-232 } \\
\text { Uranium-235 } \\
\text { Uranium-238 }\end{array}$ & $\begin{array}{l}\mathrm{pCi} / \mathrm{g} \\
\mathrm{pCi} / \mathrm{g} \\
\mathrm{pCi} / \mathrm{g} \\
\mathrm{pCi} / \mathrm{g} \\
\mathrm{pCi} i \mathrm{~g} \\
\mathrm{pCi} / \mathrm{g}\end{array}$ & $\begin{array}{r}1.2195 \\
22.2143 \\
0.6716 \\
1.2591 \\
0.2184\end{array}$ & $\begin{array}{r}0.0508 \\
21.5251 \\
0.6243 \\
1.3567\end{array}$ & $\begin{array}{r}0.0220 \\
22.0695 \\
0.6632 \\
1.3916 \\
1.4411\end{array}$ & $\begin{array}{r}0.0459 \\
28.4921 \\
0.6693 \\
1.6653 \\
0.2563\end{array}$ & $\begin{array}{r}0.0073 \\
32.4522 \\
0.6224 \\
1.6946\end{array}$ & $\begin{array}{r}33.0335 \\
0.5640 \\
1.6435 \\
\end{array}$ \\
\hline
\end{tabular}

Location $=$ ORR; Formation $=$ NOLICHUCKY; Site $=28 ; 00-05=1337 ; 05-10=1338 ; 10-15=1339 ; 15-20=1340$; 20-25=1341; 25-30=1342; Phase $=1$

$\begin{array}{llrrrrrr}\text { Cesium-137 } & \text { pCi/g } & 1.4521 & 0.4315 & 0.1465 & 0.0288 & 0.0241 & 0.0079 \\ \text { Potassium-40 } & \text { PCi/g } & 10.9102 & 12.0193 & 12.0245 & 28.8269 & 35.8351 & 35.2583 \\ \text { Radium-226 } & \text { PCi/g } & 0.8626 & 0.9214 & 0.8631 & 0.7461 & 0.5446 & 0.6036 \\ \text { Thorium-232 } & \text { pCi/g } & 1.0515 & 1.1281 & 1.2079 & 1.4183 & 1.4888 & 1.4185 \\ \text { Uranium-235 } & \text { pCi/g } & 0.1886 & 0.1687 & 0.1467 & 0.3551 & 0.1632 & 0.0079 \\ \text { Uranium-238 } & \text { pCi/g } & 2.6364 & 2.1114 & 2.3656 & 1.5654 & 3.1094 & 0.9010\end{array}$


B-86

Table B.2 (continued)

\begin{tabular}{llllllll}
\hline & \multicolumn{8}{c}{ Depth $(\mathrm{cm})$} \\
\cline { 2 - 7 } Analysis & Units & $00-05$ & $05-10$ & $10-15$ & $15-20$ & $20-25$ & $25-30$ \\
\hline
\end{tabular}

Location =ORR; Formation =NOLICHUCKY; Site=3; 00-05=1331; 05-10=1332; 10-15=1333; $15-20=1334$; $20-25=1335 ; 25-30=1336 ;$ Phase $=1$

$\begin{array}{lllrrrrr}\text { Cesium-137 } & \text { PCi/g } & 1.18164 & 0.3320 & 0.0515 & 0.0236 & 0.0261 & 0.0017 \\ \text { Potassium-40 } & \text { PCi/g } & 9.83880 & 11.2675 & 10.9431 & 11.8086 & 15.6450 & 15.6981 \\ \text { Radium-226 } & \text { PCi/g } & 0.76528 & 0.7156 & 0.6469 & 0.7372 & 0.7150 & 0.7377 \\ \text { Thorium-232 } & \text { PCi/g } & 1.00806 & 1.0064 & 0.9741 & 1.1360 & 1.2901 & 1.2811 \\ \text { Uranium-235 } & \text { pCi/g } & 0.15702 & 0.1385 & 0.0556 & 0.1362 & 0.1167 & 0.0301 \\ \text { Uranium-238 } & \text { pCi/g } & 2.51189 & 1.9898 & 1.9450 & 2.2331 & 2.4346 & 0.8476\end{array}$

Location =ORR; Formation =NOLICHUCKY; Site =31; 00-05=1373; 05-10=1374; 10-15=1375; $15-20=1376$; $20-25=1377 ; 25-30=1378 ;$ Phase $=1$

\begin{tabular}{|c|c|c|c|c|c|c|c|}
\hline $\begin{array}{l}\text { Cesium-137 } \\
\text { Potassium-40 } \\
\text { Radium-226 } \\
\text { Thorium-232 } \\
\text { Uranium-235 } \\
\text { Uranium-238 }\end{array}$ & $\begin{array}{l}p C i / g \\
p C i / g \\
p C i / g \\
p C i / g \\
p C i / g \\
p C i / g\end{array}$ & $\begin{array}{l}1.81133 \\
7.58745 \\
0.81426 \\
1.10946 \\
0.18207 \\
3.21282\end{array}$ & $\begin{array}{l}0.40512 \\
7.85760 \\
0.87903 \\
1.08215 \\
0.27899 \\
1.08908\end{array}$ & $\begin{array}{l}0.06764 \\
7.91839 \\
0.84332 \\
1.11157 \\
0.14678 \\
2.30077\end{array}$ & $\begin{array}{l}0.01823 \\
8.72471 \\
0.89600 \\
1.13255 \\
0.06268 \\
1.38541\end{array}$ & $\begin{array}{r}0.0082 \\
10.7174 \\
0.8555 \\
1.1654 \\
0.0492 \\
0.8731\end{array}$ & $\begin{array}{r}0.0093 \\
13.0808 \\
0.7552 \\
1.2004 \\
0.0811 \\
2.0346\end{array}$ \\
\hline
\end{tabular}

Location=ORR; Formation $=$ NOLICHUCKY; Site $=42 ; 00-05=1379 ; 05-10=1380 ; 10-15=1381 ; 15-20=1382 ;$ $20-25=1383 ; 25-30=1384 ;$ Phase $=1$

$\begin{array}{llrrrrrr}\text { Cesium-137 } & \text { pCi/g } & 1.1265 & 0.1807 & 0.0418 & 0.0249 & 0.0041 & 0.0093 \\ \text { Potassium-40 } & \text { pCi/g } & 12.7187 & 13.3355 & 21.0567 & 23.6743 & 20.8052 & 19.6050 \\ \text { Radium-226 } & \text { pCi/g } & 0.7755 & 0.6449 & 0.7492 & 0.5907 & 0.6654 & 0.6581 \\ \text { Thorium-232 } & \text { pCi/g } & 1.1648 & 1.1105 & 1.2794 & 1.3561 & 1.3606 & 1.2759 \\ \text { Uranium-235 } & \text { pCi/g } & 0.1788 & 0.1978 & 0.0899 & 0.0443 & 0.0432 & 0.0236 \\ \text { Uranium-238 } & \text { pCi/g } & 3.2981 & 2.1334 & 1.1212 & 2.5000 & 1.2277 & 1.6120\end{array}$

Location=ORR; Formation =NOLICHUCKY; Site $=5 ; 00-05=1349 ; 05-10=1350 ; 10-15=1351 ; 15-20=1352$; $20-25=1353 ; 25-30=1354 ;$ Phase $=1$

$\begin{array}{llllrrrr}\text { Cesium-137 } & \text { PCi/g } & 1.13769 & 0.42327 & 0.0943 & 0.0288 & 0.0096 & 0.0034 \\ \text { Potassium-40 } & \text { PCi/g } & 8.44980 & 9.81740 & 10.3635 & 11.9426 & 13.3605 & 14.0540 \\ \text { Radium-226 } & \text { PCi/g } & 0.84649 & 1.03180 & 0.7932 & 0.7644 & 0.7808 & 0.6828 \\ \text { Thorium-232 } & \text { PCi/g } & 1.09361 & 1.27041 & 1.1657 & 1.1789 & 1.2477 & 1.2289 \\ \text { Uranium-235 } & \text { PCi/g } & 0.10365 & 0.16878 & 0.0620 & 0.1338 & 0.1376 & 0.0866 \\ \text { Uranium-238 } & \text { PCi/g } & 0.86400 & 2.24251 & 1.9811 & 1.9422 & 2.3860 & 0.8546\end{array}$

Location =ROA; Formation $=$ COPPER RIDGE; Site $=33 ; 00-05=3248 ; 05-10=3249 ; 10-15=3250$; $15-20=3251 ; 20-25=3252 ; 25-30=3253 ;$ Phase $=2$

\begin{tabular}{|c|c|c|c|c|c|c|c|}
\hline $\begin{array}{l}\text { Cesium- } 137 \\
\text { Potassium-40 } \\
\text { Radium-226 } \\
\text { Thorium-232 } \\
\text { Uranium-235 }\end{array}$ & $\begin{array}{l}\mathrm{pCi} / \mathrm{g} \\
\mathrm{pCi} / \mathrm{g} \\
\mathrm{pCi} \mathrm{g} \\
\mathrm{pCi} / \mathrm{g} \\
\mathrm{pCi} / \mathrm{g}\end{array}$ & $\begin{array}{l}1.42089 \\
3.66091 \\
0.61187 \\
0.46099\end{array}$ & $\begin{array}{l}0.17370 \\
5.62405 \\
0.85530 \\
0.86471 \\
0.13936\end{array}$ & $\begin{array}{l}0.13236 \\
5.68683 \\
0.94200 \\
1.00398 \\
0.08840\end{array}$ & $\begin{array}{l}0.08078 \\
6.20255 \\
1.05538 \\
1.17719 \\
0.07543\end{array}$ & $\begin{array}{l}0.00976 \\
6.42044 \\
1.00632 \\
1.28282 \\
0.09698\end{array}$ & $\begin{array}{l}0.02394 \\
5.84363 \\
0.92647 \\
1.17226 \\
0.08271\end{array}$ \\
\hline
\end{tabular}

Location $=$ ROA; Formation $=$ COPPER RIDGE; Site $=34 ; 00-05=3334 ; 05-10=3335 ; 10-15=3336$; $15-20=3337 ; 20-25=3338 ; 25-30=3339 ;$ Phase $=2$

$\begin{array}{llllllll}\text { Cesium-137 } & \text { pCi/g } & 1.50010 & 0.39234 & 0.13031 & 0.04139 & 0.01188 & 0.02232 \\ \text { Potassium-40 } & \text { PCi/g } & 2.06069 & 3.20465 & 2.81806 & 3.69338 & 3.97714 & 4.49428 \\ \text { Radium-226 } & \text { pCi/g } & 1.20894 & 1.26975 & 1.14309 & 1.21746 & 1.22803 & 1.33746 \\ \text { Thorium-232 } & \text { PCi/g } & 0.47181 & 0.62236 & 0.62107 & 0.80169 & 0.93824 & 1.13285 \\ \text { Uranium-235 } & \text { PCi/g } & 0.20547 & 0.19351 & 0.11316 & 0.41792 & 0.09387 & 0.09821 \\ \text { Uranium-238 } & \text { pCi/g } & 2.15706 & 2.07559 & 2.18372 & 3.35191 & 1.81413 & 2.93381\end{array}$


B-87

Table B2 (continued)

\begin{tabular}{llllllll}
\hline & \multicolumn{8}{c}{ Depth $(\mathrm{cm})$} \\
\cline { 2 - 7 } Analysis & Units & $00-05$ & $05-10$ & $10-15$ & $15-20$ & $20-25$ & $25-30$ \\
\hline
\end{tabular}

Location =ROA; Formation =COPPER RIDGE; Site $=35 ; 00-05=3239 ; 05-10=3240 ; 10-15=3241$; $15-20=3242 ; 20-25=3243 ; 25-30=3244 ;$ Phase $=2$

$\begin{array}{llllllll}\text { Cesium-137 } & \text { PCi/g } & 0.74448 & 0.47548 & 0.39568 & 0.22968 & 0.11171 & 0.01546 \\ \text { Potassium-40 } & \text { PCi/g } & 2.37983 & 2.97783 & 3.11111 & 3.84590 & 3.84399 & 3.56885 \\ \text { Radium-226 } & \text { pCi/g } & 0.70666 & 0.77877 & 0.77855 & 0.82811 & 0.91289 & 0.80375 \\ \text { Thorium-232 } & \text { pCi/g } & 0.54866 & 0.59929 & 0.59707 & 0.67450 & 0.76217 & 0.71218 \\ \text { Uranium-235 } & \text { pCi/g } & \mathbf{0 . 1 3 4 2 3} & \mathbf{0 . 1 2 7 0 1} & \mathbf{0 . 0 5 5 9 6} & \mathbf{0 . 1 3 0 2 0} & & \mathbf{0 . 0 4 4 6 0}\end{array}$

Location=ROA; Formation $=$ COPPER RIDGE; Site $=39 ; 00-05=3343 ; 05-10=3344 ; 10-15=3345$; $15-20=3346 ; 20-25=3347 ; 25-30=3348 ;$ Phase $=2$

$\begin{array}{llllllll}\text { Cesium-137 } & \text { pCi/g } & 1.19964 & 0.52695 & 0.15373 & 0.03051 & 0.02256 & 0.00601 \\ \text { Potassium-40 } & \text { pCi/g } & 1.13013 & 1.60930 & 2.01373 & 2.47298 & 2.17872 & 2.05719 \\ \text { Radium-226 } & \text { PCi/g } & 0.37441 & 0.50181 & 0.57357 & 0.63343 & 0.61066 & 0.56519 \\ \text { Thorium-232 } & \text { pCi/g } & 0.28021 & 0.38252 & 0.42223 & 0.47041 & 0.44419 & 0.46148 \\ \text { Uranium-235 } & \text { pCi/g } & 0.15649 & 0.15288 & 0.14462 & 0.14204 & 0.11531 & 0.06908 \\ \text { Uranium-238 } & \text { pCi/g } & 3.03199 & 2.80025 & 2.35244 & 2.57874 & 2.62625 & 0.56791\end{array}$

Location=ROA; Formation $=$ COPPER RIDGE; Site $=40 ; 00-05=3267 ; 05-10=3268 ; 10-15=3269$; $15-20=3270 ; 20-25=3271 ; 25-30=3272 ;$ Phase $=2$

$\begin{array}{llllllll}\text { Cesium-137 } & \mathrm{pCi} / \mathrm{g} & 1.02160 & 0.30799 & 0.05902 & 0.01482 & 0.00677 & 0.00663 \\ \text { Potassium-40 } & \mathrm{pCi/g} & 1.69320 & 1.30756 & 1.47958 & 1.53804 & 2.05725 & 1.91741 \\ \text { Radium-226 } & \mathrm{pCi} / \mathrm{g} & 0.58486 & 0.53910 & 0.54432 & 0.54351 & 0.55226 & 0.55265 \\ \text { Thorium-232 } & \mathrm{pCi/g} & 0.40629 & 0.37357 & 0.40845 & 0.39573 & 0.44499 & 0.45797 \\ \text { - Uranium-235 } & \mathrm{pCi} / \mathrm{g} & \mathbf{0 . 1 4 0 1 7} & 0.10252 & \mathbf{0 . 0 3 2 6 3} & \mathbf{0 . 1 0 0 8 2} & \mathbf{0 . 0 7 7 1} & \mathbf{0 . 0 4 5 0 8}\end{array}$

Location $=R O A ;$ Formation $=$ COPPER RIDGE; Site $=41 ; 00-05=3352 ; 05-10=3353 ; 10-15=3354 ;$ $15-20=3355 ; 20-25=3356 ; 25-30=3357 ;$ Phase $=2$

$\begin{array}{llllllll}\text { Cesium-137 } & \mathrm{pCi} / \mathrm{g} & 0.88398 & 0.33666 & 0.10927 & 0.03548 & 0.02445 & 0.01198 \\ \text { Potassium-40 } & \mathrm{pCi/g} & 1.28679 & 1.71866 & 1.42272 & 1.73185 & 1.78048 & 1.44937 \\ \text { Radium-226 } & \mathrm{pCi/g} & 0.44430 & 0.54968 & 0.51597 & 0.57426 & 0.55097 & 0.42974 \\ \text { Thorium-232 } & \mathrm{pCi} / \mathrm{g} & 0.30179 & 0.41479 & 0.33370 & 0.40299 & 0.40394 & 0.31513 \\ \text { Uranium-235 } & \mathrm{pCi/g} & 0.15924 & 0.13800 & 0.15647 & 0.13517 & 0.12688 & 0.03775 \\ \text { Uranium-238 } & \mathrm{pCi/g} & 1.95675 & 1.31326 & 2.74891 & 2.63331 & \mathbf{2 . 4 3 2 8 0} & \mathbf{0 . 6 6 4 6 8}\end{array}$

Location =ROA; Formation $=$ COPPER RIDGE; Site $=42 ; 00-05=3276 ; 05-10=3277 ; 10-15=3278$; $15-20=3279 ; 20-25=3280 ; 25-30=3281 ;$ Phase $=2$

$\begin{array}{llllllll}\text { Cesium-137 } & \text { pCi/g } & 0.81581 & 0.28574 & 0.07326 & 0.05847 & 0.02060 & 0.00336 \\ \text { Potassium-40 } & \text { pCi/g } & 1.56942 & 2.56750 & 2.59056 & 2.58473 & 2.55578 & 2.78359 \\ \text { Radium-226 } & \text { pCi/g } & 0.64389 & 0.68527 & 0.79614 & 0.80461 & 0.77313 & 0.74344 \\ \text { Thorium-232 } & \text { pCi/g } & 0.49772 & 0.47805 & 0.55512 & 0.55197 & 0.55703 & 0.55041 \\ \text { Uranium-235 } & \text { pCi/g } & 0.14479 & 0.03502 & 0.13266 & 0.05985 & 0.12432 & \mathbf{0 . 0 8 7 6 5}\end{array}$

Location $=R O A ;$ Formation $=C O P P E R$ RIDGE; Site $=43 ; 00-05=3285 ; 05-10=3286 ; 10-15=3287$; $15-20=3288 ; 20-25=3289 ; 25-30=3290 ;$ Phase $=2$

$\begin{array}{llllllll}\text { Cesium-137 } & \text { pCi/g } & 1.33013 & 0.60864 & 0.13026 & 0.07920 & 0.03467 & 0.02015 \\ \text { Potassium-40 } & \text { pCi/g } & 2.38210 & 2.36627 & 2.49891 & 2.43622 & 2.50911 & 2.61555 \\ \text { Radium-226 } & \text { pCi/g } & 0.62641 & 0.63599 & 0.65419 & 0.73858 & 0.73074 & 0.70106 \\ \text { Thorium-232 } & \text { pCi/g } & 0.47328 & 0.53264 & 0.63097 & 0.61873 & 0.63939 & 0.73477 \\ \text { Uranium-235 } & \text { pCi/g } & & 0.24902 & 0.12685 & 0.27740 & 0.12333 & 0.09324\end{array}$

Location $=R O A ;$ Formation $=$ COPPER $R I D G E ;$ Site $=44 ; 00-05=3257 ; 05-10=3258 ; 10-15=3259$; $15-20=3260 ; 20-25=3261 ; 25-30=3262 ;$ Phase $=2$

$\begin{array}{llllllll}\text { Cesium-137 } & \text { PCi/g } & 0.65399 & 0.19382 & 0.03836 & 0.02781 & 0.02858 & 0.02969 \\ \text { Potassium-40 } & \text { pCi/g } & 1.58787 & 2.76000 & 2.61316 & 3.15918 & 2.91833 & 2.67472\end{array}$


B-88

Table B.2 (continued)

\begin{tabular}{|c|c|c|c|c|c|c|c|}
\hline \multirow[b]{2}{*}{ Analysis } & \multicolumn{6}{|c|}{ Depth (cm) } & \multirow[b]{2}{*}{$25-30$} \\
\hline & Units & $00-05$ & $05-10$ & $10-15$ & $15-20$ & $20-25$ & \\
\hline $\begin{array}{l}\text { Radium-226 } \\
\text { Thorium-232 } \\
\text { Uranium-235 }\end{array}$ & $\begin{array}{l}\mathrm{pCi} / \mathrm{g} \\
\mathrm{pCi} / \mathrm{g} \\
\mathrm{pCi} / \mathrm{g}\end{array}$ & $\begin{array}{l}0.64419 \\
0.63206 \\
0.14065\end{array}$ & $\begin{array}{l}0.79218 \\
0.66964 \\
0.14304\end{array}$ & $\begin{array}{l}0.78815 \\
0.62208 \\
0.12120\end{array}$ & $\begin{array}{l}0.72928 \\
0.59277 \\
0.18906\end{array}$ & $\begin{array}{l}0.70668 \\
0.63110 \\
0.08387\end{array}$ & $\begin{array}{l}0.59892 \\
0.54020 \\
0.06813\end{array}$ \\
\hline \multicolumn{8}{|c|}{$\begin{array}{l}\text { Location }=\text { ROA; Formation }=C O P P E R \text { RIDGE; Site }=45 ; 00-05=3312 ; 05-10=3313 ; 10-15=3314 ; \\
\qquad 15-20=3315 ; 20-25=3316 ; 25-30=3317 ; \text { Phase }=2\end{array}$} \\
\hline $\begin{array}{l}\text { Cesium-137 } \\
\text { Potassium-40 } \\
\text { Radium-226 } \\
\text { Thorium-232 } \\
\text { Uranium-235 } \\
\text { Uranium-238 }\end{array}$ & $\begin{array}{l}\mathrm{pCi} / \mathrm{g} \\
\mathrm{pCi} / \mathrm{g} \\
\mathrm{pCi} / \mathrm{g} \\
\mathrm{pCi} / \mathrm{g} \\
\mathrm{pCi} / \mathrm{g} \\
\mathrm{pCi} / \mathrm{g}\end{array}$ & $\begin{array}{l}0.89438 \\
2.16620 \\
0.68194 \\
0.49183 \\
0.15722 \\
2.43663\end{array}$ & $\begin{array}{l}0.43598 \\
2.38725 \\
0.78870 \\
0.50322 \\
0.22716 \\
2.01107\end{array}$ & $\begin{array}{l}0.10722 \\
2.63967 \\
1.07470 \\
0.55650 \\
0.15622 \\
2.22693\end{array}$ & $\begin{array}{l}0.03823 \\
2.80100 \\
0.80994 \\
0.51313 \\
0.10671 \\
2.14694\end{array}$ & $\begin{array}{l}0.03171 \\
2.31511 \\
0.69929 \\
0.50845 \\
0.09078 \\
2.03645\end{array}$ & $\begin{array}{l}0.01201 \\
2.73322 \\
0.69517 \\
0.55551 \\
0.03234 \\
1.10243\end{array}$ \\
\hline
\end{tabular}

Location $=R O A ;$ Formation $=C O P P E R$ RIDGE; Site $=46 ; 00-05=3303 ; 05-10=3304 ; 10-15=3305$;

$15-20=3306 ; 20-25=3307 ; 25-30=3308 ;$ Phase $=2$

$\begin{array}{llllllll}\text { Cesium-137 } & \mathrm{PCi} / \mathrm{g} & \mathbf{0 . 8 7 1 7 3} & \mathbf{0 . 5 5 3 3 3} & 0.19283 & 0.05686 & 0.01535 & 0.02846 \\ \text { Potassium-40 } & \mathrm{PCi} / \mathrm{g} & 2.50159 & 2.51479 & 2.26845 & 2.49329 & 2.32488 & 2.98673 \\ \text { Radium-226 } & \mathrm{PCi} / \mathrm{g} & \mathbf{0 . 7 1 0 1 3} & 0.75386 & 0.77877 & 0.69155 & 0.70618 & 0.79303 \\ \text { Thorium-232 } & \mathrm{PCi} / \mathrm{g} & \mathbf{0 . 7 2 2 0 2} & \mathbf{0 . 7 0 8 2 6} & 0.64636 & 0.68451 & 0.78843 & 0.96833 \\ \text { Uranium-235 } & \mathrm{pCi} / \mathrm{g} & \mathbf{0 . 1 0 2 0 4} & \mathbf{0 . 1 5 3 8 2} & 0.05957 & \mathbf{0 . 0 9 3 5 4} & \mathbf{0 . 1 3 8 9 4} & \mathbf{0 . 1 5 6 2 6}\end{array}$

Location=ROA; Formation =COPPER RIDGE; Site =47; 00-05=3294; 05-10=3295; 10-15=3296; $15-20=3297 ; 20-25=3298 ; 25-30=3299 ;$ Phase $=2$

$\begin{array}{llllllll}\text { Cesium-137 } & \mathrm{PCi} / \mathrm{g} & \mathbf{0 . 8 3 5 2 8} & 0.18813 & 0.02676 & 0.02606 & 0.02194 & 0.02398 \\ \text { Potassium-40 } & \mathrm{PCi} / \mathrm{g} & 2.85348 & 3.77000 & 2.77097 & 3.17363 & 3.17019 & 2.83175 \\ \text { Radium-226 } & \mathrm{PCi} / \mathrm{g} & \mathbf{0 . 8 7 3 0 8} & \mathbf{0 . 9 3 7 8 5} & 0.89051 & 0.86913 & 0.84645 & 0.82832 \\ \text { Thorium-232 } & \mathrm{PCi} / \mathrm{g} & 0.90364 & 0.80033 & 0.75580 & 0.73762 & 0.78887 & 0.74115 \\ \text { Uranium-235 } & \mathrm{PCi} / \mathrm{g} & \mathbf{0 . 1 0 0 9 1} & \mathbf{0 . 1 6 2 6 2} & \mathbf{0 . 1 5 5 5 3} & 0.10678 & \mathbf{0 . 1 4 4 2 7} & \mathbf{0 . 1 4 1 8 4}\end{array}$

Location=ROA; Formation =DISMAL GAP; Site =10;00-05=3178; 05-10=3179; 10-15=3180; 15-20=3181; 20-25=3182; 25-30=3183; Phase $=1$

$\begin{array}{llrrrrrr}\text { Cesium-137 } & \text { pCi/g } & 0.6479 & 0.0460 & 0.0289 & 0.0182 & 0.0259 & 0.0257 \\ \text { Potassium-40 } & \text { pCi/g } & 11.1886 & 12.2362 & 13.1197 & 17.5574 & 19.4597 & 21.8072 \\ \text { Radium-226 } & \text { pCi/g } & 0.6988 & 0.7406 & 0.7150 & 0.7095 & 0.7025 & 0.7337 \\ \text { Thorium-232 } & \text { pCi/g } & 0.9756 & 1.1513 & 1.1846 & 1.4620 & 1.5374 & 1.7462 \\ \text { Uranium-235 } & \text { pCi/g } & 0.1505 & 0.0913 & 0.0219 & 0.0350 & 0.1580 & 0.1664 \\ \text { Uranium-238 } & \text { pCi/g } & 2.2086 & 0.4811 & 2.2488 & 1.1617 & 1.9573 & 2.7254\end{array}$

Location=ROA; Formation=DISMAL GAP; Site=13;00-05=3131;05-10=3132; 10-15=3133; 15-20=3134; 20-25=3135; 25-30=3136; Phase $=1$

$\begin{array}{lllllrrr}\text { Cesium-137 } & \text { PCi/g } & 0.20342 & 0.03059 & 0.01112 & 0.0064 & 0.0494 & 0.0253 \\ \text { Potassium-40 } & \text { PCi/g } & 6.21173 & 6.58742 & 8.07113 & 11.7095 & 16.5192 & 19.2372 \\ \text { Radium-226 } & \text { PCi/g } & 0.70700 & 0.70586 & 0.71849 & 0.6952 & 0.6415 & 0.6707 \\ \text { Thorium-232 } & \text { PCi/g } & 1.01938 & 0.97115 & 1.01649 & 1.1559 & 1.2880 & 1.4293 \\ \text { Uranium-235 } & \text { PCi/g } & 0.10645 & 0.07286 & 0.12643 & 0.1399 & 0.1517 & 0.1553 \\ \text { Uranium-238 } & \text { PCi/g } & 2.28876 & 1.65555 & 2.15058 & 1.3867 & 1.0939 & 2.4900\end{array}$

Location=ROA; Formation=DISMAL GAP; Site=14;00-05=3152;05-10=3153; 10-15=3154; 15-20=3155; $20-25=3156 ; 25-30=3157 ;$ Phase $=1$

$\begin{array}{llrrrrrr}\text { Cesium-137 } & \text { pCi/g } & 1.5469 & 0.2365 & 0.0486 & 0.0053 & 0.0383 & 0.0366 \\ \text { Potassium-40 } & \text { pCi/g } & 15.9916 & 21.2335 & 20.4532 & 17.4199 & 18.2892 & 18.5685 \\ \text { Radium-226 } & \text { pCi/g } & 0.6981 & 0.6281 & 0.6715 & 0.6370 & 0.6924 & 0.5819 \\ \text { Thorium-232 } & \text { pCi/g } & 1.2680 & 1.3550 & 1.4823 & 1.4512 & 1.5986 & 1.5064 \\ \text { Uranium-235 } & \text { pCi/g } & 0.1999 & 0.2226 & 0.1907 & 0.2500 & 0.1130 & 0.2129 \\ \text { Uranium-238 } & \text { pCi/g } & 4.1361 & 3.5846 & 2.4398 & 3.9481 & 4.4876 & 3.7519\end{array}$


B-89

Table B.2 (continued)

\begin{tabular}{llllllll}
\hline & \multicolumn{8}{c}{ Depth $(\mathrm{cm})$} \\
\cline { 2 - 8 } Analysis & Units & $00-05$ & $05-10$ & $10-15$ & $15-20$ & $20-25$ & $25-30$ \\
\hline
\end{tabular}

Location=ROA; Formation $=$ DISMAL GAP; Site=17; 00-05=3022; 05-10=3023; 10-15=3024; 15-20=3025; $20-25=3026 ; 25-30=3027 ;$ Phase $=1$

$\begin{array}{lrlllrrr}\text { Cesium-137 } & \text { PCi/g } & 1.06811 & 0.41668 & 0.12426 & 0.0491 & 0.0243 & 0.0144 \\ \text { Potassium-40 } & \text { PCi/g } & 7.86085 & 8.01872 & 8.32302 & 12.3916 & 15.1041 & 17.0407 \\ \text { Radium-226 } & \text { PCi/g } & 0.74439 & 0.74809 & 0.68905 & 0.6779 & 0.7289 & 0.7326 \\ \text { Thorium-232 } & \text { PCi/g } & 1.01544 & 1.02281 & 1.05709 & 1.1309 & 1.2731 & 1.3357 \\ \text { Uranium-235 } & \text { PCi/g } & 0.08073 & 0.15287 & & 0.1279 & & 0.0391 \\ \text { Uranium-238 } & \text { PCi/g } & 1.49078 & 0.61736 & 1.98948 & 2.0866 & 2.3101 & 0.7476\end{array}$

Location=ROA; Formation=DISMAL GAP; Site=19; 00-05=3036;05-10=3037; 10-15=3038; 15-20=3039; 20-25=3040; 25-30=3041; Phase =1

\begin{tabular}{|c|c|c|c|c|c|c|c|}
\hline $\begin{array}{l}\text { Cesium-137 } \\
\text { Potassium-40 } \\
\text { Radium-226 } \\
\text { Thorium-232 } \\
\text { Uranium-235 } \\
\text { Uranium-238 }\end{array}$ & $\begin{array}{l}\mathrm{pCi} / \mathrm{g} \\
\mathrm{pCi} / \mathrm{g} \\
\mathrm{pCi} / \mathrm{g} \\
\mathrm{pCi} / \mathrm{g} \\
\mathrm{pCi} / \mathrm{g} \\
\mathrm{pCi} / \mathrm{g}\end{array}$ & $\begin{array}{l}0.77258 \\
7.65583 \\
0.98148 \\
1.22177 \\
0.10668 \\
1.22403\end{array}$ & $\begin{array}{l}0.07183 \\
9.00872 \\
1.22142 \\
1.38829 \\
0.20344 \\
2.76961\end{array}$ & $\begin{array}{l}0.02488 \\
8.31273 \\
1.13411 \\
1.21685 \\
2.52996\end{array}$ & $\begin{array}{l}0.02617 \\
9.59149 \\
0.92588 \\
1.26869 \\
0.17395 \\
2.85302\end{array}$ & $\begin{array}{l}0.01874 \\
9.03652 \\
1.00616 \\
1.33524 \\
0.10917 \\
2.96084\end{array}$ & $\begin{array}{l}0.02448 \\
9.54134 \\
0.94241 \\
1.25627 \\
0.06610 \\
2.53167\end{array}$ \\
\hline
\end{tabular}

Location=ROA; Formation =DISMAL GAP; Site $=20 ; 00-05=3050 ; 05-10=3051 ; 10-15=3052 ; 15-20=3053$; $20-25=3054 ; 25-30=3055 ;$ Phase $=1$

$\begin{array}{llllrrrr}\text { Cesium-137 } & \text { PCi/g } & 0.86373 & 0.16860 & 0.0694 & 0.0374 & 0.0177 & 0.0102 \\ \text { Potassium-40 } & \text { pCi/g } & 8.37732 & 9.11153 & 15.4059 & 18.4632 & 26.6641 & 29.0528 \\ \text { Radium-226 } & \text { PCi/g } & 0.98084 & 0.64531 & 0.6469 & 0.6153 & 0.5690 & 0.6767 \\ \text { Thorium-232 } & \text { pCi/g } & 0.94018 & 0.97575 & 1.2443 & 1.2996 & 1.4085 & 1.4203 \\ \text { Uranium-235 } & \text { pCi/g } & 0.18081 & 0.15954 & 0.1336 & 0.1782 & 0.0445 & 0.0749 \\ \text { Uranium-238 } & \text { pCi/g } & 2.96989 & 3.16245 & 1.9166 & 3.9993 & 1.1015 & 0.6548\end{array}$

Location=ROA; Formation =DISMAL GAP; Site =21;00-05=3117; 05-10=3118; $10-15=3119 ; 15-20=3120$; $20-25=3121 ; 25-30=3122 ;$ Phase $=1$

$\begin{array}{llrrrrrr}\text { Cesium-137 } & \text { PCi/g } & 0.9331 & 0.1301 & 0.02048 & 0.03094 & 0.01416 & 0.03293 \\ \text { Potassium-40 } & \text { pCi/g } & 12.7528 & 12.7725 & 9.16888 & 8.93252 & 9.76466 & 9.57555 \\ \text { Radium-226 } & \text { pCi/g } & 0.6918 & 0.8860 & 0.79204 & 0.87766 & 1.03810 & 0.89798 \\ \text { Thorium-232 } & \text { pCi/g } & 1.2858 & 1.3053 & 1.11777 & 1.17581 & 1.22282 & 1.26791 \\ \text { Uranium-235 } & \text { PCi/g } & \mathbf{0 . 1 0 2 0} & \mathbf{0 . 0 9 9 3} & \mathbf{0 . 1 8 3 9 1} & \mathbf{0 . 2 0 8 6 2} & \mathbf{0 . 2 0 4 2 6} & \mathbf{0 . 2 3 6 1 8} \\ \text { Uranium-238 } & \text { pCi/g } & \mathbf{3 . 6 1 2 2} & 1.3212 & \mathbf{3 . 1 7 4 1 8} & \mathbf{3 . 5 8 7 2 1} & \mathbf{2 . 3 3 8 4 4} & \mathbf{3 . 6 0 5 1 4}\end{array}$

Location $=R O A ;$ Formation $=D I S M A L$ GAP; Site $=22 ; 00-05=3062 ; 05-10=3063 ; 10-15=3064 ; 15-20=3065$; $20-25=3066 ; 25-30=3067 ;$ Phase $=1$

$\begin{array}{llllrrrr}\text { Cesium-137 } & \text { PCi/g } & 0.56818 & 0.11340 & 0.0243 & 0.0286 & 0.0322 & 0.0077 \\ \text { Potassium-40 } & \text { PCi/g } & 7.22849 & 8.81445 & 12.4847 & 17.6887 & 21.6703 & 21.7934 \\ \text { Radium-226 } & \text { PCi/g } & 0.72895 & 0.65960 & 0.6180 & 1.0002 & 1.0064 & 0.9894 \\ \text { Thorium-232 } & \text { PCi/g } & 0.92026 & 0.95387 & 1.0513 & 1.3405 & 1.5092 & 1.4504 \\ \text { Uranium-235 } & \text { PCi/g } & 0.13483 & 0.11169 & 0.1491 & 0.0546 & 0.3750 & 0.0671 \\ \text { Uranium-238 } & \text { pCi/g } & 3.73728 & 2.25628 & 2.3393 & 3.0799 & 3.0480 & 1.1228\end{array}$

Location $=R O A ;$ Formation $=D I S M A L$ GAP; Site $=3 ; 00-05=3103 ; 05-10=3104 ; 10-15=3105 ; 15-20=3106$; 20-25=3107; 25-30=3108; Phase $=1$

$\begin{array}{llrrrrrr}\text { Cesium-137 } & \text { PCi/g } & 0.8547 & 0.1987 & 0.0747 & 0.0327 & 0.0061 & 0.0162 \\ \text { Potassium-40 } & \text { pCi/g } & 11.5364 & 10.8670 & 10.4431 & 10.4249 & 10.9776 & 11.0646 \\ \text { Radium-226 } & \text { pCi/g } & 0.7445 & 0.9087 & 0.8165 & 0.7291 & 0.6889 & 0.6538 \\ \text { Thorium-232 } & \text { pCi/g } & 1.1137 & 1.1802 & 1.1179 & 1.1422 & 1.1728 & 1.1765 \\ \text { Uranium-235 } & \text { pCi/g } & 0.0547 & 0.1555 & 0.1333 & 0.0442 & 0.1412 & 0.1444 \\ \text { Uranium-238 } & \text { pCi/g } & 4.2823 & 1.7353 & 2.7705 & 2.4742 & 2.5445 & 2.4345\end{array}$


Table B.2 (continued)

\begin{tabular}{|c|c|c|c|c|c|c|c|}
\hline \multirow[b]{2}{*}{ Analysis } & \multicolumn{6}{|c|}{ Depth $(\mathrm{cm})$} & \multirow[b]{2}{*}{$25-30$} \\
\hline & Units & $00-05$ & $05-10$ & $10-15$ & $15-20$ & $20-25$ & \\
\hline \multicolumn{8}{|c|}{$\begin{array}{l}\text { Location=ROA; Formation }=\text { DISMAL GAP; Site }=7 ; 00-05=3089 ; 05-10=3090 ; 10-15=3091 ; 15-20=3092 ; \\
20-25=3093 ; 25-30=3094 ; \text { Phase }=1\end{array}$} \\
\hline $\begin{array}{l}\text { Cesium-137 } \\
\text { Potassium-40 } \\
\text { Radium-226 } \\
\text { Thorium-232 } \\
\text { Uranium-235 } \\
\text { Uranium-238 }\end{array}$ & $\begin{array}{l}\mathrm{pCi} / \mathrm{g} \\
\mathrm{pCi} / \mathrm{g} \\
\mathrm{pCi} / \mathrm{g} \\
\mathrm{pCi} / \mathrm{g} \\
\mathrm{pCi} / \mathrm{g} \\
\mathrm{pCi} / \mathrm{g}\end{array}$ & $\begin{array}{l}1.00018 \\
7.85868 \\
0.78784 \\
0.99946 \\
0.09426 \\
2.25179\end{array}$ & $\begin{array}{l}0.06758 \\
9.07075 \\
0.77877 \\
1.15157 \\
0.17547 \\
1.09497\end{array}$ & $\begin{array}{l}0.05618 \\
8.85627 \\
0.80245 \\
1.09878 \\
0.32156 \\
1.61468\end{array}$ & $\begin{array}{r}0.0358 \\
10.6781 \\
0.7743 \\
1.1931 \\
0.1489 \\
1.6798\end{array}$ & $\begin{array}{r}0.0033 \\
15.1970 \\
0.7757 \\
1.3321 \\
0.0679 \\
1.3074\end{array}$ & $\begin{array}{r}0.0022 \\
18.7766 \\
0.7139 \\
1.4702 \\
0.0258 \\
1.1676\end{array}$ \\
\hline \multicolumn{8}{|c|}{$\begin{array}{l}\text { Location=ROA; Formation }=\text { DISMAL GAP; Site =8; } 00-05=3076 ; 05-10=3077 ; 10-15=3078 ; 15-20=3079 ; \\
20-25=3080 ; 25-30=3081 ; \text { Phase }=1\end{array}$} \\
\hline $\begin{array}{l}\text { Cesium-137 } \\
\text { Potassium-40 } \\
\text { Radium-226 } \\
\text { Thorium-232 } \\
\text { Uranium-235 } \\
\text { Uranium-238 }\end{array}$ & $\begin{array}{l}\mathrm{pCi} / \mathrm{g} \\
\mathrm{pCi} / \mathrm{g} \\
\mathrm{pCi} / \mathrm{g} \\
\mathrm{pCi} / \mathrm{g} \\
\mathrm{pCi} / \mathrm{g} \\
\mathrm{pCi} / \mathrm{g}\end{array}$ & $\begin{array}{l}1.80437 \\
6.84697 \\
0.73428 \\
1.10390 \\
0.16511 \\
2.17199\end{array}$ & $\begin{array}{l}0.47874 \\
6.31750 \\
0.74100 \\
1.07775 \\
1.97052\end{array}$ & $\begin{array}{l}0.07930 \\
6.59872 \\
0.75145 \\
1.10967 \\
0.11434 \\
2.13584\end{array}$ & $\begin{array}{l}0.02469 \\
7.26735 \\
0.79693 \\
1.16853 \\
0.13874 \\
1.33850\end{array}$ & $\begin{array}{l}0.02150 \\
8.17710 \\
0.74925 \\
1.29059 \\
0.14543 \\
1.17409\end{array}$ & $\begin{array}{r}0.0078 \\
10.9998 \\
0.7442 \\
1.2971 \\
0.1043 \\
1.2732\end{array}$ \\
\hline \multicolumn{8}{|c|}{$\begin{array}{c}\text { Location }=R O A ; \text { Formation }=D I S M A L \text { GAP; Site }=9 ; 00-05=3008 ; 05-10=3009 ; 10-15=3010 ; 15-20=3011 ; \\
20-25=3012 ; 25-30=3013 ; \text { Phase }=1\end{array}$} \\
\hline $\begin{array}{l}\text { Cesium-137 } \\
\text { Potassium-40 } \\
\text { Radium-226 } \\
\text { Thorium-232 } \\
\text { Uranium-235 } \\
\text { Uranium-238 }\end{array}$ & $\begin{array}{l}\mathrm{pCi} / \mathrm{g} \\
\mathrm{pCi} / \mathrm{g} \\
\mathrm{pCi} / \mathrm{g} \\
\mathrm{pCi} / \mathrm{g} \\
\mathrm{pCi} / \mathrm{g} \\
\mathrm{pCi} / \mathrm{g}\end{array}$ & $\begin{array}{l}2.37360 \\
7.97527 \\
0.76762 \\
1.07995 \\
0.20495 \\
1.68335\end{array}$ & $\begin{array}{l}0.33769 \\
7.65437 \\
0.79481 \\
1.15073 \\
0.16226 \\
1.37521\end{array}$ & $\begin{array}{l}0.03726 \\
7.31478 \\
0.75858 \\
1.02602 \\
0.04140 \\
0.69534\end{array}$ & $\begin{array}{l}0.00893 \\
7.00821 \\
0.78649 \\
1.03807 \\
0.08682 \\
1.51008\end{array}$ & $\begin{array}{l}0.00427 \\
7.48968 \\
0.78544 \\
1.01271 \\
0.08396 \\
0.79716\end{array}$ & $\begin{array}{l}0.00863 \\
7.82609 \\
0.71572 \\
0.96568 \\
0.05803 \\
1.24066\end{array}$ \\
\hline
\end{tabular}

aeighted results are on an oven-dried-soil weight (dry-weight) basis. 
Table B3. Unweighted gamma screening results for soil samples

\begin{tabular}{llllllll}
\hline & \multicolumn{8}{c}{ Depth (cm) } \\
\cline { 2 - 7 } Analysis & Units & $00-05$ & $05-10$ & $10-15$ & $15-20$ & $20-25$ & $25-30$ \\
\hline
\end{tabular}

Location $=A N D ;$ Formation $=C O P P E R$ RIDGE; Site $=31 ; 00-05=2203 ; 05-10=2204 ; 10-15=2205$; $15-20=2206 ; 20-25=2207 ; 25-30=2208 ;$ Phase $=2$

$\begin{array}{llrrrrrr}\text { Cesium-137 } & \mathrm{pCi} / \mathrm{cm}^{2} & 4.369 & 2.672 & 0.766 & 0.546 & 0.294 & 0.071 \\ \text { Potassium-40 } & \mathrm{pCi} / \mathrm{cm}^{2} & 10.360 & 13.480 & 15.570 & 20.800 & 18.370 & 18.890 \\ \text { Radium-226 } & \mathrm{pCi} / \mathrm{cm}^{2} & 3.024 & 3.906 & 4.483 & 5.050 & 5.607 & 5.333 \\ \text { Thorium-232 } & \mathrm{PCi} / \mathrm{cm}^{2} & 2.111 & 3.145 & 3.789 & 3.794 & 4.402 & 4.645 \\ \text { Uranium-235 } & \mathrm{pCi} / \mathrm{cm}^{2} & 1.355 & 0.375 & & 0.621 & 0.763 & 0.490 \\ \text { Uranium-238 } & \mathrm{PCi} / \mathrm{cm}^{2} & 11.200 & 7.221 & & 20.910 & 16.448 & 8.801\end{array}$

Location $=A N D ;$ Formation $=C O P P E R$ RIDGE; Site $=32 ; 00-05=2212 ; 05-10=2213 ; 10-15=2214 ;$ $15-20=2215 ; 20-25=2216 ; 25-30=2217 ;$ Phase $=2$

$\begin{array}{llrrrrrr}\text { Cesium-137 } & \mathrm{pCi} / \mathrm{cm}^{2} & 4.809 & 1.382 & 0.797 & 0.197 & 0.026 & 0.021 \\ \text { Potassium-40 } & \mathrm{pCi} / \mathrm{cm}^{2} & 9.288 & 18.360 & 20.340 & 23.790 & 27.260 & 29.300 \\ \text { Radium-226 } & \mathrm{pCi} / \mathrm{cm}^{2} & 3.206 & 5.025 & 6.073 & 6.589 & 6.000 & 5.478 \\ \text { Thorium-232 } & \mathrm{pCi} / \mathrm{cm}^{2} & 3.874 & 4.172 & 4.717 & 4.898 & 4.758 & 4.854 \\ \text { Uranium-235 } & \mathrm{pCi} / \mathrm{cm}^{2} & 0.848 & 0.810 & 1.694 & 1.110 & 1.098 & 0.608 \\ \text { Uranium-238 } & \mathrm{pCi} / \mathrm{cm}^{2} & 13.560 & 15.103 & 16.370 & 16.680 & .18 .520 & 11.120\end{array}$

Location $=A N D ;$ Formation $=$ COPPER RIDGE; Site $=33 ; 00-05=2221 ; 05-10=2222 ; 10-15=2223$; $15-20=2224 ; 20-25=2225 ; 25-30=2226 ;$ Phase $=2$

$\begin{array}{llrrrrrr}\text { Cesium-137 } & \mathrm{pCi} / \mathrm{cm}^{2} & 5.414 & 1.699 & 0.677 & 0.398 & 0.309 & 0.048 \\ \text { Potassium-40 } & \mathrm{pCi} / \mathrm{cm}^{2} & 11.060 & 17.990 & 19.620 & 22.040 & 22.260 & 25.410 \\ \text { Radium-226 } & \mathrm{pCi} / \mathrm{cm}^{2} & 3.535 & 4.907 & 6.586 & 6.703 & 6.836 & 6.824 \\ \text { Thorium-232 } & \mathrm{pCi} / \mathrm{cm}^{2} & 2.133 & 3.190 & 3.793 & 4.063 & 4.159 & 4.618 \\ \text { Uranium-235 } & \mathrm{pCi} / \mathrm{cm}^{2} & 0.841 & 1.383 & 1.638 & 1.087 & 1.096 & 0.773 \\ \text { Uranium-238 } & \mathrm{pCi} / \mathrm{cm}^{2} & 3.799 & 15.913 & 18.260 & 7.945 & 15.316 & 12.410\end{array}$

Location=AND; Formation=COPPER RIDGE; Site $=34 ; 00-05=2230 ; 05-10=2231 ; 10-15=2232$; $15-20=2233 ; 20-25=2234 ; 25-30=2235 ;$ Phase $=2$

$\begin{array}{llrrrrrr}\text { Cesium-137 } & \mathrm{pCi} / \mathrm{cm}^{2} & 3.507 & 2.957 & 2.275 & 1.307 & 0.939 & 0.443 \\ \text { Potassium-40 } & \mathrm{PCi} / \mathrm{cm}^{2} & 16.810 & 18.030 & 20.230 & 19.290 & 23.760 & 24.440 \\ \text { Radium-226 } & \mathrm{pCi} / \mathrm{cm}^{2} & 4.608 & 5.630 & 6.125 & 6.393 & 6.708 & 6.725 \\ \text { Thorium-232 } & \mathrm{PCi} / \mathrm{cm}^{2} & 2.989 & 3.883 & 4.059 & 4.277 & 4.470 & 4.466 \\ \text { Uranium-235 } & \mathrm{pCi} / \mathrm{cm}^{2} & 0.937 & 0.999 & 0.969 & 1.006 & 0.426 & 0.477 \\ \text { Uranium-238 } & \mathrm{pCi} / \mathrm{cm}^{2} & 12.430 & 10.160 & 9.527 & 16.620 & 15.387 & 9.499\end{array}$

Location $=A N D ;$ Formation $=C O P P E R$ RIDGE; Site $=35 ; 00-05=2239 ; 05-10=2240 ; 10-15=2241$; $15-20=2242 ; 20-25=2243 ; 25-30=2244 ;$ Phase $=2$

$\begin{array}{llrrrrrr}\text { Cesium-137 } & \mathrm{pCi} / \mathrm{cm}^{2} & 3.459 & 2.299 & 0.625 & 1.003 & 0.080 & 0.037 \\ \text { Potassium-40 } & \mathrm{PCi} / \mathrm{cm}^{2} & 17.850 & 27.900 & 24.580 & 34.250 & 35.000 & 32.460 \\ \text { Radium-226 } & \mathrm{PCi} / \mathrm{cm}^{2} & 4.904 & 5.886 & 7.244 & 7.200 & 7.172 & 6.165 \\ \text { Thorium-232 } & \mathrm{PCi} / \mathrm{cm}^{2} & 5.416 & .5 .159 & 5.770 & 5.986 & 6.581 & 6.198 \\ \text { Uranium-235 } & \mathrm{pCi} / \mathrm{cm}^{2} & 1.188 & 1.333 & 2.173 & 1.600 & 0.641 & 0.450 \\ \text { Uranium-238 } & \mathrm{pCi} / \mathrm{cm}^{2} & 15.650 & 22.336 & 25.561 & 26.083 & 13.430 & 11.650\end{array}$

Location $=A N D ;$ Formation $=$ COPPER RIDGE; Site $=36 ; 00-05=2248 ; 05-10=2249 ; 10-15=2250$; $15-20=2251 ; 20-25=2252 ; 25-30=2253 ;$ Phase $=2$

$\begin{array}{llrrrrrr}\text { Cesium-137 } & \mathrm{PCi} / \mathrm{cm}^{2} & 2.670 & 1.141 & 1.046 & 0.201 & 0.228 & 0.048 \\ \text { Potassium-40 } & \mathrm{PCi} / \mathrm{cm}^{2} & 18.920 & 19.950 & 22.710 & 19.990 & 22.370 & 27.740 \\ \text { Radium-226 } & \mathrm{PCi} / \mathrm{cm}^{2} & 5.122 & 5.649 & 5.796 & 5.634 & 6.125 & 5.756 \\ \text { Thorium-232 } & \mathrm{PCi} / \mathrm{cm}^{2} & 3.189 & 3.702 & 3.826 & 3.923 & 4.603 & 4.802 \\ \text { Uranium-235 } & \mathrm{PCi} / \mathrm{cm}^{2} & 1.115 & 1.201 & 1.183 & 1.147 & 1.243 & 0.541 \\ \text { Uranium-238 } & \mathrm{pCi} / \mathrm{cm}^{2} & 4.946 & 17.528 & 19.105 & 21.482 & 14.410 & 11.160\end{array}$


Table B.3 (continued)

\begin{tabular}{llllllll}
\hline & \multicolumn{7}{c}{ Depth $(\mathrm{cm})$} \\
\cline { 2 - 7 } Analysis & Units & $00-05$ & $05-10$ & $10-15$ & $15-20$ & $20-25$ & $25-30$ \\
\hline
\end{tabular}

Location $=A N D$; Formation $=$ COPPER RIDGE; Site $=37 ; 00-05=2287 ; 05-10=2288 ; 10-15=2289$; $15-20=2290 ; 20-25=2291 ; 25-30=2292 ;$ Phase $=2$

$\begin{array}{llrrrrrr}\text { Cesium-137 } & \mathrm{PCi} / \mathrm{cm}^{2} & 3.631 & 3.619 & 3.020 & 2.096 & 0.388 & 0.062 \\ \text { Potassium-40 } & \mathrm{PCi} / \mathrm{cm}^{2} & 12.810 & 13.960 & 13.090 & 13.320 & 23.070 & 36.850 \\ \text { Radium-226 } & \mathrm{PCi} / \mathrm{cm}^{2} & 3.557 & 4.279 & 4.755 & 4.386 & 5.873 & 7.960 \\ \text { Thorium-232 } & \mathrm{PCi} / \mathrm{cm}^{2} & 2.402 & 3.040 & 2.843 & 2.717 & 3.368 & 4.640 \\ \text { Uranium-235 } & \mathrm{PCi} / \mathrm{cm}^{2} & 0.773 & 0.670 & 0.788 & 0.802 & 0.365 & 0.818 \\ \text { Uranium-238 } & \mathrm{PCi} / \mathrm{cm}^{2} & 9.687 & 20.582 & 1.482 & 9.598 & 5.934 & 14.690\end{array}$

Location $=A N D$; Formation $=C O P P E R$ RIDGE; Site $=38 ; 00-05=2296 ; 05-10=2297 ; 10-15=2298$; $15-20=2299 ; 20-25=2300 ; 25-30=2301 ;$ Phase $=2$

$\begin{array}{llrrrrrr}\text { Cesium-137 } & \mathrm{PCi} / \mathrm{cm}^{2} & 4.213 & 3.541 & 1.861 & 0.585 & 0.268 & 0.052 \\ \text { Potassium-40 } & \mathrm{PCi} / \mathrm{cm}^{2} & 20.290 & 21.860 & 27.140 & 23.350 & 28.440 & 27.360 \\ \text { Radium-226 } & \mathrm{PCi} / \mathrm{cm}^{2} & 3.177 & 3.746 & 4.697 & 4.432 & 4.932 & 4.963 \\ \text { Thorium-232 } & \mathrm{PCi} / \mathrm{cm}^{2} & 2.299 & 2.560 & 2.988 & 2.684 & 3.049 & 2.921 \\ \text { Uranium-235 } & \mathrm{PCi} / \mathrm{cm}^{2} & 0.929 & 0.895 & 0.809 & 0.207 & 0.257 & 0.430 \\ \text { Uranium-238 } & \mathrm{PCi} / \mathrm{cm}^{2} & 17.676 & 17.392 & 14.749 & 6.190 & 7.625 & 8.463\end{array}$

Location $=A N D$; Formation $=C O P P E R$ RIDGE; Site $=39 ; 00-05=2331 ; 05-10=2332 ; 10-15=2333$; $15-20=2334 ; 20-25=2335 ; 25-30=2336 ;$ Phase $=2$

$\begin{array}{llrrrrrr}\text { Cesium-137 } & \mathrm{PCi} / \mathrm{cm}_{2}^{2} & 2.446 & 2.133 & 1.172 & 0.758 & 0.197 & 0.108 \\ \text { Potassium-40 } & \mathrm{PCi} / \mathrm{cm}^{2} & 12.810 & 17.890 & 17.560 & 18.150 & 21.770 & 19.440 \\ \text { Radium-226 } & \mathrm{PCi} / \mathrm{cm}^{2} & 4.810 & 6.033 & 6.022 & 5.910 & 5.839 & 5.842 \\ \text { Thorium-232 } & \mathrm{PCi} / \mathrm{cm}_{2}^{2} & 3.400 & 4.273 & 4.381 & 4.275 & 4.402 & 4.833 \\ \text { Uranium-235 } & \mathrm{PCi} / \mathrm{cm}_{2}^{2} & 1.038 & 0.628 & 0.958 & 0.747 & 0.571 & 0.661 \\ \text { Uranium-238 } & \mathrm{PCi} / \mathrm{cm}^{2} & 22.429 & 16.467 & 16.742 & 17.175 & 21.950 & 6.915\end{array}$

Location $=A N D ;$ Formation $=C O P P E R$ RIDGE; Site $=40 ; 00-05=2322 ; 05-10=2323 ; 10-15=2324$; $15-20=2325 ; 20-25=2326 ; 25-30=2327 ;$ Phase $=2$

$\begin{array}{llrrrrrr}\text { Cesium-137 } & \mathrm{PCi} / \mathrm{cm}_{2}^{2} & 4.350 & 2.211 & 0.444 & 0.181 & 0.101 & 0.011 \\ \text { Potassium-40 } & \mathrm{PCi} / \mathrm{cm}^{2} & 19.940 & 23.920 & 26.900 & 27.640 & 25.520 & 23.010 \\ \text { Radium-226 } & \mathrm{PCi} / \mathrm{cm}^{2} & 4.837 & 5.384 & 5.457 & 5.341 & 5.523 & 5.590 \\ \text { Thorium-232 } & \mathrm{PCi} / \mathrm{cm}_{2}^{2} & 4.278 & 5.029 & 5.814 & 5.703 & 5.577 & 5.644 \\ \text { Uranium-235 } & \mathrm{pCi} / \mathrm{cm}_{2} & 0.979 & 1.020 & 0.466 & 0.586 & 0.648 & 0.401 \\ \text { Uranium-238 } & \mathrm{pCi} / \mathrm{cm}^{2} & 29.250 & 21.560 & 19.264 & 12.940 & 6.659 & 8.247\end{array}$

Location $=A N D$; Formation $=C O P P E R$ RIDGE; Site $=41 ; 00-05=2314 ; 05-10=2315 ; 10-15=2316$; $15-20=2317 ; 20-25=2318 ; 25-30=2319 ;$ Phase $=2$

\begin{tabular}{|c|c|c|c|c|c|c|c|}
\hline $\begin{array}{l}\text { Cesium-137 } \\
\text { Potassium-40 } \\
\text { Radium-226 } \\
\text { Thorium-232 } \\
\text { Uranium-235 } \\
\text { Uranium-238 }\end{array}$ & $\begin{array}{l}\mathrm{pCi} / \mathrm{cm}^{2} \\
\mathrm{p} \mathrm{Ci} / \mathrm{cm}_{2}^{2} \\
\mathrm{pCi} / \mathrm{cm}_{2}^{2} \\
\mathrm{pCi} / \mathrm{cm}_{2}^{2} \\
\mathrm{pCi} / \mathrm{cm}_{2} \\
\mathrm{pCi} / \mathrm{cm}^{2}\end{array}$ & $\begin{array}{r}5.956 \\
23.500 \\
5.192 \\
4.706 \\
1.037 \\
7.650\end{array}$ & $\begin{array}{r}4.883 \\
26.030 \\
5.801 \\
4.856 \\
1.049 \\
9.068\end{array}$ & $\begin{array}{r}2.971 \\
28.130 \\
6.430 \\
5.209 \\
1.266 \\
16.188\end{array}$ & $\begin{array}{r}0.473 \\
26.180 \\
6.251 \\
5.248 \\
0.740 \\
9.319\end{array}$ & $\begin{array}{r}0.117 \\
31.650 \\
6.270 \\
5.877 \\
0.462 \\
12.852\end{array}$ & $\begin{array}{r}0.033 \\
31.990 \\
6.482 \\
5.969 \\
0.688 \\
11.350\end{array}$ \\
\hline
\end{tabular}

Location $=A N D$; Formation $=C O P P E R$ RIDGE; Site $=42 ; 00-05=2305 ; 05-10=2306 ; 10-15=2307$; $15-20=2308 ; 20-25=2309 ; 25-30=2310$; Phase $=2$

$\begin{array}{llrrrrrr}\text { Cesium-137 } & \text { PCi/cm } 2 & 3.665 & 2.037 & 0.983 & 0.207 & 0.196 & 0.080 \\ \text { Potassium-40 } & \text { PCi/cm } & 25.270 & 24.760 & 30.050 & 31.820 & 37.130 & 38.030 \\ \text { Radium-226 } & \text { PCi/cm } & 6.961 & 8.018 & 8.252 & 7.223 & 7.787 & 7.982 \\ \text { Thorium-232 } & \mathrm{PCi} / \mathrm{cm}_{2}^{2} & 5.430 & 5.596 & 6.008 & 6.019 & 6.366 & 6.711 \\ \text { Uranium-235 } & \mathrm{PCi} / \mathrm{cm}^{2} & 1.094 & 1.163 & 0.793 & 0.623 & 0.856 & 0.725 \\ \text { Uranium-238 } & \mathrm{PCi} / \mathrm{cm}^{2} & 15.860 & 16.846 & 19.277 & 21.210 & 15.589 & 11.890\end{array}$


Table B.3 (continued)

\begin{tabular}{llllllll}
\hline & \multicolumn{8}{c}{ Depth $(\mathrm{cm})$} \\
\cline { 2 - 8 } Analysis & Units & $00-05$ & $05-10$ & $10-15$ & $15-20$ & $20-25$ & $25-30$ \\
\hline
\end{tabular}

Location $=A N D ;$ Formation $=$ DISMAL GAP; Site $=1 ; 00-05=2161 ; 05-10=2162 ; 10-15=2163 ; 15-20=2164$; $20-25=2165 ; 25-30=2166 ;$ Phase $=1$

$\begin{array}{llrrrrrr}\text { Cesium-137 } & \text { pCi/cm } & 5.245 & 1.112 & 0.216 & & 0.015 \\ \text { Potassium-40 } & \mathrm{pCi} / \mathrm{cm}^{2} & 77.220 & 115.400 & 129.100 & 161.900 & 154.600 & 162.600 \\ \text { Radium-226 } & \mathrm{pCi} / \mathrm{cm}^{2} & 2.977 & 4.256 & 3.549 & 2.972 & 3.022 & 3.145 \\ \text { Thorium-232 } & \mathrm{pCi} / \mathrm{cm}^{2} & 4.448 & 6.337 & 5.944 & 6.547 & 6.234 & 6.712 \\ \text { Uranium-235 } & \mathrm{pCi} / \mathrm{cm}^{2} & & & & 18.050 & & 0.333 \\ \text { Uranium-238 } & \mathrm{pCi} / \mathrm{cm}^{2} & & 10.640 & & 6.472\end{array}$

Location $=A N D ;$ Formation $=D I S M A L$ GAP; Site $=10 ; 00-05=2151 ; 05-10=2152 ; 10-15=2153 ; 15-20=2154$; $20-25=2155 ; 25-30=2156 ;$ Phase $=I$

$\begin{array}{llrrrrrr}\text { Cesium-137 } & \mathrm{pCi} / \mathrm{cm}^{2} & 4.999 & 2.828 & 0.504 & 0.793 & 0.275 & 0.007 \\ \text { Potassium-40 } & \mathrm{PCi} / \mathrm{cm}^{2} & 34.000 & 74.480 & 78.900 & 79.360 & 118.900 & 134.400 \\ \text { Radium-226 } & \mathrm{PCi} / \mathrm{cm}^{2} & 2.185 & 4.382 & 4.833 & 4.895 & 5.067 & 5.279 \\ \text { Thorium-232 } & \mathrm{pCi} / \mathrm{cm}^{2} & 2.797 & 5.552 & 5.899 & 5.826 & 7.666 & 8.010 \\ \text { Uranium-235 } & \mathrm{PCi} / \mathrm{cm}^{2} & 0.645 & 0.857 & 0.952 & 0.867 & 0.755 & 0.401 \\ \text { Uranium-238 } & \mathrm{pCi} / \mathrm{cm}^{2} & 11.752 & 16.760 & 6.909 & 3.823 & 9.128 & 7.821\end{array}$

Location $=A N D ;$ Formation $=$ DISMAL GAP; Site $=11 ; 00-05=2008 ; 05-10=2009 ; 10-15=2010 ; 15-20=2011$; $20-25=2012 ; 25-30=2013 ;$ Phase $=1$

$\begin{array}{llrrrrrr}\text { Cesium-137 } & \mathrm{pCi} / \mathrm{cm}^{2} & 6.389 & 2.294 & 0.921 & 0.421 & 0.177 & 0.062 \\ \text { Potassium-40 } & \mathrm{pCi} / \mathrm{cm}^{2} & 127.000 & 154.900 & 171.200 & 202.600 & 212.400 & 202.600 \\ \text { Radium-226 } & \mathrm{pCi} / \mathrm{cm}^{2} & 2.596 & 2.945 & 2.975 & 3.442 & 3.490 & 3.468 \\ \text { Thorium-232 } & \mathrm{pCi} / \mathrm{cm}^{2} & 3.703 & 4.666 & 5.410 & 6.566 & 7.296 & 7.335 \\ \text { Uranium-235 } & \mathrm{pCi} / \mathrm{cm}^{2} & 1.553 & 0.818 & 0.844 & 0.188 & 0.923 & 0.296 \\ \text { Uranium-238 } & \mathrm{pCi} / \mathrm{cm}^{2} & 13.638 & 15.897 & 16.000 & 8.982 & 18.827 & 19.190\end{array}$

Location $=A N D ;$ Formation $=D I S M A L$ GAP; Site $=12 ; 00-05=2084 ; 05-10=2085 ; 10-15=2086 ; 15-20=2087$; $20-25=2088 ; 25-30=2089 ;$ Phase $=1$

$\begin{array}{llrrrrrr}\text { Cesium-137 } & \mathrm{PCi} / \mathrm{cm}^{2} & 5.909 & 0.815 & 0.139 & 0.092 & 0.159 & 0.200 \\ \text { Potassium-40 } & \mathrm{PCi} / \mathrm{cm}^{2} & 134.800 & 156.100 & 163.400 & 169.700 & 176.300 & 172.700 \\ \text { Radium-226 } & \mathrm{PCi} / \mathrm{cm}^{2} & 3.058 & 3.829 & 3.739 & 3.725 & 3.744 & 3.479 \\ \text { Thorium-232 } & \mathrm{PCi} / \mathrm{cm}^{2} & 4.288 & 5.285 & 5.256 & 6.767 & 6.887 & 6.882 \\ \text { Uranium-235 } & \mathrm{PCi} / \mathrm{cm}^{2} & 0.573 & & 1.000 & 1.145 & 0.892 & 1.225 \\ \text { Uranium-238 } & \mathrm{PCi} / \mathrm{cm}^{2} & & & & 25.071 & 17.804 & 23.264\end{array}$

Location =AND; Formation $=$ DISMAL GAP; Site $=19 ; 00-05=2124 ; 05-10=2125 ; 10-15=2126 ; 15-20=2127 ;$ $20-25=2128 ; 25-30=2129 ;$ Phase $=1$

$\begin{array}{llrrrrrr}\text { Cesium-137 } & \mathrm{PCi} / \mathrm{cm}^{2} & 11.250 & 2.483 & 0.419 & 0.198 & 0.024 & 0.050 \\ \text { Potassium-40 } & \mathrm{PCi} / \mathrm{cm}^{2} & 55.460 & 86.830 & 97.760 & 110.100 & 108.300 & 116.400 \\ \text { Radium-226 } & \mathrm{PCi} / \mathrm{cm}^{2} & 2.994 & 4.467 & 4.426 & 3.760 & 3.415 & 3.382 \\ \text { Thorium-232 } & \mathrm{pCi} / \mathrm{cm}^{2} & 4.121 & 6.343 & 7.132 & 7.275 & 6.713 & 6.938 \\ \text { Uranium-235 } & \mathrm{PCi} / \mathrm{cm}^{2} & 0.525 & 0.914 & 0.937 & 0.596 & 0.831 & 0.359 \\ \text { Uranium-238 } & \mathrm{PCi} / \mathrm{cm}^{2} & 5.091 & 5.614 & 15.815 & 16.143 & 12.070 & 7.134\end{array}$

Location $=A N D ;$ Formation $=D I S M A L$ GAP; Site $=20 ; 00-05=2074 ; 05-10=2075 ; 10-15=2076 ; 15-20=2077$; 20-25=2078; $25-30=2079 ;$ Phase $=1$

$\begin{array}{llrrrrrr}\text { Cesium-137 } & \mathrm{pCi} / \mathrm{cm}^{2} & 6.284 & 0.356 & 0.196 & & 0.197 & \\ \text { Potassium-40 } & \mathrm{pCi} / \mathrm{cm}_{2}^{2} & 97.750 & 108.500 & 101.100 & 104.600 & 101.200 & 90.530 \\ \text { Radium-226 } & \mathrm{pCi} / \mathrm{cm}^{2} & 3.988 & 4.366 & 3.728 & 3.542 & 3.745 & 2.741 \\ \text { Thorium-232 } & \mathrm{pCi} / \mathrm{cm}^{2} & 5.858 & 6.268 & 6.481 & 6.851 & 6.898 & 6.054 \\ \text { Uranium-235 } & \mathrm{pCi} / \mathrm{cm}^{2} & & & & & 1.102 & 0.425 \\ \text { Uranium-238 } & \mathrm{pCi} / \mathrm{cm}^{2} & & & & & 19.881 & \end{array}$


Table B3 (continued)

\begin{tabular}{llllllll}
\hline & \multicolumn{8}{c}{ Depth $(\mathrm{cm})$} \\
\cline { 2 - 7 } Analysis & Units & $00-05$ & $05-10$ & $10-15$ & $15-20$ & $20-25$ & $25-30$ \\
\hline
\end{tabular}

Location =AND; Formation $=$ DISMAL GAP; Site $=21 ; 00-05=2105 ; 05-10=2106 ; 10-15=2107 ; 15-20=2108$; $20-25=2109 ; 25-30=2110 ;$ Phase $=1$

$\begin{array}{llrrrrrr}\text { Cesium-137 } & \mathrm{pCi} / \mathrm{cm}^{2} & 3.965 & 1.209 & 0.471 & 0.438 & 0.090 & 0.081 \\ \text { Potassium-40 } & \mathrm{pCi} / \mathrm{cm}^{2} & 99.460 & 136.700 & 164.800 & 156.500 & 139.900 & 151.000 \\ \text { Radium-226 } & \mathrm{pCi} / \mathrm{cm}^{2} & 2.167 & 3.439 & 3.191 & 2.751 & 2.978 & 2.741 \\ \text { Thorium-232 } & \mathrm{pCi} / \mathrm{cm}^{2} & 4.082 & 5.474 & 6.345 & 6.375 & 5.948 & 6.506 \\ \text { Uranium-235 } & \mathrm{pCi} / \mathrm{cm}^{2} & & & & 1.092 & 1.461 & 1.152 \\ \text { Uranium-238 } & \mathrm{pCi} / \mathrm{cm}^{2} & & & 31.690 & 20.803 & 21.349 & 22.795\end{array}$

Location $=A N D ;$ Formation $=D I S M A L$ GAP; Site $=22 ; 00-05=2094 ; 05-10=2095 ; 10-15=2096 ; 15-20=2097$; $20-25=2098 ; 25-30=2099 ;$ Phase $=1$

$\begin{array}{llrrrrrr}\text { Cesium-137 } & \mathrm{PCi} / \mathrm{cm}^{2} & 3.019 & 0.325 & 0.144 & 0.144 & 0.139 & 0.045 \\ \text { Potassium-40 } & \mathrm{PCi} / \mathrm{cm}^{2} & 167.000 & 162.400 & 157.800 & 136.300 & 158.100 & 176.400 \\ \text { Radium-226 } & \mathrm{PCi} / \mathrm{cm}^{2} & 3.532 & 3.781 & 3.603 & 3.243 & 3.484 & 3.378 \\ \text { Thorium-232 } & \mathrm{PCi} / \mathrm{cm}^{2} & 5.984 & 6.503 & 6.857 & 6.800 & 6.734 & 6.729 \\ \text { Uranium-235 } & \mathrm{PCi} / \mathrm{cm}^{2} & 0.889 & 0.910 & 0.882 & 0.863 & 0.909 & 0.341 \\ \text { Uranium-238 } & \mathrm{PCi} / \mathrm{cm}^{2} & 17.838 & 16.400 & 18.484 & 17.910 & 16.820 & 7.410\end{array}$

Location $=A N D ;$ Formation $=$ DISMAL GAP; Site $=3 ; 00-05=2063 ; 05-10=2064 ; 10-15=2065 ; 15-20=2066$; $20-25=2067 ; 25-30=2068 ;$ Phase $=1$

$\begin{array}{llrrrrrr}\text { Cesium-137 } & \mathrm{pCi} / \mathrm{cm}^{2} & 3.276 & 0.941 & 0.297 & 0.144 & 0.077 & \\ \text { Potassium-40 } & \mathrm{pCi} / \mathrm{cm}^{2} & 122.100 & 125.500 & 127.700 & 128.700 & 124.100 & 118.400 \\ \text { Radium-226 } & \mathrm{pCi} / \mathrm{cm}^{2} & 3.227 & 4.262 & 3.743 & 3.456 & 3.448 & 2.850 \\ \text { Thorium-232 } & \mathrm{pCi} / \mathrm{cm}^{2} & 6.423 & 7.044 & 7.002 & 7.299 & 7.031 & 7.082 \\ \text { Uranium-235 } & \mathrm{pCi} / \mathrm{cm}^{2} & 0.759 & & 0.863 & 0.306 & & 23.220 \\ \text { Uranium-238 } & \mathrm{pCi} / \mathrm{cm}^{2} & & 13.240 & & 9.655 & & 23.220\end{array}$

Location $=A N D ;$ Formation $=D I S M A L$ GAP; Site $=4 ; 00-05=2043 ; 05-10=2044 ; 10-15=2045 ; 15-20=2046$; $20-25=2047 ; 25-30=2048 ;$ Phase $=1$

$\begin{array}{llrrrrrr}\text { Cesium-137 } & \mathrm{pCi} / \mathrm{cm}^{2} & 7.805 & 0.921 & 0.313 & 0.378 & 0.404 & 0.162 \\ \text { Potassium-40 } & \mathrm{pCi} / \mathrm{cm}^{2} & 211.100 & 214.000 & 218.500 & 233.300 & 230.100 & 224.600 \\ \text { Radium-226 } & \mathrm{pCi} / \mathrm{cm}^{2} & 3.300 & 3.571 & 3.517 & 3.179 & 3.483 & 3.255 \\ \text { Thorium-232 } & \mathrm{pCi} / \mathrm{cm}_{2}^{2} & 5.803 & 5.929 & 6.255 & 6.735 & 7.397 & 6.793 \\ \text { Uranium-235 } & \mathrm{pCi} / \mathrm{cm}_{2}^{2} & & & & & & \\ \text { Uranium-238 } & \mathrm{pCi} / \mathrm{cm}^{2} & & & & & & \end{array}$

Location $=A N D ;$ Formation $=D I S M A L$ GAP; Site $=5 ; 00-05=2053 ; 05-10=2054 ; 10-15=2055 ; 15-20=2056$; $20-25=2057 ; 25-30=2058 ;$ Phase $=1$

$\begin{array}{llrrrrrr}\text { Cesium-137 } & \mathrm{pCi} / \mathrm{cm}_{2}^{2} & 3.946 & 0.560 & 0.062 & & & \\ \text { Potassium-40 } & \mathrm{pCi} / \mathrm{cm}_{2}^{2} & 234.800 & 260.000 & 276.500 & 268.400 & 235.800 & 207.000 \\ \text { Radium-226 } & \mathrm{pCi} / \mathrm{cm}^{2} & 3.146 & 3.499 & 3.477 & 3.441 & 3.385 & 3.468 \\ \text { Thorium-232 } & \mathrm{pCi} / \mathrm{cm}_{2}^{2} & 6.167 & 6.967 & 7.672 & 7.487 & 6.929 & 6.241 \\ \text { Uranium-235 } & \mathrm{PCi} / \mathrm{cm}_{2}^{2} & & 1.549 & & & 1.606 & 0.450 \\ \text { Uranium-238 } & \mathrm{pCi} / \mathrm{cm}^{2} & & & 22.730 & 22.610 & & 6.546\end{array}$

Location $=A N D ;$ Formation $=D I S M A L$ GAP; Site $=9 ; 00-05=2137 ; 05-10=2138 ; 10-15=2139 ; 15-20=2140$; $20-25=2141 ; 25-30=2142 ;$ Phase $=1$

$\begin{array}{llrrrrrr}\text { Cesium-137 } & \mathrm{PCi} / \mathrm{cm}^{2} & 7.064 & 1.246 & 0.387 & 0.046 & 0.190 & 0.020 \\ \text { Potassium-40 } & \mathrm{pCi} / \mathrm{cm}^{2} & 93.590 & 95.870 & 92.600 & 86.530 & 106.400 & 96.570 \\ \text { Radium-226 } & \mathrm{pCi} / \mathrm{cm}^{2} & 2.819 & 3.557 & 3.509 & 3.462 & 3.541 & 3.456 \\ \text { Thorium-232 } & \mathrm{pCi} / \mathrm{cm}^{2} & 4.435 & 6.244 & 6.583 & 5.740 & 7.119 & 6.540 \\ \text { Uranium-235 } & \mathrm{PCi} / \mathrm{cm}^{2} & & 2.502 & & 1.062 & 1.149 & 0.457 \\ \text { Uranium-238 } & \mathrm{pCi} / \mathrm{cm}^{2} & 6.419 & & & 19.966 & 21.260 & 10.510\end{array}$


Table B3 (continued)

\begin{tabular}{llllllll}
\hline & \multicolumn{8}{c}{ Depth (cm) } \\
\cline { 2 - 8 } Analysis & Units & $00-05$ & $05-10$ & $10-15$ & $15-20$ & $20-25$ & $25-30$ \\
\hline
\end{tabular}

Location=ORR; Formation $=$ CHEPULTEPEC; Site $=50 ; 00-05=1847 ; 05-10=1848 ; 10-15=1849$; $15-20=1850 ; 20-25=1851 ; 25-30=1852 ;$ Phase $=2$

$\begin{array}{llrrrrrr}\text { Cesium-137 } & \mathrm{pCi} / \mathrm{cm}^{2} & 5.584 & 1.059 & 0.963 & 0.565 & 0.151 & 0.083 \\ \text { Potassium-40 } & \mathrm{pCi} / \mathrm{cm}^{2} & 4.954 & 11.010 & 10.190 & 12.970 & 13.490 & 14.330 \\ \text { Radium-226 } & \mathrm{pCi} / \mathrm{cm}^{2} & 2.255 & 4.058 & 5.026 & 5.691 & 5.264 & 5.316 \\ \text { Thorium-232 } & \mathrm{pCi} / \mathrm{cm}^{2} & 2.260 & 6.018 & 4.640 & 4.922 & 5.220 & 5.405 \\ \text { Uranium-235 } & \mathrm{pCi} / \mathrm{cm}^{2} & 0.735 & 1.043 & 0.438 & 0.821 & & 0.450 \\ \text { Uranium-238 } & \mathrm{pCi} / \mathrm{cm}^{2} & 13.249 & 17.180 & 13.890 & 13.820 & 12.238 & 11.320\end{array}$

Location $=$ ORR; Formation $=$ CHEPULTEPEC; Site $=52 ; 00-05=1654 ; 05-10=1655 ; 10-15=1656$; $15-20=1657 ; 20-25=1658 ; 25-30=1659 ;$ Phase $=2$

$\begin{array}{llrrrrrr}\text { Cesium-137 } & \mathrm{pCi} / \mathrm{cm}^{2} & 4.143 & 4.188 & 1.285 & 0.492 & 0.135 & 0.039 \\ \text { Potassium-40 } & \mathrm{pCi} / \mathrm{cm}_{2}^{2} & 8.046 & 13.060 & 14.560 & 14.250 & 15.300 & 18.220 \\ \text { Radium-226 } & \mathrm{pCi} / \mathrm{cm}^{2} & 2.930 & 5.092 & 5.203 & 4.933 & 5.050 & 5.504 \\ \text { Thorium-232 } & \mathrm{PCi} / \mathrm{cm}^{2} & 3.414 & 4.374 & 4.764 & 4.024 & 4.181 & 4.401 \\ \text { Uranium-235 } & \mathrm{PCi} / \mathrm{cm}^{2} & 0.890 & 0.941 & 0.949 & 1.713 & 0.637 & 0.510 \\ \text { Uranium-238 } & \mathrm{pCi} / \mathrm{cm}^{2} & 11.851 & 13.405 & 12.829 & 9.655 & 11.223 & 8.168\end{array}$

Location=ORR; Formation $=$ CHEPULTEPEC; Site $=53 ; 00-05=1642 ; 05-10=1643 ; 10-15=1644$; $15-20=1645 ; 20-25=1646 ; 25-30=1647 ;$ Phase $=2$

$\begin{array}{llrrrrrr}\text { Cesium-137 } & \mathrm{PCi} / \mathrm{cm}^{2} & 3.263 & 1.955 & 0.681 & 0.162 & 0.144 & 0.036 \\ \text { Potassium-40 } & \mathrm{PCi} / \mathrm{cm}_{2}^{2} & 8.500 & 11.890 & 15.960 & 15.520 & 13.910 & 16.270 \\ \text { Radium-226 } & \mathrm{pCi} / \mathrm{cm}_{2}^{2} & 2.535 & 3.989 & 4.181 & 4.056 & 3.623 & 3.742 \\ \text { Thorium-232 } & \mathrm{pCi} / \mathrm{cm}^{2} & 2.588 & 2.950 & 3.154 & 3.112 & 2.710 & 2.818 \\ \text { Uranium-235 } & \mathrm{pCi} / \mathrm{cm}^{2} & 0.516 & 0.342 & 0.685 & 0.307 & 0.072 & 0.293\end{array}$

Location=ORR; Formation $=$ CHEPULTEPEC; Site $=66 ; 00-05=1821 ; 05-10=1822 ; 10-15=1823$; $15-20=1824 ; 20-25=1825 ; 25-30=1826 ;$ Phase $=2$

$\begin{array}{llrrrrrr}\text { Cesium-137 } & \mathrm{pCi} / \mathrm{cm}^{2} & 3.375 & 1.316 & 0.620 & 0.200 & 0.124 & 0.018 \\ \text { Potassium-40 } & \mathrm{pCi} / \mathrm{cm}^{2} & 13.300 & 15.200 & 16.290 & 26.010 & 32.570 & 42.060 \\ \text { Radium-226 } & \mathrm{pCi} / \mathrm{cm}^{2} & 3.737 & 4.087 & 3.825 & 5.272 & 5.297 & 5.242 \\ \text { Thorium-232 } & \mathrm{pCi} / \mathrm{cm}^{2} & 2.700 & 3.252 & 2.789 & 4.452 & 6.019 & 6.648 \\ \text { Uranium-235 } & \mathrm{pCi} / \mathrm{cm}^{2} & 1.068 & 0.617 & 0.749 & 0.777 & 0.472 & 0.315\end{array}$

Location =ORR; Formation = CHEPULTEPEC; Site $=68 ; 00-05=1787 ; 05-10=1788 ; 10-15=1789$; $15-20=1790 ; 20-25=1791 ; 25-30=1792 ;$ Phase $=2$

$\begin{array}{llrrrrrr}\text { Cesium-137 } & \mathrm{pCi} / \mathrm{cm}^{2} & 4.046 & 3.647 & 1.984 & 0.597 & 0.100 & 0.062 \\ \text { Potassium-40. } & \mathrm{pCi} / \mathrm{cm}^{2} & 10.620 & 14.090 & 13.360 & 16.550 & 16.940 & 19.210 \\ \text { Radium-226 } & \mathrm{pCi} / \mathrm{cm}^{2} & 3.338 & 4.303 & 5.089 & 4.967 & 5.168 & 5.199 \\ \text { Thorium-232 } & \mathrm{PCi} / \mathrm{cm}^{2} & 2.265 & 3.259 & 3.195 & 3.568 & 3.449 & 3.746 \\ \text { Uranium-235 } & \mathrm{pCi} / \mathrm{cm}^{2} & 0.558 & 0.968 & 0.968 & 0.765 & 0.856 & 0.212 \\ \text { Uranium-238 } & \mathrm{pCi} / \mathrm{cm}^{2} & 9.643 & 12.310 & 15.404 & 13.122 & 12.180 & 7.516\end{array}$

Location =ORR; Formation $=$ CHEPULTEPEC; Site $=73 ; 00-05=1841 ; 05-10=1842 ; 10-15=1843$; $15-20=1844 ; 20-25=1845 ; 25-30=1846 ;$ Phase $=2$

$\begin{array}{llrrrrrr}\text { Cesium-137 } & \mathrm{PCi} / \mathrm{cm}^{2} & 5.746 & 4.756 & 1.738 & 0.194 & 0.184 & 0.134 \\ \text { Potassium-40 } & \mathrm{PCi} / \mathrm{cm}_{2}^{2} & 13.290 & 21.450 & 22.040 & 40.720 & 42.860 & 46.870 \\ \text { Radium-226 } & \mathrm{PCi} / \mathrm{cm}^{2} & 2.911 & 5.545 & 4.911 & 6.583 & 5.496 & 6.519 \\ \text { Thorium-232 } & \mathrm{pCi} / \mathrm{cm}^{2} & 2.034 & 3.555 & 3.011 & 4.764 & 4.729 & 5.370 \\ \text { Uranium-235 } & \mathrm{pCi} / \mathrm{cm}^{2} & 0.447 & 1.600 & 0.449 & & 1.124 & 0.618 \\ \text { Uranium-238 } & \mathrm{PCi} / \mathrm{cm}^{2} & 10.840 & 16.400 & 15.895 & 13.452 & 19.780 & 12.530\end{array}$


Table B.3 (continued)

\begin{tabular}{llllllll}
\hline & \multicolumn{7}{c}{ Depth (cm) } \\
\cline { 2 - 7 } Analysis & Units & $00-05$ & $05-10$ & $10-15$ & $15-20$ & $20-25$ & $25-30$ \\
\hline
\end{tabular}

Location $=O R R ;$ Formation $=C H E P U L T E P E C ;$ Site $=74 ; 00-05=1773 ; 05-10=1774 ; 10-15=1775$; $15-20=1776 ; 20-25=1777 ; 25-30=1778 ;$ Phase $=2$

$\begin{array}{llrrrrrr}\text { Cesium-137 } & \mathrm{pCi} / \mathrm{cm}^{2} & 5.000 & 1.682 & 0.303 & 0.143 & 0.127 & 0.031 \\ \text { Potassium-40 } & \mathrm{pCi} / \mathrm{cm}^{2} & 11.720 & 11.950 & 15.510 & 18.430 & 20.540 & 23.220 \\ \text { Radium-226 } & \mathrm{pCi} / \mathrm{cm}^{2} & 3.083 & 3.404 & 4.567 & 4.604 & 4.729 & 4.781 \\ \text { Thorium-232 } & \mathrm{pCi} / \mathrm{cm}^{2} & 1.953 & 2.359 & 2.694 & 2.920 & 3.325 & 3.502 \\ \text { Uranium-235 } & \mathrm{pCi} / \mathrm{cm}^{2} & 0.286 & 0.696 & 0.922 & 0.566 & 0.757 & 0.173 \\ \text { Uranium-238 } & \mathrm{pCi} / \mathrm{cm}^{2} & 14.680 & 12.691 & 15.462 & 15.630 & 10.938 & 12.460\end{array}$

Location $=O R R ;$ Formation $=$ CHEPULTEPEC; Site $=77 ; 00-05=1690 ; 05-10=1691 ; 10-15=1692$; $15-20=1693 ; 20-25=1694 ; 25-30=1695 ;$ Phase $=2$

$\begin{array}{llrrrrrr}\text { Cesium-137 } & \mathrm{pCi} / \mathrm{cm}^{2} & 7.507 & 3.694 & 0.518 & 0.113 & 0.044 & 0.012 \\ \text { Potassium-40 } & \mathrm{pCi} / \mathrm{cm}^{2} & 10.370 & 13.590 & 17.450 & 19.790 & 23.470 & 20.150 \\ \text { Radium-226 } & \mathrm{pCi} / \mathrm{cm}^{2} & 3.391 & 3.953 & 5.012 & 5.137 & 5.267 & 5.505 \\ \text { Thorium-232 } & \mathrm{pCi} / \mathrm{cm}^{2} & 3.043 & 3.689 & 4.271 & 4.744 & 5.127 & 4.373 \\ \text { Uranium-235 } & \mathrm{pCi} / \mathrm{cm}^{2} & \mathbf{0 . 8 8 4} & & 0.883 & 0.682 & 0.439 & 0.447 \\ \text { Uranium-238 } & \mathrm{pCi} / \mathrm{cm}^{2} & 14.449 & 14.670 & 14.020 & 15.370 & 10.842 & 7.632\end{array}$

Location $=$ ORR; Formation $=$ CHEPULTEPEC; Site $=78 ; 00-05=1672 ; 05-10=1673 ; 10-15=1674 ;$ $15-20=1675 ; 20-25=1676 ; 25-30=1677 ;$ Phase $=2$

$\begin{array}{llrrrrrr}\text { Cesium-137 } & \mathrm{PCi} / \mathrm{cm}^{2} & 4.537 & 3.070 & 0.900 & 0.128 & 0.038 & 0.014 \\ \text { Potassium-40 } & \mathrm{PCi} / \mathrm{cm}^{2} & 14.980 & 15.190 & 16.610 & 19.380 & 16.900 & 21.940 \\ \text { Radium-226 } & \mathrm{PCi} / \mathrm{cm}^{2} & 4.716 & 4.423 & 4.568 & 4.821 & 4.738 & 5.736 \\ \text { Thorium-232 } & \mathrm{PCi} / \mathrm{cm}^{2} & 2.890 & 2.958 & 3.242 & 4.190 & 3.758 & 4.597 \\ \text { Uranium-235 } & \mathrm{pCi} / \mathrm{cm}^{2} & 0.826 & 0.876 & 0.858 & 0.823 & 0.238 & 0.460 \\ \text { Uranium-238 } & \mathrm{pCi} / \mathrm{cm}^{2} & 17.424 & 10.940 & 10.810 & 12.542 & 6.167 & 10.380\end{array}$

Location $=$ ORR; Formation $=$ CHEPULTEPEC; Site $=85 ; 00-05=1726 ; 05-10=1727 ; 10-15=1728$; $15-20=1729 ; 20-25=1730 ; 25-30=1731 ;$ Phase $=2$

$\begin{array}{llrrrrrr}\text { Cesium-137 } & \mathrm{PCi} / \mathrm{cm}^{2} & 4.605 & 2.668 & 0.615 & 0.219 & 0.139 & 0.015 \\ \text { Potassium-40 } & \mathrm{pCi} / \mathrm{cm}^{2} & 16.770 & 22.070 & 20.660 & 23.660 & 25.350 & 24.250 \\ \text { Radium-226 } & \mathrm{pCi} / \mathrm{cm}^{2} & 3.793 & 4.559 & 3.852 & 4.148 & 4.276 & 4.067 \\ \text { Thorium-232 } & \mathrm{PCi} / \mathrm{cm}^{2} & 2.861 & 3.500 & 2.919 & 3.459 & 3.620 & 3.366 \\ \text { Uranium-235 } & \mathrm{pCi} / \mathrm{cm}^{2} & 0.898 & 0.875 & 0.816 & 0.562 & 0.689 & 0.101 \\ \text { Uranium-238 } & \mathrm{pCi} / \mathrm{cm}^{2} & 8.429 & 5.773 & 13.717 & 15.510 & 8.565 & 6.518\end{array}$

Location $=O R R$; Formation $=$ CHEPULTEPEC; Site $=86 ; 00-05=1708 ; 05-10=1709 ; 10-15=1710$; $15-20=1711 ; 20-25=1712 ; 25-30=1713 ;$ Phase $=2$

$\begin{array}{llrrrrrr}\text { Cesium-137 } & \mathrm{pCi} / \mathrm{cm}^{2} & 4.733 & 1.670 & 0.819 & 0.291 & 0.123 & 0.057 \\ \text { Potassium-40 } & \mathrm{PCi} / \mathrm{cm}^{2} & 3.521 & 9.244 & 13.310 & 16.210 & 16.750 & 18.890 \\ \text { Radium-226 } & \mathrm{PCi} / \mathrm{cm}^{2} & 2.221 & 3.721 & 3.308 & 3.385 & 3.623 & 3.445 \\ \text { Thorium-232 } & \mathrm{PCi} / \mathrm{cm}^{2} & 0.502 & 1.369 & 2.116 & 2.328 & 2.772 & 2.558 \\ \text { Uranium-235 } & \mathrm{pCi} / \mathrm{cm}^{2} & 0.605 & 0.820 & 1.383 & 0.663 & 1.036 & 0.348 \\ \text { Uranium-238 } & \mathrm{PCi} / \mathrm{cm}^{2} & 12.140 & 11.950 & 16.270 & 15.210 & 10.470 & 7.805\end{array}$

Location $=O R R ;$ Formation $=$ CHEPULTEPEC; Site $=90 ; 00-05=1757 ; 05-10=1758 ; 10-15=1759$; $15-20=1760 ; 20-25=1761 ; 25-30=1762 ;$ Phase $=2$

$\begin{array}{llrrrrrr}\text { Cesium-137 } & \mathrm{pCi} / \mathrm{cm}^{2} & 5.479 & 3.047 & 0.644 & 0.359 & 0.252 & 0.105 \\ \text { Potassium-40 } & \mathrm{pCi} / \mathrm{cm}^{2} & 10.110 & 12.210 & 13.530 & 14.450 & 25.100 & 37.730 \\ \text { Radium-226 } & \mathrm{pCi} / \mathrm{cm}^{2} & 2.893 & 4.111 & 3.825 & 3.118 & 4.051 & 3.889 \\ \text { Thorium-232 } & \mathrm{pCi} / \mathrm{cm}^{2} & 3.066 & 4.114 & 3.794 & 3.159 & 4.193 & 5.508 \\ \text { Uranium-235 } & \mathrm{pCi} / \mathrm{cm}^{2} & 0.374 & 1.521 & 0.298 & 0.638 & 0.659 & 0.113 \\ \text { Uranium-238 } & \mathrm{pCi} / \mathrm{cm}^{2} & 4.853 & 12.822 & 13.283 & 6.859 & 15.300 & 5.025\end{array}$


Table B3 (continued)

\begin{tabular}{|c|c|c|c|c|c|c|c|}
\hline \multirow[b]{2}{*}{ Analysis } & \multicolumn{5}{|c|}{ Depth (cm) } & \multirow[b]{2}{*}{$20-25$} & \multirow[b]{2}{*}{$25-30$} \\
\hline & Units & $00-05$ & $05-10$ & $10-15$ & $15-20$ & & \\
\hline
\end{tabular}

Location=ORR; Formation =CHICKAMAUGA Bethel V; Site $=100 ; 00-05=1883 ; 05-10=1884 ; 10-15=1885$; $15-20=1886 ; 20-25=1887 ; 25-30=1888 ;$ Phase $=2$.

$\begin{array}{llrrrrrr}\text { Cesium-137 } & \mathrm{pCi} / \mathrm{cm}^{2} & 12.520 & 0.550 & 0.156 & 0.149 & 0.165 & 0.160 \\ \text { Potassium-40 } & \mathrm{pCi} / \mathrm{cm}^{2} & 75.810 & 165.600 & 187.800 & 191.000 & 199.200 & 175.200 \\ \text { Radium-226 } & \mathrm{pCi} / \mathrm{cm}^{2} & 2.186 & 3.860 & 3.428 & 2.861 & 3.308 & 3.046 \\ \text { Thorium-232 } & \mathrm{pCi} / \mathrm{cm}^{2} & 3.539 & 7.663 & 8.115 & 8.062 & 8.295 & 7.421 \\ \text { Uranium-235 } & \mathrm{pCi} / \mathrm{cm}^{2} & 0.944 & 1.182 & 0.965 & 0.957 & 0.995 & 0.114\end{array}$

Location=ORR; Formation =CHICKAMAUGA Bethel V; Site=101; 00-05=1920; 05-10 =1921; 10-15=1922; $15-20=1923 ; 20-25=1924 ; 25-30=1925 ;$ Phase $=2$

$\begin{array}{llrrrrrr}\text { Cesium-137 } & \mathrm{PCi} / \mathrm{cm}^{2} & 11.450 & 6.548 & 2.665 & 1.156 & 0.662 & 0.411 \\ \text { Potassium-40 } & \mathrm{PCi} / \mathrm{cm}^{2} & 105.000 & 159.900 & 154.100 & 171.700 & 170.500 & 180.600 \\ \text { Radium-226 } & \mathrm{PCi} / \mathrm{cm}^{2} & 2.598 & 3.458 & 3.604 & 3.489 & 3.504 & 3.785 \\ \text { Thorium-232 } & \mathrm{PCi} / \mathrm{cm}^{2} & 4.993 & 7.101 & 7.115 & 7.870 & 7.735 & 8.133 \\ \text { Uranium-235 } & \mathrm{PCi} / \mathrm{cm}^{2} & 1.053 & 1.208 & 1.154 & 0.431 & 0.944 & 0.246\end{array}$

Location $=O R R ;$ Formation $=$ CHICKAMAUGA Bethel V; Site $=102 ; 00-05=1914 ; 05-10=1915 ; 10-15=1916$; $15-20=1917 ; 20-25=1918 ; 25-30=1919 ;$ Phase $=2$

$\begin{array}{llrrrrrr}\text { Cesium-137 } & \mathrm{PCi} / \mathrm{cm}^{2} & 8.069 & 6.771 & 1.729 & 0.517 & 0.219 & 0.051 \\ \text { Potassium-40 } & \mathrm{PCi} / \mathrm{cm}^{2} & 51.380 & 71.770 & 84.500 & 91.910 & 96.760 & 118.600 \\ \text { Radium-226 } & \mathrm{PCi} / \mathrm{cm}^{2} & 3.085 & 4.335 & 4.837 & 4.241 & 4.502 & 4.369 \\ \text { Thorium-232 } & \mathrm{PCi} / \mathrm{cm}^{2} & 5.194 & 6.978 & 7.836 & 7.672 & 7.190 & 7.983 \\ \text { Uranium-235 } & \mathrm{PCi} / \mathrm{cm}^{2} & 0.582 & 1.143 & 0.753 & 0.934 & 0.899 & 0.721\end{array}$

Location=ORR; Formation =CHICKAMAUGA Bethel V; Site $=103 ; 00-05=1908 ; 05-10=1909 ; 10-15=1910$; $15-20=1911 ; 20-25=1912 ; 25-30=1913 ;$ Phase $=2$

$\begin{array}{llrrrrrr}\text { Cesium-137 } & \mathrm{PCi} / \mathrm{cm}^{2} & 5.958 & 0.709 & 0.578 & 0.057 & 0.234 & 0.094 \\ \text { Potassium-40 } & \mathrm{pCi} / \mathrm{cm}^{2} & 126.200 & 181.400 & 169.700 & 165.900 & 167.500 & 172.300 \\ \text { Radium-226 } & \mathrm{pCi} / \mathrm{cm}^{2} & 2.620 & 3.611 & 3.096 & 3.267 & 3.013 & 2.977 \\ \text { Thorium-232 } & \mathrm{PCi} / \mathrm{cm}^{2} & 4.180 & 7.722 & 7.461 & 7.385 & 7.784 & 7.991 \\ \text { Uranium-235 } & \mathrm{pCi} / \mathrm{cm}^{2} & 0.926 & 1.246 & 1.172 & 1.173 & 0.291 & 0.528\end{array}$

Location=ORR; Formation $=$ CHICKAMAUGA Bethel V; Site $=104 ; 00-05=1981 ; 05-10=1982 ; 10-15=1983$; $15-20=1984 ; 20-25=1985 ; 25-30=1986 ;$ Phase $=2$

$\begin{array}{llrrrrrr}\text { Cesium-137 } & \text { PCi } / \mathrm{cm}_{2}^{2} & 7.139 & 2.257 & 0.646 & 0.216 & 0.034 & 0.082 \\ \text { Potassium-40 } & \mathrm{PCi} / \mathrm{cm}^{2} & 61.890 & 131.000 & 154.300 & 167.000 & 165.100 & 159.900 \\ \text { Radium-226 } & \mathrm{pCi} / \mathrm{cm}^{2} & 2.312 & 3.780 & 3.888 & 3.995 & 3.383 & 3.109 \\ \text { Thorium-232 } & \mathrm{pCi} / \mathrm{cm}^{2} & 2.337 & 4.221 & 5.430 & 6.903 & 7.979 & 8.067 \\ \text { Uranium-235 } & \mathrm{PCi} / \mathrm{cm}_{2}^{2} & 0.765 & 0.701 & 1.010 & 0.756 & 0.963 & 0.177 \\ \text { Uranium-238 } & \mathrm{pCi} / \mathrm{cm}^{2} & & & 7.319 & 31.990 & 15.897 & \end{array}$

Location=ORR; Formation =CHICKAMAUGA Bethel V; Site $=108 ; 00-05=1987 ; 05-10=1988 ; 10-15=1989$; $15-20=1990 ; 20-25=1991 ; 25-30=1992 ;$ Phase $=2$

$\begin{array}{llrrrrrr}\text { Cesium-137 } & \mathrm{pCi} / \mathrm{cm}^{2} & 7.191 & 0.867 & 0.396 & 0.083 & 0.142 & 0.017 \\ \text { Potassium-40 } & \mathrm{pCi} / \mathrm{cm}^{2} & 47.150 & 82.150 & 127.100 & 132.600 & 133.800 & 127.700 \\ \text { Radium-226 } & \mathrm{pCi} / \mathrm{cm}^{2} & 4.887 & 5.442 & 5.281 & 4.347 & 3.847 & 3.475 \\ \text { Thorium-232 } & \mathrm{pCi} / \mathrm{cm}^{2} & 7.454 & 9.752 & 9.738 & 9.477 & 8.991 & 8.818 \\ \text { Uranium-235 } & \mathrm{pCi} / \mathrm{cm}_{2}^{2} & 1.611 & 1.422 & & 0.072 & 1.306 & 0.300 \\ \text { Uranium-238 } & \mathrm{pCi} / \mathrm{cm}^{2} & & & 21.328 & 20.277 & 17.330 & 6.348\end{array}$

Location=ORR; Formation =CHICKAMAUGA Bethel V; Site $=110 ; 00-05=4032 ; 05-10=4033 ;-10-15=4034 ;$ $15-20=4035 ; 20-25=4036 ; 25-30=4037 ;$ Phase $=2$

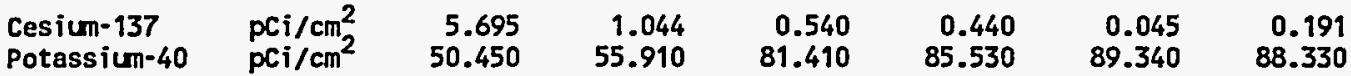


Table B.3 (continued)

\begin{tabular}{llllllll}
\hline & \multicolumn{7}{c}{ Depth $(\mathrm{cm})$} \\
\cline { 2 - 7 } Analysis & Units & $00-05$ & $05-10$ & $10-15$ & $15-20$ & $20-25$ & $25-30$ \\
\hline & & & & & & & \\
Radium-226 & $\mathrm{PCi} / \mathrm{cm}^{2}$ & 5.782 & 4.487 & 5.563 & 5.044 & 6.572 & 4.583 \\
Therium-232 & $\mathrm{PCi} / \mathrm{cm}^{2}$ & 7.744 & 7.043 & 8.816 & 8.563 & 8.512 & 8.407 \\
Uranium-235 & $\mathrm{PCi} / \mathrm{cm}^{2}$ & 1.225 & 1.437 & 1.399 & 1.664 & $\mathbf{0 . 9 4 1}$ & $\mathbf{0 . 2 2 2}$ \\
Uranium-238 & $\mathrm{PCi} / \mathrm{cm}^{2}$ & & & & 13.497 & 15.290 & 5.483
\end{tabular}

Location=ORR; Formation $=$ CHICKAMAUGA Bethel V; Site $=115 ; 00-05=4026 ; 05-10=4027 ; 10-15=4028$; $15-20=4029 ; 20-25=4030 ; 25-30=4031 ;$ Phase $=2$

$\begin{array}{llrrrrrr}\text { Cesium-137 } & \mathrm{pCi} / \mathrm{cm}^{2} & 6.913 & 1.540 & 0.491 & 0.220 & 0.218 & 0.141 \\ \text { Potassium-40 } & \mathrm{pCi} / \mathrm{cm}^{2} & 29.290 & 48.130 & 62.080 & 68.000 & 73.120 & 69.860 \\ \text { Radium-226 } & \mathrm{pCi} / \mathrm{cm}^{2} & 3.959 & 5.565 & 6.102 & 5.872 & 6.245 & 6.260 \\ \text { Thorium-232 } & \mathrm{pCi} / \mathrm{cm}^{2} & 5.486 & 8.658 & 9.685 & 9.840 & 9.788 & 8.522 \\ \text { Uranium-235 } & \mathrm{pCi} / \mathrm{cm}^{2} & 0.989 & 1.045 & 0.976 & 1.217 & 0.821 & 0.582 \\ \text { Uranium-238 } & \mathrm{pCi} / \mathrm{cm}^{2} & 20.612 & 5.564 & 18.670 & 4.567 & 19.763 & 6.411\end{array}$

Location =ORR; Formation $=$ CHICKAMAUGA Bethel V; Site $=116 ; 00-05=4000 ; 05-10=4001 ; 10-15=4002 ;$ $15-20=4003 ; 20-25=4004 ; 25-30=4005 ;$ Phase $=2$

$\begin{array}{llrrrrrr}\text { Cesium-137 } & \text { pCi } / \mathrm{cm}^{2} & 4.999 & 1.915 & 0.541 & 0.222 & 0.164 & 0.093 \\ \text { Potassium-40 } & \mathrm{pCi} / \mathrm{cm}^{2} & 65.140 & 94.250 & 108.800 & 115.800 & 141.800 & 150.000 \\ \text { Radium-226 } & \mathrm{pCi} / \mathrm{cm}^{2} & 4.079 & 4.565 & 4.837 & 4.028 & 3.750 & 3.956 \\ \text { Thorium-232 } & \mathrm{PCi} / \mathrm{cm}^{2} & 5.421 & 7.799 & 8.474 & 7.982 & 8.938 & 9.220 \\ \text { Uranium-235 } & \mathrm{pCi} / \mathrm{cm}^{2} & 0.398 & 1.134 & 1.399 & 0.897 & 0.133 & 0.328\end{array}$

Location $=$ ORR; Formation $=$ CHICKAMAUGA Bethel V; Site $=117 ; 00-05=4013 ; 05-10=4014 ; 10-15=4015$; $15-20=4016 ; 20-25=4017 ; 25-30=4018 ;$ Phase $=2$

$\begin{array}{llrrrrrr}\text { Cesium-137 } & \mathrm{PCi} / \mathrm{cm}^{2} & 4.367 & 2.908 & 1.232 & 0.319 & 0.096 & 0.042 \\ \text { Potassium-40 } & \mathrm{PCi} / \mathrm{cm}^{2} & 40.340 & 48.460 & 65.660 & 126.000 & 145.900 & 149.900 \\ \text { Radium-226 } & \mathrm{PCi} / \mathrm{cm}^{2} & 4.026 & 4.286 & 4.687 & 3.725 & 3.975 & 3.378 \\ \text { Thorium-232 } & \mathrm{PCi} / \mathrm{cm}^{2} & 5.021 & 6.052 & 7.110 & 8.365 & 8.811 & 8.465 \\ \text { Uranium-235 } & \mathrm{PCi} / \mathrm{cm}^{2} & 0.967 & 1.038 & 1.120 & 0.935 & 0.612 & 0.254\end{array}$

Location=ORR; Formation =CHICKAMAUGA Bethel V; Site=93; 00-05=1871; 05-10=1872; 10-15=1873; $15-20=1874 ; 20-25=1875 ; 25-30=1876 ;$ Phase $=2$

$\begin{array}{llrrrrrr}\text { Cesium-137 } & \mathrm{PCi} / \mathrm{cm}_{2}^{2} & 8.474 & 0.993 & 0.439 & 0.257 & 0.252 & 0.165 \\ \text { Potassium-40 } & \mathrm{PCi} / \mathrm{cm}^{2} & 73.850 & 99.450 & 116.000 & 112.800 & 126.800 & 125.300 \\ \text { Radium-226 } & \mathrm{PCi} / \mathrm{cm}^{2} & 3.939 & 4.398 & 3.621 & 3.233 & 3.447 & 3.968 \\ \text { Thorium-232 } & \mathrm{PCi} / \mathrm{cm}_{2}^{2} & 6.591 & 8.272 & 7.632 & 7.007 & 7.741 & 7.280 \\ \text { Uranium-235 } & \mathrm{PCi} / \mathrm{cm}^{2} & 1.084 & 2.156 & 0.888 & 0.885 & 0.935 & 0.668\end{array}$

Location $=$ ORR; Formation $=$ CHICKAMAUGA Bethel V; Site $=99 ; 00-05=1877 ; 05-10=1878 ; 10-15=1879$; $15-20=1880 ; 20-25=1881 ; 25-30=1882 ;$ Phase $=2$

$\begin{array}{llrrrrrr}\text { Cesium-137 } & \mathrm{pCi} / \mathrm{cm}^{2} & 8.641 & 2.230 & 1.286 & 0.235 & 0.098 & 0.051 \\ \text { Potassium-40 } & \mathrm{pCi} / \mathrm{cm}^{2} & 81.330 & 81.630 & 85.390 & 102.900 & 109.800 & 117.800 \\ \text { Radium-226 } & \mathrm{pCi} / \mathrm{cm}^{2} & 5.896 & 5.879 & 5.434 & 6.126 & 6.212 & 6.326 \\ \text { Thorium-232 } & \mathrm{pCi} / \mathrm{cm}^{2} & 8.450 & 8.812 & 8.240 & 9.208 & 9.050 & 9.464 \\ \text { Uranium-235 } & \mathrm{pCi} / \mathrm{cm}^{2} & 1.331 & 1.271 & 0.957 & 1.146 & 1.054 & 0.338\end{array}$

Location $=O R R ;$ Formation $=$ CHICKAMAUGA K-25; Site $=118 ; 00-05=4077 ; 05-10=4078 ; 10-15=4079$; $15-20=4080 ; 20-25=4081 ; 25-30=4082 ;$ Phase $=2$

$\begin{array}{llrrrrrr}\text { Cesium-137 } & \mathrm{PCj} / \mathrm{cm}^{2} & 7.869 & 1.200 & 0.156 & 0.323 & 0.280 & 0.097 \\ \text { Potassium-40 } & \mathrm{PCi} / \mathrm{cm}^{2} & 62.720 & 122.300 & 149.700 & 125.100 & 142.500 & 151.100 \\ \text { Radium-226 } & \mathrm{PCi} / \mathrm{cm}^{2} & 2.694 & 4.021 & 3.759 & 3.470 & 3.861 & 3.392 \\ \text { Thorium-232 } & \mathrm{PCi} / \mathrm{cm}^{2} & 5.157 & 8.513 & 9.713 & 8.212 & 9.603 & 9.296 \\ \text { Uranium-235 } & \mathrm{PCi} / \mathrm{cm}^{2} & 1.556 & 1.342 & 1.445 & 1.873 & 1.409 & 0.650 \\ \text { Uranium-238 } & \mathrm{pCi} / \mathrm{cm}^{2} & 19.400 & 28.450 & 25.933 & 18.400 & 29.434 & 11.220\end{array}$


Table B.3 (continued)

\begin{tabular}{llllllll}
\hline & \multicolumn{8}{c}{ Depth (cm) } \\
\cline { 2 - 7 } Analysis & Units & $00-05$ & $05-10$ & $10-15$ & $15-20$ & $20-25$ & $25-30$ \\
\hline
\end{tabular}

Location $=O R R ;$ Formation $=C H I C K A M A U G A K-25 ;$ Site $=119 ; 00-05=4180 ; 05-10=4181 ; 10-15=4182$; $15-20=4183 ; 20-25=4184 ; 25-30=4185 ;$ Phase $=2$

$\begin{array}{llrrrrrr}\text { Cesium-137 } & \mathrm{PCi} / \mathrm{cm}^{2} & 4.432 & 2.875 & 0.400 & 0.252 & 0.252 & 0.090 \\ \text { Potassium-40 } & \mathrm{PCi} / \mathrm{cm}^{2} & 37.320 & 58.160 & 84.170 & 99.440 & 127.000 & 143.000 \\ \text { Radium-226 } & \mathrm{PCi} / \mathrm{cm}^{2} & 3.206 & 4.540 & 5.987 & 5.053 & 4.622 & 3.704 \\ \text { Thorium-232 } & \mathrm{PCi} / \mathrm{cm}^{2} & 3.897 & 6.497 & 9.420 & 7.940 & 8.030 & 7.973 \\ \text { Uranium-235 } & \mathrm{PCi} / \mathrm{cm}^{2} & 1.184 & 1.685 & 1.213 & 1.239 & 1.389 & 0.368 \\ \text { Uranium-238 } & \mathrm{pCi} / \mathrm{cm}^{2} & 18.213 & 19.404 & 23.751 & 12.400 & 19.040 & 7.784\end{array}$

Location=ORR; Formation =CHICKAMAUGA K-25; Site =120;00-05=4071; 05-10=4072; 10-15=4073; $15-20=4074 ; 20-25=4075 ; 25-30=4076 ;$ Phase $=2$

$\begin{array}{llrrrrrr}\text { Cesium-137 } & \mathrm{pCi} / \mathrm{cm}^{2} & 4.635 & 2.907 & 1.015 & 0.317 & 0.235 & 0.041 \\ \text { Potassium-40 } & \mathrm{pCi} / \mathrm{cm}^{2} & 18.010 & 31.480 & 31.570 & 42.240 & 52.500 & 65.180 \\ \text { Radium-226 } & \mathrm{pCi} / \mathrm{cm}^{2} & 4.852 & 5.986 & 7.008 & 6.901 & 7.032 & 5.844 \\ \text { Thorium-232 } & \mathrm{pCi} / \mathrm{cm}^{2} & 4.569 & 6.220 & 7.461 & 8.373 & 8.530 & 8.535 \\ \text { Uranium-235 } & \mathrm{pCi} / \mathrm{cm}^{2} & 1.053 & 0.911 & 0.349 & 1.590 & 1.136 & 0.547 \\ \text { Uranium-238 } & \mathrm{pCi} / \mathrm{cm}^{2} & 14.190 & 22.350 & 11.750 & 16.090 & 25.172 & 13.120\end{array}$

Location=ORR; Formation =CHICKAMAUGA K-25; Site=121; 00-05=4186; 05-10=4187; $10-15=4188$; $15-20=4189 ; 20-25=4190 ; 25-30=4191 ;$ Phase $=2$

$\begin{array}{llrrrrrr}\text { Cesium-137 } & \mathrm{pCi} / \mathrm{cm}^{2} & 5.614 & 0.614 & 0.143 & 0.190 & 0.187 & 0.060 \\ \text { Potassium-40 } & \mathrm{pCi} / \mathrm{cm}^{2} & 34.680 & 50.880 & 57.810 & 82.380 & 146.400 & 171.900 \\ \text { Radium-226 } & \mathrm{pCi} / \mathrm{cm}^{2} & 3.917 & 5.116 & 4.640 & 4.307 & 2.751 & 2.813 \\ \text { Thorium-232 } & \mathrm{pCi} / \mathrm{cm}^{2} & 4.612 & 6.574 & 6.059 & 6.283 & 6.771 & 7.250 \\ \text { Uranium-235 } & \mathrm{pCi} / \mathrm{cm}^{2} & 0.838 & 0.525 & 0.305 & 1.014 & 0.951 & 0.243 \\ \text { Uranium-238 } & \mathrm{pCi} / \mathrm{cm}^{2} & 17.664 & 20.514 & 17.386 & 17.690 & 21.470 & 9.990\end{array}$

Location=ORR; Formation $=$ CHICKAMAUGA K-25; Site $=122 ; 00-05=4192 ; 05-10=4193 ; 10-15=4194$; $15-20=4195 ; 20-25=4196 ; 25-30=4197 ;$ Phase $=2$

$\begin{array}{llrrrrrr}\text { Cesium-137 } & \mathrm{pCi} / \mathrm{cm}^{2} & 3.200 & 2.567 & 0.747 & 0.367 & 0.113 & 0.077 \\ \text { Potassium-40 } & \mathrm{PCi} / \mathrm{cm}^{2} & 36.930 & 44.990 & 94.180 & 132.700 & 139.600 & .143 .800 \\ \text { Radium-226 } & \mathrm{PCi} / \mathrm{cm}^{2} & 3.124 & 4.065 & 4.953 & 5.386 & 5.273 & 4.168 \\ \text { Thorium-232 } & \mathrm{pCi} / \mathrm{cm}^{2} & 3.485 & 4.449 & 6.771 & 8.769 & 8.467 & 8.378 \\ \text { Uranium-235 } & \mathrm{PCi} / \mathrm{cm}^{2} & 0.772 & 0.151 & 0.700 & 1.244 & 1.183 & 0.352 \\ \text { Uranium-238 } & \mathrm{PCi} / \mathrm{cm}^{2} & 21.623 & 16.132 & 13.120 & 21.308 & 9.286 & 7.347\end{array}$

Location=ORR; Formation =CHICKAMAUGA K-25; Site $=123 ; 00-05=4210 ; 05-10=4211 ; 10-15=4212$; $15-20=4213 ; 20-25=4214 ; 25-30=4215 ;$ Phase $=2$

$\begin{array}{llrrrrrr}\text { Cesium-137 } & \mathrm{PCi} / \mathrm{cm}^{2} & 4.078 & 2.105 & 0.566 & 0.221 & 0.490 & 0.072 \\ \text { Potassium-40 } & \mathrm{PCi} / \mathrm{cm}^{2} & 42.480 & 63.660 & 76.920 & 110.800 & 99.370 & 99.250 \\ \text { Radium-226 } & \mathrm{PCi} / \mathrm{cm}^{2} & 3.425 & 3.906 & 3.817 & 3.801 & 3.500 & 3.548 \\ \text { Thorium-232 } & \mathrm{pCi} / \mathrm{cm}^{2} & 6.102 & 7.253 & 7.931 & 9.505 & 8.385 & 8.636 \\ \text { Uranium-235 } & \mathrm{PCi} / \mathrm{cm}^{2} & 0.636 & 1.053 & 1.132 & 1.081 & 0.542 & 0.380 \\ \text { Uranium-238 } & \mathrm{pCi} / \mathrm{cm}^{2} & 17.477 & 20.203 & 19.810 & 23.055 & 19.458 & 8.558\end{array}$

Location=ORR; Formation $=$ CHICKAMAUGA K-25; Site $=124 ; 00-05=4204 ; 05-10=4205 ; 10-15=4206$; $15-20=4207 ; 20-25=4208 ; 25-30=4209 ;$ Phase $=2$

$\begin{array}{llrrrrrr}\text { Cesium-137 } & \mathrm{pCi} / \mathrm{cm}^{2} & 4.176 & 3.144 & 0.771 & 0.199 & 0.227 & 0.062 \\ \text { Potassium-40 } & \mathrm{PCi} / \mathrm{cm}^{2} & 24.750 & 34.760 & 61.620 & 136.100 & 153.300 & 152.000 \\ \text { Radium-226 } & \mathrm{pCi} / \mathrm{cm}^{2} & 2.966 & 3.467 & 4.839 & 4.351 & 4.305 & 3.497 \\ \text { Thorium-232 } & \mathrm{pCi} / \mathrm{cm}^{2} & 4.101 & 6.258 & 8.797 & 8.917 & 8.942 & 8.666 \\ \text { Uranium-235 } & \mathrm{PCi} / \mathrm{cm}^{2} & 1.091 & 0.961 & 1.133 & 1.149 & 1.181 & 0.267 \\ \text { Uranium-238 } & \mathrm{pCi} / \mathrm{cm}^{2} & 16.950 & 15.130 & 19.474 & 23.255 & 26.484 & 7.506\end{array}$


Table B.3 (continued)

\begin{tabular}{llllllll}
\hline & \multicolumn{8}{c}{ Depth $(\mathrm{cm})$} \\
\cline { 2 - 7 } Analysis & Units & $00-05$ & $05-10$ & $10-15$ & $15-20$ & $20-25$ & $25-30$ \\
\hline
\end{tabular}

Location $=O R R ;$ Formation $=$ CHICKAMAUGA K-25; Site $=125 ; 00-05=4198 ; 05-10=4199 ; 10-15=4200$; $15-20=4201 ; 20-25=4202 ; 25-30=4203 ;$ Phase $=2$

$\begin{array}{llrrrrrr}\text { Cesium-137 } & \mathrm{PCi} / \mathrm{cm}^{2} & 5.157 & 1.684 & 0.587 & 0.186 & 0.207 & 0.085 \\ \text { Potassium-40 } & \mathrm{PCi} / \mathrm{cm}^{2} & 35.070 & 45.780 & 70.250 & 88.310 & 116.400 & 124.600 \\ \text { Radium-226 } & \mathrm{PCi} / \mathrm{cm}^{2} & 3.803 & 4.333 & 4.875 & 4.025 & 4.411 & 4.168 \\ \text { Thorium-232 } & \mathrm{PCi} / \mathrm{cm}^{2} & 4.592 & 6.439 & 7.061 & 7.238 & 8.052 & 8.304 \\ \text { Uranium-235 } & \mathrm{PCi} / \mathrm{cm}^{2} & 1.041 & 0.699 & 1.131 & 1.006 & 1.074 & 0.372 \\ \text { Uranium-238 } & \mathrm{PCi} / \mathrm{cm}^{2} & 15.447 & 15.913 & 19.112 & 21.500 & 35.150 & 7.414\end{array}$

Location=ORR; Formation =CHICKAMAUGA K-25; Site $=126 ; 00-05=4065 ; 05-10=4066 ; 10-15=4067$; $15-20=4068 ; 20-25=4069 ; 25-30=4070 ;$ Phase $=2$

$\begin{array}{llrrrrrr}\text { Cesium-137 } & \mathrm{pCi} / \mathrm{cm}^{2} & 6.911 & 1.569 & 0.487 & 0.216 & 0.078 & 0.043 \\ \text { Potassium-40 } & \mathrm{pCi} / \mathrm{cm}^{2} & 60.190 & 86.140 & 105.900 & 93.230 & 128.300 & 130.700 \\ \text { Radium-226 } & \mathrm{pCi} / \mathrm{cm}^{2} & 5.434 & 5.986 & 5.568 & 5.037 & 5.126 & 4.737 \\ \text { Thorium-232 } & \mathrm{pCi} / \mathrm{cm}^{2} & 7.085 & 8.887 & 8.983 & 8.168 & 9.295 & 9.454 \\ \text { Uranium-235 } & \mathrm{pCi} / \mathrm{cm}^{2} & 1.446 & 1.169 & 1.201 & 1.129 & 1.194 & 0.377 \\ \text { Uranium-238 } & \mathrm{pCi} / \mathrm{cm}^{2} & 9.252 & 8.862 & 22.261 & 20.685 & 20.524 & 6.910\end{array}$

Location=ORR; Formation $=$ CHICKAMAUGA K-25; Site $=127 ; 00-05=4241 ; 05-10=4242 ; 10-15=4243$; $15-20=4244 ; 20-25=4245 ; 25-30=4246 ;$ Phase $=2$

$\begin{array}{llrrrrrr}\text { Cesium-137 } & \mathrm{pCi} / \mathrm{cm}_{2}^{2} & 7.888 & 1.126 & 0.335 & 0.198 & 0.275 & 0.060 \\ \text { Potassium-40 } & \mathrm{pCi} / \mathrm{cm}^{2} & 76.220 & 112.700 & 117.000 & 130.400 & 117.700 & 126.100 \\ \text { Radium-226 } & \mathrm{pCi} / \mathrm{cm}^{2} & 2.653 & 4.152 & 3.745 & 3.451 & 3.103 & 3.730 \\ \text { Thorium-232 } & \mathrm{pCi} / \mathrm{cm}^{2} & 5.382 & 7.676 & 7.398 & 7.716 & 7.697 & 7.761 \\ \text { Uranium-235 } & \mathrm{pCi} / \mathrm{cm}^{2} & 1.129 & 0.789 & 0.955 & 1.113 & 1.418 & 0.566 \\ \text { Uranium-238 } & \mathrm{pCi} / \mathrm{cm}^{2} & 22.336 & 11.610 & 21.979 & 21.075 & 21.492 & 12.880\end{array}$

Location=ORR; Formation=CHICKAMAUGA K-25; Site =128; 00-05=4247;05-10=4248; $10-15=4249$; $15-20=4250 ; 20-25=4251 ; 25-30=4252 ;$ Phase $=2$

$\begin{array}{llrrrrrr}\text { Cesium-137 } & \mathrm{pCi} / \mathrm{cm}^{2} & 6.468 & 1.528 & 0.593 & 0.182 & 0.192 & 0.119 \\ \text { Potassium-40 } & \mathrm{pCi} / \mathrm{cm}_{2}^{2} & 39.320 & 66.470 & 55.080 & 89.670 & 122.800 & 110.400 \\ \text { Radium-226 } & \mathrm{pCi} / \mathrm{cm}^{2} & 3.849 & 4.593 & 4.420 & 4.668 & 4.414 & 3.710 \\ \text { Thorium-232 } & \mathrm{PCi} / \mathrm{cm}^{2} & 4.707 & 7.299 & 6.322 & 7.848 & 9.444 & 7.777 \\ \text { Uranium-235 } & \mathrm{PCi} / \mathrm{cm}^{2} & 1.229 & 1.087 & 1.047 & 1.348 & 0.803 & 0.710 \\ \text { Uranium-238 } & \mathrm{pCi} / \mathrm{cm}^{2} & 18.500 & 13.500 & 19.128 & 11.170 & 21.943 & 11.770\end{array}$

Location $=O R R ;$ Formation $=C H I C K A M A U G A$ K-25; Site $=129 ; 00-05=4059 ; 05-10=4060 ; 10-15=4061$; $15-20=4062 ; 20-25=4063 ; 25-30=4064 ;$ Phase $=2$

$\begin{array}{llrrrrrr}\text { Cesium- } 137 & \mathrm{pCi} / \mathrm{cm}^{2} & 5.763 & 1.902 & 0.745 & 0.484 & 0.274 & 0.077 \\ \text { Potassium-40 } & \mathrm{pCi} / \mathrm{cm}^{2} & 16.240 & 31.980 & 52.620 & 78.430 & 95.520 & 96.940 \\ \text { Radium-226 } & \mathrm{PCi} / \mathrm{cm}^{2} & 3.103 & 5.764 & 6.449 & 6.085 & 4.943 & 4.222 \\ \text { Thorium-232 } & \mathrm{pCi} / \mathrm{cm}^{2} & 3.172 & 5.809 & 7.469 & 9.115 & 9.305 & 9.829 \\ \text { Uranium-235 } & \mathrm{PCi} / \mathrm{cm}^{2} & 0.711 & 1.178 & 1.304 & 1.527 & 2.440 & 0.498 \\ \text { Uranium-238 } & \mathrm{pCi} / \mathrm{cm}^{2} & 18.875 & 20.772 & 28.704 & 24.439 & 23.411 & 9.509\end{array}$

Location =ORR; Formation $=$ COPPER RIDGE; Sire $=45 ; 00-05=1593 ; 05-10=1594 ; 10-15=1595$; $15-20=1596 ; 20-25=1597 ; 25-30=1598 ;$ Phase $=2$

$\begin{array}{llrrrrrr}\text { Cesium-137 } & \mathrm{pCi} / \mathrm{cm}^{2} & 6.360 & 1.194 & 0.309 & 0.083 & 0.112 & 0.063 \\ \text { Potassium-40 } & \mathrm{PCi} / \mathrm{cm}^{2} & 11.650 & 13.410 & 18.040 & 16.830 & 16.670 & 17.340 \\ \text { Radium-226 } & \mathrm{PCi} / \mathrm{cm}^{2} & 3.974 & 4.653 & 5.499 & 5.313 & 5.370 & 5.469 \\ \text { Thorium-232 } & \mathrm{PCi} / \mathrm{cm}^{2} & 2.692 & 3.249 & 3.600 & 3.926 & 4.353 & 4.567 \\ \text { Uranium-235 } & \mathrm{PCi} / \mathrm{cm}^{2} & 0.613 & 0.678 & 0.729 & 0.260 & 0.589 & 0.455\end{array}$


Table B3 (continued)

\begin{tabular}{llllllll}
\hline & \multicolumn{8}{c}{ Depth $(\mathrm{cm})$} \\
\cline { 2 - 7 } Analysis & Units & $00-05$ & $05-10$ & $10-15$ & $15-20$ & $20-25$ & $25-30$ \\
\hline
\end{tabular}

Location $=$ ORR; Formation $=$ COPPER RIDGE; Site $=51 ; 00-05=1566 ; 05-10=1567 ; 10-15=1568$; $15-20=1569 ; 20-25=1570 ; 25-30=1571 ;$ Phase $=2$

$\begin{array}{llrrrrrr}\text { Cesium-137 } & \mathrm{PCi} / \mathrm{cm}^{2} & 3.619 & 2.745 & 1.396 & 0.528 & 0.126 & 0.001 \\ \text { Potassium-40 } & \mathrm{PCi} / \mathrm{cm}^{2} & 12.050 & 16.450 & 17.660 & 19.970 & 20.430 & 20.970 \\ \text { Radium-226 } & \mathrm{PCi} / \mathrm{cm}^{2} & 3.540 & 4.564 & 5.058 & 5.352 & 5.021 & 5.185 \\ \text { Thorium-232 } & \mathrm{PCi} / \mathrm{cm}^{2} & 3.266 & 3.973 & 4.127 & 4.212 & 3.879 & 4.374 \\ \text { Uranium-235 } & \mathrm{pCi} / \mathrm{cm}^{2} & 0.388 & 0.722 & 0.864 & 1.554 & 0.784 & 0.333\end{array}$

Location $=O R R ;$ Formation $=$ COPPER RIDGE; Site $=54 ; 00-05=1630 ; 05-10=1631 ; 10-15=1632$; $15-20=1633 ; 20-25=1634 ; 25-30=1635 ;$ Phase $=2$

$\begin{array}{llrrrrrr}\text { Cesium-137 } & \mathrm{pCi} / \mathrm{cm}^{2} & 3.418 & 2.364 & 0.937 & 0.407 & 0.319 & 0.312 \\ \text { Potassium-40 } & \mathrm{PCi} / \mathrm{cm}^{2} & 14.360 & 21.450 & 23.850 & 26.690 & 28.190 & 24.320 \\ \text { Radium-226 } & \mathrm{PCi} / \mathrm{cm}^{2} & 4.360 & 6.317 & 6.812 & 6.579 & 6.832 & 6.863 \\ \text { Thorium-232 } & \mathrm{PCi} / \mathrm{cm}^{2} & 2.808 & 3.869 & 4.156 & 3.938 & 4.731 & 3.974 \\ \text { Uranium-235 } & \mathrm{PCi} / \mathrm{cm}^{2} & 0.080 & 1.257 & 0.826 & 0.214 & 0.844 & 0.466\end{array}$

Location $=O R R ;$ Formation $=C O P P E R$ RIDGE; Site $=55 ; 00-05=1560 ; 05-10=1561 ; 10-15=1562$; $15-20=1563 ; 20-25=1564 ; 25-30=1565 ;$ Phase $=2$

$\begin{array}{lllrrrrr}\text { Cesium-137 } & \mathrm{PCi} / \mathrm{cm}_{2}^{2} & 2.415 & 2.996 & 2.242 & 0.698 & 0.093 & 0.095 \\ \text { Potassium-40 } & \mathrm{PCi} / \mathrm{cm}_{2}^{2} & 7.522 & 13.170 & 20.880 & 19.310 & 20.590 & 20.440 \\ \text { Radium-226 } & \mathrm{PCi} / \mathrm{cm}^{2} & 2.651 & 4.309 & 5.465 & 5.676 & 5.724 & 5.533 \\ \text { Thorium-232 } & \mathrm{PCi} / \mathrm{cm}^{2} & 1.634 & 2.798 & 3.446 & 3.508 & 3.715 & 3.392 \\ \text { Uranium-235 } & \mathrm{PCi} / \mathrm{cm}^{2} & 0.589 & 0.783 & 0.409 & 0.801 & 0.563 & 0.339\end{array}$

Location $=O R R ;$ Formation $=C O P P E R$ RIDGE; Site $=58 ; 00-05=1519 ; 05-10=1520 ; 10-15=1521$; $15-20=1522 ; 20-25=1523 ; 25-30=1524 ;$ Phase $=2$

$\begin{array}{llrrrrrr}\text { Cesium-137 } & \mathrm{pCi} / \mathrm{cm}^{2} & 3.551 & 2.283 & 1.078 & 0.179 & 0.115 & 0.002 \\ \text { Potassium-40 } & \mathrm{pCi} / \mathrm{cm}^{2} & 15.790 & 21.110 & 23.070 & 22.990 & 22.510 & 20.540 \\ \text { Radium-226 } & \mathrm{pCi} / \mathrm{cm}^{2} & 3.488 & 5.129 & 5.316 & 5.765 & 5.385 & 5.163 \\ \text { Thorium-232 } & \mathrm{pCi} / \mathrm{cm}^{2} & 3.198 & 4.560 & 4.708 & 4.757 & 4.515 & 4.644 \\ \text { Uranium-235 } & \mathrm{pCi} / \mathrm{cm}^{2} & 0.689 & 0.849 & 0.750 & 0.557 & 0.615 & 0.456\end{array}$

Location $=O R R ;$ Formation $=C O P P E R$ RIDGE; Site $=59 ; 00-05=1513 ; 05-10=1514 ; 10-15=1515$; $15-20=1516 ; 20-25=1517 ; 25-30=1518 ;$ Phase $=2$

$\begin{array}{llrrrrrr}\text { Cesium-137 } & \mathrm{pCi} / \mathrm{cm}^{2} & 5.617 & 1.152 & 0.531 & 0.183 & 0.101 & 0.131 \\ \text { Potassium-40 } & \mathrm{PCi} / \mathrm{cm}^{2} & 12.580 & 17.160 & 15.170 & 15.760 & 21.290 & 21.210 \\ \text { Radium-226 } & \mathrm{PCi} / \mathrm{cm}^{2} & 3.646 & 5.337 & 5.189 & 5.506 & 6.024 & 6.198 \\ \text { Thorium-232 } & \mathrm{PCi} / \mathrm{cm}^{2} & 2.276 & 3.964 & 3.706 & 3.825 & 4.416 & 4.180 \\ \text { Uranium-235 } & \mathrm{pCi} / \mathrm{cm}^{2} & 0.702 & 0.463 & 1.263 & 0.387 & 0.805 & 0.360\end{array}$

Location=ORR; Formation =COPPER RIDGE; Site $=60 ; 00-05=1572 ; 05-10=1573 ; 10-15=1574$; $15-20=1575 ; 20-25=1576 ; 25-30=1577 ;$ Phase $=2$

$\begin{array}{llrrrrrr}\text { Cesium-137 } & \mathrm{pCi} / \mathrm{cm}^{2} & 3.948 & 1.230 & 0.467 & 0.221 & 0.185 & 0.182 \\ \text { Potassium-40 } & \mathrm{PCi} / \mathrm{cm}^{2} & 8.893 & 12.280 & 12.180 & 15.070 & 18.600 & 18.610 \\ \text { Radium-226 } & \mathrm{PCi} / \mathrm{cm}^{2} & 3.909 & 4.692 & 4.494 & 5.161 & 5.510 & 5.504 \\ \text { Thorium-232 } & \mathrm{PCi} / \mathrm{cm}^{2} & 3.098 & 3.968 & 4.031 & 5.325 & 6.105 & 6.128 \\ \text { Uranium-235 } & \mathrm{pCi} / \mathrm{cm}^{2} & 0.859 & 1.331 & 0.888 & 0.806 & 0.819 & 0.305\end{array}$

Location=ORR; Formation =COPPER RIDGE; Site $=62 ; 00-05=1554 ; 05-10=1555 ; 10-15=1556$; $15-20=1557 ; 20-25=1558 ; 25-30=1559 ;$ Phase $=2$

$\begin{array}{llrrrrrr}\text { Cesium-137 } & \mathrm{PCi} / \mathrm{cm}^{2} & 6.207 & 2.584 & 0.718 & 0.574 & 0.236 & 0.128 \\ \text { Potassium-40 } & \mathrm{pCi} / \mathrm{cm}^{2} & 11.640 & 12.670 & 13.270 & 16.350 & 15.850 & 13.940 \\ \text { Radium-226 } & \mathrm{pCi} / \mathrm{cm}^{2} & 3.911 & 4.801 & 5.174 & 5.194 & 5.090 & 4.466\end{array}$


B-102

Table B.3 (continued)

\begin{tabular}{|c|c|c|c|c|c|c|c|}
\hline \multirow[b]{2}{*}{ Analysis } & \multicolumn{7}{|c|}{ Depth $(\mathrm{cm})$} \\
\hline & Units & $00-05$ & $05-10$ & $10-15$ & $15-20$ & $20-25$ & $25-30$ \\
\hline $\begin{array}{l}\text { Thorium-232 } \\
\text { Uranium-235 }\end{array}$ & $\begin{array}{l}\mathrm{pCi} / \mathrm{cm}_{2}^{2} \\
\mathrm{pci} / \mathrm{cm}^{2}\end{array}$ & $\begin{array}{l}3.242 \\
0.759\end{array}$ & $\begin{array}{l}4.264 \\
0.500\end{array}$ & $\begin{array}{l}4.226 \\
0.759\end{array}$ & $\begin{array}{l}5.067 \\
0.801\end{array}$ & $\begin{array}{l}4.682 \\
0.303\end{array}$ & $\begin{array}{l}3.822 \\
0.325\end{array}$ \\
\hline
\end{tabular}

Location $=$ ORR; Formation $=$ COPPER RIDGE; Site $=64 ; 00-05=1624 ; 05-10=1625 ; 10-15=1626$; $15-20=1627 ; 20-25=1628 ; 25-30=1629 ;$ Phase $=2$

$\begin{array}{llrrrrrr}\text { Cesium-137 } & \mathrm{PCi} / \mathrm{cm}^{2} & 4.652 & 2.859 & 0.751 & 0.332 & 0.170 & 0.054 \\ \text { Potassium-40 } & \mathrm{PCi} / \mathrm{cm}^{2} & 15.680 & 22.240 & 22.400 & 27.820 & 25.720 & 40.450 \\ \text { Radium-226 } & \mathrm{PCi} / \mathrm{cm}^{2} & 3.915 & 6.486 & 6.887 & 6.897 & 6.688 & 9.077 \\ \text { Thorium-232 } & \mathrm{PCi} / \mathrm{cm}^{2} & 2.168 & 2.916 & 3.489 & 3.881 & 3.845 & 5.175 \\ \text { Uranium-235 } & \mathrm{PCi} / \mathrm{cm}^{2} & 0.641 & 0.770 & 0.493 & 0.703 & 0.399 & 0.328\end{array}$

Location =ORR; Formation $=$ COPPER RIDGE; Site $=75 ; 00-05=1584 ; 05-10=1585 ; 10-15=1586$; $15-20=1587 ; 20-25=1588 ; 25-30=1589 ;$ Phase $=2$

$\begin{array}{llrrrrrr}\text { Cesium-137 } & \mathrm{PCi} / \mathrm{cm}^{2} & 5.587 & 3.823 & 0.529 & 0.061 & 0.047 & 0.047 \\ \text { Potassium-40 } & \mathrm{PCi} / \mathrm{cm}^{2} & 14.490 & 19.750 & 18.200 & 23.800 & 19.540 & 19.510 \\ \text { Radium-226 } & \mathrm{PCi} / \mathrm{cm}^{2} & 4.454 & 5.478 & 5.593 & 5.732 & 5.643 & 5.644 \\ \text { Thorium-232 } & \mathrm{PCi} / \mathrm{cm}^{2} & 3.947 & 4.432 & 4.222 & 4.194 & 4.058 & 4.188 \\ \text { Uranium-235 } & \mathrm{PCi} / \mathrm{cm}^{2} & 0.777 & 0.147 & 0.251 & 1.214 & 0.667 & 0.299\end{array}$

Location $=O R R ;$ Formation $=C O P P E R$ RIDGE; Site $=83 ; 00-05=1636 ; 05-10=1637 ; 10-15=1638$; $15-20=1639 ; 20-25=1640 ; 25-30=1641 ;$ Phase $=2$

$\begin{array}{llrrrrrr}\text { Cesium-137 } & \mathrm{pCi} / \mathrm{cm}^{2} & 6.032 & 2.357 & 0.453 & 0.087 & 0.079 & 0.060 \\ \text { Potassium-40 } & \mathrm{PCi} / \mathrm{cm}^{2} & 13.350 & 15.770 & 19.290 & 23.200 & 23.270 & 25.080 \\ \text { Radium-226 } & \mathrm{PCi} / \mathrm{cm}^{2} & 3.680 & 4.825 & 5.953 & 6.484 & 6.590 & 6.816 \\ \text { Thorium-232 } & \mathrm{PCi} / \mathrm{cm}^{2} & 3.142 & 4.001 & 4.174 & 4.907 & 4.839 & 5.181 \\ \text { Uranium-235 } & \mathrm{pCi} / \mathrm{cm}^{2} & 0.332 & 0.528 & 0.298 & 0.407 & 0.603 & 0.841\end{array}$

Location $=O R R ;$ Formation $=$ COPPER RIDGE; Site $=91 ; 00-05=1525 ; 05-10=1526 ; 10-15=1527$; $15-20=1528 ; 20-25=1529 ; 25-30=1530 ;$ Phase $=2$

$\begin{array}{llrrrrrr}\text { Cesium-137 } & \mathrm{pCi} / \mathrm{cm}^{2} & 6.114 & 4.079 & 0.657 & 0.110 & 0.113 & 0.003 \\ \text { Potassium-40 } & \mathrm{pCi} / \mathrm{cm}^{2} & 12.500 & 17.190 & 17.320 & 19.800 & 22.030 & 26.290 \\ \text { Radium-226 } & \mathrm{PCi} / \mathrm{cm}^{2} & 3.990 & 5.152 & 6.148 & 6.265 & 6.295 & 6.531 \\ \text { Thorium-232 } & \mathrm{pCi} / \mathrm{cm}^{2} & 2.852 & 3.986 & 4.752 & 4.753 & 4.943 & 5.503 \\ \text { Uranium-235 } & \mathrm{pCi} / \mathrm{cm}^{2} & 0.764 & 0.670 & 0.653 & 0.376 & 1.206 & 0.719\end{array}$

Location =ORR; Formation $=$ DISMAL GAP; Site $=10 ; 00-05=1129 ; 05-10=1130 ; 10-15=1131 ; 15-20=1132$ $20-25=1133 ; 25-30=1134 ;$ Phase $=1$

$\begin{array}{llrrrrrr}\text { Cesium-137 } & \mathrm{PCi} / \mathrm{cm}^{2} & 4.759 & 3.682 & 1.414 & 0.873 & 0.174 & 0.082 \\ \text { Potassium-40 } & \mathrm{PCi} / \mathrm{cm}^{2} & 76.680 & 99.600 & 95.990 & 97.560 & 100.900 & 95.320 \\ \text { Radium-226 } & \mathrm{PCi} / \mathrm{cm}^{2} & 2.500 & 2.797 & 2.926 & 2.961 & 4.928 & 2.817 \\ \text { Thorium-232 } & \mathrm{PCi} / \mathrm{cm}^{2} & 4.069 & 5.306 & 5.565 & 5.801 & 6.721 & 6.130 \\ \text { Uranium-235 } & \mathrm{PCi} / \mathrm{cm}^{2} & 0.591 & 0.790 & 0.398 & 0.767 & 0.872 & 0.135 \\ \text { Uranium-238 } & \mathrm{PCi} / \mathrm{cm}^{2} & 12.321 & 15.515 & 14.191 & 14.284 & 14.644 & 2.261\end{array}$

Location $=$ ORR; Formation $=$ DISMAL GAP; Site $=11 ; 00-05=1040 ; 05-10=1041 ; 10-15=1042 ; 15-20=1043$; $20-25=1044 ; 25-30=1045 ;$ Phase $=1$

$\begin{array}{llrrrrrr}\text { Cesium-137 } & \mathrm{pCi} / \mathrm{cm}_{2}^{2} & 4.310 & 1.672 & 0.583 & 0.398 & 0.200 & 0.086 \\ \text { Potassium-40 } & \mathrm{pCi} / \mathrm{cm}^{2} & 87.030 & 106.900 & 113.000 & 97.550 & 112.200 & 120.800 \\ \text { Radium-226 } & \mathrm{pCi} / \mathrm{cm}^{2} & 2.768 & 2.947 & 2.979 & 2.949 & 2.918 & 3.400 \\ \text { Thorium-232 } & \mathrm{pCi} / \mathrm{cm}^{2} & 4.631 & 5.391 & 6.231 & 6.035 & 6.103 & 6.546 \\ \text { Uranium-235 } & \mathrm{pCi} / \mathrm{cm}^{2} & 0.429 & 0.697 & 0.524 & 0.506 & 0.476 & 0.530\end{array}$


Table B3 (continued)

\begin{tabular}{llllllll}
\hline & \multicolumn{8}{c}{ Depth $(\mathrm{cm})$} \\
\cline { 2 - 8 } Analysis & Units & $00-05$ & $05-10$ & $10-15$ & $15-20$ & $20-25$ & $25-30$ \\
\hline
\end{tabular}

Location=ORR; Fomation =DISMAL GAP; Site =19; 00-05=1093; 05-10=1094; 10-15=1095; 15-20=1096; $20-25=1097 ; 25-30=1098 ;$ Phase $=1$

$\begin{array}{llrrrrrr}\text { Cesium-137 } & \mathrm{pCi} / \mathrm{cm}^{2} & 8.065 & 0.603 & 0.126 & 0.029 & 0.048 & 0.027 \\ \text { Potassium-40 } & \mathrm{pCi} / \mathrm{cm}^{2} & 68.050 & 85.770 & 96.070 & 93.080 & 115.000 & 121.400 \\ \text { Radium-226 } & \mathrm{pCi} / \mathrm{cm}^{2} & 3.882 & 4.665 & 4.566 & 4.279 & 4.610 & 4.714 \\ \text { Thorium-232 } & \mathrm{pCi} / \mathrm{cm}^{2} & 4.830 & 6.485 & 6.457 & 6.428 & 7.356 & 7.756 \\ \text { Uranium-235 } & \mathrm{pCi} / \mathrm{cm}^{2} & 0.952 & 0.956 & 0.584 & 0.324 & 0.270 & 0.632 \\ \text { Uranium-238 } & \mathrm{pCi} / \mathrm{cm}^{2} & 16.393 & 15.355 & 4.717 & 8.440 & 3.522 & 10.930\end{array}$

Location=ORR; Formation =DISMAL GAP; Site=2; 00-05 =1183; 05-10=1184; 10-15=1185; 15-20=1186; $20-25=1187 ; 25-30=1188 ;$ Phase $=1$

$\begin{array}{llrrrrrr}\text { Cesium-137 } & \mathrm{PCi} / \mathrm{cm}^{2} & 6.188 & 1.218 & 0.427 & 0.279 & 0.182 & 0.077 \\ \text { Potassium-40 } & \mathrm{PCi} / \mathrm{cm}^{2} & 54.710 & 94.370 & 111.200 & 142.000 & 148.600 & 134.300 \\ \text { Radium-226 } & \mathrm{PCi} / \mathrm{cm}^{2} & 3.256 & 4.324 & 3.792 & 4.020 & 4.970 & 3.457 \\ \text { Thorium-232 } & \mathrm{PCi} / \mathrm{cm}^{2} & 3.648 & 5.723 & 6.086 & 7.001 & 7.637 & 8.072 \\ \text { Uranium-235 } & \mathrm{PCi} / \mathrm{cm}^{2} & 0.348 & 0.835 & 0.836 & 0.918 & 1.223 & 0.943 \\ \text { Uranium-238 } & \mathrm{PCi} / \mathrm{cm}^{2} & 11.796 & 13.721 & 13.507 & 13.390 & 15.677 & 16.343\end{array}$

Location $=O R R ;$ Formation $=D I S M A L$ GAP; Site $=22 ; 00-05=1100 ; 05-10=1101 ; 10-15=1102 ; 15-20=1103$; $20-25=1104 ; 25-30=1105 ;$ Phase $=1$

$\begin{array}{llrrrrrr}\text { Cesium-137 } & \mathrm{PCi} / \mathrm{cm}^{2} & 6.120 & 2.393 & 0.568 & 0.156 & 0.142 & 0.055 \\ \text { Potassium-40 } & \mathrm{PCi} / \mathrm{cm}^{2} & 54.530 & 99.450 & 113.400 & 147.200 & 154.600 & 152.500 \\ \text { Radium-226 } & \mathrm{PCi} / \mathrm{cm}^{2} & 2.679 & 4.334 & 4.723 & 4.330 & 3.743 & 3.081 \\ \text { Thorium-232 } & \mathrm{PCi} / \mathrm{cm}^{2} & 3.902 & 6.480 & 7.290 & 7.805 & 7.806 & 7.730 \\ \text { Uranium-235 } & \mathrm{pCi} / \mathrm{cm}^{2} & 0.720 & 0.698 & & 0.926 & & 0.289 \\ \text { Uranium-238 } & \mathrm{PCi} / \mathrm{cm}^{2} & 13.006 & 14.738 & 15.277 & 15.990 & 15.394 & 7.227\end{array}$

Location=ORR; Formation $=$ DISMAL GAP; Site $=26 ; 00-05=1207 ; 05-10=1208 ; 10-15=1209 ; 15-20=1210$; 20-25 $=1211 ; 25-30=1212 ;$ Phase $=1$

$\begin{array}{llrrrrrr}\text { Cesium-137 } & \mathrm{pCi} / \mathrm{cm}^{2} & 6.384 & 1.531 & 0.519 & 0.136 & 0.056 & 0.063 \\ \text { Potassium-40 } & \mathrm{pCi} / \mathrm{cm}^{2} & 85.340 & 93.340 & 108.600 & 113.700 & 103.800 & 127.600 \\ \text { Radium-226 } & \mathrm{pCi} / \mathrm{cm}^{2} & 3.909 & 2.895 & 3.338 & 3.495 & 3.129 & 3.269 \\ \text { Thorium-232 } & \mathrm{pCi} / \mathrm{cm}^{2} & 4.963 & 5.114 & 5.928 & 6.519 & 6.291 & 6.814 \\ \text { Uranium-235 } & \mathrm{pCi} / \mathrm{cm}^{2} & 0.820 & 0.520 & 0.856 & 0.799 & 0.826 & 0.240 \\ \text { Uranium-238 } & \mathrm{pCi} / \mathrm{cm}^{2} & 15.568 & 14.741 & 15.094 & 16.066 & 12.170 & 7.890\end{array}$

Location =ORR; Formation $=$ DISMAL GAP; Site $=27 ; 00-05=1009 ; 05-10=1010 ; 10-15=1011 ; 15-20=1012$ $20-25=1013 ; 25-30=1014 ;$ Phase $=1$

$\begin{array}{llrrrrrr}\text { Cesium-137 } & \mathrm{PCi} / \mathrm{cm}^{2} & 4.135 & 1.333 & 0.837 & 0.055 & 0.043 & 0.007 \\ \text { Potassium-40 } & \mathrm{PCi} / \mathrm{cm}^{2} & 49.690 & 59.540 & 67.120 & 76.560 & 86.630 & 99.010 \\ \text { Radium-226 } & \mathrm{PCi} / \mathrm{cm}^{2} & 2.484 & 3.472 & 3.156 & 3.755 & 3.904 & 3.875 \\ \text { Thorium-232 } & \mathrm{PCi} / \mathrm{cm}^{2} & 3.254 & 4.402 & 4.676 & 5.155 & 5.328 & 5.799 \\ \text { Uranium-235 } & \mathrm{pCi} / \mathrm{cm}^{2} & 0.536 & 0.465 & 0.653 & 0.558 & 0.588 & 0.554\end{array}$

Location=ORR; Formation=DISMAL GAP; Site=32; 00-05=1087; 05-10=1088; 10-15=1089; 15-20=1090; 20-25=1091; 25-30=1092; Phase $=1$

$\begin{array}{llrrrrrr}\text { Cesium-137 } & \mathrm{pCi} / \mathrm{cm}^{2} & 5.229 & 1.642 & 0.908 & 0.103 & 0.001 & 0.003 \\ \text { Potassium-40 } & \mathrm{pCi} / \mathrm{cm}^{2} & 76.990 & 105.300 & 112.200 & 116.100 & 130.400 & 148.800 \\ \text { Radium-226 } & \mathrm{pCi} / \mathrm{cm}^{2} & 3.274 & 3.960 & 4.944 & 4.759 & 3.796 & 3.753 \\ \text { Thorium-232 } & \mathrm{pCi} / \mathrm{cm}^{2} & 5.120 & 6.369 & 7.043 & 7.172 & 7.508 & 7.493 \\ \text { Uranium-235 } & \mathrm{pCi} / \mathrm{cm}^{2} & 0.380 & 0.345 & 0.841 & 0.392 & 0.389 & 0.321 \\ \text { Uranium-238 } & \mathrm{pCi} / \mathrm{cm}^{2} & 5.161 & 8.219 & 14.870 & 8.259 & 5.169 & 5.215\end{array}$




\section{B-104}

Table B3 (continued)

\begin{tabular}{llllllll}
\hline & \multicolumn{8}{c}{ Depth $(\mathrm{cm})$} \\
\cline { 2 - 7 } Analysis & Units & $00-05$ & $05-10$ & $10-15$ & $15-20$ & $20-25$ & $25-30$ \\
\hline
\end{tabular}

Location=ORR; Formation =DISMAL GAP; Site =33; $00-05=1109 ; 05-10=1110 ; 10-15=1111 ; 15-20=1112$; $20-25=1113 ; 25-30=1114 ;$ Phase $=1$

$\begin{array}{llrrrrrr}\text { Cesium-137 } & \mathrm{pCi} / \mathrm{cm}^{2} & 6.244 & 2.304 & 0.322 & 0.194 & 0.202 & 0.128 \\ \text { Potassium-40 } & \mathrm{PCi} / \mathrm{cm}^{2} & 44.920 & 108.000 & 131.000 & 114.500 & 118.700 & 125.600 \\ \text { Radium-226 } & \mathrm{PCi} / \mathrm{cm}^{2} & 2.859 & 4.659 & 4.027 & 3.814 & 4.718 & 4.797 \\ \text { Thorium-232 } & \mathrm{pCi} / \mathrm{cm}^{2} & 3.206 & 6.592 & 7.584 & 7.203 & 8.378 & 7.648 \\ \text { Uranium-235 } & \mathrm{pCi} / \mathrm{cm}^{2} & 0.335 & 1.100 & 0.827 & 1.053 & & 0.855 \\ \text { Uranium-238 } & \mathrm{PCi} / \mathrm{cm}^{2} & 18.409 & 21.350 & 24.996 & 19.684 & 15.260 & 15.554\end{array}$

Location=ORR; Formation $=$ DISMAL GAP; Site $=35 ; 00-05=1116 ; 05-10=1117 ; 10-15=1118 ; 15-20=1119$; $20-25=1120 ; 25-30=1121 ;$ Phase $=1$

$\begin{array}{llrrrrrr}\text { Cesium-137 } & \mathrm{pCi} / \mathrm{cm}^{2} & 4.784 & 0.668 & 0.140 & 0.128 & 0.022 & 0.130 \\ \text { Potassium-40 } & \mathrm{pCi} / \mathrm{cm}^{2} & 33.850 & 38.070 & 39.780 & 46.950 & 57.570 & 72.430 \\ \text { Radium-226 } & \mathrm{pCi} / \mathrm{cm}^{2} & 3.943 & 5.466 & 6.091 & 5.333 & 5.337 & 5.597 \\ \text { Thorium-232 } & \mathrm{pCi} / \mathrm{cm}^{2} & 4.394 & 6.303 & 6.220 & 6.354 & 6.657 & 7.148 \\ \text { Uranium-235 } & \mathrm{pCi} / \mathrm{cm}^{2} & 0.646 & 0.736 & 0.907 & 0.894 & 0.165 & 0.722 \\ \text { Uranium-238 } & \mathrm{pCi} / \mathrm{cm}^{2} & 16.810 & 13.795 & 5.152 & 27.200 & 9.461 & 16.568\end{array}$

Location=ORR; Formation =DISMAL GAP; Site $=41 ; 00-05=1028 ; 05-10=1029 ; 10-15=1030 ; 15-20=1031$; $20-25=1032 ; 25-30=1033 ;$ Phase $=1$

$\begin{array}{llrrrrrr}\text { Cesium-137 } & \mathrm{PCi} / \mathrm{cm}^{2} & 5.877 & 2.912 & 1.600 & 0.336 & 0.082 & 0.087 \\ \text { Potassium-40 } & \mathrm{PCi} / \mathrm{cm}^{2} & 30.520 & 48.050 & 54.250 & 79.970 & 73.820 & 80.450 \\ \text { Radium-226 } & \mathrm{PCi} / \mathrm{cm}^{2} & 2.148 & 3.498 & 3.372 & 2.633 & 2.342 & 3.005 \\ \text { Thorium-232 } & \mathrm{PCi} / \mathrm{cm}^{2} & 3.021 & 4.885 & 5.164 & 6.088 & 6.156 & 6.417 \\ \text { Uranium-235 } & \mathrm{PCi} / \mathrm{cm}^{2} & 0.539 & 0.638 & 0.557 & 0.604 & 0.488 & 0.527\end{array}$

Location=ORR; Formation $=$ DISMAL GAP; Site $=43 ; 00-05=1240 ; 05-10=1241 ; 10-15=1242 ; 15-20=1243$; $20-25=1244 ; 25-30=1245 ;$ Phase $=1$

$\begin{array}{llrrrrrr}\text { Cesium-137 } & \mathrm{PCi} / \mathrm{cm}^{2} & 5.571 & 1.920 & 0.434 & 0.195 & 0.172 & 0.199 \\ \text { Potassium-40 } & \mathrm{PCi} / \mathrm{cm}^{2} & 72.950 & 93.950 & 99.590 & 107.500 & 104.200 & 115.300 \\ \text { Radium-226 } & \mathrm{PCi} / \mathrm{cm}^{2} & 3.711 & 5.460 & 5.157 & 4.374 & 3.890 & 3.719 \\ \text { Thorium-232 } & \mathrm{PCi} / \mathrm{cm}^{2} & 5.417 & 6.589 & 7.005 & 7.418 & 6.849 & 6.566 \\ \text { Uranium-235 } & \mathrm{PCi} / \mathrm{cm}^{2} & 1.104 & 1.170 & & 1.022 & 1.016 & 0.957 \\ \text { Uranium-238 } & \mathrm{pCi} / \mathrm{cm}^{2} & 21.230 & 15.220 & 10.830 & 16.600 & 32.340 & 21.395\end{array}$

Location=ORR; Formation =NOLICHUCKY; Site $=13 ; 00-05=1355 ; 05-10=1356 ; 10-15=1357 ; 15-20=1358$; $20-25=1359 ; 25-30=1360 ;$ Phase $=1$

$\begin{array}{llrrrrrr}\text { Cesium-137 } & \mathrm{pCi} / \mathrm{cm}^{2} & 5.246 & 2.894 & 0.620 & 0.152 & 0.028 & 0.045 \\ \text { Potassium-40 } & \mathrm{PCi} / \mathrm{cm}^{2} & 64.080 & 81.320 & 93.600 & 94.870 & 101.300 & 127.600 \\ \text { Radium-226 } & \mathrm{PCi} / \mathrm{cm}^{2} & 3.476 & 4.343 & 4.528 & 4.719 & 4.498 & 4.509 \\ \text { Thorium-232 } & \mathrm{PCi} / \mathrm{cm}^{2} & 5.083 & 7.023 & 7.665 & 7.535 & 7.651 & 8.480 \\ \text { Uranium-235 } & \mathrm{pCi} / \mathrm{cm}^{2} & 0.546 & 0.923 & 0.952 & 0.993 & 0.981 & 0.530 \\ \text { Uranium-238 } & \mathrm{pCi} / \mathrm{cm}^{2} & 11.482 & 13.950 & 14.713 & 14.590 & 5.978 & 8.344\end{array}$

Location =ORR; Formation =NOLICHUCKY; Site $=15 ; 00-05=1307 ; 05-10=1308 ; 10-15=1309 ; 15-20=1310$; $20-25=1311 ; 25-30=1312 ;$ Phase $=1$

$\begin{array}{llrrrrrr}\text { Cesium-137 } & \mathrm{pCi} / \mathrm{cm}^{2} & 5.201 & 2.050 & 0.387 & 0.195 & 0.249 & 0.208 \\ \text { Potassium-40 } & \mathrm{pCi} / \mathrm{cm}^{2} & 29.890 & 40.970 & 47.510 & 58.740 & 72.330 & 84.280 \\ \text { Radium-226 } & \mathrm{pCi} / \mathrm{cm}^{2} & 2.978 & 4.096 & 4.614 & 4.047 & 4.181 & 4.449 \\ \text { Thorium-232 } & \mathrm{pCi} / \mathrm{cm}^{2} & 4.357 & 6.481 & 6.907 & 7.849 & 8.749 & 9.260 \\ \text { Uranium-235 } & \mathrm{pCi} / \mathrm{cm}^{2} & 1.008 & 9.101 & 1.134 & 1.543 & 1.265 & 1.280 \\ \text { Uranium-238 } & \mathrm{pCi} / \mathrm{cm}^{2} & 15.468 & 19.609 & 17.150 & 5.092 & 18.200 & 20.880\end{array}$


Table B.3 (continued)

\begin{tabular}{|c|c|c|c|c|c|c|c|}
\hline \multirow[b]{2}{*}{ Analysis } & \multicolumn{7}{|c|}{ Depth (cm) } \\
\hline & Units & 00-05 & $05-10$ & $10-15$ & $15-20$ & $20-25$ & $25-30$ \\
\hline Location $=O$ & ormatior & $\begin{array}{r}\mathrm{OLICH} \\
20\end{array}$ & $\begin{array}{l}Y ; \text { Site } \\
=1347 \text {; }\end{array}$ & $\begin{array}{l}5 ; 00-05= \\
-30=134\end{array}$ & $\begin{array}{l}\text { 3; } 05-1 \\
\text { ase }=1\end{array}$ & 10 & 843; \\
\hline $\begin{array}{l}\text { Cesium-137 } \\
\text { Potassium-40 } \\
\text { Radium-226 } \\
\text { Thorium-232 } \\
\text { Uranium-235 } \\
\text { Uranium-238 }\end{array}$ & $\begin{array}{l}\mathrm{pci} / \mathrm{cm}^{2} \\
\mathrm{pci} / \mathrm{cm}^{2} \\
\mathrm{pCi} / \mathrm{cm}^{2} \\
\mathrm{pCi} / \mathrm{cm}_{2}^{2} \\
\mathrm{pCi} / \mathrm{cm}^{2} \\
\mathrm{pCi} / \mathrm{cm}^{2}\end{array}$ & $\begin{array}{r}6.754 \\
89.360 \\
3.987 \\
6.797\end{array}$ & $\begin{array}{r}2.168 \\
101.300 \\
4.735 \\
8.466 \\
0.637 \\
4.548\end{array}$ & $\begin{array}{r}0.482 \\
19.000 \\
5.308 \\
9.270 \\
22.990\end{array}$ & $\begin{array}{r}0.158 \\
114.100 \\
5.095 \\
9.033\end{array}$ & $\begin{array}{r}75.210 \\
4.962 \\
8.650\end{array}$ & $\begin{array}{r}0.172 \\
85.820 \\
4.896 \\
8.897 \\
0.604 \\
7.154\end{array}$ \\
\hline
\end{tabular}

Location =ORR; Formation $=$ NOLICHUCKY; Site $=21 ; 00-05=1367 ; 05-10=1368 ; 10-15=1369 ; 15-20=1370$; $20-25=1371 ; 25-30=1372 ;$ Phase $=1$

$\begin{array}{llrrrrrr}\text { Cesium-137 } & \mathrm{pCi} / \mathrm{cm}^{2} & 10.650 & 0.724 & 0.165 & 0.156 & 0.092 & 0.055 \\ \text { Potassium-40 } & \mathrm{pCi} / \mathrm{cm}^{2} & 120.400 & 187.900 & 135.600 & 125.100 & 164.600 & 243.300 \\ \text { Radium-226 } & \mathrm{pCi} / \mathrm{cm}^{2} & 2.470 & 3.830 & 4.301 & 2.916 & 3.226 & 3.424 \\ \text { Thorium-232 } & \mathrm{pCi} / \mathrm{cm}^{2} & 6.099 & 10.100 & 8.872 & 9.165 & 9.904 & 10.100 \\ \text { Uranium-235 } & \mathrm{pCi} / \mathrm{cm}^{2} & 0.885 & 0.388 & -1.027 & 0.570 & 1.440 & 0.200 \\ \text { Uranium-238 } & \mathrm{pCi} / \mathrm{cm}^{2} & 15.931 & 18.495 & 16.164 & 17.040 & 15.696 & 8.715\end{array}$

Location=ORR; Formation $=$ NOLICHUCKY; Site $=23 ; 00-05=1316 ; 05-10=1317 ; 10-15=1318 ; 15-20=1319$; $20-25=1320 ; 25-30=1321 ;$ Phase $=1$

$\begin{array}{llrrrrrr}\text { Cesium-137 } & \mathrm{pCi} / \mathrm{cm}^{2} & 5.495 & 2.962 & 0.352 & 0.201 & 0.156 & 0.195 \\ \text { Potassium-40 } & \mathrm{PCi} / \mathrm{cm}_{2}^{2} & 52.660 & 63.460 & 113.200 & 136.100 & 155.100 & 150.300 \\ \text { Radium-226 } & \mathrm{PCi} / \mathrm{cm}^{2} & 3.798 & 4.033 & 3.469 & 2.755 & 3.297 & 2.868 \\ \text { Thorium-232 } & \mathrm{PCi} / \mathrm{cm}^{2} & 5.900 & 6.549 & 8.027 & 8.120 & 9.116 & 8.672 \\ \text { Uranium-235 } & \mathrm{pCi} / \mathrm{cm}^{2} & 1.116 & 1.120 & 1.209 & 1.134 & 1.278 & 1.186 \\ \text { Uranium-238 } & \mathrm{pCi} / \mathrm{cm}^{2} & 13.191 & 14.080 & 20.266 & 21.804 & 26.601 & 13.340\end{array}$

Location =ORR; Formation=NOLICHUCKY; Site $=24 ; 00-05=1361 ; 05-10=1362 ; 10-15=1363 ; 15-20=1364$; 20-25=1365; $25-30=1366 ;$ Phase $=1$

$\begin{array}{llrrrrrr}\text { Cesium-137 } & \mathrm{pCi} / \mathrm{cm}^{2} & 6.922 & 2.744 & 0.586 & 0.251 & 0.166 & 0.009 \\ \text { Potassium-40 } & \mathrm{pCi} / \mathrm{cm}^{2} & 111.200 & 160.500 & 156.700 & 180.400 & 188.500 & 183.800 \\ \text { Radium-226 } & \mathrm{pCi} / \mathrm{cm}^{2} & 3.299 & 4.234 & 3.918 & 4.073 & 4.095 & 3.638 \\ \text { Thorium-232 } & \mathrm{pCi} / \mathrm{cm}^{2} & 5.847 & 8.358 & 8.674 & 9.820 & 10.210 & 9.445 \\ \text { Uranium-235 } & \mathrm{pCi} / \mathrm{cm}^{2} & 0.876 & 0.993 & 0.951 & 1.044 & 1.015 & 0.290 \\ \text { Uranium-238 } & \mathrm{pCi} / \mathrm{cm}^{2} & 15.657 & 28.660 & 6.363 & 16.118 & 17.571 & 5.318\end{array}$

Location=ORR; Formation=NOLICHUCKY; Site =25; 00-05=1325; 05-10=1326; 10-15=1327; 15-20=1328; $20-25=1329 ; 25-30=1330 ;$ Phase $=1$

\begin{tabular}{|c|c|c|c|c|c|c|c|}
\hline $\begin{array}{l}\text { Cesium-137 } \\
\text { Potassium-40 } \\
\text { Radium-226 } \\
\text { Thorium-232 } \\
\text { Uranium-235 } \\
\text { Uranium-238 }\end{array}$ & $\begin{array}{l}\mathrm{pci} / \mathrm{cm}_{2}^{2} \\
\mathrm{pCi} / \mathrm{cm}_{2}^{2} \\
\mathrm{pCi} / \mathrm{cm}_{2}^{2} \\
\mathrm{pCi} / \mathrm{cm}_{2}^{2} \\
\mathrm{pCi} / \mathrm{cm}^{2} \\
\mathrm{pCi} / \mathrm{cm}^{2}\end{array}$ & $\begin{array}{r}6.829 \\
124.400 \\
3.761 \\
7.051 \\
1.223\end{array}$ & $\begin{array}{r}0.357 \\
151.300 \\
4.388 \\
9.536\end{array}$ & $\begin{array}{r}0.152 \\
152.500 \\
4.583 \\
9.616 \\
9.958\end{array}$ & $\begin{array}{r}0.265 \\
164.200 \\
3.857 \\
9.597 \\
1.477\end{array}$ & $\begin{array}{r}0.042 \\
186.600 \\
3.579 \\
9.744\end{array}$ & $\begin{array}{r}202.000 \\
3.449 \\
10.050\end{array}$ \\
\hline
\end{tabular}

Location =ORR; Formation $=$ NOLICHUCKY; Site $=28 ; 00-05=1337 ; 05-10=1338 ; 10-15=1339 ; 15-20=1340$; $20-25=1341 ; 25-30=1342 ;$ Phase $=1$

$\begin{array}{llrrrrrr}\text { Cesium-137 } & \mathrm{pCi} / \mathrm{cm}^{2} & 5.695 & 2.859 & 0.971 & 0.179 & 0.156 & 0.053 \\ \text { Potassium-40 } & \mathrm{pCi} / \mathrm{cm}^{2} & 42.790 & 79.640 & 79.650 & 178.900 & 233.000 & 237.500 \\ \text { Radium-226 } & \mathrm{pCi} / \mathrm{cm}^{2} & 3.383 & 6.105 & 5.717 & 4.630 & 3.541 & 4.066 \\ \text { Thorium-232 } & \mathrm{PCi} / \mathrm{cm}^{2} & 4.124 & 7.475 & 8.001 & 8.802 & 9.680 & 9.555 \\ \text { Uranium-235 } & \mathrm{PCi} / \mathrm{cm}^{2} & 0.740 & 1.118 & 0.972 & 2.204 & 1.061 & 0.053 \\ \text { Uranium-238 } & \mathrm{pCi} / \mathrm{cm}^{2} & 10.340 & 13.990 & 15.670 & 9.715 & 20.217 & 6.069\end{array}$


Table B3 (continued)

\begin{tabular}{|c|c|c|c|c|c|c|c|}
\hline \multicolumn{8}{|c|}{ Depth (cm) } \\
\hline Analysis & Units & $00-05$ & $05-10$ & $10-15$ & $15-20$ & $20-25$ & $25-30$ \\
\hline
\end{tabular}

Location =ORR; Formation $=$ NOLICHUCKY; Site $=3 ; 00-05=1331 ; 05-10=1332 ; 10-15=1333 ; 15-20=1334$; $20-25=1335 ; 25-30=1336 ;$ Phase $=1$

$\begin{array}{llrrrrrr}\text { Cesium-137 } & \mathrm{PCi} / \mathrm{cm}^{2} & 6.019 & 2.085 & 0.376 & 0.150 & 0.143 & 0.009 \\ \text { Potassium-40 } & \mathrm{PCi} / \mathrm{cm}^{2} & 50.050 & 70.760 & 79.950 & 74.760 & 85.750 & 84.440 \\ \text { Radium-226 } & \mathrm{PCi} / \mathrm{cm}^{2} & 3.893 & 4.494 & 4.726 & 4.667 & 3.919 & 3.968 \\ \text { Thorium-232 } & \mathrm{PCi} / \mathrm{cm}^{2} & 5.128 & 6.320 & 7.117 & 7.192 & 7.071 & 6.891 \\ \text { Uranium-235 } & \mathrm{PCi} / \mathrm{cm}^{2} & 0.799 & 0.870 & 0.406 & 0.862 & 0.640 & 0.162 \\ \text { Uranium-238 } & \mathrm{PCi} / \mathrm{cm}^{2} & 12.778 & 12.496 & 14.210 & 14.138 & 13.344 & 4.559\end{array}$

Location =ORR; Formation $=$ NOLICHUCKY; Site $=31 ; 00-05=1373 ; 05-10=1374 ; 10-15=1375 ; 15-20=1376$ $20-25=1377 ; 25-30=1378 ;$ Phase $=1$

$\begin{array}{llrrrrrr}\text { Cesium-137 } & \mathrm{pCi} / \mathrm{cm}^{2} & 8.026 & 2.515 & 0.424 & 0.116 & 0.057 & 0.069 \\ \text { Potassium-40 } & \mathrm{pCi} / \mathrm{cm}^{2} & 33.620 & 48.780 & 49.680 & 55.620 & 74.250 & 97.400 \\ \text { Radium-226 } & \mathrm{pCi} / \mathrm{cm}^{2} & 3.608 & 5.457 & 5.291 & 5.712 & 5.927 & 5.623 \\ \text { Thorium-232 } & \mathrm{pCi} / \mathrm{cm}_{2}^{2} & 4.916 & 6.718 & 6.974 & 7.220 & 8.074 & 8.938 \\ \text { Uranium-235 } & \mathrm{pCi} / \mathrm{cm}_{2} & 0.807 & 1.732 & 0.921 & 0.400 & 0.341 & 0.604 \\ \text { Uranium-238 } & \mathrm{pCi} / \mathrm{cm}^{2} & 14.236 & 6.761 & 14.435 & 8.832 & 6.049 & 15.150\end{array}$

Location=ORR; Formation $=$ NOLICHUCKY; Site $=42 ; 00-05=1379 ; 05-10=1380 ; 10-15=1381 ; 15-20=1382$; $20-25=1383 ; 25-30=1384 ;$ Phase $=1$

$\begin{array}{llrrrrrr}\text { Cesium-137 } & \mathrm{PCi} / \mathrm{cm}^{2} & 5.113 & 1.114 & 0.269 & 0.172 & 0.028 & 0.064 \\ \text { Potassium-40 } & \mathrm{PCi} / \mathrm{cm}^{2} & 57.730 & 82.200 & 135.500 & 163.400 & 141.600 & 135.000 \\ \text { Radium-226 } & \mathrm{PCi} / \mathrm{cm}^{2} & 3.520 & 3.975 & 4.821 & 4.077 & 4.529 & 4.532 \\ \text { Thorium-232 } & \mathrm{PCi} / \mathrm{cm}^{2} & 5.287 & 6.845 & 8.233 & 9.360 & 9.260 & 8.786 \\ \text { Uranium-235 } & \mathrm{PCi} / \mathrm{cm}^{2} & 0.812 & 1.219 & 0.579 & 0.306 & 0.294 & 0.163 \\ \text { Uranium-238 } & \mathrm{PCi} / \mathrm{cm}^{2} & 14.970 & 13.150 & 7.215 & 17.255 & \mathbf{8 . 3 5 6} & 11.100\end{array}$

Location =ORR; Formation =NOLICHUCKY; Site $=5 ; 00-05=1349 ; 05-10=1350 ; 10-15=1351 ; 15-20=1352 ;$ $20-25=1353 ; 25-30=1354 ;$ Phase $=1$

$\begin{array}{llrrrrrr}\text { Cesium-137 } & \mathrm{PCi} / \mathrm{cm}^{2} & 6.040 & 2.063 & 0.628 & 0.207 & 0.069 & 0.025 \\ \text { Potassium-40 } & \mathrm{PCi} / \mathrm{cm}^{2} & 44.860 & 47.850 & 69.000 & 85.700 & 96.650 & 103.100 \\ \text { Radium-226 } & \mathrm{PCi} / \mathrm{cm}^{2} & 4.494 & 5.029 & 5.281 & 5.485 & 5.648 & 5.009 \\ \text { Thorium-232 } & \mathrm{PCi} / \mathrm{cm}^{2} & 5.806 & 6.192 & 7.761 & 8.460 & 9.026 & 9.015 \\ \text { Uranium-235 } & \mathrm{PCi} / \mathrm{cm}^{2} & 0.550 & 0.823 & 0.413 & 0.960 & 0.995 & 0.636 \\ \text { Uranium-238 } & \mathrm{PCi} / \mathrm{cm}^{2} & 4.587 & 10.930 & 13.190 & 13.937 & 17.260 & 6.269\end{array}$

Location =ROA; Formation =COPPER RIDGE; Site $=33 ; 00-05=3248 ; 05-10=3249 ; 10-15=3250$; $15-20=3251 ; 20-25=3252 ; 25-30=3253 ;$ Phase $=2$

$\begin{array}{llrrrrrr}\text { Cesium-137 } & \mathrm{PCi} / \mathrm{cm}^{2} & 7.157 & 1.144 & 0.799 & 0.468 & 0.054 & 0.149 \\ \text { Potassium-40 } & \mathrm{PCi} / \mathrm{cm}^{2} & 18.440 & 37.040 & 34.320 & 35.950 & 35.550 & 36.400 \\ \text { Radium-226 } & \mathrm{PCi} / \mathrm{cm}^{2} & 3.082 & 5.633 & 5.685 & 6.117 & 5.572 & 5.771 \\ \text { Thorium-232 } & \mathrm{PCi} / \mathrm{cm}^{2} & 2.322 & 5.695 & 6.059 & 6.823 & 7.103 & 7.302 \\ \text { Uranium-235 } & \mathrm{PCi} / \mathrm{cm}^{2} & & 0.918 & 0.534 & 0.437 & 0.537 & 0.515\end{array}$

Location=ROA; Formation $=$ COPPER RIDGE; Site $=34 ; 00-05=3334 ; 05-10=3335 ; 10-15=3336$; $15-20=3337 ; 20-25=3338 ; 25-30=3339 ;$ Phase $=2$

$\begin{array}{llrrrrrr}\text { Cesium-137 } & \mathrm{PCi} / \mathrm{cm}_{2}^{2} & 7.316 & 2.429 & 0.953 & 0.290 & 0.080 & 0.144 \\ \text { Potassium-40 } & \mathrm{PCi} / \mathrm{cm}_{2} & 10.050 & 19.840 & 20.600 & 25.850 & 26.790 & 29.060 \\ \text { Radium-226 } & \mathrm{PCi} / \mathrm{cm}_{2} & 5.896 & 7.861 & 8.356 & 8.521 & 8.272 & 8.648 \\ \text { Thorium-232 } & \mathrm{PCi} / \mathrm{cm}_{2} & 2.301 & 3.853 & 4.540 & 5.611 & 6.320 & 7.325 \\ \text { Uranium-235 } & \mathrm{PCi} / \mathrm{cm}_{2} & 1.002 & 1.198 & 0.827 & 2.925 & 0.632 & 0.635 \\ \text { Uranium-238 } & \mathrm{PCi} / \mathrm{cm}^{2} & 10.520 & 12.850 & 15.963 & 23.460 & 12.220 & 18.970\end{array}$


Table B.3 (continued)

\begin{tabular}{llllllll}
\hline & \multicolumn{7}{c}{ Depth (cm) } \\
\cline { 2 - 7 } Analysis & Units & $00-05$ & $05-10$ & $10-15$ & $15-20$ & $20-25$ & $25-30$ \\
\hline
\end{tabular}

Location =ROA; Formation $=$ COPPER RIDGE; Site $=35 ; 00-05=3239 ; 05-10=3240 ; 10-15=3241$; $15-20=3242 ; 20-25=3243 ; 25-30=3244 ;$ Phase $=2$

$\begin{array}{llrrrrrr}\text { Cesium-137 } & \mathrm{pCi} / \mathrm{cm}^{2} & 3.779 & 2.938 & 2.564 & 1.407 & 0.675 & 0.107 \\ \text { Potassium-40 } & \mathrm{pCi} / \mathrm{cm}^{2} & 12.080 & 18.400 & 20.160 & 23.560 & 23.210 & 24.750 \\ \text { Radium-226 } & \mathrm{pCi} / \mathrm{cm}^{2} & 3.587 & 4.812 & 5.045 & 5.073 & 5.512 & 5.574 \\ \text { Thorium-232 } & \mathrm{pCi} / \mathrm{cm}^{2} & 2.785 & 3.703 & 3.869 & 4.132 & 4.602 & 4.939 \\ \text { Uranium-235 } & \mathrm{pCi} / \mathrm{cm}^{2} & 0.681 & 0.785 & 0.363 & 0.798 & & 0.309\end{array}$

Uranium-235

$\mathrm{PCi} / \mathrm{cm}^{2} \quad 0.68$

0.785

0.363

0.798

Location =ROA; Formation =COPPER RIDGE; Site =39;00-05=3343; 05-10=3344; 10-15=3345; $15-20=3346 ; 20-25=3347 ; 25-30=3348 ;$ Phase $=2$

$\begin{array}{llrrrrrr}\text { Cesium-137 } & \mathrm{pCi} / \mathrm{cm}^{2} & 6.075 & 3.353 & 1.097 & 0.237 & 0.190 & 0.053 \\ \text { Potassium-40 } & \mathrm{pCi} / \mathrm{cm}^{2} & 5.723 & 10.240 & 14.370 & 19.220 & 18.310 & 18.130 \\ \text { Radium-226 } & \mathrm{pCi} / \mathrm{cm}^{2} & 1.896 & 3.193 & 4.093 & 4.923 & 5.132 & 4.981 \\ \text { Thorium-232 } & \mathrm{pCi} / \mathrm{cm}^{2} & 1.419 & 2.434 & 3.013 & 3.656 & 3.733 & 4.067 \\ \text { Uranium-235 } & \mathrm{pCi} / \mathrm{cm}_{2}^{2} & 0.792 & 0.973 & 1.032 & 1.104 & 0.969 & 0.609 \\ \text { Uranium-238 } & \mathrm{pCi} / \mathrm{cm}^{2} & 15.354 & 17.818 & 16.787 & 20.042 & 22.071 & 5.005\end{array}$

Location =ROA; Formation $=$ COPPER RIDGE; Site $=40 ; 00-05=3267 ; 05-10=3268 ; 10-15=3269$; $15-20=3270 ; 20-25=3271 ; 25-30=3272 ;$ Phase $=2$

$\begin{array}{llllrrrr}\text { Cesium-137 } & \mathrm{pCi} / \mathrm{cm}^{2} & 6.007 & 2.123 & 0.425 & 0.108 & 0.047 & 0.050 \\ \text { Potassium-40 } & \mathrm{pCi} / \mathrm{cm}^{2} & 9.956 & 9.013 & 10.650 & 11.240 & 14.230 & 14.440 \\ \text { Radium-226 } & \mathrm{pCi} / \mathrm{cm}^{2} & 3.439 & 3.716 & 3.918 & 3.972 & 3.820 & 4.162 \\ \text { Thorium-232 } & \mathrm{pCi} / \mathrm{cm}^{2} & 2.389 & 2.575 & 2.940 & 2.892 & 3.078 & 3.449 \\ \text { Uranium-235 } & \mathrm{pCi} / \mathrm{cm}^{2} & 0.824 & 0.707 & 0.235 & 0.737 & 0.533 & 0.340\end{array}$

Location $=R O A ;$ Formation $=$ COPPER RIDGE; Site $=41 ; 00-05=3352 ; 05-10=3353 ; 10-15=3354$; $15-20=3355 ; 20-25=3356 ; 25-30=3357 ;$ Phase $=2$

$\begin{array}{llrrrrrr}\text { Cesium-137 } & \mathrm{pCi} / \mathrm{cm}^{2} & 5.539 & 2.331 & 0.800 & 0.278 & 0.203 & 0.109 \\ \text { Potassium-40 } & \mathrm{pCi} / \mathrm{cm}^{2} & 8.063 & 11.990 & 10.420 & 13.550 & 14.810 & 13.140 \\ \text { Radium-226 } & \mathrm{pCi} / \mathrm{cm}^{2} & 2.784 & 3.806 & 3.779 & 4.493 & 4.583 & 3.896 \\ \text { Thorium-232 } & \mathrm{pCi} / \mathrm{cm}^{2} & 1.891 & 2.872 & 2.444 & 3.153 & 3.360 & 2.857 \\ \text { Uranium-235 } & \mathrm{PCi} / \mathrm{cm}_{2}^{2} & 0.998 & 0.956 & 1.146 & 1.058 & 1.055 & 0.342 \\ \text { Uranium-238 } & \mathrm{pCi} / \mathrm{cm}^{2} & 12.261 & 9.093 & 20.133 & 20.603 & 20.236 & 6.026\end{array}$

Location =ROA; Formation $=$ COPPER RIDGE; Site $=42 ; 00-05=3276 ; 05-10=3277 ; 10-15=3278$; $15-20=3279 ; 20-25=3280 ; 25-30=3281 ;$ Phase $=2$

$\begin{array}{lllrrrrr}\text { Cesium-137 } & \mathrm{pCi} / \mathrm{cm}^{2} & 4.119 & 1.725 & 0.467 & 0.361 & 0.132 & 0.023 \\ \text { Potassium-40 } & \mathrm{pCi} / \mathrm{cm}^{2} & 7.924 & 15.500 & 16.520 & 15.940 & 16.380 & 19.410 \\ \text { Radium-226 } & \mathrm{pCi} / \mathrm{cm}^{2} & 3.251 & 4.137 & 5.077 & 4.962 & 4.955 & 5.184 \\ \text { Thorium-232 } & \mathrm{PCi} / \mathrm{cm}^{2} & 2.513 & 2.886 & 3.540 & 3.404 & 3.570 & 3.838 \\ \text { Uranium-235 } & \mathrm{pCi} / \mathrm{cm}^{2} & 0.731 & 0.211 & 0.846 & 0.369 & 0.797 & 0.611\end{array}$

Location $=R O A ;$ Formation $=$ COPPER RIDGE; Site $=43 ; 00-05=3285 ; 05-10=3286 ; 10-15=3287$; $15-20=3288 ; 20-25=3289 ; 25-30=3290 ;$ Phase $=2$

$\begin{array}{llrrrrrr}\text { Cesium-137 } & \text { pCi } / \mathrm{cm}^{2} & 7.192 & 4.028 & 0.898 & 0.565 & 0.248 & 0.148 \\ \text { Potassium-40 } & \mathrm{pCi} / \mathrm{cm}^{2} & 12.880 & 15.660 & 17.220 & 17.380 & 17.910 & 19.240 \\ \text { Radium-226 } & \mathrm{pCi} / \mathrm{cm}^{2} & 3.387 & 4.209 & 4.508 & 5.269 & 5.216 & 5.157 \\ \text { Thorium-232 } & \mathrm{pCi} / \mathrm{cm}^{2} & 2.559 & 3.525 & 4.348 & 4.414 & 4.564 & 5.405 \\ \text { Uranium-235 } & \mathrm{pCi} / \mathrm{cm}^{2} & & 1.648 & 0.874 & 1.979 & 0.880 & 0.686\end{array}$

Location $=R O A ;$ Formation $=$ COPPER RIDGE; Site $=44 ; 00-05=3257 ; 05-10=3258 ; 10-15=3259$; $15-20=3260 ; 20-25=3261 ; 25-30=3262 ;$ Phase $=2$

$\begin{array}{llrrrrrr}\text { Cesium-137 } & \mathrm{pCi} / \mathrm{cm}^{2} & 3.937 & 1.066 & 0.228 & 0.182 & 0.193 & 0.226 \\ \text { Potassium-40 } & \mathrm{pCi} / \mathrm{cm}^{2} & 9.559 & 15.180 & 15.530 & 20.620 & 19.690 & 20.360\end{array}$


Table B3 (continued)

\begin{tabular}{llllllll}
\hline & \multicolumn{7}{c}{ Depth $(\mathrm{cm})$} \\
\cline { 2 - 8 } Analysis & Units & $00-05$ & $05-10$ & $10-15$ & $15-20$ & $20-25$ & $25-30$ \\
\hline & & & & & & & \\
Radium-226 & $\mathrm{PCi} / \mathrm{cm}^{2}$ & 3.878 & 4.357 & 4.684 & 4.760 & 4.768 & 4.559 \\
Thorium-232 & $\mathrm{pCi} / \mathrm{cm}^{2}$ & 3.805 & 3.683 & 3.697 & 3.869 & 4.258 & 4.112 \\
Uranium-235 & $\mathrm{pCi} / \mathrm{cm}^{2}$ & 0.847 & 0.787 & 0.720 & 1.234 & 0.566 & 0.519
\end{tabular}

Location=ROA; Formation $=$ COPPER RJDGE; Site $=45 ; 00-05=3312 ; 05-10=3313 ; 10-15=3314$; 15-20=3315: $20-25=3316 ; 25-30=3317 ;$ Phase $=2$

$\begin{array}{llrrrrrr}\text { Cesium-137 } & \mathrm{PCi} / \mathrm{cm}^{2} & 4.488 & 2.639 & 0.683 & 0.261 & 0.240 & 0.088 \\ \text { Potassium-40 } & \mathrm{PCi} / \mathrm{cm}^{2} & 10.870 & 14.450 & 16.820 & 19.100 & 17.530 & 20.040 \\ \text { Radium-226 } & \mathrm{PCi} / \mathrm{cm}^{2} & 3.422 & 4.774 & 6.848 & 5.523 & 5.295 & 5.097 \\ \text { Thorium-232 } & \mathrm{PCi} / \mathrm{cm}^{2} & 2.468 & 3.046 & 3.546 & 3.499 & 3.850 & 4.073 \\ \text { Uranium-235 } & \mathrm{PCi} / \mathrm{cm}^{2} & 0.789 & 1.375 & 0.995 & 0.728 & 0.687 & 0.237 \\ \text { Uranium-238 } & \mathrm{pCi} / \mathrm{cm}^{2} & 12.227 & 12.173 & 14.190 & 14.640 & 15.420 & 8.083\end{array}$

Location $=R O A ;$ Formation $=C O P P E R$ RIDGE; Site $=46 ; 00-05=3303 ; 05-10=3304 ; 10-15=3305$; $15-20=3306 ; 20-25=3307 ; 25-30=3308 ;$ Phase $=2$

$\begin{array}{llrrrrrr}\text { Cesium-137 } & \mathrm{pCi} / \mathrm{cm}_{2}^{2} & 4.105 & 2.900 & 1.168 & 0.356 & 0.097 & 0.172 \\ \text { Potassium-40 } & \mathrm{pCi} / \mathrm{cm}^{2} & 11.780 & 13.180 & 13.740 & 15.600 & 14.670 & 18.010 \\ \text { Radium-226 } & \mathrm{pCi} / \mathrm{cm}^{2} & 3.344 & 3.951 & 4.717 & 4.327 & 4.456 & 4.782 \\ \text { Thorium-232 } & \mathrm{pCi} / \mathrm{cm}^{2} & 3.400 & 3.712 & 3.915 & 4.283 & 4.975 & 5.839 \\ \text { Uranium-235 } & \mathrm{pCi} / \mathrm{cm}^{2} & 0.481 & 0.806 & 0.361 & 0.585 & 0.877 & 0.942\end{array}$

Location $=$ ROA; Formation $=$ COPPER RIDGE; Site $=47 ; 00-05=3294 ; 05-10=3295 ; 10-15=3296$; $15-20=3297 ; 20-25=3298 ; 25-30=3299 ;$ Phase $=2$

$\begin{array}{llrrrrrr}\text { Cesium-137 } & \mathrm{pCi} / \mathrm{cm}^{2} & 3.580 & 1.011 & 0.146 & 0.162 & 0.137 & 0.154 \\ \text { Potassium-40 } & \mathrm{PCi} / \mathrm{cm}^{2} & 12.230 & 20.260 & 15.160 & 19.740 & 19.820 & 18.160 \\ \text { Radium-226 } & \mathrm{pCi} / \mathrm{cm}^{2} & 3.742 & 5.040 & 4.872 & 5.406 & 5.292 & 5.312 \\ \text { Thorium-232 } & \mathrm{PCi} / \mathrm{cm}^{2} & 3.873 & 4.301 & 4.135 & 4.588 & 4.932 & 4.753 \\ \text { Uranium-235 } & \mathrm{pCi} / \mathrm{cm}^{2} & 0.433 & 0.874 & 0.851 & 0.664 & 0.902 & 0.910\end{array}$

Location $=R O A ;$ Formation $=$ DISMAL GAP; Site $=10 ; 00-05=3178 ; 05-10=3179 ; 10-15=3180 ; 15-20=3181$; $20-25=3182 ; 25-30=3183 ;$ Phase $=1$

$\begin{array}{llrrrrrr}\text { Cesium-137 } & \mathrm{PCi} / \mathrm{cm}^{2} & 3.799 & 0.315 & 0.207 & 0.116 & 0.160 & 0.145 \\ \text { Potassium-40 } & \mathrm{PCi} / \mathrm{cm}^{2} & 65.610 & 83.720 & 93.950 & 111.700 & 120.300 & 122.600 \\ \text { Radium-226 } & \mathrm{PCi} / \mathrm{cm}^{2} & 4.098 & 5.067 & 5.120 & 4.514 & 4.343 & 4.125 \\ \text { Thorium-232 } & \mathrm{PCi} / \mathrm{cm}^{2} & 5.721 & 7.877 & 8.483 & 9.301 & 9.504 & 9.817 \\ \text { Uranium-235 } & \mathrm{PCi} / \mathrm{cm}^{2} & 0.883 & 0.625 & 0.157 & 0.223 & 0.977 & 0.936 \\ \text { Uranium-238 } & \mathrm{PCi} / \mathrm{cm}^{2} & 12.951 & 3.292 & 16.104 & 7.391 & 12.100 & 15.322\end{array}$

Location $=R O A ;$ Formation $=D I S M A L$ GAP; Site $=13 ; 00-05=3131 ; 05-10=3132 ; 10-15=3133 ; 15-20=3134$; $20-25=3135 ; 25-30=3136 ;$ Phase $=1$

$\begin{array}{llrrrrrr}\text { Cesium-137 } & \mathrm{pCi} / \mathrm{cm}^{2} & 1.249 & 0.211 & 0.078 & 0.042 & 0.274 & 0.131 \\ \text { Potassium-40 } & \mathrm{pCi} / \mathrm{cm}^{2} & 38.140 & 45.440 & 56.280 & 76.100 & 91.830 & 99.610 \\ \text { Radium-226 } & \mathrm{pCi} / \mathrm{cm}^{2} & 4.341 & 4.869 & 5.010 & 4.518 & 3.566 & 3.473 \\ \text { Thorium-232 } & \mathrm{pCi} / \mathrm{cm}_{2}^{2} & 6.259 & 6.699 & 7.088 & 7.512 & 7.160 & 7.401 \\ \text { Uranium-235 } & \mathrm{pCi} / \mathrm{cm}_{2}^{2} & 0.654 & 0.503 & 0.882 & 0.909 & 0.843 & 0.804 \\ \text { Uranium-238 } & \mathrm{pCi} / \mathrm{cm}^{2} & 14.053 & 11.420 & 14.996 & 9.012 & 6.081 & 12.893\end{array}$

Location=ROA; Formation =DISMAL GAP; Site =14; $00-05=3152 ; 05-10=3153 ; 10-15=3154 ; 15-20=3155$; $20-25=3156 ; 25-30=3157 ;$ Phase $=1$

$\begin{array}{llrrrrrr}\text { Cesium-137 } & \mathrm{pCi} / \mathrm{cm}^{2} & 6.661 & 1.273 & 0.240 & 0.028 & 0.177 & 0.181 \\ \text { Potassium-40 } & \mathrm{pCi} / \mathrm{cm}^{2} & 68.860 & 114.300 & 101.100 & 91.890 & 84.240 & 91.970 \\ \text { Radium-226 } & \mathrm{pCi} / \mathrm{cm}^{2} & 3.006 & 3.381 & 3.319 & 3.360 & 3.189 & 2.882 \\ \text { Thorium-232 } & \mathrm{pCi} / \mathrm{cm}^{2} & 5.460 & 7.294 & 7.327 & 7.655 & 7.363 & 7.461 \\ \text { Uranium-235 } & \mathrm{pCi} / \mathrm{cm}^{2} & 0.861 & 1.198 & 0.943 & 1.319 & 0.520 & 1.055 \\ \text { Uranium-238 } & \mathrm{pCi} / \mathrm{cm}^{2} & 17.810 & 19.296 & 12.060 & 20.826 & 20.670 & 18.583\end{array}$


Table B.3 (continued)

Depth (cm)

\begin{tabular}{llllllll}
\cline { 2 - 4 } Analysis & Units & $00-05$ & $05-10$ & $10-15$ & $15-20$ & $20-25$ & $25-30$
\end{tabular}

Location=ROA; Formation $=$ DISMAL GAP; Site $=17 ; 00-05=3022 ; 05-10=3023 ; 10-15=3024 ; 15-20=3025 ;$ $20-25=3026 ; 25-30=3027 ;$ Phase $=1$

$\begin{array}{llrrrrrr}\text { Cesium-137 } & \mathrm{pCi} / \mathrm{cm}^{2} & 5.840 & 2.448 & 0.803 & 0.301 & 0.143 & 0.081 \\ \text { Potassium-40 } & \mathrm{pCi} / \mathrm{cm}_{2}^{2} & 42.980 & 47.110 & 53.800 & 76.010 & 88.540 & 95.530 \\ \text { Radium-226 } & \mathrm{pCi} / \mathrm{cm}^{2} & 4.070 & 4.395 & 4.454 & 4.158 & 4.273 & 4.107 \\ \text { Thorium-232 } & \mathrm{pCi} / \mathrm{cm}^{2} & 5.552 & 6.009 & 6.833 & 6.937 & 7.463 & 7.488 \\ \text { Uranium-235 } & \mathrm{pCi} / \mathrm{cm}^{2} & 0.441 & 0.898 & & 0.784 & & .4 .219 \\ \text { Uranium-238 } & \mathrm{pCi} / \mathrm{cm}^{2} & 8.151 & 3.627 & 12.860 & 12.799 & 13.542 & 4.191\end{array}$

Location=ROA; Formation =DISMAL GAP; Site =19;00-05=3036;05-10=3037; 10-15=3038; 15-20=3039; $20-25=3040 ; 25-30=3041 ;$ Phase $=1$

$\begin{array}{llrrrrrr}\text { Cesium-137 } & \mathrm{pCi} / \mathrm{cm}^{2} & 3.421 & 0.288 & 0.131 & 0.124 & 0.085 & 0.119 \\ \text { Potassium-40 } & \mathrm{pCi} / \mathrm{cm}^{2} & 33.900 & 36.170 & 43.700 & 45.550 & 41.080 & 46.390 \\ \text { Radium-226 } & \mathrm{pCi} / \mathrm{cm}^{2} & 4.346 & 4.904 & 5.962 & 4.397 & 4.574 & 4.582 \\ \text { Thorium-232 } & \mathrm{PCi} / \mathrm{cm}^{2} & 5.410 & 5.574 & 6.397 & 6.025 & 6.070 & 6.108 \\ \text { Uranium-235 } & \mathrm{PCi} / \mathrm{cm}^{2} & 0.472 & 0.817 & & 0.826 & 0.496 & 0.321 \\ \text { Uranium-238 } & \mathrm{pCi} / \mathrm{cm}^{2} & 5.420 & 11.120 & 13.300 & 13.549 & 13.460 & 12.309\end{array}$

Location=ROA; Formation =DISMAL GAP; Site $=20 ; 00-05=3050 ; 05-10=3051 ; 10-15=3052 ; 15-20=3053$; 20-25 $=3054 ; 25-30=3055 ;$ Phase $=1$

$\begin{array}{llrrrrrr}\text { Cesium-137 } & \mathrm{pCi} / \mathrm{cm}^{2} & 4.418 & 0.974 & 0.382 & 0.203 & 0.091 & 0.053 \\ \text { Potassium-40 } & \mathrm{pCi} / \mathrm{cm}^{2} & 42.850 & 52.610 & 84.640 & 100.200 & 137.400 & 150.900 \\ \text { Radium-226 } & \mathrm{pCi} / \mathrm{cm}^{2} & 5.017 & 3.726 & 3.554 & 3.339 & 2.932 & 3.515 \\ \text { Thorium-232 } & \mathrm{pCi} / \mathrm{cm}^{2} & 4.809 & 5.634 & 6.836 & 7.053 & 7.258 & 7.377 \\ \text { Uranium-235 } & \mathrm{pCi} / \mathrm{cm}^{2} & 0.925 & 0.921 & 0.734 & 0.967 & 0.229 & 0.389 \\ \text { Uranium-238 } & \mathrm{pCi} / \mathrm{cm}^{2} & 15.191 & 18.260 & 10.530 & 21.704 & 5.676 & 3.401\end{array}$

Location=ROA; Formation $=$ DISMAL GAP; Site $=21 ; 00-05=3117 ; 05-10=3118 ; 10-15=3119 ; 15-20=3120$; $20-25=3121 ; 25-30=3122 ;$ Phase $=1$

$\begin{array}{llrrrrrr}\text { Cesium-137 } & \mathrm{PCi} / \mathrm{cm}^{2} & 4.254 & 0.630 & 0.116 & 0.157 & 0.076 & 0.169 \\ \text { Potassium-40 } & \mathrm{PCi} / \mathrm{cm}^{2} & 58.140 & 61.870 & 51.850 & 45.270 & 52.280 & 49.180 \\ \text { Radium-226 } & \mathrm{PCi} / \mathrm{cm}^{2} & 3.154 & 4.292 & 4.479 & 4.448 & 5.558 & 4.612 \\ \text { Thorium-232 } & \mathrm{PCi} / \mathrm{cm}^{2} & 5.862 & 6.323 & 6.321 & 5.959 & 6.547 & 6.512 \\ \text { Uranium-235 } & \mathrm{PCi} / \mathrm{cm}^{2} & 0.465 & 0.481 & 1.040 & 1.057 & 1.094 & 1.213 \\ \text { Uranium-238 } & \mathrm{PCi} / \mathrm{cm}^{2} & 16.468 & 6.400 & 17.950 & 18.180 & 12.520 & 18.516\end{array}$

Location $=R O A ;$ Formation $=$ DISMAL GAP; Site $=22 ; 00-05=3062 ; 05-10=3063 ; 10-15=3064 ; 15-20=3065$; $20-25=3066 ; 25-30=3067 ;$ Phase $=1$

$\begin{array}{llrrrrrr}\text { Cesium-137 } & \mathrm{PCi} / \mathrm{cm}^{2} & 3.071 & 0.669 & 0.127 & 0.135 & 0.131 & 0.030 \\ \text { Potassium-40 } & \mathrm{PCi} / \mathrm{cm}^{2} & 39.070 & 51.970 & 65.270 & 83.420 & 88.480 & 86.280 \\ \text { Radium-226 } & \mathrm{PCi} / \mathrm{cm}^{2} & 3.940 & 3.889 & 3.231 & 4.717 & 4.109 & 3.917 \\ \text { Thorium-232 } & \mathrm{PCi} / \mathrm{cm}^{2} & 4.974 & 5.624 & 5.496 & 6.322 & 6.162 & 5.742 \\ \text { Uranium-235 } & \mathrm{PCi} / \mathrm{cm}^{2} & 0.729 & 0.659 & 0.780 & 0.257 & 1.531 & 0.266 \\ \text { Uranium-238 } & \mathrm{PCi} / \mathrm{cm}^{2} & 20.200 & 13.303 & 12.230 & 14.525 & 12.445 & 4.445\end{array}$

Location=ROA; Formation $=D I S M A L$ GAP; Site $=3 ; 00-05=3103 ; 05-10=3104 ; 10-15=3105 ; 15-20=3106$; $20-25=3107 ; 25-30=3108 ;$ Phase $=1$

$\begin{array}{llrrrrrr}\text { Cesium-137 } & \mathrm{pCi} / \mathrm{cm}^{2} & 3.894 & 1.029 & 0.396 & 0.188 & 0.037 & 0.094 \\ \text { Potassium-40 } & \mathrm{pCi} / \mathrm{cm}^{2} & 52.560 & 56.280 & 55.380 & 60.110 & 65.800 & 64.020 \\ \text { Radium-226 } & \mathrm{pCi} / \mathrm{cm}^{2} & 3.392 & 4.706 & 4.330 & 4.204 & 4.129 & 3.783 \\ \text { Thorium-232 } & \mathrm{pCi} / \mathrm{cm}^{2} & 5.074 & 6.112 & 5.928 & 6.586 & 7.030 & 6.807 \\ \text { Uranium-235 } & \mathrm{pCi} / \mathrm{cm}^{2} & 0.249 & 0.805 & 0.707 & 0.255 & 0.846 & 0.836 \\ \text { Uranium-238 } & \mathrm{pCi} / \mathrm{cm}^{2} & 19.510 & 8.987 & 14.692 & 14.266 & 15.252 & 14.086\end{array}$


B-110

Table B.3 (continued)

\begin{tabular}{|c|c|c|c|c|c|c|c|}
\hline \multicolumn{8}{|c|}{ Depth (cm) } \\
\hline Analysis & Units & $00-05$ & $05-10$ & $10-15$ & $15-20$ & $20-25$ & $25-30$ \\
\hline \multicolumn{8}{|c|}{$\begin{array}{c}\text { Location }=R O A ; \text { Formation }=\text { DISMAL GAP; Site }=7 ; 00-05=3089 ; 05-10=3090 ; 10-15=3091 ; 15-20=3092 ; \\
20-25=3093 ; 25-30=3094 ; \text { Phase }=1\end{array}$} \\
\hline $\begin{array}{l}\text { Cesium-137 } \\
\text { Potassium-40 } \\
\text { Radium-226 } \\
\text { Thorium-232 } \\
\text { Uranium-235 } \\
\text { Uranium-238 }\end{array}$ & $\begin{array}{l}\mathrm{pCi} / \mathrm{cm}_{2}^{2} \\
\mathrm{pCi} / \mathrm{cm}^{2} \\
\mathrm{pCi} / \mathrm{cm}^{2} \\
\mathrm{pci} / \mathrm{cm}_{2}^{2} \\
\mathrm{pCi} / \mathrm{cm}^{2} \\
\mathrm{pCi} / \mathrm{cm}^{2}\end{array}$ & $\begin{array}{r}5.577 \\
43.820 \\
4.393 \\
5.573 \\
0.526 \\
12.556\end{array}$ & $\begin{array}{r}0.430 \\
57.690 \\
4.953 \\
7.324 \\
1.116 \\
6.964\end{array}$ & $\begin{array}{r}0.367 \\
57.920 \\
5.248 \\
7.186 \\
2.103 \\
10.560\end{array}$ & $\begin{array}{r}0.231 \\
68.970 \\
5.001 \\
7.706 \\
0.962 \\
10.850\end{array}$ & $\begin{array}{r}0.019 \\
87.960 \\
4.490 \\
7.710 \\
0.393 \\
7.567\end{array}$ & $\begin{array}{r}0.012 \\
101.600 \\
3.863 \\
7.955 \\
0.140 \\
6.318\end{array}$ \\
\hline \multicolumn{8}{|c|}{$\begin{array}{c}\text { Location=ROA; Formation }=\text { DISMAL GAP; Site }=8 ; 00-05=3076 ; 05-10=3077 ; 10-15=3078 ; 15-20=3079 ; \\
20-25=3080 ; 25-30=3081 ; \text { Phase }=1\end{array}$} \\
\hline $\begin{array}{l}\text { Cesium-137 } \\
\text { Potassium-40 } \\
\text { Radium-226 } \\
\text { Thorium-232 } \\
\text { Uranium-235 } \\
\text { Uranium-238 }\end{array}$ & $\begin{array}{l}\mathrm{pCi} / \mathrm{cm}_{2}^{2} \\
\mathrm{pCi} / \mathrm{cm}_{2} \\
\mathrm{pci} / \mathrm{cm}_{2}^{2} \\
\mathrm{pCi} / \mathrm{cm}_{2}^{2} \\
\mathrm{pCi} / \mathrm{cm}_{2} \\
\mathrm{pCi} / \mathrm{cm}^{2}\end{array}$ & $\begin{array}{r}8.006 \\
30.380 \\
3.258 \\
4.898 \\
0.733 \\
9.637\end{array}$ & $\begin{array}{r}3.085 \\
40.710 \\
4.775 \\
6.945 \\
12.698\end{array}$ & $\begin{array}{r}0.521 \\
43.380 \\
4.940 \\
7.295 \\
0.752 \\
14.041\end{array}$ & $\begin{array}{r}0.154 \\
45.450 \\
4.984 \\
7.308 \\
0.868 \\
8.371\end{array}$ & $\begin{array}{r}0.128 \\
48.850 \\
4.476 \\
7.710 \\
0.869 \\
7.014\end{array}$ & $\begin{array}{r}0.042 \\
58.750 \\
3.975 \\
6.928 \\
0.557 \\
6.800\end{array}$ \\
\hline \multicolumn{8}{|c|}{$\begin{array}{l}\text { Location=ROA; Formation }=\text { DISMAL GAP; Site =9; } 00-05=3008 ; 05-10=3009 ; 10-15=3010 ; 15-20=3011 ; \\
20-25=3012 ; 25-30=3013 ; \text { Phase }=1\end{array}$} \\
\hline $\begin{array}{l}\text { Cesium-137 } \\
\text { Potassium-40 } \\
\text { Radium-226 } \\
\text { Thorium-232 } \\
\text { Uranium-235 } \\
\text { Uranium-238 }\end{array}$ & $\begin{array}{l}\mathrm{pCi} / \mathrm{cm}_{2}^{2} \\
\mathrm{pCi} / \mathrm{cm}_{2}^{2} \\
\mathrm{pCi} / \mathrm{cm}_{2} \\
\mathrm{pCi} / \mathrm{cm}_{2} \\
\mathrm{pCi} / \mathrm{cm}_{2}^{2} \\
\mathrm{pCi} / \mathrm{cm}^{2}\end{array}$ & $\begin{array}{r}8.253 \\
27.730 \\
2.669 \\
3.755 \\
0.713 \\
5.853\end{array}$ & $\begin{array}{r}1.575 \\
35.700 \\
3.707 \\
5.367 \\
0.757 \\
6.414\end{array}$ & $\begin{array}{r}0.206 \\
40.480 \\
4.198 \\
5.678 \\
0.229 \\
3.848\end{array}$ & $\begin{array}{r}0.048 \\
37.550 \\
4.214 \\
5.562 \\
0.465 \\
8.091\end{array}$ & $\begin{array}{r}0.026 \\
45.380 \\
4.759 \\
6.136 \\
0.509 \\
4.830\end{array}$ & $\begin{array}{r}0.059 \\
53.820 \\
4.922 \\
6.641 \\
0.399 \\
8.532\end{array}$ \\
\hline
\end{tabular}

Unweighted results are on a field-moist, volumetric basis. 
Appendix C

ORGANIC ANALYSIS DATA 


\section{C-3}

Table C.1. Organic analysis results for soil samples

\begin{tabular}{|c|c|c|c|}
\hline \multirow{2}{*}{ Analysis } & \multicolumn{3}{|c|}{ A horizon } \\
\hline & Units & Result & Qualif \\
\hline \multicolumn{4}{|c|}{ Location $=A N D ;$ Fonnation $=$ COPPER RIDGE; } \\
\hline alpha-BHC & $\mu g / k g$ & 2.10 & UJ \\
\hline alpha-Chlordane & $\mu \mathrm{g} / \mathrm{kg}$ & 2.10 & $\mathbf{U}$ \\
\hline beta-BHC & $\mu g / \mathrm{kg}$ & 2.10 & $\mathbf{U}$ \\
\hline delta-BHC & $\mu \mathrm{g} / \mathrm{kg}$ & 2.10 & UJ \\
\hline gamma-Chlordane & $\mu \mathrm{g} / \mathrm{kg}$ & 2.10 & U \\
\hline Acenaphthylene & $\mu g / \mathrm{kg}$ & 41.60 & U \\
\hline Aldrin & $\mu g / \mathrm{kg}$ & 2.10 & U \\
\hline Benzo(a)anthracene & $\mu \mathrm{g} / \mathrm{kg}$ & 2.70 & $\mathrm{~J}$ \\
\hline Benzo(a)pyrene & $\mu g / \mathrm{kg}$ & 2.30 & $\mathrm{~J}$ \\
\hline Dalapon & $\mu g / \mathrm{kg}$ & 4168.00 & u \\
\hline Dicamba & $\mu \mathrm{g} / \mathrm{kg}$ & 191.00 & $\mathbf{U}$ \\
\hline Dichlorprop & $\mu g / \mathrm{kg}$ & 476.00 & UJ \\
\hline Dieldrin & $\mu \mathrm{g} / \mathrm{kg}$ & 4.20 & U \\
\hline Dinoseb & $\mu \mathrm{g} / \mathrm{kg}$ & 100.00 & U \\
\hline Endosulfan sulfate & $\mu \mathrm{g} / \mathrm{kg}$ & 4.20 & $u$ \\
\hline Endosulfan I & $\mu \mathrm{g} / \mathrm{kg}$ & 2.10 & $u$ \\
\hline Endosulfan if & $\mu g / k g$ & 4.20 & u \\
\hline Endrin & $\mu \mathrm{g} / \mathrm{kg}$ & 4.20 & U \\
\hline Endrin aldehyde & $\mu \mathrm{g} / \mathrm{kg}$ & 4.20 & U \\
\hline Endrin ketone & $\mu g / \mathbf{k g}$ & 4.20 & U \\
\hline Fluoranthene & $\mu \mathrm{g} / \mathrm{kg}$ & 4.00 & $J$ \\
\hline Heptachlor & $\mu \mathrm{g} / \mathrm{kg}$ & 2.10 & U \\
\hline Heptachlor epoxide & $\mu \mathrm{g} / \mathrm{kg}$ & 2.10 & u \\
\hline Indeno $(1,2,3-c d)$ pyrene & $\mu \mathrm{g} / \mathrm{kg}$ & 10.40 & $\vec{U}$ \\
\hline Lindane & $\mu g / \mathrm{kg}$ & 2.10 & UJ \\
\hline Methoxychlor & $\mu \mathrm{g} / \mathrm{kg}$ & 21.00 & $\mathrm{u}$ \\
\hline MCPA & $\mu \mathrm{g} / \mathrm{kg}$ & 178637.00 & US \\
\hline MCPP & $\mu \mathrm{g} / \mathrm{kg}$ & 135764.00 & US \\
\hline Phenanthrene & $\mu \mathrm{g} / \mathrm{kg}$ & 6.90 & $\mathbf{J}$ \\
\hline Pyrene & $\mu \mathrm{g} / \mathrm{kg}$ & 3.70 & $\mathbf{J}$ \\
\hline РСB-1016 & $\mu \mathrm{g} / \mathrm{kg}$ & 42.00 & $u$ \\
\hline PCB-1221 & $\mu \mathrm{g} / \mathrm{kg}$ & 84.00 & $u$ \\
\hline PCB-1232 & $\mu g / k g$ & 42.00 & $u$ \\
\hline PCB-1242 & $\mu g / k g$ & 42.00 & $u$ \\
\hline PCB- 1248 & $\mu \mathrm{g} / \mathrm{kg}$ & 42.00 & $\begin{array}{l}u \\
u\end{array}$ \\
\hline $\begin{array}{l}\text { PCB }=1254 \\
\text { PCB-1260 }\end{array}$ & $\begin{array}{l}\mu \mathrm{g} / \mathrm{kg} \\
\mu \mathrm{g} / \mathrm{kg}\end{array}$ & $\begin{array}{l}42.00 \\
42.00\end{array}$ & $\begin{array}{l}U \\
U\end{array}$ \\
\hline silvex & $\mu \mathrm{g} / \mathrm{kg}$ & 119 & $\mathbf{U}$ \\
\hline Toxaphene & $\mu \mathrm{g} / \mathrm{kg}$ & 210.00 & $\mathbf{U}$ \\
\hline $2,4-D$ & $\mu \mathrm{g} / \mathrm{kg}$ & 857.00 & U \\
\hline $2,4-D B$ & $\mu \mathrm{g} / \mathrm{kg}$ & 643.00 & U \\
\hline $2,4,5-T$ & $\mu g / \mathrm{kg}$ & 143.00 & u \\
\hline $4,4^{i}-D D D$ & $\mu \mathrm{g} / \mathrm{kg}$ & & $\mathbf{U}$ \\
\hline$\because-D D E$ & $\mu \mathrm{g} / \mathrm{kg}$ & & U \\
\hline -DDT & $\mu \mathrm{g} / \mathrm{kg}$ & 20 & UJ \\
\hline
\end{tabular}

Location $=A N D ;$ Formation $=C O P P E R$ RIDGE; Site $=32 ; A=2259 ; A$ dup=' '; Phase $=2$

\begin{tabular}{|c|c|c|}
\hline $\begin{array}{l}\text { alpha-BHC } \\
\text { alpha-Chlordane } \\
\text { beta-BHC } \\
\text { del ta-BHC } \\
\text { gamma-Chlordane } \\
\text { Acenaphthene } \\
\text { Acenaphthylene } \\
\text { Aldrin } \\
\text { Benzo(a)anthracene } \\
\text { Benzo(a)pyrene } \\
\text { Benzo(b)fluoranthene } \\
\text { Benzo(ghi)perylene } \\
\text { Benzo(k)fluoranthene } \\
\text { Dalapon } \\
\text { Dicamba } \\
\text { Dichlorprop }\end{array}$ & $\begin{array}{l}\mu g / \mathrm{kg} \\
\mu g / \mathrm{kg} \\
\mu g / \mathrm{kg} \\
\mu g / \mathrm{kg} \\
\mu g / \mathrm{kg} \\
\mu g / \mathrm{kg} \\
\mu g / \mathrm{kg} \\
\mu g / \mathrm{kg} \\
\mu g / \mathrm{kg} \\
\mu g / \mathrm{kg} \\
\mu g / \mathrm{kg} \\
\mu g / \mathrm{kg} \\
\mu g / \mathrm{kg} \\
\mu g / \mathrm{kg} \\
\mu g / \mathrm{kg} \\
\mu g / \mathrm{kg}\end{array}$ & $\begin{array}{r}2.20 \\
2.20 \\
2.20 \\
2.20 \\
2.20 \\
1.10 \\
43.20 \\
2.20 \\
1.70 \\
1.70 \\
3.50 \\
4.50 \\
1.50 \\
3400.00 \\
155.00 \\
389.00\end{array}$ \\
\hline
\end{tabular}


Table C.1 (continued)

\begin{tabular}{|c|c|c|c|c|c|}
\hline Analysis & Units & $\begin{array}{c}\text { A horizon } \\
\text { Result }\end{array}$ & Qualifier & $\begin{array}{l}\text { A horizon } \\
\text { field dup }\end{array}$ & Qualifier \\
\hline $\begin{array}{l}\text { Dieldrin } \\
\text { Dinoseb } \\
\text { Endosul fan sul fate } \\
\text { Endosul fan I } \\
\text { Endosulfan II } \\
\text { Endrin } \\
\text { Endrin aldehyde } \\
\text { Endrin ketone } \\
\text { Fluoranthene } \\
\text { Heptachlor } \\
\text { Heptachlor epoxide } \\
\text { Indeno( } 1,2,3-\text { cd)pyrene } \\
\text { Lindane } \\
\text { Methoxychlor } \\
\text { MCPA } \\
\text { MCPP } \\
\text { Phenanthrene } \\
\text { Pyrene } \\
\text { PCB-1016 } \\
\text { PCB- } 1221 \\
\text { PCB-1232 } \\
\text { PCB-1242 } \\
\text { PCB-1248 } \\
\text { PCB-1254 } \\
\text { PCB-1260 } \\
\text { Silvex } \\
\text { Toxaphene } \\
2,4-D \\
2,4-D B \\
2,4,5-T \\
4,4:-D D D \\
4,4:-D D E \\
4,4:-D D T\end{array}$ & 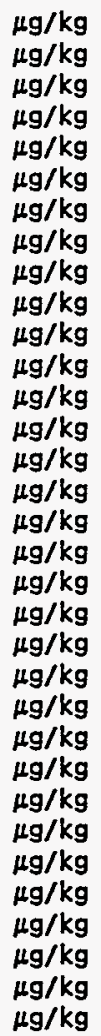 & $\begin{array}{r}4.30 \\
82.00 \\
4.30 \\
2.20 \\
4.30 \\
4.30 \\
4.30 \\
4.30 \\
3.50 \\
2.20 \\
2.20 \\
10.80 \\
2.20 \\
22.00 \\
145716.00 \\
110744.00 \\
6.30 \\
3.50 \\
43.00 \\
87.00 \\
43.00 \\
43.00 \\
43.00 \\
43.00 \\
43.00 \\
97.00 \\
220.00 \\
699.00 \\
525.00 \\
117.00 \\
4.30 \\
4.30 \\
4.30\end{array}$ & $\begin{array}{l}U \\
U \\
U \\
U \\
U \\
U \\
U \\
U \\
U \\
U \\
U \\
U \\
U J \\
U \\
U J \\
U J \\
\\
\\
U \\
U \\
U \\
U \\
U \\
U \\
U \\
U \\
U \\
U \\
U \\
U \\
U \\
U \\
U J\end{array}$ & . & \\
\hline
\end{tabular}

Location $=A N D ;$ Formation $=C O P P E R$ RIDGE; Site $=33 ; A=2262 ; A$ dup=' '; Phase $=2$

alpha-BHC
alpha-Chlordane
beta-BHC
del ta-BHC
garma-Chlordane
Acenaphthylene
Aldrin
Dalapon
Dicamba
Dichlorprop
Dieldrin
Dinoseb
Endosulfan sulfate
Endosulfan I
Endosulfan II
Endrin
Endrin aldehyde
Endrin ketone
Fluorene
Heptachlor
Heptachlor epoxide
Indeno(1,2,3-cd)pyrene
Lindane
Methoxychlor
MCPA
MCPP
Phenanthrene
PCB-1016
PCB-1221
PCB-1232
PCB-1242

$\begin{array}{lrl}\mu g / k g & 2.80 & U J \\ \mu g / k g & 2.80 & U \\ \mu g / k g & 2.80 & U \\ \mu g / k g & 2.80 & U J \\ \mu g / k g & 2.80 & U \\ \mu g / k g & 53.90 & U \\ \mu g / k g & 3.40 & \\ \mu g / k g & 3305.00 & U J \\ \mu g / k g & 151.00 & U J \\ \mu g / k g & 378.00 & U J \\ \mu g / k g & 5.50 & U \\ \mu g / k g & 79.00 & U J \\ \mu g / k g & 5.50 & U \\ \mu g / k g & 2.80 & U \\ \mu g / k g & 5.50 & U \\ \mu g / k g & 5.50 & U \\ \mu g / k g & 5.50 & U \\ \mu g / k g & 5.50 & U \\ \mu g / k g & 2.40 & J \\ \mu g / k g & 2.80 & U \\ \mu g / k g & 2.80 & U \\ \mu g / k g & 13.50 & U \\ \mu g / k g & 2.80 & U J \\ \mu g / k g & 28.00 & U \\ \mu g / k g & 141643.00 & U J \\ \mu g / k g & 107649.00 & U J \\ \mu g / k g & 1.10 & J \\ \mu g / k g & 55.00 & U \\ \mu g / k g & 110.00 & U \\ \mu g / k g & 55.00 & U \\ \mu g / k g & 55.00 & U\end{array}$




\section{C-5}

Table C.1 (continued)

\begin{tabular}{|c|c|c|c|c|c|c|}
\hline Analysis & Units & $\begin{array}{c}\text { A horizon } \\
\text { Result }\end{array}$ & Qualifier & $\begin{array}{l}\text { A horizon } \\
\text { field dup }\end{array}$ & Qualifier & \\
\hline $\begin{array}{l}\text { PCB-1248 } \\
\text { PCB- } 1254 \\
\text { PCB-1260 } \\
\text { Silvex } \\
\text { Toxaphene } \\
2,4-D \\
2,4-D B \\
2,4,5-T \\
4,4 \text {-DDD } \\
4,41-D D E \\
4,41-D D T\end{array}$ & $\begin{array}{l}\mu \mathrm{g} / \mathrm{kg} \\
\mu \mathrm{g} / \mathrm{kg} \\
\mu \mathrm{g} / \mathrm{kg} \\
\mu \mathrm{g} / \mathrm{kg} \\
\mu \mathrm{g} / \mathrm{kg} \\
\mu \mathrm{g} / \mathrm{kg} \\
\mu \mathrm{g} / \mathrm{kg} \\
\mu \mathrm{g} / \mathrm{kg} \\
\mu \mathrm{g} / \mathrm{kg} \\
\mu \mathrm{g} / \mathrm{kg} \\
\mu \mathrm{g} / \mathrm{kg}\end{array}$ & $\begin{array}{r}55.00 \\
55.00 \\
55.00 \\
94.00 \\
280.00 \\
680.00 \\
510.00 \\
113.00 \\
5.50 \\
5.50 \\
5.50\end{array}$ & 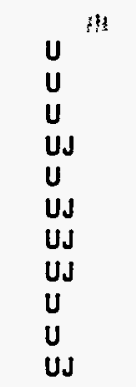 & $\therefore \because$ & . & $2 a^{2} \cos _{0}$ \\
\hline
\end{tabular}

Location=AND; Formation $=$ COPPER RIDGE; Site $=34 ; A=2265 ; A$ dup =' '; Phase $=2$

$\begin{array}{lrrl} & & \\ \text { alpha-BHC } & \mu \mathrm{g} / \mathrm{kg} & 2.20 & \mathrm{UJ} \\ \text { alpha-Chlordane } & \mu \mathrm{g} / \mathrm{kg} & 2.20 & \mathrm{U} \\ \text { beta-BHC } & \mu \mathrm{g} / \mathrm{kg} & 2.20 & \mathrm{U} \\ \text { delta-BHC } & \mu \mathrm{g} / \mathrm{kg} & 2.20 & \mathrm{UJ} \\ \text { gamm-Chlordane } & \mu \mathrm{g} / \mathrm{kg} & 2.20 & \mathrm{U} \\ \text { Acenaphthylene } & \mu \mathrm{g} / \mathrm{kg} & 43.10 & \mathrm{U} \\ \text { Aldrin } & \mu \mathrm{g} / \mathrm{kg} & 2.20 & \mathrm{U} \\ \text { Benzo(a)anthracene } & \mu \mathrm{g} / \mathrm{kg} & 1.50 & \mathrm{~J} \\ \text { Benzo(a)pyrene } & \mu \mathrm{g} / \mathrm{kg} & 2.40 & \mathrm{~J} \\ \text { Benzo(b)fluoranthene } & \mu \mathrm{g} / \mathrm{kg} & 4.50 & \mathrm{~J} \\ \text { Benzo(ghi)perylene } & \mu \mathrm{g} / \mathrm{kg} & 1.70 & \mathrm{~J} \\ \text { Benzo(k)fluoranthene } & \mu \mathrm{g} / \mathrm{kg} & 1.50 & \mathrm{~J} \\ \text { Dieldrin } & \mu \mathrm{g} / \mathrm{kg} & 4.40 & \mathrm{U} \\ \text { Endosulfan sulfate } & \mu \mathrm{g} / \mathrm{kg} & 4.40 & \mathrm{U} \\ \text { Endosulfan I } & \mu \mathrm{g} / \mathrm{kg} & 2.20 & \mathrm{U} \\ \text { Endosulfan II } & \mu \mathrm{g} / \mathrm{kg} & 4.40 & \mathrm{U} \\ \text { Endrin } & \mu \mathrm{g} / \mathrm{kg} & 4.40 & \mathrm{U} \\ \text { Endrin aldehyde } & \mu \mathrm{g} / \mathrm{kg} & 4.40 & \mathrm{U} \\ \text { Endrin ketone } & \mu \mathrm{g} / \mathrm{kg} & 4.40 & \mathrm{U} \\ \text { Fluorene } & \mu \mathrm{g} / \mathrm{kg} & 2.80 & \mathrm{~J} \\ \text { Heptachlor } & \mu \mathrm{g} / \mathrm{kg} & 2.20 & \mathrm{U} \\ \text { Heptachlor epoxide } & \mu \mathrm{g} / \mathrm{kg} & 2.20 & \mathrm{U} \\ \text { Indeno(1,2,3-cd)pyrene } & \mu \mathrm{g} / \mathrm{kg} & 10.80 & \mathrm{U} \\ \text { Lindane } & \mu \mathrm{g} / \mathrm{kg} & 2.20 & \mathrm{UJ} \\ \text { Hethoxychlor } & \mu \mathrm{g} / \mathrm{kg} & 22.00 & \mathrm{U} \\ \text { Phenanthrene } & \mu \mathrm{g} / \mathrm{kg} & 5.00 & \mathrm{~J} \\ \text { Pyrene } & \mu \mathrm{g} / \mathrm{kg} & 3.20 & \mathrm{~J} \\ \text { PCB-1016 } & \mu \mathrm{g} / \mathrm{kg} & 44.00 & \mathrm{U} \\ \text { PCB-1221 } & \mu \mathrm{g} / \mathrm{kg} & 88.00 & \mathrm{U} \\ \text { PCB-1232 } & \mu \mathrm{g} / \mathrm{kg} & 44.00 & \mathrm{U} \\ \text { PCB-1242 } & \mu \mathrm{g} / \mathrm{kg} & 44.00 & \mathrm{U} \\ \text { PCB-1248 } & \mu \mathrm{g} / \mathrm{kg} & 44.00 & \mathrm{U} \\ \text { PCB-1254 } & \mu \mathrm{g} / \mathrm{kg} & 44.00 & \mathrm{U} \\ \text { PCB-1260 } & \mu \mathrm{g} / \mathrm{kg} & 44.00 & \mathrm{U} \\ \text { Toxaphene } & \mu \mathrm{g} / \mathrm{kg} & 220.00 & \mathrm{U} \\ 4,41-D D D & \mu \mathrm{g} / \mathrm{kg} & 4.40 & \mathrm{U} \\ 4,41-D D E & \mu \mathrm{g} / \mathrm{kg} & 4.40 & \mathrm{U} \\ 4,41-D D T & \mu \mathrm{g} / \mathrm{kg} & 4.40 & \mathrm{UJ} \\ & & & \end{array}$

Location =AND; Formation=COPPER RIDGE; Site =35; $A=2268 ; A$ dup=' '; Phase=2

$\begin{array}{llrl}\text { alpha-BHC } & \mu g / k g & 1.30 & \mathrm{UJ} \\ \text { alpha-Chlordane } & \mu \mathrm{g} / \mathrm{kg} & 2.90 & \mathrm{U} \\ \text { beta-BHC } & \mu \mathrm{g} / \mathrm{kg} & 2.90 & \mathrm{U} \\ \text { del ta-BHC } & \mu \mathrm{g} / \mathrm{kg} & 2.90 & \mathrm{UJ} \\ \text { gamma-Chlordane } & \mu \mathrm{g} / \mathrm{kg} & 2.90 & \mathrm{U} \\ \text { Acenaphthylene } & \mu \mathrm{g} / \mathrm{kg} & 59.40 & \mathrm{U} \\ \text { Aldrin } & \mu \mathrm{g} / \mathrm{kg} & 2.90 & \mathrm{U} \\ \text { Anthracene } & \mu \mathrm{g} / \mathrm{kg} & 0.90 & \mathrm{~J} \\ \text { Benzo(a)anthracene } & \mu \mathrm{g} / \mathrm{kg} & 0.90 & \mathrm{~J} \\ \text { Benzo(a)pyrene } & \mu \mathrm{g} / \mathrm{kg} & 0.90 & \mathrm{~J}\end{array}$


C-6

Table C.1 (continued)

\begin{tabular}{|c|c|c|c|c|c|}
\hline Analysis & Units & $\begin{array}{c}\text { A horizon } \\
\text { Result }\end{array}$ & Qualifier & $\begin{array}{l}\text { A horizon } \\
\text { field dup }\end{array}$ & Qualifier \\
\hline $\begin{array}{l}\text { Benzo(ghi)perylene } \\
\text { Dieldrin } \\
\text { Endosulfan sul fate } \\
\text { Endosulfan I } \\
\text { Endosulfan II } \\
\text { Endrin } \\
\text { Endrin aldehyde } \\
\text { Endrin ketone } \\
\text { Heptachlor } \\
\text { Heptachlor epoxide } \\
\text { Indeno(1,2,3-cd)pyrene } \\
\text { Lindane } \\
\text { Hethoxychlor } \\
\text { Phenanthrene } \\
\text { Pyrene } \\
\text { PCB-1016 } \\
\text { PCB-1221 } \\
\text { PCB-1232 } \\
\text { PCB-1242 } \\
\text { PCB-1248 } \\
\text { PCB-1254 } \\
\text { PCB-1260 } \\
\text { TOXaphene } \\
4,4 '-D D D \\
4,4 \text {-DDE } \\
4,4 \text {-DDT }\end{array}$ & $\begin{array}{l}\mu g / \mathrm{kg} \\
\mu \mathrm{g} / \mathrm{kg} \\
\mu \mathrm{g} / \mathrm{kg} \\
\mu \mathrm{g} / \mathrm{kg} \\
\mu \mathrm{g} / \mathrm{kg} \\
\mu \mathrm{g} / \mathrm{kg} \\
\mu \mathrm{g} / \mathrm{kg} \\
\mu \mathrm{g} / \mathrm{kg} \\
\mu \mathrm{g} / \mathrm{kg} \\
\mu \mathrm{g} / \mathrm{kg} \\
\mu \mathrm{g} / \mathrm{kg} \\
\mu \mathrm{g} / \mathrm{kg} \\
\mu \mathrm{g} / \mathrm{kg} \\
\mu \mathrm{g} / \mathrm{kg} \\
\mu \mathrm{g} / \mathrm{kg} \\
\mu \mathrm{g} / \mathrm{kg} \\
\mu \mathrm{g} / \mathrm{kg} \\
\mu \mathrm{g} / \mathrm{kg} \\
\mu \mathrm{g} / \mathrm{kg} \\
\mu \mathrm{g} / \mathrm{kg} \\
\mu \mathrm{g} / \mathrm{kg} \\
\mu \mathrm{g} / \mathrm{kg} \\
\mu \mathrm{g} / \mathrm{kg} \\
\mu \mathrm{g} / \mathrm{kg} \\
\mu \mathrm{g} / \mathrm{kg} \\
\mu \mathrm{g} / \mathrm{kg}\end{array}$ & $\begin{array}{r}1.80 \\
5.90 \\
5.90 \\
2.90 \\
5.90 \\
5.90 \\
5.90 \\
5.90 \\
2.90 \\
2.90 \\
10.70 \\
2.90 \\
29.00 \\
2.10 \\
1.80 \\
59.00 \\
120.00 \\
59.00 \\
59.00 \\
59.00 \\
59.00 \\
59.00 \\
290.00 \\
5.90 \\
5.90 \\
5.90\end{array}$ & $\begin{array}{l}J \\
U \\
U \\
U \\
U \\
U \\
U \\
U \\
U \\
U \\
J \\
U J \\
U \\
J \\
J \\
U \\
U \\
U \\
U \\
U \\
U \\
U \\
U \\
U \\
U \\
U J\end{array}$ & & \\
\hline
\end{tabular}

Location=AND; Formation=COPPER RIDGE; Site $=36 ; A=2271 ; A$ dup=' ; Phase $=2$

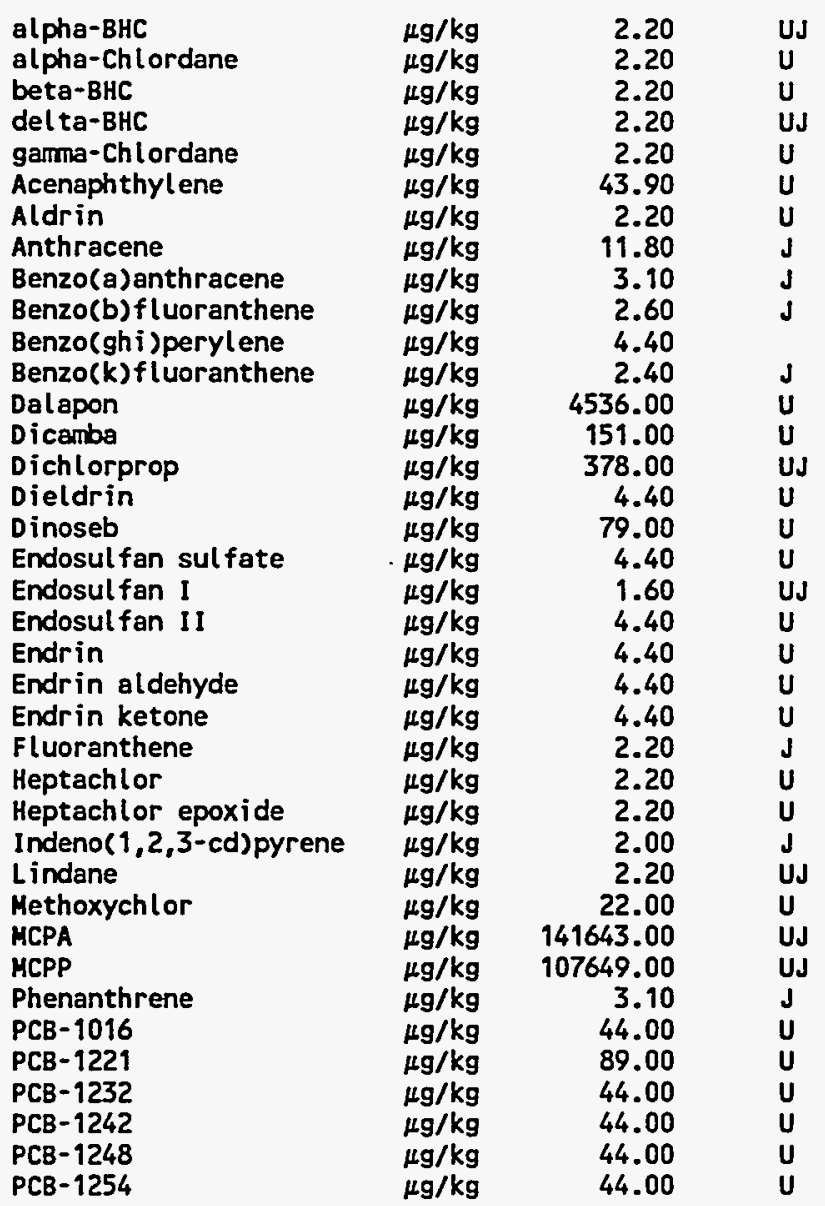


Table C.1 (continued)

\begin{tabular}{|c|c|c|c|c|c|c|}
\hline Analysis & Units & $\begin{array}{c}\text { A horizon } \\
\text { Result }\end{array}$ & Qualifier & $\begin{array}{l}\text { A horizon } \\
\text { field dup }\end{array}$ & Qualifier & \\
\hline $\begin{array}{l}\text { PCB- } 1260 \\
\text { Silvex } \\
\text { Toxaphene } \\
2,4-D \\
2,4-D B \\
2,4,5-T \\
4,41-D D D \\
4,41-D D E \\
4,41-D D T\end{array}$ & $\begin{array}{l}\mu g / \mathrm{kg} \\
\mu g / \mathrm{kg} \\
\mu g / \mathrm{kg} \\
\mu g / \mathrm{kg} \\
\mu g / \mathrm{kg} \\
\mu g / \mathrm{kg} \\
\mu g / \mathrm{kg} \\
\mu g / \mathrm{kg} \\
\mu g / \mathrm{kg}\end{array}$ & $\begin{array}{r}44.00 \\
94.00 \\
220.00 \\
680.00 \\
510.00 \\
113.00 \\
4.40 \\
4.40 \\
4.40\end{array}$ & $\begin{array}{l}U \\
U \\
U \\
U \\
U \\
U \\
U \\
U \\
U J\end{array}$ & $\therefore$ & & ristis: \\
\hline
\end{tabular}

Location $=A N D ;$ Formation $=$ COPPER RIDGE; Site $=37 ; A=2273 ; A$ dup $=$ ' '; Phase $=2$

\begin{tabular}{|c|c|c|}
\hline 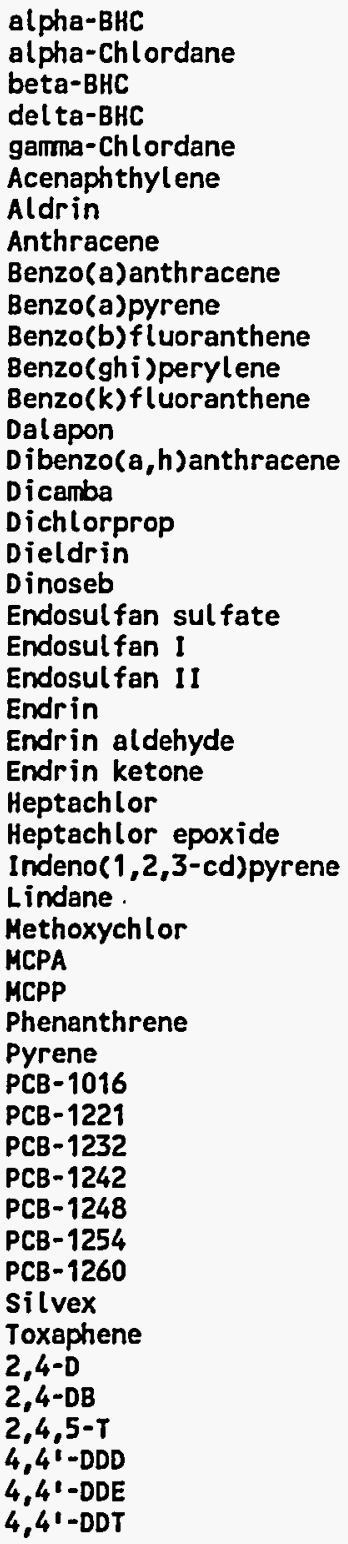 & 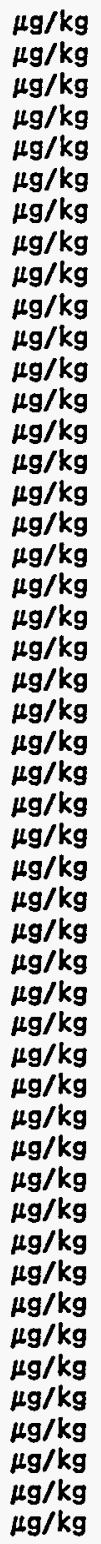 & $\begin{array}{r}2.30 \\
2.30 \\
2.30 \\
2.30 \\
2.30 \\
45.40 \\
2.30 \\
1.40 \\
1.80 \\
2.30 \\
1.40 \\
2.70 \\
1.40 \\
4580.00 \\
0.90 \\
209.00 \\
523.00 \\
4.60 \\
110.00 \\
4.60 \\
2.30 \\
4.60 \\
4.60 \\
4.60 \\
4.60 \\
2.30 \\
2.30 \\
11.80 \\
2.30 \\
23.00 \\
196293.00 \\
149182.00 \\
2.50 \\
3.90 \\
46.00 \\
93.00 \\
46.00 \\
46.00 \\
46.00 \\
46.00 \\
46.00 \\
131.00 \\
230.00 \\
942.00 \\
707.00 \\
157.00 \\
4.60 \\
4.60 \\
4.60\end{array}$ \\
\hline
\end{tabular}




\section{C-8}

Table C.1 (continued)

\begin{tabular}{|c|c|c|c|c|c|}
\hline Analysis & Units & $\begin{array}{c}\text { A horizon } \\
\text { Result }\end{array}$ & Qualifier & $\begin{array}{l}\text { A horizon } \\
\text { field dup }\end{array}$ & Qualifier \\
\hline
\end{tabular}

Location=AND; Formation $=$ COPPER RIDGE; Site $=38 ; A=2275 ; A$ dup=' '; Phase $=2$

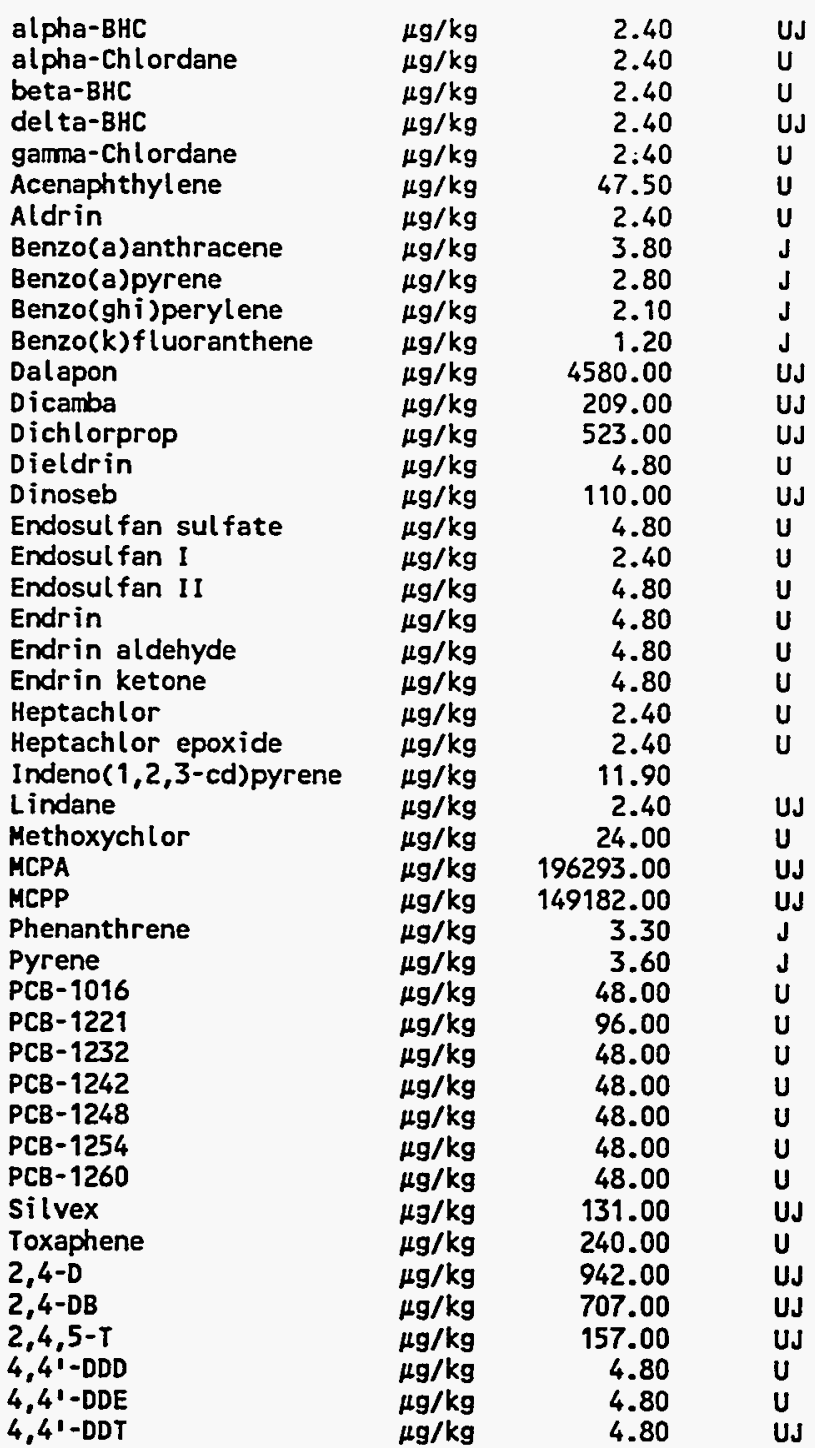

Location =AND; Formation=COPPER RIDGE; Site $=39 ; A=2277 ; A$ dup=' '; Phase $=2$

$\begin{array}{llrl}\text { alpha-BHC } & \mu \mathrm{g} / \mathrm{kg} & 2.20 & \mathrm{UJ} \\ \text { alpha-Chlordane } & \mu \mathrm{g} / \mathrm{kg} & 2.20 & \mathrm{U} \\ \text { beta-BHC } & \mu \mathrm{g} / \mathrm{kg} & 2.20 & \mathrm{U} \\ \text { delta-BHC } & \mu \mathrm{g} / \mathrm{kg} & 2.20 & \mathrm{UJ} \\ \text { gamma-Chlordane } & \mu \mathrm{g} / \mathrm{kg} & 2.20 & \mathrm{U} \\ \text { Acenaphthylene } & \mu \mathrm{g} / \mathrm{kg} & 44.90 & \mathrm{U} \\ \text { Aldrin } & \mu \mathrm{g} / \mathrm{kg} & 2.20 & \mathrm{U} \\ \text { Anthracene } & \mu \mathrm{kg} & 1.10 & \mathrm{~J} \\ \text { Benzo(a)anthracene } & \mu \mathrm{g} / \mathrm{kg} & 2.70 & \mathrm{~J} \\ \text { Benzo(a)pyrene } & \mu \mathrm{g} / \mathrm{kg} & 1.30 & \mathrm{~J} \\ \text { Benzo(b)fluoranthene } & \mu \mathrm{g} / \mathrm{kg} & 3.10 & \mathrm{~J} \\ \text { Benzo(ghi)perylene } & \mu \mathrm{g} / \mathrm{kg} & 1.80 & \mathrm{~J} \\ \text { Berizo(k)fluoranthene } & \mu \mathrm{g} / \mathrm{kg} & 1.10 & \mathrm{~J} \\ \text { Dalapon } & \mu \mathrm{g} / \mathrm{kg} & 4584.00 & \mathrm{U} \\ \text { Dicamba } & \mu \mathrm{g} / \mathrm{kg} & 209.00 & \mathrm{U}\end{array}$




\section{C-9}

Table C.1 (continued)

\begin{tabular}{|c|c|c|c|c|c|c|}
\hline Analysis & Units & $\begin{array}{c}\text { A horizon } \\
\text { Result }\end{array}$ & Qualifier & $\begin{array}{l}\text { A horizon } \\
\text { field dup }\end{array}$ & Qualifier & \\
\hline $\begin{array}{l}\text { Dichlorprop } \\
\text { Dieldrin } \\
\text { Dinoseb } \\
\text { Endosulfan sul fate } \\
\text { Endosulfan I } \\
\text { Endosulfan II } \\
\text { Endrin } \\
\text { Endrin aldehyde } \\
\text { Endrin ketone } \\
\text { Heptachlor } \\
\text { Heptachlor epoxide } \\
\text { Indeno(1,2,3-cd)pyrene } \\
\text { Lindane } \\
\text { Methoxychlor } \\
\text { MCPA } \\
\text { MCPP } \\
\text { Phenanthrene } \\
\text { Pyrene } \\
\text { PCB-1016 } \\
\text { PCB-1221 } \\
\text { PCB-1232 } \\
\text { PCB-1242 } \\
\text { PCB-1248 } \\
\text { PCB-1254 } \\
\text { PCB-1260 } \\
\text { Silvex } \\
\text { Toxaphene } \\
2,4-D \\
2,4-D B \\
2,4,5-T \\
4,41-D D D \\
4,41-D D E \\
4,4 \cdot-D D T\end{array}$ & $\begin{array}{l}\mu g / k g \\
\mu g / k g \\
\mu g / k g \\
\mu g / k g \\
\mu g / k g \\
\mu g / k g \\
\mu g / k g \\
\mu g / k g \\
\mu g / k g \\
\mu g / k g \\
\mu g / k g \\
\mu g / k g \\
\mu g / k g \\
\mu g / k g \\
\mu g / k g \\
\mu g / k g \\
\mu g / k g \\
\mu g / k g \\
\mu g / k g \\
\mu g / k g \\
\mu g / k g \\
\mu g / k g \\
\mu g / k g \\
\mu g / k g \\
\mu g / k g \\
\mu g / k g \\
\mu g / k g \\
\mu g / k g \\
\mu g / k g \\
\mu g / k g \\
\mu g / k g \\
\mu g / k g \\
\mu g / k g\end{array}$ & $\begin{array}{r}523.00 \\
4.50 \\
110.00 \\
4.50 \\
2.20 \\
4.50 \\
4.50 \\
4.50 \\
4.50 \\
2.20 \\
2.20 \\
25.60 \\
2.20 \\
22.00 \\
196293.00 \\
149182.00 \\
4.70 \\
4.70 \\
45.00 \\
90.00 \\
45.00 \\
45.00 \\
45.00 \\
45.00 \\
45.00 \\
131.00 \\
220.00 \\
942.00 \\
707.00 \\
157.00 \\
4.50 \\
4.50 \\
4.50\end{array}$ & $\begin{array}{l}U J \\
U \\
U \\
U \\
U \\
U \\
U \\
U \\
U \\
U \\
U \\
U J \\
U \\
U J \\
U J \\
U J \\
J \\
J \\
U \\
U \\
U \\
U \\
U \\
U \\
U \\
U \\
U \\
U \\
U \\
U \\
U \\
U \\
U J\end{array}$ & . & & $y \underbrace{\prime}{ }^{1}$ \\
\hline
\end{tabular}

Location=AND; Formation=COPPER RIDGE; Site=40; $A=2279 ; A$ dup=' '; Phase=2

$\begin{array}{lrrl}\text { alpha-BHC } & \mu \mathrm{g} / \mathrm{kg} & 2.20 & \mathrm{UJ} \\ \text { alpha-Chlordane } & \mu \mathrm{g} / \mathrm{kg} & 2.20 & \mathrm{U} \\ \text { beta-BHC } & \mu \mathrm{g} / \mathrm{kg} & 2.20 & \mathrm{U} \\ \text { delta-BHC } & \mu \mathrm{g} / \mathrm{kg} & 2.20 & \mathrm{UJ} \\ \text { gamma-Chlordane } & \mu \mathrm{g} / \mathrm{kg} & 2.20 & \mathrm{U} \\ \text { Acenaphthylene } & \mu \mathrm{g} / \mathrm{kg} & 42.60 & \mathrm{U} \\ \text { Aldrin } & \mu \mathrm{g} / \mathrm{kg} & 2.20 & \mathrm{U} \\ \text { Anthracene } & \mu \mathrm{g} / \mathrm{kg} & 0.90 & \mathrm{~J} \\ \text { Benzo(a)anthracene } & \mu \mathrm{g} / \mathrm{kg} & 3.00 & \mathrm{~J} \\ \text { Benzo(a)pyrene } & \mu \mathrm{g} / \mathrm{kg} & 0.60 & \mathrm{~J} \\ \text { Benzo(b)fluoranthene } & \mu \mathrm{g} / \mathrm{kg} & 2.10 & \mathrm{~J} \\ \text { Benzo(ghi)perylene } & \mu \mathrm{g} / \mathrm{kg} & 1.70 & \mathrm{~J} \\ \text { Benzo(k)fluoranthene } & \mu \mathrm{g} / \mathrm{kg} & 1.10 & \mathrm{~J} \\ \text { Dalapon } & \mu \mathrm{g} / \mathrm{kg} & 3472.00 & \mathrm{UJ} \\ \text { Dibenzo(a,h)anthracene } & \mu \mathrm{g} / \mathrm{kg} & 1.90 & \mathrm{~J} \\ \text { Dicamba } & \mu \mathrm{g} / \mathrm{kg} & 209.00 & \mathrm{U} \\ \text { Dichlorprop } & \mu \mathrm{g} / \mathrm{kg} & 523.00 & \mathrm{UJ} \\ \text { Dieldrin } & \mu \mathrm{g} / \mathrm{kg} & 4.30 & \mathrm{U} \\ \text { Dinoseb } & \mu \mathrm{g} / \mathrm{kg} & 110.00 & \mathrm{U} \\ \text { Endosulfan sulfate } & \mu \mathrm{g} / \mathrm{kg} & 4.30 & \mathrm{U} \\ \text { Endosulfan I } & \mu \mathrm{g} / \mathrm{kg} & 2.20 & \mathrm{U} \\ \text { Endosulfan II } & \mu \mathrm{g} / \mathrm{kg} & 4.30 & \mathrm{U} \\ \text { Endrin } & \mu \mathrm{g} / \mathrm{kg} & 4.30 & \mathrm{U} \\ \text { Endrin aldehyde } & \mu \mathrm{g} / \mathrm{kg} & 4.30 & \mathrm{U} \\ \text { Endrin ketone } & \mu \mathrm{g} / \mathrm{kg} & 4.30 & \mathrm{U} \\ \text { Fluoranthene } & \mu \mathrm{g} / \mathrm{kg} & 3.60 & \mathrm{~J} \\ \text { Heptachlor } & \mu \mathrm{g} / \mathrm{kg} & 2.20 & \mathrm{U} \\ \text { Heptachlor epoxide } & \mu \mathrm{g} / \mathrm{kg} & 2.20 & \mathrm{U} \\ \text { Indeno(1,2,3-cd)pyrene } & \mu \mathrm{g} / \mathrm{kg} & 8.30 & \mathrm{~J} \\ \text { Lindane } & \mu \mathrm{g} / \mathrm{kg} & 2.20 & \mathrm{UJ} \\ \text { Methoxychlor } & \mu \mathrm{g} / \mathrm{kg} & 22.00 & \mathrm{U} \\ & & & \end{array}$


C-10

Table C.1 (continued)

\begin{tabular}{|c|c|c|c|c|c|}
\hline Anolunis & Wnite & A horizon & Ouglifior & A horizon & Onolifier \\
\hline & & & & & \\
\hline MCPA & $10 \mathrm{~g} / \mathrm{kg}$ & 196293.00 & U. & & \\
\hline MCPP & $\begin{array}{l}\mu g / \mathrm{kg} \\
\mu \mathrm{g} / \mathrm{kg}\end{array}$ & 149182.00 & UJ & & \\
\hline Phenanthrene & $\mu g / \mathrm{kg}$ & 2.60 & $J$ & & \\
\hline Pyrene & $\mu \mathrm{g} / \mathrm{kg}$ & 3.40 & J & & \\
\hline PCB-1016 & $\mu \mathrm{g} / \mathrm{kg}$ & 43.00 & u & & \\
\hline PCB-1221 & $\mu \mathrm{g} / \mathrm{kg}$ & 86.00 & u & & \\
\hline PCB-1232 & $\mu \mathrm{g} / \mathrm{kg}$ & 43.00 & u & & \\
\hline PCB -1242 & $\mu \mathrm{g} / \mathrm{kg}$ & 43.00 & u & & \\
\hline PCB- 1248 & $\mu \mathrm{g} / \mathrm{kg}$ & 43.00 & $u$ & & \\
\hline PCB- 1254 & $\mu \mathrm{g} / \mathrm{kg}$ & 43.00 & u & & \\
\hline PCB-1260 & $\mu \mathrm{g} / \mathrm{kg}$ & 43.00 & u & & \\
\hline Silvex & $\mu \mathrm{g} / \mathrm{kg}$ & 131.00 & u & & \\
\hline Toxaphene & $\mu g / \mathrm{kg}$ & 220.00 & u & & \\
\hline $2,4-D$ & $\mu \mathrm{g} / \mathrm{kg}$ & 942.00 & u & & \\
\hline & $\mu \mathrm{g} / \mathrm{kg}$ & 707.00 & $u$ & & \\
\hline $2,4,5-\mathrm{T}$ & $\mu \mathrm{g} / \mathrm{kg}$ & 157.00 & $u$ & & \\
\hline $4,4-D D D$ & $\mu \mathrm{g} / \mathrm{kg}$ & 4.30 & $u$ & & \\
\hline $4,41-D D E$ & $\mu g / k g$ & 4.30 & $u$ & & \\
\hline $4,4^{1}$-DDT & $\mu \mathrm{g} / \mathrm{kg}$ & 4.30 & UJ & & \\
\hline
\end{tabular}

Location $=A N D ;$ Formation $=$ COPPER RIDGE; Site $=41 ; A=2281 ; A$ dup =' '; Phase $=2$

\begin{tabular}{|c|c|c|c|}
\hline $\begin{array}{l}\text { alpha-BHC } \\
\text { alpha-Chlordane } \\
\text { beta-BHC } \\
\text { delta-BHC } \\
\text { gamma-Chlordane } \\
\text { Acenaphthylene } \\
\text { Aldrin } \\
\text { Anthracene } \\
\text { Benzo(a)anthracene } \\
\text { Benzo(a)pyrene } \\
\text { Benzo(b)fluoranthene } \\
\text { Benzo(ghi)perylene } \\
\text { Benzo(K)fluoranthene } \\
\text { Dieldrin } \\
\text { Endosulfan sulfate } \\
\text { Endosulfan I } \\
\text { Endosulfan II } \\
\text { Endrin } \\
\text { Endrin aldehyde } \\
\text { Endrin ketone } \\
\text { Fluoranthene } \\
\text { Fluorene } \\
\text { Heptachlor } \\
\text { Heptachlor epoxide } \\
\text { Indeno(1,2,3-cd)pyrene } \\
\text { Lindane } \\
\text { Methoxychlor } \\
\text { Phenanthrene } \\
\text { Pyrene } \\
\text { PCB-1016 } \\
\text { PCB-1221 } \\
\text { PCB-1232 } \\
\text { PCB-1242 } \\
\text { PCB-1248 } \\
\text { PCB-1254 } \\
\text { PCB-1260 } \\
\text { Toxaphene } \\
4,4 \text {-DDD } \\
4,4 \text {-DDE } \\
4,4 \text {-DDT }\end{array}$ & 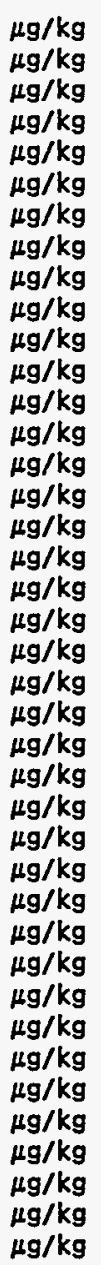 & $\begin{array}{r}2.10 \\
2.20 \\
2.10 \\
2.10 \\
2.10 \\
42.70 \\
2.10 \\
0.60 \\
1.50 \\
2.10 \\
2.80 \\
1.70 \\
1.10 \\
4.20 \\
4.20 \\
3.00 \\
4.20 \\
4.20 \\
4.20 \\
4.20 \\
3.20 \\
1.50 \\
2.10 \\
2.10 \\
19.60 \\
2.10 \\
21.00 \\
2.60 \\
3.40 \\
42.00 \\
84.00 \\
42.00 \\
42.00 \\
42.00 \\
42.00 \\
42.00 \\
210.00 \\
4.20 \\
4.20 \\
4.20\end{array}$ & $\begin{array}{l}U \\
U J \\
U \\
U \\
U \\
J \\
J \\
J \\
J \\
J \\
J \\
U \\
U \\
P \\
U \\
U \\
U \\
U \\
J \\
J \\
U \\
U\end{array}$ \\
\hline
\end{tabular}




\section{C-11}

Table C.1 (continued)

\begin{tabular}{|c|c|c|c|c|c|c|}
\hline Analysis & Units & $\begin{array}{c}\text { A horizon } \\
\text { Result }\end{array}$ & Qualifier & $\begin{array}{l}\text { A horizon } \\
\text { field dup }\end{array}$ & Qualifier & v \\
\hline \multicolumn{6}{|c|}{ Location $=A N D ;$ Formation $=$ COPPER RIDGE; Site $=42 ; A=2283 ; A$ dup=" ; Phase $=2$} & 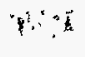 \\
\hline $\begin{array}{l}\text { alpha-BHC } \\
\text { alpha-Chlordane } \\
\text { beta-BHC } \\
\text { del ta-BHC } \\
\text { gamma-Chlordane } \\
\text { Acenaphthene } \\
\text { Acenaphthylene } \\
\text { Aldrin } \\
\text { Anthracene } \\
\text { Benzo(a)anthracene } \\
\text { Benzo(a)pyrene } \\
\text { Benzo(b)fluoranthene } \\
\text { Benzo(ghi)perylene } \\
\text { Benzo(K)fluoranthene } \\
\text { Chrysene } \\
\text { Dalapon } \\
\text { Dicamba } \\
\text { Dichlorprop } \\
\text { Dieldrin } \\
\text { Dinoseb } \\
\text { Endosulfan sul fate } \\
\text { Endosulfan I! } \\
\text { Endosulfan II } \\
\text { Endrin } \\
\text { Endrin aldehyde } \\
\text { Endrin ketone } \\
\text { Fluoranthene } \\
\text { Heptachlor } \\
\text { Heptachlor epoxide } \\
\text { Indeno(1,2,3-cd)pyrene } \\
\text { Lindane } \\
\text { Methoxychlor } \\
\text { MCPA } \\
\text { MCPP } \\
\text { Phenanthrene } \\
\text { Pyrene } \\
\text { PCB-1016 } \\
\text { PCB-1221 } \\
\text { PCB-1232 } \\
\text { PCB-1242 } \\
\text { PCB-1248 } \\
\text { PCB-1254 } \\
\text { PCB-1260 } \\
\text { Silvex } \\
\text { Toxaphene } \\
2,4-D \\
2,4-D B \\
2,4,5-T \\
4,41-D D D \\
4,41-D D E \\
4,41-D D T\end{array}$ & 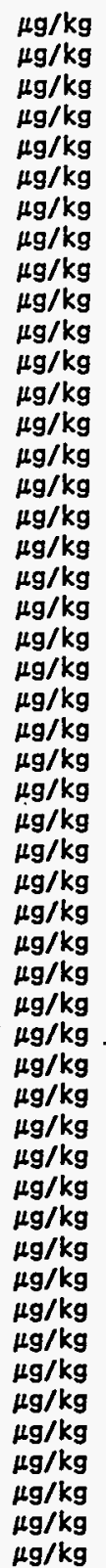 & $\begin{array}{r}2.20 \\
2.20 \\
2.20 \\
2.20 \\
2.20 \\
1.30 \\
43.10 \\
2.20 \\
0.90 \\
2.60 \\
2.20 \\
2.40 \\
2.40 \\
1.30 \\
4.30 \\
4480.00 \\
205.00 \\
512.00 \\
4.30 \\
108.00 \\
4.30 \\
2.20 \\
4.30 \\
4.30 \\
4.30 \\
4.30 \\
1.50 \\
2.20 \\
2.20 \\
17.20 \\
2.20 \\
22.00 \\
192002.00 \\
145921.00 \\
12.70 \\
1.30 \\
43.00 \\
86.00 \\
43.00 \\
43.00 \\
43.00 \\
43.00 \\
43.00 \\
128.00 \\
220.00 \\
922.00 \\
691.00 \\
154.00 \\
4.30 \\
4.30 \\
4.30\end{array}$ & $\begin{array}{l}U J \\
U \\
U J \\
U \\
J \\
U \\
U \\
J \\
J \\
J \\
J \\
J \\
J \\
J \\
U J \\
U \\
U J \\
U \\
U \\
U \\
U \\
U \\
U \\
U \\
U \\
J \\
U \\
U \\
U \\
U \\
U \\
U J \\
U J \\
J \\
J \\
U \\
U \\
U \\
U \\
U \\
U \\
U \\
U \\
U \\
U \\
U\end{array}$ & & . & \\
\hline
\end{tabular}

Location $=A N D ;$ Formation $=D I S M A L$ GAP; Site $=1 ; A=2157 ; A$ dup=' '; Phase $=1$

$\begin{array}{llrl}\text { alpha-BHC } & \mu \mathrm{g} / \mathrm{kg} & 2.50 & \mathrm{U} \\ \text { alpha-Chlordane } & \mu \mathrm{g} / \mathrm{kg} & 2.50 & \mathrm{U} \\ \text { beta-BHC } & \mu \mathrm{g} / \mathrm{kg} & 2.50 & \mathrm{U} \\ \text { del ta-BHC } & \mu \mathrm{g} / \mathrm{kg} & 2.50 & \mathrm{U} \\ \text { gamma-Chlordane } & \mu \mathrm{g} / \mathrm{kg} & 2.50 & \mathrm{U} \\ \text { Acenaphthene } & \mu \mathrm{gg} & 17.00 & \mathrm{JN} \\ \text { Acenaphthylene } & \mu \mathrm{k} / \mathrm{kg} & 36.00 & \mathrm{UJ} \\ \text { Aldrin } & \mu \mathrm{g} / \mathrm{kg} & 2.50 & \mathrm{UJ} \\ \text { Anthracene } & \mu \mathrm{gg} & 1.00 & \mathrm{UJ} \\ \text { Benzo(a)anthracene } & \mu \mathrm{g} / \mathrm{kg} & 3.80 & \mathrm{JN}\end{array}$




\section{C-12}

Table C.1 (continued)

\begin{tabular}{|c|c|c|c|c|c|}
\hline Analysis & Units & $\begin{array}{c}\text { A horizon } \\
\text { Result }\end{array}$ & Qualifier & $\begin{array}{l}\text { A horizon } \\
\text { field dup }\end{array}$ & Qualifier \\
\hline $\begin{array}{l}\text { Benzo(a)pyrene } \\
\text { Benzo(b)fluoranthene } \\
\text { Benzo(K) fluoranthene } \\
\text { Chrysene } \\
\text { Dieldrin } \\
\text { Endosulfan sulfate } \\
\text { Endosulfan ! } \\
\text { Endosul fan II } \\
\text { Endrin } \\
\text { Endrin aldehyde } \\
\text { Endrin ketone } \\
\text { Fluoranthene } \\
\text { Fluorene } \\
\text { Heptachlor } \\
\text { Heptachlor epoxide } \\
\text { Indeno(1,2,3-cd)pyrene } \\
\text { Lindane } \\
\text { Methoxychlor } \\
\text { Naphthalene } \\
\text { Phenanthrene } \\
\text { Pyrene } \\
\text { PCB-1016 } \\
\text { PCB-1221 } \\
\text { PCB-1232 } \\
\text { PCB-1242 } \\
\text { PCB-1248 } \\
\text { PCB-1254 } \\
\text { PCB-1260 } \\
\text { Toxaphene } \\
4,41-D D D \\
4,41-D D E \\
4,41-D D T\end{array}$ & $\begin{array}{l}\mu g / \mathrm{kg} \\
\mu g / \mathrm{kg} \\
\mu g / \mathrm{kg} \\
\mu g / \mathrm{kg} \\
\mu g / \mathrm{kg} \\
\mu g / \mathrm{kg} \\
\mu g / \mathrm{kg} \\
\mu g / \mathrm{kg} \\
\mu g / \mathrm{kg} \\
\mu g / \mathrm{kg} \\
\mu g / \mathrm{kg} \\
\mu g / \mathrm{kg} \\
\mu g / \mathrm{kg} \\
\mu g / \mathrm{kg} \\
\mu g / \mathrm{kg} \\
\mu g / \mathrm{kg} \\
\mu g / \mathrm{kg} \\
\mu g / \mathrm{kg} \\
\mu g / \mathrm{kg} \\
\mu g / \mathrm{kg} \\
\mu g / \mathrm{kg} \\
\mu g / \mathrm{kg} \\
\mu g / \mathrm{kg} \\
\mu g / \mathrm{kg} \\
\mu g / \mathrm{kg} \\
\mu g / \mathrm{kg} \\
\mu g / \mathrm{kg} \\
\mu g / \mathrm{kg} \\
\mu g / \mathrm{kg} \\
\mu g / \mathrm{kg} \\
\mu g / \mathrm{kg} \\
\mu g / \mathrm{kg}\end{array}$ & $\begin{array}{r}5.90 \\
3.00 \\
3.00 \\
22.00 \\
4.90 \\
4.90 \\
2.50 \\
4.90 \\
4.90 \\
4.90 \\
4.90 \\
23.00 \\
6.00 \\
2.50 \\
2.50 \\
6.00 \\
2.50 \\
25.40 \\
15.00 \\
15.00 \\
1.00 \\
49.40 \\
100.30 \\
49.40 \\
49.40 \\
49.40 \\
49.40 \\
49.40 \\
254.50 \\
4.90 \\
4.90 \\
4.90\end{array}$ & $\begin{array}{l}J N \\
U J \\
U N \\
U J \\
U J \\
U \\
U \\
U \\
U \\
U \\
U \\
J \\
U J \\
U J \\
U \\
U J \\
U J \\
U \\
U J \\
J \\
U J \\
U \\
U \\
U \\
U \\
U \\
U \\
U \\
U \\
U \\
U \\
U J\end{array}$ & & \\
\hline
\end{tabular}

Location $=A N D ;$ Formation $=D I S M A L$ GAP; Site $=10 ; A=2143 ; A$ dup $=$ ' '; Phase $=1$

\begin{tabular}{|c|c|c|}
\hline $\begin{array}{l}\text { alpha-BHC } \\
\text { alpha-Chlordane } \\
\text { beta-BHC } \\
\text { delta-BHC } \\
\text { gamma-Chlordane } \\
\text { Aldrin } \\
\text { Benzo(a)anthracene } \\
\text { Benzo(a)pyrene } \\
\text { Dieldrin } \\
\text { Endosulfan sulfate } \\
\text { Endosulfan I } \\
\text { Endosul fan II } \\
\text { Endrin } \\
\text { Endrin aldehyde } \\
\text { Endrin ketone } \\
\text { Heptachlor } \\
\text { Heptachlor epoxide } \\
\text { Lindane } \\
\text { Methoxychlor } \\
\text { PCB-1016 } \\
\text { PCB-1221 } \\
\text { PCB-1232 } \\
\text { PCB-1242 } \\
\text { PCB-1248 } \\
\text { PCB-1254 } \\
\text { PCB-1260 } \\
\text { Toxaphene } \\
4,41-D D D \\
4,4 '-D D E \\
4,41-D D T\end{array}$ & $\begin{array}{l}\mu g / k g \\
\mu g / k g \\
\mu g / k g \\
\mu g / k g \\
\mu g / k g \\
\mu g / k g \\
\mu g / k g \\
\mu g / k g \\
\mu g / k g \\
\mu g / k g \\
\mu g / k g \\
\mu g / k g \\
\mu g / k g \\
\mu g / k g \\
\mu g / k g \\
\mu g / k g \\
\mu g / k g \\
\mu g / k g \\
\mu g / k g \\
\mu g / k g \\
\mu g / k g \\
\mu g / k g \\
\mu g / k g \\
\mu g / k g \\
\mu g / k g \\
\mu g / k g \\
\mu g / k g \\
\mu g / k g \\
\mu g / k g \\
\mu g / k g\end{array}$ & $\begin{array}{r}2.50 \\
2.50 \\
2.50 \\
2.50 \\
2.50 \\
2.50 \\
3.80 \\
5.40 \\
4.80 \\
4.80 \\
2.50 \\
4.80 \\
4.80 \\
4.80 \\
4.80 \\
2.50 \\
2.50 \\
2.50 \\
24.90 \\
48.30 \\
98.00 \\
48.30 \\
48.30 \\
48.30 \\
48.30 \\
48.30 \\
248.80 \\
4.80 \\
4.80 \\
4.80\end{array}$ \\
\hline
\end{tabular}




\section{C-13}

Table C.1 (continucd)

\begin{tabular}{|c|c|c|c|c|c|c|}
\hline Analysis & Units & $\begin{array}{c}\text { A horizon } \\
\text { Result }\end{array}$ & Qualifier & $\begin{array}{l}\text { A horizon } \\
\text { field dup }\end{array}$ & Qualifier & \\
\hline \multicolumn{6}{|c|}{ Location $=A N D ;$ Formation $=$ DISMAL GAP; Sité $=11 ; A=2112 ; A$ dup=' '; Phase $=1$} & *is siti \\
\hline $\begin{array}{l}\text { alpha-8HC } \\
\text { alpha-Chlordane } \\
\text { beta-BHC } \\
\text { del ta-BHC } \\
\text { gamma-Chlordane } \\
\text { Acenaphthene } \\
\text { Acenaphthylene } \\
\text { Aldrin } \\
\text { Anthracene } \\
\text { Benzo(a)anthracene } \\
\text { Benzo(a)pyrene } \\
\text { Benzo(b)fluoranthene } \\
\text { Benzo(k)fluoranthene } \\
\text { Chrysene } \\
\text { Dieldrin } \\
\text { Endosul fan sulfate } \\
\text { Endosulfan I } \\
\text { Endosul fan II } \\
\text { Endrin } \\
\text { Endrin aldehyde } \\
\text { Endrin ketone } \\
\text { Fluoranthene } \\
\text { Fluorene } \\
\text { Heptachlor } \\
\text { Heptachlor epoxide } \\
\text { Indeno(1,2,3-cd)pyrene } \\
\text { Lindane } \\
\text { Methoxychlor } \\
\text { Naphthalene } \\
\text { Phenanthrene } \\
\text { Pyrene } \\
\text { PCB-1016 } \\
\text { PCB-1221 } \\
\text { PCB-1232 } \\
\text { PCB-1242 } \\
\text { PCB-1248 } \\
\text { PCB-1254 } \\
\text { PCB-1260 } \\
\text { Toxaphene } \\
4,44^{\prime}-D D D \\
4,4-D D E \\
4,4-D D T\end{array}$ & $\begin{array}{l}\mu \mathrm{g} / \mathrm{kg} \\
\mu \mathrm{g} / \mathrm{kg} \\
\mu \mathrm{g} / \mathrm{kg} \\
\mu \mathrm{g} / \mathrm{kg} \\
\mu \mathrm{g} / \mathrm{kg} \\
\mu \mathrm{g} / \mathrm{kg} \\
\mu \mathrm{g} / \mathrm{kg} \\
\mu \mathrm{g} / \mathrm{kg} \\
\mu \mathrm{g} / \mathrm{kg} \\
\mu \mathrm{g} / \mathrm{kg} \\
\mu \mathrm{g} / \mathrm{kg} \\
\mu \mathrm{g} / \mathrm{kg} \\
\mu \mathrm{g} / \mathrm{kg} \\
\mu \mathrm{g} / \mathrm{kg} \\
\mu \mathrm{g} / \mathrm{kg} \\
\mu \mathrm{g} / \mathrm{kg} \\
\mu \mathrm{g} / \mathrm{kg} \\
\mu \mathrm{g} / \mathrm{kg} \\
\mu \mathrm{g} / \mathrm{kg} \\
\mu \mathrm{g} / \mathrm{kg} \\
\mu \mathrm{g} / \mathrm{kg} \\
\mu \mathrm{g} / \mathrm{kg} \\
\mu \mathrm{g} / \mathrm{kg} \\
\mu \mathrm{g} / \mathrm{kg} \\
\mu \mathrm{g} / \mathrm{kg} \\
\mu \mathrm{g} / \mathrm{kg} \\
\mu \mathrm{g} / \mathrm{kg} \\
\mu \mathrm{g} / \mathrm{kg} \\
\mu \mathrm{g} / \mathrm{kg} \\
\mu \mathrm{g} / \mathrm{kg} \\
\mu \mathrm{g} / \mathrm{kg} \\
\mu \mathrm{g} / \mathrm{kg} \\
\mu \mathrm{g} / \mathrm{kg} \\
\mu \mathrm{g} / \mathrm{kg} \\
\mu \mathrm{g} / \mathrm{kg} \\
\mu \mathrm{g} / \mathrm{kg} \\
\mu \mathrm{g} / \mathrm{kg} \\
\mu \mathrm{g} / \mathrm{kg} \\
\mu \mathrm{g} / \mathrm{kg} \\
\mu \mathrm{g} / \mathrm{kg} \\
\mu \mathrm{g} / \mathrm{kg} \\
\mu \mathrm{g} / \mathrm{kg}\end{array}$ & $\begin{array}{r}2.60 \\
2.60 \\
2.60 \\
2.60 \\
2.60 \\
2.00 \\
200.00 \\
2.60 \\
2.00 \\
1.70 \\
3.00 \\
3.00 \\
3.00 \\
23.00 \\
5.00 \\
5.00 \\
2.60 \\
5.00 \\
5.00 \\
5.00 \\
5.00 \\
8.00 \\
6.00 \\
2.60 \\
2.60 \\
6.00 \\
2.60 \\
25.80 \\
15.00 \\
13.00 \\
2.00 \\
50.00 \\
101.60 \\
50.00 \\
50.00 \\
50.00 \\
50.00 \\
50.00 \\
257.80 \\
5.00 \\
5.00 \\
5.00\end{array}$ & $\begin{array}{l}U \\
U \\
U \\
U \\
U \\
U J \\
J \\
U J \\
U J \\
J N \\
U J \\
U J \\
U J \\
U J \\
U J \\
U \\
U \\
U \\
U \\
U \\
U \\
U J \\
U J \\
U J \\
U \\
U J \\
U J \\
U \\
U J \\
J N \\
U J \\
U \\
U \\
U \\
U \\
U \\
U \\
U \\
U \\
U \\
U \\
U J \\
U\end{array}$ & - & & \\
\hline
\end{tabular}

Location=AND; Formation=DISMAL GAP; Site=12; $A=2080 ; A$ dup =' '; Phase $=1$

\begin{tabular}{|c|c|c|}
\hline $\begin{array}{l}\text { alpha-BHC } \\
\text { alpha-Chlordane } \\
\text { beta-BHC } \\
\text { del ta-BHC } \\
\text { gamma-Chlordane } \\
\text { Acenaphthene } \\
\text { Acenaphthylene } \\
\text { Aldrin } \\
\text { Anthracene } \\
\text { Benzo(a)anthracene } \\
\text { Benzo(a)pyrene } \\
\text { Benzo(b)f fuoranthene } \\
\text { Benzo(k) fluoranthene } \\
\text { Chrysene } \\
\text { Dieldrin } \\
\text { Endosul fan sul fate } \\
\text { Endosul fan I } \\
\text { Endosul fan II } \\
\text { Endrin }\end{array}$ & $\begin{array}{l}\mu \mathrm{g} / \mathrm{kg} \\
\mu \mathrm{g} / \mathrm{kg} \\
\mu \mathrm{g} / \mathrm{kg} \\
\mu \mathrm{g} / \mathrm{kg} \\
\mu \mathrm{g} / \mathrm{kg} \\
\mu \mathrm{g} / \mathrm{kg} \\
\mu \mathrm{g} / \mathrm{kg} \\
\mu \mathrm{g} / \mathrm{kg} \\
\mu \mathrm{g} / \mathrm{kg} \\
\mu \mathrm{g} / \mathrm{kg} \\
\mu \mathrm{g} / \mathrm{kg} \\
\mu \mathrm{g} / \mathrm{kg} \\
\mu \mathrm{g} / \mathrm{kg} \\
\mu \mathrm{g} / \mathrm{kg} \\
\mu \mathrm{g} / \mathrm{kg} \\
\mu \mathrm{g} / \mathrm{kg} \\
\mu \mathrm{g} / \mathrm{kg} \\
\mu \mathrm{g} / \mathrm{kg} \\
\mu \mathrm{g} / \mathrm{kg}\end{array}$ & $\begin{array}{r}2.30 \\
2.30 \\
2.30 \\
2.30 \\
2.30 \\
3.20 \\
34.00 \\
2.30 \\
1.00 \\
2.10 \\
2.70 \\
3.00 \\
3.00 \\
21.00 \\
4.50 \\
4.50 \\
2.30 \\
4.50 \\
4.50\end{array}$ \\
\hline
\end{tabular}




\section{C-14}

Table C.1 (continued)

\begin{tabular}{|c|c|c|c|c|c|}
\hline Analysis & Units & $\begin{array}{c}\text { A horizon } \\
\text { Result }\end{array}$ & Qualifier & $\begin{array}{l}\text { A horizon } \\
\text { field dup }\end{array}$ & Qualifier \\
\hline $\begin{array}{l}\text { Endrin aldehyde } \\
\text { Endrin ketone } \\
\text { Fluoranthene } \\
\text { Fluorene } \\
\text { Heptachlor } \\
\text { Heptachlor epoxide } \\
\text { Indeno(1,2,3-cd)pyrene } \\
\text { Lindane } \\
\text { Methoxychlor } \\
\text { Naphthal ene } \\
\text { Phenanthrene } \\
\text { Pyrene } \\
\text { PCB-1016 } \\
\text { PCB-1221 } \\
\text { PCB-1232 } \\
\text { PCB-1242 } \\
\text { PCB-1248 } \\
\text { PCB-1254 } \\
\text { PCB-1260 } \\
\text { TOXaphene } \\
4,41-D D D \\
4,41-D D E \\
4,41-D D T\end{array}$ & $\begin{array}{l}\mu g / \mathrm{kg} \\
\mu g / \mathrm{kg} \\
\mu g / \mathrm{kg} \\
\mu g / \mathrm{kg} \\
\mu g / \mathrm{kg} \\
\mu g / \mathrm{kg} \\
\mu g / \mathrm{kg} \\
\mu g / \mathrm{kg} \\
\mu g / \mathrm{kg} \\
\mu \mathrm{g} / \mathrm{kg} \\
\mu \mathrm{g} / \mathrm{kg} \\
\mu \mathrm{g} / \mathrm{kg} \\
\mu g / \mathrm{kg} \\
\mu g / \mathrm{kg} \\
\mu \mathrm{g} / \mathrm{kg} \\
\mu \mathrm{g} / \mathrm{kg} \\
\mu \mathrm{g} / \mathrm{kg} \\
\mu \mathrm{g} / \mathrm{kg} \\
\mu \mathrm{g} / \mathrm{kg} \\
\mu \mathrm{g} / \mathrm{kg} \\
\mu \mathrm{g} / \mathrm{kg} \\
\mu \mathrm{g} / \mathrm{kg} \\
\mu \mathrm{g} / \mathrm{kg}\end{array}$ & $\begin{array}{r}4.50 \\
4.50 \\
7.00 \\
5.00 \\
2.30 \\
2.30 \\
5.00 \\
2.30 \\
23.30 \\
14.00 \\
35.00 \\
1.00 \\
45.30 \\
92.00 \\
45.30 \\
158.70 \\
45.30 \\
45.30 \\
45.30 \\
233.50 \\
4.50 \\
4.50 \\
4.50\end{array}$ & $\begin{array}{l}U \\
U \\
U J \\
U J \\
U J \\
U \\
U J \\
U J \\
U \\
U J \\
J \\
U J \\
U \\
U \\
U \\
U \\
U \\
U \\
U \\
U \\
U \\
U J\end{array}$ & & \\
\hline
\end{tabular}

Location=AND; Formation=DISMAL GAP; Site $=19 ; A=2116 ; A$ dup $=2120 ;$ Phase $=1$

\begin{tabular}{|c|c|c|c|c|c|}
\hline $\begin{array}{l}\text { alpha-BHC } \\
\text { alpha-Chlordane } \\
\text { beta-BHC } \\
\text { del ta-BHC } \\
\text { gamma-Chlordane } \\
\text { Acenaphthene } \\
\text { Acenaphthylene } \\
\text { Aldrin } \\
\text { Anthracene } \\
\text { Benzo(a)anthracene } \\
\text { Benzo(a)pyrene } \\
\text { Benzo(b)fluoranthene } \\
\text { Benzo(k)fluoranthene } \\
\text { Chrysene } \\
\text { Dieldrin } \\
\text { Endosulfan sulfate } \\
\text { Endosulfan I } \\
\text { Endosulfan II } \\
\text { Endrin } \\
\text { Endrin aldehyde } \\
\text { Endrin ketone } \\
\text { Fluoranthene } \\
\text { Fluorene } \\
\text { Heptachlor } \\
\text { Heptachlor epoxide } \\
\text { Indeno(1,2,3-cd)pyrene } \\
\text { Lindane } \\
\text { Methoxychlor } \\
\text { Naphthalene } \\
\text { Phenanthrene } \\
\text { Pyrene } \\
\text { PCB-1016 } \\
\text { PCB-1221 } \\
\text { PCB-1232 } \\
\text { PCB-1242 } \\
\text { PCB-1248 } \\
\text { PCB-1254 } \\
\text { PCB-1260 } \\
\text { Toxaphene } \\
4,41-D D D\end{array}$ & 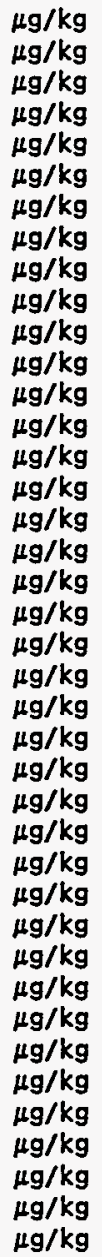 & $\begin{array}{r}2.10 \\
2.10 \\
2.10 \\
2.10 \\
2.10 \\
4.60 \\
13.00 \\
2.10 \\
1.00 \\
2.70 \\
3.70 \\
2.00 \\
2.00 \\
19.00 \\
4.10 \\
4.10 \\
2.10 \\
4.10 \\
4.10 \\
4.10 \\
4.10 \\
6.00 \\
21.00 \\
2.10 \\
2.10 \\
5.00 \\
2.10 \\
21.10 \\
12.00 \\
23.00 \\
1.00 \\
41.00 \\
83.30 \\
41.00 \\
41.00 \\
41.00 \\
41.00 \\
41.00 \\
211.40 \\
4.10\end{array}$ & $\begin{array}{l}U \\
U \\
U \\
U \\
U \\
J \\
J \\
U J \\
U J \\
J J \\
J U \\
U J \\
U J \\
U J \\
U J \\
U \\
U \\
U \\
U \\
U \\
U \\
U \\
U J \\
J J \\
U J \\
U \\
U J \\
U J \\
U J \\
U J H\end{array}$ & $\begin{array}{r}2.1 \\
2.1 \\
2.1 \\
2.1 \\
2.1 \\
3.1 \\
32.0 \\
2.1 \\
1.0 \\
2.2 \\
2.9 \\
3.0 \\
3.0 \\
19.0 \\
4.1 \\
4.1 \\
2.1 \\
4.1 \\
4.1 \\
4.1 \\
4.1 \\
6.0 \\
24.0 \\
2.1 \\
2.1 \\
5.0 \\
2.1 \\
21.2 \\
13.0 \\
20.0 \\
1.0 \\
41.1 \\
83.5 \\
41.1 \\
41.1 \\
41.1 \\
41.1 \\
41.1 \\
211.8 \\
4.1\end{array}$ & ' \\
\hline
\end{tabular}




\section{C-15}

Table C.1 (continued)

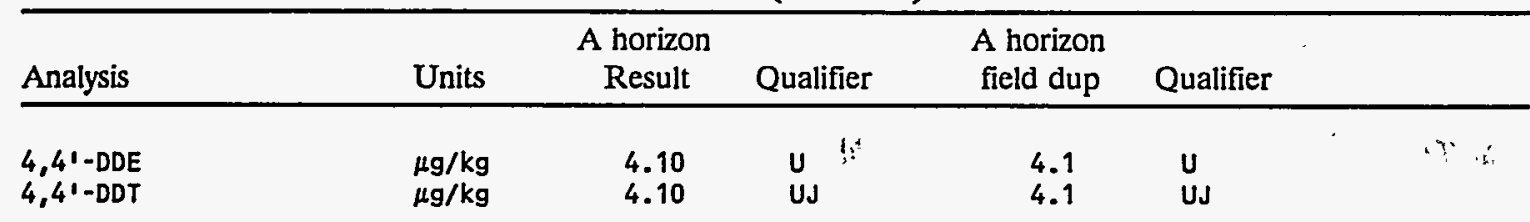

Location $=A N D ;$ Formation $=D I S M A L$ GAP; Site $=20 ; A=2070 ; A$ dup=' '; Phase $=1$

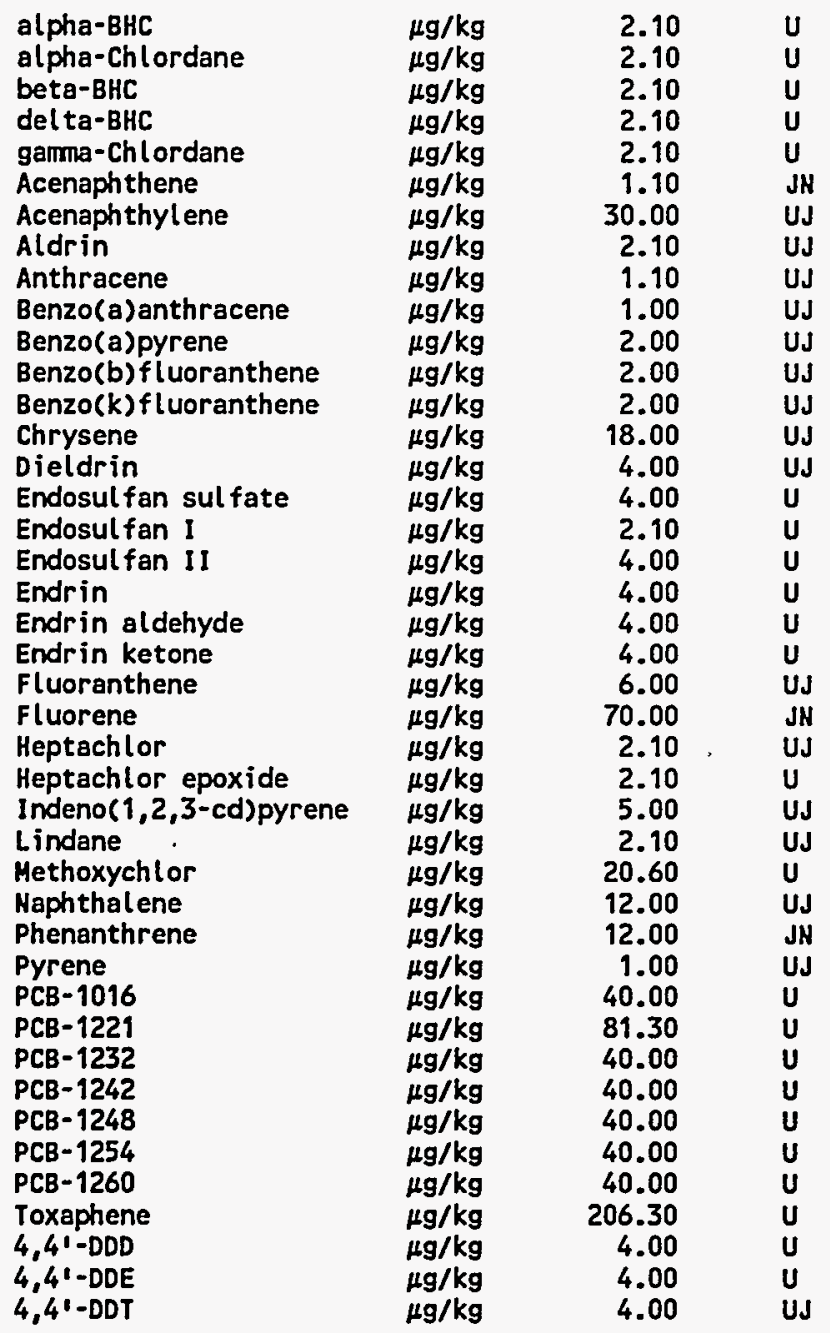

Location=AND; Formation=DISMAL GAP; Site=21; $A=2101 ; A$ dup=' '; Phase=1

\begin{tabular}{|c|c|c|}
\hline $\begin{array}{l}\text { alpha-BHC } \\
\text { alpha-Chlordane } \\
\text { beta-BHC } \\
\text { del ta-BHC } \\
\text { gama-Chlordane } \\
\text { Acenaphthene } \\
\text { Acenaphthylene } \\
\text { Aldrin } \\
\text { Anthracene } \\
\text { Benzo(a)anthracene } \\
\text { Benzo(a)pyrene } \\
\text { Benzo(b)fluoranthene } \\
\text { Benzo(k)fluoranthene } \\
\text { Chrysene } \\
\text { Dieldrin }\end{array}$ & $\begin{array}{l}\mu g / k g \\
\mu g / k g \\
\mu g / k g \\
\mu g / k g \\
\mu g / k g \\
\mu g / k g \\
\mu g / k g \\
\mu g / k g \\
\mu g / k g \\
\mu g / k g \\
\mu g / k g \\
\mu g / k g \\
\mu g / k g \\
\mu g / k g \\
\mu g / k g\end{array}$ & $\begin{array}{r}2.30 \\
2.30 \\
2.30 \\
2.30 \\
2.30 \\
5.80 \\
33.00 \\
2.30 \\
1.00 \\
1.90 \\
3.00 \\
3.00 \\
3.00 \\
20.00 \\
4.40\end{array}$ \\
\hline
\end{tabular}




\section{C-16}

Table C.1 (continued)

\begin{tabular}{|c|c|c|c|c|c|}
\hline Analysis & Units & $\begin{array}{c}\text { A horizon } \\
\text { Result }\end{array}$ & Qualifier & $\begin{array}{l}\text { A horizon } \\
\text { field dup }\end{array}$ & Qualifier \\
\hline $\begin{array}{l}\text { Endosulfan sulfate } \\
\text { Endosulfan I } \\
\text { Endosulfan II } \\
\text { Endrin } \\
\text { Endrin aldehyde } \\
\text { Endrin ketone } \\
\text { Fluoranthene } \\
\text { Fluorene } \\
\text { Heptachlor } \\
\text { Heptachlor epoxide } \\
\text { Indeno(1,2,3-cd)pyrene } \\
\text { Lindane } \\
\text { Methoxychlor } \\
\text { Haphthalene } \\
\text { Phenanthrene } \\
\text { Pyrene } \\
\text { PCB-1016 } \\
\text { PCB-1221 } \\
\text { PCB-1232 } \\
\text { PCB-1242 } \\
\text { PCB-1248 } \\
\text { PCB-1254 } \\
\text { PCB-1260 } \\
\text { Toxaphene } \\
4,4 '-D D D \\
4,41-D D E \\
4,41-D D T\end{array}$ & 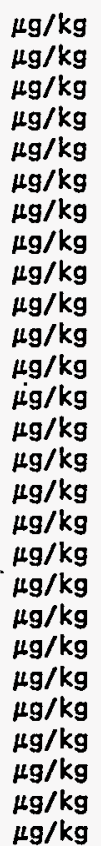 & $\begin{array}{r}4.40 \\
2.30 \\
4.40 \\
4.40 \\
4.40 \\
4.40 \\
7.00 \\
9.80 \\
2.30 \\
2.30 \\
5.00 \\
2.30 \\
22.90 \\
13.00 \\
1.00 \\
1.00 \\
44.50 \\
90.30 \\
44.50 \\
44.50 \\
44.50 \\
44.50 \\
44.50 \\
229.00 \\
4.40 \\
4.40 \\
4.40\end{array}$ & $\begin{array}{l}U \\
U \\
U \\
U \\
U \\
U \\
U J \\
J N \\
U J \\
U \\
U J \\
U J \\
U \\
U J \\
U J \\
U J \\
U \\
U \\
U \\
U \\
U \\
U \\
U \\
U \\
U \\
U \\
U J\end{array}$ & & \\
\hline
\end{tabular}

Location $=A N D$; Formation $=D I S M A L$ GAP; Site $=22 ; A=2090 ; A$ dup =' '; Phase $=1$

\begin{tabular}{|c|c|c|}
\hline $\begin{array}{l}\text { alpha-BHC } \\
\text { alpha-Chlordane } \\
\text { beta-BHC } \\
\text { delta-BHC } \\
\text { gamma-Chlordane } \\
\text { Acenaphthene } \\
\text { Aldrin } \\
\text { Benzo(a)pyrene } \\
\text { Benzo(ghi)perylene } \\
\text { Dieldrin } \\
\text { Endosulfan sulfate } \\
\text { Endosulfan I } \\
\text { Endosulfan II } \\
\text { Endrin } \\
\text { Endrin aldehyde } \\
\text { Endrin ketone } \\
\text { Heptachlor } \\
\text { Heptachlor epoxide } \\
\text { Lindane } \\
\text { Methoxychlor } \\
\text { Phenanthrene } \\
\text { Pyrene } \\
\text { PCB-1016 } \\
\text { PCB-1221 } \\
\text { PCB-1232 } \\
\text { PCB-1242 } \\
\text { PCB-1248 } \\
\text { PCB-1254 } \\
\text { PCB-1260 } \\
\text { Toxaphene } \\
4,41-D D D \\
4,41-D D E \\
4,41-D D T\end{array}$ & $\begin{array}{l}\mu g / \mathrm{kg} \\
\mu g / \mathrm{kg} \\
\mu g / \mathrm{kg} \\
\mu g / \mathrm{kg} \\
\mu g / \mathrm{kg} \\
\mu g / \mathrm{kg} \\
\mu g / \mathrm{kg} \\
\mu g / \mathrm{kg} \\
\mu g / \mathrm{kg} \\
\mu g / \mathrm{kg} \\
\mu g / \mathrm{kg} \\
\mu g / \mathrm{kg} \\
\mu g / \mathrm{kg} \\
\mu g / \mathrm{kg} \\
\mu g / \mathrm{kg} \\
\mu g / \mathrm{kg} \\
\mu g / \mathrm{kg} \\
\mu g / \mathrm{kg} \\
\mu g / \mathrm{kg} \\
\mu g / \mathrm{kg} \\
\mu g / \mathrm{kg} \\
\mu g / \mathrm{kg} \\
\mu g / \mathrm{kg} \\
\mu g / \mathrm{kg} \\
\mu g / \mathrm{kg} \\
\mu g / \mathrm{kg} \\
\mu g / \mathrm{kg} \\
\mu g / \mathrm{kg} \\
\mu g / \mathrm{kg} \\
\mu g / \mathrm{kg} \\
\mu g / \mathrm{kg} \\
\mu g / \mathrm{kg} \\
\mu g / \mathrm{kg}\end{array}$ & $\begin{array}{r}2.30 \\
2.30 \\
2.30 \\
2.30 \\
2.30 \\
19.00 \\
2.30 \\
2.10 \\
3.00 \\
4.50 \\
4.50 \\
2.30 \\
4.50 \\
4.50 \\
4.50 \\
4.50 \\
2.30 \\
2.30 \\
2.30 \\
23.10 \\
17.00 \\
0.84 \\
44.90 \\
91.20 \\
44.90 \\
44.90 \\
44.90 \\
44.90 \\
44.90 \\
231.30 \\
4.50 \\
4.50 \\
4.50\end{array}$ \\
\hline
\end{tabular}




\section{C-17}

Table C.1 (continued)

\begin{tabular}{|c|c|c|c|c|c|c|}
\hline Analysis & Units & $\begin{array}{c}\text { A horizon } \\
\text { Result }\end{array}$ & Qualifier & $\begin{array}{l}\text { A horizon } \\
\text { field dup }\end{array}$ & Qualifier & \\
\hline \multicolumn{7}{|c|}{ Location $=A N D ;$ Formation $=D I S M A L G A P ;$ Site $=3 ; A=2059 ; A$ dup $=$ ' '; Phase $=1$} \\
\hline $\begin{array}{l}\text { alpha-BHC } \\
\text { alpha-Chlordane } \\
\text { beta-BHC } \\
\text { delta-BHC } \\
\text { gamma-Chlordane } \\
\text { Aldrin } \\
\text { Benzo(a)anthracene } \\
\text { Benzo(a)pyrene } \\
\text { Dieldrin } \\
\text { Endosul fan sulfate } \\
\text { Endosulfan I } \\
\text { Endosul fan II } \\
\text { Endrin } \\
\text { Endrin aldehyde } \\
\text { Endrin ketone } \\
\text { Heptachlor } \\
\text { Heptachlor epoxide } \\
\text { Lindane } \\
\text { Methoxychlor } \\
\text { Phenanthrene } \\
\text { PCB-1016 } \\
\text { PCB-1221 } \\
\text { PCB-1232 } \\
\text { PCB-1242 } \\
\text { PCB-1248 } \\
\text { PCB-1254 } \\
\text { PCB-1260 } \\
\text { TOXaphene } \\
4,4^{\prime}-D D D \\
4,4^{\prime}-D D E \\
4,4^{\prime}-D D T\end{array}$ & 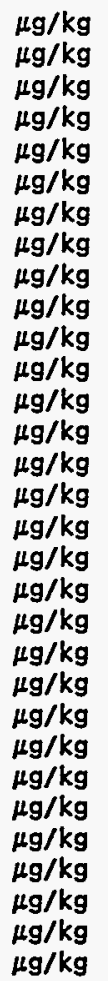 & $\begin{array}{r}2.00 \\
2.00 \\
2.00 \\
2.00 \\
2.00 \\
2.00 \\
1.70 \\
2.10 \\
3.90 \\
3.90 \\
2.00 \\
3.90 \\
3.90 \\
3.90 \\
3.90 \\
2.00 \\
2.00 \\
2.00 \\
20.30 \\
30.00 \\
39.50 \\
80.10 \\
39.50 \\
39.50 \\
39.50 \\
39.50 \\
39.50 \\
203.30 \\
3.90 \\
3.90 \\
3.90\end{array}$ & $\begin{array}{l}U \\
U \\
U \\
U \\
U \\
U J \\
J \\
J \\
U J \\
U \\
U \\
U \\
U \\
U \\
U \\
U J \\
U \\
U \\
U \\
U \\
J \\
U \\
U \\
U \\
U \\
U \\
U \\
U \\
U \\
U \\
U \\
U \\
U\end{array}$ & & & \\
\hline
\end{tabular}

Location=AND; Formation=DISMAL GAP; Site=4; $A=2039 ; A$ dup=' '; Phase =1

\begin{tabular}{|c|c|c|}
\hline $\begin{array}{l}\text { alpha-BHC } \\
\text { alpha-Chlordane } \\
\text { beta-BHC } \\
\text { del ta-BHC } \\
\text { gamma-Chlordane } \\
\text { Acenaphthene } \\
\text { Acenaphthylene } \\
\text { Aldrin } \\
\text { Anthracene } \\
\text { Benzo(a)anthracene } \\
\text { Benzo(a)pyrene } \\
\text { Benzo(b) fluoranthene } \\
\text { Benzo(k)fluoranthene } \\
\text { Chrysene } \\
\text { Dieldrin } \\
\text { Endosulfan sulfate } \\
\text { Endosulfan I } \\
\text { Endosulfan II } \\
\text { Endrin } \\
\text { Endrin aldehyde } \\
\text { Endrin ketone } \\
\text { Fluoranthene } \\
\text { Fluorene } \\
\text { Heptachlor } \\
\text { Heptachlor epoxide } \\
\text { Indeno( } 1,2,3-c d) p y r e n e \\
\text { Lindane } \\
\text { Methoxychlor } \\
\text { Kaphthalene } \\
\text { Phenanthrene }\end{array}$ & 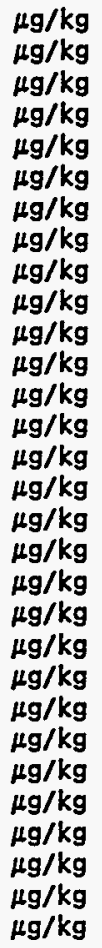 & $\begin{array}{r}1.90 \\
1.90 \\
1.90 \\
1.90 \\
1.90 \\
1.00 \\
28.00 \\
1.90 \\
1.00 \\
0.50 \\
2.00 \\
2.00 \\
2.00 \\
17.00 \\
3.70 \\
3.70 \\
1.90 \\
3.70 \\
3.70 \\
3.70 \\
3.70 \\
6.00 \\
5.00 \\
1.90 \\
1.90 \\
5.00 \\
1.90 \\
18.90 \\
11.00 \\
11.00\end{array}$ \\
\hline
\end{tabular}




\section{C-18}

Table C.1 (continued)

\begin{tabular}{lcccc}
\hline Analysis & Units & $\begin{array}{c}\text { A horizon } \\
\text { Result }\end{array}$ & Qualifier & $\begin{array}{c}\text { A horizon } \\
\text { field dup }\end{array}$ Qualifier \\
\hline & $\mu g / k g$ & 1.00 & $U J$ & \\
Pyrene & $\mu g / k g$ & 36.70 & $U$ & \\
PCB-1016 & $\mu g / k g$ & 74.50 & $U$ & \\
PCB-1221 & $\mu g / k g$ & 36.70 & $U$ & \\
PCB-1232 & $\mu g / k g$ & 36.70 & $U$ & \\
PCB-1242 & $\mu g / k g$ & 36.70 & $U$ & \\
PCB-1248 & $\mu g / k g$ & 36.70 & $U$ & \\
PCB-1254 & $\mu g / k g$ & 36.70 & $U$ & \\
PCB-1260 & $\mu g / k g$ & 189.10 & $U$ & \\
TOXaphene & $\mu g / k g$ & 3.70 & $U$ & \\
$4,4^{\prime}-D D D$ & $\mu g / k g$ & 3.70 & $U$ & \\
$4,4 '-D D E$ & $\mu g / k g$ & 3.70 & $U J$ & \\
$4,4 '-D D T$ & & &
\end{tabular}

Location=AND; Formation=DISMAL GAP; Site =5; $A=2149 ; A$ dup=' '; Phase $=1$

\begin{tabular}{|c|c|c|}
\hline 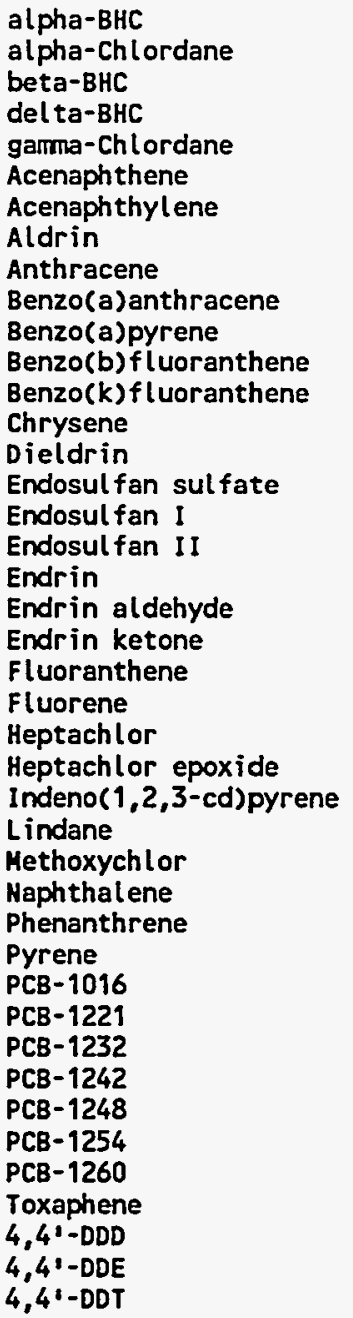 & 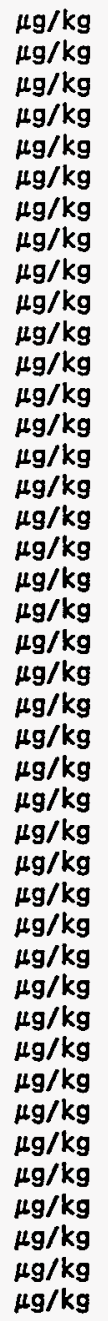 & $\begin{array}{r}1.90 \\
1.90 \\
1.90 \\
1.90 \\
1.90 \\
1.80 \\
29.00 \\
1.90 \\
1.00 \\
1.10 \\
1.60 \\
2.00 \\
2.00 \\
17.00 \\
3.80 \\
3.80 \\
1.90 \\
3.80 \\
3.80 \\
3.80 \\
3.80 \\
34.00 \\
5.00 \\
1.90 \\
1.90 \\
5.00 \\
1.90 \\
19.50 \\
11.00 \\
33.00 \\
1.00 \\
37.80 \\
76.70 \\
37.80 \\
37.80 \\
37.80 \\
37.80 \\
37.80 \\
194.60 \\
3.80 \\
3.80 \\
3.80\end{array}$ \\
\hline
\end{tabular}

Location $=A N D ;$ Formation $=D I S M A L$ GAP; Site=9; $A=2130 ; A$ dup=' '; Phase=1

$\begin{array}{llll}\text { alpha-BHC } & \mu \mathrm{g} / \mathrm{kg} & 2.00 & \mathrm{U} \\ \text { alpha-Chlordane } & \mu \mathrm{g} / \mathrm{kg} & 2.00 & \mathrm{U} \\ \text { beta-BHC } & \mu \mathrm{g} / \mathrm{kg} & 2.00 & U \\ \text { delta-BHC } & \mu \mathrm{kg} & 2.00 & \mathrm{U} \\ \text { gamma-Chlordane } & \mu \mathrm{g} / \mathrm{kg} & 2.00 & U\end{array}$


Table C.1 (continued)

\begin{tabular}{|c|c|c|c|c|c|c|}
\hline Analysis & Units & $\begin{array}{c}\text { A horizon } \\
\text { Result }\end{array}$ & Qualifier & $\begin{array}{l}\text { A horizon } \\
\text { field dup }\end{array}$ & Qualifier & \\
\hline $\begin{array}{l}\text { Acenaphthene } \\
\text { Aldrin } \\
\text { Benzo(a)anthracene } \\
\text { Dieldrin } \\
\text { Endosul fan sul fate } \\
\text { Endosul fan I } \\
\text { Endosul fan II } \\
\text { Endrin } \\
\text { Endrin aldehyde } \\
\text { Endrin ketone } \\
\text { Heptachlor } \\
\text { Heptachlor epoxide } \\
\text { Lindane } \\
\text { Methoxychlor } \\
\text { Phenanthrene } \\
\text { PCB-1016 } \\
\text { PCB-1221 } \\
\text { PCB-1232 } \\
\text { PCB-1242 } \\
\text { PCB-1248 } \\
\text { PCB-1254 } \\
\text { PCB-1260 } \\
\text { Toxaphene } \\
\text { 4,4'-DDD } \\
4,41-D D E \\
4,41-D D T\end{array}$ & $\begin{array}{l}\mu g / \mathrm{kg} \\
\mu g / \mathrm{kg} \\
\mu \mathrm{g} / \mathrm{kg} \\
\mu g / \mathrm{kg} \\
\mu g / \mathrm{kg} \\
\mu g / \mathrm{kg} \\
\mu g / \mathrm{kg} \\
\mu g / \mathrm{kg} \\
\mu g / \mathrm{kg} \\
\mu \mathrm{g} / \mathrm{kg} \\
\mu g / \mathrm{kg} \\
\mu g / \mathrm{kg} \\
\mu g / \mathrm{kg} \\
\mu g / \mathrm{kg} \\
\mu g / \mathrm{kg} \\
\mu \mathrm{g} / \mathrm{kg} \\
\mu g / \mathrm{kg} \\
\mu g / \mathrm{kg} \\
\mu \mathrm{g} / \mathrm{kg} \\
\mu \mathrm{g} / \mathrm{kg} \\
\mu g / \mathrm{kg} \\
\mu \mathrm{g} / \mathrm{kg} \\
\mu \mathrm{g} / \mathrm{kg} \\
\mu g / \mathrm{kg} \\
\mu \mathrm{g} / \mathrm{kg} \\
\mu \mathrm{g} / \mathrm{kg}\end{array}$ & $\begin{array}{r}7.60 \\
2.00 \\
2.90 \\
3.80 \\
3.80 \\
2.00 \\
3.80 \\
3.80 \\
3.80 \\
3.80 \\
2.00 \\
2.00 \\
2.00 \\
19.80 \\
33.00 \\
38.50 \\
78.10 \\
38.50 \\
38.50 \\
38.50 \\
38.50 \\
38.50 \\
198.20 \\
3.80 \\
3.80 \\
3.80\end{array}$ & $\begin{array}{l}J \\
J \\
U J \\
J \\
U J \\
U \\
U \\
U \\
U \\
U \\
U \\
U J \\
U \\
U J \\
U \\
J \\
U \\
U \\
U \\
U \\
U \\
U \\
U \\
U \\
U \\
U \\
U J\end{array}$ & & , &. \\
\hline
\end{tabular}

Location $=$ ORR; Formation $=$ CHEPULTEPEC; Site $=50 ; A=1548 ; A$ dup =' ; Phase $=2$

Acenaphthene
Acenaphthylene
Benzo(a)anthracene
Benzo(ghi)perylene
Dibenzo(a,h)anthracene
Fluoranthene
Indeno(1,2,3-cd)pyrene
Haphthalene
Phenanthrene
Pyrene

$\begin{array}{lrr}\mu \mathrm{g} / \mathrm{kg} & 0.80 & \mathrm{~J} \\ \mu \mathrm{g} / \mathrm{kg} & 75.10 & \mathrm{U} \\ \mu \mathrm{g} / \mathrm{kg} & 1.50 & \mathrm{~J} \\ \mu \mathrm{g} / \mathrm{kg} & 2.60 & \mathrm{~J} \\ \mu \mathrm{g} / \mathrm{kg} & 0.40 & \mathrm{~J} \\ \mu \mathrm{g} / \mathrm{kg} & 0.40 & \mathrm{~J} \\ \mu \mathrm{g} / \mathrm{kg} & 18.80 & \mathrm{U} \\ \mu \mathrm{g} / \mathrm{kg} & 48.40 & \mathrm{~J} \\ \mu \mathrm{g} / \mathrm{kg} & 1.50 & \mathrm{~J} \\ \mu \mathrm{g} / \mathrm{kg} & 2.60 & \mathrm{~J}\end{array}$

Location=ORR; Formation =CHEPULTEPEC; Site=53; $A=1553 ; A$ dup=' '; Phase $=2$

$\begin{array}{lrrr}\text { Acenaphthylene } & \mu \mathrm{g} / \mathrm{kg} & 74.20 & \mathrm{U} \\ \text { Benzo(a)anthracene } & \mu \mathrm{g} / \mathrm{kg} & 2.60 & \mathrm{~J} \\ \text { Benzo(a)pyrene } & \mu \mathrm{g} / \mathrm{kg} & 2.60 & \mathrm{~J} \\ \text { Benzo(ghi)perylene } & \mu \mathrm{g} / \mathrm{kg} & 2.60 & \mathrm{~J} \\ \text { Benzo(k)fluoranthene } & \mu \mathrm{g} / \mathrm{kg} & 1.10 & \mathrm{~J} \\ \text { Dibenzo(a,h)anthracene } & \mu \mathrm{g} / \mathrm{kg} & 3.70 & \mathrm{~J} \\ \text { Fluoranthene } & \mu \mathrm{g} / \mathrm{kg} & 4.10 & \mathrm{~J} \\ \text { Fluorene } & \mu \mathrm{g} / \mathrm{kg} & 0.20 & \mathrm{~J} \\ \text { Indeno( } 1,2,3-\mathrm{cd}) \text { pyrene } & \mu \mathrm{g} / \mathrm{kg} & 18.60 & \mathrm{U} \\ \text { Phenanthrene } & \mu \mathrm{g} / \mathrm{kg} & 4.80 & \mathrm{~J} \\ \text { Pyrene } & \mu \mathrm{g} / \mathrm{kg} & 3.30 & \mathrm{~J}\end{array}$

Location=ORR; Formation $=$ CHEPULTEPEC; Site $=66 ; A=1738 ; A$ dup =' '; Phase $=2$

$\begin{array}{llrl}\text { alpha-BHC } & \mu g / \mathrm{kg} & 1.80 & \mathrm{UJ} \\ \text { alpha-Chlordane } & \mu g / \mathrm{kg} & 1.80 & \mathrm{U} \\ \text { beta-BHC } & \mu g / \mathrm{kg} & 1.80 & \mathrm{UJ} \\ \text { del ta-BHC } & \mu g / \mathrm{kg} & 1.80 & \mathrm{UJ} \\ \text { gamma-Chlordane } & \mu g / \mathrm{kg} & 1.80 & \mathrm{U} \\ \text { Acenaphthylene } & \mu g / \mathrm{kg} & 76.90 & \mathrm{U} \\ \text { Aldrin } & \mu \mathrm{kg} & 1.80 & \mathrm{U} \\ \text { Benzo(a)anthracene } & \mu \mathrm{k} / \mathrm{kg} & 0.40 & \mathrm{~J}\end{array}$


C-20

Table C.1 (continued)

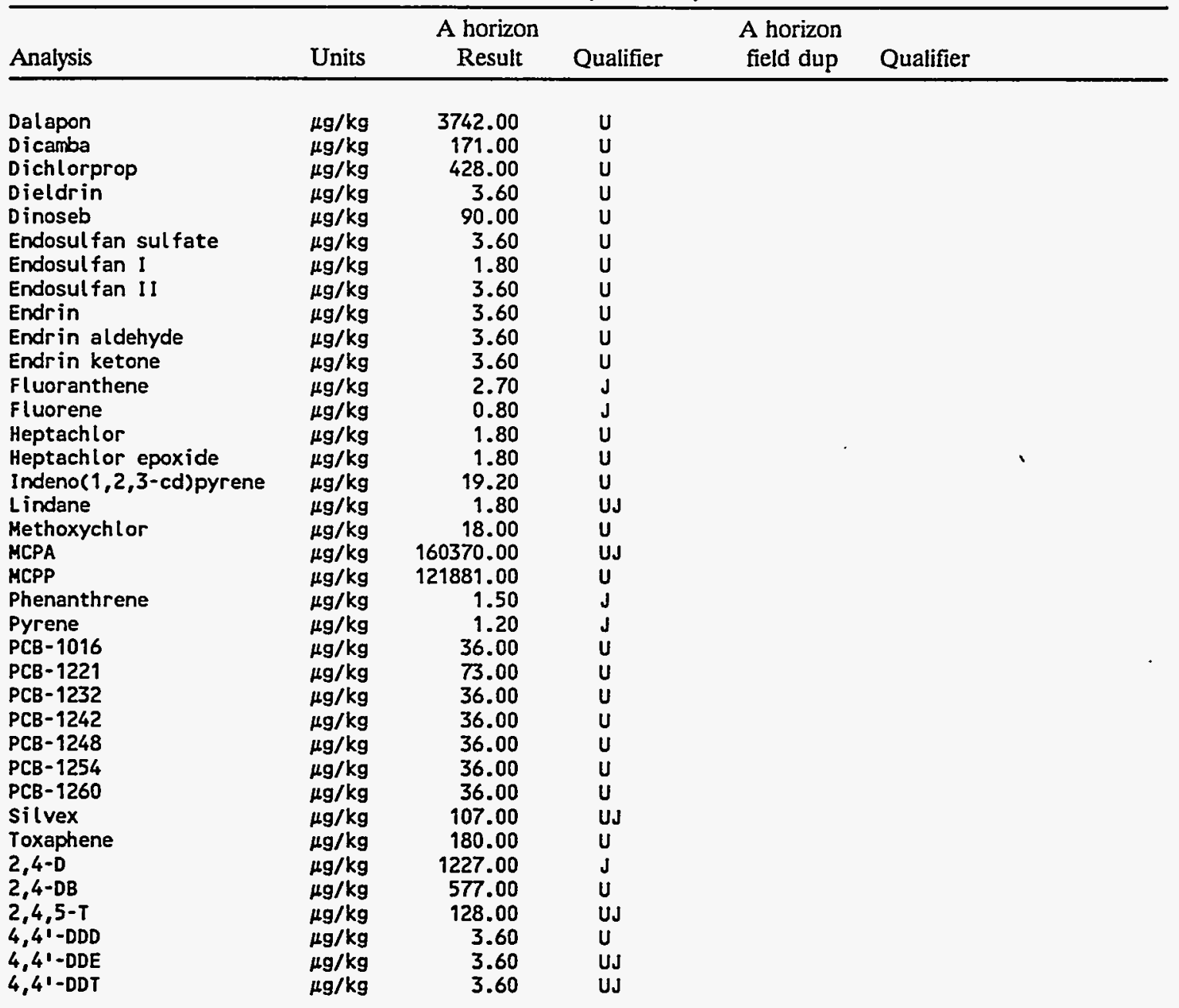

Location=ORR; Formation=CHEPULTEPEC; Site $=68 ; A=1741 ; A$ dup=' '; Phase $=2$

\begin{tabular}{|c|c|c|}
\hline $\begin{array}{l}\text { alpha-BHC } \\
\text { alpha-Chlordane } \\
\text { beta-BHC } \\
\text { delta-BHC } \\
\text { gamma-Chlordane } \\
\text { Acenaphthylene } \\
\text { Aldrin } \\
\text { Anthracene } \\
\text { Benzo(a)anthracene } \\
\text { Benzo(a)pyrene } \\
\text { Benzo(b)fluoranthene } \\
\text { Benzo(ghi)perylene } \\
\text { Benzo(k)fluoranthene } \\
\text { Dalapon } \\
\text { Dicamba } \\
\text { Dichlorprop } \\
\text { Dieldrin } \\
\text { Dinoseb } \\
\text { Endosulfan sul fate } \\
\text { Endosulfan I } \\
\text { Endosulfan II } \\
\text { Endrin } \\
\text { Endrin aldehyde } \\
\text { Endrin ketone } \\
\text { Fluoranthene } \\
\text { Fluorene } \\
\text { Heptachlor }\end{array}$ & 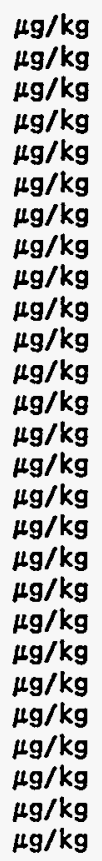 & $\begin{array}{r}1.90 \\
1.90 \\
1.90 \\
1.90 \\
1.90 \\
76.60 \\
1.90 \\
0.40 \\
1.90 \\
3.10 \\
3.40 \\
1.90 \\
1.50 \\
3850.00 \\
177.00 \\
443.00 \\
3.80 \\
93.00 \\
3.80 \\
1.90 \\
3.80 \\
3.80 \\
3.80 \\
3.80 \\
1.90 \\
0.80 \\
1.90\end{array}$ \\
\hline
\end{tabular}


C-21

Table C.1 (continued)

\begin{tabular}{|c|c|c|c|c|c|c|}
\hline Analysis & Units & $\begin{array}{c}\text { A horizon } \\
\text { Result }\end{array}$ & Qualifier & $\begin{array}{l}\text { A horizon } \\
\text { field dup }\end{array}$ & Qualifier & \\
\hline $\begin{array}{l}\text { Heptachlor epoxide } \\
\text { Indeno(1,2,3-cd)pyrene } \\
\text { Lindane } \\
\text { Methoxychlor } \\
\text { MCPA } \\
\text { MCPP } \\
\text { Phenanthrene } \\
\text { Pyrene } \\
\text { PCB-1016 } \\
\text { PCB-1221 } \\
\text { PCB-1232 } \\
\text { PCB-1242 } \\
\text { PCB-1248 } \\
\text { PCB-1254 } \\
\text { PCB-1260 } \\
\text { Silvex } \\
\text { Toxaphene } \\
2,4-D \\
2,4-D B \\
2,4,5-T \\
4,4 \text {-DDD } \\
4,4^{1}-\text { DDE } \\
4,41-D D T\end{array}$ & $\begin{array}{l}\mu g / \mathrm{kg} \\
\mu g / \mathrm{kg} \\
\mu g / \mathrm{kg} \\
\mu g / \mathrm{kg} \\
\mu g / \mathrm{kg} \\
\mu g / \mathrm{kg} \\
\mu g / \mathrm{kg} \\
\mu g / \mathrm{kg} \\
\mu g / \mathrm{kg} \\
\mu g / \mathrm{kg} \\
\mu g / \mathrm{kg} \\
\mu g / \mathrm{kg} \\
\mu g / \mathrm{kg} \\
\mu g / \mathrm{kg} \\
\mu g / \mathrm{kg} \\
\mu g / \mathrm{kg} \\
\mu \mathrm{g} / \mathrm{kg} \\
\mu g / \mathrm{kg} \\
\mu g / \mathrm{kg} \\
\mu g / \mathrm{kg} \\
\mu g / \mathrm{kg} \\
\mu g / \mathrm{kg} \\
\mu g / \mathrm{kg}\end{array}$ & $\begin{array}{r}1.90 \\
19.20 \\
1.90 \\
19.00 \\
165955.00 \\
126126.00 \\
3.40 \\
2.70 \\
38.00 \\
76.00 \\
38.00 \\
38.00 \\
38.00 \\
38.00 \\
38.00 \\
111.00 \\
190.00 \\
797.00 \\
597.00 \\
133.00 \\
3.80 \\
3.80 \\
3.80\end{array}$ & $\begin{array}{l}U H^{\prime \prime} \\
U \\
U J \\
U \\
U J \\
U \\
J \\
J \\
U \\
U \\
U \\
U \\
U \\
U \\
U \\
U J \\
U \\
U J \\
U \\
U J \\
U \\
U J \\
U J\end{array}$ & . & & $\cdots$ \\
\hline
\end{tabular}

Location $=$ ORR; Formation $=$ CHEPULTEPEC; Site $=73 ; A=1744 ; A$ dup $=$; ; Phase $=2$

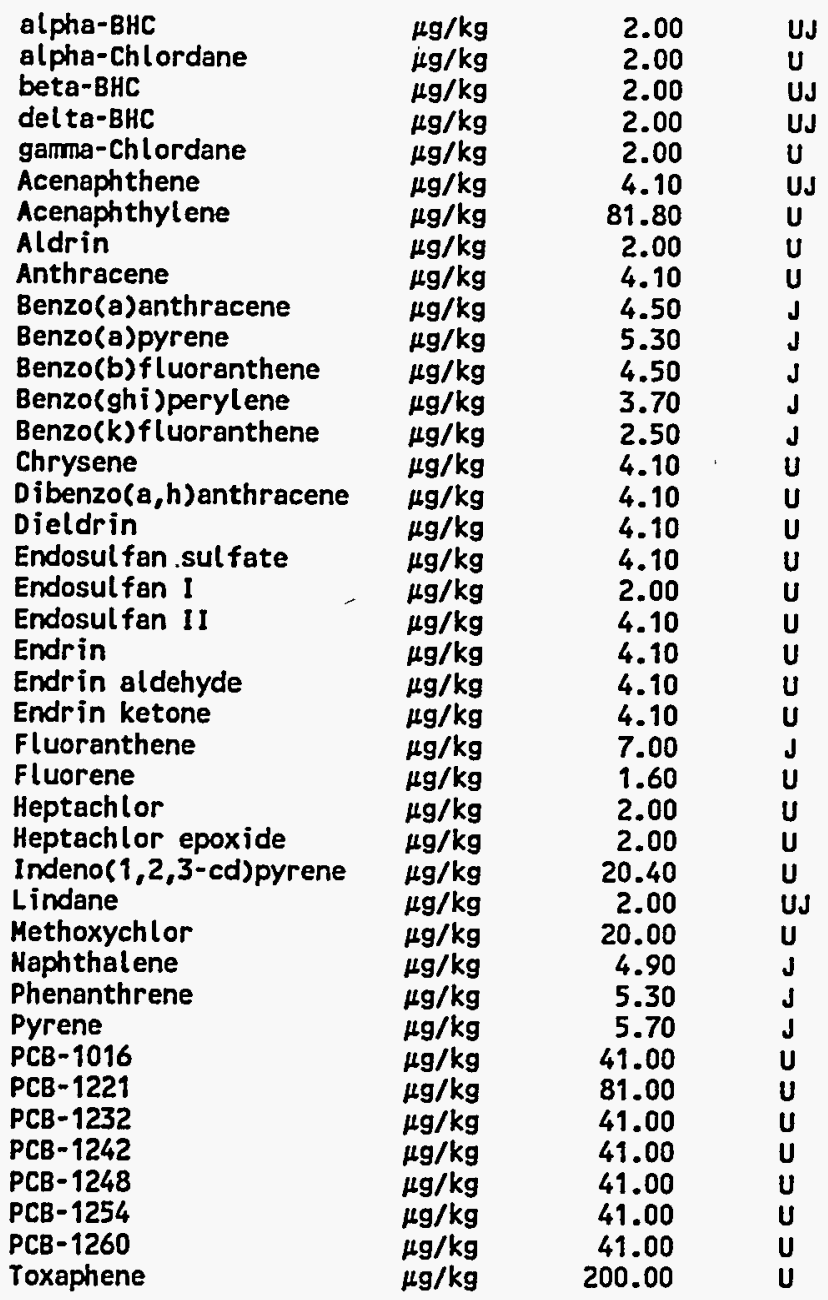


Table C.1 (continued)

\begin{tabular}{|c|c|c|c|c|c|}
\hline Analysis & Units & $\begin{array}{c}\text { A horizon } \\
\text { Result }\end{array}$ & Qualifier & $\begin{array}{l}\text { A horizon } \\
\text { field dup }\end{array}$ & Qualifier \\
\hline $\begin{array}{l}4,4^{\prime}-\mathrm{DDD} \\
4,4^{\prime}-\mathrm{DDE} \\
4,4^{\prime}-\mathrm{DDT}\end{array}$ & $\begin{array}{l}\mu \mathrm{g} / \mathrm{kg} \\
\mu \mathrm{g} / \mathrm{kg} \\
\mu \mathrm{g} / \mathrm{kg}\end{array}$ & $\begin{array}{l}4.10 \\
4.10 \\
4.10\end{array}$ & $\begin{array}{l}\text { U } \\
\text { UJ } \\
\text { UJ }\end{array}$ & . & \\
\hline
\end{tabular}

Location=ORR; Formation $=$ CHEPULTEPEC; Site $=74 ; A=1746 ; A$ dup =' '; Phase=2

$\begin{array}{lrrl} & & & \\ \text { alpha-BHC } & \mu \mathrm{g} / \mathrm{kg} & 1.90 & \mathrm{UJ} \\ \text { alpha-Chlordane } & \mu \mathrm{g} / \mathrm{kg} & 1.90 & \mathrm{U} \\ \text { beta-BHC } & \mu \mathrm{g} / \mathrm{kg} & 1.90 & \mathrm{UJ} \\ \text { delta-BHC } & \mu \mathrm{g} / \mathrm{kg} & 1.90 & \mathrm{UJ} \\ \text { gamma-Chlordane } & \mu \mathrm{g} / \mathrm{kg} & 1.90 & \mathrm{U} \\ \text { Acenaphthene } & \mu \mathrm{g} / \mathrm{kg} & 3.90 & \mathrm{U} \\ \text { Acenaphthylene } & \mu \mathrm{g} / \mathrm{kg} & 77.90 & \mathrm{U} \\ \text { Aldrin } & \mu \mathrm{g} / \mathrm{kg} & 1.90 & \mathrm{U} \\ \text { Anthracene } & \mu \mathrm{g} / \mathrm{kg} & 0.40 & \mathrm{~J} \\ \text { Benzo(a)anthracene } & \mu \mathrm{g} / \mathrm{kg} & 1.60 & \mathrm{~J} \\ \text { Benzo(a)pyrene } & \mu \mathrm{g} / \mathrm{kg} & 2.70 & \mathrm{~J} \\ \text { Benzo(b)fluoranthene } & \mu \mathrm{g} / \mathrm{kg} & 3.90 & \mathrm{U} \\ \text { Benzo(ghi)perylene } & \mu \mathrm{g} / \mathrm{kg} & 1.60 & \mathrm{~J} \\ \text { Benzo(k)fluoranthene } & \mu \mathrm{g} / \mathrm{kg} & 1.20 & \mathrm{~J} \\ \text { Chrysene } & \mu \mathrm{g} / \mathrm{kg} & 3.90 & \mathrm{U} \\ \text { Dibenzo(a,h)anthracene } & \mu \mathrm{g} / \mathrm{kg} & 0.80 & \mathrm{~J} \\ \text { Dieldrin } & \mu \mathrm{g} / \mathrm{kg} & 3.90 & \mathrm{U} \\ \text { Endosulfan sulfate } & \mu \mathrm{g} / \mathrm{kg} & 3.90 & \mathrm{U} \\ \text { Endosulfan I } & \mu \mathrm{g} / \mathrm{kg} & 1.90 & \mathrm{U} \\ \text { Endosulfan II } & \mu \mathrm{g} / \mathrm{kg} & 3.90 & \mathrm{U} \\ \text { Endrin } & \mu \mathrm{g} / \mathrm{kg} & 3.90 & \mathrm{U} \\ \text { Endrin aldehyde } & \mu \mathrm{g} / \mathrm{kg} & 3.90 & \mathrm{U} \\ \text { Endrin ketone } & \mu \mathrm{g} / \mathrm{kg} & 3.90 & \mathrm{U} \\ \text { Fluoranthene } & \mu \mathrm{g} / \mathrm{kg} & 5.10 & \\ \text { Fluorene } & \mu \mathrm{g} / \mathrm{kg} & 1.60 & \mathrm{U} \\ \text { Heptachlor } & \mu \mathrm{g} / \mathrm{kg} & 1.90 & \mathrm{U} \\ \text { Heptachlor epoxide } & \mu \mathrm{g} / \mathrm{kg} & 1.90 & \mathrm{U} \\ \text { Indeno(1,2,3-cd)pyrene } & \mu \mathrm{g} / \mathrm{kg} & 19.50 & \mathrm{U} \\ \text { Lindane } & \mu \mathrm{g} / \mathrm{kg} & 1.90 & \mathrm{UJ} \\ \text { Methoxychlor } & \mu \mathrm{g} / \mathrm{kg} & 19.00 & \mathrm{U} \\ \text { Maphthalene } & \mu \mathrm{g} / \mathrm{kg} & 4.70 & \mathrm{~J} \\ \text { Phenanthrene } & \mu \mathrm{g} / \mathrm{kg} & 3.50 & \mathrm{~J} \\ \text { Pyrene } & \mu \mathrm{g} / \mathrm{kg} & 4.30 & \\ \text { PCB-1016 } & \mu \mathrm{g} / \mathrm{kg} & 39.00 & \mathrm{U} \\ \text { PCB-1221 } & \mu \mathrm{g} / \mathrm{kg} & 78.00 & \mathrm{U} \\ \text { PCB-1232 } & \mu \mathrm{g} / \mathrm{kg} & 39.00 & \mathrm{U} \\ \text { PCB-1242 } & \mu \mathrm{g} / \mathrm{kg} & 39.00 & \mathrm{U} \\ \text { PCB-1248 } & \mu \mathrm{g} / \mathrm{kg} & 39.00 & \mathrm{U} \\ \text { PCB-1254 } & \mu \mathrm{g} / \mathrm{kg} & 39.00 & \mathrm{U} \\ \text { PCB-1260 } & \mu \mathrm{g} / \mathrm{kg} & 39.00 & \mathrm{U} \\ \text { Toxaphene } & \mu \mathrm{g} / \mathrm{kg} & 190.00 & \mathrm{U} \\ \text { 4,4'-DDD } & \mu \mathrm{g} / \mathrm{kg} & 3.90 & \mathrm{U} \\ \text { 4,4'-DDE } & 3.90 & \mathrm{UJ} \\ \text { 4,4'-DDT } & 3.90 & \mathrm{UJ} \\ & & & \end{array}$

Location $=$ ORR; Formation=CHEPULTEPEC; Site $=90 ; A=1749 ; A$ dup=' '; Phase $=2$

$\begin{array}{llrl}\text { alpha-BHC } & \mu g / \mathrm{kg} & 1.90 & \mathrm{UJ} \\ \text { alpha-Chlordane } & \mu \mathrm{g} / \mathrm{kg} & 1.90 & \mathrm{U} \\ \text { beta-BHC } & \mu \mathrm{g} / \mathrm{kg} & 1.90 & \mathrm{UJ} \\ \text { del ta-BHC } & \mu \mathrm{g} / \mathrm{kg} & 1.90 & \mathrm{UJ} \\ \text { gamma-Chlordane } & \mu \mathrm{g} / \mathrm{kg} & 1.90 & \mathrm{U} \\ \text { Acenaphthene } & \mu \mathrm{g} / \mathrm{kg} & 4.70 & \mathrm{U} \\ \text { Acenaphthylene } & \mu \mathrm{g} / \mathrm{kg} & 93.80 & \mathrm{U} \\ \text { Aldrin } & \mu \mathrm{g} / \mathrm{kg} & 1.90 & \mathrm{U} \\ \text { Anthracene } & \mu \mathrm{g} / \mathrm{kg} & 4.70 & \mathrm{U} \\ \text { Benzo(a)anthracene } & \mu \mathrm{g} / \mathrm{kg} & 1.90 & \mathrm{~J} \\ \text { Benzo(a)pyrene } & \mu \mathrm{g} / \mathrm{kg} & 3.30 & \mathrm{~J} \\ \text { Benzo(b)fluoranthene } & \mu \mathrm{g} / \mathrm{kg} & 4.70 & \mathrm{U}\end{array}$


C-23

Table C.1 (continued)

\begin{tabular}{|c|c|c|c|c|c|c|}
\hline Analysis & Units & $\begin{array}{c}\text { A horizon } \\
\text { Result }\end{array}$ & Qualifier & $\begin{array}{l}\text { A horizon } \\
\text { field dup }\end{array}$ & Qualifier & \\
\hline $\begin{array}{l}\text { Benzo(ghi)perylene } \\
\text { Benzo(k)fluoranthene } \\
\text { Chrysene } \\
\text { Dibenzo(a,h)anthracene } \\
\text { Dieldrin } \\
\text { Endosulfan sulfate } \\
\text { Endosulfan I } \\
\text { Endosulfan II } \\
\text { Endrin } \\
\text { Endrin aldehyde } \\
\text { Endrin ketone } \\
\text { Fluoranthene } \\
\text { Fluorene } \\
\text { Heptachlor } \\
\text { Heptachlor epoxide } \\
\text { Indeno( } 1,2,3-\text {-cd)pyrene } \\
\text { Lindane } \\
\text { Methoxychlor } \\
\text { Naphthalene } \\
\text { Phenanthrene } \\
\text { Pyrene } \\
\text { PCB-1016 } \\
\text { PCB-1221 } \\
\text { PCB-1232 } \\
\text { PCB-1242 } \\
\text { PCB-1248 } \\
\text { PCB-1254 } \\
\text { PCB-1260 } \\
\text { Toxaphene } \\
4,41-D D D \\
4,41-D D E \\
4,41-D D T\end{array}$ & 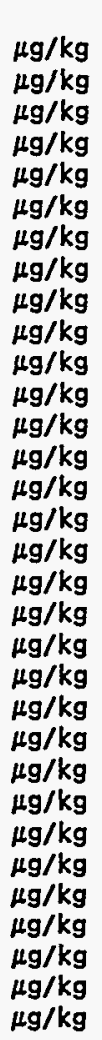 & $\begin{array}{r}3.80 \\
1.90 \\
4.70 \\
4.70 \\
3.80 \\
3.80 \\
1.90 \\
3.80 \\
3.80 \\
3.80 \\
3.80 \\
8.90 \\
4.70 \\
1.90 \\
1.90 \\
16.40 \\
1.90 \\
19.00 \\
23.50 \\
4.20 \\
8.00 \\
38.00 \\
75.00 \\
38.00 \\
38.00 \\
38.00 \\
38.00 \\
38.00 \\
190.00 \\
3.80 \\
3.80 \\
3.80\end{array}$ & $\begin{array}{l}J \\
J \\
J \\
U \\
U \\
U \\
U \\
U \\
U \\
U \\
U \\
U \\
U \\
U \\
J \\
U J \\
U \\
U \\
J \\
U \\
U \\
U \\
U \\
U \\
U \\
U \\
U \\
U \\
U J \\
U J\end{array}$ & & , & 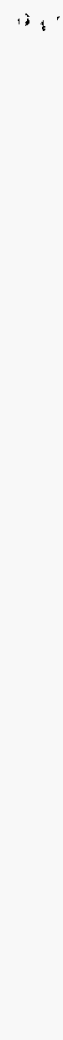 \\
\hline
\end{tabular}

Location=ORR; Formation=CHICKAMAUGA Bethel V; Site=100; $A=1906 ; A$ dup=' '; Phase =2

\begin{tabular}{|c|c|c|}
\hline $\begin{array}{l}\text { 1alpha-BHC } \\
\text { alpha-Chlordane } \\
\text { beta-BHC } \\
\text { del ta-BHC } \\
\text { gamma-Chlordane } \\
\text { Acenaphthylene } \\
\text { Aldrin } \\
\text { Anthracene } \\
\text { Benzo(a) anthracene } \\
\text { Benzo(a)pyrene } \\
\text { Benzo(b)fluoranthene } \\
\text { Benzo(k) fluoranthene } \\
\text { Chrysene } \\
\text { Dibenzo(a,h)anthracene } \\
\text { Dieldrin } \\
\text { Endosulfan sulfate } \\
\text { Endosul fan I } \\
\text { Endosul fan } 11 \\
\text { Endrin } \\
\text { Endrin aldehyde } \\
\text { Endrin ketone } \\
\text { Fluoranthene } \\
\text { Heptachlor } \\
\text { Heptachlor epoxide } \\
\text { Indeno(1,2,3-cd)pyrene } \\
\text { Lindane } \\
\text { Methoxychlor } \\
\text { Naphthalene } \\
\text { Phenanthrene } \\
\text { Pyrene } \\
\text { PCB-1016 } \\
\text { PCB-1221 }\end{array}$ & 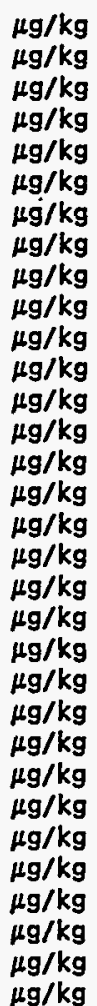 & $\begin{array}{r}2.40 \\
2.40 \\
2.40 \\
2.40 \\
2.40 \\
88.40 \\
2.40 \\
1.30 \\
7.50 \\
7.50 \\
7.10 \\
4.40 \\
6.20 \\
4.40 \\
4.60 \\
23.50 \\
2.40 \\
4.60 \\
4.60 \\
4.60 \\
4.60 \\
2.70 \\
2.40 \\
2.40 \\
5.30 \\
2.40 \\
4.60 \\
16.30 \\
20.80 \\
11.00 \\
46.00 \\
93.00\end{array}$ \\
\hline
\end{tabular}




\section{C-24}

Table C.1 (continued)

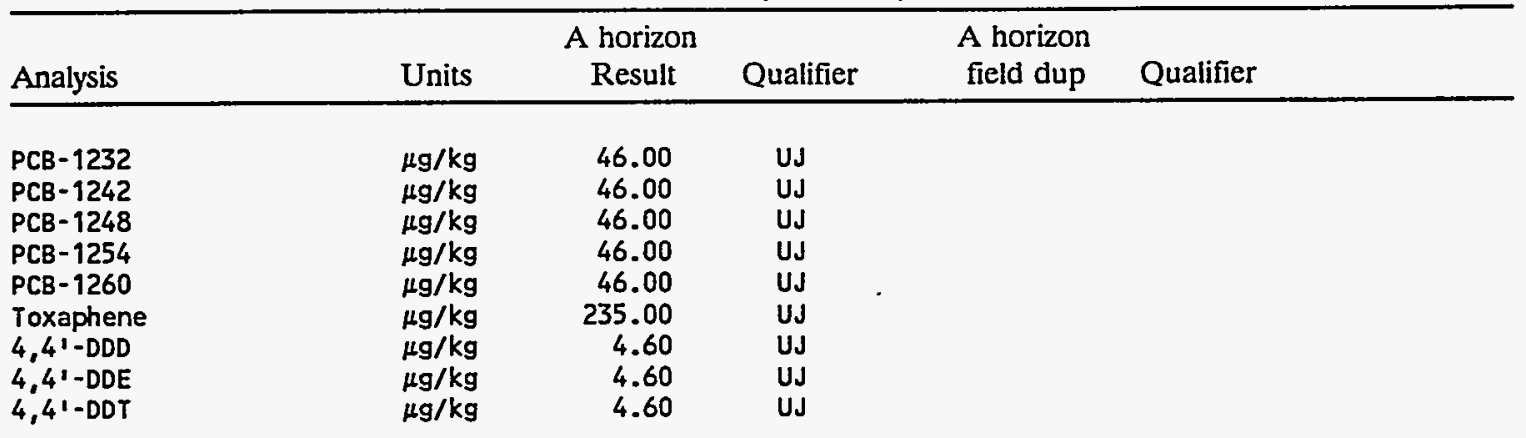

Location =ORR; Formation $=$ CHICKAMAUGA Bethel V; Site $=101 ; A=1897 ; A$ dup=' ; Phase $=2$

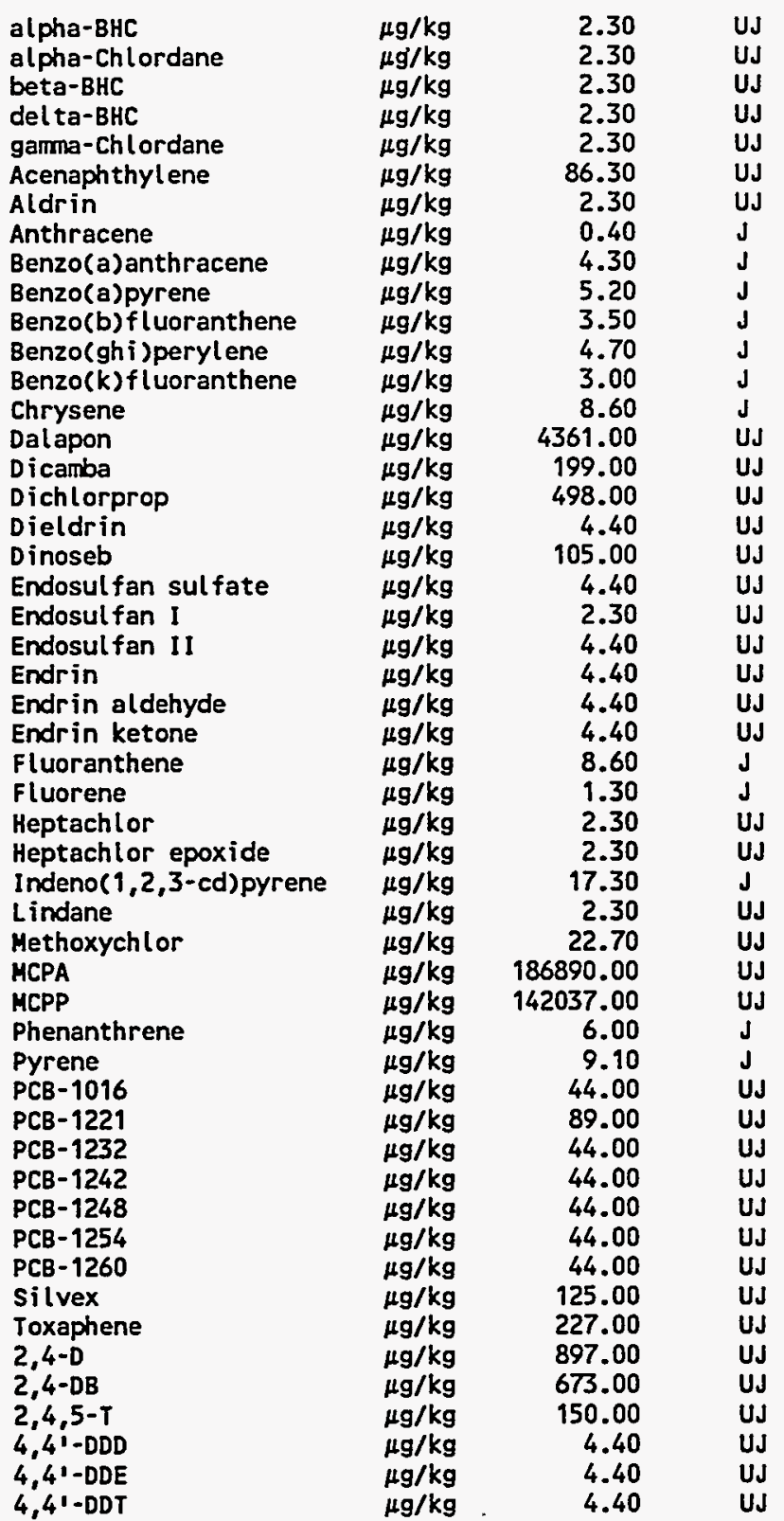




\section{C-26}

Table C.1 (continued)

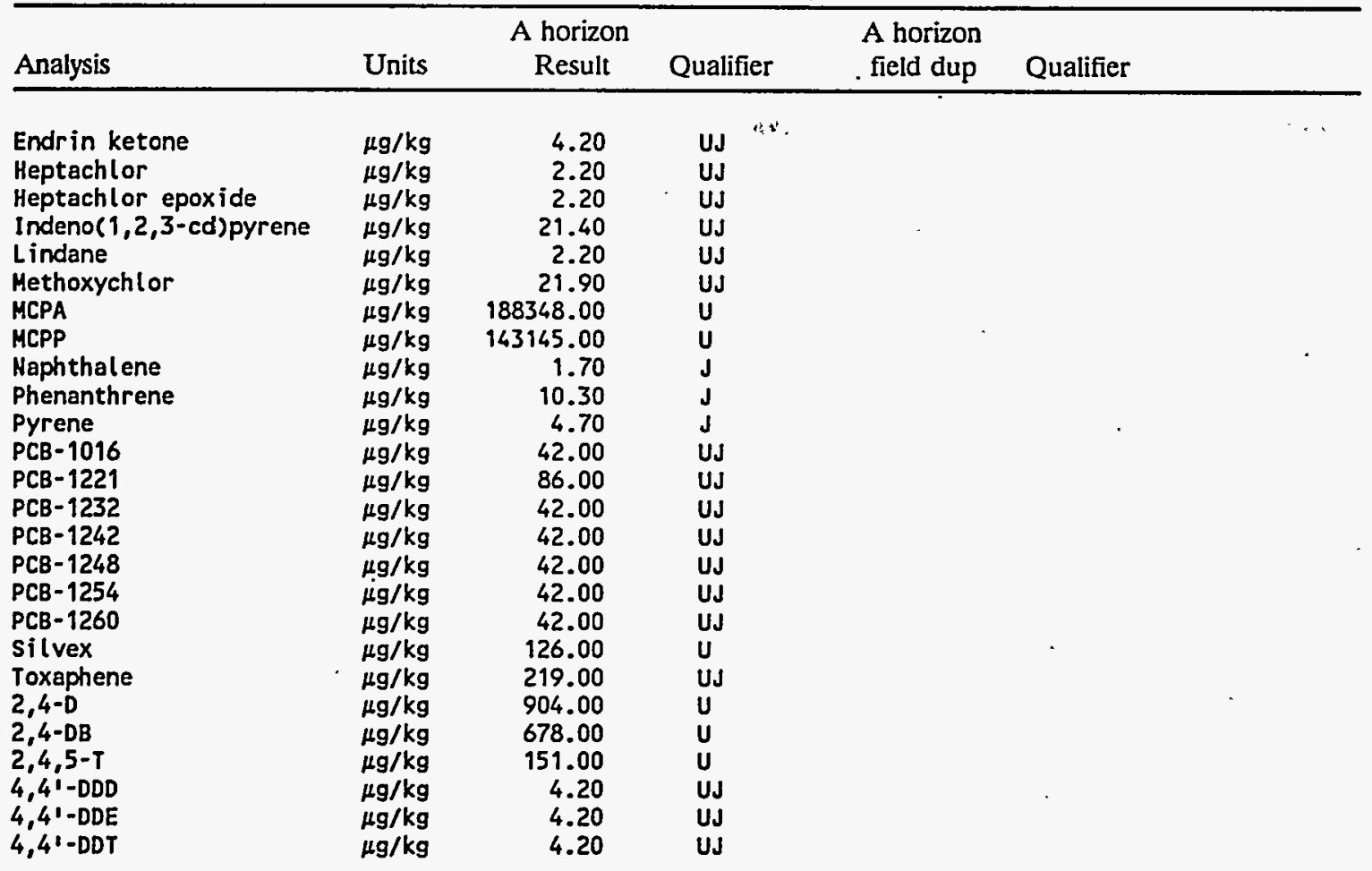

Location=ORR; Formation=CHICKAMAUGA Bethel V; Site=104; $A=1964 ; A$ dup=' '; Phase $=2$

\begin{tabular}{|c|c|c|}
\hline $\begin{array}{l}\text { alpha-BHC } \\
\text { alpha-Chlordane } \\
\text { beta-BHC } \\
\text { del ta-BHC } \\
\text { gamma-Chlordane } \\
\text { Acenaphthylene } \\
\text { Aldrin } \\
\text { Benzo(a)pyrene } \\
\text { Benzo(b)fluoranthene } \\
\text { Benzo(k) fluoranthene } \\
\text { Dieldrin } \\
\text { Endosul fan sul fate } \\
\text { Endosulfan I } \\
\text { Endosulfan II } \\
\text { Endrin } \\
\text { Endrin aldehyde } \\
\text { Endrin ketone } \\
\text { Heptachlor } \\
\text { Heptachlor epoxide } \\
\text { Indeno(1,2,3-cd)pyrene } \\
\text { Lindane } \\
\text { Methoxychlor } \\
\text { Haphthalene } \\
\text { Phenanthrene } \\
\text { PCB-1016 } \\
\text { PCB-1221 } \\
\text { PCB-1232 } \\
\text { PCB-1242 } \\
\text { PCB-1248 } \\
\text { PCB-1254 } \\
\text { PCB-1260 } \\
\text { Toxaphene } \\
4,41-D D D \\
4,41-D D E \\
4,4^{\prime}-D D T\end{array}$ & 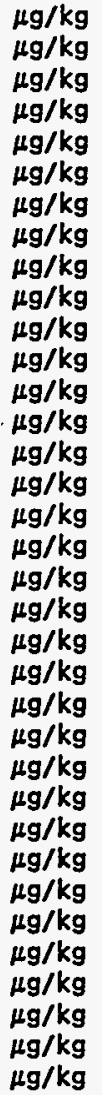 & $\begin{array}{r}2.20 \\
2.20 \\
2.20 \\
2.20 \\
2.20 \\
83.80 \\
2.20 \\
3.40 \\
2.10 \\
1.70 \\
4.20 \\
4.20 \\
2.20 \\
4.20 \\
4.20 \\
2.20 \\
2.20 \\
2.20 \\
2.20 \\
20.90 \\
2.20 \\
21.70 \\
0.80 \\
4.60 \\
22.00 \\
43.00 \\
22.00 \\
22.00 \\
22.00 \\
22.00 \\
22.00 \\
217.00 \\
4.20 \\
4.20 \\
4.20\end{array}$ \\
\hline
\end{tabular}




\section{C-25}

Table C.1 (continued)

\begin{tabular}{|c|c|c|c|c|c|}
\hline & & A horizon & & A horizon & \\
\hline Analysis & Units & Result & Qualifier & field dup & Qualifier \\
\hline
\end{tabular}

Location=ORR; Formation $=$ CHICKAMAUGA Bethel V; Site $=102 ; A=1894 ; A$ dup=' '; Phase $=2$

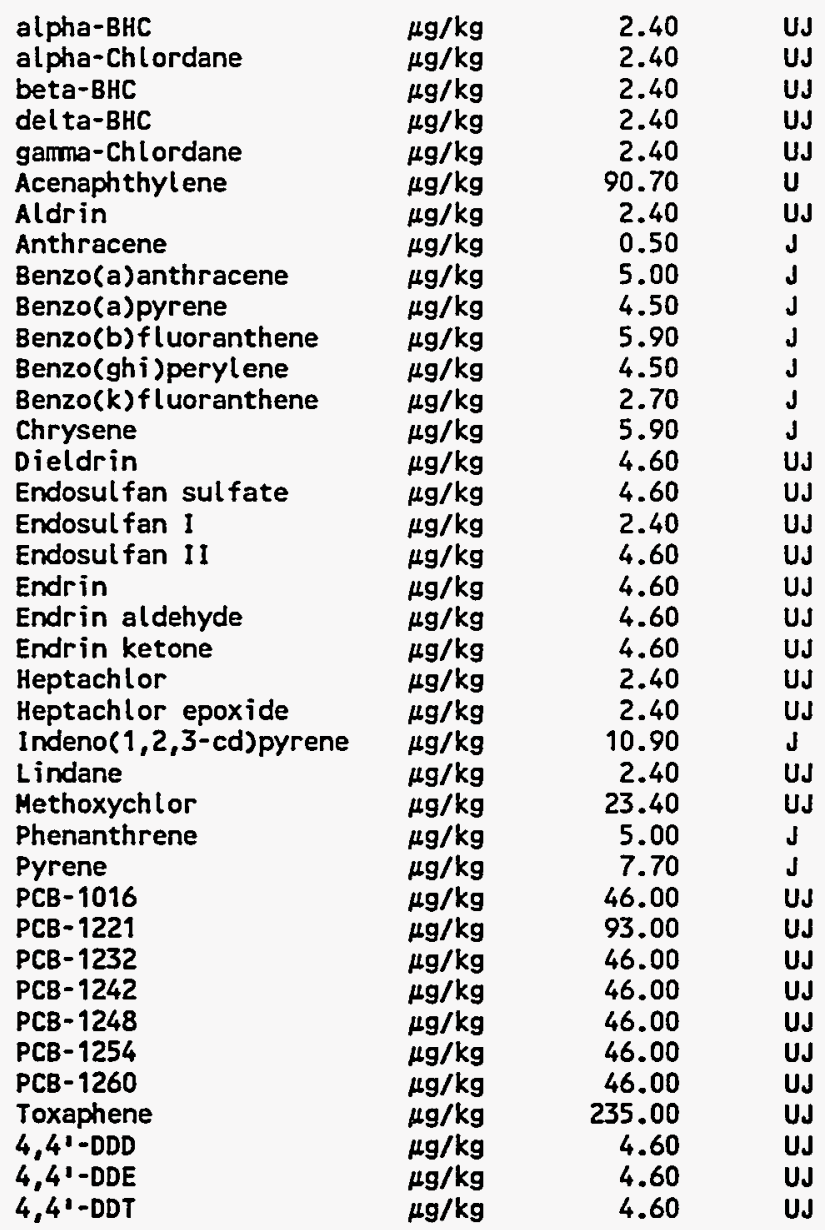

Location=ORR; Formation=CHICKAMAUGA Bethel V; Site=103; $A=1891 ; A$ dup=' '; Phase=2

\begin{tabular}{|c|c|c|}
\hline $\begin{array}{l}\text { alpha-BHC } \\
\text { alpha-Chlordane } \\
\text { beta-BHC } \\
\text { del ta-BHC } \\
\text { gamma-Chlordane } \\
\text { Acenaphthylene } \\
\text { Aldrin } \\
\text { Benzo(a)anthracene } \\
\text { Benzo(a)pyrene } \\
\text { Benzo(ghi)perylene } \\
\text { Benzo(k) fluoranthene } \\
\text { Chrysene } \\
\text { Dalapon } \\
\text { Dicamba } \\
\text { Dichlorprop } \\
\text { Dieldrin } \\
\text { Dinoseb } \\
\text { Endosulfan sulfate } \\
\text { Endosul fan I } \\
\text { Endosulfan II } \\
\text { Endrin } \\
\text { Endrin aldehyde }\end{array}$ & 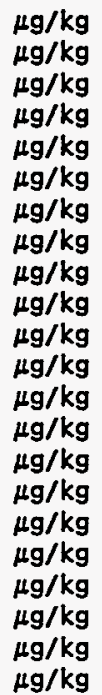 & $\begin{array}{r}2.20 \\
2.20 \\
2.20 \\
2.20 \\
2.20 \\
85.70 \\
2.20 \\
3.00 \\
3.40 \\
2.60 \\
1.70 \\
3.40 \\
4395.00 \\
201.00 \\
502.00 \\
4.20 \\
105.00 \\
4.20 \\
2.20 \\
4.20 \\
4.20 \\
4.20\end{array}$ \\
\hline
\end{tabular}


Table C.1 (continued)

\begin{tabular}{|c|c|c|c|c|c|}
\hline Analysis & Units & $\begin{array}{c}\text { A horizon } \\
\text { Result }\end{array}$ & Qualifier & $\begin{array}{l}\text { A horizon } \\
\text { field dup }\end{array}$ & Qualifier \\
\hline
\end{tabular}

Location=ORR; Formation=CHICKAMAUGA Bethel $V ;$ Site $=108 ; A=1967 ;$ A dup= " ; Phase $=2$ ", "

\begin{tabular}{|c|c|c|}
\hline 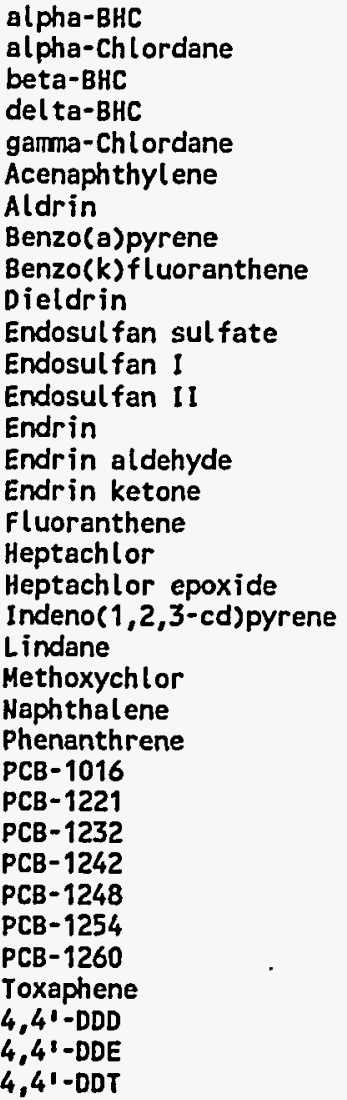 & $\begin{array}{l}\mu g / \mathrm{kg} \\
\mu g / \mathrm{kg} \\
\mu \mathrm{g} / \mathrm{kg} \\
\mu \mathrm{g} / \mathrm{kg} \\
\mu \mathrm{g} / \mathrm{kg} \\
\mu \mathrm{g} / \mathrm{kg} \\
\mu g / \mathrm{kg} \\
\mu \mathrm{g} / \mathrm{kg} \\
\mu \mathrm{g} / \mathrm{kg} \\
\mu \mathrm{g} / \mathrm{kg} \\
\mu \mathrm{g} / \mathrm{kg} \\
\mu \mathrm{g} / \mathrm{kg} \\
\mu \mathrm{g} / \mathrm{kg} \\
\mu \mathrm{g} / \mathrm{kg} \\
\mu \mathrm{g} / \mathrm{kg} \\
\mu \mathrm{g} / \mathrm{kg} \\
\mu \mathrm{g} / \mathrm{kg} \\
\mu \mathrm{g} / \mathrm{kg} \\
\mu \mathrm{g} / \mathrm{kg} \\
\mu \mathrm{g} / \mathrm{kg} \\
\mu \mathrm{g} / \mathrm{kg} \\
\mu \mathrm{g} / \mathrm{kg} \\
\mu \mathrm{g} / \mathrm{kg} \\
\mu \mathrm{g} / \mathrm{kg} \\
\mu \mathrm{g} / \mathrm{kg} \\
\mu \mathrm{g} / \mathrm{kg} \\
\mu \mathrm{g} / \mathrm{kg} \\
\mu \mathrm{g} / \mathrm{kg} \\
\mu \mathrm{g} / \mathrm{kg} \\
\mu \mathrm{g} / \mathrm{kg} \\
\mu \mathrm{g} / \mathrm{kg} \\
\mu \mathrm{g} / \mathrm{kg} \\
\mu \mathrm{g} / \mathrm{kg} \\
\mu \mathrm{g} / \mathrm{kg} \\
\mu g / \mathrm{kg}\end{array}$ & $\begin{array}{r}2.20 \\
2.20 \\
2.20 \\
2.20 \\
2.20 \\
86.30 \\
2.20 \\
0.90 \\
1.30 \\
4.30 \\
4.30 \\
2.20 \\
4.30 \\
4.30 \\
4.30 \\
4.30 \\
3.90 \\
2.20 \\
2.20 \\
21.60 \\
2.20 \\
22.10 \\
25.00 \\
3.50 \\
43.00 \\
87.00 \\
43.00 \\
43.00 \\
43.00 \\
43.00 \\
43.00 \\
221.00 \\
4.30 \\
4.30 \\
4.30\end{array}$ \\
\hline
\end{tabular}

Location=ORR; Formation=CHICKAMAUGA Bethel V; Site=110; $A=1970 ; A$ dup=' '; Phase=2

$\begin{array}{lrrl}\text { alpha-BHC } & \mu \mathrm{g} / \mathrm{kg} & 2.20 & \mathrm{UJ} \\ \text { alpha-Chlordane } & \mu \mathrm{g} / \mathrm{kg} & 2.20 & \mathrm{U} \\ \text { beta-BHC } & \mu \mathrm{g} / \mathrm{kg} & 2.20 & \mathrm{U} \\ \text { del ta-BHC } & \mu \mathrm{g} / \mathrm{kg} & 2.20 & \mathrm{U} \\ \text { gamma-Chlordane } & \mu \mathrm{g} / \mathrm{kg} & 2.20 & \mathrm{U} \\ \text { Acenaphthylene } & \mu \mathrm{g} / \mathrm{kg} & 88.50 & \mathrm{UJ} \\ \text { Aldrin } & \mu \mathrm{g} / \mathrm{kg} & 2.20 & \mathrm{U} \\ \text { Benzo(a)pyrene } & \mu \mathrm{g} / \mathrm{kg} & 3.50 & \mathrm{~J} \\ \text { Benzo(k)fluoranthene } & \mu \mathrm{g} / \mathrm{kg} & 1.80 & \mathrm{~J} \\ \text { Dibenzo(a,h)anthracene } & \mu \mathrm{g} / \mathrm{kg} & 0.40 & \mathrm{~J} \\ \text { Dieldrin } & \mu \mathrm{g} / \mathrm{kg} & 4.30 & \mathrm{U} \\ \text { Endosulfan sulfate } & \mu \mathrm{g} / \mathrm{kg} & 4.30 & \mathrm{U} \\ \text { Endosulfan I } & \mu \mathrm{g} / \mathrm{kg} & 2.20 & \mathrm{U} \\ \text { Endosulfan 11 } & \mu \mathrm{g} / \mathrm{kg} & 4.30 & \mathrm{U} \\ \text { Endrin } & \mu \mathrm{g} / \mathrm{kg} & 4.30 & \mathrm{U} \\ \text { Endrin aldehyde } & \mu \mathrm{g} / \mathrm{kg} & 4.30 & \mathrm{U} \\ \text { Endrin ketone } & \mu \mathrm{g} / \mathrm{kg} & 4.30 & \mathrm{U} \\ \text { Heptachlor } & \mu \mathrm{g} / \mathrm{kg} & 2.20 & \mathrm{U} \\ \text { Heptachlor epoxide } & \mu \mathrm{g} / \mathrm{kg} & 2.20 & \mathrm{U} \\ \text { Indeno(1,2,3-cd)pyrene } & \mu \mathrm{g} / \mathrm{kg} & 11.90 & \mathrm{~J} \\ \text { Lindane } & \mu \mathrm{g} / \mathrm{kg} & 2.20 & \mathrm{U} \\ \text { Methoxychlor } & \mu \mathrm{g} / \mathrm{kg} & 22.20 & \mathrm{U} \\ \text { Phenanthrene } & \mu \mathrm{g} / \mathrm{kg} & 4.40 & \mathrm{~J} \\ \text { PCB-1016 } & \mu \mathrm{g} / \mathrm{kg} & 43.00 & \mathrm{U} \\ \text { PCB-1221 } & \mu \mathrm{g} / \mathrm{kg} & 88.00 & \mathrm{U} \\ \text { PCB-1232 } & \mu \mathrm{g} / \mathrm{kg} & 43.00 & \mathrm{U}\end{array}$




\section{C-28}

Table C.1 (continucd)

\begin{tabular}{|c|c|c|c|c|c|}
\hline Analysis & Units & $\begin{array}{c}\text { A horizon } \\
\text { Result }\end{array}$ & Qualifier & $\begin{array}{l}\text { A horizon } \\
\text { field dup }\end{array}$ & Qualifier \\
\hline $\begin{array}{l}\text { PCB-1242 } \\
\text { PCB-1248 } \\
\text { PCB-1254 } \\
\text { PCB-1260 } \\
\text { Toxaphene } \\
4,4^{\prime}-\text {-DD } \\
4,4^{\prime}-D D E \\
4,4^{\prime}-D D T\end{array}$ & $\begin{array}{l}\mu g / \mathrm{kg} \\
\mu g / \mathrm{kg} \\
\mu g / \mathrm{kg} \\
\mu g / \mathrm{kg} \\
\mu g / \mathrm{kg} \\
\mu g / \mathrm{kg} \\
\mu g / \mathrm{kg} \\
\mu g / \mathrm{kg}\end{array}$ & $\begin{array}{r}43.00 \\
43.00 \\
43.00 \\
43.00 \\
222.00 \\
4.30 \\
4.30 \\
4.30\end{array}$ & $\begin{array}{l}U \\
U \\
U \\
U \\
U \\
U \\
U U \\
U\end{array}$ & & \\
\hline
\end{tabular}

Location=ORR; Formation=CHICKAMAUGA Bethel V; Site=115; $A=1973 ; A$ dup=' '; Phase=2

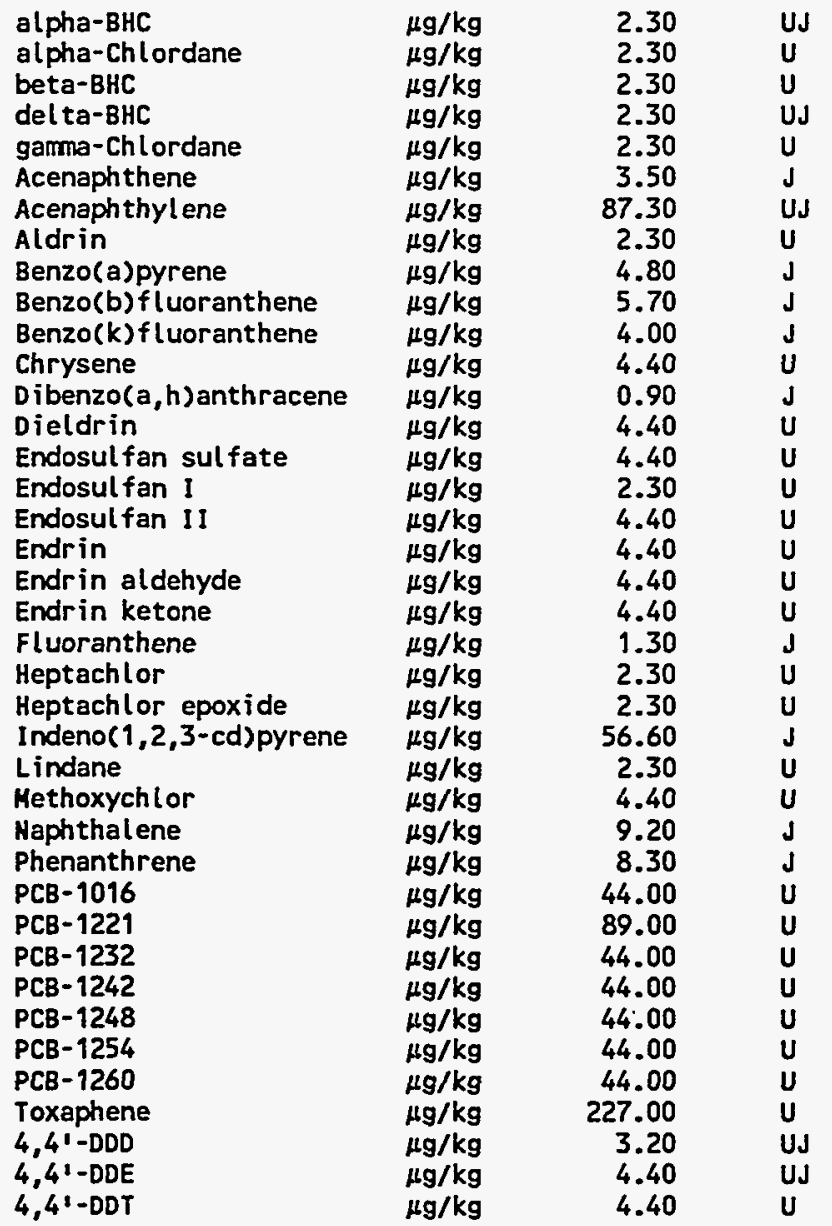

Location=ORR; Formation=CHICKAMAUGA Bethel V; Site=116; $A=1976 ; A$ dup=' '; Phase=2

$\begin{array}{llrl}\text { alpha-BHC } & \mu \mathrm{g} / \mathrm{kg} & 2.10 & \text { US } \\ \text { alpha-Chlordane } & \mu \mathrm{g} / \mathrm{kg} & 2.10 & \text { UJ } \\ \text { beta-BHC } & \mu \mathrm{g} / \mathrm{kg} & 2.10 & \text { UJ } \\ \text { delta-BHC } & \mu \mathrm{g} / \mathrm{kg} & 2.10 & \text { UJ } \\ \text { gamma-Chlordane } & \mu \mathrm{g} / \mathrm{kg} & 2.10 & \text { UJ } \\ \text { Acenaphthylene } & \mu \mathrm{g} / \mathrm{kg} & 83.10 & \text { UJ } \\ \text { Aldrin } & \mu \mathrm{g} / \mathrm{kg} & 2.10 & \text { UJ } \\ \text { Benzo(a)pyrene } & \mu \mathrm{g} / \mathrm{kg} & 4.20 & \mathrm{~J} \\ \text { Benzo(b)fluoranthene } & \mu \mathrm{g} / \mathrm{kg} & 4.20 & \mathrm{~J} \\ \text { Benzo(k)fluoranthene } & \mu \mathrm{g} / \mathrm{kg} & 2.90 & \mathrm{~J} \\ \text { Dieldrin } & \mu \mathrm{g} / \mathrm{kg} & 4.10 & \text { UJ } \\ \text { Endosulfan sulfate } & \mu \mathrm{g} / \mathrm{kg} & 4.10 & \text { UJ }\end{array}$


Table C.1 (continued)

\begin{tabular}{|c|c|c|c|c|c|c|}
\hline Analysis & Units & $\begin{array}{c}\text { A horizon } \\
\text { Result }\end{array}$ & Qualifier & $\begin{array}{l}\text { A horizon } \\
\text { field dup }\end{array}$ & Qualifier & \\
\hline $\begin{array}{l}\text { Endosulfan I } \\
\text { Endosul fan II } \\
\text { Endrin } \\
\text { Endrin aldehyde } \\
\text { Endrin ketone } \\
\text { Fluoranthene } \\
\text { Heptachlor } \\
\text { Heptachlor epoxide } \\
\text { Indeno(1,2,3-cd)pyrene } \\
\text { Lindane } \\
\text { Methoxychlor } \\
\text { Naphthalene } \\
\text { Phenanthrene } \\
\text { PCB-1016 } \\
\text { PCB-1221 } \\
\text { PCB-1232 } \\
\text { PCB-1242 } \\
\text { PCB-1248 } \\
\text { PCB-1254 } \\
\text { PCB-1260 } \\
\text { Toxaphene } \\
4,41-D D D \\
4,41-D D E \\
4,41-D D T\end{array}$ & 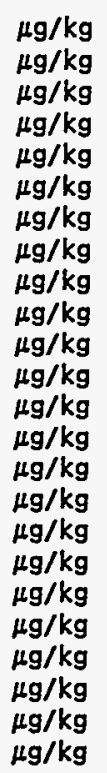 & $\begin{array}{r}2.10 \\
4.10 \\
4.10 \\
4.10 \\
4.10 \\
12.50 \\
2.10 \\
2.10 \\
5.80 \\
2.10 \\
21.40 \\
4.60 \\
11.20 \\
41.00 \\
84.00 \\
41.00 \\
41.00 \\
41.00 \\
41.00 \\
41.00 \\
214.00 \\
4.10 \\
4.10 \\
4.10\end{array}$ & $\begin{array}{l}\text { UJ : } \\
\text { UJ } \\
U J \\
U J \\
U J \\
J \\
U J \\
U J \\
J \\
U J \\
U J \\
J \\
J \\
U J \\
U J \\
U J \\
U J \\
\text { UJ } \\
\text { UJ } \\
\text { UJ } \\
\text { UJ } \\
\text { UJ } \\
\text { UJ } \\
\text { UJ }\end{array}$ & & . & \\
\hline
\end{tabular}

Location=ORR; Formation=CHICKAMAUGA Bethel V; Site $=117 ; A=1979 ; A$ dup =' '; Phase $=2$

\begin{tabular}{|c|c|c|}
\hline $\begin{array}{l}\text { alpha-BHC } \\
\text { alpha-Chlordane } \\
\text { beta-BHC } \\
\text { del ta-BHC } \\
\text { gamma-Chlordane } \\
\text { Acenaphthylene } \\
\text { Aldrin } \\
\text { Benzo(a)pyrene } \\
\text { Benzo(k) fluoranthene } \\
\text { Dieldrin } \\
\text { Endosulfan sulfate } \\
\text { Endosulfan I } \\
\text { Endosul fan II } \\
\text { Endrin } \\
\text { Endrin aldehyde } \\
\text { Endrin ketone } \\
\text { Fluoranthene } \\
\text { Fluorene } \\
\text { Heptachlor } \\
\text { Heptachlor epoxide } \\
\text { Lindane } \\
\text { Hethoxychlor } \\
\text { Naphthalene } \\
\text { Phenanthrene } \\
\text { PCB-1016 } \\
\text { PCB-1221 } \\
\text { PCB-1232 } \\
\text { PCB-1242 } \\
\text { PCB-1248 } \\
\text { PCB-1254 } \\
\text { PCB-1260 } \\
\text { Toxaphene } \\
4,41-D D D \\
4,41-D D E \\
4,4-D D T\end{array}$ & $\begin{array}{l}\mu g / k g \\
\mu g / k g \\
\mu g / k g \\
\mu g / k g \\
\mu g / k g \\
\mu g / k g \\
\mu g / k g \\
\mu g / k g \\
\mu g / k g \\
\mu g / k g \\
\mu g / k g \\
\mu g / k g \\
\mu g / k g \\
\mu g / k g \\
\mu g / k g \\
\mu g / k g \\
\mu g / k g \\
\mu g / k g \\
\mu g / k g \\
\mu g / k g \\
\mu g / k g \\
\mu g / k g \\
\mu g / k g \\
\mu g / k g \\
\mu g / k g \\
\mu g / k g \\
\mu g / k g \\
\mu g / k g \\
\mu g / k g \\
\mu g / k g \\
\mu g / k g \\
\mu g / k g \\
\mu g / k g \\
\mu g / k g \\
\mu g / k g\end{array}$ & $\begin{array}{r}2.20 \\
2.20 \\
2.20 \\
2.20 \\
2.20 \\
86.10 \\
2.20 \\
2.60 \\
0.90 \\
4.30 \\
4.30 \\
2.20 \\
4.30 \\
4.30 \\
4.30 \\
4.30 \\
6.00 \\
5.20 \\
2.20 \\
2.20 \\
2.20 \\
21.90 \\
15.10 \\
5.20 \\
43.00 \\
86.00 \\
43.00 \\
43.00 \\
43.00 \\
43.00 \\
43.00 \\
219.00 \\
4.30 \\
4.30 \\
4.30\end{array}$ \\
\hline
\end{tabular}


C-30

Table C.1 (continued)

\begin{tabular}{|c|c|c|c|c|c|}
\hline Analysis & Units & $\begin{array}{c}\text { A horizon } \\
\text { Result }\end{array}$ & Qualifier & $\begin{array}{l}\text { A horizon } \\
\text { field dup }\end{array}$ & Qualifier \\
\hline
\end{tabular}

Location=ORR; Formation $=$ CHICKAMAUGA Bethel V; Site=93; $A=1900 ; A$ dup=' '; Phase=2

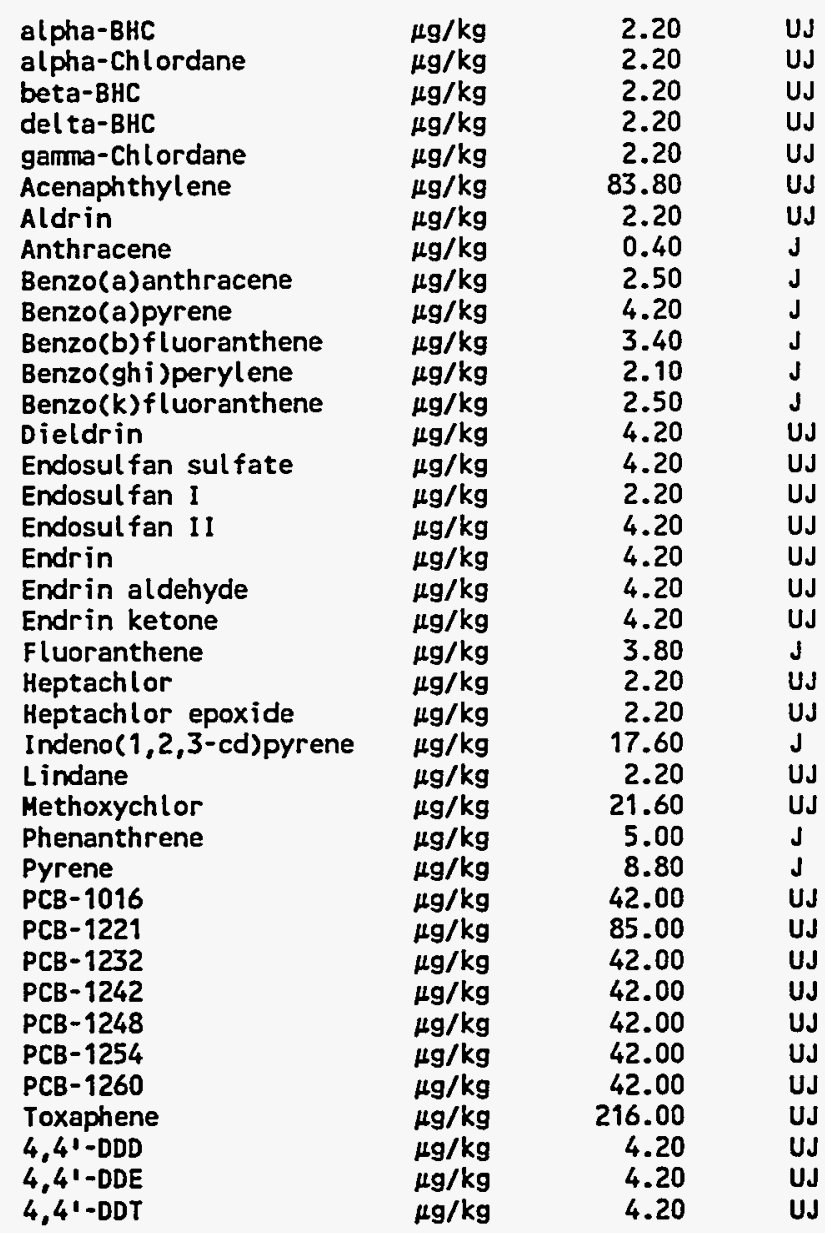

Location =ORR; Formation =CHICKAMAUGA Bethel V; Site=99; $A=1903 ; A$ dup=' '; Phase=2

$\begin{array}{lrrr}\text { alpha-BHC } & \mu \mathrm{g} / \mathrm{kg} & 2.20 & \mathrm{UJ} \\ \text { alpha-Chlordane } & \mu \mathrm{g} / \mathrm{kg} & 2.20 & \mathrm{UJ} \\ \text { beta-BHC } & \mu \mathrm{g} / \mathrm{kg} & 2.20 & \mathrm{UJ} \\ \text { delta-BHC } & \mu \mathrm{g} / \mathrm{kg} & 2.20 & \mathrm{UJ} \\ \text { gamma-Chlordane } & \mu \mathrm{g} / \mathrm{kg} & 2.20 & \mathrm{UJ} \\ \text { Acenaphthylene } & \mu \mathrm{gg} & 86.50 & \mathrm{U} \\ \text { Aldrin } & \mu \mathrm{g} / \mathrm{kg} & 2.20 & \mathrm{UJ} \\ \text { Anthracene } & \mu \mathrm{g} / \mathrm{kg} & 0.90 & \mathrm{~J} \\ \text { Benzo(a)anthracene } & \mu \mathrm{g} / \mathrm{kg} & 5.20 & \mathrm{~J} \\ \text { Benzo(a)pyrene } & \mu \mathrm{g} / \mathrm{kg} & 6.10 & \mathrm{~J} \\ \text { Benzo(b)fluoranthene } & \mu \mathrm{g} / \mathrm{kg} & 6.10 & \mathrm{~J} \\ \text { Benzo(ghi)perylene } & \mu \mathrm{g} / \mathrm{kg} & 4.30 & \mathrm{~J} \\ \text { Benzo(k)fluoranthene } & \mu \mathrm{g} / \mathrm{kg} & 3.00 & \mathrm{~J} \\ \text { Dalapon } & \mu \mathrm{g} / \mathrm{kg} & 4506.00 & \mathrm{UJ} \\ \text { Dicamba } & \mu \mathrm{g} / \mathrm{kg} & 206.00 & \mathrm{UJ} \\ \text { Dichlorprop } & \mu \mathrm{g} / \mathrm{kg} & 515.00 & \mathrm{UJ} \\ \text { Dieldrin } & \mu \mathrm{g} / \mathrm{kg} & 4.30 & \mathrm{UJ} \\ \text { Dinoseb } & \mu \mathrm{g} / \mathrm{kg} & 108.00 & \mathrm{UJ} \\ \text { Endosulfan sul fate } & \mu \mathrm{g} / \mathrm{kg} & 4.30 & \mathrm{UJ} \\ \text { Endosulfan I } & \mu \mathrm{g} / \mathrm{kg} & 2.20 & \mathrm{UJ} \\ \text { Endosul fan II } & \mu \mathrm{g} / \mathrm{kg} & 4.30 & \mathrm{UJ} \\ \text { Endrin } & \mu \mathrm{g} / \mathrm{kg} & 4.30 & \mathrm{UJ}\end{array}$


C-31

Table C.1 (continued)

\begin{tabular}{|c|c|c|c|c|c|c|}
\hline Analysis & Units & $\begin{array}{c}\text { A horizon } \\
\text { Result }\end{array}$ & Qualifier & $\begin{array}{l}\text { A horizon } \\
\text { field dup }\end{array}$ & Qualifier & \\
\hline $\begin{array}{l}\text { Endrin aldehyde } \\
\text { Endrin ketone } \\
\text { Fluoranthene } \\
\text { Heptechlor } \\
\text { Heptachlor epoxide } \\
\text { Indeno(1,2,3-cd)pyrene } \\
\text { Lindane } \\
\text { Hethoxychlor } \\
\text { MCPA } \\
\text { MCPP } \\
\text { Phenanthrene } \\
\text { Pyrene } \\
\text { PCB-1016 } \\
\text { PCB-1221 } \\
\text { PCB-1232 } \\
\text { PCB-1242 } \\
\text { PCB-1248 } \\
\text { PCB-1254 } \\
\text { PCB-1260 } \\
\text { Silvex } \\
\text { Toxaphene } \\
2,4-D \\
2,4-D B \\
2,4,5-T \\
4,42-D D D \\
4,41-D D E \\
4,41-D D T\end{array}$ & $\begin{array}{l}\mu g / k g \\
\mu g / k g \\
\mu g / k g \\
\mu g / k g \\
\mu g / k g \\
\mu g / k g \\
\mu g / k g \\
\mu g / k g \\
\mu g / k g \\
\mu g / k g \\
\mu g / k g \\
\mu g / k g \\
\mu g / k g \\
\mu g / k g \\
\mu g / k g \\
\mu g / k g \\
\mu g / k g \\
\mu g / k g \\
\mu g / k g \\
\mu g / k g \\
\mu g / k g \\
\mu g / k g \\
\mu g / k g \\
\mu g / k g \\
\mu g / k g \\
\mu g / k g \\
\mu g / k g\end{array}$ & $\begin{array}{r}4.30 \\
4.30 \\
10.80 \\
2.20 \\
2.20 \\
6.90 \\
2.20 \\
22.10 \\
193113.00 \\
146766.00 \\
6.50 \\
7.30 \\
43.00 \\
87.00 \\
43.00 \\
43.00 \\
43.00 \\
43.00 \\
43.00 \\
129.00 \\
221.00 \\
927.00 \\
695.00 \\
154.00 \\
4.30 \\
4.30 \\
4.30\end{array}$ & $\begin{array}{l}\text { UJ } \\
\text { UJ } \\
\mathbf{J} \\
\mathbf{U J} \\
\mathbf{U J} \\
\mathbf{J} \\
\mathbf{U J} \\
\mathbf{U J} \\
\mathbf{U J} \\
\mathbf{U J} \\
\mathbf{J} \\
\mathbf{J} \\
\mathbf{U J} \\
\mathbf{U J} \\
\mathbf{U J} \\
\mathbf{U J} \\
\mathbf{U J} \\
\mathbf{U J} \\
\mathbf{U J} \\
\mathbf{U J} \\
\mathbf{U J} \\
\mathbf{U J} \\
\mathbf{U J} \\
\mathbf{U J} \\
\mathbf{U J} \\
\mathbf{U J} \\
\mathbf{U J}\end{array}$ & ' & & \\
\hline
\end{tabular}

Location=ORR; Formation $=$ CHICKAMAUGA K-25; Site=118; $A=4095 ; A$ dup=' '; Phase $=2$

$\begin{array}{lrrl}\text { alpha-BHC } & \mu g / k g & 2.70 & \mathrm{UJ} \\ \text { alpha-Chlordane } & \mu \mathrm{g} / \mathrm{kg} & 2.70 & \mathrm{U} \\ \text { beta-BHC } & \mu \mathrm{g} / \mathrm{kg} & 2.70 & \mathrm{U} \\ \text { delta-BHC } & \mu \mathrm{g} / \mathrm{kg} & 2.70 & \mathrm{UJ} \\ \text { gamma-Chlordane } & \mu \mathrm{g} / \mathrm{kg} & 2.70 & \mathrm{U} \\ \text { Acenaphthene } & \mu \mathrm{g} / \mathrm{kg} & 2.40 & \mathrm{~J} \\ \text { Acenaphthylene } & \mu \mathrm{g} / \mathrm{kg} & 54.20 & \mathrm{U} \\ \text { Aldrin } & \mu \mathrm{g} / \mathrm{kg} & 2.70 & \mathrm{U} \\ \text { Anthracene } & \mu \mathrm{g} / \mathrm{kg} & 1.60 & \mathrm{~J} \\ \text { Benzo(a)anthracene } & \mu \mathrm{g} / \mathrm{kg} & 13.30 & \mathrm{~J} \\ \text { Benzo(a)pyrene } & \mu \mathrm{g} / \mathrm{kg} & 11.40 & \mathrm{~J} \\ \text { Benzo(b)fluoranthene } & \mu \mathrm{g} / \mathrm{kg} & 12.70 & \mathrm{~J} \\ \text { Benzo(ghi)perylene } & \mu \mathrm{g} / \mathrm{kg} & 11.10 & \mathrm{~J} \\ \text { Benzo(k)fluoranthene } & \mu \mathrm{g} / \mathrm{kg} & 8.70 & \mathrm{~J} \\ \text { Chrysene } & \mu \mathrm{g} / \mathrm{kg} & 15.20 & \mathrm{~J} \\ \text { Dalapon } & \mu \mathrm{g} / \mathrm{kg} & 5304.00 & \mathrm{UJ} \\ \text { Dibenzo(a,h)anthracene } & \mu \mathrm{g} / \mathrm{kg} & 0.80 & \mathrm{~J} \\ \text { Dicamba } & \mu \mathrm{g} / \mathrm{kg} & 242.00 & \mathrm{U} \\ \text { Dichlorprop } & \mu \mathrm{g} / \mathrm{kg} & 606.00 & \mathrm{UJ} \\ \text { Dieldrin } & \mu \mathrm{g} / \mathrm{kg} & 5.40 & \mathrm{U} \\ \text { Dinoseb } & \mu \mathrm{g} / \mathrm{kg} & 127.00 & \mathrm{U} \\ \text { Endosulfan sulfate } & \mu \mathrm{g} / \mathrm{kg} & 5.40 & \mathrm{U} \\ \text { Endosulfan I } & \mu \mathrm{g} / \mathrm{kg} & 2.70 & \mathrm{U} \\ \text { Endosulfan II } & \mu \mathrm{g} / \mathrm{kg} & 5.40 & \mathrm{U} \\ \text { Endrin } & \mu \mathrm{g} / \mathrm{kg} & 5.40 & \mathrm{U} \\ \text { Endrin aldehyde } & \mu \mathrm{g} / \mathrm{kg} & 5.40 & \mathrm{U} \\ \text { Endrin ketone } & \mu \mathrm{g} / \mathrm{kg} & 5.40 & \mathrm{U} \\ \text { Fluoranthene } & \mu \mathrm{g} / \mathrm{kg} & 7.00 & \mathrm{~J} \\ \text { Heptachlor } & \mu \mathrm{g} / \mathrm{kg} & 2.70 & \mathrm{U} \\ \text { Heptachlor epoxide } & \mu \mathrm{g} / \mathrm{kg} & 2.70 & \mathrm{U} \\ \text { Indeno(1,2,3-cd)pyrene } & \mu \mathrm{g} / \mathrm{kg} & 28.50 & \\ \text { Lindane } & \mu \mathrm{g} / \mathrm{kg} & 2.70 & \mathrm{UJ} \\ \text { Methoxychlor } & \mu \mathrm{g} / \mathrm{kg} & 27.00 & \mathrm{U} \\ \text { MCPA } & \mu \mathrm{g} / \mathrm{kg} & 227314.00 & \mathrm{UJ} \\ \text { MCPP } & \mu \mathrm{g} / \mathrm{kg} & 172759.00 & \mathrm{UJ} \\ \text { Naphthalene } & \mu \mathrm{g} / \mathrm{kg} & 3.00 & \mathrm{~J} \\ \text { Phenanthrene } & \mu \mathrm{g} / \mathrm{kg} & 16.50 & \mathrm{~J}\end{array}$




\section{C- -32}

Table C.1 (continued)

\begin{tabular}{|c|c|c|c|c|c|}
\hline Analysis & Units & $\begin{array}{c}\text { A horizon } \\
\text { Result }\end{array}$ & Qualifier & $\begin{array}{l}\text { A horizon } \\
\text { field dup }\end{array}$ & Qualifier \\
\hline $\begin{array}{l}\text { Pyrene } \\
\text { PCB-1016 } \\
\text { PCB-1221 } \\
\text { PCB-1232 } \\
\text { PCB-1242 } \\
\text { PCB-1248 } \\
\text { PCB-1254 } \\
\text { PCB-1260 } \\
\text { silvex } \\
\text { Toxaphene } \\
2,4-D \\
2,4-D B \\
2,4,5-T \\
4,4^{\prime} \text {-DDD } \\
4,4^{\prime} \text {-DDE } \\
4,4^{\prime} \text {-DDT }\end{array}$ & $\begin{array}{l}\mu g / \mathrm{kg} \\
\mu \mathrm{g} / \mathrm{kg} \\
\mu \mathrm{g} / \mathrm{kg} \\
\mu \mathrm{g} / \mathrm{kg} \\
\mu \mathrm{g} / \mathrm{kg} \\
\mu \mathrm{g} / \mathrm{kg} \\
\mu \mathrm{g} / \mathrm{kg} \\
\mu \mathrm{g} / \mathrm{kg} \\
\mu \mathrm{g} / \mathrm{kg} \\
\mu \mathrm{g} / \mathrm{kg} \\
\mu g / \mathrm{kg} \\
\mu \mathrm{g} / \mathrm{kg} \\
\mu \mathrm{g} / \mathrm{kg} \\
\mu \mathrm{g} / \mathrm{kg} \\
\mu g / \mathrm{kg} \\
\mu \mathrm{g} / \mathrm{kg}\end{array}$ & $\begin{array}{r}26.80 \\
54.00 \\
110.00 \\
54.00 \\
54.00 \\
54.00 \\
54.00 \\
54.00 \\
152.00 \\
270.00 \\
1091.00 \\
818.00 \\
182.00 \\
5.40 \\
5.40 \\
5.40\end{array}$ & $\begin{array}{l}J \\
U \\
U \\
U \\
U \\
U \\
U \\
U \\
U \\
U \\
U \\
U \\
U \\
U \\
U \\
U J\end{array}$ & & \\
\hline
\end{tabular}

Location=ORR; Formation=CHICKAMAUGA K-25; Site=119; $A=4127 ; A$ dup=' '; Phase $=2$

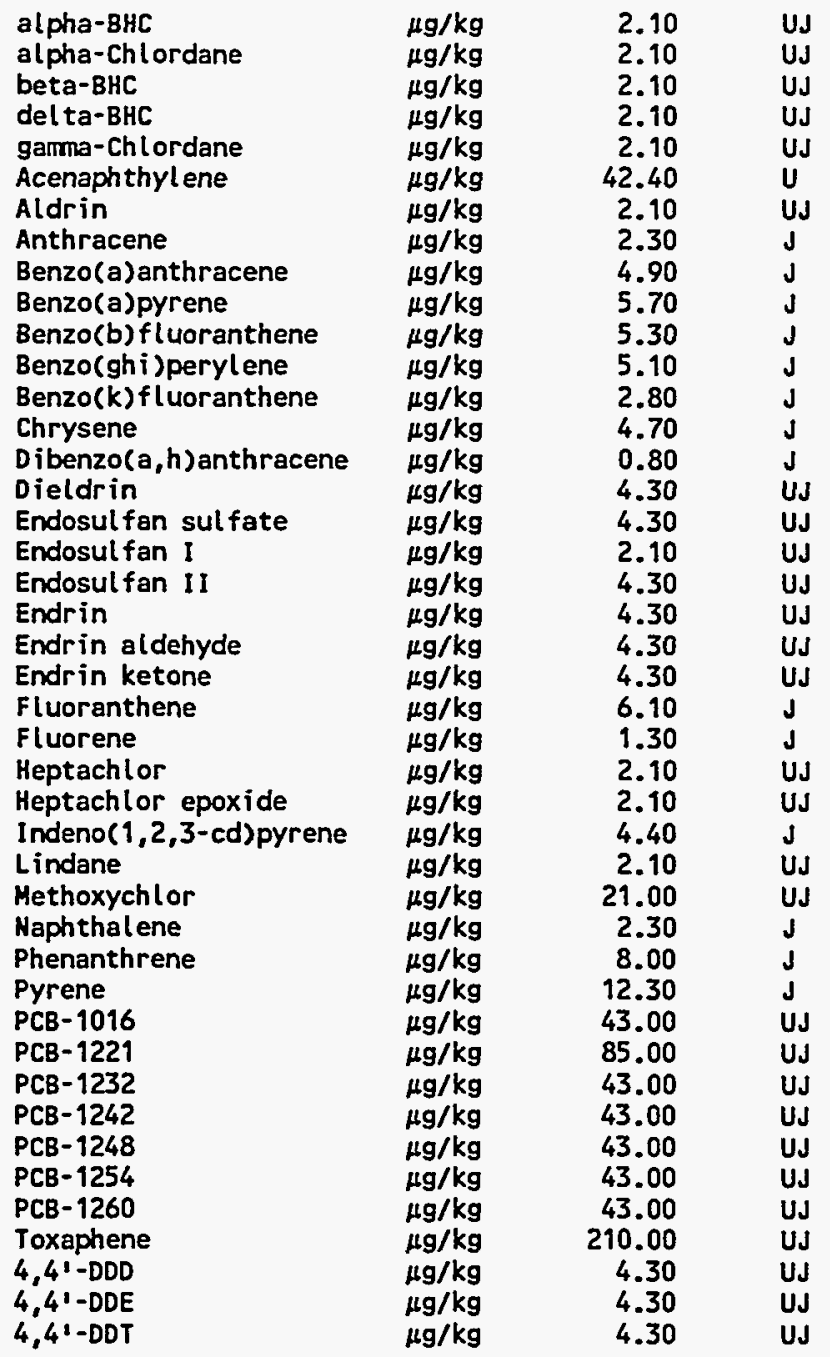




\section{C-33}

Table C.1 (continued)

\begin{tabular}{lcccc}
\hline Analysis & Anits & $\begin{array}{c}\text { A horizon } \\
\text { Result }\end{array}$ & Qualifier & $\begin{array}{c}\text { A horizon } \\
\text { field dup }\end{array}$ Qualifier \\
\hline
\end{tabular}

Location=ORR; Formation=CHICKAMAUGA K-25; Site=120; $A=4092 ; A$ dup=' '; Phase=2

\begin{tabular}{|c|c|c|}
\hline $\begin{array}{l}\text { alpha-BHC } \\
\text { alpha-Chlordane } \\
\text { beta-BHC } \\
\text { del ta-BHC } \\
\text { garma-Chlordane } \\
\text { ACenaphthylene } \\
\text { Aldrin } \\
\text { Anthracene } \\
\text { Benzo(a)anthracene } \\
\text { Benzo(a)pyrene } \\
\text { Benzo(b)fluoranthene } \\
\text { Benzo(ghi)perylene } \\
\text { Benzo(k)fluoranthene } \\
\text { Dalapon } \\
\text { Dicamba } \\
\text { Dichlorprop } \\
\text { Dieldrin } \\
\text { Dinoseb } \\
\text { Endosulfan sulfate } \\
\text { Endosulfan } 1 \\
\text { Endosulfan II } \\
\text { Endrin } \\
\text { Endrin aldehyde } \\
\text { Endrin ketone } \\
\text { Fluoranthene } \\
\text { Fluorene } \\
\text { Heptachlor } \\
\text { Heptachlor epoxide } \\
\text { Indeno(1,2,3-cd)pyrene } \\
\text { Lindane } \\
\text { Hethoxychlor } \\
\text { MCPA } \\
\text { MCPP } \\
\text { Phenanthrene } \\
\text { Pyrene } \\
\text { PCB-1016 } \\
\text { PCB-1221 } \\
\text { PCB-1232 } \\
\text { PCB-1242 } \\
\text { PCB-1248 } \\
\text { PCB-1254 } \\
\text { PCB-1260 } \\
\text { Silvex } \\
\text { Toxaphene } \\
2,4-D \\
2,4-D B \\
\text { 2,4,5-T } \\
4,4 \text {-DDD } \\
4,4 '-D D E \\
4,4 \cdot-D D T\end{array}$ & $\begin{array}{l}\mu g / k g \\
\mu g / k g \\
\mu g / k g \\
\mu g / k g \\
\mu g / k g \\
\mu g / k g \\
\mu g / k g \\
\mu g / k g \\
\mu g / k g \\
\mu g / k g \\
\mu g / k g \\
\mu g / k g \\
\mu g / k g \\
\mu g / k g \\
\mu g / k g \\
\mu g / k g \\
\mu g / k g \\
\mu g / k g \\
\mu g / k g \\
\mu g / k g \\
\mu g / k g \\
\mu g / k g \\
\mu g / k g \\
\mu g / k g \\
\mu g / k g \\
\mu g / k g \\
\mu g / k g \\
\mu g / k g \\
\mu g / k g \\
\mu g / k g \\
\mu g / k g \\
\mu g / k g \\
\mu g / k g \\
\mu g / k g \\
\mu g / k g \\
\mu g / k g \\
\mu g / k g \\
\mu g / k g \\
\mu g / k g \\
\mu g / k g \\
\mu g / k g \\
\mu g / k g \\
\mu g / k g \\
\mu g / k g \\
\mu g / k g \\
\mu g / k g \\
\mu g / k g \\
\mu g / k g \\
\mu g / k g \\
\mu g / k g\end{array}$ & $\begin{array}{r}2.40 \\
2.40 \\
2.40 \\
2.40 \\
2.40 \\
48.30 \\
2.40 \\
2.20 \\
15.70 \\
11.10 \\
10.60 \\
10.90 \\
5.80 \\
4867.00 \\
195.00 \\
488.00 \\
4.80 \\
102.00 \\
4.80 \\
2.40 \\
4.80 \\
4.80 \\
4.80 \\
4.80 \\
4.30 \\
4.60 \\
2.40 \\
2.40 \\
34.10 \\
2.40 \\
24.00 \\
183025.00 \\
139099.00 \\
12.80 \\
22.20 \\
48.00 \\
96.00 \\
48.00 \\
48.00 \\
48.00 \\
48.00 \\
48.00 \\
122.00 \\
240.00 \\
879.00 \\
659.00 \\
146.00 \\
4.80 \\
4.80 \\
4.80\end{array}$ \\
\hline
\end{tabular}

Location=ORR; Formation=CHICKAMAUGA K-25; Site=121; $A=4129 ; A$ dup=' '; Phase $=2$

$\begin{array}{llrl}\text { alpha-BHC } & \mu \mathrm{g} / \mathrm{kg} & 2.30 & \mathrm{UJ} \\ \text { alpha-Chlordane } & \mu \mathrm{g} / \mathrm{kg} & 3.60 & \mathrm{~J} \\ \text { beta-BHC } & \mu \mathrm{g} / \mathrm{kg} & 2.30 & \mathrm{UJ} \\ \text { del ta-BHC } & \mu \mathrm{g} / \mathrm{kg} & 2.30 & \mathrm{UJ} \\ \text { gamma-Chlordane } & \mu \mathrm{g} / \mathrm{kg} & 2.30 & \mathrm{UJ} \\ \text { Acenaphthene } & \mu \mathrm{g} / \mathrm{kg} & 0.90 & \mathrm{~J} \\ \text { Acenaphthylene } & \mu \mathrm{g} / \mathrm{kg} & 45.30 & \mathrm{U} \\ \text { Aldrin } & \mu \mathrm{g} / \mathrm{kg} & 2.30 & \mathrm{UJ} \\ \text { Anthracene } & \mu \mathrm{g} / \mathrm{kg} & 1.40 & \mathrm{~J} \\ \text { Benzo(a)anthracene } & \mu \mathrm{g} / \mathrm{kg} & 9.50 & \mathrm{~J} \\ \text { Benzo(a)pyrene } & \mu \mathrm{g} / \mathrm{kg} & 9.70 & \mathrm{~J}\end{array}$




\section{C-34}

Table C.1 (continued)

\begin{tabular}{|c|c|c|c|c|c|}
\hline Analysis & Units & $\begin{array}{c}\text { A horizon } \\
\text { Result }\end{array}$ & Qualifier & $\begin{array}{l}\text { A horizon } \\
\text { field dup }\end{array}$ & Qualifier \\
\hline $\begin{array}{l}\text { Benzo(b)fluoranthene } \\
\text { Benzo(ghi)perylene } \\
\text { Benzo(k)fluoranthene } \\
\text { Chrysene } \\
\text { Dieldrin } \\
\text { Endosulfan sulfate } \\
\text { Endosulfan I } \\
\text { Endosul fan II } \\
\text { Endrin } \\
\text { Endrin aldehyde } \\
\text { Endrin ketone } \\
\text { Fluoranthene } \\
\text { Fluorene } \\
\text { Heptachlor } \\
\text { Heptachlor epoxide } \\
\text { Indeno( } 1,2,3-\text {-cd)pyrene } \\
\text { Lindane } \\
\text { Methoxychlor } \\
\text { Naphthalene } \\
\text { Phenanthrene } \\
\text { Pyrene } \\
\text { PCB-1016 } \\
\text { PCB-1221 } \\
\text { PCB-1232 } \\
\text { PCB-1242 } \\
\text { PCB-1248 } \\
\text { PCB-1254 } \\
\text { PCB-1260 } \\
\text { Toxaphene } \\
4,41-D D D \\
4,41-D D E \\
4,41-D D T\end{array}$ & 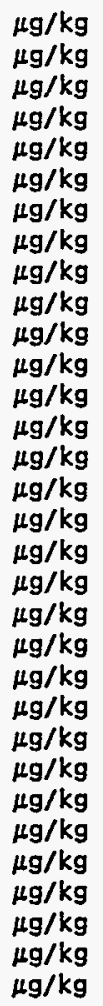 & $\begin{array}{r}11.60 \\
8.80 \\
5.40 \\
12.70 \\
4.50 \\
4.50 \\
2.30 \\
4.50 \\
4.50 \\
4.50 \\
4.50 \\
22.20 \\
0.90 \\
2.30 \\
2.30 \\
9.10 \\
2.30 \\
23.00 \\
5.40 \\
12.90 \\
24.50 \\
45.00 \\
90.00 \\
45.00 \\
45.00 \\
45.00 \\
45.00 \\
45.00 \\
230.00 \\
4.50 \\
4.50 \\
4.50\end{array}$ & $\begin{array}{l}\mathbf{J} \\
\mathbf{J} \\
\mathbf{J} \\
\mathbf{J} \\
\text { UJ } \\
\text { UJ } \\
\text { UJ } \\
\text { UJ } \\
\text { UJ } \\
\text { UJ } \\
\text { UJ } \\
\mathbf{J} \\
\mathbf{J} \\
\text { UJ } \\
\text { UJ } \\
\mathbf{J} \\
\text { UJ } \\
\text { UJ } \\
\mathbf{J} \\
\mathbf{J} \\
\mathbf{J} \\
\text { UJ } \\
\text { UJ } \\
\text { UJ } \\
\text { UJ } \\
\text { UJ } \\
\text { UJ } \\
\text { UJ } \\
\text { UJ } \\
\text { UJ } \\
\text { UJ } \\
\text { UJ }\end{array}$ & & \\
\hline
\end{tabular}

Location=ORR; Formation=CHICKAMAUGA K-25; Site=122; $A=4105 ; A$ dup=' '; Phase $=2$

$\begin{array}{lrrl}\text { alpha-BHC } & \mu \mathrm{g} / \mathrm{kg} & 2.20 & \mathrm{UJ} \\ \text { alpha-Chlordane } & \mu \mathrm{g} / \mathrm{kg} & 2.20 & \mathrm{U} \\ \text { beta-BHC } & \mu \mathrm{g} / \mathrm{kg} & 2.20 & \mathrm{U} \\ \text { delta-BHC } & \mu \mathrm{g} / \mathrm{kg} & 2.20 & \mathrm{UJ} \\ \text { gamma-Chlordane } & \mu \mathrm{g} / \mathrm{kg} & 2.20 & \mathrm{U} \\ \text { Acenaphthylene } & \mu \mathrm{g} / \mathrm{kg} & 44.40 & \mathrm{U} \\ \text { Aldrin } & \mu \mathrm{g} / \mathrm{kg} & 2.20 & \mathrm{U} \\ \text { Anthracene } & \mu \mathrm{g} / \mathrm{kg} & 2.40 & \mathrm{~J} \\ \text { Benzo(a)anthracene } & \mu \mathrm{g} / \mathrm{kg} & 5.50 & \mathrm{~J} \\ \text { Benzo(a)pyrene } & \mu \mathrm{g} / \mathrm{kg} & 5.10 & \mathrm{~J} \\ \text { Benzo(b)fluoranthene } & \mu \mathrm{g} / \mathrm{kg} & 3.80 & \mathrm{~J} \\ \text { Benzo(ghi)perylene } & \mu \mathrm{g} / \mathrm{kg} & 5.80 & \mathrm{~J} \\ \text { Benzo(k)fluoranthene } & \mu \mathrm{g} / \mathrm{kg} & 2.40 & \mathrm{~J} \\ \text { Dalapon } & \mu \mathrm{g} / \mathrm{kg} & 4766.00 & \mathrm{UJ} \\ \text { Dibenzo(a,h)anthracene } & \mu \mathrm{gg} / \mathrm{kg} & 0.70 & \mathrm{~J} \\ \text { Dicamba } & \mu \mathrm{g} / \mathrm{kg} & 218.00 & \mathrm{U} \\ \text { Dichlorprop } & \mu \mathrm{g} / \mathrm{kg} & 545.00 & \mathrm{UJ} \\ \text { Dieldrin } & \mu \mathrm{g} / \mathrm{kg} & 4.50 & \mathrm{U} \\ \text { Dinoseb } & \mu \mathrm{g} / \mathrm{kg} & 114.00 & \mathrm{U} \\ \text { Endosulfan sulfate } & \mu \mathrm{g} / \mathrm{kg} & 4.50 & \mathrm{U} \\ \text { Endosulfan I } & \mu \mathrm{g} / \mathrm{kg} & 2.20 & \mathrm{U} \\ \text { Endosulfan II } & \mu \mathrm{g} / \mathrm{kg} & 4.50 & \mathrm{U} \\ \text { Endrin } & \mu \mathrm{g} / \mathrm{kg} & 4.50 & \mathrm{U} \\ \text { Endrin aldehyde } & \mu \mathrm{g} / \mathrm{kg} & 4.50 & \mathrm{U} \\ \text { Endrin ketone } & \mu \mathrm{g} / \mathrm{kg} & 4.50 & \mathrm{U} \\ \text { Fluoranthene } & \mu \mathrm{g} / \mathrm{kg} & 6.00 & \mathrm{~J} \\ \text { Fluorene } & \mu \mathrm{g} / \mathrm{kg} & 3.80 & \mathrm{~J} \\ \text { Heptachlor } & \mu \mathrm{g} / \mathrm{kg} & 2.20 & \mathrm{U} \\ \text { Heptachlor epoxide } & \mu \mathrm{g} / \mathrm{kg} & 2.20 & \mathrm{U} \\ \text { Indeno(1,2,3-cd)pyrene } & \mu \mathrm{g} / \mathrm{kg} & 11.10 & \mathrm{U} \\ \text { Lindane } & \mu \mathrm{g} / \mathrm{kg} & 2.20 & \mathrm{UJ} \\ \text { Methoxychlor } & \mu \mathrm{g} / \mathrm{kg} & 22.00 & \mathrm{U}\end{array}$


Table C.1 (continued)

\begin{tabular}{|c|c|c|c|c|c|c|}
\hline Analysis & Units & $\begin{array}{c}\text { A horizon } \\
\text { Result }\end{array}$ & Qualifier & $\begin{array}{l}\text { A horizon } \\
\text { field dup }\end{array}$ & Qualifier & \\
\hline $\begin{array}{l}\text { MCPA } \\
\text { HCPP } \\
\text { Phenanthrene } \\
\text { Pyrene } \\
\text { PCB-1016 } \\
\text { PCB-1221 } \\
\text { PCB-1232 } \\
\text { PCB-1242 } \\
\text { PCB- } 1248 \\
\text { PCB-1254 } \\
\text { PCB-1260 } \\
\text { Silvex } \\
\text { TOxaphene } \\
2,4-D \\
2,4-D B \\
2,4,5-T \\
4,41-D D D \\
4,41-D D E \\
4,41-D D T\end{array}$ & $\begin{array}{l}\mu g / \mathrm{kg} \\
\mu g / \mathrm{kg} \\
\mu g / \mathrm{kg} \\
\mu g / \mathrm{kg} \\
\mu g / \mathrm{kg} \\
\mu g / \mathrm{kg} \\
\mu g / \mathrm{kg} \\
\mu \mathrm{g} / \mathrm{kg} \\
\mu \mathrm{g} / \mathrm{kg} \\
\mu g / \mathrm{kg} \\
\mu g / \mathrm{kg} \\
\mu g / \mathrm{kg} \\
\mu g / \mathrm{kg} \\
\mu g / \mathrm{kg} \\
\mu g / \mathrm{kg} \\
\mu g / \mathrm{kg} \\
\mu g / \mathrm{kg} \\
\mu g / \mathrm{kg} \\
\mu g / \mathrm{kg}\end{array}$ & $\begin{array}{r}204237.00 \\
155220.00 \\
4.70 \\
7.80 \\
45.00 \\
90.00 \\
45.00 \\
45.00 \\
45.00 \\
45.00 \\
45.00 \\
136.00 \\
220.00 \\
980.00 \\
735.00 \\
163.00 \\
4.50 \\
4.50 \\
4.50\end{array}$ & $\begin{array}{l}U J^{\prime}=R \\
U J \\
J \\
J \\
U \\
U \\
U \\
U \\
U \\
U \\
U \\
U \\
U \\
U \\
U \\
U \\
U \\
U \\
U J\end{array}$ & . & & $:$ \\
\hline
\end{tabular}

Location=ORR; Formation=CHICKAMAUGA $K-25 ;$ Site $=123 ; A=4131 ; A$ dup=' '; Phase $=2$

\begin{tabular}{|c|c|c|}
\hline 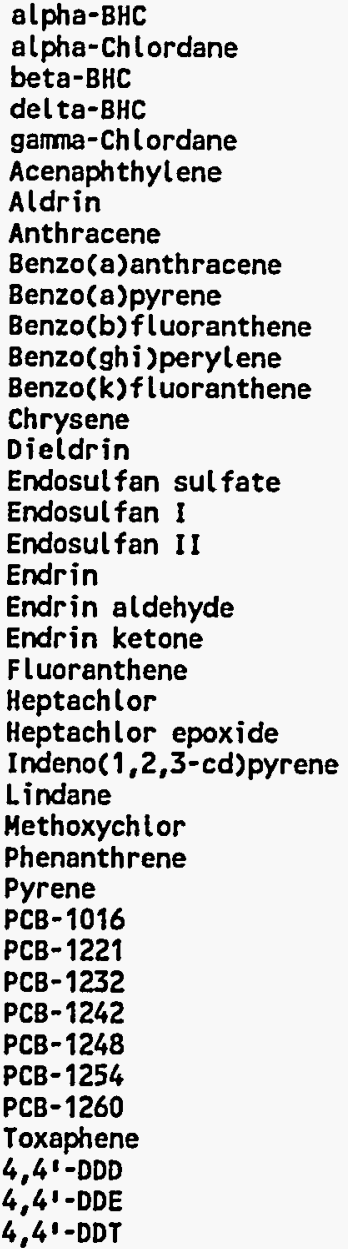 & $\begin{array}{l}\mu g / k g \\
\mu g / k g \\
\mu g / k g \\
\mu g / k g \\
\mu g / k g \\
\mu g / k g \\
\mu g / k g \\
\mu g / k g \\
\mu g / k g \\
\mu g / k g \\
\mu g / k g \\
\mu g / k g \\
\mu g / k g \\
\mu g / k g \\
\mu g / k g \\
\mu g / k g \\
\mu g / k g \\
\mu g / k g \\
\mu g / k g \\
\mu g / k g \\
\mu g / k g \\
\mu g / k g \\
\mu g / k g \\
\mu g / k g \\
\mu g / k g \\
\mu g / k g \\
\mu g / k g \\
\mu g / k g \\
\mu g / k g \\
\mu g / k g \\
\mu g / k g \\
\mu g / k g \\
\mu g / k g \\
\mu g / k g \\
\mu g / k g \\
\mu g / k g \\
\mu g / k g \\
\mu g / k g \\
\mu g / k g \\
\mu g / k g\end{array}$ & $\begin{array}{r}2.30 \\
2.30 \\
2.30 \\
2.30 \\
2.30 \\
45.30 \\
2.30 \\
0.90 \\
6.60 \\
7.70 \\
7.20 \\
5.90 \\
4.10 \\
7.70 \\
4.60 \\
4.60 \\
2.30 \\
4.60 \\
4.60 \\
4.60 \\
4.60 \\
15.20 \\
2.30 \\
2.30 \\
20.20 \\
2.30 \\
23.00 \\
9.10 \\
17.40 \\
46.00 \\
92.00 \\
46.00 \\
46.00 \\
46.00 \\
46.00 \\
46.00 \\
230.00 \\
4.60 \\
4.60 \\
4.60\end{array}$ \\
\hline
\end{tabular}


C-36

Table C.1 (continued)

\begin{tabular}{|c|c|c|c|c|c|}
\hline Analysis & Units & $\begin{array}{c}\text { A horizon } \\
\text { Result }\end{array}$ & Qualifier & $\begin{array}{l}\text { A horizon } \\
\text { field dup }\end{array}$ & Qualifier \\
\hline
\end{tabular}

Location $=O R R ;$ Formation $=C H I C K A M A U G A$ K-25; Site $=124 ; A=4115 ; A$ dup=' ' Phase $=2$

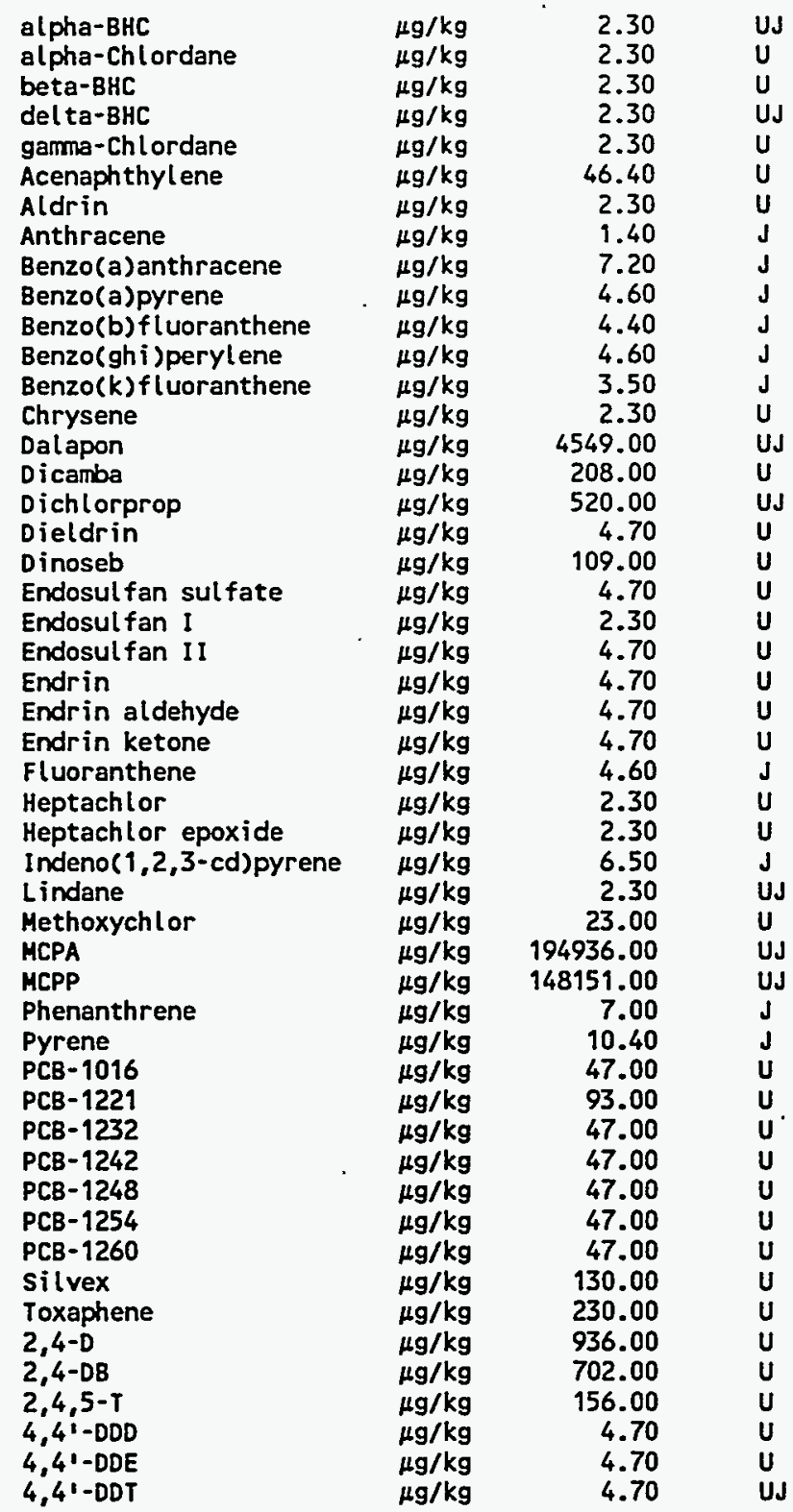

Location=ORR; Formation $=$ CHICKAMAUGA K-25; Site $=125 ; A=4133 ; A$ dup=' '; Phase $=2$

$\begin{array}{llrl}\text { alpha-BHC } & \mu \mathrm{g} / \mathrm{kg} & 2.40 & \mathrm{UJ} \\ \text { alpha-Chlordane } & \mu \mathrm{g} / \mathrm{kg} & 2.40 & \mathrm{UJ} \\ \text { beta-BHC } & \mu \mathrm{kg} & 2.40 & \mathrm{UJ} \\ \text { del ta-BHC } & \mu \mathrm{g} / \mathrm{kg} & 2.40 & \mathrm{UJ} \\ \text { gamma-Chlordane } & \mu \mathrm{g} / \mathrm{kg} & 2.40 & \mathrm{UJ} \\ \text { Acenaphthylene } & \mu \mathrm{gg} & 47.30 & \mathrm{U} \\ \text { Aldrin } & \mu \mathrm{k} / \mathrm{kg} & 2.40 & \mathrm{UJ} \\ \text { Anthracene } & \mu \mathrm{g} & 0.70 & \mathrm{~J} \\ \text { Benzo(a)anthracene } & \mu \mathrm{g} / \mathrm{kg} & 6.60 & \mathrm{~J} \\ \text { Benzo(a)pyrene } & \mu \mathrm{g} / \mathrm{kg} & 4.50 & \mathrm{~J} \\ \text { Benzo(b)fluoranthene } & \mu \mathrm{g} / \mathrm{kg} & 3.50 & \mathrm{~J}\end{array}$




\section{C-37}

Table C.1 (continued)

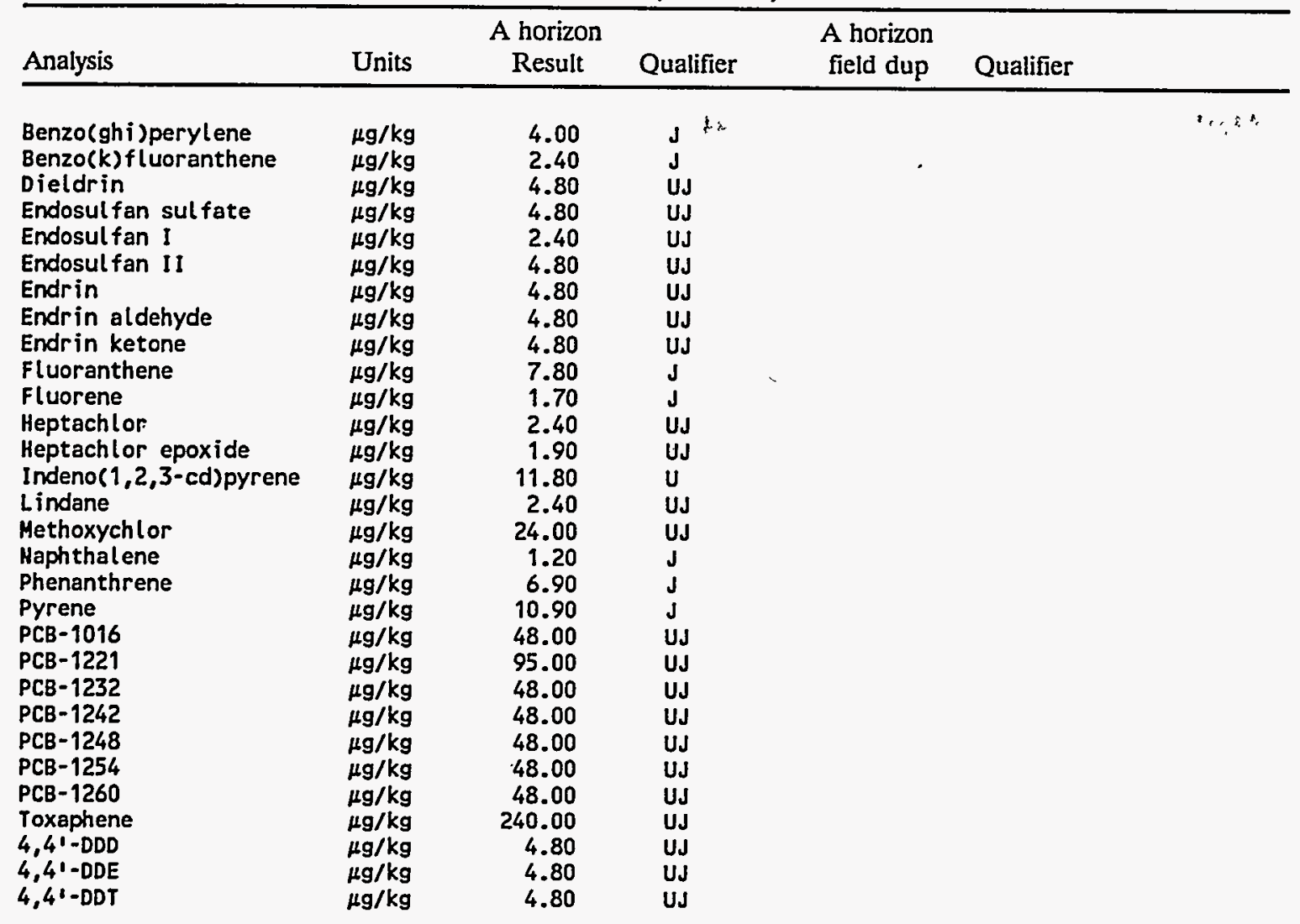

Location=ORR; Formation=CHICKAMAUGA K-25; Site=126; $A=4089 ; A$ dup=' '; Phase $=2$

\begin{tabular}{|c|c|c|}
\hline $\begin{array}{l}\text { alpha-BHC } \\
\text { Blpha-Chlordane } \\
\text { beta-BHC } \\
\text { del ta-BHC } \\
\text { gamma-Chlordane } \\
\text { Acenaphthene } \\
\text { Acenaphthylene } \\
\text { Aldrin } \\
\text { Benzo(a)anthracene } \\
\text { Benzo(a)pyrene } \\
\text { Benzo(b)fluoranthene } \\
\text { Benzo(ghi)perylene } \\
\text { Benzo(k)fluoranthene } \\
\text { Dieldrin } \\
\text { Endosulfan sul fate } \\
\text { Endosulfan I } \\
\text { Endosulfan II } \\
\text { Endrin } \\
\text { Endrin aldehyde } \\
\text { Endrin ketone } \\
\text { Fluoranthene } \\
\text { Heptachlor } \\
\text { Heptachlor epoxide } \\
\text { lndeno(1,2,3-cd)pyrene } \\
\text { Lindane } \\
\text { Methoxychlor } \\
\text { Maphthalene } \\
\text { Phenanthrene } \\
\text { Pyrene } \\
\text { PCB-1016 } \\
\text { PCB-1221 } \\
\text { PCB-1232 } \\
\text { PCB-1242 } \\
\text { PCB-1248 }\end{array}$ & $\begin{array}{l}\mu g / k g \\
\mu g / k g \\
\mu g / k g \\
\mu g / k g \\
\mu g / k g \\
\mu g / k g \\
\mu g / k g \\
\mu g / k g \\
\mu g / k g \\
\mu g / k g \\
\mu g / k g \\
\mu g / k g \\
\mu g / k g \\
\mu g / k g \\
\mu g / k g \\
\mu g / k g \\
\mu g / k g \\
\mu g / k g \\
\mu g / k g \\
\mu g / k g \\
\mu g / k g \\
\mu g / k g \\
\mu g / k g \\
\mu g / k g \\
\mu g / k g \\
\mu g / k g \\
\mu g / k g \\
\mu g / k g \\
\mu g / k g \\
\mu g / k g \\
\mu g / k g \\
\mu g / k g \\
\mu g / k g \\
\mu g / k g\end{array}$ & $\begin{array}{r}2.20 \\
2.20 \\
2.20 \\
2.20 \\
2.20 \\
1.10 \\
44.50 \\
2.20 \\
3.80 \\
3.30 \\
2.70 \\
2.20 \\
1.80 \\
4.40 \\
4.40 \\
2.20 \\
4.40 \\
4.40 \\
4.40 \\
4.40 \\
6.50 \\
2.20 \\
2.20 \\
11.10 \\
2.20 \\
22.00 \\
0.70 \\
4.20 \\
6.00 \\
44.00 \\
89.00 \\
44.00 \\
44.00 \\
44.00\end{array}$ \\
\hline
\end{tabular}




\section{C-38}

Table C.1 (continued)

\begin{tabular}{|c|c|c|c|c|c|}
\hline Analysis & Units & $\begin{array}{c}\text { A horizon } \\
\text { Result }\end{array}$ & Qualifier & $\begin{array}{l}\text { A horizon } \\
\text { field dup }\end{array}$ & Qualifier \\
\hline $\begin{array}{l}\text { PCB- } 1254 \\
\text { PCB- } 1260 \\
\text { Toxaphene } \\
4,4^{\prime} \text {-DDD } \\
4,4^{\prime} \text {-DDE } \\
4,4^{\prime} \text {-DDT }\end{array}$ & $\begin{array}{l}\mu g / \mathrm{kg} \\
\mu \mathrm{g} / \mathrm{kg} \\
\mu \mathrm{g} / \mathrm{kg} \\
\mu g / \mathrm{kg} \\
\mu g / \mathrm{kg} \\
\mu g / \mathrm{kg}\end{array}$ & $\begin{array}{r}44.00 \\
44.00 \\
220.00 \\
4.40 \\
4.40 \\
4.40\end{array}$ & $\begin{array}{l}\mathbf{U} \\
\mathbf{U} \\
\mathbf{U} \\
\mathbf{U} \\
\mathbf{U} \\
\mathbf{U} J\end{array}$ & & \\
\hline
\end{tabular}

Location=ORR; Formation $=$ CHICKAMAUGA $K-25 ;$ Site $=127 ; A=4135 ; A$ dup=' '; Phase $=2$

\begin{tabular}{|c|c|c|}
\hline $\begin{array}{l}\text { alpha-BHC } \\
\text { alpha-Chlordane } \\
\text { beta-BHC } \\
\text { delta-BHC } \\
\text { gamm-Chlordane } \\
\text { Acenaphthylene } \\
\text { Aldrin } \\
\text { Anthracene } \\
\text { Benzo(a)anthracene } \\
\text { Benzo(a)pyrene } \\
\text { Benzo(b)fluoranthene } \\
\text { Benzo(ghi)perylene } \\
\text { Benzo(k)fluoranthene } \\
\text { Chrysene } \\
\text { Dieldrin } \\
\text { Endosulfan sulfate } \\
\text { Endosulfan I } \\
\text { Endosulfan II } \\
\text { Endrin } \\
\text { Endrin aldehyde } \\
\text { Endrin ketone } \\
\text { Fluoranthene } \\
\text { Heptachlor } \\
\text { Heptachlor epoxide } \\
\text { Indeno(1,2,3-cd)pyrene } \\
\text { Lindane } \\
\text { Methoxychlor } \\
\text { Phenanthrene } \\
\text { Pyrene } \\
\text { PCB-1016 } \\
\text { PCB-1221 } \\
\text { PCB-1232 } \\
\text { PCB-1242 } \\
\text { PCB-1248 } \\
\text { PCB-1254 } \\
\text { PCB-1260 } \\
\text { Toxaphene } \\
4,44^{\prime}-D D D \\
4,4 '-D D E \\
4,4 '-D D T\end{array}$ & $\begin{array}{l}\mu g / \mathrm{kg} \\
\mu g / \mathrm{kg} \\
\mu g / \mathrm{kg} \\
\mu g / \mathrm{kg} \\
\mu g / \mathrm{kg} \\
\mu g / \mathrm{kg} \\
\mu g / \mathrm{kg} \\
\mu g / \mathrm{kg} \\
\mu g / \mathrm{kg} \\
\mu g / \mathrm{kg} \\
\mu g / \mathrm{kg} \\
\mu g / \mathrm{kg} \\
\mu g / \mathrm{kg} \\
\mu g / \mathrm{kg} \\
\mu g / \mathrm{kg} \\
\mu g / \mathrm{kg} \\
\mu g / \mathrm{kg} \\
\mu g / \mathrm{kg} \\
\mu g / \mathrm{kg} \\
\mu g / \mathrm{kg} \\
\mu g / \mathrm{kg} \\
\mu g / \mathrm{kg} \\
\mu g / \mathrm{kg} \\
\mu g / \mathrm{kg} \\
\mu g / \mathrm{kg} \\
\mu g / \mathrm{kg} \\
\mu g / \mathrm{kg} \\
\mu g / \mathrm{kg} \\
\mu g / \mathrm{kg} \\
\mu g / \mathrm{kg} \\
\mu g / \mathrm{kg} \\
\mu g / \mathrm{kg} \\
\mu g / \mathrm{kg} \\
\mu g / \mathrm{kg} \\
\mu g / \mathrm{kg} \\
\mu g / \mathrm{kg} \\
\mu g / \mathrm{kg} \\
\mu g / \mathrm{kg} \\
\mu g / \mathrm{kg} \\
\mu g / \mathrm{kg}\end{array}$ & $\begin{array}{r}2.40 \\
2.40 \\
2.40 \\
2.40 \\
2.40 \\
47.40 \\
2.40 \\
0.90 \\
3.10 \\
2.60 \\
2.80 \\
3.10 \\
1.20 \\
2.40 \\
4.80 \\
4.80 \\
2.40 \\
4.80 \\
4.80 \\
4.80 \\
4.80 \\
3.30 \\
2.40 \\
2.40 \\
11.90 \\
2.40 \\
24.00 \\
5.50 \\
4.30 \\
48.00 \\
96.00 \\
48.00 \\
48.00 \\
48.00 \\
48.00 \\
48.00 \\
240.00 \\
4.80 \\
4.80 \\
4.80\end{array}$ \\
\hline
\end{tabular}

Location $=O R R ;$ Formation $=$ CHICKAMAUGA K-25; Site $=128 ; A=4137 ;$ A dup=' '; Phase $=2$

$\begin{array}{llrl}\text { alpha-BHC } & \mu g / \mathrm{kg} & 2.10 & \mathrm{UJ} \\ \text { alpha-Chlordane } & \mu g / \mathrm{kg} & 2.10 & \mathrm{UJ} \\ \text { beta-BHC } & \mu g / \mathrm{kg} & 2.10 & \mathrm{UJ} \\ \text { delta-BHC } & \mu \mathrm{kg} & 2.10 & \mathrm{UJ} \\ \text { gamma-Chlordane } & \mu g / \mathrm{kg} & 2.10 & \mathrm{UJ} \\ \text { Acenaphthylene } & \mu g / \mathrm{kg} & 41.30 & \mathrm{U} \\ \text { Aldrin } & \mu g / \mathrm{kg} & 2.10 & \mathrm{UJ} \\ \text { Anthracene } & \mu \mathrm{kg} & 0.40 & \mathrm{~J} \\ \text { Benzo(a)anthracene } & \mu \mathrm{kg} / \mathrm{kg} & 1.20 & \mathrm{~J} \\ \text { Benzo(a)pyrene } & \mu \mathrm{kg} & 1.90 & \mathrm{~J} \\ \text { Benzo(b)fluoranthene } & \mu g / \mathrm{kg} & 1.90 & \mathrm{~J} \\ \text { Benzo(ghi)perylene } & \mu g / \mathrm{kg} & 2.10 & \mathrm{~J} \\ \text { Benzo(k)fluoranthene } & \mu \mathrm{g} / \mathrm{kg} & 1.00 & \mathrm{~J}\end{array}$


Table C.1 (continued)

\begin{tabular}{|c|c|c|c|c|c|c|c|}
\hline Analysis & Units & $\begin{array}{c}\text { A horizon } \\
\text { Result }\end{array}$ & Qualifier & $\begin{array}{l}\text { A horizon } \\
\text { field dup }\end{array}$ & Qualifier & & \\
\hline $\begin{array}{l}\text { Dieldrin } \\
\text { Endosul fan sul fate } \\
\text { Endosul fan I } \\
\text { Endosulfan II } \\
\text { Endrin } \\
\text { Endrin aldehyde } \\
\text { Endrin ketone } \\
\text { Fluoranthene } \\
\text { Fluorene } \\
\text { Heptachlor } \\
\text { Heptachlor epoxide } \\
\text { Indeno(1,2,3-cd)pyrene } \\
\text { Lindane } \\
\text { Methoxychlor } \\
\text { Naphthalene } \\
\text { Phenanthrene } \\
\text { Pyrene } \\
\text { PCB-1016 } \\
\text { PCB-1221 } \\
\text { PCB-1232 } \\
\text { PCB-1242 } \\
\text { PCB-1248 } \\
\text { PCB-1254 } \\
\text { PCB-1260 } \\
\text { TOXaphene } \\
4,41-D D D \\
4,4:-D D E \\
4,41-D D T\end{array}$ & 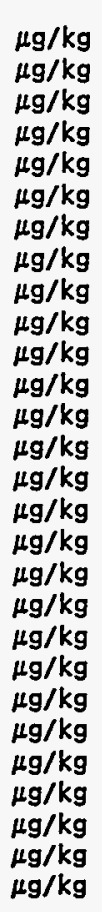 & $\begin{array}{r}4.10 \\
4.10 \\
2.10 \\
4.10 \\
4.10 \\
4.10 \\
4.10 \\
5.20 \\
0.40 \\
2.10 \\
2.10 \\
12.00 \\
2.10 \\
21.00 \\
1.40 \\
2.90 \\
3.90 \\
41.00 \\
82.00 \\
41.00 \\
41.00 \\
41.00 \\
41.00 \\
41.00 \\
210.00 \\
4.10 \\
4.10 \\
4.10\end{array}$ & $\begin{array}{l}\text { UJ : }:= \\
\text { UJ } \\
\text { UJ } \\
\text { UJ } \\
\text { UJ } \\
\text { UJ } \\
\text { UJ } \\
J \\
J \\
\text { UJ } \\
\text { UJ } \\
\text { UJ } \\
\text { UJ } \\
J \\
J \\
J \\
\text { UJ } \\
\text { UJ } \\
\text { UJ } \\
\text { UJ } \\
\text { UJ } \\
\text { UJ } \\
\text { UJ } \\
\text { UJ } \\
\text { UJ } \\
\text { UJ } \\
\text { UJ }\end{array}$ & . & & " & \\
\hline
\end{tabular}

Location=ORR; Formation =CHICKAMAUGA K-25; Site=129; $A=4086 ; A$ dup=' '; Phase=2

\begin{tabular}{|c|c|c|}
\hline $\begin{array}{l}\text { Acenaphthylene } \\
\text { Benzo(a)anthracene } \\
\text { Benzo(a)pyrene } \\
\text { Benzo(b)fluoranthene } \\
\text { Benzo(ghi)perylene } \\
\text { Benzo(k)fluoranthene } \\
\text { Dalapon } \\
\text { Dicamba } \\
\text { Dichlorprop } \\
\text { Dinoseb } \\
\text { Fluorene } \\
\text { Indeno(1,2,3-cd)pyrene } \\
\text { MCPA } \\
\text { MCPP } \\
\text { Phenanthrene } \\
\text { Pyrene } \\
\text { Silvex } \\
2,4-D \\
2,4-D B \\
2,4,5-T\end{array}$ & $\begin{array}{l}\mu g / k g \\
\mu g / k g \\
\mu g / k g \\
\mu g / k g \\
\mu g / k g \\
\mu g / k g \\
\mu g / k g \\
\mu g / k g \\
\mu g / k g \\
\mu g / k g \\
\mu g / k g \\
\mu g / k g \\
\mu g / k g \\
\mu g / k g \\
\mu g / k g \\
\mu \dot{g} / \mathbf{k g} \\
\mu g / k g \\
\mu g / k g \\
\mu g / k g \\
\mu g / k g\end{array}$ & $\begin{array}{r}41.30 \\
4.50 \\
4.10 \\
1.70 \\
2.90 \\
2.70 \\
4271.00 \\
195.00 \\
488.00 \\
102.00 \\
0.80 \\
10.30 \\
183025.00 \\
139099.00 \\
6.00 \\
10.70 \\
122.00 \\
879.00 \\
659.00 \\
146.00\end{array}$ \\
\hline
\end{tabular}

Location=ORR; Formation=COPPER RIDGE; Site $=45 ; A=1544 ; A$ dup=' '; Phase =2

$\begin{array}{llrl}\text { Acenaphthene } & \mu g / \mathbf{k g} & 1.50 & \mathrm{~J} \\ \text { Benzo(a)anthracene } & \mu \mathrm{g} / \mathrm{kg} & 2.20 & \mathrm{~J} \\ \text { Benzo(a)pyrene } & \mu \mathrm{kg} & 1.10 & \mathrm{~J} \\ \text { Benzo(ghi)perylene } & \mu \mathrm{kg} / \mathrm{kg} & 0.40 & \mathrm{~J} \\ \text { Fluoranthene } & \mu \mathrm{gg} & 1.50 & \mathrm{~J} \\ \text { Indeno(1,2,3-cd)pyrene } & \mu g / \mathrm{kg} & 18.60 & \mathrm{U} \\ \text { Phenanthrene } & \mu \mathrm{g} / \mathrm{kg} & 6.70 & \mathrm{~J} \\ \text { Pyrene } & \mu \mathrm{kg} & 3.00 & \mathrm{~J}\end{array}$


Table C.1 (continued)

\begin{tabular}{lcccc}
\hline Analysis & Units & $\begin{array}{c}\text { A horizon } \\
\text { Result }\end{array}$ & Qualifier & $\begin{array}{c}\text { A horizon } \\
\text { field dup }\end{array}$ Qualifier \\
\hline
\end{tabular}

Location=ORR; Formation $=$ COPPER RIDGE; Site $=51 ; A=1546 ; A$ dup=' '; Phase $=2$

$\begin{array}{llrl}\text { Benzo(a)anthracene } & \mu g / \mathrm{kg} & 1.20 & \mathrm{~J} \\ \text { Benzo(ghi)perylene } & \mu \mathrm{g} / \mathrm{kg} & 2.00 & \mathrm{~J} \\ \text { Benzo(k)fluoranthene } & \mu \mathrm{g} / \mathrm{kg} & 0.80 & \mathrm{~J} \\ \text { Fluoranthene } & \mu \mathrm{g} / \mathrm{kg} & 4.40 & \mathrm{~J} \\ \text { Fluorene } & \mu \mathrm{g} / \mathrm{kg} & 0.40 & \mathrm{~J} \\ \text { Indeno(1,2,3-cd)pyrene } & \mu \mathrm{g} / \mathrm{kg} & 20.00 & \mathrm{U} \\ \text { Naphthalene } & \mu \mathrm{g} / \mathrm{kg} & 10.40 & \mathrm{~J} \\ \text { Phenanthrene } & \mu \mathrm{g} / \mathrm{kg} & 2.40 & \mathrm{~J} \\ \text { Pyrene } & \mu g / \mathrm{kg} & 3.20 & \mathrm{~J}\end{array}$

Location $=O R R ;$ Formation $=$ COPPER RIDGE; Site $=54 ; A=1497 ; A$ dup $=$ ' '; Phase $=2$

\begin{tabular}{|c|c|c|}
\hline 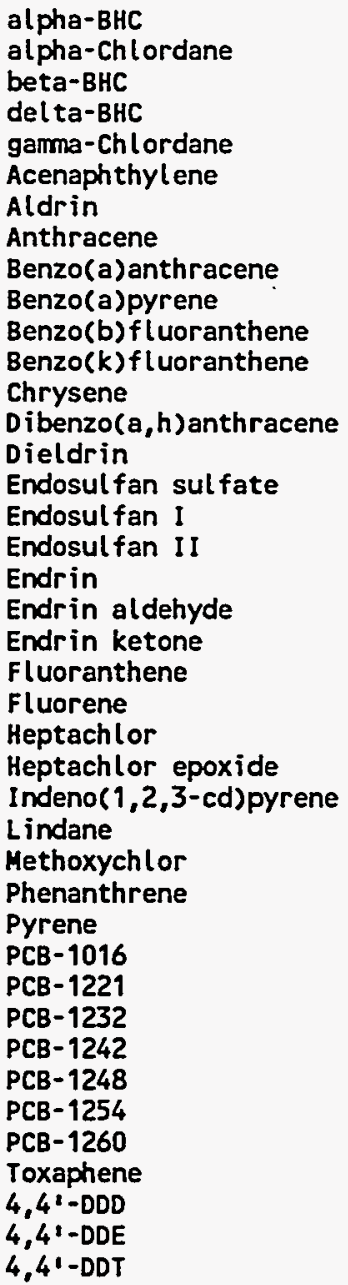 & 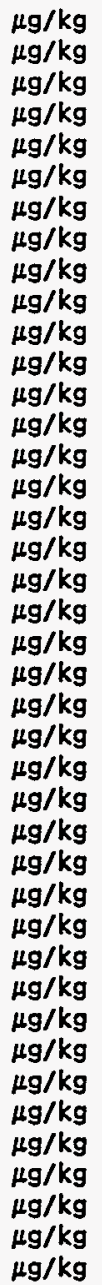 & $\begin{array}{r}1.80 \\
1.80 \\
1.80 \\
1.80 \\
1.80 \\
182.80 \\
1.80 \\
0.40 \\
1.50 \\
1.50 \\
1.10 \\
0.70 \\
2.20 \\
2.90 \\
3.70 \\
3.70 \\
1.80 \\
3.70 \\
3.70 \\
3.70 \\
3.70 \\
4.80 \\
2.20 \\
1.80 \\
1.80 \\
18.30 \\
1.80 \\
18.00 \\
2.90 \\
4.40 \\
37.00 \\
74.00 \\
37.00 \\
37.00 \\
37.00 \\
37.00 \\
37.00 \\
180.00 \\
3.70 \\
3.70 \\
3.30\end{array}$ \\
\hline
\end{tabular}

Location=ORR; Formation=COPPER RIDGE; Site $=55 ; A=1495 ; A$ dup=' '; Phase $=2$

$\begin{array}{lrrl}\text { alpha-BHC } & \mu \mathrm{g} / \mathrm{kg} & 1.90 & \mathrm{UJ} \\ \text { alpha-Chlordane } & \mu \mathrm{g} / \mathrm{kg} & 1.90 & \mathrm{U} \\ \text { beta-BHC } & \mu \mathrm{g} / \mathrm{kg} & 1.70 & \mathrm{UJ} \\ \text { del ta-BHC } & \mu \mathrm{g} / \mathrm{kg} & 1.90 & \mathrm{U} \\ \text { gamma-Chlordane } & \mu \mathrm{g} / \mathrm{kg} & 1.90 & \mathrm{U} \\ \text { Acenaphthylene } & \mu \mathrm{gg} / \mathrm{kg} & 4287.40 & \\ \text { Aldrin } & \mu \mathrm{g} / \mathrm{kg} & 1.90 & \mathrm{U}\end{array}$


C-41

Table C.1 (continued)

\begin{tabular}{|c|c|c|c|c|c|c|}
\hline Analysis & Units & $\begin{array}{c}\text { A horizon } \\
\text { Result }\end{array}$ & Qualifier & $\begin{array}{l}\text { A horizon } \\
\text { field dup }\end{array}$ & Qualifier & \\
\hline $\begin{array}{l}\text { Anthracene } \\
\text { Benzo(a)anthracene } \\
\text { Benzo(a)pyrene } \\
\text { Benzo(b)fluoranthene } \\
\text { Benzo(k)fluoranthene } \\
\text { Chrysene } \\
\text { Dibenzo(a,h)anthracene } \\
\text { Dieldrin } \\
\text { Endosulfan sul fate } \\
\text { Endosul fan I } \\
\text { Endosulfan II } \\
\text { Endrin } \\
\text { Endrin aldehyde } \\
\text { Endrin ketone } \\
\text { Fluoranthene } \\
\text { Heptachlor } \\
\text { Heptachlor epoxide } \\
\text { Indeno(1,2,3-cd)pyrene } \\
\text { Lindane } \\
\text { Methoxychlor } \\
\text { Phenanthrene } \\
\text { Pyrene } \\
\text { PCB-1016 } \\
\text { PCB-1221 } \\
\text { PCB-1232 } \\
\text { PCB-1242 } \\
\text { PCB-1248 } \\
\text { PCB-1254 } \\
\text { PCB-1260 } \\
\text { Toxaphene } \\
4,41-0 D D \\
4,4:-D D E \\
4,41-D D T\end{array}$ & 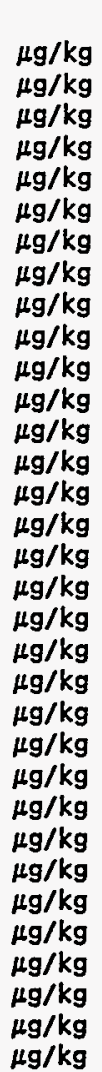 & $\begin{array}{r}0.80 \\
1.90 \\
1.90 \\
0.40 \\
1.10 \\
2.70 \\
3.80 \\
3.70 \\
3.70 \\
1.90 \\
1.80 \\
3.70 \\
3.70 \\
3.70 \\
6.50 \\
1.90 \\
1.90 \\
19.10 \\
1.90 \\
19.00 \\
3.40 \\
5.00 \\
37.00 \\
74.00 \\
37.00 \\
37.00 \\
37.00 \\
37.00 \\
37.00 \\
190.00 \\
1.70 \\
3.70 \\
3.70\end{array}$ & 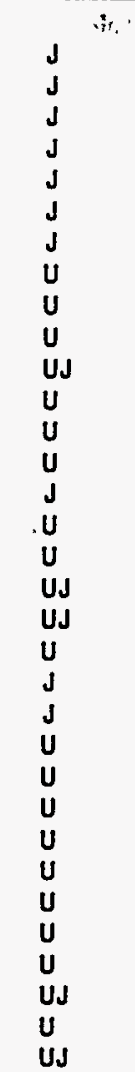 & . & . & $=\quad "$ \\
\hline
\end{tabular}

Location=ORR; Formation=COPPER RIDGE; Site=58; $A=1542 ; A$ dup= '; Phase=2

$\begin{array}{lrrr}\text { Acenaphthylene } & \mu \mathrm{g} / \mathrm{kg} & 76.50 & \mathrm{U} \\ \text { Benzo(a)anthracene } & \mu \mathrm{g} / \mathrm{kg} & 0.40 & \mathrm{~J} \\ \text { Benzo(k)fluoranthene } & \mu \mathrm{g} / \mathrm{kg} & 0.80 & \mathrm{~J} \\ \text { Fluoranthene } & \mu \mathrm{g} / \mathrm{kg} & 1.50 & \mathrm{~J} \\ \text { Indeno(1,2,3-cd)pyrene } & \mu \mathrm{g} / \mathrm{kg} & 19.10 & \mathrm{U} \\ \text { Naphthalene } & \mu \mathrm{g} / \mathrm{kg} & 20.30 & \mathrm{~J} \\ \text { Phenanthrene } & \mu \mathrm{g} / \mathrm{kg} & 1.50 & \mathrm{~J} \\ \text { Pyrene } & \mu \mathrm{g} / \mathrm{kg} & 0.40 & \mathrm{~J}\end{array}$

Location=ORR; Formation $=$ COPPER RIDGE; Site $=59 ; A=1491 ; A$ dup=' ; Phase $=2$

\begin{tabular}{|c|c|c|}
\hline $\begin{array}{l}\text { alpha-BHC } \\
\text { alpha-Chlordane } \\
\text { beta-BHC } \\
\text { del ta-BHC } \\
\text { gamma-Chlordane } \\
\text { Acenaphthene } \\
\text { Acenaphthylene } \\
\text { Aldrin } \\
\text { Anthracene } \\
\text { Benzo(a)anthracene } \\
\text { Benzo(a)pyrene } \\
\text { Benzo(b)fluoranthene } \\
\text { Benzo(ghi)perylene } \\
\text { Benzo(k)fluoranthene } \\
\text { Chrysene } \\
\text { Dibenzo(a, h)anthracene } \\
\text { Dieldrin } \\
\text { Endosulfan sulfate }\end{array}$ & 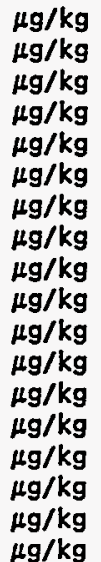 & $\begin{array}{r}1.90 \\
1.90 \\
1.90 \\
1.30 \\
1.90 \\
3.80 \\
187.60 \\
1.90 \\
14.60 \\
7.50 \\
11.30 \\
9.40 \\
10.10 \\
6.00 \\
12.00 \\
1.50 \\
3.80 \\
3.80\end{array}$ \\
\hline
\end{tabular}


C-42

Table C.1 (continued)

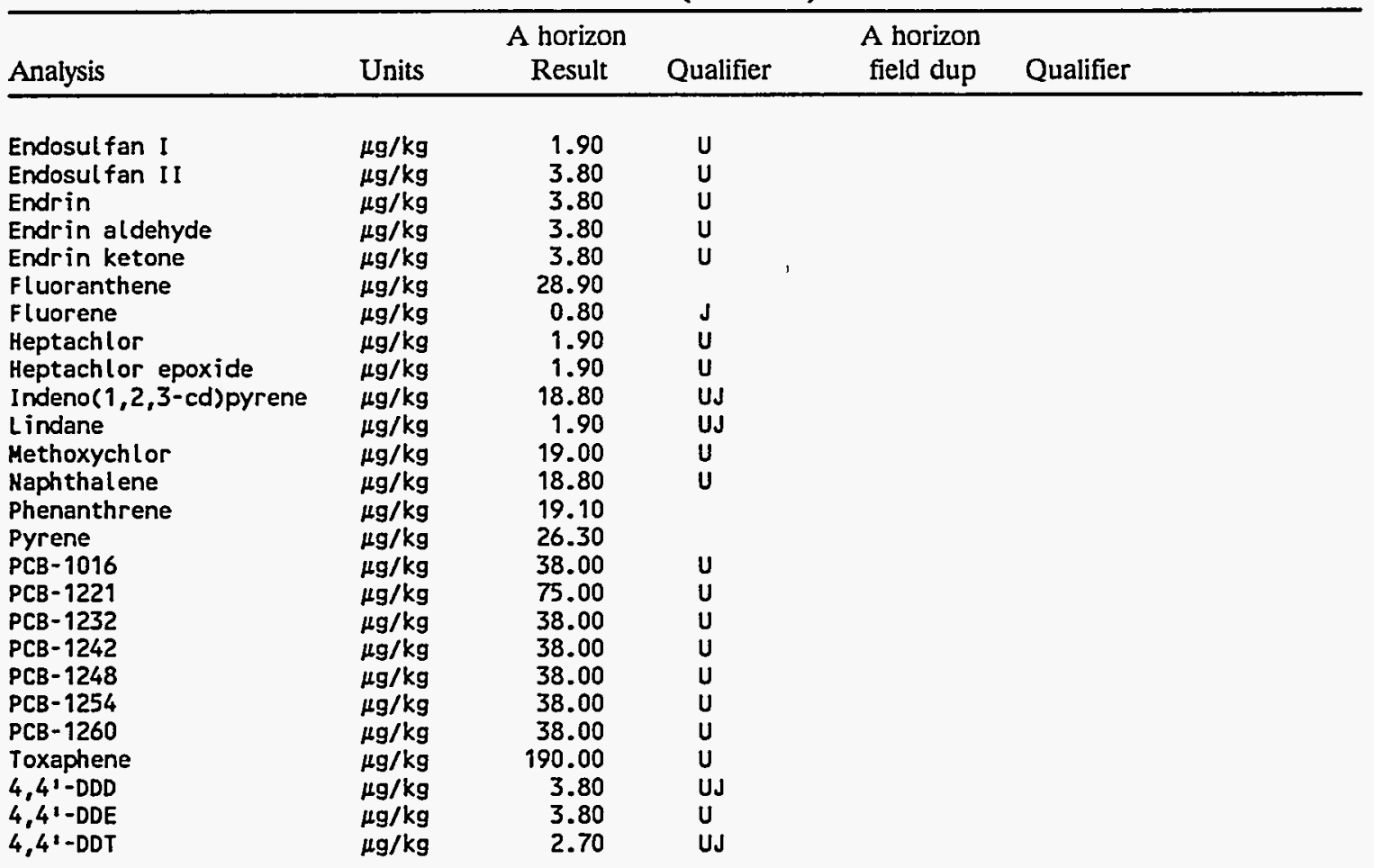

Location=ORR; Formation=COPPER RIDGE; Site $=60 ; A=1493 ; A$ dup =' '; Phase $=2$

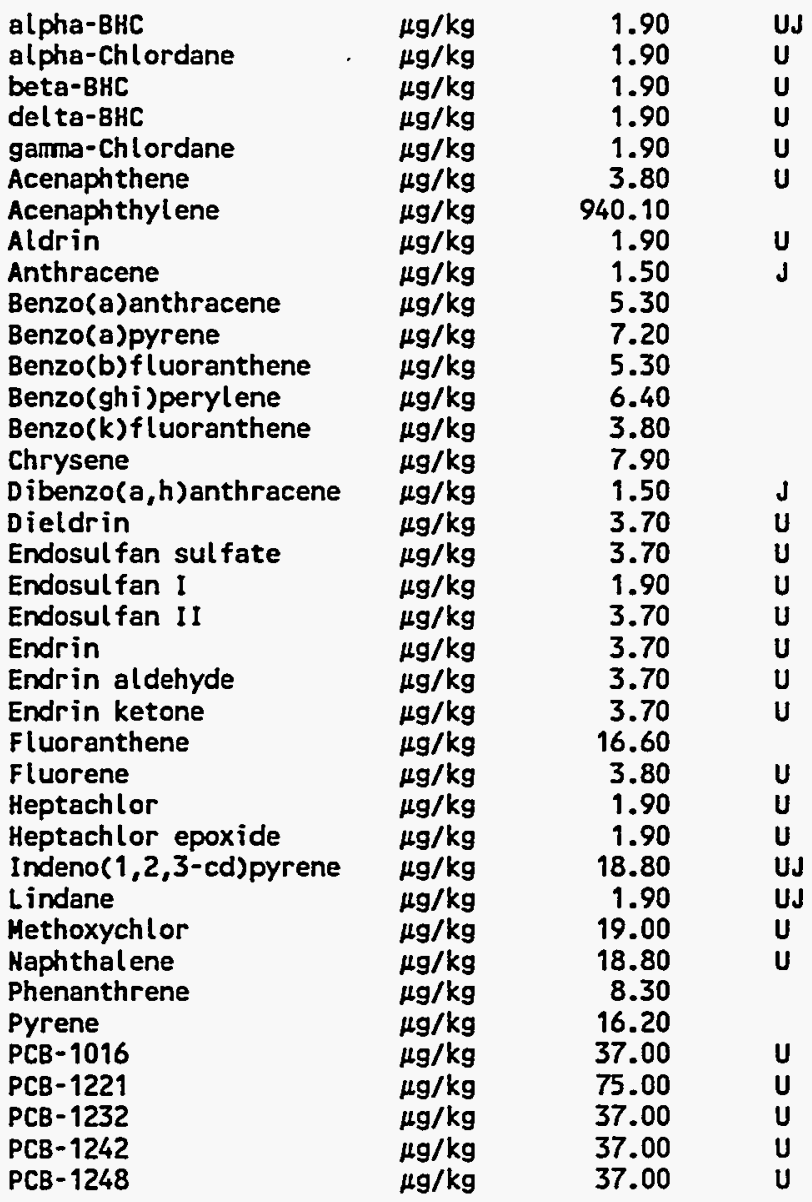




\section{C-43}

Table C.1 (continued)

\begin{tabular}{|c|c|c|c|c|c|c|}
\hline Analysis & Units & $\begin{array}{c}\text { A horizon } \\
\text { Result }\end{array}$ & Qualifier & $\begin{array}{l}\text { A horizon } \\
\text { field dup }\end{array}$ & Qualifier & \\
\hline $\begin{array}{l}\text { PCB- } 1254 \\
\text { PCB- } 1260 \\
\text { Toxaphene } \\
4,4^{\prime}-D D D \\
4,4^{\prime}-D D E \\
4,4^{\prime}-D D T\end{array}$ & $\begin{array}{l}\mu g / \mathrm{kg} \\
\mu g / \mathrm{kg} \\
\mu g / \mathrm{kg} \\
\mu g / \mathrm{kg} \\
\mu g / \mathrm{kg} \\
\mu \mathrm{g} / \mathrm{kg}\end{array}$ & $\begin{array}{r}37.00 \\
37.00 \\
190.00 \\
3.70 \\
3.70 \\
1.90\end{array}$ & $\begin{array}{l}U^{i r} \\
U \\
U \\
U J \\
U \\
U J\end{array}$ & & & 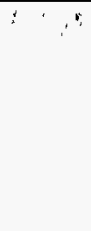 \\
\hline
\end{tabular}

Location=ORR; Formation=COPPER RIDGE; Site $=62 ; A=1480 ; A$ dup=' ; Phase=2

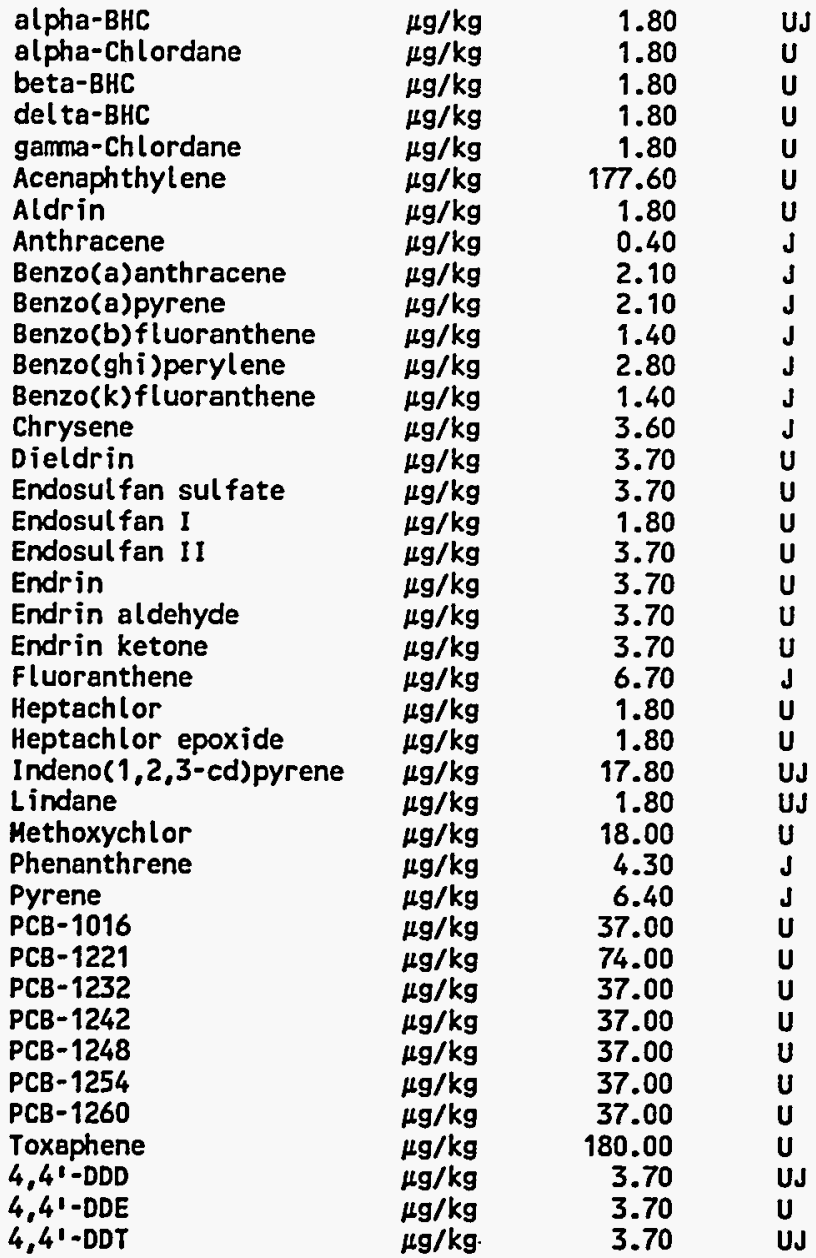

Location=ORR; Formation=COPPER RIDGE; Site $=64 ; A=1483 ; A$ dup=' '; Phase $=2$

$\begin{array}{llrl}\text { alpha-BHC } & \mu \mathrm{g} / \mathrm{kg} & 1.90 & \mathrm{UJ} \\ \text { alpha-Chlordane } & \mu \mathrm{g} / \mathrm{kg} & 1.90 & \mathrm{U} \\ \text { beta-BHC } & \mu \mathrm{g} / \mathrm{kg} & 1.90 & \mathrm{U} \\ \text { del ta-BHC } & \mu \mathrm{g} / \mathrm{kg} & 1.90 & \mathrm{U} \\ \text { gamma-Chlordane } & \mu \mathrm{g} / \mathrm{kg} & 1.90 & \mathrm{U} \\ \text { Acenaphthylene } & \mu \mathrm{g} / \mathrm{kg} & 28.40 & \mathrm{~J} \\ \text { Aldrin } & \mu \mathrm{g} / \mathrm{kg} & 1.90 & \mathrm{U} \\ \text { Benzo(a)anthracene } & \mu \mathrm{g} / \mathrm{kg} & 0.80 & \mathrm{~J} \\ \text { Benzo(a)pyrene } & \mu \mathrm{g} / \mathrm{kg} & 0.80 & \mathrm{~J} \\ \text { Benzo(ghi)perylene } & \mu \mathrm{g} / \mathrm{kg} & 1.20 & \mathrm{~J} \\ \text { Benzo(k)fluoranthene } & \mu \mathrm{g} / \mathrm{kg} & 0.40 & \mathrm{~J} \\ \text { Chrysene } & \mu \mathrm{g} / \mathrm{kg} & 1.20 & \mathrm{~J} \\ \text { Dibenzo(a, h)anthracene } & \mu \mathrm{g} / \mathrm{kg} & 0.40 & \mathrm{~J}\end{array}$


Table C.1 (continued)

\begin{tabular}{|c|c|c|c|c|c|}
\hline Analysis & Units & $\begin{array}{c}\text { A horizon } \\
\text { Result }\end{array}$ & Qualifier & $\begin{array}{l}\text { A horizon } \\
\text { field dup }\end{array}$ & Qualifier \\
\hline $\begin{array}{l}\text { Dieldrin } \\
\text { Endosulfan sulfate } \\
\text { Endosulfan I } \\
\text { Endosul fan II } \\
\text { Endrin } \\
\text { Endrin aldehyde } \\
\text { Endrin ketone } \\
\text { Fluoranthene } \\
\text { Heptachlor } \\
\text { Heptachlor epoxide } \\
\text { Indeno(1,2,3-cd)pyrene } \\
\text { Lindane } \\
\text { Methoxychlor } \\
\text { Phenanthrene } \\
\text { Pyrene } \\
\text { PCB-1016 } \\
\text { PCB-1221 } \\
\text { PCB-1232 } \\
\text { PCB-1242 } \\
\text { PCB-1248 } \\
\text { PCB-1254 } \\
\text { PCB-1260 } \\
\text { Toxaphene } \\
4,4 '-D D D \\
4,4 '-D D E \\
4,4 '-D D T\end{array}$ & 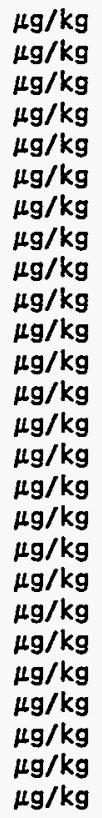 & $\begin{array}{r}3.80 \\
3.80 \\
1.90 \\
3.80 \\
3.80 \\
2.00 \\
3.80 \\
3.60 \\
1.90 \\
1.90 \\
19.70 \\
1.90 \\
19.00 \\
1.60 \\
2.80 \\
38.00 \\
77.00 \\
38.00 \\
38.00 \\
38.00 \\
38.00 \\
38.00 \\
190.00 \\
3.80 \\
3.80 \\
3.80\end{array}$ & $\begin{array}{l}U \\
U \\
U \\
U \\
U \\
U J \\
U \\
J \\
U \\
U \\
U J \\
U J \\
U \\
J \\
J \\
U \\
U \\
U \\
U \\
U \\
U \\
U \\
U \\
U \\
U \\
U \\
U \\
U \\
U\end{array}$ & & \\
\hline
\end{tabular}

Location $=O R R$; Formation =COPPER RIDGE; Site $=75 ; A=1477 ; A$ dup=' '; Phase $=2$

\begin{tabular}{|c|c|c|}
\hline $\begin{array}{l}\text { alpha-BHC } \\
\text { alpha-Chlordane } \\
\text { beta-BHC } \\
\text { del ta-BHC } \\
\text { gamma-Chlordane } \\
\text { Acenaphthene } \\
\text { Acenaphthylene } \\
\text { Aldrin } \\
\text { Anthracene } \\
\text { Benzo(a)anthracene } \\
\text { Benzo(a)pyrene } \\
\text { Benzo(b)fluoranthene } \\
\text { Benzo(ghi)perylene } \\
\text { Benzo(k)fluoranthene } \\
\text { Chrysene } \\
\text { Dibenzo(a,h)anthracene } \\
\text { Dieldrin } \\
\text { Endosul fan sul fate } \\
\text { Endosul fan I } \\
\text { Endosul fan II } \\
\text { Endrin } \\
\text { Endrin aldehyde } \\
\text { Endrin ketone } \\
\text { Fluoranthene } \\
\text { Fluorene } \\
\text { Heptachlor } \\
\text { Heptachlor epoxide } \\
\text { Indeno(1,2,3-cd)pyrene } \\
\text { Lindane } \\
\text { Methoxychlor } \\
\text { Haphthalene } \\
\text { Phenanthrene } \\
\text { Pyrene } \\
\text { PCB-1016 } \\
\text { PCB-1221 } \\
\text { PCB-1232 } \\
\text { PCB-1242 } \\
\text { PCB-1248 }\end{array}$ & 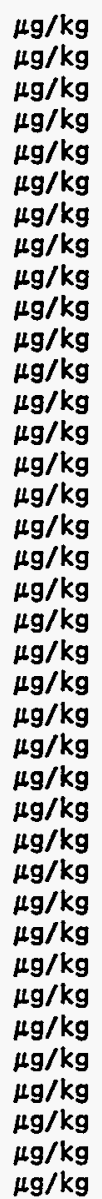 & $\begin{array}{r}2.00 \\
2.00 \\
2.00 \\
2.00 \\
2.00 \\
3.90 \\
195.30 \\
2.00 \\
0.40 \\
1.60 \\
2.00 \\
1.60 \\
2.70 \\
1.20 \\
2.70 \\
0.80 \\
4.00 \\
4.00 \\
2.00 \\
4.00 \\
4.00 \\
4.00 \\
4.00 \\
4.70 \\
3.90 \\
2.00 \\
2.00 \\
19.50 \\
2.00 \\
20.00 \\
19.50 \\
2.70 \\
4.30 \\
40.00 \\
79.00 \\
40.00 \\
40.00 \\
40.00\end{array}$ \\
\hline
\end{tabular}




\section{C-45}

Table C.1 (continued)

\begin{tabular}{lrrrrr}
\hline Analysis & Units & $\begin{array}{c}\text { A horizon } \\
\text { Result }\end{array}$ & Qualifier & $\begin{array}{c}\text { A horizon } \\
\text { field dup }\end{array}$ & Qualifier \\
\hline & & & & & \\
PCB-1254 & $\mu g / k g$ & 40.00 & $U$ & & \\
PCB-1260 & $\mu g / k g$ & 40.00 & $U$ & \\
Toxaphene & $\mu g / k g$ & 200.00 & $U$ & \\
$4,41-D D D$ & $\mu g / k g$ & 4.00 & UJ & \\
$4,41-D D E$ & $\mu g / k g$ & 4.00 & $U$ & \\
$4,41-D D T$ & $\mu g / k g$ & 4.00 & UJ &
\end{tabular}

Location=ORR; Formation=COPPER RIDGE; Site $=83 ; A=1474 ; A$ dup =' '; Phase $=2$

\begin{tabular}{|c|c|c|}
\hline $\begin{array}{l}\text { alpha-BHC } \\
\text { alpha-Chlordane } \\
\text { beta-BHC } \\
\text { del ta-BHC } \\
\text { gamma-Chlordane } \\
\text { Acenaphthene } \\
\text { Acenaphthylene } \\
\text { Aldrin } \\
\text { Anthracene } \\
\text { Benzo(a)anthracene } \\
\text { Benzo(a)pyrene } \\
\text { Benzo(b)fluoranthene } \\
\text { Benzo(ghi)perylene } \\
\text { Benzo(k)fluoranthene } \\
\text { Chrysene } \\
\text { Dibenzo(a,h)anthracene } \\
\text { Dieldrin } \\
\text { Endosul fan sul fate } \\
\text { Endosul fan I } \\
\text { Endosul fan II } \\
\text { Endrin } \\
\text { Endrin aldehyde } \\
\text { Endrin ketone } \\
\text { Fluoranthene } \\
\text { Heptachlor } \\
\text { Heptachlor epoxide } \\
\text { Indeno(1,2,3-cd)pyrene } \\
\text { Lindane } \\
\text { Methoxychlor } \\
\text { Haphthalene } \\
\text { Phenanthrene } \\
\text { Pyrene } \\
\text { PCB-1016 } \\
\text { PCB-1221 } \\
\text { PCB-1232 } \\
\text { PCB-1242 } \\
\text { PCB-1248 } \\
\text { PCB-1254 } \\
\text { PCB-1260 } \\
\text { Toxaphene } \\
4,4 \text {-DDD } \\
4,4:-D 0 E \\
4,41-D D T \\
\text { (a) }\end{array}$ & 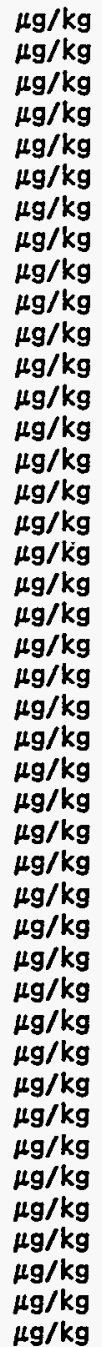 & $\begin{array}{r}2.00 \\
2.00 \\
2.00 \\
2.00 \\
2.00 \\
2.40 \\
198.40 \\
2.00 \\
0.40 \\
3.20 \\
4.00 \\
2.80 \\
4.80 \\
2.00 \\
5.20 \\
0.40 \\
4.00 \\
4.00 \\
2.20 \\
4.00 \\
4.00 \\
4.00 \\
4.00 \\
10.30 \\
2.00 \\
2.00 \\
19.80 \\
2.00 \\
20.00 \\
7.50 \\
5.20 \\
9.10 \\
40.00 \\
79.00 \\
40.00 \\
40.00 \\
40.00 \\
40.00 \\
40.00 \\
200.00 \\
4.00 \\
4.00 \\
4.00\end{array}$ \\
\hline
\end{tabular}

Location $=$ ORR; Formation $=$ COPPER RIDGE; Site $=91 ; A=1471 ; A$ dup=' '; Phase $=2$

$\begin{array}{llrl}\text { alpha-BHC } & \mu g / \mathrm{kg} & 2.00 & \mathrm{UJ} \\ \text { alpha-Chlordane } & \mu g / \mathrm{kg} & 2.00 & \mathrm{U} \\ \text { beta-BHC } & \mu g / \mathrm{kg} & 2.00 & \mathrm{U} \\ \text { del ta-BHC } & \mu g / \mathrm{kg} & 2.00 & \mathrm{U} \\ \text { gamma-Chlordane } & \mu g / \mathrm{kg} & 2.00 & \mathrm{U} \\ \text { Acenaphthene } & \mu \mathrm{kg} & 0.80 & \mathrm{~J} \\ \text { Acenaphthylene } & \mu g / \mathrm{kg} & 43.40 & \mathrm{~J} \\ \text { Aldrin } & \mu g / \mathrm{kg} & 2.00 & \mathrm{U} \\ \text { Anthracene } & \mu g / \mathrm{kg} & 0.80 & \mathrm{~J} \\ \text { Benzo(a)anthracene } & \mu g / \mathrm{kg} & 4.30 & \mathrm{~J}\end{array}$


Table C.1 (continued)

\begin{tabular}{|c|c|c|c|c|c|}
\hline Analysis & Units & $\begin{array}{c}\text { A horizon } \\
\text { Result }\end{array}$ & Qualifier & $\begin{array}{l}\text { A horizon } \\
\text { field dup }\end{array}$ & Qualifier \\
\hline $\begin{array}{l}\text { Benzo(a)pyrene } \\
\text { Benzo(b)f luoranthene } \\
\text { Benzo(ghi)perylene } \\
\text { Benzo(k)fluoranthene } \\
\text { Chrysene } \\
\text { D ibenzo(a, h) anthracene } \\
\text { Dieldrin } \\
\text { Endosul fan sulfate } \\
\text { Endosul fan II } \\
\text { Endrin } \\
\text { Endrin aldehyde } \\
\text { Endrin ketone } \\
\text { Fluoranthene } \\
\text { Fluorene } \\
\text { Heptachlor } \\
\text { Heptachlor epoxide } \\
\text { Indeno(1,2,3-cd)pyrene } \\
\text { Lindane } \\
\text { Methoxychlor } \\
\text { Naphthalene } \\
\text { Phenanthrene } \\
\text { Pyrene } \\
\text { PCB-1016 } \\
\text { PCB-1221 } \\
\text { PCB-1232 } \\
\text { PCB-1242 } \\
\text { PCB-1248 } \\
\text { PCB-1254 } \\
\text { PCB-1260 } \\
\text { Toxaphene } \\
4,4 '-D D D \\
4,41-D D E \\
4,41-D D T\end{array}$ & $\begin{array}{l}\mu g / k g \\
\mu g / k g \\
\mu g / k g \\
\mu g / k g \\
\mu g / k g \\
\mu g / k g \\
\mu g / k g \\
\mu g / k g \\
\mu g / k g \\
\mu g / k g \\
\mu g / k g \\
\mu g / k g \\
\mu g / k g \\
\mu g / k g \\
\mu g / k g \\
\mu g / k g \\
\mu g / k g \\
\mu g / k g \\
\mu g / k g \\
\mu g / k g \\
\mu g / k g \\
\mu g / k g \\
\mu g / k g \\
\mu g / k g \\
\mu g / k g \\
\mu g / k g \\
\mu g / k g \\
\mu g / k g \\
\mu g / k g \\
\mu g / k g \\
\mu g / k g \\
\mu g / k g \\
\mu g / k g\end{array}$ & $\begin{array}{r}5.10 \\
3.90 \\
5.50 \\
2.70 \\
6.60 \\
0.40 \\
4.00 \\
4.00 \\
4.00 \\
4.00 \\
4.00 \\
1.80 \\
11.30 \\
3.90 \\
2.00 \\
2.00 \\
19.50 \\
2.00 \\
20.00 \\
19.50 \\
5.50 \\
10.60 \\
40.00 \\
79.00 \\
40.00 \\
40.00 \\
40.00 \\
40.00 \\
40.00 \\
200.00 \\
4.00 \\
4.00 \\
4.00\end{array}$ & $\begin{array}{l}J \\
J \\
J \\
J \\
J \\
J \\
U \\
U \\
U \\
U \\
U \\
U J J \\
J \\
U J \\
U \\
U \\
U J \\
U J \\
U \\
U J \\
J \\
J \\
U \\
U \\
U \\
U \\
U \\
U \\
U \\
U \\
U \\
U J \\
U \\
U J J\end{array}$ & - & \\
\hline
\end{tabular}

Location =ORR; Formation=DISMAL GAP; Site $=10 ; A=1127$; dup=' '; Phase=1

\begin{tabular}{|c|c|c|c|}
\hline $\begin{array}{l}\text { alpha-BHC } \\
\text { alpha-Chlordane } \\
\text { beta-BHC } \\
\text { del ta-BHC } \\
\text { gamma-Chlordane } \\
\text { Acenaphthene } \\
\text { Acenaphthylene } \\
\text { Aldrin } \\
\text { Anthracene } \\
\text { Benzo(a)anthracene } \\
\text { Benzo(a)pyrene } \\
\text { Benzo(b)fluoranthene } \\
\text { Benzo(ghi)perylene } \\
\text { Benzo(k)fluoranthene } \\
\text { Chrysene } \\
\text { D ibenzo(a,h)anthracene } \\
\text { Dicamba } \\
\text { Dichlorprop } \\
\text { Dieldrin } \\
\text { Dinoseb } \\
\text { Endosulfan sul fate } \\
\text { Endosulfan I } \\
\text { Endosulfan II } \\
\text { Endrin } \\
\text { Endrin aldehyde } \\
\text { Endrin ketone } \\
\text { Fluorene } \\
\text { Heptachlor } \\
\text { Heptachlor epoxide } \\
\text { Indeno( } 1,2,3 \text {-cd)pyrene } \\
\text { Lindane }\end{array}$ & $\begin{array}{l}\mu g / \mathrm{kg} \\
\mu g / \mathrm{kg} \\
\mu g / \mathrm{kg} \\
\mu g / \mathrm{kg} \\
\mu g / \mathrm{kg} \\
\mu g / \mathrm{kg} \\
\mu g / \mathrm{kg} \\
\mu g / \mathrm{kg} \\
\mu g / \mathrm{kg} \\
\mu g / \mathrm{kg} \\
\mu g / \mathrm{kg} \\
\mu g / \mathrm{kg} \\
\mu g / \mathrm{kg} \\
\mu \mathrm{g} / \mathrm{kg} \\
\mu g / \mathrm{kg} \\
\mu g / \mathrm{kg} \\
\mu \mathrm{g} / \mathrm{kg} \\
\mu \mathrm{g} / \mathrm{kg} \\
\mu g / \mathrm{kg} \\
\mu g / \mathrm{kg} \\
\mu \mathrm{g} / \mathrm{kg} \\
\mu \mathrm{g} / \mathrm{kg} \\
\mu g / \mathrm{kg} \\
\mu g / \mathrm{kg} \\
\mu g / \mathrm{kg} \\
\mu g / \mathrm{kg} \\
\mu g / \mathrm{kg} \\
\mu g / \mathrm{kg} \\
\mu g / \mathrm{kg} \\
\mu g / \mathrm{kg} \\
\mu g / \mathrm{kg}\end{array}$ & $\begin{array}{r}4.40 \\
4.40 \\
4.40 \\
4.40 \\
4.40 \\
241.00 \\
307.00 \\
4.40 \\
88.00 \\
2.00 \\
3.00 \\
2.00 \\
10.00 \\
2.00 \\
20.00 \\
4.00 \\
212.00 \\
531.00 \\
8.80 \\
111.00 \\
8.80 \\
4.40 \\
8.80 \\
8.80 \\
8.80 \\
8.80 \\
28.00 \\
4.40 \\
4.40 \\
6.00 \\
4.40\end{array}$ & $\begin{array}{l}U \\
U \\
U J J \\
U \\
U \\
\text { UJN } \\
\text { UJN } \\
\text { UJ } \\
\text { UJN } \\
\text { UJN } \\
\text { UJN } \\
\text { UJN } \\
\text { UJN } \\
\text { UJN } \\
\text { UJN } \\
\text { UJN } \\
\text { UJ } \\
\text { UJ } \\
U \\
\text { UJ } \\
U \\
U \\
U \\
U \\
U \\
U \\
U \\
\text { UJN } \\
U \\
U \\
\text { UJN } \\
\text { UJ }\end{array}$ \\
\hline
\end{tabular}


Table C.1 (continued)

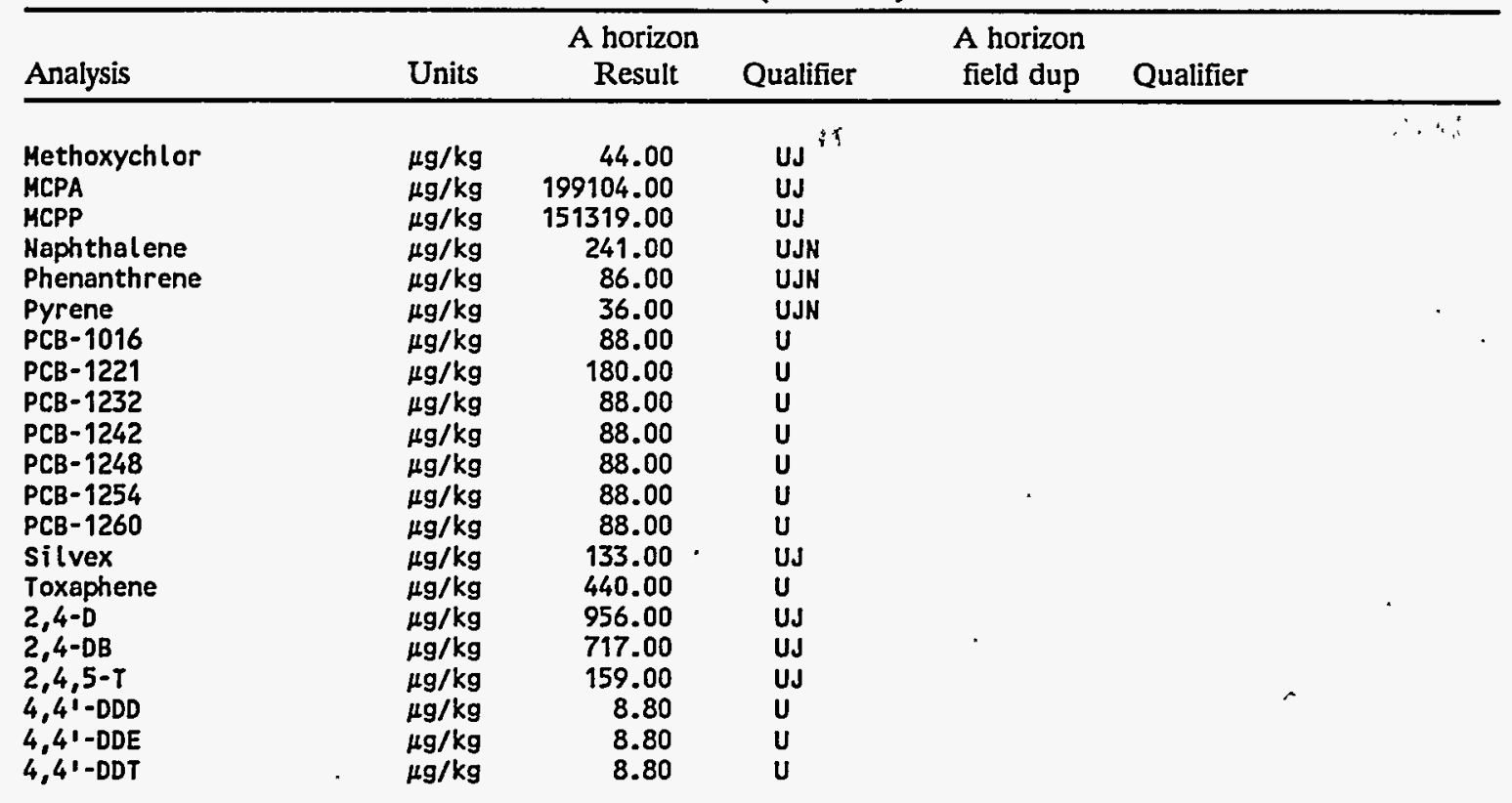

Location $=O R R ;$ Formation $=D I S M A L$ GAP; Site $=11 ; A=1080 ; A$ dup =' '; Phase $=1$

\begin{tabular}{|c|c|c|}
\hline $\begin{array}{l}\text { alpha-BHC } \\
\text { alpha-Chlordane } \\
\text { beta-BHC } \\
\text { delta-BHC } \\
\text { gamma-Chlordane } \\
\text { Acenaphthene } \\
\text { Acenaphthylene } \\
\text { Aldrin } \\
\text { Anthracene } \\
\text { Benzo(a)anthracene } \\
\text { Benzo(a)pyrene } \\
\text { Benzo(b)fluoranthene } \\
\text { Benzo(ghi)perylene } \\
\text { Benzo(k)fluoranthene } \\
\text { Chrysene } \\
\text { Dibenzo(a,h)anthracene } \\
\text { Dicamba } \\
\text { Dichlorprop } \\
\text { Dieldrin } \\
\text { Dinoseb } \\
\text { Endosulfan sulfate } \\
\text { Endosulfan I } \\
\text { Endosulfan II } \\
\text { Endrin } \\
\text { Endrin aldehyde } \\
\text { Endrin ketone } \\
\text { Fluoranthene } \\
\text { Fluorene } \\
\text { Heptachlor } \\
\text { Heptachlor epoxide } \\
\text { Indeno(1,2,3-cd)pyrene } \\
\text { Lindane } \\
\text { Methoxychlor } \\
\text { MCPA } \\
\text { MCPP } \\
\text { Haphthalene } \\
\text { Phenanthrene } \\
\text { Pyrene } \\
\text { PCB-1016 } \\
\text { PCB-1221 } \\
\text { PCB-1232 } \\
\text { PCB-1242 } \\
\text { PCB-1248 }\end{array}$ & 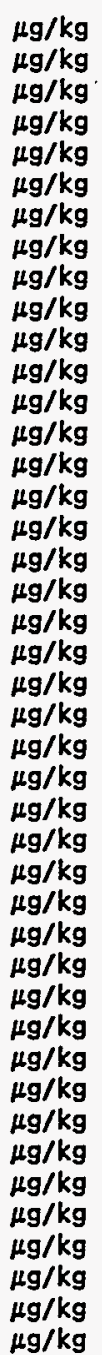 & $\begin{array}{r}4.30 \\
4.30 \\
4.30 \\
4.30 \\
4.30 \\
11593.00 \\
14814.00 \\
4.30 \\
4251.00 \\
84.00 \\
148.00 \\
116.00 \\
490.00 \\
109.00 \\
966.00 \\
193.00 \\
412.00 \\
1031.00 \\
8.60 \\
216.00 \\
8.80 \\
4.30 \\
8.60 \\
8.60 \\
8.60 \\
8.60 \\
348.00 \\
1353.00 \\
4.30 \\
4.30 \\
277.00 \\
4.30 \\
43.00 \\
386449.00 \\
293701.00 \\
11593.00 \\
4122.00 \\
1739.00 \\
86.00 \\
254.00 \\
86.00 \\
86.00 \\
86.00\end{array}$ \\
\hline
\end{tabular}




\section{C-48}

Table C.1 (continued)

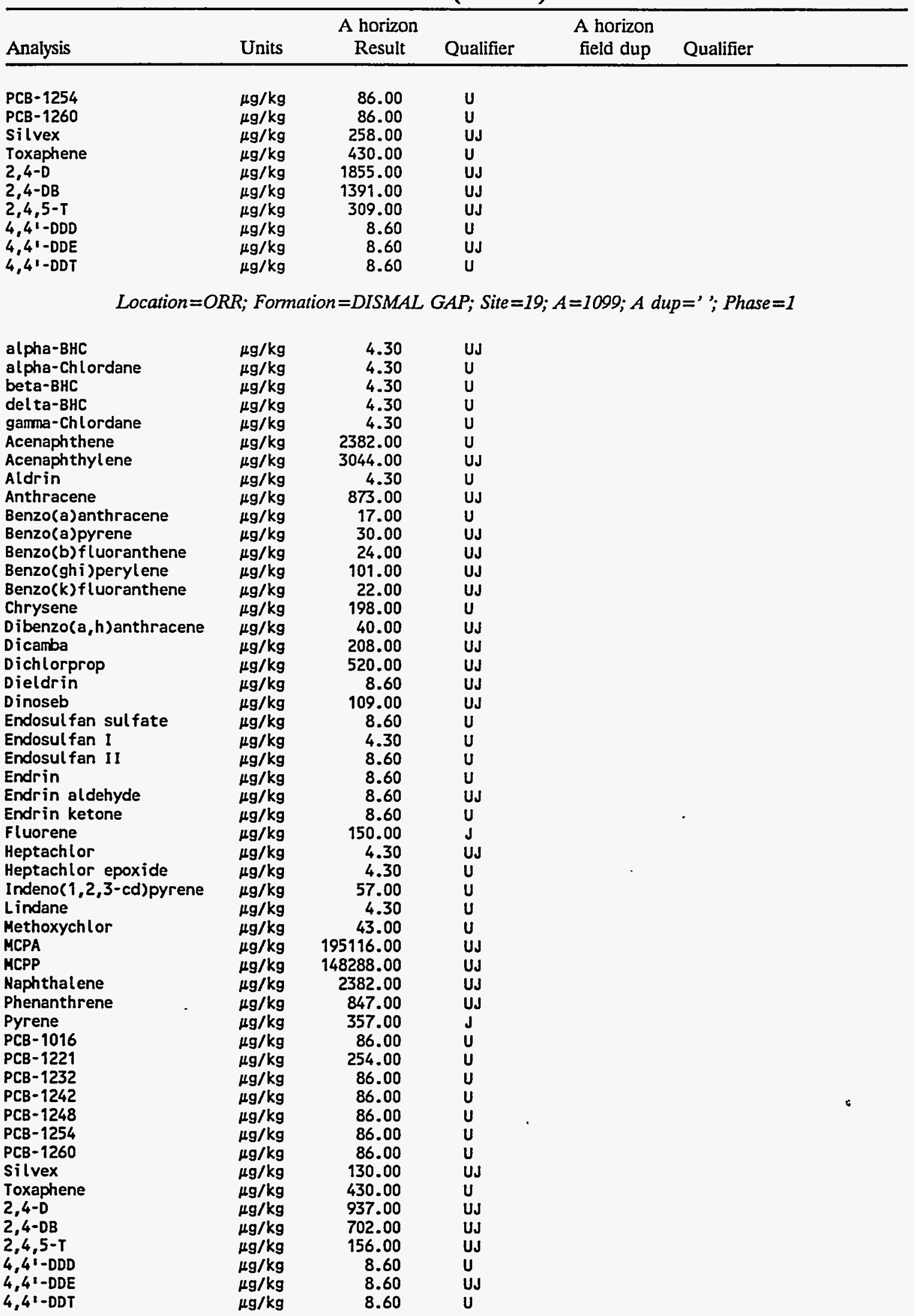


Table C.1 (continued)

\begin{tabular}{|c|c|c|c|c|c|c|}
\hline Analysis & Units & $\begin{array}{c}\text { A horizon } \\
\text { Result }\end{array}$ & Qualifier & $\begin{array}{l}\text { A horizon } \\
\text { field dup }\end{array}$ & -Qualifier & \\
\hline
\end{tabular}

alpha-BHC

alpha-Chlordane

beta-BHC

delta-BhC

gamma-Chlordane

Acenaph thene

Acenaphthylene

Aldrin

Anthracene

Benzo(a)anthracene

Benzo(a)pyrene

Benzo(b) fluoranthene

Benzo(ghi)perylene

Benzo(k)fluoranthene

Chrysene

Dalapon

Dibenzo $(a, h)$ anthracene

Dicamba

Dichlorprop

Dieldrin

Dinoseb

Endosulfan sulfate

Endosulfan I

Endosulfan II

Endrin

Endrin aldehyde

Endrin ketone

Fluorene

Heptachlor

Heptachlor epoxide

Indeno $(1,2,3-c d)$ pyrene

Lindane

Methoxychlor

MCPA

MCPP

Haph thalene

Phenanthrene

Pyrene

PCB-1016

PCB-1221

PCB-1232

PCB-1242

PCB-1248

PCB-1254

PCB-1260

silvex

Toxaphene

2,4-D

2, 4-DB

$2,4,5-T$

$4,4^{1}$-DDD

$4,4^{\prime}-D D E$

$4,4^{\circ}-$ DDT

$\begin{array}{lr}\mu g / k g & 4.10 \\ \mu g / k g & 4.10 \\ \mu g / k g & 4.10 \\ \mu g / k g & 4.10 \\ \mu g / k g & 4.10 \\ \mu g / k g & 213.00 \\ \mu g / k g & 272.00 \\ \mu g / k g & 4.10 \\ \mu g / k g & 78.00 \\ \mu g / k g & 2.00 \\ \mu g / k g & 3.00 \\ \mu g / k g & 2.00 \\ \mu g / k g & 9.00 \\ \mu g / k g & 2.00 \\ \mu g / k g & 18.00 \\ \mu g / k g & 864.00 \\ \mu g / k g & 4.00 \\ \mu g / k g & 40.00 \\ \mu g / k g & 99.00 \\ \mu g / k g & 4.10 \\ \mu g / k g & 21.00 \\ \mu g / k g & 8.20 \\ \mu g / k g & 4.10 \\ \mu g / k g & 8.20 \\ \mu g / k g & 8.20 \\ \mu g / k g & 8.20 \\ \mu g / k g & 8.20 \\ \mu g / k g & 25.00 \\ \mu g / k g & 4.10 \\ \mu g / k g & 4.10 \\ \mu g / k g & 5.00 \\ \mu g / k g & 4.10 \\ \mu g / k g & 41.00 \\ \mu g / k g & 37230.00 \\ \mu g / k g & 28295.00 \\ \mu g / k g & 213.00 \\ \mu g / k g & 76.00 \\ \mu g / k g & 32.00 \\ \mu g / k g & 82.00 \\ \mu g / k g & 160.00 \\ \mu g / k g & 82.00 \\ \mu g / k g & 82.00 \\ \mu g / k g & 82.00 \\ \mu g / k g & 82.00 \\ \mu g / k g & 82.00 \\ \mu g / k g & 25.00 \\ \mu g / k g & 410.00 \\ \mu g / k g & 179.00 \\ \mu g / k g & 134.00 \\ \mu g / k g & 30.00 \\ \mu g / k g & 8.20 \\ \mu g / k g & 8.20 \\ \mu g / k g & 8.20 \\ & \end{array}$

\begin{tabular}{|c|c|c|}
\hline UJ & 4.0 & UJ \\
\hline UJ & 4.0 & UJ \\
\hline UJ & 4.0 & UJ \\
\hline UJ & 4.0 & UJ \\
\hline U & 4.0 & U \\
\hline UJ & 213.0 & UJ \\
\hline UJ & 272.0 & UJ \\
\hline$U$ & 4.0 & $u$ \\
\hline UJ & 78.0 & UJ \\
\hline UJ & 2.0 & UJ \\
\hline UJ & 3.0 & UJ \\
\hline UJ & 2.0 & US \\
\hline UJ & 9.0 & UJ \\
\hline UJ & 2.0 & UJ \\
\hline UJ & 18.0 & UJ \\
\hline UJ & 850.0 & UJ \\
\hline UJ & 4.0 & UJ \\
\hline$U$ & 39.0 & UJ \\
\hline U & 98.0 & UJ \\
\hline UJ & 4.0 & UJ \\
\hline$U$ & 21.0 & UJ \\
\hline$U$ & & \\
\hline UJ & 4.0 & UJ \\
\hline$u$ & 8.0 & $u$ \\
\hline U & 8.0 & $u$ \\
\hline $\mathbf{u}$ & & \\
\hline $\mathbf{U}$ & 8.0 & $u$ \\
\hline UJ & 25.0 & UJ \\
\hline UJ & 4.0 & UJ \\
\hline $\mathbf{U}$ & 4.0 & $U$ \\
\hline UJ & .5 .0 & UJ \\
\hline UJ & 4.0 & US \\
\hline$u$ & 40.0 & $\mathbf{U}$ \\
\hline U & 36643.0 & UJ \\
\hline$u$ & 27849.0 & UJ \\
\hline UJ & 213.0 & UJ \\
\hline UJ & 76.0 & UJ \\
\hline UJN & 9.1 & \rfloor \\
\hline $\mathrm{u}$ & 80.0 & $\mathbf{U}$ \\
\hline $\mathbf{u}$ & 160.0 & U \\
\hline U & 80.0 & $U$ \\
\hline$U$ & 80.0 & $u$ \\
\hline$u$ & 80.0 & U \\
\hline 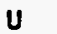 & 80.0 & U \\
\hline$U$ & 80.0 & u \\
\hline UJ & 24.0 & US \\
\hline$U$ & 400.0 & $U$ \\
\hline$U$ & 176.0 & US \\
\hline U & 132.0 & UJ \\
\hline UJ & 29.0 & UJ \\
\hline UJ & 8.0 & UJ \\
\hline UJ & 8.0 & UJ \\
\hline U & 8.0 & $\mathbf{U}$ \\
\hline
\end{tabular}

Location=ORR; Formation=DISMAL GAP; Site=22; $A=1106 ; A$ dup=' ; Phase=1

$\begin{array}{lrrl}\text { alpha-BHC } & \mu \mathrm{g} / \mathrm{kg} & 4.30 & \mathrm{UJ} \\ \text { alpha-Chlordane } & \mu \mathrm{g} / \mathrm{kg} & 4.30 & \mathrm{U} \\ \text { beta-BHC } & \mu \mathrm{g} / \mathrm{kg} & 4.30 & \mathrm{U} \\ \text { delta-BHC } & \mu \mathrm{g} / \mathrm{kg} & 4.30 & \mathrm{U} \\ \text { gamma-Chlordane } & \mu \mathrm{g} / \mathrm{kg} & 4.30 & \mathrm{U} \\ \text { Acenaphthene } & \mu \mathrm{g} / \mathrm{kg} & 2437.00 & \mathrm{UJN} \\ \text { Acenaphthylene } & \mu \mathrm{g} / \mathrm{kg} & 3114.00 & \mathrm{UJN} \\ \text { Aldrin } & \mu \mathrm{g} / \mathrm{kg} & 4.30 & \mathrm{U}\end{array}$


Table C.1 (continued)

\begin{tabular}{|c|c|c|c|c|c|}
\hline Analysis & Units & $\begin{array}{c}\text { A horizon } \\
\text { Result }\end{array}$ & Qualifier & $\begin{array}{l}\text { A horizon } \\
\text { field dup }\end{array}$ & Qualifier \\
\hline $\begin{array}{l}\text { Anthracene } \\
\text { Benzo(a)anthracene } \\
\text { Benzo(a)pyrene } \\
\text { Benzo(b)fluoranthene } \\
\text { Benzo(ghi)perylene } \\
\text { Benzo(k)fluoranthene } \\
\text { Chrysene } \\
\text { Dibenzo(a, h)anthracene } \\
\text { Dicamba } \\
\text { Dichlorprop } \\
\text { Dieldrin } \\
\text { Dinoseb } \\
\text { Endosulfan sulfate } \\
\text { Endosulfan I } \\
\text { Endosulfan II } \\
\text { Endrin } \\
\text { Endrin aldehyde } \\
\text { Endrin ketone } \\
\text { Fluorene } \\
\text { Heptachlor } \\
\text { Heptachlor epoxide } \\
\text { Indeno( } 1,2,3-\text { cd)pyrene } \\
\text { Lindane } \\
\text { Methoxychlor } \\
\text { MCPA } \\
\text { MCPP } \\
\text { Naphthalene } \\
\text { Phenanthrene } \\
\text { Pyrene } \\
\text { PCB-1016 } \\
\text { PCB-1221 } \\
\text { PCB-1232 } \\
\text { PCB-1242 } \\
\text { PCB-1248 } \\
\text { PCB-1254 } \\
\text { PCB-1260 } \\
\text { Silvex } \\
\text { Toxaphene } \\
2,4-D \\
2,4-D B \\
2,4,5-T \\
4,4 '-0 D D \\
4,4 '-D D E \\
4,4 \text {-DDT }\end{array}$ & 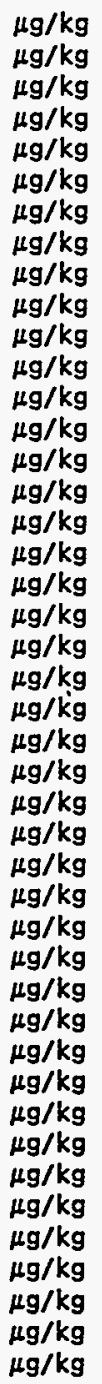 & $\begin{array}{r}894.00 \\
18.00 \\
31.00 \\
24.00 \\
103.00 \\
23.00 \\
203.00 \\
41.00 \\
216.00 \\
539.00 \\
4.30 \\
113.00 \\
8.80 \\
4.30 \\
8.80 \\
8.80 \\
8.80 \\
8.80 \\
284.00 \\
4.30 \\
4.30 \\
58.00 \\
4.30 \\
43.00 \\
202302.00 \\
153749.00 \\
2437.00 \\
867.00 \\
366.00 \\
88.00 \\
176.00 \\
88.00 \\
88.00 \\
88.00 \\
88.00 \\
88.00 \\
135.00 \\
430.00 \\
971.00 \\
728.00 \\
162.00 \\
8.80 \\
8.80 \\
4.30\end{array}$ & 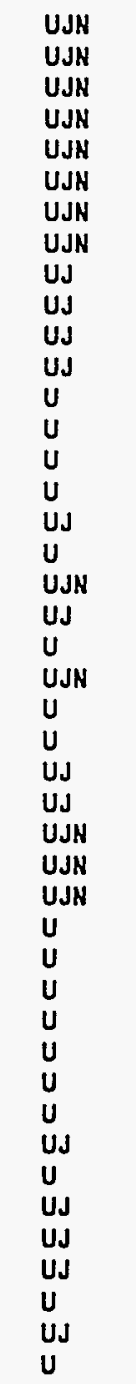 & & \\
\hline
\end{tabular}

Location=ORR; Formation=DISMAL GAP; Site $=26 ; A=1213 ; A$ dup=' '; Phase=1

\begin{tabular}{|c|c|c|}
\hline $\begin{array}{l}\text { alpha-BHC } \\
\text { alpha-Chlordane } \\
\text { beta-BHC } \\
\text { del ta-BHC } \\
\text { gamma-Chlordane } \\
\text { Aldrin } \\
\text { Dalapon } \\
\text { Dicamba } \\
\text { Dichlorprop } \\
\text { Dieldrin } \\
\text { Dinoseb } \\
\text { Endosul fan sulfate } \\
\text { Endosul fan I } \\
\text { Endosul fan II } \\
\text { Endrin } \\
\text { Endrin aldehyde } \\
\text { Endrin ketone } \\
\text { Heptachlor } \\
\text { Heptachlor epoxide } \\
\text { Lindane }\end{array}$ & $\begin{array}{l}\mu g / \mathrm{kg} \\
\mu \mathrm{g} / \mathrm{kg} \\
\mu \mathrm{g} / \mathrm{kg} \\
\mu \mathrm{g} / \mathrm{kg} \\
\mu g / \mathrm{kg} \\
\mu g / \mathrm{kg} \\
\mu g / \mathrm{kg} \\
\mu g / \mathrm{kg} \\
\mu g / \mathrm{kg} \\
\mu g / \mathrm{kg} \\
\mu g / \mathrm{kg} \\
\mu g / \mathrm{kg} \\
\mu g / \mathrm{kg} \\
\mu g / \mathrm{kg} \\
\mu g / \mathrm{kg} \\
\mu \mathrm{g} / \mathrm{kg} \\
\mu g / \mathrm{kg} \\
\mu g / \mathrm{kg} \\
\mu g / \mathrm{kg} \\
\mu g / \mathrm{kg}\end{array}$ & $\begin{array}{r}4.10 \\
4.10 \\
4.10 \\
4.10 \\
4.10 \\
4.10 \\
877.00 \\
40.00 \\
101.00 \\
8.30 \\
21.00 \\
8.30 \\
4.10 \\
8.30 \\
8.30 \\
8.30 \\
8.30 \\
4.10 \\
4.10 \\
4.10\end{array}$ \\
\hline
\end{tabular}




\section{C-51}

Table C.1 (continued)

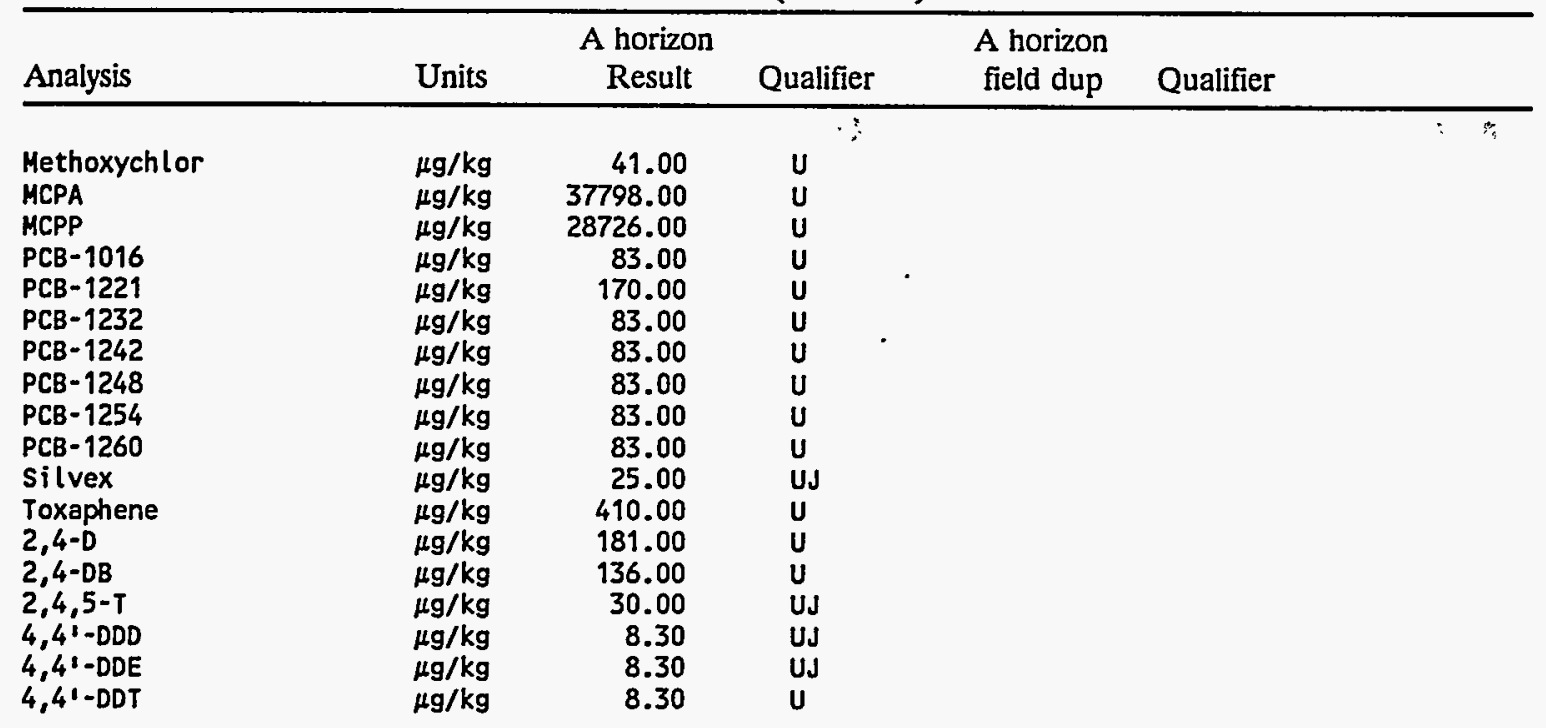

Location $=O R R ;$ Formation=DISMAL GAP; Site $=27 ; A=1072 ; A$ dup=' '; Phase $=1$

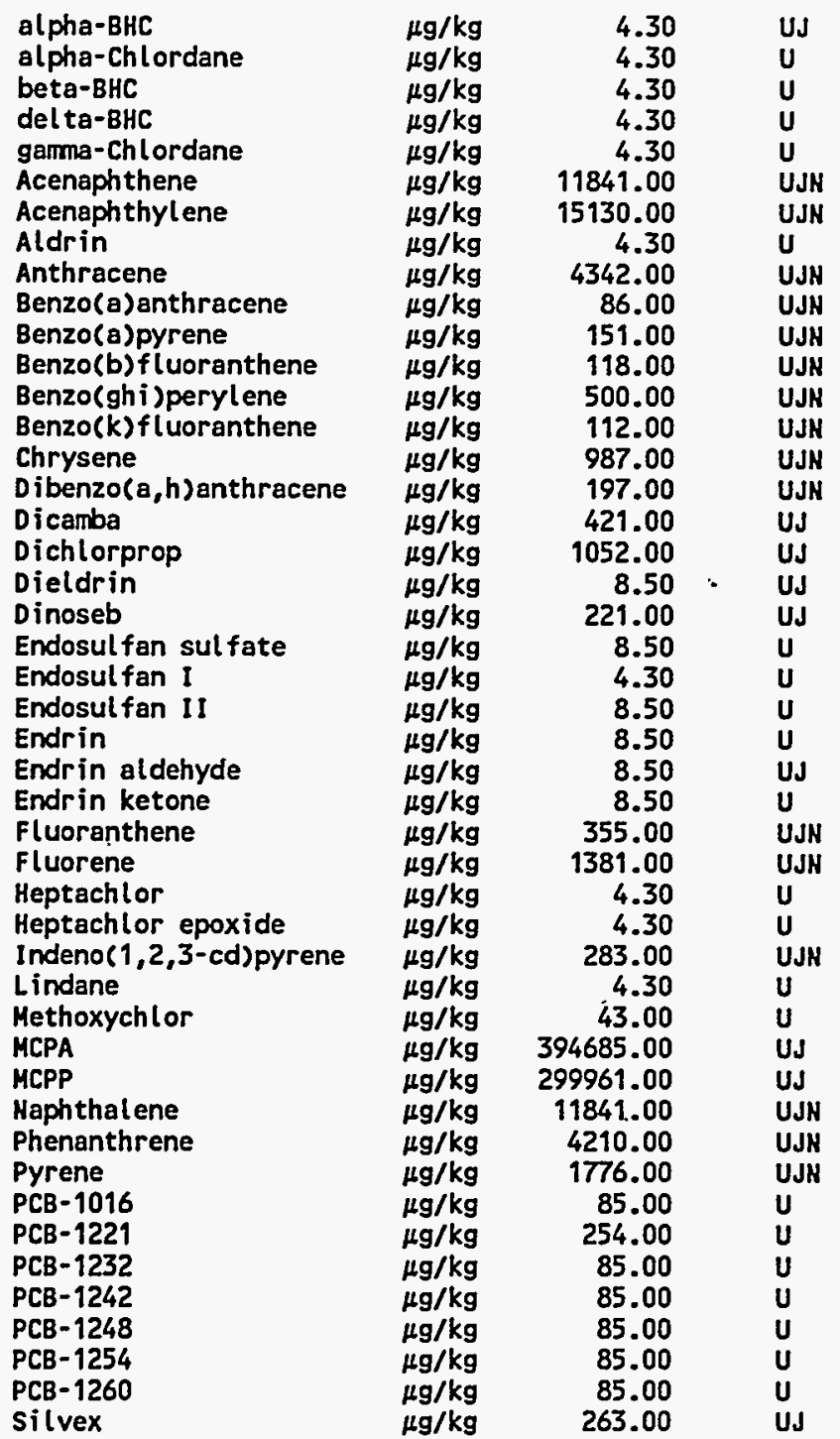


C-52

Table C.1 (continucd)

\begin{tabular}{|c|c|c|c|c|c|}
\hline Analysis & Units & $\begin{array}{c}\text { A horizon } \\
\text { Result }\end{array}$ & Qualifier & $\begin{array}{l}\text { A horizon } \\
\text { field dup }\end{array}$ & Qualifier \\
\hline $\begin{array}{l}\text { Toxaphene } \\
2,4-D \\
2,4-D B \\
2,4,5-T \\
4^{\prime}, 4^{\prime}-D D D \\
4,4^{\prime}-D D E \\
4,4^{\prime}-D D T\end{array}$ & $\begin{array}{l}\mu g / \mathrm{kg} \\
\mu g / \mathrm{kg} \\
\mu g / \mathrm{kg} \\
\mu g / \mathrm{kg} \\
\mu \mathrm{g} / \mathrm{kg} \\
\mu g / \mathrm{kg} \\
\mu g / \mathrm{kg}\end{array}$ & $\begin{array}{r}430.00 \\
1894.00 \\
1421.00 \\
316.00 \\
8.50 \\
8.50 \\
8.50\end{array}$ & $\begin{array}{l}U \\
U J \\
U J \\
U J \\
U \\
U J \\
U\end{array}$ & & \\
\hline
\end{tabular}

Location $=$ ORR; Formation $=$ DISMAL GAP; Site $=32 ; A=1107 ; A$ dup=' '; Phase $=1$

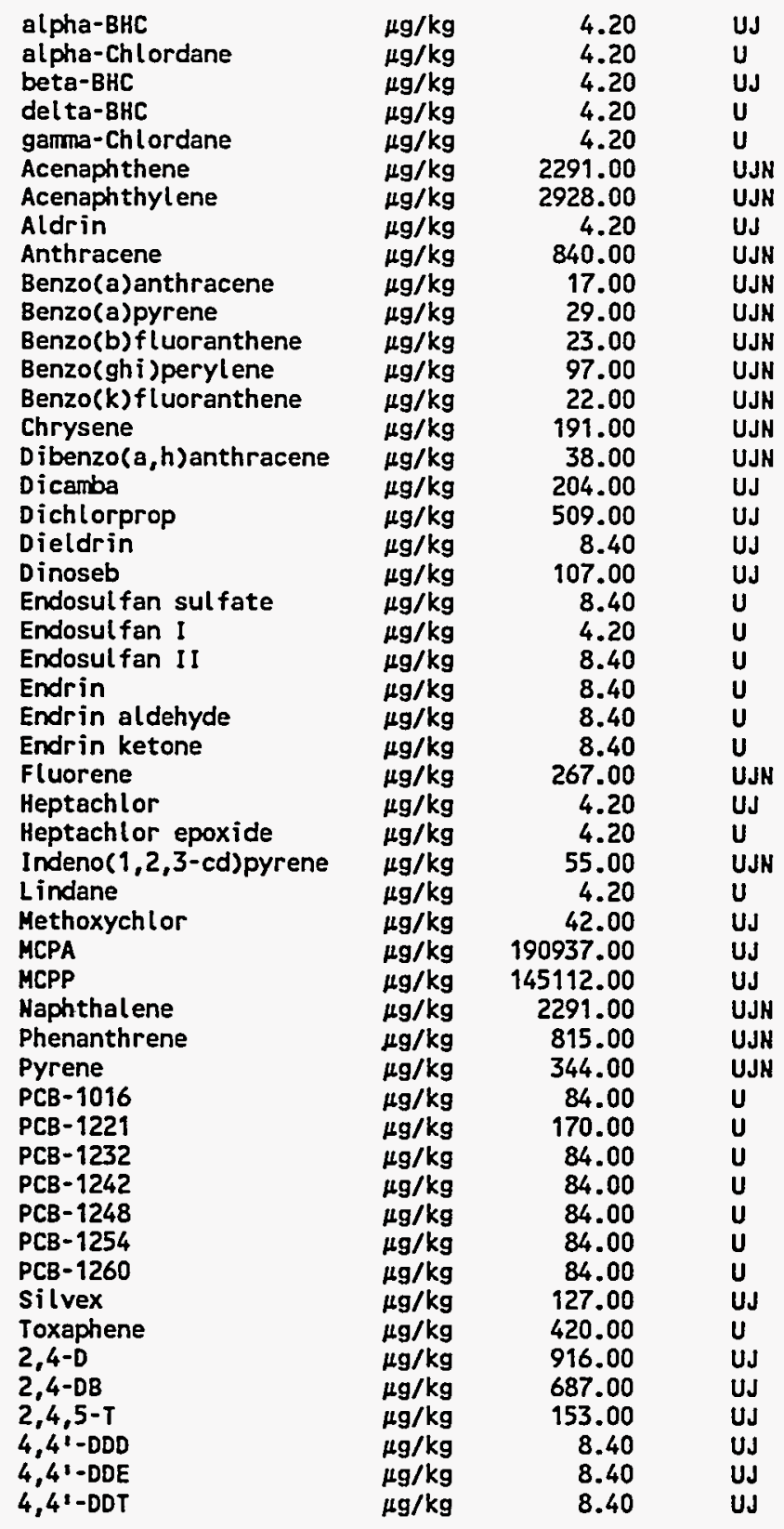




\section{C-53}

Table C.1 (continued)

\begin{tabular}{|c|c|c|c|c|c|c|}
\hline Analysis & Units & $\begin{array}{c}\text { A horizon } \\
\text { Result }\end{array}$ & Qualifier & $\begin{array}{l}\text { A horizon } \\
\text { field dup }\end{array}$ & Qualifier & \\
\hline \multicolumn{7}{|c|}{ Location=ORR; Formation =DISMAL GAP; Site $=33 ; A=1108 ; A$ dup=' '; Phase $=1$} \\
\hline 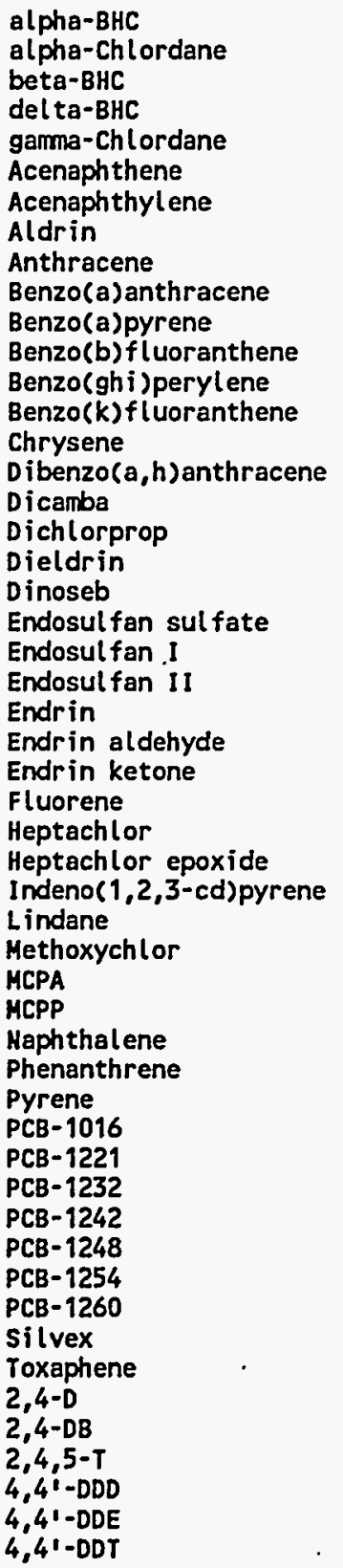 & 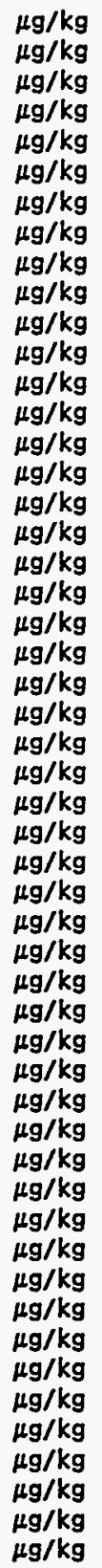 & $\begin{array}{r}4.70 \\
4.70 \\
4.70 \\
4.70 \\
4.70 \\
2568.00 \\
3281.00 \\
4.70 \\
942.00 \\
19.00 \\
33.00 \\
26.00 \\
108.00 \\
24.00 \\
214.00 \\
43.00 \\
228.00 \\
570.00 \\
9.40 \\
120.00 \\
9.40 \\
4.70 \\
9.40 \\
9.40 \\
9.40 \\
9.40 \\
300.00 \\
4.70 \\
4.70 \\
61.00 \\
4.70 \\
47.00 \\
213583.00 \\
162323.00 \\
2568.00 \\
913.00 \\
385.00 \\
94.00 \\
190.00 \\
94.00 \\
94.00 \\
94.00 \\
94.00 \\
94.00 \\
142.00 \\
470.00 \\
1025.00 \\
769.00 \\
171.00 \\
9.40 \\
9.40 \\
9.40\end{array}$ & 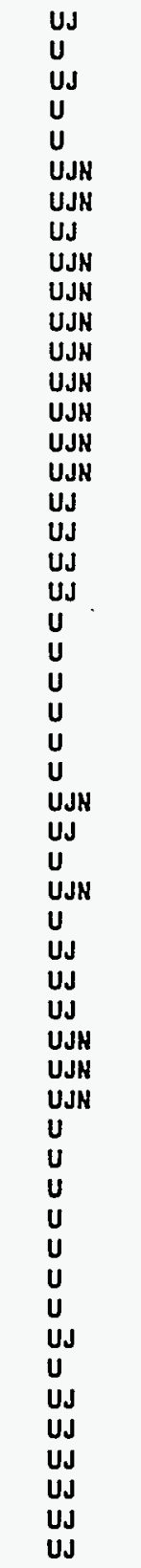 & & & \\
\hline
\end{tabular}

Location=ORR; Formation=DISMAL GAP; Site=35; $A=1115 ; A$ dup=' '; Phase=1

$\begin{array}{lrrl}\text { alpha-BHC } & \mu \mathrm{g} / \mathrm{kg} & 4.20 & \mathrm{UJ} \\ \text { alpha-Chlordane } & \mu \mathrm{g} / \mathrm{kg} & 4.20 & \mathrm{U} \\ \text { beta-BHC } & \mu \mathrm{g} / \mathrm{kg} & 4.20 & \mathrm{UJ} \\ \text { del ta-BHC } & \mu \mathrm{g} / \mathrm{kg} & 4.20 & \mathrm{U} \\ \text { gamma-Chlordane } & \mu \mathrm{g} / \mathrm{kg} & 4.20 & \mathrm{U} \\ \text { Acenaphthene } & \mu \mathrm{g} / \mathrm{kg} & 2285.00 & \mathrm{UJN} \\ \text { Acenaphthylene } & \mu \mathrm{g} / \mathrm{kg} & 2920.00 & \mathrm{UJN} \\ \text { Aldrin } & \mu \mathrm{g} / \mathrm{kg} & 4.20 & \text { UJ } \\ \text { Anthracene } & \mu \mathrm{g} / \mathrm{kg} & 838.00 & \text { UJN }\end{array}$




\section{C-54}

Table C.1 (continued)

\begin{tabular}{|c|c|c|c|c|c|}
\hline Analysis & Units & $\begin{array}{c}\text { A horizon } \\
\text { Result }\end{array}$ & Qualifier & $\begin{array}{l}\text { A horizon } \\
\text { field dup }\end{array}$ & Qualifier \\
\hline 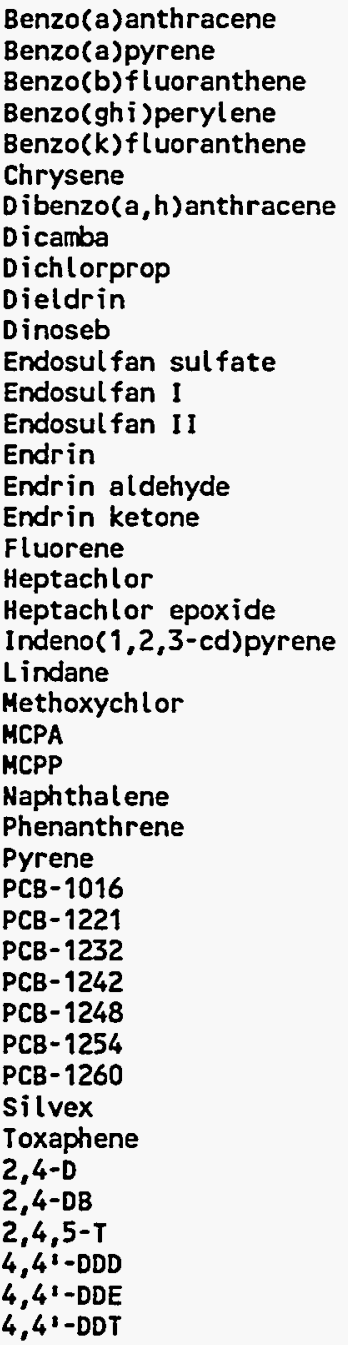 & 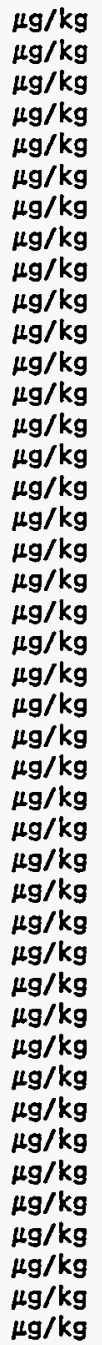 & $\begin{array}{r}17.00 \\
29.00 \\
23.00 \\
96.00 \\
22.00 \\
190.00 \\
38.00 \\
202.00 \\
504.00 \\
8.40 \\
106.00 \\
8.40 \\
4.20 \\
8.40 \\
8.40 \\
8.40 \\
8.40 \\
267.00 \\
4.20 \\
4.20 \\
55.00 \\
4.20 \\
42.00 \\
189119.00 \\
143730.00 \\
2285.00 \\
813.00 \\
343.00 \\
84.00 \\
170.00 \\
84.00 \\
84.00 \\
84.00 \\
84.00 \\
84.00 \\
126.00 \\
420.00 \\
908.00 \\
681.00 \\
151.00 \\
8.40 \\
8.40 \\
8.40\end{array}$ & $\begin{array}{l}\text { UJN } \\
\text { UJN } \\
\text { UJN } \\
\text { UJN } \\
\text { UJN } \\
\text { UJN } \\
\text { UJN } \\
\text { UJ } \\
\text { UJ } \\
\text { UJ } \\
U J \\
U \\
U \\
U \\
U \\
U \\
U \\
U J N \\
U J \\
U \\
U J N \\
U \\
U J \\
U J \\
U J \\
U J N \\
\text { UJN } \\
\text { UJN } \\
U \\
U \\
U \\
U \\
U \\
U \\
U \\
U J \\
U \\
U J \\
U J \\
U J \\
U J \\
U J \\
U J\end{array}$ & & \\
\hline
\end{tabular}

Location=ORR; Formation =DISMAL GAP; Site=41; $A=1064 ; A$ dup=' '; Phase =1

\begin{tabular}{|c|c|c|}
\hline $\begin{array}{l}\text { alpha-BHC } \\
\text { alpha-Chlordane } \\
\text { beta-BHC } \\
\text { del ta-BHC } \\
\text { gamma-Chlordane } \\
\text { Acenaphthene } \\
\text { Acenaphthylene } \\
\text { Aldrin } \\
\text { Anthracene } \\
\text { Benzo(a)anthracene } \\
\text { Benzo(a)pyrene } \\
\text { Benzo(b)fluoranthene } \\
\text { Benzo(ghi)perylene } \\
\text { Benzo(k)fluoranthene } \\
\text { Chrysene } \\
\text { Dibenzo(a, h)anthracene } \\
\text { Dicamba } \\
\text { Dichlorprop } \\
\text { Dieldrin } \\
\text { Dinoseb } \\
\text { Endosulfan sulfate }\end{array}$ & $\begin{array}{l}\mu g / \mathrm{kg} \\
\mu g / \mathrm{kg} \\
\mu g / \mathrm{kg} \\
\mu g / \mathrm{kg} \\
\mu g / \mathrm{kg} \\
\mu g / \mathrm{kg} \\
\mu g / \mathrm{kg} \\
\mu g / \mathrm{kg} \\
\mu g / \mathrm{kg} \\
\mu g / \mathrm{kg} \\
\mu g / \mathrm{kg} \\
\mu g / \mathrm{kg} \\
\mu g / \mathrm{kg} \\
\mu g / \mathrm{kg} \\
\mu g / \mathrm{kg} \\
\mu g / \mathrm{kg} \\
\mu g / \mathrm{kg} \\
\mu g / \mathrm{kg} \\
\mu g / \mathrm{kg} \\
\mu g / \mathrm{kg} \\
\mu g / \mathrm{kg}\end{array}$ & $\begin{array}{r}4.20 \\
4.20 \\
4.20 \\
4.20 \\
4.20 \\
11464.00 \\
14648.00 \\
4.20 \\
4203.00 \\
83.00 \\
146.00 \\
115.00 \\
484.00 \\
108.00 \\
955.00 \\
191.00 \\
408.00 \\
1019.00 \\
8.50 \\
214.00 \\
8.50\end{array}$ \\
\hline
\end{tabular}




\section{C-55}

Table C.1 (continued)

\begin{tabular}{|c|c|c|c|c|c|c|}
\hline Analysis & Units & $\begin{array}{c}\text { A horizon } \\
\text { Result }\end{array}$ & Qualifier & $\begin{array}{l}\text { A horizon } \\
\text { field dup }\end{array}$ & Qualifier & \\
\hline $\begin{array}{l}\text { Endosulfan I } \\
\text { Endosulfan II } \\
\text { Endrin } \\
\text { Endrin aldehyde } \\
\text { Endrin ketone } \\
\text { Fluoranthene } \\
\text { Fluorene } \\
\text { Heptachlor } \\
\text { Heptachlor epoxide } \\
\text { Indeno(1,2,3-cd)pyrene } \\
\text { Lindane } \\
\text { Methoxychlor } \\
\text { MCPA } \\
\text { MCPP } \\
\text { Naphthalene } \\
\text { Phenanthrene } \\
\text { Pyrene } \\
\text { PCB-1016 } \\
\text { PCB-1221 } \\
\text { PCB-1232 } \\
\text { PCB-1242 } \\
\text { PCB-1248 } \\
\text { PCB-1254 } \\
\text { PCB-1260 } \\
\text { Silvex } \\
\text { Toxaphene } \\
2,4-D \\
2,4-D B \\
2,4,5-T \\
4,41-D D D \\
4,41-D D E \\
4,41-D D T\end{array}$ & 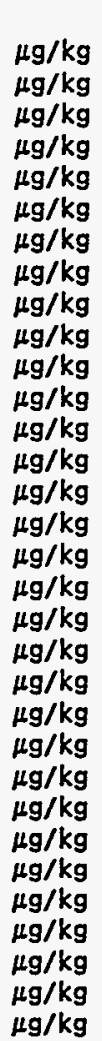 & $\begin{array}{r}4.20 \\
8.50 \\
8.50 \\
8.50 \\
8.50 \\
344.00 \\
1337.00 \\
4.20 \\
4.20 \\
274.00 \\
4.20 \\
42.00 \\
382117.00 \\
290409.00 \\
11464.00 \\
4076.00 \\
1720.00 \\
85.00 \\
169.00 \\
85.00 \\
85.00 \\
85.00 \\
85.00 \\
85.00 \\
255.00 \\
420.00 \\
1834.00 \\
1376.00 \\
306.00 \\
8.50 \\
8.50 \\
8.50\end{array}$ & $\begin{array}{l}U \\
U U \\
U \\
U J \\
U \\
U J N \\
U J H \\
U \\
U \\
U J N \\
U \\
U \\
U J \\
U J \\
U J N \\
U J N \\
U J N \\
U \\
U \\
U \\
U \\
U \\
U \\
U \\
U J \\
U \\
U S \\
U J \\
U J \\
U \\
U J \\
U \\
U S\end{array}$ & & & $\because \dot{r}$ \\
\hline
\end{tabular}

Location=ORR; Formation=DISMAL GAP; Site=43; $A=1231 ; A$ dup=' '; Phase $=1$

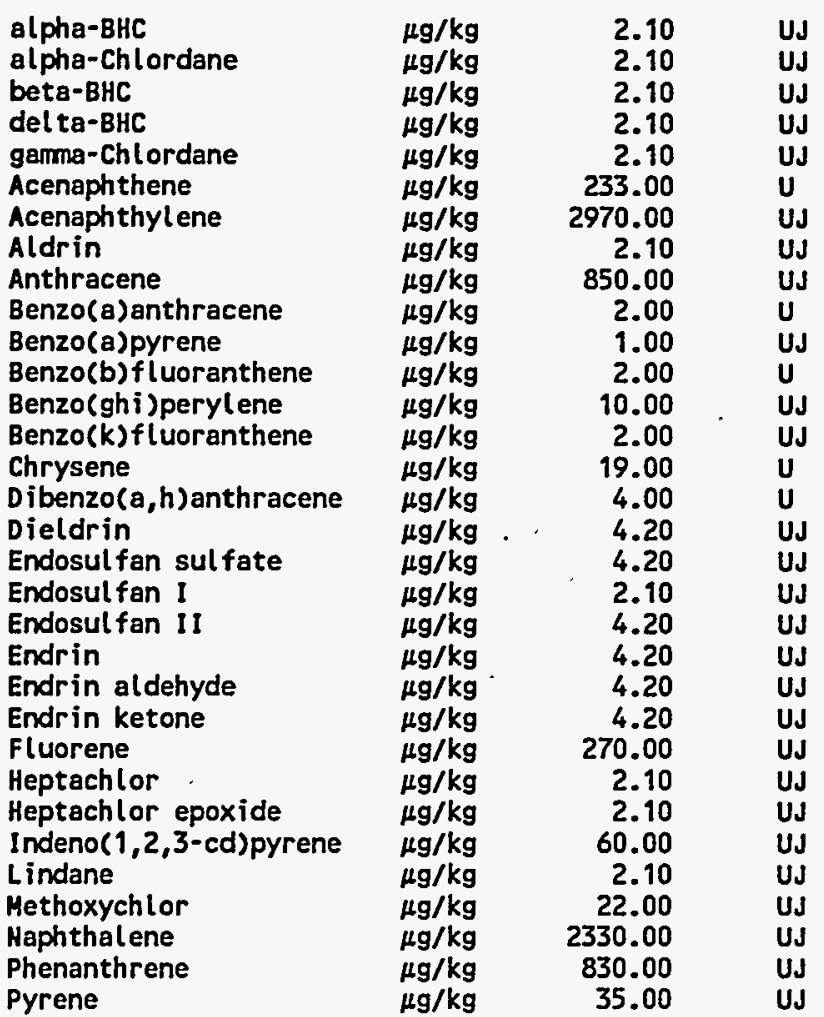


C-56

Table C.1 (continued)

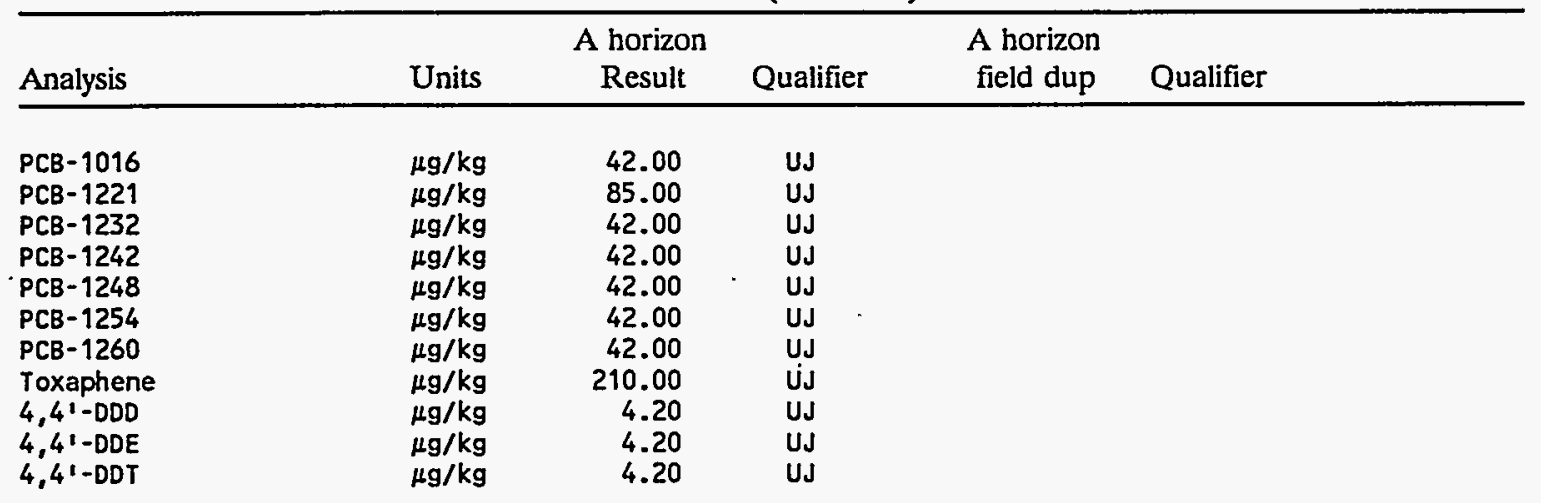

Location $=O R R ;$ Formation $=$ NOLICHUCKY; Site $=13 ; A=1299 ;$ A dup=' '; Phase $=1$

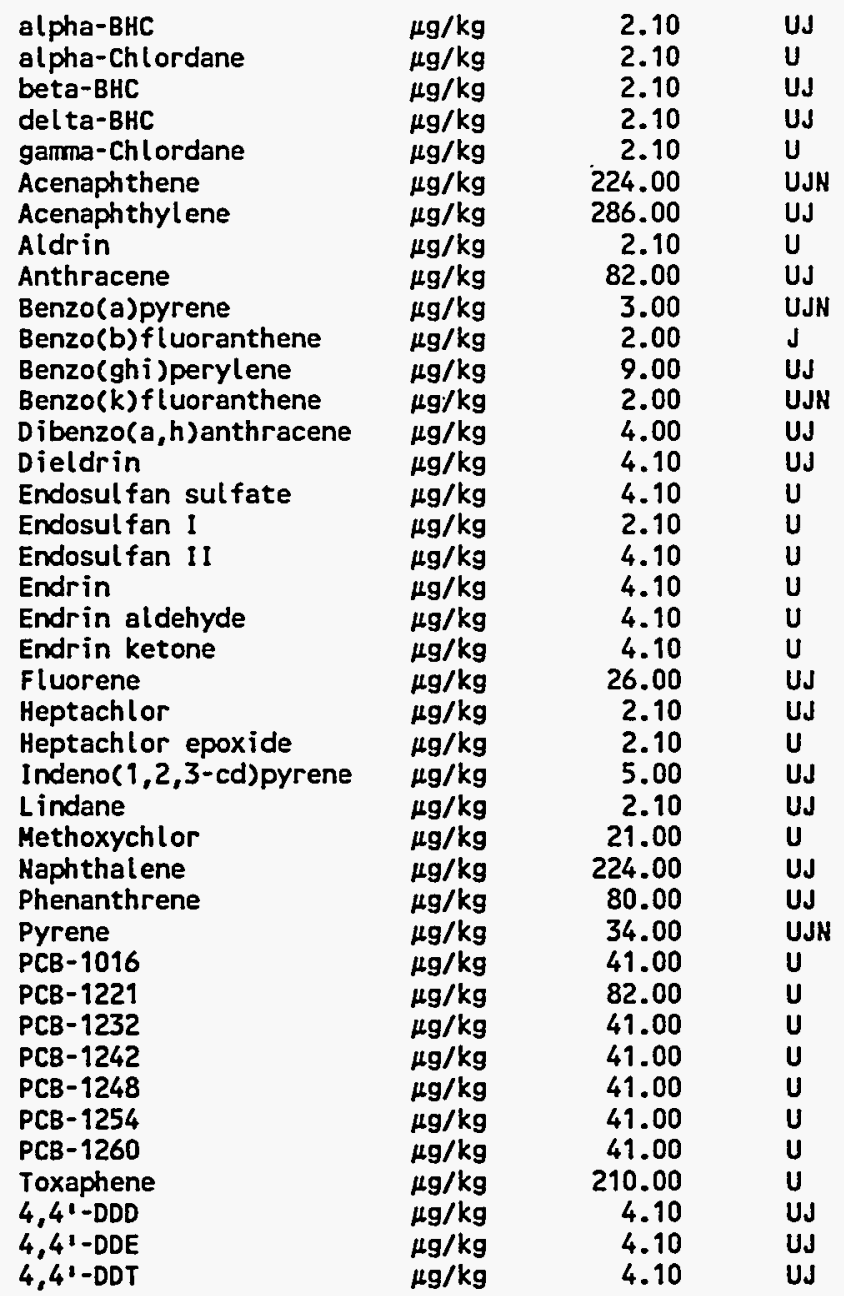

Location=ORR; Formation=NOLICHUCKY; Site $=15 ; A=1300 ; A$ dup=' '; Phase $=1$

$\begin{array}{llrl}\text { alpha-8HC } & \mu \mathrm{g} / \mathrm{kg} & 2.00 & \text { UJ } \\ \text { alpha-Chlordane } & \mu \mathrm{gg} & 2.00 & \mathrm{U} \\ \text { beta-BHC } & \mu \mathrm{kg} & 2.00 & \text { UJ } \\ \text { del ta-BHC } & \mu \mathrm{kg} / \mathrm{kg} & 2.00 & \text { UJ } \\ \text { garma-Chlordane } & \mu \mathrm{kg} & 2.00 & \mathrm{U} \\ \text { Acenaphthene } & \mu \mathrm{kg} / \mathrm{kg} & 224.00 & \text { UJ } \\ \text { Acenaphthylene } & \mu \mathrm{g} / \mathrm{kg} & 286.00 & \mathrm{UJ}\end{array}$




\section{C-57}

Table C.1 (continued)

\begin{tabular}{|c|c|c|c|c|c|c|}
\hline Analysis & Units & $\begin{array}{c}\text { A horizon } \\
\text { Result }\end{array}$ & Qualifier & $\begin{array}{l}\text { A horizon } \\
\text { field dup }\end{array}$ & Qualifier & \\
\hline $\begin{array}{l}\text { Aldrin } \\
\text { Anthracene } \\
\text { Benzo(a)pyrene } \\
\text { Benzo(b)fluoranthene } \\
\text { Benzo(ghi)perylene } \\
\text { Benzo(k)fluoranthene } \\
\text { Dibenzo(a,h)anthracene } \\
\text { Dieldrin } \\
\text { Endosul fan sul fate } \\
\text { Endosul fan I } \\
\text { Endosul fan II } \\
\text { Endrin } \\
\text { Endrin aldehyde } \\
\text { Endrin ketone } \\
\text { Fluorene } \\
\text { Heptachlor } \\
\text { Heptachlor epoxide } \\
\text { Indeno(1,2,3-cd)pyrene } \\
\text { Lindane } \\
\text { Methoxychlor } \\
\text { Naphthalene } \\
\text { Phenanthrene } \\
\text { Pyrene } \\
\text { PCB-1016 } \\
\text { PCB-1221 } \\
\text { PCB-1232 } \\
\text { PCB-1242 } \\
\text { PCB-1248 } \\
\text { PCB-1254 } \\
\text { PCB-1260 } \\
\text { Toxaphene } \\
4,41-D D D \\
4,41-D D E \\
4,41-D D T\end{array}$ & $\begin{array}{l}\mu g / \mathrm{kg} \\
\mu \mathrm{g} / \mathrm{kg} \\
\mu \mathrm{g} / \mathrm{kg} \\
\mu \mathrm{g} / \mathrm{kg} \\
\mu \mathrm{g} / \mathrm{kg} \\
\mu \mathrm{g} / \mathrm{kg} \\
\mu \mathrm{g} / \mathrm{kg} \\
\mu \mathrm{g} / \mathrm{kg} \\
\mu \mathrm{g} / \mathrm{kg} \\
\mu \mathrm{g} / \mathrm{kg} \\
\mu \mathrm{g} / \mathrm{kg} \\
\mu \mathrm{g} / \mathrm{kg} \\
\mu \mathrm{g} / \mathrm{kg} \\
\mu \mathrm{g} / \mathrm{kg} \\
\mu \mathrm{g} / \mathrm{kg} \\
\mu \mathrm{g} / \mathrm{kg} \\
\mu \mathrm{g} / \mathrm{kg} \\
\mu \mathrm{g} / \mathrm{kg} \\
\mu \mathrm{g} / \mathrm{kg} \\
\mu \mathrm{g} / \mathrm{kg} \\
\mu \mathrm{g} / \mathrm{kg} \\
\mu \mathrm{g} / \mathrm{kg} \\
\mu \mathrm{g} / \mathrm{kg} \\
\mu \mathrm{g} / \mathrm{kg} \\
\mu \mathrm{g} / \mathrm{kg} \\
\mu g / \mathrm{kg} \\
\mu \mathrm{g} / \mathrm{kg} \\
\mu \mathrm{g} / \mathrm{kg} \\
\mu \mathrm{g} / \mathrm{kg} \\
\mu \mathrm{g} / \mathrm{kg} \\
\mu \mathrm{g} / \mathrm{kg} \\
\mu g / \mathrm{kg} \\
\mu \mathrm{g} / \mathrm{kg} \\
\mu \mathrm{g} / \mathrm{kg}\end{array}$ & $\begin{array}{r}2.00 \\
82.00 \\
3.00 \\
2.00 \\
9.00 \\
2.00 \\
4.00 \\
4.10 \\
4.10 \\
2.00 \\
4.10 \\
4.10 \\
4.10 \\
4.10 \\
26.00 \\
2.60 \\
2.00 \\
5.00 \\
2.00 \\
20.00 \\
224.00 \\
80.00 \\
34.00 \\
41.00 \\
82.00 \\
41.00 \\
41.00 \\
41.00 \\
41.00 \\
41.00 \\
200.00 \\
4.10 \\
4.10 \\
4.10\end{array}$ & 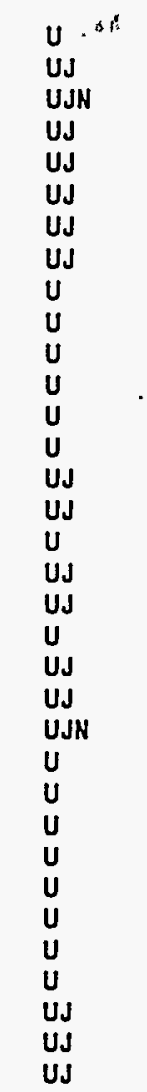 & & . & $\therefore 1 \%$ \\
\hline
\end{tabular}

Location=ORR; Formation=NOLICHUCKY; Site $=16 ; A=1301 ; A$ dup=' '; Phase $=1$

\begin{tabular}{|c|c|c|}
\hline $\begin{array}{l}\text { alpha-BHC } \\
\text { alpha-Chlordane } \\
\text { beta-BHC } \\
\text { del ta-BHC } \\
\text { gamma-Chlordane } \\
\text { Acenaphthene } \\
\text { Acenaphthylene } \\
\text { Aldrin } \\
\text { Anthracene } \\
\text { Benzo(a)pyrene } \\
\text { Benzo(b)fluoranthene } \\
\text { Benzo(ghi)perylene } \\
\text { Benzo(k)fluoranthene } \\
\text { Dibenzo(a, h)anthracene } \\
\text { Dieldrin } \\
\text { Endosul fan sul fate } \\
\text { Endosul fan I } \\
\text { Endosul fan II } \\
\text { Endrin } \\
\text { Endrin aldehyde } \\
\text { Endrin ketone } \\
\text { Fluorene } \\
\text { Heptachlor } \\
\text { Heptachlor epoxide } \\
\text { Indeno(1,2,3-cd)pyrene } \\
\text { Lindane } \\
\text { Methoxychlor } \\
\text { Naphthalene } \\
\text { Phenanthrene } \\
\text { Pyrene }\end{array}$ & $\begin{array}{l}\mu g / \mathrm{kg} \\
\mu \mathrm{g} / \mathrm{kg} \\
\mu \mathrm{g} / \mathrm{kg} \\
\mu \mathrm{g} / \mathrm{kg} \\
\mu \mathrm{ggg} \\
\mu \mathrm{kg} / \mathrm{kg} \\
\mu \mathrm{g} / \mathrm{kg} \\
\mu \mathrm{g} / \mathrm{kg} \\
\mu \mathrm{g} / \mathrm{kg} \\
\mu \mathrm{g} / \mathrm{kg} \\
\mu \mathrm{g} / \mathrm{kg} \\
\mu \mathrm{g} / \mathrm{kg} \\
\mu \mathrm{g} / \mathrm{kg} \\
\mu \mathrm{g} / \mathrm{kg} \\
\mu \mathrm{g} / \mathrm{kg} \\
\mu \mathrm{gg} / \mathrm{kg} \\
\mu \mathrm{g} / \mathrm{kg} \\
\mu \mathrm{g} / \mathrm{kg} \\
\mu \mathrm{g} / \mathrm{kg} \\
\mu \mathrm{g} / \mathrm{kg} \\
\mu \mathrm{g} / \mathrm{kg} \\
\mu \mathrm{g} / \mathrm{kg} \\
\mu \mathrm{g} / \mathrm{kg} \\
\mu \mathrm{g} / \mathrm{kg} \\
\mu \mathrm{g} / \mathrm{kg} \\
\mu \mathrm{g} / \mathrm{kg} \\
\mu \mathrm{g} / \mathrm{kg} \\
\mu \mathrm{g} / \mathrm{kg} \\
\mu g / \mathrm{kg} \\
\mu \mathrm{g} / \mathrm{kg}\end{array}$ & $\begin{array}{r}2.00 \\
2.00 \\
2.00 \\
2.00 \\
2.00 \\
216.00 \\
276.00 \\
2.00 \\
79.00 \\
1.00 \\
1.00 \\
9.00 \\
1.00 \\
4.00 \\
4.00 \\
4.00 \\
2.00 \\
4.00 \\
4.00 \\
4.00 \\
4.00 \\
25.00 \\
2.00 \\
2.00 \\
5.00 \\
2.00 \\
21.00 \\
216.00 \\
77.00 \\
32.00\end{array}$ \\
\hline
\end{tabular}




\section{C-58}

Table C.1 (continued)

\begin{tabular}{|c|c|c|c|c|c|}
\hline Analysis & Units & $\begin{array}{c}\text { A horizon } \\
\text { Result }\end{array}$ & Qualifier & $\begin{array}{l}\text { A horizon } \\
\text { field dup }\end{array}$ & Qualifier \\
\hline $\begin{array}{l}\text { PCB- } 1016 \\
\text { PCB-1221 } \\
\text { PCB-1232 } \\
\text { PCB-1242 } \\
\text { PCB-1248 } \\
\text { PCB-1254 } \\
\text { PCB-1260 } \\
\text { TOxaphene } \\
4,4^{1}-D D D \\
4,4^{1}-D D E \\
4,4^{1}-D D T\end{array}$ & $\begin{array}{l}\mu g / \mathrm{kg} \\
\mu \mathrm{g} / \mathrm{kg} \\
\mu \mathrm{g} / \mathrm{kg} \\
\mu \mathrm{g} / \mathrm{kg} \\
\mu \mathrm{g} / \mathrm{kg} \\
\mu \mathrm{g} / \mathrm{kg} \\
\mu \mathrm{g} / \mathrm{kg} \\
\mu \mathrm{g} / \mathrm{kg} \\
\mu \mathrm{g} / \mathrm{kg} \\
\mu \mathrm{g} / \mathrm{kg} \\
\mu \mathrm{g} / \mathrm{kg}\end{array}$ & $\begin{array}{r}40.00 \\
80.00 \\
40.00 \\
40.00 \\
40.00 \\
40.00 \\
40.00 \\
200.00 \\
4.00 \\
4.00 \\
4.00\end{array}$ & $\begin{array}{l}U \\
U \\
U \\
U \\
U \\
U \\
U \\
U \\
U J \\
U J \\
U J\end{array}$ & & \\
\hline
\end{tabular}

Location $=O R R ;$ Formation $=$ NOLICHUCKY; Site $=21 ; A=1296 ; A$ dup=' '; Phase $=1$

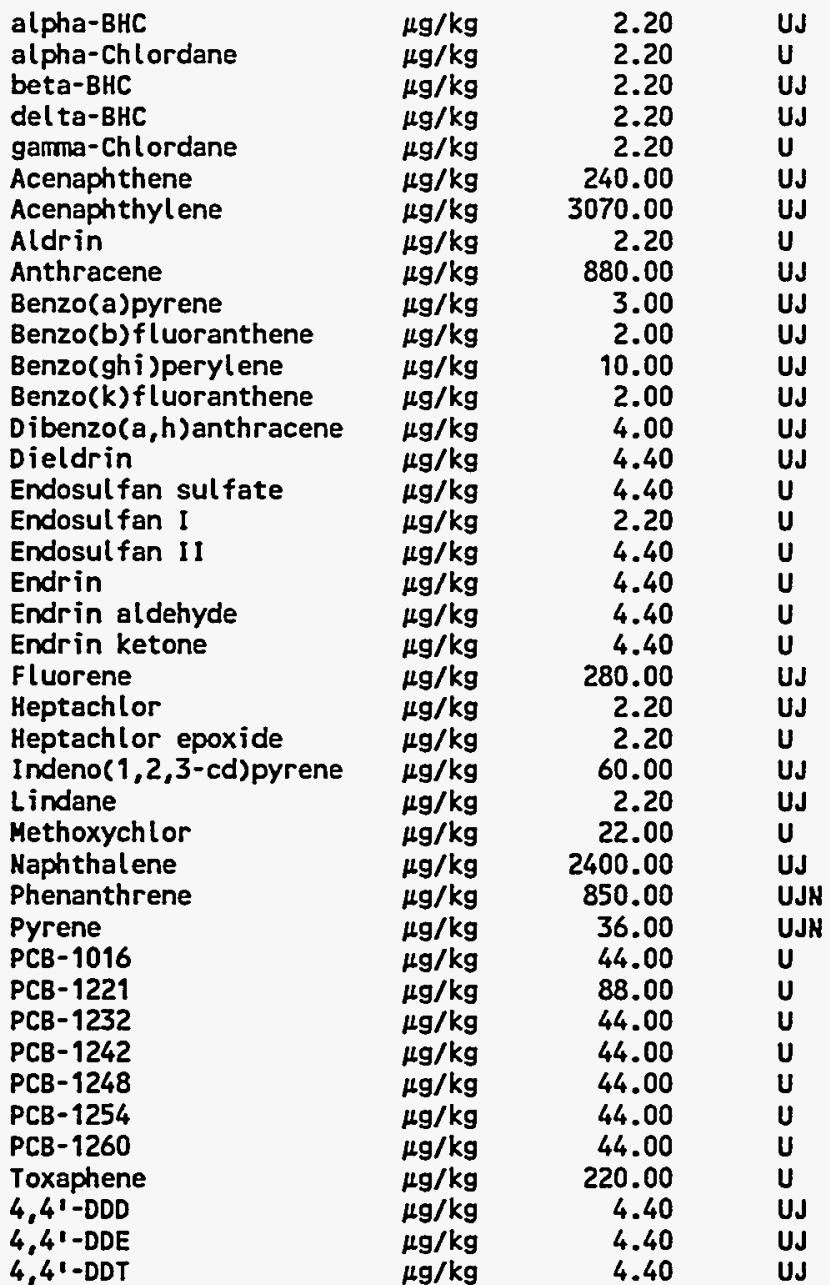

Location=ORR; Formation=NOLICHUCKY; Site $=23 ; A=1295 ;$ A dup=' '; Phase $=1$

$\begin{array}{lrrl}\text { alpha-BHC } & \mu g / \mathrm{kg} & 2.00 & \mathrm{UJ} \\ \text { alpha-Chlordane } & \mu \mathrm{g} / \mathrm{kg} & 2.00 & \mathrm{U} \\ \text { beta-BHC } & \mu \mathrm{kg} & 2.00 & \mathrm{UJ} \\ \text { del ta-BHC } & \mu g / \mathrm{kg} & 2.00 & \mathrm{UJ} \\ \text { gamma-Chlordane } & \mu g / \mathrm{kg} & 2.00 & \mathrm{U} \\ \text { Acenaphthene } & \mu g / \mathrm{kg} & 112.00 & \mathrm{~J} \\ \text { Acenaphthylene } & \mu \mathrm{g} / \mathrm{kg} & 2750.00 & \mathrm{UJ}\end{array}$




\section{C-59}

Table C.1 (continued)

\begin{tabular}{|c|c|c|c|c|c|c|}
\hline Analysis & Units & $\begin{array}{c}\text { A horizon } \\
\text { Result }\end{array}$ & Qualifier & $\begin{array}{l}\text { A horizon } \\
\text { field dup }\end{array}$ & Qualifier & \\
\hline $\begin{array}{l}\text { Aldrin } \\
\text { Anthracene } \\
\text { Benzo(a)pyrene } \\
\text { Benzo(b)fluoranthene } \\
\text { Benzo(ghi)perylene } \\
\text { Benzo(k)fluoranthene } \\
\text { Dibenzo(a,h)anthracene } \\
\text { Dieldrin } \\
\text { Endosul fan sulfate } \\
\text { Endosul fan I } \\
\text { Endosul fan II } \\
\text { Endrin } \\
\text { Endrin aldehyde } \\
\text { Endrin ketone } \\
\text { Fluorene } \\
\text { Heptachlor } \\
\text { Heptachlor epoxide } \\
\text { Indeno(1,2,3-cd)pyrene } \\
\text { Lindane } \\
\text { Hethoxychlor } \\
\text { Naphthalene } \\
\text { Phenanthrene } \\
\text { Pyrene } \\
\text { PCB-1016 } \\
\text { PCB-1221 } \\
\text { PCB-1232 } \\
\text { PCB-1242 } \\
\text { PCB-1248 } \\
\text { PCB-1254 } \\
\text { PCB-1260 } \\
\text { Toxaphene } \\
4,41-000 \\
4,41-D 0 E \\
4,41-0 D T\end{array}$ & $\begin{array}{l}\mu g / \mathrm{kg} \\
\mu g / \mathrm{kg} \\
\mu g / \mathrm{kg} \\
\mu g / \mathrm{kg} \\
\mu g / \mathrm{kg} \\
\mu g / \mathrm{kg} \\
\mu g / \mathrm{kg} \\
\mu g / \mathrm{kg} \\
\mu g / \mathrm{kg} \\
\mu \mathrm{g} / \mathrm{kg} \\
\mu g / \mathrm{kg} \\
\mu g / \mathrm{kg} \\
\mu g / \mathrm{kg} \\
\mu g / \mathrm{kg} \\
\mu g / \mathrm{kg} \\
\mu \mathrm{g} / \mathrm{kg} \\
\mu g / \mathrm{kg} \\
\mu g / \mathrm{kg} \\
\mu g / \mathrm{kg} \\
\mu g / \mathrm{kg} \\
\mu g / \mathrm{kg} \\
\mu g / \mathrm{kg} \\
\mu g / \mathrm{kg} \\
\mu g / \mathrm{kg} \\
\mu g / \mathrm{kg} \\
\mu g / \mathrm{kg} \\
\mu g / \mathrm{kg} \\
\mu g / \mathrm{kg} \\
\mu g / \mathrm{kg} \\
\mu g / \mathrm{kg} \\
\mu g / \mathrm{kg} \\
\mu g / \mathrm{kg} \\
\mu g / \mathrm{kg} \\
\mu g / \mathrm{kg}\end{array}$ & $\begin{array}{r}2.00 \\
790.00 \\
3.00 \\
2.00 \\
9.00 \\
2.00 \\
4.00 \\
4.00 \\
4.00 \\
2.00 \\
4.00 \\
4.00 \\
4.00 \\
4.00 \\
250.00 \\
2.00 \\
2.00 \\
50.00 \\
2.00 \\
20.00 \\
2150.00 \\
760.00 \\
32.00 \\
40.00 \\
79.00 \\
40.00 \\
40.00 \\
40.00 \\
40.00 \\
40.00 \\
200.00 \\
4.00 \\
4.00 \\
4.00\end{array}$ & $\begin{array}{l}U \\
U J \\
U J \\
U J \\
U J \\
U J \\
U J \\
U J \\
U \\
U \\
U \\
U \\
U \\
U \\
U J \\
U J \\
U \\
U J \\
U J \\
U \\
U J \\
U J \\
U J H \\
U \\
U \\
U \\
U \\
U \\
U \\
U \\
U \\
U J \\
U J \\
U J\end{array}$ & - & & ${ }^{\prime}$ \\
\hline
\end{tabular}

Location=ORR; Formation=NOLICHUCKY; Site=24; $A=1294 ;$ A dup=" '; Phase=1

$\begin{array}{lrrl} & & & \\ \text { alpha-BHC } & \mu g / \mathrm{kg} & 2.10 & \mathrm{UJ} \\ \text { alpha-Chlordane } & \mu \mathrm{g} / \mathrm{kg} & 2.10 & \mathrm{U} \\ \text { beta-BHC } & \mu \mathrm{g} / \mathrm{kg} & 2.10 & \mathrm{UJ} \\ \text { delta-BHC } & \mu \mathrm{g} / \mathrm{kg} & 2.10 & \mathrm{UJ} \\ \text { gamm-Chlordane } & \mu \mathrm{g} / \mathrm{kg} & 2.10 & \mathrm{U} \\ \text { Acenaphthene } & \mu \mathrm{g} / \mathrm{kg} & 230.00 & \mathrm{UJ} \\ \text { Acenaphthylene } & \mu \mathrm{g} / \mathrm{kg} & 2940.00 & \mathrm{UJ} \\ \text { Aldrin } & \mu \mathrm{g} / \mathrm{kg} & 2.10 & \mathrm{U} \\ \text { Anthracene } & \mu \mathrm{g} / \mathrm{kg} & 840.00 & \mathrm{UJ} \\ \text { Benzo(a)pyrene } & \mu \mathrm{g} / \mathrm{kg} & 3.00 & \mathrm{UJ} \\ \text { Benzo(b)fluoranthene } & \mu \mathrm{g} / \mathrm{kg} & 2.00 & \mathrm{UJ} \\ \text { Benzo(ghi)perylene } & \mu \mathrm{g} / \mathrm{kg} & 10.00 & \mathrm{UJ} \\ \text { Benzo(k)fluoranthene } & \mu \mathrm{g} / \mathrm{kg} & 2.00 & \mathrm{UJ} \\ \text { Dibenzo(a,h)anthracene } & \mu \mathrm{g} / \mathrm{kg} & 4.00 & \mathrm{UJ} \\ \text { Dieldrin } & \mu \mathrm{g} / \mathrm{kg} & 4.30 & \mathrm{UJ} \\ \text { Endosulfan sulfate } & \mu \mathrm{g} / \mathrm{kg} & 4.30 & \mathrm{U} \\ \text { Endosulfan I } & \mu \mathrm{g} / \mathrm{kg} & 2.10 & \mathrm{U} \\ \text { Endosulfan II } & \mu \mathrm{g} / \mathrm{kg} & 4.30 & \mathrm{U} \\ \text { Endrin } & \mu \mathrm{g} / \mathrm{kg} & 4.30 & \mathrm{U} \\ \text { Endrin aldehyde } & \mu \mathrm{g} / \mathrm{kg} & 4.30 & \mathrm{U} \\ \text { Endrin ketone } & \mu \mathrm{g} / \mathrm{kg} & 4.30 & \mathrm{U} \\ \text { Fluorene } & \mu \mathrm{g} / \mathrm{kg} & 270.00 & \mathrm{UJ} \\ \text { Heptachlor } & \mu \mathrm{kg} & 2.10 & \mathrm{UJ} \\ \text { Heptachlor epoxide } & \mu \mathrm{g} / \mathrm{kg} & 2.10 & \mathrm{U} \\ \text { Indeno(1,2,3-cd)pyrene } & \mu \mathrm{g} / \mathrm{kg} & 60.00 & \mathrm{UJ} \\ \text { Lindane } & \mu \mathrm{g} / \mathrm{kg} & 2.10 & \mathrm{UJ} \\ \text { Methoxychlor } & \mu \mathrm{g} / \mathrm{kg} & 21.00 & \mathrm{U} \\ \text { Maphthalene } & \mu \mathrm{g} / \mathrm{kg} & 2300.00 & \mathrm{UJ} \\ \text { Phenanthrene } & \mu \mathrm{g} / \mathrm{kg} & 820.00 & \mathrm{UJN} \\ \text { Pyrene } & \mu \mathrm{g} / \mathrm{kg} & 10.00 & \mathrm{~J} \\ & & & \\ & & & \end{array}$


Table C.1 (continued)

\begin{tabular}{|c|c|c|c|c|c|}
\hline Analysis & Units & $\begin{array}{c}\text { A horizon } \\
\text { Result }\end{array}$ & Qualifier & $\begin{array}{l}\text { A horizon } \\
\text { field dup }\end{array}$ & Qualifier \\
\hline $\begin{array}{l}\text { PCB-1016 } \\
\text { PCB-1221 } \\
\text { PCB-1232 } \\
\text { PCB-1242 } \\
\text { PCB-1248 } \\
\text { PCB-1254 } \\
\text { PCB-1260 } \\
\text { Toxaphene } \\
4,4^{\prime}-D D D \\
4,4^{\prime}-D D E \\
4,4^{\prime}-D D T\end{array}$ & $\begin{array}{l}\mu g / \mathrm{kg} \\
\mu g / \mathrm{kg} \\
\mu g / \mathrm{kg} \\
\mu g / \mathrm{kg} \\
\mu \mathrm{g} / \mathrm{kg} \\
\mu \mathrm{g} / \mathrm{kg} \\
\mu g / \mathrm{kg} \\
\mu g / \mathrm{kg} \\
\mu g / \mathrm{kg} \\
\mu g / \mathrm{kg} \\
\mu g / \mathrm{kg}\end{array}$ & $\begin{array}{r}43.00 \\
85.00 \\
43.00 \\
43.00 \\
43.00 \\
43.00 \\
43.00 \\
210.00 \\
4.30 \\
4.30 \\
4.30\end{array}$ & $\begin{array}{l}U \\
U \\
U \\
U \\
U \\
U \\
U \\
U \\
U J \\
U J \\
U J\end{array}$ & & \\
\hline
\end{tabular}

Location=ORR; Formation =NOLICHUCKY; Site $=25 ; A=1293 ; A$ dup =' '; Phase $=1$

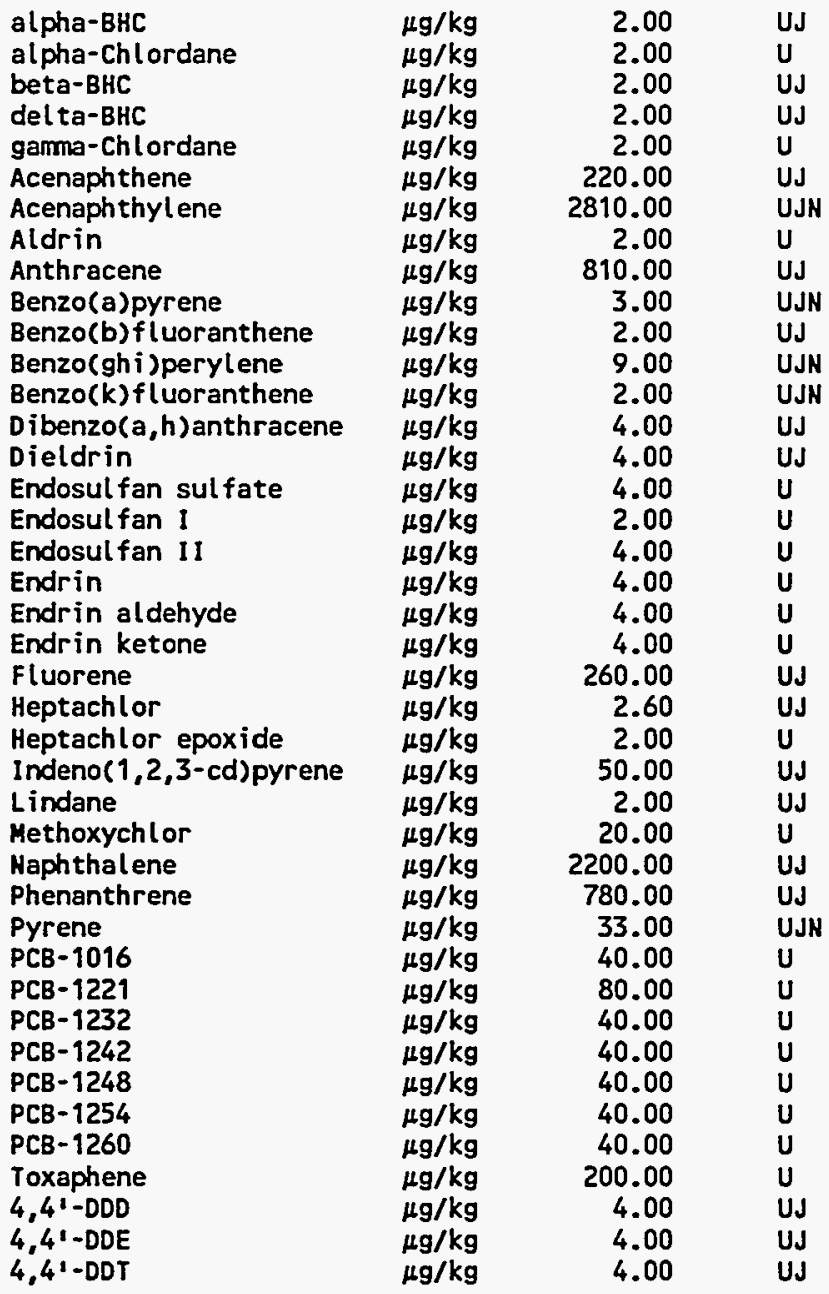

Location=ORR; Formation $=$ NOLICHUCKY; Site $=28 ; A=1292 ; A$ dup=' '; Phase $=1$

$\begin{array}{lrrl}\text { alpha-BHC } & \mu \mathrm{g} / \mathrm{kg} & 1.90 & \mathrm{UJ} \\ \text { alpha-Chlordane } & \mu \mathrm{g} / \mathrm{kg} & 1.90 & \mathrm{U} \\ \text { beta-BHC } & \mu \mathrm{g} / \mathrm{kg} & 1.90 & \mathrm{UJ} \\ \text { delta-BHC } & \mu \mathrm{g} / \mathrm{kg} & 1.90 & \mathrm{UJ} \\ \text { gamma-Chlordane } & \mu \mathrm{g} / \mathrm{kg} & 1.90 & \mathrm{U} \\ \text { Acenaphthene } & \mu \mathrm{gg} & 207.00 & \mathrm{UJ} \\ \text { Acenaphthylene } & \mu \mathrm{g} / \mathrm{kg} & 2650.00 & \text { UJN }\end{array}$


C-61

Table C1 (continucd)

\begin{tabular}{|c|c|c|c|c|c|c|}
\hline Analysis & Units & $\begin{array}{c}\text { A horizon } \\
\text { Result }\end{array}$ & Qualifier & $\begin{array}{l}\text { A horizon } \\
\text { field dup }\end{array}$ & Qualifier & \\
\hline $\begin{array}{l}\text { Aldrin } \\
\text { Anthracene } \\
\text { Benzo(a)pyrene } \\
\text { Benzo(b)fluoranthene } \\
\text { Benzo(ghi)perylene } \\
\text { Benzo(k)fluoranthene } \\
\text { Dibenzo(a,h)anthracene } \\
\text { Dieldrin } \\
\text { Endosulfan sulfate } \\
\text { Endosulfan I } \\
\text { Endosulfan II } \\
\text { Endrin } \\
\text { Endrin aldehyde } \\
\text { Endrin ketone } \\
\text { Fluorene } \\
\text { Heptachlor } \\
\text { Heptachlor epoxide } \\
\text { Indeno( } 1,2,3-\text {-Cd)pyrene } \\
\text { Lindane } \\
\text { Hethoxychlor } \\
\text { Naphthalene } \\
\text { Phenanthrene } \\
\text { Pyrene } \\
\text { PCB-1016 } \\
\text { PCB-1221 } \\
\text { PCB-1232 } \\
\text { PCB-1242 } \\
\text { PCB-1248 } \\
\text { PCB-1254 } \\
\text { PCB-1260 } \\
\text { Toxaphene } \\
4,4 '-D D D \\
4,41-D D E \\
4,41-D D T\end{array}$ & 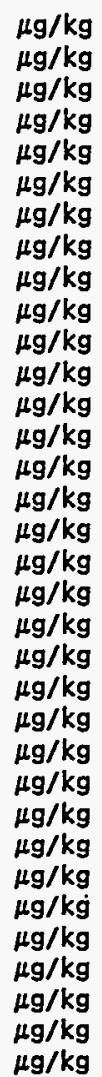 & $\begin{array}{r}1.90 \\
760.00 \\
3.00 \\
2.00 \\
9.00 \\
2.00 \\
3.00 \\
3.80 \\
3.80 \\
1.90 \\
3.80 \\
3.80 \\
3.80 \\
3.80 \\
240.00 \\
1.90 \\
1.90 \\
50.00 \\
1.90 \\
19.00 \\
2070.00 \\
740.00 \\
31.00 \\
38.00 \\
76.00 \\
38.00 \\
38.00 \\
38.00 \\
38.00 \\
38.00 \\
190.00 \\
3.80 \\
3.80 \\
3.80\end{array}$ & $\begin{array}{l}\text { U } \\
\text { UJ } \\
\text { UJ } \\
\text { UJ } \\
\text { UJ } \\
\text { UJ } \\
\text { UJ } \\
\text { UJ } \\
U \\
U \\
U \\
U \\
U \\
U \\
\text { UJ } \\
\text { UJ } \\
U \\
\text { UJ } \\
\text { UJ } \\
U \\
\text { UJ } \\
\text { UJ } \\
\text { UJN } \\
U \\
U \\
U \\
U \\
U \\
U \\
U \\
U \\
U J \\
U J \\
U J\end{array}$ & & . & $"$ \\
\hline
\end{tabular}

Location=ORR; Formation=NOLICHUCKY; Site=3; A=1297; $A$ dup=' '; Phase=1

$\begin{array}{lrrl}\text { alpha-BHC } & \mu g / \mathrm{kg} & 2.00 & \mathrm{UJ} \\ \text { alpha-Chlordane } & \mu \mathrm{g} / \mathrm{kg} & 2.00 & \mathrm{U} \\ \text { beta-BHC } & \mu \mathrm{g} / \mathrm{kg} & 2.00 & \mathrm{UJ} \\ \text { delta-BHC } & \mu \mathrm{g} / \mathrm{kg} & 2.00 & \mathrm{UJ} \\ \text { gamma-Chlordane } & \mu \mathrm{g} / \mathrm{kg} & 2.00 & \mathrm{U} \\ \text { Acenaphthene } & \mu \mathrm{g} / \mathrm{kg} & 220.00 & \mathrm{UJ} \\ \text { Acenaphthylene } & \mu \mathrm{g} / \mathrm{kg} & 2810.00 & \mathrm{UJ} \\ \text { Aldrin } & \mu \mathrm{g} / \mathrm{kg} & 2.00 & \mathrm{U} \\ \text { Anthracene } & \mu \mathrm{g} / \mathrm{kg} & 810.00 & \mathrm{UJ} \\ \text { Benzo(a)pyrene } & \mu \mathrm{g} / \mathrm{kg} & 3.00 & \mathrm{UJN} \\ \text { Benzo(b)fluoranthene } & \mu \mathrm{g} / \mathrm{kg} & 2.00 & \mathrm{UJ} \\ \text { Benzo(ghi)perylene } & \mu \mathrm{g} / \mathrm{kg} & 9.00 & \mathrm{UJ} \\ \text { Benzo(k)fluoranthene } & \mu \mathrm{g} / \mathrm{kg} & 2.00 & \mathrm{UJN} \\ \text { Dibenzo(a,h)anthracene } & \mu \mathrm{g} / \mathrm{kg} & 4.00 & \mathrm{UJ} \\ \text { Dieldrin } & \mu \mathrm{g} / \mathrm{kg} & 4.00 & \mathrm{UJ} \\ \text { Endosulfan sulfate } & \mu \mathrm{g} / \mathrm{kg} & 4.00 & \mathrm{U} \\ \text { Endosulfan I } & \mu \mathrm{g} / \mathrm{kg} & 2.00 & \mathrm{U} \\ \text { Endosulfan II } & \mu \mathrm{g} / \mathrm{kg} & 4.00 & \mathrm{U} \\ \text { Endrin } & \mu \mathrm{g} / \mathrm{kg} & 4.00 & \mathrm{U} \\ \text { Endrin aldehyde } & \mu \mathrm{g} / \mathrm{kg} & 4.00 & \mathrm{U} \\ \text { Endrin ketone } & \mu \mathrm{g} / \mathrm{kg} & 4.00 & \mathrm{U} \\ \text { Fluorene } & \mu \mathrm{g} / \mathrm{kg} & 260.00 & \mathrm{UJ} \\ \text { Heptachlor } & \mu \mathrm{g} / \mathrm{kg} & 2.00 & \mathrm{UJ} \\ \text { Heptachlor epoxide } & \mu \mathrm{g} / \mathrm{kg} & 2.00 & \mathrm{U} \\ \text { Indeno(1,2,3-cd)pyrene } & \mu \mathrm{g} / \mathrm{kg} & 50.00 & \mathrm{UJ} \\ \text { Lindane } & \mu \mathrm{g} / \mathrm{kg} & 2.00 & \mathrm{UJ} \\ \text { Hethoxychlor } & \mu \mathrm{g} / \mathrm{kg} & 20.00 & \mathrm{U} \\ \text { Naphthalene } & \mu \mathrm{g} / \mathrm{kg} & 2200.00 & \mathrm{UJ} \\ \text { Phenanthrene } & \mu \mathrm{g} / \mathrm{kg} & 780.00 & \mathrm{UJ} \\ \text { Pyrene } & \mu \mathrm{g} / \mathrm{kg} & 33.00 & \mathrm{UJN} \\ & & & \end{array}$




\section{C-62}

Table C.1 (continued)

\begin{tabular}{|c|c|c|c|c|c|}
\hline Analysis & Units & $\begin{array}{c}\text { A horizon } \\
\text { Result }\end{array}$ & Qualifier & $\begin{array}{l}\text { A horizon } \\
\text { field dup }\end{array}$ & Qualifier \\
\hline $\begin{array}{l}\text { PCB-1016 } \\
\text { PCB-1221 } \\
\text { PCB-1232 } \\
\text { PCB- } 1242 \\
\text { PCB- } 1248 \\
\text { PCB-1254 } \\
\text { PCB-1260 } \\
\text { Toxaphene } \\
4,4^{\prime}-D D D \\
4,4^{\prime}-D D E \\
4,4 !-D D T\end{array}$ & $\begin{array}{l}\mu g / \mathrm{kg} \\
\mu g / \mathrm{kg} \\
\mu g / \mathrm{kg} \\
\mu g / \mathrm{kg} \\
\mu g / \mathrm{kg} \\
\mu g / \mathrm{kg} \\
\mu g / \mathrm{kg} \\
\mu g / \mathrm{kg} \\
\mu g / \mathrm{kg} \\
\mu g / \mathrm{kg} \\
\mu g / \mathrm{kg}\end{array}$ & $\begin{array}{r}40.00 \\
81.00 \\
40.00 \\
40.00 \\
40.00 \\
40.00 \\
40.00 \\
200.00 \\
4.00 \\
4.00 \\
4.00\end{array}$ & $\begin{array}{l}U \\
U \\
U \\
U \\
U \\
U \\
U \\
U \\
U J \\
U J \\
U J\end{array}$ & & \\
\hline
\end{tabular}

Location=ORR; Formation=NOLICHUCKY; Site $=31 ; A=1303 ; A$ dup =' '; Phase $=1$

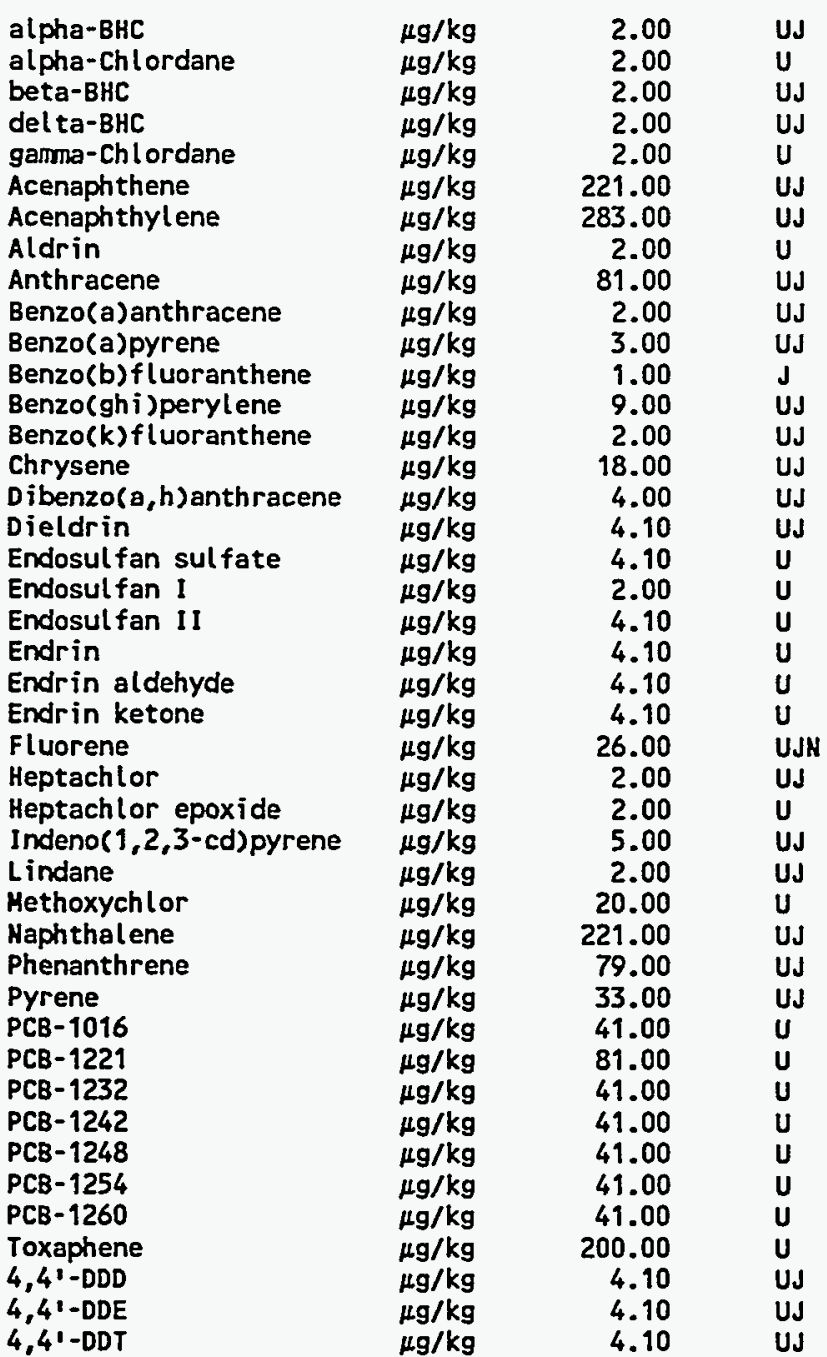

Location $=$ ORR; Formation=NOLICHUCKY; Site $=42 ; A=1302 ; A$ dup=" '; Phase $=1$

$\begin{array}{llll}\text { alpha-BHC } & \mu \mathrm{g} / \mathrm{kg} & 2.00 & \mathrm{UJ} \\ \text { alpha-Chlordane } & \mu \mathrm{g} / \mathrm{kg} & 2.00 & \mathrm{U} \\ \text { beta-BHC } & \mu \mathrm{kg} & 2.00 & \mathrm{UJ} \\ \text { delta-BHC } & \mu \mathrm{kg} / \mathrm{kg} & 2.00 & \mathrm{UJ} \\ \text { gamma-Chlordane } & \mu \mathrm{kg} & 2.00 & \mathrm{U}\end{array}$




\section{C-63}

Table C.1 (continued)

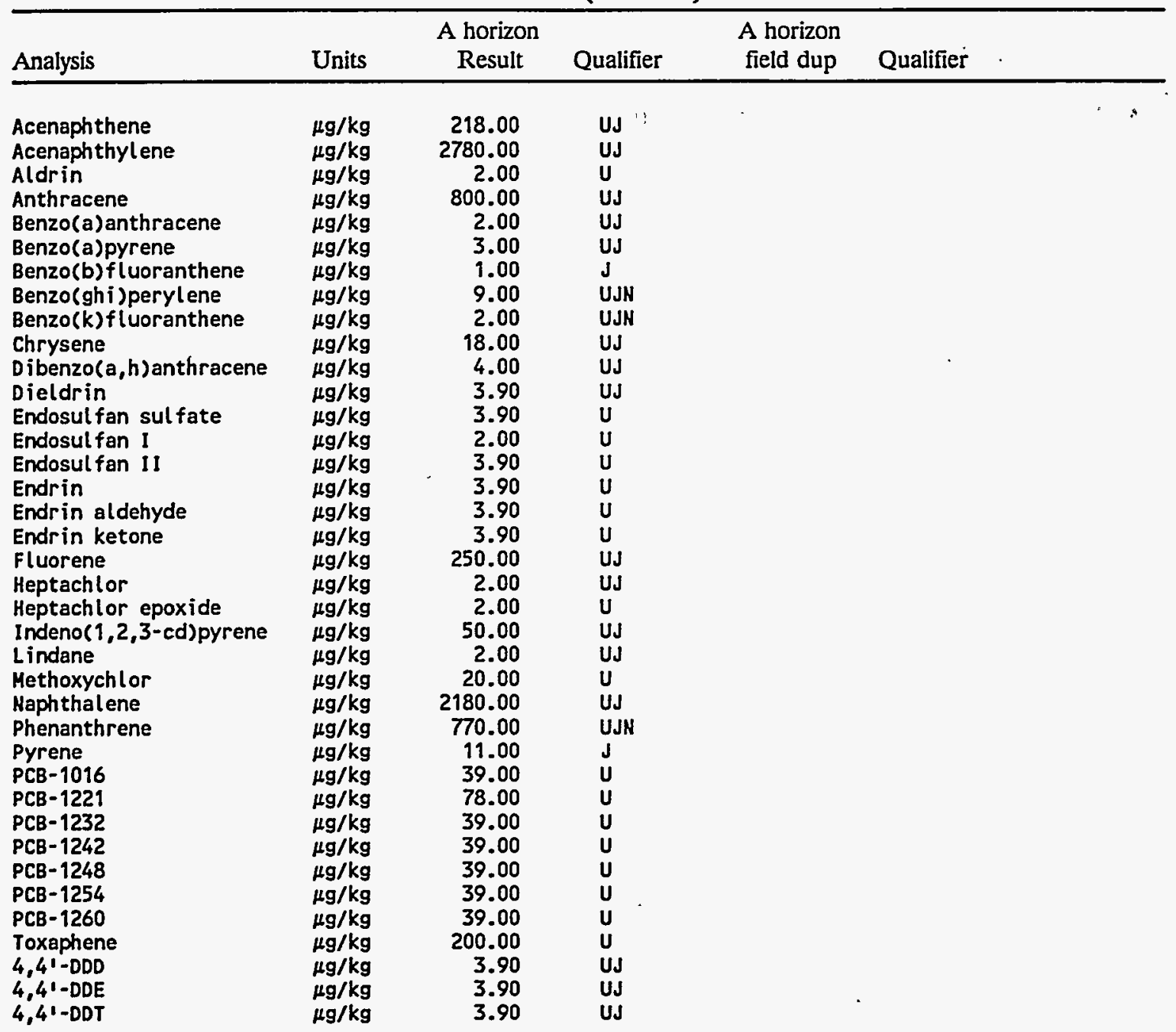

Location=ORR; Formation=NOLICHUCKY; Site $=5 ; A=1298 ; A$ dup=" '; Phase $=1$

\begin{tabular}{|c|c|c|}
\hline $\begin{array}{l}\text { alpha-BHC } \\
\text { alpha-Chlordane } \\
\text { beta-BHC } \\
\text { del ta-BHC } \\
\text { gamma-Chlordane } \\
\text { Acenaphthene } \\
\text { Acenaphthylene } \\
\text { Aldrin } \\
\text { Anthracene } \\
\text { Benzo(a)pyrene } \\
\text { Benzo(b)fluoranthene } \\
\text { Benzo(ghi)perylene } \\
\text { Benzo(k)fluoranthene } \\
\text { Dibenzo(a,h)anthracene } \\
\text { Dieldrin } \\
\text { Endosulfan sul fate } \\
\text { Endosulfan I } \\
\text { Endosulfan II } \\
\text { Endrin } \\
\text { Endrin aldehyde } \\
\text { Endrin ketone } \\
\text { Fluorene } \\
\text { Heptachlor } \\
\text { Heptachlor epoxide } \\
\text { Indeno(1,2,3-cd)pyrene } \\
\text { Lindane }\end{array}$ & 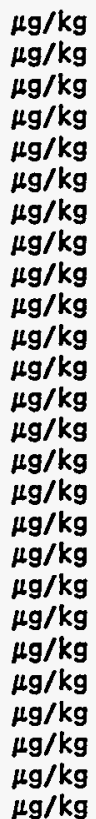 & $\begin{array}{r}1.90 \\
1.90 \\
1.90 \\
1.90 \\
1.90 \\
202.00 \\
2580.00 \\
1.90 \\
740.00 \\
3.00 \\
2.00 \\
9.00 \\
2.00 \\
3.00 \\
3.70 \\
3.70 \\
1.90 \\
3.70 \\
3.70 \\
3.70 \\
3.70 \\
240.00 \\
1.90 \\
1.90 \\
50.00 \\
1.90\end{array}$ \\
\hline
\end{tabular}




\section{C-64}

Table C.1 (continued)

\begin{tabular}{|c|c|c|c|c|c|}
\hline Analysis & Units & $\begin{array}{c}\text { A horizon } \\
\text { Result }\end{array}$ & Qualifier & $\begin{array}{l}\text { A horizon } \\
\text { field dup }\end{array}$ & Qualifier \\
\hline $\begin{array}{l}\text { Methoxychlor } \\
\text { Naphthalene } \\
\text { Phenanthrene } \\
\text { Pyrene } \\
\text { PCB-1016 } \\
\text { PCB-1221 } \\
\text { PCB-1232 } \\
\text { PCB-1242 } \\
\text { PCB-1248 } \\
\text { PCB-1254 } \\
\text { PCB-1260 } \\
\text { Toxaphene } \\
4,4^{\prime} \text {-DDD } \\
4,4^{\prime}-\text { DDE } \\
4,4^{\prime}-\text { DDT }\end{array}$ & $\begin{array}{l}\mu g / \mathbf{k g} \\
\mu g / \mathbf{k g} \\
\mu g / \mathbf{k g} \\
\mu g / \mathbf{k g} \\
\mu g / \mathbf{k g} \\
\mu g / \mathbf{k g} \\
\mu g / \mathbf{k g} \\
\mu g / \mathbf{k g} \\
\mu g / \mathbf{k g} \\
\mu g / \mathbf{k g} \\
\mu g / \mathbf{k g} \\
\mu g / \mathbf{k g} \\
\mu g / \mathbf{k g} \\
\mu g / \mathbf{k g} \\
\mu g / \mathbf{k g}\end{array}$ & $\begin{array}{r}19.00 \\
2020.00 \\
720.00 \\
30.00 \\
37.00 \\
75.00 \\
37.00 \\
37.00 \\
37.00 \\
37.00 \\
37.00 \\
190.00 \\
3.70 \\
3.70 \\
3.70\end{array}$ & $\begin{array}{l}U \\
U J \\
U J \\
U J N \\
U \\
U \\
U \\
U \\
U \\
U \\
U \\
U \\
U J \\
U J \\
U J\end{array}$ & & \\
\hline
\end{tabular}

Location=ROA; Formation=COPPER RIDGE; Site =33; $A=3203 ; A$ dup=' '; Phase $=2$

\begin{tabular}{|c|c|c|}
\hline 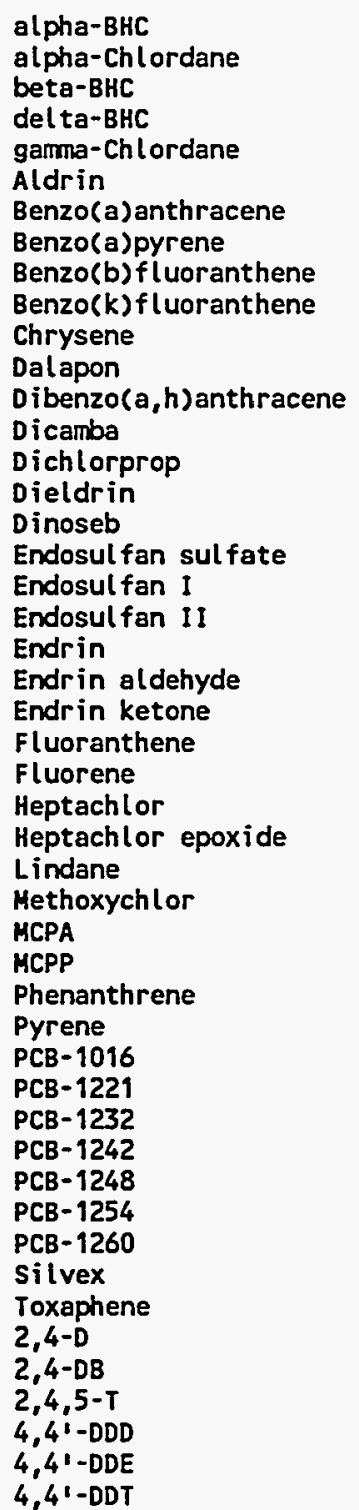 & 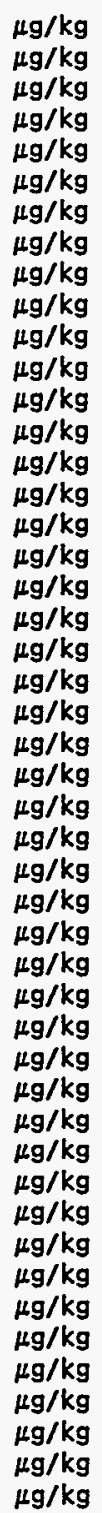 & $\begin{array}{r}2.70 \\
2.70 \\
2.70 \\
2.70 \\
2.70 \\
2.70 \\
2.10 \\
1.10 \\
1.60 \\
1.60 \\
2.70 \\
5527.20 \\
2.70 \\
254.10 \\
635.30 \\
5.20 \\
133.40 \\
5.20 \\
2.70 \\
5.20 \\
5.20 \\
5.20 \\
5.20 \\
5.90 \\
1.10 \\
2.70 \\
2.70 \\
2.70 \\
26.80 \\
238239.50 \\
181062.10 \\
4.30 \\
1.60 \\
52.10 \\
105.70 \\
52.10 \\
52.10 \\
52.10 \\
52.10 \\
52.10 \\
158.80 \\
268.30 \\
1143.50 \\
857.70 \\
190.60 \\
5.20 \\
5.20 \\
11.50\end{array}$ \\
\hline
\end{tabular}


C-65

Table C.1 (continued)

\begin{tabular}{lccc}
\hline Analysis & Units & $\begin{array}{c}\text { A horizon } \\
\text { Result }\end{array}$ Qualifier & $\begin{array}{c}\text { A horizon } \\
\text { field dup }\end{array}$ Qualifier \\
\hline Location $=$ ROA; Formation $=$ COPPER RIDGE; Site $=34 ; A=3206 ; A$ dup=' '; Phase=2
\end{tabular}

olpha-BHC

alpha-Chlordane

beta-BHC

del ta-BHC

garma-Chlordane

Acenaph thene

Acenaph thylene

Aldrin

Anthracene

Benzo(a)anthracene

Benzo(a)pyrene

Benzo(b)fluoranthene

Benzo(ghi)perylene

Benzo(k)fluoranthene

Chrysene

Dalapon

Dibenzo $(a, h)$ anthracene

Dicamba

Dichlorprop

Dieldrin

Dinoseb

Endosulfan sulfate

Endosulfan I

Endosulfan II

Endrin

Endrin aldehyde

Endrin ketone

Fluoranthene

Fluorene

Heptachlor

Heptachlor epoxide

Indeno $(1,2,3-\mathrm{cd})$ pyrene

Lindane

Methoxychlor

MCPA

MCPP

Naphthalene

Phenanthrene

Pyrene

PCB-1016

PCB-1221

PCB-1232

PCB- 1242

PCB- 1248

PCB- 1254

PCB-1260

$\mu \mathrm{g} / \mathrm{kg}$

$\mu \mathrm{g} / \mathrm{kg}$
$\mu \mathrm{g} / \mathrm{kg}$

$\mu \mathrm{g} / \mathrm{kg}$

$\mu \mathrm{g} / \mathrm{kg}$

$\mu \mathrm{g} / \mathrm{kg}$

$\mu \mathrm{g} / \mathrm{kg}$

$\mu \mathrm{g} / \mathrm{kg}$

$\mu \mathrm{g} / \mathrm{kg}$

$\mu \mathrm{g} / \mathrm{kg}$

$\mu \mathrm{g} / \mathrm{kg}$

$\mu \mathrm{g} / \mathrm{kg}$

$\mu \mathrm{g} / \mathrm{kg}$

$\mu \mathrm{g} / \mathrm{kg}$

$\mu \mathrm{g} / \mathrm{kg}$

$\mu \mathrm{g} / \mathrm{kg}$

$\mu \mathrm{g} / \mathrm{kg}$

$\mu \mathrm{g} / \mathrm{kg}$

$\mu \mathrm{g} / \mathrm{kg}$

$\mu \mathrm{g} / \mathrm{kg}$

$\mu \mathrm{g} / \mathrm{kg}$

$\mu \mathrm{g} / \mathrm{kg}$

$\mu \mathrm{g} / \mathrm{kg}$

$\mu \mathrm{g} / \mathrm{kg}$

$\mu \mathrm{g} / \mathrm{kg}$

$\mu \mathrm{g} / \mathrm{kg}$

$\mu \mathrm{g} / \mathrm{kg}$

$\mu \mathrm{g} / \mathrm{kg}$

$\mu \mathrm{g} / \mathrm{kg}$

$\mu \mathrm{g} / \mathrm{kg}$

$\mu \mathrm{g} / \mathrm{kg}$

$\mu \mathrm{g} / \mathrm{kg}$

$\mu \mathrm{g} / \mathrm{kg}$

$\mu \mathrm{g} / \mathrm{kg}$

$\mu \mathrm{g} / \mathrm{kg}$

$\mu \mathrm{g} / \mathrm{kg}$

$\mu \mathrm{g} / \mathrm{kg}$

$\mu \mathrm{g} / \mathrm{kg}$

$\mu \mathrm{g} / \mathrm{kg}$

$\mu \mathrm{g} / \mathrm{kg}$

$\mu \mathrm{g} / \mathrm{kg}$

$\mu \mathrm{g} / \mathrm{kg}$

$\mu \mathrm{g} / \mathrm{kg}$

$\mu \mathrm{g} / \mathrm{kg}$

$\mu \mathrm{g} / \mathrm{kg}$

$\mu \mathrm{g} / \mathrm{kg}$

$\mu \mathrm{g} / \mathrm{kg}$

$\mu \mathrm{g} / \mathrm{kg}$

Toxaphene

$\mu \mathrm{g} / \mathrm{kg}$

$\mu \mathrm{g} / \mathrm{kg}$

$\mu \mathrm{g} / \mathrm{kg}$

$\mu \mathrm{g} / \mathrm{kg}$

$\mu \mathrm{g} / \mathrm{kg}$

$4,41-D D D$

$4,41-D D E$

4,41-DDT

2.40

2.40

2.40

2.40

2.40

4.70

236.70

2.40

1.90

6.20

3.30

2.40

3.30

1.90

4.70

5527.20

1.90

254.10

635.30

4.60

133.40

4.60

2.40

4.60

4.60

4.60

4.60

6.20

0.50

2.40

2.40

42.60

2.40

24.00

238239.50

181062.10

23.70

10.90

6.20

46.50

94.40

46.50

46.50

46.50

46.50

46.50

158.80

239.50

1143.50

857.70

190.60

4.60

4.60

4.60

Location=ROA; Formation=COPPER RIDGE; Site =35; $A=3209 ; A$ dup=' '; Phase=2

alpha-BHC

alpha-Chlordane

beta-BHC

delta-BHC

gamma-Chlordane

Acenaphthene

Acenaphthylene

$\begin{array}{lrl}\mu \mathrm{g} / \mathrm{kg} & 2.20 & \mathrm{U} \\ \mu \mathrm{g} / \mathrm{kg} & 2.20 & \mathrm{U} \\ \mu \mathrm{g} / \mathrm{kg} & 2.20 & \mathrm{U} \\ \mu \mathrm{g} / \mathrm{kg} & 2.20 & \mathrm{U} \\ \mu \mathrm{g} / \mathrm{kg} & 2.20 & \mathrm{U} \\ \mu \mathrm{g} / \mathrm{kg} & 4.30 & \mathrm{UJ} \\ \mu \mathrm{g} / \mathrm{kg} & 154.10 & \mathrm{~J}\end{array}$




\section{C-66}

Table C.1 (continued)

\begin{tabular}{|c|c|c|c|c|c|}
\hline Analysis & Units & $\begin{array}{c}\text { A horizon } \\
\text { Result }\end{array}$ & Qualifier & $\begin{array}{l}\text { A horizon } \\
\text { field dup }\end{array}$ & Qualifier \\
\hline 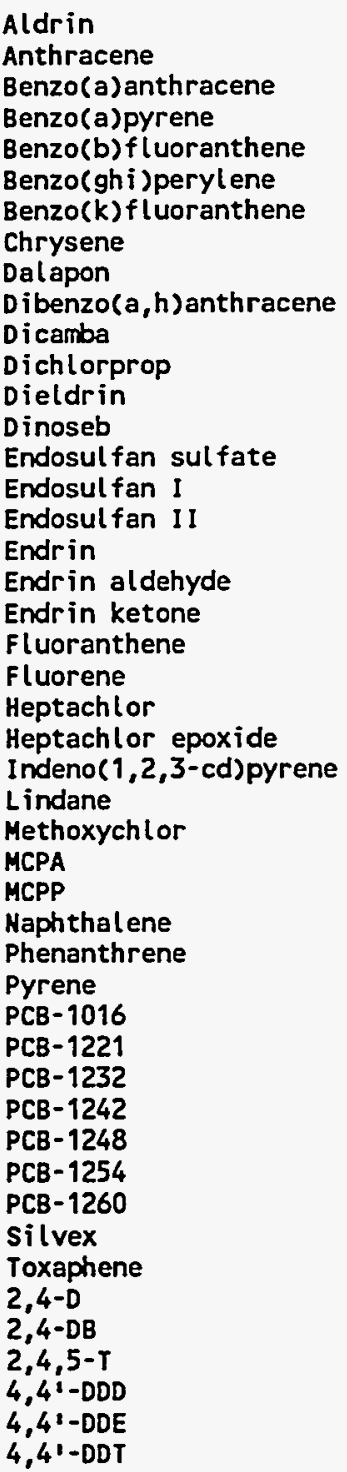 & 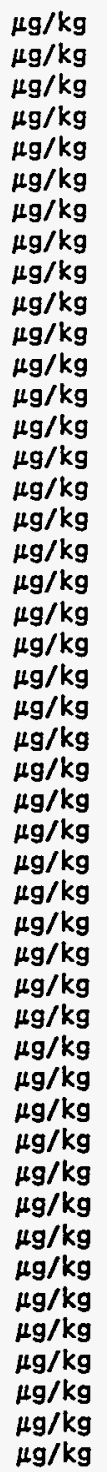 & $\begin{array}{r}2.20 \\
4.30 \\
4.70 \\
0.90 \\
4.30 \\
2.10 \\
0.90 \\
4.30 \\
4536.30 \\
4.30 \\
208.60 \\
521.40 \\
4.20 \\
109.50 \\
4.20 \\
2.20 \\
4.20 \\
4.20 \\
4.20 \\
4.20 \\
3.00 \\
4.30 \\
2.20 \\
2.20 \\
17.90 \\
2.20 \\
21.90 \\
195530.40 \\
148603.10 \\
21.30 \\
1.70 \\
2.60 \\
42.40 \\
86.20 \\
42.40 \\
42.40 \\
42.40 \\
42.40 \\
42.40 \\
130.40 \\
218.60 \\
938.50 \\
703.90 \\
156.40 \\
4.20 \\
4.20 \\
4.20\end{array}$ & $\begin{array}{l}U \\
U J \\
J \\
J \\
U J \\
J \\
J \\
U J \\
U J \\
U J \\
U \\
U \\
U \\
U J \\
U \\
U \\
U \\
U \\
U \\
U \\
U \\
J \\
U J J\end{array}$ & & . \\
\hline
\end{tabular}

Location=ROA; Formation=COPPER RIDGE; Site $=39 ; A=3214 ; A$ dup=' '; Phase =2

$\begin{array}{lrrl}\text { alpha-BHC } & \mu \mathrm{g} / \mathrm{kg} & 2.00 & \mathrm{U} \\ \text { alpha-Chlordane } & \mu \mathrm{g} / \mathrm{kg} & 2.00 & \mathrm{U} \\ \text { beta-BHC } & \mu \mathrm{g} / \mathrm{kg} & 2.00 & \mathrm{U} \\ \text { delta-BHC } & \mu \mathrm{g} / \mathrm{kg} & 2.00 & \mathrm{U} \\ \text { gamma-Chlordane } & \mu \mathrm{g} / \mathrm{kg} & 2.00 & \mathrm{U} \\ \text { Acenaphthene } & \mu \mathrm{g} / \mathrm{kg} & 4.10 & \mathrm{UJ} \\ \text { Acenaphthylene } & \mu \mathrm{g} / \mathrm{kg} & 203.80 & \mathrm{UJ} \\ \text { Aldrin } & \mu \mathrm{g} / \mathrm{kg} & 2.00 & \mathrm{U} \\ \text { Anthracene } & \mu \mathrm{g} / \mathrm{kg} & 4.10 & \mathrm{UJ} \\ \text { Benzo(a)anthracene } & \mu \mathrm{g} / \mathrm{kg} & 1.60 & \mathrm{~J} \\ \text { Benzo(a)pyrene } & \mu \mathrm{g} / \mathrm{kg} & 0.80 & \mathrm{~J} \\ \text { Benzo(b)fluoranthene } & \mu \mathrm{g} / \mathrm{kg} & 4.10 & \mathrm{UJ} \\ \text { Benzo(ghi)perylene } & \mu \mathrm{g} / \mathrm{kg} & 1.20 & \mathrm{~J} \\ \text { Benzo(k)fluoranthene } & \mu \mathrm{g} / \mathrm{kg} & 0.80 & \mathrm{~J} \\ \text { Chrysene } & \mu \mathrm{g} / \mathrm{kg} & 4.10 & \mathrm{UJ} \\ \text { Dalapon } & \mu \mathrm{g} / \mathrm{kg} & 4270.10 & \mathrm{UJ} \\ \text { Dibenzo(a,h)anthracene } & \mu \mathrm{g} / \mathrm{kg} & 4.10 & \mathrm{UJ}\end{array}$




\section{C-67}

Table C.1 (continued)

\begin{tabular}{|c|c|c|c|c|c|c|}
\hline Analysis & Units & $\begin{array}{c}\text { A horizon } \\
\text { Result }\end{array}$ & Qualifier & $\begin{array}{l}\text { A horizon } \\
\text { field dup }\end{array}$ & Qualifier & \\
\hline $\begin{array}{l}\text { Dicamba } \\
\text { Dichlorprop } \\
\text { Dieldrin } \\
\text { Dinoseb } \\
\text { Endosulfan sul fate } \\
\text { Endosulfan I } \\
\text { Endosulfan II } \\
\text { Endrin } \\
\text { Endrin aldehyde } \\
\text { Endrin ketone } \\
\text { Fluoranthene } \\
\text { Fluorene } \\
\text { Heptachlor } \\
\text { Heptachlor epoxide } \\
\text { Indeno(1,2,3-Cd)pyrene } \\
\text { Lindane } \\
\text { Methoxychlor } \\
\text { MCPA } \\
\text { MCPP } \\
\text { Haphthalene } \\
\text { Phenanthrene } \\
\text { Pyrene } \\
\text { PCB-1016 } \\
\text { PCB-1221 } \\
\text { PCB-1232 } \\
\text { PCB-1242 } \\
\text { PCB-1248 } \\
\text { PCB-1254 } \\
\text { PCB-1260 } \\
\text { Silvex } \\
\text { Toxaphene } \\
2,4-D \\
2,4-D B \\
2,4,5-T \\
4,41-D D D \\
4,41-D D E \\
4,41-D D T\end{array}$ & 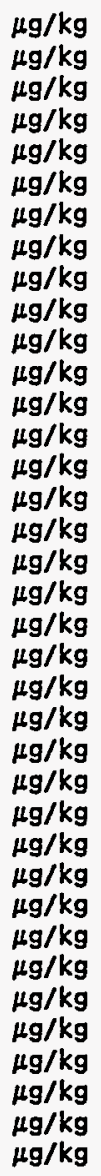 & $\begin{array}{r}196.30 \\
490.80 \\
3.90 \\
103.10 \\
3.90 \\
2.00 \\
3.90 \\
3.90 \\
3.90 \\
3.90 \\
5.30 \\
4.10 \\
2.00 \\
2.00 \\
20.40 \\
2.00 \\
20.30 \\
184054.30 \\
139881.30 \\
20.40 \\
2.40 \\
0.80 \\
39.30 \\
79.90 \\
39.30 \\
39.30 \\
39.30 \\
39.30 \\
39.30 \\
122.70 \\
202.60 \\
883.50 \\
662.60 \\
147.20 \\
3.90 \\
3.90 \\
3.90\end{array}$ & 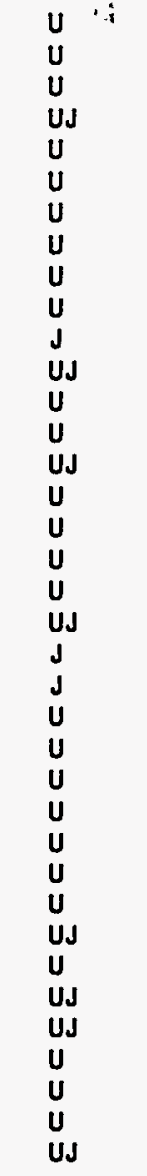 & & & 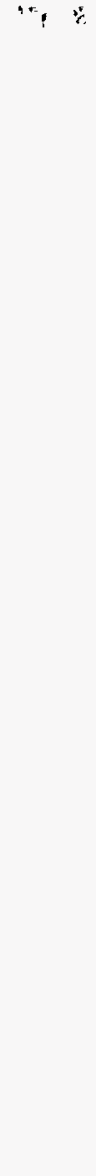 \\
\hline
\end{tabular}

Location=ROA; Formation=COPPER RIDGE; Site=40; $A=3211 ; A$ dup=' '; Phase =2

$\begin{array}{llrl} & \mu \mathrm{g} / \mathrm{kg} & 1.90 & \mathrm{U} \\ \text { alpha-BHC } & \mu \mathrm{gg} / \mathrm{kg} & 1.90 & \mathrm{U} \\ \text { alpha-Chlordane } & \mu \mathrm{g} / \mathrm{kg} & 1.90 & \mathrm{U} \\ \text { beta-BHC } & \mu \mathrm{g} / \mathrm{kg} & 1.90 & \mathrm{U} \\ \text { del ta-BHC } & \mu \mathrm{g} / \mathrm{kg} & 1.90 & \mathrm{U} \\ \text { gamma-Chlordane } & \mu \mathrm{g} / \mathrm{kg} & 3.90 & \mathrm{UJ} \\ \text { Acenaphthene } & \mu \mathrm{kg} & 1.90 & \mathrm{U} \\ \text { Aldrin } & \mu \mathrm{g} / \mathrm{kg} & 3.90 & \mathrm{UJ} \\ \text { Anthracene } & \mu \mathrm{g} / \mathrm{kg} & 6.60 & \mathrm{~J} \\ \text { Benzo(a)anthracene } & \mu \mathrm{g} / \mathrm{kg} & 1.60 & \mathrm{~J} \\ \text { Benzo(a)pyrene } & 1.20 & \mathrm{~J} \\ \text { Benzo(b)fluoranthene } & \mu \mathrm{g} / \mathrm{kg} & 2.30 & \mathrm{~J} \\ \text { Benzo(ghi)perylene } & \mu \mathrm{g} / \mathrm{kg} & 0.80 & \mathrm{~J} \\ \text { Benzo(k)fluoranthene } & \mu \mathrm{g} / \mathrm{kg} & 3.90 & \mathrm{UJ} \\ \text { Chrysene } & \mu \mathrm{g} / \mathrm{kg} & 3992.70 & \mathrm{UJ} \\ \text { Dalapon } & \mu \mathrm{g} / \mathrm{kg} & 0.80 & \mathrm{~J} \\ \text { Dibenzo(a,h)anthracene } & \mu \mathrm{g} / \mathrm{kg} & 183.60 & \mathrm{U} \\ \text { Dicamba } & \mu \mathrm{g} / \mathrm{kg} & 458.90 & \mathrm{U} \\ \text { Dichlorprop } & \mu \mathrm{g} / \mathrm{kg} & 3.70 & \mathrm{U} \\ \text { Dieldrin } & \mu \mathrm{g} / \mathrm{kg} & 96.40 & \mathrm{UJ} \\ \text { Dinoseb } & \mu \mathrm{g} / \mathrm{kg} & 3.70 & \mathrm{U} \\ \text { Endosulfan sulfate } & \mu \mathrm{g} / \mathrm{kg} & 1.90 & \mathrm{U} \\ \text { Endosulfan I } & \mu \mathrm{g} / \mathrm{kg} & 3.70 & \mathrm{U} \\ \text { Endosulfan II } & \mu \mathrm{g} / \mathrm{kg} & 3.70 & \mathrm{U} \\ \text { Endrin } & \mu \mathrm{g} / \mathrm{kg} & 3.70 & \mathrm{U} \\ \text { Endrin aldehyde } & \mu \mathrm{g} / \mathrm{kg} & 3.70 & \mathrm{U} \\ \text { Endrin ketone } & \mu \mathrm{g} / \mathrm{kg} & 4.30 & \mathrm{~J}\end{array}$




\section{C-68}

Table C.1 (continued)

\begin{tabular}{|c|c|c|c|c|c|}
\hline Analysis & Units & $\begin{array}{c}\text { A horizon } \\
\text { Result }\end{array}$ & Qualifier & $\begin{array}{l}\text { A horizon } \\
\text { field dup }\end{array}$ & Qualifier \\
\hline $\begin{array}{l}\text { Fluorene } \\
\text { Heptachlor } \\
\text { Heptachlor epoxide } \\
\text { Indeno(1,2,3-cd)pyrene } \\
\text { Lindane } \\
\text { Methoxychlor } \\
\text { MCPA } \\
\text { MCPP } \\
\text { Naphthalene } \\
\text { Phenanthrene } \\
\text { Pyrene } \\
\text { PCB-1016 } \\
\text { PCB-1221 } \\
\text { PCB-1232 } \\
\text { PCB-1242 } \\
\text { PCB-1248 } \\
\text { PCB-1254 } \\
\text { PCB-1260 } \\
\text { Silvex } \\
\text { TOxaphene } \\
2,4-D \\
2,4-D B \\
2,4,5-T \\
4,4 '-D D D \\
4,41-D D E \\
4,41-D D T\end{array}$ & 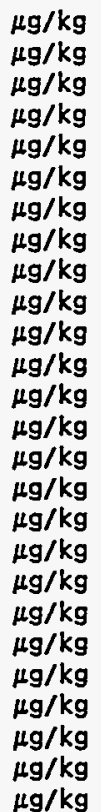 & $\begin{array}{r}3.90 \\
1.90 \\
1.90 \\
15.50 \\
1.90 \\
19.30 \\
172098.80 \\
130795.10 \\
19.40 \\
3.10 \\
3.90 \\
37.50 \\
76.10 \\
37.50 \\
37.50 \\
37.50 \\
37.50 \\
37.50 \\
114.70 \\
193.10 \\
826.10 \\
619.60 \\
137.70 \\
3.70 \\
3.70 \\
3.70\end{array}$ & $\begin{array}{l}U J \\
U \\
U \\
U J \\
U \\
U \\
P \\
U \\
U J \\
J \\
J \\
U \\
U \\
U \\
U \\
U \\
U \\
U \\
U \\
U J \\
U \\
U J \\
U J \\
U \\
U \\
U \\
U J\end{array}$ & . & \\
\hline
\end{tabular}

Location $=R O A ;$ Formation $=$ COPPER RIDGE; Site $=41 ; A=3217 ; A$ dup=' '; Phase $=2$

$\begin{array}{lrrl} & & & \\ \text { alpha-BHC } & \mu \mathrm{g} / \mathrm{kg} & 2.10 & \mathrm{U} \\ \text { alpha-Chlordane } & \mu \mathrm{g} / \mathrm{kg} & 2.10 & \mathrm{U} \\ \text { beta-BHC } & \mu \mathrm{g} / \mathrm{kg} & 2.10 & \mathrm{U} \\ \text { delta-BHC } & \mu \mathrm{g} / \mathrm{kg} & 2.10 & \mathrm{U} \\ \text { gamna-Chlordane } & \mu \mathrm{g} / \mathrm{kg} & 2.10 & \mathrm{U} \\ \text { Acenaphthene } & \mu \mathrm{g} / \mathrm{kg} & 4.20 & \mathrm{UJ} \\ \text { Acenaphthylene } & \mu \mathrm{g} / \mathrm{kg} & 207.60 & \mathrm{UJ} \\ \text { Aldrin } & \mu \mathrm{g} / \mathrm{kg} & 2.10 & \mathrm{U} \\ \text { Anthracene } & \mu \mathrm{g} / \mathrm{kg} & 1.70 & \mathrm{~J} \\ \text { Benzo(a)anthracene } & \mu \mathrm{g} / \mathrm{kg} & 1.70 & \mathrm{~J} \\ \text { Benzo(a)pyrene } & \mu \mathrm{g} / \mathrm{kg} & 1.70 & \mathrm{~J} \\ \text { Benzo(b)fluoranthene } & \mu \mathrm{g} / \mathrm{kg} & 2.10 & \mathrm{~J} \\ \text { Benzo(ghi)perylene } & \mu \mathrm{g} / \mathrm{kg} & 1.20 & \mathrm{~J} \\ \text { Benzo(k)fluoranthene } & \mu \mathrm{g} / \mathrm{kg} & 1.70 & \mathrm{~J} \\ \text { Chrysene } & \mu \mathrm{g} / \mathrm{kg} & 2.50 & \mathrm{~J} \\ \text { Dalapon } & \mu \mathrm{g} / \mathrm{kg} & 4253.40 & \mathrm{UJ} \\ \text { Dibenzo(a, h)anthracene } & \mu \mathrm{g} / \mathrm{kg} & 0.80 & \mathrm{~J} \\ \text { Dicamba } & \mu \mathrm{g} / \mathrm{kg} & 195.60 & \mathrm{U} \\ \text { Dichlorprop } & \mu \mathrm{g} / \mathrm{kg} & 488.90 & \mathrm{U} \\ \text { Dieldrin } & \mu \mathrm{g} / \mathrm{kg} & 4.10 & \mathrm{U} \\ \text { Dinoseb } & \mu \mathrm{g} / \mathrm{kg} & 102.70 & \mathrm{UJ} \\ \text { Endosulfan sulfate } & \mu \mathrm{g} / \mathrm{kg} & 4.10 & \mathrm{U} \\ \text { Endosulfan I } & \mu \mathrm{g} / \mathrm{kg} & 2.10 & \mathrm{U} \\ \text { Endosulfan II } & \mu \mathrm{g} / \mathrm{kg} & 4.10 & \mathrm{U} \\ \text { Endrin } & \mu \mathrm{g} / \mathrm{kg} & 4.10 & \mathrm{U} \\ \text { Endrin aldehyde } & \mu \mathrm{g} / \mathrm{kg} & 4.10 & \mathrm{U} \\ \text { Endrin ketone } & \mu \mathrm{g} / \mathrm{kg} & 4.10 & \mathrm{U} \\ \text { Fluoranthene } & \mu \mathrm{g} / \mathrm{kg} & 5.80 & \mathrm{~J} \\ \text { Fluorene } & \mu \mathrm{g} / \mathrm{kg} & 4.20 & \mathrm{UJ} \\ \text { Heptachlor } & \mu \mathrm{g} / \mathrm{kg} & 2.10 & \mathrm{U} \\ \text { Heptachlor epoxide } & \mu \mathrm{g} / \mathrm{kg} & 2.10 & \mathrm{U} \\ \text { Indeno(1,2,3-cd)pyrene } & \mu \mathrm{g} / \mathrm{kg} & 45.20 & \mathrm{UJ} \\ \text { Lindane } & \mu \mathrm{g} / \mathrm{kg} & 2.10 & \mathrm{U} \\ \text { Methoxychlor } & \mu \mathrm{g} / \mathrm{kg} & 21.30 & \mathrm{U} \\ \text { MCPA } & \mu \mathrm{g} / \mathrm{kg} & 183337.10 & \mathrm{U} \\ \text { MCPP } & \mu \mathrm{g} / \mathrm{kg} & 139336.20 & \mathrm{U} \\ \text { Haphthalene } & \mu \mathrm{g} / \mathrm{kg} & 20.80 & \mathrm{UJ} \\ \text { Phenanthrene } & \mu \mathrm{g} / \mathrm{kg} & 5.40 & \mathrm{~J} \\ & & & \\ & & & \\ & & & \end{array}$


Table C.1 (continued)

\begin{tabular}{|c|c|c|c|c|c|c|}
\hline - Analysis & Units & $\begin{array}{c}\text { A horizon } \\
\text { Result }\end{array}$ & Qualifier & $\begin{array}{l}\text { A horizon } \\
\text { field dup }\end{array}$ & Qualifier & \\
\hline $\begin{array}{l}\text { Pyrene } \\
\text { PCB-1016 } \\
\text { PCB-1221 } \\
\text { PCB-1232 } \\
\text { PCB-1242 } \\
\text { PCB-1248 } \\
\text { PCB-1254 } \\
\text { PCB-1260 } \\
\text { silvex } \\
\text { Toxaphene } \\
2,4-D \\
2,4-D B \\
2,4,5-T \\
4,4^{\prime}-D D D \\
4,41-D D E \\
4,4^{\prime}-D D T\end{array}$ & $\begin{array}{l}\mu \mathrm{g} / \mathrm{kg} \\
\mu \mathrm{g} / \mathrm{kg} \\
\mu \mathrm{g} / \mathrm{kg} \\
\mu \mathrm{g} / \mathrm{kg} \\
\mu \mathrm{g} / \mathrm{kg} \\
\mu \mathrm{g} / \mathrm{kg} \\
\mu g / \mathrm{kg} \\
\mu \mathrm{g} / \mathrm{kg} \\
\mu g / \mathrm{kg} \\
\mu \mathrm{g} / \mathrm{kg} \\
\mu g / \mathrm{kg} \\
\mu g / \mathrm{kg} \\
\mu g / \mathrm{kg} \\
\mu \mathrm{g} / \mathrm{kg} \\
\mu g / \mathrm{kg} \\
\mu g / \mathrm{kg}\end{array}$ & $\begin{array}{r}1.70 \\
41.40 \\
84.00 \\
41.40 \\
41.40 \\
41.40 \\
41.40 \\
41.40 \\
122.20 \\
213.10 \\
880.00 \\
660.00 \\
146.70 \\
4.10 \\
4.10 \\
4.10\end{array}$ & $\begin{array}{l}J \\
U \\
U \\
U \\
U \\
U \\
U \\
U \\
U J \\
U \\
U J \\
U J \\
U \\
U \\
U \\
U J\end{array}$ & & & $\because 4$ \\
\hline
\end{tabular}

Location=ROA; Formation=COPPER RIDGE; Site=42; $A=3235 ; A$ dup= '; Phase =2

\begin{tabular}{|c|c|c|}
\hline 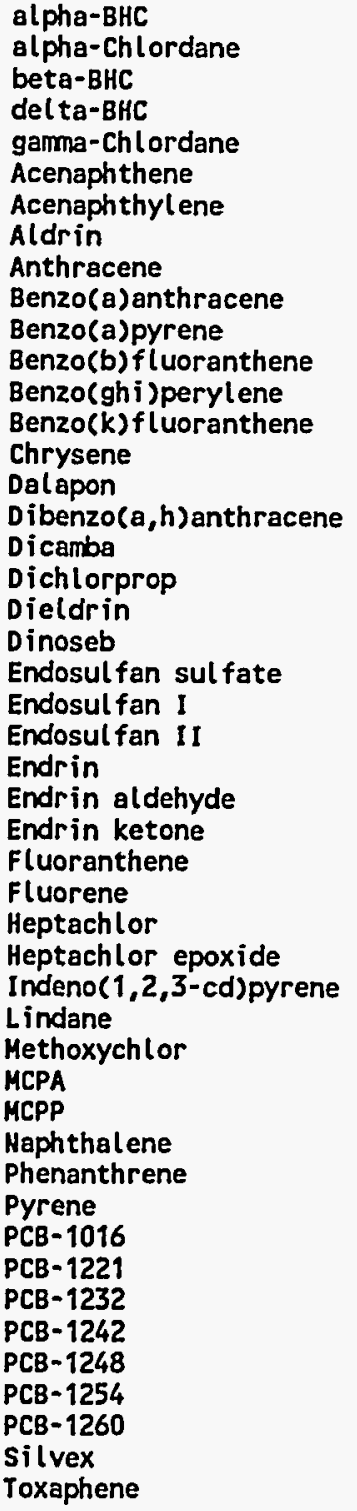 & $\begin{array}{l}\mu g / k g \\
\mu g / k g \\
\mu g / k g \\
\mu g / k g \\
\mu g / k g \\
\mu g / k g \\
\mu g / k g \\
\mu g / k g \\
\mu g / k g \\
\mu g / k g \\
\mu g / k g \\
\mu g / k g \\
\mu g / k g \\
\mu g / k g \\
\mu g / k g \\
\mu g / k g \\
\mu g / k g \\
\mu g / k g \\
\mu g / k g \\
\mu g / k g \\
\mu g / k g \\
\mu g / k g \\
\mu g / k g \\
\mu g / k g \\
\mu g / k g \\
\mu g / k g \\
\mu g / k g \\
\mu g / k g \\
\mu g / k g \\
\mu g / k g \\
\mu g / k g \\
\mu g / k g \\
\mu g / k g \\
\mu g / k g \\
\mu g / k g \\
\mu g / k g \\
\mu g / k g \\
\mu g / k g \\
\mu g / k g \\
\mu g / k g \\
\mu g / k g \\
\mu g / k g \\
\mu g / k g \\
\mu g / k g \\
\mu g / k g \\
\mu g / k g \\
\mu g / k g \\
\mu g / k g\end{array}$ & $\begin{array}{r}2.00 \\
2.00 \\
2.00 \\
2.00 \\
2.00 \\
4.00 \\
197.70 \\
2.00 \\
6.70 \\
3.20 \\
1.20 \\
4.00 \\
1.60 \\
0.80 \\
4.00 \\
4128.20 \\
0.80 \\
189.80 \\
474.50 \\
4.00 \\
99.60 \\
4.00 \\
2.00 \\
4.00 \\
4.00 \\
4.00 \\
4.00 \\
2.80 \\
4.00 \\
2.00 \\
2.00 \\
19.80 \\
2.00 \\
20.40 \\
177939.70 \\
135234.10 \\
19.80 \\
3.60 \\
2.00 \\
39.60 \\
80.50 \\
39.60 \\
39.60 \\
39.60 \\
39.60 \\
39.60 \\
118.60 \\
204.20\end{array}$ \\
\hline
\end{tabular}




\section{C-70}

Table C.1 (continued)

\begin{tabular}{|c|c|c|c|c|c|}
\hline Analysis & Units & $\begin{array}{c}\text { A horizon } \\
\text { Result }\end{array}$ & Qualifier & $\begin{array}{l}\text { A horizon } \\
\text { field dup }\end{array}$ & Qualifier \\
\hline $\begin{array}{l}2,4-D \\
2,4^{-D B} \\
2,4^{\prime}, 5-T \\
4,4^{\prime}-D D D \\
4,4^{\prime}-D D E \\
4,4^{\prime}-D D T\end{array}$ & $\begin{array}{l}\mu \mathrm{g} / \mathrm{kg} \\
\mu \mathrm{g} / \mathrm{kg} \\
\mu \mathrm{g} / \mathrm{kg} \\
\mu \mathrm{g} / \mathrm{kg} \\
\mu \mathrm{g} / \mathrm{kg} \\
\mu \mathrm{g} / \mathrm{kg}\end{array}$ & $\begin{array}{r}854.10 \\
640.60 \\
142.40 \\
4.00 \\
4.00 \\
4.00\end{array}$ & $\begin{array}{l}\text { UJ } \\
U J \\
U \\
U \\
U \\
\text { US }\end{array}$ & & \\
\hline
\end{tabular}

Location=ROA; Formation=COPPER RIDGE; Site=43; $A=3231 ; A$ dup=3233; Phase $=2$

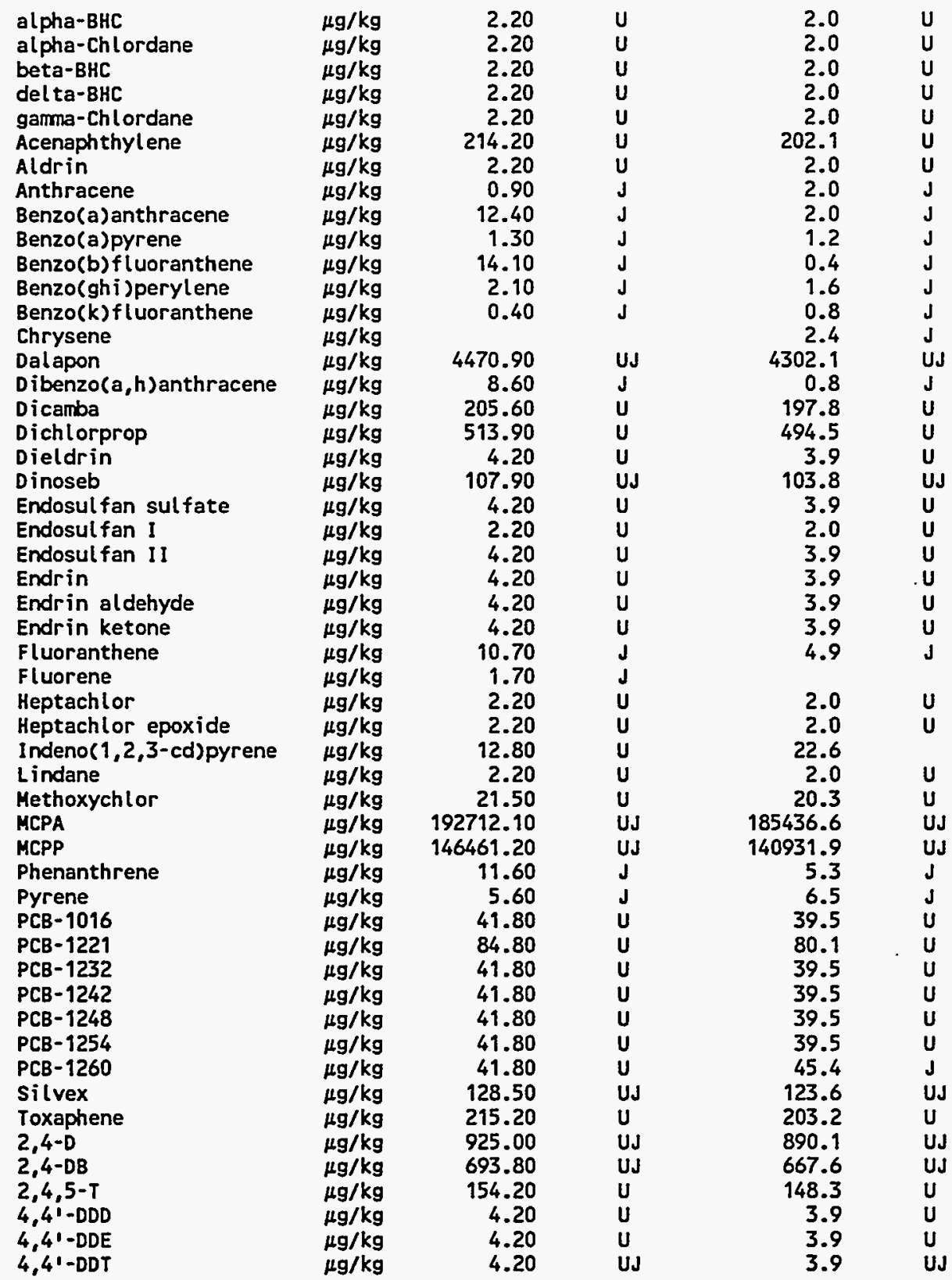




\section{C-71}

Table C.1 (continued)

\begin{tabular}{lcccc}
\hline & A horizon & A horizon \\
Analysis & Units & Result & Qualifier & $\begin{array}{c}\text { field dup } \\
\text { Qualifier }\end{array}$ \\
\hline
\end{tabular}

Location=ROA; Formation=COPPER RIDGE; Site $=44 ; A=3229 ; A$ dup=' ; Phase $=2$

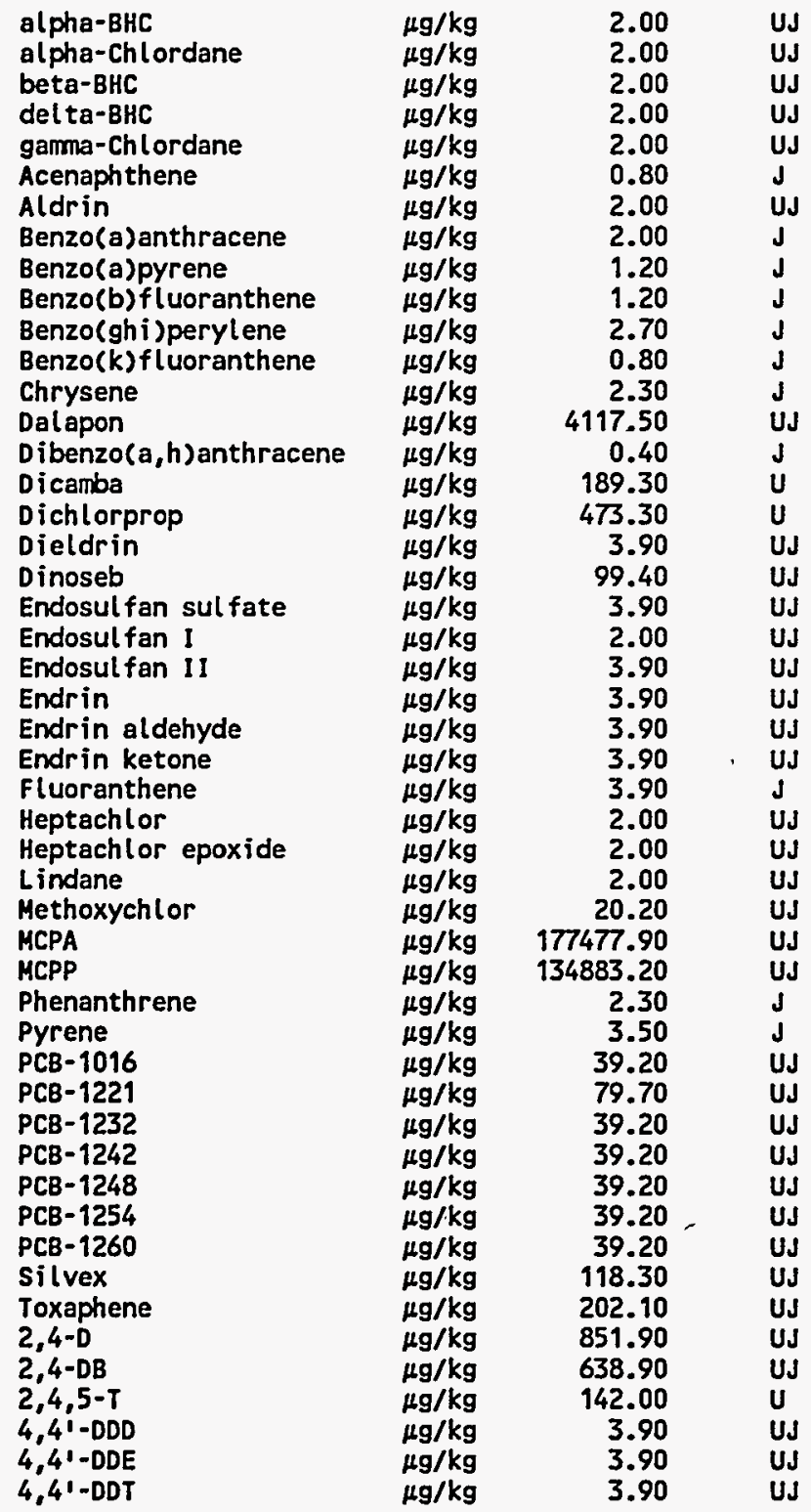

Location=ROA; Formation=COPPER RIDGE; Site =45; $A=3227 ; A$ dup=' '; Phase=2

$\begin{array}{llll}\text { alpha-BHC } & \mu g / \mathrm{kg} & 2.00 & \mathrm{U} \\ \text { alpha-Chlordane } & \mu \mathrm{kg} & 2.00 & \mathrm{U} \\ \text { beta-BHC } & \mu \mathrm{g} / \mathrm{kg} & 2.00 & \mathrm{U} \\ \text { del ta-BHC } & \mu \mathrm{kg} & 2.00 & \mathrm{U} \\ \text { gamma-Chlordane } & \mu \mathrm{kg} & 2.00 & \mathrm{U} \\ \text { Aldrin } & \mu \mathrm{kg} & 2.00 & \mathrm{U} \\ \text { Anthracene } & \mu \mathrm{kg} & 3.60 & \mathrm{~J} \\ \text { Benzo(a)anthracene } & \mu \mathrm{kg} / \mathrm{kg} & 2.80 & \mathrm{~J} \\ \text { Benzo(a)pyrene } & \mu \mathrm{kg} & 1.60 & \mathrm{~J} \\ \text { Benzo(b)fluoranthene } & \mu g / \mathrm{kg} & 1.60 & \mathrm{~J} \\ \text { Benzo(ghi)perylene } & \mu g / \mathrm{kg} & 2.80 & \mathrm{~J} \\ \text { Benzo(k)fluoranthene } & \mu g / \mathrm{kg} & 1.20 & \mathrm{~J}\end{array}$


C-72

Table C.1 (continued)

\begin{tabular}{|c|c|c|c|c|c|}
\hline Analysis & Units & $\begin{array}{c}\text { A horizon } \\
\text { Result }\end{array}$ & Qualifier & $\begin{array}{l}\text { A horizon } \\
\text { field dup }\end{array}$ & Qualifier \\
\hline $\begin{array}{l}\text { Chrysene } \\
\text { Dalapon } \\
\text { Dibenzo(a,h)anthracene } \\
\text { Dicamba } \\
\text { Dichlorprop } \\
\text { Dieldrin } \\
\text { Dinoseb } \\
\text { Endosulfan sulfate } \\
\text { Endosul fan I } \\
\text { Endosul fan II } \\
\text { Endrin } \\
\text { Endrin aldehyde } \\
\text { Endrin ketone } \\
\text { Fluoranthene } \\
\text { Heptachlor } \\
\text { Heptachlor epoxide } \\
\text { Indeno(1,2,3-Cd)pyrene } \\
\text { Lindane } \\
\text { Methoxychlor } \\
\text { MCPA } \\
\text { MCPP } \\
\text { Phenanthrene } \\
\text { Pyrene } \\
\text { PCB-1016 } \\
\text { PCB-1221 } \\
\text { PCB-1232 } \\
\text { PCB-1242 } \\
\text { PCB-1248 } \\
\text { PCB-1254 } \\
\text { PCB-1260 } \\
\text { Silvex } \\
\text { Toxaphene } \\
2,4-D \\
2,4-D B \\
2,4,5-T \\
4,4:-D D D \\
4,41-D D E \\
4,4 \text {-DDT }\end{array}$ & 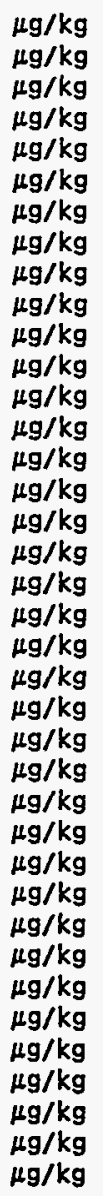 & $\begin{array}{r}3.20 \\
4161.30 \\
0.40 \\
191.30 \\
478.30 \\
4.00 \\
100.40 \\
4.00 \\
2.00 \\
4.00 \\
4.00 \\
4.00 \\
4.00 \\
4.00 \\
2.00 \\
2.00 \\
20.60 \\
2.00 \\
20.40 \\
179367.80 \\
136319.50 \\
4.40 \\
4.00 \\
39.50 \\
80.20 \\
39.50 \\
39.50 \\
39.50 \\
39.50 \\
39.50 \\
119.60 \\
203.60 \\
861.00 \\
645.70 \\
143.50 \\
4.00 \\
4.00 \\
4.00\end{array}$ & $\begin{array}{l}J \\
U J \\
J \\
U \\
U \\
U \\
U J J \\
U J \\
U \\
U \\
U \\
U \\
U \\
U \\
U \\
J \\
U \\
U \\
J \\
U \\
U \\
U \\
U \\
U \\
J \\
J \\
U \\
U \\
U \\
U \\
U \\
U \\
U \\
U \\
U \\
U \\
U J \\
U J \\
U J J \\
U J J \\
U J \\
U J\end{array}$ & & \\
\hline
\end{tabular}

Location=ROA; Formation=COPPER RIDGE; Site=46; $A=3225 ; A$ dup=' '; Phase $=2$

alpha-BHC

alpha-chlordane

beta-BHC

delta-BHC

gamma-Chlordane

Acenaph thene

Acenaphthylene

Aldrin

Anthracene

Benzo(a)anthracene

Benzo(a)pyrene

Benzo(b)f luoranthene

Benzo(ghi)perylene

Benzo(k)fluoranthene

Chrysene

Dalapon

Dibenzo $(a, h)$ anthracene

Dicamba

Dichlorprop

Dieldrin

Dinoseb

Endosulfan sulfate

Endosulfan I

Endosulfan II

Endrin

Endrin aldehyde

\begin{tabular}{|c|c|}
\hline $\begin{array}{l}\mu g / \mathrm{kg} \\
\mu \mathrm{g} / \mathrm{kg} \\
\mu \mathrm{g} / \mathrm{kg} \\
\mu \mathrm{g} / \mathrm{kg} \\
\mu \mathrm{g} / \mathrm{kg} \\
\mu \mathrm{g} / \mathrm{kg} \\
\mu \mathrm{g} / \mathrm{kg} \\
\mu g / \mathrm{kg} \\
\mu \mathrm{g} / \mathrm{kg} \\
\mu g / \mathrm{kg} \\
\mu \mathrm{g} / \mathrm{kg} \\
\mu g / \mathrm{kg} \\
\mu \mathrm{g} / \mathrm{kg} \\
\mu g / \mathrm{kg} \\
\mu g / \mathrm{kg} \\
\mu \mathrm{g} / \mathrm{kg} \\
\mu g / \mathrm{kg} \\
\mu \mathrm{g} / \mathrm{kg} \\
\mu \mathrm{g} / \mathrm{kg} \\
\mu \mathrm{g} / \mathrm{kg} \\
\mu \mathrm{g} / \mathrm{kg} \\
\mu g / \mathrm{kg} \\
\mu g / \mathrm{kg} \\
\mu g / \mathrm{kg} \\
\mu g / \mathrm{kg} \\
\mu \mathrm{g} / \mathrm{kg}\end{array}$ & $\begin{array}{r}2.20 \\
2.20 \\
2.20 \\
2.20 \\
2.20 \\
4.30 \\
212.80 \\
2.20 \\
0.40 \\
2.60 \\
0.40 \\
0.90 \\
4.30 \\
0.90 \\
4.30 \\
4351.60 \\
4.30 \\
200.10 \\
500.20 \\
4.20 \\
105.00 \\
4.20 \\
2.20 \\
4.20 \\
4.20 \\
4.20\end{array}$ \\
\hline
\end{tabular}




\section{C-73}

Table C.1 (continued)

\begin{tabular}{|c|c|c|c|c|c|c|}
\hline Analysis & Units & $\begin{array}{c}\text { A horizon } \\
\text { Result }\end{array}$ & Qualifier & $\begin{array}{l}\text { A horizon } \\
\text { field dup }\end{array}$ & Qualifier & \\
\hline $\begin{array}{l}\text { Endrin ketone } \\
\text { Fluoranthene } \\
\text { Fluorene } \\
\text { Heptachlor } \\
\text { Heptachlor epoxide } \\
\text { Indeno(1,2,3-cd)pyrene } \\
\text { Lindane } \\
\text { Methoxychlor } \\
\text { HCPA } \\
\text { HCPP } \\
\text { Naphthalene } \\
\text { Phenanthrene } \\
\text { Pyrene } \\
\text { PCB-1016 } \\
\text { PCB-1221 } \\
\text { PCB-1232 } \\
\text { PCB-1242 } \\
\text { PCB-1248 } \\
\text { PCB-1254 } \\
\text { PCB-1260 } \\
\text { Silvex } \\
\text { Toxaphene } \\
2,4-D \\
2,4-D B \\
2,4,5-T \\
4,41-D D D \\
4,41-D D E \\
4,41-D D T\end{array}$ & $\begin{array}{l}\mu g / \mathrm{kg} \\
\mu \mathrm{g} / \mathrm{kg} \\
\mu g / \mathrm{kg} \\
\mu \mathrm{g} / \mathrm{kg} \\
\mu \mathrm{g} / \mathrm{kg} \\
\mu \mathrm{g} / \mathrm{kg} \\
\mu \mathrm{g} / \mathrm{kg} \\
\mu \mathrm{g} / \mathrm{kg} \\
\mu \mathrm{g} / \mathrm{kg} \\
\mu \mathrm{g} / \mathrm{kg} \\
\mu \mathrm{g} / \mathrm{kg} \\
\mu \mathrm{g} / \mathrm{kg} \\
\mu \mathrm{g} / \mathrm{kg} \\
\mu \mathrm{g} / \mathrm{kg} \\
\mu \mathrm{g} / \mathrm{kg} \\
\mu \mathrm{g} / \mathrm{kg} \\
\mu \mathrm{g} / \mathrm{kg} \\
\mu \mathrm{g} / \mathrm{kg} \\
\mu \mathrm{g} / \mathrm{kg} \\
\mu \mathrm{g} / \mathrm{kg} \\
\mu \mathrm{g} / \mathrm{kg} \\
\mu g / \mathrm{kg} \\
\mu \mathrm{g} / \mathrm{kg} \\
\mu \mathrm{g} / \mathrm{kg} \\
\mu \mathrm{g} / \mathrm{kg} \\
\mu \mathrm{g} / \mathrm{kg} \\
\mu \mathrm{g} / \mathrm{kg} \\
\mu \mathrm{g} / \mathrm{kg}\end{array}$ & $\begin{array}{r}4.20 \\
3.00 \\
4.30 \\
2.20 \\
2.20 \\
21.30 \\
2.20 \\
21.80 \\
187569.40 \\
142552.70 \\
21.30 \\
1.30 \\
0.90 \\
42.30 \\
85.90 \\
42.30 \\
42.30 \\
42.30 \\
42.30 \\
42.30 \\
125.00 \\
217.90 \\
900.30 \\
675.20 \\
150.10 \\
4.20 \\
4.20 \\
4.20\end{array}$ & $\begin{array}{l}U \\
J \\
U \\
U \\
U \\
U J \\
U \\
U \\
U \\
U \\
U \\
J \\
J \\
U \\
U \\
U \\
U \\
U \\
U \\
U \\
U \\
U \\
U J \\
U J \\
U J \\
U J \\
U J \\
U J\end{array}$ & & & 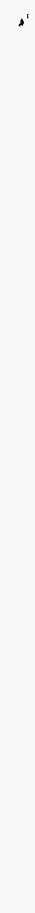 \\
\hline
\end{tabular}

Location $=R O A ;$ Formation $=$ COPPER RIDGE; Site $=47 ; A=3223 ; A$ dup=' '; Phase $=2$

\begin{tabular}{|c|c|c|}
\hline $\begin{array}{l}\text { alpha-BHC } \\
\text { alpha-Chlordane } \\
\text { beta-BHC } \\
\text { del ta-BHC } \\
\text { gamma-Chlordane } \\
\text { Acenaphthene } \\
\text { Acenaphthylene } \\
\text { Aldrin } \\
\text { Anthracene } \\
\text { Benzo(a)anthracene } \\
\text { Benzo(a)pyrene } \\
\text { Benzo(b)fluoranthene } \\
\text { Benzo(ghi)perylene } \\
\text { Benzo(k)fluoranthene } \\
\text { Chrysene } \\
\text { Dalapon } \\
\text { Dibenzo(a,h)anthracene } \\
\text { Dicamba } \\
\text { Dichlorprop } \\
\text { Dieldrin } \\
\text { Dinoseb } \\
\text { Endosulfan sulfate } \\
\text { Endosulfan I } \\
\text { Endosulfan } 11 \\
\text { Endrin } \\
\text { Endrin aldehyde } \\
\text { Endrin ketone } \\
\text { Fluoranthene } \\
\text { Fluorene } \\
\text { Heptachlor } \\
\text { Heptachlor epoxide } \\
\text { Indeno( } 1,2,3-\text { cd)pyrene } \\
\text { Lindane } \\
\text { Methoxychlor } \\
\text { MCPA } \\
\text { MCPP }\end{array}$ & 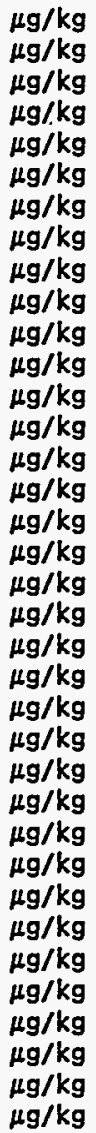 & $\begin{array}{r}2.20 \\
2.20 \\
2.20 \\
2.20 \\
2.20 \\
4.20 \\
212.20 \\
2.20 \\
0.40 \\
3.40 \\
4.20 \\
2.10 \\
1.30 \\
0.40 \\
4.20 \\
4467.50 \\
0.40 \\
205.40 \\
513.50 \\
4.20 \\
107.80 \\
4.20 \\
2.20 \\
4.20 \\
4.20 \\
4.20 \\
4.20 \\
3.40 \\
4.20 \\
2.20 \\
2.20 \\
13.20 \\
2.20 \\
21.60 \\
192563.20 \\
146348.00\end{array}$ \\
\hline
\end{tabular}


Table C.1 (continued)

\begin{tabular}{|c|c|c|c|c|c|}
\hline Analysis & Units & $\begin{array}{c}\text { A horizon } \\
\text { Result }\end{array}$ & Qualifier & $\begin{array}{l}\text { A horizon } \\
\text { field dup }\end{array}$ & Qualifier \\
\hline $\begin{array}{l}\text { Naphthalene } \\
\text { Phenanthrene } \\
\text { Pyrene } \\
\text { PCB-1016 } \\
\text { PCB-1221 } \\
\text { PCB-1232 } \\
\text { PCB-1242 } \\
\text { PCB-1248 } \\
\text { PCB-1254 } \\
\text { PCB-1260 } \\
\text { Silvex } \\
\text { ToXaphene } \\
2,4^{-D} \\
2,4^{-D B} \\
2,4^{\prime}, 5-T \\
4,4^{\prime}-D D D \\
4,4^{\prime}-D D E \\
4,4^{\prime}-D D T\end{array}$ & $\begin{array}{l}\mu g / \mathrm{kg} \\
\mu g / \mathrm{kg} \\
\mu \mathrm{g} / \mathrm{kg} \\
\mu g / \mathrm{kg} \\
\mu g / \mathrm{kg} \\
\mu \mathrm{g} / \mathrm{kg} \\
\mu g / \mathrm{kg} \\
\mu \mathrm{g} / \mathrm{kg} \\
\mu \mathrm{g} / \mathrm{kg} \\
\mu g / \mathrm{kg} \\
\mu \mathrm{g} / \mathrm{kg} \\
\mu \mathrm{g} / \mathrm{kg} \\
\mu g / \mathrm{kg} \\
\mu g / \mathrm{kg} \\
\mu \mathrm{g} / \mathrm{kg} \\
\mu \mathrm{g} / \mathrm{kg} \\
\mu g / \mathrm{kg} \\
\mu g / \mathrm{kg}\end{array}$ & $\begin{array}{r}21.20 \\
0.80 \\
0.40 \\
41.90 \\
85.10 \\
41.90 \\
41.90 \\
41.90 \\
41.90 \\
41.90 \\
128.40 \\
216.00 \\
924.30 \\
693.20 \\
154.10 \\
4.20 \\
4.20 \\
4.20\end{array}$ & $\begin{array}{l}U J \\
J \\
J \\
U \\
U \\
U \\
U \\
U \\
U \\
U \\
U J \\
U \\
U S \\
U J \\
U \\
\text { UJ } \\
\text { UJ } \\
\text { UJ }\end{array}$ & . & \\
\hline
\end{tabular}

Location=ROA; Formation=DISMAL GAP; Site=10; $A=3168 ; A$ dup=' '; Phase=1

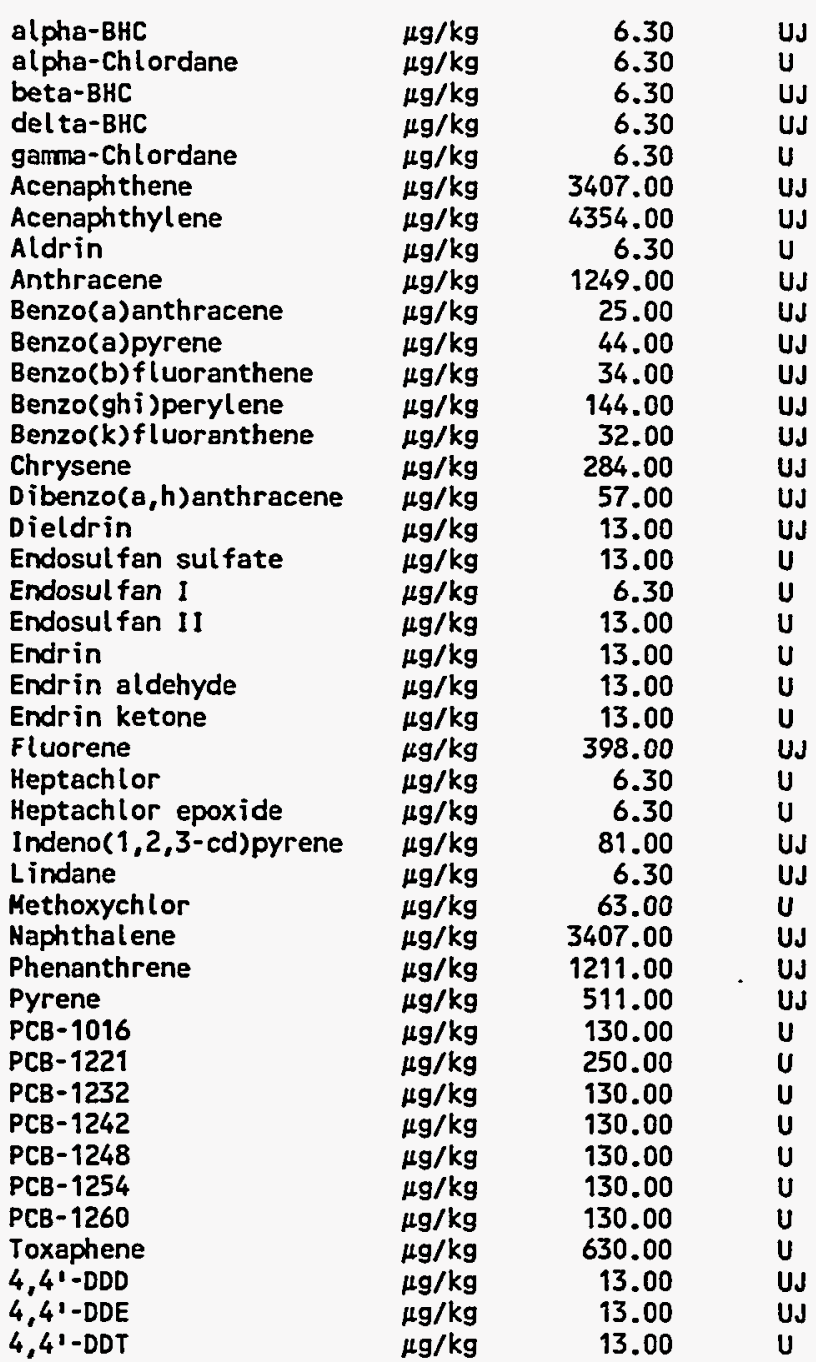




\section{C-75}

Table C.1 (continued)

\begin{tabular}{llccc}
\hline Analysis & Units & $\begin{array}{c}\text { A horizon } \\
\text { Result }\end{array}$ & Qualifier & $\begin{array}{c}\text { A horizon } \\
\text { field dup }\end{array}$ Qualifier \\
\hline
\end{tabular}

Location=ROA; Formation=DISMAL GAP; Site =13; $A=3127 ;$ dup=3139; Phase $=1$

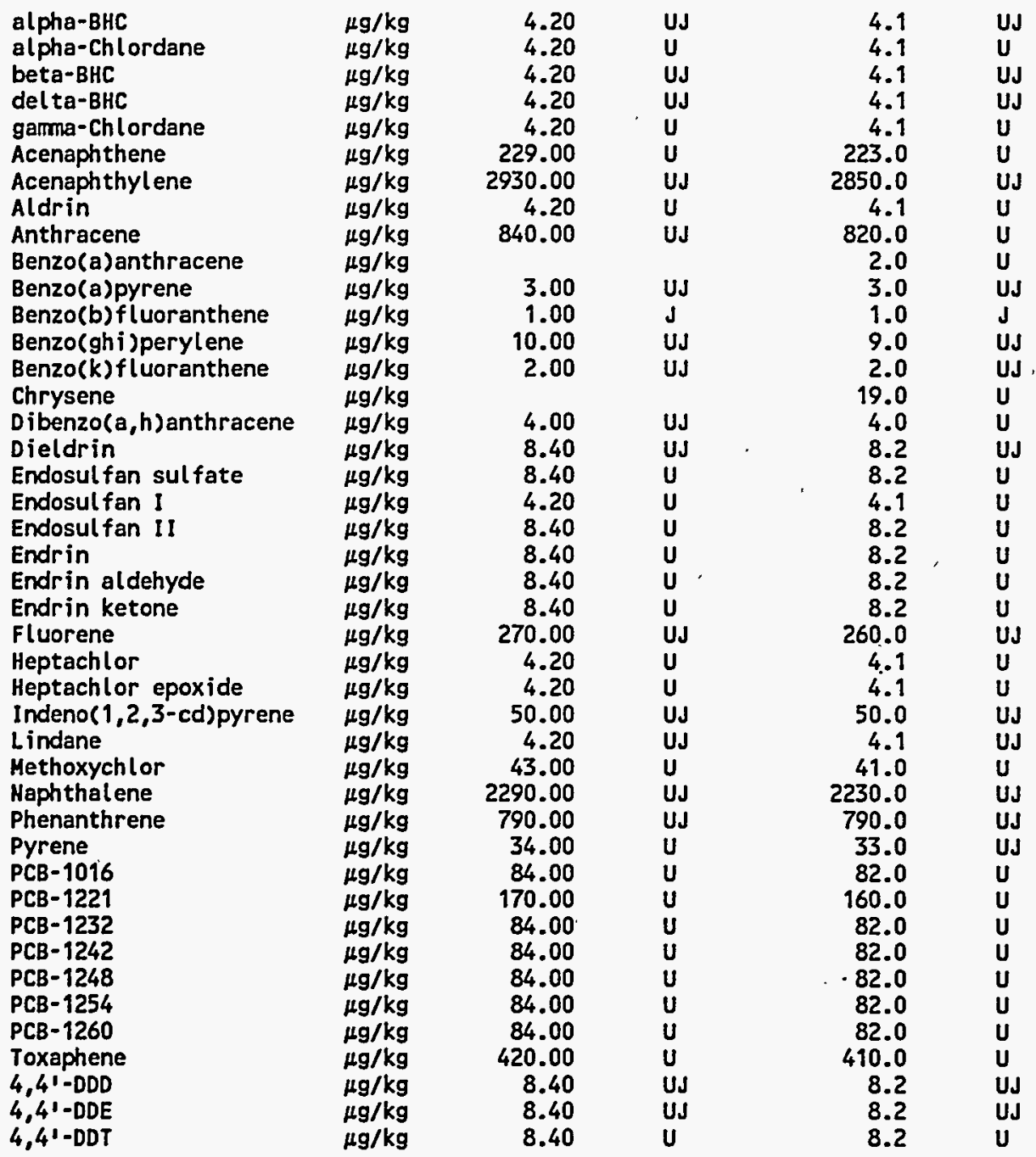

Location $=R O A ;$ Formation $=D I S M A L$ GAP; Site $=14 ; A=3148 ; A$ dup=' '; Phase $=1$

\begin{tabular}{|c|c|c|}
\hline $\begin{array}{l}\text { alpha-BHC } \\
\text { alpha-Chlordane } \\
\text { beta-BHC } \\
\text { del ta-BHC } \\
\text { gamma-Chlordane } \\
\text { Acenaphthene } \\
\text { Acenaphthylene } \\
\text { Aldrin } \\
\text { Anthracene } \\
\text { Benzo(a)pyrene } \\
\text { Benzo(b)f luoranthene } \\
\text { Benzo(ghi)perylene } \\
\text { Benzo(k)fluoranthene } \\
\text { Dibenzo(a, h)anthracene } \\
\text { Dieldrin } \\
\text { Endosulfan sul fate } \\
\text { Endosulfan I } \\
\text { Endosulfan II }\end{array}$ & $\begin{array}{l}\mu g / \mathrm{kg} \\
\mu g / \mathrm{kg} \\
\mu \mathrm{g} / \mathrm{kg} \\
\mu \mathrm{g} / \mathrm{kg} \\
\mu g / \mathrm{kg} \\
\mu g / \mathrm{kg} \\
\mu \mathrm{g} / \mathrm{kg} \\
\mu \mathrm{g} / \mathrm{kg} \\
\mu g / \mathrm{kg} \\
\mu \mathrm{g} / \mathrm{kg} \\
\mu g / \mathrm{kg} \\
\mu \mathrm{g} / \mathrm{kg} \\
\mu \mathrm{g} / \mathrm{kg} \\
\mu g / \mathrm{kg} \\
\mu g / \mathrm{kg} \\
\mu \mathrm{g} / \mathrm{kg} \\
\mu \mathrm{g} / \mathrm{kg} \\
\mu \mathrm{g} / \mathrm{kg}\end{array}$ & $\begin{array}{r}2.20 \\
2.20 \\
2.20 \\
2.20 \\
2.20 \\
240.00 \\
3070.00 \\
2.20 \\
880.00 \\
1.00 \\
2.00 \\
10.00 \\
2.00 \\
4.00 \\
4.40 \\
4.40 \\
2.20 \\
4.40\end{array}$ \\
\hline
\end{tabular}


Table C.1 (continued)

\begin{tabular}{|c|c|c|c|c|c|}
\hline Analysis & Units & $\begin{array}{l}\text { A horizon } \\
\text { Result }\end{array}$ & Qualifier & $\begin{array}{l}\text { A horizon } \\
\text { field dup }\end{array}$ & Qualifier \\
\hline $\begin{array}{l}\text { Endrin } \\
\text { Endrin aldehyde } \\
\text { Endrin ketone } \\
\text { Fluorene } \\
\text { Heptachlor } \\
\text { Heptachlor epoxide } \\
\text { Indeno(1,2,3-cd)pyrene } \\
\text { Lindane } \\
\text { Methoxychlor } \\
\text { Naphthalene } \\
\text { Phenanthrene } \\
\text { Pyrene } \\
\text { PCB-1016 } \\
\text { PCB-1221 } \\
\text { PCB-1232 } \\
\text { PCB-1242 } \\
\text { PCB-1248 } \\
\text { PCB-1254 } \\
\text { PCB-1260 } \\
\text { Toxaphene } \\
4,4^{\prime}-\text { DDD } \\
4,4^{\prime}-\text { DDE } \\
4,4^{\prime}-D D T\end{array}$ & $\begin{array}{l}\mu g / \mathrm{kg} \\
\mu g / \mathrm{kg} \\
\mu g / \mathrm{kg} \\
\mu g / \mathrm{kg} \\
\mu g / \mathrm{kg} \\
\mu g / \mathrm{kg} \\
\mu g / \mathrm{kg} \\
\mu g / \mathrm{kg} \\
\mu g / \mathrm{kg} \\
\mu g / \mathrm{kg} \\
\mu g / \mathrm{kg} \\
\mu g / \mathrm{kg} \\
\mu g / \mathrm{kg} \\
\mu g / \mathrm{kg} \\
\mu g / \mathrm{kg} \\
\mu g / \mathrm{kg} \\
\mu g / \mathrm{kg} \\
\mu g / \mathrm{kg} \\
\mu g / \mathrm{kg} \\
\mu g / \mathrm{kg} \\
\mu g / \mathrm{kg} \\
\mu g / \mathrm{kg} \\
\mu g / \mathrm{kg}\end{array}$ & $\begin{array}{r}4.40 \\
4.40 \\
4.40 \\
280.00 \\
2.20 \\
2.20 \\
60.00 \\
2.20 \\
22.00 \\
2400.00 \\
850.00 \\
36.00 \\
44.00 \\
89.00 \\
44.00 \\
44.00 \\
44.00 \\
44.00 \\
44.00 \\
220.00 \\
4.40 \\
4.40 \\
4.40\end{array}$ & $\begin{array}{l}\text { UJ } \\
\text { UJ } \\
\text { UJ } \\
\text { UJ } \\
\text { UJ } \\
\text { UJ } \\
\text { UJ } \\
\text { UJ } \\
\text { UJ } \\
\text { UJ } \\
U J \\
\text { UJ } \\
\text { UJ } \\
\text { UJ } \\
\text { UJ } \\
\text { UJ } \\
\text { UJ } \\
\text { UJ } \\
\text { UJ } \\
\text { UJ } \\
\text { UJ } \\
\text { UJ } \\
\text { UJ }\end{array}$ & & \\
\hline
\end{tabular}

Location $=$ ROA; Formation $=$ DISMAL GAP; Site $=17 ; A=3018 ; A$ dup=' ; Phase=1

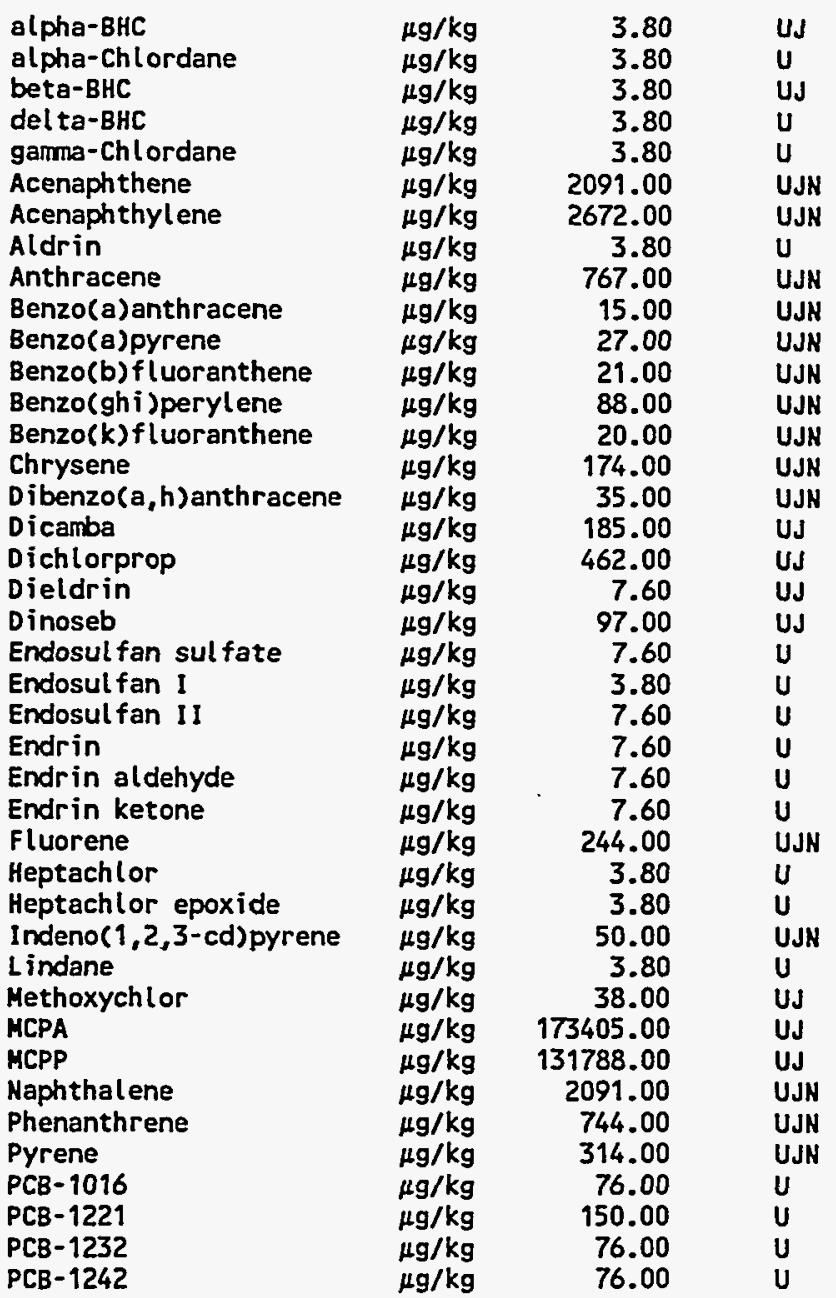




\section{C-77}

Table C.1 (continued)

\begin{tabular}{|c|c|c|c|c|c|}
\hline Analysis & Units & $\begin{array}{c}\text { A horizon } \\
\text { Result }\end{array}$ & Qualifier & $\begin{array}{l}\text { A horizon } \\
\text { field dup }\end{array}$ & Qualifier \\
\hline $\begin{array}{l}\text { PCB- } 1248 \\
\text { PCB- } 1254 \\
\text { PCB- } 1260 \\
\text { silvex } \\
\text { Toxaphene } \\
2,4-D \\
2,4-D 8 \\
2,4,5-T \\
4,4^{\prime}-D D D \\
4,4^{\prime}-D D E \\
4,4^{\prime}-D D T\end{array}$ & $\begin{array}{l}\mu g / \mathbf{k g} \\
\mu g / \mathbf{k g} \\
\mu g / \mathbf{k g} \\
\mu g / \mathbf{k g} \\
\mu g / \mathbf{k g} \\
\mu \mathrm{g} / \mathrm{kg} \\
\mu \mathrm{g} / \mathrm{kg} \\
\mu g / \mathbf{k g} \\
\mu \mathrm{g} / \mathrm{kg} \\
\mu g / \mathbf{k g} \\
\mu g / \mathbf{k g}\end{array}$ & $\begin{array}{r}76.00 \\
76.00 \\
76.00 \\
116.00 \\
380.00 \\
832.00 \\
624.00 \\
139.00 \\
7.60 \\
7.60 \\
7.60\end{array}$ & $\begin{array}{l}\mathbf{U} \\
\mathbf{U} \\
\mathbf{U} \\
\mathbf{U} \mathbf{J} \\
\mathbf{U} \\
\mathbf{U J} \\
\mathbf{U J} \\
\mathbf{U J} \\
\mathbf{U J} \\
\mathbf{U J} \\
\mathbf{U J}\end{array}$ & & \\
\hline
\end{tabular}

Location=ROA; Formation=DISMAL GAP; Site =19; $A=3032 ; A$ dup=' '; Phase $=1$

\begin{tabular}{|c|c|c|}
\hline 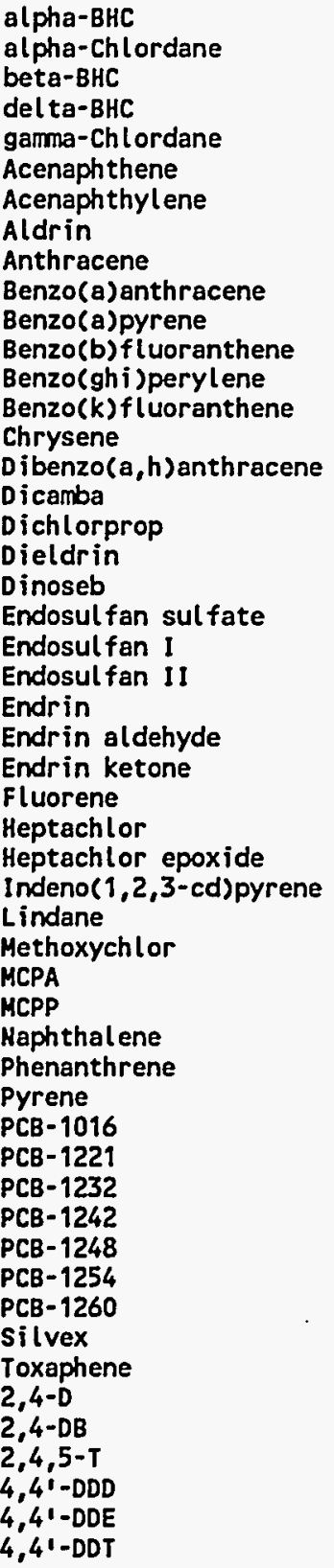 & $\begin{array}{l}\mu g / k g \\
\mu g / k g \\
\mu g / k g \\
\mu g / k g \\
\mu g / k g \\
\mu g / k g \\
\mu g / k g \\
\mu g / k g \\
\mu g / k g \\
\mu g / k g \\
\mu g / k g \\
\mu g / k g \\
\mu g / k g \\
\mu g / k g \\
\mu g / k g \\
\mu g / k g \\
\mu g / k g \\
\mu g / k g \\
\mu g / k g \\
\mu g / k g \\
\mu g / k g \\
\mu g / k g \\
\mu g / k g \\
\mu g / k g \\
\mu g / k g \\
\mu g / k g \\
\mu g / k g \\
\mu g / k g \\
\mu g / k g \\
\mu g / k g \\
\mu g / k g \\
\mu g / k g \\
\mu g / k g \\
\mu g / k g \\
\mu g / k g \\
\mu g / k g \\
\mu g / k g \\
\mu g / k g \\
\mu g / k g \\
\mu g / k g \\
\mu g / k g \\
\mu g / k g \\
\mu g / k g \\
\mu g / k g \\
\mu g / k g \\
\mu g / k g \\
\mu g / k g \\
\mu g / k g \\
\mu g / k g \\
\mu g / k g \\
\mu g / k g \\
\mu g / k g \\
\text { M }\end{array}$ & $\begin{array}{r}4.40 \\
4.30 \\
4.30 \\
4.30 \\
4.30 \\
233.00 \\
298.00 \\
4.30 \\
85.00 \\
2.00 \\
3.00 \\
2.00 \\
10.00 \\
2.00 \\
19.00 \\
4.00 \\
204.00 \\
509.00 \\
8.60 \\
107.00 \\
8.60 \\
4.30 \\
8.60 \\
8.60 \\
8.60 \\
8.60 \\
27.00 \\
4.30 \\
4.30 \\
6.00 \\
4.30 \\
43.00 \\
190824.00 \\
145027.00 \\
233.00 \\
83.00 \\
35.00 \\
86.00 \\
170.00 \\
86.00 \\
86.00 \\
86.00 \\
86.00 \\
86.00 \\
127.00 \\
430.00 \\
916.00 \\
687.00 \\
153.00 \\
8.60 \\
8.60 \\
8.60\end{array}$ \\
\hline
\end{tabular}


Table C.1 (continued)

\begin{tabular}{|c|c|c|c|c|c|}
\hline Analysis & Units & $\begin{array}{c}\text { A horizon } \\
\text { Result }\end{array}$ & Qualifier & $\begin{array}{l}\text { A horizon } \\
\text { field dup }\end{array}$ & Qualifier \\
\hline
\end{tabular}

Location $=R O A ;$ Formation $=D I S M A L$ GAP; Site $=20 ; A=3046 ; A$ dup =' '; Phase $=1$

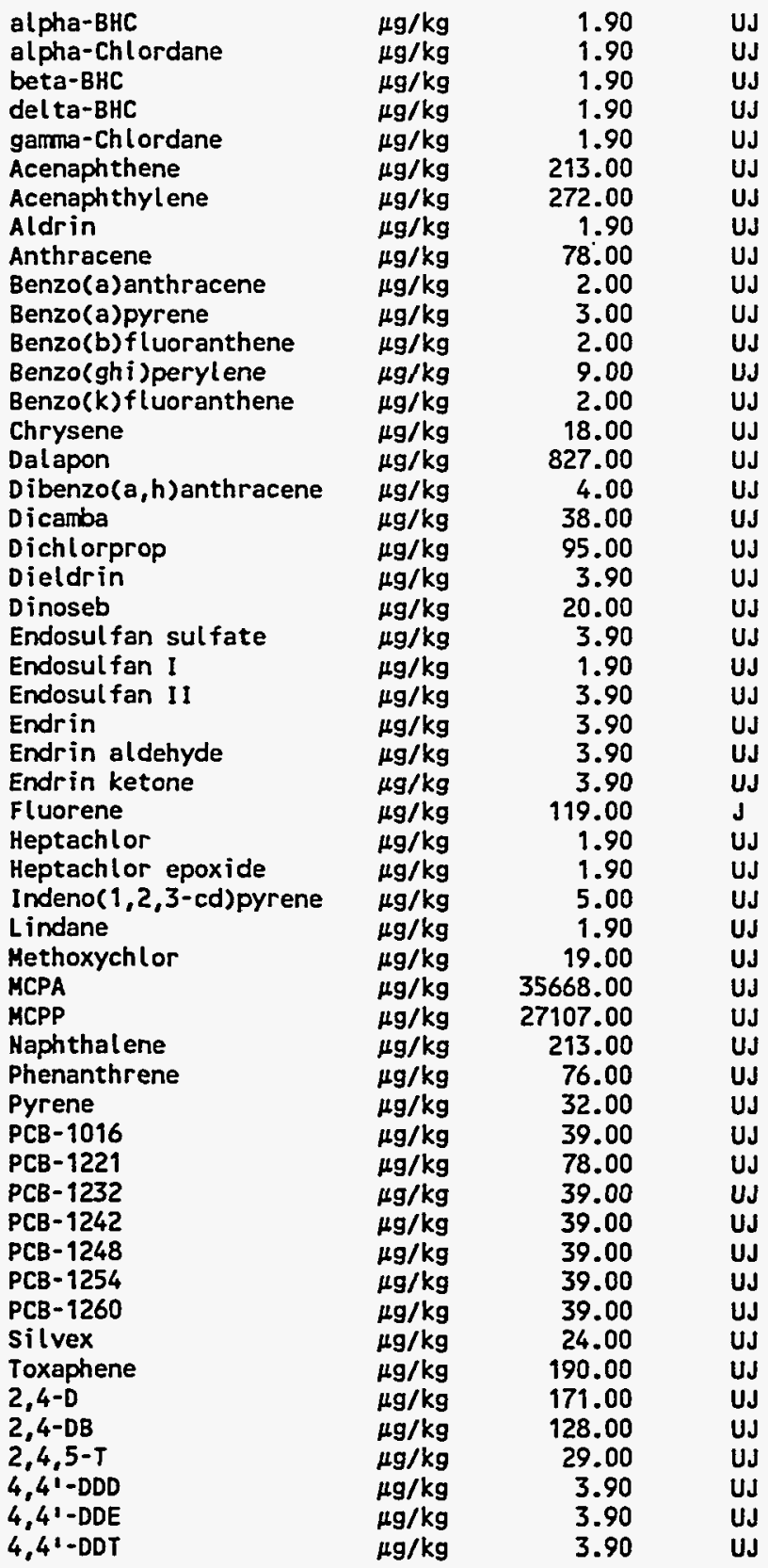

Location=ROA; Formation $=D I S M A L$ GAP; Site $=21 ; A=3113 ; A$ dup=' '; Phase $=1$

$\begin{array}{lrrr}\text { alpha-BHC } & \mu g / \mathrm{kg} & 2.10 & \text { UJ } \\ \text { alpha-Chlordane } & \mu g / \mathrm{kg} & 2.10 & \text { UJ } \\ \text { beta-BHC } & \mu g / \mathrm{kg} & 2.10 & \text { UJ } \\ \text { delta-BHC } & \mu g / \mathrm{kg} & 2.10 & \text { UJ } \\ \text { gamma-Chlordane } & \mu g / \mathrm{kg} & 2.10 & \text { UJ } \\ \text { Acenaphthene } & \mu \mathrm{g} / \mathrm{kg} & 229.00 & \text { UJ } \\ \text { Acenaphthylene } & \mu g / \mathrm{kg} & 2920.00 & \text { UJ } \\ \text { Aldrin } & \mu g / \mathrm{kg} & 2.10 & \text { UJ }\end{array}$




\section{C-79}

Table C.1 (continued)

\begin{tabular}{|c|c|c|c|c|c|c|}
\hline Analysis & Units & $\begin{array}{c}\text { A horizon } \\
\text { Result }\end{array}$ & Qualifier & $\begin{array}{l}\text { A horizon } \\
\text { field dup }\end{array}$ & Qualifier & \\
\hline $\begin{array}{l}\text { Anthracene } \\
\text { Benzo(a)anthracene } \\
\text { Benzo(a)pyrene } \\
\text { Benzo(b)fluoranthene } \\
\text { Benzo(ghi)perylene } \\
\text { Benzo(k)fluoranthene } \\
\text { Chrysene } \\
\text { Dibenzo(a, h)anthracene } \\
\text { Dieldrin } \\
\text { Endosulfan sulfate } \\
\text { Endosulfan I } \\
\text { Endosulfan II } \\
\text { Endrin } \\
\text { Endrin aldehyde } \\
\text { Endrin ketone } \\
\text { Fluorene } \\
\text { Heptachlor } \\
\text { Heptachlor epoxide } \\
\text { Indeno(1,2,3-cd)pyrene } \\
\text { Lindane } \\
\text { Methoxychlor } \\
\text { Maphthalene } \\
\text { Phenanthrene } \\
\text { Pyrene } \\
\text { PCB-1016 } \\
\text { PCB-1221 } \\
\text { PCB-1232 } \\
\text { PCB-1242 } \\
\text { PCB-1248 } \\
\text { PCB-1254 } \\
\text { PCB-1260 } \\
\text { Toxaphene } \\
4,41-D D D \\
4,41-D D E \\
4,41-D D T\end{array}$ & 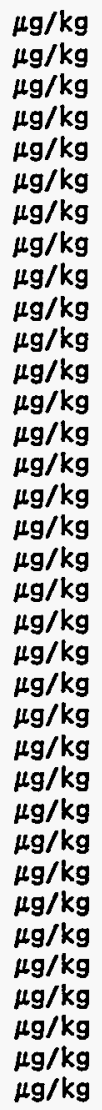 & $\begin{array}{r}840.00 \\
2.00 \\
3.00 \\
2.00 \\
10.00 \\
2.00 \\
19.00 \\
4.00 \\
4.10 \\
4.10 \\
2.10 \\
4.10 \\
4.10 \\
4.10 \\
4.10 \\
270.00 \\
2.10 \\
2.10 \\
50.00 \\
2.10 \\
21.00 \\
2290.00 \\
810.00 \\
34.00 \\
41.00 \\
82.00 \\
41.00 \\
41.00 \\
41.00 \\
41.00 \\
41.00 \\
210.00 \\
4.10 \\
4.10 \\
4.10\end{array}$ & $\begin{array}{l}\text { UJ } \\
\text { UJ } \\
\text { UJ } \\
\text { UJ } \\
\text { UJ } \\
\text { UJ } \\
\text { UJ } \\
\text { UJ } \\
\text { UJ } \\
\text { UJ } \\
\text { UJ } \\
\text { UJ } \\
\text { UJ } \\
\text { UJ } \\
\text { UJ } \\
\text { UJ } \\
\text { UJ } \\
\text { UJ } \\
\text { UJ } \\
\text { UJ } \\
\text { UJ } \\
\text { UJ } \\
\text { UJ } \\
\text { UJ } \\
\text { UJ } \\
\text { UJ } \\
\text { UJ } \\
\text { UJ } \\
\text { UJ } \\
\text { UJ } \\
\text { UJ } \\
\text { UJ } \\
\text { UJ } \\
\text { UJ } \\
\text { UJ }\end{array}$ & " & . & + \\
\hline
\end{tabular}

Location=ROA; Formation =DISMAL GAP; Site=22; $A=3058 ; A$ dup=' '; Phase=1

\begin{tabular}{|c|c|c|}
\hline $\begin{array}{l}\text { alpha-BHC } \\
\text { alpha-Chlordane } \\
\text { beta-BHC } \\
\text { delta-BHC } \\
\text { gamma-Chlordane } \\
\text { Acenaphthene } \\
\text { Acenaphthylene } \\
\text { Aldrin } \\
\text { Anthracene } \\
\text { Benzo(a)anthracene } \\
\text { Benzo(a)pyrene } \\
\text { Benzo(b)fluoranthene } \\
\text { Benzo(ghi)perylene } \\
\text { Benzo(k)fluoranthene } \\
\text { Chrysene } \\
\text { Dalapon } \\
\text { Dibenzo(a,h)anthracene } \\
\text { Dicamba } \\
\text { Dichlorprop } \\
\text { Dieldrin } \\
\text { Dinoseb } \\
\text { Endosulfan sulfate } \\
\text { Endosulfan I } \\
\text { Endosulfan II } \\
\text { Endrin } \\
\text { Endrin aldehyde } \\
\text { Endrin ketone } \\
\text { Fluorene } \\
\text { Heptachlor }\end{array}$ & 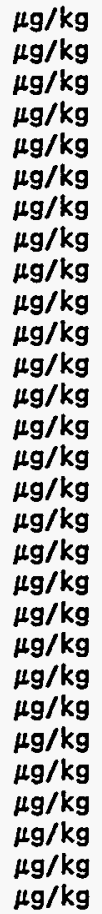 & $\begin{array}{r}3.90 \\
3.90 \\
3.90 \\
3.90 \\
3.90 \\
213.00 \\
272.00 \\
3.90 \\
78.00 \\
2.00 \\
3.00 \\
2.00 \\
9.00 \\
2.00 \\
18.00 \\
806.00 \\
4.00 \\
37.00 \\
93.00 \\
7.70 \\
19.00 \\
7.70 \\
3.90 \\
7.70 \\
7.70 \\
7.70 \\
7.70 \\
25.00 \\
3.90\end{array}$ \\
\hline
\end{tabular}


Table C.1 (continued)

\begin{tabular}{|c|c|c|c|c|c|}
\hline Analysis & Units & $\begin{array}{c}\text { A horizon } \\
\text { Result }\end{array}$ & Qualifier & $\begin{array}{l}\text { A horizon } \\
\text { field dup }\end{array}$ & Qualifier \\
\hline $\begin{array}{l}\text { Heptachlor epoxide } \\
\text { Indeno(1,2,3-cd)pyrene } \\
\text { Lindane } \\
\text { Methoxychlor } \\
\text { MCPA } \\
\text { MCPP } \\
\text { Haphthalene } \\
\text { Phenanthrene } \\
\text { Pyrene } \\
\text { PCB-1016 } \\
\text { PCB-1221 } \\
\text { PCB-1232 } \\
\text { PCB-1242 } \\
\text { PCB-1248 } \\
\text { PCB-1254 } \\
\text { PCB-1260 } \\
\text { Silvex } \\
\text { TOXaphene } \\
2,4-D \\
2,4-D B \\
2,4,5-T \\
4,4^{\prime}-D D D \\
4,4 '-D D E \\
4,4 '-D D T\end{array}$ & 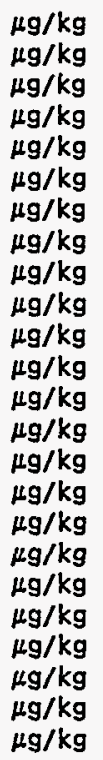 & $\begin{array}{r}3.90 \\
5.00 \\
3.90 \\
40.00 \\
34722.00 \\
26389.00 \\
496.00 \\
76.00 \\
32.00 \\
77.00 \\
150.00 \\
77.00 \\
77.00 \\
77.00 \\
77.00 \\
77.00 \\
23.00 \\
390.00 \\
167.00 \\
125.00 \\
28.00 \\
7.70 \\
7.70 \\
7.70\end{array}$ & $\begin{array}{l}\text { UJ } \\
\text { US } \\
\text { UJ } \\
\text { UJ } \\
U \\
U \\
J \\
\text { UJ } \\
\text { UJ } \\
\text { UJ } \\
\text { UJ } \\
\text { UJ } \\
\text { UJ } \\
\text { UJ } \\
\text { UJ } \\
\text { UJ } \\
\text { UJ } \\
\text { US } \\
\text { U } \\
\text { U } \\
\text { US } \\
\text { UJ } \\
\text { UJ } \\
\text { UJ }\end{array}$ & & \\
\hline
\end{tabular}

Location=ROA; Formation $=$ DISMAL GAP; Site $=3 ; A=3099 ; A$ dup=' '; Phase $=1$

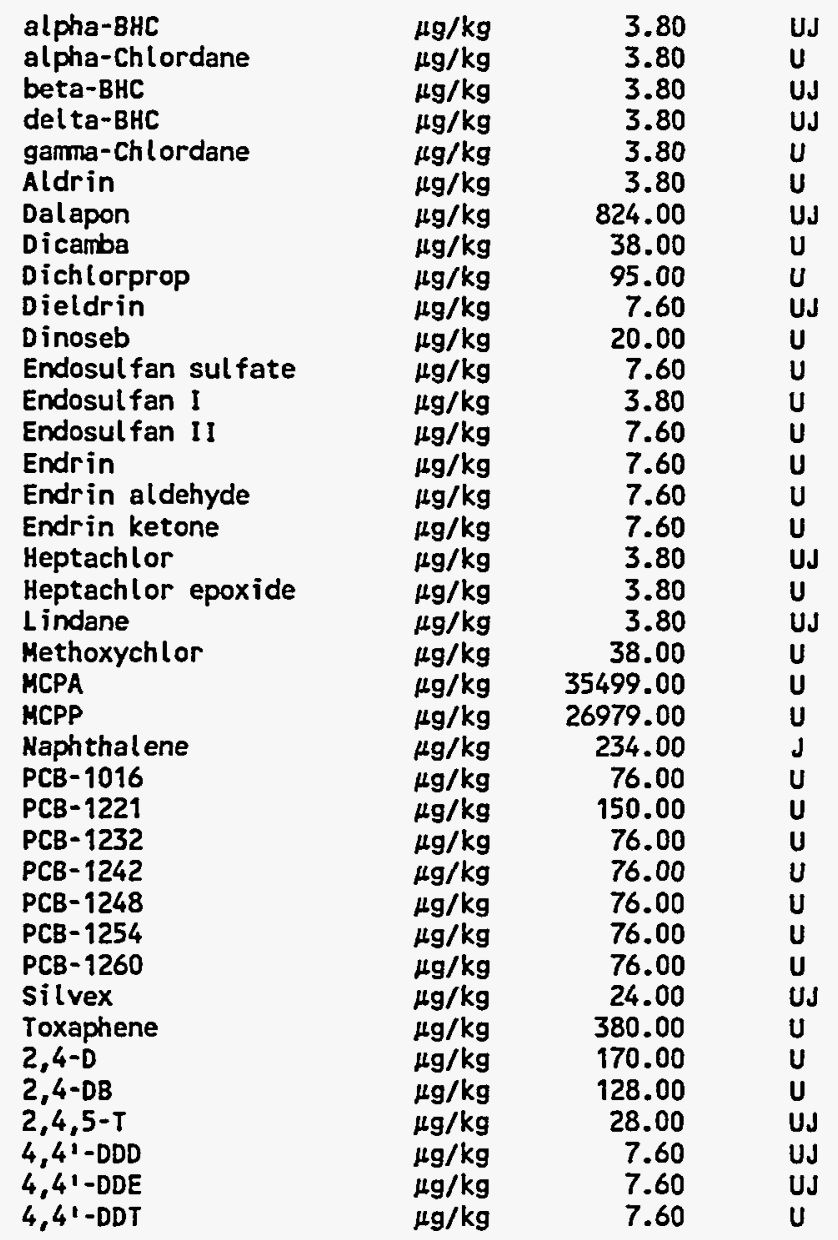


C-81

Table C.1 (continued)

\begin{tabular}{|c|c|c|c|c|c|}
\hline Analysis & Units & $\begin{array}{c}\text { A horizon } \\
\text { Result }\end{array}$ & Qualifier & $\begin{array}{l}\text { A horizon } \\
\text { field dup }\end{array}$ & Qualifier \\
\hline
\end{tabular}

Location $=$ ROA; Formation $=D I S M A L$ GAP; Site $=7 ; A=3085 ; A$ dup =' '; Phase $=1$

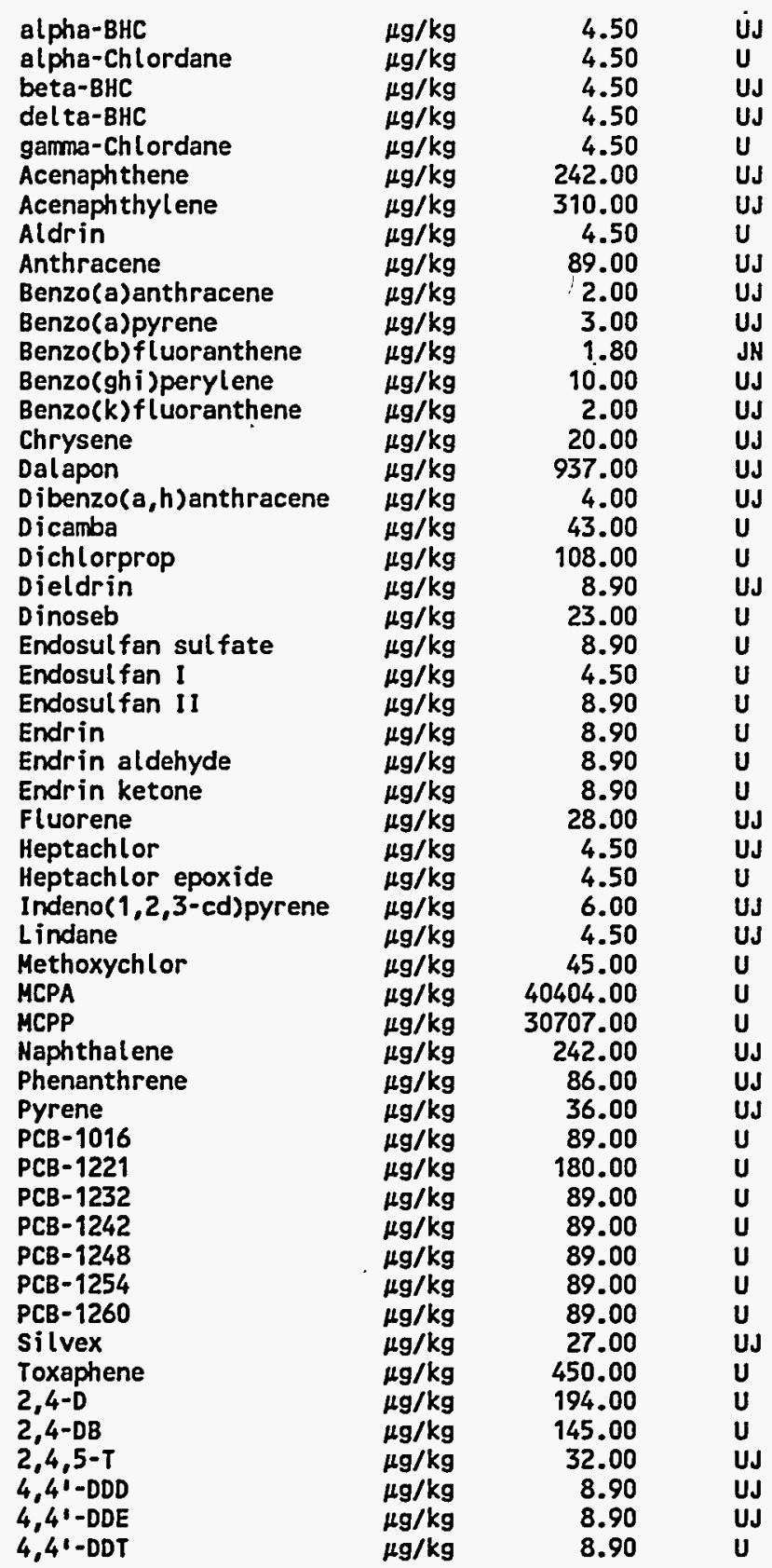

Location $=$ ROA; Formation $=$ DISMAL GAP; Site $=8 ; A=3072 ; A$ dup=' '; Phase $=1$

\begin{tabular}{|c|c|}
\hline $\begin{array}{l}\text { alpha-BHC } \\
\text { alpha-Chlordane } \\
\text { beta-BHC } \\
\text { del ta-BHC } \\
\text { gamma-Chlordane } \\
\text { Acenaphthene } \\
\text { Acenaphthylene } \\
\text { Aldrin }\end{array}$ & $\begin{array}{l}\mu g / \mathrm{kg} \\
\mu g / \mathrm{kg} \\
\mu g / \mathrm{kg} \\
\mu g / \mathrm{kg} \\
\mu g / \mathrm{kg} \\
\mu g / \mathrm{kg} \\
\mu g / \mathrm{kg} \\
\mu \mathrm{g} / \mathrm{kg}\end{array}$ \\
\hline
\end{tabular}




\section{C-82}

Table C.1 (continued)

\begin{tabular}{|c|c|c|c|c|c|}
\hline Analysis & Units & $\begin{array}{c}\text { A horizon } \\
\text { Result }\end{array}$ & Qualifier & $\begin{array}{l}\text { A horizon } \\
\text { field dup }\end{array}$ & Qualifier \\
\hline 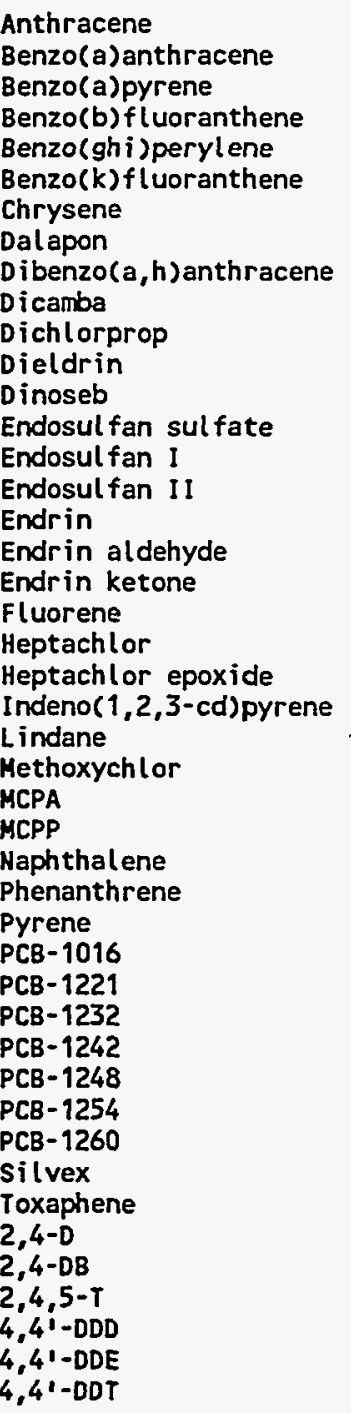 & 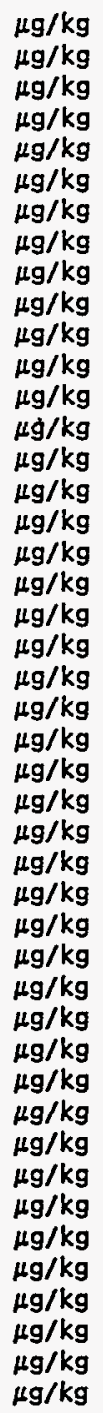 & $\begin{array}{r}84.00 \\
2.00 \\
3.00 \\
2.00 \\
10.00 \\
2.00 \\
19.00 \\
881.00 \\
4.00 \\
41.00 \\
101.00 \\
8.50 \\
21.00 \\
8.50 \\
4.40 \\
8.50 \\
8.50 \\
8.50 \\
8.50 \\
27.00 \\
4.40 \\
4.40 \\
5.00 \\
4.40 \\
44.00 \\
37989.00 \\
28872.00 \\
228.00 \\
203.00 \\
34.00 \\
85.00 \\
172.00 \\
85.00 \\
85.00 \\
85.00 \\
85.00 \\
85.00 \\
25.00 \\
436.00 \\
182.00 \\
137.00 \\
30.00 \\
8.50 \\
8.50 \\
14.00\end{array}$ & $\begin{array}{l}\text { UJ } \\
U J \\
U J \\
U J \\
U J \\
U J \\
U J \\
U J \\
U J \\
U J \\
U J \\
U J \\
U J \\
U \\
U J \\
U \\
U \\
U \\
U \\
U J \\
U J\end{array}$ & & \\
\hline
\end{tabular}

Location $=$ ROA; Formation =DISMAL GAP; Site=9; $A=3003 ; A$ dup=' '; Phase=1

$\begin{array}{lrrr}\text { alpha-BHC } & \mu \mathrm{g} / \mathrm{kg} & 4.00 & \text { UJ } \\ \text { alpha-Chlordane } & \mu \mathrm{g} / \mathrm{kg} & 4.00 & \mathrm{U} \\ \text { beta-BHC } & \mu \mathrm{g} / \mathrm{kg} & 4.00 & \mathrm{U} \\ \text { del ta-BHC } & \mu \mathrm{g} / \mathrm{kg} & 4.00 & \mathrm{U} \\ \text { gamma-Chlordane } & \mu \mathrm{g} / \mathrm{kg} & 4.00 & \mathrm{U} \\ \text { Acenaphthene } & \mu \mathrm{g} / \mathrm{kg} & 11258.00 & \text { UJN } \\ \text { Acenaphthylene } & \mu \mathrm{g} / \mathrm{kg} & 14386.00 & \text { UJN } \\ \text { Aldrin } & \mu \mathrm{g} / \mathrm{kg} & 4.00 & \mathrm{U} \\ \text { Anthracene } & \mu \mathrm{g} / \mathrm{kg} & 4128.00 & \text { UJN } \\ \text { Benzo(a)anthracene } & \mu \mathrm{g} / \mathrm{kg} & 81.00 & \text { UJN } \\ \text { Benzo(a)pyrene } & \mu \mathrm{g} / \mathrm{kg} & 144.00 & \text { UJN } \\ \text { Benzo(b)fluoranthene } & \mu \mathrm{g} / \mathrm{kg} & 113.00 & \text { UJN } \\ \text { Benzo(ghi)perylene } & \mu \mathrm{g} / \mathrm{kg} & 475.00 & \text { UJN } \\ \text { Benzo(k)fluoranthene } & \mu \mathrm{g} / \mathrm{kg} & 106.00 & \text { UJN } \\ \text { Chrysene } & \mu \mathrm{g} / \mathrm{kg} & 938.00 & \text { UJN } \\ \text { Dibenzo(a,h)anthracene } & \mu \mathrm{g} / \mathrm{kg} & 188.00 & \text { UJN } \\ \text { Dicamba } & \mu \mathrm{g} / \mathrm{kg} & 400.00 & \text { UJ } \\ \text { Dichlorprop } & \mu \mathrm{g} / \mathrm{kg} & 1001.00 & \text { UJ } \\ \text { Dieldrin } & \mu \mathrm{g} / \mathrm{kg} & 8.10 & \text { UJ }\end{array}$




\section{C-83}

Table C.1 (continued)

\begin{tabular}{|c|c|c|c|c|c|}
\hline Analysis & Units & $\begin{array}{c}\text { A horizon } \\
\text { Result }\end{array}$ & Qualifier & $\begin{array}{l}\text { A horizon } \\
\text { field dup }\end{array}$ & Qualifier \\
\hline $\begin{array}{l}\text { Dinoseb } \\
\text { Endosulfan sulfate } \\
\text { Endosulfan 1 } \\
\text { Endosulfan } 11 \\
\text { Endrin } \\
\text { Endin aldehyde } \\
\text { Endrin ketone } \\
\text { Fluoranthene } \\
\text { Fluorene } \\
\text { Heptachlor } \\
\text { Heptachlor epoxide } \\
\text { Indeno(1,2,3-cd)pyrene } \\
\text { Lindane } \\
\text { Methoxychlor } \\
\text { MCPA } \\
\text { MCPP } \\
\text { Naphthalene } \\
\text { Phenanthrene } \\
\text { Pyrene } \\
\text { PCB-1016 } \\
\text { PCB-1221 } \\
\text { PCB-1232 } \\
\text { PCB-1242 } \\
\text { PCB-1248 } \\
\text { PCB-1254 } \\
\text { PCB-1260 } \\
\text { Silvex } \\
\text { Toxaphene } \\
2,4-D \\
2,4-D B \\
2,4,5-T \\
4,4 ;-D D D \\
4,4 \text {-DDE } \\
4,4 \text {-DDT }\end{array}$ & $\begin{array}{l}\mu g / k g \\
\mu g / k g \\
\mu g / k g \\
\mu g / k g \\
\mu g / k g \\
\mu g / k g \\
\mu g / k g \\
\mu g / k g \\
\mu g / k g \\
\mu g / k g \\
\mu g / k g \\
\mu g / k g \\
\mu g / k g \\
\mu g / k g \\
\mu g / k g \\
\mu g / k g \\
\mu g / k g \\
\mu g / k g \\
\mu g / k g \\
\mu g / k g \\
\mu g / k g \\
\mu g / k g \\
\mu g / k g \\
\mu g / k g \\
\mu g / k g \\
\mu g / k g \\
\mu g / k g \\
\mu g / k g \\
\mu g / / g \\
\mu g / k g \\
\mu g / k g \\
\mu g / k g \\
\mu g / k g \\
\mu g / k g\end{array}$ & $\begin{array}{r}210.00 \\
8.10 \\
4.00 \\
8.10 \\
8.10 \\
8.10 \\
8.10 \\
338.00 \\
1313.00 \\
4.00 \\
4.00 \\
269.00 \\
4.00 \\
40.00 \\
375281.00 \\
285214.00 \\
11258.00 \\
4003.00 \\
1689.00 \\
81.00 \\
163.00 \\
81.00 \\
81.00 \\
81.00 \\
81.00 \\
81.00 \\
250.00 \\
400.00 \\
1801.00 \\
1351.00 \\
300.00 \\
8.10 \\
8.10 \\
8.10\end{array}$ & $\begin{array}{l}\text { UJ } \\
U \\
U \\
U \\
U \\
U J \\
U \\
U J N \\
U J N \\
U \\
U \\
U J N \\
U \\
U \\
U J \\
U J \\
U J N \\
U J N \\
U J N \\
U \\
U \\
U \\
U \\
U \\
U \\
U \\
U J \\
U \\
U J \\
U J \\
U J \\
U \\
U J \\
U\end{array}$ & & - \\
\hline
\end{tabular}


Appendix D

INORGANIC ANALYSIS DATA 
Table D.1. Inorganic analysis results for composite soil samples

\begin{tabular}{|c|c|c|c|c|c|c|c|c|c|}
\hline Analysis & Units & $\begin{array}{l}\text { A horizon } \\
\text { result }\end{array}$ & Qualifier & $\begin{array}{l}\text { B horizon } \\
\text { result }\end{array}$ & Qualifier & $\begin{array}{l}\text { C horizon } \\
\text { result }\end{array}$ & Qualifier & $\begin{array}{l}\text { A lab split } \\
\text { result }\end{array}$ & Qualifier \\
\hline
\end{tabular}

Location $=A N D$; Formation $=$ COPPER RIDGE; Site =31,32,36; Sample type $=$ Regular; $A=7057 ; B=7060 ; C=7063 ; A$ lab split =' '; Phase $=2$

$\begin{array}{lrrrrrrr}\text { Aluminum } & \mathrm{mg} / \mathrm{kg} & 10600.00 & & 16300.00 & & 14300.00 & \\ \text { Antimony } & \mathrm{mg} / \mathrm{kg} & 0.48 & \mathrm{UJ} & 0.43 & \mathrm{UJ} & 0.41 & \mathrm{UJ} \\ \text { Arsenic } & \mathrm{mg} / \mathrm{kg} & 9.40 & & 20.20 & & 23.30 & \\ \text { Barium } & \mathrm{mg} / \mathrm{kg} & 59.50 & & 27.40 & \mathrm{~B} & 14.50 & \mathrm{~B} \\ \text { Beryllium } & \mathrm{mg} / \mathrm{kg} & 0.61 & \mathrm{~B} & 0.55 & \mathrm{~B} & 0.48 & \mathrm{~B} \\ \text { Boron } & \mathrm{mg} / \mathrm{kg} & 4.80 & \mathrm{U} & 4.80 & \mathrm{U} & 4.80 & \mathrm{U} \\ \text { Cadnium } & \mathrm{mg} / \mathrm{kg} & 0.24 & \mathrm{U} & 0.22 & \mathrm{U} & 0.21 & \mathrm{U} \\ \text { Calcium } & \mathrm{mg} / \mathrm{kg} & 423.00 & \mathrm{~B} & 197.00 & \mathrm{~B} & 180.00 & \mathrm{~B} \\ \text { Chromium } & \mathrm{mg} / \mathrm{kg} & 19.70 & & 25.20 & & 23.70 & \\ \text { Cobalt } & \mathrm{mg} / \mathrm{kg} & 11.40 & \mathrm{~B} & 3.60 & \mathrm{~B} & 2.50 & \mathrm{~B} \\ \text { Copper } & \mathrm{mg} / \mathrm{kg} & 6.60 & & 21.80 & & 24.90 & \\ \text { Cyanide } & \mathrm{mg} / \mathrm{kg} & 1.20 & \mathrm{UJ} & 1.00 & \mathrm{UJ} & 1.00 & \mathrm{UJ} \\ \text { Iron } & \mathrm{mg} / \mathrm{kg} & 20000.00 & & 26400.00 & & 26400.00 & \\ \text { Lead } & \mathrm{mg} / \mathrm{kg} & 21.00 & & 8.80 & & 10.60 & \\ \text { Lithium } & \mathrm{mg} / \mathrm{kg} & 4.80 & \mathrm{~B} & 7.80 & \mathrm{~B} & 8.80 & \mathrm{~B} \\ \text { Magnesium } & \mathrm{mg} / \mathrm{kg} & 463.00 & \mathrm{~B} & 833.00 & \mathrm{~B} & 803.00 & \mathrm{~B} \\ \text { Manganese } & \mathrm{mg} / \mathrm{kg} & 1800.00 & \mathrm{~J} & 77.70 & \mathrm{~J} & 66.30 & \mathrm{~J} \\ \text { Mercury } & \mathrm{mg} / \mathrm{kg} & 0.12 & & 0.11 & & 0.15 & \\ \text { Holybdenum } & \mathrm{mg} / \mathrm{kg} & 2.60 & \mathrm{U} & 2.60 & \mathrm{U} & 3.00 & \mathrm{~B} \\ \text { Nickel } & \mathrm{mg} / \mathrm{kg} & 4.90 & \mathrm{U} & 11.80 & & 9.70 & \\ \text { Potassium } & \mathrm{mg} / \mathrm{kg} & 367.00 & \mathrm{~B} & 1030.00 & \mathrm{~B} & 1240.00 & \\ \text { Selenium } & \mathrm{mg} / \mathrm{kg} & 0.77 & \mathrm{~B} & 0.45 & \mathrm{~B} & 0.54 & \mathrm{~B} \\ \text { Silicon } & \mathrm{mg} / \mathrm{kg} & 368.00 & \mathrm{~J} & 481.00 & \mathrm{~J} & 555.00 & \mathrm{~J} \\ \text { Silver } & \mathrm{mg} / \mathrm{kg} & 0.96 & \mathrm{U} & 0.87 & \mathrm{U} & 0.83 & \mathrm{U} \\ \text { Sodium } & \mathrm{mg} / \mathrm{kg} & 343.00 & \mathrm{~B} & 350.00 & \mathrm{~B} & 326.00 & \mathrm{~B} \\ \text { Strontium } & \mathrm{mg} / \mathrm{kg} & 2.90 & \mathrm{~J} & 1.40 & \mathrm{~J} & 1.10 & \mathrm{~J} \\ \text { Sulfate } & \mathrm{mg} / \mathrm{kg} & 78.00 & & 52.00 & & 43.00 & \\ \text { Thallium } & \mathrm{mg} / \mathrm{kg} & 0.73 & \mathrm{U} & 0.05 & \mathrm{U} & 0.62 & \mathrm{U} \\ \text { Vanadium } & \mathrm{mg} / \mathrm{kg} & 44.20 & \mathrm{~J} & 48.80 & \mathrm{~J} & 47.90 & \mathrm{~J} \\ \text { Zinc } & \mathrm{mg} / \mathrm{kg} & 38.30 & \mathrm{~J} & 83.40 & \mathrm{~J} & 67.70 & \mathrm{~J} \\ & & & & & & \end{array}$

Location=AND; Formation=COPPER RIDGE; Site=33,34,35; Sample type $=$ Regular; $A=7046 ; B=7051 ; C=7054 ; A$ lab split=7047; Phase =2

\begin{tabular}{|c|c|c|c|c|c|c|c|c|}
\hline $\begin{array}{l}\text { Aluminum } \\
\text { Antimony } \\
\text { Arsenic } \\
\text { Barium } \\
\text { Beryllium } \\
\text { Boron } \\
\text { Cadmium } \\
\text { Calcium } \\
\text { Chromium } \\
\text { Cobalt } \\
\text { Copper } \\
\text { Cyanide } \\
\text { Iron } \\
\text { Lead } \\
\text { Lithium } \\
\text { Magnesium } \\
\text { Hanganese } \\
\text { Mercury } \\
\text { Molybdenum } \\
\text { Nickel } \\
\text { Potassium } \\
\text { Selenium } \\
\text { Silicon } \\
\text { silver } \\
\text { Sodium } \\
\text { strontium } \\
\text { sulfate } \\
\text { Thall ium } \\
\text { Vanadium } \\
\text { 2inc }\end{array}$ & $\begin{array}{l}\mathrm{mg} / \mathrm{kg} \\
\mathrm{mg} / \mathrm{kg} \\
\mathrm{mg} / \mathrm{kg} \\
\mathrm{mg} / \mathrm{kg} \\
\mathrm{mg} / \mathrm{kg} \\
\mathrm{mg} / \mathrm{kg} \\
\mathrm{mg} / \mathrm{kg} \\
\mathrm{mg} / \mathrm{kg} \\
\mathrm{mg} / \mathrm{kg} \\
\mathrm{mg} / \mathrm{kg} \\
\mathrm{mg} / \mathrm{kg} \\
\mathrm{mg} / \mathrm{kg} \\
\mathrm{mg} / \mathrm{kg} \\
\mathrm{mg} / \mathrm{kg} \\
\mathrm{mg} / \mathrm{kg} \\
\mathrm{mg} / \mathrm{kg} \\
\mathrm{mg} / \mathrm{kg} \\
\mathrm{mg} / \mathrm{kg} \\
\mathrm{mg} / \mathrm{kg} \\
\mathrm{mg} / \mathrm{kg} \\
\mathrm{mg} / \mathrm{kg} \\
\mathrm{mg} / \mathrm{kg} \\
\mathrm{mg} / \mathrm{kg} \\
\mathrm{mg} / \mathrm{kg} \\
\mathrm{mg} / \mathrm{kg} \\
\mathrm{mg} / \mathrm{kg} \\
\mathrm{mg} / \mathrm{kg} \\
\mathrm{mg} / \mathrm{kg} \\
\mathrm{mg} / \mathrm{kg} \\
\mathrm{mg} / \mathrm{kg}\end{array}$ & $\begin{array}{r}10600.00 \\
0.46 \\
7.60 \\
102.00 \\
0.53 \\
4.80 \\
0.23 \\
845.00 \\
15.10 \\
8.40 \\
7.00 \\
1.10 \\
10200.00 \\
21.50 \\
4.60 \\
537.00 \\
1280.00 \\
0.12 \\
2.60 \\
7.00 \\
546.00 \\
0.58 \\
418.00 \\
0.92 \\
374.00 \\
6.10 \\
58.00 \\
0.69 \\
23.80 \\
33.30\end{array}$ & $\begin{array}{l}\text { B } \\
\text { B } \\
\mathbf{J} \\
\mathbf{U} \\
\mathbf{U} \\
\mathbf{B} \\
\mathbf{B} \\
\mathbf{B} \\
\mathbf{J} \\
\mathbf{U} \\
\mathbf{B} \\
\mathbf{J}\end{array}$ & $\begin{array}{r}16400.00 \\
0.41 \\
11.20 \\
48.30 \\
0.48 \\
4.80 \\
0.21 \\
273.00 \\
23.30 \\
8.30 \\
17.20 \\
1.00 \\
24800.00 \\
23.70 \\
9.60 \\
692.00 \\
966.00 \\
0.12 \\
4.50 \\
9.20 \\
702.00 \\
0.66 \\
498.00 \\
0.82 \\
365.00 \\
2.40 \\
59.00 \\
0.61 \\
52.40 \\
51.40\end{array}$ & $\begin{array}{l}\mathbf{B} \\
\mathbf{B} \\
\mathbf{J} \\
\mathbf{U} \\
\mathbf{B} \\
\mathbf{J}\end{array}$ & $\begin{array}{r}18200.00 \\
0.45 \\
25.60 \\
22.80 \\
0.57 \\
4.80 \\
0.23 \\
271.00 \\
30.50 \\
3.70 \\
30.10 \\
1.10 \\
3500.00 \\
9.40 \\
9.30 \\
671.00 \\
238.00 \\
0.19 \\
3.60 \\
12.00 \\
782.00 \\
0.71 \\
435.00 \\
0.90 \\
408.00 \\
2.20 \\
26.00 \\
0.67 \\
69.10 \\
80.70\end{array}$ & $\begin{array}{l}\text { B } \\
\mathbf{B} \\
\mathbf{J} \\
\mathrm{B} \\
\mathrm{B} \\
\mathbf{B} \\
\mathbf{J} \\
\mathbf{U} \\
\mathbf{B} \\
\mathbf{J}\end{array}$ & $\begin{array}{r}11400.00 \\
0.46 \\
16.10 \\
97.30 \\
0.52 \\
4.80 \\
0.23 \\
854.00 \\
16.40 \\
11.00 \\
7.40 \\
1.20 \\
10700.00 \\
37.60 \\
6.10 \\
566.00 \\
1830.00 \\
0.12 \\
2.60 \\
7.00 \\
395.00 \\
3.60 \\
429.00 \\
0.92 \\
351.00 \\
5.60 \\
56.00 \\
2.60 \\
24.60 \\
36.20\end{array}$ \\
\hline
\end{tabular}


D-4

Table D.1 (continued)

\begin{tabular}{|c|c|c|c|c|c|c|c|c|c|}
\hline Analysis & Units & $\begin{array}{l}\text { A horizon } \\
\text { result }\end{array}$ & Qualifier & $\begin{array}{l}\text { B horizon } \\
\text { result }\end{array}$ & Qualifier & $\begin{array}{l}\mathrm{C} \text { horizon } \\
\text { result }\end{array}$ & Qualifier & $\begin{array}{l}\text { A lab split } \\
\text { result }\end{array}$ & Qualifier \\
\hline
\end{tabular}

Location $=A N D$; Formation $=C O P P E R$ RIDGE; Site $=37,38,41$; Sample type $=$ Regular; $A=7075 ; B=7078 ; C=7081 ; A$ lab split =' '; Phase $=2$

$\begin{array}{lrrrrrrr}\text { Aluminum } & \mathrm{mg} / \mathrm{kg} & 15400.00 & & 24500.00 & & 28600.00 & \\ \text { Antimony } & \mathrm{mg} / \mathrm{kg} & 0.50 & \mathrm{UJ} & 0.41 & \mathrm{UJ} & 0.43 & \text { UJ } \\ \text { Arsenic } & \mathrm{mg} / \mathrm{kg} & 14.40 & \mathrm{~J} & 26.80 & & 36.50 & \\ \text { Barium } & \mathrm{mg} / \mathrm{kg} & 156.00 & & 58.90 & & 40.60 & \mathrm{~B} \\ \text { Beryllium } & \mathrm{mg} / \mathrm{kg} & 0.95 & \mathrm{~B} & 1.00 & \mathrm{~B} & 1.20 & \\ \text { Boron } & \mathrm{mg} / \mathrm{kg} & 6.00 & \mathrm{UJ} & 4.90 & \mathrm{UJ} & 5.10 & \mathrm{UJ} \\ \text { Cacmium } & \mathrm{mg} / \mathrm{kg} & 0.25 & \mathrm{U} & 0.20 & \mathrm{U} & 0.21 & \mathrm{U} \\ \text { Calcium } & \mathrm{mg} / \mathrm{kg} & 1730.00 & & 732.00 & \mathrm{~B} & 552.00 & \mathrm{~B} \\ \text { Chromium } & \mathrm{mg} / \mathrm{kg} & 20.70 & \mathrm{~J} & 26.60 & \mathrm{~J} & 26.10 & \mathrm{~J} \\ \text { Cobalt } & \mathrm{mg} / \mathrm{kg} & 35.30 & & 23.30 & & 31.00 & \\ \text { Copper } & \mathrm{mg} / \mathrm{kg} & 13.40 & & 39.50 & & 54.20 & \\ \text { Cyanide } & \mathrm{mg} / \mathrm{kg} & 1.20 & \mathrm{UJ} & 1.00 & \mathrm{UJ} & 1.10 & \mathrm{UJ} \\ \text { Iron } & \mathrm{mg} / \mathrm{kg} & 14600.00 & & 32600.00 & & 40300.00 & \\ \text { Lead } & \mathrm{mg} / \mathrm{kg} & 45.60 & & 49.50 & & 77.90 & \mathrm{~J} \\ \text { Lithium } & \mathrm{mg} / \mathrm{kg} & 13.80 & \mathrm{~B} & 22.20 & & 22.70 & \\ \text { Magnesium } & \mathrm{mg} / \mathrm{kg} & 931.00 & \mathrm{~B} & 1010.00 & \mathrm{~B} & 928.00 & \mathrm{~B} \\ \text { Manganese } & \mathrm{mg} / \mathrm{kg} & 2560.00 & \mathrm{~J} & 786.00 & \mathrm{~J} & 1440.00 & \mathrm{~J} \\ \text { Mercury } & \mathrm{mg} / \mathrm{kg} & 0.11 & & 0.14 & & 0.21 & \\ \text { Molybdenum } & \mathrm{mg} / \mathrm{kg} & 3.20 & \mathrm{U} & 2.70 & \mathrm{U} & 2.80 & \mathrm{U} \\ \text { Mickel } & \mathrm{mg} / \mathrm{kg} & 13.50 & & 27.70 & & 33.50 & \\ \text { Potassium } & \mathrm{mg} / \mathrm{kg} & 614.00 & \mathrm{~B} & 918.00 & \mathrm{~B} & 1040.00 & \mathrm{~B} \\ \text { Selenium } & \mathrm{mg} / \mathrm{kg} & 0.82 & \mathrm{~B} & 0.70 & \mathrm{~B} & 0.69 & \mathrm{~B} \\ \text { Silicon } & \mathrm{mg} / \mathrm{kg} & 495.00 & & 580.00 & & 456.00 & \\ \text { Silver } & \mathrm{mg} / \mathrm{kg} & 1.00 & \mathrm{U} & 0.82 & \mathrm{U} & 0.86 & \mathrm{U} \\ \text { Sodium } & \mathrm{mg} / \mathrm{kg} & 423.00 & \mathrm{~B} & 410.00 & \mathrm{~B} & 400.00 & \mathrm{~B} \\ \text { Strontium } & \mathrm{mg} / \mathrm{kg} & 7.50 & \mathrm{~B} & 3.30 & \mathrm{~B} & 0.96 & \mathrm{~B} \\ \text { Sulfate } & \mathrm{mg} / \mathrm{kg} & 150.00 & & 53.90 & & 39.30 & \\ \text { Thallium } & \mathrm{mg} / \mathrm{kg} & 0.74 & \mathrm{U} & 0.61 & \mathrm{U} & 0.65 & \mathrm{~B} \\ \text { Vanadium } & \mathrm{mg} / \mathrm{kg} & 32.10 & \mathrm{~J} & 63.60 & \mathrm{~J} & 72.60 & \mathrm{~J} \\ \text { Zinc } & \mathrm{mg} / \mathrm{kg} & 67.40 & \mathrm{~J} & 141.00 & \mathrm{~J} & 170.00 & \mathrm{~J}\end{array}$

Location $=A N D$; Formation $=$ COPPER RIDGE; Site $=39,40,42$; Sample type $=$ Regular; $A=7066 ; B=7069 ; C=7072 ; A$ lab split=' '; Phase $=2$

$\begin{array}{lrrlrlrl}\text { Aluminum } & \mathrm{mg} / \mathrm{kg} & 19100.00 & & 21600.00 & & 25800.00 & \\ \text { Antimony } & \mathrm{mg} / \mathrm{kg} & 0.46 & \text { UJ } & 0.43 & \text { UJ } & 0.43 & \text { UJ } \\ \text { Arsenic } & \mathrm{mg} / \mathrm{kg} & 13.40 & \mathrm{~J} & 29.80 & & 21.20 & \mathrm{~J} \\ \text { Barium } & \mathrm{mg} / \mathrm{kg} & 196.00 & & 62.10 & & 38.90 & \mathrm{~B} \\ \text { Beryllium } & \mathrm{mg} / \mathrm{kg} & 1.00 & \mathrm{~B} & 0.70 & \mathrm{~B} & 0.66 & \mathrm{~B} \\ \text { Boron } & \mathrm{mg} / \mathrm{kg} & 5.60 & \mathrm{UJ} & 5.10 & \mathrm{UJ} & 5.20 & \mathrm{UJ} \\ \text { Cadnium } & \mathrm{mg} / \mathrm{kg} & 0.23 & \mathrm{U} & 0.21 & \mathrm{U} & 0.22 & \mathrm{U} \\ \text { Calcium } & \mathrm{mg} / \mathrm{kg} & 1050.00 & \mathrm{~B} & 754.00 & \mathrm{~B} & 654.00 & \mathrm{~B} \\ \text { Chromium } & \mathrm{mg} / \mathrm{kg} & 25.70 & \mathrm{~J} & 52.80 & \mathrm{~J} & 60.40 & \mathrm{~J} \\ \text { Cobalt } & \mathrm{mg} / \mathrm{kg} & 16.40 & & 12.30 & & 5.50 & \mathrm{~B} \\ \text { Copper } & \mathrm{mg} / \mathrm{kg} & 11.00 & & 17.00 & & 19.90 & \\ \text { Cyanide } & \mathrm{mg} / \mathrm{kg} & 1.20 & \mathrm{UJ} & 1.00 & \mathrm{UJ} & 1.10 & \mathrm{UJ} \\ \text { Iron } & \mathrm{mg} / \mathrm{kg} & 17900.00 & & 35100.00 & & 35000.00 & \\ \text { Lead } & \mathrm{mg} / \mathrm{kg} & 42.30 & & 29.70 & & 12.10 & \\ \text { Lithium } & \mathrm{mg} / \mathrm{kg} & 7.50 & \mathrm{~B} & 13.10 & \mathrm{~B} & 14.50 & \mathrm{~B} \\ \text { Magnesium } & \mathrm{mg} / \mathrm{kg} & 898.00 & \mathrm{~B} & 981.00 & \mathrm{~B} & 1090.00 & \\ \text { Manganese } & \mathrm{mg} / \mathrm{kg} & 3470.00 & \mathrm{~J} & 1230.00 & \mathrm{~J} & 500.00 & \mathrm{~J} \\ \text { Mercury } & \mathrm{mg} / \mathrm{kg} & 0.11 & & 0.16 & & 0.17 & \\ \text { Molybdenum } & \mathrm{mg} / \mathrm{kg} & 3.00 & \mathrm{U} & 2.80 & \mathrm{~B} & 2.80 & \mathrm{U} \\ \text { Mickel } & \mathrm{mg} / \mathrm{kg} & 13.20 & & 13.80 & & 17.00 & \\ \text { Potassium } & \mathrm{mg} / \mathrm{kg} & 613.00 & \mathrm{~B} & 803.00 & \mathrm{~B} & 1020.00 & \mathrm{~B} \\ \text { Selenium } & \mathrm{mg} / \mathrm{kg} & 0.88 & \mathrm{~B} & 0.72 & \mathrm{~B} & 0.66 & \mathrm{~B} \\ \text { Silicon } & \mathrm{mg} / \mathrm{kg} & 537.00 & & 484.00 & & 592.00 & \\ \text { Silver } & \mathrm{mg} / \mathrm{kg} & 0.93 & \mathrm{U} & 0.85 & \mathrm{U} & 0.87 & \mathrm{U} \\ \text { Sodium } & \mathrm{mg} / \mathrm{kg} & 463.00 & \mathrm{~B} & 373.00 & \mathrm{~B} & 386.00 & \mathrm{~B} \\ \text { Strontium } & \mathrm{mg} / \mathrm{kg} & 7.70 & \mathrm{~B} & 6.30 & \mathrm{~B} & 5.20 & \mathrm{~B} \\ \text { Sulfate } & \mathrm{mg} / \mathrm{kg} & 178.00 & & 56.80 & & 37.40 & \\ \text { Thallium } & \mathrm{mg} / \mathrm{kg} & 0.70 & \mathrm{U} & 0.64 & \mathrm{U} & 0.66 & \mathrm{U} \\ \text { Vanadium } & \mathrm{mg} / \mathrm{kg} & 40.70 & \mathrm{~J} & 67.40 & \mathrm{~J} & 67.90 & \mathrm{~J} \\ \text { Zinc } & \mathrm{mg} / \mathrm{kg} & 42.00 & \mathrm{~J} & 47.80 & \mathrm{~J} & 50.50 & \mathrm{~J}\end{array}$


D-5

Table D.1 (continued)

\begin{tabular}{|c|c|c|c|c|c|c|c|c|c|}
\hline Analysis & Units & $\begin{array}{l}\text { A horizon } \\
\text { result }\end{array}$ & Qualifier & $\begin{array}{l}\text { B horizon } \\
\text { result }\end{array}$ & Qualifier & $\begin{array}{l}C \text { horizon } \\
\text { result }\end{array}$ & Qualifier & $\begin{array}{l}\text { A lab split } \\
\text { result }\end{array}$ & Qualifier \\
\hline
\end{tabular}

Location=AND; Formation=DISMAL GAP; Site =1,20,22; Sample type=Regular; $A=7028 ; B=7031 ; C=7034 ; A$ lab split=' '; Phase $=1$

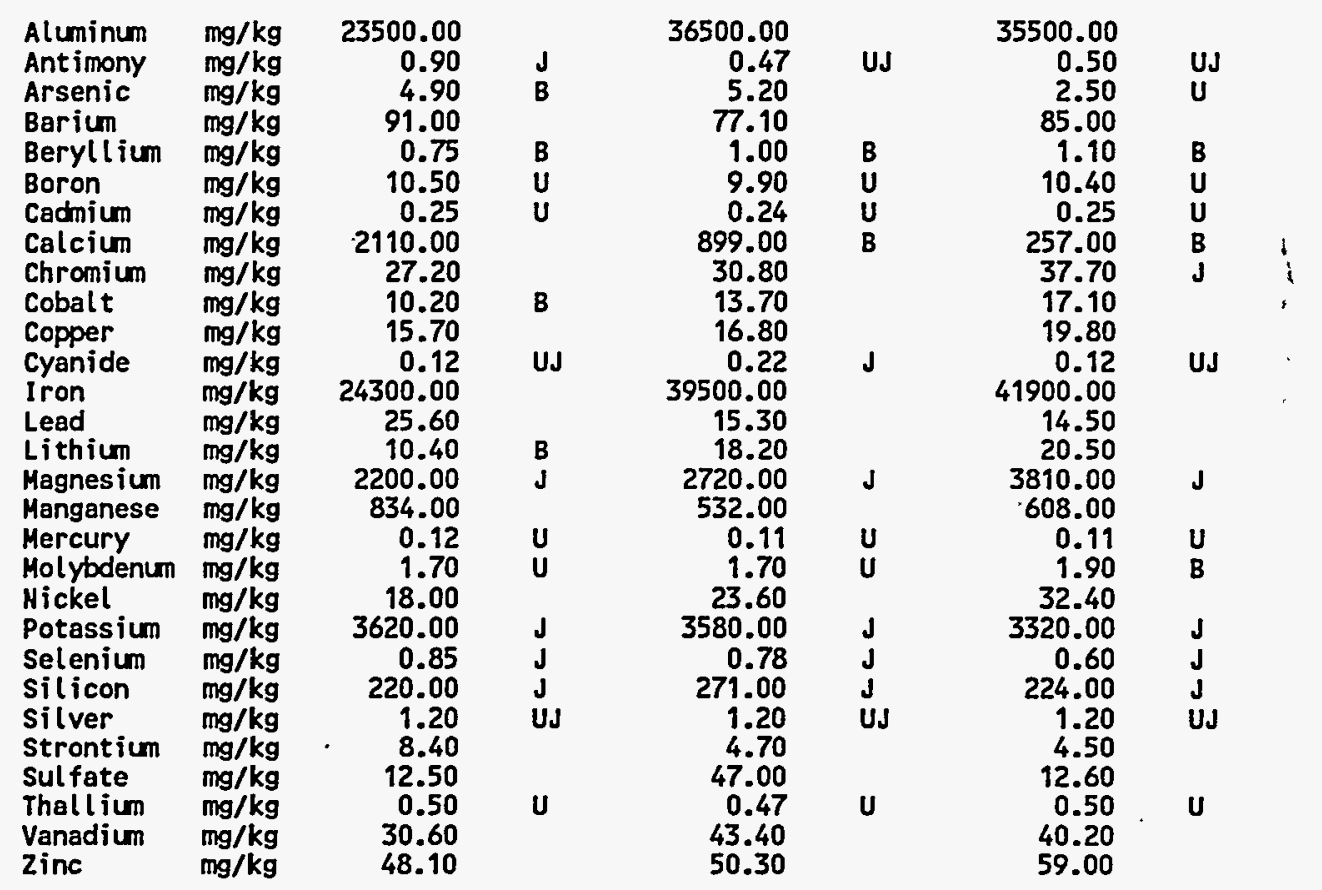

Location $=A N D ;$ Formation $=D I S M A L$ GAP; Site $=3,5,11 ;$ Sample type $=$ Regular; $A=7010 ; B=7013 ; C=7016 ; A$ lab split =' '; Phase $=1$

$\begin{array}{lrrlrrrr}\text { Aluminum } & \mathrm{mg} / \mathrm{kg} & 24100.00 & & 33400.00 & & 37200.00 & \\ \text { Antimony } & \mathrm{mg} / \mathrm{kg} & 1.00 & \mathrm{UJ} & 1.40 & \mathrm{UJ} & 1.40 & \text { UJ } \\ \text { Arsenic } & \mathrm{mg} / \mathrm{kg} & 4.10 & \mathrm{~B} & 4.50 & \mathrm{~B} & 4.60 & \mathrm{~B} \\ \text { Barium } & \mathrm{mg} / \mathrm{kg} & 74.80 & & 70.10 & & 80.10 & \\ \text { Beryllium } & \mathrm{mg} / \mathrm{kg} & 0.86 & \mathrm{~B} & 0.82 & \mathrm{~B} & 1.00 & \mathrm{~B} \\ \text { Boron } & \mathrm{mg} / \mathrm{kg} & 10.20 & \mathrm{U} & 10.20 & \mathrm{U} & 10.00 & \mathrm{U} \\ \text { Cacmium } & \mathrm{mg} / \mathrm{kg} & 0.24 & \mathrm{U} & 0.24 & \mathrm{U} & \mathbf{0 . 2 4} & \mathrm{U} \\ \text { Calcium } & \mathrm{mg} / \mathrm{kg} & 1340.00 & & 776.00 & \mathrm{~B} & 832.00 & \mathrm{~B} \\ \text { Chromium } & \mathrm{mg} / \mathrm{kg} & 29.30 & & 38.80 & & 40.10 & \\ \text { Cobalt } & \mathrm{mg} / \mathrm{kg} & 14.10 & & 9.60 & \mathrm{~B} & 9.80 & \mathrm{~B} \\ \text { Copper } & \mathrm{mg} / \mathrm{kg} & 16.30 & & 21.50 & \mathrm{~J} & 22.40 & \\ \text { Cyanide } & \mathrm{mg} / \mathrm{kg} & 0.12 & \mathrm{UJ} & 0.12 & \mathrm{UJ} & 0.12 & \mathrm{UJ} \\ \text { Iron } & \mathrm{mg} / \mathrm{kg} & 30700.00 & & 41000.00 & & 41800.00 & \\ \text { Lead } & \mathrm{mg} / \mathrm{kg} & 24.60 & & 19.40 & & 21.90 & \\ \text { Lithium } & \mathrm{mg} / \mathrm{kg} & 10.10 & \mathrm{~B} & 16.30 & & 19.60 & \\ \text { Magnesium } & \mathrm{mg} / \mathrm{kg} & 3230.00 & \mathrm{~J} & 2920.00 & \mathrm{~J} & 3650.00 & \mathrm{~J} \\ \text { Manganese } & \mathrm{mg} / \mathrm{kg} & 572.00 & & 207.00 & & 216.00 & \\ \text { Mercury } & \mathrm{mg} / \mathrm{kg} & 0.11 & \mathrm{U} & 0.11 & \mathrm{U} & 0.12 & \mathrm{U} \\ \text { Molybdenum } & \mathrm{mg} / \mathrm{kg} & 1.70 & \mathrm{U} & 1.80 & \mathrm{~B} & 1.70 & \mathrm{U} \\ \text { Mickel } & \mathrm{mg} / \mathrm{kg} & 23.00 & & 22.50 & & 27.20 & \\ \text { Potassium } & \mathrm{mg} / \mathrm{kg} & 5090.00 & \mathrm{~J} & 4430.00 & \mathrm{~J} & 5890.00 & \mathrm{~J} \\ \text { Selenium } & \mathrm{mg} / \mathrm{kg} & 0.61 & \mathrm{~B} & 0.53 & \mathrm{~B} & 0.50 & \mathrm{~B} \\ \text { Silicon } & \mathrm{mg} / \mathrm{kg} & 211.00 & \mathrm{~J} & 268.00 & \mathrm{~J} & 188.00 & \mathrm{~J} \\ \text { Silver } & \mathrm{mg} / \mathrm{kg} & 1.20 & \mathrm{UJ} & 1.20 & \mathrm{UJ} & 1.20 & \mathrm{UJ} \\ \text { Strontium } & \mathrm{mg} / \mathrm{kg} & 6.40 & & 5.20 & & 6.00 & \\ \text { Sulfate } & \mathrm{mg} / \mathrm{kg} & 11.40 & \mathrm{~B} & 39.30 & & 15.30 & \\ \text { Thallium } & \mathrm{mg} / \mathrm{kg} & 0.49 & \mathrm{U} & 0.48 & \mathrm{U} & 0.48 & \mathrm{U} \\ \text { Vanadium } & \mathrm{mg} / \mathrm{kg} & 31.10 & & 43.80 & & 43.80 & \\ \text { Zinc } & \mathrm{mg} / \mathrm{kg} & 54.90 & & 52.70 & & 62.20 & \end{array}$


D-6

Table D.1 (continued)

\begin{tabular}{|c|c|c|c|c|c|c|c|c|c|}
\hline Analysis & Units & $\begin{array}{l}\text { A horizon } \\
\text { result }\end{array}$ & Oualifier & $\begin{array}{l}\text { B horizon } \\
\text { result }\end{array}$ & Qualifier & $\begin{array}{l}C \text { horizon } \\
\text { result }\end{array}$ & Qualifier & $\begin{array}{l}\text { A lab split } \\
\text { result }\end{array}$ & Qualifier \\
\hline
\end{tabular}

Location $=A N D ;$ Formation $=D I S M A L$ GAP; Site $=4,12,21$; Sample type $=$ Regular; $A=7019 ; B=7022 ; C=7025 ; A$ lab split=' '; Phase $=1$

\begin{tabular}{|c|c|c|c|c|c|c|c|}
\hline $\begin{array}{l}\text { Aluminum } \\
\text { Antimony } \\
\text { Arsenic } \\
\text { Barium } \\
\text { Beryl lium } \\
\text { Boron } \\
\text { Cadnium } \\
\text { Calcium } \\
\text { Chromium } \\
\text { Cobalt } \\
\text { Copper } \\
\text { Cyanide } \\
\text { Iron } \\
\text { Lead } \\
\text { Lithium } \\
\text { Magnesium } \\
\text { Manganese } \\
\text { Mercury } \\
\text { Molybdenum } \\
\text { Nickel } \\
\text { Potassium } \\
\text { Selenium } \\
\text { Sil icon } \\
\text { Silver } \\
\text { Strontium } \\
\text { Sulfate } \\
\text { Thal I ium } \\
\text { Vanadium } \\
\text { Zinc }\end{array}$ & $\begin{array}{l}\mathrm{mg} / \mathrm{kg} \\
\mathrm{mg} / \mathrm{kg} \\
\mathrm{mg} / \mathrm{kg} \\
\mathrm{mg} / \mathrm{kg} \\
\mathrm{mg} / \mathrm{kg} \\
\mathrm{mg} / \mathrm{kg} \\
\mathrm{mg} / \mathrm{kg} \\
\mathrm{mg} / \mathrm{kg} \\
\mathrm{mg} / \mathrm{kg} \\
\mathrm{mg} / \mathrm{kg} \\
\mathrm{mg} / \mathrm{kg} \\
\mathrm{mg} / \mathrm{kg} \\
\mathrm{mg} / \mathrm{kg} \\
\mathrm{mg} / \mathrm{kg} \\
\mathrm{mg} / \mathrm{kg} \\
\mathrm{mg} / \mathrm{kg} \\
\mathrm{mg} / \mathrm{kg} \\
\mathrm{mg} / \mathrm{kg} \\
\mathrm{mg} / \mathrm{kg} \\
\mathrm{mg} / \mathrm{kg} \\
\mathrm{mg} / \mathrm{kg} \\
\mathrm{mg} / \mathrm{kg} \\
\mathrm{mg} / \mathrm{kg} \\
\mathrm{mg} / \mathrm{kg} \\
\mathrm{mg} / \mathrm{kg} \\
\mathrm{mg} / \mathrm{kg} \\
\mathrm{mg} / \mathrm{kg} \\
\mathrm{mg} / \mathrm{kg} \\
\mathrm{mg} / \mathrm{kg}\end{array}$ & $\begin{array}{r}22300.00 \\
1.40 \\
3.10 \\
76.50 \\
0.94 \\
10.30 \\
0.25 \\
1300.00 \\
22.60 \\
13.30 \\
16.40 \\
0.27 \\
24100.00 \\
36.40 \\
10.40 \\
2770.00 \\
576.00 \\
0.11 \\
1.70 \\
21.00 \\
3940.00 \\
0.76 \\
228.00 \\
1.20 \\
5.60 \\
14.40 \\
0.49 \\
27.80 \\
50.50\end{array}$ & $\begin{array}{l}\mathbf{B} \\
\mathbf{J} \\
\mathbf{U} \\
\mathbf{U} \\
\mathbf{J} \\
\mathbf{J} \\
\mathbf{J} \\
\mathbf{U} \mathbf{J}\end{array}$ & $\begin{array}{r}35800.00 \\
1.30 \\
2.30 \\
67.30 \\
1.20 \\
9.90 \\
0.23 \\
776.00 \\
29.30 \\
7.30 \\
19.80 \\
0.12 \\
38200.00 \\
16.80 \\
20.50 \\
3050.00 \\
111.00 \\
0.11 \\
1.60 \\
25.30 \\
4140.00 \\
0.68 \\
208.00 \\
1.20 \\
3.60 \\
47.10 \\
0.47 \\
41.00 \\
45.60\end{array}$ & $\begin{array}{l}J \\
U \\
\text { U } \\
\\
\mathbf{J} \\
\mathbf{B} \\
\mathbf{J} \\
\mathbf{U} J\end{array}$ & $\begin{array}{r}15.90 \\
3490.00 \\
627.00 \\
0.11 \\
1.70 \\
24.80 \\
5410.00 \\
0.50 \\
198.00 \\
1.20 \\
2.50 \\
29.50 \\
0.48 \\
39.50 \\
51.90\end{array}$ & $\begin{array}{l}\mathbf{U} \\
\mathbf{U} \\
\mathbf{J} \\
\mathbf{B} \\
\mathbf{J} \\
\mathbf{U} \mathbf{J}\end{array}$ \\
\hline
\end{tabular}

Location $=A N D ;$ Formation $=D I S M A L$ GAP; Site $=9,10,19 ;$ Sample type $=$ Field duplicate; $A=7037 ; B=7040 ; C=7043 ; A$ lab split=' '; Phase $=1$

\begin{tabular}{lrrrrrrr} 
Aluminum & $\mathrm{mg} / \mathrm{kg}$ & 22000.00 & & 34900.00 & & 45000.00 & \\
Antimony & $\mathrm{mg} / \mathrm{kg}$ & 0.50 & $\mathrm{UJ}$ & 0.62 & $\mathrm{UJ}$ & 0.72 & UJ \\
Arsenic & $\mathrm{mg} / \mathrm{kg}$ & 4.90 & & 5.60 & & 6.80 & \\
Barium & $\mathrm{mg} / \mathrm{kg}$ & 80.60 & & 90.50 & & 137.00 & \\
Beryllium & $\mathrm{mg} / \mathrm{kg}$ & 0.75 & $\mathrm{~B}$ & 0.83 & $\mathrm{~B}$ & 1.20 & $\mathrm{~B}$ \\
Boron & $\mathrm{mg} / \mathrm{kg}$ & 10.40 & $\mathrm{U}$ & 10.00 & $\mathrm{U}$ & 10.00 & $\mathrm{U}$ \\
Cactnium & $\mathrm{mg} / \mathrm{kg}$ & 0.24 & $\mathrm{U}$ & 0.24 & $\mathrm{U}$ & 0.24 & $\mathrm{U}$ \\
Calcium & $\mathrm{mg} / \mathrm{kg}$ & 920.00 & $\mathrm{~B}$ & 658.00 & $\mathrm{~B}$ & 289.00 & $\mathrm{~B}$ \\
Chromium & $\mathrm{mg} / \mathrm{kg}$ & 43.50 & & 51.60 & $\mathrm{~J}$ & 60.30 & $\mathrm{~J}$ \\
Cobalt & $\mathrm{mg} / \mathrm{kg}$ & 11.00 & $\mathrm{~B}$ & 18.40 & & 23.20 & \\
Copper & $\mathrm{mg} / \mathrm{kg}$ & 11.10 & & 18.20 & & 60.00 & \\
Cyanide & $\mathrm{mg} / \mathrm{kg}$ & 0.12 & $\mathrm{U}$ & 0.12 & $\mathrm{UJ}$ & 0.12 & $\mathrm{UJ}$ \\
Iron & $\mathrm{mg} / \mathrm{kg}$ & 22600.00 & & 39000.00 & & 42400.00 & \\
Lead & $\mathrm{mg} / \mathrm{kg}$ & 30.30 & & 23.90 & & 34.90 & \\
Lithium & $\mathrm{mg} / \mathrm{kg}$ & 11.40 & $\mathrm{~B}$ & 21.70 & & 33.50 & \\
Magnesium & $\mathrm{mg} / \mathrm{kg}$ & 2590.00 & $\mathrm{~J}$ & 2820.00 & $\mathrm{~J}$ & 3640.00 & $\mathrm{~J}$ \\
Manganese & $\mathrm{mg} / \mathrm{kg}$ & 814.00 & & 536.00 & & 1330.00 & \\
Mercury & $\mathrm{mg} / \mathrm{kg}$ & 0.12 & & 0.11 & $\mathrm{U}$ & 0.11 & $\mathrm{U}$ \\
Molybdenum & $\mathrm{mg} / \mathrm{kg}$ & 1.70 & $\mathrm{U}$ & 2.00 & $\mathrm{~B}$ & 1.70 & $\mathrm{~B}$ \\
Nickel & $\mathrm{mg} / \mathrm{kg}$ & 20.90 & & 24.80 & & 35.50 & \\
Potassium & $\mathrm{mg} / \mathrm{kg}$ & 3150.00 & $\mathrm{~J}$ & 3230.00 & $\mathrm{~J}$ & 3660.00 & $\mathrm{~J}$ \\
Selenium & $\mathrm{mg} / \mathrm{kg}$ & 0.76 & $\mathrm{~B}$ & 0.71 & $\mathrm{~B}$ & 0.47 & $\mathrm{U}$ \\
Silicon & $\mathrm{mg} / \mathrm{kg}$ & 205.00 & $\mathrm{~J}$ & 226.00 & $\mathrm{~J}$ & 281.00 & $\mathrm{~J}$ \\
Silver & $\mathrm{mg} / \mathrm{kg}$ & 1.20 & $\mathrm{UJ}$ & 1.20 & $\mathrm{UJ}$ & 1.20 & $\mathrm{UJ}$ \\
Strontium & $\mathrm{mg} / \mathrm{kg}$ & 4.80 & & 3.90 & & 3.70 & \\
Sulfate & $\mathrm{mg} / \mathrm{kg}$ & 17.60 & & 37.90 & & 9.90 & $\mathrm{~B}$ \\
Thallium & $\mathrm{mg} / \mathrm{kg}$ & 0.49 & $\mathrm{U}$ & $0 . .47$ & $\mathrm{U}$ & 0.47 & $\mathrm{U}$ \\
Vanadium & $\mathrm{mg} / \mathrm{kg}$ & 30.50 & & 51.60 & & 48.60 & \\
Zinc & $\mathrm{mg} / \mathrm{kg}$ & 44.90 & & 54.70 & & 70.70 & \\
\hline & & & & & &
\end{tabular}


Table D.1 (continued)

\begin{tabular}{|c|c|c|c|c|c|c|c|c|c|}
\hline Analysis & Units & $\begin{array}{l}\text { A horizon } \\
\text { result }\end{array}$ & Qualifier & $\begin{array}{l}\text { B horizon } \\
\text { result }\end{array}$ & Qualifier & $\begin{array}{l}C \text { horizon } \\
\text { result }\end{array}$ & Qualifier & $\begin{array}{l}\text { A lab split } \\
\text { result }\end{array}$ & Qualifier \\
\hline
\end{tabular}

Location $=A N D$; Formation $=D I S M A L$ GAP; Site $=9,10,19 ;$ Sample type $=$ Regular; $A=7001 ; B=7004 ; C=7007 ; A$ lab split=' '; Phase=1

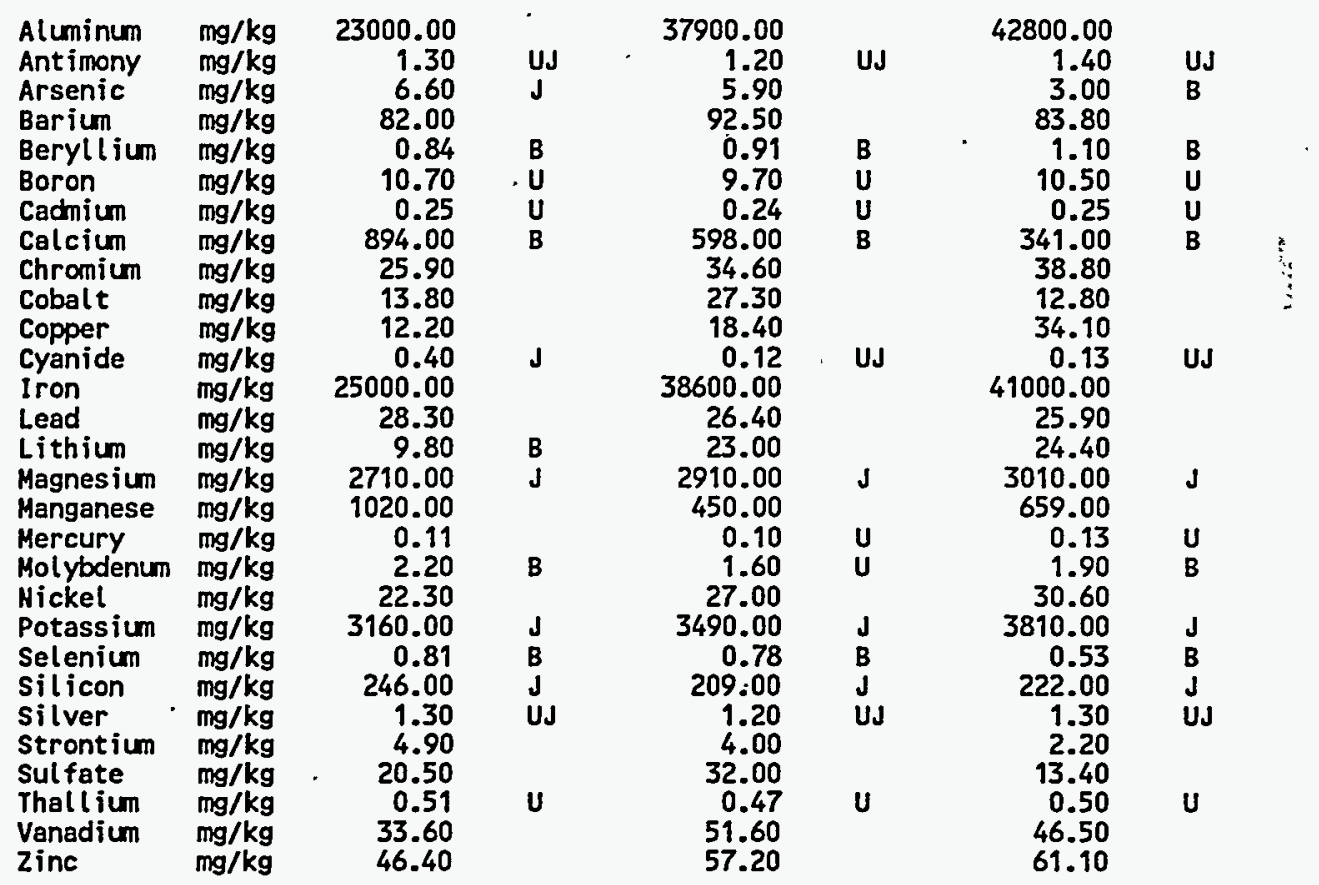

Location $=$ ORR; Formation $=$ CHEPULTEPEC; Site $=50,66,73$; Sample type $=$ Field duplicate; $A=5173 ; B=5178 ; C=5184 ; A$ lab split $=5174$ Phase $=2$

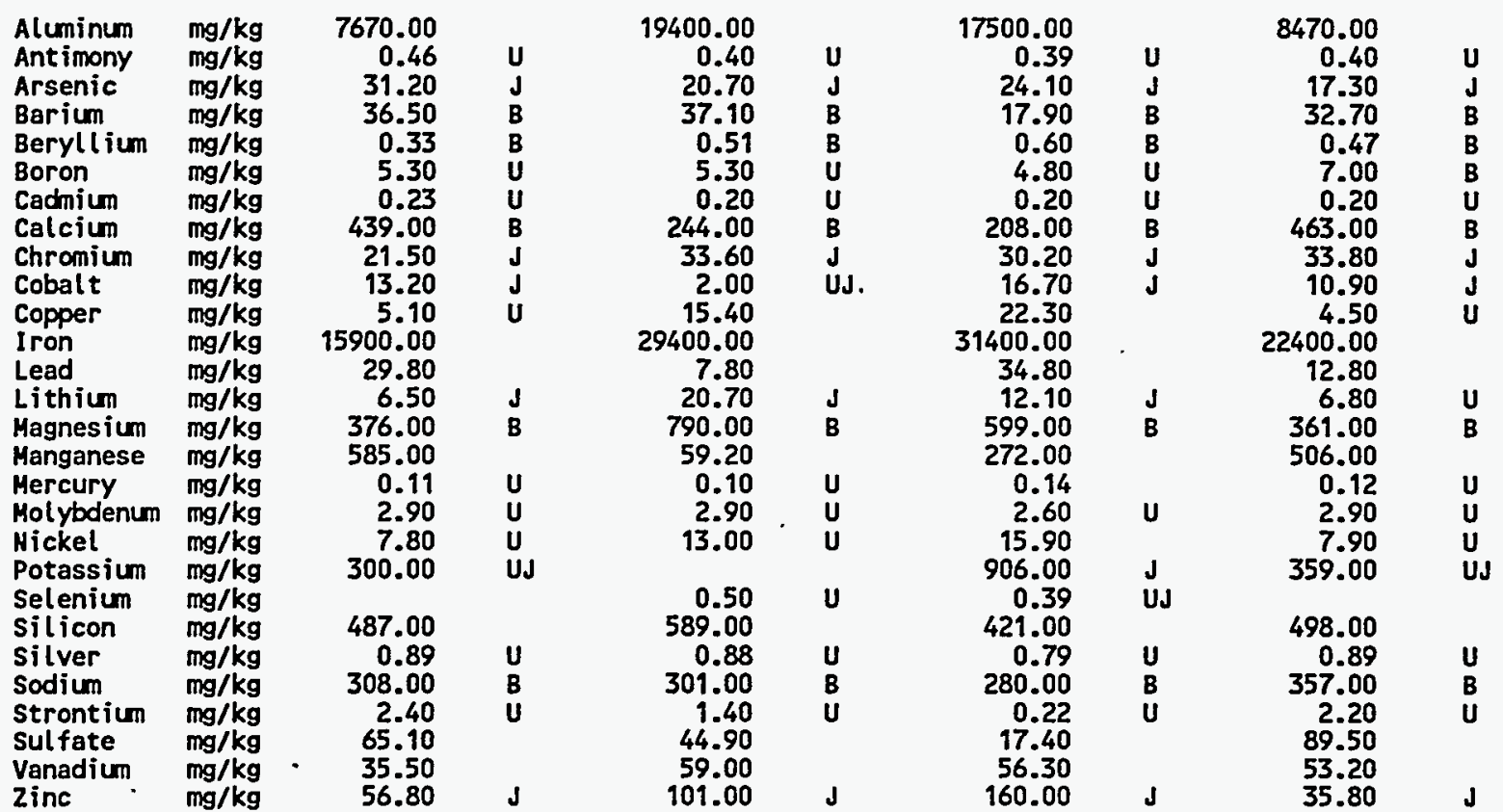


D-8

Table D.1 (continued)

\begin{tabular}{|c|c|c|c|c|c|c|c|c|c|}
\hline Analysis & Units & $\begin{array}{l}\text { A horizon } \\
\text { result }\end{array}$ & Qualifier & $\begin{array}{l}\text { B horizon } \\
\text { result }\end{array}$ & Qualifier & $\begin{array}{l}\mathrm{C} \text { horizon } \\
\text { result }\end{array}$ & Qualifier & $\begin{array}{l}\text { A lab split } \\
\text { result }\end{array}$ & Qualifier \\
\hline
\end{tabular}

Location=ORR; Formation $=C H E P U L T E P E C$; Site $=50,66,73$; Sample type $=$ Regular; $A=5165 ; B=5170 ; C=5181 ; A$ lab split $=5166 ;$ Phase $=2$

\begin{tabular}{|c|c|c|c|c|c|c|c|c|}
\hline t & $\begin{array}{l}\mathrm{mg} / \mathrm{kg} \\
\mathrm{mg} / \mathrm{kg} \\
\mathrm{mg} / \mathrm{kg} \\
\mathrm{mg} / \mathrm{kg} \\
\mathrm{mg} / \mathrm{kg} \\
\mathrm{mg} / \mathrm{kg} \\
\mathrm{mg} / \mathrm{kg} \\
\mathrm{mg} / \mathrm{kg} \\
\mathrm{mg} / \mathrm{kg} \\
\mathrm{mg} / \mathrm{kg} \\
\mathrm{mg} / \mathrm{kg} \\
\mathrm{mg} / \mathrm{kg} \\
\mathrm{mg} / \mathrm{kg} \\
\mathrm{mg} / \mathrm{kg} \\
\mathrm{mg} / \mathrm{kg} \\
\mathrm{mg} / \mathrm{kg} \\
\mathrm{mg} / \mathrm{kg} \\
\mathrm{mg} / \mathrm{kg} \\
\mathrm{mg} / \mathrm{kg} \\
\mathrm{mg} / \mathrm{kg} \\
\mathrm{mg} / \mathrm{kg} \\
\mathrm{mg} / \mathrm{kg} \\
\mathrm{mg} / \mathrm{kg} \\
\mathrm{mg} / \mathrm{kg} \\
\mathrm{mg} / \mathrm{kg} \\
\mathrm{mg} / \mathrm{kg} \\
\mathrm{mg} / \mathrm{kg}\end{array}$ & $\begin{array}{r}8790.00 \\
0.44 \\
7.10 \\
31.60 \\
0.55 \\
5.50 \\
0.22 \\
390.00 \\
26.90 \\
9.80 \\
3.60 \\
30000.00 \\
10.60 \\
5.20 \\
280.00 \\
766.00 \\
0.11 \\
3.00 \\
8.10 \\
218.00 \\
508.00 \\
0.91 \\
339.00 \\
1.80 \\
68.90 \\
61.20 \\
35.50\end{array}$ & $\begin{array}{l}\mathbf{U} \\
\mathbf{J} \\
\mathrm{B} \\
\mathrm{B} \\
\mathbf{U} \\
\mathbf{U} \\
\mathrm{B} \\
\mathrm{J} \\
\mathrm{J} \\
\mathbf{U}\end{array}$ & $\begin{array}{r}19700.00 \\
0.40 \\
21.50 \\
54.20 \\
0.58 \\
5.00 \\
0.20 \\
325.00 \\
30.40 \\
1.60 \\
19.20 \\
31400.00 \\
9.60 \\
18.50 \\
768.00 \\
59.00 \\
0.09 \\
2.70 \\
14.70 \\
1040.00 \\
410.00 \\
0.83 \\
273.00 \\
2.00 \\
51.70 \\
62.70 \\
133.00\end{array}$ & $\begin{array}{l}B \\
U \\
U \\
B \\
J \\
\text { UJ }\end{array}$ & $\begin{array}{r}19400.00 \\
0.40 \\
30.60 \\
31.70 \\
0.75 \\
5.60 \\
0.20 \\
181.00 \\
33.10 \\
16.10 \\
27.90 \\
37500.00 \\
11.60 \\
17.10 \\
689.00 \\
306.00 \\
0.11 \\
2.70 \\
21.40 \\
1080.00 \\
535.00 \\
0.82 \\
313.00 \\
0.20 \\
18.10 \\
59.90 \\
203.00\end{array}$ & $\begin{array}{l}\text { U } \\
\text { J } \\
\text { B } \\
\text { B } \\
\text { B } \\
\text { U } \\
\text { B } \\
\text { J } \\
\text { J }\end{array}$ & $\begin{array}{r}7450.00 \\
0.43 \\
13.00 \\
37.80 \\
0.28 \\
5.30 \\
0.22 \\
468.00 \\
13.30 \\
12.70 \\
4.70 \\
10200.00 \\
14.60 \\
5.90 \\
342.00 \\
639.00 \\
0.10 \\
2.90 \\
7.50 \\
316.00 \\
507.00 \\
0.89 \\
294.00 \\
2.30 \\
73.50 \\
23.50 \\
37.30\end{array}$ \\
\hline
\end{tabular}

Location $=O R R ;$ Formation $=C H E P U L T E P E C$; Site $=52,53,78$; Sample type $=$ Regular;

$A=5127 ; B=5130 ; C=5133 ; A$ lab split =' '; Phase $=2$

$\begin{array}{lrrrrrrr}\text { Aluminum } & \mathrm{mg} / \mathrm{kg} & 8440.00 & & 15600.00 & & 16300.00 & \\ \text { Antimony } & \mathrm{mg} / \mathrm{kg} & 0.42 & \mathrm{U} & 0.42 & \mathrm{U} & 0.43 & \mathrm{U} \\ \text { Arsenic } & \mathrm{mg} / \mathrm{kg} & 15.60 & & 24.40 & & 180.00 & \\ \text { Barium } & \mathrm{mg} / \mathrm{kg} & 58.90 & & 28.40 & \mathrm{~B} & 12.80 & \mathrm{~B} \\ \text { Beryllium } & \mathrm{mg} / \mathrm{kg} & 0.51 & \mathrm{U} & 0.64 & \mathrm{U} & 0.77 & \mathrm{U} \\ \text { Boron } & \mathrm{mg} / \mathrm{kg} & 5.00 & \mathrm{U} & 5.20 & \mathrm{U} & 5.20 & \mathrm{U} \\ \text { Cadmium } & \mathrm{mg} / \mathrm{kg} & 0.21 & \mathrm{U} & 0.21 & \mathrm{U} & 0.21 & \mathrm{U} \\ \text { Calcium } & \mathrm{mg} / \mathrm{kg} & 404.00 & \mathrm{~B} & 447.00 & \mathrm{~B} & 234.00 & \mathrm{~B} \\ \text { Chromium } & \mathrm{mg} / \mathrm{kg} & 15.30 & \mathrm{~J} & 27.80 & \mathrm{~J} & 26.40 & \mathrm{~J} \\ \text { Cobalt } & \mathrm{mg} / \mathrm{kg} & 11.30 & & 6.10 & \mathrm{U} & 3.00 & \mathrm{~B} \\ \text { Copper } & \mathrm{mg} / \mathrm{kg} & 5.10 & \mathrm{U} & 15.70 & & 29.30 & \mathrm{U} \\ \text { Cyanide } & \mathrm{mg} / \mathrm{kg} & 1.00 & \mathrm{U} & 1.00 & \mathrm{U} & 1.00 & \mathrm{U} \\ \text { Iron } & \mathrm{mg} / \mathrm{kg} & 18400.00 & & 33000.00 & \mathrm{~J} & 34400.00 & \mathrm{~J} \\ \text { Lead } & \mathrm{mg} / \mathrm{kg} & 18.00 & & 7.50 & & 13.90 & \\ \text { Lithium } & \mathrm{mg} / \mathrm{kg} & 2.50 & \mathrm{U} & 5.80 & \mathrm{~B} & 7.40 & \mathrm{~B} \\ \text { Magnesium } & \mathrm{mg} / \mathrm{kg} & 341.00 & \mathrm{~B} & 672.00 & \mathrm{~B} & 890.00 & \mathrm{~B} \\ \text { Manganese } & \mathrm{mg} / \mathrm{kg} & 1250.00 & & 325.00 & & 60.30 & \\ \text { Mercury } & \mathrm{mg} / \mathrm{kg} & 0.15 & \mathrm{~J} & 0.10 & \mathrm{~J} & 0.23 & \mathrm{~J} \\ \text { Molybdenum } & \mathrm{mg} / \mathrm{kg} & 2.70 & \mathrm{U} & 2.80 & \mathrm{U} & 2.80 & \mathrm{U} \\ \text { Mickel } & \mathrm{mg} / \mathrm{kg} & 6.30 & \mathrm{U} & 9.20 & \mathrm{U} & 16.40 & \\ \text { Potassium } & \mathrm{mg} / \mathrm{kg} & 267.00 & \mathrm{U} & 945.00 & \mathrm{~B} & 1530.00 & \\ \text { Selenium } & \mathrm{mg} / \mathrm{kg} & 0.50 & \mathrm{~J} & 0.42 & \mathrm{~B} & 0.75 & \mathrm{~B} \\ \text { Silicon } & \mathrm{mg} / \mathrm{kg} & 554.00 & \mathrm{~J} & 602.00 & \mathrm{~J} & 596.00 & \mathrm{~J} \\ \text { Silver } & \mathrm{mg} / \mathrm{kg} & 0.84 & \mathrm{U} & 0.87 & \mathrm{U} & 0.86 & \mathrm{U} \\ \text { Sodium } & \mathrm{mg} / \mathrm{kg} & 334.00 & \mathrm{~B} & 334.00 & \mathrm{~B} & 388.00 & \mathrm{~B} \\ \text { Strontium } & \mathrm{mg} / \mathrm{kg} & 2.90 & \mathrm{~B} & 3.70 & \mathrm{~B} & 1.10 & \mathrm{~B} \\ \text { Sulfate } & \mathrm{mg} / \mathrm{kg} & 60.50 & & 25.00 & & 10.80 & \mathrm{U} \\ \text { Thallium } & \mathrm{mg} / \mathrm{kg} & 0.42 & \mathrm{U} & 0.42 & \mathrm{U} & 0.43 & \mathrm{U} \\ \text { Vanadium } & \mathrm{mg} / \mathrm{kg} & 35.30 & \mathrm{~J} & 59.80 & \mathrm{~J} & 54.00 & \mathrm{~J} \\ \text { Zinc } & \mathrm{mg} / \mathrm{kg} & 30.50 & \mathrm{~J} & 85.10 & \mathrm{~J} & 177.00 & \mathrm{~J}\end{array}$


D-10

Table D.1 (continued)

\begin{tabular}{|c|c|c|c|c|c|c|c|c|c|}
\hline Analysis & Units & $\begin{array}{l}\text { A horizon } \\
\text { result }\end{array}$ & Qualifier & $\begin{array}{l}\text { B horizon } \\
\text { result }\end{array}$ & Qualifier & $\begin{array}{l}\mathrm{C} \text { horizon } \\
\text { result }\end{array}$ & Qualifier & $\begin{array}{l}\text { A lab split } \\
\text { result }\end{array}$ & Qualifier \\
\hline
\end{tabular}

Location=ORR; Formation $=$ CHEPULTEPEC; Site $=77,85,86$; Sample type $=$ Regular; $A=5136 ; B=5139 ; C=5142 ; A$ lab split $=$ '; Phase $=2$

$\begin{array}{lrrrrrrr}\text { Aluminum } & \mathrm{mg} / \mathrm{kg} & 9230.00 & & 20600.00 & & 18400.00 & \\ \text { Antimony } & \mathrm{mg} / \mathrm{kg} & 0.43 & \mathrm{U} & 0.44 & \mathrm{U} & 0.45 & \mathrm{U} \\ \text { Arsenic } & \mathrm{mg} / \mathrm{kg} & 7.00 & & 13.70 & & 15.10 & \\ \text { Barium } & \mathrm{mg} / \mathrm{kg} & 101.00 & & 41.10 & \mathrm{~B} & 119.00 & \\ \text { Beryllium } & \mathrm{mg} / \mathrm{kg} & 0.88 & \mathrm{U} & 1.50 & \mathrm{U} & 1.80 & \mathrm{U} \\ \text { Boron } & \mathrm{mg} / \mathrm{kg} & 5.00 & \mathrm{U} & 5.20 & \mathrm{U} & 5.40 & \mathrm{U} \\ \text { Cadmium } & \mathrm{mg} / \mathrm{kg} & 0.22 & \mathrm{U} & 0.22 & \mathrm{U} & 0.22 & \mathrm{U} \\ \text { Calcium } & \mathrm{mg} / \mathrm{kg} & 585.00 & \mathrm{~B} & 979.00 & \mathrm{~B} & 447.00 & \mathrm{~B} \\ \text { Chromium } & \mathrm{mg} / \mathrm{kg} & 11.70 & \mathrm{~J} & 26.50 & \mathrm{~J} & 24.90 & \mathrm{~J} \\ \text { Cobalt } & \mathrm{mg} / \mathrm{kg} & 16.40 & & 4.80 & \mathrm{U} & 31.20 & \\ \text { Copper } & \mathrm{mg} / \mathrm{kg} & 5.90 & \mathrm{U} & 20.60 & & 26.80 & \\ \text { Cyanide } & \mathrm{mg} / \mathrm{kg} & 1.00 & \mathrm{U} & 1.10 & \mathrm{U} & 1.10 & \mathrm{U} \\ \text { Iron } & \mathrm{mg} / \mathrm{kg} & 14200.00 & \mathrm{~J} & 35100.00 & \mathrm{~J} & 36400.00 & \mathrm{~J} \\ \text { Lead } & \mathrm{mg} / \mathrm{kg} & 20.70 & & 10.80 & & 17.60 & \\ \text { Lithium } & \mathrm{mg} / \mathrm{kg} & 3.10 & \mathrm{U} & 12.40 & \mathrm{~B} & 17.50 & \mathrm{~B} \\ \text { Magnesium } & \mathrm{mg} / \mathrm{kg} & 441.00 & \mathrm{~B} & 1230.00 & & 895.00 & \mathrm{~B} \\ \text { Manganese } & \mathrm{mg} / \mathrm{kg} & 2050.00 & & 138.00 & & 1880.00 & \\ \text { Mercury } & \mathrm{mg} / \mathrm{kg} & 0.18 & \mathrm{~J} & 0.14 & \mathrm{~J} & 0.12 & \mathrm{~J} \\ \text { Molybdenum } & \mathrm{mg} / \mathrm{kg} & 2.70 & \mathrm{U} & 2.80 & \mathrm{U} & 2.90 & \mathrm{U} \\ \text { Mickel } & \mathrm{mg} / \mathrm{kg} & 10.50 & \mathrm{U} & 22.00 & & 39.60 & \\ \text { Potassium } & \mathrm{mg} / \mathrm{kg} & 305.00 & \mathrm{U} & 1490.00 & & 1410.00 & \\ \text { Selenium } & \mathrm{mg} / \mathrm{kg} & 0.56 & \mathrm{~J} & 0.48 & \mathrm{~J} & 0.56 & \mathrm{~J} \\ \text { Silicon } & \mathrm{mg} / \mathrm{kg} & 592.00 & \mathrm{~J} & 700.00 & \mathrm{~J} & 683.00 & \mathrm{~J} \\ \text { Silver } & \mathrm{mg} / \mathrm{kg} & 0.83 & \mathrm{U} & 0.86 & \mathrm{U} & 0.89 & \mathrm{U} \\ \text { Sodium } & \mathrm{mg} / \mathrm{kg} & 331.00 & \mathrm{~B} & 331.00 & \mathrm{~B} & 340.00 & \mathrm{~B} \\ \text { Strontium } & \mathrm{mg} / \mathrm{kg} & 4.10 & \mathrm{~B} & 7.20 & \mathrm{~B} & 2.70 & \mathrm{~B} \\ \text { Sulfate } & \mathrm{mg} / \mathrm{kg} & 79.90 & & 36.40 & & 12.30 & \\ \text { Thallium } & \mathrm{mg} / \mathrm{kg} & 0.43 & \mathrm{U} & 0.44 & \mathrm{U} & 0.45 & \mathrm{U} \\ \text { Vanadium } & \mathrm{mg} / \mathrm{kg} & 29.20 & \mathrm{~J} & 56.80 & \mathrm{~J} & 58.40 & \mathrm{~J} \\ \text { Zinc } & \mathrm{mg} / \mathrm{kg} & 75.30 & \mathrm{~J} & 348.00 & \mathrm{~J} & 409.00 & \mathrm{~J}\end{array}$

Location=ORR; Formation=CHICKAMAUGA; Bethel V Site=101,102,103; Sample type=Regular; $A=5196 ; B=5199 ; C=5202 ; A$ lab split $=$ ' '; Phase $=2$

$\begin{array}{lrrlrrrr}\text { Aluminum } & \mathrm{mg} / \mathrm{kg} & 18000.00 & & 28300.00 & & 30900.00 & \\ \text { Ant imony } & \mathrm{mg} / \mathrm{kg} & 0.45 & \mathrm{UJ} & 0.44 & \text { UJ } & 0.43 & \text { UJ } \\ \text { Arsenic } & \mathrm{mg} / \mathrm{kg} & 5.70 & \mathrm{~J} & 11.20 & \mathrm{~J} & 16.90 & \mathrm{~J} \\ \text { Barium } & \mathrm{mg} / \mathrm{kg} & 103.00 & & 142.00 & & 140.00 & \\ \text { Beryllium } & \mathrm{mg} / \mathrm{kg} & 1.20 & & 1.90 & & 2.20 & \\ \text { Cadmium } & \mathrm{mg} / \mathrm{kg} & 0.23 & \mathrm{UJ} & 0.22 & \mathrm{UJ} & 0.21 & \text { UJ } \\ \text { Calcium } & \mathrm{mg} / \mathrm{kg} & 3500.00 & & 3640.00 & & 6840.00 & \\ \text { Chromium } & \mathrm{mg} / \mathrm{kg} & 23.40 & \mathrm{~J} & 30.40 & \mathrm{~J} & 29.40 & \mathrm{~J} \\ \text { Cobalt } & \mathrm{mg} / \mathrm{kg} & 15.20 & & 11.60 & & 31.90 & \\ \text { Copper } & \mathrm{mg} / \mathrm{kg} & 20.20 & & 27.70 & & 34.10 & \\ \text { Cyanide } & \mathrm{mg} / \mathrm{kg} & 1.10 & \mathrm{U} & 1.10 & \mathrm{U} & 1.10 & \mathrm{U} \\ \text { Iron } & \mathrm{mg} / \mathrm{kg} & 31000.00 & & 50100.00 & & 51000.00 & \\ \text { Lead } & \mathrm{mg} / \mathrm{kg} & 33.30 & \mathrm{~J} & 25.60 & \mathrm{~J} & 50.10 & \mathrm{~J} \\ \text { Magnesium } & \mathrm{mg} / \mathrm{kg} & 2200.00 & & 3060.00 & & 4540.00 & \\ \text { Manganese } & \mathrm{mg} / \mathrm{kg} & 1510.00 & \mathrm{~J} & 539.00 & \mathrm{~J} & 667.00 & \mathrm{~J} \\ \text { Mercury } & \mathrm{mg} / \mathrm{kg} & 0.21 & & 0.10 & \mathrm{U} & 0.09 & \mathrm{U} \\ \text { Nickel } & \mathrm{mg} / \mathrm{kg} & 18.50 & & 28.80 & & 39.10 & \\ \text { Potassium } & \mathrm{mg} / \mathrm{kg} & 2220.00 & & 2370.00 & & 2210.00 & \\ \text { Selenium } & \mathrm{mg} / \mathrm{kg} & 0.95 & \mathrm{~B} & 0.97 & \mathrm{~B} & 0.92 & \mathrm{~B} \\ \text { Silver } & \mathrm{mg} / \mathrm{kg} & 0.89 & \mathrm{U} & 0.88 & \mathrm{U} & 0.86 & \mathrm{U} \\ \text { Sodium } & \mathrm{mg} / \mathrm{kg} & 377.00 & \mathrm{~B} & 433.00 & \mathrm{~B} & 409.00 & \mathrm{~B} \\ \text { Sulfate } & \mathrm{mg} / \mathrm{kg} & 83.20 & & 95.70 & & 73.70 & \\ \text { Thallium } & \mathrm{mg} / \mathrm{kg} & 0.68 & \mathrm{U} & 0.66 & \mathrm{U} & 0.64 & \mathrm{U} \\ \text { Vanadium } & \mathrm{mg} / \mathrm{kg} & 28.60 & & 36.00 & & 34.20 & \\ \text { Zinc } & \mathrm{mg} / \mathrm{kg} & 51.30 & \mathrm{~J} & 63.60 & \mathrm{~J} & 99.00 & \mathrm{~J}\end{array}$

Location=ORR; Formation=CHICKAMAUGA; Bethel V Site=104,108,110; Sample type=Regular; $A=5205 ; B=5208 ; C=5211 ; A$ lab split=' '; Phase $=2$

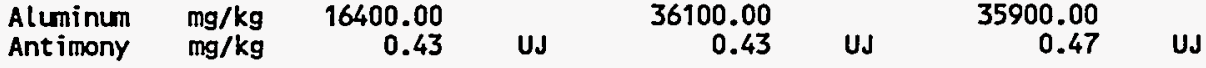


D-11

Table D.1 (continued)

\begin{tabular}{|c|c|c|c|c|c|c|c|c|c|}
\hline Analysis & Units & $\begin{array}{c}\text { A horizon } \\
\text { result }\end{array}$ & Qualifier & $\begin{array}{l}\text { B horizon } \\
\text { result }\end{array}$ & Qualifier & $\begin{array}{l}\text { C horizon } \\
\text { result }\end{array}$ & Qualifier & $\begin{array}{l}\text { A lab split } \\
\text { result }\end{array}$ & Qualifier \\
\hline $\begin{array}{l}\text { Arsenic } \\
\text { Barium } \\
\text { Beryllium } \\
\text { Boron } \\
\text { Cadmium } \\
\text { Calcium } \\
\text { Chromium } \\
\text { Cobalt } \\
\text { Copper } \\
\text { Cyanide } \\
\text { Iron } \\
\text { Lead } \\
\text { Lithium } \\
\text { Magnesium } \\
\text { Manganese } \\
\text { Mercury } \\
\text { Molybdenum } \\
\text { Nickel } \\
\text { Potassium } \\
\text { Selenium } \\
\text { silicon } \\
\text { silver } \\
\text { Sodium } \\
\text { Strontium } \\
\text { Sulfate } \\
\text { Thallium } \\
\text { Vanadium } \\
\text { Zinc }\end{array}$ & $\begin{array}{l}\mathrm{mg} / \mathrm{kg} \\
\mathrm{mg} / \mathrm{kg} \\
\mathrm{mg} / \mathrm{kg} \\
\mathrm{mg} / \mathrm{kg} \\
\mathrm{mg} / \mathrm{kg} \\
\mathrm{mg} / \mathrm{kg} \\
\mathrm{mg} / \mathrm{kg} \\
\mathrm{mg} / \mathrm{kg} \\
\mathrm{mg} / \mathrm{kg} \\
\mathrm{mg} / \mathrm{kg} \\
\mathrm{mg} / \mathrm{kg} \\
\mathrm{mg} / \mathrm{kg} \\
\mathrm{mg} / \mathrm{kg} \\
\mathrm{mg} / \mathrm{kg} \\
\mathrm{mg} / \mathrm{kg} \\
\mathrm{mg} / \mathrm{kg} \\
\mathrm{mg} / \mathrm{kg} \\
\mathrm{mg} / \mathrm{kg} \\
\mathrm{mg} / \mathrm{kg} \\
\mathrm{mg} / \mathrm{kg} \\
\mathrm{mg} / \mathrm{kg} \\
\mathrm{mg} / \mathrm{kg} \\
\mathrm{mg} / \mathrm{kg} \\
\mathrm{mg} / \mathrm{kg} \\
\mathrm{mg} / \mathrm{kg} \\
\mathrm{mg} / \mathrm{kg} \\
\mathrm{mg} / \mathrm{kg} \\
\mathrm{mg} / \mathrm{kg}\end{array}$ & $\begin{array}{r}7.00 \\
72.50 \\
1.10 \\
4.70 \\
0.21 \\
2020.00 \\
44.70 \\
18.70 \\
11.40 \\
1.10 \\
43000.00 \\
32.40 \\
10.20 \\
1180.00 \\
740.00 \\
0.16 \\
2.60 \\
12.30 \\
1140.00 \\
0.56 \\
512.00 \\
0.86 \\
414.00 \\
5.60 \\
64.00 \\
0.65 \\
48.00 \\
37.70\end{array}$ & $\begin{array}{l}B \\
\mathbf{J} \\
\mathbf{U} \\
\mathbf{B} \\
\mathbf{J} \\
\mathbf{U} \\
\mathbf{J} \\
\mathbf{J}\end{array}$. & $\begin{array}{r}5.60 \\
125.00 \\
1.60 \\
4.80 \\
0.21 \\
2590.00 \\
44.50 \\
13.30 \\
21.20 \\
1.10 \\
55400.00 \\
25.60 \\
29.80 \\
2490.00 \\
291.00 \\
0.11 \\
2.60 \\
21.60 \\
2700.00 \\
0.62 \\
549.00 \\
0.86 \\
384.00 \\
7.80 \\
75.00 \\
0.65 \\
55.90 \\
58.60\end{array}$ & $\begin{array}{l}B \\
\mathbf{J} \\
\mathbf{U} \\
B \\
\mathbf{J} \\
\mathbf{U} \\
\mathbf{J} \\
\mathbf{J}\end{array}$ & $\begin{array}{r}4.30 \\
160.00 \\
1.80 \\
4.80 \\
0.23 \\
5960.00 \\
38.60 \\
14.50 \\
25.60 \\
1.10 \\
55000.00 \\
40.00 \\
33.70 \\
3130.00 \\
645.00 \\
0.11 \\
2.60 \\
26.20 \\
2890.00 \\
0.47 \\
468.00 \\
0.93 \\
485.00 \\
15.00 \\
22.00 \\
0.70 \\
47.70 \\
70.40\end{array}$ & $\begin{array}{l}\text { UJ } \\
J \\
U \\
B \\
J\end{array}$ & & \\
\hline
\end{tabular}

Location=ORR; Formation=CHICKAMAUGA Bethel V; Site=115,116,117; Sample type=Regular; $A=5216 ; B=5222 ; C=5225 ; A$ lab split $=5217 ;$ Phase $=2$

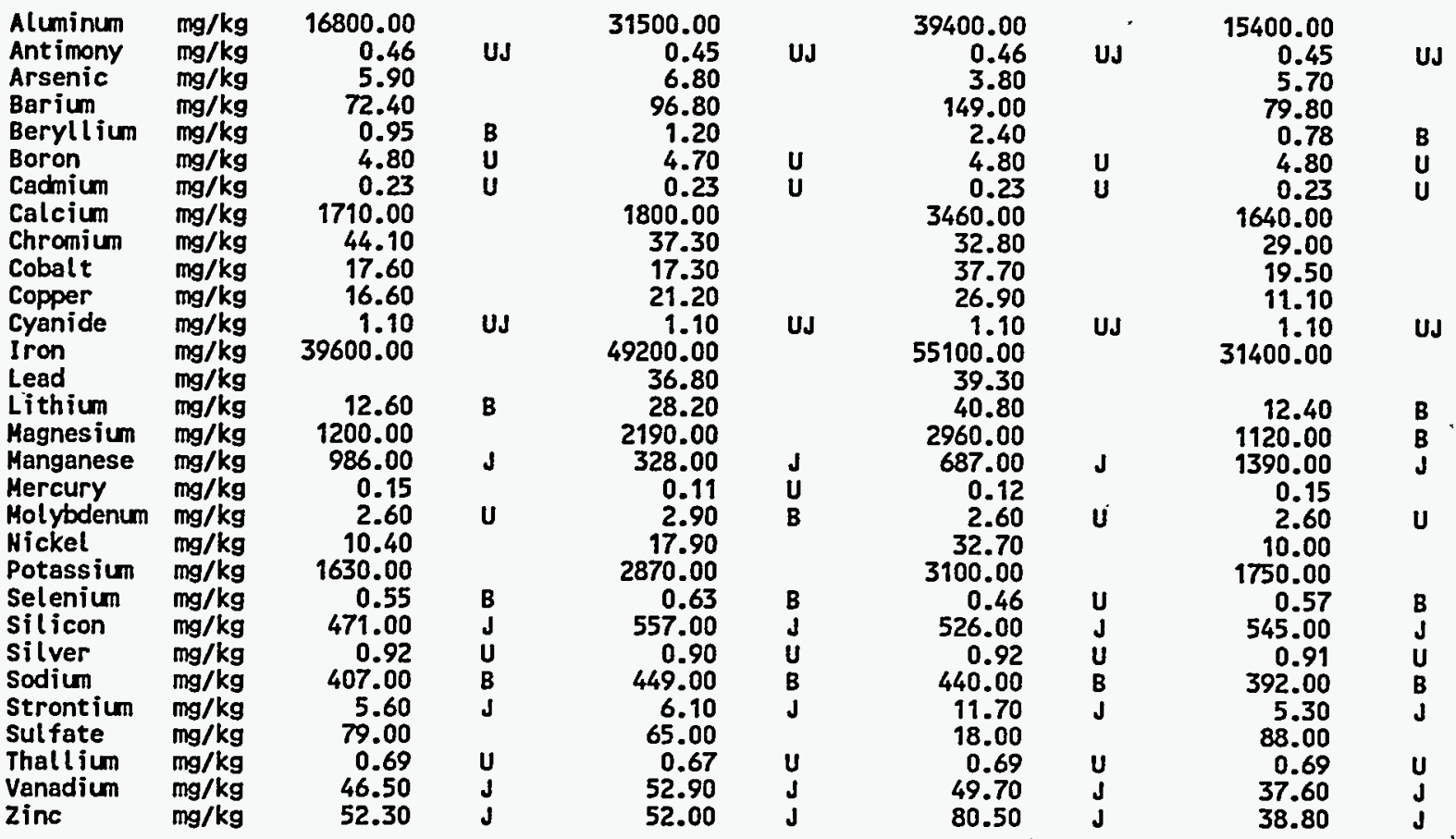

Location=ORR; Formation=CHICKAMAUGA Bethel V; Site=93,99,100; Sample type=Regular; $A=5187 ; B=5190 ; C=5193 ; A$ lab split=' '; Phase $=2$

$\begin{array}{lrrlrlrl}\text { Aluminum } & \mathrm{mg} / \mathrm{kg} & 15700.00 & & 24100.00 & & 28300.00 & \\ \text { Antimony } & \mathrm{mg} / \mathrm{kg} & 0.45 & \text { UJ } & 0.44 & \text { UJ } & 0.48 & \mathrm{~J} \\ \text { Arsenic } & \mathrm{mg} / \mathrm{kg} & 6.60 & \mathrm{~J} & 5.80 & \mathrm{~J} & 5.50 & \mathrm{~J} \\ \text { Barium } & \mathrm{mg} / \mathrm{kg} & 70.60 & & 94.60 & & 133.00 & \end{array}$


D-12

Table D.1 (continued)

\begin{tabular}{|c|c|c|c|c|c|c|c|c|c|}
\hline Analysis & Units & $\begin{array}{l}\text { A horizon } \\
\text { result }\end{array}$ & Qualifier & $\begin{array}{l}\text { B horizon } \\
\text { result }\end{array}$ & Qualifier & $\begin{array}{l}\mathrm{C} \text { horizon } \\
\text { result }\end{array}$ & Qualifier & $\begin{array}{l}\text { A lab split } \\
\text { result }\end{array}$ & Qualifier \\
\hline $\begin{array}{l}\text { Beryll ium } \\
\text { Cadmium } \\
\text { Calcium } \\
\text { Chromium } \\
\text { Cobalt } \\
\text { Copper } \\
\text { Cyanide } \\
\text { Iron } \\
\text { Lead } \\
\text { Magnesium } \\
\text { Manganese } \\
\text { Mercury } \\
\text { Mickel } \\
\text { Potassium } \\
\text { Selenium } \\
\text { Silver } \\
\text { Sodium } \\
\text { Sulfate } \\
\text { Thall ium } \\
\text { Vanadium } \\
\text { Zinc }\end{array}$ & $\begin{array}{l}\mathrm{mg} / \mathrm{kg} \\
\mathrm{mg} / \mathrm{kg} \\
\mathrm{mg} / \mathrm{kg} \\
\mathrm{mg} / \mathrm{kg} \\
\mathrm{mg} / \mathrm{kg} \\
\mathrm{mg} / \mathrm{kg} \\
\mathrm{mg} / \mathrm{kg} \\
\mathrm{mg} / \mathrm{kg} \\
\mathrm{mg} / \mathrm{kg} \\
\mathrm{mg} / \mathrm{kg} \\
\mathrm{mg} / \mathrm{kg} \\
\mathrm{mg} / \mathrm{kg} \\
\mathrm{mg} / \mathrm{kg} \\
\mathrm{mg} / \mathrm{kg} \\
\mathrm{mg} / \mathrm{kg} \\
\mathrm{mg} / \mathrm{kg} \\
\mathrm{mg} / \mathrm{kg} \\
\mathrm{mg} / \mathrm{kg} \\
\mathrm{mg} / \mathrm{kg} \\
\mathrm{mg} / \mathrm{kg} \\
\mathrm{mg} / \mathrm{kg}\end{array}$ & $\begin{array}{r}0.94 \\
0.22 \\
1000.00 \\
34.80 \\
22.10 \\
21.70 \\
1.10 \\
35300.00 \\
42.00 \\
1210.00 \\
928.00 \\
0.13 \\
14.10 \\
1350.00 \\
1.00 \\
0.88 \\
378.00 \\
181.00 \\
0.67 \\
30.90 \\
46.20\end{array}$ & $\begin{array}{l}\text { U } \\
\text { J } \\
\text { J }\end{array}$ & $\begin{array}{r}1.20 \\
0.22 \\
1400.00 \\
26.80 \\
12.30 \\
25.00 \\
1.10 \\
41800.00 \\
6.80 \\
1760.00 \\
395.00 \\
0.10 \\
23.30 \\
1810.00 \\
1.00 \\
0.91 \\
402.00 \\
85.40 \\
0.66 \\
35.60 \\
62.20\end{array}$ & $\begin{array}{l}U \\
J \\
J \\
U\end{array}$ & $\begin{array}{r}1.40 \\
0.23 \\
3160.00 \\
32.30 \\
16.10 \\
30.30 \\
1.10 \\
50200.00 \\
32.60 \\
2600.00 \\
701.00 \\
0.11 \\
30.90 \\
1870.00 \\
0.97 \\
0.92 \\
352.00 \\
62.20 \\
0.68 \\
38.80 \\
84.30\end{array}$ & $\begin{array}{l}\mathrm{U} \\
\mathrm{J} \\
\mathrm{J} \\
\mathrm{U}\end{array}$ & & \\
\hline
\end{tabular}

Location=ORR; Formation $=$ CHICKAMAUGA $K-25$; Site=118,122,124; Sample type =Regular; $A=5240 ; B=5246 ; C=5249 ; A$ lab split $=5241 ;$ Phase $=2$

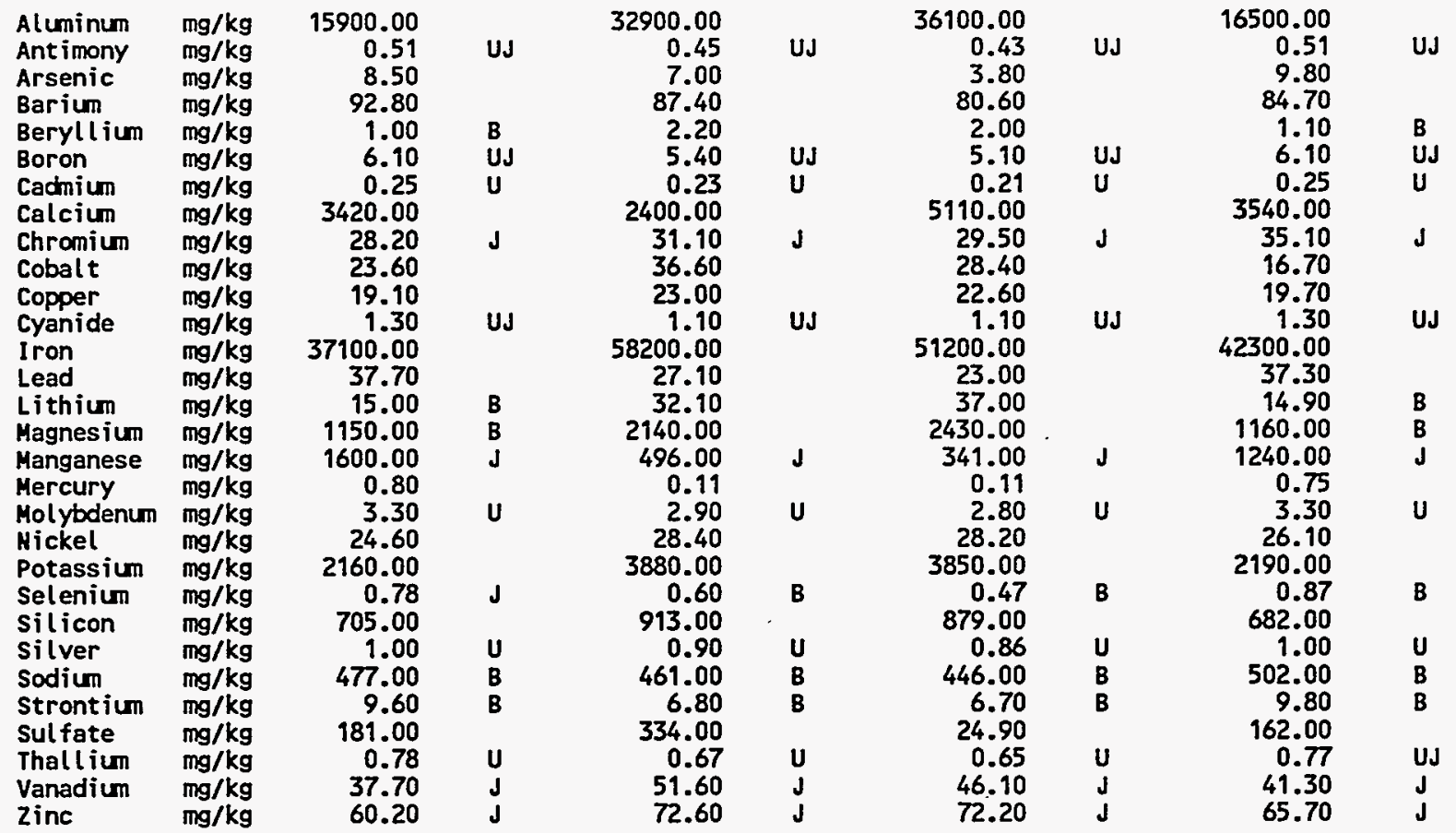

Location=ORR; Formation =CHICKAMAUGA K-25; Site=119,123,127; Sample type=Regular; $A=5252 ; B=5257 ; C=5260 ; A$ lab split $=5253 ;$ Phase $=2$

\begin{tabular}{|c|c|c|c|c|c|c|c|c|c|}
\hline $\begin{array}{l}\text { Aluminum } \\
\text { Antimony } \\
\text { Arsenic } \\
\text { Barium } \\
\text { Beryllium } \\
\text { Boron } \\
\text { Cadmium } \\
\text { Calcium } \\
\text { Chromium } \\
\text { Cobalt }\end{array}$ & $\begin{array}{l}\mathrm{mg} / \mathrm{kg} \\
\mathrm{mg} / \mathrm{kg} \\
\mathrm{mg} / \mathrm{kg} \\
\mathrm{mg} / \mathrm{kg} \\
\mathrm{mg} / \mathrm{kg} \\
\mathrm{mg} / \mathrm{kg} \\
\mathrm{mg} / \mathrm{kg} \\
\mathrm{mg} / \mathrm{kg} \\
\mathrm{mg} / \mathrm{kg} \\
\mathrm{mg} / \mathrm{kg}\end{array}$ & $\begin{array}{r}22400.00 \\
0.48 \\
8.70 \\
99.70 \\
1.40 \\
5.70 \\
0.24 \\
1430.00 \\
39.80 \\
19.40\end{array}$ & $\begin{array}{l}\text { UJ } \\
\text { U }\end{array}$ & $\begin{array}{r}40300.00 \\
0.43 \\
11.10 \\
133.00 \\
1.90 \\
5.20 \\
0.22 \\
1530.00 \\
39.00 \\
10.80\end{array}$ & $\begin{array}{l}U_{U} \\
\mathbf{U}\end{array}$ & $\begin{array}{r}40500.00 \\
0.44 \\
10.80 \\
158.00 \\
1.90 \\
5.30 \\
0.22 \\
1840.00 \\
36.00 \\
21.50\end{array}$ & $\underset{\mathbf{U}}{\mathbf{U}}$ & $\begin{array}{r}21300.00 \\
0.48 \\
9.40 \\
96.30 \\
1.30 \\
5.70 \\
0.24 \\
1410.00 \\
29.80 \\
18.30\end{array}$ & $\bigcup_{U}^{U J}$ \\
\hline
\end{tabular}


Table D.1 (continued)

\begin{tabular}{|c|c|c|c|c|c|c|c|c|c|}
\hline Analysis & Units & $\begin{array}{l}\text { A horizon } \\
\text { result }\end{array}$ & Qualifier & $\begin{array}{l}\text { B horizon } \\
\text { result }\end{array}$ & Qualifier & $\begin{array}{l}C \text { horizon } \\
\text { result }\end{array}$ & Qualifier & $\begin{array}{l}\text { A lab split } \\
\text { result }\end{array}$ & Qualifier \\
\hline $\begin{array}{l}\text { Copper } \\
\text { Cyanide } \\
\text { Iron } \\
\text { Lead } \\
\text { Lithium } \\
\text { Magnesium } \\
\text { Manganese } \\
\text { Mercury } \\
\text { Molybdenum } \\
\text { Hickel } \\
\text { Potassium } \\
\text { Selenium } \\
\text { Silicon } \\
\text { Silver } \\
\text { Sodium } \\
\text { Strontium } \\
\text { Sulfate } \\
\text { Thall ium } \\
\text { Vanadium } \\
\text { Zinc }\end{array}$ & $\begin{array}{l}\mathrm{mg} / \mathrm{kg} \\
\mathrm{mg} / \mathrm{kg} \\
\mathrm{mg} / \mathrm{kg} \\
\mathrm{mg} / \mathrm{kg} \\
\mathrm{mg} / \mathrm{kg} \\
\mathrm{mg} / \mathrm{kg} \\
\mathrm{mg} / \mathrm{kg} \\
\mathrm{mg} / \mathrm{kg} \\
\mathrm{mg} / \mathrm{kg} \\
\mathrm{mg} / \mathrm{kg} \\
\mathrm{mg} / \mathrm{kg} \\
\mathrm{mg} / \mathrm{kg} \\
\mathrm{mg} / \mathrm{kg} \\
\mathrm{mg} / \mathrm{kg} \\
\mathrm{mg} / \mathrm{kg} \\
\mathrm{mg} / \mathrm{kg} \\
\mathrm{mg} / \mathrm{kg} \\
\mathrm{mg} / \mathrm{kg} \\
\mathrm{mg} / \mathrm{kg} \\
\mathrm{mg} / \mathrm{kg}\end{array}$ & $\begin{array}{r}11.30 \\
1.20 \\
39600.00 \\
31.30 \\
20.50 \\
1350.00 \\
2350.00 \\
0.65 \\
3.10 \\
19.30 \\
2230.00 \\
0.83 \\
660.00 \\
0.96 \\
452.00 \\
40.80 \\
319.00 \\
0.71 \\
43.20 \\
58.40\end{array}$ & $\begin{array}{l}\text { B } \\
\text { U } \\
\text { B } \\
B \\
\text { U } \\
\mathbf{J} \\
\mathbf{J}\end{array}$ & $\begin{array}{r}15.00 \\
1.10^{.} \\
56600.00 \\
15.50 \\
42.30 \\
2880.00 \\
612.00 \\
0.17 \\
2.80 \\
22.00 \\
4660.00 \\
1.00 \\
802.00 \\
0.87 \\
497.00 \\
63.10 \\
169.00 \\
0.65 \\
55.30 \\
89.70\end{array}$ & $\begin{array}{c}\text { 'UJ U } \\
\text { J } \\
\text { U } \\
\text { B } \\
\text { U } \\
\text { B }\end{array}$ & $\begin{array}{r}16.30 \\
1.10 \\
57400.00 \\
15.50 \\
49.90 \\
2670.00 \\
2240.00 \\
0.16 \\
2.90 \\
24.20 \\
3900.00 \\
0.69 \\
782.00 \\
0.89 \\
454.00 \\
44.50 \\
255.00 \\
0.66 \\
53.70 \\
71.20\end{array}$ & $\begin{array}{l}\mathbf{B} \\
\mathbf{U} \\
\mathbf{B} \\
\mathbf{B}\end{array}$ & $\begin{array}{r}11.00 \\
1.20 \\
37800.00 \\
31.90 \\
21.10 \\
1390.00 \\
2210.00 \\
0.61 \\
3.10 \\
19.90 \\
2320.00 \\
1.10 \\
732.00 \\
0.95 \\
440.00 \\
39.20 \\
395.00 \\
0.72 \\
40.70 \\
57.60\end{array}$ & $\begin{array}{l}\text { B U J } \\
\text { B } \\
\text { J } \\
\text { U } \\
\text { B } \\
\text { U } \\
B \\
B \\
\text { U } \\
\text { J } \\
\text { J }\end{array}$ \\
\hline
\end{tabular}

Location=ORR; Formation=CHICKAMAUGA $K-25$; Site $=120,126,129 ;$ Sample type $=$ Regular; $A=5228 ; B=5234 ; C=5237 ; A$ lab split $=5229 ;$ Phase $=2$

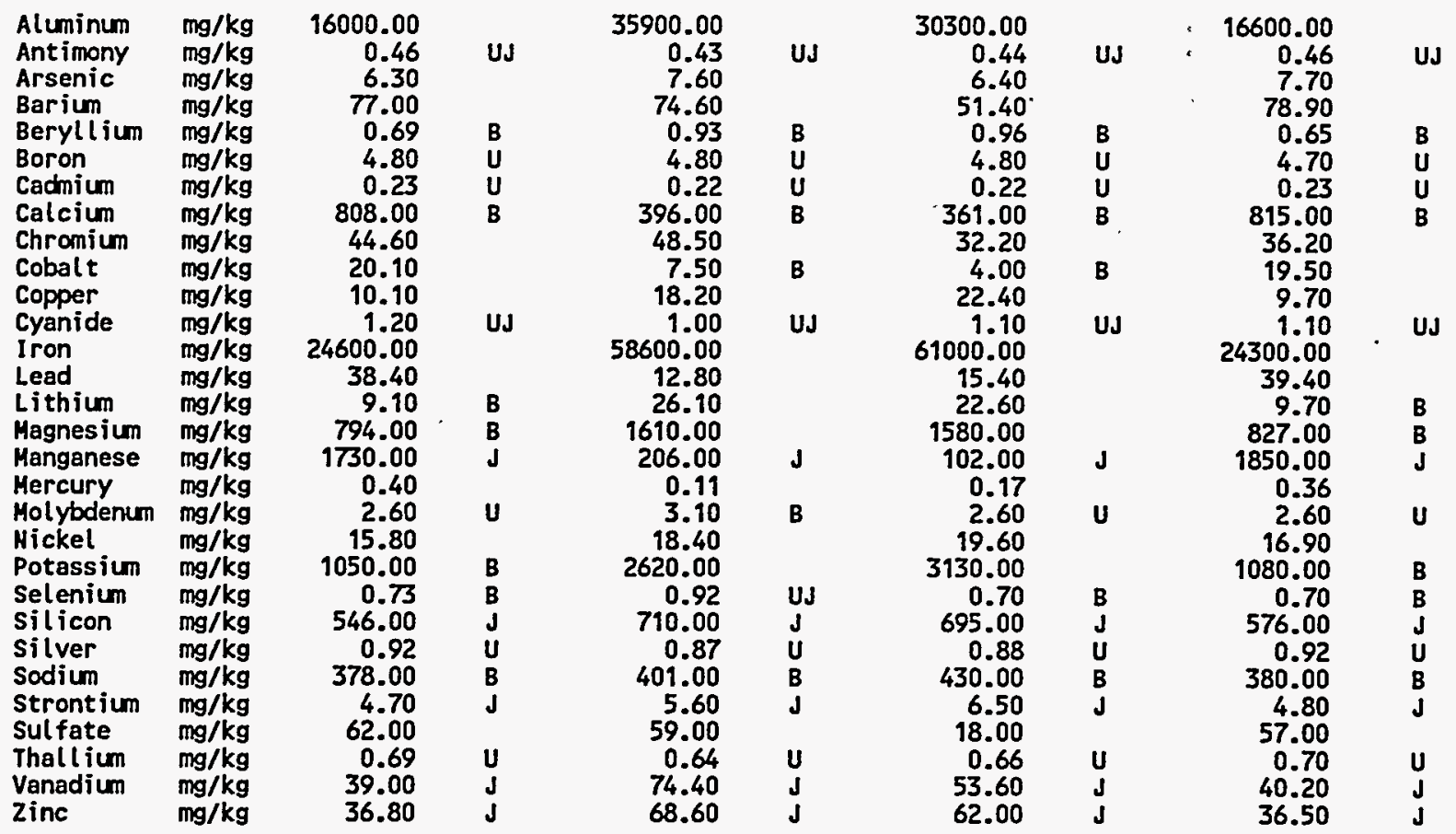

Location $=O R R ;$ Formation $=$ CHICKAMAUGA K-25; Site=121,125,128; Sample type=Regular; $A=5263 ; B=5268 ; C=5271 ; A$ lab split $=5264$ Phase $=2$

\begin{tabular}{|c|c|c|c|c|c|c|c|c|}
\hline $\begin{array}{l}\text { Aluminum } \\
\text { Antimony } \\
\text { Arsenic } \\
\text { Barium } \\
\text { Beryllium } \\
\text { Boron } \\
\text { Cadmium } \\
\text { Calcium } \\
\text { Chromium } \\
\text { Cobalt } \\
\text { Copper }\end{array}$ & $\begin{array}{l}\mathrm{mg} / \mathbf{k g} \\
\mathrm{mg} / \mathbf{k g} \\
\mathrm{mg} / \mathbf{k g} \\
\mathrm{mg} / \mathbf{k g} \\
\mathrm{mg} / \mathbf{k g} \\
\mathrm{mg} / \mathbf{k g} \\
\mathrm{mg} / \mathbf{k g} \\
\mathrm{mg} / \mathbf{k g} \\
\mathrm{mg} / \mathbf{k g} \\
\mathrm{mg} / \mathbf{k g} \\
\mathrm{mg} / \mathbf{k g}\end{array}$ & $\begin{array}{r}13100.00 \\
0.44 \\
6.20 \\
51.20 \\
0.77 \\
5.30 \\
0.22 \\
862.00 \\
31.40 \\
21.50 \\
8.10\end{array}$ & $\begin{array}{l}\text { B } \\
\text { UJ } \\
\mathbf{U} \\
\mathbf{B} \\
\mathbf{J}\end{array}$ & $\begin{array}{r}30800.00 \\
0.44 \\
5.10 \\
73.70 \\
1.10 \\
5.30 \\
0.22 \\
1390.00 \\
23.30 \\
8.70 \\
16.40\end{array}$ & $\begin{array}{l}\text { B } \\
\text { UJ } \\
\mathbf{U}\end{array}$ & $\begin{array}{r}31300.00 \\
0.41 \\
8.10 \\
59.60 \\
1.10 \\
5.00 \\
0.21 \\
638.00 \\
21.50 \\
16.90 \\
15.90\end{array}$ & $\begin{array}{l}\text { UJ } \\
\mathbf{U} \\
\mathbf{B} \\
\mathbf{J}\end{array}$ & $\begin{array}{r}12800.00 \\
0.44 \\
5.40 \\
51.00 \\
0.69 \\
5.30 \\
0.22 \\
852.00 \\
18.80 \\
17.30 \\
7.80\end{array}$ \\
\hline
\end{tabular}


Table D.1 (continued)

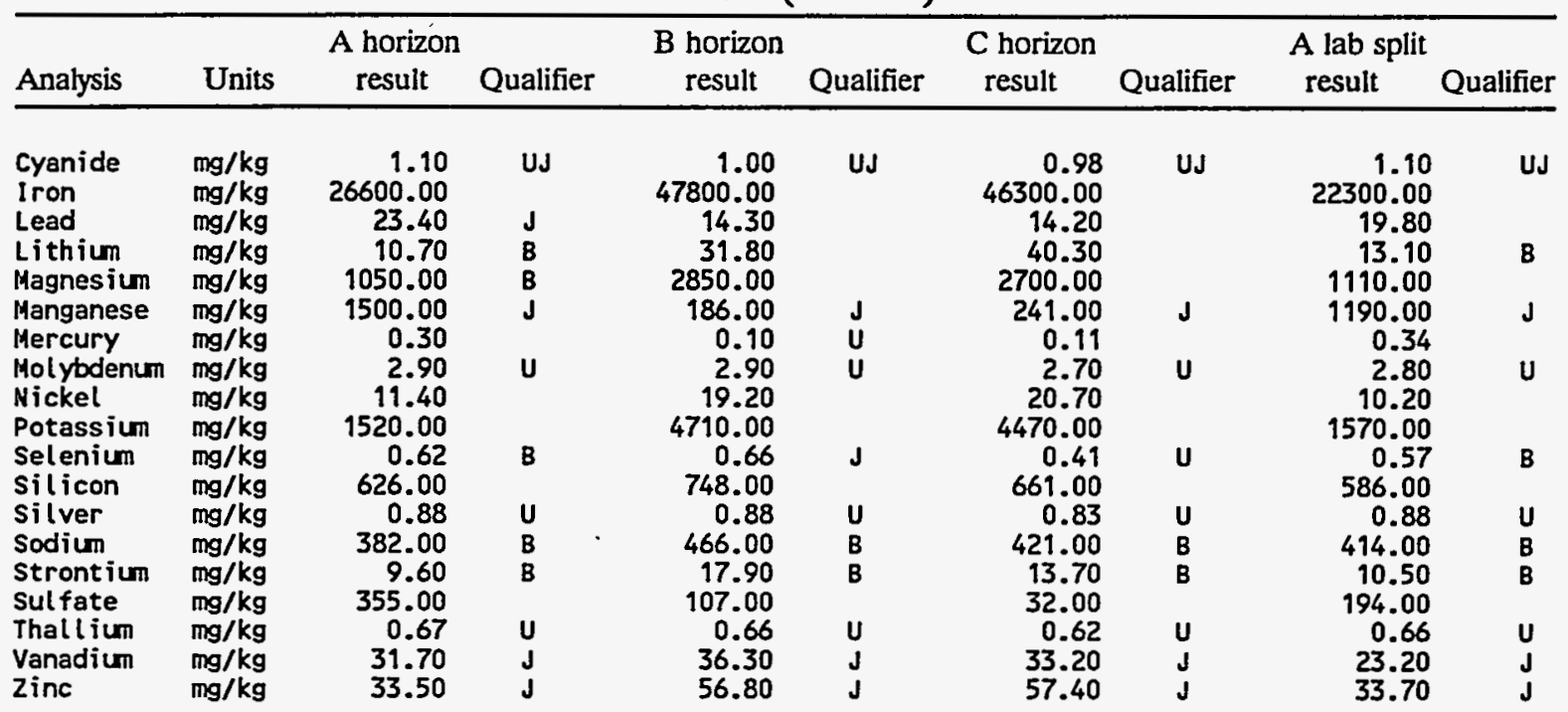

Location $=O R R ;$ Formation $=C O P P E R$ RIDGE; Site $=45,60,75 ;$ Sample type $=$ Regular; $A=5109 ; B=5112 ; C=5115 ; A$ lab split=' '; Phase=2

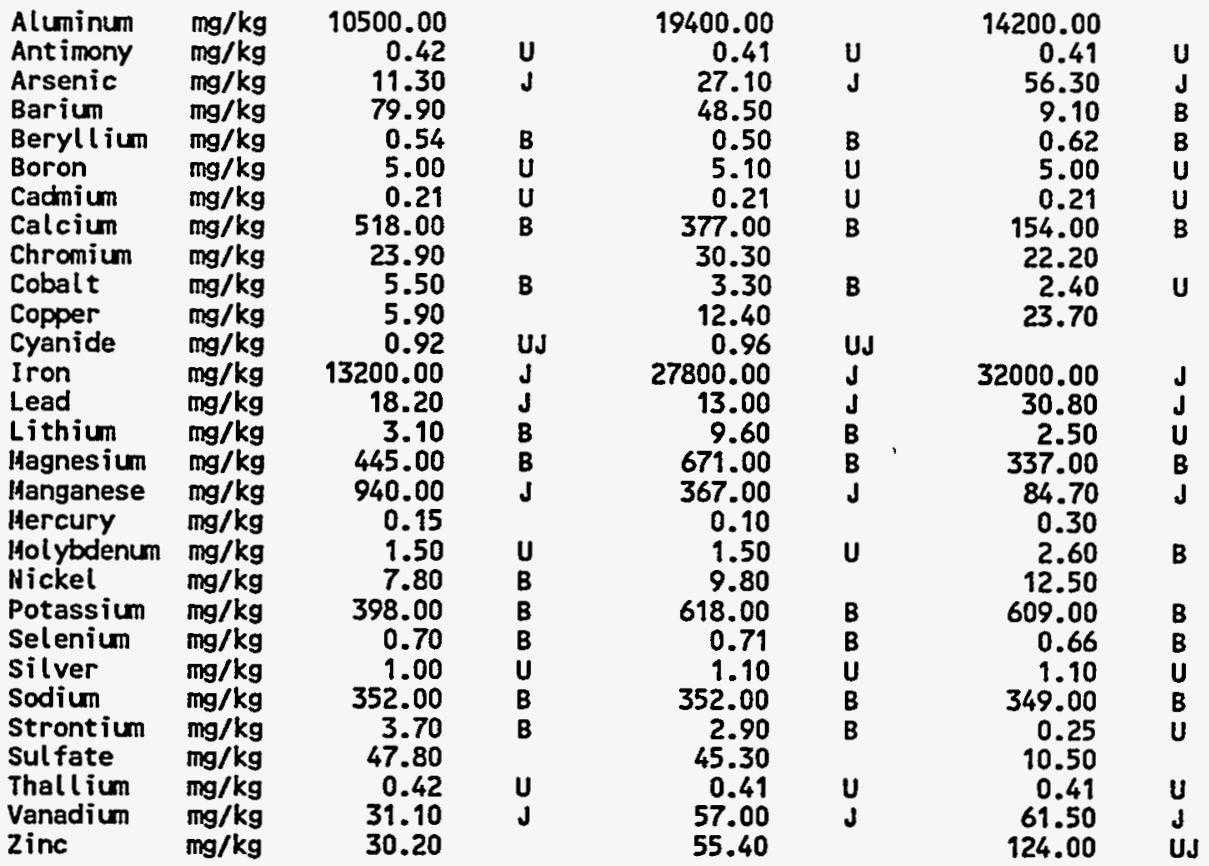

Location=ORR; Formation =COPPER RIDGE; Site $=51,55,62$; Sample type $=$ Regular; $A=5100 ; B=5104 ; C=5106 ; A$ lab split =' '; Phase $=2$

$\begin{array}{llrlrlrl}\text { Aluminum } & \mathrm{mg} / \mathrm{kg} & 9780.00 & & 12400.00 & & 14300.00 & \\ \text { Antinony } & \mathrm{mg} / \mathrm{kg} & 0.43 & \mathrm{U} & 0.43 & \mathrm{U} & 0.45 & \mathrm{U} \\ \text { Arsenic } & \mathrm{mg} / \mathrm{kg} & 17.70 & \mathrm{~J} & 38.90 & \mathrm{~J} & 58.40 & \mathrm{~J} \\ \text { Barium } & \mathrm{mg} / \mathrm{kg} & 62.90 & & 36.30 & \mathrm{~B} & 10.00 & \mathrm{~B} \\ \text { Berylium } & \mathrm{mg} / \mathrm{kg} & 0.51 & \mathrm{~B} & 0.49 & \mathrm{~B} & 0.81 & \mathrm{~B} \\ \text { Boron } & \mathrm{mg} / \mathrm{kg} & 4.90 & \mathrm{U} & 5.20 & \mathrm{U} & 5.40 & \mathrm{U} \\ \text { Cadnium } & \mathrm{mg} / \mathrm{kg} & 0.21 & \mathrm{U} & 0.22 & \mathrm{U} & 0.22 & \mathrm{U} \\ \text { Calcium } & \mathrm{mg} / \mathrm{kg} & 398.00 & \mathrm{~B} & 447.00 & \mathrm{~B} & 219.00 & \mathrm{~B} \\ \text { Chromium } & \mathrm{mg} / \mathrm{kg} & 10.50 & & 26.20 & & 32.00 & \\ \text { Cobalt } & \mathrm{mg} / \mathrm{kg} & 6.40 & \mathrm{~B} & 1.80 & \mathrm{U} & 4.20 & \mathrm{~B} \\ \text { Copper } & \mathrm{mg} / \mathrm{kg} & 5.40 & & 14.60 & & 31.70 & \\ \text { Cyanide } & \mathrm{mg} / \mathrm{kg} & 1.20 & \mathrm{UJ} & 1.10 & \mathrm{UJ} & 0.99 & \mathrm{UJ} \\ \text { Iron } & \mathrm{mg} / \mathrm{kg} & 9700.00 & \mathrm{~J} & 29000.00 & \mathrm{~J} & 39800.00 & \mathrm{~J}\end{array}$


D-15

Table D.1 (continued)

\begin{tabular}{|c|c|c|c|c|c|c|c|c|c|}
\hline Analysis & Units & $\begin{array}{l}\text { A horizon } \\
\text { result }\end{array}$ & Qualifier & $\begin{array}{l}\text { B horizon } \\
\text { result }\end{array}$ & Qualifier & $\begin{array}{l}\mathrm{C} \text { horizon } \\
\text { result }\end{array}$ & Qualifier & $\begin{array}{l}\text { A lab split } \\
\text { result }\end{array}$ & Qualifier \\
\hline $\begin{array}{l}\text { Lead } \\
\text { Lithium } \\
\text { Magnesium } \\
\text { Manganese } \\
\text { Mercury } \\
\text { Molybdenum } \\
\text { Hickel } \\
\text { Potassium } \\
\text { Selenium } \\
\text { Silver } \\
\text { Sodium } \\
\text { Strontium } \\
\text { Sulfate } \\
\text { Thallium } \\
\text { Vanadium } \\
\text { Zinc }\end{array}$ & $\begin{array}{l}\mathrm{mg} / \mathrm{kg} \\
\mathrm{mg} / \mathrm{kg} \\
\mathrm{mg} / \mathrm{kg} \\
\mathrm{mg} / \mathrm{kg} \\
\mathrm{mg} / \mathrm{kg} \\
\mathrm{mg} / \mathrm{kg} \\
\mathrm{mg} / \mathrm{kg} \\
\mathrm{mg} / \mathrm{kg} \\
\mathrm{mg} / \mathrm{kg} \\
\mathrm{mg} / \mathrm{kg} \\
\mathrm{mg} / \mathrm{kg} \\
\mathrm{mg} / \mathrm{kg} \\
\mathrm{mg} / \mathrm{kg} \\
\mathrm{mg} / \mathrm{kg} \\
\mathrm{mg} / \mathrm{kg} \\
\mathrm{mg} / \mathrm{kg}\end{array}$ & $\begin{array}{r}25.10 \\
2.80 \\
411.00 \\
943.00 \\
0.14 \\
1.40 \\
7.40 \\
274.00 \\
0.60 \\
1.00 \\
360.00 \\
2.70 \\
44.20 \\
0.43 \\
21.70 \\
29.20\end{array}$ & $\begin{array}{l}\text { J } \\
B \\
B \\
J \\
\text { U } \\
B \\
B \\
B \\
U \\
B \\
B \\
\text { U } \\
\text { J }\end{array}$ & $\begin{array}{r}18.50 \\
4.20 \\
385.00 \\
73.60 \\
0.11 \\
2.90 \\
7.60 \\
428.00 \\
0.89 \\
1.10 \\
371.00 \\
2.90 \\
51.00 \\
0.43 \\
57.80 \\
67.10\end{array}$ & $\begin{array}{l}\text { J } \\
B \\
B \\
J \\
B \\
B \\
B \\
B \\
U \\
B \\
B \\
\text { U } \\
\text { J }\end{array}$ & $\begin{array}{r}39.20 \\
4.00 \\
345.00 \\
106.00 \\
0.30 \\
3.00 \\
14.80 \\
707.00 \\
1.00 \\
1.10 \\
358.00 \\
0.68 \\
17.20 \\
0.45 \\
81.30 \\
148.00\end{array}$ & $\begin{array}{l}\text { J } \\
B \\
B \\
\text { J } \\
\text { B } \\
\text { B } \\
B \\
U \\
B \\
U \\
U \\
\text { J }\end{array}$ & 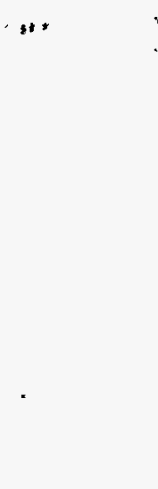 & "xos \\
\hline
\end{tabular}

Location=ORR; Formation=COPPER RIDGE; Site =54,64,83; Sample type $=$ Regular; $A=5118 ; B=5121 ; C=5124 ; A$ lab split=' '; Phase $=2$

$\begin{array}{lrrrrrrr}\text { Aluminum } & \mathrm{mg} / \mathrm{kg} & 10300.00 & & 21200.00 & & 23400.00 & \\ \text { Antimony } & \mathrm{mg} / \mathrm{kg} & 0.43 & \mathrm{U} & 0.41 & \mathrm{U} & 0.44 & \mathrm{U} \\ \text { Arsenic } & \mathrm{mg} / \mathrm{kg} & 25.00 & & 512.00 & & 655.00 & \\ \text { Barium } & \mathrm{mg} / \mathrm{kg} & 71.00 & & 43.60 & & 14.10 & \mathrm{~B} \\ \text { Beryllium } & \mathrm{mg} / \mathrm{kg} & 0.54 & \mathrm{U} & 0.76 & \mathrm{U} & 0.90 & \mathrm{U} \\ \text { Boron } & \mathrm{mg} / \mathrm{kg} & 5.20 & \mathrm{U} & 5.10 & \mathrm{U} & 5.40 & \mathrm{U} \\ \text { Cadmium } & \mathrm{mg} / \mathrm{kg} & 0 . .22 & \mathrm{U} & 0.20 & \mathrm{UJ} & 0.22 & \mathrm{U} \\ \text { Calcium } & \mathrm{mg} / \mathrm{kg} & 529.00 & \mathrm{~B} & 259.00 & \mathrm{~B} & 185.00 & \mathrm{U} \\ \text { Chromium } & \mathrm{mg} / \mathrm{kg} & 13.10 & \mathrm{~J} & 25.70 & \mathrm{~J} & 27.50 & \mathrm{~J} \\ \text { Cobalt } & \mathrm{mg} / \mathrm{kg} & 19.10 & & 5.10 & \mathrm{U} & 3.60 & \mathrm{U} \\ \text { Copper } & \mathrm{mg} / \mathrm{kg} & 10.90 & \mathrm{U} & 28.10 & & 33.50 & \\ \text { Cyanide } & \mathrm{mg} / \mathrm{kg} & 1.10 & \mathrm{U} & 1.00 & \mathrm{U} & 1.00 & \mathrm{U} \\ \text { Iron } & \mathrm{mg} / \mathrm{kg} & 11500.00 & \mathrm{~J} & 33900.00 & \mathrm{~J} & 42000.00 & \mathrm{~J} \\ \text { Lead } & \mathrm{mg} / \mathrm{kg} & 165.00 & \mathrm{~J} & 22.10 & & 31.20 & \\ \text { Lithium } & \mathrm{mg} / \mathrm{kg} & 3.80 & \mathrm{U} & 7.80 & \mathrm{~B} & 6.60 & \mathrm{~B} \\ \text { Magnesium } & \mathrm{mg} / \mathrm{kg} & 487.00 & \mathrm{~B} & 739.00 & \mathrm{~B} & 722.00 & \mathrm{~B} \\ \text { Manganese } & \mathrm{mg} / \mathrm{kg} & 1530.00 & & 120.00 & & 91.80 & \\ \text { Mercury } & \mathrm{mg} / \mathrm{kg} & 0.18 & \mathrm{~J} & 0.10 & \mathrm{~J} & 0.15 & \mathrm{~J} \\ \text { Molybdenum } & \mathrm{mg} / \mathrm{kg} & 2.80 & \mathrm{U} & 4.90 & \mathrm{~B} & 6.20 & \mathrm{~B} \\ \text { Nickel } & \mathrm{mg} / \mathrm{kg} & 11.00 & \mathrm{U} & 17.60 & & 21.30 & \\ \text { Potassium } & \mathrm{mg} / \mathrm{kg} & 413.00 & \mathrm{~B} & 727.00 & \mathrm{~B} & 1040.00 & \mathrm{~B} \\ \text { Selenium } & \mathrm{mg} / \mathrm{kg} & 0.56 & \mathrm{~B} & 0.69 & \mathrm{~B} & 0.70 & \mathrm{~J} \\ \text { Silicon } & \mathrm{mg} / \mathrm{kg} & 633.00 & \mathrm{~J} & 663.00 & \mathrm{~J} & 637.00 & \mathrm{~J} \\ \text { Silver } & \mathrm{mg} / \mathrm{kg} & 0.86 & \mathrm{U} & 0.86 & \mathrm{U} & 0.89 & \mathrm{U} \\ \text { Sodium } & \mathrm{mg} / \mathrm{kg} & 359.00 & \mathrm{U} & 361.00 & \mathrm{~B} & 350.00 & \mathrm{~B} \\ \text { Strontium } & \mathrm{mg} / \mathrm{kg} & 3.70 & \mathrm{~B} & 1.50 & \mathrm{~B} & 0.45 & \mathrm{U} \\ \text { Sulfate } & \mathrm{mg} / \mathrm{kg} & 132.00 & & 39.00 & & 11.10 & \mathrm{U} \\ \text { Thallium } & \mathrm{mg} / \mathrm{kg} & 0.43 & \mathrm{U} & 0.41 & \mathrm{~B} & 0.44 & \mathrm{U} \\ \text { Vanadium } & \mathrm{mg} / \mathrm{kg} & 26.00 & \mathrm{~J} & 66.00 & \mathrm{~J} & 74.30 & \mathrm{~J} \\ \text { Zinc } & \mathrm{mg} / \mathrm{kg} & 41.30 & \mathrm{~J} & 90.40 & \mathrm{~J} & 133.00 & \mathrm{~J}\end{array}$

Location=ORR; Formation=COPPER RIDGE; Site=58,59,91; Sample type=Regular; $A=5091 ; B=5094 ; C=5097 ; A$ lab split =' '; Phase $=2$

$\begin{array}{lrrrrrrr}\text { Aluminum } & \mathrm{mg} / \mathrm{kg} & 11600.00 & & 16400.00 & & 21300.00 & \\ \text { Antimony } & \mathrm{mg} / \mathrm{kg} & 0.46 & \mathrm{U} & 0.45 & \mathrm{U} & 0.48 & \mathrm{U} \\ \text { Arsenic } & \mathrm{mg} / \mathrm{kg} & 67.10 & \mathrm{~J} & 72.80 & \mathrm{~J} & 97.40 & \mathrm{~J} \\ \text { Barium } & \mathrm{mg} / \mathrm{kg} & 74.40 & & 32.60 & \mathrm{~B} & 14.20 & \mathrm{~B} \\ \text { Beryllium } & \mathrm{mg} / \mathrm{kg} & 0.57 & \mathrm{~B} & 0.79 & \mathrm{~B} & 0.96 & \mathrm{~B} \\ \text { Boron } & \mathrm{mg} / \mathrm{kg} & 5.50 & \mathrm{U} & 5.60 & \mathrm{U} & 5.90 & \mathrm{U} \\ \text { Cadnium } & \mathrm{mg} / \mathrm{kg} & 0.23 & \mathrm{U} & 0.23 & \mathrm{U} & 0.24 & \mathrm{U} \\ \text { Calcium } & \mathrm{mg} / \mathrm{kg} & 594.00 & \mathrm{~B} & 216.00 & \mathrm{~B} & 151.00 & \mathrm{~B} \\ \text { Chromium } & \mathrm{mg} / \mathrm{kg} & 17.20 & & 36.30 & & 35.70 & \\ \text { Cobalt } & \mathrm{mg} / \mathrm{kg} & 5.40 & \mathrm{~B} & 2.50 & \mathrm{U} & 2.00 & \mathrm{U} \\ \text { Copper } & \mathrm{mg} / \mathrm{kg} & 7.80 & & 23.60 & & 35.90 & \\ \text { Cyanide } & \mathrm{mg} / \mathrm{kg} & 1.10 & \mathrm{UJ} & 0.84 & \mathrm{UJ} & 0.99 & \mathrm{UJ} \\ \text { Iron } & \mathrm{mg} / \mathrm{kg} & 13900.00 & \mathrm{~J} & 41700.00 & \mathrm{~J} & 53900.00 & \mathrm{~J} \\ \text { Lead } & \mathrm{mg} / \mathrm{kg} & 28.20 & \mathrm{~J} & 22.10 & \mathrm{~J} & 34.90 & \mathrm{~J} \\ \text { Lithium } & \mathrm{mg} / \mathrm{kg} & 2.80 & \mathrm{U} & 5.10 & \mathrm{~B} & 4.90 & \mathrm{~B}\end{array}$


D-16

Table D.1 (continued)

\begin{tabular}{lrrrrrrrr}
\hline Analysis & Units & $\begin{array}{c}\text { A horizon } \\
\text { result }\end{array}$ & Qualifier & $\begin{array}{c}\text { B horizon } \\
\text { result }\end{array}$ & Qualifier & $\begin{array}{c}\text { C horizon } \\
\text { result }\end{array}$ & Qualifier & $\begin{array}{c}\text { A lab split } \\
\text { result }\end{array}$ \\
Qualifier \\
\hline Magnesium & $\mathrm{mg} / \mathrm{kg}$ & 517.00 & $\mathrm{~B}$ & 550.00 & $\mathrm{~B}$ & 495.00 & $\mathrm{~B}$ & \\
Manganese & $\mathrm{mg} / \mathrm{kg}$ & 959.00 & $\mathrm{~J}$ & 116.00 & $\mathrm{~J}$ & 88.70 & $\mathrm{~J}$ & \\
Mercury & $\mathrm{mg} / \mathrm{kg}$ & 0.16 & & 0.12 & & 0.28 & & \\
Molybdenum & $\mathrm{mg} / \mathrm{kg}$ & 1.80 & $\mathrm{~B}$ & 4.50 & $\mathrm{~B}$ & 4.30 & $\mathrm{~B}$ & \\
Nickel & $\mathrm{mg} / \mathrm{kg}$ & 8.10 & $\mathrm{~B}$ & 13.20 & & 15.80 & & \\
Potassium & $\mathrm{mg} / \mathrm{kg}$ & 416.00 & $\mathrm{~B}$ & 661.00 & $\mathrm{~B}$ & 904.00 & $\mathrm{~B}$ & \\
Selenium & $\mathrm{mg} / \mathrm{kg}$ & 0.70 & $\mathrm{~B}$ & 1.00 & $\mathrm{~B}$ & 1.30 & & \\
Silver & $\mathrm{mg} / \mathrm{kg}$ & 1.20 & $\mathrm{U}$ & 1.20 & $\mathrm{U}$ & 1.20 & $\mathrm{U}$ & \\
Sodium & $\mathrm{mg} / \mathrm{kg}$ & 379.00 & $\mathrm{~B}$ & 345.00 & $\mathrm{~B}$ & 380.00 & $\mathrm{~B}$ & \\
Strontium & $\mathrm{mg} / \mathrm{kg}$ & 4.10 & $\mathrm{~B}$ & 1.40 & $\mathrm{U}$ & 0.52 & $\mathrm{U}$ & \\
Sulfate & $\mathrm{mg} / \mathrm{kg}$ & 57.30 & & 45.30 & & 12.40 & $\mathrm{U}$ & \\
Thallium & $\mathrm{mg} / \mathrm{kg}$ & 0.46 & $\mathrm{U}$ & 0.45 & $\mathrm{U}$ & 0.48 & $\mathrm{U}$ & \\
Vanadium & $\mathrm{mg} / \mathrm{kg}$ & 27.80 & $\mathrm{~J}$ & 74.20 & $\mathrm{~J}$ & 101.00 & $\mathrm{~J}$ & \\
Zinc & $\mathrm{mg} / \mathrm{kg}$ & 40.90 & & 103.00 & & 146.00 & &
\end{tabular}

Location $=O R R ;$ Formation $=D I S M A L$ GAP; Site $=10,33,35 ;$ Sample type $=$ Regular; $A=5019 ; B=5022 ; C=5025 ; A$ lab split $=$ '; Phase $=1$

\begin{tabular}{|c|c|c|c|c|c|c|}
\hline $\begin{array}{l}\text { Aluminum } \\
\text { Antimony } \\
\text { Arsenic } \\
\text { Barium } \\
\text { Beryllium } \\
\text { Boron } \\
\text { Cadmium } \\
\text { Calcium } \\
\text { Chromium } \\
\text { Cobalt } \\
\text { Copper } \\
\text { Cyanide } \\
\text { Iron } \\
\text { Lead } \\
\text { Lithium } \\
\text { Magnesium } \\
\text { Manganese } \\
\text { Mercury } \\
\text { Molybdenum } \\
\text { Nickel } \\
\text { Osmiun } \\
\text { Potassium } \\
\text { Selenium } \\
\text { Sil icon } \\
\text { Silver } \\
\text { Strontium } \\
\text { Sulfate } \\
\text { Thallium } \\
\text { Vanadium } \\
\text { Zinc }\end{array}$ & $\begin{array}{l}\mathrm{mg} / \mathrm{kg} \\
\mathrm{mg} / \mathrm{kg} \\
\mathrm{mg} / \mathrm{kg} \\
\mathrm{mg} / \mathrm{kg} \\
\mathrm{mg} / \mathrm{kg} \\
\mathrm{mg} / \mathrm{kg} \\
\mathrm{mg} / \mathrm{kg} \\
\mathrm{mg} / \mathrm{kg} \\
\mathrm{mg} / \mathrm{kg} \\
\mathrm{mg} / \mathrm{kg} \\
\mathrm{mg} / \mathrm{kg} \\
\mathrm{mg} / \mathrm{kg} \\
\mathrm{mg} / \mathrm{kg} \\
\mathrm{mg} / \mathrm{kg} \\
\mathrm{mg} / \mathrm{kg} \\
\mathrm{mg} / \mathrm{kg} \\
\mathrm{mg} / \mathrm{kg} \\
\mathrm{mg} / \mathrm{kg} \\
\mathrm{mg} / \mathrm{kg} \\
\mathrm{mg} / \mathrm{kg} \\
\mathrm{mg} / \mathrm{kg} \\
\mathrm{mg} / \mathrm{kg} \\
\mathrm{mg} / \mathrm{kg} \\
\mathrm{mg} / \mathrm{kg} \\
\mathrm{mg} / \mathrm{kg} \\
\mathrm{mg} / \mathrm{kg} \\
\mathrm{mg} / \mathrm{kg} \\
\mathrm{mg} / \mathrm{kg} \\
\mathrm{mg} / \mathrm{kg} \\
\mathrm{mg} / \mathrm{kg}\end{array}$ & $\begin{array}{r}16900.00 \\
0.22 \\
7.30 \\
77.20 \\
0.55 \\
21.10 \\
0.22 \\
991.00 \\
22.50 \\
11.30 \\
15.00 \\
0.44 \\
33900.00 \\
17.00 \\
15.20 \\
2330.00 \\
791.00 \\
0.34 \\
9.00 \\
19.50 \\
14.00 \\
1960.00 \\
0.68 \\
488.00 \\
2.00 \\
6.10 \\
57.60 \\
0.79 \\
37.70 \\
45.30\end{array}$ & $\begin{array}{l}\text { UJ } \\
\mathbf{U} \\
\mathbf{J} \\
\mathbf{U}\end{array}$ & $\begin{array}{r}25600.00 \\
0.23 \\
8.00 \\
89.00 \\
0.39 \\
21.20 \\
0.23 \\
892.00 \\
36.90 \\
7.10 \\
20.50 \\
0.46 \\
34600.00 \\
15.00 \\
22.80 \\
3170.00 \\
212.00 \\
0.17 \\
9.30 \\
20.20 \\
14.70 \\
2280.00 \\
0.48 \\
401.00 \\
2.00 \\
7.00 \\
75.30 \\
0.61 \\
40.00 \\
49.70\end{array}$ & $\begin{array}{l}\mathbf{B} \\
\mathbf{J} \\
\mathbf{U} \\
\mathbf{U} \\
\mathbf{B} \\
\mathbf{J} \\
\mathbf{J} \\
\mathbf{J} \\
\mathbf{B}\end{array}$ & $\begin{array}{r}42600.00 \\
0.31 \\
10.70 \\
123.00 \\
0.97 \\
34.00 \\
0.31 \\
1290.00 \\
57.30 \\
16.80 \\
29.90 \\
0.63 \\
52000.00 \\
25.30 \\
33.00 \\
5190.00 \\
462.00 \\
0.21 \\
12.80 \\
30.20 \\
19.90 \\
3800.00 \\
0.62 \\
623.00 \\
2.80 \\
10.50 \\
200.00 \\
0.31 \\
60.10 \\
69.80\end{array}$ \\
\hline
\end{tabular}

Location $=O R R ;$ Formation $=D I S M A L$ GAP; Site $=11,27,41 ;$ Sample type $=$ Regular; $A=5001 ; B=5004 ; C=5007 ; A$ lab split =' '; Phase $=1$

$\begin{array}{ll}\text { Aluminum } & \mathrm{mg} / \mathrm{kg} \\ \text { Antimony } & \mathrm{mg} / \mathrm{kg} \\ \text { Arsenic } & \mathrm{mg} / \mathrm{kg} \\ \text { Barium } & \mathrm{mg} / \mathrm{kg} \\ \text { Beryltium } & \mathrm{mg} / \mathrm{kg} \\ \text { Boron } & \mathrm{mg} / \mathrm{kg} \\ \text { Cadnium } & \mathrm{mg} / \mathrm{kg} \\ \text { Calcium } & \mathrm{mg} / \mathrm{kg} \\ \text { Chromium } & \mathrm{mg} / \mathrm{kg} \\ \text { Cobalt } & \mathrm{mg} / \mathrm{kg} \\ \text { Copper } & \mathrm{mg} / \mathrm{kg} \\ \text { Cyanide } & \mathrm{mg} / \mathrm{kg} \\ \text { Iron } & \mathrm{mg} / \mathrm{kg} \\ \text { Lead } & \mathrm{mg} / \mathrm{kg} \\ \text { Lithium } & \mathrm{mg} / \mathrm{kg} \\ \text { Magnesium } & \mathrm{mg} / \mathrm{kg} \\ \text { Manganese } & \mathrm{mg} / \mathrm{kg}\end{array}$

18500.00$$
0.23
$$$$
5.50
$$

88.60

0.62
19.80

19.80
0.23

1050.00

19.40

12.20

16.80

0.12

25500.00

35.40

14.00

2090.00

1020.00
34600.00

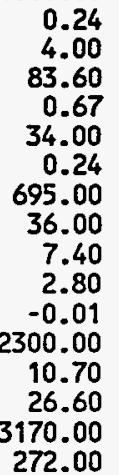

38100.00

UJ
$\mathbf{B}$
$\mathbf{B}$
$\mathbf{J}$
$\mathbf{U}$
$\mathbf{B}$
$\mathbf{J}$
$\mathbf{U J}$
$\mathbf{J}$
$\mathbf{B}$
$\mathbf{J}$

$\begin{array}{rl}0.23 & \text { UJ } \\ 18.30 & \mathrm{~B} \\ 82.70 & \\ 1.00 & \mathrm{~B} \\ 33.40 & \mathrm{~J} \\ 0.23 & \mathrm{U} \\ 606.00 & \\ 45.50 & \\ 6.30 & \mathrm{~B} \\ 35.50 & \mathrm{~J} \\ -0.04 & \text { UJ } \\ 43300.00 & \mathrm{~J} \\ 12.50 & \mathrm{~B} \\ 28.90 & \\ 3980.00 & \mathrm{~J} \\ 85.00 & \end{array}$


D-17

Table D.1 (continued)

\begin{tabular}{|c|c|c|c|c|c|c|c|c|c|}
\hline Analysis & Units & $\begin{array}{c}\text { A horizon } \\
\text { result }\end{array}$ & Qualifier & $\begin{array}{l}\text { B horizon } \\
\text { result }\end{array}$ & Qualifier & $\begin{array}{l}\text { C horizon } \\
\text { result }\end{array}$ & Qualifier & $\begin{array}{l}\text { A lab split } \\
\text { result }\end{array}$ & Qualifier \\
\hline $\begin{array}{l}\text { Mercury } \\
\text { Molybdenum } \\
\text { Nickel } \\
\text { Osmium } \\
\text { Potassium } \\
\text { Selenium } \\
\text { silicon } \\
\text { silver } \\
\text { Strontium } \\
\text { Sulfate } \\
\text { Thallium } \\
\text { Vanadium } \\
\text { Zinc }\end{array}$ & $\begin{array}{l}\mathrm{mg} / \mathrm{kg} \\
\mathrm{mg} / \mathrm{kg} \\
\mathrm{mg} / \mathrm{kg} \\
\mathrm{mg} / \mathrm{kg} \\
\mathrm{mg} / \mathrm{kg} \\
\mathrm{mg} / \mathrm{kg} \\
\mathrm{mg} / \mathrm{kg} \\
\mathrm{mg} / \mathrm{kg} \\
\mathrm{mg} / \mathrm{kg} \\
\mathrm{mg} / \mathrm{kg} \\
\mathrm{mg} / \mathrm{kg} \\
\mathrm{mg} / \mathrm{kg} \\
\mathrm{mg} / \mathrm{kg}\end{array}$ & $\begin{array}{r}0.31 \\
9.50 \\
19.50 \\
14.80 \\
1890.00 \\
0.60 \\
486.00 \\
2.10 \\
7.00 \\
163.00 \\
0.23 \\
31.60 \\
42.50\end{array}$ & $\begin{array}{l}\mathbf{J} \\
\mathbf{U} \\
\mathbf{U} \mathbf{J} \\
\mathbf{J} \\
\mathbf{U} \\
\mathbf{J} \\
\mathbf{U} \\
\mathbf{J} \\
\mathbf{U}\end{array}$ & $\begin{array}{r}0.17 \\
9.80 \\
23.30 \\
15.20 \\
2380.00 \\
0.33 \\
383.00 \\
2.20 \\
5.90 \\
141.00 \\
0.24 \\
4.80 \\
55.60\end{array}$ & $\begin{array}{l}\mathbf{J} \\
\mathbf{U} \\
\mathbf{U J} \\
\mathbf{J} \\
\mathbf{U} \\
\mathbf{J} \\
\mathbf{U} \\
\mathbf{J} \\
\mathbf{U} \\
\mathrm{B}\end{array}$ & $\begin{array}{r}0.06 \\
9.50 \\
26.30 \\
14.80 \\
2940.00 \\
0.23 \\
442.00 \\
2.10 \\
5.90 \\
135.00 \\
0.32 \\
44.70 \\
59.80\end{array}$ & $\begin{array}{l}\mathbf{J} \\
\mathbf{U} \\
\mathbf{U} \mathbf{J} \\
\mathbf{J} \\
\mathbf{U} \\
\mathbf{J} \\
\mathbf{U} \\
\mathbf{J} \\
\mathbf{B}\end{array}$ & 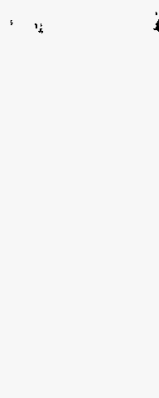 & $\therefore \therefore$ \\
\hline
\end{tabular}

Location $=O R R ;$ Formation $=D I S M A L$ GAP; Site $=19,22,32 ;$ Sample type $=$ Regular; $A=5010 ; B=5013 ; C=5016 ; A$ lab split =' '; Phase $=1$

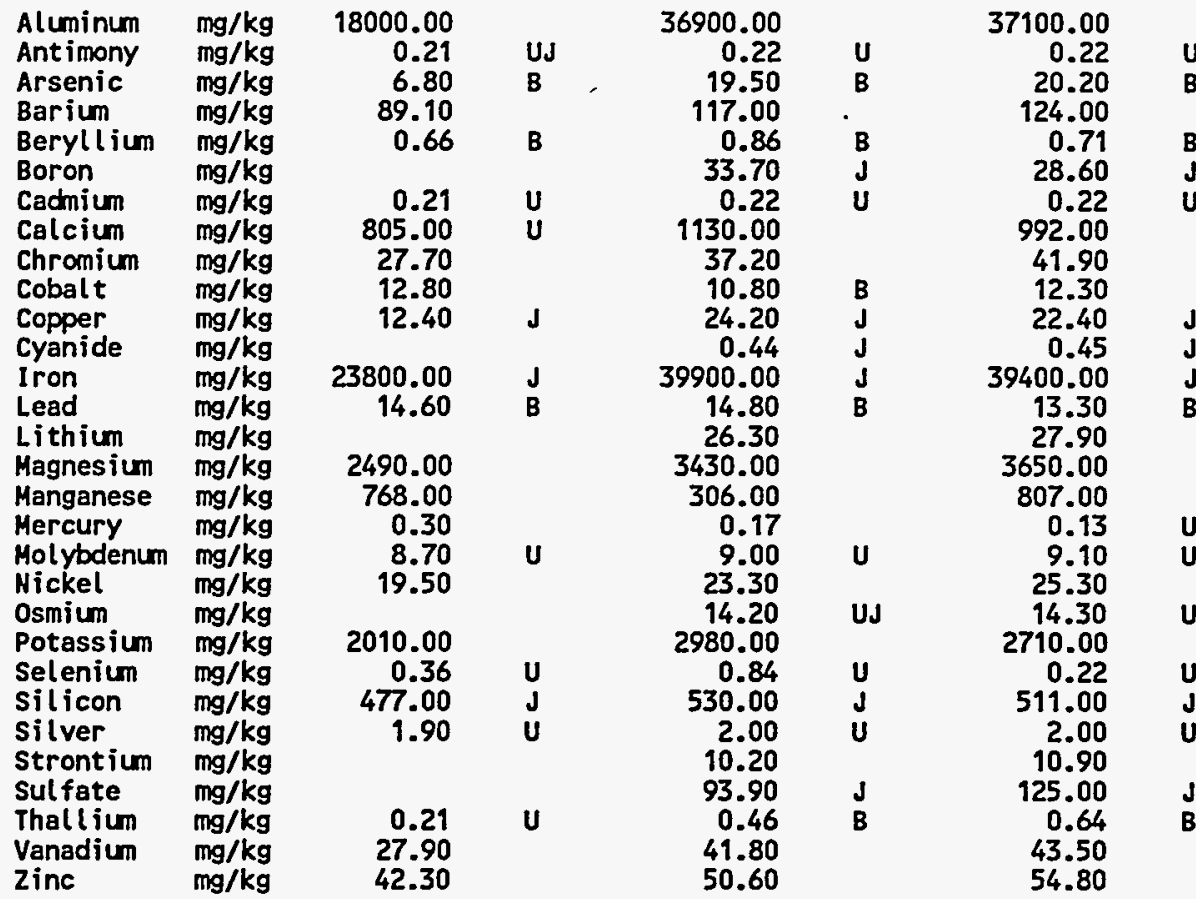

Location $=O R R ;$ Formation $=D I S M A L$ GAP; Site $=2,26,43 ;$ Sample type $=$ Field duplicate; $A=5037 ; B=5040 ; C=5043 ; A$ lab split $=$ ' ' Phase $=1$

$\begin{array}{lrrrrrrr}\text { Aluminum } & \mathrm{mg} / \mathrm{kg} & 20400.00 & & 26800.00 & & 35900.00 & \\ \text { Antimony } & \mathrm{mg} / \mathrm{kg} & 0.23 & \mathrm{U} & 0.23 & \mathrm{U} & 0.24 & \mathrm{U} \\ \text { Arsenic } & \mathrm{mg} / \mathrm{kg} & 5.30 & & 4.90 & & 6.10 & \\ \text { Barium } & \mathrm{mg} / \mathrm{kg} & 104.00 & & 100.00 & & 112.00 & \\ \text { Beryllium } & \mathrm{mg} / \mathrm{kg} & 1.10 & \mathrm{~B} & 1.30 & & 1.50 & \\ \text { Boron } & \mathrm{mg} / \mathrm{kg} & 9.80 & \mathrm{U} & 11.80 & & 20.70 & \\ \text { Cactmium } & \mathrm{mg} / \mathrm{kg} & 0.23 & \mathrm{U} & 0.23 & \mathrm{U} & 0.24 & \mathrm{U} \\ \text { Calcium } & \mathrm{mg} / \mathrm{kg} & 788.00 & \mathrm{U} & 801.00 & \mathrm{U} & 837.00 & \\ \text { Chromium } & \mathrm{mg} / \mathrm{kg} & 29.00 & & 34.30 & & 46.80 & \\ \text { Cobalt } & \mathrm{mg} / \mathrm{kg} & 13.70 & & 13.90 & & 7.70 & \mathrm{~B} \\ \text { Copper } & \mathrm{mg} / \mathrm{kg} & 13.30 & & 16.70 & & 22.00 & \\ \text { Cyanide } & \mathrm{mg} / \mathrm{kg} & 0.12 & \mathrm{UJ} & 0.12 & \mathrm{UJ} & 0.12 & \mathrm{UJ} \\ \text { Iron } & \mathrm{mg} / \mathrm{kg} & 24000.00 & & 31800.00 & & 38000.00 & \\ \text { Lead } & \mathrm{mg} / \mathrm{kg} & 18.90 & & 7.80 & & 12.30 & \\ \text { Lithium } & \mathrm{mg} / \mathrm{kg} & 12.70 & & 14.20 & & 20.70 & \\ \text { Magnesium } & \mathrm{mg} / \mathrm{kg} & 3390.00 & & 3260.00 & & 5100.00 & \\ \text { Manganese } & \mathrm{mg} / \mathrm{kg} & 970.00 & & 399.00 & & 180.00 & \\ \text { Mercury } & \mathrm{mg} / \mathrm{kg} & 0.40 & & 0.15 & \mathrm{U} & 0.15 & \mathrm{U}\end{array}$




\section{D-18}

Table D.1 (continued)

\begin{tabular}{|c|c|c|c|c|c|c|c|c|c|}
\hline Analysis & Units & $\begin{array}{l}\text { A horizon } \\
\text { result }\end{array}$ & Qualifier & $\begin{array}{l}\text { B horizon } \\
\text { result }\end{array}$ & Qualifier & $\begin{array}{l}\mathrm{C} \text { horizon } \\
\text { result }\end{array}$ & Qualifier & $\begin{array}{l}\text { A lab split } \\
\text { result }\end{array}$ & Qualifier \\
\hline $\begin{array}{l}\text { Molybdenum } \\
\text { Nickel } \\
\text { Potassium } \\
\text { Selenium } \\
\text { Sil icon } \\
\text { Silver } \\
\text { Strontium } \\
\text { Sulfate } \\
\text { Thal lium } \\
\text { Vanadium } \\
\text { Zinc }\end{array}$ & $\begin{array}{l}\mathrm{mg} / \mathrm{kg} \\
\mathrm{mg} / \mathrm{kg} \\
\mathrm{mg} / \mathrm{kg} \\
\mathrm{mg} / \mathrm{kg} \\
\mathrm{mg} / \mathrm{kg} \\
\mathrm{mg} / \mathrm{kg} \\
\mathrm{mg} / \mathrm{kg} \\
\mathrm{mg} / \mathrm{kg} \\
\mathrm{mg} / \mathrm{kg} \\
\mathrm{mg} / \mathrm{kg} \\
\mathrm{mg} / \mathrm{kg}\end{array}$ & $\begin{array}{r}9.50 \\
25.30 \\
2130.00 \\
1.20 \\
461.00 \\
2.10 \\
6.60 \\
111.00 \\
0.28 \\
27.90 \\
53.60\end{array}$ & $\begin{array}{l}u \\
u\end{array}$ & $\begin{array}{r}9.50 \\
24.60 \\
2550.00 \\
1.20 \\
559.00 \\
2.10 \\
8.20 \\
72.40 \\
0.23 \\
35.10 \\
48.60\end{array}$ & $\begin{array}{l}u \\
u\end{array}$ & $\begin{array}{r}9.80 \\
33.30 \\
3170.00 \\
1.20 \\
489.00 \\
2.10 \\
9.80 \\
79.50 \\
0.38 \\
39.60 \\
62.70\end{array}$ & $\begin{array}{l}u \\
u\end{array}$ & & \\
\hline
\end{tabular}

Location=ORR; Formation $=D I S M A L$ GAP; Site=2,26,43; Sample type=Regular; $A=5028 ; B=5031 ; C=5034 ; A$ lab split =' '; Phase $=1$

\begin{tabular}{|c|c|c|c|c|c|c|}
\hline $\begin{array}{l}\text { Aluminum } \\
\text { Ant imony } \\
\text { Arsenic } \\
\text { Barium } \\
\text { Beryllium } \\
\text { Boron } \\
\text { Cadnium } \\
\text { Calcium } \\
\text { Chromium } \\
\text { Cobalt } \\
\text { Copper } \\
\text { Cyanide } \\
\text { Iron } \\
\text { Lead } \\
\text { Lithium } \\
\text { Magnesium } \\
\text { Manganese } \\
\text { Mercury } \\
\text { Molybdenum } \\
\text { Nickel } \\
\text { Potassium } \\
\text { Selenium } \\
\text { silicon } \\
\text { silver } \\
\text { strontium } \\
\text { Sulfate } \\
\text { Thallium } \\
\text { Vanadium } \\
\text { Zinc }\end{array}$ & $\begin{array}{l}\mathrm{mg} / \mathrm{kg} \\
\mathrm{mg} / \mathrm{kg} \\
\mathrm{mg} / \mathrm{kg} \\
\mathrm{mg} / \mathrm{kg} \\
\mathrm{mg} / \mathrm{kg} \\
\mathrm{mg} / \mathrm{kg} \\
\mathrm{mg} / \mathrm{kg} \\
\mathrm{mg} / \mathrm{kg} \\
\mathrm{mg} / \mathrm{kg} \\
\mathrm{mg} / \mathrm{kg} \\
\mathrm{mg} / \mathrm{kg} \\
\mathrm{mg} / \mathrm{kg} \\
\mathrm{mg} / \mathrm{kg} \\
\mathrm{mg} / \mathrm{kg} \\
\mathrm{mg} / \mathrm{kg} \\
\mathrm{mg} / \mathrm{kg} \\
\mathrm{mg} / \mathrm{kg} \\
\mathrm{mg} / \mathrm{kg} \\
\mathrm{mg} / \mathrm{kg} \\
\mathrm{mg} / \mathrm{kg} \\
\mathrm{mg} / \mathrm{kg} \\
\mathrm{mg} / \mathrm{kg} \\
\mathrm{mg} / \mathrm{kg} \\
\mathrm{mg} / \mathrm{kg} \\
\mathrm{mg} / \mathrm{kg} \\
\mathrm{mg} / \mathrm{kg} \\
\mathrm{mg} / \mathrm{kg} \\
\mathrm{mg} / \mathrm{kg} \\
\mathrm{mg} / \mathrm{kg}\end{array}$ & $\begin{array}{r}44300.00 \\
0.21 \\
5.80 \\
212.00 \\
2.20 \\
16.40 \\
0.21 \\
1860.00 \\
32.10 \\
36.70 \\
30.10 \\
0.11 \\
49000.00 \\
19.80 \\
27.00 \\
7430.00 \\
2220.00 \\
0.23 \\
9.20 \\
56.70 \\
5390.00 \\
1.00 \\
697.00 \\
2.00 \\
16.80 \\
28.00 \\
0.44 \\
54.00 \\
108.00\end{array}$ & $\begin{array}{l}\mathbf{U} \\
\mathbf{J}\end{array}$ & $\begin{array}{r}30300.00 \\
0.23 \\
6.80 \\
101.00 \\
1.20 \\
9.80 \\
0.23 \\
708.00 \\
45.10 \\
8.40 \\
18.30 \\
0.12 \\
34600.00 \\
8.40 \\
15.70 \\
3430.00 \\
285.00 \\
0.15 \\
9.60 \\
25.20 \\
2980.00 \\
1.20 \\
868.00 \\
2.10 \\
7.00 \\
151.00 \\
0.40 \\
40.10 \\
52.00\end{array}$ & $\begin{array}{l}\mathbf{U} \\
\mathbf{U} \\
\mathbf{U} \\
\mathbf{B} \\
\text { UJ }\end{array}$ & $\begin{array}{r}41200.00 \\
0.24 \\
6.70 \\
110.00 \\
1.60 \\
13.90 \\
0.24 \\
729.00 \\
35.30 \\
24.00 \\
35.30 \\
0.12 \\
38900.00 \\
9.90 \\
22.90 \\
4530.00 \\
699.00 \\
0.15 \\
9.80 \\
35.20 \\
3150.00 \\
1.20 \\
503.00 \\
2.10 \\
9.40 \\
85.50 \\
0.45 \\
41.40 \\
62.00\end{array}$ \\
\hline
\end{tabular}

Location $=O R R ;$ Formation $=$ NOLICHUCKY; Site $=15,23,25 ;$ Sample type $=$ Regular; $A=5055 ; B=5058 ; C=5061 ; A$ lab split=' '; Phase $=1$

$\begin{array}{ll}\text { Aluminum } & \mathrm{mg} / \mathrm{kg} \\ \text { Antimony } & \mathrm{mg} / \mathrm{kg} \\ \text { Arsenic } & \mathrm{mg} / \mathrm{kg} \\ \text { Barium } & \mathrm{mg} / \mathrm{kg} \\ \text { Beryll lium } & \mathrm{mg} / \mathrm{kg} \\ \text { Boron } & \mathrm{mg} / \mathrm{kg} \\ \text { Cacdnium } & \mathrm{mg} / \mathrm{kg} \\ \text { Calcium } & \mathrm{mg} / \mathrm{kg} \\ \text { Chromium } & \mathrm{mg} / \mathrm{kg} \\ \text { Cobalt } & \mathrm{mg} / \mathrm{kg} \\ \text { Copper } & \mathrm{mg} / \mathrm{kg} \\ \text { Cyanide } & \mathrm{mg} / \mathrm{kg} \\ \text { Iron } & \mathrm{mg} / \mathrm{kg} \\ \text { Lead } & \mathrm{mg} / \mathrm{kg} \\ \text { Lithium } & \mathrm{mg} / \mathrm{kg} \\ \text { Magnesium } & \mathrm{mg} / \mathrm{kg} \\ \text { Manganese } & \mathrm{mg} / \mathrm{kg} \\ \text { Mercury } & \mathrm{mg} / \mathrm{kg} \\ \text { Molybdenum } & \mathrm{mg} / \mathrm{kg} \\ \text { Hickel } & \mathrm{mg} / \mathrm{kg} \\ \text { Potassium } & \mathrm{mg} / \mathrm{kg} \\ \text { Selenium } & \mathrm{mg} / \mathrm{kg} \\ & \end{array}$

25100.00
0.49
6.30
59.70
0.73
9.30
0.22
422.00
29.90
11.10
12.20
0.11
2880.00
20.40
15.50
2010.00
405.00
0.18
1.50
16.50
2950.00
0.74

$\begin{array}{lr} & 38800.00 \\ \text { B } & 0.80 \\ & 6.80 \\ \text { B } & 76.00 \\ \text { UJ } & 0.91 \\ \text { U } & 10.20 \\ \text { U } & 0.24 \\ & 550.00 \\ & 45.30 \\ & 7.30 \\ & 19.30 \\ \text { UJ } & 0.12 \\ & 45900.00 \\ & 9.30 \\ & 26.90 \\ & 2460.00 \\ & 128.00 \\ & 0.12 \\ & 2.00 \\ \text { U } & 17.50 \\ & 3660.00 \\ & 1.00\end{array}$

B
B J
$\mathbf{U}$
$\mathbf{U}$
B
UJ

U
B
B

$\begin{array}{rl}38200.00 & \\ 0.60 & B \\ 5.20 & \\ 71.20 & \\ 1.10 & B \\ 10.00 & \text { UJ } \\ 0.24 & \text { U } \\ 451.00 & \text { U } \\ 38.10 & \\ 6.90 & B \\ 20.40 & \\ 0.12 & \text { UJ } \\ 45700.00 & \\ 11.70 & \\ 19.90 & \\ 2900.00 & \\ 133.00 & \\ 0.11 & \text { U } \\ 1.70 & \text { U } \\ 18.70 & \\ 5070.00 & \\ 0.88 & \text { B }\end{array}$




\section{D-19}

Table D.1 (continued)

\begin{tabular}{|c|c|c|c|c|c|c|c|c|c|}
\hline Analysis & Units & $\begin{array}{l}\text { A horizon } \\
\text { result }\end{array}$ & Qualifier & $\begin{array}{l}\text { B horizon } \\
\text { result }\end{array}$ & Qualifier & $\begin{array}{l}C \text { horizon } \\
\text { result }\end{array}$ & Qualifier & $\begin{array}{l}\text { A lab split } \\
\text { result }\end{array}$ & lit Qualifier \\
\hline $\begin{array}{l}\text { Silicon } \\
\text { silver } \\
\text { Strontium } \\
\text { sulfate } \\
\text { Thallium } \\
\text { Vanadium } \\
\text { Zinc }\end{array}$ & $\begin{array}{l}\mathrm{mg} / \mathrm{kg} \\
\mathrm{mg} / \mathrm{kg} \\
\mathrm{mg} / \mathrm{kg} \\
\mathrm{mg} / \mathrm{kg} \\
\mathrm{mg} / \mathrm{kg} \\
\mathrm{mg} / \mathrm{kg} \\
\mathrm{mg} / \mathrm{kg}\end{array}$ & $\begin{array}{r}203.00 \\
1.10 \\
3.20 \\
18.90 \\
0.45 \\
35.20 \\
36.80\end{array}$ & Ju & $\begin{array}{r}218.00 \\
1.20 \\
3.80 \\
44.50 \\
0.59 \\
54.10 \\
44.10\end{array}$ & Ju & $\begin{array}{r}217.00 \\
1.20 \\
3.70 \\
47.40 \\
0.48 \\
42.80 \\
39.40\end{array}$ & $\underset{U J U}{J}$ & $=3$ & and \\
\hline
\end{tabular}

Location $=$ ORR; Formation $=$ NOLICHUCKY; Site $=16,28,42 ;$ Sample type $=$ Regular; $A=5064 ; B=5067 ; C=5070 ; A$ lab split $=$ ' '; Phase $=1$

\begin{tabular}{|c|c|c|c|c|c|c|c|}
\hline $\begin{array}{l}\text { Aluminum } \\
\text { Ant imony } \\
\text { Arsenic } \\
\text { Barium } \\
\text { Beryll ium } \\
\text { Boron } \\
\text { Cadmium } \\
\text { Calcium } \\
\text { Chromium } \\
\text { Cobalt } \\
\text { Copper } \\
\text { Cyanide } \\
\text { Iron } \\
\text { Lead } \\
\text { Lithium } \\
\text { Magnesium } \\
\text { Manganese } \\
\text { Mercury } \\
\text { Molybdenun } \\
\text { Nickel } \\
\text { Potassium } \\
\text { Selenium } \\
\text { Silicon } \\
\text { Silver } \\
\text { Strontium } \\
\text { Sulfate } \\
\text { Thall ium } \\
\text { Vanadium } \\
\text { Zinc }\end{array}$ & $\begin{array}{l}\mathrm{mg} / \mathrm{kg} \\
\mathrm{mg} / \mathrm{kg} \\
\mathrm{mg} / \mathrm{kg} \\
\mathrm{mg} / \mathrm{kg} \\
\mathrm{mg} / \mathrm{kg} \\
\mathrm{mg} / \mathrm{kg} \\
\mathrm{mg} / \mathrm{kg} \\
\mathrm{mg} / \mathrm{kg} \\
\mathrm{mg} / \mathrm{kg} \\
\mathrm{mg} / \mathrm{kg} \\
\mathrm{mg} / \mathrm{kg} \\
\mathrm{mg} / \mathrm{kg} \\
\mathrm{mg} / \mathrm{kg} \\
\mathrm{mg} / \mathrm{kg} \\
\mathrm{mg} / \mathrm{kg} \\
\mathrm{mg} / \mathrm{kg} \\
\mathrm{mg} / \mathrm{kg} \\
\mathrm{mg} / \mathrm{kg} \\
\mathrm{mg} / \mathrm{kg} \\
\mathrm{mg} / \mathrm{kg} \\
\mathrm{mg} / \mathrm{kg} \\
\mathrm{mg} / \mathrm{kg} \\
\mathrm{mg} / \mathrm{kg} \\
\mathrm{mg} / \mathrm{kg} \\
\mathrm{mg} / \mathrm{kg} \\
\mathrm{mg} / \mathrm{kg} \\
\mathrm{mg} / \mathrm{kg} \\
\mathrm{mg} / \mathrm{kg} \\
\mathrm{mg} / \mathrm{kg}\end{array}$ & $\begin{array}{r}21300.00 \\
0.47 \\
0.46 \\
67.60 \\
0.85 \\
9.90 \\
0.23 \\
594.00 \\
0.46 \\
14.10 \\
11.00 \\
0.12 \\
32100.00 \\
0.23 \\
7.60 \\
1730.00 \\
657.00 \\
0.19 \\
1.60 \\
15.20 \\
3010.00 \\
0.46 \\
291.00 \\
1.20 \\
5.00 \\
17.90 \\
0.46 \\
33.20 \\
33.90\end{array}$ & $\begin{array}{l}\mathbf{B} \\
\mathbf{U} \mathbf{J} \\
\mathbf{U} \\
\mathbf{U} \\
\mathbf{U}\end{array}$ & $\begin{array}{r}30500.00 \\
0.70 \\
0.48 \\
78.80 \\
1.10 \\
10.10 \\
0.24 \\
901.00 \\
0.48 \\
12.70 \\
18.90 \\
0.12 \\
51800.00 \\
0.24 \\
19.70 \\
2360.00 \\
291.00 \\
0.11 \\
1.70 \\
20.20 \\
3520.00 \\
0.48 \\
215.00 \\
1.20 \\
5.60 \\
43.10 \\
0.48 \\
42.80 \\
38.30\end{array}$ & $\begin{array}{l}B \\
U \mathbf{J} \\
\mathbf{U} \\
\mathbf{B} \\
\mathbf{U}\end{array}$ & $\begin{array}{r}34300.00 \\
0.55 \\
5.20 \\
85.20 \\
1.10 \\
10.10 \\
0.24 \\
1160.00 \\
39.30 \\
18.60 \\
21.00 \\
0.12 \\
44700.00 \\
11.50 \\
20.20 \\
3140.00 \\
364.00 \\
0.11 \\
1.70 \\
22.30 \\
4880.00 \\
0.47 \\
487.00 \\
1.20 \\
7.60 \\
37.00 \\
0.47 \\
40.80 \\
38.50\end{array}$ & $\begin{array}{l}\mathbf{B} \\
\mathbf{U} \\
\mathbf{U} \\
\mathbf{B}\end{array}$ \\
\hline
\end{tabular}

Location=ORR; Formation=NOLICHUCKY; Site=3,13,24; Sample type=Regular; $A=5082 ; B=5085 ; C=5088 ; A$ lab split=' '; Phase $=1$

\begin{tabular}{|c|c|c|c|c|c|c|c|}
\hline $\begin{array}{l}\text { Aluminum } \\
\text { Antimony } \\
\text { Arsenic } \\
\text { Barium } \\
\text { Beryllium } \\
\text { Boron } \\
\text { Cadnium } \\
\text { Calcium } \\
\text { Chromium } \\
\text { Cobalt } \\
\text { Copper } \\
\text { Cyanide } \\
\text { Iron } \\
\text { Lead } \\
\text { Lithium } \\
\text { Magnesium } \\
\text { Manganese } \\
\text { Mercury } \\
\text { Molybdenum } \\
\text { Hickel } \\
\text { Potassium } \\
\text { Selenium } \\
\text { Silicon } \\
\text { Silver } \\
\text { Strontium }\end{array}$ & $\begin{array}{l}\mathrm{mg} / \mathrm{kg} \\
\mathrm{mg} / \mathrm{kg} \\
\mathrm{mg} / \mathrm{kg} \\
\mathrm{mg} / \mathrm{kg} \\
\mathrm{mg} / \mathrm{kg} \\
\mathrm{mg} / \mathrm{kg} \\
\mathrm{mg} / \mathrm{kg} \\
\mathrm{mg} / \mathrm{kg} \\
\mathrm{mg} / \mathrm{kg} \\
\mathrm{mg} / \mathrm{kg} \\
\mathrm{mg} / \mathrm{kg} \\
\mathrm{mg} / \mathrm{kg} \\
\mathrm{mg} / \mathrm{kg} \\
\mathrm{mg} / \mathrm{kg} \\
\mathrm{mg} / \mathrm{kg} \\
\mathrm{mg} / \mathrm{kg} \\
\mathrm{mg} / \mathrm{kg} \\
\mathrm{mg} / \mathrm{kg} \\
\mathrm{mg} / \mathrm{kg} \\
\mathrm{mg} / \mathrm{kg} \\
\mathrm{mg} / \mathrm{kg} \\
\mathrm{mg} / \mathrm{kg} \\
\mathrm{mg} / \mathrm{kg} \\
\mathrm{mg} / \mathrm{kg} \\
\mathrm{mg} / \mathrm{kg}\end{array}$ & $\begin{array}{r}21800.00 \\
0.47 \\
6.40 \\
106.00 \\
0.81 \\
9.80 \\
0.23 \\
952.00 \\
26.40 \\
17.50 \\
12.70 \\
0.12 \\
28500.00 \\
17.20 \\
11.10 \\
2410.00 \\
935.00 \\
0.19 \\
1.60 \\
20.00 \\
3230.00 \\
0.63 \\
185.00 \\
1.20 \\
6.10\end{array}$ & $\begin{array}{l}B \\
\text { US } \\
U \\
B\end{array}$ & $\begin{array}{r}42100.00 \\
0.80 \\
6.70 \\
107.00 \\
1.20 \\
10.20 \\
0.24 \\
1360.00 \\
36.80 \\
40.10 \\
25.50 \\
0.12 \\
40600.00 \\
18.20 \\
27.80 \\
3300.00 \\
761.00 \\
0.12 \\
1.70 \\
23.90 \\
4340.00 \\
0.66 \\
274.00 \\
1.20 \\
8.70\end{array}$ & $\begin{array}{l}\text { B } \\
\text { UJ } \\
\mathbf{U}\end{array}$ & $\begin{array}{r}46700.00 \\
1.00 \\
8.80 \\
86.80 \\
1.40 \\
10.20 \\
0.23 \\
1320.00 \\
72.80 \\
12.70 \\
30.20 \\
0.12 \\
37700.00 \\
47.60 \\
32.10 \\
3830.00 \\
320.00 \\
0.12 \\
1.70 \\
27.00 \\
5560.00 \\
1.10 \\
238.00 \\
1.20 \\
7.60\end{array}$ & $\begin{array}{l}\mathbf{B} \\
\mathbf{J} \\
\text { UJ }\end{array}$ \\
\hline
\end{tabular}


D-20

Table D.1 (continued)

\begin{tabular}{|c|c|c|c|c|c|c|c|c|c|}
\hline Analysis & Units & $\begin{array}{l}\text { A horizon } \\
\text { result }\end{array}$ & Qualifier & $\begin{array}{l}\text { B horizon } \\
\text { result }\end{array}$ & Qualifier & $\begin{array}{l}C \text { horizon } \\
\text { result }\end{array}$ & Qualifier & $\begin{array}{l}\text { A lab split } \\
\text { result }\end{array}$ & Qualifier \\
\hline $\begin{array}{l}\text { Sulfate } \\
\text { Thal I ium } \\
\text { Vanadium } \\
\text { Zinc }\end{array}$ & $\begin{array}{l}\mathrm{mg} / \mathrm{kg} \\
\mathrm{mg} / \mathrm{kg} \\
\mathrm{mg} / \mathrm{kg} \\
\mathrm{mg} / \mathrm{kg}\end{array}$ & $\begin{array}{r}14.10 \\
0.47 \\
32.10 \\
40.70\end{array}$ & $U$ & $\begin{array}{r}344.00 \\
0.49 \\
46.30 \\
46.80\end{array}$ & U & $\begin{array}{r}35.10 \\
0.68 \\
43.50 \\
51.60\end{array}$ & B & & \\
\hline
\end{tabular}

Location $=O R R ;$ Formation $=$ NOLICHUCKY; Site $=5,21,31 ;$ Sample type $=$ Regular; $A=5073 ; B=5076 ; C=5079 ; A$ lab split=' '; Phase $=1$

\begin{tabular}{|c|c|c|c|c|c|c|}
\hline $\begin{array}{l}\text { Aluminum } \\
\text { Antimony } \\
\text { Arsenic } \\
\text { Barium } \\
\text { Beryllium } \\
\text { Boron } \\
\text { Cadmium } \\
\text { Calcium } \\
\text { Chromium } \\
\text { Cobalt } \\
\text { Copper } \\
\text { Cyanide } \\
\text { Iron } \\
\text { Lead } \\
\text { Lithium } \\
\text { Magnesium } \\
\text { Manganese } \\
\text { Mercury } \\
\text { Molybdenum } \\
\text { Nickel } \\
\text { Potassium } \\
\text { Selenium } \\
\text { Sil icon } \\
\text { Silver } \\
\text { Strontium } \\
\text { Sulfate } \\
\text { Thall ium } \\
\text { Vanadium } \\
\text { Zine }\end{array}$ & $\begin{array}{l}\mathrm{mg} / \mathrm{kg} \\
\mathrm{mg} / \mathrm{kg} \\
\mathrm{mg} / \mathrm{kg} \\
\mathrm{mg} / \mathrm{kg} \\
\mathrm{mg} / \mathrm{kg} \\
\mathrm{mg} / \mathrm{kg} \\
\mathrm{mg} / \mathrm{kg} \\
\mathrm{mg} / \mathrm{kg} \\
\mathrm{mg} / \mathrm{kg} \\
\mathrm{mg} / \mathrm{kg} \\
\mathrm{mg} / \mathrm{kg} \\
\mathrm{mg} / \mathrm{kg} \\
\mathrm{mg} / \mathrm{kg} \\
\mathrm{mg} / \mathrm{kg} \\
\mathrm{mg} / \mathrm{kg} \\
\mathrm{mg} / \mathrm{kg} \\
\mathrm{mg} / \mathrm{kg} \\
\mathrm{mg} / \mathrm{kg} \\
\mathrm{mg} / \mathrm{kg} \\
\mathrm{mg} / \mathrm{kg} \\
\mathrm{mg} / \mathrm{kg} \\
\mathrm{mg} / \mathrm{kg} \\
\mathrm{mg} / \mathrm{kg} \\
\mathrm{mg} / \mathrm{kg} \\
\mathrm{mg} / \mathrm{kg} \\
\mathrm{mg} / \mathrm{kg} \\
\mathrm{mg} / \mathrm{kg} \\
\mathrm{mg} / \mathrm{kg} \\
\mathrm{mg} / \mathrm{kg}\end{array}$ & $\begin{array}{r}20800.00 \\
0.47 \\
5.80 \\
75.40 \\
0.76 \\
9.90 \\
0.23 \\
498.00 \\
27.70 \\
15.90 \\
11.00 \\
0.12 \\
23000.00 \\
15.30 \\
10.90 \\
1940.00 \\
733.00 \\
0.18 \\
1.60 \\
17.80 \\
2640.00 \\
0.56 \\
328.00 \\
1.20 \\
4.40 \\
25.40 \\
0.47 \\
29.40 \\
40.60\end{array}$ & $\begin{array}{l}\text { B } \\
\text { UJ } \\
U \\
B\end{array}$ & $\begin{array}{r}29300.00 \\
0.59 \\
5.90 \\
86.20 \\
0.84 \\
10.00 \\
0.24 \\
370.00 \\
30.70 \\
8.70 \\
14.90 \\
0.12 \\
33400.00 \\
9.80 \\
21.80 \\
2870.00 \\
173.00 \\
0.11 \\
1.70 \\
22.30 \\
3330.00 \\
0.62 \\
294.00 \\
1.20 \\
5.00 \\
58.90 \\
0.48 \\
41.30 \\
49.50\end{array}$ & $\begin{array}{l}B \\
U J \\
U \\
U \\
B \\
B\end{array}$ & $\begin{array}{r}33700.00 \\
0.62 \\
11.00 \\
81.30 \\
1.10 \\
9.70 \\
0.24 \\
339.00 \\
80.10 \\
27.30 \\
29.70 \\
0.12 \\
39200.00 \\
49.60 \\
23.30 \\
3740.00 \\
689.00 \\
0.10 \\
1.60 \\
30.90 \\
4600.00 \\
1.10 \\
246.00 \\
1.20 \\
3.40 \\
34.90 \\
0.72 \\
38.70 \\
50.70\end{array}$ \\
\hline
\end{tabular}

Location=ROA; Formation $=$ COPPER RIDGE; Site =33,35,44; Sample.type $=$ Regular; $A=6046 ; B=6049 ; C=6052 ; A$ lab split =' '; Phase $=2$

\begin{tabular}{|c|c|c|c|c|c|c|}
\hline $\begin{array}{l}\text { Aluminum } \\
\text { Ant imony } \\
\text { Arsenic } \\
\text { Barium } \\
\text { Beryllium } \\
\text { Cadmium } \\
\text { Calcium } \\
\text { Chromium } \\
\text { Cobalt } \\
\text { Copper } \\
\text { Cyanide } \\
\text { I ron } \\
\text { Lead } \\
\text { Lithium } \\
\text { Magnesium } \\
\text { Manganese } \\
\text { Mercury } \\
\text { Molybdenum } \\
\text { Nickel } \\
\text { Potassium } \\
\text { Selenium } \\
\text { Silver } \\
\text { Sodium } \\
\text { Strontium } \\
\text { Sulfate } \\
\text { Thallium } \\
\text { Vanadium } \\
\text { Zinc }\end{array}$ & $\begin{array}{l}\mathrm{mg} / \mathrm{kg} \\
\mathrm{mg} / \mathrm{kg} \\
\mathrm{mg} / \mathrm{kg} \\
\mathrm{mg} / \mathrm{kg} \\
\mathrm{mg} / \mathrm{kg} \\
\mathrm{mg} / \mathrm{kg} \\
\mathrm{mg} / \mathrm{kg} \\
\mathrm{mg} / \mathrm{kg} \\
\mathrm{mg} / \mathrm{kg} \\
\mathrm{mg} / \mathrm{kg} \\
\mathrm{mg} / \mathrm{kg} \\
\mathrm{mg} / \mathrm{kg} \\
\mathrm{mg} / \mathrm{kg} \\
\mathrm{mg} / \mathrm{kg} \\
\mathrm{mg} / \mathrm{kg} \\
\mathrm{mg} / \mathrm{kg} \\
\mathrm{mg} / \mathrm{kg} \\
\mathrm{mg} / \mathrm{kg} \\
\mathrm{mg} / \mathrm{kg} \\
\mathrm{mg} / \mathrm{kg} \\
\mathrm{mg} / \mathrm{kg} \\
\mathrm{mg} / \mathrm{kg} \\
\mathrm{mg} / \mathrm{kg} \\
\mathrm{mg} / \mathrm{kg} \\
\mathrm{mg} / \mathrm{kg} \\
\mathrm{mg} / \mathrm{kg} \\
\mathrm{mg} / \mathrm{kg} \\
\mathrm{mg} / \mathrm{kg}\end{array}$ & $\begin{array}{r}9290.00 \\
0.44 \\
10.30 \\
72.00 \\
0.51 \\
0.22 \\
716.00 \\
12.20 \\
7.80 \\
7.40 \\
1.00 \\
11100.00 \\
21.20 \\
5.30 \\
454.00 \\
1050.00 \\
0.12 \\
1.60 \\
7.00 \\
376.00 \\
0.44 \\
1.10 \\
383.00 \\
4.60 \\
28.10 \\
0.44 \\
23.40 \\
42.80\end{array}$ & $\begin{array}{l}U \\
U \\
\text { B } \\
U \\
U \\
B \\
B\end{array}$ & $\begin{array}{r}18000.00 \\
0.45 \\
25.30 \\
39.30 \\
0.58 \\
0.22 \\
432.00 \\
28.50 \\
6.40 \\
14.50 \\
1.00 \\
25200.00 \\
22.30 \\
17.10 \\
787.00 \\
222.00 \\
0.12 \\
2.00 \\
14.60 \\
744.00 \\
0.74 \\
1.10 \\
428.00 \\
2.60 \\
124.00 \\
0.45 \\
50.60 \\
64.90\end{array}$ & $\begin{array}{l}\mathbf{B} \\
\mathbf{B} \\
\mathbf{U} \\
\mathbf{B} \\
\mathbf{B} \\
\mathbf{J} \\
\mathbf{U} \\
\mathbf{J}\end{array}$ & $\begin{array}{r}23200.00 \\
0.47 \\
36.10 \\
16.20 \\
0.73 \\
0.23 \\
337.00 \\
47.10 \\
4.50 \\
22.30 \\
1.10 \\
49100.00 \\
31.60 \\
22.60 \\
627.00 \\
94.80 \\
0.25 \\
4.00 \\
17.70 \\
692.00 \\
0.63 \\
1.20 \\
415.00 \\
1.20 \\
4.50 \\
0.47 \\
98.90 \\
95.80\end{array}$ \\
\hline
\end{tabular}


Table D.1 (continued)

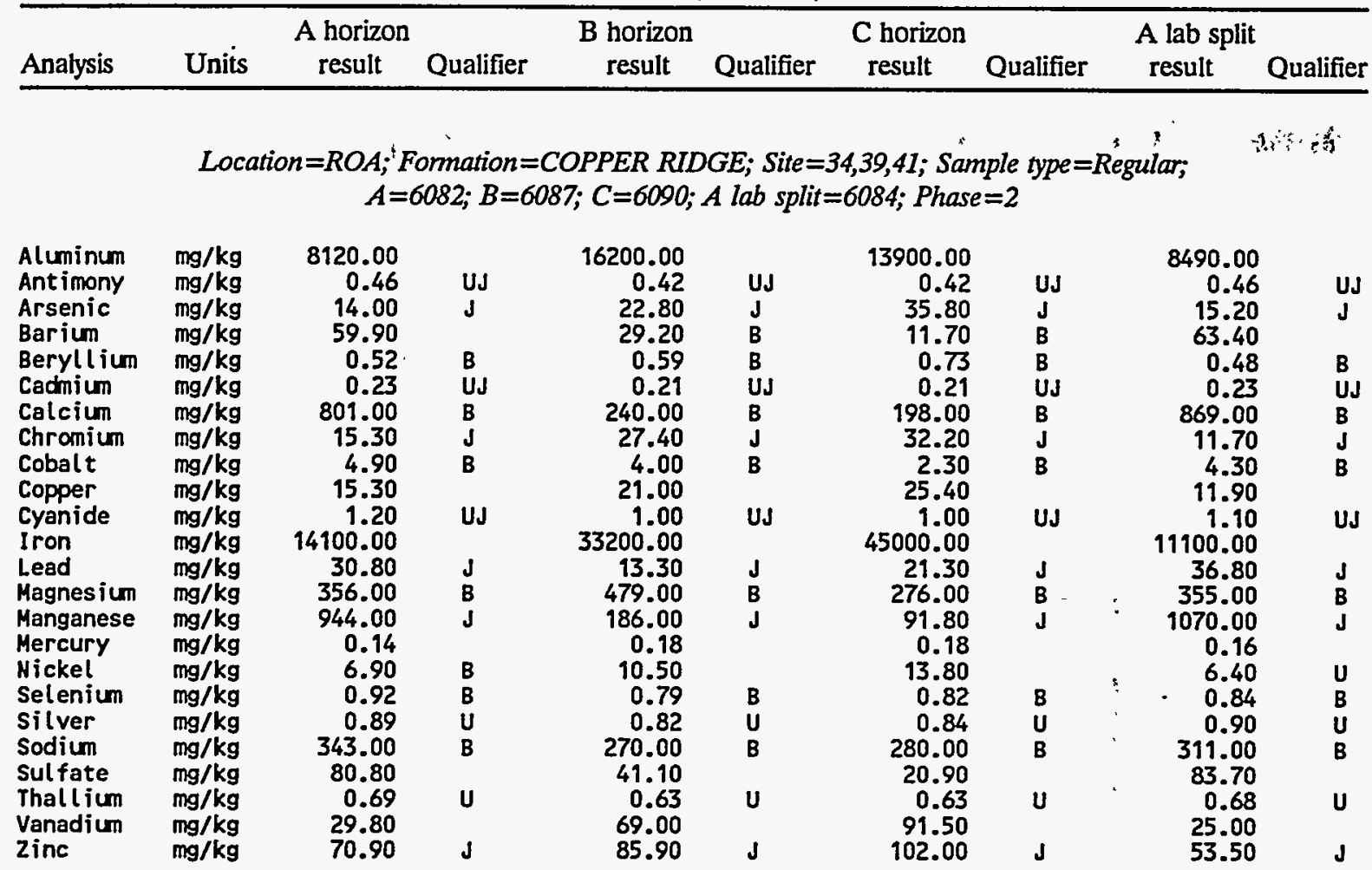

Location $=R O A ;$ Formation $=$ COPPER RIDGE; Site $=40,42,43$; Sample type $=$ Regular; $A=6055 ; B=6058 ; C=6061 ; A$ lab Split =' '; Phase $=2$

$\begin{array}{lrrrrrrr}\text { Aluminum } & \mathrm{mg} / \mathrm{kg} & 8410.00 & & 13600.00 & & 17000.00 & \\ \text { Antimony } & \mathrm{mg} / \mathrm{kg} & 0.43 & \mathrm{U} & 0.44 & \mathrm{U} & 0.46 & \mathrm{U} \\ \text { Arsenic } & \mathrm{mg} / \mathrm{kg} & 6.20 & & 11.20 & & 24.40 & \\ \text { Barium } & \mathrm{mg} / \mathrm{kg} & 37.40 & \mathrm{~B} & 28.70 & \mathrm{~B} & 16.30 & \mathrm{~B} \\ \text { Beryllium } & \mathrm{mg} / \mathrm{kg} & 0.34 & \mathrm{~B} & 0.31 & \mathrm{~B} & 0.39 & \mathrm{~B} \\ \text { Boron } & \mathrm{mg} / \mathrm{kg} & & 5.30 & \mathrm{U} & & \\ \text { Cadmium } & \mathrm{mg} / \mathrm{kg} & 0.21 & \mathrm{U} & 0.22 & \mathrm{U} & 0.23 & \mathrm{U} \\ \text { Calcium } & \mathrm{mg} / \mathrm{kg} & 280.00 & \mathrm{~B} & 272.00 & \mathrm{~B} & 172.00 & \mathrm{~B} \\ \text { Chromium } & \mathrm{mg} / \mathrm{kg} & 11.90 & \mathrm{~J} & 19.00 & \mathrm{~J} & 30.70 & \mathrm{~J} \\ \text { Cobalt } & \mathrm{mg} / \mathrm{kg} & 5.00 & \mathrm{U} & 2.40 & \mathrm{U} & 1.70 & \mathrm{U} \\ \text { Copper } & \mathrm{mg} / \mathrm{kg} & 2.70 & \mathrm{U} & 8.90 & & 9.90 & \\ \text { Cyanide } & \mathrm{mg} / \mathrm{kg} & 1.00 & \mathrm{UJ} & 1.00 & \mathrm{U} & 1.10 & \mathrm{UJ} \\ \text { Iron } & \mathrm{mg} / \mathrm{kg} & 13600.00 & \mathrm{~J} & 16700.00 & \mathrm{~J} & 31600.00 & \mathrm{~J} \\ \text { Lead } & \mathrm{mg} / \mathrm{kg} & 13.50 & & 6.50 & & 9.70 & \mathrm{~J} \\ \text { Lithium } & \mathrm{mg} / \mathrm{kg} & 2.60 & \mathrm{~B} & 6.60 & \mathrm{~B} & 7.70 & \mathrm{~B} \\ \text { Magnesium } & \mathrm{mg} / \mathrm{kg} & 379.00 & \mathrm{~B} & 527.00 & \mathrm{~B} & 543.00 & \mathrm{~B} \\ \text { Manganese } & \mathrm{mg} / \mathrm{kg} & 369.00 & & 53.70 & & 41.70 & \\ \text { Mercury } & \mathrm{mg} / \mathrm{kg} & 0.12 & & 0.20 & & 0.30 & \\ \text { Molybdenum } & \mathrm{mg} / \mathrm{kg} & 1.50 & \mathrm{U} & 1.50 & \mathrm{U} & 1.90 & \mathrm{~B} \\ \text { Hickel } & \mathrm{mg} / \mathrm{kg} & 5.90 & \mathrm{U} & 6.10 & \mathrm{U} & 8.00 & \mathrm{U} \\ \text { Potassium } & \mathrm{mg} / \mathrm{kg} & 261.00 & \mathrm{~B} & 396.00 & \mathrm{~B} & 434.00 & \mathrm{~B} \\ \text { Selenium } & \mathrm{mg} / \mathrm{kg} & 0.49 & \mathrm{~B} & 0.44 & \mathrm{U} & 0.65 & \mathrm{~B} \\ \text { Silver } & \mathrm{mg} / \mathrm{kg} & 1.10 & \mathrm{U} & 1.10 & \mathrm{U} & 1.20 & \mathrm{U} \\ \text { Sodium } & \mathrm{mg} / \mathrm{kg} & 371.00 & \mathrm{~B} & 352.00 & \mathrm{~B} & 392.00 & \mathrm{~B} \\ \text { Strontium } & \mathrm{mg} / \mathrm{kg} & 2.30 & \mathrm{~B} & 2.00 & \mathrm{~B} & 1.20 & \mathrm{~B} \\ \text { Sulfate } & \mathrm{mg} / \mathrm{kg} & 36.80 & \mathrm{~J} & 36.00 & \mathrm{~J} & 20.30 & \mathrm{~J} \\ \text { Thallium } & \mathrm{mg} / \mathrm{kg} & 0.43 & \mathrm{U} & 0.44 & \mathrm{U} & 0.46 & \mathrm{U} \\ \text { Vanadium } & \mathrm{mg} / \mathrm{kg} & 19.20 & \mathrm{~J} & 37.20 & \mathrm{~J} & 68.70 & \mathrm{~J} \\ \text { Zinc } & \mathrm{mg} / \mathrm{kg} & 36.60 & & 29.30 & & 39.30 & \\ & & & & & & \end{array}$

Location=ROA; Formation $=$ COPPER RIDGE; Site $=45,46,47 ;$ Sample type $=$ Field duplicate; $A=6076 ; B=6079 ; C=6067 ; A$ lab split =' '; Phase $=2$

\begin{tabular}{|c|c|c|c|c|c|c|}
\hline $\begin{array}{l}\text { Aluminum } \\
\text { Antimony } \\
\text { Arsenic }\end{array}$ & $\begin{array}{l}\mathrm{mg} / \mathrm{kg} \\
\mathrm{mg} / \mathrm{kg} \\
\mathrm{mg} / \mathrm{kg}\end{array}$ & $\begin{array}{r}10700.00 \\
0.42 \\
8.30\end{array}$ & $\mathbf{U}$ & $\begin{array}{r}14300.00 \\
0.41 \\
13.10\end{array}$ & $\mathbf{U}$ & $\begin{array}{r}15500.00 \\
2.20 \\
20.20\end{array}$ \\
\hline
\end{tabular}


D-22

Table D.1 (continued)

\begin{tabular}{|c|c|c|c|c|c|c|c|c|c|}
\hline Analysis & Units & $\begin{array}{l}\text { A horizon } \\
\text { result }\end{array}$ & Qualifier & $\begin{array}{l}\text { B horizon } \\
\text { result }\end{array}$ & Qualifier & $\begin{array}{l}C \text { horizon } \\
\text { result }\end{array}$ & Qualifier & $\begin{array}{l}\text { A lab split } \\
\text { result }\end{array}$ & Qualifier \\
\hline $\begin{array}{l}\text { Barium } \\
\text { Beryll ium } \\
\text { Boron } \\
\text { Cadmium } \\
\text { Calcium } \\
\text { Chromium } \\
\text { Cobalt } \\
\text { Copper } \\
\text { Cyanide } \\
\text { Iron } \\
\text { Lead } \\
\text { Lithium } \\
\text { Magnesium } \\
\text { Manganese } \\
\text { Mercury } \\
\text { Molybdenum } \\
\text { Nickel } \\
\text { Potassium } \\
\text { Selenium } \\
\text { silicon } \\
\text { Silver } \\
\text { Sodium } \\
\text { Strontium } \\
\text { Sulfate } \\
\text { Thallium } \\
\text { Vanadium } \\
\text { Zinc }\end{array}$ & $\begin{array}{l}\mathrm{mg} / \mathrm{kg} \\
\mathrm{mg} / \mathrm{kg} \\
\mathrm{mg} / \mathrm{kg} \\
\mathrm{mg} / \mathrm{kg} \\
\mathrm{mg} / \mathrm{kg} \\
\mathrm{mg} / \mathrm{kg} \\
\mathrm{mg} / \mathrm{kg} \\
\mathrm{mg} / \mathrm{kg} \\
\mathrm{mg} / \mathrm{kg} \\
\mathrm{mg} / \mathrm{kg} \\
\mathrm{mg} / \mathrm{kg} \\
\mathrm{mg} / \mathrm{kg} \\
\mathrm{mg} / \mathrm{kg} \\
\mathrm{mg} / \mathrm{kg} \\
\mathrm{mg} / \mathrm{kg} \\
\mathrm{mg} / \mathrm{kg} \\
\mathrm{mg} / \mathrm{kg} \\
\mathrm{mg} / \mathrm{kg} \\
\mathrm{mg} / \mathrm{kg} \\
\mathrm{mg} / \mathrm{kg} \\
\mathrm{mg} / \mathrm{kg} \\
\mathrm{mg} / \mathrm{kg} \\
\mathrm{mg} / \mathrm{kg} \\
\mathrm{mg} / \mathrm{kg} \\
\mathrm{mg} / \mathrm{kg} \\
\mathrm{mg} / \mathrm{kg} \\
\mathrm{mg} / \mathrm{kg}\end{array}$ & $\begin{array}{r}87.10 \\
0.50 \\
5.00 \\
0.21 \\
562.00 \\
13.40 \\
6.90 \\
5.40 \\
1.10 \\
9030.00 \\
14.60 \\
3.10 \\
478.00 \\
1270.00 \\
0.10 \\
2.70 \\
6.40 \\
290.00 \\
0.42 \\
621.00 \\
0.84 \\
349.00 \\
4.20 \\
120.00 \\
0.42 \\
21.60 \\
24.50\end{array}$ & $\begin{array}{l}B \\
U \\
U \\
B \\
J \\
U \\
U \\
U J \\
J \\
\\
B \\
B \\
\\
U \\
U \\
U \\
U \\
U \\
J \\
U \\
B \\
B \\
\\
U \\
J \\
J\end{array}$ & $\begin{array}{r}45.40 \\
0.42 \\
4.90 \\
0.20 \\
312.00 \\
29.50 \\
1.90 \\
10.50 \\
1.10 \\
23300.00 \\
7.20 \\
5.60 \\
479.00 \\
74.50 \\
0.12 \\
2.70 \\
7.60 \\
430.00 \\
0.49 \\
596.00 \\
0.82 \\
314.00 \\
2.70 \\
52.60 \\
0.41 \\
51.50 \\
22.90\end{array}$ & 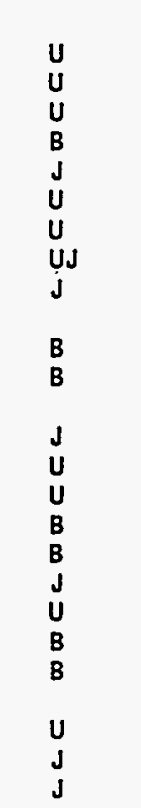 & $\begin{array}{r}23.90 \\
0.46 \\
5.30 \\
0.22 \\
313.00 \\
32.10 \\
1.10 \\
14.40 \\
1.10 \\
30500.00 \\
8.30 \\
6.60 \\
474.00 \\
51.40 \\
0.23 \\
3.20 \\
9.30 \\
419.00 \\
0.59 \\
591.00 \\
0.89 \\
389.00 \\
2.40 \\
11.10 \\
0.44 \\
70.90 \\
26.30\end{array}$ & $\begin{array}{l}B \\
B \\
U \\
U \\
B \\
J \\
U \\
U J \\
J \\
B \\
B \\
J \\
B \\
B \\
B \\
J \\
U \\
B \\
B \\
U \\
U \\
J \\
J\end{array}$ & . & \\
\hline
\end{tabular}

Location $=R O A ;$ Formation $=$ COPPER RIDGE; Site $=45,46,47$; Sample type $=$ Regular; $A=6073 ; B=6070 ; C=6064 ; A$ lab split=' '; Phase $=2$

$\begin{array}{lrrrrrrr}\text { Aluminum } & \mathrm{mg} / \mathrm{kg} & 10900.00 & & 13700.00 & & 13700.00 & \\ \text { Antimony } & \mathrm{mg} / \mathrm{kg} & 0.41 & \mathrm{U} & 0.42 & \mathrm{U} & 2.20 & \mathrm{U} \\ \text { Arsenic } & \mathrm{mg} / \mathrm{kg} & 7.20 & & 11.10 & & 26.50 & \\ \text { Barium } & \mathrm{mg} / \mathrm{kg} & 83.00 & & 57.60 & & 18.50 & \mathrm{~B} \\ \text { Beryllium } & \mathrm{mg} / \mathrm{kg} & 0.49 & \mathrm{~B} & 0.36 & \mathrm{~B} & 0.45 & \mathrm{~B} \\ \text { Boron } & \mathrm{mg} / \mathrm{kg} & 5.00 & \mathrm{U} & 5.00 & \mathrm{U} & 5.30 & \mathrm{U} \\ \text { Cadnium } & \mathrm{mg} / \mathrm{kg} & 0.21 & \mathrm{U} & 0.21 & \mathrm{~J} & 0.22 & \mathrm{U} \\ \text { Calcium } & \mathrm{mg} / \mathrm{kg} & 510.00 & \mathrm{~B} & 304.00 & \mathrm{~B} & 300.00 & \mathrm{~B} \\ \text { Chromium } & \mathrm{mg} / \mathrm{kg} & 12.80 & \mathrm{~J} & 20.10 & \mathrm{~J} & 35.40 & \mathrm{~J} \\ \text { Cobalt } & \mathrm{mg} / \mathrm{kg} & 7.40 & \mathrm{~B} & 3.10 & \mathrm{~B} & 1.30 & \mathrm{U} \\ \text { Copper } & \mathrm{mg} / \mathrm{kg} & 5.50 & & 8.00 & & 12.30 & \\ \text { Cyanide } & \mathrm{mg} / \mathrm{kg} & 1.00 & \mathrm{UJ} & 1.00 & \mathrm{UJ} & 1.10 & \mathrm{UJ} \\ \text { Iron } & \mathrm{mg} / \mathrm{kg} & 9700.00 & \mathrm{~J} & 16600.00 & \mathrm{~J} & 28900.00 & \mathrm{~J} \\ \text { Lead } & \mathrm{mg} / \mathrm{kg} & 17.00 & & 7.70 & & 10.30 & \\ \text { Lithium } & \mathrm{mg} / \mathrm{kg} & 3.20 & \mathrm{~B} & 6.30 & \mathrm{~B} & 4.50 & \mathrm{~B} \\ \text { Magnesium } & \mathrm{mg} / \mathrm{kg} & 455.00 & \mathrm{~B} & 480.00 & \mathrm{~B} & 389.00 & \mathrm{~B} \\ \text { Manganese } & \mathrm{mg} / \mathrm{kg} & 1450.00 & & 133.00 & & 65.60 & \\ \text { Mercury } & \mathrm{mg} / \mathrm{kg} & 0.10 & \mathrm{U} & 0.10 & \mathrm{U} & 0.20 & \mathrm{~J} \\ \text { Molybdenum } & \mathrm{mg} / \mathrm{kg} & 2.70 & \mathrm{U} & 2.70 & \mathrm{U} & 2.90 & \mathrm{U} \\ \text { Mickel } & \mathrm{mg} / \mathrm{kg} & 6.10 & \mathrm{~B} & 7.10 & \mathrm{~B} & 7.50 & \mathrm{~B} \\ \text { Potassium } & \mathrm{mg} / \mathrm{kg} & 321.00 & \mathrm{~B} & 315.00 & \mathrm{~B} & 414.00 & \mathrm{~B} \\ \text { Selenium } & \mathrm{mg} / \mathrm{kg} & 0.41 & \mathrm{U} & 0.54 & \mathrm{~B} & 0.48 & \mathrm{~B} \\ \text { Silicon } & \mathrm{mg} / \mathrm{kg} & 538.00 & \mathrm{~J} & 565.00 & \mathrm{~J} & 602.00 & \mathrm{~J} \\ \text { Silver } & \mathrm{mg} / \mathrm{kg} & 0.83 & \mathrm{U} & 0.84 & \mathrm{U} & 0.89 & \mathrm{U} \\ \text { Sodium } & \mathrm{mg} / \mathrm{kg} & 324.00 & \mathrm{~B} & 364.00 & \mathrm{~B} & 345.00 & \mathrm{~B} \\ \text { Strontium } & \mathrm{mg} / \mathrm{kg} & 3.80 & \mathrm{~B} & 2.50 & \mathrm{~B} & 2.10 & \mathrm{~B} \\ \text { Sulfate } & \mathrm{mg} / \mathrm{kg} & 94.20 & & 67.20 & & 11.10 & \mathrm{U} \\ \text { Thallium } & \mathrm{mg} / \mathrm{kg} & 0.41 & \mathrm{U} & 0.42 & \mathrm{U} & 0.44 & \mathrm{U} \\ \text { Vanadium } & \mathrm{mg} / \mathrm{kg} & 24.10 & \mathrm{~J} & 39.40 & \mathrm{~J} & 68.10 & \mathrm{~J} \\ \text { Zinc } & \mathrm{mg} / \mathrm{kg} & 24.90 & \mathrm{~J} & 21.10 & \mathrm{~J} & 21.90 & \mathrm{~J}\end{array}$

Location $=R O A ;$ Formation $=D I S M A L$ GAP; Site $=10,13,14 ;$ Sample type $=$ Field duplicate; $A=6037 ; B=6040 ; C=6043 ; A$ lab split=' '; Phase $=1$

\begin{tabular}{|c|c|c|c|c|c|c|}
\hline $\begin{array}{l}\text { Aluminum } \\
\text { Antimony } \\
\text { Arsenic } \\
\text { Barium }\end{array}$ & $\begin{array}{l}\mathrm{mg} / \mathrm{kg} \\
\mathrm{mg} / \mathrm{kg} \\
\mathrm{mg} / \mathrm{kg} \\
\mathrm{mg} / \mathrm{kg}\end{array}$ & $\begin{array}{r}17500.00 \\
0.26 \\
6.90 \\
179.00\end{array}$ & UJ & $\begin{array}{r}33300.00 \\
0.23 \\
7.10 \\
90.70\end{array}$ & UJ & $\begin{array}{r}28000.00 \\
0.22 \\
6.70 \\
103.00\end{array}$ \\
\hline
\end{tabular}


Table D.1 (continued)

\begin{tabular}{|c|c|c|c|c|c|c|c|c|c|}
\hline Analysis & Units & $\begin{array}{l}\text { A horizon } \\
\text { result }\end{array}$ & Qualifier & $\begin{array}{l}\text { B horizon } \\
\text { result }\end{array}$ & Qualifier & $\begin{array}{l}C \text { horizon } \\
\text { result }\end{array}$ & Qualifier & $\begin{array}{l}\text { A lab split } \\
\text { result }\end{array}$ & Qualifier \\
\hline $\begin{array}{l}\text { Beryll ium } \\
\text { Boron } \\
\text { Cadmium } \\
\text { Calcium } \\
\text { Chromium } \\
\text { Cobalt } \\
\text { Copper } \\
\text { Cyanide } \\
\text { Iron } \\
\text { Lead } \\
\text { Lithium } \\
\text { Magnesium } \\
\text { Manganese } \\
\text { Mercury } \\
\text { Molybdenum } \\
\text { Nickel } \\
\text { Potassium } \\
\text { Selenium } \\
\text { sil icon } \\
\text { silver } \\
\text { Strontium } \\
\text { Sulfate } \\
\text { Thall ium } \\
\text { Vanadium } \\
\text { Zinc }\end{array}$ & $\begin{array}{l}\mathrm{mg} / \mathrm{kg} \\
\mathrm{mg} / \mathbf{k g} \\
\mathrm{mg} / \mathbf{k g} \\
\mathrm{mg} / \mathrm{kg} \\
\mathrm{mg} / \mathrm{kg} \\
\mathrm{mg} / \mathrm{kg} \\
\mathrm{mg} / \mathrm{kg} \\
\mathrm{mg} / \mathrm{kg} \\
\mathrm{mg} / \mathrm{kg} \\
\mathrm{mg} / \mathbf{k g} \\
\mathrm{mg} / \mathbf{k g} \\
\mathrm{mg} / \mathbf{k g} \\
\mathrm{mg} / \mathbf{k g} \\
\mathrm{mg} / \mathbf{k g} \\
\mathrm{mg} / \mathbf{k g} \\
\mathrm{mg} / \mathbf{k g} \\
\mathrm{mg} / \mathbf{k g} \\
\mathrm{mg} / \mathbf{k g} \\
\mathrm{mg} / \mathbf{k g} \\
\mathrm{mg} / \mathbf{k g} \\
\mathrm{mg} / \mathbf{k g} \\
\mathrm{mg} / \mathbf{k g} \\
\mathrm{mg} / \mathbf{k g} \\
\mathrm{mg} / \mathbf{k g} \\
\mathrm{mg} / \mathbf{k g}\end{array}$ & $\begin{array}{r}1.00 \\
10.40 \\
0.26 \\
1990.00 \\
32.40 \\
40.10 \\
7.70 \\
0.40 \\
30500.00 \\
15.20 \\
11.60 \\
2000.00 \\
4470.00 \\
0.15 \\
10.10 \\
23.20 \\
1840.00 \\
1.30 \\
462.00 \\
2.20 \\
8.30 \\
103.00 \\
0.38 \\
37.60 \\
51.90\end{array}$ & $\begin{array}{l}u \\
u\end{array}$ & $\begin{array}{r}1.30 \\
13.10 \\
0.23 \\
800.00 \\
47.70 \\
4.30 \\
16.90 \\
0.12 \\
45200.00 \\
7.40 \\
25.40 \\
3170.00 \\
125.00 \\
0.15 \\
9.40 \\
26.90 \\
3310.00 \\
1.20 \\
613.00 \\
2.10 \\
6.70 \\
130.00 \\
0.37 \\
45.60 \\
57.40\end{array}$ & $\begin{array}{l}\text { B } \\
\text { UJ }\end{array}$ & $\begin{array}{r}1.60 \\
22.10 \\
0.22 \\
606.00 \\
42.30 \\
13.30 \\
20.90 \\
0.11 \\
41100.00 \\
13.30 \\
25.60 \\
4750.00 \\
574.00 \\
0.15 \\
8.40 \\
38.90 \\
3480.00 \\
1.10 \\
530.00 \\
1.80 \\
6.50 \\
74.00 \\
0.27 \\
34.80 \\
72.60\end{array}$ & $\begin{array}{l}u \\
u\end{array}$ & $\therefore$ & 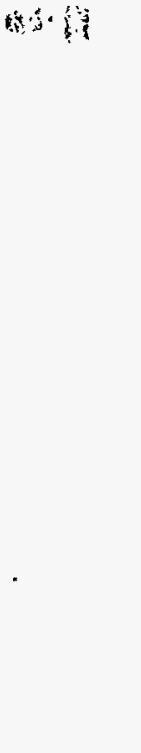 \\
\hline
\end{tabular}

Location $=R O A ;$ Formation $=D I S M A L$ GAP; Site $=10,13,14 ;$ Sample type $=$ Regular; $A=6028 ; B=6031 ; C=6034 ; A$ lab split=' '; Phase $=1$

\begin{tabular}{|c|c|c|c|c|c|c|c|}
\hline $\begin{array}{l}\text { Aluminum } \\
\text { Antimony } \\
\text { Arsenic } \\
\text { Barium } \\
\text { Beryllium } \\
\text { Boron } \\
\text { Cadnium } \\
\text { Calcium } \\
\text { Chromium } \\
\text { Cobalt } \\
\text { Copper } \\
\text { Cyanide } \\
\text { Iron } \\
\text { Lead } \\
\text { Lithium } \\
\text { Magnesium } \\
\text { Manganese } \\
\text { Hercury } \\
\text { Molybdenum } \\
\text { Nickel } \\
\text { Potassium } \\
\text { Selenium } \\
\text { silicon } \\
\text { silver } \\
\text { Strontium } \\
\text { Sulfate } \\
\text { Thallium } \\
\text { Vanadium } \\
\text { Zinc }\end{array}$ & $\begin{array}{l}\mathrm{mg} / \mathrm{kg} \\
\mathrm{mg} / \mathrm{kg} \\
\mathrm{mg} / \mathrm{kg} \\
\mathrm{mg} / \mathrm{kg} \\
\mathrm{mg} / \mathrm{kg} \\
\mathrm{mg} / \mathrm{kg} \\
\mathrm{mg} / \mathrm{kg} \\
\mathrm{mg} / \mathrm{kg} \\
\mathrm{mg} / \mathrm{kg} \\
\mathrm{mg} / \mathrm{kg} \\
\mathrm{mg} / \mathrm{kg} \\
\mathrm{mg} / \mathrm{kg} \\
\mathrm{mg} / \mathrm{kg} \\
\mathrm{mg} / \mathrm{kg} \\
\mathrm{mg} / \mathrm{kg} \\
\mathrm{mg} / \mathrm{kg} \\
\mathrm{mg} / \mathrm{kg} \\
\mathrm{mg} / \mathrm{kg} \\
\mathrm{mg} / \mathrm{kg} \\
\mathrm{mg} / \mathrm{kg} \\
\mathrm{mg} / \mathrm{kg} \\
\mathrm{mg} / \mathrm{kg} \\
\mathrm{mg} / \mathrm{kg} \\
\mathrm{mg} / \mathrm{kg} \\
\mathrm{mg} / \mathrm{kg} \\
\mathrm{mg} / \mathrm{kg} \\
\mathrm{mg} / \mathrm{kg} \\
\mathrm{mg} / \mathrm{kg} \\
\mathrm{mg} / \mathrm{kg}\end{array}$ & $\begin{array}{r}17700.00 \\
0.25 \\
3.90 \\
182.00 \\
1.10 \\
26.00 \\
0.25 \\
2020.00 \\
25.60 \\
31.60 \\
12.00 \\
0.13 \\
28100.00 \\
15.00 \\
12.60 \\
2420.00 \\
2420.00 \\
0.15 \\
9.80 \\
24.10 \\
2090.00 \\
1.30 \\
549.00 \\
2.10 \\
8.90 \\
32.10 \\
0.36 \\
32.20 \\
55.10\end{array}$ & $\begin{array}{l}\mathbf{U} \\
\mathbf{U}\end{array}$ & $\begin{array}{r}29300.00 \\
0.20 \\
7.20 \\
82.10 \\
1.20 \\
12.20 \\
0.20 \\
758.00 \\
46.00 \\
7.40 \\
18.00 \\
0.12 \\
40900.00 \\
10.90 \\
23.20 \\
2950.00 \\
212.00 \\
0.15 \\
8.70 \\
23.80 \\
2750.00 \\
1.00 \\
592.00 \\
1.90 \\
5.70 \\
107.00 \\
0.37 \\
41.80 \\
51.20\end{array}$ & $\begin{array}{l}\mathbf{U} \\
\mathbf{J}\end{array}$ & $\begin{array}{r}27300.00 \\
0.22 \\
11.80 \\
93.20 \\
1.40 \\
16.90 \\
0.22 \\
582.00 \\
48.30 \\
8.70 \\
17.80 \\
0.11 \\
37000.00 \\
7.40 \\
24.70 \\
4620.00 \\
236.00 \\
0.15 \\
9.00 \\
34.90 \\
3490.00 \\
1.10 \\
458.00 \\
2.00 \\
5.70 \\
28.40 \\
0.38 \\
35.10 \\
61.30\end{array}$ & $\begin{array}{l}\mathbf{U} \\
\mathbf{J}\end{array}$ \\
\hline
\end{tabular}

Location=ROA; Formation=DISMAL GAP; Site $=3,7,21 ;$ Sample type $=$ Regular; $A=6019 ; B=6022 ; C=6025 ; A$ lab split ='; Phase $=1$

$\begin{array}{lrrrrrrr}\text { Aluminum } & \mathrm{mg} / \mathrm{kg} & 16500.00 & & 18400.00 & & 25000.00 & \\ \text { Antimony } & \mathrm{mg} / \mathrm{kg} & 0.22 & \mathrm{U} & 0.19 & \mathrm{U} & 0.22 & \mathrm{U} \\ \text { Arsenic } & \mathrm{mg} / \mathrm{kg} & 5.70 & & 5.90 & & 6.20 & \\ \text { Barium } & \mathrm{mg} / \mathrm{kg} & 73.20 & & 60.60 & & 62.30 & \\ \text { Beryllium } & \mathrm{mg} / \mathrm{kg} & 0.82 & \mathrm{~B} & 0.82 & \mathrm{~B} & 1.10 & \mathrm{~B} \\ \text { Boron } & \mathrm{mg} / \mathrm{kg} & 14.00 & & 9.30 & \mathrm{U} & 12.80 & \\ \text { Cadmium } & \mathrm{mg} / \mathrm{kg} & 0.22 & \mathrm{U} & 0.19 & \mathrm{U} & 0.22 & \mathrm{U}\end{array}$


Table D.1 (continued)

\begin{tabular}{|c|c|c|c|c|c|c|c|c|c|}
\hline Analysis & Units & $\begin{array}{l}\text { A horizon } \\
\text { result }\end{array}$ & Qualifier & $\begin{array}{l}\text { B horizon } \\
\text { result }\end{array}$ & Qualifier & $\begin{array}{l}C \text { horizon } \\
\text { result }\end{array}$ & Qualifier & $\begin{array}{l}\text { A lab split } \\
\text { result }\end{array}$ & Qualifier \\
\hline $\begin{array}{l}\text { Calcium } \\
\text { Chromium } \\
\text { Cobalt } \\
\text { Copper } \\
\text { Cyanide } \\
\text { Iron } \\
\text { Lead } \\
\text { Lithium } \\
\text { Magnesium } \\
\text { Manganese } \\
\text { Mercury } \\
\text { Molybdenum } \\
\text { Mickel } \\
\text { Potassium } \\
\text { Selenium } \\
\text { Silicon } \\
\text { Silver } \\
\text { Strontium } \\
\text { Sulfate } \\
\text { Thallium } \\
\text { Vanadium } \\
\text { Zinc }\end{array}$ & $\begin{array}{l}\mathrm{mg} / \mathrm{kg} \\
\mathrm{mg} / \mathrm{kg} \\
\mathrm{mg} / \mathrm{kg} \\
\mathrm{mg} / \mathrm{kg} \\
\mathrm{mg} / \mathrm{kg} \\
\mathrm{mg} / \mathrm{kg} \\
\mathrm{mg} / \mathrm{kg} \\
\mathrm{mg} / \mathrm{kg} \\
\mathrm{mg} / \mathrm{kg} \\
\mathrm{mg} / \mathrm{kg} \\
\mathrm{mg} / \mathrm{kg} \\
\mathrm{mg} / \mathrm{kg} \\
\mathrm{mg} / \mathrm{kg} \\
\mathrm{mg} / \mathrm{kg} \\
\mathrm{mg} / \mathrm{kg} \\
\mathrm{mg} / \mathrm{kg} \\
\mathrm{mg} / \mathrm{kg} \\
\mathrm{mg} / \mathrm{kg} \\
\mathrm{mg} / \mathrm{kg} \\
\mathrm{mg} / \mathrm{kg} \\
\mathrm{mg} / \mathrm{kg} \\
\mathrm{mg} / \mathrm{kg}\end{array}$ & $\begin{array}{r}857.00 \\
33.80 \\
17.80 \\
8.90 \\
0.48 \\
25900.00 \\
23.90 \\
10.90 \\
2010.00 \\
1380.00 \\
0.15 \\
9.00 \\
17.40 \\
1700.00 \\
1.10 \\
445.00 \\
2.00 \\
4.60 \\
78.20 \\
0.22 \\
31.30 \\
40.60\end{array}$ & $\begin{array}{l}\mathbf{u} \\
\mathbf{u}\end{array}$ & $\begin{array}{r}464.00 \\
32.40 \\
20.40 \\
7.60 \\
0.11 \\
26000.00 \\
15.90 \\
11.60 \\
1560.00 \\
786.00 \\
0.15 \\
9.10 \\
14.10 \\
1330.00 \\
0.96 \\
486.00 \\
2.00 \\
3.30 \\
119.00 \\
0.48 \\
33.30 \\
34.10\end{array}$ & $\begin{array}{l}\text { U } \\
\text { UJ }\end{array}$ & $\begin{array}{r}337.00 \\
38.20 \\
5.80 \\
18.10 \\
0.11 \\
41600.00 \\
14.10 \\
17.40 \\
2180.00 \\
152.00 \\
0.15 \\
9.80 \\
19.80 \\
2260.00 \\
1.10 \\
438.00 \\
2.20 \\
3.10 \\
44.30 \\
0.33 \\
34.80 \\
42.70\end{array}$ & $\begin{array}{l}\text { U } \\
\text { B } \\
\text { UJ }\end{array}$ & & \\
\hline
\end{tabular}

Location=ROA; Formation $=D I S M A L$ GAP; Site=8,20,22; Sample type=Regular; $A=6010 ; B=6013 ; C=6016 ; A$ lab split =' ; Phase $=1$

\begin{tabular}{|c|c|c|c|c|c|c|}
\hline $\begin{array}{l}\text { Aluminum } \\
\text { Antimony } \\
\text { Arsenic } \\
\text { Barium } \\
\text { Beryllium } \\
\text { Boron } \\
\text { Cadnium } \\
\text { Calcium } \\
\text { Chromium } \\
\text { Cobalt } \\
\text { Copper } \\
\text { Cyanide } \\
\text { I ron } \\
\text { Lead } \\
\text { Lithium } \\
\text { Magnesium } \\
\text { Manganese } \\
\text { Mercury } \\
\text { Molybdenum } \\
\text { Nickel } \\
\text { Osmium } \\
\text { Potassium } \\
\text { Selenium } \\
\text { Sil icon } \\
\text { Silver } \\
\text { Strontium } \\
\text { Sulfate } \\
\text { Thall ium } \\
\text { Vanadium } \\
\text { Zinc }\end{array}$ & $\begin{array}{l}\mathrm{mg} / \mathrm{kg} \\
\mathrm{mg} / \mathrm{kg} \\
\mathrm{mg} / \mathrm{kg} \\
\mathrm{mg} / \mathrm{kg} \\
\mathrm{mg} / \mathrm{kg} \\
\mathrm{mg} / \mathrm{kg} \\
\mathrm{mg} / \mathrm{kg} \\
\mathrm{mg} / \mathrm{kg} \\
\mathrm{mg} / \mathrm{kg} \\
\mathrm{mg} / \mathrm{kg} \\
\mathrm{mg} / \mathrm{kg} \\
\mathrm{mg} / \mathrm{kg} \\
\mathrm{mg} / \mathrm{kg} \\
\mathrm{mg} / \mathrm{kg} \\
\mathrm{mg} / \mathrm{kg} \\
\mathrm{mg} / \mathrm{kg} \\
\mathrm{mg} / \mathrm{kg} \\
\mathrm{mg} / \mathrm{kg} \\
\mathrm{mg} / \mathrm{kg} \\
\mathrm{mg} / \mathrm{kg} \\
\mathrm{mg} / \mathrm{kg} \\
\mathrm{mg} / \mathrm{kg} \\
\mathrm{mg} / \mathrm{kg} \\
\mathrm{mg} / \mathrm{kg} \\
\mathrm{mg} / \mathrm{kg} \\
\mathrm{mg} / \mathrm{kg} \\
\mathrm{mg} / \mathrm{kg} \\
\mathrm{mg} / \mathrm{kg} \\
\mathrm{mg} / \mathrm{kg} \\
\mathrm{mg} / \mathrm{kg}\end{array}$ & $\begin{array}{r}13300.00 \\
0.22 \\
6.60 \\
57.60 \\
0.37 \\
53.00 \\
0.22 \\
801.00 \\
27.90 \\
8.90 \\
14.10 \\
0.42 \\
26600.00 \\
17.80 \\
14.30 \\
1180.00 \\
783.00 \\
0.21 \\
9.00 \\
11.80 \\
14.10 \\
1060.00 \\
0.53 \\
516.00 \\
2.00 \\
4.40 \\
57.50 \\
0.22 \\
33.70 \\
34.70\end{array}$ & $\begin{array}{l}\mathbf{B} \\
\mathbf{J} \\
\mathbf{U} \\
\mathbf{U} \\
\mathbf{B} \\
\mathbf{J} \\
\mathbf{U} \mathbf{J} \\
\mathbf{J} \\
\mathbf{B} \\
\mathbf{U}\end{array}$ & $\begin{array}{r}26400.00 \\
0.22 \\
10.00 \\
64.00 \\
0.33 \\
40.00 \\
0.22 \\
473.00 \\
52.40 \\
3.80 \\
19.80 \\
0.44 \\
37900.00 \\
11.90 \\
24.90 \\
1910.00 \\
132.00 \\
0.18 \\
8.90 \\
15.40 \\
14.00 \\
1750.00 \\
0.61 \\
588.00 \\
2.00 \\
5.00 \\
145.00 \\
0.22 \\
44.30 \\
42.70\end{array}$ & $\begin{array}{l}\text { UJ } \\
B \\
B \\
J \\
U \\
U \\
B \\
J \\
U J \\
J \\
B\end{array}$ & $\begin{array}{r}24300.00 \\
0.22 \\
7.50 \\
63.50 \\
0.53 \\
33.00 \\
0.22 \\
369.00 \\
44.00 \\
6.10 \\
23.70 \\
0.44 \\
37900.00 \\
10.80 \\
26.70 \\
3120.00 \\
83.80 \\
0.14 \\
9.10 \\
26.70 \\
14.00 \\
2320.00 \\
0.38 \\
397.00 \\
2.00 \\
3.80 \\
36.50 \\
0.22 \\
35.20 \\
49.20\end{array}$ \\
\hline
\end{tabular}

Location=ROA; Formation=DISMAL GAP; Site=9,17,19; Sample type=Regular; $A=6004 ; B=6001 ; C=6007 ; A$ lab split=' '; Phase $=1$

$\begin{array}{ll}\text { Aluminum } & \mathrm{mg} / \mathrm{kg} \\ \text { Antimony } & \mathrm{mg} / \mathrm{kg} \\ \text { Arsenic } & \mathrm{mg} / \mathrm{kg} \\ \text { Barium } & \mathrm{mg} / \mathrm{kg} \\ \text { Beryllium } & \mathrm{mg} / \mathrm{kg} \\ \text { Boron } & \mathrm{mg} / \mathrm{kg} \\ \text { Cadmium } & \mathrm{mg} / \mathrm{kg} \\ \text { Calcium } & \mathrm{mg} / \mathrm{kg} \\ \text { Chromium } & \mathrm{mg} / \mathrm{kg} \\ \text { Cobalt } & \mathrm{mg} / \mathrm{kg} \\ \text { Copper } & \mathrm{mg} / \mathrm{kg}\end{array}$

$\begin{array}{rlr}14500.00 & & 20600.00 \\ 0.21 & \text { UJ } & 0.21 \\ 5.80 & \text { B } & 5.80 \\ 78.30 & & 69.40 \\ 0.55 & \text { B } & 0.46 \\ 38.90 & \text { J } & 16.00 \\ 0.21 & \text { U } & 0.21 \\ 526.00 & \text { U } & 605.00 \\ 20.20 & & 26.50 \\ 37.00 & & 20.30 \\ 12.00 & \text { J } & 13.40\end{array}$

$\begin{array}{rl}23700.00 & \\ 0.21 & \text { UJ } \\ 7.10 & \mathrm{~B} \\ 73.20 & \\ 0.53 & \mathrm{~B} \\ 36.60 & \mathrm{~J} \\ 0.21 & \mathrm{U} \\ 487.00 & \mathrm{U} \\ 29.60 & \\ 27.00 & \\ 38.90 & \mathrm{~J}\end{array}$




\section{D-25}

Table D.1 (continued)

\begin{tabular}{|c|c|c|c|c|c|c|c|c|c|}
\hline Analysis & Units & $\begin{array}{l}\text { A horizon } \\
\text { result }\end{array}$ & Qualifier & $\begin{array}{l}\text { B horizon } \\
\text { result }\end{array}$ & Qualifier & $\begin{array}{l}C \text { horizon } \\
\text { result }\end{array}$ & Qualifier & $\begin{array}{l}\text { A lab split } \\
\text { result }\end{array}$ & Qualifier \\
\hline $\begin{array}{l}\text { Cyanide } \\
\text { Iron } \\
\text { Lead } \\
\text { Lithium } \\
\text { Magnesium } \\
\text { Manganese } \\
\text { Mercury } \\
\text { Molybdenum } \\
\text { Mickel } \\
\text { Osmium } \\
\text { Potassium } \\
\text { Selenium } \\
\text { Silicon } \\
\text { Silver } \\
\text { Sodium } \\
\text { Strontium } \\
\text { Sulfate } \\
\text { Thallium } \\
\text { Vanadium } \\
\text { Zinc }\end{array}$ & $\begin{array}{l}\mathrm{mg} / \mathrm{kg} \\
\mathrm{mg} / \mathrm{kg} \\
\mathrm{mg} / \mathrm{kg} \\
\mathrm{mg} / \mathrm{kg} \\
\mathrm{mg} / \mathrm{kg} \\
\mathrm{mg} / \mathrm{kg} \\
\mathrm{mg} / \mathrm{kg} \\
\mathrm{mg} / \mathrm{kg} \\
\mathrm{mg} / \mathrm{kg} \\
\mathrm{mg} / \mathrm{kg} \\
\mathrm{mg} / \mathrm{kg} \\
\mathrm{mg} / \mathrm{kg} \\
\mathrm{mg} / \mathrm{kg} \\
\mathrm{mg} / \mathrm{kg} \\
\mathrm{mg} / \mathrm{kg} \\
\mathrm{mg} / \mathrm{kg} \\
\mathrm{mg} / \mathrm{kg} \\
\mathrm{mg} / \mathrm{kg} \\
\mathrm{mg} / \mathrm{kg} \\
\mathrm{mg} / \mathrm{kg}\end{array}$ & $\begin{array}{r}0.43 \\
20700.00 \\
48.20 \\
13.70 \\
1200.00 \\
2360.00 \\
0.16 \\
8.70 \\
16.20 \\
13.50 \\
818.00 \\
1.20 \\
474.00 \\
1.90 \\
301.00 \\
3.50 \\
78.40 \\
0.27 \\
29.30 \\
36.30\end{array}$ & $\begin{array}{l}\text { U } \\
\text { UJ } \\
\text { J } \\
U \\
U \\
\text { J } \\
\text { B }\end{array}$ & $\begin{array}{r}0.42 \\
26600.00 \\
15.50 \\
19.20 \\
1700.00 \\
774.00 \\
0.15 \\
8.60 \\
18.70 \\
13.40 \\
1280.00 \\
0.44 \\
422.00 \\
1.90 \\
332.00 \\
4.50 \\
159.00 \\
0.42 \\
36.20 \\
36.20\end{array}$ & $\begin{array}{l}U \\
U \\
\text { UJ } \\
\text { B } \\
\text { J } \\
U \\
U \\
\text { J } \\
\text { B }\end{array}$ & $\begin{array}{r}0.42 \\
37000.00 \\
38.90 \\
31.20 \\
2570.00 \\
963.00 \\
0.16 \\
8.60 \\
26.70 \\
13.50 \\
2100.00 \\
0.21 \\
373.00 \\
1.90 \\
305.00 \\
4.20 \\
59.70 \\
0.59 \\
35.20 \\
48.30\end{array}$ & $\begin{array}{l}\text { U } \\
\text { UJ } \\
U \\
J \\
U \\
U \\
J \\
B\end{array}$ & . & 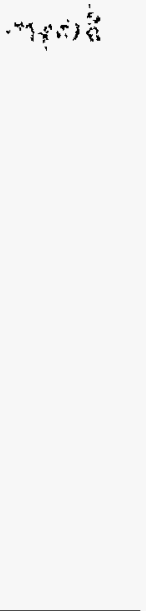 \\
\hline
\end{tabular}


Appendix E

RADIONUCLIDE ANALYSIS DATA 
, 
Table E1. Radionuclide analysis results for composite soil samples

\begin{tabular}{|c|c|c|c|c|c|c|c|}
\hline Analysis & Units & $\begin{array}{l}\text { A horizon } \\
\text { Result }\end{array}$ & Qualifier & $\begin{array}{l}\text { B horizon } \\
\text { Result }\end{array}$ & Qualifier & $\begin{array}{l}\text { C horizon } \\
\text { Result }\end{array}$ & Qualifie \\
\hline \multicolumn{8}{|c|}{$\begin{array}{c}\text { Location }=A N D ; \text { Formation }=C O P P E R \text { RIDGE; Site }=31,32,36 ; \text { Sample type }=\text { Regular; } A=7058 ; B=7061 ; \\
\text { Phase }=2\end{array}$} \\
\hline $\begin{array}{l}\text { Americium-241 } \\
\text { Barium-133 } \\
\text { Cesium-137 } \\
\text { Chromium-51 } \\
\text { Cobalt }-57 \\
\text { Cobalt }-60 \\
\text { Curium-243 } \\
\text { Curium-244 } \\
\text { Curium-245 } \\
\text { Curium-247 } \\
\text { Europium-152 } \\
\text { Europium-154 } \\
\text { Hafnium- } 181 \\
\text { Iridium-192 } \\
\text { Neptunium-237 }\end{array}$ & $\begin{array}{l}\mathrm{pCi} / \mathrm{g} \\
\mathrm{pCi} / \mathrm{g} \\
\mathrm{pCi} / \mathrm{g} \\
\mathrm{pCi} / \mathrm{g} \\
\mathrm{pCi} / \mathrm{g} \\
\mathrm{pCi} / \mathrm{g} \\
\mathrm{pCi} / \mathrm{g} \\
\mathrm{pCi} / \mathrm{g} \\
\mathrm{pCi} / \mathrm{g} \\
\mathrm{pCi} \\
\mathrm{pCi} / \mathrm{g} \\
\mathrm{pCi} / \mathrm{g} \\
\mathrm{pCi} / \mathrm{g} \\
\mathrm{pCi} / \mathrm{g} \\
\mathrm{pCi} / \mathrm{g}\end{array}$ & $\begin{array}{l}0.0756 \\
0.0316 \\
0.5240 \\
0.5000 \\
0.0172 \\
0.0284 \\
0.1460 \\
0.0685 \\
0.2080 \\
0.0280 \\
0.1500 \\
0.0326 \\
0.0509 \\
0.0308 \\
0.1150\end{array}$ & $\begin{array}{l}U \\
U \\
U \\
U \\
U \\
U \\
U J \\
U \\
U \\
U \\
U \\
U \\
U \\
J\end{array}$ & $\begin{array}{l}0.09 \\
0.03 \\
0.03 \\
0.63 \\
0.02 \\
0.03\end{array}$ & $\begin{array}{l}\mathbf{U} \\
\mathbf{U} \\
\mathbf{U} \\
\mathbf{U} \\
\mathbf{U} \\
\mathbf{U}\end{array}$ & $\begin{array}{l}0.0480 \\
0.0212 \\
0.0203 \\
0.3940 \\
0.0130 \\
0.1950\end{array}$ & $\begin{array}{l}U \\
U \\
U \\
U \\
U \\
U\end{array}$ \\
\hline $\begin{array}{l}\text { Neptunium-237 (gamma) } \\
\text { Niobium- } 95 \\
\text { Plutonium-238 } \\
\text { Plutonium- } 239 / 240\end{array}$ & $\begin{array}{l}\mathrm{pCi} / \mathrm{g} \\
\mathrm{pCi} / \mathrm{g} \\
\mathrm{pCi} / \mathrm{g} \\
\mathrm{pCi} / \mathrm{g}\end{array}$ & $\begin{array}{l}3.3700 \\
0.0624 \\
0.0831 \\
0.0665\end{array}$ & $\begin{array}{l}\text { U } \\
U \\
U J \\
U J U\end{array}$ & $\begin{array}{l}3.70 \\
0.07\end{array}$ & $\underset{\mathbf{U}}{\mathbf{U}}$ & $\begin{array}{l}2.4700 \\
0.0496\end{array}$ & $\underset{U}{U}$ \\
\hline $\begin{array}{l}\text { Potassium- } 40 \\
\text { Radium- } 226 \\
\text { Ruthenium }-103 \\
\text { Stront ium-90 }\end{array}$ & $\begin{array}{l}\mathrm{pCi} / \mathrm{g} \\
\mathrm{pCi} / \mathrm{g} \\
\mathrm{pCi} / \mathrm{g} \\
\mathrm{pCi} / \mathrm{g}\end{array}$ & $\begin{array}{l}2.2000 \\
1.0400 \\
0.0485 \\
0.9230\end{array}$ & $\begin{array}{l}U \\
U\end{array}$ & $\begin{array}{l}4.23 \\
1.15 \\
0.06\end{array}$ & $U$ & $\begin{array}{l}5.0300 \\
0.9610 \\
0.0370\end{array}$ & $u$ \\
\hline $\begin{array}{l}\text { Thorium-228 } \\
\text { Thorium-230 } \\
\text { Thorium-232 } \\
\text { Thorium-234 } \\
\text { Total Uranium }\end{array}$ & $\begin{array}{l}\mathrm{pCi} / \mathrm{g} \\
\mathrm{pCi} / \mathrm{g} \\
\mathrm{pCi} / \mathrm{g} \\
\mathrm{pCi} / \mathrm{g} \\
\mathrm{pCi} / \mathrm{g}\end{array}$ & $\begin{array}{l}0.6620 \\
0.7320 \\
0.6270 \\
0.8590 \\
0.5280\end{array}$ & $\begin{array}{l}J \\
J \\
J \\
U J\end{array}$ & $\begin{array}{l}0.99 \\
1.20 \\
1.13 \\
2.47\end{array}$ & $\begin{array}{l}\mathbf{J} \\
\mathbf{J} \\
\mathbf{J} \\
\mathbf{J}\end{array}$ & $\begin{array}{l}1.0300 \\
1.1700 \\
0.9100 \\
0.5820\end{array}$ & $\begin{array}{l}\mathbf{J} \\
\mathbf{J} \\
\mathbf{J} \\
\mathrm{UJ}\end{array}$ \\
\hline $\begin{array}{l}\text { Uranium-233/234 } \\
\text { Uranium-235 } \\
\text { Uranium-235 (gamma) } \\
\text { Uranium-236 } \\
\text { Uranium-238 } \\
\text { Uranium-238 (gamma) } \\
\text { Zinc-65 } \\
\text { Zirconium-95 }\end{array}$ & $\begin{array}{l}\mathrm{pCi} / \mathrm{g} \\
\mathrm{pCi} / \mathrm{g} \\
\mathrm{pCi} / \mathrm{g} \\
\mathrm{pCi} i / g \\
\mathrm{pCi} / \mathrm{g} \\
\mathrm{pCi} / \mathrm{g} \\
\mathrm{pCi} / \mathrm{g} \\
\mathrm{pCi} / \mathrm{g}\end{array}$ & $\begin{array}{r}0.9790 \\
0.0799 \\
0.1490 \\
0.0254 \\
1.2000 \\
16.1000 \\
0.0623 \\
0.0670\end{array}$ & $\begin{array}{l}J \\
J \\
U \\
U J \\
J \\
U \\
U \\
U\end{array}$ & $\begin{array}{r}1.52 \\
0.09 \\
0.15 \\
0.01 \\
1.65 \\
18.70 \\
0.06 \\
0.08\end{array}$ & $\begin{array}{l}J \\
J \\
U \\
U J \\
J \\
U \\
U \\
U\end{array}$ & $\begin{array}{r}1.3600 \\
0.0389 \\
0.1040 \\
0.0122 \\
1.4200 \\
13.1000 \\
0.0447 \\
0.0535\end{array}$ & $\begin{array}{l}\mathbf{J} \\
\mathbf{U} \mathbf{J} \\
\mathbf{U} \\
\mathbf{U} \mathbf{J} \\
\mathbf{U} \\
\mathbf{U} \\
\mathbf{U} \\
\mathbf{U}\end{array}$ \\
\hline
\end{tabular}


Table E.1 (continued)

\begin{tabular}{|c|c|c|c|c|c|c|c|c|c|}
\hline & & A horizon & & $\mathrm{B}$ horizon & & $\mathrm{C}$ horizon & & A lab split & \\
\hline Analysis & Units & Result & Qualifier & Result & Qualifier & Result & Qualifier & Result & Qualifier \\
\hline
\end{tabular}

Location=AND; Formation=COPPER RIDGE; Site=33; Sample type=Regular; $A=7084 ; B=$ '; $C=$ ' '; $A$ lab split =' '; Phase $=2$
Technetium- 99
$\mathrm{pCi} / \mathrm{g} \quad 1.2800 \quad \mathrm{U}$

Location=AND; Formation=COPPER RIDGE; Site=33,34,35; Sample type=Regular; $A=7048 ; B=7052 ; C=7055 ; A$ lab split $=7049$; Phase $=2$

\begin{tabular}{|c|c|c|c|c|c|c|c|c|}
\hline $\begin{array}{l}\text { Americium-241 } \\
\text { Barium-133 } \\
\text { Cesium-137 } \\
\text { Chromium-51 } \\
\text { Cobalt-57 } \\
\text { Cobalt-60 } \\
\text { Curium-243 } \\
\text { Curium-244 } \\
\text { Curium-245 } \\
\text { Curium-247 } \\
\text { Europium-152 } \\
\text { Europium-154 } \\
\text { Hafnium-181 } \\
\text { Iridium-192 } \\
\text { Neptunium-237 } \\
\text { Neptunium-237 (gamma) } \\
\text { Niobium-95 } \\
\text { Plutonium-238 } \\
\text { Plutonium-239/240 } \\
\text { Potassium-40 } \\
\text { Radium-226 } \\
\text { Ruthenium-103 } \\
\text { Strontium-90 } \\
\text { Thorium-228 } \\
\text { Thorium-230 } \\
\text { Thorium-232 } \\
\text { Thorium-234 } \\
\text { Total Uranium } \\
\text { Uranium-233/234 } \\
\text { Uranium-235 } \\
\text { Uranium-235 (gamma) } \\
\text { Uranium-236 } \\
\text { Uranium-238 } \\
\text { Uranium-238 (gamma) } \\
\text { 2inc-65 } \\
\text { 2irconium-95 }\end{array}$ & $\begin{array}{l}\mathrm{pCi} / \mathrm{g} \\
\mathrm{pCi} / \mathrm{g} \\
\mathrm{pCi} / \mathrm{g} \\
\mathrm{pCi} / \mathrm{g} \\
\mathrm{pCi} / \mathrm{g} \\
\mathrm{pCi} / \mathrm{g} \\
\mathrm{pCi} / \mathrm{g} \\
\mathrm{pCi} / \mathrm{g} \\
\mathrm{pCi} / \mathrm{g} \\
\mathrm{pCi} / \mathrm{g} \\
\mathrm{pCi} / \mathrm{g} \\
\mathrm{pCi} / \mathrm{g} \\
\mathrm{pCi} / \mathrm{g} \\
\mathrm{pCi} / \mathrm{g} \\
\mathrm{pCi} / \mathrm{g} \\
\mathrm{pCi} / \mathrm{g} \\
\mathrm{pCi} / \mathrm{g} \\
\mathrm{pCi} / \mathrm{g} \\
\mathrm{pCi} / \mathrm{g} \\
\mathrm{pCi} / \mathrm{g} \\
\mathrm{pCi} / \mathrm{g} \\
\mathrm{pCi} / \mathrm{g} \\
\mathrm{pCi} / \mathrm{g} \\
\mathrm{pCi} / \mathrm{g} \\
\mathrm{pCi} / \mathrm{g} \\
\mathrm{pCi} / \mathrm{g} \\
\mathrm{pCi} / \mathrm{g} \\
\mathrm{pCi} / \mathrm{g} \\
\mathrm{pCi} / \mathrm{g} \\
\mathrm{pCi} / \mathrm{g} \\
\mathrm{pCi} / \mathrm{g} \\
\mathrm{pCi} / \mathrm{g} \\
\mathrm{pCi} / \mathrm{g} \\
\mathrm{pCi} / \mathrm{g} \\
\mathrm{pCi} \mathrm{g} \\
\mathrm{pCi} / \mathrm{g}\end{array}$ & $\begin{array}{l}0.0496 \\
0.0154 \\
0.0142 \\
0.2580 \\
0.0101 \\
0.0144 \\
0.0816 \\
0.0078 \\
0.1230 \\
0.0149 \\
0.0793 \\
0.0193 \\
0.0257 \\
0.0167 \\
0.0594 \\
2.0600 \\
0.0371 \\
0.0477 \\
0.0331 \\
5.3300 \\
0.9200 \\
0.0240 \\
0.8660 \\
0.9070 \\
1.2500 \\
0.8390 \\
1.2300 \\
0.4160 \\
1.0900 \\
0.1110 \\
0.0794 \\
0.0119 \\
1.4100 \\
9.0200 \\
0.0331 \\
0.0389\end{array}$ & $\begin{array}{l}U \\
U \\
U \\
U \\
U \\
U \\
U \\
U J \\
U \\
U \\
U \\
U \\
U \\
U \\
U J \\
U \\
U \\
U J \\
U J \\
\\
U \\
U \\
J \\
J \\
J \\
J \\
J \\
J \\
J \\
U \\
U J \\
J \\
U \\
U \\
U\end{array}$ & $\begin{array}{l}0.14 \\
0.03 \\
0.04 \\
0.03 \\
\\
2.98 \\
0.05 \\
\\
6.78 \\
1.01 \\
0.03\end{array}$ & $\begin{array}{l}J \\
J \\
U \\
U S J \\
J \\
U \\
U \\
U \\
U\end{array}$ & $\begin{array}{r}0.1700 \\
0.0360 \\
0.0556 \\
0.0355 \\
\\
3.9200 \\
0.0726 \\
\\
4.0600 \\
1.0900 \\
0.0548 \\
1.1700 \\
1.3500 \\
1.0700 \\
1.0000 \\
\\
1.3300 \\
0.0504 \\
0.1690 \\
0.0401 \\
1.2600 \\
18.7000 \\
0.0710 \\
0.0811\end{array}$ & $\begin{array}{l}\mathbf{J} \\
\mathbf{J} \\
\mathbf{U} \\
\mathbf{U} \mathbf{J} \\
\mathbf{J} \\
\mathbf{U} \\
\mathbf{U} \\
\mathbf{U}\end{array}$ & $\begin{array}{r}0.0715 \\
0.0248 \\
0.5750 \\
0.4540 \\
0.0147 \\
0.0241 \\
0.1200 \\
0.3340 \\
0.1790 \\
0.0228 \\
0.1260 \\
0.0279 \\
0.0407 \\
0.0269 \\
0.0700 \\
2.9900 \\
0.0589 \\
0.1410 \\
0.0344 \\
3.0700 \\
1.0400 \\
0.0406 \\
0.8130 \\
0.7260 \\
0.8480 \\
0.6180 \\
0.6280 \\
1.9900 \\
0.9510 \\
0.0539 \\
0.0116 \\
0.0293 \\
1.1500 \\
15.5000 \\
0.0498 \\
0.0593\end{array}$ \\
\hline
\end{tabular}


Table E1 (continued)

\begin{tabular}{|c|c|c|c|c|c|c|c|c|c|}
\hline Analysis & Units & $\begin{array}{l}\text { A horizon } \\
\text { Result }\end{array}$ & Qualifier & $\begin{array}{c}\text { B horizon } \\
\text { Result }\end{array}$ & Qualifier & $\begin{array}{c}\text { C horizon } \\
\text { Result }\end{array}$ & Qualifier & $\begin{array}{c}\text { A lab split } \\
\text { Result }\end{array}$ & Qualifier \\
\hline
\end{tabular}

Location=AND; Formation=COPPER RIDGE; Site $=34$; Sample type $=$ Regular; $A=7085 ; B=$ ' '; $C=$ ' '; $A$ lab split=' '; Phase $=2$

Technetium-99

$\mathrm{pCi} / \mathrm{g} \quad 3.3500$

Location=AND; Formation=COPPER RIDGE; Site $=36 ;$ Sample type $=$ Regular; $A=7086 ; B==^{\prime} ; C==^{\prime}$; A lab split=' '; Phase $=2$

Technetium-99 $\quad \mathbf{p C i / g} \quad 3.5400$

Location $=A N D ;$ Formation $=$ COPPER RIDGE; Site $=37,38,41 ;$ Sample type $=$ Regular; $A=7076 ; B=7079 ; C=7082 ; A$ lab split $='$ '; Phase $=2$

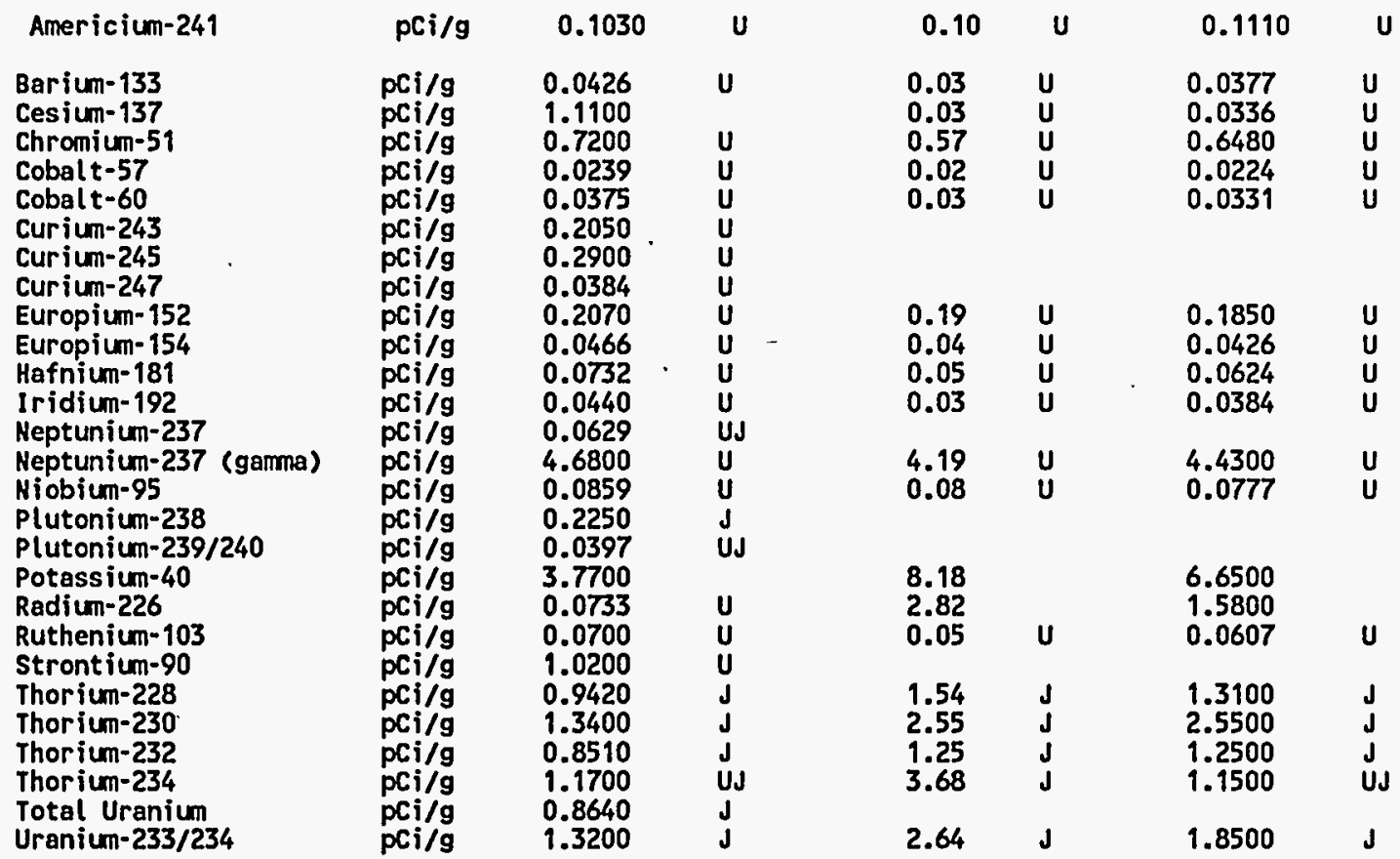




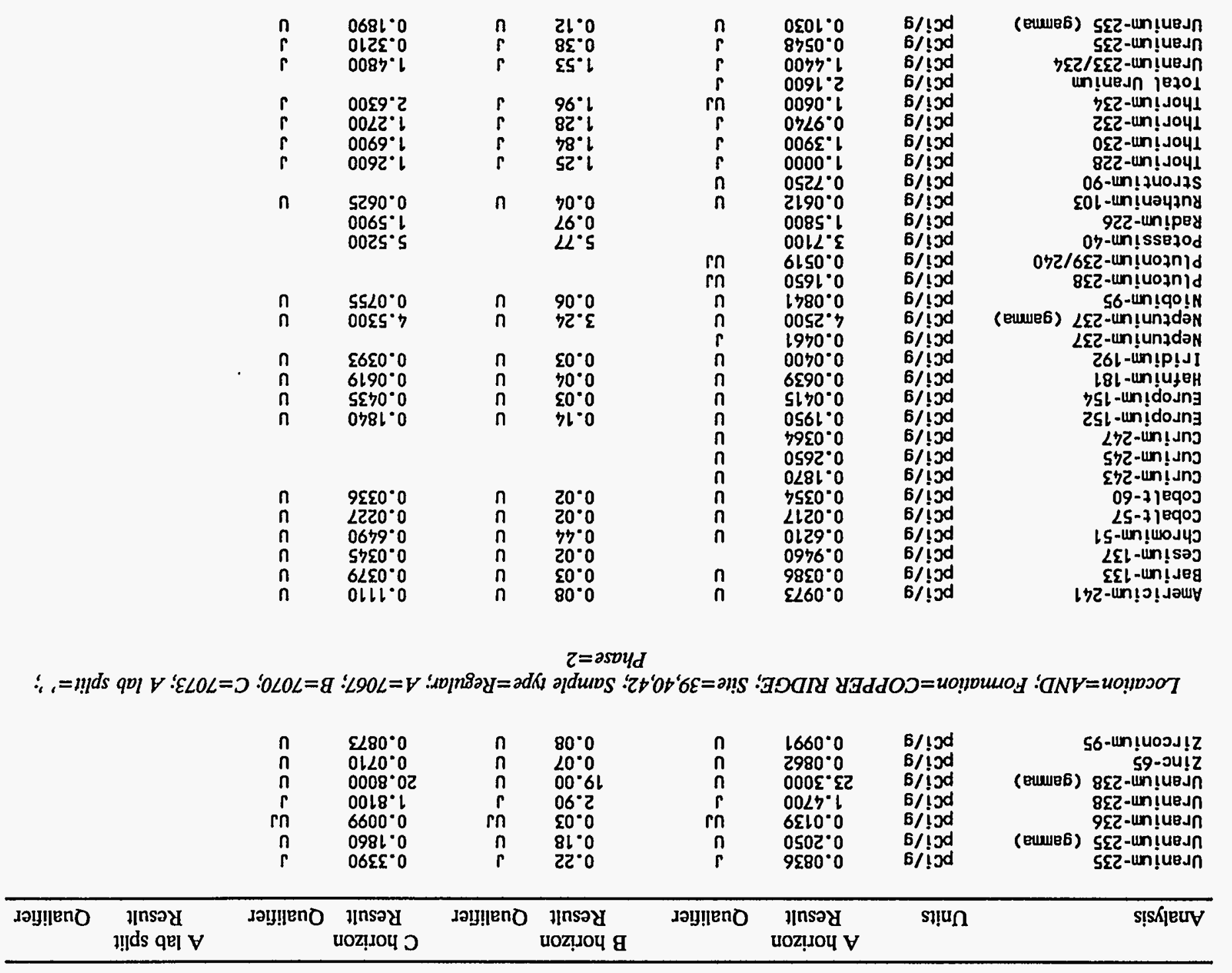

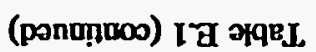


Table E.1 (continued)

\begin{tabular}{|c|c|c|c|c|c|c|c|c|c|}
\hline Analysis & Units & $\begin{array}{c}\text { A horizon } \\
\text { Result }\end{array}$ & Qualifier & $\begin{array}{c}\text { B horizon } \\
\text { Result }\end{array}$ & Qualifier & $\begin{array}{c}C \text { horizon } \\
\text { Result }\end{array}$ & Qualifier & $\begin{array}{l}\text { A lab split } \\
\text { Result }\end{array}$ & Qualifier \\
\hline $\begin{array}{l}\text { Uranium-236 } \\
\text { Uranium-238 } \\
\text { Uranium-238 (gamma) } \\
\text { Zinc-65 } \\
\text { Zirconium-95 }\end{array}$ & $\begin{array}{l}\mathrm{pCi} / \mathrm{g} \\
\mathrm{pCi} / \mathrm{g} \\
\mathrm{pCi} / \mathrm{g} \\
\mathrm{pCi} / \mathrm{g} \\
\mathrm{pCi} / \mathrm{g}\end{array}$ & $\begin{array}{r}0.0348 \\
1.5100 \\
19.8000 \\
0.0826 \\
0.0916\end{array}$ & $\begin{array}{l}\mathbf{U J} \\
\mathbf{J} \\
\mathbf{U} \\
\mathbf{U} \\
\mathbf{U}\end{array}$ & $\begin{array}{r}0.01 \\
1.42 \\
15.10 \\
0.05 \\
0.06\end{array}$ & $\begin{array}{l}U J \\
J \\
U \\
U \\
U\end{array}$ & $\begin{array}{r}0.0100 \\
1.5400 \\
20.3000 \\
0.0751 \\
0.0872\end{array}$ & $\begin{array}{l}\mathbf{U J} \\
\mathbf{J} \\
\mathbf{U} \\
\mathbf{U} \\
\mathbf{U}\end{array}$ & & \\
\hline
\end{tabular}

Location=AND; Formation=DISMAL GAP; Site=1,20,22; Sample type=Regular; $A=7029 ; B=7032 ; C=7035 ; A$ lab split =' ; Phase $=1$

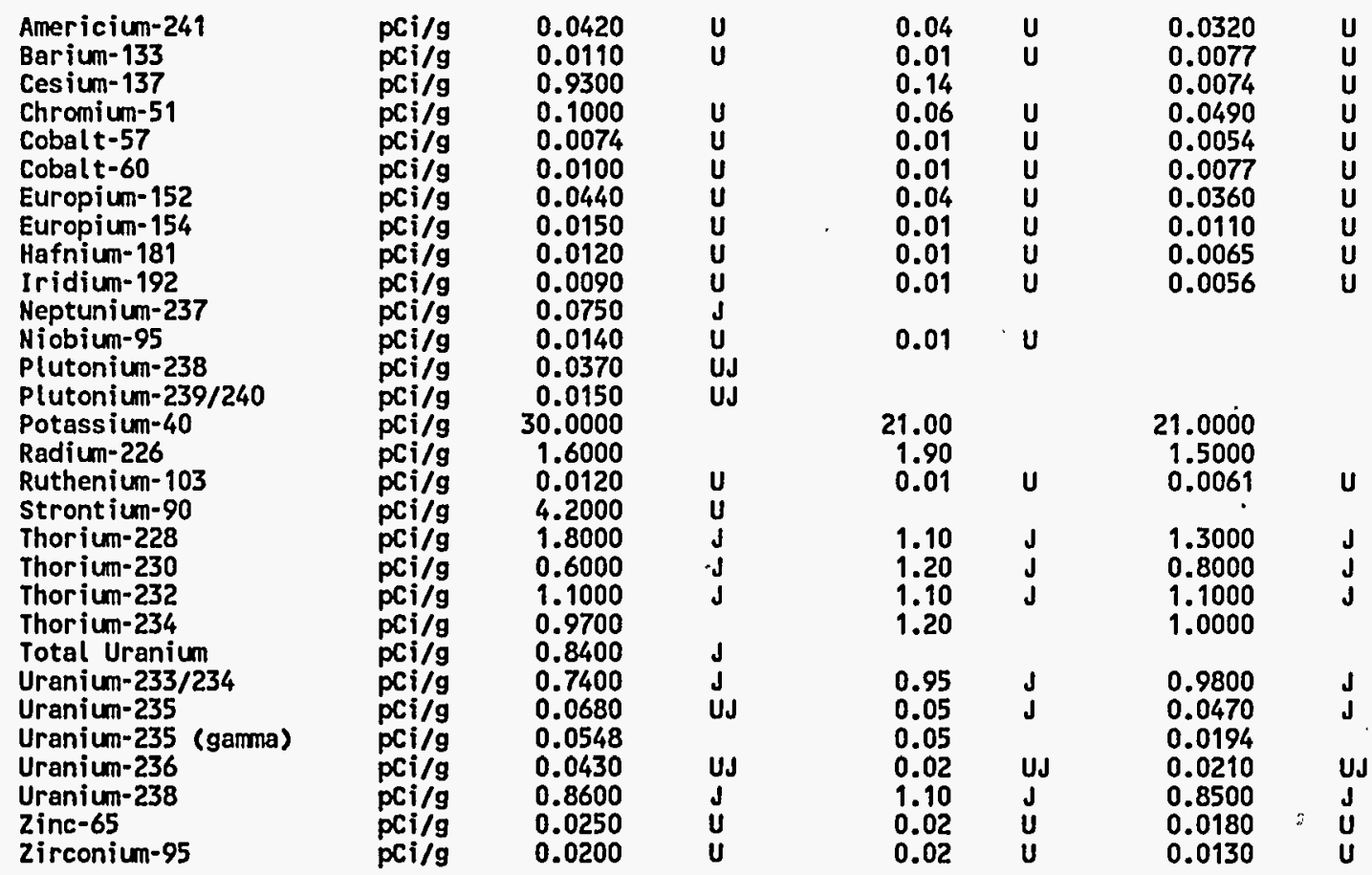


Table E1 (continued)

\begin{tabular}{|c|c|c|c|c|c|c|c|c|c|}
\hline Analysis & Units & $\begin{array}{c}\text { A horizon } \\
\text { Result }\end{array}$ & Qualifier & $\begin{array}{l}\text { B horizon } \\
\text { Result }\end{array}$ & Qualifier & $\begin{array}{c}C \text { horizon } \\
\text { Result }\end{array}$ & Qualifier & $\begin{array}{l}\text { A lab split } \\
\text { Result }\end{array}$ & Qualifier \\
\hline
\end{tabular}

Location=AND; Formation=DISMAL GAP; Site=3; Sample type =Regular; $A=7087 ; B=$ ' '; $C=$ ' '; $A$ lab split=' '; Phase $=2$

Technetium-99 $\quad$ pCi/g $\quad 6.3200$

Location=AND; Formation=DISMAL GAP; Site=3,5,11; Sample type=Regular; $A=7011 ; B=7014 ; C=7017 ; A$ lab split=' '; Phase =1

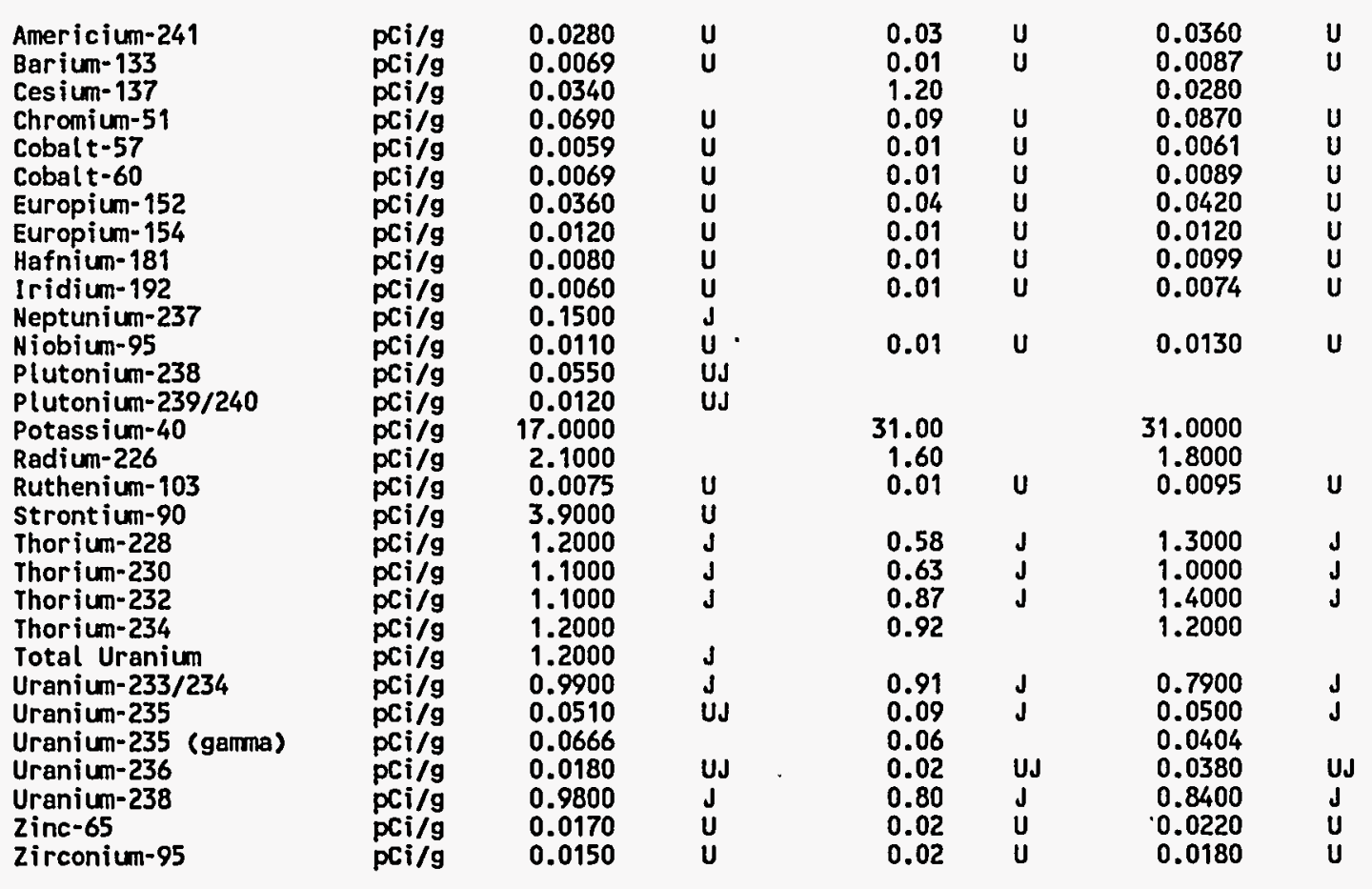


Table E1 (continued)

\begin{tabular}{|c|c|c|c|c|c|c|c|c|c|}
\hline Analysis & Units & $\begin{array}{l}\text { A horizon } \\
\text { Result }\end{array}$ & Qualifier & $\begin{array}{l}\text { B horizon } \\
\text { Result }\end{array}$ & Qualifier & $\begin{array}{c}C \text { horizon } \\
\text { Result }\end{array}$ & Qualifier & $\begin{array}{c}\text { A lab split } \\
\text { Result }\end{array}$ & Qualifier \\
\hline
\end{tabular}

Location=AND; Formation=DISMAL GAP; Site=4; Sample type=Regular; $A=7088 ; B=$ ' ' $C=$ ' '; $A$ lab split=' '; Phase=2

Technetium-99 pci/g $\quad 4.6400$

Location=AND; Formation=DISMAL GAP; Site=4; Sample type=Field Duplicate; $A=7089 ; B=$ ' '; $C=$ ' '; $A$ lab split=' '; Phase=2

$\begin{array}{llll}\text { Technetium-99 } & \text { pCi/g } & 0.7750 \quad U\end{array}$

Location=AND; Formation=DISMAL GAP; Site=4,12,21; Sample type=Regular; $A=7020 ; B=7023 ; C=7026 ; A$ lab split=' '; Phase=1

Americium-241
Barium-133
Cesium-137
Chromium-51
Cobalt -57
Cobalt -60
Europium-152
Europium-154
Hafnium-181
Iridium-192
Neptunium-237
Niobium-95
Plutonium-238
Plutonium-239/240
Potassium-40
Radium-226
Ruthenium-103
Stront ium-90
Thorium-228
Thorium-230
Thorium-232
Thorium-234
Total Uranium
Uranium-233/234
Uranium-235
Uranium-235 (gamma)
Uranium-236
Uranium-238
2inc-65
2irconium-95

\begin{tabular}{|c|c|}
\hline 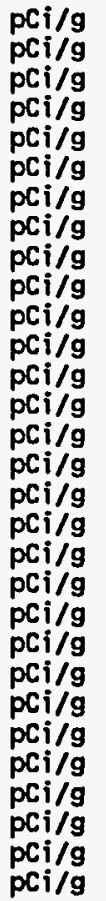 & $\begin{array}{r}0.0300 \\
0.0073 \\
0.0074 \\
0.0730 \\
0.0060 \\
0.0082 \\
0.0370 \\
0.0120 \\
0.0084 \\
0.0063 \\
0.0700 \\
0.0110 \\
0.0580 \\
0.0580 \\
21.0000 \\
1.9000 \\
0.0077 \\
3.9000 \\
1.0000 \\
0.8010 \\
1.1000 \\
1.2000 \\
0.8600 \\
1.1000 \\
0.0450 \\
0.0660 \\
0.0190 \\
0.9200 \\
0.0190 \\
0.0170\end{array}$ \\
\hline
\end{tabular}

$\begin{array}{llll}0.03 & U & 0.0390 & U \\ 0.01 & U & 0.0100 & U \\ 0.01 & & 1.1000 & \\ 0.05 & U & 0.0620 & U \\ 0.01 & U & 0.0064 & U \\ 0.01 & U & 0.0083 & U \\ 0.04 & U & 0.0420 & U \\ 0.01 & U & 0.0130 & U \\ 0.01 & U & 0.0086 & U \\ 0.01 & U & 0.0071 & U \\ 0.01 & U & 0.0086 & U \\ & & & \\ 33.00 & & 15.0000 & \\ 1.60 & & 1.6000 & \\ 0.01 & U & 0.0079 & U \\ 1.20 & J & 0.7900 & J \\ 0.81 & J & 0.6800 & J \\ 1.10 & J & 0.7100 & J \\ 1.00 & & 0.9100 & \\ 0.85 & J & 0.8000 & J \\ 0.02 & U J & 0.0320 & U J \\ 0.07 & & 0.0366 & \\ 0.02 & U J & 0.0310 & U J \\ 0.93 & J & 0.8100 & J \\ 0.02 & U & 0.0190 & U \\ 0.02 & U & 0.0140 & U\end{array}$


Table E.1 (continued)

\begin{tabular}{|c|c|c|c|c|c|c|c|c|c|}
\hline Analysis & Units & $\begin{array}{l}\text { A horizon } \\
\text { Result }\end{array}$ & Qualifier & $\begin{array}{l}\text { B horizon } \\
\text { Result }\end{array}$ & Qualifier & $\begin{array}{l}\mathrm{C} \text { horizon } \\
\text { Result }\end{array}$ & Qualifier & $\begin{array}{l}\text { A lab split } \\
\text { Result }\end{array}$ & Qualifier \\
\hline
\end{tabular}

Location=AND; Formation=DISMAL GAP; Site=9,10,19; Sample type=Regular; $A=7002 ; B=7005 ; C=7008 ; A$ lab split=' '; Phase=1

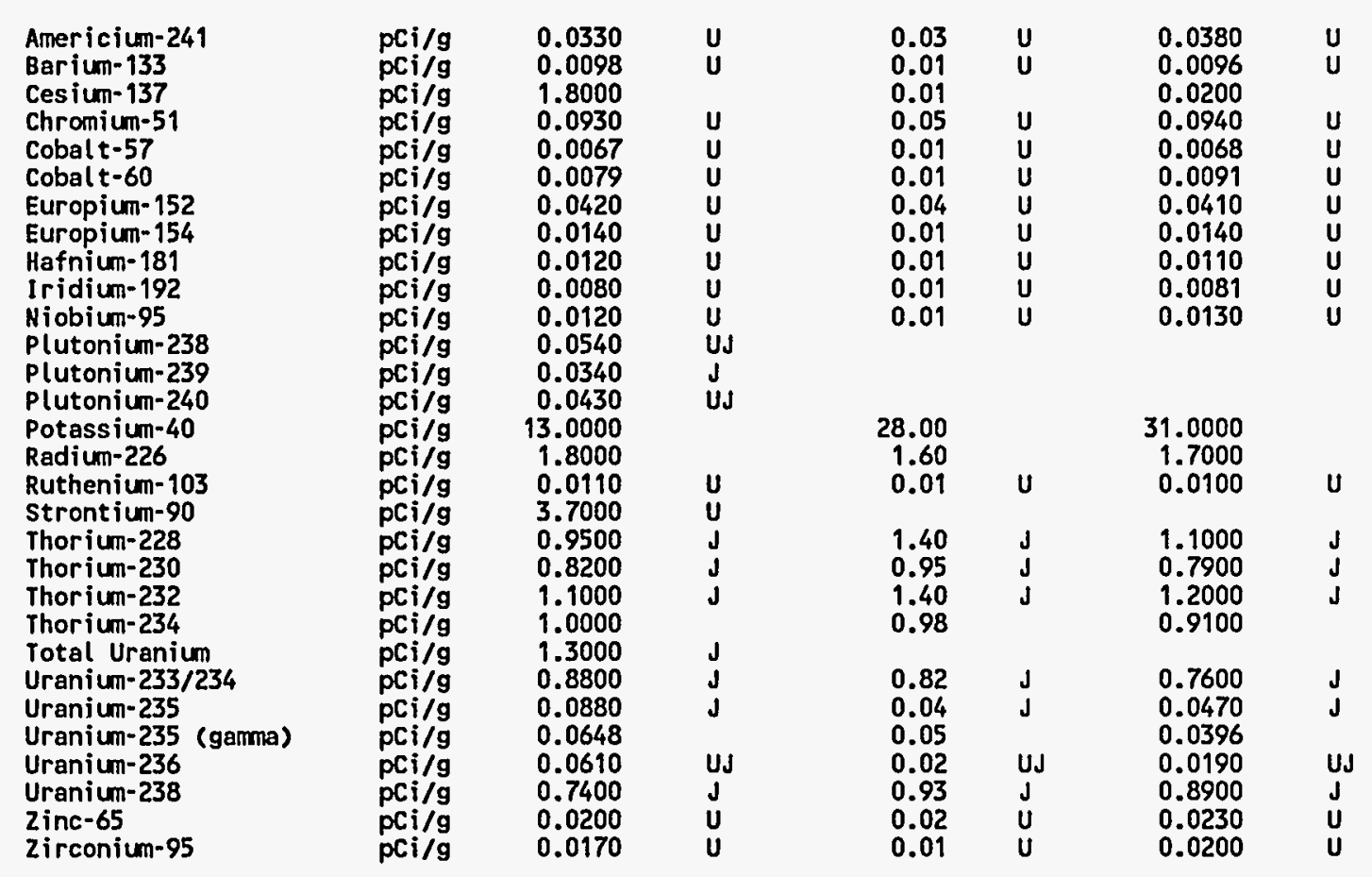


Tabke E1 (continued)

\begin{tabular}{|c|c|c|c|c|c|c|c|c|c|}
\hline Analysis & Units & $\begin{array}{c}\text { A horizon } \\
\text { Result }\end{array}$ & Qualifier & $\begin{array}{c}\text { B horizon } \\
\text { Result }\end{array}$ & Qualifier & $\begin{array}{c}\mathrm{C} \text { horizon } \\
\text { Result }\end{array}$ & Qualifier & $\begin{array}{l}\text { A lab split } \\
\text { Result }\end{array}$ & Qualifier \\
\hline
\end{tabular}

Location=AND; Formation=DISMAL GAP; Site=9,10,19; Sample type=Field Duplicate; $A=7038 ; B=7041 ; C=7044 ; A$ lab split=' '; Phase $=1$

\begin{tabular}{|c|c|c|c|c|c|c|}
\hline $\begin{array}{l}\text { Americium-241 } \\
\text { Barium-133 } \\
\text { Cesium-137 } \\
\text { Chromium-51 } \\
\text { Cobalt-57 } \\
\text { Cobalt-60 } \\
\text { Curium-243 } \\
\text { Curium-245 } \\
\text { Curium-247 } \\
\text { Europium-152 } \\
\text { Europium-154 } \\
\text { Hafnium-181 } \\
\text { Iridium-192 } \\
\text { Neptunium-237 } \\
\text { Niobium-95 } \\
\text { Plutonium-238 } \\
\text { Plutonium-239/240 } \\
\text { Potassium-40 } \\
\text { Radium-226 } \\
\text { Ruthenium-103 } \\
\text { Stront ium-90 } \\
\text { Thorium-228 } \\
\text { Thorium-230 } \\
\text { Thorium-232 } \\
\text { Thorium-234 } \\
\text { Total Uranium } \\
\text { Uranium-233/234 } \\
\text { Uranium-235 } \\
\text { Uranium-235 (gamma) } \\
\text { Uranium-236 } \\
\text { Uranium-238 } \\
\text { Zinc-65 } \\
\text { Zirconium-95 }\end{array}$ & $\begin{array}{l}\mathrm{pCi} / \mathrm{g} \\
\mathrm{pCi} / \mathrm{g} \\
\mathrm{pCi} / \mathrm{g} \\
\mathrm{pCi} / \mathrm{g} \\
\mathrm{pCi} / \mathrm{g} \\
\mathrm{pCi} / \mathrm{g} \\
\mathrm{pCi} / \mathrm{g} \\
\mathrm{pCi} / \mathrm{g} \\
\mathrm{pCi} / \mathrm{g} \\
\mathrm{pCi} / \mathrm{g} \\
\mathrm{pCi} / \mathrm{g} \\
\mathrm{pCi} / \mathrm{g} \\
\mathrm{pCi} / \mathrm{g} \\
\mathrm{pCi} / \mathrm{g} \\
\mathrm{pCi} / \mathrm{g} \\
\mathrm{pCi} / \mathrm{g} \\
\mathrm{pCi} / \mathrm{g} \\
\mathrm{pCi} / \mathrm{g} \\
\mathrm{pCi} / \mathrm{g} \\
\mathrm{pCi} / \mathrm{g} \\
\mathrm{pCi} / \mathrm{g} \\
\mathrm{pCi} / \mathrm{g} \\
\mathrm{pCi} / \mathrm{g} \\
\mathrm{pCi} / \mathrm{g} \\
\mathrm{pCi} / \mathrm{g} \\
\mathrm{pCi} / \mathrm{g} \\
\mathrm{pCi} / \mathrm{g} \\
\mathrm{pCi} / \mathrm{g} \\
\mathrm{pCi} / \mathrm{g} \\
\mathrm{pCi} / \mathrm{g} \\
\mathrm{pCi} / \mathrm{g} \\
\mathrm{pCi} / \mathrm{g} \\
\mathrm{pCi} / \mathrm{g}\end{array}$ & $\begin{array}{l}0.0300 \\
0.0086 \\
1.3000 \\
0.0510 \\
0.0058 \\
0.0071 \\
0.0460 \\
0.0770 \\
0.0080 \\
0.0370 \\
0.0120 \\
0.0074 \\
0.0060 \\
0.0750 \\
0.0076 \\
0.0350 \\
0.0440 \\
13.0000 \\
1.6000 \\
0.0066 \\
1.6000 \\
0.9600 \\
1.8000 \\
0.8000 \\
0.8400 \\
1.0000 \\
0.9400 \\
0.0480 \\
0.0470 \\
0.0210 \\
0.8800 \\
0.0180 \\
0.0130\end{array}$ & $\begin{array}{l}U \\
U \\
U \\
U \\
U \\
U \\
U \\
U \\
U \\
U \\
U \\
U \\
J \\
U \\
J \\
U J \\
\\
U \\
U \\
J \\
J \\
J \\
J \\
J \\
U J \\
U J \\
J \\
U \\
U\end{array}$ & $\begin{array}{r}0.04 \\
0.01 \\
0.01 \\
0.00 \\
0.01 \\
\\
16.00 \\
1.90 \\
0.01\end{array}$ & $\begin{array}{l}\text { UJ } \\
J \\
U \\
U\end{array}$ & $\begin{array}{r}0.0380 \\
0.0120 \\
0.0062 \\
0.0053 \\
0.0077 \\
20.0000 \\
1.9000 \\
0.0057 \\
1.0000 \\
0.9800 \\
1.2000 \\
1.1000\end{array}$ \\
\hline
\end{tabular}


Table E.1 (continued)

\begin{tabular}{|c|c|c|c|c|c|c|c|c|c|}
\hline & & A horizon & & B horizon & & $\mathrm{C}$ horizon & & A lab split & \\
\hline Analysis & Units & Result & Qualifier & Result & Qualifier & Result & Qualifier & Resuit & Qualifier \\
\hline
\end{tabular}

Location=ORR; Formation=CHEPULTEPEC; Site=50,66,73; Sample type=Regular; $A=5167 ; B=5171 ; C=5182 ; A$ lab split $=5168 ;$ Phase $=2$

\begin{tabular}{|c|c|c|c|c|c|c|c|c|}
\hline $\begin{array}{l}\text { Americium-241 } \\
\text { Barium-133 } \\
\text { Cesium-137 } \\
\text { Chromium-51 } \\
\text { Cobal t-57 } \\
\text { Cobalt-60 } \\
\text { Curium-243 } \\
\text { Curium-245 } \\
\text { Curium-247 } \\
\text { Europium-152 } \\
\text { Europium-154 } \\
\text { Hafnium-181 } \\
\text { Iridium-192 } \\
\text { Neptunium-237 } \\
\text { Neptunium-237 (gamma) } \\
\text { Niobium-95 } \\
\text { Plutonium-238 } \\
\text { Plutonium-239/240 } \\
\text { Potassium-40 } \\
\text { Radium-226 } \\
\text { Ruthenium-103 } \\
\text { Strontium-90 } \\
\text { Thorium-228 } \\
\text { Thorium-230 } \\
\text { Thorium-232 } \\
\text { Thorium-234 } \\
\text { Total Uranium } \\
\text { Uranium-233/234 } \\
\text { Uranium-235 } \\
\text { Uranium-235 (gamma) } \\
\text { Uranium-236 } \\
\text { Uranium-238 } \\
\text { Uranium-238 (gamma) } \\
\text { Zinc-65 } \\
\text { Zirconium-95 }\end{array}$ & $\begin{array}{l}\mathrm{pCi} / \mathrm{g} \\
\mathrm{pCi} / \mathrm{g} \\
\mathrm{pCi} / \mathrm{g} \\
\mathrm{pCi} / \mathrm{g} \\
\mathrm{pCi} / \mathrm{g} \\
\mathrm{pCi} / \mathrm{g} \\
\mathrm{pCi} / \mathrm{g} \\
\mathrm{pCi} / \mathrm{g} \\
\mathrm{pCi} / \mathrm{g} \\
\mathrm{pCi} / \mathrm{g} \\
\mathrm{pCi} / \mathrm{g} \\
\mathrm{pCi} / \mathrm{g} \\
\mathrm{pCi} / \mathrm{g} \\
\mathrm{pCi} / \mathrm{g} \\
\mathrm{pCi} / \mathrm{g} \\
\mathrm{pCi} / \mathrm{g} \\
\mathrm{pCi} / \mathrm{g} \\
\mathrm{pCi} / \mathrm{g} \\
\mathrm{pCi} / \mathrm{g} \\
\mathrm{pCi} / \mathrm{g} \\
\mathrm{pCi} / \mathrm{g} \\
\mathrm{pCi} / \mathrm{g} \\
\mathrm{pCi} / \mathrm{g} \\
\mathrm{pCi} / \mathrm{g} \\
\mathrm{pCi} / \mathrm{g} \\
\mathrm{pCi} / \mathrm{g} \\
\mathrm{pCi} / \mathrm{g} \\
\mathrm{pCi} / \mathrm{g} \\
\mathrm{pCi} / \mathrm{g} \\
\mathrm{pCi} / \mathrm{g} \\
\mathrm{pCi} / \mathrm{g} \\
\mathrm{pCi} / \mathrm{g} \\
\mathrm{pCi} / \mathrm{g} \\
\mathrm{pCi} / \mathrm{g} \\
\mathrm{pCi} / \mathrm{g}\end{array}$ & $\begin{array}{l}0.1160 \\
0.0423 \\
0.9800 \\
0.2530 \\
0.0206 \\
0.0421 \\
0.2030 \\
0.2800 \\
0.0411 \\
0.2360 \\
0.0429 \\
0.0386 \\
0.0309 \\
0.0321 \\
4.5500 \\
0.0399 \\
0.0360 \\
0.0377 \\
3.7100 \\
1.2500 \\
0.0356 \\
1.2100 \\
0.8990 \\
1.0900 \\
1.3100 \\
1.1700 \\
0.9720 \\
1.2300 \\
0.2170 \\
0.1790 \\
0.0267 \\
1.1600 \\
7.9000 \\
0.0879 \\
0.0673\end{array}$ & $\begin{array}{l}U \\
U \\
U \\
U \\
U \\
U \\
U U \\
U \\
U U \\
U \\
U \\
U \\
J \\
U \\
U \\
U J \\
J \\
\\
U \\
U \\
J \\
J \\
J \\
U J \\
J \\
J \\
J \\
U \\
U J \\
J \\
U \\
U \\
U\end{array}$ & $\begin{array}{l}0.21 \\
0.04 \\
0.03 \\
0.02 \\
3.99 \\
0.04 \\
\\
\\
9.46 \\
6.55 \\
0.03 \\
1.12 \\
1.56 \\
1.19 \\
1.00 \\
1.42 \\
0.18 \\
0.17 \\
0.01 \\
1.63 \\
6.61 \\
0.07 \\
0.06\end{array}$ & $\begin{array}{l}\mathbf{J} \\
\mathbf{J} \\
\mathbf{U} \\
\mathbf{U} \mathbf{J} \\
\mathbf{J} \\
\mathbf{U} \\
\mathbf{U} \\
\mathbf{U}\end{array}$ & $\begin{array}{l}0.1100 \\
0.0228 \\
0.0401 \\
0.0227 \\
2.4000 \\
0.0508 \\
\\
11.0000 \\
1.3200 \\
0.0394 \\
1.4600 \\
1.4900 \\
1.3800 \\
0.7630 \\
1.6900 \\
0.0809 \\
0.1450 \\
0.0186 \\
1.8200 \\
1.8800 \\
0.0444 \\
0.0581\end{array}$ & $\begin{array}{l}\mathbf{J} \\
\mathbf{J} \\
\mathbf{U} \\
\mathbf{U} \mathbf{J} \\
\mathbf{J} \\
\mathbf{U} \\
\mathbf{U} \\
\mathbf{U}\end{array}$ & $\begin{array}{l}0.1020 \\
0.0380 \\
0.9410 \\
0.2280 \\
0.0179 \\
0.0343 \\
0.1760 \\
0.2530 \\
0.0357 \\
0.2070 \\
0.0380 \\
0.0334 \\
0.0265 \\
0.0750 \\
3.9900 \\
0.0353 \\
0.1840 \\
0.0415 \\
3.2500 \\
1.1400 \\
0.0286 \\
0.7700 \\
0.6230 \\
0.7580 \\
0.6180 \\
0.9550 \\
5.5600 \\
1.1600 \\
0.1870 \\
0.1600 \\
0.0269 \\
1.2600 \\
6.6900 \\
0.0741 \\
0.0624\end{array}$ \\
\hline
\end{tabular}


Table E.1 (continued)

\begin{tabular}{|c|c|c|c|c|c|c|c|c|c|}
\hline Analusis & Units & A horizon & Oualifier & B horizon & Oualifier & C horizon & Oualifier & A lab split & Oualifier \\
\hline
\end{tabular}

Location=ORR; Formation=CHEPULTEPEC; Site=50,66,73; Sample type=Field Duplicate; $A=5175 ; B=5179 ; C=5185 ;$

$A$ lab split $=5176 ;$ Phase $=2$

Americium-241
Barium-133
Cesium-137
Chromium-51
Cobalt-57
Cobalt-60
Curium-243
Curium-245
Curium-247
Europium-152
Europium-154
Hafnium-181
Iridium-192
Neptunium-237
Neptunium-237 (gamma)
Niobium-95
Plutonium-238
Plutonium-239/240
Potassium-40
Radium-226
Ruthenium-103
Strontium-90
Thorium-228
Thorium-230
Thorium-232
Thorium-234
Total Uranium
Uranium-233/234
Uranium-235
Uranium-235 (gamma)
Uranium-236
Uranium-238
Uranium-238 (gamma)
Zinc-65
Zirconium-95

$\begin{array}{lll} & & \\ \mathrm{pCi} / \mathrm{g} & 0.1030 & \mathrm{U} \\ \mathrm{pCi} / \mathrm{g} & 0.0395 & \mathrm{U} \\ \mathrm{pCi} / \mathrm{g} & 1.1800 & \\ \mathrm{pCi} / \mathrm{g} & 0.2400 & \mathrm{U} \\ \mathrm{pCi} / \mathrm{g} & 0.0185 & \mathrm{U} \\ \mathrm{pCi} / \mathrm{g} & 0.0356 & \mathrm{U} \\ \mathrm{pCi} / \mathrm{g} & 0.1880 & \mathrm{U} \\ \mathrm{pCi} / \mathrm{g} & 0.2570 & \mathrm{U} \\ \mathrm{pCi} / \mathrm{g} & 0.0372 & \mathrm{U} \\ \mathrm{pCi} / \mathrm{g} & 0.2000 & \mathrm{U} \\ \mathrm{pCi} / \mathrm{g} & 0.0386 & \mathrm{U} \\ \mathrm{pCi} / \mathrm{g} & 0.0357 & \mathrm{U} \\ \mathrm{pCi} / \mathrm{g} & 0.0276 & \mathrm{U} \\ \mathrm{pCi} / \mathrm{g} & 0.0713 & \mathrm{~J} \\ \mathrm{pCi} / \mathrm{g} & 4.2600 & \mathrm{U} \\ \mathrm{pCi} / \mathrm{g} & 0.0366 & \mathrm{U} \\ \mathrm{pCi} / \mathrm{g} & 0.0513 & \mathrm{~J} \\ \mathrm{pCi} / \mathrm{g} & 0.0193 & \mathrm{UJ} \\ \mathrm{pCi} / \mathrm{g} & 2.9800 & \\ \mathrm{pCi} / \mathrm{g} & 1.1800 & \\ \mathrm{pCi} / \mathrm{g} & 0.0341 & \mathrm{U} \\ \mathrm{pCi} / \mathrm{g} & 0.7600 & \mathrm{U} \\ \mathrm{pCi/g} & 0.6530 & \mathrm{~J} \\ \mathrm{pCi} / \mathrm{g} & 0.9060 & \mathrm{~J} \\ \mathrm{pCi} / \mathrm{g} & 0.5560 & \mathrm{~J} \\ \mathrm{pCi} / \mathrm{g} & 2.6900 & \mathrm{~J} \\ \mathrm{pCi} / \mathrm{g} & 1.8500 & \mathrm{~J} \\ \mathrm{pCi} / \mathrm{g} & 1.0500 & \mathrm{~J} \\ \mathrm{pCi} / \mathrm{g} & 0.0638 & \mathrm{~J} \\ \mathrm{pCi} / \mathrm{g} & 0.1720 & \mathrm{U} \\ \mathrm{pCi} / \mathrm{g} & 0.0270 & \mathrm{UJ} \\ \mathrm{pCi} / \mathrm{g} & 1.3500 & \mathrm{~J} \\ \mathrm{pCi} / \mathrm{g} & 6.7900 & \mathrm{U} \\ \mathrm{pCi} / \mathrm{g} & 0.0768 & \mathrm{U} \\ \mathrm{pCi} / \mathrm{g} & 0.0598 & \mathrm{U} \\ & & \end{array}$

$\begin{array}{ll}0.10 & U \\ 0.04 & U \\ 0.03 & U \\ 0.21 & U \\ 0.02 & U \\ 0.03 & U \\ & \\ & \\ 0.20 & U \\ 0.04 & U \\ 0.03 & U \\ 0.02 & U \\ 3.87 & U \\ 0.03 & U \\ & \\ 9.03 & \\ 1.65 & \\ 0.03 & U \\ & \\ 1.26 & J \\ 1.63 & J \\ 1.27 & J \\ 2.18 & J \\ 1.46 & J \\ 0.09 & J \\ 0.16 & U \\ 0.03 & U J \\ 1.77 & J \\ 6.41 & U \\ 0.08 & U \\ 0.06 & U\end{array}$

0.0807
0.0270
0.0251
0.6120
0.0175
0.0263


0.1390
0.0324
0.0516
0.0312
3.4700
0.0771

11.2000
1.5100
0.0513
1.0700
1.5300
1.1200
2.2800
1.8800
0.0725
0.1440
0.0462
1.9000
22.3000
0.0608
0.0766

\begin{tabular}{|c|c|}
\hline $\mathbf{U}$ & 0.0927 \\
\hline $\begin{array}{l}\mathbf{U} \\
\mathbf{U}\end{array}$ & $\begin{array}{l}0.0351 \\
1.2000\end{array}$ \\
\hline U & 0.2080 \\
\hline U & 0.0202 \\
\hline $\mathrm{U}$ & $\begin{array}{l}0.0304 \\
0.1740\end{array}$ \\
\hline & 0.2690 \\
\hline & 0.0307 \\
\hline$U$ & 0.1780 \\
\hline$U$ & 0.0430 \\
\hline U & 0.0311 \\
\hline$U$ & 0.0230 \\
\hline & \\
\hline U & 4.3100 \\
\hline U & $\begin{array}{l}0.0337 \\
0.0732\end{array}$ \\
\hline
\end{tabular}


Table E1 (continued)

\begin{tabular}{|c|c|c|c|c|c|c|c|c|c|}
\hline Analysis & Units & $\begin{array}{c}\text { A horizon } \\
\text { Result }\end{array}$ & Qualifier & $\begin{array}{l}\text { B horizon } \\
\text { Result }\end{array}$ & Qualifier & $\begin{array}{c}\text { C horizon } \\
\text { Result }\end{array}$ & Qualifier & $\begin{array}{l}\text { A lab split } \\
\text { Result }\end{array}$ & Qualifier \\
\hline
\end{tabular}

Location=ORR; Formation=CHEPULTEPEC; Site=52,53,78; Sample type =Regular; $A=5128 ; B=5131 ; C=5134 ; A$ lab split $=$ ' '; Phase $=2$

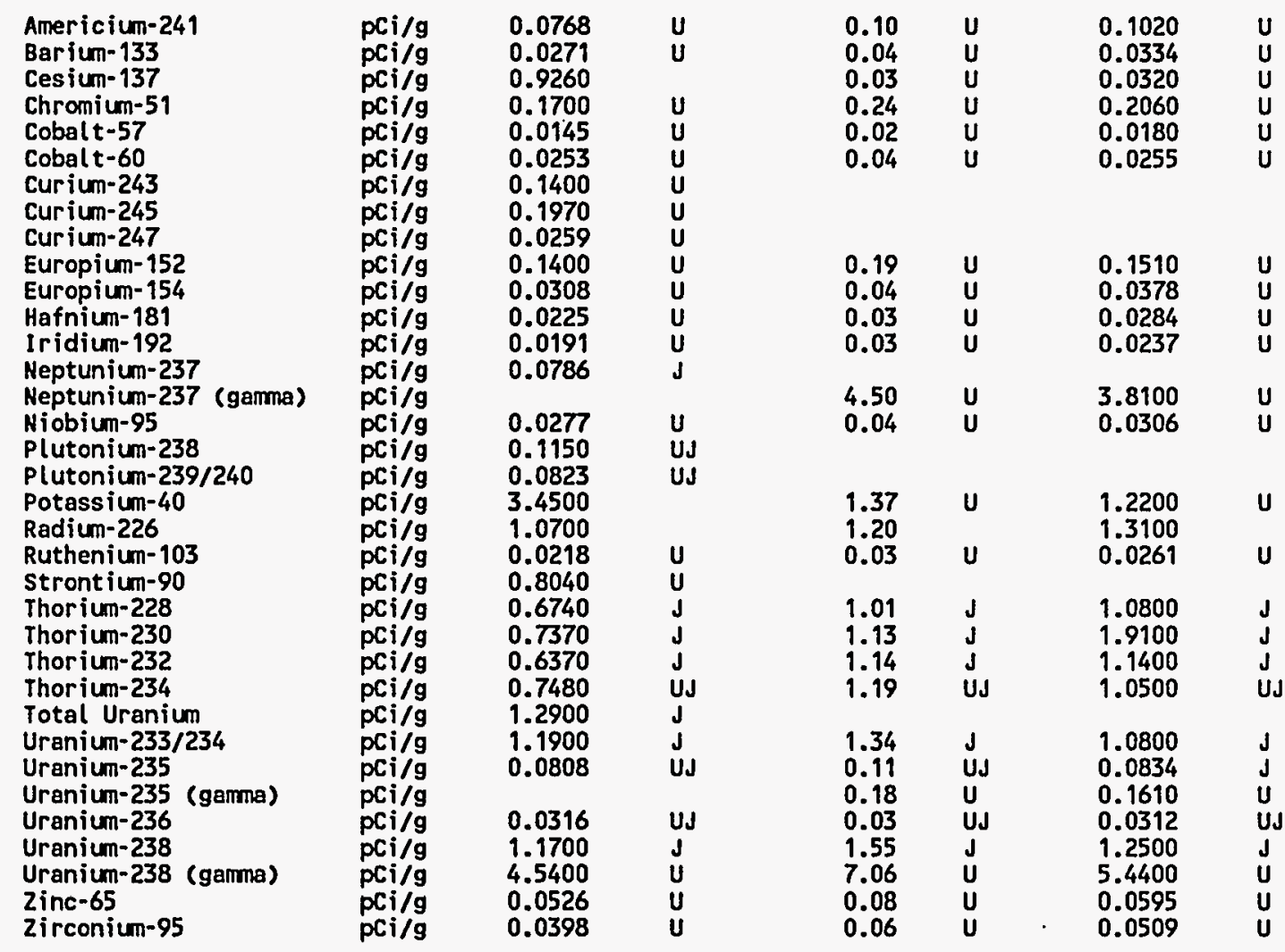


Tabłe E1 (continued)

\begin{tabular}{lccccc}
\hline & A horizon & B horizon & \multicolumn{2}{c}{ horizon } & A lab split \\
Analysis & Units & Result & Qualifier & $\begin{array}{c}\text { Result } \\
\text { Result }\end{array}$ Qualifier & Qualifier \\
\hline
\end{tabular}

Location=ORR; Fornation=CHEPULTEPEC; Sile $=68 ;$ Sample type=Regular; $A=4272 ; B={ }^{\prime}$ '; $C=$ ' ; $A$ lab split =' '; Phase $=2$

Technetium-99 $\quad$ pCi/g $\quad 1.1400 \quad \mathrm{U}$

Location=ORR; Formation=CHEPULTEPEC; Site=68,74,90; Sample type $=$ Regular; $A=5156 ; B=5160 ; C=5163 ; A$ lab split $=5157 ;$

\begin{tabular}{lll} 
Americium-241 & pCi/g & 0.0752 \\
Barium-133 & pCi/g & 0.0273 \\
Cesium-137 & pCi/g & 1.0200 \\
Chromium-51 & pCi/g & 0.1600 \\
Cobalt-57 & pCi/g & 0.0140 \\
Cobalt-60 & pCi/g & 0.0230 \\
Curium-243 & pCi/g & 0.1300 \\
Curium-245 & pCi/g & 0.1910 \\
Curium-247 & pCi/g & 0.0221 \\
Europium-152 & pCi/g & 0.1290 \\
Europium-154 & pCi/g & 0.0293 \\
Hafnium-181 & pCi/g & 0.0218 \\
Iridium-192 & pCi/g & 0.0183 \\
Neptunium-237 & pCi/g & 0.0863 \\
Neptunium-237 (gamma) & pCi/g & 3.1900 \\
Niobium-95 & pCi/g & 0.0263 \\
plutonium-238 & pCi/g & 0.1070 \\
plutonium-239/240 & pCi/g & 0.0322 \\
Potassium-40 & pCi/g & 3.4500 \\
Radium-226 & pCi/g & 0.0546 \\
Ruthenium-103 & pCi/g & 0.0188 \\
Strontium-90 & pCi/g & 0.8720 \\
Thorium-228 & pCi/g & 0.4070 \\
Thorium-230 & pCi/g & 0.6670 \\
Thorium-232 & pCi/g & 0.4970 \\
Thorium-234 & pCi/g & 0.7130 \\
Uranium-233/234 & pCi/g & 1.2000 \\
Uranium-235 & pCi/g & 0.0783 \\
Uranium-235 (gamma) & pCi/g & 0.1310 \\
Uranium-236 & pCi/g & 0.0469 \\
Uranium-238 & pCi/g & 1.2000 \\
Uranium-238 (gamma) & pCi/g & 4.4600 \\
2inc-65 & pCi/g & 0.0461 \\
Zirconium-95 & pCi/g & 0.0394 \\
\hline & &
\end{tabular}
Phase $=2$

$U$
$U$
$U$
$U$
$U$
$U$
$U$
$U$
$U$
$U$
$U$
$J$
$U$
$U$
$J$
$U J$
$U$
$U$
$U$
$J$
$J$
$J$
$U J$
$J$
$J$
$U$
$U J$
$J$
$U$
$U$
$U$

$\begin{array}{ll}0.09 & U \\ 0.03 & U \\ 0.03 & U \\ 0.21 & U \\ 0.02 & U \\ 0.03 & U \\ & \\ & \\ 0.17 & U \\ 0.04 & U \\ 0.03 & U \\ 0.02 & U \\ 3.94 & U \\ 0.03 & U \\ & \\ 13.10 & \\ 1.30 & \\ 0.03 & U \\ 1.17 & J \\ 1.28 & J \\ 1.38 & J \\ 1.06 & U J \\ 1.17 & J \\ 7.72 & J \\ 0.17 & U \\ 0.04 & U J \\ 1.42 & J \\ 5.80 & U \\ 0.07 & U \\ 0.05 & U \\ & \end{array}$

0.0682
0.0232
0.0217
0.1400
0.0126
0.0203


0.1220
0.0267
0.0193
0.0161
2.8100
0.0210

10.8000
1.4500
0.0183
1.3900
1.2600
1.3900
0.9650
1.4000
0.0413
0.1410
0.0412
1.2800
5.2000
0.0452
0.0332

$\begin{array}{ll}0.0766 & U \\ 0.0286 & U \\ 1.0600 & \\ 0.1730 & U \\ 0.0146 & U \\ 0.0230 & U \\ 0.1400 & U \\ 0.1980 & U \\ 0.0363 & U \\ 0.1370 & U \\ 0.0308 & U \\ 0.0233 & U \\ 0.0197 & U \\ 0.0707 & J \\ 3.1800 & U \\ 0.0232 & U \\ 0.1160 & J \\ 0.1170 & U J \\ 2.3400 & \\ 1.0500 & \\ 0.0224 & U \\ 0.9710 & U \\ 0.5840 & J \\ 0.7000 & J \\ 0.6250 & J \\ 1.0900 & U J \\ 1.0700 & J \\ 0.0718 & J \\ 0.1740 & U \\ 0.0358 & U J \\ 1.1600 & J \\ 4.7200 & U \\ 0.0503 & U \\ 0.0388 & U\end{array}$


Table E1 (continued)

\begin{tabular}{|c|c|c|c|c|c|c|c|c|c|}
\hline & & A horizon & & $\mathrm{B}$ horizon & & $\mathrm{C}$ horizon & & A lab split & \\
\hline Analysis & Units & Result & Qualifier & Result & Qualifier & Result & Qualifier & Result & Qualifier \\
\hline
\end{tabular}

Location=ORR; Formation=CHEPULTEPEC; Site=73; Sample type $=$ Regular; $A=4273 ; B=$ ' ' $C=$ ' ' $A$ lab split =' '; Phase $=2$

Technetium-99 $\quad$ pCi/g $1.0900 . \quad$ U

Location=ORR; Formation=CHEPULTEPEC; Site $=74$; Sample type $=$ Regular; $A=4274 ; B=$ ' ; $C=$ ' '; $A$ lab split=' '; Phase $=2$

Technetium-99 $\quad$ pCi/g $\quad 1.0200 \quad$ U

Location=ORR; Formation=CHEPULTEPEC; Site=77; Sample type =Regular; $A=4276 ; B=$ ' '; $C=$ ' '; $A$ lab split=' '; Phase $=2$

$\begin{array}{llll}\text { Technetium-99 } & \text { pci/g } & 0.9990 \quad \text { U }\end{array}$

Location $=$ ORR; Formation $=$ CHEPULTEPEC; Site $=77,85,86 ;$ Sample type $=$ Regular; $A=5137 ; B=5140 ; C=5143 ; A$ lab split $=$ ' '; Phase $=2$

\begin{tabular}{|c|c|c|c|c|c|c|}
\hline $\begin{array}{l}\text { Americium-241 } \\
\text { Barium-133 } \\
\text { Cesium-137 } \\
\text { Chromium-51 } \\
\text { Cobalt }-57 \\
\text { Cobalt-60 } \\
\text { Curium-243 } \\
\text { Curium-245 } \\
\text { Curium-247 } \\
\text { Europium-152 } \\
\text { Europium-154 } \\
\text { Hafnium-181 } \\
\text { Iridium-192 } \\
\text { Neptunium-237 } \\
\text { Heptunium-237 (gamma) } \\
\text { Niobium-95 } \\
\text { Plutonium-238 } \\
\text { Plutonium-239/240 } \\
\text { Potassium-40 }\end{array}$ & $\begin{array}{l}\mathrm{pCi} / \mathrm{g} \\
\mathrm{pCi} / \mathrm{g} \\
\mathrm{pCi} / \mathrm{g} \\
\mathrm{pCi} / \mathrm{g} \\
\mathrm{pCi} / \mathrm{g} \\
\mathrm{pCi} / \mathrm{g} \\
\mathrm{pCi} / \mathrm{g} \\
\mathrm{pCi} / \mathrm{g} \\
\mathrm{pCi} / \mathrm{g} \\
\mathrm{pCi} / \mathrm{g} \\
\mathrm{pCi} / \mathrm{g} \\
\mathrm{pCi} / \mathrm{g} \\
\mathrm{pCi} / \mathrm{g} \\
\mathrm{pCi} / \mathrm{g} \\
\mathrm{pCi} / \mathrm{g} \\
\mathrm{pCi} / \mathrm{g} \\
\mathrm{pCi} / \mathrm{g} \\
\mathrm{pCi} / \mathrm{g} \\
\mathrm{pCi} / \mathrm{g}\end{array}$ & $\begin{array}{l}0.0786 \\
0.0305 \\
1.0900 \\
0.1730 \\
0.0154 \\
0.0267 \\
0.1460 \\
0.2090 \\
0.2720 \\
0.1470 \\
0.0321 \\
0.0258 \\
0.0204 \\
0.0459 \\
3.3800 \\
0.0270 \\
0.0837 \\
0.0204 \\
2.0500\end{array}$ & $\begin{array}{l}\mathbf{U} \\
\mathbf{U} \\
\mathbf{U} \\
\mathbf{U} \\
\mathbf{U} \\
\mathbf{U} \\
\mathbf{U} \\
\mathbf{U} \\
\mathbf{U} \\
\mathbf{U} \\
\mathbf{U} \\
\mathbf{U} \\
\mathbf{U} \mathbf{J} \\
\mathbf{U} \\
\mathbf{U} \\
\mathbf{U} \mathbf{J} \\
\mathbf{U J}\end{array}$ & $\begin{array}{l}0.15 \\
0.04 \\
0.02 \\
0.20 \\
\\
3.60 \\
0.03\end{array}$ & $\begin{array}{l}U \\
U \\
U \\
U \\
U \\
U\end{array}$ & $\begin{array}{l}0.1050 \\
0.0253 \\
0.0178 \\
0.0150 \\
2.6300 \\
0.0227\end{array}$ \\
\hline
\end{tabular}


Table E1 (continued)

\begin{tabular}{|c|c|c|c|c|c|c|c|c|c|}
\hline Analysis & Units & $\begin{array}{c}\text { A horizon } \\
\text { Result }\end{array}$ & Qualifier & $\begin{array}{c}\text { B horizon } \\
\text { Result }\end{array}$ & Qualifier & $\begin{array}{c}\text { C horizon } \\
\text { Result }\end{array}$ & Qualifier & $\begin{array}{l}\text { A lab split } \\
\text { Result }\end{array}$ & Qualifier \\
\hline $\begin{array}{l}\text { Radium-226 } \\
\text { Ruthenium-103 } \\
\text { Strontium-90 } \\
\text { Thorium-228 } \\
\text { Thorium-230 } \\
\text { Thorium-232 } \\
\text { Thorium-234 } \\
\text { Total Uranium } \\
\text { Uranium-233/234 } \\
\text { Uranium-235 } \\
\text { Uranium-235 (ganma) } \\
\text { Uranium-236 } \\
\text { Uranium-238 } \\
\text { Uranium-238 (gamma) } \\
\text { Zinc-65 } \\
\text { Zirconium-95 }\end{array}$ & 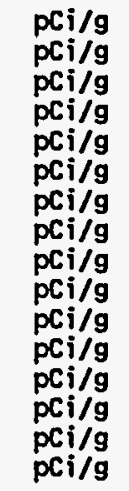 & $\begin{array}{l}0.8590 \\
0.0242 \\
0.8210 \\
0.5480 \\
0.8570 \\
0.5390 \\
1.1200 \\
2.2600 \\
1.1100 \\
0.0834 \\
0.1820 \\
0.0312 \\
0.9460 \\
5.1800 \\
0.0558 \\
0.0428\end{array}$ & $\begin{array}{l}U \\
U \\
J \\
J \\
J \\
U J J .5 \\
J \\
J \\
J \\
U \\
U J \\
J \\
J \\
U \\
U \\
U\end{array}$ & $\begin{array}{l}0.83 \\
0.02 \\
1.00 \\
0.97 \\
1.01 \\
0.80 \\
1.69 \\
0.11 \\
0.14 \\
0.03 \\
1.89 \\
4.17 \\
0.04 \\
0.05\end{array}$ & $\begin{array}{l}U \\
J \\
J \\
J \\
U J ~ \\
J \\
J \\
U \\
U J \\
J \\
J \\
U \\
U \\
U\end{array}$ & $\begin{array}{l}0.8850 \\
0.0159 \\
1.1000 \\
1.1300 \\
1.1000 \\
0.7000 \\
0.9720 \\
0.2110 \\
0.0950 \\
0.0331 \\
0.9610 \\
3.0100 \\
0.0441 \\
0.0329\end{array}$ & $\begin{array}{l}\mathbf{U} \\
\mathbf{J} \\
\mathbf{J} \\
\mathbf{J} \\
\mathbf{U} \mathbf{J} \\
\mathbf{J} \\
\mathbf{J} \\
\mathbf{U} \\
\mathbf{U} \mathbf{J} \\
\mathbf{J} \\
\mathbf{U} \\
\mathbf{U} \\
\mathbf{U}\end{array}$ & & \\
\hline
\end{tabular}

Location=ORR; Formation=CHEPULTEPEC; Site=77,85,86; Sample type=Field Duplicate; $A=5146 ; B=5149 ; C=5152 ; A$ lab split $=$ ' ';

Americium-241
Barium-133
Cesium-137
Chromium-51
Cobal -57
Cobal -60
Curium-243
Curium-245
Curium-247
Europium-152
Europium- 154
Hafnium-181
Iridium-192
Neptunium-237
Neptunium-237 (gamma)
Miobium-95
Plutonium-238
Plutonium-239/240
Potassium- 40
Radium-226
Ruthenium- 103

\begin{tabular}{|c|c|}
\hline $\begin{array}{l}p C i / g \\
p C i / g \\
p C i / g \\
p C i / g \\
p C i / g \\
p C i / g \\
p C i / g \\
p C i / g \\
p C i / g \\
p C i / g \\
p C i / g \\
p C i / g \\
p C i / g \\
p C i / g \\
p C i / g \\
p C i / g \\
p C i / g \\
p C i / g \\
p C i / g \\
p C i / g \\
p C i / g\end{array}$ & $\begin{array}{l}0.0646 \\
0.0259 \\
0.8300 \\
0.1600 \\
0.0136 \\
0.0218 \\
0.1290 \\
0.1830 \\
0.0228 \\
0.1390 \\
0.0289 \\
0.0218 \\
0.0181 \\
0.1580 \\
2.8400 \\
0.0207 \\
0.1060 \\
0.0331 \\
3.4500 \\
0.8400 \\
0.0212\end{array}$ \\
\hline
\end{tabular}

$\begin{array}{llll}0.06 & U & 0.0739 & U \\ 0.02 & U & 0.0291 & U \\ 0.02 & U & 0.0279 & U \\ 0.13 & U & 0.1750 & U \\ 0.01 & U & 0.0150 & U \\ 0.02 & U & 0.0271 & U \\ & & & \\ & & & \\ 0.12 & U & 0.1460 & U \\ 0.02 & U & 0.0318 & U \\ 0.02 & U & 0.0247 & U \\ 0.02 & U & 0.0200 & U \\ 2.60 & U J & & \\ 0.02 & U & 0.0288 & U \\ & & & \\ 7.68 & & 10.6000 & U \\ 1.10 & & 1.1600 & \\ 0.02 & U & 0.0223 & U\end{array}$


Table E.1 (continued)

\begin{tabular}{|c|c|c|c|c|c|c|c|c|c|}
\hline Analysis & Units & $\begin{array}{c}\text { A horizon } \\
\text { Result }\end{array}$ & Qualifier & $\begin{array}{c}\text { B horizon } \\
\text { Result }\end{array}$ & Qualifier & $\begin{array}{c}C \text { horizon } \\
\text { Result }\end{array}$ & Qualifier & $\begin{array}{l}\text { A lab split } \\
\text { Result }\end{array}$ & Qualifier \\
\hline $\begin{array}{l}\text { Stront ium-90 } \\
\text { Thorium-228 } \\
\text { Thorium-230 } \\
\text { Thor ium-232 } \\
\text { Thorium-234 } \\
\text { Total Uranium } \\
\text { Uranium-233/234 } \\
\text { Uranium-235 } \\
\text { Uranium-235 (gamma) } \\
\text { Uranium-236 } \\
\text { Uranium-238 } \\
\text { Uranium-238 (gamma) } \\
\text { Zinc-65 } \\
\text { Zirconium-95 }\end{array}$ & $\begin{array}{l}\mathrm{pCi} i / g \\
\mathrm{pCi} / \mathrm{g} \\
\mathrm{pCi} / \mathrm{g} \\
\mathrm{pCi} / \mathrm{g} \\
\mathrm{pCi} / \mathrm{g} \\
\mathrm{pCi} / \mathrm{g} \\
\mathrm{pCi/g} \\
\mathrm{pCi} / \mathrm{g} \\
\mathrm{pCi} / \mathrm{g} \\
\mathrm{pCi} / \mathrm{g} \\
\mathrm{pCi} / \mathrm{g} \\
\mathrm{pCi} \mathrm{g} \\
\mathrm{pCi} / \mathrm{g} \\
\mathrm{pCi} / \mathrm{g}\end{array}$ & $\begin{array}{l}0.7730 \\
0.5390 \\
0.6710 \\
0.4870 \\
0.0865 \\
2.5000 \\
0.8680 \\
0.0307 \\
0.1640 \\
0.0306 \\
0.9390 \\
4.0800 \\
0.0462 \\
0.0357\end{array}$ & $\begin{array}{l}U \\
J \\
J \\
J \\
U J \\
J \\
J \\
U J \\
U \\
U J \\
J \\
U \\
U \\
U\end{array}$ & $\begin{array}{l}0.87 \\
0.97 \\
0.83 \\
0.85 \\
0.98 \\
0.08 \\
0.15 \\
0.04 \\
1.12 \\
3.70 \\
0.04 \\
0.04\end{array}$ & $\begin{array}{l}J \\
J \\
J \\
U J \\
J \\
J \\
U \\
U J \\
J \\
U \\
U \\
U\end{array}$ & $\begin{array}{l}1.0200 \\
1.2500 \\
1.0900 \\
0.8930 \\
1.0500 \\
0.1140 \\
0.1570 \\
0.0263 \\
0.9570 \\
4.8900 \\
0.0561 \\
0.0454\end{array}$ & $\begin{array}{l}J \\
J \\
J \\
U J \\
J \\
J \\
u \\
U J \\
J \\
u \\
u \\
U\end{array}$ & & \\
\hline
\end{tabular}

Location=ORR; Formation=CHEPULTEPEC; Site =78; Sample type $=$ Regular; $A=4277 ; B=$ ' '; $C=$ ' '; $A$ lab split=' '; Phase $=2$

Technetium-99

$\mathrm{PCi} / \mathrm{g} \quad 1.0200 \quad \mathrm{U}$

Location=ORR; Formation=CHEPULTEPEC; Site=86; Sample type=Regular; $A=4278 ; B=$ '; $C=$ ' '; $A$ lab split $=4279 ;$ Phase $=2$

$\begin{array}{rrrrrr}\text { Technetium-99 } & \mathrm{pCi} / \mathrm{g} & \mathbf{1 . 0 8 0 0} & \mathrm{U} & \mathbf{1 . 0 3 0 0} & \mathrm{U}\end{array}$

Location=ORR; Formation=CHICKAMAUGA Bethel V; Site=99; Sample type=Regular; $A=4280 ; B=$ '; $C=$ ' '; $A$ lab split=' ', Phase $=2$

Technetium-99 $\quad \mathrm{pCi} / \mathrm{g} \quad 2.5600$

Location=ORR; Formation $=$ CHICKAMAUGA Bethel V; Site $=101 ;$ Sample type=Regular; $A=4281 ; B=$ ' ; C=' '; $A$ lab split=4282;

Phase $=2$
Technetium-99
$\mathrm{pCi} / \mathrm{g}$
1.5300
1.5000

Location=ORR; Formation=CHICKAMAUGA Bethel V; Site=93,99,100; Sample type=Regular; $A=5188 ; B=5191 ; C=5194$ A lab split =' '; Phase $=2$

$\begin{array}{llllllll}\text { Americium-241 } & \mathrm{PCi} / \mathrm{g} & 0.0917 & \mathrm{U} & 0.08 & \mathrm{U} & 0.0697 & \mathrm{U} \\ \text { Barium-133 } & \mathrm{pCi} / \mathrm{g} & 0.0349 & \mathrm{U} & 0.03 & \mathrm{U} & 0.0238 & U \\ \text { Cesium-137 } & \mathrm{pCi} / \mathrm{g} & 1.2500 & & 0.03 & \mathrm{U} & 0.0231 & \mathrm{U}\end{array}$


Table E.1 (continued)

\begin{tabular}{|c|c|c|c|c|c|c|c|c|c|}
\hline Analysis & Units & $\begin{array}{c}\text { A horizon } \\
\text { Result }\end{array}$ & Qualifier & $\begin{array}{c}\text { B horizon } \\
\text { Result }\end{array}$ & Qualifier & $\begin{array}{c}C \text { horizon } \\
\text { Result }\end{array}$ & Qualifier & $\begin{array}{c}\text { A lab split } \\
\text { Result }\end{array}$ & Qualifier \\
\hline $\begin{array}{l}\text { Chromium-51 } \\
\text { Cobalt-57 } \\
\text { Cobal t-60 } \\
\text { Europium-152 } \\
\text { Europium-154 } \\
\text { Hafnium-181 } \\
\text { Iridium-192 } \\
\text { Neptunium-237 (gamma) } \\
\text { Niobium-95 } \\
\text { Potassium-40 } \\
\text { Radium-226 } \\
\text { Ruthenium-103 } \\
\text { Thorium-228 } \\
\text { Thorium-230 } \\
\text { Thorium-232 } \\
\text { Thorium-234 } \\
\text { Uranium-233/234 } \\
\text { Uranium-235 } \\
\text { Uranium-235 (gamma) } \\
\text { Uranium-236 } \\
\text { Uranium-238 } \\
\text { Uranium-238 (garma) } \\
\text { 2inc-65 } \\
\text { Zirconium-95 }\end{array}$ & $\begin{array}{l}\text { pCi/g } \\
\text { pCi/g } \\
\text { pCi/g } \\
\text { pCi/g } \\
\text { pCi/g } \\
\text { pCi/g } \\
\text { pCi/g } \\
\text { pCi/g } \\
\text { pCi/g } \\
\text { pCi/g } \\
\text { pCi/g } \\
\text { pCi/g } \\
\text { pCi/g } \\
\text { pCi/g } \\
\text { pCi/g } \\
\text { pCi/g } \\
\text { pCi/g } \\
\text { pCi/g } \\
\text { pCi/g } \\
\text { pCi/g } \\
\text { pCi/g } \\
\text { pCi/g } \\
\text { pCi/g } \\
\text { pCi/g }\end{array}$ & $\begin{array}{r}1.0200 \\
0.0235 \\
0.0350 \\
0.1740 \\
0.0428 \\
0.0877 \\
0.0426 \\
4.3300 \\
0.1190 \\
15.4000 \\
0.7470 \\
0.0860 \\
1.3200 \\
1.0300 \\
1.3000 \\
0.9620 \\
1.0900 \\
0.1360 \\
0.1760 \\
0.0117 \\
1.1900 \\
6.3100 \\
0.0846 \\
0.1110\end{array}$ & $\begin{array}{l}U \\
U \\
U \\
U \\
U \\
U \\
U U \\
U \\
U \\
\\
U \\
J \\
J \\
J \\
U J \\
J \\
J \\
U \\
U J \\
J \\
U \\
U \\
U\end{array}$ & $\begin{array}{r}0.87 \\
0.02 \\
0.03 \\
0.14 \\
0.03 \\
0.07 \\
0.04 \\
3.02 \\
0.10 \\
24.30 \\
0.77 \\
0.07 \\
1.48 \\
1.25 \\
1.33 \\
0.83 \\
0.87 \\
0.14 \\
0.13 \\
0.03 \\
1.04 \\
35.10 \\
0.08 \\
0.09\end{array}$ & $\begin{array}{l}U \\
U \\
U \\
U \\
U \\
U \\
U \\
U \\
U \\
\\
U \\
J \\
J \\
J \\
U J \\
J \\
J \\
U \\
U S \\
J \\
U \\
U \\
U\end{array}$ & $\begin{array}{r}0.7220 \\
0.0176 \\
0.0262 \\
0.1270 \\
0.3160 \\
0.0586 \\
0.0313 \\
3.1000 \\
0.0922 \\
23.0000 \\
0.7880 \\
0.0606 \\
1.4900 \\
0.9660 \\
1.4800 \\
0.7040 \\
0.9080 \\
0.4630 \\
0.1270 \\
0.0477 \\
0.6870 \\
4.5400 \\
0.0695 \\
0.0845\end{array}$ & $\begin{array}{l}U \\
U \\
U \\
U \\
U \\
U \\
U \\
U \\
U \\
\\
U \\
J \\
J \\
J \\
U J \\
J \\
J \\
U \\
U J \\
J \\
U \\
U \\
U\end{array}$ & & . \\
\hline
\end{tabular}

Location=ORR; Formation=CHICKAMAUGA Bethel V; Site=101,102,103; Sample type=Regular; $A=5197 ; B=5200 ; C=5203$; A lab split $=$ ' '; Phase $=2$

Americium-241
Barium-133
Cesium-137
Chromium-51
Cobalt -57
Cobalt -60
Curium-243
Curium-245
Curium-247
Europium-152
Europium-154
Hafnium-181
Iridium-192
Neptunium-237

$\begin{array}{ll}\mathrm{pCi} / \mathrm{g} & 0.0068 \\ \mathrm{pCi} / \mathrm{g} & 0.0187 \\ \mathrm{pCi} / \mathrm{g} & 2.0900 \\ \mathrm{pCi} / \mathrm{g} & 1.0700 \\ \mathrm{pCi} / \mathrm{g} & 0.0123 \\ \mathrm{pCi} / \mathrm{g} & 0.0170 \\ \mathrm{pCi} / \mathrm{g} & 0.0880 \\ \mathrm{pCi} / \mathrm{g} & 0.1280 \\ \mathrm{pCi} / \mathrm{g} & 0.0172 \\ \mathrm{pCi/g} & 0.0781 \\ \mathrm{pCi} / \mathrm{g} & 0.0209 \\ \mathrm{pCi} / \mathrm{g} & 0.0732 \\ \mathrm{pCi} / \mathrm{g} & 0.0290 \\ \mathrm{pCi} / \mathrm{g} & 0.0770\end{array}$

$\begin{array}{ll}0.05 & U \\ 0.01 & U \\ 0.06 & \\ 0.77 & U \\ 0.01 & U \\ 0.01 & U \\ 0.07 & U \\ 0.11 & U \\ 0.01 & U \\ 0.06 & U \\ 0.02 & U \\ 0.05 & U \\ 0.02 & U\end{array}$

0.0330

0.0084

0.0080

0.5120

0.0081

0.0071

0.0488

0.0864

0.0067

0.0473

0.0138

0.0312

Neptunium-237

pCi/g

0.0770

0.02 U


Table E1 (continued)

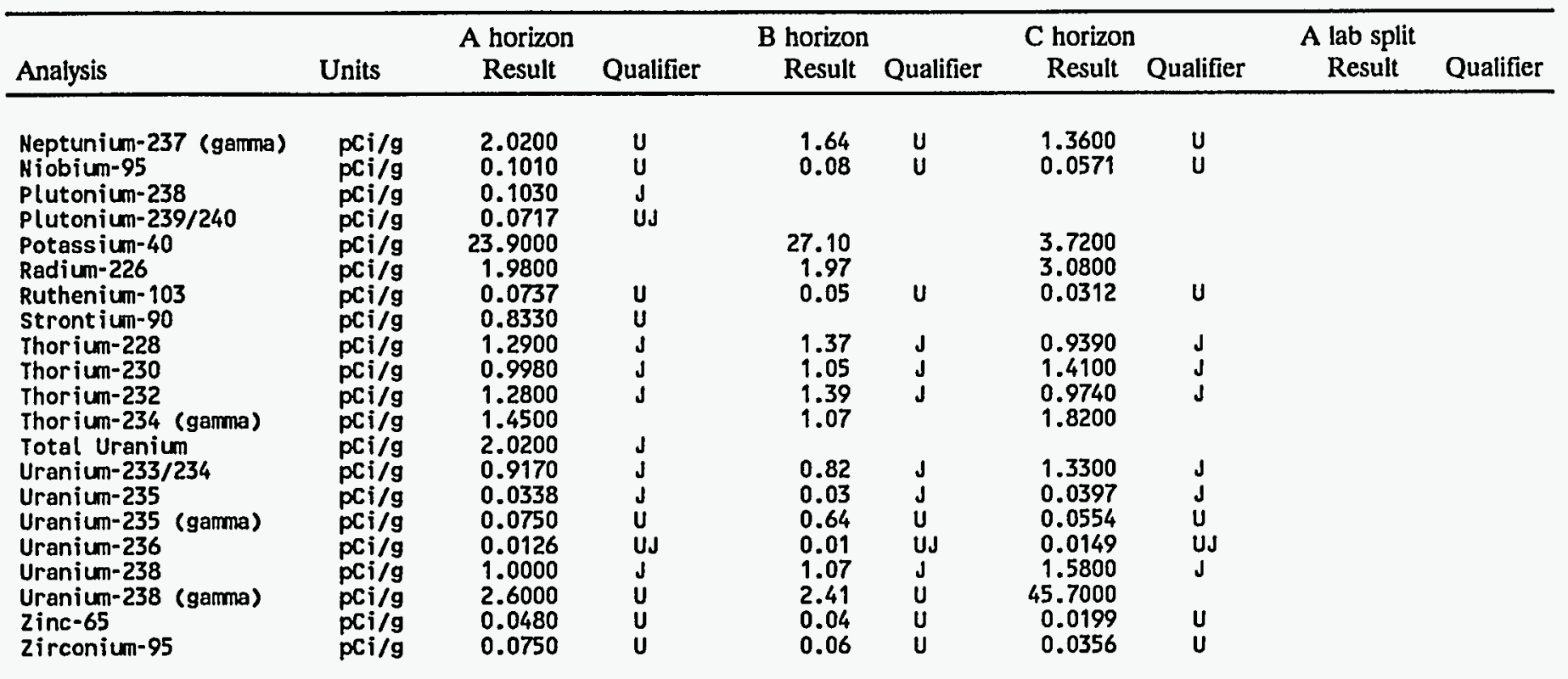

Location=ORR; Formation=CHICKAMAUGA Bethel V; Site=102; Sample type=Regular; $A=4283 ; B=$ ' ; $C=$ ' '; $A$ lab split=' '; Phase $=2$

$\begin{array}{llll}\text { Technetium-99 } & \text { pci/g } & 1.8200 & U\end{array}$

Location=ORR; Formation=CHICKAMAUGA Bethel V; Site=103; Sample type =Regular; $A=4284 ; B=$ ' '; $C=$ ' '; $A$ lab split=' '; Phase $=2$

$\begin{array}{llll}\text { Technetium-99 } & \text { pCi/g } & 1.4200 & \text { U }\end{array}$


Table E1 (continued)

\begin{tabular}{|c|c|c|c|c|c|c|c|c|c|}
\hline Analysis & Units & $\begin{array}{c}\text { A horizon } \\
\text { Result }\end{array}$ & Qualifier & $\begin{array}{c}\text { B horizon } \\
\text { Result }\end{array}$ & Qualifier & $\begin{array}{c}\text { C horizon } \\
\text { Result }\end{array}$ & Qualifier & $\begin{array}{l}\text { A lab split } \\
\text { Result }\end{array}$ & Qualifier \\
\hline
\end{tabular}

Location=ORR; Formation=CHICKAMAUGA Bethel V; Site=104,108,110; Sample type=Regular; $A=5206 ; B=5209 ; C=5212$;

A lab split=' '; Phase $=2$

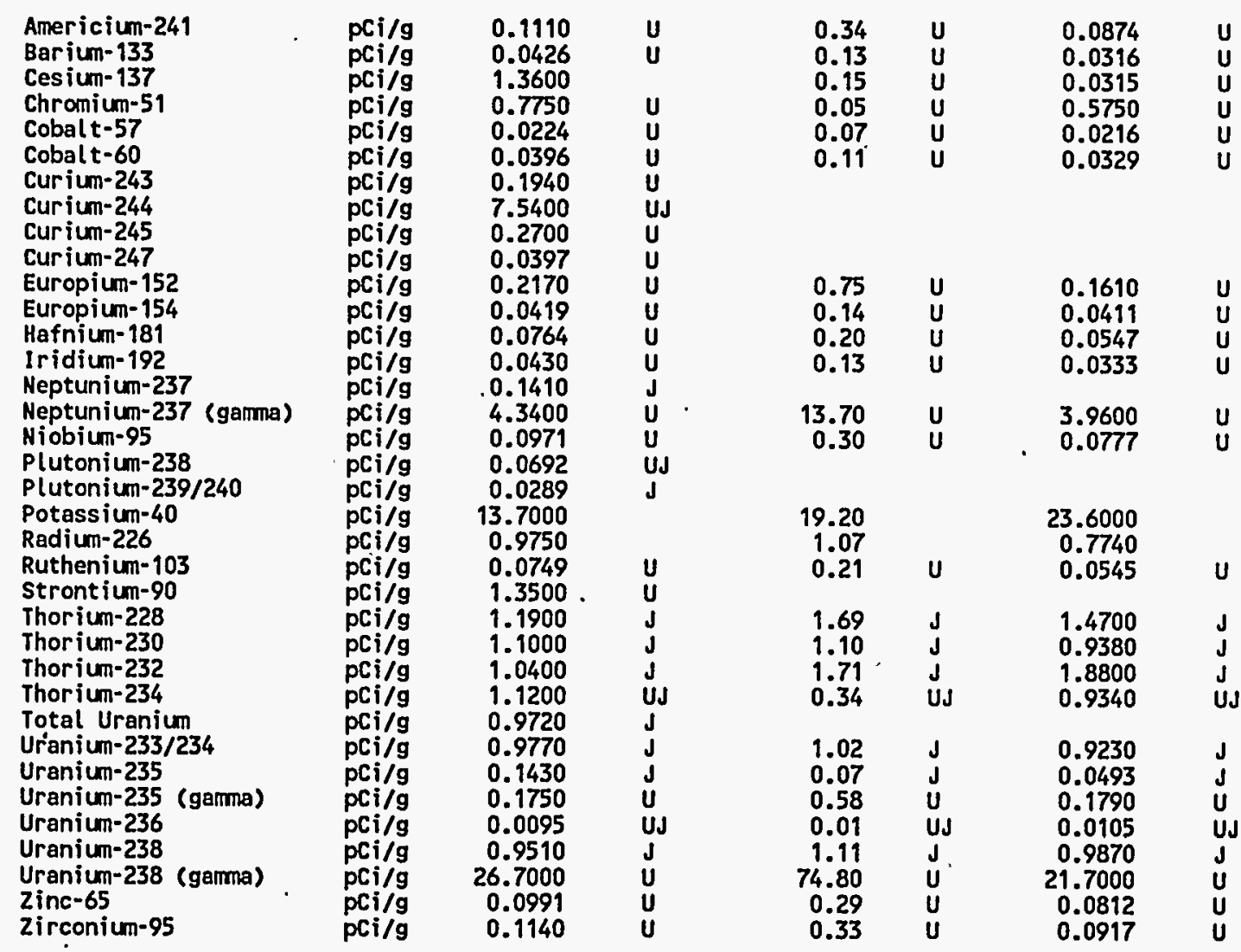


Table E1 (continued)

\begin{tabular}{|c|c|c|c|c|c|c|c|c|c|}
\hline Analysis & Units & $\begin{array}{c}\text { A horizon } \\
\text { Result }\end{array}$ & Qualifier & $\begin{array}{c}\text { B horizon } \\
\text { Result }\end{array}$ & Qualifier & $\begin{array}{c}\text { C horizon } \\
\text { Result }\end{array}$ & Qualifier & $\begin{array}{l}\text { A lab split } \\
\text { Result }\end{array}$ & Qualifier \\
\hline
\end{tabular}

Location=ORR; Formation=CHICKAMAUGA Bethel V; Site=115,116,117; Sample type=Regular; $A=5218 ; B=5223 ; C=5226 ;$ A lab split $=5219 ;$ Phase $=2$

\begin{tabular}{|c|c|c|c|c|c|c|c|c|}
\hline $\begin{array}{l}\text { Americium-241 } \\
\text { Barium-133 } \\
\text { Cesium-137 } \\
\text { Chromium-51 } \\
\text { Cobalt-57 } \\
\text { Cobalt-60 } \\
\text { Curium-243 } \\
\text { Curium-244 } \\
\text { Curium-245 } \\
\text { Curium-247 } \\
\text { Europium-152 } \\
\text { Europium-154 } \\
\text { Hafnium-181 } \\
\text { Iridium-192 } \\
\text { Neptunium-237 } \\
\text { Neptunium-237 (gamma) } \\
\text { Niobium-95 } \\
\text { Plutonium-238 } \\
\text { Plutonium-239/240 } \\
\text { Potassium-40 } \\
\text { Radium-226 } \\
\text { Ruthenium-103 } \\
\text { Strontium-90 } \\
\text { Thorium-228 } \\
\text { Thorium-230 } \\
\text { Thorium-232 } \\
\text { Thorium-234 } \\
\text { Total Uranium } \\
\text { Uranium-233/234 } \\
\text { Uranium-235 } \\
\text { Uranium-235 (gamma) } \\
\text { Uranium-236 } \\
\text { Uranium-238 } \\
\text { Uranium-238 (gamma) } \\
\text { Zinc-65 } \\
\text { Zirconium-95 }\end{array}$ & 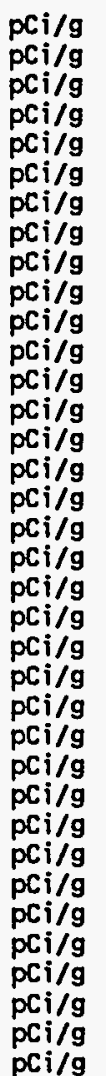 & $\begin{array}{r}0.1110 \\
0.0395 \\
0.9770 \\
0.7290 \\
0.0218 \\
0.0431 \\
0.2350 \\
0.0683 \\
0.2560 \\
0.0385 \\
0.2050 \\
0.0415 \\
0.0713 \\
0.0412 \\
0.0672 \\
4.3100 \\
0.0945 \\
0.0915 \\
0.0765 \\
10.7000 \\
1.0000 \\
0.0729 \\
1.0500 \\
1.1400 \\
1.0200 \\
1.2500 \\
1.1400 \\
0.2690 \\
1.1400 \\
0.0850 \\
0.1750 \\
0.0106 \\
1.1600 \\
25.1000 \\
0.0926 \\
0.1040\end{array}$ & $\begin{array}{l}U \\
U \\
U \\
U \\
U \\
U \\
U \\
U J \\
U \\
U \\
U \\
U \\
U \\
U \\
U \\
U \\
J \\
U \\
U \\
U J \\
U \\
J\end{array}$ & $\begin{array}{r} \\
0.14 \\
0.04 \\
0.05 \\
0.03 \\
3.67 \\
0.03 \\
\\
20.80 \\
0.82 \\
0.05\end{array}$ & $\begin{array}{l}J \\
J \\
J \\
\text { UJ } \\
J \\
\mathbf{U} \\
\mathbf{U} \\
\mathbf{U}\end{array}$ & $\begin{array}{r}1.9000 \\
1.0700 \\
1.5600 \\
1.1300 \\
1.1000 \\
0.2380 \\
0.1870 \\
0.0117 \\
1.1100 \\
28.3000 \\
0.1070 \\
0.1130\end{array}$ & $\begin{array}{l}J \\
J \\
\mathbf{U} \\
\mathbf{U} J \\
J \\
\mathbf{U} \\
\mathbf{U} \\
\mathbf{U}\end{array}$ & $\begin{array}{r}0.1000 \\
0.0389 \\
0.8980 \\
0.6440 \\
0.0200 \\
0.0346 \\
0.1780 \\
0.0300 \\
0.2410 \\
0.0354 \\
0.1920 \\
0.0383 \\
0.0683 \\
0.0395 \\
0.0828 \\
3.8500 \\
0.0878 \\
0.1330 \\
0.0377 \\
10.2000 \\
0.8690 \\
0.0656 \\
0.9770 \\
1.5800 \\
1.1900 \\
1.5600 \\
1.0100 \\
1.7000 \\
1.0100 \\
0.1430 \\
0.1550 \\
0.0097 \\
1.0900 \\
22.1000 \\
0.0773 \\
0.0916\end{array}$ \\
\hline
\end{tabular}


Table E1 (continued)

\begin{tabular}{|c|c|c|c|c|c|c|c|c|c|}
\hline Analysis & Units & $\begin{array}{c}\text { A horizon } \\
\text { Result }\end{array}$ & Oualifier & $\begin{array}{c}\text { B horizon } \\
\text { Result }\end{array}$ & Oualifier & $\begin{array}{c}\mathrm{C} \text { horizon } \\
\text { Result }\end{array}$ & Oualifier & A lab split & Oualifier \\
\hline
\end{tabular}

Location=ORR; Formation=CHICKAMAUGA Bethel V; Site=116; Sample type=Regular; $A=4285 ; B=$ '; C=' '; $A$ lab split=' '; Phase $=2$

Technetium-99 $\quad \mathrm{pCi} / \mathrm{g} \quad 2.0300$

Location=ORR; Fornation=CHICKAMAUGA Bethel V; Site=117; Sample type=Regular; $A=4286 ; B=$ ' ' C=' '; $A$ lab split=' '; Phase $=2$

Technetium-99 $\quad \mathrm{pCi} / \mathrm{g} \quad 1.5000 \quad \mathrm{U}$

Location=ORR; Formation=CHICKAMAUGA K-25; Site=118,122,124; Sample type=Regular; $A=5242 ; B=5247 ; C=5250$; A lab split=5243; Phase $=2$

Americium-241
Barium-133
Cesium-137
Chromium-51
Cobalt-57
Cobalt-60
Curium-243
Curium-244
Curium-245
Curium-247
Europium-152
Europium-154
Hafnium-181
Iridium-192
Neptunium-237
Neptunium-237 (gamma)
Niobium-95
Plutonium-238
Plutonium-239/240
Potassium-40
Radium-226
Ruthenium-103
Strontium-90
Thorium-228
Thorium-230
Thorium-232
Thorium-234
Total Uranium

\begin{tabular}{|c|c|}
\hline $\begin{array}{l}\mathrm{pCi} / \mathrm{g} \\
\mathrm{pCi} / \mathrm{g} \\
\mathrm{pCi} / \mathrm{g} \\
\mathrm{pCi} / \mathrm{g} \\
\mathrm{pCi} / \mathrm{g} \\
\mathrm{pCi} / \mathrm{g} \\
\mathrm{pCi} / \mathrm{g} \\
\mathrm{pCi} / \mathrm{g} \\
\mathrm{pCi} / \mathrm{g} \\
\mathrm{pCi} / \mathrm{g} \\
\mathrm{pCi} / \mathrm{g} \\
\mathrm{pCi} / \mathrm{g} \\
\mathrm{pCi} / \mathrm{g} \\
\mathrm{pCi} / \mathrm{g} \\
\mathrm{pCi} / \mathrm{g} \\
\mathrm{pCi} / \mathrm{g} \\
\mathrm{pCi} / \mathrm{g} \\
\mathrm{pCi} / \mathrm{g} \\
\mathrm{pCi} / \mathrm{g} \\
\mathrm{pCi} / \mathrm{g} \\
\mathrm{pCi} / \mathrm{g} \\
\mathrm{pCi} / \mathrm{g} \\
\mathrm{pCi} / \mathrm{g} \\
\mathrm{pCi} / \mathrm{g} \\
\mathrm{pCi} / \mathrm{g} \\
\mathrm{pCi} / \mathrm{g} \\
\mathrm{pCi} / \mathrm{g} \\
\mathrm{pCi} / \mathrm{g}\end{array}$ & $\begin{array}{r}0.0816 \\
0.0319 \\
1.3100 \\
0.5700 \\
0.0184 \\
0.0278 \\
0.1580 \\
0.1220 \\
0.2200 \\
0.0292 \\
0.1470 \\
0.0353 \\
0.0571 \\
0.0331 \\
0.0807 \\
3.5700 \\
0.0694 \\
0.0598 \\
0.0317 \\
12.0000 \\
0.9280 \\
0.0549 \\
0.9950 \\
1.7200 \\
0.9750 \\
1.4900 \\
1.1900 \\
0.1600\end{array}$ \\
\hline
\end{tabular}

$\begin{array}{lll}0.07 & U & 0.0861 \\ 0.02 & U & 0.0284 \\ 0.03 & U & 0.0268 \\ 0.12 & U & 0.5560 \\ 0.02 & U & 0.0178 \\ 0.02 & U & 0.0265 \\ & & \\ & & \\ & & \\ 0.12 & U & 0.1250 \\ 0.03 & U & 0.0336 \\ 0.04 & U & 0.0520 \\ 0.03 & U & 0.0309 \\ 2.97 & U & 3.3700 \\ 0.06 & U & 0.0685 \\ & & \\ 24.80 & & 1.4500 \\ 0.92 & & 0.8590 \\ 0.04 & U & 0.0511 \\ 1.59 & J & 2.6100 \\ 1.13 & J & 1.4800 \\ 1.74 & J & 2.4700 \\ 0.72 & U J & 0.3120\end{array}$

$\begin{array}{cc}U & 0.0721 \\ U & 0.0241 \\ U & 1.3500 \\ U & 0.4710 \\ U & 0.0151 \\ U & 0.0215 \\ & 0.1220 \\ & A \\ & 0.1840 \\ U & 0.0229 \\ U & 0.1230 \\ U & 0.0287 \\ U & 0.0453 \\ & 0.0260 \\ U & 0.0845 \\ U & 2.9800 \\ & 0.0599 \\ & 0.0688 \\ U & 0.0373 \\ & 12.4000 \\ U & 0.9770 \\ & 0.0438 \\ J & 0.6110 \\ J & 1.2100 \\ J & 1.1300 \\ J & 1.2500 \\ U J & 0.7170 \\ & 1.1600\end{array}$


Table E.1 (continued)

\begin{tabular}{|c|c|c|c|c|c|c|c|c|c|}
\hline Analysis & Units & $\begin{array}{c}\text { A horizon } \\
\text { Result }\end{array}$ & Qualifier & $\begin{array}{c}\text { B horizon } \\
\text { Result }\end{array}$ & Qualifier & $\begin{array}{c}\text { C horizon } \\
\text { Result }\end{array}$ & Qualifier & $\begin{array}{l}\text { A lab split } \\
\text { Result }\end{array}$ & Qualifier \\
\hline $\begin{array}{l}\text { Uranium-233/234 } \\
\text { Uranium-235 } \\
\text { Uranium-235 (gamma) } \\
\text { Uranium-236 } \\
\text { Uranium-238 } \\
\text { Uranium-238 (gamma) } \\
\text { Zinc-65 } \\
\text { Zirconium-95 }\end{array}$ & $\begin{array}{l}\mathrm{pCi} / \mathrm{g} \\
\mathrm{pCi} / \mathrm{g} \\
\mathrm{pCi} / \mathrm{g} \\
\mathrm{pCi} / \mathrm{g} \\
\mathrm{pCi} / \mathrm{g} \\
\mathrm{pCi} / \mathrm{g} \\
\mathrm{pCi} / \mathrm{g} \\
\mathrm{pCi} / \mathrm{g}\end{array}$ & $\begin{array}{r}1.3400 \\
0.1780 \\
0.1740 \\
0.0234 \\
1.3900 \\
19.4000 \\
0.0691 \\
0.0773\end{array}$ & $\begin{array}{l}J \\
J \\
U \\
U J \\
J \\
U \\
U \\
U\end{array}$ & $\begin{array}{r}1.19 \\
0.31 \\
0.12 \\
0.01 \\
1.15 \\
17.30 \\
0.06 \\
0.07\end{array}$ & $\begin{array}{l}J \\
J \\
U \\
U J \\
J \\
U \\
U \\
U\end{array}$ & $\begin{array}{r}1.0200 \\
0.0730 \\
0.1430 \\
0.0427 \\
1.2500 \\
19.3000 \\
0.0655 \\
0.0791\end{array}$ & $\begin{array}{l}J \\
J \\
U \\
U J \\
J \\
U \\
U \\
U\end{array}$ & $\begin{array}{l}1.4700 \\
0.0664 \\
0.1010 \\
0.0241 \\
1.3600 \\
1.5700 \\
0.0517 \\
0.0634\end{array}$ & $\begin{array}{l}J \\
J \\
U \\
U J \\
J \\
u \\
u \\
U\end{array}$ \\
\hline
\end{tabular}

Location=ORR; Formation=CHICKAMAUGA K-25; Site=119; Sample type=Regular; $A=4287 ; B=$ ' '; C=' '; $A$ lab split=' '; Phase =2

Technetium-99 $\quad$ pCi/g $\quad 1.7500$

Location=ORR; Formation=CHICKAMAUGA K-25; Site=119,123,127; Sample type=Regular; $A=5254 ; B=5258 ; C=5261 ;$ $A$ lab split $=5255 ;$ Phase $=2$

\begin{tabular}{|c|c|c|c|c|c|c|c|c|}
\hline $\begin{array}{l}\text { Americium-241 } \\
\text { Barium-133 } \\
\text { Cesium-137 } \\
\text { Chromium-51 } \\
\text { Cobalt }-57 \\
\text { Cobalt- }-60 \\
\text { Curium-243 } \\
\text { Curium-244 } \\
\text { Curium-245 } \\
\text { Curium-247 } \\
\text { Europium-152 } \\
\text { Europium-154 } \\
\text { Hafnium-181 } \\
\text { Iridium-192 } \\
\text { Neptunium-237 } \\
\text { Neptunium-237 (gamma) } \\
\text { Niobium- } 95 \\
\text { Plutonium-238 } \\
\text { Plutonium-239/240 } \\
\text { Potassium-40 } \\
\text { Radium-226 } \\
\text { Ruthenium-103 }\end{array}$ & $\begin{array}{l}p C i / g \\
p C i / g \\
p C i / g \\
p C i / g \\
p C i / g \\
p C i / g \\
p C i / g \\
p C i / g \\
p C i / g \\
p C i / g \\
p C i / g \\
p C i / g \\
p C i / g \\
p C i / g \\
p C i / g \\
p C i / g \\
p C i / g \\
p C i / g \\
p C i / g \\
p C i / g \\
p C i / g \\
p C i / g\end{array}$ & $\begin{array}{l}0.1120 \\
0.0411 \\
1.6800 \\
0.6500 \\
0.0219 \\
0.0423 \\
0.1940 \\
0.0607 \\
0.2620 \\
0.0405 \\
0.2120 \\
0.0421 \\
0.0724 \\
0.0407 \\
0.1130 \\
4.2400 \\
0.0879 \\
0.0554 \\
0.0384 \\
13.3000 \\
0.9830 \\
0.0675\end{array}$ & $\begin{array}{l}U \\
U \\
U \\
U \\
U \\
U \\
U J \\
U J \\
U \\
U \\
U \\
U \\
U \\
U \\
J \\
U \\
U \\
U J \\
U J\end{array}$ & $\begin{array}{l}0.16 \\
0.04 \\
0.05 \\
0.03 \\
\\
4.04 \\
0.08 \\
\\
\\
26.10 \\
0.94 \\
0.05\end{array}$ & $\begin{array}{l}U \\
U \\
U \\
U \\
U \\
U\end{array}$ & $\begin{array}{r}0.5000 \\
0.0963 \\
0.1760 \\
0.1020 \\
9.9100 \\
0.2450 \\
\\
63.4000 \\
3.0400 \\
0.1780\end{array}$ & $\begin{array}{l}\mathbf{U} \\
\mathbf{U} \\
\mathbf{U} \\
\mathbf{U} \\
\mathbf{U} \\
\mathbf{U}\end{array}$ & $\begin{array}{r}0.1190 \\
0.0418 \\
1.7200 \\
0.6890 \\
0.0234 \\
0.0370 \\
0.2040 \\
0.0914 \\
0.2790 \\
0.0407 \\
0.2070 \\
0.0453 \\
0.0701 \\
0.0433 \\
0.1470 \\
4.6500 \\
0.0855 \\
0.0947 \\
0.0379 \\
13.8000 \\
0.9920 \\
0.0702\end{array}$ \\
\hline
\end{tabular}


Table E1 (continued)

\begin{tabular}{|c|c|c|c|c|c|c|c|c|c|}
\hline Analysis & Units & $\begin{array}{c}\text { A horizon } \\
\text { Result }\end{array}$ & Qualifier & $\begin{array}{c}\text { B horizon } \\
\text { Result }\end{array}$ & Qualifier & $\begin{array}{c}\text { C horizon } \\
\text { Result }\end{array}$ & Qualifier & $\begin{array}{l}\text { A lab split } \\
\text { Result }\end{array}$ & Qualifier \\
\hline $\begin{array}{l}\text { Strontium-90 } \\
\text { Thorium-228 } \\
\text { Thorium-230 } \\
\text { Thorium-232 } \\
\text { Thorium-234 (gamma) } \\
\text { Total Uranium } \\
\text { Uranium-233/234 } \\
\text { Uranium-235 } \\
\text { Uranium-235 (gamma) } \\
\text { Uranium-236 } \\
\text { Uranium-238 } \\
\text { Uranium-238 (gamma) } \\
\text { 2inc-65 } \\
\text { Zirconium-95 }\end{array}$ & $\begin{array}{l}\mathrm{pCi} / \mathrm{g} \\
\mathrm{pCi} / \mathrm{g} \\
\mathrm{pCi} / \mathrm{g} \\
\mathrm{pCi} / \mathrm{g} \\
\mathrm{pCi} / \mathrm{g} \\
\mathrm{pCi} / \mathrm{g} \\
\mathrm{pCi} / \mathrm{g} \\
\mathrm{pCi} / \mathrm{g} \\
\mathrm{pCi} / \mathrm{g} \\
\mathrm{pCi} / \mathrm{g} \\
\mathrm{pCi} / \mathrm{g} \\
\mathrm{pCi} / \mathrm{g} \\
\mathrm{pCi} / \mathrm{g} \\
\mathrm{pCi} / \mathrm{g}\end{array}$ & $\begin{array}{r}1.0400 \\
1.3500 \\
1.1500 \\
1.2900 \\
1.1300 \\
0.3270 \\
1.4600 \\
0.0757 \\
0.1820 \\
0.0238 \\
1.4000 \\
22.6000 \\
0.0940 \\
0.1020\end{array}$ & $\begin{array}{l}u \\
J \\
J \\
J \\
U \\
J \\
J \\
U \\
J \\
J \\
U \\
U \\
U\end{array}$ & $\begin{array}{l}1.46 \\
1.40 \\
2.77 \\
0.88 \\
1.13 \\
0.06 \\
0.16 \\
0.01 \\
1.38 \\
20.90 \\
0.08 \\
0.09\end{array}$ & $\begin{array}{l}J \\
U J \\
J \\
U \\
J \\
J \\
U \\
U J \\
J \\
U \\
U \\
U\end{array}$ & $\begin{array}{r}1.9000 \\
1.2300 \\
1.9000 \\
2.0200 \\
1.2100 \\
0.1070 \\
0.4290 \\
0.0019 \\
1.3800 \\
16.0000 \\
0.2310 \\
0.2690\end{array}$ & $\begin{array}{l}J \\
J \\
J \\
U \\
J \\
J \\
U \\
J \\
J \\
U \\
U \\
U\end{array}$ & $\begin{array}{r}0.7450 \\
1.0200 \\
1.2600 \\
1.4200 \\
1.2700 \\
0.9880 \\
1.4900 \\
0.0797 \\
0.2220 \\
0.0095 \\
1.3500 \\
22.0000 \\
0.0943 \\
0.1040\end{array}$ & $\begin{array}{l}\mathbf{U} \\
\mathbf{J} \\
\mathbf{J} \\
\mathbf{J} \\
\mathbf{U} \\
\mathbf{J} \\
\mathbf{J} \\
\mathbf{U} \\
\mathbf{U J} \\
\mathbf{J} \\
\mathbf{U} \\
\mathbf{U} \\
\mathbf{U}\end{array}$ \\
\hline
\end{tabular}

Location=ORR; Formation=CHICKAMAUGA K-25; Site=120,126,129; Sample type=Regular; $A=5230 ; B=5235 ; C=5238$; A lab split $=5231$; Phase $=2$

Americium-241
Barium-133
Cesium-137
Chromium-51
Cobalt -57
Cobalt -60
Curium-243
Curium-244
Curium-245
Curium-247
Europium-152
Europium-154
Hafnium- 181
Iridium-192
Neptunium-237
Neptunium-237 (gamma)
Niobium-95
Plutonium-238
Plutonium-239/240
Potassium-40
Radium-226
Ruthenium-103
Strontium-90

\begin{tabular}{|c|c|}
\hline 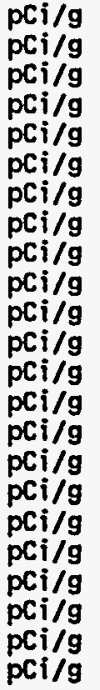 & $\begin{array}{l}0.0621 \\
0.0225 \\
0.9710 \\
0.3830 \\
0.0135 \\
0.0196 \\
0.1110 \\
0.0296 \\
0.1610 \\
0.0215 \\
0.1170 \\
0.0257 \\
0.0395 \\
0.0242 \\
0.1240 \\
2.6300 \\
0.0423 \\
0.2030 \\
0.0777 \\
0.3060 \\
0.9250 \\
0.0391 \\
0.7250\end{array}$ \\
\hline
\end{tabular}

$\begin{array}{ll}0.09 & U \\ 0.03 & U \\ 0.03 & U \\ 0.58 & U \\ 0.02 & U \\ 0.03 & U \\ & \\ & \\ 0.15 & U \\ 0.04 & U \\ 0.05 & U \\ 0.03 & U \\ 3.64 & U \\ 0.07 & U \\ & \\ 17.40 & \\ 1.05 & \\ 0.06 & U\end{array}$

0.0741

0.0234

0.2020

0.0142

0.0238

$U$
$\mathbf{U}$
$\mathbf{U}$
$\mathbf{U}$
$\mathbf{U}$
$\mathbf{U}$

0.0725

0.0248
1.0100

1.0100
0.1350

0.1350
0.0150

0.0219

0.1250

0.7950

0.1850

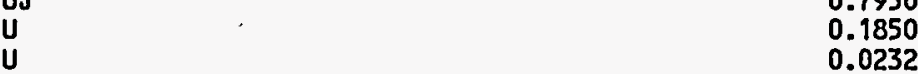

\begin{tabular}{llllll}
$U$ & 0.15 & $U$ & 0.1190 & $U$ & 0.0232 \\
\hline & 0.04 & $U$ & 0.0291240
\end{tabular}

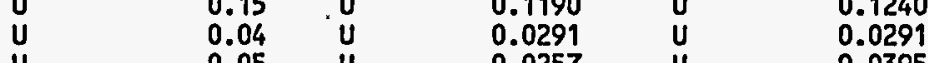

Ruthenium-103

0.7250

0.0257

0.0190

0.0395

0.0258

0.0620
3.0900

0.0528

0.0648

0.0406

6.7200

26. 1000

0.9790

6.7200
0.9800

$\mathbf{U}$

U

0.0235 
Table E.1 (continued)

\begin{tabular}{|c|c|c|c|c|c|c|c|c|c|}
\hline Analysis & Units & $\begin{array}{c}\text { A horizon } \\
\text { Result }\end{array}$ & Qualifier & $\begin{array}{c}\text { B horizon } \\
\text { Result }\end{array}$ & Qualifier & $\begin{array}{c}\text { C horizon } \\
\text { Result }\end{array}$ & Qualifier & $\begin{array}{c}\text { A lab split } \\
\text { Result }\end{array}$ & Qualifier \\
\hline $\begin{array}{l}\text { Thorium-228 } \\
\text { Thorium-230 } \\
\text { Thorium-232 } \\
\text { Thorium-234 } \\
\text { Total Uranium } \\
\text { Uranium-233/234 } \\
\text { Uranium-235 } \\
\text { Uranium-235 (gamma) } \\
\text { Uranium-236 } \\
\text { Uranium-238 } \\
\text { Uranium-238 (garma) } \\
\text { Zinc-65 } \\
\text { Zirconium-95 }\end{array}$ & $\begin{array}{l}\mathrm{pCi} i / g \\
\mathrm{pCi} i / g \\
\mathrm{pCi} i / g \\
\mathrm{pCi} / \mathrm{g} \\
\mathrm{pCi} / \mathrm{g} \\
\mathrm{pCi} \mathrm{g} \\
\mathrm{pCi} / \mathrm{g} \\
\mathrm{pCi} / \mathrm{g} \\
\mathrm{pCi} / \mathrm{g} \\
\mathrm{pCi} / \mathrm{g} \\
\mathrm{pCi} / \mathrm{g} \\
\mathrm{pCi} / \mathrm{g} \\
\mathrm{pCi} / \mathrm{g}\end{array}$ & $\begin{array}{r}0.6050 \\
0.8180 \\
0.5860 \\
0.8450 \\
1.8500 \\
0.8380 \\
0.0438 \\
0.1430 \\
0.0109 \\
0.9940 \\
12.4000 \\
0.0466 \\
0.0552\end{array}$ & $\begin{array}{l}J \\
J \\
J \\
U J \\
J \\
J \\
J \\
U \\
U J \\
J \\
U \\
U \\
U\end{array}$ & $\begin{array}{r}1.52 \\
1.06 \\
1.46 \\
0.93 \\
1.36 \\
0.13 \\
0.14 \\
0.05 \\
1.29 \\
19.40 \\
0.07 \\
0.08\end{array}$ & $\begin{array}{l}J \\
J \\
J \\
U J \\
J \\
J \\
U \\
U J \\
J \\
U \\
U \\
U\end{array}$ & $\begin{array}{l}1.8100 \\
1.0600 \\
1.7500 \\
0.8110 \\
1.2700 \\
0.1040 \\
0.1180 \\
0.0142 \\
1.2600 \\
6.5700 \\
0.0569 \\
0.0478\end{array}$ & $\begin{array}{l}J \\
J \\
J \\
U J \\
J \\
J \\
U \\
U J \\
J \\
U \\
U \\
U\end{array}$ & $\begin{array}{r}1.1600 \\
1.2200 \\
1.0900 \\
1.9000 \\
2.2200 \\
1.2800 \\
0.0492 \\
0.1260 \\
0.0123 \\
1.4300 \\
12.8000 \\
0.0482 \\
0.0580\end{array}$ & $\begin{array}{l}J \\
J \\
J \\
J \\
J \\
J \\
J \\
U \\
U J \\
J \\
U \\
U \\
U\end{array}$ \\
\hline
\end{tabular}

Location=ORR; Formation=CHICKAMAUGA K-25; Site=121; Sample type =Regular; $A=4288 ; B=$ ' '; C=' '; $A$ lab split=' '; Phase=2

Technetium-99

$\mathrm{pCi} / \mathrm{g} \quad \mathbf{1 . 3 9 0 0}$

Location=ORR; Formation =CHICKAMAUGA K-25; Site=121,125,128; Sample type=Regular; $A=5265 ; B=5269 ; C=5272 ;$ A lab split $=5266 ;$ Phase $=2$

\begin{tabular}{|c|c|c|c|c|c|c|c|c|}
\hline $\begin{array}{l}\text { Americium-241 } \\
\text { Barium-133 } \\
\text { Cesium-137 } \\
\text { Chromium-51 } \\
\text { Cobalt-57 } \\
\text { Cobalt-60 } \\
\text { Curium-243 } \\
\text { Curium-244 } \\
\text { Curium-245 } \\
\text { Curium-247 } \\
\text { Europium-152 } \\
\text { Europium-154 } \\
\text { Hafnium-181 } \\
\text { Iridium-192 } \\
\text { Neptunium-237 } \\
\text { Neptunium-237 (gamma) } \\
\text { Niobium-95 } \\
\text { Plutonium-238 } \\
\text { Plutonium-239/240 }\end{array}$ & $\begin{array}{l}\mathrm{pCi} / \mathrm{g} \\
\mathrm{pCi} / \mathrm{g} \\
\mathrm{pCi} / \mathrm{g} \\
\mathrm{pCi} / \mathrm{g} \\
\mathrm{pCi} / \mathrm{g} \\
\mathrm{pCi} / \mathrm{g} \\
\mathrm{pCi} / \mathrm{g} \\
\mathrm{pCi} / \mathrm{g} \\
\mathrm{pCi} / \mathrm{g} \\
\mathrm{pCi} / \mathrm{g} \\
\mathrm{pCi} / \mathrm{g} \\
\mathrm{pCi} / \mathrm{g} \\
\mathrm{pCi} / \mathrm{g} \\
\mathrm{pCi} / \mathrm{g} \\
\mathrm{pCi} / \mathrm{g} \\
\mathrm{pCi} / \mathrm{g} \\
\mathrm{pCi} / \mathrm{g} \\
\mathrm{pCi} / \mathrm{g} \\
\mathrm{pCi} / \mathrm{g}\end{array}$ & $\begin{array}{l}0.0769 \\
0.0289 \\
0.6610 \\
0.4520 \\
0.0183 \\
0.0285 \\
0.1450 \\
0.3080 \\
0.2210 \\
0.0263 \\
0.1510 \\
0.0349 \\
0.0468 \\
0.0290 \\
0.1050 \\
3.5900 \\
0.0641 \\
0.0777 \\
0.0167\end{array}$ & $\begin{array}{l}U \\
U \\
U \\
U \\
U J \\
U \\
U \\
U \\
U \\
U \\
U \\
U \\
U \\
J \\
U \\
U \\
U \\
J \\
U J\end{array}$ & $\begin{array}{l}0.09 \\
0.03 \\
0.02 \\
0.52 \\
0.02 \\
0.03\end{array}$ & $\begin{array}{l}\mathbf{U} \\
\mathbf{U} \\
\mathbf{U} \\
\mathbf{U} \\
\mathbf{U} \\
\mathbf{U}\end{array}$ & $\begin{array}{l}0.0924 \\
0.0327 \\
0.0336 \\
0.5820 \\
0.0215 \\
0.0350\end{array}$ & $\begin{array}{l}U \\
U \\
U \\
U \\
U \\
U\end{array}$ & $\begin{array}{l}0.1040 \\
0.0376 \\
0.5780 \\
0.6640 \\
0.0203 \\
0.0334 \\
0.1860 \\
0.0380 \\
0.2530 \\
0.0343 \\
0.1820 \\
0.0401 \\
0.0644 \\
0.0389 \\
0.0721 \\
4.1200 \\
0.0841 \\
0.1040 \\
0.0216\end{array}$ \\
\hline
\end{tabular}


Table E.1 (continued)

\begin{tabular}{|c|c|c|c|c|c|c|c|c|c|}
\hline Analysis & Units & $\begin{array}{c}\text { A horizon } \\
\text { Result }\end{array}$ & Qualifier & $\begin{array}{c}\text { B horizon } \\
\text { Result }\end{array}$ & Qualifier & $\begin{array}{c}C \text { horizon } \\
\text { Result }\end{array}$ & Qualifier & $\begin{array}{c}\text { A lab split } \\
\text { Result }\end{array}$ & Qualifier \\
\hline $\begin{array}{l}\text { Potassium-40 } \\
\text { Radium-226 } \\
\text { Ruthenium-103 } \\
\text { strontium-90 } \\
\text { Thorium-228 } \\
\text { Thorium-230 } \\
\text { Thorium-232 } \\
\text { Thorium-234 (gamma) } \\
\text { Total Uranium } \\
\text { Uranium-233/234 } \\
\text { Uranium-235 } \\
\text { Uranium-235 (garma) } \\
\text { Uranium-236 } \\
\text { Uranium-238 } \\
\text { Uranium-238 (gamma) } \\
\text { Zinc-65 } \\
\text { Zirconium-95 }\end{array}$ & $\begin{array}{l}p C i / g \\
p C i / g \\
p C i / g \\
p C i / g \\
p C i / g \\
p C i / g \\
p C i / g \\
p C i / g \\
p C i / g \\
p C i / g \\
p C i / g \\
p C i / g \\
p C i / g \\
p C i / g \\
p C i / g \\
p C i / g \\
p C i / g\end{array}$ & $\begin{array}{l}8.6700 \\
0.7420 \\
0.0469 \\
0.7740 \\
1.1400 \\
0.8400 \\
1.0800 \\
0.8090 \\
0.9040 \\
1.0400 \\
0.0120 \\
0.1440 \\
0.0383 \\
1.0400 \\
4.2100 \\
0.0700 \\
0.0707\end{array}$ & $\begin{array}{l}U \\
U \\
J \\
J \\
J \\
U \\
J \\
U J J \\
U \\
U J \\
J \\
U \\
U \\
U\end{array}$ & $\begin{array}{r}23.80 \\
0.90 \\
0.05 \\
1.55 \\
1.09 \\
1.38 \\
0.78 \\
1.12 \\
0.05 \\
0.15 \\
0.01 \\
1.22 \\
19.40 \\
0.08 \\
0.08\end{array}$ & $\begin{array}{l}\mathbf{U} \\
\mathbf{J} \\
\mathbf{J} \\
\mathbf{J} \\
\mathbf{U} \\
\mathbf{J} \\
\mathbf{J} \\
\mathbf{U} \\
\mathbf{U} \mathbf{J} \\
\mathbf{J} \\
\mathbf{U} \\
\mathbf{U} \\
\mathbf{U}\end{array}$ & $\begin{array}{r}24.6000 \\
0.7790 \\
0.0547 \\
1.7800 \\
1.2600 \\
0.8900 \\
0.9470 \\
1.1500 \\
0.0409 \\
0.1700 \\
0.0434 \\
1.1200 \\
22.2000 \\
0.0896 \\
0.0894\end{array}$ & $\begin{array}{l}U \\
J \\
J \\
J \\
U \\
J \\
J \\
J \\
U \\
U J \\
J \\
U \\
U \\
U\end{array}$ & $\begin{array}{l}7.2400 \\
0.9330 \\
0.0649 \\
0.9530 \\
0.9730 \\
0.9550 \\
0.8270 \\
1.0700 \\
0.7410 \\
0.9690 \\
0.0455 \\
0.1690 \\
0.0170 \\
0.8670 \\
A \\
0.0785 \\
0.0947\end{array}$ & $\begin{array}{l}J \\
J \\
U \\
U J \\
J \\
U \\
U \\
U\end{array}$ \\
\hline
\end{tabular}

Location=ORR; Formation=CHICKAMAUGA K-25; Site=124; Sample type=Regular; $A=4289 ; B=$ ' ; $C=$ ' '; $A$ lab split =' '; Phase=2

Technetium-99 $\quad$ pCi/g $\quad 1.9300$

Location=ORR; Formation=CHICKAMAUGA K-25; Site=127; Sample type=Regular; $A=4290 ; B=$ ' ; $C=$ ' '; $A$ lab split=' '; Phase =2

$\begin{array}{lll}\text { Technetiun-99 } & \mathrm{pCi} / \mathrm{g} \quad 0.9960 \quad \mathrm{U}\end{array}$

Location=ORR; Formation $=$ CHICKAMAUGA K-25; Site $=128 ;$ Sample type $=$ Regular; $A=4291 ; B=$ ' ' $C=$ ' '; $A$ lab split=' '; Phase $=2$

Technetium-99 pCi/g $\quad 1.1600 \quad U$

Location=ORR; Formation=CHICKAMAUGA K-25; Site=129; Sample type=Regular; $A=4292 ; B=$ ' ' C=' '; $A$ lab split=' '; Phase=2

$\begin{array}{llll}\text { Technetium-99 } & \text { pCi/g } & 1.2400 & U\end{array}$ 
Table E1 (continued)

\begin{tabular}{|c|c|c|c|c|c|c|c|c|c|}
\hline Analysis & Units & $\begin{array}{c}\text { A horizon } \\
\text { Result }\end{array}$ & Qualifier & $\begin{array}{l}\text { B horizon } \\
\text { Result }\end{array}$ & Qualifier & $\begin{array}{c}C \text { horizon } \\
\text { Result }\end{array}$ & Qualifier & $\begin{array}{l}\text { A lab split } \\
\text { Result }\end{array}$ & Qualifier \\
\hline
\end{tabular}

Location=ORR; Formation=COPPER RIDGE; Site=45,60,75; Sample type=Regular; $A=5110 ; B=5113 ; C=5116 ; A$ lab split=' ';

Phase $=2$

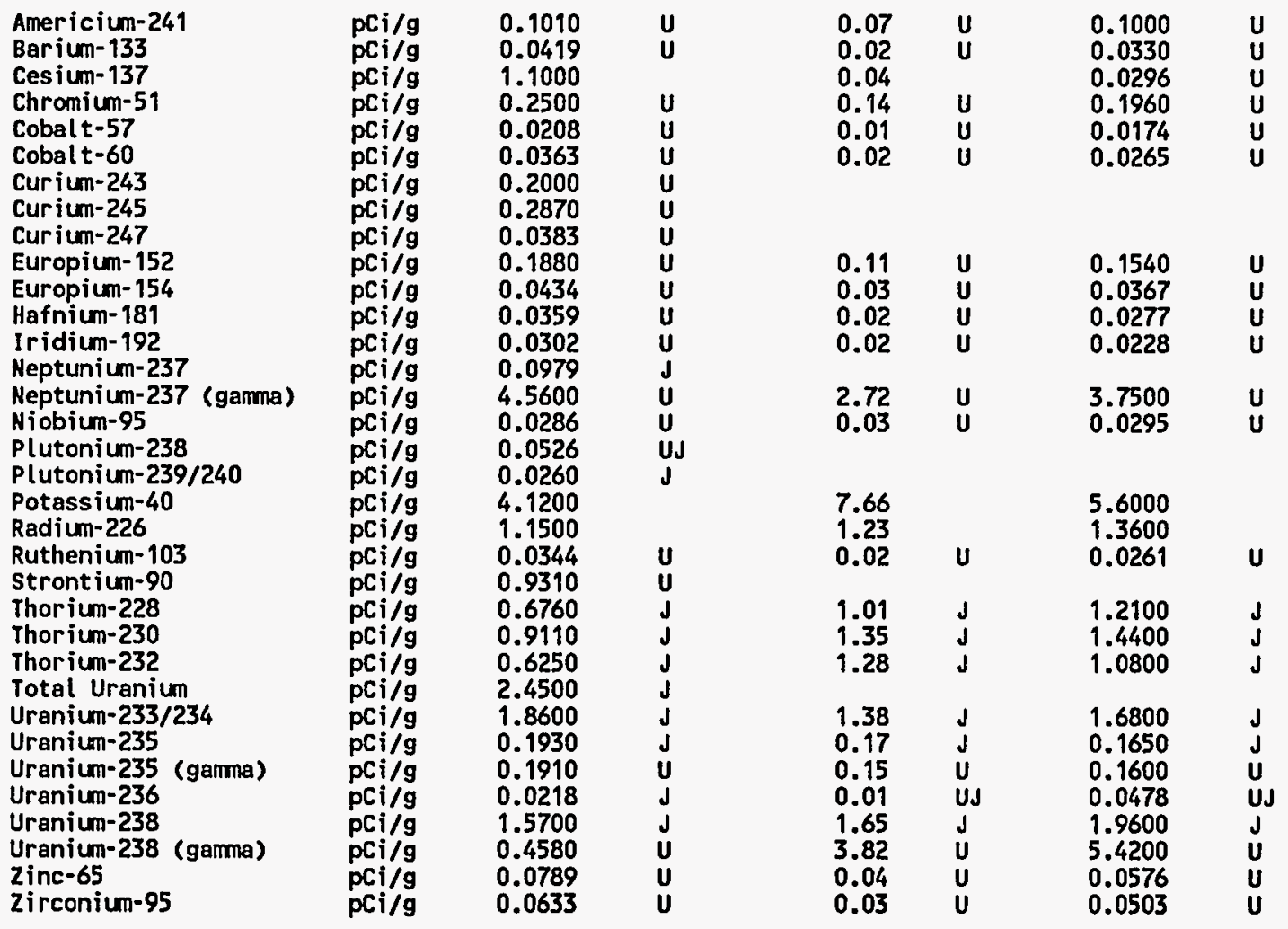




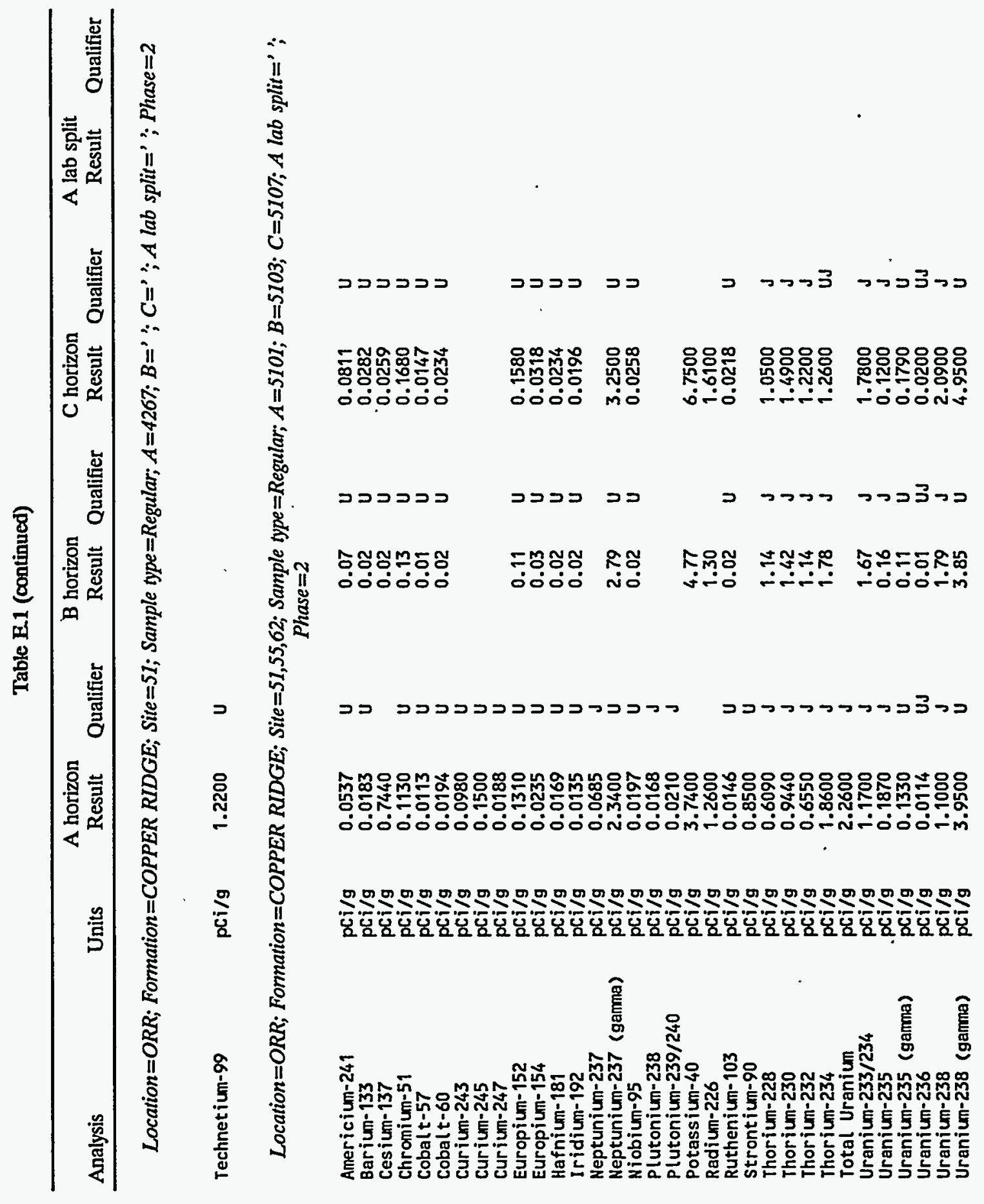


Table E1 (continued)

\begin{tabular}{|c|c|c|c|c|c|c|c|c|c|}
\hline Analysis & Units & $\begin{array}{c}\text { A horizon } \\
\text { Result }\end{array}$ & Qualifier & $\begin{array}{c}\text { B horizon } \\
\text { Result }\end{array}$ & Qualifier & $\begin{array}{c}C \text { horizon } \\
\text { Result }\end{array}$ & Qualifier & $\begin{array}{c}\text { A lab split } \\
\text { Result }\end{array}$ & Qualifier \\
\hline $\begin{array}{l}\text { Zinc-65 } \\
\text { Zirconium-95 }\end{array}$ & $\begin{array}{l}\mathrm{pCi} / \mathrm{g} \\
\mathrm{pCi} / \mathrm{g}\end{array}$ & $\begin{array}{l}0.0399 \\
0.0315\end{array}$ & $\begin{array}{l}U \\
U\end{array}$ & $\begin{array}{l}0.04 \\
0.03\end{array}$ & 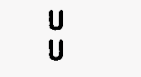 & $\begin{array}{l}0.0522 \\
0.0426\end{array}$ & $\begin{array}{l}\mathbf{U} \\
\mathbf{U}\end{array}$ & & \\
\hline
\end{tabular}

Location=ORR; Formation=COPPER RIDGE; Site=54; Sample type=Regular; $A=4268 ; B=$ ' '; $C=$ ' ' $A$ lab split=' '; Phase =2

Technetium-99 pci/g $\quad 1.0200 \quad U$

Location=ORR; Formation=COPPER RIDGE; Site=54,64,83; Sample type=Regular; $A=5119 ; B=5122 ; C=5125 ; A$ lab split $=$ ' '; Phase $=2$

$\begin{array}{lll}\text { Americium-241 } & \text { pCi/g } & 0.0889 \\ \text { Barium-133 } & \text { pCi/g } & 0.0318 \\ \text { Cesium-137 } & \text { pCi/g } & 0.9320 \\ \text { Chromium-51 } & p C i / g & 0.1900 \\ \text { Cobalt-57 } & \mathrm{pCi/g} & 0.0167 \\ \text { Cobalt-60 } & \mathrm{pCi/g} & 0.0259 \\ \text { Curium-243 } & \mathrm{pCi/g} & 0.1550 \\ \text { Curium-245 } & \mathrm{pCi/g} & 0.2190 \\ \text { Curium-247 } & \mathrm{pCi/g} & 0.0287 \\ \text { Europium-152 } & \mathrm{pCi/g} & 0.1630 \\ \text { Europium-154 } & \mathrm{pCi/g} & 0.0354 \\ \text { Hafnium-181 } & \mathrm{pCi/g} & 0.0257 \\ \text { Iridium-192 } & \mathrm{pCi/g} & 0.0229 \\ \text { Neptunium-237 } & \mathrm{pCi/g} & 0.0672 \\ \text { Neptunium-237 (gamma) } & \mathrm{pCi/g} & 3.7600 \\ \text { Niobium-95 } & \mathrm{pCi/g} & 0.0313 \\ \text { Plutonium-238 } & \mathrm{pCi/g} & 0.0298 \\ \text { Plutonium-239/240 } & \mathrm{pCi/g} & 0.0397 \\ \text { Potassium-40 } & \mathrm{pCi/g} & 4.2600 \\ \text { Radium-226 } & \mathrm{pCi/g} & 1.3700 \\ \text { Ruthenium-103 } & \mathrm{pCi/g} & 0.0244 \\ \text { Strontium-90 } & \mathrm{pCi/g} & 1.0500 \\ \text { Thorium-228 } & \mathrm{pCi/g} & 0.7860 \\ \text { Thorium-230 } & \mathrm{pCi/g} & 1.1900 \\ \text { Thorium-232 } & \mathrm{pCi/g} & 0.7730 \\ \text { Thorium-234 } & \mathrm{pCi/g} & 2.1800 \\ \text { Total Uranium } & \mathrm{pCi/g} & 3.3000 \\ \text { Uranium-233/234 } & \mathrm{pCi/g} & 1.4000\end{array}$

$\begin{array}{llll}0.07 & U & 0.0980 & U \\ 0.02 & U & 0.0377 & U \\ 0.02 & U & 0.0356 & U \\ 0.15 & U & 0.2320 & U \\ 0.01 & U & 0.0199 & U \\ 0.02 & U & 0.0338 & U \\ & & & \\ 0.14 & U & 0.1940 & U \\ 0.03 & U & 0.0415 & U \\ 0.02 & U & 0.0323 & U \\ 0.02 & U & 0.0262 & U \\ 0.03 & U & 4.3100 & U \\ 3.03 & U & 0.0372 & U \\ & & & \\ 7.94 & & 5.2800 & \\ 1.74 & & 1.5600 & \\ 0.02 & U & 0.0298 & U \\ 1.24 & J & 1.1000 & J \\ 1.72 & J & 1.7500 & J \\ 1.11 & J & 1.1800 & J \\ 2.31 & J & 1.1300 & U J \\ 2.02 & J & 1.7500 & J\end{array}$


Table E1 (continued)

\begin{tabular}{|c|c|c|c|c|c|c|c|c|c|}
\hline Analysis & Units & $\begin{array}{c}\text { A horizon } \\
\text { Result }\end{array}$ & Qualifier & $\begin{array}{c}\text { B horizon } \\
\text { Result }\end{array}$ & Qualifier & $\begin{array}{c}\mathbf{C} \text { horizon } \\
\text { Result }\end{array}$ & Qualifier & $\begin{array}{l}\text { A lab split } \\
\text { Result }\end{array}$ & Qualifier \\
\hline $\begin{array}{l}\text { Uranium-235 } \\
\text { Uranium-235 (gamma) } \\
\text { Uranium-236 } \\
\text { Uranium-238 } \\
\text { Uranium-238 (gamma) } \\
\text { Zinc-65 } \\
\text { Zirconium-95 }\end{array}$ & $\begin{array}{l}\mathrm{pCj} / \mathrm{g} \\
\mathrm{pCi} / \mathrm{g} \\
\mathrm{pCi} / \mathrm{g} \\
\mathrm{pCi} / \mathrm{g} \\
\mathrm{pCj} / \mathrm{g} \\
\mathrm{pCi} / \mathrm{g} \\
\mathrm{pCi} / \mathrm{g}\end{array}$ & $\begin{array}{l}0.0562 \\
0.1400 \\
0.0093 \\
1.4600 \\
5.4100 \\
0.0600 \\
0.0434\end{array}$ & $\begin{array}{l}J \\
U \\
U J \\
J \\
U \\
U \\
U\end{array}$ & $\begin{array}{l}0.11 \\
0.16 \\
0.01 \\
2.09 \\
4.91 \\
0.05 \\
0.04\end{array}$ & $\begin{array}{l}J \\
U \\
U J J \\
J \\
U \\
U \\
U\end{array}$ & $\begin{array}{l}0.2130 \\
0.2730 \\
0.0312 \\
1.7600 \\
6.3700 \\
0.0730 \\
0.0587\end{array}$ & $\begin{array}{l}J \\
U J \\
J \\
U \\
U \\
U\end{array}$ & & \\
\hline
\end{tabular}

Location=ORR; Formation=COPPER RIDGE; Site=58,59,91; Sample type=Regular; $A=5092 ; B=5095 ; C=5098 ; A$ lab split=' '; Phase $=2$

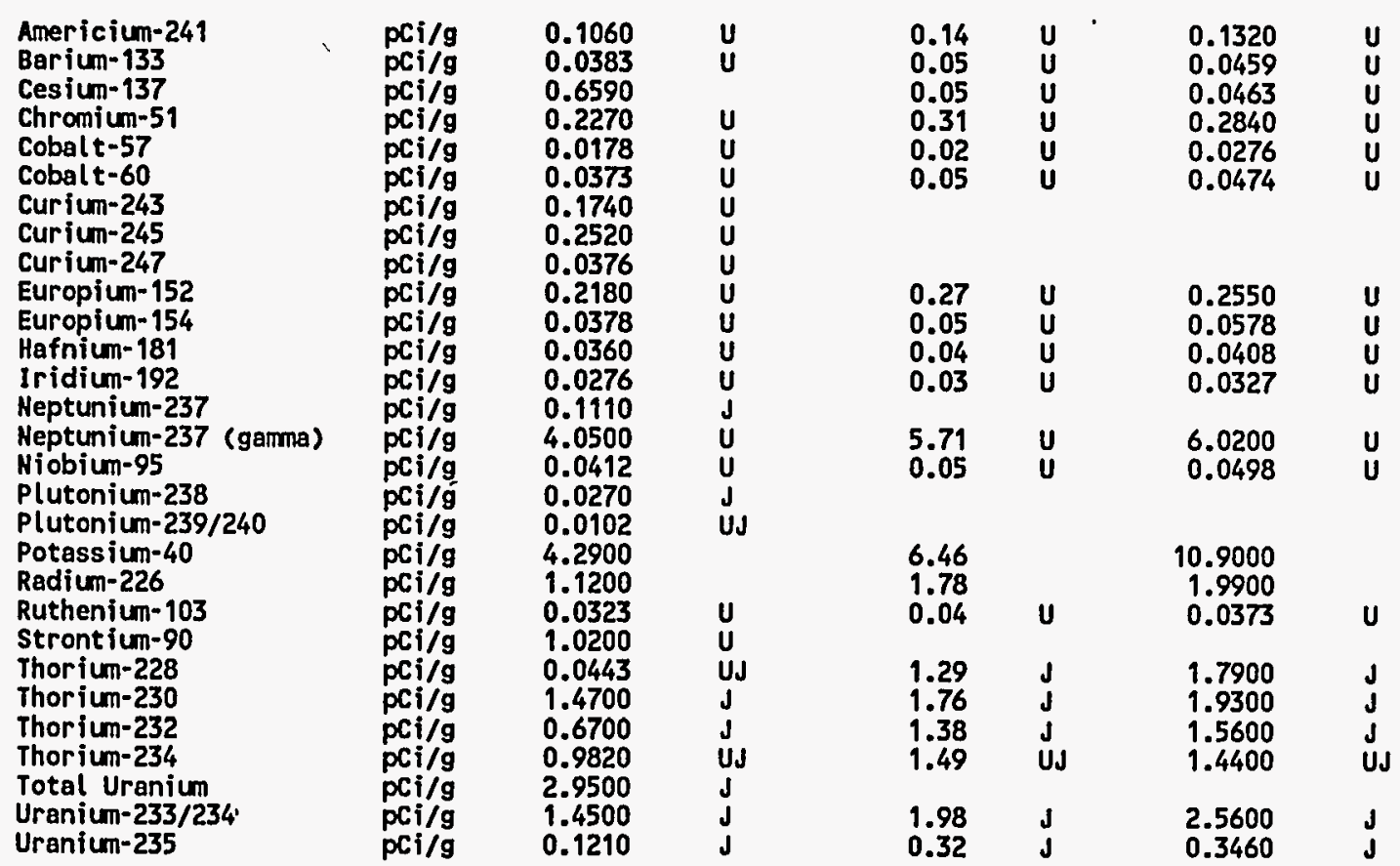


Table E.1 (continued)

\begin{tabular}{|c|c|c|c|c|c|c|c|c|c|}
\hline Analysis & Units & $\begin{array}{c}\text { A horizon } \\
\text { Result }\end{array}$ & Qualifier & $\begin{array}{l}\text { B horizon } \\
\text { Result }\end{array}$ & Qualifier & $\begin{array}{c}C \text { horizon } \\
\text { Result }\end{array}$ & Qualifier & $\begin{array}{c}\text { A lab split } \\
\text { Result }\end{array}$ & Qualifier \\
\hline $\begin{array}{l}\text { Uranium-235 (gamma) } \\
\text { Uranium-236 } \\
\text { Uranium-238 } \\
\text { Uranium-238 (gamma) } \\
\text { Zinc- } 65 \\
\text { Zirconium-95 }\end{array}$ & $\begin{array}{l}\mathrm{pCi} / \mathrm{g} \\
\mathrm{pCi} / \mathrm{g} \\
\mathrm{pCi} / \mathrm{g} \\
\mathrm{pCi} / \mathrm{g} \\
\mathrm{pCi} / \mathrm{g} \\
\mathrm{pCi} / \mathrm{g}\end{array}$ & $\begin{array}{l}0.1620 \\
0.0302 \\
1.4400 \\
6.5400 \\
0.0767 \\
0.0633\end{array}$ & $\begin{array}{l}\mathbf{U} \\
\mathbf{U J} \\
\mathbf{J} \\
\mathbf{U} \\
\mathbf{U} \\
\mathbf{U}\end{array}$ & $\begin{array}{l}0.24 \\
0.03 \\
1.84 \\
8.17 \\
0.10 \\
0.08\end{array}$ & $\begin{array}{l}U \\
U J \\
J \\
U \\
U \\
U\end{array}$ & $\begin{array}{l}0.2370 \\
0.0431 \\
2.7100 \\
7.2200 \\
0.0942 \\
0.0781\end{array}$ & $\begin{array}{l}U \\
U J \\
J \\
U \\
U \\
U\end{array}$ & & \\
\hline
\end{tabular}

Location=ORR; Formation=COPPER RIDGE; Site=60; Sample type=Regular; $A=4269 ; B=$ ' ; $C=$ '; $A$ lab split=' '; Phase=2

Technetium-99 $\quad$ pci/g $\quad 1.0600 \quad$ U

Location=ORR; Formation=COPPER RIDGE; Site=62; Sample type=Regular; $A=4270 ; B=$ ' ' $C=$ ' '; lab split=' '; Phase =2

Technetium-99 pci/g $\quad 1.5400 \quad U$

Location=ORR; Formation=COPPER RIDGE; Site=64; Sample type=Regular; $A=4271 ; B=$ ' ; $C=$ ' '; lab split=' '; Phase=2

Technetium-99 $\quad \mathrm{pCi} / \mathrm{g} \quad 1.6400 \quad \mathrm{U}$

Location=ORR; Formation=COPPER RIDGE; Site=74; Sample type=Regular; $A=4275 ; B=$ ' '; $C=$ ' '; $A$ lab split=' '; Phase $=2$

Technetium-99 $\quad$ pCi/g $\quad 1.2300 \quad U$ 


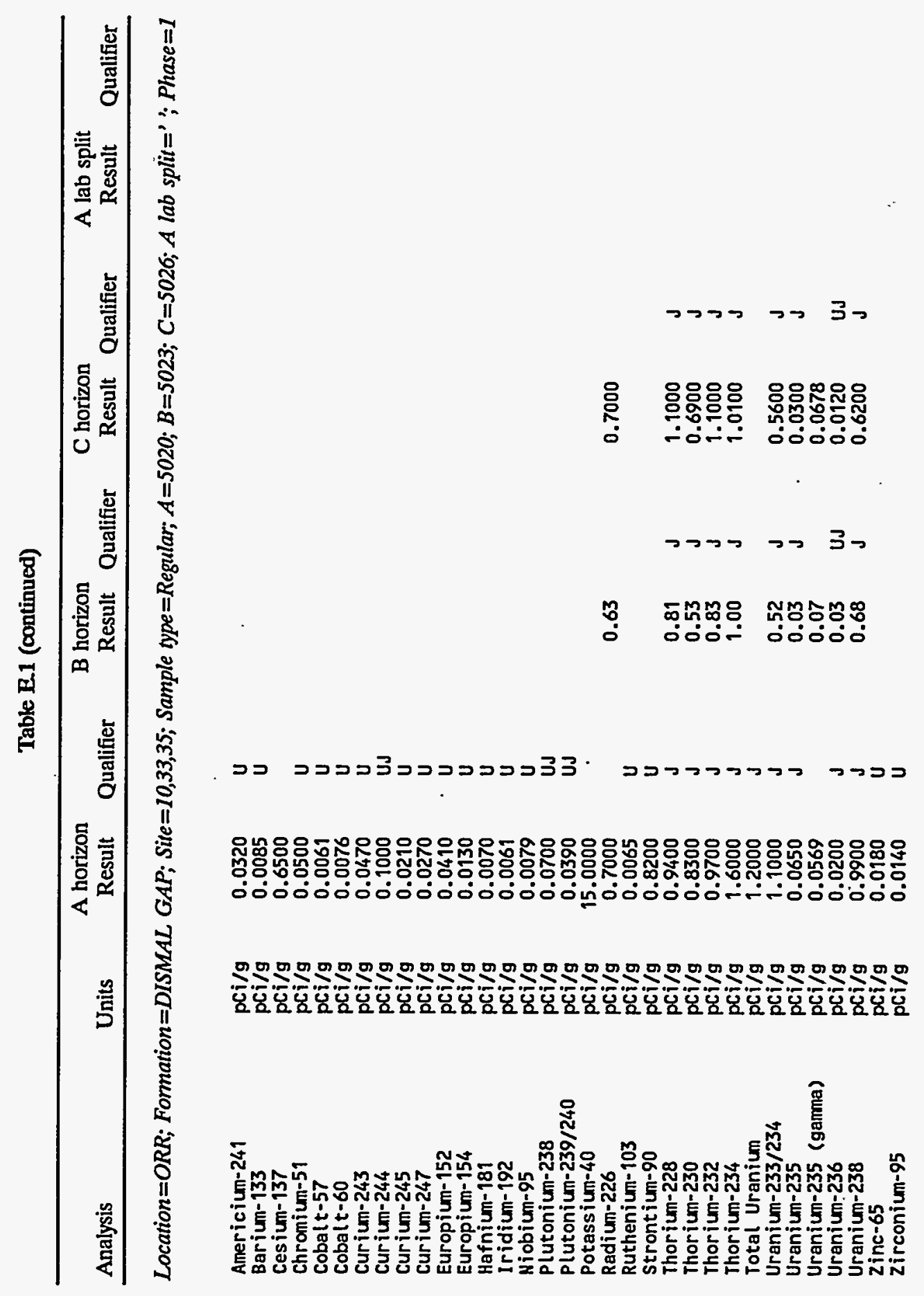


Table E.1 (continued)

\begin{tabular}{|c|c|c|c|c|c|c|c|c|c|}
\hline & & A horizon & & B horizon & & $\mathrm{C}$ horizon & & A lab split & \\
\hline Analysis & Units & Result & Qualifier & Result & Qualifier & Result & Qualifier & Result & Qualifier \\
\hline
\end{tabular}

Location=ORR; Formation=DISMAL GAP; Site =11,27,41; Sample type =Regular; $A=5002 ; B=5005 ; C=5008 ; A$ lab split $=$ ' ; Phase $=1$

\begin{tabular}{|c|c|c|c|c|c|c|}
\hline $\begin{array}{l}\text { Americium-241 } \\
\text { Barium-133 } \\
\text { Cesium-137 } \\
\text { Chromium-51 } \\
\text { Cobalt-57 } \\
\text { Cobalt-60 } \\
\text { Curium-243 } \\
\text { Curium-244 } \\
\text { Curium-245 } \\
\text { Curium-247 } \\
\text { Europium-152 } \\
\text { Europium-154 } \\
\text { Hafnium-181 } \\
\text { Iridium-192 } \\
\text { Miobium-95 } \\
\text { Plutonium-238 } \\
\text { Plutonium-239/240 } \\
\text { Potassium-40 } \\
\text { Radium-226 } \\
\text { Ruthenium-103 } \\
\text { Strontium-90 } \\
\text { Thorium-228 } \\
\text { Thorium-230 } \\
\text { Thorium-232 } \\
\text { Thorium-234 } \\
\text { Total Uranium } \\
\text { Uranium-233/234 } \\
\text { Uranium-235 } \\
\text { Uranium-235 (gamma) } \\
\text { Uranium-236 } \\
\text { Uranium-238 } \\
\text { Zinc-65 } \\
\text { Zirconium-95 }\end{array}$ & $\begin{array}{l}\mathrm{pCi} / \mathrm{g} \\
\mathrm{pCi} / \mathrm{g} \\
\mathrm{pCi} / \mathrm{g} \\
\mathrm{pCi} / \mathrm{g} \\
\mathrm{pCi} / \mathrm{g} \\
\mathrm{pCi} / \mathrm{g} \\
\mathrm{pCi} / \mathrm{g} \\
\mathrm{pCi} / \mathrm{g} \\
\mathrm{pCi} / \mathrm{g} \\
\mathrm{pCi} / \mathrm{g} \\
\mathrm{pCi} / \mathrm{g} \\
\mathrm{pCi} / \mathrm{g} \\
\mathrm{pCi} / \mathrm{g} \\
\mathrm{pCi} / \mathrm{g} \\
\mathrm{pCi} / \mathrm{g} \\
\mathrm{pCi} / \mathrm{g} \\
\mathrm{pCi} / \mathrm{g} \\
\mathrm{pCi} / \mathrm{g} \\
\mathrm{pCi} / \mathrm{g} \\
\mathrm{pCi} / \mathrm{g} \\
\mathrm{pCi} / \mathrm{g} \\
\mathrm{pCi} / \mathrm{g} \\
\mathrm{pCi} / \mathrm{g} \\
\mathrm{pCi} / \mathrm{g} \\
\mathrm{pCi} / \mathrm{g} \\
\mathrm{pCi} / \mathrm{g} \\
\mathrm{pCi} / \mathrm{g} \\
\mathrm{pCi} / \mathrm{g} \\
\mathrm{pCi} / \mathrm{g} \\
\mathrm{pCi} / \mathrm{g} \\
\mathrm{pCi} / \mathrm{g} \\
\mathrm{pCi} / \mathrm{g} \\
\mathrm{pCi} / \mathrm{g}\end{array}$ & $\begin{array}{r}0.0510 \\
0.0140 \\
0.9000 \\
0.0870 \\
0.0084 \\
0.0120 \\
0.0750 \\
3.5000 \\
0.1100 \\
0.0140 \\
0.0660 \\
0.0180 \\
0.0120 \\
0.0100 \\
0.0140 \\
0.1100 \\
0.0290 \\
14.0000 \\
0.8600 \\
0.0110 \\
1.1000 \\
0.5000 \\
0.3100 \\
0.4100 \\
1.5000 \\
2.5000 \\
1.0000 \\
0.0410 \\
0.1198 \\
0.0330 \\
1.1000 \\
0.0290 \\
0.0220\end{array}$ & $\begin{array}{l}U \\
U \\
U \\
U \\
U J \\
U \\
U \\
U \\
U \\
U \\
U \\
U \\
U \\
U \\
U \\
U J \\
J\end{array}$ & $\begin{array}{l}0.04 \\
0.01 \\
0.03 \\
0.07 \\
0.01 \\
0.01 \\
0.06 \\
0.11 \\
0.01 \\
0.06 \\
0.02 \\
0.01 \\
0.01 \\
0.01 \\
0.08 \\
0.03 \\
22.00 \\
0.92 \\
0.01 \\
1.00 \\
3.40 \\
2.30 \\
3.20 \\
1.10 \\
0.12 \\
0.74 \\
0.07 \\
0.07 \\
0.06 \\
0.63 \\
0.03 \\
0.02\end{array}$ & $\begin{array}{l}\text { U } \\
\text { NA } \\
J \\
J \\
J \\
J \\
J \\
J \\
\text { UJ }\end{array}$ & $\begin{array}{r}0.1100 \\
0.0100 \\
0.0570 \\
0.0180 \\
0.0095 \\
0.0080 \\
0.0110 \\
0.0910 \\
0.0210 \\
28.0000 \\
0.7900 \\
0.0087 \\
0.9600 \\
2.0000 \\
0.9400 \\
2.3000 \\
1.2000 \\
0.0690 \\
0.5400 \\
0.0390 \\
0.0527 \\
0.0140 \\
0.5300 \\
0.0270 \\
0.0200\end{array}$ \\
\hline
\end{tabular}


Table E1 (continued)

\begin{tabular}{lcccccc}
\hline & A horizon & B horizon & C horizon & A lab split \\
Analysis & Units & Result & Qualifier & Result & Qualifier & Result \\
Qualifier & Result & Qualifier \\
\hline
\end{tabular}

Location=ORR; Formation=DISMAL GAP; Site=19,22,32; Sample type=Regular; $A=5011 ; B=5014 ; C=5017 ; A$ lab split =' '; Phase =I

Americium-241

Barium-133

Cesium-137

Cobal t-57

Cobal $t-60$

Curium-243

Curium-244

Cur ium-245

Curium-247

Europium-152

Europium-154

Iridium-192

Niobium-95

Plutonium-238

Plutonium-239/240

Potassium- 40

Radium-226

Ruthenium-103

Stront ium: 90

Thor ium-228

Thorium-230

Thor ium-232

Thor ium-234

Total Uranium

Uranium-233/234

Uranium-235

Uranium-235 (gamma)

Uranium-236

Uranium-238

Zinc-65

Zirconium-95

\begin{tabular}{|c|c|}
\hline $\begin{array}{l}\mathrm{pCi} / \mathrm{g} \\
\mathrm{pCi} / \mathrm{g} \\
\mathrm{pCi} / \mathrm{g} \\
\mathrm{pCi} / \mathrm{g} \\
\mathrm{pCi} / \mathrm{g} \\
\mathrm{pCi} / \mathrm{g} \\
\mathrm{pCi} / \mathrm{g} \\
\mathrm{pCi} / \mathrm{g} \\
\mathrm{pCi} / \mathrm{g} \\
\mathrm{pCi} / \mathrm{g} \\
\mathrm{pCi} / \mathrm{g} \\
\mathrm{pCi} / \mathrm{g} \\
\mathrm{pCi} / \mathrm{g} \\
\mathrm{pCi} / \mathrm{g} \\
\mathrm{pCi} / \mathrm{g} \\
\mathrm{pCi} / \mathrm{g} \\
\mathrm{pCi} / \mathrm{g} \\
\mathrm{pCi} / \mathrm{g} \\
\mathrm{pCi} \mathrm{g} \\
\mathrm{pCi} \mathrm{g} \\
\mathrm{pCi} / \mathrm{g} \\
\mathrm{pCi} / \mathrm{g} \\
\mathrm{pCi} \mathrm{g} \\
\mathrm{pCi} / \mathrm{g} \\
\mathrm{pCi} \\
\mathrm{pCi} / \mathrm{g} \\
\mathrm{pCi} \\
\mathrm{pCi} \\
\mathrm{pCi} / \mathrm{g} \\
\mathrm{pCi} \\
\mathrm{pCi} / \mathrm{g} \\
\mathrm{pCi} / \mathrm{g} \\
\mathrm{pCi} / \mathrm{g}\end{array}$ & $\begin{array}{r}0.0400 \\
0.0110 \\
0.5400 \\
0.0660 \\
0.0077 \\
0.0098 \\
0.0600 \\
0.0870 \\
0.1000 \\
0.0100 \\
0.0540 \\
0.0160 \\
0.0091 \\
0.0076 \\
0.0110 \\
0.0690 \\
0.0300 \\
17.0000 \\
0.8200 \\
0.0083 \\
0.8800 \\
0.7700 \\
0.6500 \\
0.7300 \\
1.7000 \\
0.2300 \\
0.6100 \\
0.0540 \\
0.0698 \\
0.0350 \\
0.7500 \\
0.0230 \\
0.0170\end{array}$ \\
\hline
\end{tabular}

$\begin{array}{ll}0.01 & U \\ 0.01 & U \\ 0.01 & U \\ 0.08 & U \\ 0.00 & U \\ 0.01 & U \\ 0.04 & U \\ & \\ 0.06 & U \\ 0.01 & U \\ 0.04 & U \\ 0.01 & U \\ 0.01 & U \\ 0.01 & U \\ 82000.00 & U \\ 0.10 & J \\ 0.03 & J \\ 0.19 & U \\ 1.10 & U \\ 0.01 & U \\ 1.00 & N A \\ 0.53 & J \\ 0.36 & J \\ 0.44 & J \\ 0.23 & U \\ 0.83 & J \\ 3.20 & J \\ 0.24 & J \\ 0.17 & J \\ 2.60 & J \\ 0.02 & U \\ 0.01 & U \\ & \end{array}$

$\begin{array}{ll}0.0430 & U \\ 0.0110 & U \\ 0.0260 & \\ 0.0710 & U \\ 0.0084 & U \\ 0.0120 & U \\ 0.0640 & U \\ & \\ 0.1100 & U \\ 0.0110 & U \\ 0.0570 & U \\ 0.0180 & U \\ 0.0096 & U \\ 0.0081 & U \\ 0.0120 & U \\ 0.0700 & U J \\ 0.0200 & U J \\ 22.0000 & \\ 0.7700 & \\ 0.0089 & U \\ 1.0000 & \text { NA } \\ 0.0710 & U J \\ 0.1900 & J \\ 0.1800 & J \\ 1.2000 & \\ 1.3000 & J \\ 0.5800 & J \\ 0.0500 & J \\ 0.0244 & \\ 0.0130 & \text { UJ } \\ 0.5800 & J \\ 0.0260 & U \\ 0.0200 & U\end{array}$


Table E.1 (continued)

\begin{tabular}{|c|c|c|c|c|c|c|c|c|c|}
\hline Analysis & Units & $\begin{array}{l}\text { A horizon } \\
\text { Result }\end{array}$ & Qualifier & $\begin{array}{c}\text { B horizon } \\
\text { Result }\end{array}$ & Qualifier & $\begin{array}{l}C \text { horizon } \\
\text { Result }\end{array}$ & Qualifier & $\begin{array}{l}\text { A lab split } \\
\text { Result }\end{array}$ & Qualifier \\
\hline
\end{tabular}

Location=ORR; Formation=DISMAL GAP; Site=2; Sample type=Regular; $A=4255 ; B=$ '; $C=$ ' '; $A$ lab split $=$ ' '; Phase $=2$

Technetium-99 $\quad \mathrm{pCi} / \mathrm{g} \quad 1.7500 \quad$ U

Location=ORR; Formation=DISMAL GAP; Site=2,26,43; Sample type=Regular; $A=5029 ; B=5032 ; C=5035 ; A$ lab split $=$ ' ; Phase $=1$

\begin{tabular}{|c|c|c|c|c|c|c|}
\hline $\begin{array}{l}\text { Americium-241 } \\
\text { Barium-133 } \\
\text { Cesium-137 } \\
\text { Chromium-51 } \\
\text { Cobalt-57 } \\
\text { Cobal }-60 \\
\text { Curium-243 } \\
\text { Curium-244 } \\
\text { Curium-245 } \\
\text { Curium-247 } \\
\text { Europium-152 } \\
\text { Europium-154 } \\
\text { Hafnium-181 } \\
\text { Iridium-192 } \\
\text { Niobium-95 } \\
\text { Plutonium-238 } \\
\text { Plutonium-239/240 } \\
\text { Potassium-40 } \\
\text { Radium-226 } \\
\text { Ruthenium-103 } \\
\text { Strontium-90 } \\
\text { Thorium-228 } \\
\text { Thorium-230 } \\
\text { Thorium-232 } \\
\text { Thorium-234 } \\
\text { Total Uranium } \\
\text { Uranium-233/234 } \\
\text { Uranium-235 } \\
\text { Uranium-235 (gamma) } \\
\text { Uranium-236 } \\
\text { Uranium-238 } \\
\text { Zinc-65 } \\
\text { Zirconium-95 }\end{array}$ & 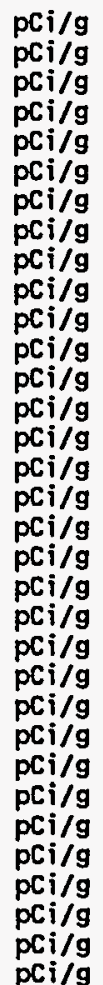 & $\begin{array}{r}0.0380 \\
0.0097 \\
0.7900 \\
0.0590 \\
0.0070 \\
0.0093 \\
0.0530 \\
1.9000 \\
0.0930 \\
0.0088 \\
0.0460 \\
0.0150 \\
0.0082 \\
0.0068 \\
0.0091 \\
0.0830 \\
0.0190 \\
18.0000 \\
0.7000 \\
0.0075 \\
0.9900 \\
0.7800 \\
0.7000 \\
0.7300 \\
1.9000 \\
6.5000 \\
1.4000 \\
0.1500 \\
0.0861 \\
0.0360 \\
1.7000 \\
0.0210 \\
0.0160\end{array}$ & $\begin{array}{l}\mathbf{U} \\
\mathbf{U} \\
\mathbf{U} \\
\mathbf{U} \\
\mathbf{U} \mathbf{J} \\
\mathbf{U} \\
\mathbf{U} \\
\mathbf{U} \\
\mathbf{U} \\
\mathbf{U} \\
\mathbf{U} \\
\mathbf{U} \\
\mathbf{J} \\
\mathbf{U} J\end{array}$ & $\begin{array}{r}16.00 \\
0.81 \\
0.01 \\
\\
0.85 \\
0.64 \\
0.99 \\
1.80\end{array}$ & $\begin{array}{l}U \\
U \\
U \\
U \\
U\end{array}$ & $\begin{array}{r}12.0000 \\
0.7700 \\
0.0077 \\
\\
1.2000 \\
0.8700 \\
1.1000 \\
1.3000\end{array}$ \\
\hline
\end{tabular}


Table E1 (continued)

\begin{tabular}{lcccccc}
\hline & A horizon & & B horizon & C horizon & A lab split \\
Analysis & Units & Result & Qualifier & Result & Qualifier & Result \\
Qualifier & Result & Qualifier \\
\hline
\end{tabular}

Location=ORR; Formation=DISMAL GAP; Site=2,26,43; Sample type=Field Duplicate; $A=5038 ; B=5041 ; C=5044 ; A$ lab split=' '; Phase $=1$

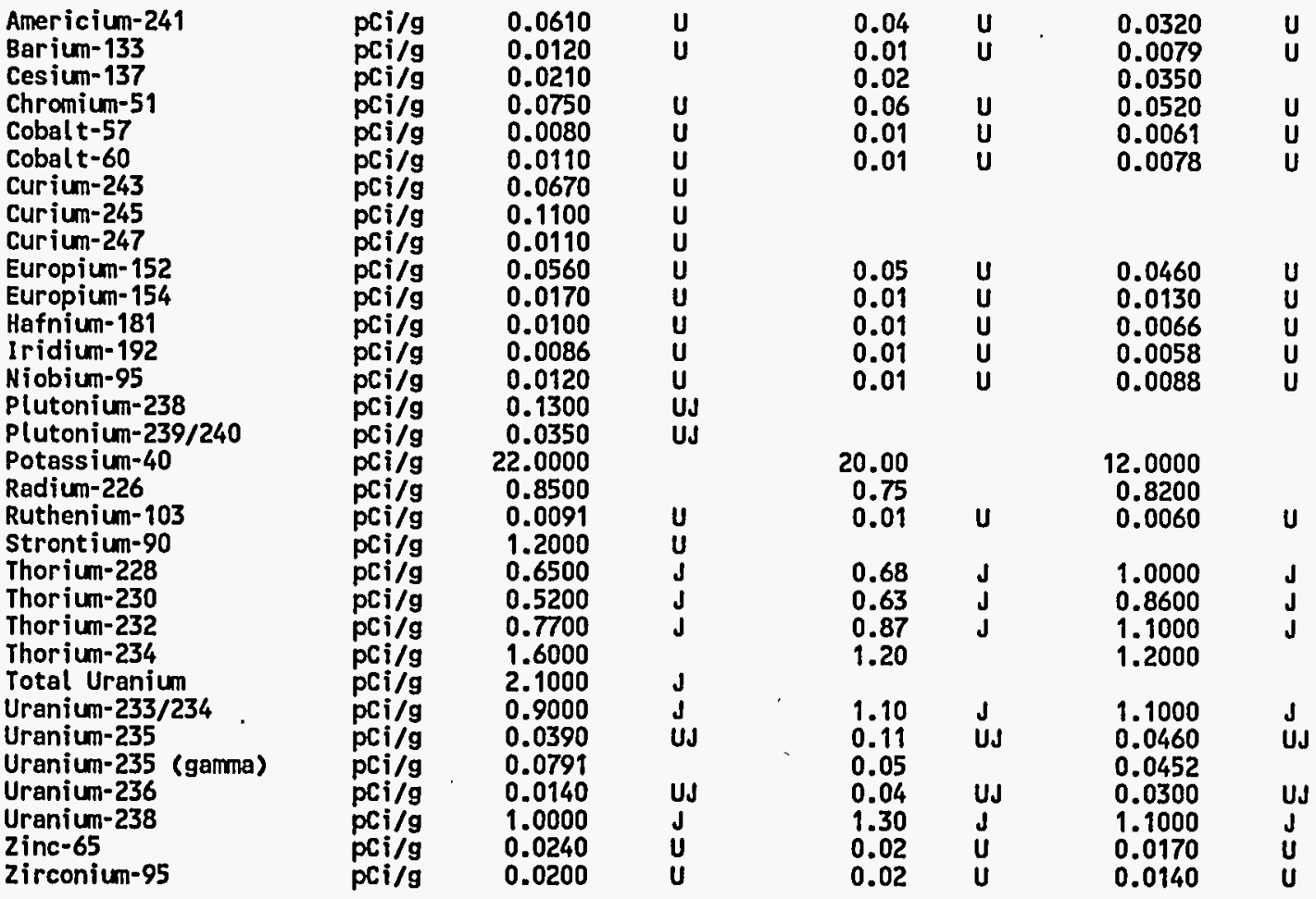

Location=ORR; Formation=DISMAL GAP; Site=26; Sample type =Regular; $A=4260 ; B=$ ' ; $C=$ ' ' $A$ lab split=' '; Phase=2

$\begin{array}{lll}\text { Technetium- } 99 & \text { pCi } / g & 1.8000\end{array}$ 
E-38

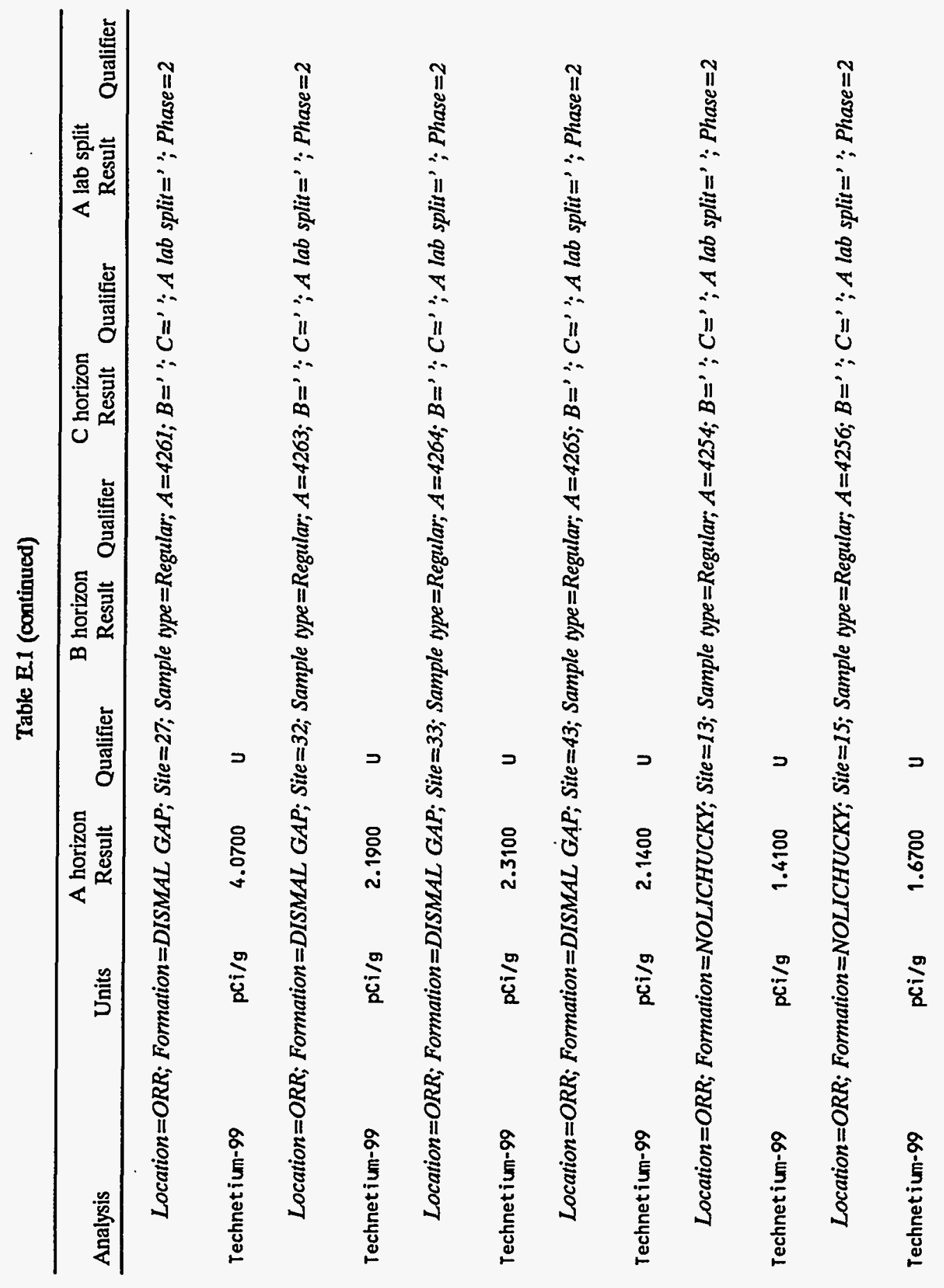


Table E.1 (continued)

\begin{tabular}{|c|c|c|c|c|c|c|c|c|c|}
\hline Analysis & Units & $\begin{array}{c}\text { A horizon } \\
\text { Result }\end{array}$ & Qualifier & $\begin{array}{l}\text { B horizon } \\
\text { Result }\end{array}$ & Qualifier & $\begin{array}{c}C \text { horizon } \\
\text { Result }\end{array}$ & Qualifier & $\begin{array}{c}\text { A lab split } \\
\text { Result }\end{array}$ & Qualifier \\
\hline
\end{tabular}

Location=ORR; Formation=NOLICHUCKY; Site=15,23,25; Sample type=Regular; $A=5056 ; B=5059 ; C=5062 ; A$ lab split =' '; Phase $=1$

Americium-241
Barium-133
Cesium-137
Chromium-51
Cobalt -57
Cobalt-60
Curium-243
Curium-245
Curium-247
Europium-152
Europium-154
P(utonium-238
Plutonium-239/240
Potassium-40
Radium-226
Ruthenium-103
Strontium-90
Thorium-228
Thorium-230
Thorium-232
Thorium-234
Total Uranium
Uranium-233/234
Uranium-235
Uranium-235 (gamma)
Uranium-236
Uranium-238
2inc-65
Zirconium-95
Tium 95

\begin{tabular}{|c|c|}
\hline $\begin{array}{l}p C i / g \\
p C i / g \\
p C i / g \\
p C i / g \\
p C i / g \\
p C i / g \\
p C i / g \\
p C i / g \\
p C i / g \\
p C i / g \\
p C i / g \\
p C i / g \\
p C i / g \\
p C i / g \\
p C i / g \\
p C i / g \\
p C i / g \\
p C i / g \\
p C i / g \\
p C i / g \\
p C i / g \\
p C i / g \\
p C i / g \\
p C i / g \\
p C i / g \\
p C i / g \\
p C i / g \\
p C i / g \\
p C i / g\end{array}$ & $\begin{array}{r}0.0240 \\
0.0064 \\
0.3800 \\
0.0390 \\
0.0041 \\
0.0055 \\
0.0350 \\
0.0560 \\
0.0060 \\
0.0350 \\
0.0088 \\
0.1200 \\
0.0280 \\
14.0000 \\
0.8200 \\
0.0050 \\
0.6300 \\
2.2000 \\
1.2000 \\
2.0000 \\
1.4000 \\
0.7500 \\
2.9000 \\
0.1300 \\
0.0684 \\
0.0200 \\
3.5000 \\
0.0120 \\
0.0100\end{array}$ \\
\hline
\end{tabular}

$\begin{array}{llll}0.05 & U & 0.0380 & U \\ 0.01 & U & 0.0098 & U \\ 0.01 & & 0.0098 & U \\ 0.08 & U & 0.0600 & U \\ 0.01 & U & 0.0074 & U \\ 0.01 & U & 0.0099 & U \\ & & & \\ & & & \\ 0.06 & U & 0.0460 & U \\ 0.02 & U & 0.0160 & U \\ & & & \\ 18.00 & & 28.0000 & \\ 1.10 & & 0.7800 & \\ 0.01 & U & 0.0074 & U \\ & & & \\ 1.80 & J & 1.5000 & J \\ 0.95 & J & 0.7000 & J \\ 1.70 & J & 1.4000 & J \\ 1.20 & & 1.1000 & \\ 2.30 & \text { UJ } & 2.3000 & U J \\ 0.09 & U J & 0.0670 & U J \\ 0.05 & & 0.0605 & \\ 0.01 & U J & 0.0270 & U J \\ 2.40 & U J & 2.4000 & U J \\ 0.03 & U & 0.0220 & U \\ 0.02 & U & 0.0180 & U \\ & & & \end{array}$


Table E.1 (continued)

\begin{tabular}{|c|c|c|c|c|c|c|c|c|c|}
\hline Analysis & Units & $\begin{array}{c}\text { A horizon } \\
\text { Result }\end{array}$ & Qualifier & $\begin{array}{c}\text { B horizon } \\
\text { Result }\end{array}$ & Qualifier & $\begin{array}{l}C \text { horizon } \\
\text { Result }\end{array}$ & Qualifier & $\begin{array}{l}\text { A lab split } \\
\text { Result }\end{array}$ & Qualifier \\
\hline
\end{tabular}

Location=ORR; Formation=NOLICHUCKY; Site=16,28,42; Sample type=Regular; $A=5065 ; B=5068 ; C=5071 ; A$ lab split=' '; Phase $=1$

\begin{tabular}{|c|c|c|c|c|c|c|}
\hline $\begin{array}{l}\text { Americium-241 } \\
\text { Barium-133 } \\
\text { Cesium-137 } \\
\text { Chromium-51 } \\
\text { Cobalt-57 } \\
\text { Cobalt-60 } \\
\text { Curium-243 } \\
\text { Curium-245 } \\
\text { Curium-247 } \\
\text { Europium-152 } \\
\text { Europium-154 } \\
\text { Neptunium-237 } \\
\text { Plutonium-238 } \\
\text { Plutonium-239/240 } \\
\text { Potassium-40 } \\
\text { Radium-226 } \\
\text { Ruthenium-103 } \\
\text { Strontium-90 } \\
\text { Thorium-228 } \\
\text { Thorium-230 } \\
\text { Thorium-232 } \\
\text { Thorium-234 } \\
\text { Total Uranium } \\
\text { Uranium-233/234 } \\
\text { Uranium-235 } \\
\text { Uranium-235 (gamma) } \\
\text { Uranium-236 } \\
\text { Uranium-238 } \\
\text { 2inc-65 } \\
\text { 2irconium-95 }\end{array}$ & 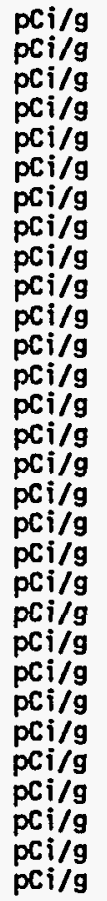 & $\begin{array}{r}0.0220 \\
0.0062 \\
0.5100 \\
0.0370 \\
0.0043 \\
0.0060 \\
0.0340 \\
0.0580 \\
0.0056 \\
0.0370 \\
0.0093 \\
0.0770 \\
0.1200 \\
0.0650 \\
16.0000 \\
0.6700 \\
0.0049 \\
0.7600 \\
1.2000 \\
0.8500 \\
1.3000 \\
1.5000 \\
1.5000 \\
2.4000 \\
0.1000 \\
0.0901 \\
0.0320 \\
2.5000 \\
0.0140 \\
0.0100\end{array}$ & $\begin{array}{l}U \\
U \\
U \\
U \\
U \\
U \\
U \\
U \\
U \\
U \\
U \\
U \\
J \\
U J \\
U J \\
U J \\
\\
U \\
U \\
J \\
J \\
J\end{array}$ & $\begin{array}{r}16.00 \\
0.35 \\
0.00 \\
1.60 \\
1.10 \\
1.50 \\
1.30 \\
\\
2.10 \\
0.09 \\
0.08 \\
0.03 \\
2.20 \\
0.01 \\
0.01\end{array}$ & $\begin{array}{l}U \\
U \\
U \\
U \\
U \\
U\end{array}$ & $\begin{array}{r}24.0000 \\
1.1000 \\
0.0046 \\
\\
1.7000 \\
0.9500 \\
1.6000 \\
0.9800 \\
2.5000 \\
0.0930 \\
0.0327 \\
0.0150 \\
2.3000 \\
0.0140 \\
0.0100\end{array}$ \\
\hline
\end{tabular}

Location=ORR; Formation $=$ NOLICHUCKY; Site=21; Sample type=Regular; $A=4257 ; B=$ ' '; C=' '; $A$ lab split $=$ ' '; Phase=2

Technetium-99 $\quad$ pCi/g $\quad 2.7900$ 
Table E1 (continued)

\begin{tabular}{|c|c|c|c|c|c|c|c|c|c|}
\hline & & A horizon & & $\mathbf{B}$ horizon & & $\mathrm{C}$ horizon & & A lab split & \\
\hline Analysis & Units & Result & Qualifier & Result & Qualifier & Result & Qualifier & Result & Qualifier \\
\hline
\end{tabular}

Location=ORR; Formation=NOLICHUCKY; Site=24; Sample type=Regular; $A=4258 ; B=$ ' '; $C=$ ' ' $A$ lab split $=4259 ;$ Phase $=2$

$\begin{array}{rlllll}\text { Technetiun-99 } & \text { pCi/g } & 1.5000 & \text { U } & 2.0400 & U\end{array}$

Location=ORR; Formation=NOLICHUCKY; Site=28; Sample type=Regular; $A=4262 ; B=$ ' ; $C=$ ' ' $A$ lab split =' '; Phase =2

$\begin{array}{lll}\text { Technetium-99 } & \text { pCi/g } & 2.2000 \quad U\end{array}$

Location=ORR; Formation $=$ NOLICHUCKY; Site=3,13,24; Sample type=Regular; $A=5083 ; B=5086 ; C=5089 ; A$ lab split =' '; Phase =1

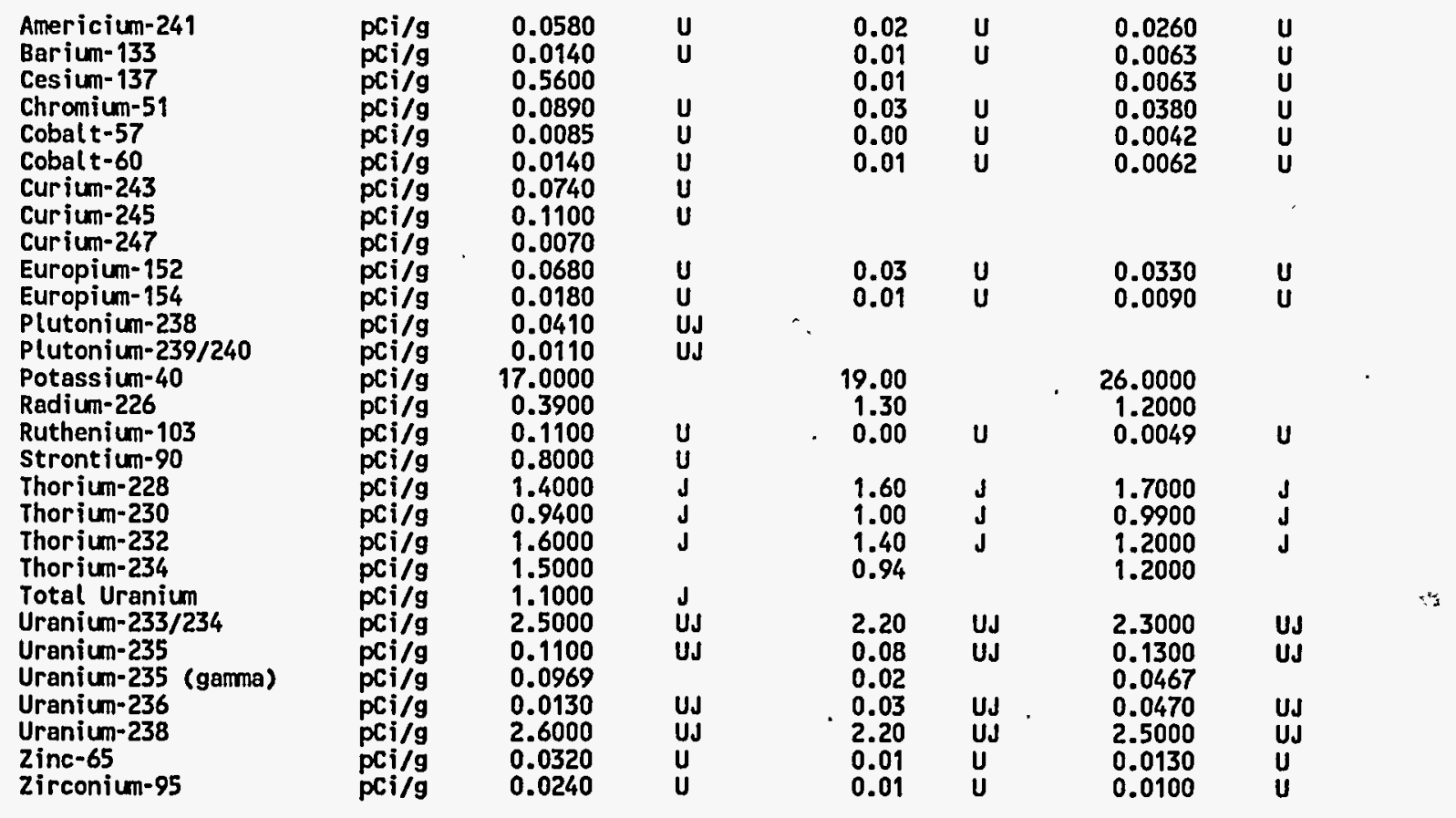


Table E1 (continucd)

\begin{tabular}{|c|c|c|c|c|c|c|c|c|c|}
\hline & & A horizon & & B horizon & & $\mathrm{C}$ horizon & & A lab split & \\
\hline Analysis & Units & Result & Qualifier & Result & Qualifier & Result & Qualifier & Result & Qualifier \\
\hline
\end{tabular}

Location=ORR; Formation=NOLICHUCKY; Site=5; Sample type=Regular; $A=4253 ; B=$ ' '; $C=$ ' '; $A$ lab split=' '; Phase=2

Technetium-99 pci/g $\quad 1.7100 \quad U$

Location=ORR; Formation=NOLICHUCKY; Site=5,21,31; Sample type=Regular; $A=5074 ; B=5077 ; C=5080 ; A$ lab split=' '; Phase =1

\begin{tabular}{|c|c|c|c|c|c|c|}
\hline $\begin{array}{l}\text { Americium-241 } \\
\text { Barium-133 } \\
\text { Cesium-137 } \\
\text { Chromium-51 } \\
\text { Cobalt }-57 \\
\text { Cobalt-60 } \\
\text { Curium-243 } \\
\text { Curium-245 } \\
\text { Curium-247 } \\
\text { Europium-152 } \\
\text { Europium-154 } \\
\text { Neptunium-237 } \\
\text { Plutonium-238 } \\
\text { Plutonium-239/240 } \\
\text { Potassium-40 } \\
\text { Radium-226 } \\
\text { Ruthenium-103 } \\
\text { Stront ium-90 } \\
\text { Thorium-228 } \\
\text { Thorium-230 } \\
\text { Thorium-232 } \\
\text { Thorium-234 } \\
\text { Total Uranium } \\
\text { Uranium-233/234 } \\
\text { Uranium-235 } \\
\text { Uranium-235 (gamma) } \\
\text { Uranium-236 } \\
\text { Uranium-238 } \\
\text { Zinc-65 } \\
\text { Zirconium-95 }\end{array}$ & $\begin{array}{l}\mathrm{pCi} / \mathrm{g} \\
\mathrm{pCi} / \mathrm{g} \\
\mathrm{pCi} j / \mathrm{g} \\
\mathrm{pCi} / \mathrm{g} \\
\mathrm{pCi} / \mathrm{g} \\
\mathrm{pCi} / \mathrm{g} \\
\mathrm{pCi} / \mathrm{g} \\
\mathrm{pCi} / \mathrm{g} \\
\mathrm{pCi} / \mathrm{g} \\
\mathrm{pCi} / \mathrm{g} \\
\mathrm{pCi} / \mathrm{g} \\
\mathrm{pCi} i / g \\
\mathrm{pCi} / \mathrm{g} \\
\mathrm{pCi} / \mathrm{g} \\
\mathrm{pCi} / \mathrm{g} \\
\mathrm{pCi} / \mathrm{g} \\
\mathrm{pCi} / \mathrm{g} \\
\mathrm{pCi} / \mathrm{g} \\
\mathrm{pCi} / \mathrm{g} \\
\mathrm{pCi} / \mathrm{g} \\
\mathrm{pCi} / \mathrm{g} \\
\mathrm{pCi} / \mathrm{g} \\
\mathrm{pCi} / \mathrm{g} \\
\mathrm{pCi} / \mathrm{g} \\
\mathrm{pCi} / \mathrm{g} \\
\mathrm{pCi} / \mathrm{g} \\
\mathrm{pCi} / \mathrm{g} \\
\mathrm{pCi} / \mathrm{g} \\
\mathrm{pCi} / \mathrm{g} \\
\mathrm{pCi} i / g\end{array}$ & $\begin{array}{l}0.0270 \\
0.0069 \\
0.7100 \\
0.0420 \\
0.0044 \\
0.0059 \\
0.0380 \\
0.0580 \\
0.0053 \\
0.0340 \\
0.0091 \\
0.2300 \\
0.0630 \\
0.0430 \\
14.0000 \\
1.4000 \\
0.0053 \\
0.6700 \\
1.4000 \\
0.9100 \\
1.2000 \\
1.3000 \\
1.4000 \\
2.5000 \\
0.1200 \\
0.0432 \\
0.0140 \\
2.8000 \\
0.0130 \\
0.0100\end{array}$ & $\begin{array}{l}U \\
U \\
J \\
\text { UJ } \\
\text { UJ }\end{array}$ & $\begin{array}{l}2.10 \\
0.04 \\
0.04 \\
0.01 \\
2.30 \\
0.01 \\
0.01\end{array}$ & $\begin{array}{l}\text { UJ } \\
\text { UJ } \\
U \\
U \\
U\end{array}$ & $\begin{array}{l}2.5000 \\
0.1700 \\
0.0541 \\
0.0130 \\
2.8000 \\
0.0130 \\
0.0099\end{array}$ \\
\hline
\end{tabular}




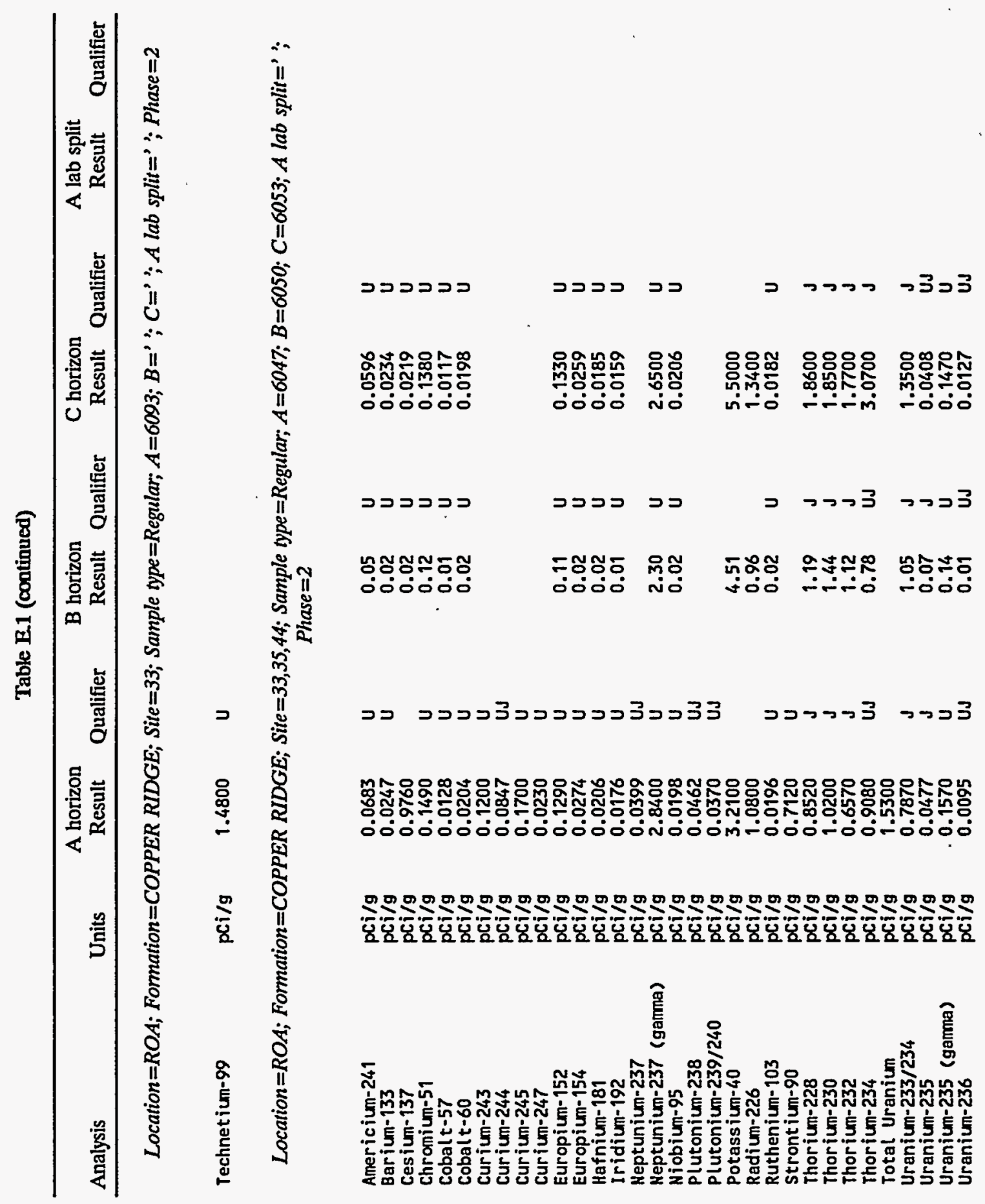


Table E.1 (continued)

\begin{tabular}{|c|c|c|c|c|c|c|c|c|c|}
\hline Analysis & Units & $\begin{array}{l}\text { A horizon } \\
\text { Result }\end{array}$ & Qualifier & $\begin{array}{c}\text { B horizon } \\
\text { Result }\end{array}$ & Qualifier & $\begin{array}{c}\mathrm{C} \text { horizon } \\
\text { Result }\end{array}$ & Qualifier & $\begin{array}{c}\text { A lab split } \\
\text { Result }\end{array}$ & Qualifier \\
\hline $\begin{array}{l}\text { Uranium-238 } \\
\text { Uranium-238 (gamma) } \\
\text { Zinc- } 65 \\
\text { Zirconium-95 }\end{array}$ & $\begin{array}{l}\mathrm{pCi} / \mathrm{g} \\
\mathrm{pCi} / \mathrm{g} \\
\mathrm{pCi} / \mathrm{g} \\
\mathrm{pCi} / \mathrm{g}\end{array}$ & $\begin{array}{l}0.7870 \\
4.2600 \\
0.0422 \\
0.0380\end{array}$ & $\begin{array}{l}\mathbf{J} \\
\mathbf{U} \\
\mathbf{U} \\
\mathbf{U}\end{array}$ & $\begin{array}{l}1.04 \\
3.47 \\
0.04 \\
0.03\end{array}$ & $\begin{array}{l}J \\
U \\
U \\
U\end{array}$ & $\begin{array}{l}1.5500 \\
4.0000 \\
0.0442 \\
0.0371\end{array}$ & $\begin{array}{l}J \\
U \\
U \\
U\end{array}$ & & \\
\hline
\end{tabular}

Location=ROA; Formation=COPPER RIDGE; Site=34; Sample type=Regular; $A=6094 ; B=$ ' '; $C=$ ' '; $A$ lab split=' '; Phase=2

Technetium-99 pCi/g $\quad 1.3400 \quad U$

Location=ROA; Formation=COPPER RIDGE; Site=34,39,41; Sample type=Regular; $A=6083 ; B=6088 ; C=6091 ; A$ lab split $=6085 ;$ Phase $=2$

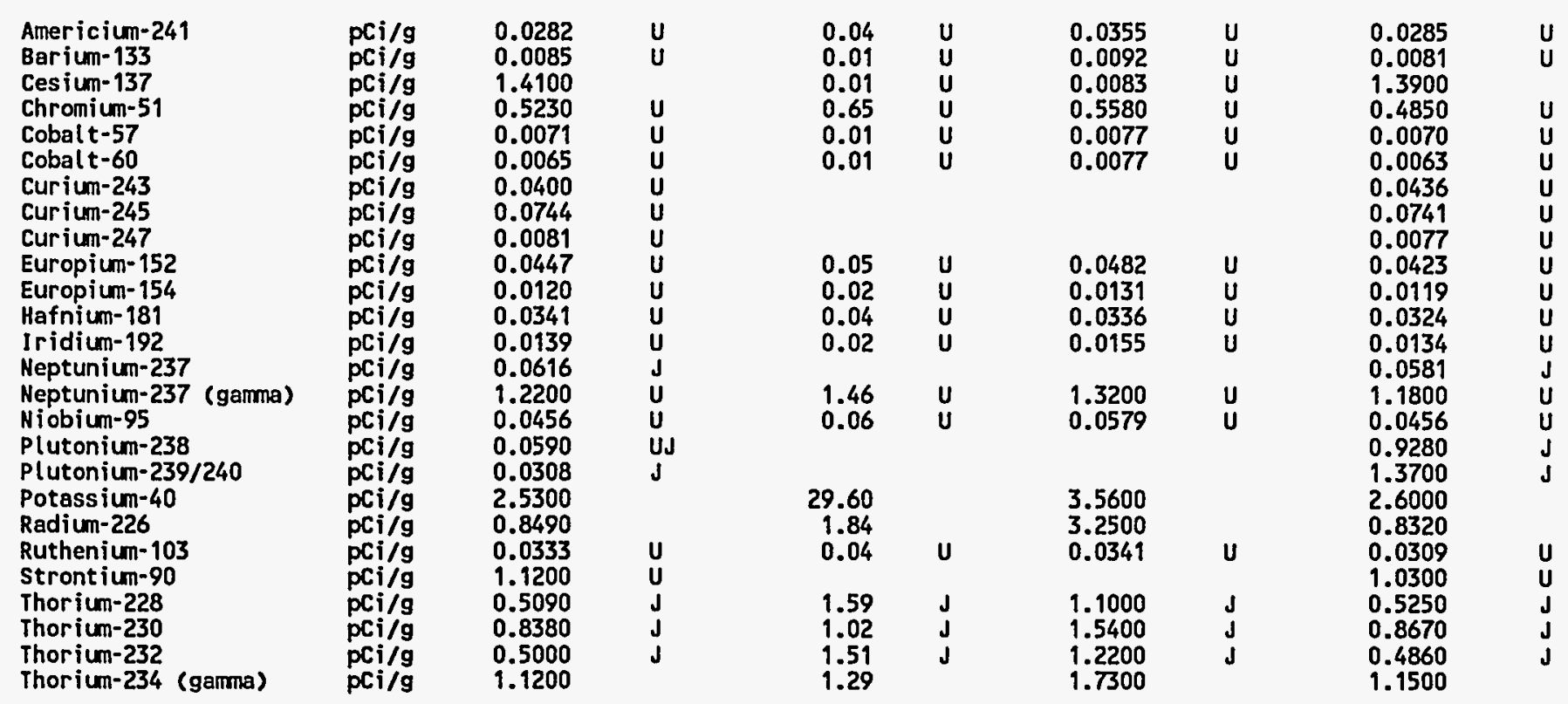


Table E1 (continued)

\begin{tabular}{|c|c|c|c|c|c|c|c|c|c|}
\hline Analysis & Units & $\begin{array}{c}\text { A horizon } \\
\text { Result }\end{array}$ & Qualifier & $\begin{array}{c}\text { B horizon } \\
\text { Result }\end{array}$ & Qualifier & $\begin{array}{c}C \text { horizon } \\
\text { Result }\end{array}$ & Qualifier & $\begin{array}{c}\text { A lab split } \\
\text { Result }\end{array}$ & Qualifier \\
\hline $\begin{array}{l}\text { Total Uranium } \\
\text { Uranium-233/234 } \\
\text { Uranium-235 } \\
\text { Uranium-235 (gamma) } \\
\text { Uranium-236 } \\
\text { Uranium-238 } \\
\text { Uranium-238 (gamma) } \\
\text { Zinc-65 } \\
\text { Zirconium-95 }\end{array}$ & $\begin{array}{l}\mathrm{pCi} / \mathrm{g} \\
\mathrm{pCi} / \mathrm{g} \\
\mathrm{pCi} / \mathrm{g} \\
\mathrm{pCi} / \mathrm{g} \\
\mathrm{pCi} / \mathrm{g} \\
\mathrm{pCi} / \mathrm{g} \\
\mathrm{pCi} / \mathrm{g} \\
\mathrm{pCi} / \mathrm{g} \\
\mathrm{pCi} / \mathrm{g}\end{array}$ & $\begin{array}{r}1.3000 \\
0.9600 \\
0.0309 \\
0.0500 \\
0.0097 \\
1.0000 \\
18.5000 \\
0.0179 \\
0.0313\end{array}$ & $\begin{array}{l}J \\
J \\
U J \\
U \\
U J \\
J \\
U \\
U \\
U\end{array}$ & $\begin{array}{r}0.84 \\
0.06 \\
0.05 \\
0.01 \\
0.96 \\
25.40 \\
0.03 \\
0.05\end{array}$ & $\begin{array}{l}J \\
J \\
U \\
U J \\
J \\
U \\
U \\
U\end{array}$ & $\begin{array}{r}1.4000 \\
0.0706 \\
0.1270 \\
0.0132 \\
1.6400 \\
20.7000 \\
0.0203 \\
0.0393\end{array}$ & $\begin{array}{l}J \\
J \\
\text { UJ } \\
J \\
\mathbf{U} \\
\mathbf{U} \\
\mathbf{U}\end{array}$ & $\begin{array}{r}1.0500 \\
0.7350 \\
0.0284 \\
0.0470 \\
0.0106 \\
0.8940 \\
17.4000 \\
0.0170 \\
0.0287\end{array}$ & $\begin{array}{l}J \\
J \\
J \\
U \\
U J \\
J \\
U \\
U \\
U\end{array}$ \\
\hline
\end{tabular}

Location=ROA; Formation=COPPER RIDGE; Site=35; Sample type=Regular; $A=6095 ; B=$ ' ; $C=$ ' '; $A$ lab split =' '; Phase =2

Technetium-99 $\quad \mathrm{pci} / \mathrm{g} \quad 1.1100 \quad \mathrm{U}$

Location=ROA; Formation=COPPER RIDGE; Site=40,42,43; Sample type=Regular; $A=6056 ; B=6059 ; C=6062 ; A$ lab split=' '; Phase $=2$

\begin{tabular}{|c|c|c|c|c|c|c|}
\hline $\begin{array}{l}\text { Americium-241 } \\
\text { Barium-133 } \\
\text { Cesium-137 } \\
\text { Chromium-51 } \\
\text { Cobalt-57 } \\
\text { Cobalt-60 } \\
\text { Curium-243 } \\
\text { Curium-245 } \\
\text { Curium-247 } \\
\text { Europium-152 } \\
\text { Europium-154 } \\
\text { Hafnium-181 } \\
\text { Iridium-192 } \\
\text { Heptunium-237 } \\
\text { Heptunium-237 (gamma) } \\
\text { Niobium-95 } \\
\text { Potassium-40 } \\
\text { Radium-226 } \\
\text { Ruthenium-103 . } \\
\text { Strontium-90 } \\
\text { Thorium-228 }\end{array}$ & $\begin{array}{l}\cdot \mathrm{pCi} / \mathrm{g} \\
\mathrm{pCi} / \mathrm{g} \\
\mathrm{pCi} / \mathrm{g} \\
\mathrm{pCi} / \mathrm{g} \\
\mathrm{pCi} / \mathrm{g} \\
\mathrm{pCi} / \mathrm{g} \\
\mathrm{pCi} / \mathrm{g} \\
\mathrm{pCi} / \mathrm{g} \\
\mathrm{pCi} / \mathrm{g} \\
\mathrm{pCi} / \mathrm{g} \\
\mathrm{pCi} / \mathrm{g} \\
\mathrm{pCi} / \mathrm{g} \\
\mathrm{pCi} / \mathrm{g} \\
\mathrm{pCi} / \mathrm{g} \\
\mathrm{pCi} / \mathrm{g} \\
\mathrm{pCi} i / g \\
\mathrm{pCi} / \mathrm{g} \\
\mathrm{pCi} i / g \\
\mathrm{pCi} i / g \\
\mathrm{pCi} i \mathrm{~g} \\
\mathrm{pCi} i / g\end{array}$ & $\begin{array}{l}0.0550 \\
0.0208 \\
0.8050 \\
0.1220 \\
0.0101 \\
0.0166 \\
0.0975 \\
0.1370 \\
0.0184 \\
0.0985 \\
0.0215 \\
0.0173 \\
0.0145 \\
0.0660 \\
2.2200 \\
0.0177 \\
2.2600 \\
0.7240 \\
0.0157 \\
0.7960 \\
0.4930\end{array}$ & $\begin{array}{l}U \\
U \\
U \\
U \\
U \\
U \\
U \\
U \\
U \\
U \\
U \\
J \\
U \\
U\end{array}$ & $\begin{array}{l}0.08 \\
0.02 \\
0.01 \\
0.01 \\
1.83 \\
0.01 \\
2.75 \\
0.77 \\
0.01\end{array}$ & $\begin{array}{l}\mathbf{U} \\
\mathbf{U} \\
\mathbf{U} \\
\mathbf{U} \\
\mathrm{U} \\
\mathbf{U}\end{array}$ & $\begin{array}{l}0.0820 \\
0.0161 \\
0.0116 \\
0.0097 \\
1.8100 \\
0.0135 \\
2.7900 \\
0.7970 \\
0.0111\end{array}$ \\
\hline
\end{tabular}


Table E1 (continued)

\begin{tabular}{|c|c|c|c|c|c|c|c|c|c|}
\hline Analysis & Units & $\begin{array}{c}\text { A horizon } \\
\text { Result }\end{array}$ & Qualifier & $\begin{array}{l}\text { B horizon } \\
\text { Result }\end{array}$ & Qualifier & $\begin{array}{c}\text { C horizon } \\
\text { Result }\end{array}$ & Qualifier & $\begin{array}{l}\text { A lab split } \\
\text { Result }\end{array}$ & Qualifier \\
\hline $\begin{array}{l}\text { Thorium-230 } \\
\text { Thorium-232 } \\
\text { Thorium-234 } \\
\text { Total Uranium } \\
\text { Uranium-233/234 } \\
\text { Uranium-235 } \\
\text { Uranium-235 (gamma) } \\
\text { Uranium-236 } \\
\text { Uranium-238 } \\
\text { Uranium-238 (gamma) } \\
\text { Zinc-65 } \\
\text { Zirconium-95 }\end{array}$ & $\begin{array}{l}\mathrm{pCi} / \mathrm{g} \\
\mathrm{pCi} / \mathrm{g} \\
\mathrm{pCi} / \mathrm{g} \\
\mathrm{pCi} / \mathrm{g} \\
\mathrm{pCi} / \mathrm{g} \\
\mathrm{pCi} i / g \\
\mathrm{pCi} i / g \\
\mathrm{pCi} / \mathrm{g} \\
\mathrm{pCi} / \mathrm{g} \\
\mathrm{pCi} i / g \\
\mathrm{pCi} / \mathrm{g} \\
\mathrm{pCi} / \mathrm{g}\end{array}$ & $\begin{array}{l}0.6920 \\
0.4270 \\
0.8550 \\
3.2100 \\
3.2100 \\
0.0175 \\
0.1510 \\
0.0087 \\
0.6370 \\
3.5300 \\
0.0379 \\
0.0287\end{array}$ & $\begin{array}{l}J \\
J \\
U J \\
J \\
J \\
U \\
U J \\
J \\
U \\
U \\
U\end{array}$ & $\begin{array}{l}0.77 \\
0.51 \\
0.54 \\
0.82 \\
0.06 \\
0.10 \\
0.03 \\
0.83 \\
2.98 \\
0.03 \\
0.03\end{array}$ & $\begin{array}{l}J \\
J \\
U J \\
J \\
J \\
U \\
U J \\
J \\
U \\
U \\
U\end{array}$ & $\begin{array}{l}1.1600 \\
1.0800 \\
0.7530 \\
0.9040 \\
0.0302 \\
0.1010 \\
0.0113 \\
1.2000 \\
2.7100 \\
0.0269 \\
0.0217\end{array}$ & $\begin{array}{l}J \\
J \\
U J \\
J \\
J \\
U \\
U J \\
J \\
U \\
U \\
U\end{array}$ & & \\
\hline
\end{tabular}

Location=ROA; Formation=COPPER RIDGE; Site=45,46,47; Sample type=Regular; $A=6074 ; B=6071 ; C=6065 ; A$ lab split =' '; Phase $=2$

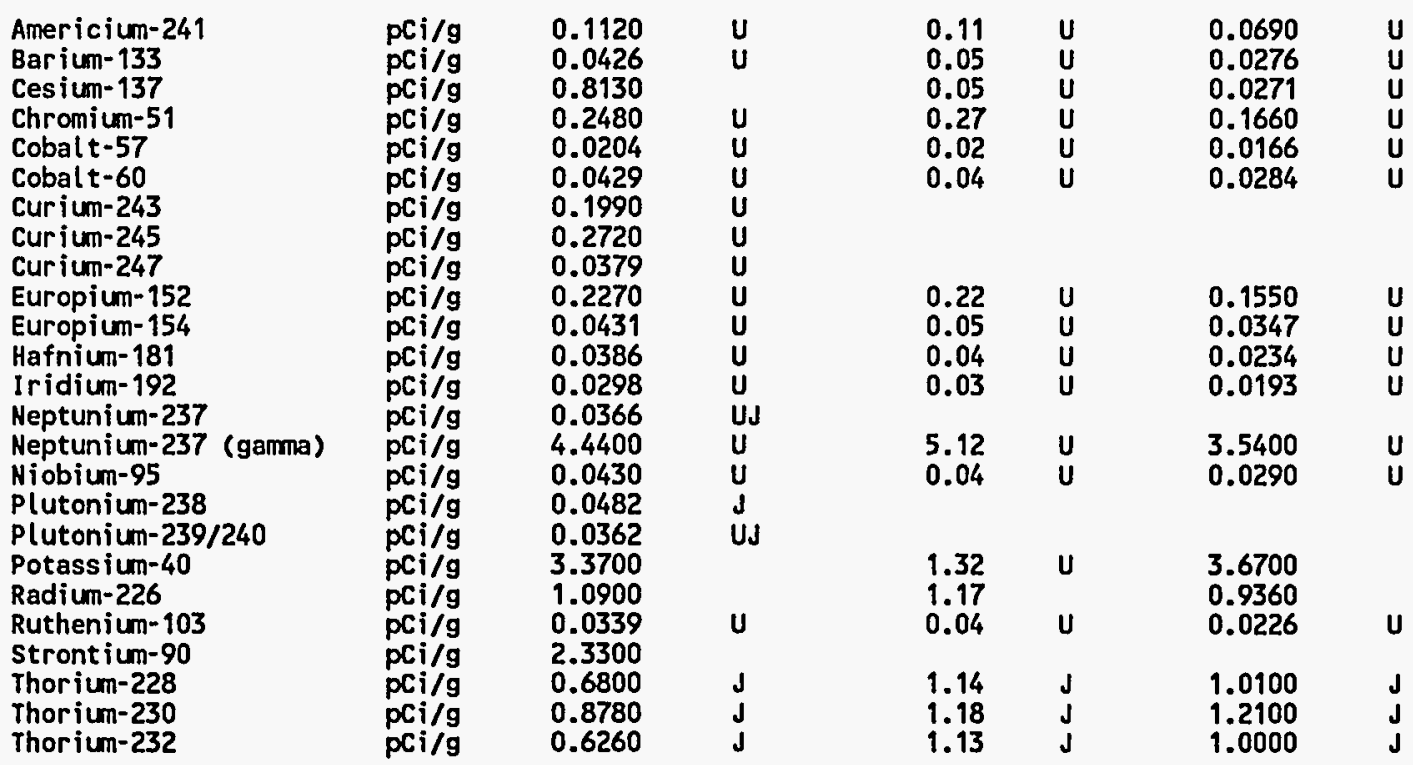


Table E1 (continued)

\begin{tabular}{|c|c|c|c|c|c|c|c|c|c|}
\hline Analysis & Units & $\begin{array}{c}\text { A horizon } \\
\text { Result }\end{array}$ & Qualifier & $\begin{array}{c}\text { B horizon } \\
\text { Result }\end{array}$ & Qualifier & $\begin{array}{c}\text { C horizon } \\
\text { Result }\end{array}$ & Qualifier & $\begin{array}{c}\text { A lab split } \\
\text { Result }\end{array}$ & Qualifier \\
\hline $\begin{array}{l}\text { Thoriun-234 } \\
\text { Total Uranium } \\
\text { Uranium-233/234 } \\
\text { Uranium-235 } \\
\text { Uranium-235 (gamma) } \\
\text { Uranium-236 } \\
\text { Uranium-238 } \\
\text { Uranium-238 (gamma) } \\
\text { Zinc-65 } \\
\text { Zirconium-95 }\end{array}$ & $\begin{array}{l}\text { pCi/g } \\
\text { pCi/g } \\
\text { pCi } / / 9 \\
\text { pCi/g } \\
\text { pCi/g } \\
\text { pCi/g } \\
\text { pCi/g } \\
\text { pCi/g } \\
\text { pCi/g } \\
\text { pCi/g }\end{array}$ & $\begin{array}{l}1.0300 \\
2.2500 \\
1.1700 \\
0.0941 \\
0.1790 \\
0.0104 \\
1.0200 \\
6.9800 \\
0.0832 \\
0.0689\end{array}$ & $\begin{array}{l}U J \\
J \\
J \\
J \\
U \\
U J \\
J \\
U \\
U \\
U\end{array}$ & $\begin{array}{l}0.21 \\
7.63 \\
0.09 \\
0.07\end{array}$ & $\begin{array}{l}\mathbf{U} \\
\mathbf{U} \\
\mathbf{U}\end{array}$ & $\begin{array}{l}0.8030 \\
1.0300 \\
0.9360 \\
0.1450 \\
0.0157 \\
1.3400 \\
5.2700 \\
0.0549 \\
0.0461\end{array}$ & $\begin{array}{l}\text { UJ } \\
J \\
J \\
U \\
J \\
J \\
U \\
U \\
U\end{array}$ & & \\
\hline
\end{tabular}

Location=ROA; Formation=COPPER RIDGE; Site=45,46,47; Sample type=Field Duplicate; $A=6077 ; B=6080 ; C=6068 ; A$ lab split $=$ ' '; Phase $=2$

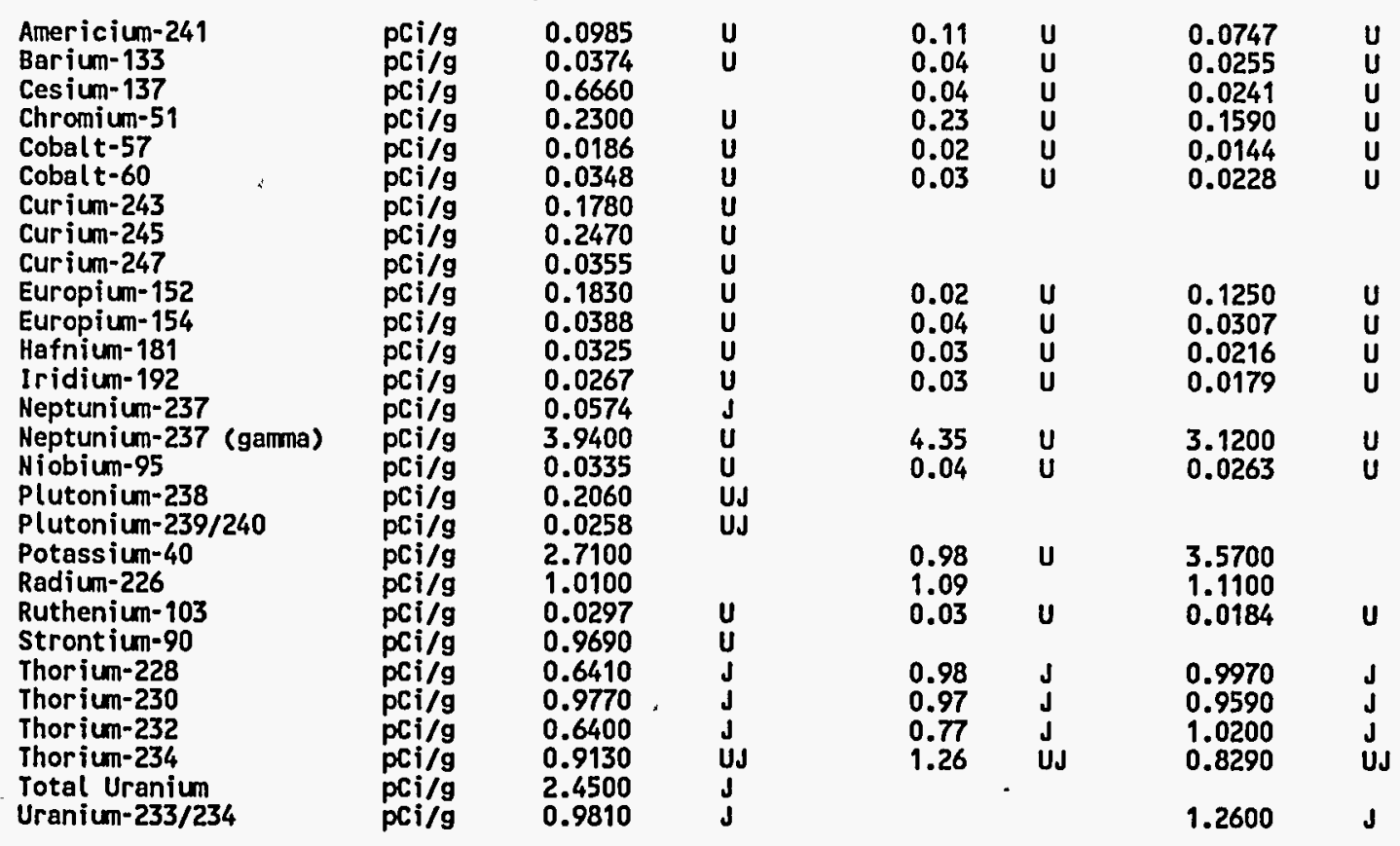


Table E.1 (continued)

\begin{tabular}{|c|c|c|c|c|c|c|c|c|c|}
\hline Analysis & Units & $\begin{array}{c}\text { A horizon } \\
\text { Result }\end{array}$ & Qualifier & $\begin{array}{c}\text { B horizon } \\
\text { Result }\end{array}$ & Qualifier & $\begin{array}{c}\mathrm{C} \text { horizon } \\
\text { Result }\end{array}$ & Qualifier & $\begin{array}{c}\text { A lab split } \\
\text { Result }\end{array}$ & Qualifier \\
\hline $\begin{array}{l}\text { Uranium-235 } \\
\text { Uranium-235 (gamma) } \\
\text { Uranium-236 } \\
\text { Uranium-238 } \\
\text { Uranium-238 (gamma) } \\
\text { Zinc-65 } \\
\text { Zirconium-95 }\end{array}$ & $\begin{array}{l}\mathrm{pCi} / \mathrm{g} \\
\mathrm{pCi} / \mathrm{g} \\
\mathrm{pCi} / \mathrm{g} \\
\mathrm{pCi} / \mathrm{g} \\
\mathrm{pCi} / \mathrm{g} \\
\mathrm{pCi} / \mathrm{g} \\
\mathrm{pCi} / \mathrm{g}\end{array}$ & $\begin{array}{l}0.0648 \\
0.1580 \\
0.0108 \\
1.0400 \\
6.2300 \\
0.0727 \\
0.0580\end{array}$ & $\begin{array}{l}J \\
U \\
U J \\
J \\
U \\
U \\
U\end{array}$ & $\begin{array}{l}0.16 \\
\\
6.09 \\
0.07 \\
0.06\end{array}$ & $\begin{array}{l}U \\
U \\
U\end{array}$ & $\begin{array}{l}0.1000 \\
0.1080 \\
0.0369 \\
1.3600 \\
4.4500 \\
0.0462 \\
0.0406\end{array}$ & $\begin{array}{l}J \\
U \\
U J \\
J \\
U \\
U \\
U\end{array}$ & & \\
\hline
\end{tabular}

Location=ROA; Formation =DISMAL GAP; Site=10,13,14; Sample type=Regular; $A=6029 ; B=6032 ; C=6035 ; A$ lab split $=$ ' ' Phase $=1$

\begin{tabular}{|c|c|c|c|c|c|c|}
\hline $\begin{array}{l}\text { Americium-241 } \\
\text { Barium-133 } \\
\text { Cesium-137 } \\
\text { Chromium-51 } \\
\text { Cobalt-57 } \\
\text { Cobalt-60 } \\
\text { Curium-243 } \\
\text { Curium-245 } \\
\text { Curium-247 } \\
\text { Europium-152 } \\
\text { Europium-154 } \\
\text { Hafnium-181 } \\
\text { Iridium-192 } \\
\text { Niobium-95 } \\
\text { Plutonium-238 } \\
\text { Plutonium-239/240 } \\
\text { Potassium-40 } \\
\text { Radium-226 } \\
\text { Ruthenium-103 } \\
\text { Strontium-90 } \\
\text { Thorium-228 } \\
\text { Thorium-230 } \\
\text { Thorium-232 } \\
\text { Thorium-234 } \\
\text { Total Uranium } \\
\text { Uranium-233/234 } \\
\text { Uranium-235 } \\
\text { Uranium-235 (garma) } \\
\text { Uranium-236 } \\
\text { Uranium-238 } \\
\text { Zinc-65 } \\
\text { Zirconium-95 }\end{array}$ & $\begin{array}{l}\mathrm{pCi} i g \\
\mathrm{pCi} / g \\
\mathrm{pCi} / \mathrm{g} \\
\mathrm{pCi} / \mathrm{g} \\
\mathrm{pCi} / \mathrm{g} \\
\mathrm{pCi} / \mathrm{g} \\
\mathrm{pCi} / \mathrm{g} \\
\mathrm{pCi} / \mathrm{g} \\
\mathrm{pCi} / \mathrm{g} \\
\mathrm{pCi} / \mathrm{g} \\
\mathrm{pCi} / \mathrm{g} \\
\mathrm{pCi} / \mathrm{g} \\
\mathrm{pCi} / \mathrm{g} \\
\mathrm{pCi} / \mathrm{g} \\
\mathrm{pCi} / \mathrm{g} \\
\mathrm{pCi} / \mathrm{g} \\
\mathrm{pCi} / \mathrm{g} \\
\mathrm{pCi} / \mathrm{g} \\
\mathrm{pCi} / \mathrm{g} \\
\mathrm{pCi} / \mathrm{g} \\
\mathrm{pCi} / \mathrm{g} \\
\mathrm{pCi} / \mathrm{g} \\
\mathrm{pCi} / \mathrm{g} \\
\mathrm{pCi} / \mathrm{g} \\
\mathrm{pCi} / \mathrm{g} \\
\mathrm{pCi} / \mathrm{g} \\
\mathrm{pCi} / \mathrm{g} \\
\mathrm{pCi} / \mathrm{g} \\
\mathrm{pCi} / \mathrm{g} \\
\mathrm{pCi} / \mathrm{g} \\
\mathrm{pCi} / \mathrm{g} \\
\mathrm{pCi} / \mathrm{g}\end{array}$ & $\begin{array}{l}0.0520 \\
0.0110 \\
0.8700 \\
0.0640 \\
0.0067 \\
0.0080 \\
0.0560 \\
0.0910 \\
0.0099 \\
0.0460 \\
0.0140 \\
0.0089 \\
0.0075 \\
0.0093 \\
0.0590 \\
0.0490 \\
9.5000 \\
0.7200 \\
0.0081 \\
1.2000 \\
0.9400 \\
0.7300 \\
0.9800 \\
0.9400 \\
2.5000 \\
0.8300 \\
0.0900 \\
0.0757 \\
0.0320 \\
0.8200 \\
0.0180 \\
0.0150\end{array}$ & $\begin{array}{l}U \\
U \\
U \\
U \\
U \\
U \\
U \\
U \\
U \\
U \\
U \\
U \\
U \\
U J \\
U J \\
\\
U \\
U \\
J \\
J \\
J\end{array}$ & $\begin{array}{r}0.04 \\
0.01 \\
0.01 \\
0.01 \\
0.01 \\
\\
17.00 \\
0.71 \\
0.01 \\
1.40 \\
0.90 \\
1.40 \\
0.88 \\
\\
0.93 \\
0.05 \\
0.07 \\
0.05 \\
1.00 \\
0.02 \\
0.01\end{array}$ & $\begin{array}{l}U \\
U \\
U \\
U \\
U\end{array}$ & $\begin{array}{l} \\
0.0370 \\
0.0120 \\
0.0065 \\
0.0057 \\
0.0077 \\
\\
\\
22.0000 \\
0.6200 \\
0.0048 \\
1.1000 \\
0.6500 \\
1.2000 \\
0.7800 \\
1.0000 \\
0.1500 \\
0.0280 \\
0.1100 \\
0.8900 \\
0.0170 \\
0.0130\end{array}$ \\
\hline
\end{tabular}


Table E1 (continued)

\begin{tabular}{|c|c|c|c|c|c|c|c|c|c|}
\hline Analysis & Units & $\begin{array}{l}\text { A horizon } \\
\text { Result }\end{array}$ & Qualifier & $\begin{array}{l}\text { B horizon } \\
\text { Result }\end{array}$ & Oualifier & $\begin{array}{c}\mathrm{C} \text { horizon } \\
\text { Result }\end{array}$ & Oualifier & A lab split & Opalifier \\
\hline
\end{tabular}

Location=ROA; Formation=DISMAL GAP; Site=10,13,14; Sample type=Field Duplicate; $A=6038 ; B=6041 ; C=6044 ; A$ lab split =' "; Phase $=1$

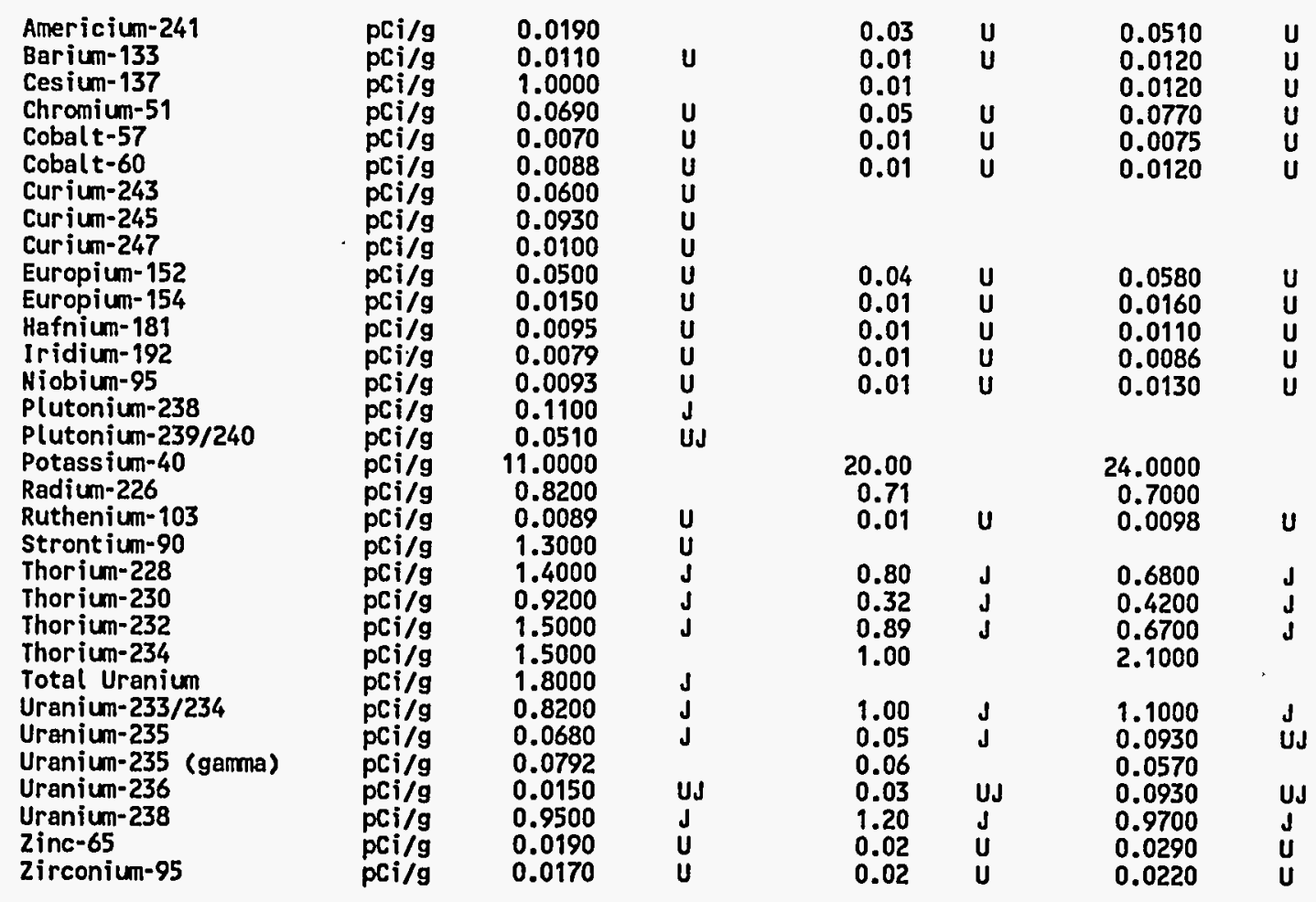

Location=ROA; Formation=DISMAL GAP; Site $=19 ;$ Sample type $=$ Regular; $A=6096 ; B=$ '; $C=$ ' '; lab split=' '; Phase $=2$

$\begin{array}{lll}\text { Technetium-99 } & \text { pci/g } & 1.2100 \quad U\end{array}$ 
Table E.1 (continued)

\begin{tabular}{|c|c|c|c|c|c|c|c|c|c|}
\hline Analysis & Units & $\begin{array}{c}\text { A horizon } \\
\text { Result }\end{array}$ & Qualifier & $\begin{array}{l}\text { B horizon } \\
\text { Result }\end{array}$ & Qualifier & $\begin{array}{c}\mathrm{C} \text { horizon } \\
\text { Result }\end{array}$ & Qualifier & $\begin{array}{l}\text { A lab split } \\
\text { Result }\end{array}$ & Qualifier \\
\hline
\end{tabular}

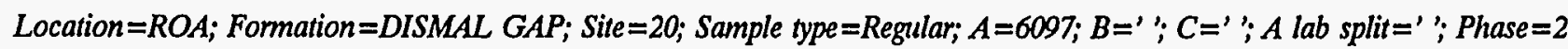

$\begin{array}{lll}\text { Technetium-99 } & \text { pCi/g } & 0.9320 \quad U\end{array}$

Location=ROA; Formation=DISMAL GAP; Site=21; Sample type=Regular; $A=6098 ; B=$ ' '; $C=$ ' '; $A$ lab split =' '; Phase $=2$

Technetium-99 $\quad$ pCi/g $1.3800 \quad \mathrm{U}$

Location=ROA; Formation=DISMAL GAP; Site=3,7,21; Sample type=Regular; $A=6020 ; B=6023 ; C=6026 ; A$ lab split=' '; Phase=1

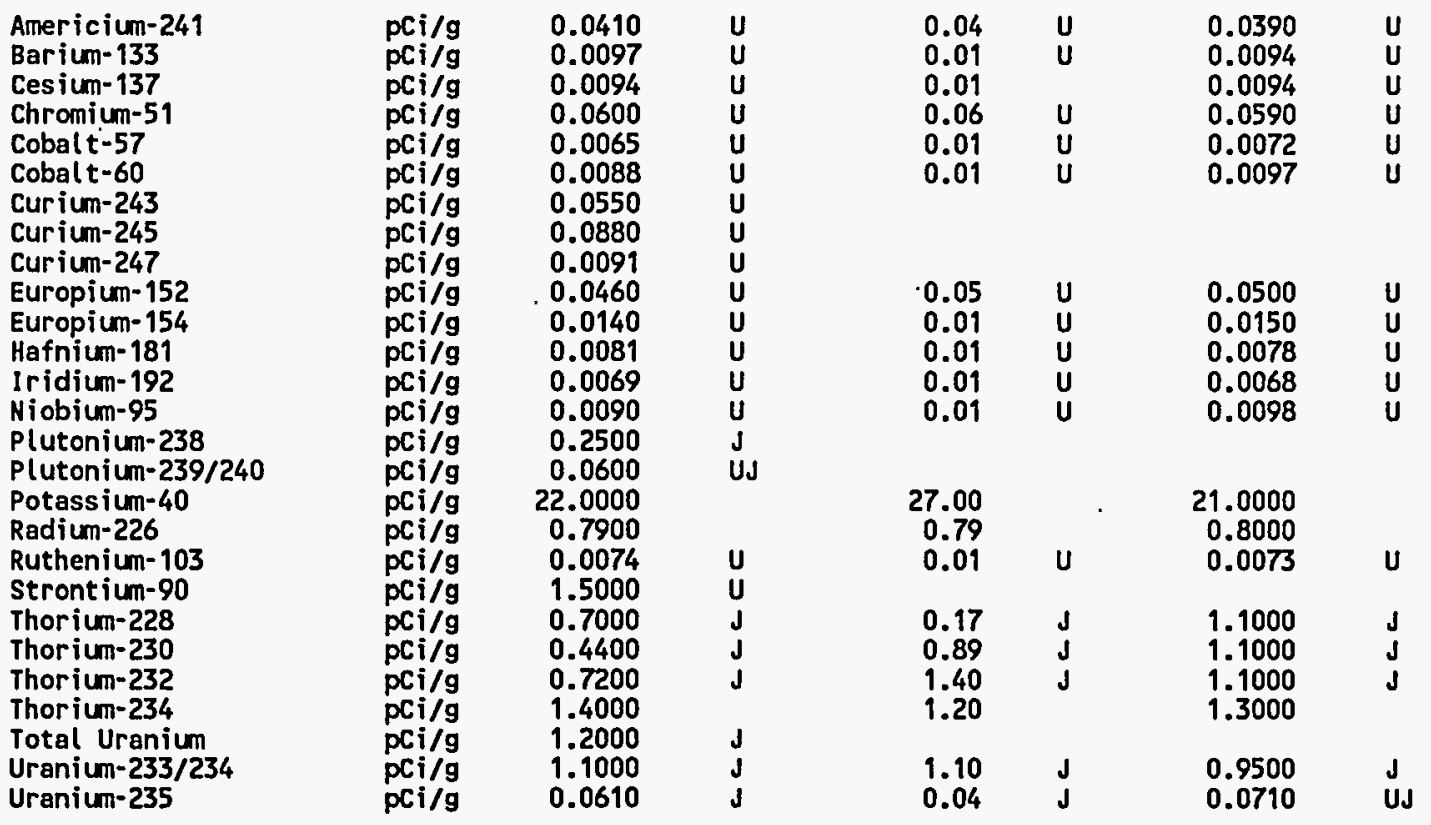


Table E1 (continued)

\begin{tabular}{|c|c|c|c|c|c|c|c|c|c|}
\hline Analysis & Units & $\begin{array}{c}\text { A horizon } \\
\text { Result }\end{array}$ & Qualifier & $\begin{array}{c}\text { B horizon } \\
\text { Result }\end{array}$ & Qualifier & $\begin{array}{c}C \text { horizon } \\
\text { Result }\end{array}$ & Qualifier & $\begin{array}{c}\text { A lab split } \\
\text { Result }\end{array}$ & Qualifier \\
\hline $\begin{array}{l}\text { Uranium-235 (gamma) } \\
\text { Uranium-236 } \\
\text { Uranium-238 } \\
\text { Zinc-65 } \\
\text { Zirconium-95 }\end{array}$ & $\begin{array}{l}\mathrm{pCi} / \mathrm{g} \\
\mathrm{pCi} / \mathrm{g} \\
\mathrm{pCi} / \mathrm{g} \\
\mathrm{pCi} / \mathrm{g} \\
\mathrm{pCi} / \mathrm{g}\end{array}$ & $\begin{array}{l}0.0907 \\
0.0840 \\
1.2000 \\
0.0210 \\
0.0160\end{array}$ & $\begin{array}{l}U J \\
\mathbf{J} \\
U \\
U\end{array}$ & $\begin{array}{l}0.03 \\
0.03 \\
1.00 \\
0.02 \\
0.02\end{array}$ & $\begin{array}{l}\text { UJ } \\
\mathbf{J} \\
\mathbf{U} \\
\mathbf{U}\end{array}$ & $\begin{array}{l}0.0708 \\
0.0700 \\
1.2000 \\
0.0220 \\
0.0170\end{array}$ & $\begin{array}{l}\text { UJ } \\
J \\
U \\
U\end{array}$ & & \\
\hline
\end{tabular}

Location=ROA; Formation=DISMAL GAP; Site=8,20,22; Sample type=Regular; $A=6011 ; B=6014 ; C=6017 ; A$ lab split=' ; Phase=1

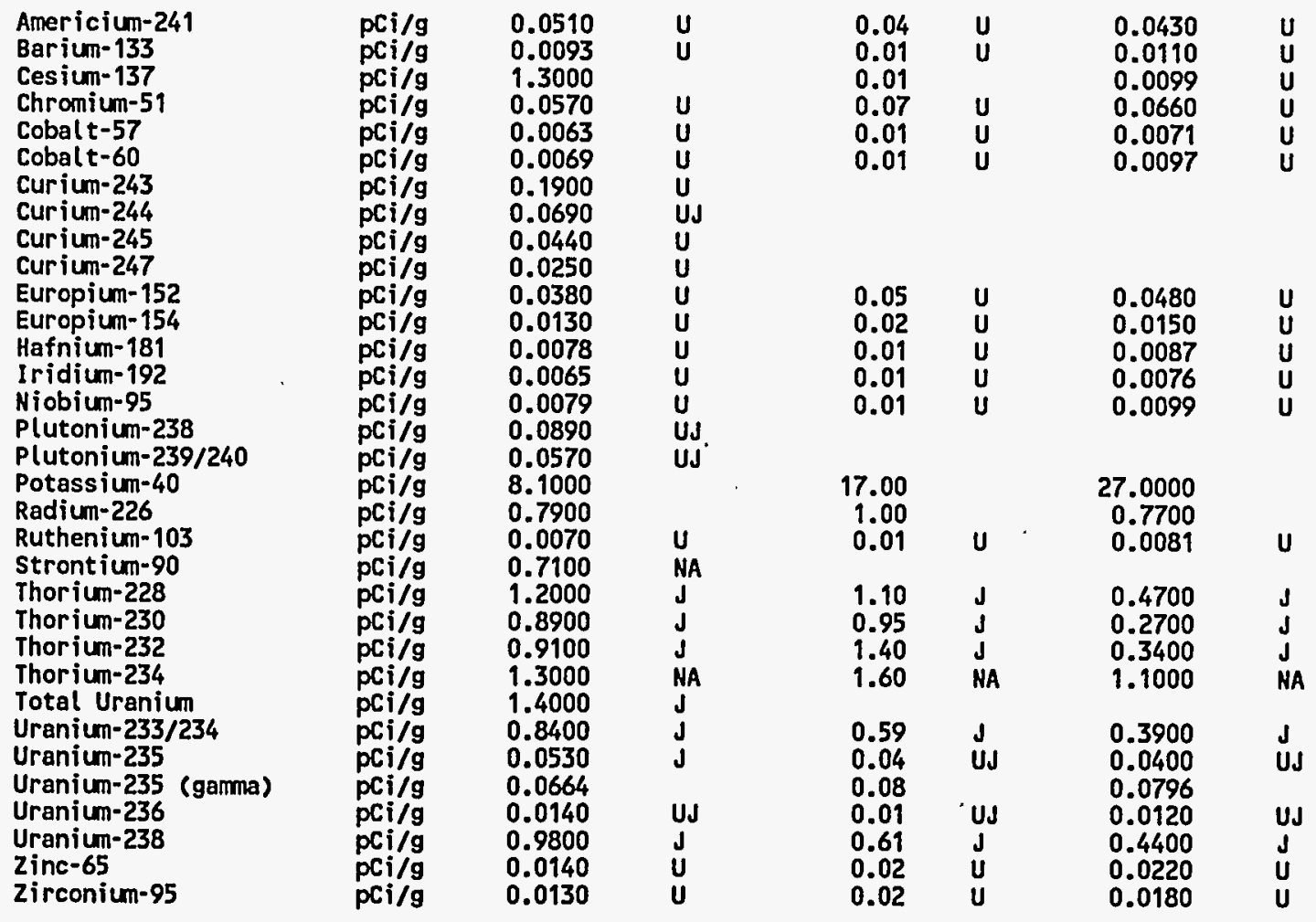


Table E.1 (continued)

\begin{tabular}{|c|c|c|c|c|c|c|c|c|c|}
\hline Analysis & Units & $\begin{array}{c}\text { A horizon } \\
\text { Result }\end{array}$ & Qualifier & $\begin{array}{c}\text { B horizon } \\
\text { Result }\end{array}$ & Qualifier & $\begin{array}{l}\text { C horizon } \\
\text { Result }\end{array}$ & Qualifier & $\begin{array}{l}\text { A lab split } \\
\text { Result }\end{array}$ & Qualifier \\
\hline \multicolumn{10}{|c|}{ Location $=R O A ;$ Formation $=D I S M A L G A P ;$ Site $=9,17,19 ;$ Sample type $=$ Regular; $A=6005 ; B=6002 ; C=6008 ; A$ lab split=' '; Phase $=1$} \\
\hline $\begin{array}{l}\text { Americium-241 } \\
\text { Barium-133 } \\
\text { Cesium-137 } \\
\text { Chromium-51 } \\
\text { Cobal }-57 \\
\text { Cobal t-60 } \\
\text { Curium-243 } \\
\text { Curium-244 } \\
\text { Curium-245 } \\
\text { Curium-247 } \\
\text { Europium-152 } \\
\text { Europium-154 } \\
\text { Hafnium-181 } \\
\text { Iridium-192 } \\
\text { Niobium-95 } \\
\text { Plutonium-238 } \\
\text { Plutonium-239/240 } \\
\text { Potassium-40 } \\
\text { Radium-226 } \\
\text { Ruthenium-103 } \\
\text { Strontium-90 } \\
\text { Thorium-228 } \\
\text { Thorium-230 } \\
\text { Thorium-232 } \\
\text { Thorium-234 } \\
\text { Total Uranium } \\
\text { Uranium-233/234 } \\
\text { Uranium-235 } \\
\text { Uranium-235 (gamma) } \\
\text { Uranium-236 } \\
\text { Uranium-238 } \\
\text { Zinc-65 } \\
\text { Zirconium-95 }\end{array}$ & $\begin{array}{l}\mathrm{pCi} / \mathrm{g} \\
\mathrm{pCi} / \mathrm{g} \\
\mathrm{pCi} / \mathrm{g} \\
\mathrm{pCi} / \mathrm{g} \\
\mathrm{pCi} / \mathrm{g} \\
\mathrm{pCi} / \mathrm{g} \\
\mathrm{pCi} / \mathrm{g} \\
\mathrm{pCi} / \mathrm{g} \\
\mathrm{pCi} / \mathrm{g} \\
\mathrm{pCi} / \mathrm{g} \\
\mathrm{pCi} / \mathrm{g} \\
\mathrm{pCi} / \mathrm{g} \\
\mathrm{pCi} / \mathrm{g} \\
\mathrm{pCi} / \mathrm{g} \\
\mathrm{pCi} / \mathrm{g} \\
\mathrm{pCi} / \mathrm{g} \\
\mathrm{pCi} / \mathrm{g} \\
\mathrm{pCi} / \mathrm{g} \\
\mathrm{pCi} / \mathrm{g} \\
\mathrm{pCi} / \mathrm{g} \\
\mathrm{pCi} / \mathrm{g} \\
\mathrm{pCi} / \mathrm{g} \\
\mathrm{pCi} / \mathrm{g} \\
\mathrm{pCi} / \mathrm{g} \\
\mathrm{pCi} / \mathrm{g} \\
\mathrm{pCi} / \mathrm{g} \\
\mathrm{pCi} / \mathrm{g} \\
\mathrm{pCi} / \mathrm{g} \\
\mathrm{pCi} / \mathrm{g} \\
\mathrm{pCi} / \mathrm{g} \\
\mathrm{pCi} / \mathrm{g} \\
\mathrm{pCi} / \mathrm{g} \\
\mathrm{pCi} / \mathrm{g}\end{array}$ & $\begin{array}{l}0.0440 \\
0.0110 \\
0.9000 \\
0.0690 \\
0.0070 \\
0.0084 \\
0.0610 \\
0.9100 \\
0.0950 \\
0.0110 \\
0.0510 \\
0.0150 \\
0.0096 \\
0.0080 \\
0.0091 \\
0.1100 \\
0.0440 \\
8.3000 \\
1.0000 \\
0.0086 \\
1.2000 \\
0.9700 \\
0.9600 \\
0.9800 \\
1.7000 \\
0.0660 \\
1.0000 \\
0.0510 \\
0.0747 \\
0.0160 \\
0.9300 \\
0.0180 \\
0.0160\end{array}$ & $\begin{array}{l}\mathbf{U} \\
\mathbf{U} \\
\mathbf{U} \\
\mathbf{U} \\
\mathbf{U} \\
\mathbf{U} \\
\mathbf{U} \mathbf{J} \\
\mathbf{U} \\
\mathbf{U} \\
\mathbf{U} \\
\mathbf{U} \\
\mathbf{U} \\
\mathbf{U} \\
\mathbf{U} \\
\mathbf{J} \\
\mathbf{U} \mathbf{J}\end{array}$ & $\begin{array}{l}0.04 \\
0.01 \\
0.02 \\
0.06 \\
0.01 \\
0.01 \\
0.05 \\
0.83 \\
0.09 \\
0.01 \\
0.05 \\
0.02 \\
0.01 \\
0.01 \\
0.01 \\
0.10 \\
0.07 \\
13.00 \\
1.00 \\
0.01 \\
1.20 \\
1.40 \\
1.10 \\
1.20 \\
1.90 \\
0.45 \\
0.55 \\
0.04 \\
0.11 \\
0.01 \\
0.69 \\
0.02 \\
0.02 \\
\end{array}$ & $\begin{array}{l}U \\
U \\
U \\
U \\
U \\
U \\
U J \\
U \\
U \\
U \\
U \\
U \\
U \\
U \\
J \\
U J \\
\\
U \\
N A \\
J \\
J \\
J \\
J \\
J \\
J \\
U J \\
J \\
U \\
U \\
\end{array}$ & $\begin{array}{r}0.0620 \\
0.0120 \\
0.0110 \\
0.0760 \\
0.0083 \\
0.0110 \\
0.0680 \\
\\
0.1100 \\
0.0110 \\
0.0560 \\
0.0180 \\
0.0100 \\
0.0088 \\
0.0260 \\
0.0950 \\
0.0250 \\
23.0000 \\
0.9400 \\
0.0095 \\
1.2000 \\
0.5600 \\
0.4200 \\
0.6100 \\
1.3000 \\
1.3000 \\
0.5200 \\
0.0500 \\
0.0642 \\
0.0130 \\
0.6200 \\
0.0260 \\
0.0200 \\
\end{array}$ & $\begin{array}{l}U \\
U U \\
U U \\
U U \\
U U \\
U \\
U U \\
U \\
U \\
U \\
U \\
U \\
U \\
U J \\
J \\
\\
U \\
N A \\
J \\
J \\
J \\
J \\
J \\
U J \\
U J \\
J \\
U \\
U \\
\end{array}$ & & \\
\hline
\end{tabular}


Table E2. Tritium analysis results for noncomposited soil samples

\begin{tabular}{|c|c|c|c|c|c|c|c|c|c|}
\hline Location & Formation & Site & Horizon & Units & $\begin{array}{c}\text { Sample } \\
\text { id }\end{array}$ & Phase & Field Duplicate & $\begin{array}{l}\text { A horizon } \\
\text { Result }\end{array}$ & Qualifier \\
\hline $\begin{array}{l}\text { AND } \\
\text { AND } \\
\text { AND } \\
\text { AND } \\
\text { ORR } \\
\text { ORR } \\
\text { ORR } \\
\text { ORR } \\
\text { ORR } \\
\text { ORR } \\
\text { ORR } \\
\text { ORR } \\
\text { ORR } \\
\text { ORR } \\
\text { ORR } \\
\text { ORR } \\
\text { ORR } \\
\text { ORR } \\
\text { ORR } \\
\text { ORR } \\
\text { ORR } \\
\text { ORR } \\
\text { ORR } \\
\text { ORR } \\
\text { ORR } \\
\text { ORR } \\
\text { ORR } \\
\text { ORR } \\
\text { ORR } \\
\text { ORR } \\
\text { ORR } \\
\text { ORR } \\
\text { ORR } \\
\text { ORR } \\
\text { ORR } \\
\text { ORR } \\
\text { ORR } \\
\text { ORR } \\
\text { ORR } \\
\text { ORR } \\
\text { ORR } \\
R O A \\
\text { ROA } \\
\text { ROA }\end{array}$ & 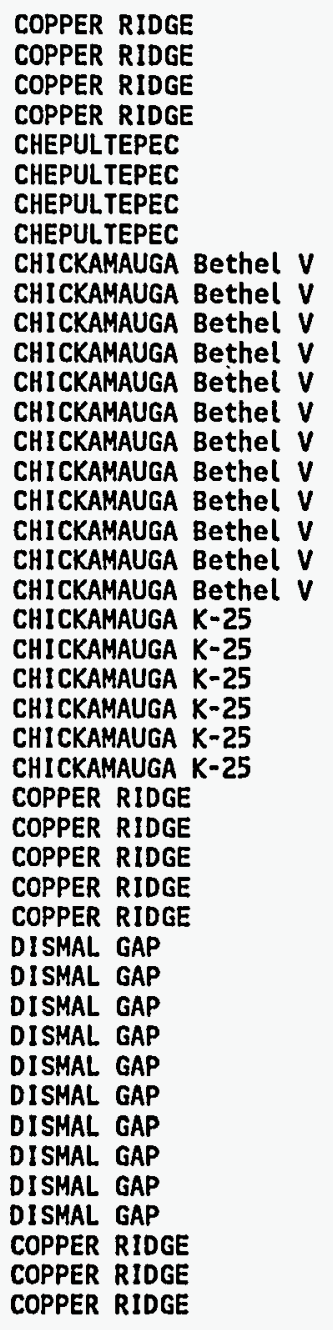 & $\begin{array}{l}32 \\
33 \\
34 \\
35 \\
50 \\
66 \\
74 \\
85 \\
93 \\
99 \\
100 \\
101 \\
102 \\
103 \\
104 \\
108 \\
110 \\
115 \\
116 \\
117 \\
118 \\
120 \\
122 \\
124 \\
126 \\
129 \\
62 \\
64 \\
75 \\
83 \\
91 \\
11 \\
2 \\
2 \\
22 \\
26 \\
27 \\
32 \\
33 \\
35 \\
41 \\
34 \\
40 \\
41\end{array}$ & $\begin{array}{l}A \\
A \\
A \\
A \\
A \\
A \\
A \\
A \\
A \\
A \\
A \\
A \\
A \\
A \\
A \\
A \\
A \\
A \\
A \\
A \\
A \\
A \\
A \\
A \\
A \\
A \\
A \\
A \\
A \\
A \\
A \\
A \\
A \\
A \\
A \\
A \\
A \\
A \\
A \\
A \\
A \\
A \\
A \\
A\end{array}$ & 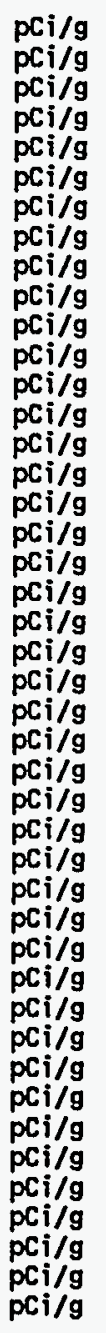 & $\begin{array}{l}2260 \\
2263 \\
2266 \\
2269 \\
1549 \\
1739 \\
1747 \\
1607 \\
4216 \\
4217 \\
4218 \\
4219 \\
4220 \\
4221 \\
1965 \\
1968 \\
1971 \\
1974 \\
1977 \\
1980 \\
4096 \\
4093 \\
4106 \\
4116 \\
4090 \\
4087 \\
1481 \\
1484 \\
1478 \\
1475 \\
1472 \\
1079 \\
1189 \\
1198 \\
1123 \\
1214 \\
1071 \\
1124 \\
1125 \\
1126 \\
1063 \\
3330 \\
3327 \\
3328\end{array}$ & $\begin{array}{l}2 \\
2 \\
2 \\
2 \\
2 \\
2 \\
2 \\
2 \\
2 \\
2 \\
2 \\
2 \\
2 \\
2 \\
2 \\
2 \\
2 \\
2 \\
2 \\
2 \\
2 \\
2 \\
2 \\
2 \\
2 \\
2 \\
2 \\
2 \\
2 \\
2 \\
2 \\
1 \\
1 \\
1 \\
1 \\
1 \\
1 \\
1 \\
1 \\
2 \\
2 \\
2\end{array}$ & FIELD DUPLICATE & $\begin{array}{l}0.049 \\
0.048 \\
0.061 \\
0.060 \\
0.100 \\
0.100 \\
0.100 \\
0.200 \\
0.130 \\
0.110 \\
0.140 \\
0.120 \\
0.220 \\
0.200 \\
0.120 \\
0.110 \\
0.130 \\
0.120 \\
0.090 \\
0.110 \\
0.092 \\
0.071 \\
0.072\end{array}$ & $\begin{array}{l}U J \\
U J \\
U J \\
U J \\
U J \\
U J \\
U J \\
U J \\
U J \\
U J \\
U J \\
J \\
J \\
J \\
U J \\
U J \\
U J \\
U J \\
U J \\
U J \\
U J \\
U J \\
U J \\
U J \\
U J \\
U J \\
J \\
J \\
J \\
J \\
U J\end{array}$ \\
\hline
\end{tabular}


Tabłe E2 (continued)

\begin{tabular}{|c|c|c|c|c|c|c|c|c|c|}
\hline Location & Formation & Site & Horizon & Units & $\begin{array}{c}\text { Sample } \\
\text { id }\end{array}$ & Phase & Field Duplicate & $\begin{array}{c}\text { A horizon } \\
\text { Result }\end{array}$ & Qualifier \\
\hline $\begin{array}{l}\text { ROA } \\
\text { ROA } \\
\text { ROA } \\
\text { ROA } \\
\text { ROA } \\
\text { ROA } \\
\text { ROA } \\
\end{array}$ & $\begin{array}{l}\text { DISMAL GAP } \\
\text { DISMAL GAP } \\
\text { DISMAL GAP } \\
\text { DISMAL GAP } \\
\text { DISMAL GAP } \\
\text { DISMAL GAP } \\
\text { DISMAL GAP }\end{array}$ & $\begin{array}{l}17 \\
20 \\
22 \\
3 \\
7 \\
8 \\
9 \\
\end{array}$ & $\begin{array}{l}A \\
A \\
A \\
A \\
A \\
A \\
A\end{array}$ & $\begin{array}{l}\mathrm{pCi} / \mathrm{g} \\
\mathrm{pCi} / \mathrm{g} \\
\mathrm{pCi} / \mathrm{g} \\
\mathrm{pCi} / \mathrm{g} \\
\mathrm{pCi} / \mathrm{g} \\
\mathrm{pCi} / \mathrm{g} \\
\mathrm{pCi} / \mathrm{g}\end{array}$ & $\begin{array}{l}3016 \\
3045 \\
3057 \\
3098 \\
3084 \\
3071 \\
3004 \\
\end{array}$ & $\begin{array}{l}1 \\
1 \\
1 \\
1 \\
1 \\
1 \\
1\end{array}$ & & $\begin{array}{l}0.046 \\
0.048 \\
0.041 \\
0.040 \\
0.075 \\
0.040 \\
0.053\end{array}$ & $\begin{array}{l}\text { UJ } \\
\text { US } \\
\text { US } \\
\text { US } \\
\text { US } \\
\text { US } \\
\text { UJ }\end{array}$ \\
\hline
\end{tabular}


Appendix F

RELATION OF SAMPLE NUMBERS TO LABORATORY SAMPLE DELIVERY GROUPS (SDGs) 


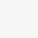

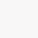


Table F.1. Relation of sample numbers to laboratory sample delivery groups (SDGs)

\begin{tabular}{|c|c|}
\hline SDG number & Sample number \\
\hline & Inorganics \\
\hline 042260 & $5001,5004,5007$ \\
\hline $\begin{array}{l}0422260 \\
0430260 / 0508260 / 05111260\end{array}$ & $\begin{array}{l}5001,5004,5007,5010,5013,5016,5019,5022,5025 \text {, } \\
6001,6004,6007,6010,6013,6016\end{array}$ \\
\hline 0430260 & $6001,6004,6007$ \\
\hline 0508260 & $5010,5013,5016,5019,5022,5025$ \\
\hline 0511260 & $6010,6013,6016$ \\
\hline 0514260 & $5028,5031,5034,5037,5040,5043,6019,6022,6025$ \\
\hline 519260 & $3144,6028,6031,6034,6037,6040,6043$ \\
\hline 0727260/0728260/0729260 & $\begin{array}{l}5055,5058,5061,5064,5067,5070,5073,5076,5079 \\
5082,5085,5088\end{array}$ \\
\hline $0722260 / 0723260$ & $\begin{array}{l}\text { 7001, 7001D, 7001, 7004, 7007, 7010, 7013, 7016, 7019, } \\
7022,7025,7028,7031,7034,7037 \mathrm{FD}, a \text { 7040FD, 7043FD }\end{array}$ \\
\hline 0803260 & 1468 \\
\hline 0909260/0915260 & $6046,6049,6052,6055,6058,6061$ \\
\hline $0924260 / 1002260 / 1009260$. & $\begin{array}{l}5091,5094,5097,1502,5100,5104,5106,5109,5112 \text {, } \\
5115\end{array}$ \\
\hline $1015260 / 1016260 / 1020260 / 1023260$ & $\begin{array}{l}6064,6067,6070 ; 6073,6076,6079,5118,5121,5124, \\
5127,5130,5133,5136,5139,5142,5145,5148,5151 \text {, } \\
1735\end{array}$ \\
\hline $1030260 / 1109260 / 1113260$ & $\begin{array}{l}5154,5155,5159,5162,5165,5166,5170,5173,5174 \\
5178,5181,5184\end{array}$ \\
\hline $1118260 / 1120260 / 1124260$ & $\begin{array}{l}3361,3367,6082,6084,6087,6090,5187,5190,5193 \\
5196,5199,5202\end{array}$ \\
\hline $1204260 / 1209260 / 1211260$ & $\begin{array}{l}5205,5208,5211,5216,5217,5222,5225,7046,7047 \\
7051,7054,7057,7060,7063,5228,5229,5234,5237\end{array}$ \\
\hline $1216260 / 1217260 / 1218260 /$ & $\begin{array}{l}5240,5241,5246,5249,7066,7069,7072,7075,7078 \\
7081,5252,5253,5257,5260,5263,5264,5268,5271\end{array}$ \\
\hline \multirow[t]{2}{*}{0108260} & $4229,4230,4236,4238,4239$ \\
\hline & Pesticides/PCBs \\
\hline 0523260 & $\begin{array}{l}1296,1292,1298,1300,1301,1302,1293,1294,1297 \\
1299,1303,1295\end{array}$ \\
\hline 0508260 & $1181,1190,1201 \mathrm{FD}, 1213$ \\
\hline 0511260 & $3058,3099,3072,3085$ \\
\hline 042260 & $3018,1064,1072,1080,3003$ \\
\hline 0424260 & $1099,1106,1107,1108,1115$ \\
\hline 0430260 & 1127,3032 \\
\hline
\end{tabular}


Table F.1 (continued)

\begin{tabular}{|c|c|}
\hline SDG number & Sample number \\
\hline 0514260 & $\begin{array}{l}\text { 3113, 3046, 1231, 3148, 3113RE, } b \text { 3046RE, 1231RE, } \\
\text { 3148RE }\end{array}$ \\
\hline 0519260 & $3127,3139,3168$ \\
\hline 0722260 & $\begin{array}{l}2070,2059,2157,2090,2101,2120,2116,2080,2112 \text {, } \\
2143,2130,2149,2039\end{array}$ \\
\hline 0828260 & $3223,3225,3227,3229,3231,3235,3233-F D$ \\
\hline 0827260 & $3203,3206,3211,3214,3217,3209$ \\
\hline $0911260 / 0916260$ & $\begin{array}{l}1471,1474,1477,1480,1483,1487,1491,1493,1495 \text {, } \\
1497\end{array}$ \\
\hline $1015260 / 1016260 / 1020260 / 1023260$ & $1744,1746,1749,1738,1741,1734$ \\
\hline $1118260 / 1120260 / 1124260$ & $3358,3364,1891,1894,1897,1900,1903,1906$ \\
\hline $1204260 / 1209260 / 1211260$ & $\begin{array}{l}1964,1967,1970,1973,1976,1979,2257,2259,2262 \text {, } \\
2265,2268,2271,2273,2275,2277,2279,2281,2283 \text {, } \\
4086,4089,4092,4095,4105,4115,4124\end{array}$ \\
\hline $1216260 / 1217260 / 1218260$ & $4127,4129,4131,4133,4135,4137$ \\
\hline \multirow[t]{2}{*}{0108260} & 4227,4233 \\
\hline & Chlorinated Herbicides \\
\hline 0508260 & $1190,1201 F D, 1213$ \\
\hline 0042260 & $1072,1080,3003,1064$ \\
\hline 0424260 & $1099,1106,1107,1108,1115,3018$ \\
\hline 0430260 & 1127,3032 \\
\hline 0511260 & $3046,3058,3085,3099,3072$ \\
\hline 0828260 & $3223,3225,3227,3229,3231,3235,3223-F D$ \\
\hline 0827260 & $3203,3206,3211,3214,3217,3209$ \\
\hline $1015260 / 1016260 / 1020260 / 1023260$ & $1738,1741,1734$ \\
\hline $1118260 / 1120260 / 1124260$ & $3359,3365,1891,1897,1903,1964,1970,1976$ \\
\hline $\begin{array}{c}1204260 / 1209260 / 1211260 \\
\mid\end{array}$ & $\begin{array}{l}2257,2259,2262,2265,2268,2271,2273,2275,2277 \\
2279,2281,2283,4086,4092,4095,4105,4115,4124\end{array}$ \\
\hline 0108260 & 4228,4235 \\
\hline
\end{tabular}


Table F.1 (continued)

\begin{tabular}{|c|c|}
\hline SDG number & Sample number \\
\hline \multicolumn{2}{|c|}{ Polynuclear Aromatic Hydorcarbons } \\
\hline 0422260 & $\begin{array}{l}1064,1072,1080,3003,1009,1106,1107,1108,1115 \text {, } \\
3018\end{array}$ \\
\hline 0430260 & 1127,3032 \\
\hline 0508260 & $1190,1201 F D, 1213$ \\
\hline 0511260 & $3046,3058,3099,3072,3085$ \\
\hline 0514260 & 1231,3113 \\
\hline 0519260 & $3127,3139,3148,3168$ \\
\hline 0523260 & $\begin{array}{l}1292,1293,1294,1295,1296,1297,1298,1299,1300 \text {, } \\
1301,1302,1303\end{array}$ \\
\hline 0722260 & $\begin{array}{l}\text { 2157RE, 2101RE, 2070RE, 2080RE, 2039RE, 2120RE, } \\
2116 R E, 2112 R E, 2143 R E, 2130 R E, 2149 R E, 2059 R E \text {, } \\
2090\end{array}$ \\
\hline $072260 / 0723260$ & $\begin{array}{l}2116,2112,2143,2059,2090,2070,2080,2039,2120 \text {, } \\
2157,2149,2130,2101\end{array}$ \\
\hline 0828260 & $3223,3225,3227,3229,3231,3235,3233-F D$ \\
\hline 0827260 & $3203,3206,3211,3214,3217,3209$ \\
\hline $0911260 / 0916260$ & $\begin{array}{l}1471,1474,1477,1480,1483,1487,1491,1493,1495, \\
1497\end{array}$ \\
\hline $0924260 / 1002260 / 1009260$ & $1542,1544,1546,1548,1551,1553$ \\
\hline $1015260 / 1016260 / 1020260 / 1023260$ & $1744,1746,1749,1738,1741,1734$ \\
\hline $1118260 / 1120260 / 1124260$ & $3360,3366,1891,1894,1897,1900,1903,1906$ \\
\hline $1204260 / 1209260 / 1211260$ & $\begin{array}{l}1964,1967,1970,1973,1976,1979,2257,2259,2262 \text {, } \\
2265,2268,2271,2273,2275,2277,2279,2281,2283 \text {, } \\
4086,4089,4092,4095,4105,4115,4124\end{array}$ \\
\hline $1216260 / 1217260 / 1218260$ & $4127,4129,4131,4133,4135,4137$ \\
\hline 0108260 & 4226,4234 \\
\hline \multicolumn{2}{|c|}{ Uranium Isotopes by Alpha Spectroscopy } \\
\hline 2684 & 1469 \\
\hline 2633 & $\begin{array}{l}7002,7011,7020,7029,7005,7008,7014,7017,7023, \\
7026,7032,7035\end{array}$ \\
\hline 2391 & $5020,5023,5026$ \\
\hline 2419 & $5029,5038,6020,5032,5041,6023,5035,5044,6026$ \\
\hline 2423 & $6029,6038,6032,6041,6035,6044$ \\
\hline 2638 & 7038FD, 7041FD, 7044FD \\
\hline
\end{tabular}


Table F.1 (continued)

\begin{tabular}{|c|c|}
\hline SDG number & Sample number \\
\hline 2658 & $\begin{array}{l}5056,5065,5074,5083,5059,5062,5068,5071,5077 \text {, } \\
5080,5086,5089\end{array}$ \\
\hline 2847 & $6047,6050,6053$ \\
\hline 2924 & $5098,5092,5095$ \\
\hline 2970 & $5101,5103,5107$ \\
\hline 21003 & $5110,5113,5116$ \\
\hline 21046 & $5119,5122,5125$ \\
\hline 21366 & $5242,5243,5247,5250$ \\
\hline 21299 & $5206,5209,5212$ \\
\hline 21345 & $\begin{array}{l}7048,7049,7058,5230,5231,7052,7055,7061,7064 \\
5235,5238\end{array}$ \\
\hline 21123 & $5156,5157,5160,5163$ \\
\hline 21058 & $5128,5131,5134$ \\
\hline 21034 & $6074,6077,6065,6068,6071,6080$ \\
\hline 2878 & $6056,6059,6062$ \\
\hline 21081 & $5137,5146,5140,5143,5149,5152$ \\
\hline 21383 & $5254,5255,5265,5266,5258,5261,5269,5272$ \\
\hline 21328 & $5218,5219,5223,5226$ \\
\hline 21377 & $7067,7070,7073,7076,7079,7082$ \\
\hline 30044 & 4240 \\
\hline 21169 & $5167,5168,5175,5176,5171,5179$ \\
\hline 21205 & 5182,5185 \\
\hline 21247 & $5188,5191,5194$ \\
\hline 21262 & $5197,5200,5203$ \\
\hline 21232 & $6083,6085,6088,6091$ \\
\hline \multicolumn{2}{|c|}{ Neptunium-237 by Alpha Spectroscopy } \\
\hline 2658 & $5056,5065,5074,5083$ \\
\hline 2391 & 5020 \\
\hline 2684 & 1469 \\
\hline 2419 & $5029,5038,6020$ \\
\hline 2633 & $7002,7011,7020,7029$ \\
\hline 2638 & 7038FD \\
\hline 2423 & 6029,6038 \\
\hline
\end{tabular}


F-7

Table F.1 (continued)

\begin{tabular}{|c|c|}
\hline SDG number & Sample number \\
\hline 2970 & 5101 \\
\hline 21046 & 5119 \\
\hline 21366 & 5242,5243 \\
\hline 21299 & 5206 \\
\hline 21345 & $7048,7049,7058,5230,5231$ \\
\hline 21123 & 5156,5157 \\
\hline 21003 & 5110 \\
\hline 21058 & 5128 \\
\hline 21034 & 6074,6077 \\
\hline 2878 & 6056 \\
\hline 21081 & 5137,5146 \\
\hline 21383 & $5254,5255,5265,5266$ \\
\hline 21328 & 5218,5219 \\
\hline 2924 & 5092 \\
\hline 21377 & 7067,7076 \\
\hline 30044 & 4240 \\
\hline 21169 & $5167,5168,5175,5176$ \\
\hline 2847 & 6047 \\
\hline 21262 & 5197 \\
\hline 21232 & 6083,6085 \\
\hline \multicolumn{2}{|c|}{ Plutonium Isotopes by Alpha Spectroscopy } \\
\hline 2419 & $5029,5038,6020,5032,5041,6023,5035,5044,6026$ \\
\hline 2658 & $5056,5065,5074,5083$ \\
\hline 2638 & 7038FD \\
\hline 2391 & 5020 \\
\hline 2633 & $7002,7011,7020,7029$ \\
\hline 2684 & 1469 \\
\hline 2423 & 6029,6038 \\
\hline 2970 & 5101 \\
\hline 21046 & 5119 \\
\hline 21366 & 5242,5243 \\
\hline 21299 & 5206 \\
\hline
\end{tabular}


Table F.1 (continued)

\begin{tabular}{|c|c|}
\hline SDG number & Sample number \\
\hline 21345 & $7048,7049,7058,5230,5231$ \\
\hline 21123 & 5156,5157 \\
\hline 21003 & 5110 \\
\hline 21058 & 5128 \\
\hline 21034 & 6074,6077 \\
\hline 2878 & 6056 \\
\hline 21081 & 5137,5146 \\
\hline 21383 & $5254,5255,5265,5266$ \\
\hline 21328 & 5218,5219 \\
\hline 2924 & 5092 \\
\hline 21377 & 7067, 7076 \\
\hline 30044 & 4240 \\
\hline 21169 & $5167,5168,5175,5176$ \\
\hline 2847 & 6047 \\
\hline 21262 & 5197 \\
\hline 21232 & 6083,6085 \\
\hline \multicolumn{2}{|c|}{ Thorium Isotopes by Alpha Spectroscopy } \\
\hline 2684 & 1469 \\
\hline 2638 & 7038FD, 7041FD, 7044FD \\
\hline 2633 & $\begin{array}{l}\text { 7002, 7011, 7020, 7029, 7005, 7008, 7014, 7017, 7023, } \\
7026,7032,7035\end{array}$ \\
\hline 2658 & $\begin{array}{l}5056,5065,5074,5083,5059,5062,5068,5071,5077 \text {, } \\
5080,5086,5089\end{array}$ \\
\hline 2419 & $5029,5038,6020,5032,5041,6023,5035,5044,6026$ \\
\hline 2423 & $6029,6038,6032,6041,6035,6044$ \\
\hline 2970 & $5101,5103,5107$ \\
\hline 21046 & $5119,5122,5125$ \\
\hline 21366 & $5242,5243,5247,5250$ \\
\hline 21299 & $5206,5209,5212$ \\
\hline 21345 & $\begin{array}{l}7048,7049,7058,5230,5231,7052,7055,7061,7064 \\
5235,5238\end{array}$ \\
\hline 21123 & $5156,5157,5160,5163$ \\
\hline 21003 & $5110,5113,5116$ \\
\hline 21058 & $5128,5131,5134$ \\
\hline
\end{tabular}


Table F.1 (continued)

\begin{tabular}{|c|c|}
\hline SDG number & Sample number \\
\hline 21034 & $6074,6077,6065,6068,6071,6080,6071$ \\
\hline 2878 & $6056,6059,6062$ \\
\hline 21081 & $5137,5146,5140,5143,5149,5152$ \\
\hline 21383 & $5254,5255,5265,5266,5258,5261,5269,5272,5261$ \\
\hline 21328 & $5128,5129,5223,5226$ \\
\hline 2924 & $5092,5095,5098$ \\
\hline 21377 & $7067,7070,7073,7076,7079,7082$ \\
\hline 30044 & 4240 \\
\hline 21169 & $5167,5168,5175,5176,5171,5179$ \\
\hline 2847 & $6047,6050,6053$ \\
\hline 21247 & $5188,5191,5194$ \\
\hline 21205 & 5182,5185 \\
\hline 21262 & $5197,5200,5203$ \\
\hline 21232 & $6083,6085,6088,6091$ \\
\hline \multicolumn{2}{|c|}{ Strontium-89/90 by Gas Flow Proportional Counter } \\
\hline 2419 & $5029,5038,6020$ \\
\hline 2423 & 6029,6038 \\
\hline 2633 & $7002,7011,7020,7029$ \\
\hline 2638 & 7038FD \\
\hline 2658 & $5056,5065,5074,5083$ \\
\hline 2684 & 1469 \\
\hline 2970 & 5101 \\
\hline 21046 & 5119 \\
\hline 21366 & 5242,5243 \\
\hline 21299 & 5206 \\
\hline 21345 & $7048,7049,7058,5230,5231$ \\
\hline 21123 & 5156,5157 \\
\hline 21003 & 5110 \\
\hline 21058 & 5128 \\
\hline 21034 & 6074,6077 \\
\hline 2878 & 6056 \\
\hline 21081 & 5137,5146 \\
\hline
\end{tabular}


F-10

Table F.1 (continued)

\begin{tabular}{|c|c|}
\hline SDG number & Sample number \\
\hline 21328 & 5218,5219 \\
\hline 21383 & $5254,5255,5265,5266$ \\
\hline 2924 & 5092 \\
\hline 21377 & 7067,7076 \\
\hline 30044 & 4240 \\
\hline 21169 & $5167,5168,5175,5176$ \\
\hline 2847 & 6047 \\
\hline 21262 & 5197 \\
\hline \multirow[t]{2}{*}{21232} & 6083,6085 \\
\hline & Gamma Spectroscopy \\
\hline 2638 & 7038FD, 7041FD \\
\hline 2684 & 1469 \\
\hline 2658 & $\begin{array}{l}5056,5065,5074,5083,5059,5062,5068,5071,5077 \text {, } \\
5080,5086,5089\end{array}$ \\
\hline 2423 & $6029,6038,6032,6041,6035,6044$ \\
\hline 2633 & $\begin{array}{l}\text { 7002, 7011, 7020, 7029, 7005, 7008, 7014, 7014, 7017, } \\
7023,7026,7032,7035\end{array}$ \\
\hline 2419 & $5029,5038,6020,5032,5041,6023,5035,5044,6026$ \\
\hline 2970 & $5101,5103,5107$ \\
\hline 21046 & $5119,5122,5125$ \\
\hline 21366 & $5242,5243,5247,5250$ \\
\hline 21299 & $5206,5209,5212$ \\
\hline 21345 & $\begin{array}{l}7048,7049,7058,5230,5231,7052,7055,7061,5235 \text {, } \\
5238,7064\end{array}$ \\
\hline 21123 & $5156,5157,5160,5163$ \\
\hline 21003 & $5110,5113,5116$ \\
\hline 21058 & $5128,5131,5134$ \\
\hline 21205 & 5128,5185 \\
\hline 21247 & $5188,5191,5194$ \\
\hline 21034 & $6074,6077,6065,6068,6071,6080$ \\
\hline 2878 & $6056,6059,6062$ \\
\hline 21081 & $5137,5146,5140,5143,5149,5152$ \\
\hline 21383 & $5254,5255,5265,5266,5258,5261,5269,5272$ \\
\hline 21328 & $5218,5219,5232,5226$ \\
\hline
\end{tabular}


F-11

Table F.1 (continued)

\begin{tabular}{|c|c|}
\hline SDG number & Sample number \\
\hline 2924 & $5092,5093,5098$ \\
\hline 21377 & $7067,7070,7073,7076,7079,7082$ \\
\hline 30044 & 4240 \\
\hline 21169 & $5167,5168,5175,5176,5171,5179$ \\
\hline 2847 & $6047,6050,6053$ \\
\hline 21262 & $5197,5200,5203$ \\
\hline 21232 & $6083,6058,6088,6091$ \\
\hline \multicolumn{2}{|c|}{ Total Uranium by Pulse Laser Phosphorimetry } \\
\hline 2423 & $6029,6038,6020$ \\
\hline 2684 & 1469 \\
\hline 2658 & $5056,5065,5074,5083$ \\
\hline 2638 & $7038 \mathrm{FD}$ \\
\hline 2633 & $7002,7011,7020,7029$ \\
\hline 2970 & 5101 \\
\hline 21046 & 5119 \\
\hline 21366 & 5242,5243 \\
\hline 21299 & 5206 \\
\hline 21345 & $7048,7049,7058,5230,5231$ \\
\hline 21123 & 5156,5157 \\
\hline 21003 & 5110 \\
\hline 21058 & 5128 \\
\hline 21034 & 6074,6077 \\
\hline 2878 & 6056 \\
\hline 21081 & 5137,5146 \\
\hline 21383 & $5254,5255,5265,5266$ \\
\hline 21328 & 5218,5219 \\
\hline 2924 & 5092 \\
\hline 21377 & 7067,7076 \\
\hline 30044 & 4240 \\
\hline 21169 & $5167,5168,5175,5176$ \\
\hline 2847 & 6047 \\
\hline 21262 & 5197 \\
\hline
\end{tabular}


F-12

Table F.1 (continued)

\begin{tabular}{|c|c|}
\hline SDG number & Sample number \\
\hline 21232 & 6083,6085 \\
\hline \multicolumn{2}{|c|}{ Curium-243 by Alpha Spectroscopy } \\
\hline 2970 & 5101 \\
\hline 21046 & 5119 \\
\hline 21366 & 5242,5243 \\
\hline 21299 & 5206 \\
\hline 21345 & $7048,7049,7058,5230,5231$ \\
\hline 21123 & 5156,5157 \\
\hline 21003 & 5110 \\
\hline 21058 & 5128 \\
\hline 21034 & 6074,6077 \\
\hline 2878 & 6056 \\
\hline 21081 & 5137,5146 \\
\hline 21383 & $5254,5255,5265,5266$ \\
\hline 21328 & 5218,5219 \\
\hline 2924 & 5092 \\
\hline 21377 & 7067, 7076 \\
\hline 21169 & $5167,5168,5175,5176$ \\
\hline 30044 & 4240 \\
\hline 21262 & 5197 \\
\hline 21232 & 6083,6085 \\
\hline 2847 & 6047 \\
\hline \multicolumn{2}{|r|}{ Technetium-99 } \\
\hline 2419 & $5029,5038,6020$ \\
\hline 2684 & 1469 \\
\hline $2419 / 2423$ & $6029,6038,5029,5038,6020$ \\
\hline $2638 / 2633 / 2658$ & $7002,7011,7020,7029,7038 \mathrm{FD}, 5056,5065,5074,5083$ \\
\hline 30584 & $\begin{array}{l}4253,4254,4255,4256,4257,4258,4259,4260,4261 \\
4262,4263,4264,4265,4266\end{array}$ \\
\hline \multirow[t]{2}{*}{30625} & $\begin{array}{l}4267,4268,4269,4270,4271,4272,4273,4274,4275 \\
4276,4277,4278,4279,6093\end{array}$ \\
\hline & $\begin{array}{l}\text { 6094, 6095, 6096, 6097, 6098, 7084, 7085, 7086, 7087, } \\
7088,7088-F D\end{array}$ \\
\hline
\end{tabular}




\section{F-13}

Table F.1 (continued)

\begin{tabular}{ll}
\hline SDG number & Sample number \\
\hline 30670 & $4280,4281,4282,4283,4284,4285,4286,4287,4288$, \\
& $4289,4290,4291,4292$ \\
& \multicolumn{1}{c}{ Tritium } \\
2369 & $1063,1071,1079,1122,1125,3004,3031,1123,1124$, \\
& $1126,1128,3016$ \\
2391 & $1180,1189,1198 \mathrm{FD}, 1214$ \\
2399 & $3071,3084,3098,3045,3057,3082$ \\
30044 & $4216,4217,4218,4219,4220,4221$ \\
2970 & 1549 \\
21299 & $1965,1968,1971,1974,1977,1980$ \\
21345 & $4087,4090,4093,4096,4106,4116,2260,2263,2266$, \\
21003 & 2269 \\
21081 & 1607 \\
21205 & 1747,1739 \\
21247 & $3327,3328,3329,3330$ \\
2858 & $1892,1895,1898,1901,1904,1907$ \\
\hline
\end{tabular}

$a_{\mathrm{FD}}=$ field duplicate sample.

$b_{\mathrm{RE}}=$ reanalyzed sample. 
Appendix G

SUMMARY OF STATISTICALLY TREATED DATA AND THE SIGNIFICANCE LEVEL OF DIFFERENCES IN THE DATA 
.

, 


\section{NOTE ON SIGNIFICANCE LEVELS}

Chi-square likelihood ratio tests for comparing formation-locations are discussed in Sect. 5.2.3. The following notation is used in Tables G.3 through G.5 to denote significance levels for these tests:

P-ALL for testing that all formation-locations and all sampling areas are the same against the alternative that they may all differ.

P-DG for testing that all Dismal Gap locations are the same against the alternative that they may all differ.

P-CR for testing that all Copper Ridge locations are the same against the alternative that they may all differ.

P-ORR for testing that all ORR formation-locations are the same against the alternative that they may all differ.

P-CHICK for testing that the two Chickamauga locations are the same against the alternative that they may differ.

P-DGN for testing that the ORR Dismal Gap and Nolichucky formations are the same against the alternative that they may differ.

P-CRC for testing that the ORR Copper Ridge and Chepultepec formations are the same against the alternative that they may differ.

P-GRP for testing that on the ORR, the Chickamauga, Knox, and Conasauga groups are all the same, against the alternative that these groups may differ, although formations within them may not. 


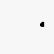

- 


\section{G-5}

Table G.1. Summary statistics for NAA data ${ }^{a}$

(Estimates and confidence bounds are in milligrams per kilogram.)

\begin{tabular}{|c|c|c|c|c|c|c|c|c|}
\hline Horizon & $\begin{array}{l}\text { Formation- } \\
\text { location }\end{array}$ & $\mathbf{N}$ & I & D & Median & UCB95 & X95 & LTB9595 \\
\hline \multicolumn{9}{|c|}{ ALUMNUM } \\
\hline $\begin{array}{l}A \\
A \\
A \\
A \\
A \\
A \\
A \\
A \\
A \\
A\end{array}$ & $\begin{array}{l}\text { DG-AND } \\
\text { DG-ROA } \\
\text { DG-ORR } \\
\text { NL-ORR } \\
\text { CHI -BV } \\
\text { CHI-K25 } \\
\text { CHE-ORR } \\
\text { CR-ORR } \\
\text { CR-AND } \\
\text { CR-ROA }\end{array}$ & $\begin{array}{l}4 \\
4 \\
4 \\
4 \\
4 \\
4 \\
4 \\
4 \\
4 \\
4\end{array}$ & $\begin{array}{l}0 \\
0 \\
0 \\
0 \\
0 \\
0 \\
0 \\
0 \\
0 \\
0\end{array}$ & $\begin{array}{l}4 \\
4 \\
4 \\
4 \\
4 \\
4 \\
4 \\
4 \\
4 \\
3\end{array}$ & $\begin{array}{l}68000 \\
53600 \\
31000 \\
53400 \\
39400 \\
31400 \\
17000 \\
23100 \\
25700 \\
2820\end{array}$ & $\begin{array}{r}169000 \\
133000 \\
76900 \\
132000 \\
97700 \\
78000 \\
42100 \\
57200 \\
63800 \\
7030\end{array}$ & $\begin{array}{r}419000 \\
330000 \\
191000 \\
329000 \\
243000 \\
194000 \\
105000 \\
142000 \\
158000 \\
17400\end{array}$ & $\begin{array}{r}159000 \\
125000 \\
72300 \\
124000 \\
91800 \\
73300 \\
39600 \\
53800 \\
60000 \\
6590\end{array}$ \\
\hline $\begin{array}{l}\text { B } \\
\text { B } \\
\text { B } \\
\text { B } \\
\text { B } \\
\text { B } \\
\text { B } \\
\text { B } \\
\text { B } \\
\text { B }\end{array}$ & $\begin{array}{l}\text { DG-AND } \\
\text { DG-ROA } \\
\text { DG-ORR } \\
\text { NL-ORR } \\
\text { CHI -BV } \\
\text { CHI-K25 } \\
\text { CHE-ORR } \\
\text { CR-ORR } \\
\text { CR-AND } \\
\text { CR-ROA }\end{array}$ & $\begin{array}{l}4 \\
4 \\
4 \\
4 \\
4 \\
4 \\
4 \\
4 \\
4 \\
4\end{array}$ & $\begin{array}{l}0 \\
0 \\
0 \\
0 \\
0 \\
0 \\
0 \\
0 \\
0 \\
0\end{array}$ & $\begin{array}{l}4 \\
4 \\
4 \\
4 \\
4 \\
4 \\
4 \\
4 \\
4 \\
4\end{array}$ & $\begin{array}{r}92300 \\
78900 \\
79100 \\
89900 \\
80100 \\
113000 \\
59400 \\
52200 \\
53600 \\
42700\end{array}$ & $\begin{array}{r}105000 \\
89500 \\
89700 \\
102000 \\
90800 \\
128000 \\
67400 \\
59200 \\
60800 \\
48400\end{array}$ & $\begin{array}{r}119000 \\
101000 \\
102000 \\
116000 \\
103000 \\
145000 \\
76400 \\
67100 \\
69000 \\
54900\end{array}$ & $\begin{array}{r}104000 \\
88800 \\
89000 \\
101000 \\
90100 \\
127000 \\
66800 \\
58700 \\
60300 \\
48000\end{array}$ \\
\hline $\begin{array}{l}c \\
c \\
c \\
c \\
c \\
c \\
c \\
c \\
c \\
c\end{array}$ & $\begin{array}{l}\text { DG-AND } \\
\text { DG-ROA } \\
\text { DG-ORR } \\
\text { NL-ORR } \\
\text { CHI-BV } \\
\text { CHI-K25 } \\
\text { CHE-ORR } \\
\text { CR-ORR } \\
\text { CR-AND } \\
\text { CR-ROA }\end{array}$ & $\begin{array}{l}4 \\
4 \\
4 \\
4 \\
4 \\
4 \\
4 \\
4 \\
3 \\
4\end{array}$ & $\begin{array}{l}0 \\
0 \\
0 \\
0 \\
0 \\
0 \\
0 \\
0 \\
0 \\
0\end{array}$ & $\begin{array}{l}4 \\
4 \\
4 \\
4 \\
4 \\
4 \\
4 \\
4 \\
3 \\
4\end{array}$ & $\begin{array}{r}102000 \\
98900 \\
94000 \\
95800 \\
94600 \\
112000 \\
73400 \\
88100 \\
69800 \\
56000\end{array}$ & $\begin{array}{r}140000 \\
136000 \\
129000 \\
131000 \\
130000 \\
153000 \\
101000 \\
121000 \\
101000 \\
76800\end{array}$ & $\begin{array}{l}192000 \\
186000 \\
177000 \\
180000 \\
178000 \\
210000 \\
138000 \\
166000 \\
131000 \\
105000\end{array}$ & $\begin{array}{r}137000 \\
133000 \\
126000 \\
129000 \\
127000 \\
150000 \\
98500 \\
118000 \\
89500 \\
75200\end{array}$ \\
\hline
\end{tabular}

\section{ANTIMONY}

$\begin{array}{lllllllll}\text { A } & \text { DG-AND } & 4 & 0 & 4 & 0.439 & 0.500 & 0.569 & 0.496 \\ \text { A } & \text { DG-ROA } & 4 & 0 & 4 & 0.532 & 0.606 & 0.690 & 0.601 \\ \text { A } & \text { DG-ORR } & 4 & 0 & 4 & 0.474 & 0.540 & 0.615 & 0.536 \\ \text { A } & \text { HL-ORR } & 4 & 0 & 4 & 0.509 & 0.580 & 0.661 & 0.575 \\ \text { A } & \text { CHI-BV } & 4 & 0 & 4 & 0.576 & 0.656 & 0.748 & 0.651 \\ \text { A } & \text { CHI-K25 } & 4 & 0 & 4 & 0.708 & 0.807 & 0.919 & 0.800 \\ \text { A } & \text { CHE-ORR } & 4 & 0 & 4 & 0.611 & 0.696 & 0.793 & 0.690 \\ \text { A } & \text { CR-ORR } & 4 & 0 & 4 & 0.865 & 0.986 & 1.120 & 0.977 \\ \text { A } & \text { CR-AND } & 3 & 0 & 3 & 0.764 & 0.888 & 0.991 & 0.846 \\ \text { A } & \text { CR-ROA } & 4 & 0 & 4 & 0.749 & 0.853 & 0.971 & 0.845 \\ \text { B } & \text { DG-AND } & 4 & 0 & 3 & 0.331 & 0.467 & 0.653 & 0.454 \\ \text { B } & \text { DG-ROA } & 4 & 0 & 4 & 0.528 & 0.741 & 1.040 & 0.723 \\ \text { B } & \text { DG-ORR } & 4 & 1 & 3 & 0.267 & 0.377 & 0.526 & 0.364 \\ \text { B } & \text { NL-ORR } & 4 & 0 & 4 & 0.426 & 0.598 & 0.839 & 0.583 \\ \text { B } & \text { CHI-BV } & 4 & 0 & 4 & 0.377 & 0.530 & 0.744 & 0.517 \\ \text { B } & \text { CHI-K25 } & 3 & 0 & 3 & 0.707 & 1.050 & 1.390 & 0.922 \\ \text { B } & \text { CHE-ORR } & 4 & 0 & 4 & 0.979 & 1.370 & 1.930 & 1.340 \\ \text { B } & \text { CR-ORR } & 4 & 0 & 4 & 1.420 & 1.990 & 2.790 & 1.940 \\ \text { B } & \text { CR-AND } & 4 & 0 & 4 & 1.130 & 1.580 & 2.220 & 1.540 \\ \text { B } & \text { CR-ROA } & 4 & 0 & 4 & 0.985 & 1.380 & 1.940 & 1.350 \\ \text { C } & \text { DG-AND } & 4 & 0 & 2 & 0.123 & 0.170 & 0.213 & 0.154 \\ \text { C } & \text { DG-ROA } & 4 & 1 & 3 & 0.333 & 0.440 & 0.578 & 0.430 \\ \text { C } & \text { DG-ORR } & 4 & 1 & 3 & 0.261 & 0.346 & 0.453 & 0.336 \\ \text { C } & \text { NL-ORR } & 4 & 0 & 4 & 0.367 & 0.484 & 0.638 & 0.475 \\ \text { C } & \text { CHI-BV } & 4 & 0 & 4 & 0.447 & 0.589 & 0.776 & 0.578 \\ \text { C } & \text { CHI-K25 } & 4 & 0 & 4 & 0.620 & 0.817 & 1.080 & 0.801 \\ \text { C } & \text { CHE-ORR } & 4 & 0 & 4 & 0.935 & 1.230 & 1.620 & 1.210 \\ \text { C } & \text { CR-ORR } & 4 & 0 & 4 & 1.910 & 2.510 & 3.310 & 2.470 \\ \text { C } & \text { CR-AND } & 4 & 0 & 4 & 0.940 & 1.240 & 1.630 & 1.210 \\ \text { C } & \text { CR-ROA } & 4 & 0 & 4 & 1.490 & 1.970 & 2.590 & 1.930 \\ & & & & & & & & \end{array}$


G-6

Table G.1 (continued)

\begin{tabular}{|c|c|c|c|c|c|c|c|c|}
\hline Horizon & $\begin{array}{l}\text { Formation- } \\
\text { location }\end{array}$ & $\mathbf{N}$ & I & $\mathrm{D}$ & Median & UCB95 & X95 & LTB9595 \\
\hline \multicolumn{9}{|c|}{ ARSENIC } \\
\hline $\begin{array}{l}A \\
A \\
A \\
A \\
A \\
A \\
A \\
A \\
A \\
A\end{array}$ & $\begin{array}{l}\text { DG-AND } \\
\text { DG-ROA } \\
\text { DG-ORR } \\
\text { NL-ORR } \\
\text { CHI -BV } \\
\text { CHI-K25 } \\
\text { CHE-ORR } \\
\text { CR-ORR } \\
\text { CR-AND } \\
\text { CR-ROA }\end{array}$ & $\begin{array}{l}4 \\
4 \\
4 \\
4 \\
4 \\
4 \\
4 \\
4 \\
3 \\
4\end{array}$ & $\begin{array}{l}0 \\
0 \\
0 \\
0 \\
0 \\
0 \\
0 \\
0 \\
0 \\
0\end{array}$ & $\begin{array}{l}4 \\
4 \\
4 \\
4 \\
4 \\
4 \\
4 \\
4 \\
3 \\
4\end{array}$ & $\begin{array}{r}4.67 \\
5.68 \\
5.18 \\
5.49 \\
8.32 \\
8.54 \\
7.85 \\
20.30 \\
14.60 \\
10.60\end{array}$ & $\begin{array}{r}5.76 \\
7.00 \\
6.38 \\
6.76 \\
10.30 \\
10.50 \\
9.68 \\
25.00 \\
18.60 \\
13.00\end{array}$ & $\begin{array}{r}7.09 \\
8.63 \\
7.86 \\
8.33 \\
12.60 \\
13.00 \\
11.90 \\
30.80 \\
22.20 \\
16.00\end{array}$ & $\begin{array}{r}5.68 \\
6.91 \\
6.29 \\
6.67 \\
10.10 \\
10.40 \\
9.54 \\
24.60 \\
17.20 \\
12.80\end{array}$ \\
\hline $\begin{array}{l}\text { B } \\
\text { B } \\
\text { B } \\
\text { B } \\
\text { B } \\
\text { B } \\
\text { B } \\
\text { B } \\
\text { B } \\
\text { B }\end{array}$ & $\begin{array}{l}\text { DG-AND } \\
\text { DG-ROA } \\
\text { DG-ORR } \\
\text { NL-ORR } \\
\text { CHI-BV } \\
\text { CHI-K25 } \\
\text { CHE-ORR } \\
\text { CR-ORR } \\
\text { CR-AND } \\
\text { CR-ROA }\end{array}$ & $\begin{array}{l}4 \\
4 \\
4 \\
4 \\
4 \\
3 \\
4 \\
4 \\
4 \\
4\end{array}$ & $\begin{array}{l}0 \\
0 \\
0 \\
0 \\
0 \\
0 \\
0 \\
0 \\
0 \\
0\end{array}$ & $\begin{array}{l}4 \\
4 \\
4 \\
4 \\
4 \\
3 \\
4 \\
4 \\
4 \\
4\end{array}$ & $\begin{array}{r}5.73 \\
6.53 \\
5.12 \\
6.96 \\
11.30 \\
9.82 \\
20.60 \\
48.40 \\
12.90 \\
16.20\end{array}$ & $\begin{array}{r}7.56 \\
8.62 \\
6.76 \\
9.18 \\
14.90 \\
13.50 \\
27.20 \\
63.90 \\
17.00 \\
21.30\end{array}$ & $\begin{array}{r}9.97 \\
11.40 \\
8.92 \\
12.10 \\
19.60 \\
17.10 \\
35.90 \\
84.40 \\
22.40 \\
28.10\end{array}$ & $\begin{array}{r}7.42 \\
8.46 \\
6.63 \\
9.01 \\
14.60 \\
12.20 \\
26.70 \\
62.70 \\
16.70 \\
20.90\end{array}$ \\
\hline $\begin{array}{l}C \\
C \\
C \\
C \\
C \\
C \\
C \\
C \\
C \\
C\end{array}$ & $\begin{array}{l}\text { DG-AND } \\
\text { DG-ROA } \\
\text { DG-ORR } \\
\text { NL-ORR } \\
\text { CHI -BV } \\
\text { CHI-K25 } \\
\text { CHE-ORR } \\
\text { CR-ORR } \\
\text { CR-AND } \\
\text { CR-ROA }\end{array}$ & $\begin{array}{l}4 \\
4 \\
4 \\
4 \\
4 \\
4 \\
4 \\
4 \\
4 \\
4\end{array}$ & $\begin{array}{l}0 \\
0 \\
0 \\
0 \\
0 \\
0 \\
0 \\
0 \\
0 \\
0\end{array}$ & $\begin{array}{l}4 \\
4 \\
4 \\
4 \\
4 \\
4 \\
4 \\
4 \\
4 \\
4\end{array}$ & $\begin{array}{r}3.93 \\
5.61 \\
4.63 \\
6.05 \\
11.80 \\
8.00 \\
23.60 \\
79.20 \\
27.80 \\
32.70\end{array}$ & $\begin{array}{r}4.92 \\
7.02 \\
5.80 \\
7.58 \\
14.80 \\
10.00 \\
29.50 \\
99.10 \\
34.80 \\
40.90\end{array}$ & $\begin{array}{r}6.16 \\
8.78 \\
7.25 \\
9.48 \\
18.50 \\
12.50 \\
36.90 \\
124.00 \\
43.60 \\
51.20\end{array}$ & $\begin{array}{r}4.85 \\
6.92 \\
5.71 \\
7.47 \\
14.60 \\
9.87 \\
29.10 \\
97.70 \\
34.30 \\
40.30\end{array}$ \\
\hline
\end{tabular}

\section{BARIUM}

$\begin{array}{rlrlrrrrr}\text { A } & \text { DG-AND } & 4 & 0 & 4 & 404.0 & 1150 & 3300 & 1080 \\ \text { A } & \text { DG-ROA } & 4 & 0 & 4 & 3450.0 & 9860 & 28200 & 9210 \\ \text { A } & \text { DG-ORR } & 4 & 0 & 4 & 123.0 & 350 & 1000 & 327 \\ \text { A } & \text { HL-ORR } & 4 & 0 & 4 & 1280.0 & 3650 & 10400 & 3410 \\ \text { A } & \text { CHI-BV } & 4 & 0 & 4 & 7950.0 & 22700 & 64900 & 21200 \\ \text { A } & \text { CHI-K25 } & 4 & 0 & 4 & 202.0 & 578 & 1650 & 540 \\ \text { A } & \text { CHE-ORR } & 4 & 0 & 4 & 380.0 & 1080 & 3100 & 1010 \\ \text { A } & \text { CR-ORR } & 4 & 0 & 4 & 265.0 & 756 & 2160 & 706 \\ \text { A } & \text { CR-AND } & 4 & 0 & 4 & 307.0 & 876 & 2500 & 818 \\ \text { A } & \text { CR-ROA } & 4 & 0 & 4 & 67.5 & 193 & 551 & 180 \\ & & & & & & & & \\ \text { B } & \text { DG-AND } & 4 & 0 & 4 & 375.0 & 744 & 1480 & 711 \\ \text { B } & \text { DG-ROA } & 4 & 0 & 4 & 7010.0 & 13900 & 27600 & 13300 \\ \text { B } & \text { DG-ORR } & 4 & 0 & 4 & 235.0 & 465 & 923 & 445 \\ \text { B } & \text { NL-ORR } & 4 & 0 & 4 & 806.0 & 1600 & 3170 & 1530 \\ \text { B } & \text { CHI-BV } & 4 & 0 & 4 & 6160.0 & 12200 & 24300 & 11700 \\ \text { B } & \text { CHI-K25 } & 4 & 0 & 4 & 317.0 & 628 & 1250 & 601 \\ \text { B } & \text { CHE-ORR } & 4 & 0 & 4 & 251.0 & 498 & 988 & 476 \\ \text { B } & \text { CR-ORR } & 4 & 0 & 4 & 82.3 & 163 & 324 & 156 \\ \text { B } & \text { CR-AND } & 4 & 0 & 4 & 118.0 & 234 & 463 & 223 \\ \text { B } & \text { CR-ROA } & 4 & 0 & 4 & 123.0 & 244 & 484 & 233 \\ & & & & & & & & \\ \text { C } & \text { DG-AND } & 4 & 0 & 4 & 333.0 & 765 & 1760 & 725 \\ \text { C } & \text { DG-ROA } & 4 & 0 & 4 & 4130.0 & 9500 & 21800 & 9000 \\ \text { C } & \text { DG-ORR } & 4 & 0 & 4 & 244.0 & 559 & 1280 & 530 \\ \text { C } & \text { NL-ORR } & 4 & 0 & 4 & 2810.0 & 6450 & 14800 & 6110 \\ \text { C } & \text { CHI-BV } & 4 & 0 & 4 & 2920.0 & 6710 & 15400 & 6350 \\ \text { C } & \text { CHI-K25 } & 4 & 0 & 4 & 354.0 & 814 & 1870 & 771 \\ \text { C } & \text { CHE-ORR } & 4 & 0 & 4 & 510.0 & 1170 & 2690 & 1110 \\ \text { C } & \text { CR-ORR } & 4 & 0 & 4 & 124.0 & 286 & 657 & 271 \\ \text { C } & \text { CR-AND } & 4 & 0 & 4 & 149.0 & 343 & 788 & 325 \\ \text { C } & \text { CR-ROA } & 4 & 0 & 4 & 71.6 & 164 & 378 & 156\end{array}$


Table G.1 (continued)

\begin{tabular}{|c|c|c|c|c|c|c|c|c|}
\hline Horizon & $\begin{array}{l}\text { Formation- } \\
\text { location }\end{array}$ & $\mathbf{N}$ & I & $\mathrm{D}$ & Median & UCB95 & X95 & LTB959 \\
\hline \multicolumn{9}{|c|}{ CERIUM } \\
\hline $\begin{array}{l}A \\
A \\
A \\
A \\
A \\
A \\
A \\
A \\
A \\
A\end{array}$ & $\begin{array}{l}\text { DG-AND } \\
\text { DG-ROA } \\
\text { DG-ORR } \\
\text { NL-ORR } \\
\text { CHI-BV } \\
\text { CHI-K25 } \\
\text { CHE-ORR } \\
\text { CR-ORR } \\
\text { CR-AND } \\
\text { CR-ROA }\end{array}$ & $\begin{array}{l}3 \\
4 \\
4 \\
4 \\
4 \\
4 \\
4 \\
4 \\
4 \\
4\end{array}$ & $\begin{array}{l}0 \\
0 \\
0 \\
0 \\
0 \\
0 \\
0 \\
0 \\
0 \\
0\end{array}$ & $\begin{array}{l}3 \\
4 \\
4 \\
4 \\
4 \\
4 \\
4 \\
4 \\
4 \\
4\end{array}$ & $\begin{array}{l}55.8 \\
57.8 \\
48.0 \\
54.6 \\
71.0 \\
63.4 \\
32.0 \\
46.1 \\
56.1 \\
34.5\end{array}$ & $\begin{array}{l}68.4 \\
68.9 \\
57.1 \\
65.1 \\
84.6 \\
75.5 \\
38.2 \\
54.9 \\
66.8 \\
41.1\end{array}$ & $\begin{array}{r}79.3 \\
82.1 \\
68.1 \\
77.5 \\
101.0 \\
90.0 \\
45.5 \\
65.4 \\
79.6 \\
: 49.0\end{array}$ & $\begin{array}{l}64.1 \\
68.1 \\
56.5 \\
64.3 \\
83.6 \\
74.7 \\
37.7 \\
54.2 \\
66.0 \\
40.7\end{array}$ \\
\hline $\begin{array}{l}\text { B } \\
\text { B } \\
\text { B } \\
\text { B } \\
\text { B } \\
\text { B } \\
\text { B } \\
\text { B } \\
\text { B } \\
\text { B }\end{array}$ & $\begin{array}{l}\text { DG-AND } \\
\text { DG-ROA } \\
\text { DG-ORR } \\
\text { NL-ORR } \\
\text { CHI-BV } \\
\text { CHI-K25 } \\
\text { CHE-ORR } \\
\text { CR-ORR } \\
\text { CR-AND } \\
\text { CR-ROA }\end{array}$ & $\begin{array}{l}4 \\
4 \\
4 \\
4 \\
4 \\
4 \\
4 \\
4 \\
4 \\
4\end{array}$ & $\begin{array}{l}0 \\
0 \\
0 \\
0 \\
0 \\
0 \\
0 \\
0 \\
0 \\
0\end{array}$ & $\begin{array}{l}4 \\
4 \\
4 \\
4 \\
4 \\
4 \\
4 \\
4 \\
4 \\
4\end{array}$ & $\begin{array}{l}70.4 \\
69.2 \\
65.5 \\
64.3 \\
89.3 \\
76.9 \\
30.6 \\
35.0 \\
32.3 \\
23.2\end{array}$ & $\begin{array}{r}90.4 \\
88.8 \\
84.0 \\
82.6 \\
115.0 \\
98.7 \\
39.2 \\
45.0 \\
41.4 \\
29.7\end{array}$ & $\begin{array}{r}116.0 \\
114.0 \\
108.0 \\
106.0 \\
147.0 \\
127.0 \\
50.3 \\
57.7 \\
53.1 \\
38.2\end{array}$ & $\begin{array}{r}88.9 \\
87.4 \\
82.7 \\
81.2 \\
113.0 \\
97.1 \\
38.6 \\
44.2 \\
40.7 \\
29.3\end{array}$ \\
\hline $\begin{array}{l}c \\
C \\
c \\
C \\
C \\
C \\
C \\
C \\
C \\
C\end{array}$ & $\begin{array}{l}\text { DG-AND } \\
\text { DG-ROA } \\
\text { DG-ORR } \\
\text { NL-ORR } \\
\text { CHI-BV } \\
\text { CHI-K25 } \\
\text { CHE-ORR } \\
\text { CR-ORR } \\
\text { CR-AND } \\
\text { CR-ROA }\end{array}$ & $\begin{array}{l}4 \\
4 \\
4 \\
3 \\
4 \\
4 \\
4 \\
4 \\
4 \\
4\end{array}$ & $\begin{array}{l}0 \\
0 \\
0 \\
0 \\
0 \\
0 \\
0 \\
0 \\
0 \\
0\end{array}$ & $\begin{array}{l}4 \\
4 \\
4 \\
3 \\
4 \\
4 \\
4 \\
4 \\
4 \\
4\end{array}$ & $\begin{array}{r}90.8 \\
81.0 \\
64.0 \\
95.4 \\
107.0 \\
90.5 \\
34.6 \\
46.8 \\
35.3 \\
29.9\end{array}$ & $\begin{array}{r}118.0 \\
105.0 \\
82.9 \\
129.0 \\
138.0 \\
117.0 \\
44.8 \\
60.6 \\
45.7 \\
38.7\end{array}$ & $\begin{array}{r}152.0 \\
136.0 \\
107.0 \\
160.0 \\
179.0 \\
152.0 \\
58.0 \\
78.4 \\
59.1 \\
50.1\end{array}$ & $\begin{array}{r}115.0 \\
103.0 \\
81.4 \\
117.0 \\
136.0 \\
115.0 \\
44.0 \\
59.5 \\
44.9 \\
38.0\end{array}$ \\
\hline
\end{tabular}

\section{CESIUM}

$\begin{array}{lllllllll}\text { A } & \text { DG-AND } & 3 & 0 & 3 & 3.15 & 3.67 & 4.10 & 3.49 \\ \text { A } & \text { DG-ROA } & 4 & 0 & 4 & 2.89 & 3.30 & 3.77 & 3.27 \\ \text { A } & \text { DG-ORR } & 4 & 0 & 4 & 2.68 & 3.06 & 3.50 & 3.04 \\ \text { A } & \text { NL-ORR } & 4 & 0 & 4 & 2.95 & 3.37 & 3.85 & 3.34 \\ \text { A } & \text { CHI-BV } & 4 & 0 & 4 & 2.58 & 2.94 & 3.36 & 2.92 \\ \text { A } & \text { CHI-K25 } & 4 & 0 & 4 & 3.11 & 3.55 & 4.05 & 3.52 \\ \text { A } & \text { CHE-ORR } & 4 & 0 & 4 & 1.42 & 1.62 & 1.85 & 1.61 \\ \text { A } & \text { CR-ORR } & 4 & 0 & 4 & 2.09 & 2.39 & 2.73 & 2.37 \\ \text { A } & \text { CR-AND } & 4 & 0 & 4 & 2.02 & 2.31 & 2.64 & 2.29 \\ \text { A } & \text { CR-ROA } & 4 & 0 & 4 & 1.88 & 2.14 & 2.45 & 2.12 \\ & & & & & & & & \\ \text { B } & \text { DG-AND } & 4 & 0 & 4 & 4.78 & 5.37 & 6.04 & 5.33 \\ \text { B } & \text { DG-ROA } & 4 & 0 & 4 & 4.44 & 4.99 & 5.60 & 4.95 \\ \text { B } & \text { DG-ORR } & 4 & 0 & 4 & 4.59 & 5.15 & 5.79 & 5.11 \\ \text { B } & \text { HL-ORR } & 4 & 0 & 4 & 5.17 & 5.81 & 6.53 & 5.77 \\ \text { B } & \text { CHI-BV } & 4 & 0 & 4 & 5.63 & 6.33 & 7.11 & 6.28 \\ \text { B } & \text { CHI-K25 } & 4 & 0 & 4 & 7.28 & 8.18 & 9.19 & 8.12 \\ \text { B } & \text { CHE-ORR } & 4 & 0 & 4 & 4.88 & 5.48 & 6.16 & 5.44 \\ \text { B } & \text { CR-ORR } & 4 & 0 & 4 & 4.85 & 5.45 & 6.12 & 5.41 \\ \text { B } & \text { CR-AND } & 4 & 0 & 4 & 3.96 & 4.45 & 5.00 & 4.42 \\ \text { B } & \text { CR-ROA } & 4 & 0 & 4 & 2.88 & 3.24 & 3.64 & 3.22 \\ & \text { CR-R } & & & & & & & \\ \text { C } & \text { DG-AND } & 4 & 0 & 4 & 4.41 & 4.92 & 5.49 & 4.89 \\ \text { C } & \text { DG-ROA } & 4 & 0 & 4 & 5.50 & 6.14 & 6.85 & 6.10 \\ \text { C } & \text { DG-ORR } & 4 & 0 & 4 & 4.51 & 5.03 & 5.61 & 4.99 \\ \text { C } & \text { NL-ORR } & 3 & 0 & 3 & 6.81 & 7.73 & 8.48 & 7.42 \\ \text { C } & \text { CHI-BV } & 4 & 0 & 4 & 6.06 & 6.76 & 7.54 & 6.71 \\ \text { C } & \text { CHI-K25 } & 4 & 0 & 4 & 7.38 & 8.24 & 9.19 & 8.18 \\ \text { C } & \text { CHE-ORR } & 4 & 0 & 4 & 4.67 & 5.21 & 5.82 & 5.18 \\ \text { C } & \text { CR-ORR } & 4 & 0 & 4 & 4.93 & 5.50 & 6.13 & 5.46 \\ \text { C } & \text { CR-AND } & 4 & 0 & 4 & 4.14 & 4.61 & 5.15 & 4.58 \\ \text { C } & \text { CR-ROA } & 4 & 0 & 4 & 4.14 & 4.62 & 5.15 & 4.59 \\ & & & & & & & & \end{array}$


G-8

Table G.1 (continued)

\begin{tabular}{|c|c|c|c|c|c|c|c|c|}
\hline Horizon & $\begin{array}{l}\text { Formation- } \\
\text { location }\end{array}$ & $\mathbf{N}$ & I & D & Median & UCB95 & X95 & LTB959: \\
\hline \multicolumn{9}{|c|}{ CHROMIUM } \\
\hline $\begin{array}{l}A \\
A \\
A \\
A \\
A \\
A \\
A \\
A \\
A \\
A\end{array}$ & $\begin{array}{l}\text { DG-AND } \\
\text { DG-ROA } \\
\text { DG-ORR } \\
\text { NL-ORR } \\
\text { CHI -BV } \\
\text { CHI - K25 } \\
\text { CHE-ORR } \\
\text { CR-ORR } \\
\text { CR-AND } \\
\text { CR-ROA }\end{array}$ & $\begin{array}{l}3 \\
4 \\
4 \\
4 \\
4 \\
4 \\
4 \\
4 \\
4 \\
4\end{array}$ & $\begin{array}{l}0 \\
0 \\
0 \\
0 \\
0 \\
0 \\
0 \\
0 \\
0 \\
0\end{array}$ & $\begin{array}{l}3 \\
4 \\
4 \\
4 \\
4 \\
4 \\
4 \\
4 \\
4 \\
4\end{array}$ & $\begin{array}{l}63.1 \\
65.8 \\
60.4 \\
67.6 \\
51.7 \\
48.6 \\
28.9 \\
37.1 \\
33.3 \\
30.3\end{array}$ & $\begin{array}{l}72.2 \\
73.9 \\
67.9 \\
75.9 \\
58.1 \\
54.6 \\
32.5 \\
41.7 \\
37.4 \\
34.1\end{array}$ & $\begin{array}{l}79.7 \\
83.1 \\
76.3 \\
85.3 \\
65.3 \\
61.4 \\
36.5 \\
46.9 \\
42.0 \\
38.3\end{array}$ & $\begin{array}{l}69.2 \\
73.3 \\
67.4 \\
75.3 \\
57.7 \\
54.2 \\
32.2 \\
41.4 \\
37.1 \\
33.8\end{array}$ \\
\hline $\begin{array}{l}\text { B } \\
\text { B } \\
\text { B } \\
\text { B } \\
\text { B } \\
\text { B } \\
\text { B } \\
\text { B } \\
\text { B } \\
\text { B }\end{array}$ & $\begin{array}{l}\text { DG-AND } \\
\text { DG-ROA } \\
\text { DG-ORR } \\
\text { NL-ORR } \\
\text { CHI -BV } \\
\text { CHI -K25 } \\
\text { CHE-ORR } \\
\text { CR-ORR } \\
\text { CR-AND } \\
\text { CR-ROA }\end{array}$ & $\begin{array}{l}4 \\
4 \\
4 \\
4 \\
4 \\
4 \\
4 \\
4 \\
4 \\
4\end{array}$ & $\begin{array}{l}0 \\
0 \\
0 \\
0 \\
0 \\
0 \\
0 \\
0 \\
0 \\
0\end{array}$ & $\begin{array}{l}4 \\
4 \\
4 \\
4 \\
4 \\
4 \\
4 \\
4 \\
4 \\
4\end{array}$ & $\begin{array}{l}84.2 \\
79.0 \\
83.8 \\
85.6 \\
72.7 \\
68.9 \\
56.5 \\
50.3 \\
51.3 \\
34.6\end{array}$ & $\begin{array}{l}95.7 \\
89.9 \\
95.3 \\
97.4 \\
82.7 \\
78.3 \\
64.3 \\
57.2 \\
58.4 \\
39.3\end{array}$ & $\begin{array}{r}109.0 \\
102.0 \\
108.0 \\
111.0 \\
94.0 \\
89.1 \\
73.1 \\
65.1 \\
66.4 \\
44.7\end{array}$ & $\begin{array}{l}94.9 \\
89.1 \\
94.5 \\
96.6 \\
82.0 \\
77.7 \\
63.8 \\
56.7 \\
57.9 \\
39.0\end{array}$ \\
\hline $\begin{array}{l}c \\
c \\
C \\
C \\
c \\
C \\
C \\
C \\
C \\
C\end{array}$ & $\begin{array}{l}\text { DG-AND } \\
\text { DG-ROA } \\
\text { DG-ORR } \\
\text { NL-ORR } \\
\text { CHI-BV } \\
\text { CHI -K25 } \\
\text { CHE-ORR } \\
\text { CR-ORR } \\
\text { CR-AND } \\
\text { CR-ROA }\end{array}$ & $\begin{array}{l}4 \\
4 \\
4 \\
3 \\
4 \\
4 \\
4 \\
4 \\
4 \\
4\end{array}$ & $\begin{array}{l}0 \\
0 \\
0 \\
0 \\
0 \\
0 \\
0 \\
0 \\
0 \\
0\end{array}$ & $\begin{array}{l}4 \\
4 \\
4 \\
3 \\
4 \\
4 \\
4 \\
4 \\
4 \\
4\end{array}$ & $\begin{array}{r}84.8 \\
92.7 \\
83.7 \\
103.0 \\
74.0 \\
69.6 \\
72.9 \\
70.7 \\
53.5 \\
52.9\end{array}$ & $\begin{array}{r}97.3 \\
106.0 \\
96.1 \\
121.0 \\
85.0 \\
79.9 \\
83.7 \\
81.1 \\
61.4 \\
60.7\end{array}$ & $\begin{array}{r}112.0 \\
122.0 \\
110.0 \\
135.0 \\
97.5 \\
91.7 \\
96.0 \\
93.1 \\
70.4 \\
69.6\end{array}$ & $\begin{array}{r}96.4 \\
105.0 \\
95.2 \\
115.0 \\
84.2 \\
79.2 \\
82.9 \\
80.4 \\
60.8 \\
60.1\end{array}$ \\
\hline \multicolumn{9}{|c|}{ COBALT } \\
\hline $\begin{array}{l}A \\
A \\
A \\
A \\
A \\
A \\
A \\
A \\
A \\
A\end{array}$ & $\begin{array}{l}\text { DG-AND } \\
\text { DG-ROA } \\
\text { DG-ORR } \\
\text { NL-ORR } \\
\text { CHI-BV } \\
\text { CHI-K25 } \\
\text { CHE-ORR } \\
\text { CR-ORR } \\
\text { CR-AND } \\
\text { CR-ROA }\end{array}$ & $\begin{array}{l}3 \\
4 \\
4 \\
4 \\
4 \\
4 \\
4 \\
4 \\
4 \\
4\end{array}$ & $\begin{array}{l}0 \\
0 \\
0 \\
0 \\
0 \\
0 \\
0 \\
0 \\
0 \\
0\end{array}$ & $\begin{array}{l}3 \\
4 \\
4 \\
4 \\
4 \\
4 \\
4 \\
4 \\
4 \\
4\end{array}$ & $\begin{array}{r}13.90 \\
18.90 \\
15.30 \\
15.20 \\
17.60 \\
17.70 \\
11.30 \\
8.29 \\
15.30 \\
5.84\end{array}$ & $\begin{array}{r}19.60 \\
25.40 \\
20.60 \\
20.50 \\
23.80 \\
23.80 \\
15.30 \\
11.20 \\
20.70 \\
7.86\end{array}$ & $\begin{array}{l}25.20 \\
34.30 \\
27.80 \\
27.60 \\
32.00 \\
32.10 \\
20.60 \\
15.00 \\
27.80 \\
10.60\end{array}$ & $\begin{array}{l}17.60 \\
24.90 \\
20.20 \\
20.10 \\
23.30 \\
23.40 \\
15.00 \\
10.90 \\
20.30 \\
7.71\end{array}$ \\
\hline $\begin{array}{l}\text { B } \\
\text { B } \\
\text { B } \\
\text { B } \\
\text { B } \\
\text { B } \\
\text { B } \\
\text { B } \\
\text { B } \\
\text { B }\end{array}$ & $\begin{array}{l}\text { DG-AND } \\
\text { DG-ROA } \\
\text { DG-ORR } \\
\text { HL-ORR } \\
\text { CHI-BV } \\
\text { CHI-K25 } \\
\text { CHE-ORR } \\
\text { CR-ORR } \\
\text { CR-AND } \\
\text { CR-ROA }\end{array}$ & $\begin{array}{l}4 \\
4 \\
4 \\
4 \\
4 \\
4 \\
4 \\
4 \\
4 \\
4\end{array}$ & $\begin{array}{l}0 \\
0 \\
0 \\
0 \\
0 \\
0 \\
0 \\
0 \\
0 \\
0\end{array}$ & $\begin{array}{l}4 \\
4 \\
4 \\
4 \\
4 \\
4 \\
4 \\
4 \\
4 \\
4\end{array}$ & $\begin{array}{r}16.10 \\
15.70 \\
12.50 \\
17.00 \\
17.10 \\
12.20 \\
4.59 \\
4.63 \\
8.66 \\
4.19\end{array}$ & $\begin{array}{r}23.00 \\
22.40 \\
17.90 \\
24.30 \\
24.40 \\
17.50 \\
6.56 \\
6.62 \\
12.40 \\
5.98\end{array}$ & $\begin{array}{r}32.90 \\
32.00 \\
25.60 \\
34.70 \\
34.90 \\
25.00 \\
9.38 \\
9.46 \\
17.70 \\
8.54\end{array}$ & $\begin{array}{r}22.50 \\
21.90 \\
17.50 \\
23.70 \\
23.80 \\
17.10 \\
6.41 \\
6.47 \\
12.10 \\
5.84\end{array}$ \\
\hline $\begin{array}{l}c \\
C \\
C \\
C \\
C \\
C \\
C \\
C \\
C \\
C\end{array}$ & $\begin{array}{l}\text { DG-AND } \\
\text { DG-ROA } \\
\text { DG-ORR } \\
\text { NL-ORR } \\
\text { CHI-BV } \\
\text { CHI-K25 } \\
\text { CHE-ORR } \\
\text { CR-ORR } \\
\text { CR-AND } \\
\text { CR-ROA }\end{array}$ & $\begin{array}{l}4 \\
4 \\
4 \\
3 \\
4 \\
4 \\
4 \\
4 \\
4 \\
4\end{array}$ & $\begin{array}{l}0 \\
0 \\
0 \\
0 \\
0 \\
0 \\
0 \\
0 \\
0 \\
0\end{array}$ & $\begin{array}{l}4 \\
4 \\
4 \\
3 \\
4 \\
4 \\
4 \\
4 \\
4 \\
4\end{array}$ & $\begin{array}{r}18.70 \\
15.20 \\
15.10 \\
21.20 \\
23.90 \\
13.70 \\
8.77 \\
4.78 \\
7.45 \\
5.07\end{array}$ & $\begin{array}{r}29.10 \\
23.60 \\
23.40 \\
35.20 \\
37.20 \\
21.40 \\
13.60 \\
7.44 \\
11.60 \\
7.89\end{array}$ & $\begin{array}{l}45.30 \\
36.70 \\
36.40 \\
51.20 \\
57.80 \\
33.20 \\
21.20 \\
11.60 \\
18.00 \\
12.30\end{array}$ & $\begin{array}{r}28.30 \\
22.90 \\
22.70 \\
30.00 \\
36.10 \\
20.70 \\
13.20 \\
7.22 \\
11.20 \\
7.66\end{array}$ \\
\hline
\end{tabular}


Table G.1 (continued)

\begin{tabular}{|c|c|c|c|c|c|c|c|c|}
\hline Horizon & $\begin{array}{l}\text { Formation- } \\
\text { location }\end{array}$ & $\mathbf{N}$ & I & $\mathrm{D}$ & Median & UCB95 & $\times 95$ & LTB9595 \\
\hline \multicolumn{9}{|c|}{ EUROPIUM } \\
\hline $\begin{array}{l}A \\
A \\
A \\
A \\
A \\
A \\
A \\
A \\
A \\
A\end{array}$ & $\begin{array}{l}\text { DG-AND } \\
\text { DG-ROA } \\
\text { DG-ORR } \\
\text { NL-ORR } \\
\text { CHI-BV } \\
\text { CHI-K25 } \\
\text { CHE-ORR } \\
\text { CR-ORR } \\
\text { CR-AND } \\
\text { CR-ROA }\end{array}$ & $\begin{array}{l}3 \\
4 \\
4 \\
4 \\
4 \\
4 \\
4 \\
4 \\
4 \\
4\end{array}$ & $\begin{array}{l}0 \\
0 \\
0 \\
0 \\
0 \\
0 \\
0 \\
0 \\
0 \\
0\end{array}$ & $\begin{array}{l}3 \\
4 \\
4 \\
4 \\
4 \\
4 \\
4 \\
4 \\
4 \\
4\end{array}$ & $\begin{array}{l}0.606 \\
0.613 \\
0.585 \\
0.577 \\
0.998 \\
0.812 \\
0.539 \\
0.581 \\
0.743 \\
0.480\end{array}$ & $\begin{array}{l}0.756 \\
0.743 \\
0.709 \\
0.699 \\
1.210 \\
0.984 \\
0.653 \\
0.703 \\
0.901 \\
0.581\end{array}$ & $\begin{array}{l}0.889 \\
0.900 \\
0.859 \\
0.847 \\
1.460 \\
1.190 \\
0.791 \\
0.852 \\
1.090 \\
0.704\end{array}$ & $\begin{array}{l}0.704 \\
0.733 \\
0.700 \\
0.690 \\
1.190 \\
0.971 \\
0.645 \\
0.694 \\
0.889 \\
0.574\end{array}$ \\
\hline $\begin{array}{l}\text { B } \\
\text { B } \\
\text { B } \\
\text { B } \\
\text { B } \\
\text { B } \\
\text { B } \\
\text { B } \\
\text { B } \\
\text { B }\end{array}$ & $\begin{array}{l}\text { DG-AND } \\
\text { DG-ROA } \\
\text { DG-ORR } \\
\text { NL-ORR } \\
\text { CHI-BV } \\
\text { CHI-K25 } \\
\text { CHE-ORR } \\
\text { CR-ORR } \\
\text { CR-AND } \\
\text { CR-ROA }\end{array}$ & $\begin{array}{l}4 \\
4 \\
4 \\
4 \\
4 \\
4 \\
4 \\
4 \\
4 \\
4\end{array}$ & $\begin{array}{l}0 \\
0 \\
0 \\
0 \\
0 \\
0 \\
0 \\
0 \\
0 \\
0\end{array}$ & $\begin{array}{l}4 \\
4 \\
4 \\
4 \\
4 \\
4 \\
4 \\
4 \\
4 \\
4\end{array}$ & $\begin{array}{l}0.713 \\
0.693 \\
0.718 \\
0.746 \\
1.330 \\
1.120 \\
0.389 \\
0.385 \\
0.380 \\
0.338\end{array}$ & $\begin{array}{l}0.870 \\
0.845 \\
0.875 \\
0.910 \\
1.620 \\
1.370 \\
0.475 \\
0.469 \\
0.464 \\
0.412\end{array}$ & $\begin{array}{l}1.060 \\
1.030 \\
1.070 \\
1.110 \\
1.970 \\
1.670 \\
0.579 \\
0.572 \\
0.566 \\
0.503\end{array}$ & $\begin{array}{l}0.859 \\
0.834 . \\
0.864 \\
0.898 \\
1.600 \\
1.350 \\
0.468 \\
0.463 \\
0.458 \\
0.407\end{array}$ \\
\hline $\begin{array}{l}c \\
c \\
c \\
c \\
c \\
c \\
c \\
c \\
c \\
c\end{array}$ & $\begin{array}{l}\text { DG-AND } \\
\text { DG-ROA } \\
\text { DG-ORR } \\
\text { NL-ORR } \\
\text { CHI-BV } \\
\text { CHI-K25 } \\
\text { CHE-ORR } \\
\text { CR-ORR } \\
\text { CR-AND } \\
\text { CR-ROA }\end{array}$ & $\begin{array}{l}4 \\
4 \\
4 \\
3 \\
4 \\
4 \\
4 \\
4 \\
4 \\
4\end{array}$ & $\begin{array}{l}0 \\
0 \\
0 \\
0 \\
0 \\
0 \\
0 \\
0 \\
0 \\
0\end{array}$ & $\begin{array}{l}4 \\
4 \\
4 \\
3 \\
4 \\
4 \\
4 \\
4 \\
4 \\
4\end{array}$ & $\begin{array}{l}1.180 \\
0.836 \\
0.733 \\
0.833 \\
1.700 \\
1.230 \\
0.413 \\
0.392 \\
0.342 \\
0.333\end{array}$ & $\begin{array}{l}1.440 \\
1.020 \\
0.893 \\
1.050 \\
2.070 \\
1.500 \\
0.503 \\
0.477 \\
0.417 \\
0.405\end{array}$ & $\begin{array}{l}1.760 \\
1.240 \\
1.090 \\
1.240 \\
2.530 \\
1.830 \\
0.613 \\
0.581 \\
0.508 \\
0.494\end{array}$ & $\begin{array}{l}1.420 \\
1.000 \\
0.881 \\
0.972 \\
2.050 \\
1.480 \\
0.496 \\
0.471 \\
0.412 \\
0.400\end{array}$ \\
\hline \multicolumn{9}{|c|}{ GAIIIUM } \\
\hline $\begin{array}{l}A \\
A \\
A \\
A \\
A \\
A \\
A \\
A \\
A \\
A\end{array}$ & $\begin{array}{l}\text { DG-AND } \\
\text { DG-ROA } \\
\text { DG-ORR } \\
\text { HL-ORR } \\
\text { CHI -BV } \\
\text { CHI-K25 } \\
\text { CHE-ORR } \\
\text { CR-ORR } \\
\text { CR-AND } \\
\text { CR-ROA }\end{array}$ & $\begin{array}{l}4 \\
4 \\
4 \\
4 \\
4 \\
4 \\
4 \\
4 \\
3 \\
4\end{array}$ & $\begin{array}{l}0 \\
0 \\
0 \\
0 \\
0 \\
0 \\
0 \\
0 \\
0 \\
0\end{array}$ & $\begin{array}{l}4 \\
4 \\
4 \\
4 \\
4 \\
4 \\
4 \\
4 \\
3 \\
4\end{array}$ & $\begin{array}{r}13.30 \\
13.60 \\
12.00 \\
15.40 \\
16.00 \\
14.70 \\
7.45 \\
5.44 \\
11.70 \\
17.00\end{array}$ & $\begin{array}{r}17.10 \\
17.50 \\
15.40 \\
19.80 \\
20.50 \\
18.80 \\
9.57 \\
7.00 \\
15.60 \\
21.80\end{array}$ & $\begin{array}{r}21.90 \\
22.40 \\
19.80 \\
25.40 \\
26.30 \\
24.20 \\
12.30 \\
8.99 \\
19.30 \\
28.00\end{array}$ & $\begin{array}{r}16.80 \\
17.20 \\
15.20 \\
19.40 \\
20.20 \\
18.50 \\
9.41 \\
6.88 \\
14.20 \\
21.40\end{array}$ \\
\hline $\begin{array}{l}\mathrm{B} \\
\mathrm{B} \\
\mathrm{B} \\
\mathrm{B} \\
\mathrm{B} \\
\mathrm{B} \\
\mathrm{B} \\
\mathrm{B} \\
\mathrm{B} \\
\mathrm{B}\end{array}$ & $\begin{array}{l}\text { DG-AHD } \\
\text { DG-ROA } \\
\text { DG-ORR } \\
\text { ML-ORR } \\
\text { CHI-BV } \\
\text { CHI-K25 } \\
\text { CHE-ORR } \\
\text { CR-ORR } \\
\text { CR-AND } \\
\text { CR-ROA }\end{array}$ & $\begin{array}{l}4 \\
4 \\
4 \\
4 \\
4 \\
3 \\
4 \\
4 \\
4 \\
4\end{array}$ & $\begin{array}{l}0 \\
0 \\
0 \\
0 \\
0 \\
0 \\
0 \\
0 \\
0 \\
0\end{array}$ & $\begin{array}{l}4 \\
4 \\
4 \\
4 \\
4 \\
3 \\
4 \\
4 \\
4 \\
4\end{array}$ & $\begin{array}{l}15.60 \\
18.20 \\
20.10 \\
21.90 \\
17.50 \\
34.20 \\
15.00 \\
14.40 \\
13.30 \\
16.20\end{array}$ & $\begin{array}{l}18.80 \\
22.00 \\
24.20 \\
26.40 \\
21.20 \\
42.40 \\
18.10 \\
17.30 \\
16.00 \\
19.50\end{array}$ & $\begin{array}{l}22.60 \\
26.50 \\
29.20 \\
31.90 \\
25.50 \\
49.70 \\
21.80 \\
20.90 \\
19.30 \\
23.60\end{array}$ & $\begin{array}{l}18.50 \\
21.70 \\
23.90 \\
26.10 \\
20.90 \\
39.60 \\
17.90 \\
17.10 \\
15.80 \\
19.30\end{array}$ \\
\hline $\begin{array}{l}c \\
C \\
c \\
c \\
C \\
C \\
C \\
C \\
C \\
C\end{array}$ & $\begin{array}{l}\text { DG-AND } \\
\text { DG-ROA } \\
\text { DG-ORR } \\
\text { HL-ORR } \\
\text { CHI-BV } \\
\text { CHI-K25 } \\
\text { CHE-ORR } \\
\text { CR-ORR } \\
\text { CR-AND } \\
\text { CR-ROA }\end{array}$ & $\begin{array}{l}4 \\
4 \\
4 \\
4 \\
4 \\
4 \\
4 \\
4 \\
4 \\
4\end{array}$ & $\begin{array}{l}0 \\
0 \\
0 \\
0 \\
0 \\
0 \\
0 \\
0 \\
0 \\
0\end{array}$ & $\begin{array}{l}4 \\
4 \\
4 \\
4 \\
4 \\
4 \\
4 \\
4 \\
4 \\
4\end{array}$ & $\begin{array}{l}19.70 \\
29.00 \\
22.00 \\
28.70 \\
21.10 \\
32.80 \\
14.40 \\
15.90 \\
12.20 \\
18.90\end{array}$ & $\begin{array}{l}24.60 \\
36.20 \\
27.50 \\
35.80 \\
26.30 \\
41.00 \\
18.00 \\
19.90 \\
15.20 \\
23.60\end{array}$ & $\begin{array}{l}30.80 \\
45.20 \\
34.40 \\
44.80 \\
32.90 \\
51.20 \\
22.50 \\
24.90 \\
19.00 \\
29.50\end{array}$ & $\begin{array}{l}24.30 \\
35.70 \\
27.10 \\
35.30 \\
25.90 \\
40.40 \\
17.80 \\
19.60 \\
15.00 \\
23.30\end{array}$ \\
\hline
\end{tabular}


G-10

Table G.1 (continued)

\begin{tabular}{|c|c|c|c|c|c|c|c|c|}
\hline Horizon & $\begin{array}{l}\text { Formation- } \\
\text { location }\end{array}$ & $\mathbf{N}$ & I & $\mathrm{D}$ & Median & UCB95 & X95 & LTB9595 \\
\hline \multicolumn{9}{|c|}{ GOLD } \\
\hline $\begin{array}{l}\text { A } \\
\text { A }\end{array}$ & $\begin{array}{l}\text { DG-AND } \\
\text { REMAINDER }\end{array}$ & $\begin{array}{r}4 \\
35\end{array}$ & $\begin{array}{l}1 \\
0\end{array}$ & $\begin{array}{l}0 \\
0\end{array}$ & : & : & : & - \\
\hline $\begin{array}{l}\text { B } \\
\text { B } \\
\text { B }\end{array}$ & $\begin{array}{l}\text { DG-ROA } \\
\text { CR-AND } \\
\text { REMAINDER }\end{array}$ & $\begin{array}{r}4 \\
4 \\
31\end{array}$ & $\begin{array}{l}0 \\
0 \\
0\end{array}$ & $\begin{array}{l}1 \\
2 \\
0\end{array}$ & $\begin{array}{l}.000969 \\
.005300 \\
.\end{array}$ & $\begin{array}{c}0.01400 \\
0.03840 \\
.\end{array}$ & $\begin{array}{c}0.02750 \\
0.15000 \\
.\end{array}$ & $\begin{array}{l}0.00220 \\
0.01250 \\
.\end{array}$ \\
\hline $\begin{array}{l}\mathrm{C} \\
\mathrm{C} \\
\mathrm{C} \\
\mathrm{C}\end{array}$ & $\begin{array}{l}\text { CHE-ORR } \\
\text { CR-ORR } \\
\text { CR-ROA } \\
\text { REMAINDER }\end{array}$ & $\begin{array}{c}4 \\
4 \\
4 \\
28\end{array}$ & $\begin{array}{l}0 \\
0 \\
0 \\
0\end{array}$ & $\begin{array}{l}2 \\
1 \\
2 \\
0\end{array}$ & $\begin{array}{l}.003070 \\
.004780 \\
.001470 \\
.\end{array}$ & $\begin{array}{l}0.00426 \\
0.00691 \\
0.00208 \\
.\end{array}$ & $\begin{array}{c}0.00502 \\
0.00781 \\
0.00241 \\
.\end{array}$ & $\begin{array}{c}0.00342 \\
0.00546 \\
0.00157 \\
.\end{array}$ \\
\hline
\end{tabular}

\section{HAFNIUM}

$\begin{array}{llrlrrrrr}\text { A } & \text { DG-AND } & 3 & 0 & 3 & 9.54 & 10.70 & 11.60 & 10.30 \\ \text { A } & \text { DG-ROA } & 4 & 0 & 4 & 10.60 & 11.70 & 12.90 & 11.60 \\ \text { A } & \text { DG-ORR } & 4 & 0 & 4 & 9.80 & 10.80 & 11.90 & 10.70 \\ \text { A } & \text { NL-ORR } & 4 & 0 & 4 & 10.00 & 11.00 & 12.20 & 11.00 \\ \text { A } & \text { CHI-BV } & 4 & 0 & 4 & 10.00 & 11.10 & 12.20 & 11.00 \\ \text { A } & \text { CHI-K25 } & 4 & 0 & 4 & 9.64 & 10.60 & 11.70 & 10.60 \\ \text { A } & \text { CHE-ORR } & 4 & 0 & 4 & 7.39 & 8.15 & 8.99 & 8.10 \\ \text { A } & \text { CR-ORR } & 4 & 0 & 4 & 7.38 & 8.15 & 8.99 & 8.09 \\ \text { A } & \text { CR-AND } & 4 & 0 & 4 & 7.56 & 8.34 & 9.21 & 8.29 \\ \text { A } & \text { CR-ROA } & 4 & 0 & 4 & 5.86 & 6.46 & 7.13 & 6.42 \\ \text { B } & \text { DG-AND } & 4 & 0 & 4 & 8.08 & 9.78 & 11.80 & 9.65 \\ \text { B } & \text { DG-ROA } & 4 & 0 & 4 & 8.73 & 10.60 & 12.80 & 10.40 \\ \text { B } & \text { DG-ORR } & 4 & 0 & 4 & 6.73 & 8.14 & 9.86 & 8.04 \\ \text { B } & \text { HL-ORR } & 4 & 0 & 4 & 6.67 & 8.07 & 9.76 & 7.97 \\ \text { B } & \text { CHI-BV } & 4 & 0 & 4 & 6.41 & 7.75 & 9.38 & 7.66 \\ \text { B } & \text { CHI-K25 } & 4 & 0 & 4 & 5.46 & 6.60 & 7.99 & 6.52 \\ \text { B } & \text { CHE-ORR } & 4 & 0 & 4 & 4.75 & 5.75 & 6.96 & 5.68 \\ \text { B } & \text { CR-ORR } & 4 & 0 & 4 & 5.52 & 6.67 & 8.08 & 6.59 \\ \text { B } & \text { CR-AND } & 4 & 0 & 4 & 6.03 & 7.30 & 8.83 & 7.21 \\ \text { B } & \text { CR-ROA } & 4 & 0 & 4 & 4.08 & 4.94 & 5.98 & 4.88 \\ \text { C } & \text { DG-AND } & 4 & 0 & 4 & 6.18 & 7.24 & 8.48 & 7.16 \\ \text { C } & \text { DG-ROA } & 4 & 0 & 4 & 6.14 & 7.19 & 8.43 & 7.12 \\ \text { C } & \text { DG-ORR } & 4 & 0 & 4 & 6.16 & 7.21 & 8.45 & 7.14 \\ \text { C } & \text { NL-ORR } & 3 & 0 & 3 & 6.58 & 7.89 & 9.02 & 7.45 \\ \text { C } & \text { CHI-BV } & 4 & 0 & 4 & 5.16 & 6.04 & 7.07 & 5.98 \\ \text { C } & \text { CHI-K25 } & 4 & 0 & 4 & 4.68 & 5.48 & 6.42 & 5.43 \\ \text { C } & \text { CHE-ORR } & 4 & 0 & 4 & 2.64 & 3.09 & 3.62 & 3.05 \\ \text { C } & \text { CR-ORR } & 4 & 0 & 4 & 2.85 & 3.34 & 3.91 & 3.30 \\ \text { C } & \text { CR-AND } & 4 & 0 & 4 & 4.74 & 5.55 & 6.50 & 5.49 \\ \text { C } & \text { CR-ROA } & 4 & 0 & 4 & 3.71 & 4.34 & 5.09 & 4.30\end{array}$

\section{IRON}

$\begin{array}{lllllllll}\text { A } & \text { DG-AND } & 3 & 0 & 3 & 29800 & 35600 & 40600 & 33700 \\ \text { A } & \text { DG-ROA } & 4 & 0 & 4 & 28500 & 33300 & 38800 & 32900 \\ \text { A } & \text { DG-ORR } & 4 & 0 & 4 & 26400 & 30800 & 35900 & 30500 \\ \text { A } & \text { NL-ORR } & 4 & 0 & 4 & 29300 & 34100 & 39800 & 33800 \\ \text { A } & \text { CHI-BV } & 4 & 0 & 4 & 30900 & 36100 & 42100 & 35700 \\ \text { A } & \text { CHI-K25 } & 4 & 0 & 4 & 28900 & 33700 & 39400 & 33400 \\ \text { A } & \text { CHE-ORR } & 4 & 0 & 4 & 11700 & 13700 & 15900 & 13500 \\ \text { A } & \text { CR-ORR } & 4 & 0 & 4 & 15900 & 18500 & 21600 & 18300 \\ \text { A } & \text { CR-AND } & 4 & 0 & 4 & 14600 & 17000 & 19800 & 16800 \\ \text { A } & \text { CR-ROA } & 4 & 0 & 4 & 13800 & 16100 & 18700 & 15900 \\ & & & & & & & & \\ \text { B } & \text { DG-AND } & 4 & 0 & 4 & 45500 & 50000 & 55000 & 49700 \\ \text { B } & \text { DG-ROA } & 4 & 0 & 4 & 39500 & 43500 & 47800 & 43200 \\ \text { B } & \text { DG-ORR } & 4 & 0 & 4 & 39000 & 42900 & 47200 & 42700 \\ \text { B } & \text { NL-ORR } & 4 & 0 & 4 & 47000 & 51700 & 56800 & 51300 \\ \text { B } & \text { CHI-BV } & 4 & 0 & 4 & 53900 & 59300 & 65200 & 58900 \\ \text { B } & \text { CHI-K25 } & 4 & 0 & 4 & 55600 & 61200 & 67300 & 60800\end{array}$


G-11

Table G.1 (continued)

\begin{tabular}{|c|c|c|c|c|c|c|c|c|}
\hline Horizon & $\begin{array}{l}\text { Formation- } \\
\text { location }\end{array}$ & $\mathbf{N}$ & I & D & Median & UCB95 & X95 & LTB9595 \\
\hline $\begin{array}{l}\text { B } \\
\text { B } \\
\text { B } \\
\text { B }\end{array}$ & $\begin{array}{l}\text { CHE-ORR } \\
\text { CR-ORR } \\
\text { CR-AND } \\
\text { CR-ROA }\end{array}$ & $\begin{array}{l}4 \\
4 \\
4 \\
4\end{array}$ & $\begin{array}{l}0 \\
0 \\
0 \\
0\end{array}$ & $\begin{array}{l}4 \\
4 \\
4 \\
4\end{array}$ & $\begin{array}{l}33500 \\
33300 \\
26600 \\
21600\end{array}$ & $\begin{array}{l}36800 \\
36600 \\
29200 \\
23800\end{array}$ & $\begin{array}{l}40500 \\
40300 \\
32100 \\
26100\end{array}$ & $\begin{array}{l}36600 \\
36400 \\
29000 \\
23600\end{array}$ \\
\hline $\begin{array}{l}c \\
c \\
C \\
C \\
c \\
c \\
C \\
C \\
C \\
C\end{array}$ & $\begin{array}{l}\text { DG-AND } \\
\text { DG-ROA } \\
\text { DG-ORR } \\
\text { NL-ORR } \\
\text { CHI-BV } \\
\text { CHI-K25 } \\
\text { CHE-ORR } \\
\text { CR-ORR } \\
\text { CR-AND } \\
\text { CR-ROA }\end{array}$ & $\begin{array}{l}4 \\
4 \\
4 \\
3 \\
4 \\
4 \\
4 \\
4 \\
4 \\
4\end{array}$ & $\begin{array}{l}0 \\
0 \\
0 \\
0 \\
0 \\
0 \\
0 \\
0 \\
0 \\
0\end{array}$ & $\begin{array}{l}4 \\
4 \\
4 \\
3 \\
4 \\
4 \\
4 \\
4 \\
4 \\
4\end{array}$ & $\begin{array}{l}50100 \\
51300 \\
40200 \\
58600 \\
57000 \\
55300 \\
35500 \\
45900 \\
32100 \\
41600\end{array}$ & $\begin{array}{l}54800 \\
56000 \\
43900 \\
64900 \\
62200 \\
60400 \\
38700 \\
50100 \\
35100 \\
45500\end{array}$ & $\begin{array}{l}59800 \\
61200 \\
48000 \\
70000 \\
68000 \\
66000 \\
42300 \\
54700 \\
38300 \\
49700\end{array}$ & $\begin{array}{l}54400 \\
55700 \\
43700 \\
62800 \\
61900 \\
60100 \\
38500 \\
49800 \\
34900 \\
45200\end{array}$ \\
\hline \multicolumn{9}{|c|}{ LANTHANUM } \\
\hline $\begin{array}{l}A \\
A \\
A \\
A \\
A \\
A \\
A \\
A \\
A \\
A\end{array}$ & $\begin{array}{l}\text { DG-AND } \\
\text { DG-ROA } \\
\text { DG-ORR } \\
\text { NL-ORR } \\
\text { CHI-BV } \\
\text { CHI-K25 } \\
\text { CHE-ORR } \\
\text { CR-ORR } \\
\text { CR-AND } \\
\text { CR-ROA }\end{array}$ & $\begin{array}{l}4 \\
4 \\
4 \\
4 \\
4 \\
4 \\
4 \\
4 \\
3 \\
4\end{array}$ & $\begin{array}{l}0 \\
0 \\
0 \\
0 \\
0 \\
0 \\
0 \\
0 \\
0 \\
0\end{array}$ & $\begin{array}{l}4 \\
4 \\
4 \\
4 \\
4 \\
4 \\
4 \\
4 \\
3 \\
4\end{array}$ & $\begin{array}{l}31.8 \\
29.9 \\
30.5 \\
32.1 \\
37.6 \\
32.0 \\
17.3 \\
21.9 \\
24.7 \\
17.3\end{array}$ & $\begin{array}{l}37.1 \\
35.0 \\
35.7 \\
37.6 \\
43.9 \\
37.4 \\
20.2 \\
24.7 \\
29.6 \\
20.2\end{array}$ & $\begin{array}{l}43.4 \\
40.9 \\
41.8 \\
43.9 \\
51.3 \\
43.7 \\
23.7 \\
28.8 \\
33.8 \\
23.6\end{array}$ & $\begin{array}{l}36.7 \\
34.6 \\
35.3 \\
37.2 \\
43.5 \\
37.0 \\
20.0 \\
24.4 \\
28.0 \\
20.0\end{array}$ \\
\hline $\begin{array}{l}\text { B } \\
\text { B } \\
\text { B } \\
\text { B } \\
\text { B } \\
\text { B } \\
\text { B } \\
\text { B } \\
\text { B } \\
\text { B }\end{array}$ & $\begin{array}{l}\text { DG-AND } \\
\text { DG-ROA } \\
\text { DG-ORR } \\
\text { NL-ORR } \\
\text { CHI-BV } \\
\text { CHI-K25 } \\
\text { CHE-ORR } \\
\text { CR-ORR } \\
\text { CR-AND } \\
\text { CR-ROA }\end{array}$ & $\begin{array}{l}4 \\
4 \\
4 \\
4 \\
4 \\
3 \\
4 \\
4 \\
4 \\
4\end{array}$ & $\begin{array}{l}0 \\
0 \\
0 \\
0 \\
0 \\
0 \\
0 \\
0 \\
0 \\
0\end{array}$ & $\begin{array}{l}4 \\
4 \\
4 \\
4 \\
4 \\
3 \\
4 \\
4 \\
4 \\
4\end{array}$ & $\begin{array}{l}39.1 \\
36.5 \\
41.4 \\
40.4 \\
48.3 \\
43.6 \\
22.0 \\
28.7 \\
11.9 \\
14.0\end{array}$ & $\begin{array}{l}46.8 \\
43.7 \\
49.5 \\
48.3 \\
57.8 \\
53.6 \\
26.3 \\
34.4 \\
14.3 \\
16.7\end{array}$ & $\begin{array}{l}55.9 \\
52.2 \\
59.2 \\
57.8 \\
69.1 \\
62.4 \\
31.4 \\
41.1 \\
17.1 \\
20.0\end{array}$ & $\begin{array}{l}46.2 \\
43.1 \\
48.9 \\
47.7 \\
57.1 \\
50.2 \\
25.9 \\
33.9 \\
14.1 \\
16.5\end{array}$ \\
\hline $\begin{array}{l}\text { C } \\
\text { C } \\
C \\
C \\
C \\
C \\
C \\
C \\
C \\
C\end{array}$ & $\begin{array}{l}\text { DG-AND } \\
\text { DG-ROA } \\
\text { DG-ORR } \\
\text { KL-ORR } \\
\text { CHI-BV } \\
\text { CHI-K25 } \\
\text { CHE-ORR } \\
\text { CR-ORR } \\
\text { CR-AND } \\
\text { CR-ROA }\end{array}$ & $\begin{array}{l}4 \\
4 \\
4 \\
4 \\
4 \\
4 \\
4 \\
4 \\
4 \\
4\end{array}$ & $\begin{array}{l}0 \\
0 \\
0 \\
0 \\
0 \\
0 \\
0 \\
0 \\
0 \\
0\end{array}$ & $\begin{array}{l}4 \\
4 \\
4 \\
4 \\
4 \\
4 \\
4 \\
4 \\
4 \\
4\end{array}$ & $\begin{array}{l}43.2 \\
43.2 \\
41.0 \\
46.7 \\
52.5 \\
45.1 \\
24.6 \\
38.2 \\
19.5 \\
19.9\end{array}$ & $\begin{array}{l}49.7 \\
49.7 \\
47.2 \\
53.7 \\
60.5 \\
51.9 \\
28.3 \\
43.9 \\
22.5 \\
22.9\end{array}$ & $\begin{array}{l}57.2 \\
57.2 \\
54.4 \\
61.9 \\
69.6 \\
59.8 \\
32.6 \\
50.6 \\
25.8 \\
26.3\end{array}$ & $\begin{array}{l}49.3 \\
49.2 \\
46.8 \\
53.2 \\
59.9 \\
51.5 \\
28.1 \\
43.5 \\
22.2 \\
22.6\end{array}$ \\
\hline \multicolumn{9}{|c|}{ LUTETIUM } \\
\hline $\begin{array}{l}A \\
A \\
A \\
A \\
A \\
A \\
A \\
A \\
A \\
A\end{array}$ & $\begin{array}{l}\text { DG-AND } \\
\text { DG-ROA } \\
\text { DG-ORR } \\
\text { NL-ORR } \\
\text { CHI-BV } \\
\text { CHI-K25 } \\
\text { CHE-ORR } \\
\text { CR-ORR } \\
\text { CR-AND } \\
\text { CR-ROA }\end{array}$ & $\begin{array}{l}3 \\
4 \\
4 \\
4 \\
4 \\
4 \\
4 \\
4 \\
4 \\
4\end{array}$ & $\begin{array}{l}0 \\
0 \\
0 \\
0 \\
0 \\
0 \\
0 \\
0 \\
0 \\
0\end{array}$ & $\begin{array}{l}3 \\
4 \\
4 \\
4 \\
4 \\
4 \\
4 \\
4 \\
4 \\
4\end{array}$ & $\begin{array}{l}0.320 \\
0.364 \\
0.340 \\
0.351 \\
0.272 \\
0.340 \\
0.257 \\
0.271 \\
0.264 \\
0.186\end{array}$ & $\begin{array}{l}0.377 \\
0.420 \\
0.392 \\
0.404 \\
0.314 \\
0.392 \\
0.296 \\
0.312 \\
0.304 \\
0.215\end{array}$ & $\begin{array}{l}0.425 \\
0.484 \\
0.452 \\
0.466 \\
0.361 \\
0.452 \\
0.341 \\
0.360 \\
0.350 \\
0.247\end{array}$ & $\begin{array}{l}0.358 \\
0.416 \\
0.388 \\
0.400 \\
0.311 \\
0.388 \\
0.293 \\
0.309 \\
0.301 \\
0.213\end{array}$ \\
\hline $\begin{array}{l}B \\
B \\
B \\
B\end{array}$ & $\begin{array}{l}\text { DG-AND } \\
\text { DG-ROA } \\
\text { DG-ORR } \\
N L-O R R\end{array}$ & $\begin{array}{l}4 \\
4 \\
4 \\
4\end{array}$ & $\begin{array}{l}0 \\
0 \\
0 \\
0\end{array}$ & $\begin{array}{l}4 \\
4 \\
4 \\
4\end{array}$ & $\begin{array}{l}0.319 \\
0.372 \\
0.292 \\
0.230\end{array}$ & $\begin{array}{l}0.399 \\
0.465 \\
0.365 \\
0.288\end{array}$ & $\begin{array}{l}0.499 \\
0.581 \\
0.456 \\
0.361\end{array}$ & $\begin{array}{l}0.393 \\
0.458 \\
0.360 \\
0.284\end{array}$ \\
\hline
\end{tabular}




\section{G-12}

Table G.1 (continued)

\begin{tabular}{|c|c|c|c|c|c|c|c|c|}
\hline Horizon & $\begin{array}{l}\text { Formation- } \\
\text { location }\end{array}$ & $\mathbf{N}$ & I & $\mathrm{D}$ & Median & UCB95 & $\mathrm{X} 95$ & LTB9595 \\
\hline $\begin{array}{l}\text { B } \\
\text { B } \\
\text { B } \\
\text { B } \\
8 \\
\text { B }\end{array}$ & $\begin{array}{l}\text { CHI -BV } \\
\text { CHI-K25 } \\
\text { CHE-ORR } \\
\text { CR-ORR } \\
\text { CR-AND } \\
\text { CR-ROA }\end{array}$ & $\begin{array}{l}4 \\
4 \\
4 \\
4 \\
4 \\
4\end{array}$ & $\begin{array}{l}0 \\
0 \\
0 \\
0 \\
0 \\
0\end{array}$ & $\begin{array}{l}4 \\
4 \\
4 \\
4 \\
4 \\
4\end{array}$ & $\begin{array}{l}0.378 \\
0.381 \\
0.218 \\
0.211 \\
0.212 \\
0.138\end{array}$ & $\begin{array}{l}0.473 \\
0.476 \\
0.273 \\
0.264 \\
0.266 \\
0.173\end{array}$ & $\begin{array}{l}0.592 \\
0.595 \\
0.341 \\
0.330 \\
0.332 \\
0.217\end{array}$ & $\begin{array}{l}0.466 \\
0.469 \\
0.269 \\
0.260 \\
0.262 \\
0.171\end{array}$ \\
\hline $\begin{array}{l}\mathrm{C} \\
\mathrm{C} \\
\mathrm{C} \\
\mathrm{C} \\
\mathrm{C} \\
\mathrm{C} \\
\mathrm{C} \\
\mathrm{C} \\
\mathrm{C} \\
\mathrm{C}\end{array}$ & $\begin{array}{l}\text { DG-AND } \\
\text { DG-ROA } \\
\text { DG-ORR } \\
\text { NL-ORR } \\
\text { CHI-BV } \\
\text { CHI-K25 } \\
\text { CHE-ORR } \\
\text { CR-ORR } \\
\text { CR-AND } \\
\text { CR-ROA }\end{array}$ & $\begin{array}{l}4 \\
4 \\
4 \\
3 \\
4 \\
4 \\
4 \\
4 \\
4 \\
4\end{array}$ & $\begin{array}{l}0 \\
0 \\
0 \\
0 \\
0 \\
0 \\
0 \\
0 \\
0 \\
0\end{array}$ & $\begin{array}{l}4 \\
4 \\
4 \\
3 \\
4 \\
4 \\
4 \\
4 \\
4 \\
4\end{array}$ & $\begin{array}{l}0.342 \\
0.287 \\
0.262 \\
0.402 \\
0.418 \\
0.361 \\
0.146 \\
0.186 \\
0.168 \\
0.157\end{array}$ & $\begin{array}{l}0.420 \\
0.353 \\
0.322 \\
0.510 \\
0.513 \\
0.444 \\
0.180 \\
0.229 \\
0.207 \\
0.193\end{array}$ & $\begin{array}{l}0.516 \\
0.433 \\
0.396 \\
0.606 \\
0.630 \\
0.545 \\
0.221 \\
0.281 \\
0.254 \\
0.237\end{array}$ & $\begin{array}{l}0.414 \\
0.348 \\
0.318 \\
0.472 \\
0.506 \\
0.438 \\
0.177 \\
0.226 \\
0.204 \\
0.191\end{array}$ \\
\hline \multicolumn{9}{|c|}{ MAGNESIUM } \\
\hline $\begin{array}{l}\mathbf{A} \\
\mathbf{A} \\
\mathbf{A} \\
\mathbf{A} \\
\mathbf{A} \\
\mathbf{A} \\
\mathbf{A} \\
\mathbf{A} \\
\mathbf{A} \\
\mathbf{A}\end{array}$ & $\begin{array}{l}\text { DG-AND } \\
\text { DG-ROA } \\
\text { DG-ORR } \\
\text { NL-ORR } \\
\text { CHI-BV } \\
\text { CHI-K25 } \\
\text { CHE-ORR } \\
\text { CR-ORR } \\
\text { CR-AND } \\
\text { CR-ROA }\end{array}$ & $\begin{array}{l}4 \\
4 \\
4 \\
4 \\
4 \\
4 \\
4 \\
4 \\
4 \\
4\end{array}$ & $\begin{array}{l}0 \\
0 \\
0 \\
0 \\
1 \\
0 \\
1 \\
0 \\
0 \\
0\end{array}$ & $\begin{array}{l}4 \\
4 \\
4 \\
4 \\
3 \\
4 \\
3 \\
4 \\
3 \\
3\end{array}$ & $\begin{array}{l}5940 \\
4200 \\
3290 \\
4440 \\
3090 \\
2300 \\
1240 \\
1430 \\
2090 \\
324\end{array}$ & $\begin{array}{r}10600 \\
7510 \\
5880 \\
7930 \\
5530 \\
4110 \\
2220 \\
2550 \\
3890 \\
581\end{array}$ & $\begin{array}{r}18900 \\
13400 \\
10500 \\
14200 \\
9850 \\
7340 \\
3970 \\
4550 \\
6650 \\
1030\end{array}$ & $\begin{array}{r}10200 \\
7210 \\
5650 \\
7610 \\
5280 \\
3950 \\
2130 \\
2450 \\
3460 \\
557\end{array}$ \\
\hline $\begin{array}{l}\text { B } \\
\text { B } \\
\text { B } \\
\text { B } \\
\text { B } \\
\text { B } \\
\text { B } \\
\text { B } \\
\text { B } \\
\text { B }\end{array}$ & $\begin{array}{l}\text { DG-AND } \\
\text { DG-ROA } \\
\text { DG-ORR } \\
\text { NL-ORR } \\
\text { CHI-BV } \\
\text { CHI-K25 } \\
\text { CHE-ORR } \\
\text { CR-ORR } \\
\text { CR-AND } \\
\text { CR-ROA }\end{array}$ & $\begin{array}{l}4 \\
4 \\
4 \\
4 \\
4 \\
4 \\
4 \\
4 \\
4 \\
4\end{array}$ & $\begin{array}{l}0 \\
0 \\
0 \\
0 \\
0 \\
0 \\
0 \\
0 \\
0 \\
0\end{array}$ & $\begin{array}{l}4 \\
4 \\
4 \\
4 \\
4 \\
4 \\
4 \\
4 \\
4 \\
4\end{array}$ & $\begin{array}{l}7710 \\
6420 \\
8180 \\
7930 \\
7010 \\
8620 \\
4670 \\
2940 \\
2940 \\
2210\end{array}$ & $\begin{array}{l}8880 \\
7390 \\
9410 \\
9130 \\
8070 \\
9920 \\
5370 \\
3390 \\
3380 \\
2540\end{array}$ & $\begin{array}{r}10200 \\
8510 \\
10800 \\
10500 \\
9300 \\
11400 \\
6190 \\
3900 \\
3890 \\
2930\end{array}$ & $\begin{array}{l}8800 \\
7320 \\
9330 \\
9040 \\
8000 \\
9830 \\
5330 \\
3360 \\
3350 \\
2520\end{array}$ \\
\hline $\begin{array}{l}\mathbf{C} \\
\mathrm{C} \\
\mathrm{C} \\
\mathrm{C} \\
\mathrm{C} \\
\mathrm{C} \\
\mathrm{C} \\
\mathrm{C} \\
\mathrm{C} \\
\mathrm{C}\end{array}$ & $\begin{array}{l}\text { DG-AND } \\
\text { DG-ROA } \\
\text { DG-ORR } \\
\text { NL-ORR } \\
\text { CHI-BV } \\
\text { CHI-K25 } \\
\text { CHE-ORR } \\
\text { CR-ORR } \\
\text { CR-AND } \\
\text { CR-ROA }\end{array}$ & $\begin{array}{l}4 \\
4 \\
4 \\
4 \\
4 \\
4 \\
4 \\
4 \\
3 \\
4\end{array}$ & $\begin{array}{l}0 \\
0 \\
0 \\
0 \\
0 \\
0 \\
0 \\
0 \\
0 \\
0\end{array}$ & $\begin{array}{l}4 \\
4 \\
4 \\
4 \\
4 \\
4 \\
4 \\
4 \\
3 \\
4\end{array}$ & $\begin{array}{l}8390 \\
8610 \\
8790 \\
8420 \\
8560 \\
8730 \\
5650 \\
5180 \\
3620 \\
2530\end{array}$ & $\begin{array}{r}11700 \\
12000 \\
12300 \\
11700 \\
11900 \\
12200 \\
7880 \\
7220 \\
5310 \\
3530\end{array}$ & $\begin{array}{l}16300 \\
16700 \\
17100 \\
16400 \\
16600 \\
17000 \\
11000 \\
10100 \\
7030 \\
4920\end{array}$ & $\begin{array}{r}11400 \\
11700 \\
12000 \\
11500 \\
11700 \\
11900 \\
7700 \\
7060 \\
4700 \\
3450\end{array}$ \\
\hline
\end{tabular}

\section{MANGANESE}

$\begin{array}{lllllrrrr}\text { A } & \text { DG-AND } & 4 & 0 & 4 & 701.0 & 2220 & 7030 & 2050 \\ \text { A } & \text { DG-ROA } & 4 & 0 & 4 & 960.0 & 3040 & 9630 & 2810 \\ \text { A } & \text { DG-ORR } & 4 & 0 & 4 & 490.0 & 1550 & 4920 & 1430 \\ \text { A } & \text { NL-ORR } & 4 & 0 & 4 & 608.0 & 1920 & 6100 & 1780 \\ \text { A } & \text { CHI-BV } & 4 & 0 & 4 & 869.0 & 2750 & 8710 & 2540 \\ \text { A } & \text { CHI-K25 } & 4 & 0 & 4 & 968.0 & 3060 & 9700 & 2830 \\ \text { A } & \text { CHE-ORR } & 4 & 0 & 4 & 807.0 & 2560 & 8100 & 2360 \\ \text { A } & \text { CR-ORR } & 4 & 0 & 4 & 1030.0 & 3250 & 10300 & 3000 \\ \text { A } & \text { CR-AND } & 4 & 0 & 4 & 1920.0 & 6090 & 19300 & 5630 \\ \text { A } & \text { CR-ROA } & 4 & 0 & 3 & 59.0 & 188 & 592 & 173 \\ \text { B } & \text { DG-AND } & 4 & 0 & 4 & 210.0 & 320 & 487 & 311 \\ 8 & \text { DG-ROA } & 4 & 0 & 4 & 309.0 & 471 & 717 & 458\end{array}$




\section{G-13}

Table G.1 (continued)

\begin{tabular}{|c|c|c|c|c|c|c|c|c|}
\hline Horizon & $\begin{array}{l}\text { Formation- } \\
\text { location }\end{array}$ & $\mathbf{N}$ & I & D & Median & UCB95 & X95 & LTB9595 \\
\hline 1. 1.1. & 得! & & $i s$ & & $: \quad Q$ & & $\therefore$ \{tok" & $f$ \\
\hline $\begin{array}{l}\text { B } \\
\text { B } \\
\text { B } \\
\text { B } \\
\text { B } \\
\text { B } \\
\text { B } \\
\text { B }\end{array}$ & $\begin{array}{l}\text { DG-ORR } \\
\text { NL-ORR } \\
\text { CHI-BV } \\
\text { CHI-K25 } \\
\text { CHE-ORR } \\
\text { CR-ORR } \\
\text { CR-AND } \\
\text { CR-ROA }\end{array}$ & $\begin{array}{l}4 \\
4 \\
4 \\
4 \\
4 \\
4 \\
4 \\
4\end{array}$ & $\begin{array}{l}0 \\
0 \\
0 \\
0 \\
0 \\
0 \\
0 \\
0\end{array}$ & $\begin{array}{l}4 \\
4 \\
4 \\
4 \\
4 \\
4 \\
4 \\
4\end{array}$ & $\begin{array}{r}231.0 \\
271.0 \\
346.0 \\
332.0 \\
89.9 \\
136.0 \\
367.0 \\
149.0\end{array}$ & $\begin{array}{l}352 \\
413 \\
528 \\
505 \\
137 \\
208 \\
559 \\
227\end{array}$ & $\begin{array}{l}536 \\
629 \\
804 \\
770 \\
209 \\
316 \\
851 \\
345\end{array}$ & $\begin{array}{l}342 \\
401 \\
514 \\
491 \\
133 \\
202 \\
544 \\
220\end{array}$ \\
\hline $\begin{array}{l}\text { C } \\
C \\
C \\
C \\
C \\
C \\
C \\
C \\
C \\
C\end{array}$ & $\begin{array}{l}\text { DG-AND } \\
\text { DG-ROA } \\
\text { DG-ORR } \\
\text { HL-ORR } \\
\text { CHI-BV } \\
\text { CHI-K25 } \\
\text { CHE-ORR } \\
\text { CR-ORR } \\
\text { CR-AND } \\
\text { CR-ROA }\end{array}$ & $\begin{array}{l}4 \\
4 \\
4 \\
4 \\
4 \\
4 \\
4 \\
4 \\
4 \\
4\end{array}$ & $\begin{array}{l}0 \\
0 \\
0 \\
0 \\
0 \\
0 \\
0 \\
0 \\
0 \\
0\end{array}$ & $\begin{array}{l}4 \\
4 \\
4 \\
4 \\
4 \\
4 \\
4 \\
4 \\
4 \\
4\end{array}$ & $\begin{array}{l}722.0 \\
260.0 \\
443.0 \\
394.0 \\
571.0 \\
412.0 \\
218.0 \\
112.0 \\
273.0 \\
69.8\end{array}$ & $\begin{array}{r}1360 \\
490 \\
837 \\
744 \\
1080 \\
777 \\
412 \\
211 \\
516 \\
132\end{array}$ & $\begin{array}{r}2570 \\
924 \\
1580 \\
1400 \\
2030 \\
1470 \\
777 \\
398 \\
973 \\
249\end{array}$ & $\begin{array}{r}1310 \\
470 \\
803 \\
713 \\
1030 \\
746 \\
395 \\
203 \\
495 \\
126\end{array}$ \\
\hline \multicolumn{9}{|c|}{ MERCURY } \\
\hline $\begin{array}{l}A \\
A\end{array}$ & $\begin{array}{l}\text { CHI -K25 } \\
\text { REMAINDER }\end{array}$ & $\begin{array}{r}4 \\
35\end{array}$ & $\begin{array}{l}1 \\
0\end{array}$ & $\begin{array}{l}0 \\
0\end{array}$ & . & . & . & $\dot{.}$ \\
\hline B & REMAINDER & 40 & 0 & 0 & - & - & - & . \\
\hline $\begin{array}{l}\mathbf{C} \\
\mathbf{C} \\
\mathbf{C} \\
\mathbf{C}\end{array}$ & $\begin{array}{l}\text { DG-AND } \\
\text { NL-ORR } \\
\text { CR-ROA } \\
\text { REMAINDER }\end{array}$ & $\begin{array}{r}4 \\
3 \\
4 \\
28\end{array}$ & $\begin{array}{l}1 \\
0 \\
0 \\
0\end{array}$ & $\begin{array}{l}0 \\
1 \\
1 \\
0\end{array}$ & . & . & : & : \\
\hline
\end{tabular}

POTASSIUM

$\begin{array}{lllllrrrr}\text { A } & \text { DG-AHD } & 4 & 0 & 4 & 26500 & 31800 & 38200 & 31400 \\ \text { A } & \text { DG-ROA } & 4 & 0 & 4 & 11200 & 13400 & 16200 & 13300 \\ \text { A } & \text { DG-ORR } & 4 & 0 & 4 & 15100 & 18200 & 21800 & 17900 \\ \text { A } & \text { HL-ORR } & 4 & 0 & 4 & 16000 & 19300 & 23200 & 19000 \\ \text { A } & \text { CHI-BV } & 4 & 0 & 4 & 13600 & 16300 & 19600 & 16100 \\ \text { A } & \text { CHI-K25 } & 4 & 0 & 4 & 10100 & 12100 & 14500 & 11900 \\ \text { A } & \text { CHE-ORR } & 4 & 0 & 4 & 3020 & 3630 & 4360 & 3590 \\ \text { A } & \text { CR-ORR } & 4 & 0 & 4 & 3630 & 4360 & 5240 & 4310 \\ \text { A } & \text { CR-AND } & 3 & 0 & 3 & 4610 & 5700 & 6650 & 5320 \\ \text { A } & \text { CR-ROA } & 4 & 0 & 4 & 3210 & 3860 & 4630 & 3810 \\ & & & & & & & & \\ \text { B } & \text { DG-AND } & 4 & 0 & 4 & 28300 & 43800 & 67900 & 42500 \\ \text { B } & \text { DG-ROA } & 4 & 0 & 4 & 15200 & 23600 & 36500 & 22900 \\ \text { B } & \text { DG-ORR } & 4 & 0 & 4 & 21100 & 32700 & 50700 & 31700 \\ \text { B } & \text { NL-ORR } & 4 & 0 & 4 & 21300 & 32900 & 51000 & 31900 \\ \text { B } & \text { CHI-BV } & 4 & 0 & 4 & 22000 & 34100 & 52800 & 33000 \\ \text { B } & \text { CHI-K25 } & 3 & 0 & 3 & 23900 & 39600 & 57300 & 33600 \\ \text { B } & \text { CHE-ORR } & 4 & 0 & 4 & 7950 & 12300 & 19100 & 11900 \\ \text { B } & \text { CR-ORR } & 4 & 0 & 4 & 5060 & 7840 & 12100 & 7600 \\ \text { B } & \text { CR-AHD } & 4 & 0 & 3 & 2380 & 3690 & 5700 & 3570 \\ \text { B } & \text { CR-ROA } & 4 & 0 & 4 & 2810 & 4350 & 6740 & 4220 \\ & & & & & & & & \\ \text { C } & \text { DG-AND } & 4 & 0 & 4 & 31600 & 36000 & 41000 & 35700 \\ \text { C } & \text { DG-ROA } & 4 & 0 & 4 & 24800 & 28200 & 32200 & 28000 \\ \text { C } & \text { DG-ORR } & 4 & 0 & 4 & 22600 & 25800 & 29400 & 25600 \\ \text { C } & \text { NL-ORR } & 4 & 0 & 4 & 30600 & 34900 & 39800 & 34600 \\ \text { C } & \text { CHI-BV } & 4 & 0 & 4 & 24000 & 27300 & 31100 & 27100 \\ \text { C } & \text { CHI-K25 } & 4 & 0 & 4 & 27800 & 31600 & 36000 & 31400 \\ \text { C } & \text { CHE-ORR } & 4 & 0 & 4 & 10300 & 11800 & 13400 & 11700 \\ \text { C } & \text { CR-ORR } & 4 & 0 & 4 & 7730 & 8810 & 10000 & 8740 \\ \text { C } & \text { CR-AND } & 4 & 0 & 4 & 6660 & 7590 & 8650 & 7530 \\ \text { C } & \text { CR-ROA } & 4 & 0 & 4 & 3880 & 4420 & 5030 & 4380\end{array}$


Table G.1 (continued)

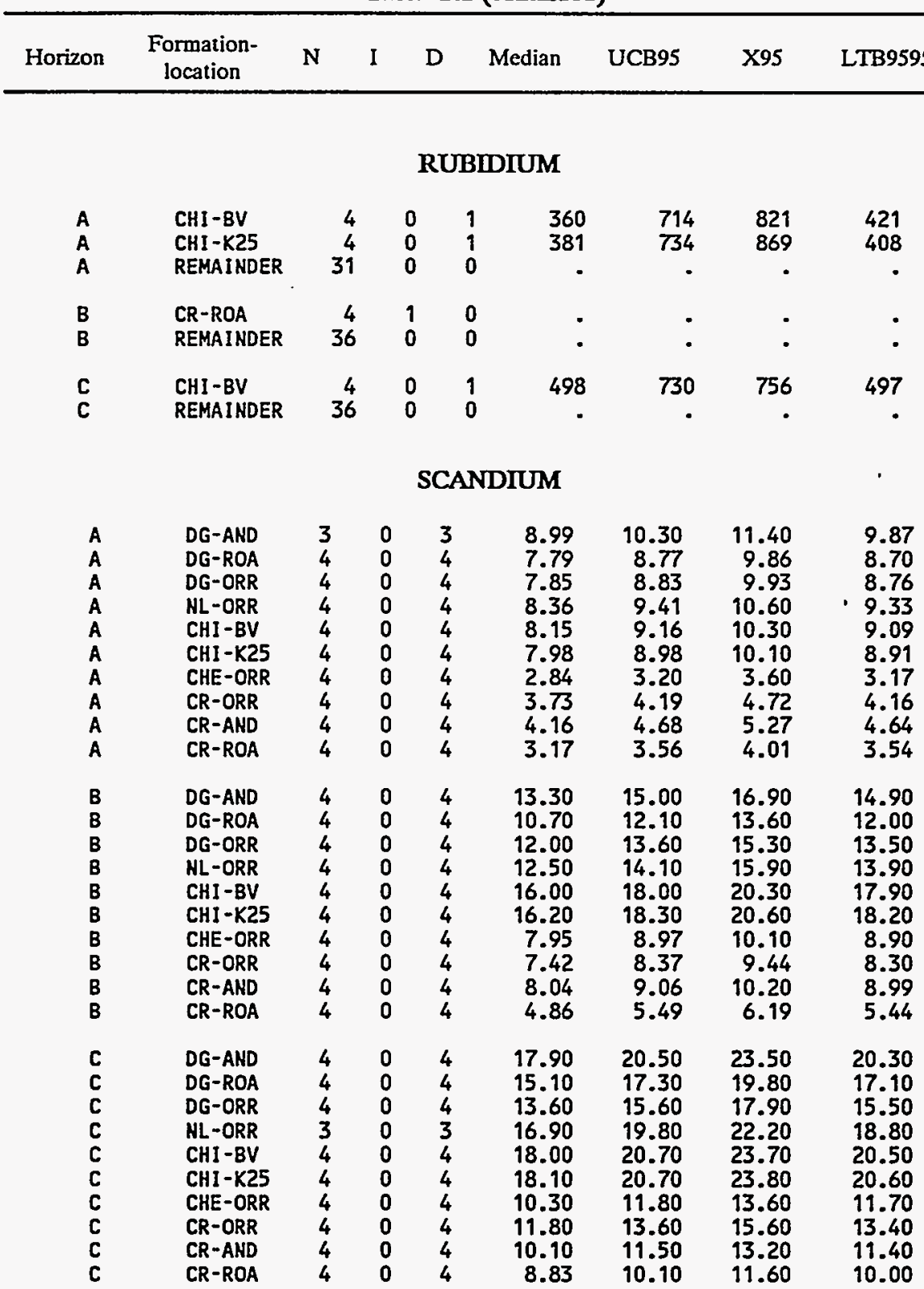

SILVER

$\begin{array}{lllllllll}\text { A } & \text { DG-AND } & 3 & 0 & 3 & 2.220 & 2.95 & 3.62 & 2.69 \\ \text { A } & \text { DG-ROA } & 4 & 0 & 4 & 1.870 & 2.39 & 3.05 & 2.35 \\ \text { A } & \text { DG-ORR } & 4 & 0 & 4 & 1.230 & 1.57 & 2.01 & 1.55 \\ \text { A } & \text { NL-ORR } & 4 & 0 & 4 & 1.030 & 1.31 & 1.68 & 1.29 \\ \text { A } & \text { CHI-BV } & 4 & 0 & 4 & 1.660 & 2.12 & 2.70 & 2.08 \\ \text { A } & \text { CHI-K25 } & 4 & 0 & 4 & 1.210 & 1.54 & 1.97 & 1.51 \\ \text { A } & \text { CHE-ORR } & 4 & 0 & 4 & 0.919 & 1.17 & 1.50 & 1.15 \\ \text { A } & \text { CR-ORR } & 4 & 0 & 4 & 1.230 & 1.57 & 2.00 & 1.54 \\ \text { A } & \text { CR-AND } & 4 & 0 & 4 & 1.540 & 1.97 & 2.51 & 1.93 \\ \text { A } & \text { CR-ROA } & 4 & 0 & 4 & 0.824 & 1.05 & 1.34 & 1.04 \\ \text { B } & \text { DG-AND } & 4 & 0 & 4 & 2.780 & 3.80 & 5.19 & 3.72 \\ \text { B } & \text { DG-ROA } & 4 & 0 & 4 & 2.150 & 2.93 & 4.01 & 2.87 \\ \text { B } & \text { DG-ORR } & 4 & 0 & 4 & 1.230 & 1.68 & 2.30 & 1.65 \\ \text { B } & \text { NL-ORR } & 4 & 0 & 4 & 1.220 & 1.66 & 2.27 & 1.63 \\ \text { B } & \text { CHI-8V } & 4 & 0 & 4 & 2.170 & 2.96 & 4.05 & 2.90 \\ \text { B } & \text { CHI-K25 } & 4 & 0 & 4 & 2.720 & 3.72 & 5.08 & 3.64 \\ \text { B } & \text { CHE-ORR } & 4 & 0 & 4 & 1.450 & 1.98 & 2.71 & 1.94 \\ \text { B } & \text { CR-ORR } & 4 & 0 & 4 & 2.220 & 3.03 & 4.14 & 2.97\end{array}$


G-15

Table G.1 (continued)

\begin{tabular}{|c|c|c|c|c|c|c|c|c|}
\hline Horizon & $\begin{array}{l}\text { Formation- } \\
\text { location }\end{array}$ & $\mathbf{N}$ & I & $\mathrm{D}$ & Median & UCB95 & X95 & LTB9595 \\
\hline 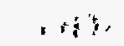 & $\Delta t_{4}$ & & \multicolumn{2}{|c|}{$\ldots$} & . & & $\frac{1}{2} i^{3}$ & $i$ \\
\hline $\begin{array}{l}\text { B } \\
\text { B }\end{array}$ & $\begin{array}{l}\text { CR-AND } \\
\text { CR-ROA }\end{array}$ & $\begin{array}{l}4 \\
4\end{array}$ & $\begin{array}{l}0 \\
0\end{array}$ & $\begin{array}{l}4 \\
4\end{array}$ & $\begin{array}{l}1.890 \\
1.310\end{array}$ & $\begin{array}{l}2.59 \\
1.79\end{array}$ & $\begin{array}{l}3.54 \\
2.45\end{array}$ & $\begin{array}{l}2.54 \\
1.76\end{array}$ \\
\hline $\begin{array}{l}C \\
C \\
C \\
C \\
C \\
C \\
C \\
C \\
C \\
C\end{array}$ & $\begin{array}{l}\text { DG-AND } \\
\text { DG-ROA } \\
\text { DG-ORR } \\
\text { NL-ORR } \\
\text { CHI -BV } \\
\text { CHI -K25 } \\
\text { CHE-ORR } \\
\text { CR-ORR } \\
\text { CR-AND } \\
\text { CR-ROA }\end{array}$ & $\begin{array}{l}4 \\
4 \\
4 \\
3 \\
4 \\
4 \\
4 \\
4 \\
4 \\
4\end{array}$ & $\begin{array}{l}0 \\
0 \\
0 \\
0 \\
0 \\
0 \\
0 \\
0 \\
0 \\
0\end{array}$ & $\begin{array}{l}4 \\
4 \\
4 \\
3 \\
4 \\
4 \\
4 \\
4 \\
4 \\
4\end{array}$ & $\begin{array}{l}2.820 \\
2.600 \\
1.640 \\
1.950 \\
2.230 \\
2.260 \\
1.610 \\
1.880 \\
2.200 \\
1.430\end{array}$ & $\begin{array}{l}3.38 \\
3.13 \\
1.97 \\
2.41 \\
2.68 \\
2.72 \\
1.93 \\
2.26 \\
2.64 \\
1.72\end{array}$ & $\begin{array}{l}4.07 \\
3.76 \\
2.37 \\
2.82 \\
3.23 \\
3.27 \\
2.32 \\
2.72 \\
3.18 \\
2.07\end{array}$ & $\begin{array}{l}3.34 \\
3.09 \\
1.95 \\
2.26 \\
2.65 \\
2.69 \\
1.91 \\
2.23 \\
2.61 \\
1.70\end{array}$ \\
\hline \multicolumn{9}{|c|}{ SODIUM } \\
\hline $\begin{array}{l}\mathbf{A} \\
\mathbf{A} \\
\mathbf{A} \\
\mathbf{A} \\
\mathbf{A} \\
\mathbf{A} \\
\mathbf{A} \\
\mathbf{A} \\
\mathbf{A} \\
\mathbf{A}\end{array}$ & $\begin{array}{l}\text { DG-AND } \\
\text { DG-ROA } \\
\text { DG-ORR } \\
\text { NL-ORR } \\
\text { CHI -BV } \\
\text { CHI-K25 } \\
\text { CHE-ORR } \\
\text { CR-ORR } \\
\text { CR-AND } \\
\text { CR-ROA }\end{array}$ & $\begin{array}{l}4 \\
4 \\
4 \\
4 \\
4 \\
4 \\
4 \\
4 \\
3 \\
4\end{array}$ & $\begin{array}{l}0 \\
0 \\
0 \\
0 \\
0 \\
0 \\
0 \\
0 \\
0 \\
0\end{array}$ & $\begin{array}{l}4 \\
4 \\
4 \\
4 \\
4 \\
4 \\
4 \\
4 \\
3 \\
4\end{array}$ & $\begin{array}{r}828 \\
540 \\
1020 \\
404 \\
1430 \\
784 \\
391 \\
434 \\
526 \\
404\end{array}$ & $\begin{array}{r}1030 \\
672 \\
1270 \\
503 \\
1770 \\
976 \\
486 \\
540 \\
677 \\
502\end{array}$ & $\begin{array}{r}1280 \\
836 \\
1580 \\
626 \\
2210 \\
1210 \\
605 \\
672 \\
814 \\
625\end{array}$ & $\begin{array}{r}1010 \\
662 \\
1250 \\
496 \\
1750 \\
962 \\
479 \\
532 \\
625 \\
495\end{array}$ \\
\hline $\begin{array}{l}\text { B } \\
\text { B } \\
\text { B } \\
\text { B } \\
\text { B } \\
\text { B } \\
\text { B } \\
\text { B } \\
\text { B } \\
\text { B }\end{array}$ & $\begin{array}{l}\text { DG-AND } \\
\text { DG-ROA } \\
\text { DG-ORR } \\
\text { NL-ORR } \\
\text { CHI-BV } \\
\text { CHI-K25 } \\
\text { CHE-ORR } \\
\text { CR-ORR } \\
\text { CR-AND } \\
\text { CR-ROA }\end{array}$ & $\begin{array}{l}4 \\
4 \\
4 \\
4 \\
4 \\
3 \\
4 \\
4 \\
4 \\
4\end{array}$ & $\begin{array}{l}0 \\
0 \\
0 \\
0 \\
0 \\
0 \\
0 \\
0 \\
0 \\
0\end{array}$ & $\begin{array}{l}4 \\
4 \\
4 \\
4 \\
4 \\
3 \\
4 \\
4 \\
4 \\
4\end{array}$ & $\begin{array}{r}624 \\
472 \\
964 \\
345 \\
2020 \\
1330 \\
212 \\
248 \\
104 \\
200\end{array}$ & $\begin{array}{r}998 \\
755 \\
1540 \\
551 \\
3230 \\
2290 \\
339 \\
396 \\
167 \\
319\end{array}$ & $\begin{array}{r}1590 \\
1210 \\
2460 \\
880 \\
5160 \\
3410 \\
541 \\
633 \\
267 \\
511\end{array}$ & $\begin{array}{r}967 \\
731 \\
1490 \\
534 \\
3130 \\
1930 \\
328 \\
384 \\
162 \\
310\end{array}$ \\
\hline $\begin{array}{l}c \\
c \\
c \\
c \\
c \\
c \\
c \\
c \\
c \\
c\end{array}$ & $\begin{array}{l}\text { DG-AKD } \\
\text { DG-ROA } \\
\text { DG-ORR } \\
\text { NL-ORR } \\
\text { CHI-BV } \\
\text { CHI-K25 } \\
\text { CHE-ORR } \\
\text { CR-ORR } \\
\text { CR-AHD } \\
\text { CR-ROA }\end{array}$ & $\begin{array}{l}4 \\
4 \\
4 \\
4 \\
4 \\
4 \\
4 \\
4 \\
4 \\
4\end{array}$ & $\begin{array}{l}0 \\
0 \\
0 \\
0 \\
0 \\
0 \\
0 \\
0 \\
0 \\
0\end{array}$ & $\begin{array}{l}4 \\
4 \\
4 \\
4 \\
4 \\
4 \\
4 \\
4 \\
4 \\
4\end{array}$ & $\begin{array}{r}806 \\
599 \\
1080 \\
383 \\
2170 \\
1540 \\
147 \\
150 \\
183 \\
162\end{array}$ & $\begin{array}{r}1080 \\
801 \\
1440 \\
512 \\
2910 \\
2060 \\
197 \\
201 \\
245 \\
216\end{array}$ & $\begin{array}{r}1440 \\
1070 \\
1920 \\
685 \\
3880 \\
2750 \\
263 \\
269 \\
327 \\
289\end{array}$ & $\begin{array}{r}1060 \\
786 \\
1410 \\
503 \\
2850 \\
2020 \\
193 \\
197 \\
240 \\
212\end{array}$ \\
\hline \multicolumn{9}{|c|}{ TERBIUM } \\
\hline $\begin{array}{l}\mathbf{A} \\
\mathbf{A} \\
\mathbf{A} \\
\mathbf{A} \\
\mathbf{A} \\
\mathbf{A} \\
\mathbf{A} \\
\mathbf{A} \\
\mathbf{A} \\
\mathbf{A}\end{array}$ & $\begin{array}{l}\text { DG-AND } \\
\text { DG-ROA } \\
\text { DG-ORR } \\
\text { NL-ORR } \\
\text { CHI-BV } \\
\text { CHI-K25 } \\
\text { CHE-ORR } \\
\text { CR-ORR } \\
\text { CR-AND } \\
\text { CR-ROA }\end{array}$ & $\begin{array}{l}3 \\
4 \\
4 \\
4 \\
4 \\
4 \\
4 \\
4 \\
4 \\
4\end{array}$ & $\begin{array}{l}0 \\
0 \\
0 \\
0 \\
0 \\
0 \\
0 \\
0 \\
0 \\
0\end{array}$ & $\begin{array}{l}3 \\
4 \\
4 \\
4 \\
4 \\
4 \\
4 \\
4 \\
4 \\
4\end{array}$ & $\begin{array}{l}0.352 \\
0.493 \\
0.535 \\
0.355 \\
0.917 \\
0.722 \\
0.547 \\
0.486 \\
0.496 \\
0.465\end{array}$ & $\begin{array}{l}0.475 \\
0.639 \\
0.692 \\
0.460 \\
1.190 \\
0.935 \\
0.708 \\
0.630 \\
0.642 \\
0.602\end{array}$ & $\begin{array}{l}0.591 \\
0.827 \\
0.896 \\
0.595 \\
1.540 \\
1.210 \\
0.917 \\
0.816 \\
0.832 \\
0.780\end{array}$ & $\begin{array}{l}0.432 \\
0.628 \\
0.680 \\
0.452 \\
1.170 \\
0.919 \\
0.696 \\
0.619 \\
0.631 \\
0.592\end{array}$ \\
\hline $\begin{array}{l}\text { B } \\
\text { B } \\
\text { B } \\
\text { B } \\
\text { B } \\
\text { B }\end{array}$ & $\begin{array}{l}\text { DG-AND } \\
\text { DG-ROA } \\
\text { DG-ORR } \\
\text { NL-ORR } \\
\text { CHI -BV } \\
\text { CHI-K25 }\end{array}$ & $\begin{array}{l}4 \\
4 \\
4 \\
4 \\
4 \\
4\end{array}$ & $\begin{array}{l}0 \\
0 \\
1 \\
0 \\
0 \\
0\end{array}$ & $\begin{array}{l}4 \\
4 \\
3 \\
2 \\
3 \\
4\end{array}$ & $\begin{array}{l}0.319 \\
0.532 \\
0.581 \\
0.300 \\
1.050 \\
0.718\end{array}$ & $\begin{array}{l}0.441 \\
0.735 \\
0.804 \\
0.437 \\
1.450 \\
0.991\end{array}$ & $\begin{array}{l}0.609 \\
1.010 \\
1.110 \\
0.573 \\
1.990 \\
1.370\end{array}$ & $\begin{array}{l}0.431 \\
0.718 \\
0.782 \\
0.390 \\
1.410 \\
0.968\end{array}$ \\
\hline
\end{tabular}


G-16

Table G.1 (continued)

\begin{tabular}{|c|c|c|c|c|c|c|c|c|}
\hline Horizon & $\begin{array}{l}\text { Formation- } \\
\text { location }\end{array}$ & $\mathbf{N}$ & I & $\mathrm{D}$ & Median & UCB95 & $\mathrm{X} 95$ & LTB9595 \\
\hline $\begin{array}{l}\text { B } \\
\text { B } \\
\text { B } \\
\text { B }\end{array}$ & $\begin{array}{l}\text { CHE-ORR } \\
\text { CR-ORR } \\
\text { CR-AND } \\
\text { CR-ROA }\end{array}$ & $\begin{array}{l}4 \\
4 \\
4 \\
4\end{array}$ & $\begin{array}{l}1 \\
0 \\
0 \\
0\end{array}$ & $\begin{array}{l}3 \\
3 \\
4 \\
4\end{array}$ & $\begin{array}{l}0.405 \\
0.307 \\
0.281 \\
0.283\end{array}$ & $\begin{array}{l}0.561 \\
0.445 \\
0.388 \\
0.391\end{array}$ & $\begin{array}{l}0.773 \\
0.585 \\
0.535 \\
0.540\end{array}$ & $\begin{array}{l}0.544 \\
0.395 \\
0.378 \\
0.382\end{array}$ \\
\hline $\begin{array}{l}c \\
C \\
C \\
C \\
C \\
C \\
C \\
c \\
C \\
C\end{array}$ & $\begin{array}{l}\text { DG-AND } \\
\text { DG-ROA } \\
\text { DG-ORR } \\
\text { HL-ORR } \\
\text { CHI-BV } \\
\text { CHI-K25 } \\
\text { CHE-ORR } \\
\text { CR-ORR } \\
\text { CR-AND } \\
\text { CR-ROA }\end{array}$ & $\begin{array}{l}4 \\
4 \\
4 \\
3 \\
4 \\
4 \\
4 \\
4 \\
4 \\
4\end{array}$ & $\begin{array}{l}0 \\
0 \\
1 \\
0 \\
0 \\
0 \\
0 \\
0 \\
0 \\
0\end{array}$ & $\begin{array}{l}4 \\
4 \\
3 \\
2 \\
4 \\
4 \\
4 \\
3 \\
2 \\
2\end{array}$ & $\begin{array}{l}0.717 \\
0.467 \\
0.282 \\
0.364 \\
1.560 \\
0.576 \\
0.299 \\
0.283 \\
0.318 \\
0.243\end{array}$ & $\begin{array}{l}0.922 \\
0.601 \\
0.367 \\
0.501 \\
2.010 \\
0.742 \\
0.385 \\
0.376 \\
0.433 \\
0.325\end{array}$ & $\begin{array}{l}1.190 \\
0.773 \\
0.467 \\
0.603 \\
2.580 \\
0.954 \\
0.496 \\
0.469 \\
0.526 \\
0.403\end{array}$ & $\begin{array}{l}0.904 \\
0.589 \\
0.352 \\
0.433 \\
1.970 \\
0.727 \\
0.378 \\
0.348 \\
0.384 \\
0.299\end{array}$ \\
\hline \multicolumn{9}{|c|}{ THORIUM-232 } \\
\hline $\begin{array}{l}A \\
A \\
A \\
A \\
A \\
A \\
A \\
A \\
A \\
A\end{array}$ & $\begin{array}{l}\text { DG-AND } \\
\text { DG-ROA } \\
\text { DG-ORR } \\
\text { NL-ORR } \\
\text { CHI-BV } \\
\text { CHI-K25 } \\
\text { CHE-ORR } \\
\text { CR-ORR } \\
\text { CR-AND } \\
\text { CR-ROA }\end{array}$ & $\begin{array}{l}3 \\
4 \\
4 \\
4 \\
4 \\
4 \\
4 \\
4 \\
4 \\
4\end{array}$ & $\begin{array}{l}0 \\
0 \\
0 \\
0 \\
0 \\
0 \\
0 \\
0 \\
0 \\
0\end{array}$ & $\begin{array}{l}3 \\
4 \\
4 \\
4 \\
4 \\
4 \\
4 \\
4 \\
4 \\
4\end{array}$ & $\begin{array}{r}10.10 \\
10.90 \\
9.74 \\
11.70 \\
10.00 \\
9.91 \\
5.18 \\
6.51 \\
6.53 \\
5.11\end{array}$ & $\begin{array}{r}11.10 \\
11.80 \\
10.60 \\
12.70 \\
10.90 \\
10.80 \\
5.62 \\
7.06 \\
7.08 \\
5.54\end{array}$ & $\begin{array}{r}11.90 \\
12.80 \\
11.50 \\
13.70 \\
11.80 \\
11.70 \\
6.10 \\
7.65 \\
7.68 \\
6.01\end{array}$ & $\begin{array}{r}10.80 \\
11.80 \\
10.50 \\
12.60 \\
10.80 \\
10.70 \\
5.59 \\
7.02 \\
7.04 \\
5.51\end{array}$ \\
\hline $\begin{array}{l}\text { B } \\
\text { B } \\
\text { B } \\
\text { B } \\
\text { B } \\
\text { B } \\
\text { B } \\
\text { B } \\
\text { B } \\
\text { B }\end{array}$ & $\begin{array}{l}\text { DG-AND } \\
\text { DG-ROA } \\
\text { DG-ORR } \\
\text { NL-ORR } \\
\text { CHI-BV } \\
\text { CHI-K25 } \\
\text { CHE-ORR } \\
\text { CR-ORR } \\
\text { CR-AND } \\
\text { CR-ROA }\end{array}$ & $\begin{array}{l}4 \\
4 \\
4 \\
4 \\
4 \\
4 \\
4 \\
4 \\
4 \\
4\end{array}$ & $\begin{array}{l}0 \\
0 \\
0 \\
0 \\
0 \\
0 \\
0 \\
0 \\
0 \\
0\end{array}$ & $\begin{array}{l}4 \\
4 \\
4 \\
4 \\
4 \\
4 \\
4 \\
4 \\
4 \\
4\end{array}$ & $\begin{array}{r}13.30 \\
13.30 \\
12.30 \\
15.00 \\
14.10 \\
14.60 \\
9.91 \\
10.30 \\
9.15 \\
6.64\end{array}$ & $\begin{array}{r}14.70 \\
14.60 \\
13.50 \\
16.40 \\
15.50 \\
16.10 \\
10.90 \\
11.30 \\
10.10 \\
7.30\end{array}$ & $\begin{array}{r}16.10 \\
16.10 \\
14.80 \\
18.10 \\
17.00 \\
17.70 \\
12.00 \\
12.40 \\
11.10 \\
8.03\end{array}$ & $\begin{array}{r}14.60 \\
14.50 \\
13.40 \\
16.30 \\
15.40 \\
16.00 \\
10.80 \\
11.20 \\
10.00 \\
7.26\end{array}$ \\
\hline $\begin{array}{l}\text { C } \\
C \\
C \\
C \\
C \\
C \\
C \\
C \\
C \\
C\end{array}$ & $\begin{array}{l}\text { DG-AND } \\
\text { DG-ROA } \\
\text { DG-ORR } \\
\text { NL-ORR } \\
\text { CHI-BV } \\
\text { CHI-K25 } \\
\text { CHE-ORR } \\
\text { CR-ORR } \\
\text { CR-AND } \\
\text { CR-ROA }\end{array}$ & $\begin{array}{l}4 \\
4 \\
4 \\
3 \\
4 \\
4 \\
4 \\
4 \\
4 \\
4\end{array}$ & $\begin{array}{l}0 \\
0 \\
0 \\
0 \\
0 \\
0 \\
0 \\
0 \\
0 \\
0\end{array}$ & $\begin{array}{l}4 \\
4 \\
4 \\
3 \\
4 \\
4 \\
4 \\
4 \\
4 \\
4\end{array}$ & $\begin{array}{l}13.20 \\
15.00 \\
12.30 \\
17.60 \\
13.90 \\
14.40 \\
10.20 \\
13.30 \\
10.80 \\
11.40\end{array}$ & $\begin{array}{l}14.60 \\
16.60 \\
13.60 \\
19.80 \\
15.40 \\
16.00 \\
11.30 \\
14.80 \\
12.00 \\
12.60\end{array}$ & $\begin{array}{l}16.20 \\
18.30 \\
15.10 \\
21.60 \\
17.10 \\
17.70 \\
12.50 \\
16.30 \\
13.30 \\
13.90\end{array}$ & $\begin{array}{l}14.50 \\
16.50 \\
13.50 \\
19.10 \\
15.30 \\
15.90 \\
11.20 \\
14.70 \\
11.90 \\
12.50\end{array}$ \\
\hline
\end{tabular}

\section{TITANIUM}

$\begin{array}{rlrrrrrrr}\text { A } & \text { DG-AND } & 4 & 0 & 4 & 5280 & 11900 & 26700 & 11200 \\ \text { A } & \text { DG-ROA } & 4 & 1 & 3 & 8080 & 18200 & 40900 & 17200 \\ \text { A } & \text { DG-ORR } & 4 & 0 & 4 & 3280 & 7380 & 16600 & 6980 \\ \text { A } & \text { HL-ORR } & 4 & 0 & 4 & 7010 & 15800 & 35500 & 14900 \\ \text { A } & \text { CHI-BV } & 4 & 0 & 4 & 5570 & 12500 & 28200 & 11800 \\ \text { A } & \text { CHI-K25 } & 4 & 0 & 4 & 4440 & 10000 & 22500 & 9460 \\ \text { A } & \text { CHE-ORR } & 4 & 0 & 4 & 4120 & 9270 & 20800 & 8770 \\ \text { A } & \text { CR-ORR } & 4 & 0 & 4 & 4410 & 9910 & 22300 & 9380 \\ \text { A } & \text { CR-AND } & 4 & 0 & 4 & 5150 & 11600 & 26000 & 10900 \\ \text { A } & \text { CR-ROA } & 4 & 0 & 3 & 783 & 1770 & 3960 & 1670 \\ & & & & & & & & \\ \text { B } & \text { DG-AND } & 4 & 0 & 4 & 5750 & 8660 & 13100 & 8430 \\ \text { B } & \text { DG-ROA } & 4 & 0 & 3 & 2470 & 3750 & 5610 & 3620 \\ \text { B } & \text { DG-ORR } & 4 & 0 & 4 & 5460 & 8220 & 12400 & 8000 \\ \text { B } & \text { NL-ORR } & 4 & 0 & 4 & 7460 & 11200 & 16900 & 10900\end{array}$


G-17

Table G.1 (continued)

\begin{tabular}{rcrrrrrrr}
\hline Horizon & $\begin{array}{c}\text { Formation- } \\
\text { location }\end{array}$ & N & I & D & Median & UCB95 & X95 & LTB9595 \\
\hline & & & & & & & & \\
\hline & CHI-BV & 4 & 0 & 4 & 5070 & 7640 & 11500 & 7430 \\
B & CHI-K25 & 4 & 0 & 4 & 5840 & 8800 & 13300 & 8560 \\
B & CHE-ORR & 4 & 0 & 4 & 4130 & 6220 & 9370 & 6050 \\
B & CR-ORR & 4 & 0 & 4 & 4580 & 6900 & 10400 & 6710 \\
B & CR-ORR & & \\
B & CR-AND & 4 & 0 & 4 & 4590 & 6920 & 10400 & 6730 \\
B & CR-ROA & 4 & 0 & 4 & 4160 & 6260 & 9430 & 6090 \\
C & DG-AND & 4 & 0 & 4 & 4750 & 6790 & 9700 & 6630 \\
C & DG-ROA & 4 & 1 & 3 & 6910 & 9880 & 14100 & 9640 \\
C & DG-ORR & 4 & 0 & 4 & 4680 & 6690 & 9560 & 6530 \\
C & ML-ORR & 4 & 0 & 4 & 8310 & 11900 & 17000 & 11600 \\
C & CHI-BV & 4 & 0 & 4 & 4700 & 6710 & 9590 & 6550 \\
C & CHI-K25 & 4 & 0 & 4 & 5540 & 7910 & 11300 & 7730 \\
C & CHE-ORR & 4 & 0 & 4 & 3170 & 4530 & 6480 & 4420 \\
C & CR-ORR & 4 & 0 & 4 & 3320 & 4740 & 6770 & 4630 \\
C & CR-AND & 3 & 0 & 3 & 4300 & 6490 & 8780 & 5690 \\
C & CR-ROA & 4 & 0 & 4 & 3130 & 4470 & 6380 & 4360
\end{tabular}

URANIUM-235

$\begin{array}{lllllllll}\text { A } & \text { OG-AND } & 4 & 0 & 4 & 0.0225 & 0.0240 & 0.0256 & 0.0239 \\ \text { A } & \text { DG-ROA } & 4 & 0 & 4 & 0.0228 & 0.0243 & 0.0259 & 0.0242 \\ \text { A } & \text { DG-ORR } & 4 & 0 & 4 & 0.0269 & 0.0287 & 0.0306 & 0.0286 \\ \text { A } & \text { NL-ORR } & 4 & 0 & 4 & 0.0255 & 0.0271 & 0.0289 & 0.0270 \\ \text { A } & \text { CHI-BV } & 4 & 0 & 4 & 0.0252 & 0.0268 & 0.0286 & 0.0267 \\ \text { A } & \text { CHI-K25 } & 4 & 0 & 4 & 0.0315 & 0.0336 & 0.0358 & 0.0334 \\ \text { A } & \text { CHE-ORR } & 4 & 0 & 4 & 0.0255 & 0.0272 & 0.0290 & 0.0271 \\ \text { A } & \text { CR-ORR } & 4 & 0 & 4 & 0.0313 & 0.0334 & 0.0356 & 0.0332 \\ \text { A } & \text { CR-AND } & 4 & 0 & 4 & 0.0286 & 0.0305 & 0.0325 & 0.0304 \\ \text { A } & \text { CR-ROA } & 4 & 0 & 4 & 0.0219 & 0.0233 & 0.0248 & 0.0232 \\ & & & & & & & & \\ \text { B } & \text { DG-AND } & 4 & 0 & 4 & 0.0233 & 0.0252 & 0.0273 & 0.0251 \\ \text { B } & \text { DG-ROA } & 4 & 0 & 4 & 0.0238 & 0.0257 & 0.0279 & 0.0256 \\ \text { B } & \text { DG-ORR } & 4 & 0 & 4 & 0.0214 & 0.0232 & 0.0252 & 0.0231 \\ \text { B } & \text { NL-ORR } & 4 & 0 & 4 & 0.0222 & 0.0240 & 0.0260 & 0.0239 \\ \text { B } & \text { CHI-BV } & 4 & 0 & 4 & 0.0236 & 0.0256 & 0.0277 & 0.0254 \\ \text { B } & \text { CHI-K25 } & 4 & 0 & 4 & 0.0273 & 0.0296 & 0.0321 & 0.0295 \\ \text { B } & \text { CHE-ORR } & 4 & 0 & 4 & 0.0306 & 0.0331 & 0.0359 & 0.0330 \\ \text { B } & \text { CR-ORR } & 4 & 0 & 4 & 0.0366 & 0.0397 & 0.0430 & 0.0395 \\ \text { B } & \text { CR-AND } & 4 & 0 & 4 & 0.0382 & 0.0414 & 0.0449 & 0.0412 \\ \text { B } & \text { CR-ROA } & 4 & 0 & 4 & 0.0275 & 0.0298 & 0.0323 & 0.0297 \\ \text { C } & \text { DG-AND } & 4 & 0 & 4 & 0.0221 & 0.0245 & 0.0272 & 0.0244 \\ \text { C } & \text { DG-ROA } & 4 & 0 & 4 & 0.0214 & 0.0237 & 0.0263 & 0.0236 \\ \text { C } & \text { DG-ORR } & 4 & 0 & 4 & 0.0190 & 0.0210 & 0.0233 & 0.0209 \\ \text { C } & \text { HL-ORR } & 4 & 0 & 4 & 0.0205 & 0.0227 & 0.0252 & 0.0226 \\ \text { C } & \text { CHI-BV } & 4 & 0 & 4 & 0.0223 & 0.0247 & 0.0274 & 0.0246 \\ \text { C } & \text { CHI-K25 } & 4 & 0 & 4 & 0.0249 & 0.0276 & 0.0305 & 0.0274 \\ \text { C } & \text { CHE-ORR } & 4 & 0 & 4 & 0.0340 & 0.0377 & 0.0418 & 0.0374 \\ \text { C } & \text { CR-ORR } & 4 & 0 & 4 & 0.0449 & 0.0497 & 0.0551 & 0.0494 \\ \text { C } & \text { CR-AND } & 4 & 0 & 4 & 0.0405 & 0.0448 & 0.0497 & 0.0445 \\ \text { C } & \text { CR-ROA } & 4 & 0 & 4 & 0.0324 & 0.0359 & 0.0398 & 0.0357 \\ & & & & & & & & \end{array}$

\section{URANIUM-238}

$\begin{array}{lllllllll}\text { A } & \text { DG-AND } & 4 & 0 & 4 & 2.78 & 3.10 & 3.46 & 3.08 \\ \text { A } & \text { DG-ROA } & 4 & 0 & 4 & 3.18 & 3.55 & 3.96 & 3.52 \\ \text { A } & \text { DG-ORR } & 4 & 0 & 4 & 3.99 & 4.45 & 4.96 & 4.42 \\ \text { A } & \text { NL-ORR } & 4 & 0 & 4 & 3.84 & 4.29 & 4.78 & 4.25 \\ \text { A } & \text { CHI-BV } & 4 & 0 & 4 & 3.60 & 4.02 & 4.48 & 3.99 \\ \text { A } & \text { CHI-K25 } & 4 & 0 & 4 & 4.67 & 5.20 & 5.81 & 5.17 \\ \text { A } & \text { CHE-ORR } & 4 & 0 & 4 & 4.11 & 4.59 & 5.12 & 4.55 \\ \text { A } & \text { CR-ORR } & 4 & 0 & 4 & 4.52 & 5.04 & 5.62 & 5.00 \\ \text { A } & \text { CR-AND } & 3 & 0 & 3 & 4.82 & 5.47 & 6.00 & 5.25 \\ \text { A } & \text { CR-ROA } & 4 & 0 & 4 & 3.23 & 3.60 & 4.02 & 3.57\end{array}$


G-18

Table G.1 (continued)

\begin{tabular}{|c|c|c|c|c|c|c|c|c|}
\hline Horizon & $\begin{array}{l}\text { Formation- } \\
\text { location }\end{array}$ & $\mathbf{N}$ & $\mathrm{I}$ & $\mathrm{D}$ & Median & UCB95 & $\times 95$ & LTB9595 \\
\hline $\begin{array}{l}\text { B } \\
\text { B } \\
\text { B } \\
\text { B } \\
\text { B } \\
\text { B } \\
\text { B } \\
\text { B } \\
\text { B } \\
\text { B }\end{array}$ & $\begin{array}{l}\text { DG-AND } \\
\text { DG-ROA } \\
\text { DG-ORR } \\
\text { NL-ORR } \\
\text { CHI-BV } \\
\text { CHI-K25 } \\
\text { CHE-ORR } \\
\text { CR-ORR } \\
\text { CR-AND } \\
\text { CR-ROA }\end{array}$ & $\begin{array}{l}4 \\
4 \\
4 \\
4 \\
4 \\
3 \\
4 \\
4 \\
4 \\
4\end{array}$ & $\begin{array}{l}0 \\
0 \\
0 \\
0 \\
0 \\
0 \\
0 \\
0 \\
0 \\
0\end{array}$ & $\begin{array}{l}4 \\
4 \\
4 \\
4 \\
4 \\
3 \\
4 \\
4 \\
4 \\
4\end{array}$ & $\begin{array}{l}2.94 \\
3.49 \\
3.28 \\
3.15 \\
3.23 \\
4.16 \\
4.14 \\
5.22 \\
6.52 \\
3.33\end{array}$ & $\begin{array}{l}3.59 \\
4.27 \\
4.02 \\
3.86 \\
3.95 \\
5.25 \\
5.07 \\
6.39 \\
7.98 \\
4.08\end{array}$ & $\begin{array}{l}4.40 \\
5.23 \\
4.92 \\
4.72 \\
4.84 \\
6.23 \\
6.21 \\
7.82 \\
9.77 \\
4.99\end{array}$ & $\begin{array}{l}3.54 \\
4.21 \\
3.96 \\
3.81 \\
3.90 \\
4.88 \\
5.00 \\
6.30 \\
7.88 \\
4.02\end{array}$ \\
\hline $\begin{array}{l}C \\
C \\
C \\
C \\
C \\
C \\
C \\
C \\
C \\
C\end{array}$ & $\begin{array}{l}\text { DG-AND } \\
\text { DG-ROA } \\
\text { DG-ORR } \\
\text { NL-ORR } \\
\text { CHI-BV } \\
\text { CHI-K25 } \\
\text { CHE-ORR } \\
\text { CR-ORR } \\
\text { CR-AND } \\
\text { CR-ROA }\end{array}$ & $\begin{array}{l}4 \\
4 \\
4 \\
4 \\
4 \\
4 \\
4 \\
4 \\
4 \\
4\end{array}$ & $\begin{array}{l}0 \\
0 \\
0 \\
0 \\
0 \\
0 \\
0 \\
0 \\
0 \\
0\end{array}$ & $\begin{array}{l}4 \\
4 \\
4 \\
4 \\
4 \\
4 \\
4 \\
4 \\
4 \\
4\end{array}$ & $\begin{array}{l}2.67 \\
3.22 \\
2.69 \\
3.36 \\
3.14 \\
3.59 \\
4.35 \\
4.95 \\
5.42 \\
5.04\end{array}$ & $\begin{array}{l}3.23 \\
3.90 \\
3.26 \\
4.07 \\
3.80 \\
4.35 \\
5.27 \\
6.00 \\
6.57 \\
6.10\end{array}$ & $\begin{array}{l}3.92 \\
4.73 \\
3.96 \\
4.93 \\
4.61 \\
5.27 \\
6.39 \\
7.27 \\
7.96 \\
7.40\end{array}$ & $\begin{array}{l}3.19 \\
3.85 \\
3.22 \\
4.02 \\
3.75 \\
4.30 \\
5.21 \\
5.93 \\
6.48 \\
6.03\end{array}$ \\
\hline \multicolumn{9}{|c|}{ VANADIUM } \\
\hline $\begin{array}{l}A \\
A \\
A \\
A \\
A \\
A \\
A \\
A \\
A \\
A\end{array}$ & $\begin{array}{l}\text { DG-AND } \\
D G-R O A \\
D G-O R R \\
N L-O R R \\
C H I-B V \\
C H I-K 25 \\
C H E-O R R \\
C R-O R R \\
C R-A N D \\
C R-R O A\end{array}$ & $\begin{array}{l}4 \\
4 \\
4 \\
4 \\
4 \\
4 \\
4 \\
4 \\
4 \\
4\end{array}$ & $\begin{array}{l}0 \\
0 \\
0 \\
0 \\
0 \\
0 \\
0 \\
0 \\
0 \\
0\end{array}$ & $\begin{array}{l}4 \\
4 \\
4 \\
4 \\
4 \\
4 \\
4 \\
4 \\
4 \\
3\end{array}$ & $\begin{array}{r}75.40 \\
64.40 \\
35.40 \\
57.00 \\
52.20 \\
44.20 \\
32.50 \\
40.70 \\
40.60 \\
6.35\end{array}$ & $\begin{array}{r}168.0 \\
144.0 \\
79.1 \\
127.0 \\
116.0 \\
98.7 \\
72.5 \\
90.8 \\
90.7 \\
14.2\end{array}$ & $\begin{array}{r}375.0 \\
320.0 \\
176.0 \\
284.0 \\
260.0 \\
220.0 \\
162.0 \\
203.0 \\
202.0 \\
31.6\end{array}$ & $\begin{array}{r}159.0 \\
136.0 \\
74.8 \\
120.0 \\
110.0 \\
93.4 \\
68.6 \\
85.9 \\
85.8 \\
13.4\end{array}$ \\
\hline $\begin{array}{l}\text { B } \\
\text { B } \\
\text { B } \\
\text { B } \\
\text { B } \\
\text { B } \\
\text { B } \\
\text { B } \\
\text { B } \\
\text { B }\end{array}$ & $\begin{array}{l}\text { DG-AND } \\
\text { DG-ROA } \\
\text { DG-ORR } \\
\text { HL-ORR } \\
\text { CHI-BV } \\
\text { CHI-K25 } \\
\text { CHE-ORR } \\
\text { CR-ORR } \\
\text { CR-AND } \\
\text { CR-ROA }\end{array}$ & $\begin{array}{l}4 \\
4 \\
4 \\
4 \\
4 \\
4 \\
4 \\
4 \\
4 \\
4\end{array}$ & $\begin{array}{l}0 \\
0 \\
0 \\
0 \\
0 \\
0 \\
0 \\
0 \\
0 \\
0\end{array}$ & $\begin{array}{l}4 \\
4 \\
4 \\
4 \\
4 \\
4 \\
4 \\
4 \\
4 \\
4\end{array}$ & $\begin{array}{r}101.00 \\
85.40 \\
86.30 \\
93.10 \\
96.60 \\
129.00 \\
91.40 \\
89.90 \\
85.90 \\
77.70\end{array}$ & $\begin{array}{r}111.0 \\
93.8 \\
94.7 \\
102.0 \\
106.0 \\
142.0 \\
100.0 \\
98.6 \\
94.2 \\
85.3\end{array}$ & $\begin{array}{r}122.0 \\
103.0 \\
104.0 \\
112.0 \\
116.0 \\
156.0 \\
110.0 \\
108.0 \\
103.0 \\
93.6\end{array}$ & $\begin{array}{r}110.0 \\
93.2 \\
94.1 \\
102.0 \\
105.0 \\
141.0 \\
99.7 \\
98.0 \\
93.7 \\
84.8\end{array}$ \\
\hline $\begin{array}{l}C \\
C \\
C \\
C \\
C \\
C \\
C \\
C \\
C \\
C\end{array}$ & $\begin{array}{l}\text { DG-AND } \\
\text { DG-ROA } \\
\text { DG-ORR } \\
\text { NL-ORR } \\
\text { CHI-BV } \\
\text { CHI-K25 } \\
\text { CHE-ORR } \\
\text { CR-ORR } \\
\text { CR-AND } \\
\text { CR-ROA }\end{array}$ & $\begin{array}{l}4 \\
4 \\
4 \\
4 \\
4 \\
4 \\
4 \\
4 \\
3 \\
4\end{array}$ & $\begin{array}{l}0 \\
0 \\
0 \\
0 \\
0 \\
0 \\
0 \\
0 \\
0 \\
1\end{array}$ & $\begin{array}{l}4 \\
4 \\
4 \\
4 \\
4 \\
4 \\
4 \\
4 \\
3 \\
3\end{array}$ & $\begin{array}{r}104.00 \\
92.70 \\
93.90 \\
91.90 \\
104.00 \\
117.00 \\
104.00 \\
130.00 \\
97.50 \\
98.30\end{array}$ & $\begin{array}{l}140.0 \\
125.0 \\
127.0 \\
124.0 \\
141.0 \\
158.0 \\
141.0 \\
175.0 \\
138.0 \\
133.0\end{array}$ & $\begin{array}{l}189.0 \\
169.0 \\
171.0 \\
168.0 \\
190.0 \\
214.0 \\
190.0 \\
236.0 \\
178.0 \\
179.0\end{array}$ & $\begin{array}{l}137.0 \\
123.0 \\
124.0 \\
122.0 \\
138.0 \\
155.0 \\
138.0 \\
172.0 \\
123.0 \\
130.0\end{array}$ \\
\hline
\end{tabular}


G-19

.Table G.1 (continued)

\begin{tabular}{|c|c|c|c|c|c|c|c|c|}
\hline Horizon & $\begin{array}{l}\text { Formation- } \\
\text { location }\end{array}$ & $\mathbf{N}$ & I & D & Median & UCB95 & X95 & LTB9595 \\
\hline$\because \quad 3$ & $A: *$ & & & & $\mathrm{~s}$ & & $\therefore y=$ & is. \\
\hline \multicolumn{9}{|c|}{ YTIERBIUM } \\
\hline $\begin{array}{l}A \\
A \\
A \\
A \\
A \\
A \\
A \\
A \\
A \\
A\end{array}$ & $\begin{array}{l}\text { DG-AND } \\
\text { DG-ROA } \\
\text { DG-ORR } \\
\text { NL-ORR } \\
\text { CHI -BV } \\
\text { CHI -K25 } \\
\text { CHE-ORR } \\
\text { CR-ORR } \\
\text { CR-AND } \\
\text { CR-ROA }\end{array}$ & $\begin{array}{l}3 \\
4 \\
4 \\
4 \\
4 \\
4 \\
4 \\
4 \\
4 \\
4\end{array}$ & $\begin{array}{l}0 \\
0 \\
0 \\
0 \\
0 \\
0 \\
0 \\
0 \\
0 \\
0\end{array}$ & $\begin{array}{l}3 \\
4 \\
4 \\
4 \\
4 \\
4 \\
4 \\
4 \\
4 \\
4\end{array}$ & $\begin{array}{l}2.600 \\
3.800 \\
1.900 \\
2.900 \\
3.130 \\
2.960 \\
2.630 \\
2.300 \\
2.360 \\
1.660\end{array}$ & $\begin{array}{l}3.18 \\
4.53 \\
2.26 \\
3.46 \\
3.72 \\
3.53 \\
3.13 \\
2.74 \\
2.80 \\
1.97\end{array}$ & $\begin{array}{l}3.69 \\
5.39 \\
2.69 \\
4.11 \\
4.43 \\
4.20 \\
3.73 \\
3.26 \\
3.34 \\
2.35\end{array}$ & $\begin{array}{l}2.980 \\
4.470 \\
2.240 \\
3.420 \\
3.680 \\
3.490 \\
3.100 \\
2.710 \\
2.770 \\
1.950\end{array}$ \\
\hline $\begin{array}{l}\text { B } \\
\text { B } \\
\text { B } \\
\text { B } \\
\text { B } \\
\text { B } \\
\text { B } \\
\text { B } \\
\text { B } \\
\text { B }\end{array}$ & $\begin{array}{l}\text { DG-AND } \\
\text { DG-ROA } \\
\text { DG-ORR } \\
\text { HL-ORR } \\
\text { CHI-BV } \\
\text { CHI-K25 } \\
\text { CHE-ORR } \\
\text { CR-ORR } \\
\text { CR-AND } \\
\text { CR-ROA }\end{array}$ & $\begin{array}{l}4 \\
4 \\
4 \\
4 \\
4 \\
4 \\
4 \\
4 \\
4 \\
4\end{array}$ & $\begin{array}{l}0 \\
1 \\
1 \\
0 \\
0 \\
0 \\
1 \\
0 \\
0 \\
1\end{array}$ & $\begin{array}{l}4 \\
3 \\
3 \\
3 \\
2 \\
4 \\
3 \\
3 \\
4 \\
2\end{array}$ & $\begin{array}{l}3.320 \\
2.230 \\
1.440 \\
1.280 \\
0.583 \\
2.560 \\
2.010 \\
2.110 \\
1.440 \\
1.180\end{array}$ & $\begin{array}{l}5.67 \\
3.88 \\
2.53 \\
2.30 \\
1.23 \\
4.38 \\
3.48 \\
3.72 \\
2.46 \\
2.06\end{array}$ & $\begin{array}{l}9.69 \\
6.51 \\
4.21 \\
3.74 \\
1.70 \\
7.47 \\
5.85 \\
6.16 \\
4.21 \\
3.43\end{array}$ & $\begin{array}{l}5.430 \\
3.630 \\
2.320 \\
2.020 \\
0.786 \\
4.190 \\
3.260 \\
3.400 \\
2.360 \\
1.900\end{array}$ \\
\hline $\begin{array}{l}c \\
C \\
C \\
c \\
c \\
C \\
C \\
C \\
C \\
C\end{array}$ & $\begin{array}{l}\text { DG-AND } \\
\text { DG-ROA } \\
\text { DG-ORR } \\
\text { NL-ORR } \\
\text { CHI-BV } \\
\text { CHI -K25 } \\
\text { CHE-ORR } \\
\text { CR-ORR } \\
\text { CR-AND } \\
\text { CR-ROA }\end{array}$ & $\begin{array}{l}4 \\
4 \\
4 \\
3 \\
4 \\
4 \\
4 \\
4 \\
4 \\
4\end{array}$ & $\begin{array}{l}0 \\
0 \\
1 \\
0 \\
0 \\
0 \\
1 \\
0 \\
0 \\
1\end{array}$ & $\begin{array}{l}2 \\
3 \\
2 \\
3 \\
4 \\
4 \\
3 \\
3 \\
3 \\
3\end{array}$ & $\begin{array}{l}1.270 \\
1.680 \\
1.310 \\
2.360 \\
1.040 \\
2.400 \\
1.180 \\
2.260 \\
0.633 \\
0.723\end{array}$ & $\begin{array}{l}2.69 \\
3.22 \\
2.54 \\
4.77 \\
1.92 \\
4.43 \\
2.25 \\
4.33 \\
1.23 \\
1.34\end{array}$ & $\begin{array}{l}4.31 \\
5.71 \\
4.46 \\
8.01 \\
3.55 \\
8.17 \\
4.02 \\
7.70 \\
2.15 \\
2.46\end{array}$ & $\begin{array}{l}2.000 \\
2.880 \\
2.230 \\
3.790 \\
1.830 \\
4.220 \\
2.040 \\
3.890 \\
1.070 \\
1.270\end{array}$ \\
\hline \multicolumn{9}{|c|}{ ZINC } \\
\hline $\begin{array}{l}\mathbf{A} \\
\mathbf{A} \\
\mathbf{A} \\
\mathbf{A} \\
\mathbf{A} \\
\mathbf{A} \\
\mathbf{A} \\
\mathbf{A}\end{array}$ & $\begin{array}{l}\text { DG-AHD } \\
\text { DG-ROA } \\
\text { NL-ORR } \\
\text { CHI-K25 } \\
\text { CHE-ORR } \\
\text { CR-ORR } \\
\text { CR-AHD } \\
\text { CR-ROA }\end{array}$ & $\begin{array}{l}3 \\
4 \\
2 \\
4 \\
3 \\
4 \\
4 \\
4\end{array}$ & $\begin{array}{l}0 \\
0 \\
0 \\
0 \\
0 \\
0 \\
0 \\
0\end{array}$ & $\begin{array}{l}2 \\
4 \\
2 \\
4 \\
3 \\
4 \\
4 \\
4\end{array}$ & $\begin{array}{l}52.9 \\
77.1 \\
62.6 \\
73.5 \\
61.3 \\
65.7 \\
68.3 \\
43.2\end{array}$ & $\begin{array}{r}75.3 \\
104.0 \\
95.8 \\
99.3 \\
86.9 \\
88.8 \\
92.2 \\
58.3\end{array}$ & $\begin{array}{r}96.6 \\
141.0 \\
114.0 \\
134.0 \\
112.0 \\
120.0 \\
125.0 \\
78.8\end{array}$ & $\begin{array}{r}66.5 \\
101.0 \\
73.1 \\
96.4 \\
77.1 \\
86.3 \\
89.6 \\
56.6\end{array}$ \\
\hline $\begin{array}{l}\text { B } \\
\text { B } \\
\text { B } \\
\text { B } \\
\text { B } \\
\text { B } \\
\text { B } \\
\text { B }\end{array}$ & $\begin{array}{l}\text { DG-AHD } \\
\text { DG-ROA } \\
\text { NL-ORR } \\
\text { CHI-K25 } \\
\text { CHE-ORR } \\
\text { CR-ORR } \\
\text { CR-AND } \\
\text { CR-ROA }\end{array}$ & $\begin{array}{l}4 \\
4 \\
2 \\
4 \\
3 \\
4 \\
4 \\
4\end{array}$ & $\begin{array}{l}0 \\
0 \\
0 \\
0 \\
0 \\
0 \\
0 \\
0\end{array}$ & $\begin{array}{l}4 \\
4 \\
2 \\
4 \\
3 \\
4 \\
4 \\
4\end{array}$ & $\begin{array}{r}96.4 \\
76.1 \\
73.9 \\
162.0 \\
183.0 \\
133.0 \\
113.0 \\
50.8\end{array}$ & $\begin{array}{r}125.0 \\
98.4 \\
106.0 \\
209.0 \\
247.0 \\
172.0 \\
146.0 \\
65.7\end{array}$ & $\begin{array}{r}161.0 \\
127.0 \\
124.0 \\
270.0 \\
307.0 \\
222.0 \\
189.0 \\
84.9\end{array}$ & $\begin{array}{r}122.0 \\
96.2 \\
84.5 \\
204.0 \\
223.0 \\
168.0 \\
143.0 \\
64.2\end{array}$ \\
\hline $\begin{array}{l}\mathrm{C} \\
\mathrm{C} \\
\mathrm{C} \\
\mathrm{C} \\
\mathrm{C} \\
\mathrm{C} \\
\mathrm{C} \\
\mathrm{C} \\
\mathrm{C}\end{array}$ & $\begin{array}{l}\text { DG-AND } \\
\text { OG-ROA } \\
\text { HL-ORR } \\
\text { CHI -BV } \\
\text { CHI -K25 } \\
\text { CHE-ORR } \\
\text { CR-ORR } \\
\text { CR-AND } \\
\text { CR-ROA } \\
\end{array}$ & $\begin{array}{l}4 \\
4 \\
2 \\
1 \\
4 \\
3 \\
4 \\
4 \\
4\end{array}$ & $\begin{array}{l}0 \\
0 \\
0 \\
0 \\
0 \\
0 \\
0 \\
0 \\
0\end{array}$ & $\begin{array}{l}2 \\
4 \\
2 \\
1 \\
4 \\
3 \\
4 \\
4 \\
4\end{array}$ & $\begin{array}{r}50.7 \\
102.0 \\
113.0 \\
120.0 \\
128.0 \\
257.0 \\
251.0 \\
141.0 \\
71.2 \\
\end{array}$ & $\begin{array}{r}74.1 \\
147.0 \\
189.0 \\
248.0 \\
184.0 \\
391.0 \\
361.0 \\
203.0 \\
102.0\end{array}$ & $\begin{array}{l}105.0 \\
212.0 \\
234.0 \\
248.0 \\
265.0 \\
531.0 \\
520.0 \\
292.0 \\
147.0\end{array}$ & $\begin{array}{r}70.4 \\
142.0 \\
136.0 \\
118.0 \\
178.0 \\
339.0 \\
349.0 \\
196.0 \\
98.9\end{array}$ \\
\hline
\end{tabular}

${ }^{a} \mathrm{~N}=$ number of observations, possibly averages over replicates at sites; $\mathrm{I}=$ number of interval censored observations (see text); $D=$ number of true detects (see text); UCB95 = 95\% upper confidence bound for median; X95 = estimate of 95th percentile; LTB9595 = 95\% lower confidence bound for 95th percentile; REMAINDER refers to the remaining observations-no detects. 
Table G.2. Summary statistics for ICP/MS data ${ }^{a}$ (Estimates and confidence bounds are in milligrams per kilogram.)

\begin{tabular}{|c|c|c|c|c|c|c|c|c|}
\hline Horizon & $\begin{array}{l}\text { Formation- } \\
\text { location }\end{array}$ & $\mathbf{N}$ & I & D & Median & UCB95 & X95 & LTB9595 \\
\hline \multicolumn{9}{|c|}{ ALUMTNUM } \\
\hline $\begin{array}{l}A \\
A \\
A \\
A \\
A \\
A \\
A \\
A \\
A \\
A\end{array}$ & $\begin{array}{l}\text { DG-AHD } \\
\text { DG-ROA } \\
\text { DG-ORR } \\
\text { HL-ORR } \\
\text { CHI-BV } \\
\text { CHI-K25 } \\
\text { CHE-ORR } \\
\text { CR-ORR } \\
\text { CR-AND } \\
\text { CR-ROA }\end{array}$ & $\begin{array}{l}4 \\
3 \\
3 \\
4 \\
4 \\
4 \\
4 \\
4 \\
4 \\
4\end{array}$ & $\begin{array}{l}0 \\
0 \\
0 \\
0 \\
0 \\
0 \\
0 \\
0 \\
0 \\
0\end{array}$ & $\begin{array}{l}4 \\
3 \\
3 \\
4 \\
4 \\
4 \\
4 \\
4 \\
4 \\
4\end{array}$ & $\begin{array}{r}20000 \\
17100 \\
19700 \\
13700 \\
17800 \\
18200 \\
8540 \\
9330 \\
13700 \\
9190\end{array}$ & $\begin{array}{r}22800 \\
19900 \\
22900 \\
15700 \\
20300 \\
20800 \\
9740 \\
10600 \\
15600 \\
10500\end{array}$ & $\begin{array}{l}26000 \\
22300 \\
25600 \\
17900 \\
23100 \\
23700 \\
11100 \\
12100 \\
17800 \\
12000\end{array}$ & $\begin{array}{r}22600 \\
19000 \\
21800 \\
15500 \\
20100 \\
20600 \\
9650 \\
10500 \\
15500 \\
10400\end{array}$ \\
\hline $\begin{array}{l}B \\
B \\
B \\
B \\
B \\
B \\
B \\
B \\
B \\
B\end{array}$ & $\begin{array}{l}\text { DG-AND } \\
\text { DG-ROA } \\
\text { DG-ORR } \\
\text { HL-ORR } \\
\text { CHI-BV } \\
\text { CHI-K25 } \\
\text { CHE-ORR } \\
\text { CR-ORR } \\
\text { CR-AND } \\
\text { CR-ROA }\end{array}$ & $\begin{array}{l}4 \\
3 \\
3 \\
4 \\
4 \\
4 \\
4 \\
4 \\
4 \\
4\end{array}$ & $\begin{array}{l}0 \\
0 \\
0 \\
0 \\
0 \\
0 \\
0 \\
0 \\
0 \\
0\end{array}$ & $\begin{array}{l}4 \\
3 \\
3 \\
4 \\
4 \\
4 \\
4 \\
4 \\
4 \\
4\end{array}$ & $\begin{array}{l}30800 \\
26100 \\
33600 \\
23000 \\
34400 \\
35100 \\
16900 \\
12800 \\
18700 \\
15400\end{array}$ & $\begin{array}{l}36700 \\
32000 \\
41200 \\
27400 \\
41100 \\
41900 \\
20200 \\
15300 \\
22300 \\
18300\end{array}$ & $\begin{array}{l}43700 \\
37100 \\
47800 \\
32600 \\
48900 \\
49900 \\
24000 \\
18200 \\
26500 \\
21900\end{array}$ & $\begin{array}{l}36200 \\
30000 \\
38600 \\
27100 \\
40600 \\
41400 \\
19900 \\
15100 \\
22000 \\
18100\end{array}$ \\
\hline $\begin{array}{l}c \\
c \\
c \\
c \\
c \\
c \\
c \\
c \\
c \\
C\end{array}$ & $\begin{array}{l}\text { DG-AHD } \\
\text { DG-ROA } \\
\text { DG-ORR } \\
\text { NL-ORR } \\
\text { CHI-BV } \\
\text { CHI-K25 } \\
\text { CHE-ORR } \\
\text { CR-ORR } \\
\text { CR-AND } \\
\text { CR-ROA }\end{array}$ & $\begin{array}{l}4 \\
3 \\
3 \\
4 \\
4 \\
4 \\
4 \\
4 \\
4 \\
4\end{array}$ & $\begin{array}{l}0 \\
0 \\
0 \\
0 \\
0 \\
0 \\
0 \\
0 \\
0 \\
0\end{array}$ & $\begin{array}{l}4 \\
3 \\
3 \\
4 \\
4 \\
4 \\
4 \\
4 \\
4 \\
4\end{array}$ & $\begin{array}{l}33400 \\
30100 \\
38500 \\
24300 \\
37400 \\
33000 \\
17100 \\
11800 \\
20100 \\
14000\end{array}$ & $\begin{array}{l}40700 \\
37800 \\
48400 \\
29600 \\
45500 \\
40300 \\
20800 \\
14400 \\
24500 \\
17000\end{array}$ & $\begin{array}{l}49600 \\
44700 \\
57200 \\
36100 \\
55500 \\
49000 \\
25400 \\
17600 \\
29900 \\
20700\end{array}$ & $\begin{array}{l}40100 \\
35100 \\
45000 \\
29200 \\
44900 \\
39700 \\
20500 \\
14200 \\
24200 \\
16800\end{array}$ \\
\hline
\end{tabular}

ANTIMONY

\begin{tabular}{|c|c|c|c|c|c|c|c|c|}
\hline A & $\begin{array}{l}\text { CR-ORR } \\
\text { REMAINDER }\end{array}$ & $\begin{array}{r}4 \\
34\end{array}$ & $\begin{array}{l}0 \\
0\end{array}$ & $\begin{array}{l}1 \\
0\end{array}$ & 0.184 & $\begin{array}{c}0.283 \\
.\end{array}$ & 0.281 & $\begin{array}{c}0.192 \\
.\end{array}$ \\
\hline & $\begin{array}{l}\text { CHE-ORR } \\
\text { REMAINDER }\end{array}$ & $\begin{array}{r}4 \\
34\end{array}$ & $\begin{array}{l}1 \\
0\end{array}$ & $\begin{array}{l}0 \\
0\end{array}$ & : & : & - & : \\
\hline & $\begin{array}{l}\text { CR-ROA } \\
\text { REMAINDER }\end{array}$ & $\begin{array}{r}4 \\
34\end{array}$ & $\begin{array}{l}1 \\
0\end{array}$ & $\begin{array}{l}0 \\
0\end{array}$ & • & $\dot{\bullet}$ & : & • \\
\hline
\end{tabular}

ARSENIC

$\begin{array}{lllllrrrr}\text { A } & \text { DG-AND } & 4 & 0 & 4 & 3.15 & 3.82 & 4.64 & 3.77 \\ \text { A } & \text { DG-ROA } & 3 & 0 & 3 & 4.11 & 5.14 & 6.06 & 4.79 \\ \text { A } & \text { DG-ORR } & 3 & 0 & 3 & 3.50 & 4.37 & 5.16 & 4.07 \\ \text { A } & \text { NL-ORR } & 4 & 0 & 4 & 3.94 & 4.78 & 5.80 & 4.72 \\ \text { A } & \text { CHI-BV } & 4 & 0 & 4 & 5.22 & 6.34 & 7.70 & 6.26 \\ \text { A } & \text { CHI-K25 } & 4 & 0 & 4 & 5.36 & 6.51 & 7.91 & 6.43 \\ \text { A } & \text { CHE-ORR } & 4 & 0 & 4 & 6.88 & 8.35 & 10.10 & 8.24 \\ \text { A } & \text { CR-ORR } & 4 & 0 & 4 & 14.60 & 17.70 & 21.60 & 17.50 \\ \text { A } & \text { CR-AND } & 4 & 0 & 4 & 12.50 & 15.20 & 18.50 & 15.00 \\ \text { A } & \text { CR-ROA } & 4 & 0 & 4 & 7.63 & 9.26 & 11.20 & 9.14 \\ \text { B } & \text { DG-AND } & 4 & 0 & 4 & 3.43 & 4.26 & 5.29 & 4.20 \\ \text { B } & \text { DG-ROA } & 3 & 0 & 3 & 4.50 & 5.77 & 6.93 & 5.33 \\ \text { B } & \text { DG-ORR } & 3 & 0 & 3 & 3.57 & 4.58 & 5.50 & 4.23 \\ \text { B } & \text { HL-ORR } & 4 & 0 & 4 & 3.84 & 4.77 & 5.92 & 4.69 \\ \text { B } & \text { CHI-BV } & 4 & 0 & 4 & 5.11 & 6.35 & 7.88 & 6.26 \\ \text { B } & \text { CHI-K25 } & 4 & 0 & 4 & 5.27 & 6.54 & 8.12 & 6.44\end{array}$


G-21

Table G.2 (continued)

\begin{tabular}{|c|c|c|c|c|c|c|c|c|}
\hline Horizon & $\begin{array}{l}\text { Formation- } \\
\text { location }\end{array}$ & $\mathbf{N}$ & I & D & Median & UCB95 & X95 & LTB9595 \\
\hline$\therefore \because \frac{1}{n}$ & $\because$ & & & & . & & $\therefore$ & \\
\hline $\begin{array}{l}\text { B } \\
\text { B } \\
\text { B } \\
\text { B }\end{array}$ & $\begin{array}{l}\text { CHE-ORR } \\
\text { CR-ORR } \\
\text { CR-AND } \\
\text { CR-ROA }\end{array}$ & $\begin{array}{l}4 \\
4 \\
4 \\
4\end{array}$ & $\begin{array}{l}0 \\
0 \\
0 \\
0\end{array}$ & $\begin{array}{l}4 \\
4 \\
4 \\
4\end{array}$ & $\begin{array}{l}17.40 \\
30.70 \\
16.90 \\
13.90\end{array}$ & $\begin{array}{l}21.60 \\
38.20 \\
21.00 \\
17.30\end{array}$ & $\begin{array}{l}26.80 \\
47.40 \\
26.10 \\
21.50\end{array}$ & $\begin{array}{l}21.30 \\
37.60 \\
20.70 \\
17.00\end{array}$ \\
\hline $\begin{array}{l}C \\
C \\
C \\
c \\
c \\
C \\
C \\
C \\
C \\
C\end{array}$ & $\begin{array}{l}\text { DG-AND } \\
\text { DG-ROA } \\
\text { DG-ORR } \\
\text { NL-ORR } \\
\text { CHI-BV } \\
\text { CHI-K25 } \\
\text { CHE-ORR } \\
\text { CR-ORR } \\
\text { CR-AND } \\
\text { CR-ROA }\end{array}$ & $\begin{array}{l}4 \\
3 \\
3 \\
4 \\
4 \\
4 \\
4 \\
4 \\
4 \\
4\end{array}$ & $\begin{array}{l}0 \\
0 \\
0 \\
0 \\
0 \\
0 \\
0 \\
0 \\
0 \\
0\end{array}$ & $\begin{array}{l}4 \\
3 \\
3 \\
4 \\
4 \\
4 \\
4 \\
4 \\
4 \\
4\end{array}$ & $\begin{array}{r}2.78 \\
3.15 \\
3.06 \\
3.03 \\
5.64 \\
4.15 \\
20.40 \\
43.60 \\
20.70 \\
18.90\end{array}$ & $\begin{array}{r}3.56 \\
4.19 \\
4.07 \\
3.89 \\
7.23 \\
5.31 \\
26.10 \\
55.90 \\
26.50 \\
24.30\end{array}$ & $\begin{array}{r}4.56 \\
5.17 \\
5.02 \\
4.98 \\
9.26 \\
6.80 \\
33.50 \\
71.60 \\
33.90 \\
31.10\end{array}$ & $\begin{array}{r}3.50 \\
3.83 \\
3.72 \\
3.82 \\
7.11 \\
5.22 \\
25.70 \\
54.90 \\
26.00 \\
23.80\end{array}$ \\
\hline \multicolumn{9}{|c|}{ BARIUM } \\
\hline $\begin{array}{l}A \\
A \\
A \\
A \\
A \\
A \\
A \\
A \\
A \\
A\end{array}$ & $\begin{array}{l}\text { DG-AND } \\
\text { DG-ROA } \\
\text { DG-ORR } \\
\text { NL-ORR } \\
\text { CHI-BV } \\
\text { CHI-K25 } \\
\text { CHE-ORR } \\
\text { CR-ORR } \\
\text { CR-AND } \\
\text { CR-ROA }\end{array}$ & $\begin{array}{l}4 \\
3 \\
3 \\
4 \\
4 \\
4 \\
4 \\
4 \\
4 \\
4\end{array}$ & $\begin{array}{l}0 \\
0 \\
0 \\
0 \\
0 \\
0 \\
0 \\
0 \\
0 \\
0\end{array}$ & $\begin{array}{l}4 \\
3 \\
3 \\
4 \\
4 \\
4 \\
4 \\
4 \\
4 \\
4\end{array}$ & $\begin{array}{r}76.80 \\
81.70 \\
94.20 \\
62.70 \\
80.10 \\
74.40 \\
55.70 \\
70.10 \\
107.00 \\
52.70\end{array}$ & $\begin{array}{r}102.0 \\
113.0 \\
130.0 . \\
83.0 \\
106.0 \\
98.5 \\
73.7 \\
92.7 \\
141.0 \\
69.8\end{array}$ & $\begin{array}{r}134.0 \\
143.0 \\
165.0 \\
110.0 \\
140.0 \\
130.0 \\
97.6 \\
123.0 \\
187.0 \\
92.4\end{array}$ & $\begin{array}{r}99.6 \\
102.0 \\
117.0 \\
81.4 \\
104.0 \\
96.6 \\
72.3 \\
91.0 \\
138.0 \\
68.4\end{array}$ \\
\hline $\begin{array}{l}\text { B } \\
\text { B } \\
\text { B } \\
\text { B } \\
\text { B } \\
\text { B } \\
\text { B } \\
\text { B } \\
\text { B } \\
\text { B }\end{array}$ & $\begin{array}{l}\text { DG-AND } \\
\text { DG-ROA } \\
\text { DG-ORR } \\
\text { NL-ORR } \\
\text { CHI-BV } \\
\text { CHI-K25 } \\
\text { CHE-ORR } \\
\text { CR-ORR } \\
\text { CR-AND } \\
\text { CR-ROA }\end{array}$ & $\begin{array}{l}4 \\
3 \\
3 \\
4 \\
4 \\
4 \\
4 \\
4 \\
4 \\
4\end{array}$ & $\begin{array}{l}0 \\
0 \\
0 \\
0 \\
0 \\
0 \\
0 \\
0 \\
0 \\
0\end{array}$ & $\begin{array}{l}4 \\
3 \\
3 \\
4 \\
4 \\
4 \\
4 \\
4 \\
4 \\
4\end{array}$ & $\begin{array}{r}69.90 \\
88.10 \\
104.00 \\
68.80 \\
125.00 \\
84.60 \\
38.20 \\
31.20 \\
38.90 \\
32.80\end{array}$ & $\begin{array}{r}88.1 \\
115.0 \\
136.0 \\
86.7 \\
157.0 \\
107.0 \\
48.2 \\
39.3 \\
49.1 \\
41.3\end{array}$ & $\begin{array}{r}111.0 \\
140.0 \\
165.0 \\
109.0 \\
198.0 \\
134.0 \\
60.7 \\
49.5 \\
61.8 \\
52.1\end{array}$ & $\begin{array}{r}86.7 \\
106.0 \\
125.0 \\
85.3 \\
154.0 \\
105.0 \\
47.4 \\
38.7 \\
48.3 \\
40.7\end{array}$ \\
\hline $\begin{array}{l}\text { C } \\
\text { C } \\
\text { C } \\
\text { C } \\
\text { C } \\
\text { C } \\
\text { C } \\
\text { C } \\
\text { C } \\
\text { C }\end{array}$ & $\begin{array}{l}\text { DG-AND } \\
\text { DG-ROA } \\
\text { DG-ORR } \\
\text { NL-ORR } \\
\text { CHI-BV } \\
\text { CHI-K25 } \\
\text { CHE-ORR } \\
\text { CR-ORR } \\
\text { CR-AND } \\
\text { CR-ROA }\end{array}$ & $\begin{array}{l}4 \\
3 \\
3 \\
4 \\
4 \\
4 \\
4 \\
4 \\
4 \\
4\end{array}$ & $\begin{array}{l}0 \\
0 \\
0 \\
0 \\
0 \\
0 \\
0 \\
0 \\
0 \\
0\end{array}$ & $\begin{array}{l}4 \\
3 \\
3 \\
4 \\
4 \\
4 \\
4 \\
4 \\
4 \\
4\end{array}$ & $\begin{array}{r}72.90 \\
82.10 \\
125.00 \\
63.70 \\
164.00 \\
75.90 \\
18.70 \\
8.24 \\
25.80 \\
12.60\end{array}$ & $\begin{array}{r}99.4 \\
118.0 \\
179.0 \\
86.9 \\
224.0 \\
104.0 \\
25.5 \\
11.2 \\
35.2 \\
17.2\end{array}$ & $\begin{array}{l}136.0 \\
153.0 \\
232.0 \\
119.0 \\
306.0 \\
141.0 \\
34.8 \\
15.3 \\
48.0 \\
23.5\end{array}$ & $\begin{array}{r}97.3 \\
105.0 \\
159.0 \\
85.0 \\
220.0 \\
101.0 \\
25.0 \\
11.0 \\
34.5 \\
16.9\end{array}$ \\
\hline \multicolumn{9}{|c|}{ BERYLLIUM } \\
\hline $\begin{array}{l}A \\
A \\
A \\
A \\
A \\
A \\
A \\
A \\
A \\
A\end{array}$ & $\begin{array}{l}\text { DG-AND } \\
\text { DG-ROA } \\
\text { DG-ORR } \\
\text { HL-ORR } \\
\text { CHI-BV } \\
\text { CHI-K25 } \\
\text { CHE-ORR } \\
\text { CR-ORR } \\
\text { CR-AND } \\
\text { CR-ROA }\end{array}$ & $\begin{array}{l}4 \\
3 \\
3 \\
4 \\
4 \\
4 \\
4 \\
4 \\
4 \\
4\end{array}$ & $\begin{array}{l}0 \\
0 \\
0 \\
0 \\
0 \\
0 \\
0 \\
0 \\
0 \\
0\end{array}$ & $\begin{array}{l}4 \\
3 \\
3 \\
4 \\
4 \\
4 \\
4 \\
4 \\
4 \\
4\end{array}$ & $\begin{array}{l}0.832 \\
.0 .669 \\
0.752 \\
0.547 \\
0.973 \\
0.809 \\
0.406 \\
0.474 \\
0.628 \\
0.440\end{array}$ & $\begin{array}{l}1.020 \\
0.851 \\
0.956 \\
0.673 \\
1.200 \\
0.996 \\
0.500 \\
0.584 \\
0.773 \\
0.541\end{array}$ & $\begin{array}{l}1.260 \\
1.010 \\
1.140 \\
0.829 \\
1.470 \\
1.230 \\
0.615 \\
0.719 \\
0.952 \\
0.666\end{array}$ & $\begin{array}{l}1.010 \\
0.788 \\
0.885 \\
0.664 \\
1.180 \\
0.982 \\
0.493 \\
0.576 \\
0.762 \\
0.534\end{array}$ \\
\hline
\end{tabular}


G-22

Table G.2 (continued)

\begin{tabular}{ccccccccc}
\hline Horizon & $\begin{array}{c}\text { Formation- } \\
\text { location }\end{array}$ & N & I & D & Median & UCB95 & X95 & LTB9595 \\
\hline & & & & & & & & \\
B & DG-AND & 4 & 0 & 4 & 1.050 & 1.400 & 1.870 & 1.370 \\
B & DG-ROA & 3 & 0 & 3 & 0.741 & 1.040 & 1.330 & 0.930 \\
B & DG-ORR & 3 & 0 & 3 & 0.870 & 1.220 & 1.560 & 1.090 \\
B & NL-ORR & 4 & 0 & 4 & 0.641 & 0.858 & 1.150 & 0.840 \\
B & CHI-BV & 4 & 0 & 4 & 1.500 & 2.000 & 2.680 & 1.960 \\
B & CHI-K25 & 4 & 0 & 4 & 1.200 & 1.610 & 2.160 & 1.580 \\
B & CHE-ORR & 4 & 0 & 4 & 0.473 & 0.634 & 0.848 & 0.620 \\
B & CR-ORR & 4 & 0 & 4 & 0.289 & 0.387 & 0.518 & 0.379 \\
B & CR-AND & 4 & 0 & 4 & 0.399 & 0.534 & 0.714 & 0.522 \\
B & CR-ROA & 4 & 1 & 2 & 0.256 & 0.349 & 0.458 & 0.332 \\
C & DG-AND & 4 & 0 & 4 & 1.170 & 1.520 & 1.980 & 1.490 \\
C & DG-ROA & 3 & 0 & 3 & 0.909 & 1.230 & 1.540 & 1.120 \\
C & DG-ORR & 3 & 0 & 3 & 1.070 & 1.450 & 1.810 & 1.310 \\
C & NL-ORR & 4 & 0 & 4 & 0.791 & 1.030 & 1.340 & 1.010 \\
C & CHI-BV & 4 & 0 & 4 & 2.070 & 2.700 & 3.510 & 2.640 \\
C & CHI-K25 & 4 & 0 & 4 & 1.280 & 1.670 & 2.180 & 1.640 \\
C & CHE-ORR & 4 & 0 & 4 & 0.643 & 0.838 & 1.090 & 0.822 \\
C & CR-ORR & 4 & 0 & 4 & 0.346 & 0.450 & 0.587 & 0.442 \\
C & CR-AND & 4 & 0 & 4 & 0.466 & 0.607 & 0.791 & 0.595 \\
C & CR-ROA & 4 & 0 & 2 & 0.240 & 0.320 & 0.407 & 0.303
\end{tabular}

\section{CADMTUM}

$\begin{array}{lllll}\text { A } & \text { REMAINDER } & 38 & 0 & 0 \\ \text { B } & \text { REMAINDER } & 38 & 0 & 0 \\ \text { C } & \text { REMAINDER } & 38 & 0 & 0\end{array}$

\section{CHROMIUM}

$\begin{array}{lllllllll}\text { A } & \text { DG-AND } & 4 & 0 & 4 & 25.9 & 29.9 & 34.4 & 29.6 \\ \text { A } & \text { DG-ROA } & 3 & 0 & 3 & 33.0 & 38.8 & 43.8 & 36.8 \\ \text { A } & \text { DG-ORR } & 3 & 0 & 3 & 30.2 & 35.5 & 40.0 & 33.7 \\ \text { A } & \text { NL-ORR } & 4 & 0 & 4 & 29.3 & 33.8 & 38.9 & 33.4 \\ \text { A } & \text { CHI-BV } & 4 & 0 & 4 & 32.2 & 37.1 & 42.7 & 36.7 \\ \text { A } & \text { CHI-K25 } & 4 & 0 & 4 & 29.1 & 33.5 & 38.6 & 33.2 \\ \text { A } & \text { CHE-ORR } & 4 & 0 & 4 & 13.2 & 15.3 & 17.6 & 15.1 \\ \text { A } & \text { CR-ORR } & 4 & 0 & 4 & 16.4 & 18.9 & 21.8 & 18.8 \\ \text { A } & \text { CR-AND } & 4 & 0 & 4 & 24.2 & 27.9 & 32.1 & 27.6 \\ \text { A } & \text { CR-ROA } & 4 & 0 & 4 & 14.5 & 16.7 & 19.2 & 16.5 \\ & & & & & & & & \\ \text { B } & \text { DG-AND } & 4 & 0 & 4 & 34.2 & 38.8 & 44.0 & 38.5 \\ \text { B } & \text { DG-ROA } & 3 & 0 & 3 & 47.1 & 54.5 & 60.6 & 52.0 \\ \text { B } & \text { DG-ORR } & 3 & 0 & 3 & 39.6 & 45.8 & 50.9 & 43.7 \\ \text { B } & \text { NL-ORR } & 4 & 0 & 4 & 34.5 & 39.1 & 44.4 & 38.8 \\ \text { B } & \text { CHI-BV } & 4 & 0 & 4 & 38.4 & 43.5 & 49.3 & 43.1 \\ \text { B } & \text { CHI-K25 } & 4 & 0 & 4 & 35.6 & 40.3 & 45.7 & 40.0 \\ \text { B } & \text { CHE-ORR } & 4 & 0 & 4 & 31.9 & 36.2 & 41.0 & 35.9 \\ \text { B } & \text { CR-ORR } & 4 & 0 & 4 & 28.4 & 32.2 & 36.6 & 32.0 \\ \text { B } & \text { CR-AND } & 4 & 0 & 4 & 28.1 & 31.8 & 36.1 & 31.5 \\ \text { B } & \text { CR-ROA } & 4 & 0 & 4 & 25.5 & 28.9 & 32.7 & 28.6 \\ & & & & & & & \\ \text { C } & \text { DG-AND } & 4 & 0 & 4 & 31.2 & 35.2 & 39.7 & 34.9 \\ \text { C } & \text { DG-ROA } & 3 & 0 & 3 & 45.3 & 52.0 & 57.6 & 49.7 \\ \text { C } & \text { DG-ORR } & 3 & 0 & 3 & 43.0 & 49.4 & 54.7 & 47.3 \\ \text { C } & \text { HL-ORR } & 4 & 0 & 4 & 35.7 & 40.3 & 45.4 & 39.9 \\ \text { C } & \text { CHI-BV } & 4 & 0 & 4 & 36.9 & 41.7 & 47.0 & 41.3 \\ \text { C } & \text { CHI-K25 } & 4 & 0 & 4 & 30.5 & 34.5 & 38.9 & 34.2 \\ \text { C } & \text { CHE-ORR } & 4 & 0 & 4 & 29.5 & 33.3 & 37.5 & 33.0 \\ \text { C } & \text { CR-ORR } & 4 & 0 & 4 & 27.2 & 30.6 & 34.6 & 30.4 \\ \text { C } & \text { CR-AND } & 4 & 0 & 4 & 29.9 & 33.7 & 38.0 & 33.4 \\ \text { C } & \text { CR-ROA } & 4 & 0 & 4 & 31.2 & 35.2 & 39.7 & 34.9 \\ & & & & & & & & \end{array}$


Table G.2 (continued)

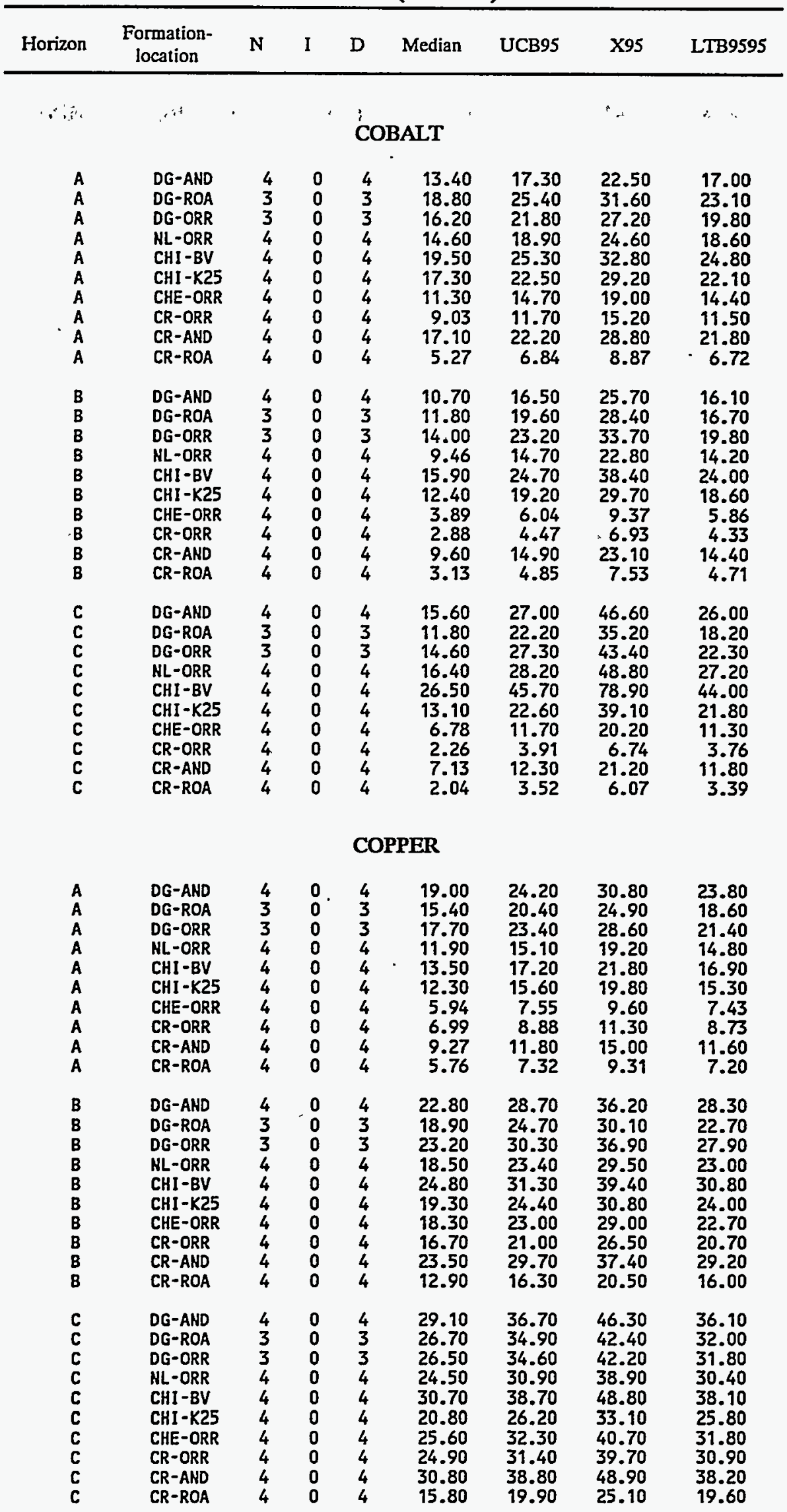


G-24

Table G.2 (continued)

\begin{tabular}{|c|c|c|c|c|c|c|c|c|}
\hline Horizon & $\begin{array}{l}\text { Formation- } \\
\text { location }\end{array}$ & $\mathbf{N}$ & I & D & Median & UCB95 & X95 & LTB9595 \\
\hline \multicolumn{9}{|c|}{ LEAD } \\
\hline $\begin{array}{l}A \\
A \\
A \\
A \\
A \\
A \\
A \\
A \\
A \\
A\end{array}$ & $\begin{array}{l}\text { DG-AND } \\
\text { DG-ROA } \\
\text { DG-ORR } \\
\text { NL-ORR } \\
\text { CHI-BV } \\
\text { CHI-K25 } \\
\text { CHE-ORR } \\
\text { CR-ORR } \\
\text { CR-AND } \\
\text { CR-ROA }\end{array}$ & $\begin{array}{l}4 \\
3 \\
3 \\
4 \\
4 \\
4 \\
4 \\
4 \\
4 \\
4\end{array}$ & $\begin{array}{l}0 \\
0 \\
0 \\
0 \\
0 \\
0 \\
0 \\
0 \\
0 \\
0\end{array}$ & $\begin{array}{l}4 \\
3 \\
3 \\
4 \\
4 \\
4 \\
4 \\
4 \\
4 \\
4\end{array}$ & $\begin{array}{l}28.6 \\
24.1 \\
17.0 \\
18.2 \\
39.8 \\
31.4 \\
17.6 \\
26.9 \\
36.1 \\
20.6\end{array}$ & $\begin{array}{l}33.3 \\
28.8 \\
20.3 \\
21.2 \\
46.4 \\
36.6 \\
20.5 \\
31.4 \\
42.0 \\
24.0\end{array}$ & $\begin{array}{l}38.8 \\
32.7 \\
23.2 \\
24.7 \\
54.1 \\
42.7 \\
23.9 \\
36.6 \\
49.0 \\
28.0\end{array}$ & $\begin{array}{l}32.9 \\
27.2 \\
19.2 \\
21.0 \\
45.9 \\
36.2 \\
20.3 \\
31.0 \\
41.6 \\
23.8\end{array}$ \\
\hline $\begin{array}{l}\text { B } \\
\text { B } \\
\text { B } \\
\text { B } \\
\text { B } \\
\text { B } \\
\text { B } \\
B \\
B \\
B\end{array}$ & $\begin{array}{l}\text { DG-AND } \\
\text { DG-ROA } \\
\text { DG-ORR } \\
\text { NL-ORR } \\
\text { CHI-BV } \\
\text { CHI-K25 } \\
\text { CHE-ORR } \\
\text { CR-ORR } \\
\text { CR-AND } \\
\text { CR-ROA }\end{array}$ & $\begin{array}{l}4 \\
3 \\
3 \\
4 \\
4 \\
4 \\
4 \\
4 \\
4 \\
4\end{array}$ & $\begin{array}{l}0 \\
0 \\
0 \\
0 \\
0 \\
0 \\
0 \\
0 \\
0 \\
0\end{array}$ & $\begin{array}{l}4 \\
3 \\
3 \\
4 \\
4 \\
4 \\
4 \\
4 \\
4 \\
4\end{array}$ & $\begin{array}{l}20.7 \\
17.8 \\
16.2 \\
12.1 \\
28.8 \\
19.0 \\
12.1 \\
15.7 \\
16.5 \\
11.8\end{array}$ & $\begin{array}{l}27.4 \\
24.6 \\
22.4 \\
16.1 \\
38.1 \\
25.1 \\
16.0 \\
20.7 \\
21.9 \\
15.6\end{array}$ & $\begin{array}{l}36.3 \\
31.2 \\
28.4 \\
21.3 \\
50.5 \\
33.3 \\
21.2 \\
27.5 \\
29.0 \\
20.7\end{array}$ & $\begin{array}{l}26.9 \\
22.2 \\
20.2 \\
15.7 \\
37.4 \\
24.6 \\
15.7 \\
20.3 \\
21.4 \\
15.3\end{array}$ \\
\hline $\begin{array}{l}\text { C } \\
C \\
C \\
C \\
C \\
C \\
C \\
C \\
C \\
C\end{array}$ & $\begin{array}{l}\text { DG-AND } \\
\text { DG-ROA } \\
\text { DG-ORR } \\
\text { NL-ORR } \\
\text { CHI-BV } \\
\text { CHI-K25 } \\
\text { CHE-ORR } \\
\text { CR-ORR } \\
\text { CR-AND } \\
\text { CR-ROA }\end{array}$ & $\begin{array}{l}4 \\
3 \\
3 \\
4 \\
4 \\
4 \\
4 \\
4 \\
4 \\
4\end{array}$ & $\begin{array}{l}0 \\
0 \\
0 \\
0 \\
0 \\
0 \\
0 \\
0 \\
0 \\
0\end{array}$ & $\begin{array}{l}4 \\
3 \\
3 \\
4 \\
4 \\
4 \\
4 \\
4 \\
4 \\
4\end{array}$ & $\begin{array}{l}25.5 \\
15.0 \\
18.4 \\
15.8 \\
43.6 \\
19.9 \\
20.3 \\
27.9 \\
21.8 \\
15.1\end{array}$ & $\begin{array}{l}36.1 \\
22.5 \\
27.5 \\
22.4 \\
61.7 \\
28.2 \\
28.7 \\
39.5 \\
30.8 \\
21.4\end{array}$ & $\begin{array}{l}51.1 \\
30.1 \\
36.9 \\
31.7 \\
87.3 \\
39.9 \\
40.6 \\
56.0 \\
43.6 \\
30.3\end{array}$ & $\begin{array}{l}35.2 \\
19.7 \\
24.2 \\
21.8 \\
60.2 \\
27.5 \\
28.0 \\
38.6 \\
30.1 \\
20.9\end{array}$ \\
\hline
\end{tabular}

MANGANESE

$\begin{array}{lllllrrrr}\text { A } & \text { DG-AND } & 4 & 0 & 4 & 717.0 & 945 & 1250 & 928 \\ \text { A } & \text { DG-ROA } & 3 & 0 & 3 & 1370.0 & 1880 & 2370 & 1700 \\ \text { A } & \text { DG-ORR } & 3 & 0 & 3 & 954.0 & 1310 & 1660 & 1190 \\ \text { A } & \text { NL-ORR } & 4 & 0 & 4 & 749.0 & 987 & 1300 & 969 \\ \text { A } & \text { CHI--BV } & 4 & 0 & 4 & 1080.0 & 1430 & 1880 & 1400 \\ \text { A } & \text { CHI-K25 } & 4 & 0 & 4 & 1430.0 & 1880 & 2480 & 1840 \\ \text { A } & \text { CHE-ORR } & 4 & 0 & 4 & 936.0 & 1230 & 1630 & 1210 \\ \text { A } & \text { CR-ORR } & 4 & 0 & 4 & 1380.0 & 1820 & 2400 & 1790 \\ \text { A } & \text { CR-AND } & 4 & 0 & 4 & 2360.0 & 3110 & 4100 & 3050 \\ \text { A } & \text { CR-ROA } & 4 & 0 & 4 & 825.0 & 1090 & 1430 & 1070 \\ \text { B } & \text { DG-AND } & 4 & 0 & 4 & 241.0 & 396 & 652 & 383 \\ \text { B } & \text { DG-ROA } & 3 & 0 & 3 & 341.0 & 605 & 922 & 504 \\ \text { B } & \text { DG-ORR } & 3 & 0 & 3 & 353.0 & 627 & 955 & 522 \\ \text { B } & \text { NL-ORR } & 4 & 0 & 4 & 206.0 & 339 & 557 & 327 \\ \text { B } & \text { CHI-BV } & 4 & 0 & 4 & 447.0 & 735 & 1210 & 710 \\ \text { B } & \text { CHI-K25 } & 4 & 0 & 4 & 266.0 & 437 & 719 & 422 \\ \text { B } & \text { CHE-ORR } & 4 & 0 & 4 & 113.0 & 186 & 305 & 179 \\ \text { B } & \text { CR-ORR } & 4 & 0 & 4 & 93.9 & 154 & 254 & 149 \\ \text { B } & \text { CR-AND } & 4 & 0 & 4 & 334.0 & 549 & 902 & 530 \\ \text { B } & \text { CR-ROA } & 4 & 0 & 4 & 108.0 & 178 & 292 & 172 \\ & \text { CR } & & & & & & & \\ \text { C } & \text { DG-AND } & 4 & 0 & 4 & 647.0 & 1220 & 2310 & 1170 \\ \text { C } & \text { DG-ROA } & 3 & 0 & 3 & 223.0 & 465 & 797 & 368 \\ \text { C } & \text { DG-ORR } & 3 & 0 & 3 & 418.0 & 871 & 1490 & 689 \\ \text { C } & \text { NL-ORR } & 4 & 0 & 4 & 374.0 & 707 & 1340 & 677 \\ \text { C } & \text { CHI-BV } & 4 & 0 & 4 & 760.0 & 1440 & 2710 & 1370 \\ \text { C } & \text { CHI-K25 } & 4 & 0 & 4 & 342.0 & 646 & 1220 & 618 \\ \text { C } & \text { CHE-ORR } & 4 & 0 & 4 & 177.0 & 334 & 631 & 320 \\ \text { C } & \text { CR-ORR } & 4 & 0 & 4 & 87.8 & 166 & 314 & 159 \\ \text { C } & \text { CR-AND } & 4 & 0 & 4 & 257.0 & 485 & 916 & 464 \\ \text { C } & \text { CR-ROA } & 4 & 0 & 4 & 56.0 & 106 & 200 & 101 \\ & & & & & & & & \end{array}$


G-25

Table G.2 (continued)

\begin{tabular}{|c|c|c|c|c|c|c|c|c|}
\hline Horizon & $\begin{array}{c}\text { Formation- } \\
\text { location }\end{array}$ & $\mathbf{N}$ & I & D & Median & UCB95 & X95 & LTB959 \\
\hline$\therefore y$ & 垱" & : & & & XKEL & & 站 & $\therefore$ \\
\hline $\begin{array}{l}A \\
A \\
A \\
A \\
A \\
A \\
A \\
A \\
A \\
A\end{array}$ & $\begin{array}{l}\text { DG-AND } \\
\text { DG-ROA } \\
\text { DG-ORR } \\
\text { NL-ORR } \\
\text { CHI-BV } \\
\text { CHI-K25 } \\
\text { CHE-ORR } \\
\text { CR-ORR } \\
\text { CR-AND } \\
\text { CR-ROA }\end{array}$ & $\begin{array}{l}4 \\
3 \\
3 \\
4 \\
4 \\
4 \\
4 \\
4 \\
4 \\
4\end{array}$ & $\begin{array}{l}0 \\
0 \\
0 \\
0 \\
0 \\
0 \\
0 \\
0 \\
0 \\
0\end{array}$ & $\begin{array}{l}4 \\
3 \\
3 \\
4 \\
4 \\
4 \\
4 \\
4 \\
4 \\
4\end{array}$ & $\begin{array}{r}18.90 \\
16.10 \\
20.80 \\
12.20 \\
12.60 \\
19.20 \\
7.27 \\
7.33 \\
9.39 \\
4.80\end{array}$ & $\begin{array}{r}23.90 \\
21.10 \\
27.30 \\
15.50 \\
15.90 \\
24.30 \\
9.19 \\
9.26 \\
11.90 \\
6.06\end{array}$ & $\begin{array}{l}30.20 \\
25.70 \\
33.20 \\
19.50 \\
20.10 \\
30.60 \\
11.60 \\
11.70 \\
15.00 \\
7.66\end{array}$ & $\begin{array}{r}23.50 \\
19.30 \\
25.00 \\
15.20 \\
15.60 \\
23.90 \\
9.04 \\
9.11 \\
11.70 \\
5.97\end{array}$ \\
\hline $\begin{array}{l}\text { B } \\
\text { B } \\
\text { B } \\
\text { B } \\
\text { B } \\
\text { B } \\
\text { B } \\
\text { B } \\
\text { B } \\
\text { B }\end{array}$ & $\begin{array}{l}\text { DG-AND } \\
\text { DG-ROA } \\
\text { DG-ORR } \\
\text { NL-ORR } \\
\text { CHI-BV } \\
\text { CHI-K25 } \\
\text { CHE-ORR } \\
\text { CR-ORR } \\
\text { CR-AND } \\
\text { CR-ROA }\end{array}$ & $\begin{array}{l}4 \\
3 \\
3 \\
4 \\
4 \\
4 \\
4 \\
4 \\
4 \\
4\end{array}$ & $\begin{array}{l}0 \\
0 \\
0 \\
0 \\
0 \\
0 \\
0 \\
0 \\
0 \\
0\end{array}$ & $\begin{array}{l}4 \\
3 \\
3 \\
4 \\
4 \\
4 \\
4 \\
4 \\
4 \\
4\end{array}$ & $\begin{array}{r}21.40 \\
19.70 \\
22.40 \\
16.20 \\
22.80 \\
22.20 \\
11.30 \\
7.94 \\
13.20 \\
7.87\end{array}$ & $\begin{array}{l}28.40 \\
27.20 \\
31.00 \\
21.40 \\
30.20 \\
29.40 \\
15.00 \\
10.50 \\
17.50 \\
10.40\end{array}$ & $\begin{array}{l}37.60 \\
34.60 \\
39.40 \\
28.40 \\
40.00 \\
39.00 \\
19.90 \\
14.00 \\
23.20 \\
13.80\end{array}$ & $\begin{array}{l}27.80 \\
24.50 \\
27.90 \\
21.00 \\
29.60 \\
28.90 \\
14.70 \\
10.30 \\
17.20 \\
10.20\end{array}$ \\
\hline $\begin{array}{l}\text { C } \\
\text { C } \\
\text { C } \\
\text { C } \\
C \\
C \\
C \\
C \\
C \\
C\end{array}$ & $\begin{array}{l}\text { DG-AND } \\
\text { DG-ROA } \\
\text { DG-ORR } \\
\text { NL-ORR } \\
\text { CHI-BV } \\
\text { CHI-K25 } \\
\text { CHE-ORR } \\
\text { CR-ORR } \\
\text { CR-AND } \\
\text { CR-ROA }\end{array}$ & $\begin{array}{l}4 \\
3 \\
3 \\
4 \\
4 \\
4 \\
4 \\
4 \\
4 \\
4\end{array}$ & $\begin{array}{l}0 \\
0 \\
0 \\
0 \\
0 \\
0 \\
0 \\
0 \\
0 \\
0\end{array}$ & $\begin{array}{l}4 \\
3 \\
3 \\
4 \\
4 \\
4 \\
4 \\
4 \\
4 \\
4\end{array}$ & $\begin{array}{r}23.60 \\
28.00 \\
26.60 \\
19.20 \\
33.80 \\
23.30 \\
18.20 \\
11.40 \\
17.00 \\
7.61\end{array}$ & $\begin{array}{l}31.30 \\
38.80 \\
36.80 \\
25.50 \\
44.80 \\
31.00 \\
24.20 \\
15.20 \\
22.50 \\
10.10\end{array}$ & $\begin{array}{l}41.60 \\
49.20 \\
46.80 \\
33.80 \\
59.50 \\
41.10 \\
32.10 \\
20.10 \\
29.90 \\
13.40\end{array}$ & $\begin{array}{r}30.70 \\
34.90 \\
33.20 \\
25.00 \\
44.00 \\
30.40 \\
23.70 \\
14.90 \\
22.10 \\
9.90\end{array}$ \\
\hline
\end{tabular}

\section{SEIENIUM}

$\begin{array}{lllllllll}\text { A } & \text { DG-AND } & 4 & 0 & 3 & 0.366 & 0.469 & 0.595 & 0.449 \\ \text { A } & \text { DG-ROA } & 3 & 1 & 2 & 0.391 & 0.522 & 0.635 & 0.462 \\ \text { A } & \text { DG-ORR } & 3 & 1 & 1 & 0.346 & 0.464 & 0.562 & 0.408 \\ \text { A } & \text { NL-ORR } & 4 & 0 & 4 & 0.315 & 0.401 & 0.511 & 0.384 \\ \text { A } & \text { CHE-ORR } & 2 & 0 & 2 & 0.673 & 0.949 & 1.090 & 0.752 \\ \text { A } & \text { CR-ORR } & 1 & 0 & 1 & 0.680 & 1.100 & 1.100 & 0.665 \\ \text { A } & \text { CR-ROA } & 1 & 0 & 1 & 0.475 & 0.772 & 0.772 & 0.464 \\ & & & & & & & & \\ \text { B } & \text { DG-AND } & 4 & 0 & 4 & 0.346 & 0.424 & 0.521 & 0.410 \\ \text { B } & \text { DG-ROA } & 3 & 0 & 3 & 0.584 & 0.739 & 0.879 & 0.673 \\ \text { B } & \text { DG-ORR } & 3 & 1 & 0 & 0.220 & 0.293 & 0.331 & 0.247 \\ \text { B } & \text { HL-ORR } & 4 & 0 & 4 & 0.417 & 0.512 & 0.628 & 0.495 \\ \text { B } & \text { CHE-ORR } & 2 & 0 & 2 & 0.721 & 0.963 & 1.090 & 0.793 \\ \text { B } & \text { CR-ORR } & 1 & 0 & 1 & 0.930 & 1.400 & 1.400 & 0.913 \\ \text { B } & \text { CR-ROA } & 1 & 0 & 1 & 0.485 & 0.731 & 0.731 & 0.476 \\ & & & & & & & & \\ \text { C } & \text { DG-AND } & 4 & 0 & 2 & 0.248 & 0.341 & 0.440 & 0.311 \\ \text { C } & \text { DG-ROA } & 3 & 0 & 2 & 0.239 & 0.340 & 0.424 & 0.287 \\ \text { C } & \text { DG-ORR } & 3 & 0 & 1 & 0.265 & 0.386 & 0.471 & 0.319 \\ \text { C } & \text { NL-ORR } & 4 & 0 & 3 & 0.306 & 0.411 & 0.543 & 0.383 \\ \text { C } & \text { CHE-ORR } & 2 & 0 & 2 & 0.629 & 0.945 & 1.120 & 0.707 \\ \text { C } & \text { CR-ORR } & 1 & 0 & 1 & 0.500 & 0.888 & 0.888 & 0.482 \\ \text { C } & \text { CR-ROA } & 1 & 0 & 1 & 0.575 & 1.020 & 1.020 & 0.554\end{array}$


G-26

Table G.2 (continued)

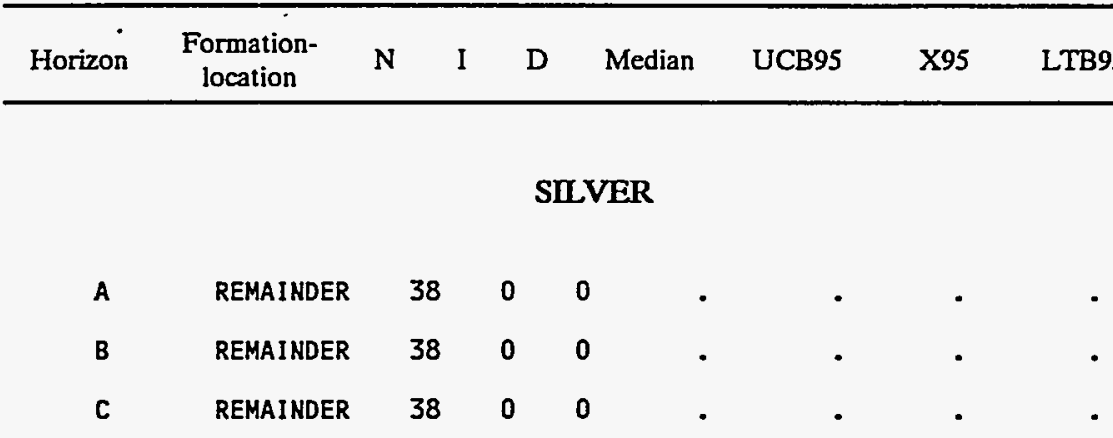

THALLIUM

$\begin{array}{lllllllll}\text { A } & \text { CHI-BV } & 4 & 0 & 1 & 0.198 & 0.274 & 0.319 & 0.232 \\ \text { A } & \text { CHI-K25 } & 4 & 0 & 2 & 0.225 & 0.297 & 0.363 & 0.263 \\ \text { A } & \text { CR-ORR } & 4 & 0 & 1 & 0.169 & 0.240 & 0.273 & 0.195 \\ \text { A } & \text { CR-AND } & 4 & 1 & 2 & 0.267 & 0.347 & 0.430 & 0.313 \\ \text { A } & \text { REMAINDER } & 22 & 0 & 0 & \cdot & \cdot & \cdot & \cdot \\ & & & & & & & \\ \text { B } & \text { DG-AND } & 4 & 1 & 1 & 0.208 & 0.253 & 0.281 & 0.231 \\ \text { B } & \text { DG-ROA } & 3 & 0 & 2 & 0.239 & 0.286 & 0.322 & 0.266 \\ \text { B } & \text { DG-ORR } & 3 & 1 & 2 & 0.243 & 0.292 & 0.327 & 0.269 \\ \text { B } & \text { NL-ORR } & 4 & 0 & 2 & 0.213 & 0.252 & 0.286 & 0.240 \\ \text { B } & \text { CHI-BV } & 4 & 0 & 3 & 0.250 & 0.291 & 0.336 & 0.285 \\ \text { B } & \text { CHI-K25 } & 4 & 0 & 4 & 0.284 & 0.329 & 0.382 & 0.324 \\ \text { B } & \text { CHE-ORR } & 4 & 1 & 3 & 0.253 & 0.296 & 0.341 & 0.287 \\ B & \text { CR-ORR } & 4 & 0 & 2 & 0.224 & 0.264 & 0.302 & 0.255 \\ \text { B } & \text { CR-AND } & 4 & 0 & 4 & 0.316 & 0.367 & 0.426 & 0.361 \\ \text { B } & \text { CR-ROA } & 4 & 1 & 2 & 0.237 & 0.278 & 0.319 & 0.269 \\ & & & & & & & & \\ \text { C } & \text { DG-AND } & 4 & 0 & 1 & 0.201 & 0.257 & 0.288 & 0.225 \\ \text { C } & \text { DG-ROA } & 3 & 0 & 3 & 0.245 & 0.302 & 0.351 & 0.281 \\ \text { C } & \text { DG-ORR } & 3 & 0 & 3 & 0.270 & 0.332 & 0.387 & 0.309 \\ \text { C } & \text { NL-ORR } & 4 & 0 & 2 & 0.218 & 0.268 & 0.312 & 0.252 \\ \text { C } & \text { CHI-BV } & 4 & 0 & 2 & 0.230 & 0.282 & 0.331 & 0.269 \\ \text { C } & \text { CHI-K25 } & 4 & 0 & 4 & 0.295 & 0.353 & 0.423 & 0.346 \\ \text { C } & \text { CHE-ORR } & 4 & 0 & 3 & 0.252 & 0.304 & 0.361 & 0.296 \\ \text { C } & \text { CR-ORR } & 4 & 0 & 2 & 0.231 & 0.282 & 0.332 & 0.270 \\ \text { C } & \text { CR-AND } & 4 & 0 & 4 & 0.332 & 0.398 & 0.476 & 0.390 \\ \text { C } & \text { CR-ROA } & 4 & 0 & 2 & 0.211 & 0.259 & 0.303 & 0.245\end{array}$

ZINC

$\begin{array}{lllllrrrr}\text { A } & \text { DG-AND } & 4 & 0 & 4 & 46.2 & 59.6 & 76.8 & 58.5 \\ \text { A } & \text { DG-ROA } & 3 & 0 & 3 & 38.5 & 51.7 & 64.0 & 47.0 \\ \text { A } & \text { DG-ORR } & 3 & 0 & 3 & 41.3 & 55.4 & 68.6 & 50.4 \\ \text { A } & \text { NL-ORR } & 4 & 0 & 4 & 25.5 & 32.8 & 42.3 & 32.3 \\ \text { A } & \text { CHI-BV } & 4 & 0 & 4 & 36.2 & 46.6 & 60.1 & 45.8 \\ \text { A } & \text { CHI-K25 } & 4 & 0 & 4 & 39.2 & 50.5 & 65.1 & 49.6 \\ \text { A } & \text { CHE-ORR } & 4 & 0 & 4 & 34.8 & 44.9 & 57.9 & 44.1 \\ \text { A } & \text { CR-ORR } & 4 & 0 & 4 & 24.4 & 31.5 & 40.6 & 30.9 \\ \text { A } & \text { CR-AND } & 4 & 0 & 4 & 39.9 & 51.4 & 66.3 & 50.6 \\ \text { A } & \text { CR-ROA } & 4 & 0 & 4 & 24.6 & 31.7 & 40.9 & 31.2 \\ & & & & & & & & \\ \text { B } & \text { DG-AND } & 4 & 0 & 4 & 46.2 & 62.0 & 83.4 & 60.8 \\ \text { B } & \text { DG-ROA } & 3 & 0 & 3 & 41.6 & 58.5 & 75.2 & 52.5 \\ \text { B } & \text { DG-ORR } & 3 & 0 & 3 & 50.5 & 71.0 & 91.2 & 63.7 \\ \text { B } & \text { HL-ORR } & 4 & 0 & 4 & 31.4 & 42.2 & 56.8 & 41.4 \\ \text { B } & \text { CHI-BV } & 4 & 0 & 4 & 50.3 & 67.6 & 90.9 & 66.3 \\ \text { B } & \text { CHI-K25 } & 4 & 0 & 4 & 59.9 & 80.5 & 108.0 & 78.8 \\ \text { B } & \text { CHE-ORR } & 4 & 0 & 4 & 101.0 & 135.0 & 182.0 & 132.0 \\ \text { B } & \text { CR-ORR } & 4 & 0 & 4 & 49.9 & 67.1 & 90.1 & 65.7 \\ \text { B } & \text { CR-AND } & 4 & 0 & 4 & 58.9 & 79.2 & 106.0 & 77.6 \\ \text { B } & \text { CR-ROA } & 4 & 0 & 4 & 35.2 & 47.3 & 63.6 & 46.4 \\ & & & & & & & & \\ \text { C } & \text { DG-AND } & 4 & 0 & 4 & 47.1 & 62.7 & 83.5 & 61.4 \\ \text { C } & \text { DG-ROA } & 3 & 0 & 3 & 49.5 & 68.9 & 87.8 & 62.0 \\ \text { C } & \text { DG-ORR } & 3 & 0 & 3 & 52.4 & 73.0 & 93.0 & 65.6\end{array}$




\section{G-27}

Table G.2 (continued)

\begin{tabular}{rlrrrrrrr}
\hline Horizon & $\begin{array}{c}\text { Formation- } \\
\text { location }\end{array}$ & N & I & D & Median & UCB95 & X95 & LTB9595 \\
\hline & & & & & & & & \\
C & NL-ORR & 4 & 0 & 4 & 32.6 & 43.4 & 57.9 & 42.6 \\
C & CHI-BV & 4 & 0 & 4 & 75.3 & 100.0 & 134.0 & 98.3 \\
C & CHI-K25 & 4 & 0 & 4 & 54.5 & 72.6 & 96.7 & 71.2 \\
C & CHE-ORR & 4 & 0 & 4 & 164.0 & 218.0 & 290.0 & 214.0 \\
C & CR-ORR & 4 & 0 & 4 & 88.4 & 118.0 & 157.0 & 115.0 \\
C & CR-AND & 4 & 0 & 4 & 75.6 & 101.0 & 134.0 & 98.8 \\
C & CR-ROA & 4 & 0 & 4 & 39.0 & 51.9 & 69.2 & 50.9 \\
\hline
\end{tabular}

$a_{N}=$ number of observations, possibly averages over replicates at sites; $I=$ number of interval censored observations (see text); $D=$ number of true detects (see text); UCB95 $=95 \%$ upper confidence bound for median; X95 = estimate of 95 th percentile; LTB9595 $=95 \%$ lower confidence bound for 95th percentile; REMAINDER refers to the remaining observations-no detects. 
Table G.3. Significance levels for comparing inorganics ${ }^{a}$

(Selected analytes were deleted because of insufficient detection.)

\begin{tabular}{|c|c|c|c|c|c|c|c|c|c|c|c|}
\hline Analysis $\mathrm{H}$ & izon & $\mathbf{N}$ & $\mathrm{D}$ & P-AIL & P-DG & P-CR & P-ORR & P-CHICK & P-DGN & P-CRC & P-GRP \\
\hline $\begin{array}{l}\text { Aluminum } \\
\text { Aluminum } \\
\text { Aluminum }\end{array}$ & $\begin{array}{l}A \\
B \\
C\end{array}$ & $\begin{array}{l}40 \\
40 \\
40\end{array}$ & $\begin{array}{l}40 \\
40 \\
40\end{array}$ & $\begin{array}{l}.0000 \\
.0000 \\
.0000\end{array}$ & $\begin{array}{l}.0008 \\
.0013 \\
.0001\end{array}$ & $\begin{array}{l}.0013 \\
.0922 \\
.1204\end{array}$ & $\begin{array}{l}.0000 \\
.0000 \\
.0000\end{array}$ & $\begin{array}{l}.9974 \\
.1320 \\
.7900\end{array}$ & $\begin{array}{l}.4820 \\
.2864 \\
.7825\end{array}$ & $\begin{array}{l}.0357 \\
.4391 \\
.9180\end{array}$ & $\begin{array}{l}.0000 \\
.0000 \\
.0000\end{array}$ \\
\hline $\begin{array}{l}\text { Arsenic } \\
\text { Arsenic } \\
\text { Arsenic }\end{array}$ & $\begin{array}{l}\text { A } \\
\text { B } \\
\text { C }\end{array}$ & $\begin{array}{l}39 \\
38 \\
39\end{array}$ & $\begin{array}{l}39 \\
37 \\
37\end{array}$ & $\begin{array}{l}.0000 \\
.0000 \\
.0000\end{array}$ & $\begin{array}{l}.2008 \\
.0255 \\
.0044\end{array}$ & $\begin{array}{l}.0002 \\
.0039 \\
.0275\end{array}$ & $\begin{array}{l}.0000 \\
.0000 \\
.0000\end{array}$ & $\begin{array}{l}.3517 \\
.8421 \\
.8035\end{array}$ & $\begin{array}{l}.9558 \\
.4869 \\
.0655\end{array}$ & $\begin{array}{l}.0008 \\
.0127 \\
.0513\end{array}$ & $\begin{array}{l}.0000 \\
.0000 \\
.0000\end{array}$ \\
\hline $\begin{array}{l}\text { Barium } \\
\text { Barium } \\
\text { Barium }\end{array}$ & $\begin{array}{l}\text { A } \\
\text { B } \\
\text { C }\end{array}$ & $\begin{array}{l}40 \\
40 \\
40\end{array}$ & $\begin{array}{l}40 \\
40 \\
40\end{array}$ & $\begin{array}{l}.1073 \\
.0000 \\
.0000\end{array}$ & $\begin{array}{l}.6577 \\
.0601 \\
.3493\end{array}$ & $\begin{array}{l}.0189 \\
.1774 \\
.0148\end{array}$ & $\begin{array}{l}.2108 \\
.0000 \\
.0000\end{array}$ & $\begin{array}{l}.8701 \\
.1000 \\
.0327\end{array}$ & $\begin{array}{l}.2270 \\
.4117 \\
.2875\end{array}$ & $\begin{array}{l}.1969 \\
.4305 \\
.0049\end{array}$ & $\begin{array}{l}.1323 \\
.0000 \\
.0000\end{array}$ \\
\hline $\begin{array}{l}\text { Beryll ium } \\
\text { Beryl lium } \\
\text { Beryl lium }\end{array}$ & $\begin{array}{l}\text { A } \\
B \\
C\end{array}$ & $\begin{array}{l}40 \\
40 \\
40\end{array}$ & $\begin{array}{l}37 \\
36 \\
37\end{array}$ & $\begin{array}{l}.0000 \\
.0000 \\
.0000\end{array}$ & $\begin{array}{l}.3360 \\
.1433 \\
.2024\end{array}$ & $\begin{array}{l}.0213 \\
.1569 \\
.3085\end{array}$ & $\begin{array}{l}.0000 \\
.0000 \\
.0000\end{array}$ & $\begin{array}{l}.5341 \\
.9781 \\
.1284\end{array}$ & $\begin{array}{l}.9679 \\
.1408 \\
.4744\end{array}$ & $\begin{array}{l}.0763 \\
.7586 \\
.1733\end{array}$ & $\begin{array}{l}.0000 \\
.0000 \\
.0000\end{array}$ \\
\hline $\begin{array}{l}\text { Calcium } \\
\text { Calcium } \\
\text { Calcium }\end{array}$ & $\begin{array}{l}\text { A } \\
\text { B } \\
\text { C }\end{array}$ & $\begin{array}{l}35 \\
34 \\
34\end{array}$ & $\begin{array}{l}35 \\
34 \\
34\end{array}$ & $\begin{array}{l}.0001 \\
.0000 \\
.0000\end{array}$ & $\begin{array}{l}.9639 \\
.9169 \\
.0491\end{array}$ & $\begin{array}{l}.0919 \\
.5150 \\
.1227\end{array}$ & $\begin{array}{l}.0000 \\
.0000 \\
.0000\end{array}$ & $\begin{array}{l}.2674 \\
.0430 \\
.0003\end{array}$ & $\begin{array}{l}.1029 \\
.7081 \\
.4311\end{array}$ & $\begin{array}{l}.6405 \\
.1426 \\
.2942\end{array}$ & $\begin{array}{l}.0000 \\
.0000 \\
.0000\end{array}$ \\
\hline $\begin{array}{l}\text { Chromium } \\
\text { Chromium } \\
\text { Chromium }\end{array}$ & $\begin{array}{l}A \\
B \\
C\end{array}$ & $\begin{array}{l}39 \\
39 \\
40\end{array}$ & $\begin{array}{l}38 \\
39 \\
40\end{array}$ & $\begin{array}{l}.0000 \\
.1152 \\
.0019\end{array}$ & $\begin{array}{l}.6438 \\
.8241 \\
.3715\end{array}$ & $\begin{array}{l}.0107 \\
.3101 \\
.4125\end{array}$ & $\begin{array}{l}.0000 \\
.4152 \\
.0001\end{array}$ & $\begin{array}{l}.7641 \\
.9810 \\
.4205\end{array}$ & $\begin{array}{l}.4253 \\
.9582 \\
.2767\end{array}$ & $\begin{array}{l}.7173 \\
.9340 \\
.6486\end{array}$ & $\begin{array}{l}.0000 \\
.0823 \\
.0000\end{array}$ \\
\hline $\begin{array}{l}\text { Cobalt } \\
\text { Cobalt } \\
\text { Cobalt }\end{array}$ & $\begin{array}{l}\text { A } \\
\text { B } \\
\text { C }\end{array}$ & $\begin{array}{l}40 \\
40 \\
40\end{array}$ & $\begin{array}{l}38 \\
31 \\
33\end{array}$ & $\begin{array}{l}.0000 \\
.0000 \\
.0001\end{array}$ & $\begin{array}{l}.0824 \\
.7194 \\
.8067\end{array}$ & $\begin{array}{l}.0002 \\
.0003 \\
.0083\end{array}$ & $\begin{array}{l}.0076 \\
.0000 \\
.0012\end{array}$ & $\begin{array}{l}.8173 \\
.8774 \\
.3911\end{array}$ & $\begin{array}{l}.9829 \\
.3007 \\
.7297\end{array}$ & $\begin{array}{l}.1114 \\
.5433 \\
.0182\end{array}$ & $\begin{array}{l}.0014 \\
.0000 \\
.0010\end{array}$ \\
\hline $\begin{array}{l}\text { Copper } \\
\text { Copper } \\
\text { Copper }\end{array}$ & $\begin{array}{l}\text { A } \\
\text { B } \\
\text { C }\end{array}$ & $\begin{array}{l}40 \\
39 \\
40\end{array}$ & $\begin{array}{l}34 \\
38 \\
39\end{array}$ & $\begin{array}{l}.0000 \\
.0347 \\
.0338\end{array}$ & $\begin{array}{l}.1679 \\
.0862 \\
.5866\end{array}$ & $\begin{array}{l}.0738 \\
.0057 \\
.0022\end{array}$ & $\begin{array}{l}.0000 \\
.5382 \\
.0970\end{array}$ & $\begin{array}{l}.0958 \\
.1324 \\
.0274\end{array}$ & $\begin{array}{l}.1256 \\
.7468 \\
.4426\end{array}$ & $\begin{array}{l}.0559 \\
.5625 \\
.0633\end{array}$ & $\begin{array}{l}.0000 \\
.4964 \\
.6539\end{array}$ \\
\hline $\begin{array}{l}\text { Iron } \\
\text { Iron } \\
\text { Iron }\end{array}$ & $\begin{array}{l}\text { A } \\
B \\
\text { C }\end{array}$ & $\begin{array}{l}40 \\
40 \\
40\end{array}$ & $\begin{array}{l}40 \\
40 \\
40\end{array}$ & $\begin{array}{l}.0000 \\
.0000 \\
.0000\end{array}$ & $\begin{array}{l}.4468 \\
.1911 \\
.4203\end{array}$ & $\begin{array}{l}.0737 \\
.0054 \\
.0743\end{array}$ & $\begin{array}{l}.0000 \\
.0000 \\
.0001\end{array}$ & $\begin{array}{l}.2517 \\
.2555 \\
.8421\end{array}$ & $\begin{array}{l}.6789 \\
.2289 \\
.7186\end{array}$ & $\begin{array}{l}.1870 \\
.8212 \\
.0405\end{array}$ & $\begin{array}{l}.0000 \\
.0000 \\
.0000\end{array}$ \\
\hline $\begin{array}{l}\text { Lead } \\
\text { Lead } \\
\text { Lead }\end{array}$ & $\begin{array}{l}\text { A } \\
\text { B } \\
\text { C }\end{array}$ & $\begin{array}{l}38 \\
39 \\
39\end{array}$ & $\begin{array}{l}38 \\
39 \\
39\end{array}$ & $\begin{array}{l}.0638 \\
.0531 \\
.1319\end{array}$ & $\begin{array}{l}.4463 \\
.2013 \\
.6057\end{array}$ & $\begin{array}{l}.0466 \\
.0243 \\
.0768\end{array}$ & $\begin{array}{l}.0208 \\
.0960 \\
.0601\end{array}$ & $\begin{array}{l}.6766 \\
.4901 \\
.0177\end{array}$ & $\begin{array}{l}.6098 \\
.9863 \\
.1822\end{array}$ & $\begin{array}{l}.0075 \\
.0301 \\
.1781\end{array}$ & $\begin{array}{l}.0556 \\
.1212 \\
.3855\end{array}$ \\
\hline $\begin{array}{l}\text { Lithium } \\
\text { Lithium } \\
\text { Lithium }\end{array}$ & $\begin{array}{l}A \\
B \\
C\end{array}$ & $\begin{array}{l}36 \\
37 \\
37\end{array}$ & $\begin{array}{l}29 \\
36 \\
34\end{array}$ & $\begin{array}{l}.0000 \\
.0000 \\
.0000\end{array}$ & $\begin{array}{l}.1174 \\
.7490 \\
.4819\end{array}$ & $\begin{array}{l}.0002 \\
.0161 \\
.0001\end{array}$ & $\begin{array}{l}.0000 \\
.0000 \\
.0000\end{array}$ & $\begin{array}{l}.4630 \\
.6565 \\
.9191\end{array}$ & $\begin{array}{l}.0929 \\
.7285 \\
.4866\end{array}$ & $\begin{array}{l}.1008 \\
.0298 \\
.0003\end{array}$ & $\begin{array}{l}.0000 \\
.0000 \\
.0000\end{array}$ \\
\hline $\begin{array}{l}\text { Magnesium } \\
\text { Magnesium } \\
\text { Magnesium }\end{array}$ & $\begin{array}{l}\text { A } \\
\text { B } \\
\text { C }\end{array}$ & $\begin{array}{l}40 \\
40 \\
40\end{array}$ & $\begin{array}{l}40 \\
40 \\
40\end{array}$ & $\begin{array}{l}.0000 \\
.0000 \\
.0000\end{array}$ & $\begin{array}{l}.0009 \\
.0015 \\
.0620\end{array}$ & $\begin{array}{l}.0076 \\
.0022 \\
.0001\end{array}$ & $\begin{array}{l}.0000 \\
.0000 \\
.0000\end{array}$ & $\begin{array}{l}.1318 \\
.9434 \\
.0290\end{array}$ & $\begin{array}{l}.0322 \\
.1689 \\
.0993\end{array}$ & $\begin{array}{l}.1550 \\
.0101 \\
.0026\end{array}$ & $\begin{array}{l}.0000 \\
.0000 \\
.0000\end{array}$ \\
\hline $\begin{array}{l}\text { Manganese } \\
\text { Manganese } \\
\text { Manganese }\end{array}$ & $\begin{array}{l}A \\
B \\
C\end{array}$ & $\begin{array}{l}40 \\
40 \\
40\end{array}$ & $\begin{array}{l}40 \\
40 \\
40\end{array}$ & $\begin{array}{l}.0011 \\
.0510 \\
.0193\end{array}$ & $\begin{array}{l}.0078 \\
.8868 \\
.5073\end{array}$ & $\begin{array}{l}.0026 \\
.0070 \\
.0307\end{array}$ & $\begin{array}{l}.0541 \\
.0985 \\
.0575\end{array}$ & $\begin{array}{l}.0936 \\
.7692 \\
.3226\end{array}$ & $\begin{array}{l}.1235 \\
.9131 \\
.9122\end{array}$ & $\begin{array}{l}.5841 \\
.6683 \\
.1890\end{array}$ & $\begin{array}{l}.0616 \\
.0111 \\
.0180\end{array}$ \\
\hline $\begin{array}{l}\text { Mercury } \\
\text { Mercury } \\
\text { Mercury }\end{array}$ & $\begin{array}{l}A \\
B \\
C\end{array}$ & $\begin{array}{l}40 \\
40 \\
40\end{array}$ & $\begin{array}{l}31 \\
20 \\
24\end{array}$ & $\begin{array}{l}.0000 \\
.0004 \\
.0000\end{array}$ & $\begin{array}{l}.0000 \\
.0018 \\
.0144\end{array}$ & $\begin{array}{l}.0337 \\
.1083 \\
.0741\end{array}$ & $\begin{array}{l}.0000 \\
.0039 \\
.0000\end{array}$ & $\begin{array}{l}.0000 \\
.0104 \\
.0396\end{array}$ & $\begin{array}{l}.0004 \\
.0013 \\
.8944\end{array}$ & $\begin{array}{l}.1679 \\
.8062 \\
.0056\end{array}$ & $\begin{array}{l}.0016 \\
.6109 \\
.0000\end{array}$ \\
\hline $\begin{array}{l}\text { Molybdenum } \\
\text { Molybdenum }\end{array}$ & $\begin{array}{l}\text { B } \\
\text { C }\end{array}$ & $\begin{array}{l}37 \\
37\end{array}$ & $\begin{array}{l}11 \\
11\end{array}$ & $\begin{array}{l}.2365 \\
.0010\end{array}$ & $\begin{array}{l}1.000 \\
1.000\end{array}$ & $\begin{array}{l}.0847 \\
.1520\end{array}$ & $\begin{array}{l}.1834 \\
.0002\end{array}$ & $\begin{array}{l}.8057 \\
1.000\end{array}$ & $\begin{array}{l}.9997 \\
1.000\end{array}$ & $\begin{array}{l}.1706 \\
.0154\end{array}$ & $\begin{array}{l}.0608 \\
.0001\end{array}$ \\
\hline $\begin{array}{l}\text { Nickel } \\
\text { Nickel } \\
\text { Nickel }\end{array}$ & $\begin{array}{l}\text { A } \\
\text { B } \\
\text { C }\end{array}$ & $\begin{array}{l}40 \\
40 \\
40\end{array}$ & $\begin{array}{l}30 \\
35 \\
38\end{array}$ & $\begin{array}{l}.0000 \\
.0000 \\
.0004\end{array}$ & $\begin{array}{l}.1962 \\
.2591 \\
.9179\end{array}$ & $\begin{array}{l}.0107 \\
.0348 \\
.1370\end{array}$ & $\begin{array}{l}.0000 \\
.0002 \\
.0341\end{array}$ & $\begin{array}{l}.1886 \\
.8335 \\
.1276\end{array}$ & $\begin{array}{l}.1049 \\
.6299 \\
.4252\end{array}$ & $\begin{array}{l}.1988 \\
.6924 \\
.2856\end{array}$ & $\begin{array}{l}.0000 \\
.0000 \\
.0175\end{array}$ \\
\hline $\begin{array}{l}\text { Potassium } \\
\text { Potassium } \\
\text { Potassium }\end{array}$ & $\begin{array}{l}\text { A } \\
B \\
C\end{array}$ & $\begin{array}{l}39 \\
39 \\
39\end{array}$ & $\begin{array}{l}34 \\
39 \\
39\end{array}$ & $\begin{array}{l}.0000 \\
.0000 \\
.0000\end{array}$ & $\begin{array}{l}.0000 \\
.0000 \\
.0002\end{array}$ & $\begin{array}{l}.0175 \\
.0016 \\
.0001\end{array}$ & $\begin{array}{l}.0000 \\
.0000 \\
.0000\end{array}$ & $\begin{array}{l}.6133 \\
.0015 \\
.0016\end{array}$ & $\begin{array}{l}.1488 \\
.0148 \\
.0007\end{array}$ & $\begin{array}{l}.0037 \\
.0001 \\
.0023\end{array}$ & $\begin{array}{l}.0000 \\
.0000 \\
.0000\end{array}$ \\
\hline $\begin{array}{l}\text { Selenium } \\
\text { Selenium } \\
\text { Selenium }\end{array}$ & $\begin{array}{l}\text { A } \\
\text { B } \\
\text { C }\end{array}$ & $\begin{array}{l}38 \\
39 \\
39\end{array}$ & $\begin{array}{l}27 \\
28 \\
25\end{array}$ & $\begin{array}{l}.0021 \\
.0008 \\
.0000\end{array}$ & $\begin{array}{l}.0036 \\
.0006 \\
.0001\end{array}$ & $\begin{array}{l}.0023 \\
.0974 \\
.2597\end{array}$ & $\begin{array}{l}.0113 \\
.0001 \\
.0000\end{array}$ & $\begin{array}{l}.8721 \\
.6019 \\
.5137\end{array}$ & $\begin{array}{l}.0181 \\
.0004 \\
.0000\end{array}$ & $\begin{array}{l}.1515 \\
.0031 \\
.0330\end{array}$ & $\begin{array}{l}.0262 \\
.0617 \\
.2522\end{array}$ \\
\hline silicon & A & 32 & 32 & .0000 & .0000 & .0183 & .0000 & .0316 & .0000 & .2262 & .0002 \\
\hline
\end{tabular}


Table G.3 (continued)

\begin{tabular}{|c|c|c|c|c|c|c|c|c|c|c|c|}
\hline Analysis & Horizon & $\mathbf{N}$ & $\mathbf{D}$ & P-ALL & P-DG & P-CR & P-ORR & P-CHICK & P-DGN & P-CRC & P-GRP \\
\hline $\begin{array}{ll} & \text { silicon } \\
\text { silicon. } & \text { silicon }\end{array}$ & $\stackrel{B}{C}$ & $\begin{array}{l}32 \\
32\end{array}$ & $\begin{array}{r}32 \\
32\end{array}$ & $\begin{array}{l}.0000 \\
.0000\end{array}$ & $\begin{array}{l}.0000 \\
.0000\end{array}$ & $\begin{array}{l}.2060 \\
.3368\end{array}$ & $\begin{array}{l}.0000 \\
.0000\end{array}$ & $\begin{array}{l}.0044 \\
.0042\end{array}$ & $\begin{array}{l}.0000 \\
.0000\end{array}$ & $\begin{array}{l}.5131 \\
.6241\end{array}$ & $\begin{array}{r}.0000 \\
.0003\end{array}$ \\
\hline $\begin{array}{l}\text { Sodium } \\
\text { Sodium } \\
\text { Sodium }\end{array}$ & $\begin{array}{l}\text { A } \\
\text { B } \\
\text { C }\end{array}$ & $\begin{array}{l}25 \\
25 \\
25\end{array}$ & $\begin{array}{l}23 \\
24 \\
24\end{array}$ & $\begin{array}{l}.0015 \\
.0007 \\
.0099\end{array}$ & $\begin{array}{l}1.000 \\
1.000 \\
1.000\end{array}$ & $\begin{array}{l}.0981 \\
.3744 \\
.6896\end{array}$ & $\begin{array}{l}.0019 \\
.0002 \\
.0079\end{array}$ & $\begin{array}{l}.1337 \\
.1534 \\
.5264\end{array}$ & $\begin{array}{l}1.000 \\
1.000 \\
1.000\end{array}$ & $\begin{array}{l}.0873 \\
.0680 \\
.2107\end{array}$ & $\begin{array}{l}.0009 \\
.0001 \\
.0010\end{array}$ \\
\hline $\begin{array}{l}\text { Strontium } \\
\text { Strontium } \\
\text { Strontium }\end{array}$ & $\begin{array}{l}\text { A } \\
\text { B } \\
\text { C }\end{array}$ & $\begin{array}{l}36 \\
37 \\
37\end{array}$ & $\begin{array}{l}34 \\
34 \\
31\end{array}$ & $\begin{array}{l}.0004 \\
.0001 \\
.0000\end{array}$ & $\begin{array}{l}.2929 \\
.2037 \\
.0647\end{array}$ & $\begin{array}{l}.1656 \\
.4723 \\
.0000\end{array}$ & $\begin{array}{l}.0000 \\
.0000 \\
.0000\end{array}$ & $\begin{array}{l}.0303 \\
.0760 \\
.9377\end{array}$ & $\begin{array}{l}.0651 \\
.3514 \\
.1653\end{array}$ & $\begin{array}{l}.1620 \\
.2706 \\
.0078\end{array}$ & $\begin{array}{l}.0009 \\
.0000 \\
.0000\end{array}$ \\
\hline $\begin{array}{l}\text { Sulfate } \\
\text { Sulfate }\end{array}$ & $\begin{array}{l}\text { A } \\
\text { B }\end{array}$ & $\begin{array}{l}39 \\
40\end{array}$ & $\begin{array}{l}39 \\
40\end{array}$ & $\begin{array}{l}.0000 \\
.0005\end{array}$ & $\begin{array}{l}.0000 \\
.0005\end{array}$ & $\begin{array}{l}.0745 \\
.6491\end{array}$ & $\begin{array}{l}.0000 \\
.0021\end{array}$ & $\begin{array}{l}.0326 \\
.0599\end{array}$ & $\begin{array}{l}.0000 \\
.3579\end{array}$ & $\begin{array}{l}.5937 \\
.9335\end{array}$ & $\begin{array}{l}.0002 \\
.0007\end{array}$ \\
\hline $\begin{array}{l}\text { Thall ium } \\
\text { Thal l ium } \\
\text { Thal l ium }\end{array}$ & $\begin{array}{l}\mathbf{A} \\
\mathbf{B} \\
\mathbf{C}\end{array}$ & $\begin{array}{l}38 \\
38 \\
38\end{array}$ & $\begin{array}{l}2 \\
5 \\
8\end{array}$ & $\begin{array}{l}.6741 \\
.8626 \\
.0820\end{array}$ & $\begin{array}{l}.6354 \\
.4175 \\
.4808\end{array}$ & $\begin{array}{l}.1466 \\
.6219 \\
.1077\end{array}$ & $\begin{array}{l}.8130 \\
.8422 \\
.0794\end{array}$ & $\begin{array}{l}1.000 \\
1.000 \\
1.000\end{array}$ & $\begin{array}{l}.3296 \\
.8994 \\
.0684\end{array}$ & $\begin{array}{l}1.000 \\
.4403 \\
.3435\end{array}$ & $\begin{array}{l}.5211 \\
.4873 \\
.0624\end{array}$ \\
\hline Sulfate & c & 40 & 35 & .0000 & .0000 & .0019 & .0000 & .6366 & .0021 & .4277 & .0000 \\
\hline $\begin{array}{l}\text { Vanadium } \\
\text { Vanadium } \\
\text { Vanadium }\end{array}$ & $\begin{array}{l}\text { A } \\
\text { B } \\
\text { C }\end{array}$ & $\begin{array}{l}40 \\
39 \\
40\end{array}$ & $\begin{array}{l}40 \\
39 \\
40\end{array}$ & $\begin{array}{l}.0111 \\
.0005 \\
.0000\end{array}$ & $\begin{array}{l}.6029 \\
.4041 \\
.0139\end{array}$ & $\begin{array}{l}.0050 \\
.0733 \\
.0295\end{array}$ & $\begin{array}{l}.0679 \\
.0007 \\
.0000\end{array}$ & $\begin{array}{l}.9834 \\
.1038 \\
.3769\end{array}$ & $\begin{array}{l}.6531 \\
.2217 \\
.2136\end{array}$ & $\begin{array}{l}.2860 \\
.8019 \\
.0027\end{array}$ & $\begin{array}{l}.0115 \\
.0002 \\
.0000\end{array}$ \\
\hline $\begin{array}{l}\text { Zine } \\
\text { Zine } \\
\text { Zine }\end{array}$ & $\begin{array}{l}\text { A } \\
\text { B } \\
\text { C }\end{array}$ & $\begin{array}{l}40 \\
40 \\
40 \\
\end{array}$ & $\begin{array}{l}40 \\
40 \\
39\end{array}$ & $\begin{array}{l}.6155 \\
.0017 \\
.0001 \\
\end{array}$ & $\begin{array}{l}.4195 \\
.5332 \\
.7204\end{array}$ & $\begin{array}{l}.4519 \\
.0279 \\
.0076\end{array}$ & $\begin{array}{l}.3643 \\
.0028 \\
.0000\end{array}$ & $\begin{array}{l}.8914 \\
.4098 \\
.3329\end{array}$ & $\begin{array}{l}.1170 \\
.5154 \\
.1944 \\
\end{array}$ & $\begin{array}{l}.5165 \\
.0696 \\
.2553\end{array}$ & $\begin{array}{l}.2764 \\
.0010 \\
.0000\end{array}$ \\
\hline
\end{tabular}

${ }^{a} \mathrm{~N}=$ number of observations; $\mathrm{D}=$ number of detects. See "Note on Significance Levels" at beginning of this appendix for definitions of significance levels P-ALL through P-GRP. 


\section{G-30}

Table G.4. Significance levels for comparing PAHs (Dismal Gap and Nolichucky data excluded)

\begin{tabular}{|c|c|c|c|c|c|c|c|c|}
\hline Analysis & $\mathbf{N}$ & $\mathrm{D}$ & P-ALL & P-CR & P-ORR & P-CHICK & P-CRC & P-GRP \\
\hline Acenaphthene & 25 & 11 & 0.0960 & 0.3413 & 0.0517 & 0.0223 & 0.1437 & 0.5664 \\
\hline Acenaphthylene & 61 & 5 & 0.0033 & 0.0074 & 0.0023 & 1.000 & 0.0181 & 0.0116 \\
\hline Anthracene & 44 & 39 & 0.2485 & 0.4729 & 0.2316 & 0.1345 & 0.2230 & 0.7280 \\
\hline Benzo [a] anthracene & 60 & 60 & 0.0001 & 0.1210 & 0.0001 & 0.3603 & 0.5492 & 0.0000 \\
\hline Benzo [a] pyrene & 61 & 60 & 0.0000 & 0.0073 & 0.0515 & 0.1662 & 0.4871 & 0.0684 \\
\hline Benzo [b] fluoranthene & 52 & 47 & 0.0050 & 0.3834 & 0.0443 & 0.9141 & 0.4641 & 0.0231 \\
\hline Benzo $[g, h, i]$ perylene & 53 & 52 & 0.0051 & 0.2631 & 0.0748 & 0.2602 & 0.7158 & 0.0635 \\
\hline Benzo $[k]$ f luoranthene & 61 & 61 & 0.0000 & 0.1393 & 0.0076 & 0.2458 & 0.6917 & 0.0054 \\
\hline Chrysene & 36 & 23 & 0.0311 & 0.0955 & 0.1427 & 0.8623 & 0.0732 & 0.3339 \\
\hline D ibenzo $[a, h]$ anthracene & 33 & 27 & 0.9138 & 0.8686 & 0.7798 & 0.7161 & 0.9920 & 0.6199 \\
\hline Fluoranthene & 56 & 56 & 0.0610 & 0.0875 & 0.0925 & 0.2968 & 0.0395 & 0.5540 \\
\hline Fluorene & 34 & 20 & 0.0158 & 0.1654 & 0.0101 & 0.2484 & 0.1152 & 0.0222 \\
\hline Indeno $[1,2,3-c, d]$ pyrene & 64 & 27 & 0.2847 & 0.0681 & 0.2109 & 0.5841 & 0.2298 & 0.2499 \\
\hline Naphthalene & 32 & 19 & 0.1016 & 0.3923 & 0.0526 & 0.0267 & 0.8000 & 0.2584 \\
\hline Phenanthrene & 67 & 67 & 0.0026 & 0.5975 & 0.0090 & 0.7508 & 0.3534 & 0.0049 \\
\hline Pyrene & 59 & 59 & 0.0000 & 0.0138 & 0.0046 & 0.3421 & 0.2464 & 0.0045 \\
\hline
\end{tabular}

${ }^{a} \mathbf{N}=$ number of observations; D = number of detects. See "Note on Significance Levels" at beginning of this appendix for definitions of significance levels P-ALL through P-GRP. 
Table G.5. Significance levels for comparisons of 'selected ${ }^{a}$ radionuclides (by type of analysis)

\begin{tabular}{|c|c|c|c|c|c|c|c|c|c|c|c|}
\hline Analysis & izon & $\mathbf{N}$ & D & P-ALL & P-DG & P-CR & P-ORR & P-CHICK & P-DGN & P-CRC & P-GRP \\
\hline \multicolumn{12}{|c|}{ AIPHA } \\
\hline $\begin{array}{l}\text { Neptunium-237 } \\
\text { Plutonium-238 } \\
\text { Plutonium-239/24 } \\
\text { Radium-226 } \\
\text { Radium-226 } \\
\text { Radium-226 } \\
\text { Thorium-228 } \\
\text { Thorium-228 } \\
\text { Thorium-228 } \\
\text { Thorium-230 } \\
\text { Thorium-230 } \\
\text { Thorium-230 } \\
\text { Thorium-232 } \\
\text { Thorium-232 } \\
\text { Thorium-232 } \\
\text { Total Uranium } \\
\text { Uranium-233/234 } \\
\text { Uranium-233/234 } \\
\text { Uranium-233/234 } \\
\text { Uranium-235 } \\
\text { Uranium-235 } \\
\text { Uranium-235 } \\
\text { Uranium-238 } \\
\text { Uranium-238 } \\
\text { Uranium-238 }\end{array}$ & $\begin{array}{l}A \\
A \\
A \\
A \\
B \\
C \\
A \\
B \\
C \\
A \\
B \\
C \\
A \\
B \\
C \\
A \\
A \\
B \\
C \\
A \\
B \\
C \\
A \\
B \\
C\end{array}$ & $\begin{array}{l}29 \\
38 \\
38 \\
40 \\
40 \\
40 \\
40 \\
40 \\
40 \\
40 \\
40 \\
40 \\
40 \\
40 \\
40 \\
38 \\
40 \\
39 \\
40 \\
40 \\
39 \\
40 \\
40 \\
39 \\
40\end{array}$ & $\begin{array}{r}23 \\
9 \\
7 \\
38 \\
39 \\
40 \\
39 \\
40 \\
39 \\
40 \\
39 \\
40 \\
40 \\
40 \\
40 \\
37 \\
40 \\
39 \\
40 \\
29 \\
32 \\
29 \\
40 \\
39 \\
40\end{array}$ & $\begin{array}{l}.0942 \\
.0075 \\
.0736 \\
.1201 \\
.0212 \\
.0221 \\
.0013 \\
.2339 \\
.0700 \\
.0037 \\
.0473 \\
.0000 \\
.0000 \\
.2009 \\
.1281 \\
.2438 \\
.1374 \\
.0029 \\
.0000 \\
.0084 \\
.0215 \\
.0342 \\
.0000 \\
.0002 \\
.0000\end{array}$ & $\begin{array}{l}1.000 \\
.0068 \\
.3350 \\
.0223 \\
.0031 \\
.0016 \\
.2394 \\
.3850 \\
.2298 \\
.0139 \\
.4295 \\
.0696 \\
.0045 \\
.5377 \\
.2541 \\
.3682 \\
.9967 \\
.1616 \\
.1540 \\
.4178 \\
.4823 \\
.9956 \\
.3195 \\
.1986 \\
.2419\end{array}$ & $\begin{array}{l}.1020 \\
.0077 \\
.0716 \\
.0720 \\
.4751 \\
.5727 \\
.0154 \\
.9371 \\
.9823 \\
.2130 \\
.1362 \\
.6691 \\
.0247 \\
.3934 \\
.8969 \\
.1372 \\
.4057 \\
.0055 \\
.0141 \\
.0006 \\
.2400 \\
.1941 \\
.0000 \\
.0009 \\
.0348\end{array}$ & $\begin{array}{l}.3136 \\
.0235 \\
.3805 \\
.6498 \\
.0249 \\
.0523 \\
.0002 \\
.4273 \\
.0344 \\
.0008 \\
.0388 \\
.0003 \\
.0000 \\
.1466 \\
.2569 \\
.2986 \\
.1222 \\
.0349 \\
.0000 \\
.0512 \\
.0627 \\
.0318 \\
.0323 \\
.0087 \\
.0000\end{array}$ & $\begin{array}{l}.9793 \\
.9635 \\
.6793 \\
.6498 \\
.6121 \\
.9598 \\
.6625 \\
.9447 \\
.2878 \\
.9050 \\
.9692 \\
.5068 \\
.3421 \\
.2406 \\
.6125 \\
.5574 \\
.2585 \\
.1320 \\
.5381 \\
.1222 \\
.7454 \\
.3751 \\
.1652 \\
.2907 \\
.2578\end{array}$ & $\begin{array}{l}1.000 \\
.2395 \\
.1852 \\
.8503 \\
.5117 \\
.2954 \\
.0178 \\
.1127 \\
.0078 \\
.0013 \\
.1355 \\
.0528 \\
.0000 \\
.0693 \\
.0776 \\
.7756 \\
.0593 \\
.7710 \\
.0016 \\
.9693 \\
.6232 \\
.6193 \\
.0235 \\
.7214 \\
.0018\end{array}$ & $\begin{array}{l}.3293 \\
.0077 \\
.8842 \\
.2967 \\
.8167 \\
.3240 \\
.0620 \\
.7555 \\
.8687 \\
.0257 \\
.2680 \\
.5515 \\
.4984 \\
.7807 \\
.9175 \\
.5032 \\
.1007 \\
.1497 \\
.0142 \\
.0757 \\
.4017 \\
.0681 \\
.0368 \\
.2924 \\
.0037\end{array}$ & $\begin{array}{l}.0832 \\
.1006 \\
.1861 \\
.3674 \\
.0024 \\
.0116 \\
.0004 \\
.3173 \\
.1360 \\
.0363 \\
.0155 \\
.0001 \\
.0001 \\
.1702 \\
.2038 \\
.0739 \\
.4590 \\
.0214 \\
.0003 \\
.0600 \\
.0089 \\
.0188 \\
.4380 \\
.0014 \\
.0001\end{array}$ \\
\hline \multicolumn{12}{|c|}{ BETA } \\
\hline Technetium-99 & A & 46 & 9 & .0010 & .0011 & .0036 & .0447 & .7280 & .2997 & 1.000 & .0062 \\
\hline \multicolumn{12}{|c|}{ GAMMA } \\
\hline $\begin{array}{l}\text { Cesium-137 } \\
\text { Cesium-137 } \\
\text { Cesium-137 } \\
\text { Potassium-40 } \\
\text { Potass ium-40 } \\
\text { Potassium-40 } \\
\text { Uranium-235 } \\
\text { Uranium-235 } \\
\text { Uranium-235 }\end{array}$ & $\begin{array}{l}A \\
B \\
C \\
A \\
B \\
C \\
A \\
B \\
C\end{array}$ & $\begin{array}{l}40 \\
39 \\
39 \\
40 \\
36 \\
36 \\
39 \\
39 \\
40\end{array}$ & $\begin{array}{r}37 \\
16 \\
4 \\
40 \\
36 \\
36 \\
16 \\
15 \\
18\end{array}$ & $\begin{array}{l}.1313 \\
.0812 \\
.0277 \\
.0000 \\
.0000 \\
.0000 \\
.0970 \\
.7760 \\
.0208\end{array}$ & $\begin{array}{l}.1259 \\
.2877 \\
.0418 \\
.0060 \\
.3252 \\
.7842 \\
.2040 \\
.6458 \\
.0463\end{array}$ & $\begin{array}{l}.8531 \\
.1263 \\
1.000 \\
.0665 \\
.8216 \\
.0663 \\
1.000 \\
1.000 \\
.0596\end{array}$ & $\begin{array}{l}.8041 \\
.2715 \\
.0813 \\
.0000 \\
.0001 \\
.0000 \\
.8745 \\
.6640 \\
.0095\end{array}$ & $\begin{array}{l}.7671 \\
.1893 \\
1.000 \\
.0102 \\
.9845 \\
.0024 \\
.9998 \\
1.000 \\
1.000\end{array}$ & $\begin{array}{l}.8635 \\
.2404 \\
.0166 \\
.6614 \\
.5517 \\
.3416 \\
.5033 \\
.0825 \\
.6945\end{array}$ & $\begin{array}{l}.8176 \\
.2965 \\
1.000 \\
.1189 \\
.1099 \\
.1266 \\
1.000 \\
1.000 \\
.0285\end{array}$ & $\begin{array}{l}.3423 \\
.3303 \\
.1320 \\
.0000 \\
.0000 \\
.0001 \\
.5056 \\
.8961 \\
.0059\end{array}$ \\
\hline
\end{tabular}

\section{TRIIIUM}

Tritium $\begin{array}{llllll}A & 50 & 12 & .0009 & .2047 & .8898\end{array}$ $.0012 \quad .0008$ $1.000 \quad .9079$ .0117

${ }^{a} \mathrm{~N}=$ number of observations; $\mathrm{D}=$ number of detects. See "Note on Significance Levels" at beginning of this appendix for definitions of significance levels P-ALL through P-GRP. 
Table G.6. Comparisons of horizons for inorganics (Estimates and standard errors are in milligrams per kilogram.)

\begin{tabular}{cccc}
$\begin{array}{c}\text { Formation- } \\
\text { location }\end{array}$ & $\begin{array}{c}\text { Difference } \\
\text { estimate }\end{array}$ & $\begin{array}{c}\text { Standard } \\
\text { error }\end{array}$ & $\begin{array}{c}\text { Significance } \\
\text { level }\end{array}$ \\
\hline
\end{tabular}

Analysis=Aluminum Comparison: A-B Area-differences $\mathrm{P}=\mathbf{. 0 0 0 2}$

$\begin{array}{lrll}\text { DG-AND } & -12425.00 & 2071.01 & .0001 \\ \text { DG-ROA } & -8700.00 & 2071.01 & .0001 \\ \text { DG-ORR } & -9975.00 & 2071.01 & .0001 \\ \text { NL-ORR } & -12925.00 & 2071.01 & .0001 \\ \text { CHI-BV } & -13450.00 & 2071.01 & .0001 \\ \text { CHI-K25 } & -18150.00 & 2071.01 & .0001 \\ \text { CHE-ORR } & -10037.50 & 2071.01 & .0001 \\ \text { CR-ORR } & -6805.00 & 2071.01 & .0010 \\ \text { CR-AND } & -5675.00 & 2071.01 & .0061 \\ \text { CR-ROA } & -6248.75 & 2071.01 & .0026\end{array}$

Analysis=Aluminum Comparison: B-C Area-differences $P=.0355$

$\begin{array}{lrrr}\text { DG-AND } & -3450.00 & 1731.09 & .0463 \\ \text { DG-ROA } & -987.50 & 1731.09 & .5684 \\ \text { DG-ORR } & -7675.00 & 1731.09 & .0001 \\ \text { NL-ORR } & -3050.00 & 1731.09 & .0781 \\ \text { CHI-BV } & -3625.00 & 1731.09 & .0363 \\ \text { CHI-K25 } & 425.00 & 1731.09 & .8061 \\ \text { CHE-ORR } & 862.50 & 1731.09 & .6183 \\ \text { CR-ORR } & -950.00 & 1731.09 & .5832 \\ \text { CR-AND } & -2025.00 & 1731.09 & .2421 \\ \text { CR-ROA } & -1725.00 & 1731.09 & .3190\end{array}$

Analysis=Aluminum Comparison: A-C Area-differences $\mathbf{P}=\mathbf{. 0 0 0 1}$

$\begin{array}{lrrr}\text { DG-AND } & -15875.00 & 1995.80 & .0001 \\ \text { DG-ROA } & -9687.50 & 1995.80 & .0001 \\ \text { DG-ORR } & -17650.00 & 1995.80 & .0001 \\ \text { NL-ORR } & -15975.00 & 1995.80 & .0001 \\ \text { CHI-BV } & -17075.00 & 1995.80 & .0001 \\ \text { CHI-K25 } & -17725.00 & 1995.80 & .0001 \\ \text { CHE-ORR } & -9175.00 & 1995.80 & .0001 \\ \text { CR-ORR } & -7755.00 & 1995.80 & .0001 \\ \text { CR-AND } & -7700.00 & 1995.80 & .0001 \\ \text { CR-ROA } & -7973.75 & 1995.80 & .0001\end{array}$

Analysis=Arsenic Comparison: A-B Area-differences $\mathbf{P}=.0001$

$\begin{array}{lrrr}\text { DG-AND } & 0.30 & 1.97 & .8785 \\ \text { DG-ROA } & -1.34 & 1.96 & .4950 \\ \text { DG-ORR } & -3.05 & 1.96 & .1197 \\ \text { NL-ORR } & -0.30 & 2.26 & .8945 \\ \text { CHI-BV } & -1.08 & 1.96 & .5834 \\ \text { CHI-K25 } & 0.05 & 1.96 & .9796 \\ \text { CHE-ORR } & -9.28 & 1.96 & .0001 \\ \text { CR-ORR } & -14.23 & 2.26 & .0001 \\ \text { CR-AND } & -9.74 & 1.96 & .0001 \\ \text { CR-ROA } & -8.14 & 1.96 & .0001\end{array}$




\section{G-33}

Table G.6 (continued)

\begin{tabular}{|c|c|c|c|}
\hline $\begin{array}{l}\text { Formation- } \\
\text { location }\end{array}$ & $\begin{array}{c}\text { Differenice } \\
\text { estimate }\end{array}$ & $\begin{array}{l}\text { Standard } \\
\text { error }\end{array}$ & $\begin{array}{l}\text { Significance } \\
\text { level }\end{array}$ \\
\hline Analysis $=$ Arsenic & Compárison: B-C & \multicolumn{2}{|c|}{ Area-differences $\ddot{\mathrm{P}}=2523$} \\
\hline $\begin{array}{l}\text { DG-AND } \\
\text { DG-ROA } \\
\text { DG-ORR } \\
\text { NL-ORR } \\
\text { CHI-BV } \\
\text { CHI-K25 } \\
\text { CHE-ORR } \\
\text { CR-ORR } \\
\text { CR-AND } \\
\text { CR-ROA }\end{array}$ & $\begin{array}{r}0.26 \\
-0.30 \\
-4.56 \\
-1.87 \\
-0.28 \\
0.43 \\
-38.04 \\
-24.43 \\
-4.65 \\
-12.06\end{array}$ & $\begin{array}{l}11.23 \\
11.22 \\
11.22 \\
12.96 \\
11.22 \\
11.22 \\
11.22 \\
12.96 \\
11.22 \\
11.22\end{array}$ & $\begin{array}{l}.9814 \\
.9787 \\
.6843 \\
.8855 \\
.9805 \\
.9698 \\
.0007 \\
.0594 \\
.6786 \\
.2824\end{array}$ \\
\hline
\end{tabular}

Analysis $=$ Arsenic Comparison: A-C Area-differences $P=.0186$

$\begin{array}{lrrr}\text { DG-AND } & 0.57 & 11.15 & .9589 \\ \text { DG-ROA } & -1.64 & 11.15 & .8833 \\ \text { DG-ORR } & -7.61 & 11.15 & .4948 \\ \text { NL-ORR } & -2.17 & 12.88 & .8664 \\ \text { CHI-BV } & -1.35 & 11.15 & .9036 \\ \text { CHI-K25 } & 0.48 & 11.15 & .9660 \\ \text { CHE-ORR } & -47.31 & 11.15 & .0001 \\ \text { CR-ORR } & -38.67 & 12.88 & .0027 \\ \text { CR-AND } & -14.39 & 11.15 & .1970 \\ \text { CR-ROA } & -20.20 & 11.15 & .0701\end{array}$

Analysis $=$ Barium

DG-AND
DG-ROA
DG-ORR
ML-ORR
CHI-BV
CHI-K25
CHE-ORR
CR-ORR
CR-AND
CR-ROA

Analysis $=$ Barium

DG-AND
DG-ROA
DG-ORR
HL-ORR
CHI-BV
CHI-K25
CHE-ORR
CR-ORR
CR-AND
CR-ROA

Analysis=Barium

DG-AND
DG-ROA
DG-ORR
NL-ORR
CHI-BV
CHI-K25
CHE-ORR
CR-ORR
CR-AND
CR-ROA

Comparison: A-B Area-differences $\mathrm{P}=\mathbf{. 0 0 0 1}$

$\begin{array}{rrr}4.40 & 12.36 & .7218 \\ 27.30 & 12.36 & .0271 \\ 5.70 & 12.36 & .6446 \\ -9.83 & 12.36 & .4265 \\ -34.05 & 12.36 & .0059 \\ -13.23 & 12.36 & .2845 \\ 26.40 & 12.36 & .0326 \\ 31.80 & 12.36 & .0101 \\ 78.61 & 12.36 & .0001 \\ 26.85 & 12.36 & .0298\end{array}$

Comparison: B-C Area-differences $\mathbf{P}=\mathbf{. 0 0 0 1}$

$\begin{array}{rll}-8.35 & 9.24 & .3663 \\ -4.18 & 9.24 & .6515 \\ -12.65 & 9.24 & .1711 \\ 5.88 & 9.24 & .5250 \\ -30.90 & 9.24 & .0008 \\ 4.78 & 9.24 & .6054 \\ -9.23 & 9.24 & .3183 \\ 28.40 & 9.24 & .0021 \\ 19.98 & 9.24 & .0307 \\ 20.83 & 9.24 & .0243\end{array}$

Comparison: A-C Area-differences $\mathbf{P}=\mathbf{0 0 0 1}$

$\begin{array}{rrr}-3.95 & 13.06 & .7624 \\ 23.13 & 13.06 & .0767 \\ -6.95 & 13.06 & .5947 \\ -3.95 & 13.06 & .7624 \\ -64.95 & 13.06 & .0001 \\ -8.45 & 13.06 & .5178 \\ 17.18 & 13.06 & .1886 \\ 60.20 & 13.06 & .0001 \\ 98.59 & 13.06 & .0001 \\ 47.68 & 13.06 & .0003\end{array}$


Table G.6 (continued)

\begin{tabular}{cccc}
$\begin{array}{c}\text { Formation- } \\
\text { location }\end{array}$ & $\begin{array}{c}\text { Difference } \\
\text { estimate }\end{array}$ & $\begin{array}{c}\text { Standard } \\
\text { error }\end{array}$ & $\begin{array}{c}\text { Significance } \\
\text { level }\end{array}$ \\
\hline Analysis=Beryllium & Comparison: A-B & Area-differences $\mathbf{P}=\mathbf{0 0 0 1}$ \\
& & & \\
DG-AND & -0.14 & 0.09 & .1127 \\
DG-ROA & -0.02 & 0.09 & .8386 \\
DG-ORR & 0.08 & 0.09 & .3670 \\
NL-ORR & -0.23 & 0.09 & .0088 \\
CHI-BV & -0.45 & 0.09 & .0001 \\
CHI-K25 & -0.58 & 0.09 & .0001 \\
CHE-ORR & -0.17 & 0.12 & .1519 \\
CR-ORR & -0.03 & 0.10 & .7871 \\
CR-AND & 0.09 & 0.09 & .3016 \\
CR-ROA & 0.01 & 0.09 & .8689
\end{tabular}

Analysis=Beryllium Comparison: B-C Area-differences $\mathbf{P}=.0091$

$\begin{array}{llll}\text { DG-AND } & -0.22 & 0.09 & .0169 \\ \text { DG-ROA } & -0.20 & 0.09 & .0263 \\ \text { DG-ORR } & -0.27 & 0.09 & .0032 \\ \text { NL-ORR } & -0.16 & 0.09 & .0710 \\ \text { CHI-BV } & -0.48 & 0.09 & .0001 \\ \text { CHI-K25 } & 0.04 & 0.09 & .6368 \\ \text { CHE-ORR } & -0.04 & 0.13 & .7531 \\ \text { CR-ORR } & -0.23 & 0.10 & .0269 \\ \text { CR-AND } & -0.05 & 0.09 & .6171 \\ \text { CR-ROA } & -0.13 & 0.09 & .1419\end{array}$

Analysis=Beryllium Comparison: A-C Area-differences $\mathbf{P}=\mathbf{. 0 0 0 1}$

$\begin{array}{llll}\text { DG-AND } & -0.35 & 0.11 & .0009 \\ \text { DG-ROA } & -0.22 & 0.11 & .0401 \\ \text { DG-ORR } & -0.19 & 0.11 & .0768 \\ \text { NL-ORR } & -0.39 & 0.11 & .0003 \\ \text { CHI-BV } & -0.92 & 0.11 & .0001 \\ \text { CHI-K25 } & -0.54 & 0.11 & .0001 \\ \text { CHE-ORR } & -0.21 & 0.15 & .1523 \\ \text { CR-ORR } & -0.26 & 0.12 & .0357 \\ \text { CR-AND } & 0.04 & 0.11 & .6797 \\ \text { CR-ROA } & -0.12 & 0.11 & .2778\end{array}$

Analysis $=$ Boron

DG-AND

DG-ROA

DG-ORR

NL-ORR

CHI-BV

CHI-K25

CHE-ORR

CR-ORR

CR-AND

CR-ROA

Analysis $=$ Boron
Comparison: A-B Area-differences $\mathrm{P}=.0002$

$\begin{array}{rll}0.21 & 6.17 & .9726 \\ 12.55 & 2.30 & .0001 \\ -5.30 & 2.67 & .0469 \\ -0.21 & 5.84 & .9718 \\ 0.00 & 3.78 & 1.000 \\ 0.13 & 2.83 & .9623 \\ -1.47 & 2.58 & .5685 \\ -0.05 & 2.79 & .9856 \\ 0.19 & 2.77 & .9459 \\ 0.03 & 5.46 & .9963\end{array}$

Comparison: B-C Area-differences $\mathrm{P}=\mathbf{6 9 5 8}$

$\begin{array}{rll}-0.11 & 5.30 & .9831 \\ -7.08 & 2.59 & .0063 \\ -3.92 & 2.53 & .1222 \\ 0.06 & 5.29 & .9902 \\ -0.03 & 4.15 & .9952 \\ 0.06 & 3.00 & .9836 \\ -1.01 & 2.80 & .7185 \\ -0.09 & 3.05 & .9777 \\ -0.04 & 2.97 & .9901 \\ -0.18 & 6.01 & .9768\end{array}$




\section{G-35}

Table G.6 (continued)

\begin{tabular}{cccc}
\hline $\begin{array}{c}\text { Formation- } \\
\text { location }\end{array}$ & $\begin{array}{c}\text { Difference } \\
\text { estimate }\end{array}$ & $\begin{array}{c}\text { Standard } \\
\text { error }\end{array}$ & $\begin{array}{c}\text { Significance } \\
\text { level }\end{array}$ \\
\hline Analysis=Boron & Comparison: A-C & Area-differences P=.0024 \\
& & & \\
DG-AND & 0.10 & 6.26 & .9872 \\
DG-ROA & 5.13 & 2.22 & .0209 \\
DG-ORR & -12.25 & 2.72 & .0001 \\
NL-ORR & -0.14 & 5.70 & .9804 \\
CHI-BV & -0.03 & 3.81 & .9947 \\
CHI-K25 & 0.20 & 2.82 & .9447 \\
CHE-ORR & -2.36 & 2.53 & .3507 \\
CR-ORR & -0.14 & 2.83 & .9619 \\
CR-AND & 0.15 & 2.79 & .9568 \\
CR-ROA & -0.15 & 5.58 & .9786
\end{tabular}

Analysis $=$ Calcium Comparison: A-B Area-differences $P=.0001$

$\begin{array}{lrrr}\text { DG-AND } & 644.50 & 151.53 & .0001 \\ \text { DG-ROA } & 1226.00 & 303.06 & .0001 \\ \text { DG-ORR } & 355.00 & 303.06 & .2414 \\ \text { NL-ORR } & -140.00 & 214.29 & .5136 \\ \text { CHI-BV } & -308.75 & 151.53 & .0416 \\ \text { CHI-K25 } & 213.13 & 151.53 & .1596 \\ \text { CHE-ORR } & -67.13 & 151.53 & .6578 \\ \text { CR-ORR } & 185.00 & 151.53 & .2221 \\ \text { CR-AND } & 524.13 & 151.53 & .0005 \\ \text { CR-ROA } & 278.75 & 151.53 & .0658\end{array}$

Analysis $=$ Calcium

DG-AND

DG-ROA

DG-ORR

NL-ORR

CHI-BV

CHI -K25

CHE-ORR

CR-ORR

CR-AND

CR-ROA

Analysis $=$ Calcium

DG-AND

DG-ROA

DG-ORR

NL-ORR

CHI -BV

CHI-K25

CHE-ORR

CR-ORR

CR-AND

CR-ROA

Comparison: B-C

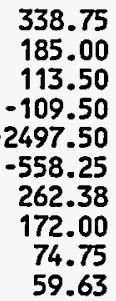

275.12

550.24

389.08

389.08

275.12

275.12

275.12

317.68

275.12

275.12

Comparison: A-C

$\begin{array}{rrr}983.25 & 255.59 & .0001 \\ 1411.00 & 511.17 & . .0058 \\ 389.33 & 295.13 & .1871 \\ -368.00 & 511.17 & .4716 \\ -2806.25 & 255.59 & .0001 \\ -345.13 & 255.59 & .1769 \\ 195.25 & 255.59 & .4449 \\ 328.67 & 295.13 & .2654 \\ 598.88 & 255.59 & .0191 \\ 338.38 & 255.59 & .1855\end{array}$

Analysis=Chromium

Comparison: A-B

Area-differences $\mathrm{P}=\mathbf{0 0 1 7}$

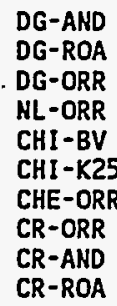

$$
\begin{array}{r}
-7.05 \\
-11.81 \\
-12.41 \\
-9.60 \\
0.11 \\
-2.49 \\
-14.58 \\
-13.45 \\
-11.51 \\
-12.25
\end{array}
$$

2.84

2.84

2.84

3.28

2.84

2.84

2.86

2.84

2.84
2.84

.2182

.7367

.7705

.7784

.0001

.0424

.3402

.588 ?

.8284

CR-ROA

$-12.25$

2.84

$$
\begin{aligned}
& .0132 \\
& .0001 \\
& .0001 \\
& .0035 \\
& .9684 \\
& .3816 \\
& .0001 \\
& .0001 \\
& .0001 \\
& .0001
\end{aligned}
$$




\section{G-36}

Table G.6 (continued)

\begin{tabular}{cccc}
\hline $\begin{array}{c}\text { Formation- } \\
\text { location }\end{array}$ & $\begin{array}{c}\text { Difference } \\
\text { estimate }\end{array}$ & $\begin{array}{c}\text { Standard } \\
\text { error }\end{array}$ & $\begin{array}{c}\text { Significance } \\
\text { level }\end{array}$ \\
\hline
\end{tabular}

Analysis $=$ Chromium Comparison: B-C Area-differences $\mathbf{P}=\mathbf{. 0 0 0 1}$

$\begin{array}{lrrr}\text { DG-AND } & -3.06 & 4.12 & .4573 \\ \text { DG-ROA } & 0.26 & 4.12 & .9492 \\ \text { DG-ORR } & -8.99 & 4.12 & .0292 \\ \text { NL-ORR } & -26.07 & 4.76 & .0001 \\ \text { CHI-BV } & 1.48 & 4.12 & .7204 \\ \text { CHI-K25 } & 5.68 & 4.12 & .1684 \\ \text { CHE-ORR } & 2.63 & 4.12 & .5241 \\ \text { CR-ORR } & 0.28 & 4.12 & .9468 \\ \text { CR-AND } & -3.20 & 4.12 & .4374 \\ \text { CR-ROA } & -11.01 & 4.12 & .0075\end{array}$

Analysis=Chromium Comparison: A-C Area-differences $\mathbf{P}=\mathbf{. 0 0 0 1}$

$\begin{array}{lrrr}\text { DG-AND } & -10.11 & 4.35 & .0200 \\ \text { DG-ROA } & -11.55 & 4.35 & .0079 \\ \text { DG-ORR } & -21.40 & 4.35 & .0001 \\ \text { NL-ORR } & -35.67 & 5.02 & .0001 \\ \text { CHI-BV } & 1.59 & 4.35 & .7149 \\ \text { CHI-K25 } & 3.19 & 4.35 & .4633 \\ \text { CHE-ORR } & -12.00 & 4.36 & .0059 \\ \text { CR-ORR } & -13.18 & 4.35 & .0024 \\ \text { CR-AND } & -14.71 & 4.35 & .0007 \\ \text { CR-ROA } & -23.26 & 4.35 & .0001\end{array}$

Analysis=Cobalt Comparison: A-B Area-differences $P=.1829$

$\begin{array}{lrrr}\text { DG-AND } & -0.86 & 3.73 & .8171 \\ \text { DG-ROA } & 12.30 & 3.73 & .0010 \\ \text { DG-ORR } & 6.26 & 3.73 & .0931 \\ \text { NL-ORR } & -2.55 & 3.73 & .4942 \\ \text { CHI-BV } & 5.01 & 3.73 & .1790 \\ \text { CHI-K25 } & 3.65 & 3.73 & .3278 \\ \text { CHE-ORR } & 8.92 & 3.76 & .0178 \\ \text { CR-ORR } & 7.02 & 3.75 & .0615 \\ \text { CR-AND } & 6.33 & 3.73 & .0899 \\ \text { CR-ROA } & 2.46 & 3.81 & .5187\end{array}$

Analysis=Cobalt Comparison: B-C Area-differences $\mathbf{P}=.1959$

$\begin{array}{lrrr}\text { DG-AND } & -1.61 & 4.79 & .7361 \\ \text { DG-ROA } & 0.11 & 4.79 & .9812 \\ \text { DG-ORR } & -3.70 & 4.79 & .4394 \\ \text { NL-ORR } & 0.83 & 4.79 & .8631 \\ \text { CHI-BV } & -11.43 & 4.79 & .0170 \\ \text { CHI-K25 } & -1.80 & 4.79 & .7068 \\ \text { CHE-ORR } & -14.91 & 4.81 & .0019 \\ \text { CR-ORR } & -0.07 & 4.84 & .9892 \\ \text { CR-AND } & 1.20 & 4.79 & .8020 \\ \text { CR-ROA } & 1.11 & 4.86 & .8189\end{array}$

Analysis $=$ Cobalt

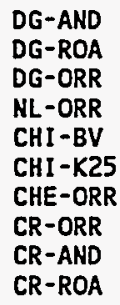

Comparison: A-C

$$
\begin{array}{r}
-2.48 \\
12.41 \\
2.56 \\
-1.73 \\
-6.41 \\
1.85 \\
-5.92 \\
7.02 \\
7.53 \\
3.59
\end{array}
$$




\section{G-37}

Table G.6 (continued)

\begin{tabular}{|c|c|c|c|}
\hline $\begin{array}{l}\text { Formation- } \\
\text { location }\end{array}$ & $\begin{array}{l}\text { Difference } \\
\text { estimate }\end{array}$ & $\begin{array}{l}\text { Standard } \\
\text { error }\end{array}$ & $\begin{array}{l}\text { Significance } \\
\text { level }\end{array}$ \\
\hline Analysis $=C o \dot{p} p \dot{e}$ & Comparison: A-B & \multicolumn{2}{|c|}{ Area-differences $\mathrm{P}=\mathbf{0 0 0 1}$} \\
\hline $\begin{array}{l}\text { DG-AND } \\
\text { DG-ROA } \\
\text { DG-ORR } \\
\text { NL-ORR } \\
\text { CHI-BV } \\
\text { CHI-K25 } \\
\text { CHE-ORR } \\
\text { CR-ORR } \\
\text { CR-AND } \\
\text { CR-ROA }\end{array}$ & $\begin{array}{r}-4.09 \\
-3.35 \\
-4.37 \\
-7.93 \\
-6.99 \\
-6.05 \\
-13.36 \\
-12.69 \\
-14.33 \\
-6.39\end{array}$ & $\begin{array}{l}2.05 \\
2.05 \\
2.37 \\
2.05 \\
2.05 \\
2.05 \\
2.12 \\
2.10 \\
2.05 \\
2.13\end{array}$ & $\begin{array}{l}.0465 \\
.1028 \\
.0656 \\
.0001 \\
.0007 \\
.0032 \\
.0001 \\
.0001 \\
.0001 \\
.0027\end{array}$ \\
\hline Analysis=Copper & Comparison: B-C & \multicolumn{2}{|c|}{ Area-differences $\mathrm{P}=\mathbf{3 2 5 9}$} \\
\hline $\begin{array}{l}\text { DG-AND } \\
\text { DG-ROA } \\
\text { DG-ORR } \\
\text { NL-ORR } \\
\text { CHI-BV } \\
\text { CHI-K25 } \\
\text { CHE-ORR } \\
\text { CR-ORR } \\
\text { CR-AND } \\
\text { CR-ROA }\end{array}$ & $\begin{array}{r}-9.89 \\
-10.45 \\
-6.25 \\
-5.68 \\
-5.45 \\
-1.15 \\
-5.01 \\
-11.53 \\
-8.40 \\
-4.95\end{array}$ & $\begin{array}{l}2.93 \\
2.93 \\
3.39 \\
2.93 \\
2.93 \\
2.93 \\
3.26 \\
2.93 \\
2.93 \\
2.96\end{array}$ & $\begin{array}{l}.0008 \\
.0004 \\
.0651 \\
.0531 \\
.0632 \\
.0951 \\
.1242 \\
.0001 \\
.0042 \\
.0941\end{array}$ \\
\hline
\end{tabular}

Analysis=Copper Comparison: A-C Area-differences $\mathbf{P}=\mathbf{0 1 0 0}$

$\begin{array}{llll}\text { DG-AND } & -13.98 & 3.43 & .0001 \\ \text { DG-ROA } & -13.80 & 3.43 & .0001 \\ \text { DG-ORR } & -12.64 & 3.43 & .0002 \\ \text { NL-ORR } & -13.60 & 3.43 & .0001 \\ \text { CHI-BV } & -12.44 & 3.43 & .0003 \\ \text { CHI-K25 } & -7.20 & 3.43 & .0358 \\ \text { CHE-ORR } & -18.46 & 3.86 & .0001 \\ \text { CR-ORR } & -24.91 & 3.51 & .0001 \\ \text { CR-AND } & -22.73 & 3.43 & .0001 \\ \text { CR-ROA } & -11.14 & 3.44 & .0012\end{array}$

Analysis=Cyanide

DG-AHD DG-ROA DG-ORR

HL-ORR

Analysis $=$ Cyanide

DG-AND

DG-ROA

DG-ORR

NL-ORR

Analysis $=$ Cyanide

DG-AND

DG-ROA

DG-ORR

NL-ORR
Comparison: A-B Area-differences $P=.1157$

$\begin{array}{rrr}0.06 & 0.05 & .2940 \\ 0.29 & 0.06 & .0001 \\ 0.01 & 0.06 & .8330 \\ -0.00 & 0.06 & .9833\end{array}$

Comparison: B-C Area-differences $\mathrm{P}=.7807$

$\begin{array}{rrr}0.07 & 0.04 & .0895 \\ 0.00 & 0.08 & .9770 \\ -0.06 & 0.03 & .0894 \\ -0.00 & 0.06 & 1.000\end{array}$

Comparison: A-C Area-differences $P=.0071$

$\begin{array}{rrr}0.11 & 0.05 & .0244 \\ 0.30 & 0.06 & .0001 \\ -0.06 & 0.05 & .2668 \\ -0.00 & 0.06 & .9824\end{array}$




\section{G-38}

Table G.6 (continued)

\begin{tabular}{ccccc}
\hline $\begin{array}{c}\text { Formation- } \\
\text { location }\end{array}$ & $\begin{array}{c}\text { Difference } \\
\text { estimate }\end{array}$ & $\begin{array}{c}\text { Standard } \\
\text { error }\end{array}$ & $\begin{array}{c}\text { Sign } \\
\text { lent }\end{array}$ \\
\hline Analysis=Iron & Comparison: A-B & Area-differences \\
& & & & \\
DG-AND & -13650.00 & 2787.30 & .0001 \\
DG-ROA & -7762.50 & 2787.30 & .0054 \\
DG-ORR & -7575.00 & 2787.30 & .0066 \\
NL-ORR & -14825.00 & 2787.30 & .0001 \\
CHI-BV & -12925.00 & 2787.30 & .0001 \\
CHI-K25 & -23475.00 & 2787.30 & .0001 \\
CHE-ORR & -18687.50 & 2787.30 & .0001 \\
CR-ORR & -21025.00 & 2787.30 & .0001 \\
CR-AND & -13987.50 & 2787.30 & .0001 \\
CR-ROA & -12096.25 & 2787.30 & .0001
\end{tabular}

Analysis $=$ Iron

DG-AND
DG-ROA
DG-ORR
$N L-O R R$
CHI-BV
CHI-K25
CHE-ORR
CR-ORR
CR-AND
CR-ROA

Analysis $=$ Iron

DG-AND

DG-ROA

$D G-O R R$

NL-ORR

CHI - BV

CHI $-K 25$

CHE-ORR

CR-ORR

CR-AND

CR-ROA

Analysis $=$ Lead

DG-AND

$D G-R O A$

DG-ORR

NL-ORR

CHI-BV

CHI -K25

CHE-ORR

CR-ORR

CR-AND

CR-ROA

Analysis $=$ Lead

DG-AND

DG-ROA

DG-ORR

NL-ORR

CHI-BV

$\mathrm{CHI}-\mathrm{K} 25$

CHE-ORR

CR-ORR

CR-AND

CR-ROA
Comparison: B-C Area-differences $\mathrm{P}=.0001$

$\begin{array}{rrr}-3325.00 & 2385.76 & .1634 \\ -5500.00 & 2385.76 & .0211 \\ -5787.50 & 2385.76 & .0153 \\ 1100.00 & 2385.76 & .6447 \\ -3700.00 & 2385.76 & .1209 \\ 1325.00 & 2385.76 & .5786 \\ -962.50 & 2385.76 & .6866 \\ -8825.00 & 2385.76 & .0002 \\ -4450.00 & 2385.76 & .0621 \\ -15087.50 & 2385.76 & .0001\end{array}$

Comparison: A-C Area-differences $\mathbf{P}=.0002$

$\begin{array}{lll}-16975.00 & 3050.83 & .0001 \\ -13262.50 & 3050.83 & .0001 \\ -13362.50 & 3050.83 & .0001 \\ -13725.00 & 3050.83 & .0001 \\ -16625.00 & 3050.83 & .0001 \\ -22150.00 & 3050.83 & .0001 \\ -19650.00 & 3050.83 & .0001 \\ -29850.00 & 3050.83 & .0001 \\ -18437.50 & 3050.83 & .0001 \\ -27183.75 & 3050.83 & .0001\end{array}$

Comparison: A-B Area-differences $P=.4906$

$\begin{array}{rrr}9.81 & 10.37 & .3441 \\ 13.14 & 10.37 & .2052 \\ 9.44 & 10.37 & .3628 \\ 5.20 & 11.97 & .6641 \\ 16.57 & 11.97 & .1665 \\ 14.98 & 10.37 & .1487 \\ 7.65 & 10.37 & .4607 \\ 40.20 & 10.37 & .0001 \\ 6.69 & 10.37 & .5190 \\ 8.69 & 10.37 & .4022\end{array}$

Comparison: B-C Area-differences $P=.0118$

$\begin{array}{rrr}-2.32 & 5.99 & .6987 \\ -5.43 & 5.18 & .2953 \\ -3.40 & 5.18 & .5119 \\ -23.87 & 5.99 & .0001 \\ -16.80 & 5.18 & .0012 \\ 0.40 & 5.18 & .9385 \\ -15.45 & 5.18 & .0029 \\ -15.10 & 5.18 & .0036 \\ 0.43 & 5.18 & .9347 \\ -5.59 & 5.18 & .2810\end{array}$




\section{G-39}

Table G.6 (continued)

\begin{tabular}{cccc}
\hline $\begin{array}{c}\text { Formation- } \\
\text { location }\end{array}$ & $\begin{array}{c}\text { Difference } \\
\text { estimate }\end{array}$ & $\begin{array}{c}\text { Standard } \\
\text { error }\end{array}$ & $\begin{array}{c}\text { Significance } \\
\text { level }\end{array}$ \\
\hline Analysis=Lead ${ }^{*}$ & Comparison: A-C & Area-differences $\mathbf{P}=5296$ \\
DG-AND & 4.23 & 14.02 & .7626 \\
DG-ROA & 7.71 & 12.14 & .5252 \\
DG-ORR & 6.04 & 12.14 & .6189 \\
NL-ORR & -18.67 & 14.02 & .1830 \\
CHI-BV & -5.00 & 14.02 & .7213 \\
CHI-K25 & 15.38 & 12.14 & .2053 \\
CHE-ORR & -7.80 & 12.14 & .5205 \\
CR-ORR & 25.10 & 12.14 & .0387 \\
CR-AND & 7.11 & 12.14 & .5579 \\
CR-ROA & 3.10 & 12.14 & .7984
\end{tabular}

Analysis=Lithium Comparison: A-B Area-differences $\mathbf{P}=.0001$

$\begin{array}{lrrr}\text { DG-AND } & -8.96 & 1.83 & .0001 \\ \text { DG-ROA } & -9.00 & 2.08 & .0001 \\ \text { DG-ORR } & -5.10 & 2.12 & .0160 \\ \text { NL-ORR } & -12.78 & 1.83 & .0001 \\ \text { CHI-BV } & -17.65 & 2.59 & .0001 \\ \text { CHI-K25 } & -18.81 & 1.83 & .0001 \\ \text { CHE-ORR } & -8.26 & 1.94 & .0001 \\ \text { CR-ORR } & -4.35 & 1.86 & .0197 \\ \text { CR-AND } & -5.31 & 1.83 & .0038 \\ \text { CR-ROA } & -6.20 & 2.12 & .0034\end{array}$

Analysis=Lithium Comparison: B-C Area-differences $\mathbf{P}=\mathbf{0 6 3 3}$

$\begin{array}{lrrr}\text { DG-AND } & -1.90 & 2.08 & .3603 \\ \text { DG-ROA } & -5.11 & 2.08 & .0138 \\ \text { DG-ORR } & -5.24 & 2.08 & .0117 \\ \text { NL-ORR } & 0.18 & 2.08 & .9329 \\ \text { CHI-BV } & -8.25 & 2.94 & .0050 \\ \text { CHI-K25 } & -4.38 & 2.08 & .0352 \\ \text { CHE-ORR } & -2.10 & 2.45 & .3912 \\ \text { CR-ORR } & 2.44 & 2.08 & .2410 \\ \text { CR-AND } & -0.65 & 2.08 & .7543 \\ \text { CR-ROA } & -2.07 & 2.40 & .3889\end{array}$

Analysis=Lithium Comparison: A-C Area-differences $\mathbf{P}=\mathbf{. 0 0 0 1}$

$\begin{array}{llll}\text { DG-AND } & -10.86 & 2.71 & .0001 \\ \text { DG-ROA } & -14.43 & 2.96 & .0001 \\ \text { DG-ORR } & -11.55 & 3.13 & .0002 \\ \text { HL-ORR } & -12.60 & 2.71 & .0001 \\ \text { CHI-BV } & -25.90 & 3.83 & .0001 \\ \text { CHI-K25 } & -23.19 & 2.71 & .0001 \\ \text { CHE-ORR } & -8.95 & 2.92 & .0021 \\ \text { CR-ORR } & -1.87 & 2.73 & .4948 \\ \text { CR-AND } & -5.96 & 2.71 & .0276 \\ \text { CR-ROA } & -8.27 & 3.13 & .0082\end{array}$

Analysis=Magnesium Comparison: A-B Area-differences P=.0056

$\begin{array}{lrll}\text { DG-AND } & -176.25 & 244.55 & .4711 \\ \text { DG-ROA } & -407.50 & 244.55 & .0957 \\ \text { DG-ORR } & -198.75 & 244.55 & .4164 \\ \text { NL-ORR } & -725.00 & 244.55 & .0030 \\ \text { CHI-BV } & -937.50 & 244.55 & .0001 \\ \text { CHI-K25 } & -1266.13 & 244.55 & .0001 \\ \text { CHE-ORR } & -457.81 & 244.55 & .0612 \\ \text { CR-ORR } & -121.25 & 244.55 & .6200 \\ \text { CR-AND } & -168.13 & 244.55 & .4918 \\ \text { CR-ROA } & -154.38 & 244.55 & .5279\end{array}$


Table G.6 (continued)

\begin{tabular}{cccc}
$\begin{array}{c}\text { Formation- } \\
\text { location }\end{array}$ & $\begin{array}{c}\text { Difference } \\
\text { estimate }\end{array}$ & $\begin{array}{c}\text { Standard } \\
\text { error }\end{array}$ & $\begin{array}{c}\text { Significance } \\
\text { level }\end{array}$ \\
\hline
\end{tabular}

Anatysis=Magnesium Comparison: B-C Area-differences $\mathbf{P}=.0001$

$\begin{array}{lrll}\text { DG-AND } & -680.00 & 150.62 & .0001 \\ \text { DG-ROA } & -1081.25 & 150.62 & .0001 \\ \text { DG-ORR } & -1130.00 & 150.62 & .0001 \\ \text { NL-ORR } & -655.00 & 150.62 & .0001 \\ \text { CHI-BV } & -932.50 & 150.62 & .0001 \\ \text { CHI-K25 } & 25.00 & 150.62 & .8682 \\ \text { CHE-ORR } & 84.75 & 150.62 & .5737 \\ \text { CR-ORR } & 111.50 & 150.62 & .4591 \\ \text { CR-AND } & 6.00 & 150.62 & .9682 \\ \text { CR-ROA } & 98.75 & 150.62 & .5121\end{array}$

Analysis=Magnesium Comparison: A-C Area-differences $\mathrm{P}=\mathbf{. 0 0 0 1}$

$\begin{array}{lrrr}\text { DG-AND } & -856.25 & 269.44 & .0015 \\ \text { DG-ROA } & -1488.75 & 269.44 & .0001 \\ \text { DG-ORR } & -1328.75 & 269.44 & .0001 \\ \text { NL-ORR } & -1380.00 & 269.44 & .0001 \\ \text { CHI-BV } & -1870.00 & 269.44 & .0001 \\ \text { CHI-K25 } & -1241.13 & 269.44 & .0001 \\ \text { CHE-ORR } & -373.06 & 269.44 & .1662 \\ \text { CR-ORR } & -9.75 & 269.44 & .9711 \\ \text { CR-AND } & -162.13 & 269.44 & .5474 \\ \text { CR-ROA } & -55.63 & 269.44 & .8364\end{array}$

Analysis=Manganese Comparison: A-B Area-differences $P=.0011$

$\begin{array}{lrll}\text { DG-AND } & 389.00 & 246.03 & .1138 \\ \text { DG-ROA } & 1526.88 & 246.03 & .0001 \\ \text { DG-ORR } & 760.50 & 246.03 & .0020 \\ \text { NL-ORR } & 344.25 & 246.03 & .1617 \\ \text { CHI-BV } & 703.25 & 246.03 & .0043 \\ \text { CHI-K25 } & 1333.75 & 246.03 & .0001 \\ \text { CHE-ORR } & 974.33 & 246.03 & .0001 \\ \text { CR-ORR } & 923.85 & 246.03 & .0002 \\ \text { CR-AND } & 1581.33 & 246.03 & .0001 \\ \text { CR-ROA } & 805.14 & 246.03 & .0011\end{array}$

Analysis=Manganese Comparison: B-C Area-differences $\mathrm{P}=.4209$

$\begin{array}{lrrr}\text { DG-AND } & -275.63 & 227.61 & .2259 \\ \text { DG-ROA } & 64.18 & 227.61 & .7780 \\ \text { DG-ORR } & -165.38 & 227.61 & .4675 \\ \text { NL-ORR } & -38.25 & 227.61 & .8665 \\ \text { CHI-BV } & -286.75 & 227.61 & .2077 \\ \text { CHI-K25 } & -356.00 & 227.61 & .1178 \\ \text { CHE-ORR } & -501.80 & 227.61 & .0275 \\ \text { CR-ORR } & 76.35 & 227.61 & .7373 \\ \text { CR-AND } & 203.85 & 227.61 & .3705 \\ \text { CR-ROA } & 69.66 & 227.61 & .7596\end{array}$

Analysis = Manganese Comparison: A-C Area-differences $\mathbf{P}=\mathbf{0 0 0 1}$

$\begin{array}{lrll}\text { DG-AND } & 113.38 & 244.80 & .6433 \\ \text { DG-ROA } & 1591.05 & 244.80 & .0001 \\ \text { DG-ORR } & 595.13 & 244.80 & .0151 \\ \text { NL-ORR } & 306.00 & 244.80 & .2113 \\ \text { CHI-BV } & 416.50 & 244.80 & .0889 \\ \text { CHI-K25 } & 977.75 & 244.80 & .0001 \\ \text { CHE-ORR } & 472.53 & 244.80 & .0536 \\ \text { CR-ORR } & 1000.20 & 244.80 & .0001 \\ \text { CR-AND } & 1785.18 & 244.80 & .0001 \\ \text { CR-ROA } & 874.80 & 244.80 & .0004\end{array}$


G-41

Table G.6 (continued)

\begin{tabular}{cccc}
\hline $\begin{array}{c}\text { Formation- } \\
\text { location }\end{array}$ & $\begin{array}{c}\text { Difference } \\
\text { estimate }\end{array}$ & $\begin{array}{c}\text { Standard } \\
\text { error }\end{array}$ & $\begin{array}{c}\text { Significance } \\
\text { level }\end{array}$ \\
\hline Analysis=Mercury & Comparison- A-B & Arca-differences P=.0001 \\
DG-AND & 0.03 & 0.05 & .5308 \\
DG-ROA & 0.04 & 0.04 & .3203 \\
DG-ORR & 0.18 & 0.03 & .0001 \\
NL-ORR & 0.13 & 0.04 & .0004 \\
CHI-BV & 0.11 & 0.04 & .0016 \\
CHI-K25 & 0.42 & 0.03 & .0001 \\
CHE-ORR & 0.03 & 0.03 & .4657 \\
CR-ORR & 0.05 & 0.03 & .1079 \\
CR-AND & -0.02 & 0.03 & .4277 \\
CR-ROA & -0.04 & 0.03 & .2669.
\end{tabular}

Analysis=Mercury Comparison: B-C Area-differences $P=.0001$

$\begin{array}{lrrr}\text { DG-AND } & -0.00 & 0.09 & .9733 \\ \text { DG-ROA } & 0.01 & 0.03 & .6606 \\ \text { DG-ORR } & 0.10 & 0.03 & .0010 \\ \text { NL-ORR } & 0.00 & 0.09 & . .9735 \\ \text { CHI-BV } & -0.05 & 0.03 & .1598 \\ \text { CHI-K25 } & -0.02 & 0.02 & .2686 \\ \text { CHE-ORR } & -0.07 & 0.02 & .0017 \\ \text { CR-ORR } & -0.15 & 0.02 & . .0001 \\ \text { CR-AND } & -0.05 & 0.02 & .0226 \\ \text { CR-ROA } & -0.09 & 0.02 & .0001\end{array}$

Analysis=Mercury Comparison: A-C Area-differences $P=.0001$

$\begin{array}{llll}\text { DG-AND } & 0.02 & 0.05 & .7026 \\ \text { DG-ROA } & 0.05 & 0.05 & .3577 \\ \text { DG-ORR } & 0.24 & 0.05 & .0001 \\ \text { NL-ORR } & 0.13 & 0.04 & .0024 \\ \text { CHI-BV } & 0.08 & 0.04 & .0557 \\ \text { CHI-K25 } & 0.39 & 0.04 & .0001 \\ \text { CHE-ORR } & -0.05 & 0.04 & .2444 \\ \text { CR-ORR } & -0.10 & 0.04 & .0112 \\ \text { CR-AND } & -0.07 & 0.04 & .0688 \\ \text { CR-ROA } & -0.12 & 0.04 & .0018\end{array}$

Analysis $=$ Molybdenum Comparison: A-B Area-differences $P=.0360$

$\begin{array}{lccc}\text { DG-AND } & -0.40 & 0.44 & .3658 \\ \text { DG-ROA } & -5.67 & . & . \\ \text { DG-ORR } & -6.09 & . & . \\ \text { NL-ORR } & -0.94 & 0.46 & .0421 \\ \text { CHI-BV } & -1.44 & 1.70 & .3960 \\ \text { CHI-K25 } & -1.59 & 1.45 & .2712 \\ \text { CHE-ORR } & -1.39 & 1.91 & .4660 \\ \text { CR-ORR } & -2.17 & 0.25 & .0001 \\ \text { CR-AND } & -2.11 & 0.38 & .0001 \\ \text { CR-ROA } & -0.94 & 0.54 & .0859\end{array}$

Analysis $=$ Molybdenum Comparison: B-C Area-differences $\mathrm{P}=.0077$

$\begin{array}{lrcl}\text { DG-AND } & -0.18 & 0.39 & .6405 \\ \text { DG-ROA } & -4.62 & . & . \\ \text { DG-ORR } & -5.16 & . & - \\ \text { HL-ORR } & 0.75 & 0.56 & .1829 \\ \text { CHI-BV } & 1.41 & 1.10 & .2012 \\ \text { CHI-K25 } & 1.51 & 0.94 & .1068 \\ \text { CHE-ORR } & -0.01 & 0.57 & .9855 \\ \text { CR-ORR } & -0.66 & 0.30 & .0290 \\ \text { CR-AHD } & 0.26 & 0.38 & .4948 \\ \text { CR-ROA } & -1.68 & 0.45 & .0002\end{array}$




\section{G-42}

Table G.6 (continued)

\begin{tabular}{cccc}
\hline $\begin{array}{c}\text { Formation- } \\
\text { location }\end{array}$ & $\begin{array}{c}\text { Difference } \\
\text { estimate }\end{array}$ & $\begin{array}{c}\text { Standard } \\
\text { error }\end{array}$ & $\begin{array}{c}\text { Significance } \\
\text { level }\end{array}$ \\
\hline
\end{tabular}

Analysis=Molybdenum Comparison: A-C Area-differences $\mathbf{P}=\mathbf{0 2 7 6}$

\begin{tabular}{lrrl} 
DG-AND & -0.52 & 0.44 & .2336 \\
DG-ROA & -4.93 & \multicolumn{1}{c}{} \\
DG-ORR & -5.52 & \multicolumn{1}{c}{$\cdot$} & \multicolumn{1}{c}{} \\
NL-ORR & -0.05 & 1.71 & .9767 \\
CHI-BV & -0.00 & 71.12 & 1.000 \\
CHI-K25 & 0.02 & 86.72 & .9998 \\
CHE-ORR & -1.33 & 1.18 & .2574 \\
CR-ORR & -2.71 & 0.30 & .0001 \\
CR-AND & -1.78 & 0.69 & .0096 \\
CR-ROA & -2.12 & 0.42 & .0001
\end{tabular}

Analysis $=$ Nickel Comparison: A-B Area-differences $\mathrm{P}=\mathbf{. 0 1 7 1}$

$\begin{array}{lrrr}\text { DG-AND } & -3.43 & 1.99 & .0852 \\ \text { DG-ROA } & -1.13 & 1.99 & .5718 \\ \text { DG-ORR } & 1.95 & 1.99 & .3270 \\ \text { NL-ORR } & -3.60 & 1.99 & .0704 \\ \text { CHI-BV } & -9.13 & 1.99 & .0001 \\ \text { CHI-K25 } & -3.98 & 1.99 & .0457 \\ \text { CHE-ORR } & -6.38 & 2.92 & .0287 \\ \text { CR-ORR } & -4.08 & 2.05 & .0473 \\ \text { CR-AND } & -6.50 & 2.02 & .0013 \\ \text { CR-ROA } & -4.84 & 2.24 & .0306\end{array}$

Analysis=Nickel Comparison: B-C Area-differences $P=.0001$

$\begin{array}{lrll}\text { DG-AND } & -5.04 & 1.83 & .0059 \\ \text { DG-ROA } & -9.14 & 1.83 & .0001 \\ \text { DG-ORR } & -6.09 & 1.83 & .0009 \\ \text { NL-ORR } & -3.75 & 1.83 & .0405 \\ \text { CHI-BV } & -9.33 & 1.83 & .0001 \\ \text { CHI-K25 } & -1.18 & 1.83 & .5209 \\ \text { CHE-ORR } & -17.40 & 2.08 & .0001 \\ \text { CR-ORR } & -4.05 & 1.83 & .0269 \\ \text { CR-AND } & -2.43 & 1.83 & .1852 \\ \text { CR-ROA } & -2.94 & 2.04 & .1502\end{array}$

Analysis=Nickel Comparison: A-C Area-differences $\mathrm{P}=\mathbf{0 0 1 8}$

$\begin{array}{lrrr}\text { DG-AND } & -8.46 & 2.93 & .0038 \\ \text { DG-ROA } & -10.26 & 2.93 & .0005 \\ \text { DG-ORR } & -4.14 & 2.93 & .1576 \\ \text { NL-ORR } & -7.35 & 2.93 & .0121 \\ \text { CHI-BV } & -18.45 & 2.93 & .0001 \\ \text { CHI-K25 } & -5.15 & 2.93 & .0786 \\ \text { CHE-ORR } & -19.35 & 3.10 & .0001 \\ \text { CR-ORR } & -8.45 & 3.01 & .0050 \\ \text { CR-AND } & -9.04 & 2.95 & .0022 \\ \text { CR-ROA } & -7.33 & 3.12 & .0187\end{array}$

Analysis=Potassium Comparison: A-B Area-differences $\mathbf{P}=\mathbf{. 0 0 0 1}$

$\begin{array}{lrrr}\text { DG-AND } & 73.75 & 212.77 & .7289 \\ \text { DG-ROA } & -461.75 & 212.77 & .0300 \\ \text { DG-ORR } & -196.25 & 212.77 & .3564 \\ \text { NL-ORR } & -755.00 & 212.77 & .0004 \\ \text { CHI-BV } & -837.50 & 212.77 & .0001 \\ \text { CHI-K25 } & -2202.50 & 212.77 & .0001 \\ \text { CHE-ORR } & -954.21 & 217.42 & .0001 \\ \text { CR-ORR } & -233.25 & 212.77 & .2730 \\ \text { CR-AND } & -347.13 & 212.77 & .1028 \\ \text { CR-ROA } & -214.41 & 246.09 & .3836\end{array}$




\section{$\mathrm{G}-43$}

Table G.6 (continued)

\begin{tabular}{cccc}
\hline $\begin{array}{c}\text { Formation- } \\
\text { location }\end{array}$ & $\begin{array}{c}\text { Difference } \\
\text { estimate }\end{array}$ & $\begin{array}{c}\text { Standard } \\
\text { error }\end{array}$ & $\begin{array}{c}\text { Signific } \\
\text { lev }\end{array}$ \\
\hline Analysis=Potassium & Comparison: B-C & Area-differénces \\
DG-AND & -711.25 & 180.51 & .0001 \\
DG-ROA & -693.75 & 180.51 & .0001 \\
DG-ORR & -551.25 & 180.51 & .0023 \\
NL-ORR & -1315.00 & 180.51 & .0001 \\
CHI-BV & -80.00 & 180.51 & .6576 \\
CHI-K25 & 130.00 & 180.51 & .4714 \\
CHE-ORR & -128.25 & 180.51 & .4774 \\
CR-ORR & -206.50 & 180.51 & .2526 \\
CR-AND & -157.25 & 180.51 & .3837 \\
CR-ROA & -10.00 & 208.44 & .9617
\end{tabular}

Analysis=Potassium Comparison: A-C Area-differences $\mathbf{P}=\mathbf{0 0 0 1}$

$\begin{array}{lrll}\text { DG-AND } & -637.50 & 239.35 & .0077 \\ \text { DG-ROA } & -1155.50 & 239.35 & .0001 \\ \text { DG-ORR } & -747.50 & 239.35 & .0018 \\ \text { NL-ORR } & -2070.00 & 239.35 & .0001 \\ \text { CHI-BV } & -917.50 & 239.35 & .0001 \\ \text { CHI-K25 } & -2072.50 & 239.35 & .0001 \\ \text { CHE-ORR } & -1083.71 & 243.45 & .0001 \\ \text { CR-ORR } & -439.75 & 239.35 & .0662 \\ \text { CR-AND } & -504.38 & 239.35 & .0351 \\ \text { CR-ROA } & -224.27 & 276.72 & .4177\end{array}$

Anałysis=Silicon Comparison: A-B

Area-differénces $\mathrm{P}=\mathbf{0 0 8 1}$

$\begin{array}{lrrr}\text { DG-AND } & -20.00 & 28.23 & .4786 \\ \text { DG-ROA } & -39.50 & 28.23 & .1617 \\ \text { DG-ORR } & 0.63 & 28.23 & .9823 \\ \text { NL-ORR } & 1.50 & 28.23 & .9576 \\ \text { CHI-BV } & -43.00 & 39.92 & .2814 \\ \text { CHI-K25 } & -154.13 & 28.23 & .0001 \\ \text { CHE-ORR } & -61.63 & 28.23 & .0290 \\ \text { CR-ORR } & -30.00 & 56.46 & .5951 \\ \text { CR-AND } & -54.88 & 28.23 & .0519 \\ \text { CR-ROA } & -1.00 & 56.46 & .9859\end{array}$

Analysis $=$ Silicon

DG-AND
DG-ROA
DG-ORR
NL-ORR
CHI-BV
CHI-K25
CHE-ORR
CR-ORR
CR-AND
CR-ROA

Anatysis=Silicon

Comparison: B-C$$
\text { Area-differences } \mathrm{P}=5950
$$

25.75
99.13
-11.13
-46.75
56.00
39.00
13.88
26.00
1.25
-16.00

$\begin{array}{ll}42.60 & .5456 \\ 42.60 & .0200 \\ 42.60 & .7940 \\ 42.60 & .2725 \\ 60.25 & .3527 \\ 42.60 & .3600 \\ 42.60 & .7447 \\ 85.21 & .7603 \\ 42.60 & .9766 \\ 85.21 & .8511\end{array}$

Comparison: A-C Area-differences $\mathbf{P}=\mathbf{0} 0250$

$\begin{array}{lrrr}\text { DG-AND } & 5.75 & 31.63 & .8558 \\ \text { DG-ROA } & 59.63 & 31.63 & .0594 \\ \text { DG-ORR } & -10.50 & 31.63 & .7399 \\ \text { NL-ORR } & -45.25 & 31.63 & .1526 \\ \text { CHI-BV } & 13.00 & 44.73 & .7713 \\ \text { CHI-K25 } & -115.13 & 31.63 & .0003 \\ \text { CHE-ORR } & -47.75 & 31.63 & .1311 \\ \text { CR-ORR } & -4.00 & 63.26 & .9496 \\ \text { CR-AND } & -53.63 & 31.63 & .0900 \\ \text { CR-ROA } & -17.00 & 63.26 & .7881\end{array}$




\section{G-44}

Table G.6 (continued)

\begin{tabular}{cccc}
\hline $\begin{array}{c}\text { Formation- } \\
\text { location }\end{array}$ & $\begin{array}{c}\text { Difference } \\
\text { estimate }\end{array}$ & $\begin{array}{c}\text { Standard } \\
\text { error }\end{array}$ & $\begin{array}{c}\text { Significa } \\
\text { leve }\end{array}$ \\
\hline Analysis=Sodium & Comparison: A-B & Area-differences \\
& & & \\
DG-AND & 0.00 & 0.00 &. \\
DG-ROA & -121.59 & 391926 & .9998 \\
DG-ORR & 0.00 & 0.00 &. \\
NL-ORR & 0.00 & 0.00 &. \\
CHI-BV & -24.88 & 16.78 & .1383 \\
CHI-K25 & -28.13 & 16.78 & .0938 \\
CHE-ORR & 4.25 & 16.78 & .8001 \\
CR-ORR & -1.39 & 17.63 & .9371 \\
CR-AND & 23.38 & 16.78 & .1637 \\
CR-ROA & 7.13 & 16.78 & .6712
\end{tabular}

Analysis $=$ Sodium

DG-AND
DG-ROA
DG-ORR
NL-ORR
CHI-BV
CHI-K25
CHE-ORR
CR-ORR
CR-AND
CR-ROA

Analysis $=$ Sodium
Comparison: B-C Area-differences $\mathbf{P}=.8503$

$$
\begin{array}{r}
0.00 \\
-99.87 \\
0.00 \\
0.00 \\
-4.50 \\
18.50 \\
-11.75 \\
-2.00 \\
-5.50 \\
-16.25
\end{array}
$$

$\begin{array}{rl}0.00 & . \\ 336833 & .9998 \\ 0.00 & . \\ 0.00 & . \\ 16.48 & .7848 \\ 16.48 & .2615 \\ 16.48 & .4758 \\ 16.48 & .9034 \\ 16.48 & .7385 \\ 16.48 & .3240\end{array}$

Comparison: A-C Area-differences $\mathbf{P}=\mathbf{6 4 9 5}$

$$
\begin{array}{r}
0.00 \\
-99.04 \\
0.00 \\
0.00 \\
-29.38 \\
-9.63 \\
-7.50 \\
-4.72 \\
17.88 \\
-9.13
\end{array}
$$

0.00
378275
0.00
0.00
16.44
16.44
16.44
17.52
16.44
16.44

.9998

0740

.5583

.6483

.7875

.2769

.5789

Analysis=Strontium Comparison: A-B Area-differences $\mathbf{P}=.0063$

$\begin{array}{lrrr}\text { DG-AND } & 1.95 & 1.76 & .2674 \\ \text { DG-ROA } & 0.53 & 1.76 & .7652 \\ \text { DG-ORR } & 1.43 & 2.03 & .4802 \\ \text { NL-ORR } & -1.10 & 1.76 & .5315 \\ \text { CHI-BV } & -1.43 & 2.49 & .5666 \\ \text { CHI-K25 } & -7.23 & 1.76 & .0001 \\ \text { CHE-ORR } & -0.81 & 1.81 & .6570 \\ \text { CR-ORR } & 1.54 & 1.76 & .3806 \\ \text { CR-AND } & 2.64 & 1.76 & .1336 \\ \text { CR-ROA } & 1.23 & 2.03 & .5435\end{array}$

Analysis=Strontium Comparison: B-C Area-differences $\mathbf{P}=.0004$

$\begin{array}{lrrr}\text { DG-AND } & 0.38 & 1.37 & .7846 \\ \text { DG-ROA } & 0.45 & 1.37 & .7430 \\ \text { DG-ORR } & -1.55 & 1.37 & .2587 \\ \text { NL-ORR } & 0.20 & 1.37 & .8841 \\ \text { CHI-BV } & -6.40 & 1.94 & .0010 \\ \text { CHI-K25 } & 5.50 & 1.37 & .0001 \\ \text { CHE-ORR } & 2.30 & 1.40 & .1010 \\ \text { CR-ORR } & 1.77 & 1.38 & .1986 \\ \text { CR-AND } & 0.99 & 1.37 & .4729 \\ \text { CR-ROA } & 0.85 & 1.58 & .5917\end{array}$


Table G.6 (continued)

\begin{tabular}{cccc}
\hline $\begin{array}{c}\text { Formation- } \\
\text { location }\end{array}$ & $\begin{array}{c}\text { Difference } \\
\text { estimate }\end{array}$ & $\begin{array}{c}\text { Standard } \\
\text { error }\end{array}$ & $\begin{array}{r}\text { Sign } \\
\text { le }\end{array}$ \\
\hline Analysis=Vanadium & Comparison: B-C & Area-differences \\
& & & \\
DG-AND & 2.19 & 3.26 & .5023 \\
DG-ROA & 4.34 & 3.26 & .1834 \\
DG-ORR & -8.23 & 3.77 & .0288 \\
NL-ORR & 4.68 & 3.26 & .1516 \\
CHI-BV & 2.50 & 3.26 & .4433 \\
CHI-K25 & 7.75 & 3.26 & .0175 \\
CHE-ORR & 3.78 & 3.26 & .2470 \\
CR-ORR & -15.78 & 3.26 & .0001 \\
CR-AND & -6.33 & 3.26 & .0524 \\
CR-ROA & -31.59 & 3.26 & .0001
\end{tabular}

Analysis $=$ Vanadium Comparison: A-C Area-differences $\mathbf{P}=\mathbf{0 0 0 1}$

$\begin{array}{lrll}\text { DG-AHD } & -12.38 & 4.61 & .0073 \\ \text { DG-ROA } & -2.74 & 4.61 & .5528 \\ \text { DG-ORR } & -12.66 & 4.61 & .0060 \\ \text { HL-ORR } & -8.98 & 4.61 & .0516 \\ \text { CHI-BV } & -5.21 & 4.61 & .2583 \\ \text { CHI-K25 } & -9.53 & 4.61 & .0389 \\ \text { CHE-ORR } & -26.81 & 4.61 & .0001 \\ \text { CR-ORR } & -52.88 & 4.61 & .0001 \\ \text { CR-AND } & -29.08 & 4.61 & .0001 \\ \text { CR-ROA } & -58.94 & 4.61 & .0001\end{array}$

Analysis $=$ Zinc

DG-AND

DG-ROA

NL-ORR

CHI $-\mathrm{BV}$

CHI - K25

CHE-ORR

CR-ORR

CR-AND.

CR-ROA

Comparison: A-

$$
\begin{array}{r}
-1.35 \\
-0.55 \\
1.18 \\
-6.68 \\
-13.91 \\
-24.13 \\
-97.04 \\
-43.58 \\
-35.29 \\
-8.95
\end{array}
$$

4.61

.0001

gnificance level

Analysis $=$ Zinc

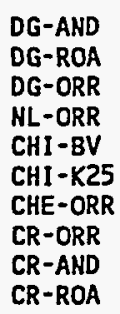

Analysis $=$ Zinc
Comparison: B-C Area-differences $\mathrm{P}=\mathbf{. 0 0 0 1}$

$\begin{array}{rll}-8.61 & 8.97 & .3369 \\ -9.96 & 8.97 & .2666 \\ -10.14 & 8.97 & .2583 \\ -0.38 & 8.97 & .9666 \\ -24.45 & 8.97 & .0064 \\ 6.23 & 8.97 & .4876 \\ -72.08 & 8.97 & .0001 \\ -53.42 & 9.71 & .0001 \\ -11.33 & 8.97 & .2067 \\ -14.78 & 8.97 & .0994\end{array}$

Comparison: A-C Area-differences $\mathrm{P}=\mathbf{0 0 0 1}$

$\begin{array}{lrrr}\text { DG-AND } & -9.96 & 19.73 & .6136 \\ \text { DG-ROA } & -10.51 & 19.73 & .5941 \\ \text { DG-ORR } & -8.96 & 19.73 & .6496 \\ \text { NL-ORR } & -7.05 & 19.73 & .7208 \\ \text { CHI-BV } & -38.36 & 19.73 & .0518 \\ \text { CHI-K25 } & -17.90 & 19.73 & .3643 \\ \text { CHE-ORR } & -169.11 & 19.73 & .0001 \\ \text { CR-ORR } & -94.59 & 20.67 & .0001 \\ \text { CR-AND } & -46.61 & 19.73 & .0181 \\ \text { CR-ROA } & -23.73 & 19.73 & .2292\end{array}$


G-45

Table G.6 (continued)

\begin{tabular}{cccc}
$\begin{array}{c}\text { Formation- } \\
\text { location }\end{array}$ & $\begin{array}{c}\text { Difference } \\
\text { estimate }\end{array}$ & $\begin{array}{c}\text { Standard } \\
\text { error }\end{array}$ & $\begin{array}{c}\text { Significance } \\
\text { level }\end{array}$ \\
\hline
\end{tabular}

Analysis=Strontium Comparison: A-C Area-differences $\mathbf{P}=\mathbf{0 0 0 1}$

$\begin{array}{lrrr}\text { DG-AND } & 2.33 & 0.84 & .0059 \\ \text { DG-ROA } & 0.98 & 0.84 & .2478 \\ \text { DG-ORR } & -0.40 & 0.97 & .6813 \\ \text { NL-ORR } & -0.90 & 0.84 & .2860 \\ \text { CHI-BV } & -7.83 & 1.19 & .0001 \\ \text { CHI-K25 } & -1.73 & 0.84 & .0409 \\ \text { CHE-ORR } & 1.53 & 0.88 & .0810 \\ \text { CR-ORR } & 3.31 & 0.85 & .0001 \\ \text { CR-AND } & 3.62 & 0.84 & .0001 \\ \text { CR-ROA } & 2.08 & 0.97 & .0325\end{array}$

Analysis=Sulfate

DG-AND
DG-ROA
DG-ORR
NL-ORR
CHI-BV
CHI-K25
CHE-ORR
CR-ORR
CR-AND
CR-ROA

Analysis=Sulfate

DG-AND

DG-ROA

DG-ORR

NL-ORR

$\mathrm{CHI}-\mathrm{BV}$

CHI - K25

CHE-ORR

CR-ORR

CR-AND

CR-ROA
Analysis $=$ Sulfate

DG-AND

DG-ROA

DG-ORR

NL-ORR

CHI-BV

CHI $-\mathrm{K} 25$

CHE-ORR

CR-ORR

CR-AND

CR-ROA

Comparison: A-B Area-differences $\mathbf{P}=.0225$

-27.75
-64.96
-12.63
-103.55
22.65
48.38
25.4
25.18
60.33
-1.69

$$
\begin{array}{ll}
34.90 & .4265 \\
34.90 & .0627 \\
40.30 & .7539 \\
34.90 & .0030 \\
34.90 & .5163 \\
34.90 & .1657 \\
34.90 & .4665 \\
34.90 & .4707 \\
34.90 & .0839 \\
34.90 & .9614
\end{array}
$$

Comparison: B-C Area-differences $\mathrm{P}=\mathbf{2 7 3 2}$

$\begin{array}{rrr}24.83 & 32.89 & .4504 \\ 87.45 & 32.89 & .0078 \\ -30.15 & 32.89 & .3593 \\ 84.03 & 32.89 & .0106 \\ 36.30 & 32.89 & .2697 \\ 84.78 & 32.89 & .0099 \\ 33.84 & 32.90 & .3037 \\ 35.29 & 32.91 & .2837 \\ 19.00 & 32.89 & .5635 \\ 52.44 & 32.90 & .1110\end{array}$

Comparison: A-C Area-differences $\mathrm{P}=\mathbf{0 0 0 1}$

$\begin{array}{rll}-2.93 & 20.33 & .8856 \\ 22.49 & 20.33 & .2686 \\ -42.47 & 23.47 & .0704 \\ -19.53 & 20.33 & .3368 \\ 58.95 & 20.33 & .0037 \\ 133.15 & 20.33 & .0001 \\ 59.24 & 20.35 & .0036 \\ 60.38 & 20.36 & .0030 \\ 79.33 & 20.33 & .0001 \\ 50.67 & 20.34 & .0127\end{array}$

Analysis $=$ Vanadium Comparison: A-B Area-differences $\mathbf{P}=\mathbf{0 0 0 1}$

$\begin{array}{lrrr}\text { DG-AND } & -14.56 & 3.69 & .0001 \\ \text { DG-ROA } & -7.08 & 3.69 & .0549 \\ \text { DG-ORR } & -4.28 & 4.26 & .3142 \\ \text { NL-ORR } & -13.65 & 3.69 & .0002 \\ \text { CHI-BV } & -7.71 & 3.69 & .0364 \\ \text { CHI-K25 } & -17.28 & 3.69 & .0001 \\ \text { CHE-ORR } & -30.59 & 3.69 & .0001 \\ \text { CR-ORR } & -37.10 & 3.69 & .0001 \\ \text { CR-AND } & -22.75 & 3.69 & .0001 \\ \text { CR-ROA } & -27.35 & 3.69 & .0001\end{array}$




\section{G-47}

Table G.7. Comparisons of horizons for selected radionuclides

(Estimates and standard errors are in picocuries per gram.)

\begin{tabular}{cccc}
\hline $\begin{array}{c}\text { Formation- } \\
\text { location }\end{array}$ & $\begin{array}{c}\text { Difference } \\
\text { estimate }\end{array}$ & $\begin{array}{c}\text { Standard } \\
\text { error }\end{array}$ & $\begin{array}{c}\text { Significance } \\
\text { level }\end{array}$ \\
\hline
\end{tabular}

Analysis $=$ CESIUM-137 Type $=$ GAMMA Comparison: A-B Area-differences $P=.0348$

$\begin{array}{llll}\text { DG-AND } & 0.29 & 0.24 & .2322 \\ \text { DG-ROA } & 0.78 & 0.24 & .0014 \\ \text { DG-ORR } & 0.44 & 0.28 & .1117 \\ \text { HL-ORR } & 0.53 & 0.24 & .0285 \\ \text { CHI-BV } & 1.37 & 0.24 & .0001 \\ \text { CHI-K25 } & 1.15 & 0.24 & .0001 \\ \text { CHE-ORR } & 0.98 & 0.24 & .0001 \\ \text { CR-ORR } & 0.84 & 0.24 & .0005 \\ \text { CR-AND } & 0.45 & 0.24 & .0600 \\ \text { CR-ROA } & 0.97 & 0.24 & .0001\end{array}$

Analysis $=$ CESIUM-137 Type $=$ GAMMA Comparison: $B-C$ Area-differences $P=9698$

$\begin{array}{lrrr}\text { DG-AND } & 0.05 & 0.15 & .7106 \\ \text { DG-ROA } & 0.00 & 0.15 & .9740 \\ \text { DG-ORR } & -0.08 & 0.17 & .6426 \\ \text { HL-ORR } & 0.01 & 0.15 & .9635 \\ \text { CHI-BV } & 0.03 & 0.15 & .8548 \\ \text { CHI-K25 } & -0.01 & 0.15 & .9546 \\ \text { CHE-ORR } & 0.00 & 0.15 & .9819 \\ \text { CR-ORR } & 0.00 & 0.15 & .9824 \\ \text { CR-AND } & 0.25 & 0.15 & .0921 \\ \text { CR-ROA } & 0.00 & 0.15 & .9896\end{array}$

Analysis=CESIUM-137 Type=GAMMA Comparison: A-C Area-differences $P=.0242$

$\begin{array}{llll}\text { DG-AND } & 0.34 & 0.22 & .1256 \\ \text { DG-ROA } & 0.78 & 0.22 & .0005 \\ \text { DG-ORR } & 0.37 & 0.26 & .1582 \\ \text { HL-ORR } & 0.54 & 0.22 & .0168 \\ \text { CHI-BV } & 1.40 & 0.22 & .0001 \\ \text { CHI-K25 } & 1.14 & 0.22 & .0001 \\ \text { CHE-ORR } & 0.99 & 0.22 & .0001 \\ \text { CR-ORR } & 0.84 & 0.22 & .0002 \\ \text { CR-AND } & 0.70 & 0.22 & .0017 \\ \text { CR-ROA } & 0.97 & 0.22 & .0001\end{array}$

Analysis=POTASSIUM-40 Type=GAMMA Comparison: A-B Area-differences $P=.0557$

$\begin{array}{lrrr}\text { DG-AND } & -6.50 & 2.54 & .0106 \\ \text { DG-ROA } & -6.71 & 2.54 & .0083 \\ \text { DG-ORR } & -3.00 & 3.60 & .4041 \\ \text { HL-ORR } & -1.50 & 2.54 & .5553 \\ \text { CHI-BV } & -6.99 & 2.54 & .0060 \\ \text { CHI-K25 } & -12.92 & 2.54 & .0001 \\ \text { CHE-ORR } & -7.23 & 2.94 & .0138 \\ \text { CR-ORR } & -2.61 & 2.54 & .3056 \\ \text { CR-AND } & -2.77 & 2.54 & .2760 \\ \text { CR-ROA } & -9.61 & 2.94 & .0011\end{array}$

Analysis=POTASSIUM-40 Type=GAMMA Comparison: B-C Area-differences $P=.0111$

$\begin{array}{lrll}\text { DG-AND } & 3.63 & 4.08 & .3742 \\ \text { DG-ROA } & -4.63 & 4.08 & .2569 \\ \text { DG-ORR } & 0.00 & 5.77 & 1.000 \\ \text { HL-ORR } & -8.50 & 4.08 & .0372 \\ \text { CHI-BV } & 4.87 & 4.08 & .2325 \\ \text { CHI-K25 } & -15.60 & 4.71 & .0009 \\ \text { CHE-ORR } & 0.22 & 5.77 & .9692 \\ \text { CR-ORR } & -0.43 & 4.08 & .9170 \\ \text { CR-AND } & .0 .93 & 4.08 & .8206 \\ \text { CR-ROA } & 8.34 & 4.71 & .0767\end{array}$


Table G.7 (continued)

\begin{tabular}{cccc}
\hline $\begin{array}{c}\text { Formation- } \\
\text { location }\end{array}$ & $\begin{array}{c}\text { Difference } \\
\text { estimate }\end{array}$ & $\begin{array}{c}\text { Standard } \\
\text { error }\end{array}$ & $\begin{array}{c}\text { Significance } \\
\text { level }\end{array}$ \\
\hline
\end{tabular}

Analysis=POTASSIUM-40 Type=GAMMA Comparison: A-C Area-differences $\mathbf{P}=.0002$

$\begin{array}{lrll}\text { DG-AND } & -2.88 & 4.00 & .4721 \\ \text { DG-ROA } & -11.34 & 4.00 & .0046 \\ \text { DG-ORR } & -3.67 & 4.62 & .4271 \\ \text { NL-ORR } & -10.00 & 4.00 & .0124 \\ \text { CHI-BV } & -2.12 & 4.00 & .5964 \\ \text { CHI-K25 } & -28.63 & 4.62 & .0001 \\ \text { CHE-ORR } & -7.72 & 5.65 & .1723 \\ \text { CR-ORR } & -3.03 & 4.00 & .4485 \\ \text { CR-AND } & -1.85 & 4.00 & .6445 \\ \text { CR-ROA } & -1.10 & 4.00 & .7835\end{array}$

Analysis=RADIUM-226 Type=ALPHA Comparison: A-B Area-differences $\mathbf{P}=\mathbf{2 6 1 4}$

$\begin{array}{lrrr}\text { DG-AND } & 0.11 & 0.30 & .7099 \\ \text { DG-ROA } & -0.04 & 0.30 & .9013 \\ \text { DG-ORR } & 0.06 & 0.31 & .8585 \\ \text { NL-ORR } & -0.17 & 0.30 & .5796 \\ \text { CHI-BV } & 0.00 & 0.30 & .9918 \\ \text { CHI-K25 } & -0.02 & 0.30 & .9492 \\ \text { CHE-ORR } & -0.98 & 0.30 & .0011 \\ \text { CR-ORR } & -0.29 & 0.30 & .3417 \\ \text { CR-AND } & -0.58 & 0.30 & .0558 \\ \text { CR-ROA } & -0.25 & 0.30 & .4021\end{array}$

Analysis=RADIUM-226 Type=ALPHA Comparison: B-C Area-differences $\mathbf{P}=\mathbf{4 2 0 4}$

$\begin{array}{lrll}\text { DG-AND } & 0.04 & 0.31 & .9027 \\ \text { DG-ROA } & 0.08 & 0.31 & .7879 \\ \text { DG-ORR } & -0.03 & 0.32 & .9181 \\ \text { NL-ORR } & 0.00 & 0.31 & .9935 \\ \text { CHI-BV } & -0.28 & 0.31 & .3578 \\ \text { CHI-K25 } & -0.46 & 0.31 & .1315 \\ \text { CHE-ORR } & 0.59 & 0.31 & .0535 \\ \text { CR-ORR } & -0.12 & 0.31 & .7016 \\ \text { CR-AND } & 0.18 & 0.31 & .5507 \\ \text { CR-ROA } & -0.43 & 0.31 & .1653\end{array}$

Analysis=RADIUM-226 Type $=$ ALPHA Comparison: A-C Area-differences $P=.4828$

$\begin{array}{llll}\text { DG-AND } & 0.15 & 0.27 & .5821 \\ \text { DG-ROA } & 0.05 & 0.27 & .8689 \\ \text { DG-ORR } & 0.03 & 0.27 & .9269 \\ \text { NL-ORR } & -0.17 & 0.27 & .5450 \\ \text { CHI -8V } & -0.28 & 0.27 & .3063 \\ \text { CHI-K25 } & -0.48 & 0.27 & .0772 \\ \text { CHE-ORR } & -0.39 & 0.27 & .1508 \\ \text { CR-ORR } & -0.41 & 0.27 & .1374 \\ \text { CR-AND } & -0.40 & 0.27 & .1467 \\ \text { CR-ROA } & -0.68 & 0.27 & .0128\end{array}$

Analysis $=$ THORIUM-228 Type $=$ ALPHA Comparison: A-B Area-differences $P=\mathbf{2 0 7 9}$

$\begin{array}{lrll}\text { DG-AND } & 0.18 & 0.25 & .4678 \\ \text { DG-ROA } & 0.07 & 0.25 & .7869 \\ \text { DG-ORR } & -0.65 & 0.25 & .0098 \\ \text { NL-ORR } & -0.05 & 0.25 & .8413 \\ \text { CHI-BV } & -0.22 & 0.25 & .3891 \\ \text { CHI-K25 } & -0.38 & 0.25 & .1252 \\ \text { CHE-ORR } & -0.46 & 0.25 & .0641 \\ \text { CR-ORR } & -0.65 & 0.25 & .0096 \\ \text { CR-AND } & -0.36 & 0.25 & .1503 \\ \text { CR-ROA } & -0.50 & 0.25 & .0434\end{array}$




\section{G-49}

Table G.7 (continued)

\begin{tabular}{cccc}
\hline $\begin{array}{c}\text { Formation- } \\
\text { location }\end{array}$ & $\begin{array}{c}\text { Difference } \\
\text { estimate }\end{array}$ & $\begin{array}{c}\text { Standard } \\
\text { error }\end{array}$ & $\begin{array}{c}\text { Significance } \\
\text { level }\end{array}$ \\
\hline
\end{tabular}

is

Analysis=THORIUM-228 Type=ALPHA Comparison: B-C Area-differences $\mathbf{P}=\mathbf{3 3 7 3}$

$\begin{array}{lrll}\text { DG-AND } & -0.05 & 0.21 & .7982 \\ \text { DG-ROA } & 0.19 & 0.21 & .3611 \\ \text { DG-ORR } & 0.32 & 0.21 & .1224 \\ \text { NL-ORR } & 0.03 & 0.21 & .9031 \\ \text { CHI-BV } & 0.06 & 0.21 & .7878 \\ \text { CHI-K25 } & -0.50 & 0.21 & .0159 \\ \text { CHE-ORR } & -0.12 & 0.21 & .5491 \\ \text { CR-ORR } & -0.12 & 0.21 & .5671 \\ \text { CR-AND } & 0.02 & 0.21 & .9156 \\ \text { CR-ROA } & -0.15 & 0.21 & .4620\end{array}$

Analysis $=$ THORTUM-228 Type $=$ ALPHA Comparison: A-C Area-differences $P=.0002$

$\begin{array}{lrll}\text { DG-AND } & 0.13 & 0.20 & .5250 \\ \text { DG-ROA } & 0.26 & 0.20 & .2081 \\ \text { DG-ORR } & -0.33 & 0.20 & .1052 \\ \text { KL-ORR } & -0.03 & 0.20 & .9018 \\ \text { CHI-BV } & -0.16 & 0.20 & .4303 \\ \text { CHI-K25 } & -0.88 & 0.20 & .0001 \\ \text { CHE-ORR } & -0.59 & 0.20 & .0039 \\ \text { CR-ORR } & -0.76 & 0.20 & .0002 \\ \text { CR-AND } & -0.34 & 0.20 & .0958 \\ \text { CR-ROA } & -0.66 & 0.20 & .0012\end{array}$

Analysis=THORIUM-230 Type=ALPHA Comparison: A-B Area-differences $\mathbf{P}=\mathbf{3 8 7 3}$

$\begin{array}{llll}\text { DG-AND } & -0.05 & 0.19 & .7907 \\ \text { DG-ROA } & -0.11 & 0.19 & .5714 \\ \text { DG-ORR } & -0.36 & 0.19 & .0637 \\ \text { NL-ORR } & -0.03 & 0.19 & .8759 \\ \text { CHI-BV } & -0.02 & 0.19 & .9295 \\ \text { CHI-K25 } & -0.03 & 0.20 & .8699 \\ \text { CHE-ORR } & -0.47 & 0.19 & .0155 \\ \text { CR-ORR } & -0.43 & 0.19 & .0240 \\ \text { CR-AND } & -0.50 & 0.19 & .0090 \\ \text { CR-ROA } & -0.20 & 0.19 & .2901\end{array}$

Analysis=THORIUM-230 Type=ALPHA Comparison: B-C Area-differences $P=.0461$

$\begin{array}{llll}\text { DG-AND } & 0.16 & 0.16 & .2991 \\ \text { DG-ROA } & 0.31 & 0.16 & .0504 \\ \text { DG-ORR } & 0.29 & 0.16 & .0686 \\ \text { NL-ORR } & 0.12 & 0.16 & .4432 \\ \text { CHI-BV } & -0.02 & 0.16 & .8945 \\ \text { CHI-K25 } & -0.20 & 0.17 & .2515 \\ \text { CHE-ORR } & -0.22 & 0.16 & .1533 \\ \text { CR-ORR } & -0.09 & 0.16 & .5652 \\ \text { CR-AND } & -0.06 & 0.16 & .6991 \\ \text { CR-ROA } & -0.33 & 0.16 & .0337\end{array}$

Analysis=THORIUM-230 Type=ALPHA Comparison: A-C Area-differences $P=.0001$

$\begin{array}{lrrr}\text { DG-AND } & 0.11 & 0.15 & .4592 \\ \text { DG-ROA } & 0.20 & 0.15 & .1898 \\ \text { DG-ORR } & -0.07 & 0.15 & .6362 \\ \text { NL-ORR } & 0.09 & 0.15 & .5502 \\ \text { CHI-BV } & -0.04 & 0.15 & .8021 \\ \text { CHI-K25 } & -0.21 & 0.15 & .1554 \\ \text { CHE-ORR } & -0.69 & 0.15 & .0001 \\ \text { CR-ORR } & -0.52 & 0.15 & .0005 \\ \text { CR-AND } & -0.56 & 0.15 & .0002 \\ \text { CR-ROA } & -0.54 & 0.15 & .0004\end{array}$


Table G.7 (continued)

\begin{tabular}{cccc}
\hline $\begin{array}{c}\text { Formation- } \\
\text { location }\end{array}$ & $\begin{array}{c}\text { Difference } \\
\text { estimate }\end{array}$ & $\begin{array}{c}\text { Standard } \\
\text { error }\end{array}$ & $\begin{array}{c}\text { Significance } \\
\text { level }\end{array}$ \\
\hline
\end{tabular}

Analysis=THORIUM-232 Type $=$ ALPHA Comparison: A-B Area-differences $P=\mathbf{3 4 3 4}$

$\begin{array}{lrll}\text { DG-AND } & -0.06 & 0.24 & .8160 \\ \text { DG-ROA } & -0.32 & 0.24 & .1707 \\ \text { DG-ORR } & -0.64 & 0.24 & .0072 \\ \text { ML-ORR } & 0.03 & 0.24 & .9158 \\ \text { CHI-BV } & -0.14 & 0.24 & .5501 \\ \text { CHI-K25 } & -0.71 & 0.24 & .0027 \\ \text { CHE-ORR } & -0.54 & 0.24 & .0234 \\ \text { CR-ORR } & -0.55 & 0.24 & .0207 \\ \text { CR-AND } & -0.41 & 0.24 & .0829 \\ \text { CR-ROA } & -0.47 & 0.24 & .0475\end{array}$

Anatysis=THORIUM-232 Type=ALPHA Comparison: B-C Area-differences $\mathbf{P}=.1332$

$\begin{array}{lrrr}\text { DG-AND } & 0.02 & 0.17 & .9289 \\ \text { DG-ROA } & 0.54 & 0.17 & .0013 \\ \text { DG-ORR } & 0.18 & 0.17 & .2842 \\ \text { NL-ORR } & 0.13 & 0.17 & .4570 \\ \text { CHI-BV } & -0.08 & 0.17 & .6511 \\ \text { CHI-K25 } & 0.09 & 0.17 & .6130 \\ \text { CHE-ORR } & -0.05 & 0.17 & .7587 \\ \text { CR-ORR } & -0.03 & 0.17 & .8467 \\ \text { CR-AND } & 0.08 & 0.17 & .6341 \\ \text { CR-ROA } & -0.25 & 0.17 & .1383\end{array}$

Analysis=THORIUM-232 Type=ALPHA Comparison: A-C Area-differences $\mathbf{P}=.0033$

$\begin{array}{lrrr}\text { DG-AND } & -0.04 & 0.20 & .8426 \\ \text { DG-ROA } & 0.22 & 0.20 & .2832 \\ \text { DG-ORR } & -0.46 & 0.20 & .0239 \\ \text { NL-ORR } & 0.15 & 0.20 & .4566 \\ \text { CHI-BV } & -0.22 & 0.20 & .2810 \\ \text { CHI-K25 } & -0.62 & 0.20 & .0020 \\ \text { CHE-ORR } & -0.59 & 0.20 & .0036 \\ \text { CR-ORR } & -0.58 & 0.20 & .0040 \\ \text { CR-AND } & -0.33 & 0.20 & .1016 \\ \text { CR-ROA } & -0.72 & 0.20 & .0004\end{array}$

Analysis $=$ THORIUM-234 Type=BETA Comparison: A-B Area-differences $P=\mathbf{0 0 0 1}$

$\begin{array}{lccc}\text { DG-AND } & 0.02 & 0.22 & .9289 \\ \text { DG-ROA } & 0.09 & 0.26 & .7182 \\ \text { DG-ORR } & 0.70 & 0.22 & .0017 \\ \text { NL-ORR } & 0.32 & 0.22 & .1597 \\ \text { CHI-BV } & 0.23 & 0.49 & .6416 \\ \text { CHI-K25 } & 0.46 & 0.49 & .3419 \\ \text { CHE-ORR } & -0.19 & 0.40 & .6286 \\ \text { CR-ORR } & -0.03 & 0.31 & .9192 \\ \text { CR-AND } & -1.56 & 0.25 & .0001 \\ \text { CR-ROA } & 0.09 & 0.49 & .8554\end{array}$

Analysis $=$ THORIUM-234 Type=BETA Comparison: B-C Area-differences $\mathbf{P}=\mathbf{. 0 0 4 7}$

$\begin{array}{lrrr}\text { DG-AND } & 0.02 & 0.33 & .9434 \\ \text { DG-ROA } & 0.00 & 0.39 & 1.000 \\ \text { DG-ORR } & -0.23 & 0.33 & .4845 \\ \text { NL-ORR } & 0.04 & 0.33 & .9048 \\ \text { CHI-BV } & -0.12 & 0.50 & .8030 \\ \text { CHI-K25 } & 0.15 & 0.57 & .7995 \\ \text { CHE-ORR } & 0.03 & 0.44 & .9503 \\ \text { CR-ORR } & 1.21 & 0.47 & .0090 \\ \text { CR-AND } & 1.17 & 0.35 & .0009 \\ \text { CR-ROA } & -1.08 & 0.43 & .0118\end{array}$


G-51

Table G.7 (continued)

\begin{tabular}{cccc}
\hline $\begin{array}{c}\text { Formation- } \\
\text { location }\end{array}$ & $\begin{array}{c}\text { Difference } \\
\text { estimate }\end{array}$ & $\begin{array}{c}\text { Standard } \\
\text { error }\end{array}$ & $\begin{array}{c}\text { Significance } \\
\text { level }\end{array}$ \\
\hline
\end{tabular}

$i$

Analysis=THORIUM-234 Type=BETA Comparison: A-C Area-differences $P=.0001$

$\begin{array}{lrrr}\text { DG-AND } & 0.04 & 0.21 & .8323 \\ \text { DG-ROA } & 0.09 & 0.24 & .6957 \\ \text { DG-ORR } & 0.47 & 0.21 & .0222 \\ \text { NL-ORR } & 0.36 & 0.21 & .0858 \\ \text { CHI-BV } & 0.09 & 0.67 & .8884 \\ \text { CHI-K25 } & 0.54 & 0.42 & .1992 \\ \text { CHE-ORR } & -0.17 & 0.38 & .6412 \\ \text { CR-ORR } & 1.07 & 0.31 & .0005 \\ \text { CR-AND } & -0.68 & 0.26 & .0076 \\ \text { CR-ROA } & -1.13 & 0.26 & .0001\end{array}$

Analysis $=$ URANIUM-233/234 Type=AIPHA Comparison: A-B Area-differences $\mathbf{P}=\mathbf{. 1 8 0 5}$

$\begin{array}{lrrr}\text { DG-AND } & 0.02 & 0.29 & .9521 \\ \text { DG-ROA } & 0.14 & 0.29 & .6306 \\ \text { DG-ORR } & -0.46 & 0.29 & .1121 \\ \text { NL-ORR } & 0.24 & 0.29 & .4186 \\ \text { CHI-BV } & 0.12 & 0.29 & .6827 \\ \text { CHI-K25 } & 0.04 & 0.29 & .9019 \\ \text { CHE-ORR } & -0.21 & 0.29 & .4628 \\ \text { CR-ORR } & -0.29 & 0.29 & .3150 \\ \text { CR-AND } & -0.52 & 0.29 & .0740 \\ \text { CR-ROA } & 0.71 & 0.34 & .0342\end{array}$

Analysis=URANIUM-233/234 Type=ALPHA Comparison: B-C Area-differences $P=.0567$

$\begin{array}{lrll}\text { DG-AND } & 0.04 & 0.21 & .8382 \\ \text { DG-ROA } & 0.07 & 0.21 & .7232 \\ \text { DG-ORR } & 0.73 & 0.21 & .0004 \\ \text { NL-ORR } & -0.10 & 0.21 & .6246 \\ \text { CHI-BV } & -0.17 & 0.21 & .4156 \\ \text { CHI-K25 } & 0.04 & 0.21 & .8571 \\ \text { CHE-ORR } & 0.00 & 0.21 & .9923 \\ \text { CR-ORR } & -0.18 & 0.21 & .3873 \\ \text { CR-AND } & 0.21 & 0.21 & .3248 \\ \text { CR-ROA } & -0.32 & 0.24 & .1901\end{array}$

Analysis=URANIUM-233/234 Type $=$ ALPHA Comparison: A-C Area-differences $P=2651$

$\begin{array}{lrll}\text { DG-AND } & 0.06 & 0.23 & .7948 \\ \text { DG-ROA } & 0.21 & 0.23 & .3542 \\ \text { DG-ORR } & 0.27 & 0.23 & .2419 \\ \text { HL-ORR } & 0.13 & 0.23 & .5625 \\ \text { CHI-BV } & -0.05 & 0.23 & .8267 \\ \text { CHI-K25 } & 0.07 & 0.23 & .7505 \\ \text { CHE-ORR } & -0.21 & 0.23 & .3587 \\ \text { CR-ORR } & -0.47 & 0.23 & .0406 \\ \text { CR-AND } & -0.32 & 0.23 & .1720 \\ \text { CR-ROA } & 0.28 & 0.23 & .2245\end{array}$

Analysis=URANIUM-235 Type=ALPHA Comparison: A-B Area-differences $P=2395$

$\begin{array}{lrrr}\text { DG-AND } & -0.01 & 0.53 & .9814 \\ \text { DG-ROA } & 0.02 & 0.53 & .9670 \\ \text { DG-ORR } & -0.04 & 0.53 & .9393 \\ \text { NL-ORR } & 0.01 & 0.53 & .9895 \\ \text { CHI-BV } & 0.00 & 0.53 & .9931 \\ \text { CHI-K25 } & -0.07 & 0.53 & .8943 \\ \text { CHE-ORR } & -1.93 & 0.53 & .0003 \\ \text { CR-ORR } & -0.05 & 0.53 & .9230 \\ \text { CR-AND } & -0.12 & 0.53 & .8270 \\ \text { CR-ROA } & -0.03 & 0.61 & .9551\end{array}$




\section{G-52}

Table G.7 (continued)

\begin{tabular}{cccc}
\hline $\begin{array}{c}\text { Formation- } \\
\text { location }\end{array}$ & $\begin{array}{c}\text { Difference } \\
\text { estimate }\end{array}$ & $\begin{array}{c}\text { Standard } \\
\text { error }\end{array}$ & $\begin{array}{c}\text { Significance } \\
\text { level }\end{array}$ \\
\hline
\end{tabular}

Analysis=URANIUM-235 Type=ALPHA Comparison: B-C Area-differences $\mathbf{P}=\mathbf{2 4 3 3}$

$\begin{array}{lrrr}\text { DG-AND } & 0.01 & 0.54 & .9859 \\ \text { DG-ROA } & -0.01 & 0.54 & .9833 \\ \text { DG-ORR } & 0.06 & 0.54 & .9125 \\ \text { NL-ORR } & 0.00 & 0.54 & .9945 \\ \text { CHI-BV } & -0.10 & 0.54 & .8587 \\ \text { CHI-K25 } & 0.06 & 0.54 & .9150 \\ \text { CHE-ORR } & 1.92 & 0.54 & .0003 \\ \text { CR-ORR } & -0.02 & 0.54 & .9695 \\ \text { CR-AND } & 0.01 & 0.54 & .9874 \\ \text { CR-ROA } & 0.02 & 0.62 & .9703\end{array}$

Analysis=URANIUM-235 Type=ALPHA Comparison: A-C Area-differences P=3855

$\begin{array}{lrll}\text { DG-AND } & -0.00 & 0.05 & .9551 \\ \text { DG-ROA } & 0.01 & 0.05 & .8307 \\ \text { DG-ORR } & 0.02 & 0.05 & .7192 \\ \text { NL-ORR } & 0.01 & 0.05 & .8362 \\ \text { CHI-BV } & -0.09 & 0.05 & .0793 \\ \text { CHI-K25 } & -0.01 & 0.05 & .7988 \\ \text { CHE-ORR } & -0.01 & 0.05 & .8246 \\ \text { CR-ORR } & -0.07 & 0.05 & .1658 \\ \text { CR-AND } & -0.11 & 0.05 & .0375 \\ \text { CR-ROA } & -0.12 & 0.05 & .0221\end{array}$

Analysis=URANIUM-235 Type=GAMMA Comparison: A-B Area-differences $\mathbf{P}=\mathbf{9 9 3 8}$

$\begin{array}{lrll}\text { DG-AND } & 0.01 & 0.01 & .6541 \\ \text { DG-ROA } & 0.01 & 0.01 & .6032 \\ \text { DG-ORR } & 0.02 & 0.02 & .2926 \\ \text { NL-ORR } & 0.03 & 0.01 & .0411 \\ \text { CHI-BV } & -0.02 & 0.48 & .9587 \\ \text { CHI-K25 } & 0.01 & 2.82 & .9979 \\ \text { CHE-ORR } & -0.01 & 8.38 & .9992 \\ \text { CR-ORR } & 0.01 & 2.48 & .9972 \\ \text { CR-AND } & -0.04 & 0.19 & .8396 \\ \text { CR-ROA } & -0.00 & 0.05 & .9586\end{array}$

Analysis $=$ URANIUM-235 Type $=$ GAMMA Comparison: B-C Area-differences $\mathbf{P}=.0001$

$\begin{array}{lrrr}\text { DG-AND } & 0.02 & 0.01 & .1078 \\ \text { DG-ROA } & 0.01 & 0.01 & .5974 \\ \text { DG-ORR } & 0.02 & 0.01 & .2126 \\ \text { HL-ORR } & -0.00 & 0.01 & .9037 \\ \text { CHI-BV } & 0.03 & 0.64 & .9566 \\ \text { CHI-K25 } & -0.00 & 8.57 & 1.000 \\ \text { CHE-ORR } & 0.01 & 75.25 & .9999 \\ \text { CR-ORR } & -0.13 & 0.02 & .0001 \\ \text { CR-AND } & 0.01 & 5.31 & .9986 \\ \text { CR-ROA } & -0.08 & 0.02 & .0001\end{array}$

Analysis=URANIUM-235 Type $=$ GAMMA Comparison: A-C Area-differences $P=.0001$

$\begin{array}{lrrr}\text { DG-AND } & 0.02 & 0.01 & .0241 \\ \text { DG-ROA } & 0.01 & 0.01 & .2280 \\ \text { DG-ORR } & 0.04 & 0.01 & .0009 \\ \text { HL-ORR } & 0.03 & 0.01 & .0155 \\ \text { CHI-BV } & 0.01 & 0.14 & .9422 \\ \text { CHI-K25 } & 0.01 & 45.24 & .9999 \\ \text { CHE-ORR } & 0.00 & 56.07 & 1.000 \\ \text { CR-ORR } & -0.14 & 0.02 & .0001 \\ \text { CR-AND } & -0.03 & 0.26 & .9098 \\ \text { CR-ROA } & -0.09 & 0.02 & .0001\end{array}$


G-53

Table G.7 (continued)

\begin{tabular}{cccc}
\hline $\begin{array}{c}\text { Formation- } \\
\text { location }\end{array}$ & $\begin{array}{c}\text { Difference } \\
\text { estimate }\end{array}$ & $\begin{array}{c}\text { Standard } \\
\text { error }\end{array}$ & $\begin{array}{c}\text { Significance } \\
\text { level }\end{array}$ \\
\hline
\end{tabular}

s.

Analysis=URANIUM-238 Type=ALPHA Comparison: A-B Area-differences $P=.0820$

$\begin{array}{lrrr}\text { DG-AND } & -0.08 & 0.19 & .6735 \\ \text { DG-ROA } & 0.15 & 0.19 & .4405 \\ \text { DG-ORR } & -0.28 & 0.19 & .1465 \\ \text { NL-ORR } & 0.24 & 0.19 & .2221 \\ \text { CHI-BV } & 0.01 & 0.19 & .9524 \\ \text { CHI-K25 } & -0.03 & 0.19 & .8718 \\ \text { CHE-ORR } & -0.42 & 0.19 & .0314 \\ \text { CR-ORR } & -0.45 & 0.19 & .0196 \\ \text { CR-AND } & -0.48 & 0.19 & .0123 \\ \text { CR-ROA } & -0.15 & 0.22 & .4892\end{array}$

Analysis $=$ URANIUM-238 Type $=$ ALPHA Comparison: B-C Area-differences $P=.0034$

$\begin{array}{lrll}\text { DG-AND } & 0.10 & 0.19 & .5889 \\ \text { DG-ROA } & 0.05 & 0.19 & .7766 \\ \text { DG-ORR } & 0.64 & 0.19 & .0006 \\ \text { NL-ORR } & -0.10 & 0.19 & .5820 \\ \text { CHI-BV } & -0.04 & 0.19 & .8458 \\ \text { CHI-K25 } & 0.01 & 0.19 & .9677 \\ \text { CHE-ORR } & 0.21 & 0.19 & .2645 \\ \text { CR-ORR } & -0.29 & 0.19 & .1203 \\ \text { CR-AND } & 0.34 & 0.19 & .0662 \\ \text { CR-ROA } & -0.52 & 0.21 & .0151\end{array}$

Analysis $=U$ RANIUM-238 Type $=$ ALPHA Comparison: A-C Area-differences $P=.0001$

$\begin{array}{llll}\text { DG-AND } & 0.02 & 0.12 & .8804 \\ \text { DG-ROA } & 0.20 & 0.12 & .1062 \\ \text { DG-ORR } & 0.36 & 0.12 & .0042 \\ \text { NL-ORR } & 0.13 & 0.12 & .2835 \\ \text { CHI-BV } & -0.02 & 0.12 & .8441 \\ \text { CHI-K25 } & -0.02 & 0.12 & .8496 \\ \text { CHE-ORR } & -0.21 & 0.12 & .0942 \\ \text { CR-ORR } & -0.74 & 0.12 & .0001 \\ \text { CR-AND } & -0.14 & 0.12 & .2527 \\ \text { CR-ROA } & -0.58 & 0.12 & .0001\end{array}$




\section{G-54}

Table G.8. Summary statistics for ORR inorganics -overallab (Estimates and confidence bounds are in milligrams per kilogram.)

\begin{tabular}{|c|c|c|c|c|c|c|c|}
\hline Horizon & $\mathbf{N}$ & I & $\mathrm{D}$ & Median & UCB95 & X95 & LTB9595 \\
\hline \multicolumn{8}{|c|}{ Aluminum } \\
\hline \multirow[t]{2}{*}{$\begin{array}{l}A \\
B \\
C\end{array}$} & $\begin{array}{l}24 \\
24 \\
24\end{array}$ & $\begin{array}{l}0 \\
0 \\
0\end{array}$ & $\begin{array}{l}24 \\
24 \\
24\end{array}$ & $\begin{array}{l}14900 \\
26500 \\
28500\end{array}$ & $\begin{array}{l}17000 \\
29600 \\
32200\end{array}$ & $\begin{array}{l}27800 \\
45500 \\
51800\end{array}$ & $\begin{array}{l}22900 \\
38400 \\
42900\end{array}$ \\
\hline & & & & Antimom & & & \\
\hline \multirow[t]{2}{*}{$\begin{array}{l}\text { A } \\
B \\
C\end{array}$} & $\begin{array}{l}24 \\
24 \\
24\end{array}$ & $\begin{array}{l}0 \\
0 \\
0\end{array}$ & $\begin{array}{l}1 \\
4 \\
5\end{array}$ & $\begin{array}{l}0.0812 \\
0.1280 \\
0.1860\end{array}$ & $\begin{array}{l}0.622 \\
0.365 \\
0.365\end{array}$ & $\begin{array}{l}0.338 \\
0.808 \\
0.785\end{array}$ & $\begin{array}{l}0.172 \\
0.413 \\
0.466\end{array}$ \\
\hline & & & & Arsenic & · & & \\
\hline \multirow[t]{2}{*}{$\begin{array}{l}\text { A } \\
\text { B } \\
\text { C }\end{array}$} & $\begin{array}{l}23 \\
22 \\
23\end{array}$ & $\begin{array}{l}0 \\
0 \\
0\end{array}$ & $\begin{array}{l}23 \\
22 \\
22\end{array}$ & $\begin{array}{c}9.04 \\
11.20 \\
13.00\end{array}$ & $\begin{array}{l}11.1 \\
14.6 \\
18.8\end{array}$ & $\begin{array}{l}24.4 \\
38.7 \\
75.4\end{array}$ & $\begin{array}{l}17.8 \\
25.8 \\
42.8\end{array}$ \\
\hline & & & & Barium & & & \\
\hline \multirow[t]{2}{*}{$\begin{array}{l}\text { A } \\
\text { B } \\
\text { C }\end{array}$} & $\begin{array}{l}24 \\
24 \\
24\end{array}$ & $\begin{array}{l}0 \\
0 \\
0\end{array}$ & $\begin{array}{l}24 \\
24 \\
24\end{array}$ & $\begin{array}{l}74.8 \\
70.2 \\
56.0\end{array}$ & $\begin{array}{l}84.0 \\
82.5 \\
78.1\end{array}$ & $\begin{array}{l}132 \\
156 \\
285\end{array}$ & $\begin{array}{l}110 \\
121 \\
171\end{array}$ \\
\hline & & & & Beryllium & & & \\
\hline \multirow[t]{2}{*}{$\begin{array}{l}\text { A } \\
\text { B } \\
\text { C }\end{array}$} & $\begin{array}{l}24 \\
24 \\
24\end{array}$ & $\begin{array}{l}0 \\
0 \\
0\end{array}$ & $\begin{array}{l}21 \\
21 \\
21\end{array}$ & $\begin{array}{l}0.704 \\
0.884 \\
1.080\end{array}$ & $\begin{array}{l}0.81 \\
1.05 \\
1.26\end{array}$ & $\begin{array}{l}1.38 \\
1.98 \\
2.24\end{array}$ & $\begin{array}{l}1.11 \\
1.53 \\
1.78\end{array}$ \\
\hline & & & & Boron & & & \\
\hline \multirow[t]{2}{*}{$\begin{array}{l}\text { A } \\
\text { B } \\
\text { C }\end{array}$} & $\begin{array}{l}21 \\
22 \\
22\end{array}$ & $\begin{array}{l}2 \\
1 \\
1\end{array}$ & $\begin{array}{l}1 \\
4 \\
5\end{array}$ & $\begin{array}{l}1.55 \\
1.64 \\
2.19\end{array}$ & $\begin{array}{l}5.48 \\
6.18 \\
6.50\end{array}$ & $\begin{array}{l}11.1 \\
28.9 \\
33.9\end{array}$ & $\begin{array}{c}5.13 \\
9.76 \\
12.10\end{array}$ \\
\hline & & & & Cadmiun & & & \\
\hline \multirow[t]{2}{*}{$\begin{array}{l}\text { A } \\
\text { B } \\
\text { C }\end{array}$} & $\begin{array}{l}24 \\
24 \\
24\end{array}$ & $\begin{array}{l}0 \\
0 \\
0\end{array}$ & $\begin{array}{l}0 \\
0 \\
0\end{array}$ & - & - & - & - \\
\hline & & & & Calcium & & & \\
\hline \multirow[t]{2}{*}{$\begin{array}{l}\text { A } \\
\text { B } \\
\text { C }\end{array}$} & $\begin{array}{l}21 \\
21 \\
21\end{array}$ & $\begin{array}{l}0 \\
0 \\
0\end{array}$ & $\begin{array}{l}21 \\
21 \\
21\end{array}$ & $\begin{array}{l}893 \\
798 \\
829\end{array}$ & $\begin{array}{l}1140 \\
1070 \\
1280\end{array}$ & $\begin{array}{l}2700 \\
3000 \\
6110\end{array}$ & $\begin{array}{l}1860 \\
1930 \\
3130\end{array}$ \\
\hline & & & & Chromiun & & & \\
\hline \multirow[t]{2}{*}{$\begin{array}{l}\mathbf{A} \\
\mathrm{B} \\
\mathbf{C}\end{array}$} & $\begin{array}{l}23 \\
23 \\
24\end{array}$ & $\begin{array}{l}1 \\
0 \\
0\end{array}$ & $\begin{array}{l}22 \\
23 \\
24\end{array}$ & $\begin{array}{l}23.3 \\
33.3 \\
35.2\end{array}$ & $\begin{array}{l}27.0 \\
35.6 \\
39.2\end{array}$ & $\begin{array}{l}46.7 \\
45.6 \\
60.0\end{array}$ & $\begin{array}{l}37.3 \\
41.2 \\
50.7\end{array}$ \\
\hline & & & & Cobalt & & & \\
\hline \multirow[t]{2}{*}{$\begin{array}{l}\text { A } \\
B \\
C\end{array}$} & $\begin{array}{l}24 \\
24 \\
24\end{array}$ & $\begin{array}{l}0 \\
1 \\
0\end{array}$ & $\begin{array}{l}24 \\
18 \\
20\end{array}$ & $\begin{array}{c}13.80 \\
6.78 \\
9.39\end{array}$ & $\begin{array}{c}15.80 \\
9.52 \\
14.10\end{array}$ & $\begin{array}{l}27.5 \\
34.0 \\
65.1\end{array}$ & $\begin{array}{l}22.1 \\
20.0 \\
34.5\end{array}$ \\
\hline & & & & Copper & & & \\
\hline $\begin{array}{l}A \\
B \\
C\end{array}$ & $\begin{array}{l}24 \\
23 \\
24\end{array}$ & $\begin{array}{l}1 \\
0 \\
0\end{array}$ & $\begin{array}{l}20 \\
23 \\
23\end{array}$ & $\begin{array}{c}9.89 \\
19.30 \\
25.40\end{array}$ & $\begin{array}{l}11.9 \\
20.9 \\
27.6\end{array}$ & $\begin{array}{l}24.3 \\
28.0 \\
38.3\end{array}$ & $\begin{array}{l}18.2 \\
24.9 \\
33.7\end{array}$ \\
\hline
\end{tabular}


Table G.8 (continued)

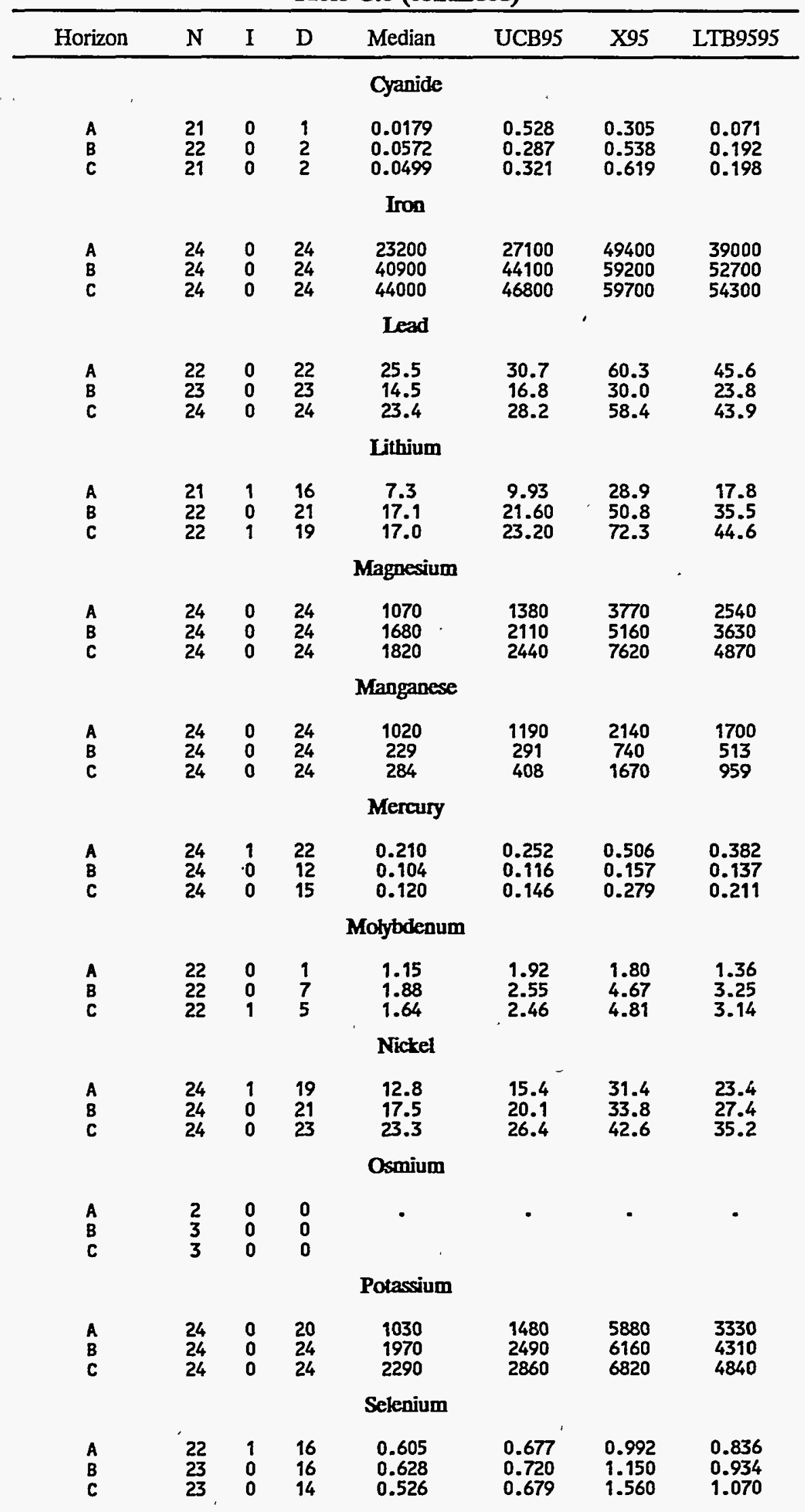


Table G.8 (continued)

\begin{tabular}{|c|c|c|c|c|c|c|c|}
\hline Horizon & $N$ & I & $\mathrm{D}$ & Median & UCB95 & $\mathrm{X} 95$ & LTB9595 \\
\hline \multirow{3}{*}{$\begin{array}{l}\text { A } \\
\text { B } \\
\text { C }\end{array}$} & & & & Silicon & & & \\
\hline & $\begin{array}{l}19 \\
19 \\
19\end{array}$ & $\begin{array}{l}0 \\
0 \\
0\end{array}$ & $\begin{array}{l}19 \\
19 \\
19\end{array}$ & $\begin{array}{l}468 \\
504 \\
507\end{array}$ & $\begin{array}{l}538 \\
592 \\
586\end{array}$ & $\begin{array}{c}859 \\
1020 \\
950\end{array}$ & $\begin{array}{l}694 \\
798 \\
762\end{array}$ \\
\hline & & & & Silver & & & \\
\hline \multirow[t]{2}{*}{$\begin{array}{l}A \\
B \\
C\end{array}$} & $\begin{array}{l}24 \\
24 \\
24\end{array}$ & $\begin{array}{l}0 \\
0 \\
0\end{array}$ & $\begin{array}{l}0 \\
0 \\
0\end{array}$ & - & . & - & - \\
\hline & & & & Sodium & & & \\
\hline \multirow[t]{2}{*}{$\begin{array}{l}A \\
B \\
C\end{array}$} & $\begin{array}{l}16 \\
16 \\
16\end{array}$ & $\begin{array}{l}0 \\
0 \\
0\end{array}$ & $\begin{array}{l}15 \\
16 \\
16\end{array}$ & $\begin{array}{l}372 \\
383 \\
383\end{array}$ & $\begin{array}{l}391 \\
407 \\
406\end{array}$ & $\begin{array}{l}456 \\
490 \\
484\end{array}$ & $\begin{array}{l}421 \\
446 \\
443\end{array}$ \\
\hline & & & & Strontium & & & \\
\hline \multirow[t]{2}{*}{$\begin{array}{l}\text { A } \\
\text { B } \\
\text { C }\end{array}$} & $\begin{array}{l}21 \\
22 \\
22\end{array}$ & $\begin{array}{l}0 \\
0 \\
0\end{array}$ & $\begin{array}{l}19 \\
19 \\
16\end{array}$ & $\begin{array}{l}5.00 \\
5.06 \\
2.44\end{array}$ & $\begin{array}{l}6.48 \\
7.02 \\
5.02\end{array}$ & $\begin{array}{l}16.1 \\
23.0 \\
61.7\end{array}$ & $\begin{array}{l}10.8 \\
13.8 \\
19.8\end{array}$ \\
\hline & & & & Sulfate & & & \\
\hline \multirow[t]{2}{*}{$\begin{array}{l}A \\
B \\
C\end{array}$} & $\begin{array}{l}23 \\
24 \\
24\end{array}$ & $\begin{array}{l}0 \\
0 \\
1\end{array}$ & $\begin{array}{l}23 \\
24 \\
20\end{array}$ & $\begin{array}{l}70.3 \\
75.4 \\
31.4\end{array}$ & $\begin{array}{l}92.8 \\
92.9 \\
45.0\end{array}$ & $\begin{array}{l}267 \\
210 \\
178\end{array}$ & $\begin{array}{l}174 \\
152 \\
102\end{array}$ \\
\hline & & & & Thallium & & & \\
\hline A & 22 & 0 & 1 & 0.0449 & 1.420 & 0.428 & 0.148 \\
\hline B & 22 & 0 & 4 & 0.3010 & 0.420 & 0.604 & 0.459 \\
\hline \multirow[t]{2}{*}{$\mathrm{C}$} & 22 & 1 & 6 & 0.3780 & 0.463 & 0.684 & 0.549 \\
\hline & & & & Vanadiun & & & \\
\hline \multirow[t]{2}{*}{$\begin{array}{l}\text { A } \\
\text { B } \\
\text { C }\end{array}$} & $\begin{array}{l}24 \\
23 \\
24\end{array}$ & $\begin{array}{l}0 \\
0 \\
0\end{array}$ & $\begin{array}{l}24 \\
23 \\
24\end{array}$ & $\begin{array}{l}32.5 \\
51.0 \\
50.7\end{array}$ & $\begin{array}{l}34.9 \\
55.2 \\
55.4\end{array}$ & $\begin{array}{l}46.2 \\
74.2 \\
78.2\end{array}$ & $\begin{array}{l}41.4 \\
65.8 \\
68.3\end{array}$ \\
\hline & & & & Zinc & & & \\
\hline $\begin{array}{l}\text { A } \\
\text { B } \\
\text { C }\end{array}$ & $\begin{array}{l}24 \\
24 \\
24\end{array}$ & $\begin{array}{l}0 \\
0 \\
0\end{array}$ & $\begin{array}{l}24 \\
24 \\
23\end{array}$ & $\begin{array}{l}42.0 \\
66.4 \\
81.7\end{array}$ & $\begin{array}{l}46.5 \\
76.4 \\
98.8\end{array}$ & $\begin{array}{l}69.2 \\
132.0 \\
205.0\end{array}$ & $\begin{array}{c}59.1 \\
107.0 \\
153.0\end{array}$ \\
\hline
\end{tabular}

${ }^{a} \mathrm{~N}=$ number of observations, possibly averages over replicates at sites; $\mathrm{I}=$ number of interval censored observations (see text); D = number of true detects (see text); UCB95 $=95 \%$ upper confidence bound for median; X95 = estimate of 95th percentile; LTB9595 $=95 \%$ lower confidence bound for 95th percentile; REMAINDER refers to the remaining observations-no detects.

${ }^{b}$ May be inappropriate when areas have different median analyte concentrations. Because of the statistical sampling design, it is important not to use combined results when the data from the different formation-locations are significantly different, because the error estimates will be inflated. If P-ORR $<0.01$ or P-GRP $<0.01$ ORR overall results should not be used. If P-CHICK $<.01$, or P-DGN 0.01, or P-CRC $<0.01$, then neither group nor the overall ORR results should be used. If P-CHICK $>0.01$ but P-DGN $<0.01$, one might be justified in combining the Chickamauga data, but not as it has been done here, because the Dismal Gap and Nolichucky data are also combined. How to combine the data is complex, and should be done on an ad hoc, analyte-by-analyte basis. 
Table G.9. Summary statistics for ORR radionuclides-overal1 ${ }^{a b}$

(Estimates and confidence bounds are in picocuries per gram)

\begin{tabular}{|c|c|c|c|c|c|c|c|}
\hline Horizon & $\mathbf{N}$ & I & $\mathrm{D}$ & Median & UCB95 & $\mathrm{X} 95$ & LTB9595 \\
\hline \multicolumn{8}{|c|}{ Cesium-137 (Gamma) } \\
\hline $\begin{array}{l}\text { A } \\
\text { B } \\
\text { C }\end{array}$ & $\begin{array}{l}23 \\
23 \\
23\end{array}$ & $\begin{array}{l}0 \\
0 \\
0\end{array}$ & $\begin{array}{r}23 \\
6 \\
2\end{array}$ & $\begin{array}{l}0.82200 \\
0.00697 \\
0.00007\end{array}$ & $\begin{array}{l}0.9870 \\
0.0185 \\
0.0516\end{array}$ & $\begin{array}{l}1.5400 \\
0.0911 \\
0.0751\end{array}$ & $\begin{array}{l}1.26000 \\
0.03550 \\
0.00506\end{array}$ \\
\hline \multicolumn{8}{|c|}{ Curium-247 (Gamma) } \\
\hline $\begin{array}{l}\text { A } \\
\text { B } \\
\text { C }\end{array}$ & $\begin{array}{r}23 \\
3 \\
3\end{array}$ & $\begin{array}{l}0 \\
0 \\
0\end{array}$ & $\begin{array}{l}2 \\
0 \\
0\end{array}$ & $\begin{array}{c}0.00552 \\
:\end{array}$ & $\begin{array}{c}0.00649 \\
:\end{array}$ & $\begin{array}{c}0.00716 \\
:\end{array}$ & $\begin{array}{c}0.00579 \\
\vdots\end{array}$ \\
\hline \multicolumn{8}{|c|}{ Neptunium-237 (Alpha) } \\
\hline $\begin{array}{l}\text { A } \\
\text { B }\end{array}$ & $\begin{array}{r}17 \\
1\end{array}$ & $\begin{array}{l}2 \\
0\end{array}$ & $\begin{array}{r}14 \\
0\end{array}$ & 0.0885 & $\begin{array}{c}0.102 \\
.\end{array}$ & 0.155 & $\begin{array}{c}0.126 \\
.\end{array}$ \\
\hline \multicolumn{8}{|c|}{ Plutonium-238 (Alpha) } \\
\hline $\begin{array}{l}\mathrm{A} \\
\mathrm{B} \\
\mathrm{C}\end{array}$ & $\begin{array}{r}23 \\
2 \\
2\end{array}$ & $\begin{array}{l}7 \\
0 \\
0\end{array}$ & $\begin{array}{l}6 \\
2 \\
0\end{array}$ & $\begin{array}{c}0.0494 \\
0.0853 \\
.\end{array}$ & $\begin{array}{c}0.0626 \\
0.0991 \\
.\end{array}$ & $\begin{array}{l}0.125 \\
0.105 \\
.\end{array}$ & $\begin{array}{l}0.0907 \\
0.0838 \\
.\end{array}$ \\
\hline \multicolumn{8}{|c|}{ Plutonium-239/240 (Alpha) } \\
\hline $\begin{array}{l}\text { A } \\
\text { B } \\
\text { C }\end{array}$ & $\begin{array}{r}22 \\
2 \\
2\end{array}$ & $\begin{array}{l}5 \\
0 \\
0\end{array}$ & $\begin{array}{l}6 \\
1 \\
0\end{array}$ & $\begin{array}{c}0.0231 \\
\vdots\end{array}$ & $\begin{array}{c}0.028 \\
:\end{array}$ & $\begin{array}{c}0.0454 \\
:\end{array}$ & 0.0346 \\
\hline \multicolumn{8}{|c|}{ Potassium-40 (Gamma) } \\
\hline $\begin{array}{l}A \\
B \\
C\end{array}$ & $\begin{array}{l}24 \\
21 \\
20\end{array}$ & $\begin{array}{l}0 \\
0 \\
0\end{array}$ & $\begin{array}{l}24 \\
21 \\
20\end{array}$ & $\begin{array}{r}8.82 \\
14.90 \\
16.10\end{array}$ & $\begin{array}{l}11.1 \\
.17 .8 \\
20.8\end{array}$ & $\begin{array}{l}27.4 \\
33.9 \\
50.6\end{array}$ & $\begin{array}{l}19.2 \\
25.7 \\
34.1\end{array}$ \\
\hline \multicolumn{8}{|c|}{ Radium-226 (Alpha) } \\
\hline $\begin{array}{l}\text { A } \\
\text { B } \\
\text { C }\end{array}$ & $\begin{array}{l}24 \\
24 \\
24\end{array}$ & $\begin{array}{l}1 \\
0 \\
0\end{array}$ & $\begin{array}{l}23 \\
23 \\
24\end{array}$ & $\begin{array}{l}0.924 \\
1.080 \\
1.140\end{array}$ & $\begin{array}{l}1.03 \\
1.26 \\
1.31\end{array}$ & $\begin{array}{l}1.57 \\
2.28 \\
2.24\end{array}$ & $\begin{array}{l}1.33 \\
1.81 \\
1.81\end{array}$ \\
\hline \multicolumn{8}{|c|}{ Strontium-90 (Beta) } \\
\hline A & 22 & 0 & 1 & 0.27 & 1.49 & 0.823 . & 0.501 \\
\hline \multicolumn{8}{|c|}{ Technetium-99 (Beta) } \\
\hline $\mathbf{A}$ & 35 & 0 & 6 & 0.684 & 1.17 & 2.32 & 1.6 \\
\hline \multicolumn{8}{|c|}{ Thorium-228 (Alpha) } \\
\hline $\begin{array}{l}\text { A } \\
\text { B } \\
\text { C }\end{array}$ & $\begin{array}{l}24 \\
24 \\
24\end{array}$ & $\begin{array}{l}0 \\
0 \\
0\end{array}$ & $\begin{array}{l}23 \\
24 \\
23\end{array}$ & $\begin{array}{l}0.824 \\
1.290 \\
1.270\end{array}$ & $\begin{array}{l}1.06 \\
1.45 \\
1.59\end{array}$ & $\begin{array}{l}2.83 \\
2.30 \\
3.88\end{array}$ & $\begin{array}{l}1.91 \\
1.92 \\
2.71\end{array}$ \\
\hline \multicolumn{8}{|c|}{ Thorium-230 (Alpha) } \\
\hline $\begin{array}{l}\text { A } \\
\text { B } \\
\text { C }\end{array}$ & $\begin{array}{l}24 \\
24 \\
24\end{array}$ & $\begin{array}{l}0 \\
0 \\
0\end{array}$ & $\begin{array}{l}24 \\
23 \\
24\end{array}$ & $\begin{array}{l}0.895 \\
1.080 \\
1.080\end{array}$ & $\begin{array}{l}0.99 \\
1.23 \\
1.26\end{array}$ & $\begin{array}{l}1.47 \\
2.02 \\
2.29\end{array}$ & $\begin{array}{l}1.26 \\
1.66 \\
1.81\end{array}$ \\
\hline \multicolumn{8}{|c|}{ Thorium-232 (Alpha) } \\
\hline $\begin{array}{l}A \\
B \\
C\end{array}$ & $\begin{array}{l}24 \\
24 \\
24\end{array}$ & $\begin{array}{l}0 \\
0 \\
0\end{array}$ & $\begin{array}{l}24 \\
24 \\
24\end{array}$ & $\begin{array}{l}0.917 \\
1.320 \\
1.270\end{array}$ & $\begin{array}{l}1.05 \\
1.50 \\
1.49\end{array}$ & $\begin{array}{l}1.75 \\
2.44 \\
2.79\end{array}$ & $\begin{array}{l}1.43 \\
2.01 \\
2.18\end{array}$ \\
\hline \multicolumn{8}{|c|}{ Thorium-234 (Beta) } \\
\hline $\begin{array}{l}A \\
B \\
C\end{array}$ & $\begin{array}{l}20 \\
20 \\
20\end{array}$ & $\begin{array}{l}2 \\
1 \\
1\end{array}$ & $\begin{array}{r}10 \\
9 \\
8\end{array}$ & $\begin{array}{l}1.070 \\
0.703 \\
0.769 .\end{array}$ & $\begin{array}{l}1.310 \\
0.998 \\
0.964\end{array}$ & $\begin{array}{l}2.39 \\
2.50 \\
1.67\end{array}$ & $\begin{array}{l}1.77 \\
1.55 \\
1.24\end{array}$ \\
\hline
\end{tabular}


Table G.9 (continued)

\begin{tabular}{|c|c|c|c|c|c|c|c|}
\hline Horizon & $\mathbf{N}$ & I & $\mathrm{D}$ & Median & UCB95 & $\mathrm{X} 95$ & LTB9595 \\
\hline \multicolumn{8}{|c|}{ Thorium-234 (Gamma) } \\
\hline $\begin{array}{l}\text { A } \\
\text { B } \\
\text { C }\end{array}$ & $\begin{array}{l}3 \\
3 \\
3\end{array}$ & $\begin{array}{l}0 \\
0 \\
0\end{array}$ & $\begin{array}{l}1 \\
1 \\
1\end{array}$ & $\begin{array}{l}0.909 \\
0.739 \\
1.010\end{array}$ & $\begin{array}{l}1.68 \\
1.20 \\
2.27\end{array}$ & $\begin{array}{l}1.76 \\
1.24 \\
2.49\end{array}$ & $\begin{array}{l}0.893 \\
0.728 \\
0.989\end{array}$ \\
\hline \multicolumn{8}{|c|}{ Total Uranium (Alpha) } \\
\hline A & 22 & 0 & 22 & 1.430 & 1.810 & 4.26 & 2.980 \\
\hline B & 2 & 0 & 2 & 0.316 & 0.972 & 1.55 & 0.276 \\
\hline C & 2 & 0 & 2 & 0.299 & 1.650 & 3.35 & 0.244 \\
\hline \multicolumn{8}{|c|}{ Tritium (Tritium) } \\
\hline A & 33 & 3 & 9 & 0.0275 & 0.0381 & 0.0978 & 0.0656 \\
\hline \multicolumn{8}{|c|}{ Uranium-233/234 (Alpha) } \\
\hline $\begin{array}{l}\text { A } \\
\text { B } \\
\text { C }\end{array}$ & $\begin{array}{l}24 \\
24 \\
24\end{array}$ & $\begin{array}{l}0 \\
0 \\
0\end{array}$ & $\begin{array}{l}24 \\
24 \\
24\end{array}$ & $\begin{array}{l}1.16 \\
1.19 \\
1.14\end{array}$ & $\begin{array}{l}1.24 \\
1.35 \\
1.30\end{array}$ & $\begin{array}{l}1.64 \\
2.16 \\
2.12\end{array}$ & $\begin{array}{l}1.47 \\
1.79 \\
1.75\end{array}$ \\
\hline \multicolumn{8}{|c|}{ Uranium-235 (Alpha) } \\
\hline $\begin{array}{l}\text { A } \\
\text { B } \\
\text { C }\end{array}$ & $\begin{array}{l}24 \\
24 \\
24\end{array}$ & $\begin{array}{l}3 \\
1 \\
1\end{array}$ & $\begin{array}{l}19 \\
21 \\
21\end{array}$ & $\begin{array}{l}0.0721 \\
0.1030 \\
0.0767\end{array}$ & $\begin{array}{l}0.0871 \\
0.1530 \\
0.1010\end{array}$ & $\begin{array}{l}0.179 \\
0.698 \\
0.291\end{array}$ & $\begin{array}{l}0.134 \\
0.381 \\
0.191\end{array}$ \\
\hline \multicolumn{8}{|c|}{ Uranium-235 (Gamma) } \\
\hline $\begin{array}{l}\text { A } \\
\text { B } \\
\text { C }\end{array}$ & $\begin{array}{l}23 \\
23 \\
24\end{array}$ & $\begin{array}{l}0 \\
0 \\
0\end{array}$ & $\begin{array}{l}8 \\
7 \\
9\end{array}$ & $\begin{array}{l}0.0725 \\
0.0503 \\
0.0497\end{array}$ & $\begin{array}{l}0.0850 \\
0.0661 \\
0.0672\end{array}$ & $\begin{array}{l}0.117 \\
0.107 \\
0.132\end{array}$ & $\begin{array}{l}0.0940 \\
0.0748 \\
0.0901\end{array}$ \\
\hline \multicolumn{8}{|c|}{ Uranium-236 (Alpha) } \\
\hline $\begin{array}{l}\text { A } \\
\text { B } \\
\text { C }\end{array}$ & $\begin{array}{l}24 \\
24 \\
24\end{array}$ & $\begin{array}{l}1 \\
0 \\
0\end{array}$ & $\begin{array}{l}2 \\
1 \\
1\end{array}$ & $\begin{array}{l}0.007310 \\
0.000004 \\
.\end{array}$ & $\begin{array}{l}0.0136 \\
3.5600 \\
.\end{array}$ & $\begin{array}{c}0.0207 \\
0.0134 \\
.\end{array}$ & $\begin{array}{l}0.013500 \\
0.000305 \\
.\end{array}$ \\
\hline \multicolumn{8}{|c|}{ Uranium-238 (Alpha) } \\
\hline $\begin{array}{l}A \\
B \\
C\end{array}$ & $\begin{array}{l}24 \\
24 \\
24\end{array}$ & $\begin{array}{l}0 \\
0 \\
0\end{array}$ & $\begin{array}{l}24 \\
24 \\
24\end{array}$ & $\begin{array}{l}1.18 \\
1.28 \\
1.18\end{array}$ & $\begin{array}{l}1.25 \\
1.43 \\
1.35\end{array}$ & $\begin{array}{l}1.57 \\
2.18 \\
2.30\end{array}$ & $\begin{array}{l}1.44 \\
1.84 \\
1.87\end{array}$ \\
\hline \multicolumn{8}{|c|}{ Uranium-238 (Gamma) } \\
\hline $\begin{array}{l}A \\
B \\
C\end{array}$ & $\begin{array}{l}16 \\
16 \\
16 \\
\end{array}$ & $\begin{array}{l}0 \\
0 \\
0\end{array}$ & $\begin{array}{l}0 \\
0 \\
1\end{array}$ & 0.0341 & $274^{\circ}$ & 12.9 & 0.727 \\
\hline
\end{tabular}

of interval censored observations (see text); $\mathrm{D}=$ number of true detects (see text); UCB95 $=95 \%$ upper confidence bound for median; X95 = estimate of 95th percentile; LTB9595 $=95 \%$ lower confidence bound for 95th percentile; REMAINDER refers to the remaining observations-no detects.

${ }^{b}$ May be inappropriate when areas have different median analyte concentrations. Because of the statistical sampling design, it is important not to use combined results when the data from the different formation-locations are significantly different, because the error estimates will be inflated. If P-ORR $<0.01$ or P-GRP $<0.01$ ORR overall results should not be used. If P-CHICK $<.01$, or P-DGN 0.01 , or P-CRC $<0.01$, then neither group nor the overall ORR results should be used. If P-CHICK $>0.01$ but P-DGN $<0.01$, one might be justified in combining the Chickamauga data, but not as it has been done here, because the Dismal Gap and Nolichucky data are also combined. How to combine the data is complex, and should be done on an ad hoc, analyte-by-analyte basis. 
Table G.10. Summary statistics for ORR PAHs-overall ${ }^{a, b}$ (Estimates and confidence bounds are in micrograms per kilogram.)

\begin{tabular}{lrrrrrrr}
\hline \multicolumn{1}{c}{ Analysis } & N & I & D & Median & UCB95 & X95 & LTB9595 \\
\hline Acenaphthene & 14 & 0 & 8 & 1.420 & 1.89 & 3.31 & 2.21 \\
Acenaphthylene & 41 & 0 & 4 & 0.715 & 28.40 & 227.00 & 45.00 \\
Anthracene & 27 & 0 & 25 & 0.880 & 1.16 & 3.51 & 2.31 \\
Benzo[a] anthracene & 37 & 0 & 37 & 3.080 & 3.87 & 12.30 & 8.69 \\
Benzo[a]pyrene & 39 & 0 & 39 & 3.740 & 4.43 & 10.70 & 8.28 \\
Benzo[b] fluoranthene & 32 & 0 & 30 & 3.580 & 4.45 & 12.00 & 8.64 \\
Benzo[g,h,i]perylene & 32 & 0 & 32 & 3.500 & 4.26 & 10.60 & 7.85 \\
Benzo[k] fluoranthene & 40 & 0 & 40 & 2.040 & 2.43 & 6.03 & 4.64 \\
Chrysene & 23 & 0 & 17 & 4.080 & 5.36 & 13.90 & 9.26 \\
Dibenzo[a,h] anthracene & 19 & 0 & 16 & 0.909 & 1.24 & 3.16 & 1.99 \\
Fluoranthene & 38 & 0 & 38 & 5.280 & 6.56 & 20.10 & 14.40 \\
Fluorene & 21 & 0 & 14 & 0.994 & 1.44 & 4.40 & 2.60 \\
Indeno[1,2,3-c,d]pyrene & 42 & 0 & 16 & 9.200 & 11.70 & 26.70 & 20.30 \\
Naphthalene & 24 & 0 & 19 & 4.790 & 7.27 & 31.30 & 17.10 \\
Phenanthrene & 43 & 0 & 43 & 5.220 & 6.13 & 14.90 & 11.70 \\
Pyrene & 37 & 0 & 37 & 6.470 & 8.14 & 26.20 & 18.40 \\
\hline & & & & &
\end{tabular}

${ }^{a} \mathrm{~N}=$ number of observations, possibly averages over replicates at sites; $\mathrm{I}=$ number of interval censored observations (see text); D = number of true detects (see text); UCB95 $=95 \%$ upper confidence bound for median; X95 = estimate of 95th percentile; LTB9595 $=95 \%$ lower confidence bound for 95th percentile; REMAINDER refers to the remaining observations-no detects.

${ }^{b}$ May be inappropriate when areas have different median analyte concentrations. Because of the statistical sampling design, it is important not to use combined results when the data from the different formation-locations are significantly different, because the error estimates will be inflated. If P-ORR $<0.01$ or P-GRP $<0.01$ ORR overall results should not be used. If P-CHICK $<.01$, or P-DGN 0.01, or P-CRC $<0.01$, then neither group nor the overall ORR results should be used. If P-CHICK $>0.01$ but P-DGN $<0.01$, one might be justified in combining the Chickamauga data, but not as it has been done here, because - the Dismal Gap and Nolichucky data are also combined. How to combine the data is complex, and should be done on an ad hoc, analyte-by-analyte basis. 
Appendix $\mathrm{H}$

NEUTRON ACTIVATION ANALYSIS (NAA) DATA 

H-3

Table H1. NAA analysis results for composite soil samples

\begin{tabular}{|c|c|c|c|c|c|c|c|c|c|}
\hline Analysis & Units & $\begin{array}{l}\text { A horizon } \\
\text { result }\end{array}$ & Qualifier & $\begin{array}{l}\text { B horizon } \\
\text { result }\end{array}$ & Qualifier & $\begin{array}{l}\mathrm{C} \text { horizon } \\
\text { result }\end{array}$ & Qualifier & $\begin{array}{l}\text { A lab split } \\
\text { result }\end{array}$ & Qualifier \\
\hline
\end{tabular}

Location=AND; Formation=COPPER RIDGE; Site=31,32,36; Sample type=Regular; $A=7059 ; B=7062 ; C=7065 ; A$ lab split $=$ '

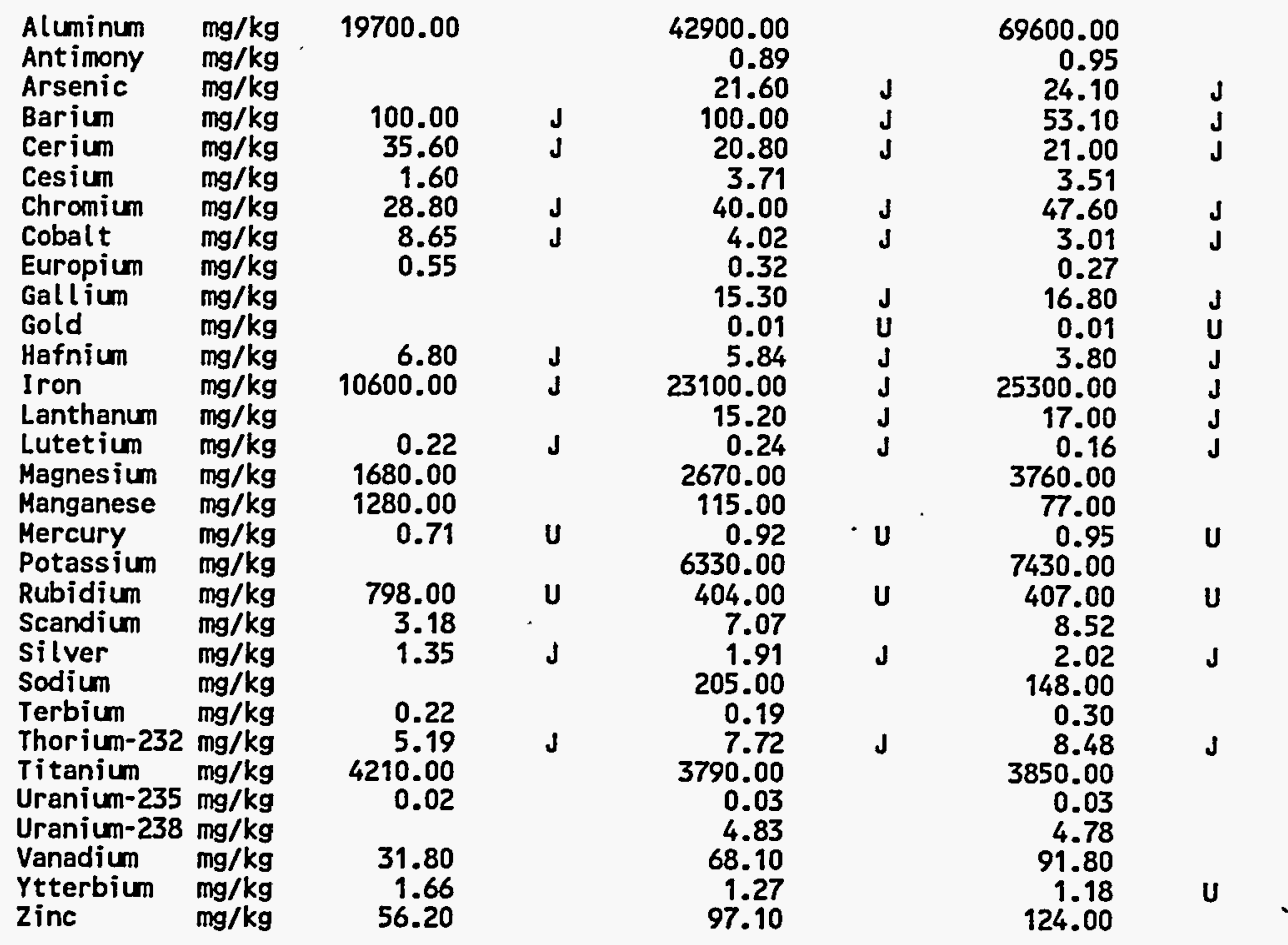

Location =AND; Formation=COPPER RIDGE; Site=33,34,35; Sample type=Regular; $A=7050 ; B=7053 ; C=7056 ; A$ lab split=' '

\begin{tabular}{|c|c|c|c|c|c|c|}
\hline $\begin{array}{l}\text { Aluminum } \\
\text { Antimony } \\
\text { Arsenic } \\
\text { Barjum } \\
\text { Cerium } \\
\text { Cesium } \\
\text { Chromium } \\
\text { Cobalt } \\
\text { Europium } \\
\text { Gal lium } \\
\text { Gold } \\
\text { Hafnium } \\
\text { Iron } \\
\text { Lanthanum } \\
\text { Lutetium } \\
\text { Magnesium } \\
\text { Manganese } \\
\text { Mercury } \\
\text { Potassium } \\
\text { Rubidjum } \\
\text { Scandium } \\
\text { Silver } \\
\text { Sodium } \\
\text { Terbium } \\
\text { Thorium-232 } \\
\text { Titanium } \\
\text { Uranium-235 } \\
\text { Uranium-238 } \\
\text { Vanadium } \\
\text { Ytterbium } \\
\text { Zinc }\end{array}$ & 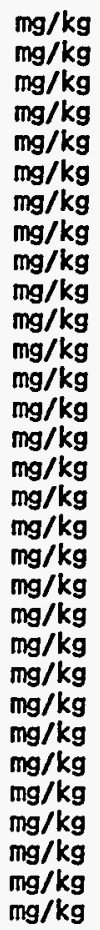 & $\begin{array}{r}22300.00 \\
0.87 \\
15.60 \\
495.00 \\
41.10 \\
1.66 \\
32.80 \\
9.39 \\
0.50 \\
7.45 \\
0.01 \\
7.74 \\
15500.00 \\
17.00 \\
0.23 \\
2960.00 \\
1440.00 \\
0.73 \\
3730.00 \\
1430.00 \\
3.63 \\
1.41 \\
435.00 \\
0.44 \\
6.50 \\
5160.00 \\
0.03 \\
4.45 \\
39.10 \\
1.88 \\
57.10\end{array}$ & $\begin{array}{l}\mathbf{J} \\
\mathbf{J} \\
\mathbf{J} \\
\mathbf{J} \\
\mathbf{J} \\
\mathbf{J} \\
\mathbf{U} \\
\mathbf{J} \\
\mathbf{J} \\
\mathbf{J} \\
\mathbf{J}\end{array}$ & $\begin{array}{r}59900.00 \\
0.89 \\
20.10 \\
76.30 \\
24.70 \\
3.78 \\
44.80 \\
5.58 \\
0.31 \\
15.80 \\
0.01 \\
5.20 \\
23900.00 \\
15.10 \\
0.16 \\
2240.00 \\
303.00 \\
0.82 \\
5360.00 \\
498.00 \\
6.89 \\
1.73 \\
256.00 \\
0.22 \\
7.94 \\
4720.00 \\
0.03 \\
3.54 \\
86.80 \\
1.02 \\
96.80\end{array}$ & $\begin{array}{l}\mathbf{J} \\
\mathbf{U} \\
\mathbf{J} \\
\mathbf{J} \\
\mathbf{J} \\
\mathbf{J}\end{array}$ & $\begin{array}{r}66800.00 \\
1.16 \\
30.90 \\
80.50 \\
26.60 \\
4.10 \\
52.40 \\
4.15 \\
0.25 \\
16.00 \\
0.01 \\
4.18 \\
33400.00 \\
20.90 \\
0.13 \\
3350.00 \\
121.00 \\
0.96 \\
5690.00 \\
628.00 \\
8.78 \\
2.01 \\
168.00 \\
0.37 \\
10.20 \\
3980.00 \\
0.03 \\
4.04 \\
97.00 \\
2.17 \\
127.00\end{array}$ \\
\hline
\end{tabular}




\section{H-4}

Table H.1 (continued)

\begin{tabular}{|c|c|c|c|c|c|c|c|c|c|}
\hline Analysis & Units & $\begin{array}{l}\text { A horizon } \\
\text { result }\end{array}$ & Qualifier & $\begin{array}{l}\text { B horizon } \\
\text { result }\end{array}$ & Qualifier & $\begin{array}{l}\mathrm{C} \text { horizon } \\
\text { result }\end{array}$ & Qualifier & $\begin{array}{l}\text { A lab split } \\
\text { result }\end{array}$ & Qualifier \\
\hline
\end{tabular}

Location $=A N D ;$ Formation $=$ COPPER RIDGE; Site=37,38,41; Sample type=Regular; $A=7077 ; B=7080 ; C=7083 ; A$ lab split=" '

\begin{tabular}{|c|c|c|c|c|c|c|}
\hline $\begin{array}{l}\text { Aluminum } \\
\text { Antimony } \\
\text { Arsenic } \\
\text { Barium } \\
\text { Cerium } \\
\text { Cesium } \\
\text { Chromium } \\
\text { Cobalt } \\
\text { Europium } \\
\text { Gallium } \\
\text { Gold } \\
\text { Hafnium } \\
\text { Iron } \\
\text { Lanthanum } \\
\text { Lutetium } \\
\text { Magnesium } \\
\text { Manganese } \\
\text { Mercury } \\
\text { Potassium } \\
\text { Rubidium } \\
\text { Scandium } \\
\text { Silver } \\
\text { Sodium } \\
\text { Terbium } \\
\text { Thorium-232 } \\
\text { Titanium } \\
\text { Uranium-235 } \\
\text { Uranium-238 } \\
\text { Vanadium } \\
\text { Ytterbium } \\
\text { Zinc }\end{array}$ & $\begin{array}{l}\mathrm{mg} / \mathrm{kg} \\
\mathrm{mg} / \mathrm{kg} \\
\mathrm{mg} / \mathrm{kg} \\
\mathrm{mg} / \mathrm{kg} \\
\mathrm{mg} / \mathrm{kg} \\
\mathrm{mg} / \mathrm{kg} \\
\mathrm{mg} / \mathrm{kg} \\
\mathrm{mg} / \mathrm{kg} \\
\mathrm{mg} / \mathrm{kg} \\
\mathrm{mg} / \mathrm{kg} \\
\mathrm{mg} / \mathrm{kg} \\
\mathrm{mg} / \mathrm{kg} \\
\mathrm{mg} / \mathrm{kg} \\
\mathrm{mg} / \mathrm{kg} \\
\mathrm{mg} / \mathrm{kg} \\
\mathrm{mg} / \mathrm{kg} \\
\mathrm{mg} / \mathrm{kg} \\
\mathrm{mg} / \mathrm{kg} \\
\mathrm{mg} / \mathrm{kg} \\
\mathrm{mg} / \mathrm{kg} \\
\mathrm{mg} / \mathrm{kg} \\
\mathrm{mg} / \mathrm{kg} \\
\mathrm{mg} / \mathrm{kg} \\
\mathrm{mg} / \mathrm{kg} \\
\mathrm{mg} / \mathrm{kg} \\
\mathrm{mg} / \mathrm{kg} \\
\mathrm{mg} / \mathrm{kg} \\
\mathrm{mg} / \mathrm{kg} \\
\mathrm{mg} / \mathrm{kg} \\
\mathrm{mg} / \mathrm{kg} \\
\mathrm{mg} / \mathrm{kg}\end{array}$ & $\begin{array}{r}31600.00 \\
0.60 \\
14.30 \\
707.00 \\
80.70 \\
2.28 \\
33.60 \\
46.10 \\
1.08 \\
14.50 \\
0.01 \\
7.06 \\
15700.00 \\
26.40 \\
0.30 \\
2440.00 \\
2360.00 \\
0.94 \\
4950.00 \\
2120.00 \\
4.84 \\
1.79 \\
520.00 \\
0.64 \\
6.78 \\
5740.00 \\
0.03 \\
4.96 \\
44.90 \\
2.20 \\
101.00\end{array}$ & $\begin{array}{l}\mathbf{J} \\
\mathbf{U} \\
\mathbf{J} \\
\mathbf{J} \\
\mathbf{J} \\
\mathbf{J}\end{array}$ & $\begin{array}{r}58900.00 \\
5.14 \\
3.03 \\
167.00 \\
59.70 \\
4.23 \\
46.10 \\
25.60 \\
0.50 \\
9.11 \\
0.19 \\
5.89 \\
30500.00 \\
5.76 \\
0.20 \\
3470.00 \\
755.00 \\
0.95 \\
189.00 \\
614.00 \\
11.00 \\
1.93 \\
6.88 \\
0.36 \\
11.00 \\
4690.00 \\
0.05 \\
17.20 \\
89.30 \\
2.03 \\
200.00\end{array}$ & $\begin{array}{l}\mathbf{J} \\
\mathbf{J} \\
\mathbf{J} \\
\mathbf{J}\end{array}$ & $\begin{array}{r}1.45 \\
38.00 \\
1260.00 \\
91.80 \\
4.78 \\
55.30 \\
35.30 \\
0.56 \\
10.30 \\
0.01 \\
5.22 \\
39600.00 \\
27.70 \\
0.18 \\
1460.00 \\
1.39 \\
8380.00 \\
998000.00 \\
16.60 \\
2.87 \\
208.00 \\
0.53 \\
14.10 \\
0.06 \\
7.89 \\
0.08 \\
276.00\end{array}$ \\
\hline
\end{tabular}

Location =AND; Formation=COPPER RIDGE; Site=39,40,42; Sample type=Regular; $A=7068 ; B=7071 ; C=7074 ; A$ lab split=' '

\begin{tabular}{|c|c|c|c|c|c|c|}
\hline $\begin{array}{l}\text { Aluminum } \\
\text { Antimony } \\
\text { Arsenic } \\
\text { Barium } \\
\text { Cerium } \\
\text { Cesium } \\
\text { Chromium } \\
\text { Cobalt } \\
\text { Europium } \\
\text { Gallium } \\
\text { Gold } \\
\text { Hafnium } \\
\text { Iron } \\
\text { Lanthanum } \\
\text { Lutetium } \\
\text { Magnesium } \\
\text { Manganese } \\
\text { Mercury } \\
\text { Potassium } \\
\text { Rubidium } \\
\text { Scandium } \\
\text { Silver } \\
\text { Sodium } \\
\text { Terbium } \\
\text { Thorium-23 } \\
\text { Titanium } \\
\text { Uranium-23 } \\
\text { Ulanim-23 }\end{array}$ & $\begin{array}{l}\mathrm{mg} / \mathrm{kg} \\
\mathrm{mg} / \mathrm{kg} \\
\mathrm{mg} / \mathrm{kg} \\
\mathrm{mg} / \mathrm{kg} \\
\mathrm{mg} / \mathrm{kg} \\
\mathrm{mg} / \mathrm{kg} \\
\mathrm{mg} / \mathrm{kg} \\
\mathrm{mg} / \mathrm{kg} \\
\mathrm{mg} / \mathrm{kg} \\
\mathrm{mg} / \mathrm{kg} \\
\mathrm{mg} / \mathrm{kg} \\
\mathrm{mg} / \mathrm{kg} \\
\mathrm{mg} / \mathrm{kg} \\
\mathrm{mg} / \mathrm{kg} \\
\mathrm{mg} / \mathrm{kg} \\
\mathrm{mg} / \mathrm{kg} \\
\mathrm{mg} / \mathrm{kg} \\
\mathrm{mg} / \mathrm{kg} \\
\mathrm{mg} / \mathrm{kg} \\
\mathrm{mg} / \mathrm{kg} \\
\mathrm{mg} / \mathrm{kg} \\
\mathrm{mg} / \mathrm{kg} \\
\mathrm{mg} / \mathrm{kg} \\
\mathrm{mg} / \mathrm{kg} \\
\mathrm{mg} / \mathrm{kg} \\
\mathrm{mg} / \mathrm{kg} \\
\mathrm{mg} / \mathrm{kg} \\
\mathrm{mg} / \mathrm{kg} \\
\mathrm{mg} / \mathrm{kg} \\
\mathrm{mg} / \mathrm{kg} \\
\mathrm{mg} / \mathrm{kg}\end{array}$ & $\begin{array}{r}31500.00 \\
0.85 \\
14.00 \\
253.00 \\
83.70 \\
2.77 \\
38.60 \\
14.80 \\
1.02 \\
14.70 \\
0.01 \\
8.79 \\
17400.00 \\
33.70 \\
0.33 \\
3410.00 \\
3150.00 \\
0.88 \\
5300.00 \\
2490.00 \\
5.37 \\
1.65 \\
643.00 \\
0.98 \\
7.93 \\
5620.00 \\
0.03 \\
5.07 \\
48.90 \\
4.49 \\
67.00\end{array}$ & $\begin{array}{l}\mathbf{J} \\
\mathbf{U} \\
\mathbf{J} \\
\mathbf{J} \\
\mathbf{J} \\
\mathbf{J} \\
\mathbf{U}\end{array}$ & $\begin{array}{r}54600.00 \\
0.40 \\
20.90 \\
151.00 \\
35.30 \\
4.15 \\
84.10 \\
9.79 \\
0.41 \\
14.10 \\
0.00 \\
7.39 \\
29600.00 \\
15.30 \\
0.27 \\
3580.00 \\
688.00 \\
1.04 \\
5510.00 \\
1150.00 \\
7.78 \\
2.02 \\
330.00 \\
0.42 \\
10.40 \\
5300.00 \\
0.04 \\
6.16 \\
103.00 \\
1.65 \\
86.50\end{array}$ & $\begin{array}{l}\mathbf{J} \\
\mathbf{J} \\
\mathbf{J} \\
\mathbf{J}\end{array}$ & $\begin{array}{r}73200.00 \\
0.49 \\
21.20 \\
92.10 \\
30.20 \\
4.25 \\
59.20 \\
6.99 \\
0.36 \\
7.89 \\
0.00 \\
6.08 \\
31700.00 \\
14.70 \\
0.22 \\
3760.00 \\
411.00 \\
1.01 \\
5570.00 \\
495.00 \\
8.26 \\
2.00 \\
217.00 \\
0.41 \\
11.20 \\
5190.00 \\
0.04 \\
5.65 \\
104.00 \\
1.79 \\
90.80\end{array}$ \\
\hline
\end{tabular}




\section{H-5}

Table H.1 (continued)

\begin{tabular}{|c|c|c|c|c|c|c|c|c|c|}
\hline Analysis & Units & $\begin{array}{l}\text { A horizon } \\
\text { result }\end{array}$ & Qualifier & $\begin{array}{l}\text { B horizon } \\
\text { result }\end{array}$ & Qualifier & $\begin{array}{l}\text { C horizon } \\
\text { result }\end{array}$ & Qualifier & $\begin{array}{l}\text { A lab split } \\
\text { result }\end{array}$ & Qualifier \\
\hline
\end{tabular}

Location=AND; Formation=DISMAL GAP; Site=1,20,22; Sample type=Regular; $\quad \therefore$ $A=7030 ; B=7033 ; C=7036 ; A$ lab split=' '

\begin{tabular}{|c|c|c|c|c|c|c|}
\hline $\begin{array}{l}\text { Aluminum } \\
\text { Antimony } \\
\text { Arsenic } \\
\text { Barium } \\
\text { Cerium } \\
\text { Cesium } \\
\text { Chromium } \\
\text { Cobalt } \\
\text { Europium } \\
\text { Gallium } \\
\text { Gold } \\
\text { Hafnium } \\
\text { Iron } \\
\text { Lanthanum } \\
\text { Lutetium } \\
\text { Magnesium } \\
\text { Manganese } \\
\text { Mercury } \\
\text { Potassium } \\
\text { Rubidium } \\
\text { Scandium } \\
\text { Silver } \\
\text { Sodium } \\
\text { Terbium } \\
\text { Thorium-232 } \\
\text { Titanium } \\
\text { Uranium-235 } \\
\text { Uranium-238 } \\
\text { Vanadium } \\
\text { Ytterbium } \\
\text { Zinc }\end{array}$ & 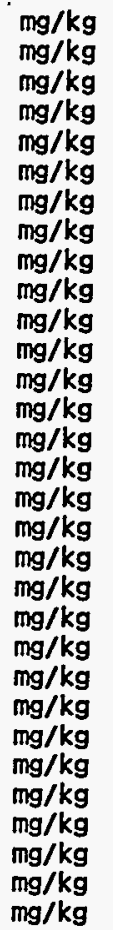 & $\begin{array}{r}67900.00 \\
0.42 \\
5.54 \\
323.00 \\
54.70 \\
3.26 \\
62.40 \\
10.30 \\
0.61 \\
16.20 \\
0.01 \\
9.58 \\
31300.00 \\
31.40 \\
0.36 \\
5610.00 \\
877.00 \\
1.29 \\
22300.00 \\
1050.00 \\
9.00 \\
2.20 \\
703.00 \\
0.24 \\
10.40 \\
5270.00 \\
0.02 \\
3.40 \\
83.80 \\
2.87 \\
101.00\end{array}$ & $\begin{array}{l}\mathbf{J} \\
\mathbf{U} \\
\mathbf{J} \\
\mathrm{J} \\
\mathbf{J} \\
\mathbf{J}\end{array}$ & $\begin{array}{r}93300.00 \\
0.49 \\
5.35 \\
336.00 \\
91.10 \\
4.59 \\
89.30 \\
30.30 \\
0.91 \\
10.40 \\
0.01 \\
15.10 \\
43600.00 \\
41.10 \\
0.39 \\
7960.00 \\
307.00 \\
1.77 \\
25000.00 \\
856.00 \\
12.90 \\
3.10 \\
615.00 \\
0.49 \\
14.80 \\
5470.00 \\
0.02 \\
2.83 \\
100.00 \\
3.21 \\
128.00\end{array}$ & $\begin{array}{l}\mathbf{J} \\
\mathbf{U} \\
\mathbf{J} \\
\mathbf{J} \\
\mathbf{J} \\
\mathbf{J}\end{array}$ & $\begin{array}{r}101000.00 \\
0.16 \\
3.44 \\
307.00 \\
111.00 \\
4.02 \\
79.80 \\
20.00 \\
1.71 \\
17.00 \\
0.01 \\
7.66 \\
47300.00 \\
47.40 \\
0.34 \\
7710.00 \\
865.00 \\
1.52 \\
29800.00 \\
1000.00 \\
16.20 \\
2.80 \\
1600.00 \\
1.52 \\
12.50 \\
5370.00 \\
0.02 \\
2.21 \\
105.00 \\
1.18 \\
25.10\end{array}$ \\
\hline
\end{tabular}

Location=AND; Formation=DISMAL GAP; Site=3,5,11; Sample type=Regular; $A=7012 ; B=7015 ; C=7018 ; A$ lab split=" '

\begin{tabular}{|c|c|c|c|c|c|c|}
\hline $\begin{array}{l}\text { Aluminum } \\
\text { Antimony } \\
\text { Arsenic } \\
\text { Barium } \\
\text { Cerium } \\
\text { Cesium } \\
\text { Chromium } \\
\text { Cobalt } \\
\text { Europium } \\
\text { Gallium } \\
\text { Gold } \\
\text { Hafnium } \\
\text { Iron } \\
\text { Lanthanum } \\
\text { Lutetium } \\
\text { Magnesium } \\
\text { Manganese } \\
\text { Mercury } \\
\text { Potassium } \\
\text { Rubidium } \\
\text { Scandium } \\
\text { Silver } \\
\text { Sodium } \\
\text { Terbium } \\
\text { Thorium-232 } \\
\text { Titanium } \\
\text { Uranium-235 } \\
\text { Uranium-238 } \\
\text { Vanadium } \\
\text { Ytterbium } \\
\text { Zinc }\end{array}$ & $\begin{array}{l}\mathrm{mg} / \mathrm{kg} \\
\mathrm{mg} / \mathrm{kg} \\
\mathrm{mg} / \mathrm{kg} \\
\mathrm{mg} / \mathrm{kg} \\
\mathrm{mg} / \mathrm{kg} \\
\mathrm{mg} / \mathrm{kg} \\
\mathrm{mg} / \mathrm{kg} \\
\mathrm{mg} / \mathrm{kg} \\
\mathrm{mg} / \mathrm{kg} \\
\mathrm{mg} / \mathrm{kg} \\
\mathrm{mg} / \mathrm{kg} \\
\mathrm{mg} / \mathrm{kg} \\
\mathrm{mg} / \mathrm{kg} \\
\mathrm{mg} / \mathrm{kg} \\
\mathrm{mg} / \mathrm{kg} \\
\mathrm{mg} / \mathrm{kg} \\
\mathrm{mg} / \mathrm{kg} \\
\mathrm{mg} / \mathrm{kg} \\
\mathrm{mg} / \mathrm{kg} \\
\mathrm{mg} / \mathrm{kg} \\
\mathrm{mg} / \mathrm{kg} \\
\mathrm{mg} / \mathrm{kg} \\
\mathrm{mg} / \mathrm{kg} \\
\mathrm{mg} / \mathrm{kg} \\
\mathrm{mg} / \mathrm{kg} \\
\mathrm{mg} / \mathrm{kg} \\
\mathrm{mg} / \mathrm{kg} \\
\mathrm{mg} / \mathrm{kg} \\
\mathrm{mg} / \mathrm{kg} \\
\mathrm{mg} / \mathrm{kg} \\
\mathrm{mg} / \mathrm{kg}\end{array}$ & $\begin{array}{r}34.00 \\
6030.00 \\
581.00 \\
34600.00 \\
766.00\end{array}$ & $\underset{\mathbf{U}}{\mathbf{J}}$ & $\begin{array}{r}107000.00 \\
0.47 \\
7.46 \\
402.00 \\
64.50 \\
5.28 \\
90.20 \\
14.10 \\
0.60 \\
14.70 \\
0.01 \\
5.60 \\
53600.00 \\
42.10 \\
0.25 \\
8810.00 \\
194.00 \\
1.53 \\
35300.00 \\
601.00 \\
13.90 \\
2.82 \\
840.00 \\
0.12 \\
14.40 \\
5870.00 \\
0.02 \\
3.30 \\
112.00 \\
2.73 \\
56.80\end{array}$ & $\begin{array}{l}\mathbf{J} \\
\mathbf{U} \\
\mathbf{J} \\
\mathbf{J} \\
\mathbf{J} \\
\mathbf{J}\end{array}$ & $\begin{array}{r}115000.00 \\
0.08 \\
3.77 \\
313.00 \\
80.80 \\
5.47 \\
95.50 \\
12.90 \\
0.92 \\
21.20 \\
0.01 \\
4.39 \\
52400.00 \\
42.90 \\
0.26 \\
10700.00 \\
464.00 \\
1.71 \\
35900.00 \\
752.00 \\
19.00 \\
3.13 \\
1390.00 \\
0.48 \\
14.60 \\
4170.00 \\
0.02 \\
2.69 \\
114.00 \\
3.15 \\
102.00\end{array}$ \\
\hline
\end{tabular}


Table H.1 (continued)

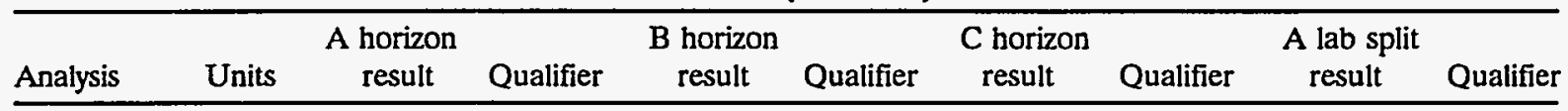

Location $=A N D ;$ Formation $=D I S M A L$ GAP; Site $=4,12,21 ;$ Sample type $=$ Regular; $A=7021 ; B=7024 ; C=7027 ; A$ lab split $=$ '

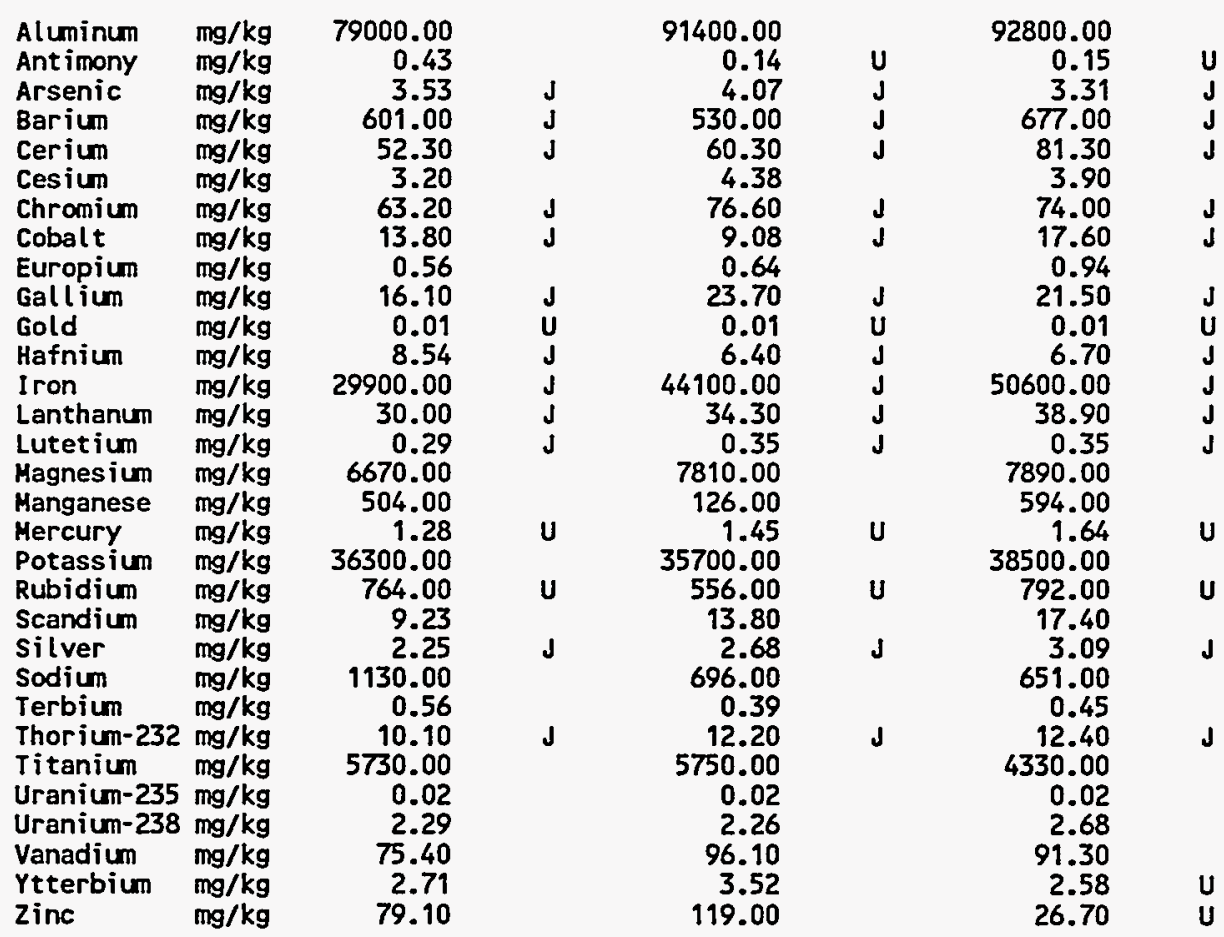

Location $=A N D ;$ Formation $=D I S M A L$ GAP; Site=9,10,19; Sample type=Regular; $A=7003 ; B=7006 ; C=7009 ; A$ lab split=" '

\begin{tabular}{|c|c|c|c|c|c|c|}
\hline $\begin{array}{l}\text { Aluminum } \\
\text { Antimony } \\
\text { Arsenic } \\
\text { Barium } \\
\text { Cerium } \\
\text { Cesium } \\
\text { Chromium } \\
\text { Cobalt } \\
\text { Europium } \\
\text { Gallium } \\
\text { Gold } \\
\text { Hafnium } \\
\text { Iron } \\
\text { Lanthanum } \\
\text { Lutetium } \\
\text { Magnesium } \\
\text { Manganese } \\
\text { Mercury } \\
\text { Potassium } \\
\text { Rubidium } \\
\text { Scandium } \\
\text { Silver } \\
\text { Sodium } \\
\text { Terbium } \\
\text { Thorium-23 } \\
\text { Titanium } \\
\text { Uranium-23 } \\
\text { Uranium-23 } \\
\text { Vanadium } \\
\text { Ytterbium } \\
\text { Zinc }\end{array}$ & $\begin{array}{l}\mathrm{mg} / \mathrm{kg} \\
\mathrm{mg} / \mathrm{kg} \\
\mathrm{mg} / \mathrm{kg} \\
\mathrm{mg} / \mathrm{kg} \\
\mathrm{mg} / \mathrm{kg} \\
\mathrm{mg} / \mathrm{kg} \\
\mathrm{mg} / \mathrm{kg} \\
\mathrm{mg} / \mathrm{kg} \\
\mathrm{mg} / \mathrm{kg} \\
\mathrm{mg} / \mathrm{kg} \\
\mathrm{mg} / \mathrm{kg} \\
\mathrm{mg} / \mathrm{kg} \\
\mathrm{mg} / \mathrm{kg} \\
\mathrm{mg} / \mathrm{kg} \\
\mathrm{mg} / \mathrm{kg} \\
\mathrm{mg} / \mathrm{kg} \\
\mathrm{mg} / \mathrm{kg} \\
\mathrm{mg} / \mathrm{kg} \\
\mathrm{mg} / \mathrm{kg} \\
\mathrm{mg} / \mathrm{kg} \\
\mathrm{mg} / \mathrm{kg} \\
\mathrm{mg} / \mathrm{kg} \\
\mathrm{mg} / \mathrm{kg} \\
\mathrm{mg} / \mathrm{kg} \\
\mathrm{mg} / \mathrm{kg} \\
\mathrm{mg} / \mathrm{kg} \\
\mathrm{mg} / \mathrm{kg} \\
\mathrm{mg} / \mathrm{kg} \\
\mathrm{mg} / \mathrm{kg} \\
\mathrm{mg} / \mathrm{kg} \\
\mathrm{mg} / \mathrm{kg}\end{array}$ & $\begin{array}{r}56000.00 \\
0.45 \\
5.72 \\
280.00 \\
59.80 \\
2.73 \\
61.30 \\
16.00 \\
0.65 \\
16.20 \\
0.01 \\
10.60 \\
2660.00 \\
31.50 \\
0.34 \\
4880.00 \\
913.00 \\
1.27 \\
16700.00 \\
685.00 \\
8.47 \\
2.32 \\
541.00 \\
0.34 \\
9.68 \\
5480.00 \\
0.02 \\
3.28 \\
65.50 \\
2.36 \\
21.30\end{array}$ & $\begin{array}{l}\mathbf{J} \\
\mathbf{U} \\
\mathbf{J} \\
\mathbf{J} \\
\mathbf{J} \\
\mathbf{J}\end{array}$ & $\begin{array}{r}76400.00 \\
0.36 \\
6.25 \\
247.00 \\
69.50 \\
4.74 \\
81.00 \\
20.00 \\
0.80 \\
11.70 \\
0.01 \\
8.05 \\
41000.00 \\
39.20 \\
0.32 \\
6420.00 \\
311.00 \\
1.36 \\
20200.00 \\
522.00 \\
12.50 \\
2.47 \\
422.00 \\
0.46 \\
12.10 \\
5870.00 \\
0.03 \\
3.29 \\
96.10 \\
3.92 \\
93.90\end{array}$ & $\begin{array}{l}\mathbf{J} \\
\mathbf{U} \\
\mathbf{J} \\
\mathbf{J} \\
\mathbf{J} \\
\mathbf{J}\end{array}$ & $\begin{array}{r}104000.00 \\
0.34 \\
5.17 \\
244.00 \\
82.10 \\
4.55 \\
100.00 \\
22.90 \\
1.11 \\
21.20 \\
0.01 \\
6.22 \\
50100.00 \\
39.80 \\
0.36 \\
7090.00 \\
755.00 \\
0.49 \\
24800.00 \\
974.00 \\
18.50 \\
0.95 \\
284.00 \\
1.15 \\
13.30 \\
5470.00 \\
0.02 \\
3.23 \\
109.00 \\
3.31 \\
121.00\end{array}$ \\
\hline
\end{tabular}


H-7

Table H.1 (continued)

\begin{tabular}{|c|c|c|c|c|c|c|c|c|c|}
\hline Analysis & Units & $\begin{array}{c}\text { A horizon } \\
\text { result }\end{array}$ & Qualifier & $\begin{array}{l}\text { B horizon } \\
\text { result }\end{array}$ & Qualifier & $\begin{array}{l}\mathrm{C} \text { horizon } \\
\text { result }\end{array}$ & Qualifier & $\begin{array}{l}\text { A lab split } \\
\text { result }\end{array}$ & Qualifier \\
\hline
\end{tabular}

\begin{tabular}{|c|c|c|c|c|c|c|}
\hline $\begin{array}{l}\text { Aluminum } \\
\text { Antimony } \\
\text { Arsenic } \\
\text { Barium } \\
\text { Cerium } \\
\text { Cesium } \\
\text { Chromium } \\
\text { Cobalt } \\
\text { Europium } \\
\text { Gallium } \\
\text { Gold } \\
\text { Hafnium } \\
\text { Iron } \\
\text { Lanthanum } \\
\text { Lutetium } \\
\text { Magnesium } \\
\text { Manganese } \\
\text { Mercury } \\
\text { Potassium } \\
\text { Rubidium } \\
\text { Scandium } \\
\text { Silver } \\
\text { Sodium } \\
\text { Terbium } \\
\text { Thorium-232 } \\
\text { Titanium } \\
\text { Uranium-235 } \\
\text { Uranium-238 } \\
\text { Vanadium } \\
\text { Ytterbium } \\
\text { Zinc }\end{array}$ & $\begin{array}{l}\mathrm{mg} / \mathrm{kg} \\
\mathrm{mg} / \mathrm{kg} \\
\mathrm{mg} / \mathrm{kg} \\
\mathrm{mg} / \mathrm{kg} \\
\mathrm{mg} / \mathrm{kg} \\
\mathrm{mg} / \mathrm{kg} \\
\mathrm{mg} / \mathrm{kg} \\
\mathrm{mg} / \mathrm{kg} \\
\mathrm{mg} / \mathrm{kg} \\
\mathrm{mg} / \mathrm{kg} \\
\mathrm{mg} / \mathrm{kg} \\
\mathrm{mg} / \mathrm{kg} \\
\mathrm{mg} / \mathrm{kg} \\
\mathrm{mg} / \mathrm{kg} \\
\mathrm{mg} / \mathrm{kg} \\
\mathrm{mg} / \mathrm{kg} \\
\mathrm{mg} / \mathrm{kg} \\
\mathrm{mg} / \mathrm{kg} \\
\mathrm{mg} / \mathrm{kg} \\
\mathrm{mg} / \mathrm{kg} \\
\mathrm{mg} / \mathrm{kg} \\
\mathrm{mg} / \mathrm{kg} \\
\mathrm{mg} / \mathrm{kg} \\
\mathrm{mg} / \mathrm{kg} \\
\mathrm{mg} / \mathrm{kg} \\
\mathrm{mg} / \mathrm{kg} \\
\mathrm{mg} / \mathrm{kg} \\
\mathrm{mg} / \mathrm{kg} \\
\mathrm{mg} / \mathrm{kg} \\
\mathrm{mg} / \mathrm{kg} \\
\mathrm{mg} / \mathrm{kg}\end{array}$ & $\begin{array}{r}58900.00 \\
0.48 \\
5.73 \\
225.00 \\
61.90 \\
3.24 \\
66.00 \\
21.80 \\
0.65 \\
11.60 \\
0.00 \\
10.60 \\
30100.00 \\
32.00 \\
0.31 \\
6140.00 \\
964.00 \\
1.29 \\
18500.00 \\
923.00 \\
9.05 \\
2.12 \\
524.00 \\
0.30 \\
9.93 \\
5810.00 \\
0.02 \\
3.33 \\
74.50 \\
2.17 \\
20.40\end{array}$ & $\begin{array}{l}\mathbf{J} \\
\mathbf{J} \\
\mathbf{J} \\
\mathbf{J} \\
\mathbf{J} \\
\mathbf{J}\end{array}$ & $\begin{array}{r}82400.00 \\
0.50 \\
6.99 \\
306.00 \\
69.40 \\
5.13 \\
81.90 \\
14.70 \\
0.70 \\
20.80 \\
0.01 \\
7.69 \\
42200.00 \\
39.50 \\
0.28 \\
6510.00 \\
205.00 \\
1.47 \\
20400.00 \\
386.00 \\
12.70 \\
2.64 \\
423.00 \\
0.44 \\
12.30 \\
5990.00 \\
0.03 \\
3.75 \\
97.20 \\
3.98 \\
106.00\end{array}$ & $\begin{array}{l}\mathbf{J} \\
\boldsymbol{U} \\
\mathbf{J} \\
\mathbf{J} \\
\mathbf{J} \\
\mathbf{J}\end{array}$ & $\begin{array}{r}97100.00 \\
0.17 \\
5.96 \\
135.00 \\
104.00 \\
4.30 \\
83.10 \\
31.20 \\
1.55 \\
17.80 \\
0.01 \\
6.73 \\
50500.00 \\
48.10 \\
0.52 \\
8120.00 \\
1520.00 \\
0.76 \\
23700.00 \\
1370.00 \\
19.60 \\
3.69 \\
300.00 \\
0.47 \\
13.70 \\
5050.00 \\
0.03 \\
3.13 \\
103.00 \\
0.70 \\
163.00\end{array}$ \\
\hline
\end{tabular}

Location $=O R R ;$ Formation $=$ CHEPULTEPEC; Site $=50,66,73 ;$ Sample type $=$ Regular; $A=5169 ; B=5172 ; C=5183 ; A$ lab split $=5215$

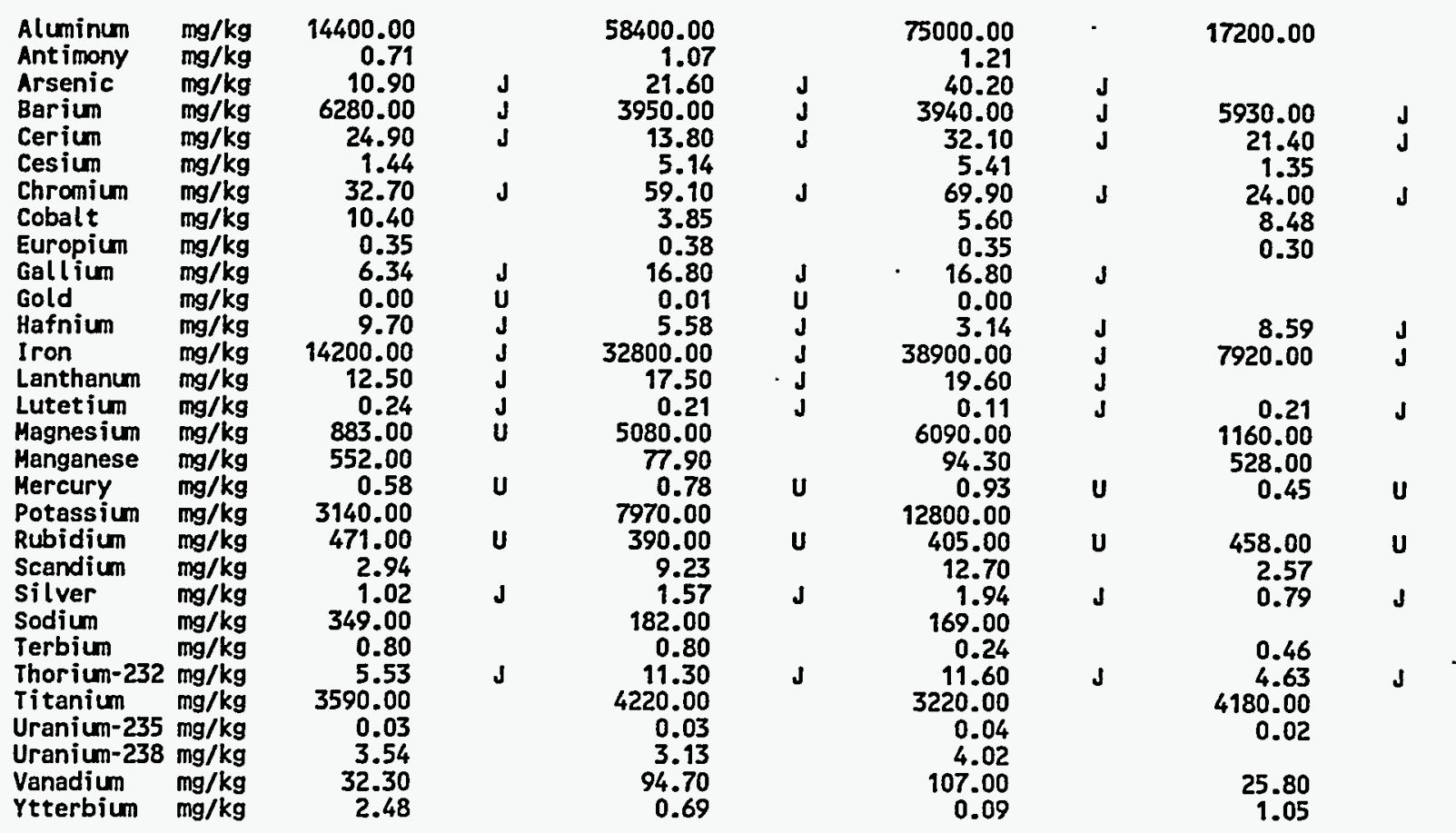


$\mathrm{H}-8$

Table H.1 (continued)

\begin{tabular}{|c|c|c|c|c|c|c|c|c|c|}
\hline Analysis & Units & $\begin{array}{c}\text { A horizon } \\
\text { result }\end{array}$ & Qualifier & $\begin{array}{l}\text { B horizon } \\
\text { result }\end{array}$ & Qualifier & $\begin{array}{l}C \text { horizon } \\
\text { result }\end{array}$ & Qualifier & $\begin{array}{l}\text { A lab split } \\
\text { result }\end{array}$ & Qualifier \\
\hline
\end{tabular}

Location $=$ ORR; Formation $=$ CHEPULTEPEC; Site $=50,66,73$; Sample type $=$ Field Duplicate; $A=5177 ; B=5180 ; C=5186 ; A$ lab split $=5214$

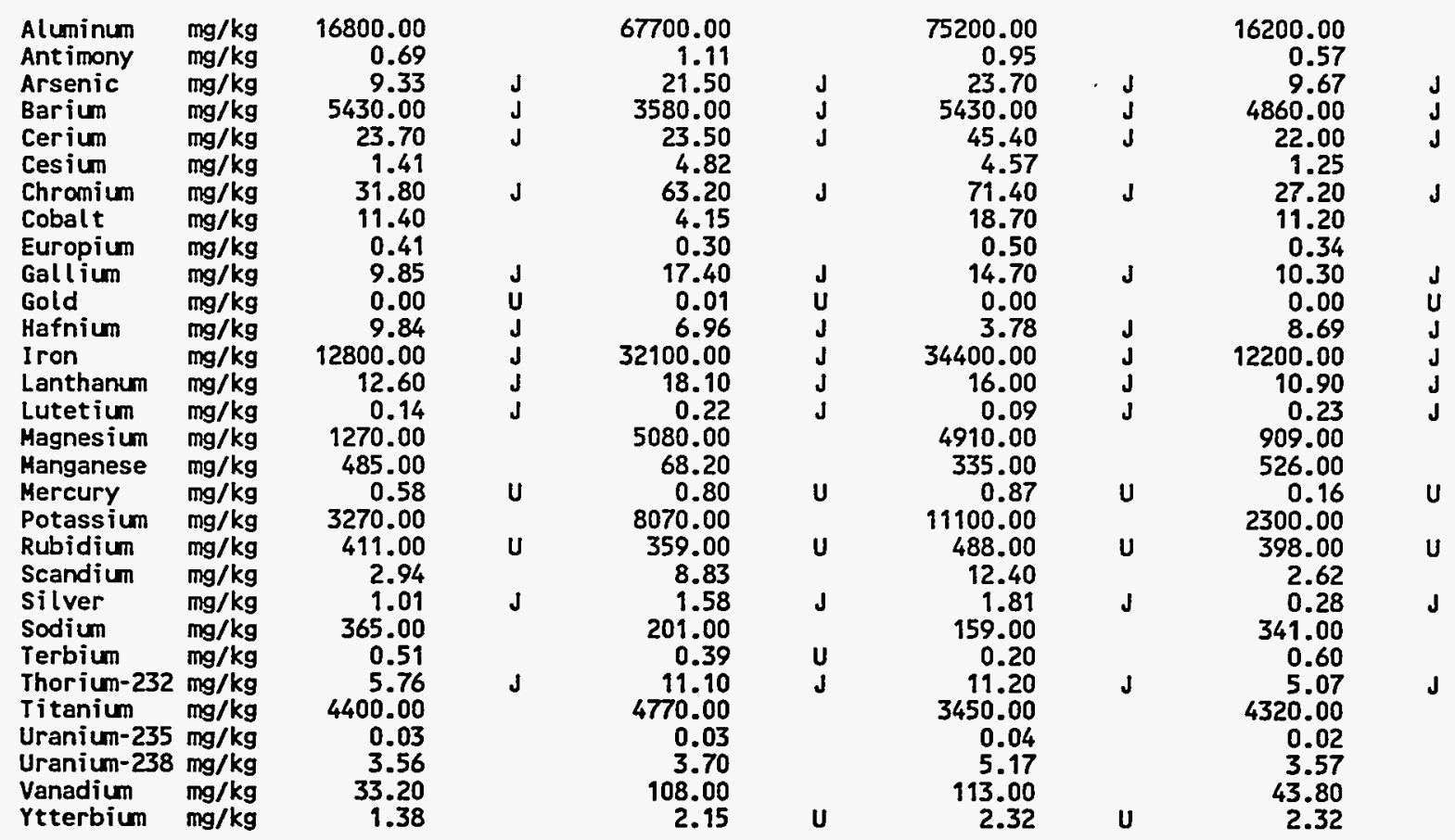

Location $=O R R ;$ Formation $=C H E P U L T E P E C ;$ Site $=52,53,78$; Sample type $=$ Regular; $A=5129 ; B=5132 ; C=5135 ; A$ lab split $=$ "'

\begin{tabular}{|c|c|c|c|c|c|c|}
\hline $\begin{array}{l}\text { Aluminum } \\
\text { Antimony } \\
\text { Arsenic } \\
\text { Barium } \\
\text { Cerium } \\
\text { Cesium } \\
\text { Chromium } \\
\text { Cobalt } \\
\text { Europium } \\
\text { Gallium } \\
\text { Gold } \\
\text { Hafnium } \\
\text { Iron } \\
\text { Lanthanum } \\
\text { Lutetium } \\
\text { Magnesium } \\
\text { Manganese } \\
\text { Mercury } \\
\text { Potassium } \\
\text { Rubidium } \\
\text { Scandium } \\
\text { Silver } \\
\text { Sodium } \\
\text { Terbium } \\
\text { Thorium-232 } \\
\text { Titanium } \\
\text { Uranium-235 } \\
\text { Uranium-238 } \\
\text { Vanadium } \\
\text { Ytterbium } \\
\text { Zinc }\end{array}$ & $\begin{array}{l}\mathrm{mg} / \mathrm{kg} \\
\mathrm{mg} / \mathrm{kg} \\
\mathrm{mg} / \mathrm{kg} \\
\mathrm{mg} / \mathrm{kg} \\
\mathrm{mg} / \mathrm{kg} \\
\mathrm{mg} / \mathrm{kg} \\
\mathrm{mg} / \mathrm{kg} \\
\mathrm{mg} / \mathrm{kg} \\
\mathrm{mg} / \mathrm{kg} \\
\mathrm{mg} / \mathrm{kg} \\
\mathrm{mg} / \mathrm{kg} \\
\mathrm{mg} / \mathrm{kg} \\
\mathrm{mg} / \mathrm{kg} \\
\mathrm{mg} / \mathrm{kg} \\
\mathrm{mg} / \mathrm{kg} \\
\mathrm{mg} / \mathrm{kg} \\
\mathrm{mg} / \mathrm{kg} \\
\mathrm{mg} / \mathrm{kg} \\
\mathrm{mg} / \mathrm{kg} \\
\mathrm{mg} / \mathrm{kg} \\
\mathrm{mg} / \mathrm{kg} \\
\mathrm{mg} / \mathrm{kg} \\
\mathrm{mg} / \mathrm{kg} \\
\mathrm{mg} / \mathrm{kg} \\
\mathrm{mg} / \mathrm{kg} \\
\mathrm{mg} / \mathrm{kg} \\
\mathrm{mg} / \mathrm{kg} \\
\mathrm{mg} / \mathrm{kg} \\
\mathrm{mg} / \mathrm{kg} \\
\mathrm{mg} / \mathrm{kg} \\
\mathrm{mg} / \mathrm{kg}\end{array}$ & $\begin{array}{r}15400.00 \\
0.59 \\
7.95 \\
102.00 \\
32.60 \\
1.31 \\
26.10 \\
7.89 \\
0.46 \\
2.76 \\
0.00 \\
7.49 \\
9870.00 \\
15.60 \\
0.24 \\
1450.00 \\
905.00 \\
0.61 \\
2880.00 \\
661.00 \\
2.72 \\
0.90 \\
385.00 \\
0.44 \\
4.94 \\
3840.00 \\
0.03 \\
4.01 \\
32.00 \\
2.60 \\
44.60\end{array}$ & $\begin{array}{l}\mathbf{J} \\
\mathbf{U} \\
\mathbf{J} \\
\mathbf{J} \\
\mathbf{J} \\
\mathbf{J}\end{array}$ & $\begin{array}{r}48700.00 \\
0.99 \\
27.10 \\
94.20 \\
20.50 \\
4.05 \\
64.10 \\
2.98 \\
0.31 \\
13.60 \\
0.00 \\
5.18 \\
33600.00 \\
17.60 \\
0.20 \\
3670.00 \\
68.00 \\
0.77 \\
6720.00 \\
416.00 \\
6.21 \\
1.27 \\
213.00 \\
0.29 \\
9.01 \\
4290.00 \\
0.03 \\
5.08 \\
80.80 \\
2.26 \\
126.00\end{array}$ & $\begin{array}{l}\mathbf{J} \\
\mathbf{U} \\
\mathbf{J} \\
\mathbf{J} \\
\mathbf{J} \\
\mathbf{J}\end{array}$ & $\begin{array}{r}69900.00 \\
1.09 \\
41.70 \\
419.00 \\
19.50 \\
4.52 \\
145.00 \\
4.96 \\
0.31 \\
19.30 \\
0.00 \\
2.83 \\
39300.00 \\
26.60 \\
0.14 \\
6590.00 \\
88.00 \\
0.89 \\
12400.00 \\
1710.00 \\
10.20 \\
1.52 \\
163.00 \\
0.33 \\
10.50 \\
3490.00 \\
0.04 \\
5.60 \\
117.00 \\
2.34 \\
282.00\end{array}$ \\
\hline
\end{tabular}


H-9

Table H.1 (continued)

\begin{tabular}{|c|c|c|c|c|c|c|c|c|c|}
\hline Analysis & Units & $\begin{array}{l}\text { A horizon } \\
\text { result }\end{array}$ & Qualifier & $\begin{array}{l}\text { B horizon } \\
\text { result }\end{array}$ & Qualifier & $\begin{array}{l}C \text { horizon } \\
\text { result }\end{array}$ & Qualifier & $\begin{array}{l}\text { A lab split } \\
\text { result }\end{array}$ & Qualifier \\
\hline
\end{tabular}

$\because$ Location $=$ ORR; Formation $=$ CHEPULTEPEC; Site $=68,74,90 ;$ Sample type $=$ Regular; $\quad \because \cdots, 413$ $A=5158 ; B=5161 ; C=5164 ; A$ lab split $=$ "

\begin{tabular}{|c|c|c|c|c|c|c|}
\hline $\begin{array}{l}\text { Aluminum } \\
\text { Ant imony } \\
\text { Arsenic } \\
\text { Barium } \\
\text { Cerium } \\
\text { Cesium } \\
\text { Chromium } \\
\text { Cobalt }\end{array}$ & $\begin{array}{l}\mathrm{mg} / \mathrm{kg} \\
\mathrm{mg} / \mathrm{kg} \\
\mathrm{mg} / \mathrm{kg} \\
\mathrm{mg} / \mathrm{kg} \\
\mathrm{mg} / \mathrm{kg} \\
\mathrm{mg} / \mathrm{kg} \\
\mathrm{mg} / \mathrm{kg} \\
\mathrm{mg} / \mathrm{kg} \\
\mathrm{mg} / \mathrm{kg} \\
\mathrm{mg} / \mathrm{kg} \\
\mathrm{mg} / \mathrm{kg} \\
\mathrm{mg} / \mathrm{kg} \\
\mathrm{mg} / \mathrm{kg} \\
\mathrm{mg} / \mathrm{kg} \\
\mathrm{mg} / \mathrm{kg} \\
\mathrm{mg} / \mathrm{kg} \\
\mathrm{mg} / \mathrm{kg} \\
\mathrm{mg} / \mathrm{kg} \\
\mathrm{mg} / \mathrm{kg} \\
\mathrm{mg} / \mathrm{kg} \\
\mathrm{mg} / \mathrm{kg} \\
\mathrm{mg} / \mathrm{kg} \\
\mathrm{mg} / \mathrm{kg} \\
\mathrm{mg} / \mathrm{kg} \\
\mathrm{mg} / \mathrm{kg} \\
\mathrm{mg} / \mathrm{kg} \\
\mathrm{mg} / \mathrm{kg} \\
\mathrm{mg} / \mathrm{kg} \\
\mathrm{mg} / \mathrm{kg} \\
\mathrm{mg} / \mathrm{kg} \\
\mathrm{mg} / \mathrm{kg}\end{array}$ & $\begin{array}{r}15700.00 \\
0.59 \\
7.39 \\
148.00 \\
24.90 \\
1.39 \\
36.60 \\
12.20 \\
0.41 \\
10.10 \\
0.00 \\
7.01 \\
14600.00 \\
13.00 \\
0.23 \\
945.00 \\
446.00 \\
0.66 \\
2660.00 \\
453.00 \\
2.68 \\
0.98 \\
355.00 \\
0.33 \\
5.29 \\
4390.00 \\
0.02 \\
4.49 \\
31.80 \\
2.84 \\
40.60\end{array}$ & $\begin{array}{l}\mathbf{J} \\
\mathbf{U} \\
\mathbf{J} \\
\mathbf{J} \\
\mathbf{J} \\
\mathbf{J}\end{array}$ & $\begin{array}{r}6960.00 \\
1.01 \\
20.80 \\
94.10 \\
21.00 \\
5.38 \\
54.40 \\
3.06 \\
0.33 \\
20.90 \\
0.01 \\
4.12 \\
33400.00 \\
20.70 \\
0.15 \\
5600.00 \\
63.40 \\
0.88 \\
9250.00 \\
458.00 \\
8.14 \\
1.46 \\
200.00 \\
0.31 \\
10.70 \\
4100.00 \\
0.03 \\
4.68 \\
106.00 \\
2.25 \\
132.00\end{array}$ & $\begin{array}{l}\mathbf{J} \\
\mathbf{U} \\
\mathbf{J} \\
\mathbf{J} \\
\mathbf{J} \\
\mathbf{J}\end{array}$ & $\begin{array}{r}87900.00 \\
1.11 \\
20.30 \\
111.00 \\
26.70 \\
6.39 \\
65.20 \\
3.54 \\
0.36 \\
20.40 \\
0.01 \\
2.85 \\
40700.00 \\
25.80 \\
0.14 \\
5980.00 \\
67.50 \\
1.04 \\
11600.00 \\
587.00 \\
11.30 \\
1.72 \\
165.00 \\
0.22 \\
12.70 \\
3450.00 \\
0.03 \\
4.56 \\
116.00 \\
0.60 \\
169.00\end{array}$ \\
\hline
\end{tabular}

Location=ORR; Formation=CHEPULTEPEC; Site=77,85,86; Sample type=Regular; $A=5138 ; B=5141 ; C=5144 ; A$ lab split =' '

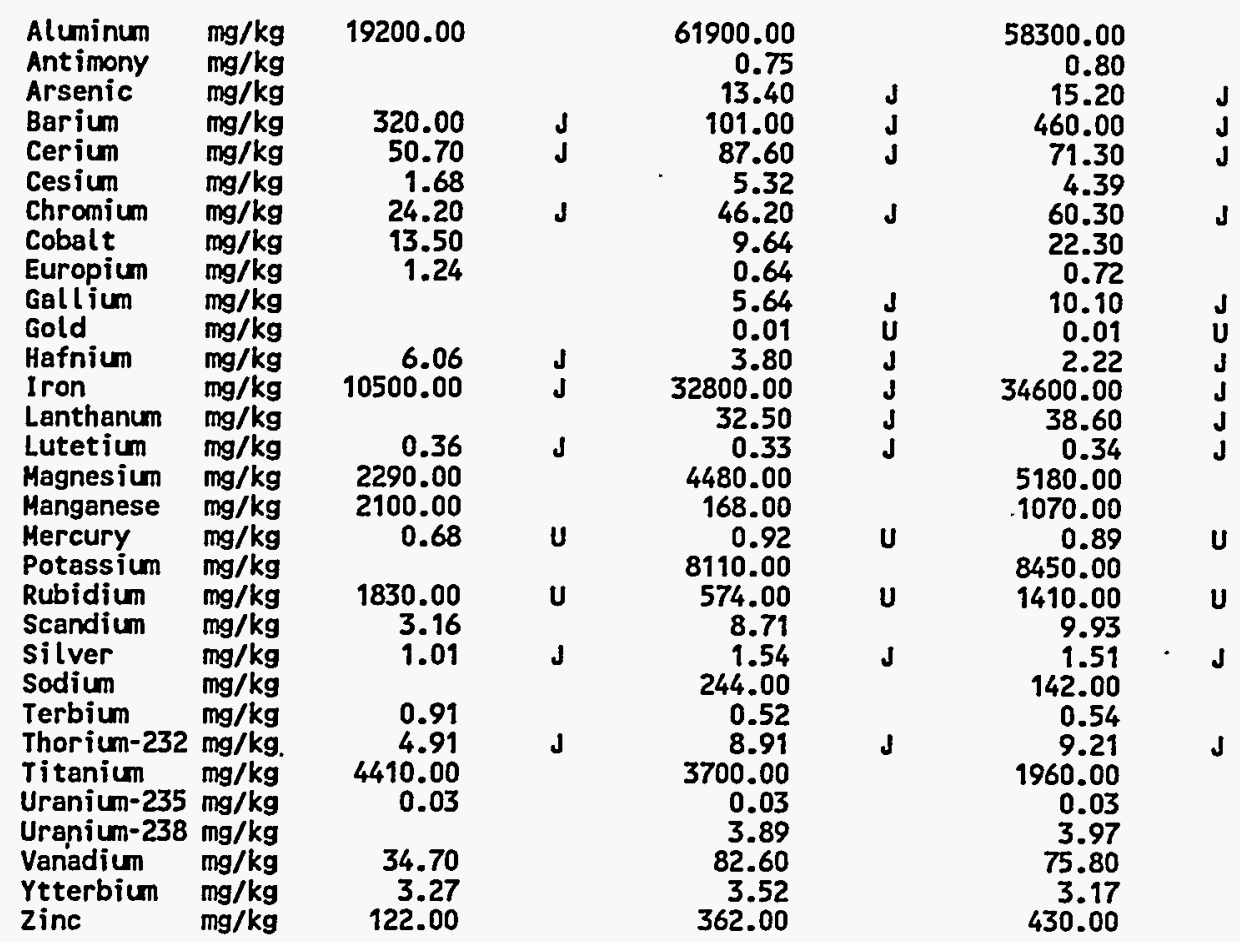


H-10

Table H.1 (continued)

\begin{tabular}{|c|c|c|c|c|c|c|c|c|c|}
\hline Analysis & Units & $\begin{array}{l}\text { A horizon } \\
\text { result }\end{array}$ & Qualifier & $\begin{array}{l}\text { B horizon } \\
\text { result }\end{array}$ & Qualifier & $\begin{array}{l}\mathrm{C} \text { horizon } \\
\text { result }\end{array}$ & Qualifier & $\begin{array}{l}\text { A lab split } \\
\text { result }\end{array}$ & Qualifier \\
\hline
\end{tabular}

Location =ORR; Formation $=$ CHEPULTEPEC; Site $=77,85,86 ;$ Sample type $=$ Field Duplicate; $A=5147 ; B=5150 ; C=5153 ; A$ lab split='

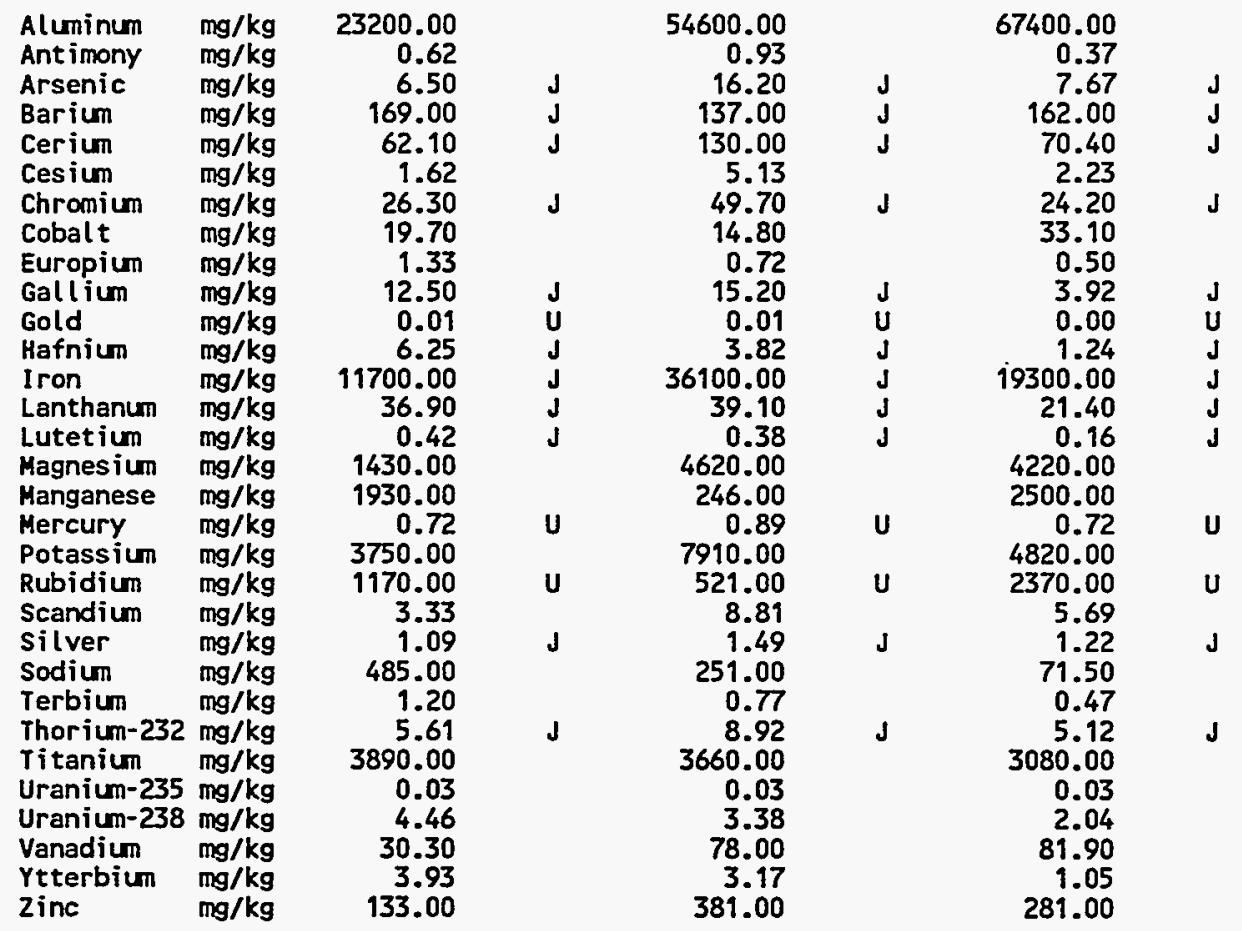

Location=ORR; Formation $=$ CHICKAMAUGA Bethel V; Site=101,102,103; Sample type=Regular; $A=5198 ; B=5201 ; C=5204 ; A$ lab split=' '

\begin{tabular}{|c|c|c|c|c|c|c|}
\hline $\begin{array}{l}\text { Aluminum } \\
\text { Antimony } \\
\text { Arsenic } \\
\text { Barium } \\
\text { Cerium } \\
\text { Cesium } \\
\text { Chromium } \\
\text { Cobalt } \\
\text { Europium } \\
\text { Gallium } \\
\text { Gold } \\
\text { Hafnium } \\
\text { Iron } \\
\text { Lanthanum } \\
\text { Lutetium } \\
\text { Magnesium } \\
\text { Manganese } \\
\text { Hercury } \\
\text { Potassium } \\
\text { Rubidium } \\
\text { Scandium } \\
\text { Silver } \\
\text { Sodium } \\
\text { Terbium } \\
\text { Thorium-232 } \\
\text { Titanium } \\
\text { Uranium-235 } \\
\text { Uranium-238 } \\
\text { Vanadium } \\
\text { Ytterbium }\end{array}$ & $\begin{array}{l}\mathrm{mg} / \mathrm{kg} \\
\mathrm{mg} / \mathrm{kg} \\
\mathrm{mg} / \mathrm{kg} \\
\mathrm{mg} / \mathrm{kg} \\
\mathrm{mg} / \mathrm{kg} \\
\mathrm{mg} / \mathrm{kg} \\
\mathrm{mg} / \mathrm{kg} \\
\mathrm{mg} / \mathrm{kg} \\
\mathrm{mg} / \mathrm{kg} \\
\mathrm{mg} / \mathrm{kg} \\
\mathrm{mg} / \mathrm{kg} \\
\mathrm{mg} / \mathrm{kg} \\
\mathrm{mg} / \mathrm{kg} \\
\mathrm{mg} / \mathrm{kg} \\
\mathrm{mg} / \mathrm{kg} \\
\mathrm{mg} / \mathrm{kg} \\
\mathrm{mg} / \mathrm{kg} \\
\mathrm{mg} / \mathrm{kg} \\
\mathrm{mg} / \mathrm{kg} \\
\mathrm{mg} / \mathrm{kg} \\
\mathrm{mg} / \mathrm{kg} \\
\mathrm{mg} / \mathrm{kg} \\
\mathrm{mg} / \mathrm{kg} \\
\mathrm{mg} / \mathrm{kg} \\
\mathrm{mg} / \mathrm{kg} \\
\mathrm{mg} / \mathrm{kg} \\
\mathrm{mg} / \mathrm{kg} \\
\mathrm{mg} / \mathrm{kg} \\
\mathrm{mg} / \mathrm{kg} \\
\mathrm{mg} / \mathrm{kg}\end{array}$ & $\begin{array}{r}45900.00 \\
0.68 \\
9.64 \\
10600.00 \\
96.40 \\
3.79 \\
53.90 \\
18.60 \\
1.32 \\
11.70 \\
0.01 \\
9.14 \\
36100.00 \\
48.00 \\
0.42 \\
3540.00 \\
1260.00 \\
1.01 \\
25800.00 \\
562.00 \\
11.20 \\
2.04 \\
2320.00 \\
0.91 \\
19.00 \\
4920.00 \\
0.02 \\
3.86 \\
50.40 \\
2.52\end{array}$ & $\begin{array}{l}\mathbf{J} \\
\mathbf{U} \\
\mathbf{J} \\
\mathbf{J} \\
\mathbf{J} \\
\mathbf{J}\end{array}$ & $\begin{array}{r}86200.00 \\
0.43 \\
13.30 \\
7670.00 \\
93.50 \\
5.59 \\
71.00 \\
15.80 \\
2.42 \\
17.50 \\
0.01 \\
4.78 \\
55500.00 \\
61.10 \\
0.58 \\
9030.00 \\
499.00 \\
1.10 \\
26000.00 \\
706.00 \\
17.40 \\
2.33 \\
2520.00 \\
3.59 \\
13.10 \\
4530.00 \\
0.02 \\
2.72 \\
98.00 \\
3.27\end{array}$ & $\begin{array}{l}\mathbf{J} \\
\mathbf{U} \\
\mathbf{J} \\
\mathbf{J} \\
\mathbf{J} \\
\mathbf{J}\end{array}$ & $\begin{array}{r}96900.00 \\
0.64 \\
23.80 \\
10700.00 \\
117.00 \\
6.08 \\
75.40 \\
36.30 \\
1.86 \\
13.10 \\
0.01 \\
4.31 \\
56500.00 \\
56.50 \\
0.37 \\
10800.00 \\
645.00 \\
1.08 \\
26000.00 \\
1110.00 \\
19.20 \\
2.25 \\
3190.00 \\
1.36 \\
13.90 \\
3980.00 \\
0.02 \\
2.82 \\
105.00 \\
0.45\end{array}$ \\
\hline
\end{tabular}


H-11

Table H.1 (continued)

\begin{tabular}{|c|c|c|c|c|c|c|c|c|c|}
\hline Analysis & Units & $\begin{array}{l}\text { A horizon } \\
\text { result }\end{array}$ & Qualifier & $\begin{array}{l}\text { B horizon } \\
\text { result }\end{array}$ & Qualifier & $\begin{array}{l}\mathrm{C} \text { horizon } \\
\text { result }\end{array}$ & Qualifier & $\begin{array}{l}\text { A lab split } \\
\text { result }\end{array}$ & Qualifier \\
\hline
\end{tabular}

Location=ORR; Formation=CHICKAMAUGA Bethel V; Site=104,108,110; Sample type=Regular; :"s; $A=5207 ; B=5210 ; C=5213 ; A$ lab split=' '

\begin{tabular}{|c|c|c|c|c|c|c|}
\hline $\begin{array}{l}\text { Aluminum } \\
\text { Antimony } \\
\text { Arsenic } \\
\text { Barium } \\
\text { Cerium } \\
\text { Cesium } \\
\text { Chromium } \\
\text { Cobalt } \\
\text { Europium } \\
\text { Gallium } \\
\text { Gold } \\
\text { Hafnium } \\
\text { Iron } \\
\text { Lenthanum } \\
\text { Lutetium } \\
\text { Magnesium } \\
\text { Manganese } \\
\text { Mercury } \\
\text { Potassium } \\
\text { Rubidium } \\
\text { Scandium } \\
\text { Silver } \\
\text { Sodium } \\
\text { Terbium } \\
\text { Thorium-232 } \\
\text { Titanium } \\
\text { Uranium-235 } \\
\text { Uranium-238 } \\
\text { Vanadium } \\
\text { Ytterbium }\end{array}$ & 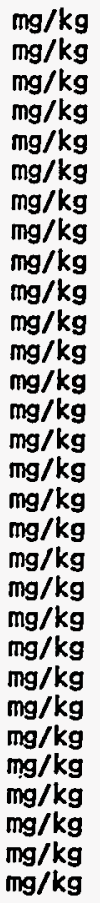 & $\begin{array}{r}35300.00 \\
0.41 \\
7.55 \\
5120.00 \\
55.20 \\
1.91 \\
47.60 \\
14.30 \\
0.91 \\
13.90 \\
0.01 \\
11.30 \\
24700.00 \\
28.80 \\
0.23 \\
2680.00 \\
459.00 \\
0.66 \\
10300.00 \\
443.00 \\
6.17 \\
1.27 \\
1080.00 \\
0.77 \\
8.60 \\
5530.00 \\
0.03 \\
2.91 \\
44.40 \\
2.51\end{array}$ & $\begin{array}{l}\mathbf{J} \\
\text { U } \\
\mathbf{J} \\
\mathbf{J} \\
\mathbf{J} \\
\mathbf{J}\end{array}$ & $\begin{array}{r}87500.00 \\
0.40 \\
9.49 \\
5430.00 \\
92.50 \\
5.88 \\
78.40 \\
14.60 \\
1.19 \\
20.90 \\
0.01 \\
6.91 \\
56800.00 \\
43.30 \\
0.22 \\
7630.00 \\
199.00 \\
1.00 \\
18100.00 \\
532.00 \\
15.60 \\
2.07 \\
1800.00 \\
1.32 \\
14.20 \\
5100.00 \\
0.02 \\
3.81 \\
102.00 \\
0.11\end{array}$ & $\begin{array}{l}\mathbf{J} \\
\mathbf{U} \\
\mathbf{J} \\
\mathbf{J} \\
\mathbf{J} \\
\mathbf{J}\end{array}$ & $\begin{array}{r}80500.00 \\
0.19 \\
7.72 \\
6580.00 \\
98.40 \\
5.82 \\
76.50 \\
16.70 \\
1.70 \\
22.00 \\
0.01 \\
5.99 \\
58500.00 \\
50.60 \\
0.48 \\
7350.00 \\
549.00 \\
1.05 \\
20100.00 \\
616.00 \\
17.20 \\
2.14 \\
1900.00 \\
1.98 \\
14.70 \\
4940.00 \\
0.02 \\
2.51 \\
93.50 \\
3.41\end{array}$ \\
\hline
\end{tabular}

Location=ORR; Formation=CHICKAMAUGA Bethel V; Site=115,116,117; Sample type=Regular; $A=5220 ; B=5224 ; C=5227 ; A$ lab split $=5221$

\begin{tabular}{|c|c|c|c|c|c|c|c|c|}
\hline $\begin{array}{l}\text { Aluminum } \\
\text { Antimony } \\
\text { Arsenic } \\
\text { Barium } \\
\text { Cerium } \\
\text { Cesium } \\
\text { Chromium } \\
\text { Cobalt } \\
\text { Europium } \\
\text { Gallium } \\
\text { Gold } \\
\text { Hafnium } \\
\text { Iron } \\
\text { Lanthanum } \\
\text { Lutetium } \\
\text { Magnesium } \\
\text { Manganese } \\
\text { Hercury } \\
\text { Potassium } \\
\text { Rubidium } \\
\text { Scandium } \\
\text { silver } \\
\text { Sodium } \\
\text { Terbium } \\
\text { Thorium-232 } \\
\text { Titanium } \\
\text { Uranium-235 } \\
\text { Uranium-238 } \\
\text { Vanadium } \\
\text { Ytterbium } \\
\text { Zinc }\end{array}$ & $\begin{array}{l}\mathrm{mg} / \mathrm{kg} \\
\mathrm{mg} / \mathrm{kg} \\
\mathrm{mg} / \mathrm{kg} \\
\mathrm{mg} / \mathrm{kg} \\
\mathrm{mg} / \mathrm{kg} \\
\mathrm{mg} / \mathrm{kg} \\
\mathrm{mg} / \mathrm{kg} \\
\mathrm{mg} / \mathrm{kg} \\
\mathrm{mg} / \mathrm{kg} \\
\mathrm{mg} / \mathrm{kg} \\
\mathrm{mg} / \mathrm{kg} \\
\mathrm{mg} / \mathrm{kg} \\
\mathrm{mg} / \mathrm{kg} \\
\mathrm{mg} / \mathrm{kg} \\
\mathrm{mg} / \mathrm{kg} \\
\mathrm{mg} / \mathrm{kg} \\
\mathrm{mg} / \mathrm{kg} \\
\mathrm{mg} / \mathrm{kg} \\
\mathrm{mg} / \mathrm{kg} \\
\mathrm{mg} / \mathrm{kg} \\
\mathrm{mg} / \mathrm{kg} \\
\mathrm{mg} / \mathrm{kg} \\
\mathrm{mg} / \mathrm{kg} \\
\mathrm{mg} / \mathrm{kg} \\
\mathrm{mg} / \mathrm{kg} \\
\mathrm{mg} / \mathrm{kg} \\
\mathrm{mg} / \mathrm{kg} \\
\mathrm{mg} / \mathrm{kg} \\
\mathrm{mg} / \mathrm{kg} \\
\mathrm{mg} / \mathrm{kg} \\
\mathrm{mg} / \mathrm{kg}\end{array}$ & $\begin{array}{r}35400.00 \\
0.59 \\
7.67 \\
6560.00 \\
60.40 \\
2.01 \\
47.80 \\
14.50 \\
0.74 \\
16.50 \\
0.01 \\
9.76 \\
29300.00 \\
31.70 \\
0.35 \\
3500.00 \\
788.00 \\
0.73 \\
7760.00 \\
579.00 \\
6.93 \\
1.41 \\
984.00 \\
1.38 \\
9.54 \\
6190.00 \\
0.02 \\
3.18 \\
57.80 \\
3.54\end{array}$ & $\begin{array}{l}\mathbf{J} \\
\mathbf{U} \\
\mathbf{J} \\
\mathbf{J} \\
\mathbf{J} \\
\mathbf{J}\end{array}$ & $\begin{array}{r}82700.00 \\
0.20 \\
14.90 \\
5600.00 \\
87.80 \\
6.17 \\
73.10 \\
20.20 \\
0.96 \\
20.90 \\
0.01 \\
7.06 \\
56300.00 \\
46.00 \\
0.54 \\
6480.00 \\
277.00 \\
1.06 \\
20200.00 \\
533.00 \\
16.40 \\
2.16 \\
1790.00 \\
0.53 \\
15.50 \\
5600.00 \\
0.03 \\
3.38 \\
105.00 \\
2.65\end{array}$ & $\begin{array}{l}\mathbf{U} \\
\mathbf{U} \\
\mathbf{J} \\
\mathbf{U}\end{array}$ & $\begin{array}{r}118000.00 \\
0.69 \\
11.90 \\
203.00 \\
140.00 \\
6.32 \\
67.60 \\
33.60 \\
1.94 \\
25.90 \\
0.01 \\
4.97 \\
57100.00 \\
52.20 \\
0.45 \\
9190.00 \\
640.00 \\
1.25 \\
22800.00 \\
666.00 \\
17.30 \\
2.27 \\
1590.00 \\
1.00 \\
13.10 \\
5990.00 \\
0.02 \\
4.54 \\
124.00 \\
3.26 \\
120.00\end{array}$ & $\begin{array}{l}\mathbf{J} \\
\mathbf{U} \\
\mathbf{J} \\
\mathbf{J} \\
\mathbf{J} \\
\mathbf{J}\end{array}$ & $\begin{array}{r}34000.00 \\
0.63 \\
7.93 \\
14700.00 \\
50.60 \\
1.90 \\
49.90 \\
16.20 \\
0.75 \\
17.60 \\
0.01 \\
8.40 \\
30100.00 \\
34.50 \\
0.26 \\
2410.00 \\
1730.00 \\
0.70 \\
7980.00 \\
1260.00 \\
6.48 \\
1.38 \\
1050.00 \\
1.04 \\
9.11 \\
6770.00 \\
0.03 \\
3.39 \\
61.40 \\
4.48\end{array}$ \\
\hline
\end{tabular}


H-12

Table H.1 (continued)

\begin{tabular}{|c|c|c|c|c|c|c|c|c|c|}
\hline Analysis & Units & $\begin{array}{c}\text { A horizon } \\
\text { result }\end{array}$ & Qualifier & $\begin{array}{l}\text { B horizon } \\
\text { result }\end{array}$ & Qualifier & $\begin{array}{l}C \text { horizon } \\
\text { result }\end{array}$ & Qualifier & $\begin{array}{l}\text { A lab split } \\
\text { result }\end{array}$ & Qualifier \\
\hline
\end{tabular}

Location=ORR; Formation =CHICKAMAUGA Bethel V; Site=93,99,100; Sample type=Regular; $A=5189 ; B=5192 ; C=5195 ; A$ lab split=' '

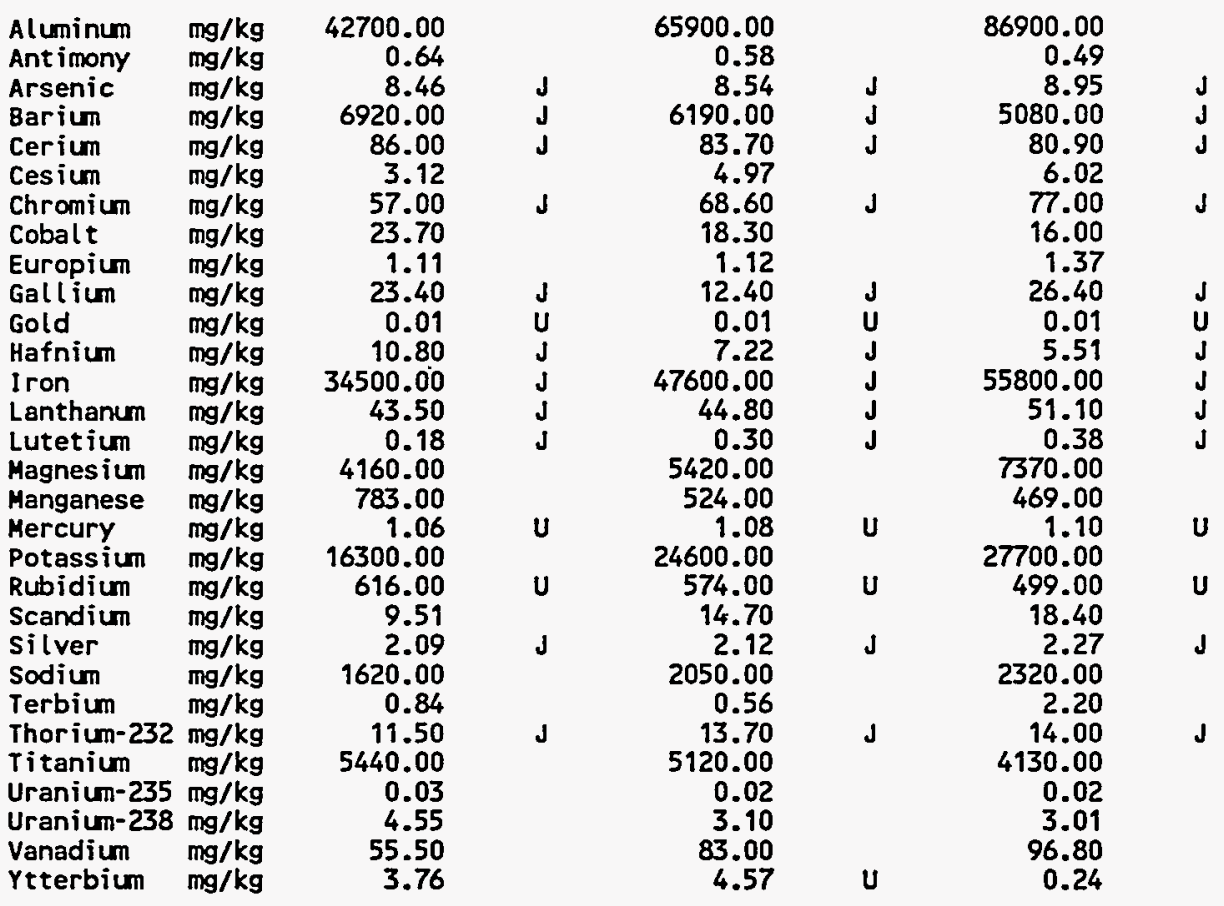

Location=ORR; Formation=CHICKAMAUGA K-25; Site=118,122,124; Sample type=Regular; $A=5244 ; B=5248 ; C=5251 ; A$ lab split $=5245$

\begin{tabular}{|c|c|c|c|c|c|c|c|c|}
\hline $\begin{array}{l}\text { Aluminum } \\
\text { Antimony } \\
\text { Arsenic } \\
\text { Barium } \\
\text { Cerium } \\
\text { Cesium } \\
\text { Chromium } \\
\text { Cobalt } \\
\text { Europium } \\
\text { Gallium } \\
\text { Gold } \\
\text { Hafnium } \\
\text { Iron } \\
\text { Lanthanum } \\
\text { Lutetium } \\
\text { Magnesium } \\
\text { Manganese } \\
\text { Mercury } \\
\text { Potassium } \\
\text { Rubidium } \\
\text { Scandium } \\
\text { Silver } \\
\text { Sodium } \\
\text { Terbium } \\
\text { Thorium-232 } \\
\text { Titanium } \\
\text { Uranium-235 } \\
\text { Uranium-238 } \\
\text { Vanadium } \\
\text { Ytterbium } \\
\text { Zinc }\end{array}$ & $\begin{array}{l}\mathrm{mg} / \mathrm{kg} \\
\mathrm{mg} / \mathrm{kg} \\
\mathrm{mg} / \mathrm{kg} \\
\mathrm{mg} / \mathrm{kg} \\
\mathrm{mg} / \mathrm{kg} \\
\mathrm{mg} / \mathrm{kg} \\
\mathrm{mg} / \mathrm{kg} \\
\mathrm{mg} / \mathrm{kg} \\
\mathrm{mg} / \mathrm{kg} \\
\mathrm{mg} / \mathrm{kg} \\
\mathrm{mg} / \mathrm{kg} \\
\mathrm{mg} / \mathrm{kg} \\
\mathrm{mg} / \mathrm{kg} \\
\mathrm{mg} / \mathrm{kg} \\
\mathrm{mg} / \mathrm{kg} \\
\mathrm{mg} / \mathrm{kg} \\
\mathrm{mg} / \mathrm{kg} \\
\mathrm{mg} / \mathrm{kg} \\
\mathrm{mg} / \mathrm{kg} \\
\mathrm{mg} / \mathrm{kg} \\
\mathrm{mg} / \mathrm{kg} \\
\mathrm{mg} / \mathrm{kg} \\
\mathrm{mg} / \mathrm{kg} \\
\mathrm{mg} / \mathrm{kg} \\
\mathrm{mg} / \mathrm{kg} \\
\mathrm{mg} / \mathrm{kg} \\
\mathrm{mg} / \mathrm{kg} \\
\mathrm{mg} / \mathrm{kg} \\
\mathrm{mg} / \mathrm{kg} \\
\mathrm{mg} / \mathrm{kg} \\
\mathrm{mg} / \mathrm{kg}\end{array}$ & $\begin{array}{r}65800.00 \\
0.91 \\
10.30 \\
166.00 \\
67.50 \\
3.48 \\
55.80 \\
15.80 \\
0.88 \\
13.10 \\
0.01 \\
7.43 \\
37800.00 \\
35.00 \\
0.30 \\
3350.00 \\
1080.00 \\
0.22 \\
12600.00 \\
1690.00 \\
10.00 \\
2.25 \\
1050.00 \\
0.54 \\
11.10 \\
5790.00 \\
0.03 \\
5.00 \\
75.90 \\
2.43 \\
112.00\end{array}$ & $\begin{array}{l}\mathbf{J} \\
\mathbf{U} \\
\mathbf{J} \\
\mathbf{J} \\
\mathbf{J} \\
\mathbf{J}\end{array}$ & $\begin{array}{r}116000.00 \\
320.00 \\
93.00 \\
6.98 \\
68.10 \\
19.50 \\
1.16\end{array}$ & $\mathbf{J}$ & $\begin{array}{r}121000.00 \\
0.59 \\
7.33 \\
390.00 \\
155.00 \\
7.09 \\
69.90 \\
27.30 \\
1.39 \\
29.90 \\
0.01 \\
4.43 \\
51800.00 \\
47.50 \\
0.42 \\
8470.00 \\
359.00\end{array}$ & $\begin{array}{l}\mathbf{J} \\
\mathbf{U} \\
\mathbf{J} \\
\mathbf{J} \\
\mathbf{J} \\
\mathbf{J}\end{array}$ & $\begin{array}{r}66.50 \\
3.84 \\
52.80 \\
15.10 \\
0.84 \\
17.90 \\
0.01 \\
7.48 \\
35800.00 \\
34.40 \\
0.31\end{array}$ \\
\hline
\end{tabular}


Table H.1 (continued)

\begin{tabular}{|c|c|c|c|c|c|c|c|c|c|}
\hline Analysis & Units & $\begin{array}{l}\text { A horizon } \\
\text { result }\end{array}$ & Qualifier & $\begin{array}{l}\text { B horizon } \\
\text { result }\end{array}$ & Qualifier & $\begin{array}{l}\mathrm{C} \text { horizon } \\
\text { result }\end{array}$ & Qualifier & $\begin{array}{l}\text { A lab split } \\
\text { result }\end{array}$ & Qualifier \\
\hline
\end{tabular}

Location=ORR; Formation=CHICKAMAUGA K-25; Site=119,123,127; Sample type =Regular; $A=5256 ; B=5259 ; C=5262 ; A$ lab split $=$ ',

\begin{tabular}{|c|c|c|c|c|c|c|}
\hline $\begin{array}{l}\text { Aluminum } \\
\text { Antimony } \\
\text { Arsenic } \\
\text { Barium } \\
\text { Cerium } \\
\text { Cesium } \\
\text { Chromium } \\
\text { Cobalt } \\
\text { Europium } \\
\text { Gallium } \\
\text { Gold } \\
\text { Hafnium } \\
\text { Iron } \\
\text { Lanthanum } \\
\text { Lutetium } \\
\text { Magnesium } \\
\text { Manganese } \\
\text { Mercury } \\
\text { Potassium } \\
\text { Rubidium } \\
\text { Scandium } \\
\text { Silver } \\
\text { Sodium } \\
\text { Terbium } \\
\text { Thorium-232 } \\
\text { Titanium } \\
\text { Uranium-235 } \\
\text { Uranium-238 } \\
\text { Vanadium } \\
\text { Ytterbium } \\
\text { Zine }\end{array}$ & $\begin{array}{l}\mathrm{mg} / \mathrm{kg} \\
\mathrm{mg} / \mathrm{kg} \\
\mathrm{mg} / \mathrm{kg} \\
\mathrm{mg} / \mathrm{kg} \\
\mathrm{mg} / \mathrm{kg} \\
\mathrm{mg} / \mathrm{kg} \\
\mathrm{mg} / \mathrm{kg} \\
\mathrm{mg} / \mathrm{kg} \\
\mathrm{mg} / \mathrm{kg} \\
\mathrm{mg} / \mathrm{kg} \\
\mathrm{mg} / \mathrm{kg} \\
\mathrm{mg} / \mathrm{kg} \\
\mathrm{mg} / \mathrm{kg} \\
\mathrm{mg} / \mathrm{kg} \\
\mathrm{mg} / \mathrm{kg} \\
\mathrm{mg} / \mathrm{kg} \\
\mathrm{mg} / \mathrm{kg} \\
\mathrm{mg} / \mathrm{kg} \\
\mathrm{mg} / \mathrm{kg} \\
\mathrm{mg} / \mathrm{kg} \\
\mathrm{mg} / \mathrm{kg} \\
\mathrm{mg} / \mathrm{kg} \\
\mathrm{mg} / \mathrm{kg} \\
\mathrm{mg} / \mathrm{kg} \\
\mathrm{mg} / \mathrm{kg} \\
\mathrm{mg} / \mathrm{kg} \\
\mathrm{mg} / \mathrm{kg} \\
\mathrm{mg} / \mathrm{kg} \\
\mathrm{mg} / \mathrm{kg} \\
\mathrm{mg} / \mathrm{kg} \\
\mathrm{mg} / \mathrm{kg}\end{array}$ & $\begin{array}{r}56200.00 \\
0.73 \\
9.85 \\
350.00 \\
70.40 \\
3.57 \\
55.10 \\
18.50 \\
0.90 \\
14.00 \\
0.01 \\
8.89 \\
3500.00 \\
34.80 \\
0.34 \\
4980.00 \\
2070.00 \\
1.10 \\
13000.00 \\
690.00 \\
9.05 \\
0.48 \\
847.00 \\
0.83 \\
10.30 \\
6940.00 \\
0.03 \\
5.47 \\
65.60 \\
2.60 \\
74.30\end{array}$ & $\begin{array}{l}\mathbf{J} \\
U \\
\mathbf{J} \\
\mathbf{J} \\
\mathbf{J}\end{array}$ & $\begin{array}{r}129000.00 \\
0.71 \\
9.59 \\
330.00 \\
92.20 \\
7.45 \\
73.00 \\
11.70 \\
1.60 \\
50.40 \\
0.01 \\
5.45 \\
54900.00 \\
49.70 \\
0.45 \\
7580.00 \\
562.00 \\
1.39 \\
26500.00 \\
1780.00 \\
16.80 \\
2.87 \\
1470.00 \\
0.58 \\
15.10 \\
5690.00 \\
0.03 \\
3.91 \\
145.00 \\
2.70 \\
194.00\end{array}$ & $\begin{array}{l}\mathbf{J} \\
\mathbf{U} \\
\mathbf{J} \\
\mathbf{J} \\
\mathbf{J} \\
\mathbf{J}\end{array}$ & $\begin{array}{r}24200.00 \\
0.71 \\
9.62 \\
71.80 \\
92.50 \\
7.47 \\
73.20 \\
11.80 \\
1.61 \\
50.50 \\
0.01 \\
5.46 \\
55100.00 \\
49.90 \\
0.46 \\
1690.00 \\
392.00 \\
1.39 \\
26600.00 \\
428.00 \\
16.80 \\
2.88 \\
1470.00 \\
0.58 \\
15.20 \\
1280.00 \\
0.03 \\
3.92 \\
25.70 \\
2.71 \\
195.00\end{array}$ \\
\hline
\end{tabular}

Location=ORR; Formation=CHICKAMAUGA K-25; Site=120,126,129; Sample type=Regular; $A=5232 ; B=5236 ; C=5239 ; A$ lab split $=5233$

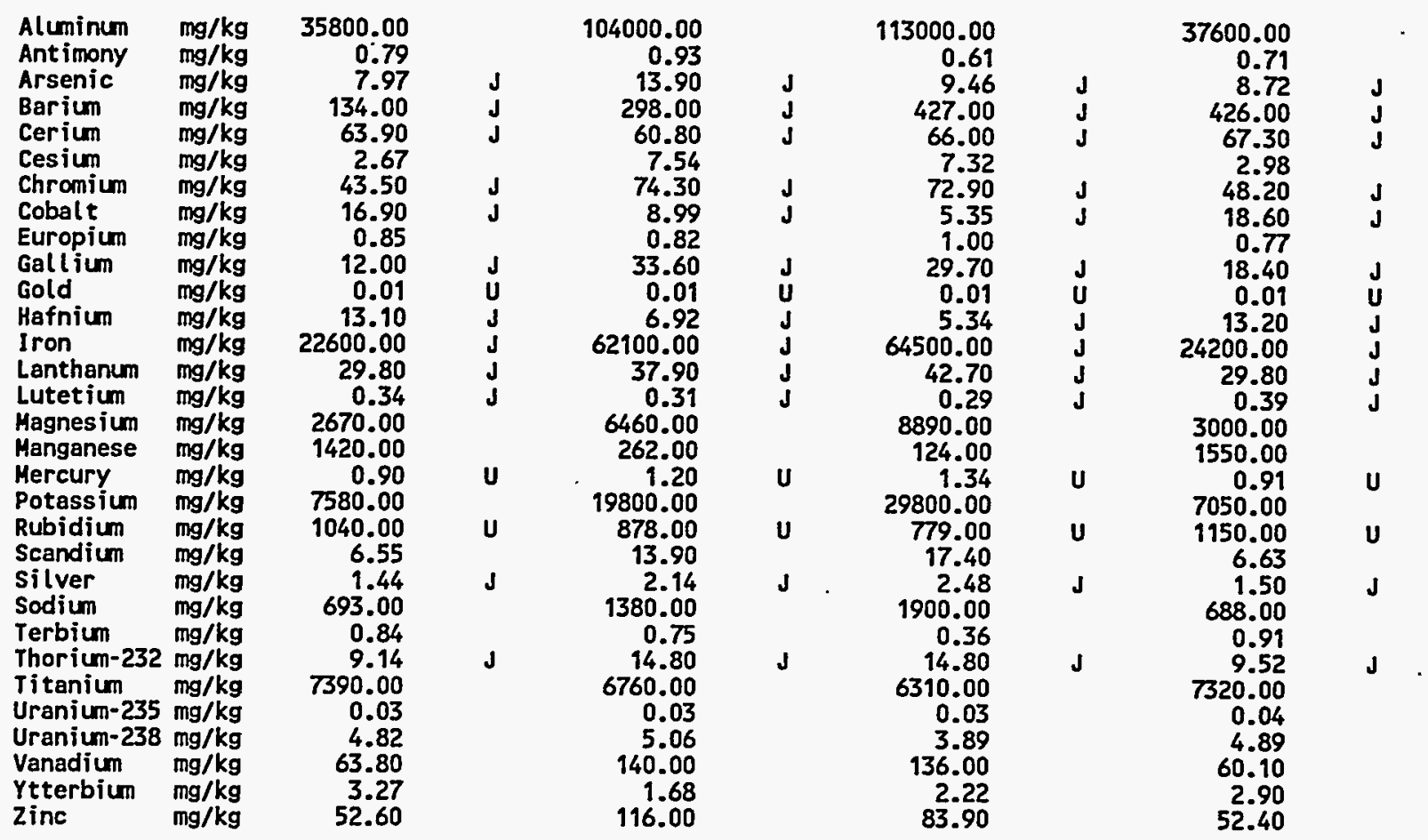




\section{$\mathrm{H}-14$}

Table H.1 (continued)

\begin{tabular}{|c|c|c|c|c|c|c|c|c|c|}
\hline Analysis & Units & $\begin{array}{c}\text { A horizon } \\
\text { result }\end{array}$ & Qualifier & $\begin{array}{l}\text { B horizon } \\
\text { result }\end{array}$ & Qualifier & $\begin{array}{l}\mathrm{C} \text { horizon } \\
\text { result }\end{array}$ & Qualifier & $\begin{array}{l}\text { A lab split } \\
\text { result }\end{array}$ & Qualifier \\
\hline
\end{tabular}

Location=ORR; Formation $=$ CHICKAMAUGA K-25; Site=121,125,128; Sample type =Regular;

$$
A=5267 ; B=5270 ; C=5273 ; A \text { lab split=' }
$$

\begin{tabular}{|c|c|c|c|c|c|c|}
\hline $\begin{array}{l}\text { Aluminum } \\
\text { Ant imony } \\
\text { Arsenic } \\
\text { Barium } \\
\text { Cerium } \\
\text { Cesium } \\
\text { Chromium } \\
\text { Cobalt } \\
\text { Europium } \\
\text { Gallium } \\
\text { Gold } \\
\text { Hafnium } \\
\text { Iron } \\
\text { Lanthanum } \\
\text { Lutetium } \\
\text { Magnesium } \\
\text { Manganese } \\
\text { Mercury } \\
\text { Potassium } \\
\text { Rubidium } \\
\text { Scandium } \\
\text { Si tver } \\
\text { Sodium } \\
\text { Terbium } \\
\text { Thorium-23 } \\
\text { Titanium } \\
\text { Uranium-23 } \\
\text { Uranium-23 } \\
\text { Vanadium } \\
\text { Ytterbium } \\
\text { Zinc }\end{array}$ & $\begin{array}{l}\mathrm{mg} / \mathrm{kg} \\
\mathrm{mg} / \mathrm{kg} \\
\mathrm{mg} / \mathrm{kg} \\
\mathrm{mg} / \mathrm{kg} \\
\mathrm{mg} / \mathrm{kg} \\
\mathrm{mg} / \mathrm{kg} \\
\mathrm{mg} / \mathrm{kg} \\
\mathrm{mg} / \mathrm{kg} \\
\mathrm{mg} / \mathrm{kg} \\
\mathrm{mg} / \mathrm{kg} \\
\mathrm{mg} / \mathrm{kg} \\
\mathrm{mg} / \mathrm{kg} \\
\mathrm{mg} / \mathrm{kg} \\
\mathrm{mg} / \mathrm{kg} \\
\mathrm{mg} / \mathrm{kg} \\
\mathrm{mg} / \mathrm{kg} \\
\mathrm{mg} / \mathrm{kg} \\
\mathrm{mg} / \mathrm{kg} \\
\mathrm{mg} / \mathrm{kg} \\
\mathrm{mg} / \mathrm{kg} \\
\mathrm{mg} / \mathrm{kg} \\
\mathrm{mg} / \mathrm{kg} \\
\mathrm{mg} / \mathrm{kg} \\
\mathrm{mg} / \mathrm{kg} \\
\mathrm{mg} / \mathrm{kg} \\
\mathrm{mg} / \mathrm{kg} \\
\mathrm{mg} / \mathrm{kg} \\
\mathrm{mg} / \mathrm{kg} \\
\mathrm{mg} / \mathrm{kg} \\
\mathrm{mg} / \mathrm{kg} \\
\mathrm{mg} / \mathrm{kg}\end{array}$ & $\begin{array}{r}7180.00 \\
0.54 \\
5.87 \\
103.00 \\
52.20 \\
2.53 \\
40.70 \\
19.30 \\
0.70 \\
14.00 \\
0.01 \\
9.89 \\
23200.00 \\
29.00 \\
0.36 \\
593.00 \\
264.00 \\
0.96 \\
8820.00 \\
298.00 \\
6.70 \\
1.87 \\
619.00 \\
0.64 \\
8.97 \\
1320.00 \\
0.03 \\
3.96 \\
12.40 \\
3.51 \\
63.70\end{array}$ & $\begin{array}{l}\mathbf{J} \\
U \\
\vec{J} \\
\mathbf{J} \\
\mathbf{J} \\
\mathbf{J}\end{array}$ & $\begin{array}{r}104000.00 \\
0.54 \\
7.11 \\
320.00 \\
67.20 \\
7.17 \\
60.90 \\
10.90 \\
1.05 \\
23.60 \\
0.01 \\
4.97 \\
51300.00 \\
44.00 \\
0.39 \\
12400.00 \\
269.00 \\
1.38 \\
25900.00 \\
1360.00 \\
16.60 \\
2.92 \\
1170.00 \\
0.75 \\
14.10 \\
5320.00 \\
0.03 \\
3.64 \\
107.00 \\
2.03 \\
164.00\end{array}$ & $\begin{array}{l}\mathbf{J} \\
\mathbf{U} \\
\mathbf{J} \\
\mathbf{J} \\
\mathbf{J} \\
\mathbf{J}\end{array}$ & $\begin{array}{r}473000.0 \\
0.5 \\
6.1 \\
1320.0 \\
70.8 \\
7.6 \\
63.0 \\
20.7 \\
1.0 \\
25.8 \\
0.0 \\
3.7 \\
50900.0 \\
40.9 \\
0.3 \\
45700.0 \\
1650.0 \\
0.5 \\
28000.0 \\
3330.0 \\
18.5 \\
1.0 \\
1040.00 \\
0.6 \\
13.60 \\
21600.00 \\
0.0 \\
3.7 \\
446.00 \\
1.80 \\
141.00\end{array}$ \\
\hline
\end{tabular}

Location=ORR; Formation=COPPER RIDGE; Site=45,60,75; Sample type=Regular; $A=5111 ; B=5114 ; C=5117 ; A$ lab split $=$ '

\begin{tabular}{|c|c|c|c|c|c|c|}
\hline $\begin{array}{l}\text { Aluminum } \\
\text { Ant imony } \\
\text { Arsenic } \\
\text { Barium } \\
\text { Cerium } \\
\text { Cesium } \\
\text { Chronium } \\
\text { Cobalt } \\
\text { Europium } \\
\text { Gallium } \\
\text { Gold } \\
\text { Hafnium } \\
\text { Iron } \\
\text { Lanthanum } \\
\text { Lutetium } \\
\text { Magnesium } \\
\text { Manganese }\end{array}$ & $\begin{array}{l}\mathrm{mg} / \mathrm{kg} \\
\mathrm{mg} / \mathrm{kg} \\
\mathrm{mg} / \mathrm{kg} \\
\mathrm{mg} / \mathrm{kg} \\
\mathrm{mg} / \mathrm{kg} \\
\mathrm{mg} / \mathrm{kg} \\
\mathrm{mg} / \mathrm{kg} \\
\mathrm{mg} / \mathrm{kg} \\
\mathrm{mg} / \mathrm{kg} \\
\mathrm{mg} / \mathrm{kg} \\
\mathrm{mg} / \mathrm{kg} \\
\mathrm{mg} / \mathrm{kg} \\
\mathrm{mg} / \mathrm{kg} \\
\mathrm{mg} / \mathrm{kg} \\
\mathrm{mg} / \mathrm{kg} \\
\mathrm{mg} / \mathrm{kg} \\
\mathrm{mg} / \mathrm{kg} \\
\mathrm{mg} / \mathrm{kg} \\
\mathrm{mg} / \mathrm{kg} \\
\mathrm{mg} / \mathrm{kg} \\
\mathrm{mg} / \mathrm{kg} \\
\mathrm{mg} / \mathrm{kg} \\
\mathrm{mg} / \mathrm{kg} \\
\mathrm{mg} / \mathrm{kg} \\
\mathrm{mg} / \mathrm{kg} \\
\mathrm{mg} / \mathrm{kg} \\
\mathrm{mg} / \mathrm{kg} \\
\mathrm{mg} / \mathrm{kg} \\
\mathrm{mg} / \mathrm{kg} \\
\mathrm{mg} / \mathrm{kg} \\
\mathrm{mg} / \mathrm{kg}\end{array}$ & $\begin{array}{r}18800.00 \\
0.77 \\
15.90 \\
202.00 \\
41.70 \\
2.16 \\
32.90 \\
5.44 \\
0.54 \\
3.34 \\
0.01 \\
7.23 \\
14700.00 \\
20.50 \\
0.28 \\
660.00 \\
797.00 \\
0.67 \\
3110.00 \\
750.00 \\
3.45 \\
1.03 \\
392.00 \\
0.46 \\
6.02 \\
4150.00 \\
0.03 \\
4.58 \\
34.30 \\
2.78 \\
58.90\end{array}$ & $\begin{array}{l}\mathbf{J} \\
\mathbf{U} \\
\mathbf{J} \\
\mathbf{J} \\
\mathbf{J} \\
\mathbf{J}\end{array}$ & $\begin{array}{r}43600.00 \\
1.15 \\
29.50 \\
113.00 \\
28.00 \\
4.18 \\
50.30 \\
4.29 \\
0.32 \\
12.60 \\
0.01 \\
6.09 \\
27700.00 \\
20.40 \\
0.21 \\
2750.00 \\
135.00 \\
0.76 \\
3710.00 \\
400.00 \\
6.23 \\
1.26 \\
240.00 \\
0.26 \\
9.68 \\
4750.00 \\
0.03 \\
4.80 \\
78.20 \\
2.57 \\
99.40\end{array}$ & $\begin{array}{l}J \\
U \\
J \\
J \\
J \\
J\end{array}$ & $\begin{array}{r}72400.00 \\
1.8 \\
84.90 \\
81.20 \\
41.30 \\
4.1 \\
74.50 \\
4.2 \\
0.34 \\
19.30 \\
0.01 \\
2.28 \\
41400.00 \\
35.80 \\
0.10 \\
4810.00 \\
87.10 \\
0.94 \\
6270.00 \\
430.00 \\
10.60 \\
1.67 \\
128.00 \\
0.19 \\
12.10 \\
2800.00 \\
0.04 \\
2.96 \\
108.00 \\
2.10 \\
249.00\end{array}$ \\
\hline
\end{tabular}




\section{$\mathrm{H}-15$}

Table H.1 (continued)

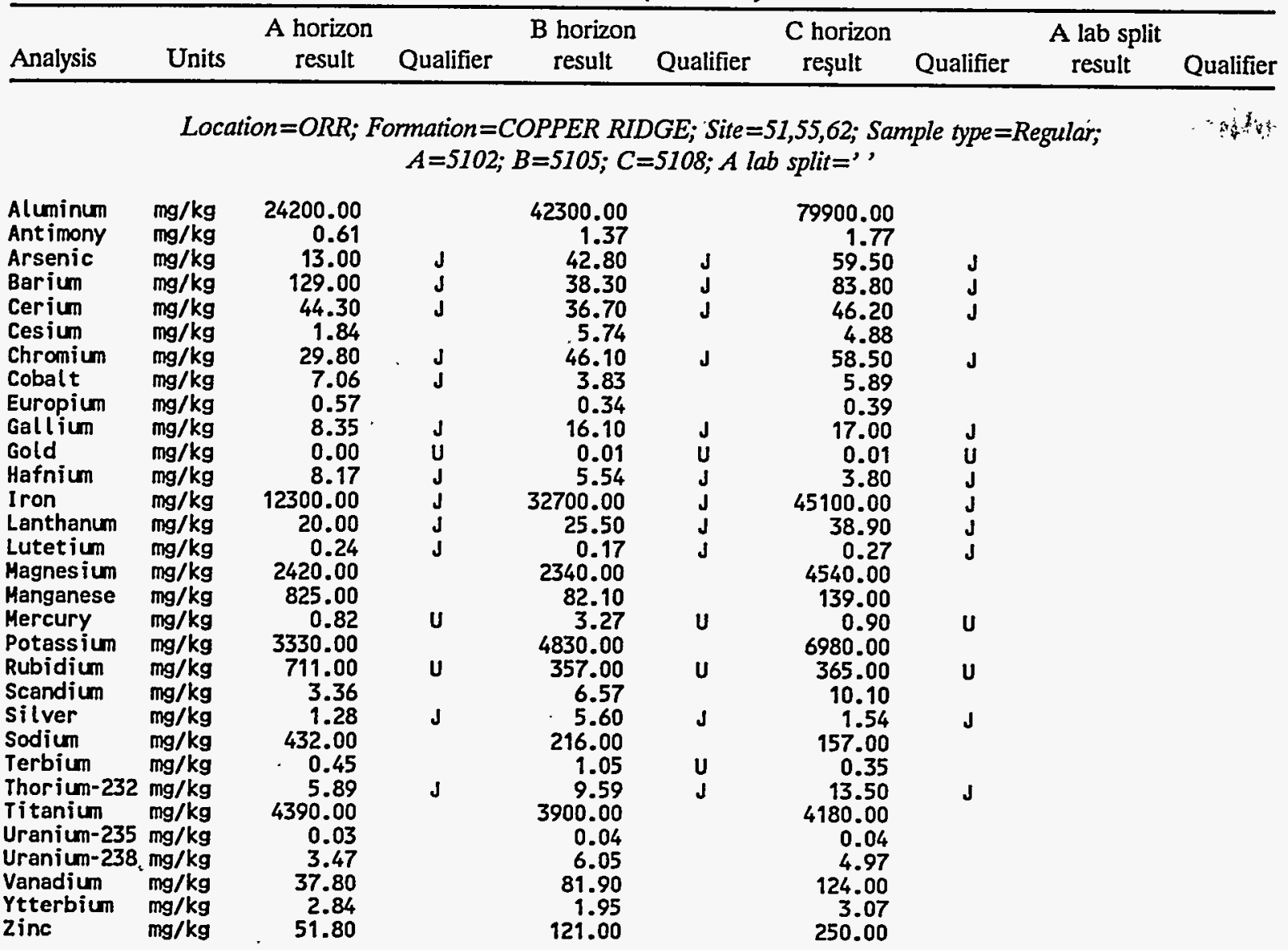

Location=ORR; Formation=COPPER RIDGE; Site=54,64,83; Sample type=Regular; $A=5120 ; B=5123 ; C=5126 ; A$ lab split=' '

\begin{tabular}{|c|c|c|c|c|c|c|}
\hline $\begin{array}{l}\text { Aluminum } \\
\text { Antimony } \\
\text { Arsenic } \\
\text { Barium } \\
\text { Cerium } \\
\text { Cesium } \\
\text { Chromium } \\
\text { Cobalt } \\
\text { Europium } \\
\text { Gallium } \\
\text { Gold } \\
\text { Hafnium } \\
\text { Iron } \\
\text { Lanthanum } \\
\text { Lutetium } \\
\text { Magnesium } \\
\text { Manganese } \\
\text { Mercury } \\
\text { Potassium } \\
\text { Rubidium } \\
\text { Scandium } \\
\text { Silver } \\
\text { Sodium } \\
\text { Terbium } \\
\text { Thorium-232 } \\
\text { Titanium } \\
\text { Uranium-235 } \\
\text { Uranium-238 } \\
\text { Vanadium } \\
\text { Ytterbium } \\
\text { Zinc }\end{array}$ & $\begin{array}{l}\mathrm{mg} / \mathrm{kg} \\
\mathrm{mg} / \mathrm{kg} \\
\mathrm{mg} / \mathrm{kg} \\
\mathrm{mg} / \mathrm{kg} \\
\mathrm{mg} / \mathrm{kg} \\
\mathrm{mg} / \mathrm{kg} \\
\mathrm{mg} / \mathrm{kg} \\
\mathrm{mg} / \mathrm{kg} \\
\mathrm{mg} / \mathrm{kg} \\
\mathrm{mg} / \mathrm{kg} \\
\mathrm{mg} / \mathrm{kg} \\
\mathrm{mg} / \mathrm{kg} \\
\mathrm{mg} / \mathrm{kg} \\
\mathrm{mg} / \mathrm{kg} \\
\mathrm{mg} / \mathrm{kg} \\
\mathrm{mg} / \mathrm{kg} \\
\mathrm{mg} / \mathrm{kg} \\
\mathrm{mg} / \mathrm{kg} \\
\mathrm{mg} / \mathrm{kg} \\
\mathrm{mg} / \mathrm{kg} \\
\mathrm{mg} / \mathrm{kg} \\
\mathrm{mg} / \mathrm{kg} \\
\mathrm{mg} / \mathrm{kg} \\
\mathrm{mg} / \mathrm{kg} \\
\mathrm{mg} / \mathrm{kg} \\
\mathrm{mg} / \mathrm{kg} \\
\mathrm{mg} / \mathrm{kg} \\
\mathrm{mg} / \mathrm{kg} \\
\mathrm{mg} / \mathrm{kg} \\
\mathrm{mg} / \mathrm{kg} \\
\mathrm{mg} / \mathrm{kg}\end{array}$ & $\begin{array}{r}23800.00 \\
0.83 \\
20.50 \\
558.00 \\
49.10 \\
2.13 \\
32.60 \\
16.90 \\
0.55 \\
3.56 \\
0.01 \\
6.37 \\
13400.00 \\
23.00 \\
0.24 \\
1490.00 \\
1420.00 \\
0.72 \\
4310.00 \\
1650.00 \\
3.93 \\
1.10 \\
464.00 \\
0.50 \\
6.47 \\
4380.00 \\
0.03 \\
5.63 \\
43.10 \\
1.75 \\
83.90\end{array}$ & $\begin{array}{l}\mathbf{J} \\
\mathbf{U} \\
\mathbf{J} \\
\mathbf{J} \\
\mathbf{J} \\
\mathbf{J}\end{array}$ & $\begin{array}{r}63300.00 \\
1.40 \\
54.70 \\
98.90 \\
38.30 \\
4.35 \\
48.00 \\
6.47 \\
0.49 \\
15.30 \\
0.01 \\
4.90 \\
33700.00 \\
46.40 \\
0.20 \\
3260.00 \\
192.00 \\
0.86 \\
5990.00 \\
439.00 \\
8.09 \\
1.49 \\
271.00 \\
0.34 \\
9.98 \\
4820.00 \\
0.04 \\
4.52 \\
88.50 \\
2.95 \\
169.00\end{array}$ & $\begin{array}{l}\mathbf{J} \\
\mathbf{U} \\
\mathbf{J} \\
\mathbf{J} \\
\mathbf{J} \\
\mathbf{J}\end{array}$ & $\begin{array}{r}92200.00 \\
1.69 \\
72.80 \\
318.00 \\
54.90 \\
4.77 \\
66.20 \\
5.17 \\
0.50 \\
22.90 \\
0.01 \\
2.36 \\
44300.00 \\
36.60 \\
0.19 \\
5730.00 \\
120.00 \\
1.02 \\
9410.00 \\
1350.00 \\
12.70 \\
1.83 \\
151.00 \\
0.35 \\
12.20 \\
2800.00 \\
0.05 \\
6.36 \\
126.00 \\
2.91 \\
263.00\end{array}$ \\
\hline
\end{tabular}


H-16

Table H.1 (continued)

\begin{tabular}{|c|c|c|c|c|c|c|c|c|c|}
\hline Analysis & Units & $\begin{array}{l}\text { A horizon } \\
\text { result }\end{array}$ & Qualifier & $\begin{array}{l}\text { B horizon } \\
\text { result }\end{array}$ & Qualifier & $\begin{array}{l}C \text { horizon } \\
\text { result }\end{array}$ & Qualifier & $\begin{array}{l}\text { A lab split } \\
\text { result }\end{array}$ & Qualifier \\
\hline
\end{tabular}

Location $=O R R ;$ Formation $=$ COPPER RIDGE; Site $=58,59,91$; Sample type $=$ Regular; $A=5093 ; B=5096$; $C=5099 ;$ A lab split=' '

\begin{tabular}{|c|c|c|c|c|c|c|}
\hline $\begin{array}{l}\text { Aluminum } \\
\text { Antimony } \\
\text { Arsenic } \\
\text { Barium } \\
\text { Cerium } \\
\text { Cesium } \\
\text { Chromium } \\
\text { Cobalt } \\
\text { Europium } \\
\text { Gallium } \\
\text { Gold } \\
\text { Hafnium } \\
\text { Iron } \\
\text { Lanthanum } \\
\text { Lutetium } \\
\text { Magnesium } \\
\text { Manganese } \\
\text { Mercury } \\
\text { Potassium } \\
\text { Rubidium } \\
\text { Scandium } \\
\text { Silver } \\
\text { Sodium } \\
\text { Terbium } \\
\text { Thorium-232 } \\
\text { Titanium } \\
\text { Uranium-235 } \\
\text { Uranium-238 } \\
\text { Vanadium } \\
\text { Ytterbium } \\
\text { Zinc }\end{array}$ & $\begin{array}{l}\mathrm{mg} / \mathrm{kg} \\
\mathrm{mg} / \mathrm{kg} \\
\mathrm{mg} / \mathrm{kg} \\
\mathrm{mg} / \mathrm{kg} \\
\mathrm{mg} / \mathrm{kg} \\
\mathrm{mg} / \mathrm{kg} \\
\mathrm{mg} / \mathrm{kg} \\
\mathrm{mg} / \mathrm{kg} \\
\mathrm{mg} / \mathrm{kg} \\
\mathrm{mg} / \mathrm{kg} \\
\mathrm{mg} / \mathrm{kg} \\
\mathrm{mg} / \mathrm{kg} \\
\mathrm{mg} / \mathrm{kg} \\
\mathrm{mg} / \mathrm{kg} \\
\mathrm{mg} / \mathrm{kg} \\
\mathrm{mg} / \mathrm{kg} \\
\mathrm{mg} / \mathrm{kg} \\
\mathrm{mg} / \mathrm{kg} \\
\mathrm{mg} / \mathrm{kg} \\
\mathrm{mg} / \mathrm{kg} \\
\mathrm{mg} / \mathrm{kg} \\
\mathrm{mg} / \mathrm{kg} \\
\mathrm{mg} / \mathrm{kg} \\
\mathrm{mg} / \mathrm{kg} \\
\mathrm{mg} / \mathrm{kg} \\
\mathrm{mg} / \mathrm{kg} \\
\mathrm{mg} / \mathrm{kg} \\
\mathrm{mg} / \mathrm{kg} \\
\mathrm{mg} / \mathrm{kg} \\
\mathrm{mg} / \mathrm{kg} \\
\mathrm{mg} / \mathrm{kg}\end{array}$ & $\begin{array}{r}26100.00 \\
1.44 \\
40.00 \\
338.00 \\
49.60 \\
2.26 \\
59.20 \\
7.26 \\
0.68 \\
8.85 \\
0.00 \\
7.89 \\
26300.00 \\
21.00 \\
0.34 \\
1740.00 \\
1190.00 \\
0.98 \\
3890.00 \\
941.00 \\
4.24 \\
1.56 \\
451.00 \\
0.55 \\
7.81 \\
4730.00 \\
0.03 \\
4.66 \\
49.00 \\
2.04 \\
73.00\end{array}$ & $\begin{array}{l}\mathbf{J} \\
\mathbf{U} \\
\mathrm{J} \\
\mathrm{J} \\
\mathrm{J} \\
\mathrm{J}\end{array}$ & $\begin{array}{r}63400.00 \\
1.82 \\
79.70 \\
107.00 \\
38.30 \\
5.32 \\
57.60 \\
4.34 \\
0.41 \\
13.80 \\
0.01 \\
5.60 \\
40300.00 \\
28.20 \\
0.27 \\
3570.00 \\
162.00 \\
1.31 \\
6110.00 \\
337.00 \\
9.16 \\
2.29 \\
269.00 \\
0.34 \\
12.00 \\
4940.00 \\
0.04 \\
5.65 \\
115.00 \\
2.37 \\
154.00\end{array}$ & $\begin{array}{l}\mathbf{J} \\
U \\
\mathbf{J} \\
\mathbf{J} \\
\mathbf{J} \\
\mathbf{J}\end{array}$ & $\begin{array}{r}113000.00 \\
2.45 \\
107.00 \\
111.00 \\
45.70 \\
6.17 \\
86.60 \\
4.07 \\
0.36 \\
8.60 \\
0.01 \\
3.23 \\
53500.00 \\
41.60 \\
0.24 \\
5750.00 \\
108.00 \\
1.48 \\
8690.00 \\
403.00 \\
14.40 \\
2.66 \\
169.00 \\
0.48 \\
15.80 \\
3690.00 \\
0.05 \\
6.42 \\
168.00 \\
2.73 \\
243.00\end{array}$ \\
\hline
\end{tabular}

Location=ORR; Formation $=D I S M A L$ GAP; Site $=10,33,35$; Sample type $=$ Regular; $A=5021 ; B=5024 ; C=5027 ; A$ lab split=' '

\begin{tabular}{|c|c|c|c|c|c|c|}
\hline $\begin{array}{l}\text { Aluminum } \\
\text { Antimony } \\
\text { Arsenic } \\
\text { Barium } \\
\text { Cerium } \\
\text { Cesium } \\
\text { Chromium } \\
\text { Cobalt } \\
\text { Europium } \\
\text { Gallium } \\
\text { Gold } \\
\text { Hafnium } \\
\text { Iron } \\
\text { Lanthanum } \\
\text { Lutetium } \\
\text { Magnesium } \\
\text { Manganese } \\
\text { Mercury } \\
\text { Potassium } \\
\text { Rubidium } \\
\text { Scandium } \\
\text { Silver } \\
\text { Sodium } \\
\text { Terbium } \\
\text { Thorium-232 } \\
\text { Titanium } \\
\text { Uranium-235 } \\
\text { Uranium-238 } \\
\text { Vanadium } \\
\text { Ytterbium }\end{array}$ & $\begin{array}{l}\mathrm{mg} / \mathrm{kg} \\
\mathrm{mg} / \mathrm{kg} \\
\mathrm{mg} / \mathrm{kg} \\
\mathrm{mg} / \mathrm{kg} \\
\mathrm{mg} / \mathrm{kg} \\
\mathrm{mg} / \mathrm{kg} \\
\mathrm{mg} / \mathrm{kg} \\
\mathrm{mg} / \mathrm{kg} \\
\mathrm{mg} / \mathrm{kg} \\
\mathrm{mg} / \mathrm{kg} \\
\mathrm{mg} / \mathrm{kg} \\
\mathrm{mg} / \mathrm{kg} \\
\mathrm{mg} / \mathrm{kg} \\
\mathrm{mg} / \mathrm{kg} \\
\mathrm{mg} / \mathrm{kg} \\
\mathrm{mg} / \mathrm{kg} \\
\mathrm{mg} / \mathrm{kg} \\
\mathrm{mg} / \mathrm{kg} \\
\mathrm{mg} / \mathrm{kg} \\
\mathrm{mg} / \mathrm{kg} \\
\mathrm{mg} / \mathrm{kg} \\
\mathrm{mg} / \mathrm{kg} \\
\mathrm{mg} / \mathrm{kg} \\
\mathrm{mg} / \mathrm{kg} \\
\mathrm{mg} / \mathrm{kg} \\
\mathrm{mg} / \mathrm{kg} \\
\mathrm{mg} / \mathrm{kg} \\
\mathrm{mg} / \mathrm{kg} \\
\mathrm{mg} / \mathrm{kg} \\
\mathrm{mg} / \mathrm{kg}\end{array}$ & $\begin{array}{r}49100.00 \\
0.48 \\
5.34 \\
138.00 \\
43.00 \\
2.55 \\
55.50 \\
12.30 \\
0.56 \\
8.93 \\
0.01 \\
9.67 \\
25800.00 \\
28.30 \\
0.35 \\
5300.00 \\
607.00 \\
0.60 \\
14600.00 \\
440.00 \\
7.38 \\
1.15 \\
2710.00 \\
0.35 \\
9.40 \\
5230.00 \\
0.03 \\
4.14 \\
56.50 \\
1.47\end{array}$ & $\begin{array}{l}\mathbf{J} \\
\mathbf{U} \\
\mathbf{J} \\
\mathbf{J} \\
\mathbf{J} \\
\mathbf{J}\end{array}$ & $\begin{array}{r}77900.00 \\
0.20 \\
5.33 \\
254.00 \\
55.10 \\
4.22 \\
78.20 \\
9.60 \\
0.54 \\
16.60 \\
0.01 \\
7.42 \\
38200.00 \\
37.40 \\
0.29 \\
6800.00 \\
173.00 \\
0.77 \\
19600.00 \\
423.00 \\
10.90 \\
1.52 \\
2260.00 \\
0.40 \\
11.90 \\
5740.00 \\
0.02 \\
3.11 \\
85.50 \\
2.38\end{array}$ & $\begin{array}{l}\mathbf{J} \\
\mathbf{J}\end{array}$ & $\begin{array}{r}90700.00 \\
0.33 \\
4.93 \\
243.00 \\
55.90 \\
4.36 \\
80.30 \\
11.70 \\
0.54 \\
24.80 \\
0.01 \\
5.59 \\
37900.00 \\
36.60 \\
0.29 \\
7810.00 \\
264.00 \\
0.74 \\
21400.00 \\
391.00 \\
12.70 \\
1.50 \\
2890.00 \\
0.26 \\
12.00 \\
5260.00 \\
0.02 \\
1.48 \\
93.70 \\
1.51\end{array}$ \\
\hline
\end{tabular}


$\mathrm{H}-17$

Table H.1 (continued)

\begin{tabular}{|c|c|c|c|c|c|c|c|c|c|}
\hline Analysis & Units & $\begin{array}{l}\text { A horizon } \\
\text { result }\end{array}$ & Qualifier & $\begin{array}{l}\text { B horizon } \\
\text { result }\end{array}$ & Qualifier & $\begin{array}{l}\text { C horizon } \\
\text { result }\end{array}$ & Qualifier & $\begin{array}{l}\text { A lab split } \\
\text { result }\end{array}$ & Qualifier \\
\hline \multicolumn{9}{|c|}{$\begin{array}{l}\text { Location=ORR; Fonnation }=D I S M A L G A P ; \text { Site }=11,27,41 ; \text { Sample type=Regular; } \\
\qquad A=5003 ; B=5006 ; C=5009 ; A \text { lab split=' }\end{array}$} & ․ \\
\hline $\begin{array}{l}\text { Aluminum } \\
\text { Antimony } \\
\text { Arsenic } \\
\text { Barium } \\
\text { Cerium } \\
\text { Cesium } \\
\text { Chromium } \\
\text { Cobalt } \\
\text { Europium } \\
\text { Gallium } \\
\text { Gold } \\
\text { Hafnium } \\
\text { Iron } \\
\text { Lanthanum } \\
\text { Lutetium } \\
\text { Magnesium } \\
\text { Manganese } \\
\text { Mercury } \\
\text { Potassium } \\
\text { Rubidium } \\
\text { Scandium } \\
\text { Silver } \\
\text { Sodium } \\
\text { Terbium } \\
\text { Thorium-232 } \\
\text { Titanium } \\
\text { Uranium-235 } \\
\text { Uranium-238 } \\
\text { Vanadium } \\
\text { Ytterbium }\end{array}$ & $\begin{array}{l}\mathrm{mg} / \mathrm{kg} \\
\mathrm{mg} / \mathrm{kg} \\
\mathrm{mg} / \mathrm{kg} \\
\mathrm{mg} / \mathrm{kg} \\
\mathrm{mg} / \mathrm{kg} \\
\mathrm{mg} / \mathrm{kg} \\
\mathrm{mg} / \mathrm{kg} \\
\mathrm{mg} / \mathrm{kg} \\
\mathrm{mg} / \mathrm{kg} \\
\mathrm{mg} / \mathrm{kg} \\
\mathrm{mg} / \mathrm{kg} \\
\mathrm{mg} / \mathrm{kg} \\
\mathrm{mg} / \mathrm{kg} \\
\mathrm{mg} / \mathrm{kg} \\
\mathrm{mg} / \mathrm{kg} \\
\mathrm{mg} / \mathrm{kg} \\
\mathrm{mg} / \mathrm{kg} \\
\mathrm{mg} / \mathrm{kg} \\
\mathrm{mg} / \mathrm{kg} \\
\mathrm{mg} / \mathrm{kg} \\
\mathrm{mg} / \mathrm{kg} \\
\mathrm{mg} / \mathrm{kg} \\
\mathrm{mg} / \mathrm{kg} \\
\mathrm{mg} / \mathrm{kg} \\
\mathrm{mg} / \mathrm{kg} \\
\mathrm{mg} / \mathrm{kg} \\
\mathrm{mg} / \mathrm{kg} \\
\mathrm{mg} / \mathrm{kg} \\
\mathrm{mg} / \mathrm{kg} \\
\mathrm{mg} / \mathrm{kg}\end{array}$ & $\begin{array}{r}45200.00 \\
0.52 \\
5.49 \\
142.00 \\
41.90 \\
2.31 \\
62.90 \\
15.30 \\
0.49 \\
11.60 \\
0.01 \\
9.69 \\
27100.00 \\
27.70 \\
0.24 \\
4060.00 \\
627.00 \\
0.61 \\
13000.00 \\
425.00 \\
7.20 \\
1.18 \\
535.00 \\
0.35 \\
9.41 \\
5030.00 \\
0.03 \\
3.87 \\
48.60 \\
1.94\end{array}$ & $\begin{array}{l}\mathbf{J} \\
\mathbf{J} \\
\mathbf{J} \\
\mathbf{J} \\
\mathbf{J} \\
\mathbf{J} \\
\mathbf{U} \\
\mathbf{J} \\
\mathbf{J} \\
\mathbf{J} \\
\mathbf{J}\end{array}$ & $\begin{array}{r}77000.00 \\
0.36 \\
4.44 \\
245.00 \\
54.80 \\
4.55 \\
84.50 \\
8.77 \\
0.52 \\
22.10 \\
0.01 \\
6.66 \\
36700.00 \\
38.20 \\
0.30 \\
8050.00 \\
129.00 \\
0.24 \\
19700.00 \\
248.00 \\
11.10 \\
0.49 \\
549.00 \\
0.58 \\
11.40 \\
5410.00 \\
0.02 \\
3.96 \\
84.10 \\
2.46\end{array}$ & $\begin{array}{l}\mathbf{J} \\
\mathbf{J} \\
\mathbf{J} \\
\mathbf{J} \\
\mathbf{J} \\
\mathbf{J} \\
\mathbf{U} \\
\mathbf{J} \\
\mathbf{J} \\
\mathbf{J} \\
\mathbf{J}\end{array}$ & $\begin{array}{r}103000.00 \\
0.28 \\
4.02 \\
267.00 \\
66.90 \\
4.89 \\
95.80 \\
9.62 \\
0.65 \\
22.30 \\
0.01 \\
5.98 \\
45200.00 \\
45.50 \\
0.25 \\
10600.00 \\
117.00 \\
0.82 \\
26500.00 \\
312.00 \\
15.10 \\
1.70 \\
466.00 \\
0.27 \\
12.90 \\
5480.00 \\
0.02 \\
3.39 \\
109.00 \\
1.78\end{array}$ & $\begin{array}{l}\mathbf{J} \\
\mathbf{J} \\
\mathbf{J} \\
\mathbf{J} \\
\mathbf{J} \\
\mathbf{J} \\
\mathbf{U} \\
\mathbf{J} \\
\mathbf{J} \\
\mathbf{J} \\
\mathbf{J}\end{array}$ & 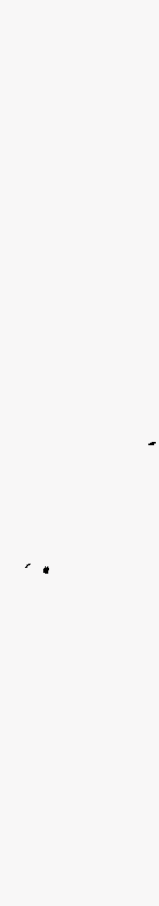 & · \\
\hline
\end{tabular}

Location=ORR; Formation $=D I S M A L$ GAP; Site=19,22,32; Sample type=Regular; $A=5012 ; B=5015 ; C=5018 ; A$ lab split=" '

\begin{tabular}{|c|c|c|c|c|c|c|}
\hline $\begin{array}{l}\text { Aluminum } \\
\text { Antimony } \\
\text { Arsenic } \\
\text { Barium } \\
\text { Cerium } \\
\text { Cesium } \\
\text { Chromium } \\
\text { Cobalt } \\
\text { Europium } \\
\text { Gallium } \\
\text { Gold } \\
\text { Hafnium } \\
\text { Iron } \\
\text { Lanthanum } \\
\text { Lutetium } \\
\text { Magnesium } \\
\text { Manganese } \\
\text { Mercury } \\
\text { Potassium } \\
\text { Rubidium } \\
\text { Scandium } \\
\text { Silver } \\
\text { Sodium } \\
\text { Terbium } \\
\text { Thorium-232 } \\
\text { Titanium } \\
\text { Uranium-235 } \\
\text { Uranium-238 } \\
\text { Vanadium } \\
\text { Ytterbium }\end{array}$ & $\begin{array}{l}\mathrm{mg} / \mathrm{kg} \\
\mathrm{mg} / \mathrm{kg} \\
\mathrm{mg} / \mathrm{kg} \\
\mathrm{mg} / \mathrm{kg} \\
\mathrm{mg} / \mathrm{kg} \\
\mathrm{mg} / \mathrm{kg} \\
\mathrm{mg} / \mathrm{kg} \\
\mathrm{mg} / \mathrm{kg} \\
\mathrm{mg} / \mathrm{kg} \\
\mathrm{mg} / \mathrm{kg} \\
\mathrm{mg} / \mathrm{kg} \\
\mathrm{mg} / \mathrm{kg} \\
\mathrm{mg} / \mathrm{kg} \\
\mathrm{mg} / \mathrm{kg} \\
\mathrm{mg} / \mathrm{kg} \\
\mathrm{mg} / \mathrm{kg} \\
\mathrm{mg} / \mathrm{kg} \\
\mathrm{mg} / \mathrm{kg} \\
\mathrm{mg} / \mathrm{kg} \\
\mathrm{mg} / \mathrm{kg} \\
\mathrm{mg} / \mathrm{kg} \\
\mathrm{mg} / \mathrm{kg} \\
\mathrm{mg} / \mathrm{kg} \\
\mathrm{mg} / \mathrm{kg} \\
\mathrm{mg} / \mathrm{kg} \\
\mathrm{mg} / \mathrm{kg} \\
\mathrm{mg} / \mathrm{kg} \\
\mathrm{mg} / \mathrm{kg} \\
\mathrm{mg} / \mathrm{kg} \\
\mathrm{mg} / \mathrm{kg}\end{array}$ & $\begin{array}{r}8330.00 \\
0.45 \\
4.84 \\
31.80 \\
56.70 \\
3.17 \\
61.40 \\
15.70 \\
0.73 \\
13.80 \\
0.01 \\
9.91 \\
26900.00 \\
34.50 \\
0.43 \\
1140.00 \\
148.00 \\
0.65 \\
16500.00 \\
172.00 \\
8.51 \\
1.26 \\
888.00 \\
0.92 \\
10.50 \\
834.00 \\
0.03 \\
3.33 \\
9.92 \\
1.91\end{array}$ & $\begin{array}{l}\mathbf{J} \\
\mathbf{U} \\
\mathbf{J} \\
\mathbf{J} \\
\mathbf{J} \\
\mathbf{J}\end{array}$ & $\begin{array}{r}90400.00 \\
0.39 \\
5.69 \\
231.00 \\
78.80 \\
4.80 \\
82.50 \\
22.50 \\
0.92 \\
20.20 \\
0.01 \\
6.24 \\
41900.00 \\
41.50 \\
0.20 \\
9000.00 \\
379.00 \\
0.78 \\
21200.00 \\
447.00 \\
13.00 \\
1.56 \\
1040.00 \\
0.69 \\
13.00 \\
5050.00 \\
0.02 \\
2.74 \\
89.80 \\
0.82\end{array}$ & $\begin{array}{l}\mathbf{J} \\
\mathbf{U} \\
\mathbf{J} \\
\mathbf{J} \\
\mathrm{J} \\
\mathbf{J}\end{array}$ & $\begin{array}{r}94000.00 \\
0.28 \\
4.61 \\
196.00 \\
72.10 \\
4.39 \\
80.10 \\
13.40 \\
1.00 \\
23.00 \\
0.01 \\
5.83 \\
40000.00 \\
41.30 \\
0.23 \\
7790.00 \\
771.00 \\
0.78 \\
22200.00 \\
1140.00 \\
14.40 \\
1.58 \\
1260.00 \\
0.32 \\
12.00 \\
3460.00 \\
0.02 \\
3.03 \\
92.30 \\
1.72\end{array}$ \\
\hline
\end{tabular}


H-18

Table H.1 (continued)

\begin{tabular}{lcccccccc}
\hline & A horizon & \multicolumn{3}{c}{ B horizon } & & C horizon & & A lab split \\
Analysis & Units & result & Qualifier & $\begin{array}{c}\text { result } \\
\text { Result }\end{array}$ & Qualifier & result & Qualifier & resulif \\
\hline
\end{tabular}

Location=ORR; Formation =DISMAL GAP; Site=2,26,43; Sample type=Regular; $A=5030 ; B=5033 ; C=5036 ; A$ lab split=' '

\begin{tabular}{|c|c|c|c|c|c|c|}
\hline $\begin{array}{l}\text { Aluminum } \\
\text { Ant imony } \\
\text { Arsenic } \\
\text { Barium } \\
\text { Cerium } \\
\text { Cesium } \\
\text { Chromium } \\
\text { Cobalt } \\
\text { Europium } \\
\text { Gal lium } \\
\text { Gold } \\
\text { Hafnium } \\
\text { Iron } \\
\text { Lanthanum } \\
\text { Lutetium } \\
\text { Magnesium } \\
\text { Manganese } \\
\text { Mercury } \\
\text { Potassium } \\
\text { Rubidium } \\
\text { Scandium } \\
\text { Silver } \\
\text { Sodium } \\
\text { Terbium } \\
\text { Thorium-232 } \\
\text { Titanium } \\
\text { Uranium-235 } \\
\text { Uranium-238 } \\
\text { Vanadium } \\
\text { Ytterbium }\end{array}$ & $\begin{array}{l}\mathrm{mg} / \mathrm{kg} \\
\mathrm{mg} / \mathrm{kg} \\
\mathrm{mg} / \mathrm{kg} \\
\mathrm{mg} / \mathrm{kg} \\
\mathrm{mg} / \mathrm{kg} \\
\mathrm{mg} / \mathrm{kg} \\
\mathrm{mg} / \mathrm{kg} \\
\mathrm{mg} / \mathrm{kg} \\
\mathrm{mg} / \mathrm{kg} \\
\mathrm{mg} / \mathrm{kg} \\
\mathrm{mg} / \mathrm{kg} \\
\mathrm{mg} / \mathrm{kg} \\
\mathrm{mg} / \mathrm{kg} \\
\mathrm{mg} / \mathrm{kg} \\
\mathrm{mg} / \mathrm{kg} \\
\mathrm{mg} / \mathbf{k g} \\
\mathrm{mg} / \mathrm{kg} \\
\mathrm{mg} / \mathrm{kg} \\
\mathrm{mg} / \mathbf{k g} \\
\mathrm{mg} / \mathrm{kg} \\
\mathrm{mg} / \mathrm{kg} \\
\mathrm{mg} / \mathrm{kg} \\
\mathrm{mg} / \mathrm{kg} \\
\mathrm{mg} / \mathrm{kg} \\
\mathrm{mg} / \mathrm{kg} \\
\mathrm{mg} / \mathrm{kg} \\
\mathrm{mg} / \mathrm{kg} \\
\mathrm{mg} / \mathrm{kg} \\
\mathrm{mg} / \mathrm{kg} \\
\mathrm{mg} / \mathrm{kg}\end{array}$ & $\begin{array}{r}52000.00 \\
0.51 \\
4.53 \\
546.00 \\
56.00 \\
2.84 \\
64.20 \\
19.00 \\
0.60 \\
15.20 \\
0.01 \\
10.10 \\
26400.00 \\
32.80 \\
0.43 \\
4120.00 \\
1060.00 \\
0.70 \\
17300.00 \\
1080.00 \\
8.48 \\
1.33 \\
836.00 \\
0.42 \\
9.74 \\
5900.00 \\
0.03 \\
4.74 \\
56.10 \\
1.47\end{array}$ & $\begin{array}{l}\mathrm{J} \\
\mathrm{J} \\
\mathrm{J}\end{array}$ & $\begin{array}{r}76200.00 \\
0.26 \\
6.23 \\
159.00 \\
53.60 \\
4.64 \\
77.70 \\
11.40 \\
0.65 \\
15.90 \\
0.01 \\
7.32 \\
37200.00 \\
40.40 \\
0.32 \\
10400.00 \\
217.00 \\
1.01 \\
21200.00 \\
1240.00 \\
11.70 \\
2.04 \\
716.00 \\
0.41 \\
12.40 \\
5820.00 \\
0.02 \\
2.60 \\
94.30 \\
2.62\end{array}$ & $\begin{array}{l}\mathbf{J} \\
\mathbf{J} \\
\mathbf{J} \\
\mathbf{J} \\
\mathbf{J} \\
\mathbf{J} \\
\mathbf{U} \\
\mathbf{J} \\
\mathbf{J} \\
\mathbf{J} \\
\mathbf{J}\end{array}$ & $\begin{array}{r}84500.00 \\
0.19 \\
4.95 \\
246.00 \\
70.00 \\
4.57 \\
87.50 \\
50.40 \\
0.94 \\
19.10 \\
0.01 \\
7.14 \\
42700.00 \\
44.40 \\
0.25 \\
8490.00 \\
2900.00 \\
0.97 \\
22000.00 \\
1750.00 \\
14.20 \\
1.93 \\
843.00 \\
0.39 \\
13.40 \\
4340.00 \\
0.02 \\
3.48 \\
76.20 \\
1.69\end{array}$ \\
\hline
\end{tabular}

Location=ORR; Formation=DISMAL GAP; Site=2,26,43; Sample type=Field Duplicate; $A=5039 ; B=5042 ; C=5045 ; A$ lab split=' '

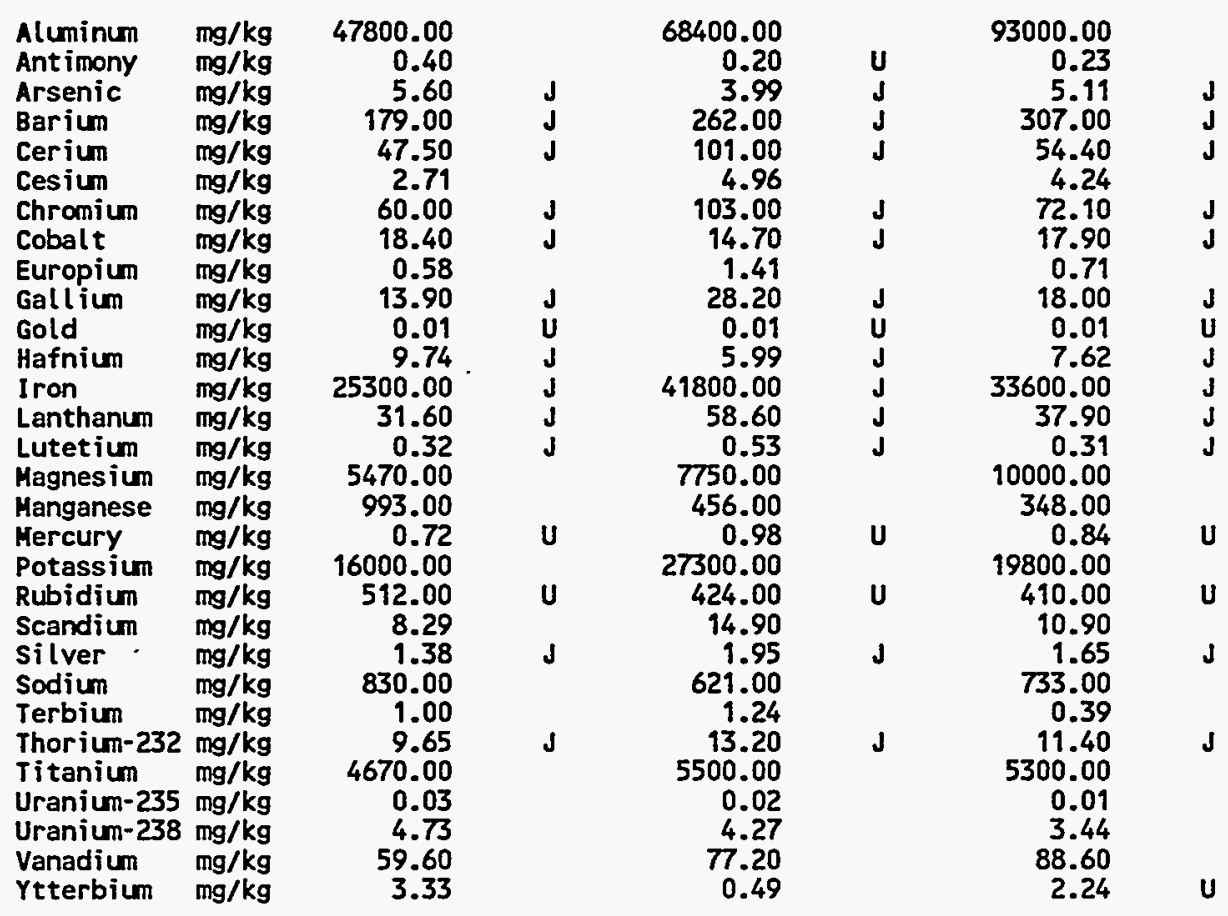


Table H.1 (continued)

\begin{tabular}{|c|c|c|c|c|c|c|c|c|c|}
\hline Analysis & Units & $\begin{array}{l}\text { A horizon } \\
\text { result }\end{array}$ & Qualifier & $\begin{array}{l}\text { B horizon } \\
\text { result }\end{array}$ & Qualifier & $\begin{array}{l}\mathrm{C} \text { horizon } \\
\text { result }\end{array}$ & Qualifier & $\begin{array}{l}\text { A lab split } \\
\text { result }\end{array}$ & Qualifier \\
\hline
\end{tabular}

Location=ORR; Formation=NOLICHUCKY; Site=15,23,25; Sample type=Regular; $A=5057 ; B=5060 ; C=5063 ; A$ lab split=' ,

\begin{tabular}{|c|c|c|c|c|c|c|}
\hline $\begin{array}{l}\text { Aluminum } \\
\text { Antimony } \\
\text { Arsenic } \\
\text { Barium } \\
\text { Cerium } \\
\text { Cesium } \\
\text { Chromium } \\
\text { Cobalt } \\
\text { Europium } \\
\text { Gallium } \\
\text { Gold } \\
\text { Hafnium } \\
\text { Iron } \\
\text { Lanthanum } \\
\text { Lutetium } \\
\text { Magnesium } \\
\text { Manganese } \\
\text { Mercury } \\
\text { Potassium } \\
\text { Rubidium } \\
\text { Scandium } \\
\text { Silver } \\
\text { Sodium } \\
\text { Terbium } \\
\text { Thorium-232 } \\
\text { Titanium } \\
\text { Uranium-235 } \\
\text { Uranium-238 } \\
\text { Vanadium } \\
\text { Ytterbium }\end{array}$ & $\begin{array}{l}\mathrm{mg} / \mathrm{kg} \\
\mathrm{mg} / \mathrm{kg} \\
\mathrm{mg} / \mathrm{kg} \\
\mathrm{mg} / \mathrm{kg} \\
\mathrm{mg} / \mathrm{kg} \\
\mathrm{mg} / \mathrm{kg} \\
\mathrm{mg} / \mathrm{kg} \\
\mathrm{mg} / \mathrm{kg} \\
\mathrm{mg} / \mathrm{kg} \\
\mathrm{mg} / \mathrm{kg} \\
\mathrm{mg} / \mathrm{kg} \\
\mathrm{mg} / \mathrm{kg} \\
\mathrm{mg} / \mathrm{kg} \\
\mathrm{mg} / \mathrm{kg} \\
\mathrm{mg} / \mathrm{kg} \\
\mathrm{mg} / \mathrm{kg} \\
\mathrm{mg} / \mathrm{kg} \\
\mathrm{mg} / \mathrm{kg} \\
\mathrm{mg} / \mathrm{kg} \\
\mathrm{mg} / \mathrm{kg} \\
\mathrm{mg} / \mathrm{kg} \\
\mathrm{mg} / \mathrm{kg} \\
\mathrm{mg} / \mathrm{kg} \\
\mathrm{mg} / \mathrm{kg} \\
\mathrm{mg} / \mathrm{kg} \\
\mathrm{mg} / \mathrm{kg} \\
\mathrm{mg} / \mathrm{kg} \\
\mathrm{mg} / \mathrm{kg} \\
\mathrm{mg} / \mathrm{kg} \\
\mathrm{mg} / \mathrm{kg}\end{array}$ & $\begin{array}{r}57100.00 \\
0.62 \\
6.83 \\
195.00 \\
49.00 \\
3.43 \\
75.10 \\
12.60 \\
0.55 \\
13.70 \\
0.01 \\
9.17 \\
34200.00 \\
32.70 \\
0.37 \\
4730.00 \\
494.00 \\
0.74 \\
16100.00 \\
382.00 \\
8.74 \\
1.41 \\
356.00 \\
0.21 \\
11.70 \\
4920.00 \\
0.03 \\
4.51 \\
67.00 \\
3.19\end{array}$ & $\begin{array}{l}\mathbf{J} \\
\mathbf{U} \\
\mathbf{J} \\
\mathbf{J} \\
\mathrm{J} \\
\mathbf{J}\end{array}$ & $\begin{array}{r}90500.00 \\
0.41 \\
6.69 \\
115.00 \\
51.30 \\
6.56 \\
90.10 \\
9.49 \\
0.79 \\
28.00 \\
0.01 \\
5.94 \\
47400.00 \\
38.70 \\
0.22 \\
7360.00 \\
134.00 \\
0.90 \\
20900.00 \\
364.00 \\
12.70 \\
1.81 \\
298.00 \\
0.37 \\
15.10 \\
5060.00 \\
0.02 \\
2.69 \\
99.70 \\
2.48\end{array}$ & $\begin{array}{l}\mathbf{J} \\
\mathbf{U} \\
\mathbf{J} \\
\mathbf{J} \\
\mathbf{J} \\
\mathbf{J}\end{array}$ & $\begin{array}{r}89000.00 \\
0.33 \\
5.60 \\
183.00 \\
78.30 \\
5.67 \\
93.40 \\
13.50 \\
0.73 \\
41.00 \\
0.01 \\
5.02 \\
52800.00 \\
43.00 \\
0.36 \\
7970.00 \\
243.00 \\
0.34 \\
28400.00 \\
403.00 \\
15.10 \\
2.13 \\
343.00 \\
0.43 \\
15.80 \\
4610.00 \\
0.02 \\
3.20 \\
84.60 \\
1.41\end{array}$ \\
\hline
\end{tabular}

Location =ORR; Formation =NOLICHUCKY; Site=16,28,42; Sample type=Regular; $A=5066 ; B=5069 ; C=5072 ; A$ lab split=' '

$\begin{array}{ll}\text { Aluminum } & \mathrm{mg} / \mathrm{kg} \\ \text { Antimony } & \mathrm{mg} / \mathrm{kg} \\ \text { Arsenic } & \mathrm{mg} / \mathrm{kg} \\ \text { Barium } & \mathrm{mg} / \mathrm{kg} \\ \text { Cerium } & \mathrm{mg} / \mathrm{kg} \\ \text { Cesium } & \mathrm{mg} / \mathrm{kg} \\ \text { Chromium } & \mathrm{mg} / \mathrm{kg} \\ \text { Cobalt } & \mathrm{mg} / \mathrm{kg} \\ \text { Europium } & \mathrm{mg} / \mathrm{kg} \\ \text { Gallium } & \mathrm{mg} / \mathrm{kg} \\ \text { Gold } & \mathrm{mg} / \mathrm{kg} \\ \text { Hafnium } & \mathrm{mg} / \mathrm{kg} \\ \text { Iron } & \mathrm{mg} / \mathrm{kg} \\ \text { Lanthanum } & \mathrm{mg} / \mathrm{kg} \\ \text { Lutetium } & \mathrm{mg} / \mathrm{kg} \\ \text { Magnesium } & \mathrm{mg} / \mathrm{kg} \\ \text { Manganese } & \mathrm{mg} / \mathrm{kg} \\ \text { Mercury } & \mathrm{mg} / \mathrm{kg} \\ \text { Potassium } & \mathrm{mg} / \mathrm{kg} \\ \text { Rubidium } & \mathrm{mg} / \mathrm{kg} \\ \text { Scandium } & \mathrm{mg} / \mathrm{kg} \\ \text { Silver } & \mathrm{mg} / \mathrm{kg} \\ \text { Sodium } & \mathrm{mg} / \mathrm{kg} \\ \text { Terbium } & \mathrm{mg} / \mathrm{kg} \\ \text { Thorium-232 } & \mathrm{mg} / \mathrm{kg} \\ \text { Titanium } & \mathrm{mg} / \mathrm{kg} \\ \text { Uranium-235 } & \mathrm{mg} / \mathrm{kg} \\ \text { Uranium-238 } & \mathrm{mg} / \mathrm{kg} \\ \text { Vanadium } & \mathrm{mg} / \mathrm{kg} \\ \text { Ytterbium } & \mathrm{mg} / \mathrm{kg} \\ \text { Zinc } & \mathrm{mg} / \mathrm{kg} \\ & \end{array}$

42800.00

0.48

5.96

186.00

48.60

2.36

75.90

14.10

0.61

16.40

0.01

10.80

30900.00

32.30

0.33

3730.00

579.00

0.79

16000.00

366.00

8.47

1.50

367.00

0.40
11.90

4920.00

0.03

3.11

50.00

2.29
72700.00

0.31

7.98

108.00

56.80

4.32

85.30

18.40

0.62

15.00

0.01

7.23

49100.00

40.80

0.15

6660.00

387.00

0.97

20100.00

401.00

12.10

1.89

337.00

0.39

15.80

5510.00

0.02

3.04

83.70

0.28
85200.00

$\begin{array}{rr}0.34 & \\ 5.57 & \mathrm{~J} \\ 7860.00 & \mathrm{~J} \\ 73.00 & \mathrm{~J} \\ 6.28 & \\ 90.80 & \mathrm{~J} \\ 25.00 & \mathrm{~J} \\ 0.60 & \\ 21.50 & \mathrm{~J} \\ 0.01 & \mathrm{U} \\ 5.96 & \mathrm{~J} \\ 54700.00 & \mathrm{~J} \\ 40.10 & \mathrm{~J} \\ 0.29 & \mathrm{~J}\end{array}$

7840.00

655.00

$1.23 \quad U$

28100.00

$661.00 \quad U$

14.10

1.18

0.29
14.40

9880.00

0.02

2.84

2.40 
$\mathrm{H}-20$

Table H.1 (continued)

\begin{tabular}{|c|c|c|c|c|c|c|c|c|c|}
\hline Analysis & Units & $\begin{array}{l}\text { A horizon } \\
\text { result }\end{array}$ & Qualifier & $\begin{array}{l}\text { B horizon } \\
\text { result }\end{array}$ & Qualifier & $\begin{array}{l}\mathrm{C} \text { horizon } \\
\text { result }\end{array}$ & Qualifier & $\begin{array}{l}\text { A lab split } \\
\text { result }\end{array}$ & Qualifier \\
\hline
\end{tabular}

Location=ORR; Formation=NOLICHUCKY; Site=3,13,24; Sample type=Regular; $A=5084 ; B=5087 ; C=5090 ; A$ lab split $=$ '

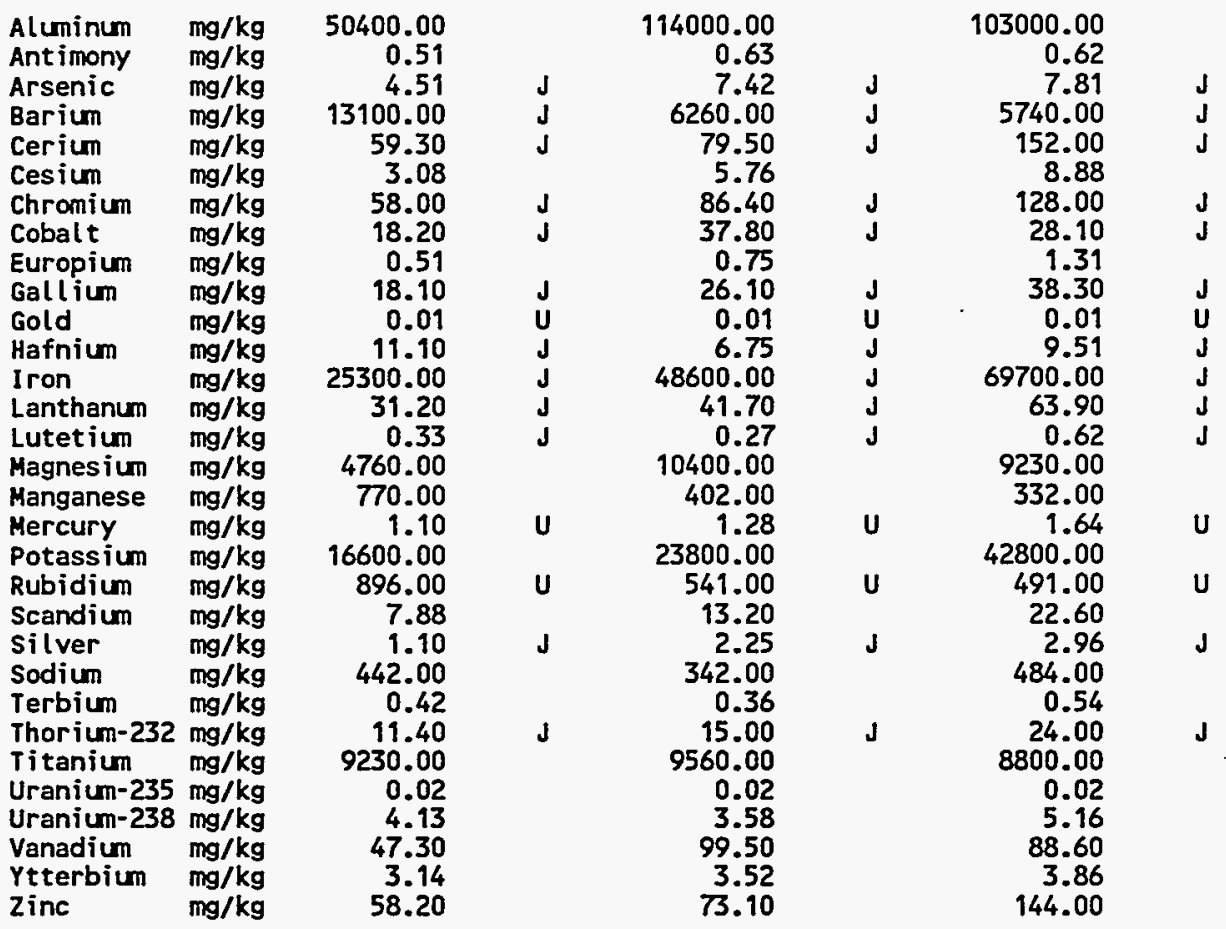

Location $=O R R$; Formation $=$ NOLICHUCKY; Site $=5,21,31$; Sample type=Regular; $A=5075 ; B=5078 ; C=5081 ; A$ lab split=' '

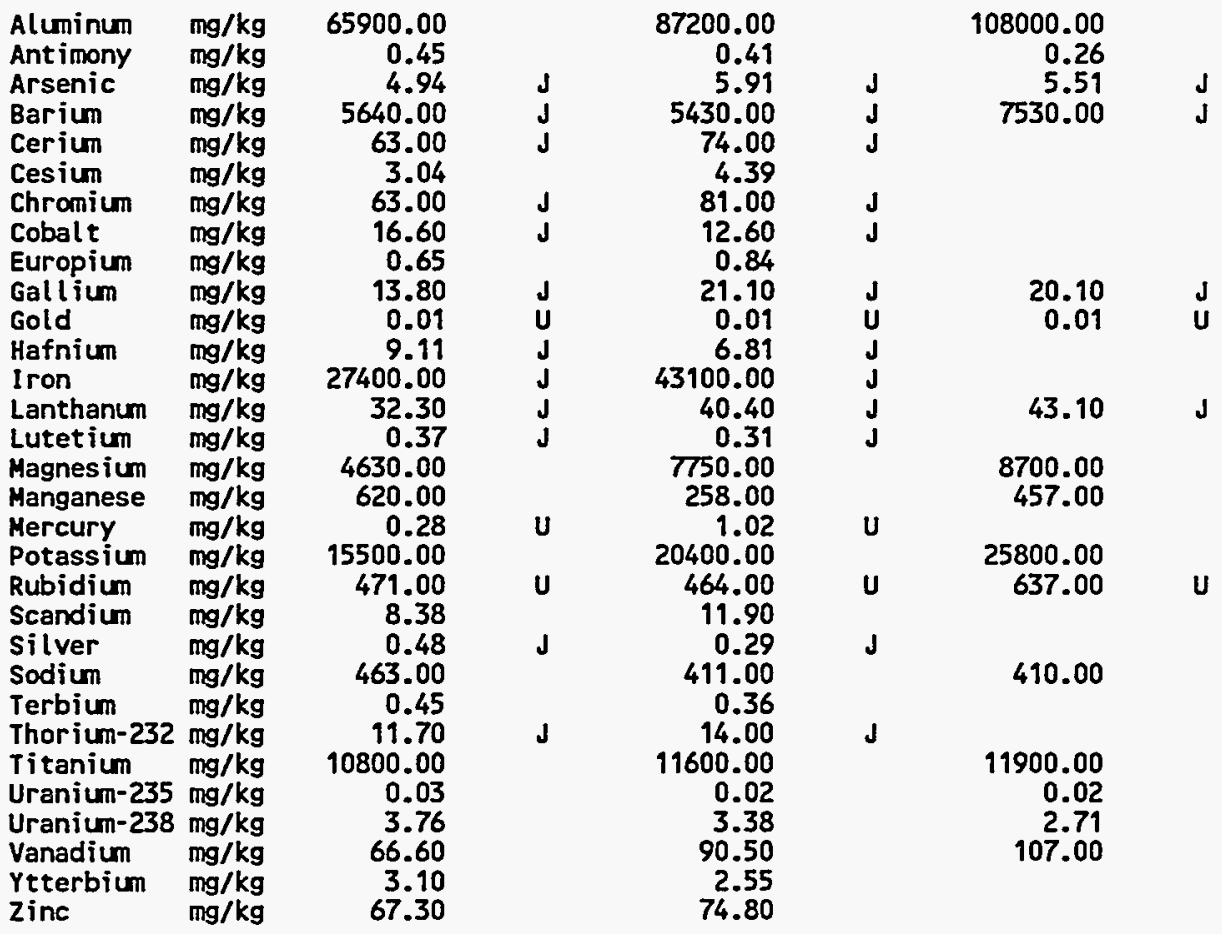


Table H.1 (continued)

\begin{tabular}{|c|c|c|c|c|c|c|c|c|c|}
\hline Analysis & Units & $\begin{array}{l}\text { A horizon } \\
\text { result }\end{array}$ & Qualifier & $\begin{array}{l}\text { B horizon } \\
\text { result }\end{array}$ & Qualifier & $\begin{array}{l}\text { C horizon } \\
\text { result }\end{array}$ & Qualifier & $\begin{array}{l}\text { A lab split } \\
\text { result }\end{array}$ & Qualifier \\
\hline \multicolumn{9}{|c|}{$\begin{array}{l}\text { Location }=\text { ROA; Formation }=\text { COPPER RIDGE; Site=33,35,44; Sample type=Regular; } \\
\qquad A=6048 ; B=6051 ; C=6054 ; A \text { lab split=" }\end{array}$} & roth \\
\hline $\begin{array}{l}\text { Aluminum } \\
\text { Antimony } \\
\text { Arsenic } \\
\text { Barium } \\
\text { Cerium } \\
\text { Cesium } \\
\text { Chromium } \\
\text { Cobalt } \\
\text { Europium } \\
\text { Gallium } \\
\text { Gold } \\
\text { Hafnium } \\
\text { Iron } \\
\text { Lanthanum } \\
\text { Lutetium } \\
\text { Magnesium } \\
\text { Manganese } \\
\text { Mercury } \\
\text { Potassium } \\
\text { Rubidium } \\
\text { Scandium } \\
\text { Silver } \\
\text { Sodium } \\
\text { Terbium } \\
\text { Thorium-232 } \\
\text { Titanium } \\
\text { Uranium-235 } \\
\text { Uranium-238 } \\
\text { Vanadium } \\
\text { Ytterbium } \\
\text { Zinc }\end{array}$ & 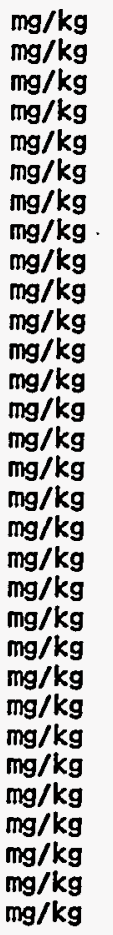 & $\begin{array}{r}12.00 \\
0.73 \\
13.10 \\
1.68 \\
38.40 \\
2.00 \\
29.90 \\
10.30 \\
0.60 \\
16.50 \\
0.01 \\
5.24 \\
16900.00 \\
19.70 \\
0.20 \\
16.20 \\
0.06 \\
0.71 \\
4340.00 \\
\\
3.61 \\
1.18 \\
426.00 \\
0.47 \\
5.48 \\
6.31 \\
0.02 \\
2.86 \\
0.05 \\
2.25 \\
55.20\end{array}$ & $\begin{array}{l}\mathbf{J} \\
\mathbf{J} \\
\mathbf{J} \\
\mathbf{J} \\
\mathbf{J} \\
\mathbf{J} \\
\mathbf{U} \\
\mathbf{J} \\
\mathbf{J} \\
\mathbf{J} \\
\mathbf{J} \\
\mathbf{U} \\
\mathbf{U} \\
\mathbf{U}\end{array}$ & $\begin{array}{r}45100.00 \\
0.99 \\
20.50 \\
164.00 \\
33.20 \\
3.53 \\
46.70 \\
8.42 \\
0.48 \\
15.90 \\
0.01 \\
5.37 \\
27800.00 \\
19.40 \\
0.21 \\
2520.00 \\
209.00 \\
0.94 \\
4780.00 \\
401.00 \\
7.54 \\
1.67 \\
313.00 \\
0.31 \\
8.77 \\
4240.00 \\
0.03 \\
4.48 \\
77.70 \\
2.32 \\
92.50\end{array}$ & $\begin{array}{l}J \\
J \\
J \\
J \\
J \\
J \\
U \\
J \\
J \\
J \\
J\end{array}$ & $\begin{array}{r}95700.00 \\
1.76 \\
38.20 \\
110.00 \\
46.00 \\
4.84 \\
61.00 \\
8.71 \\
0.48 \\
24.60 \\
0.00 \\
3.90 \\
49300.00 \\
26.60 \\
0.21 \\
3460.00 \\
107.00 \\
0.39 \\
4610.00 \\
471.00 \\
12.40 \\
1.88 \\
169.00 \\
0.29 \\
13.20 \\
4480.00 \\
0.04 \\
5.58 \\
146.00 \\
1.34 \\
122.00\end{array}$ & $\begin{array}{l}\mathbf{J} \\
\mathbf{J} \\
\mathbf{J} \\
\mathbf{J} \\
\mathbf{J} \\
\mathbf{J} \\
\mathbf{J} \\
\mathbf{J} \\
\mathbf{J} \\
\mathbf{J}\end{array}$ & & . \\
\hline
\end{tabular}

Location=ROA; Formation=COPPER RIDGE; Site=34,39,41; Sample type=Regular; $A=6086 ; B=6089 ; C=6092 ; A$ lab split=' '

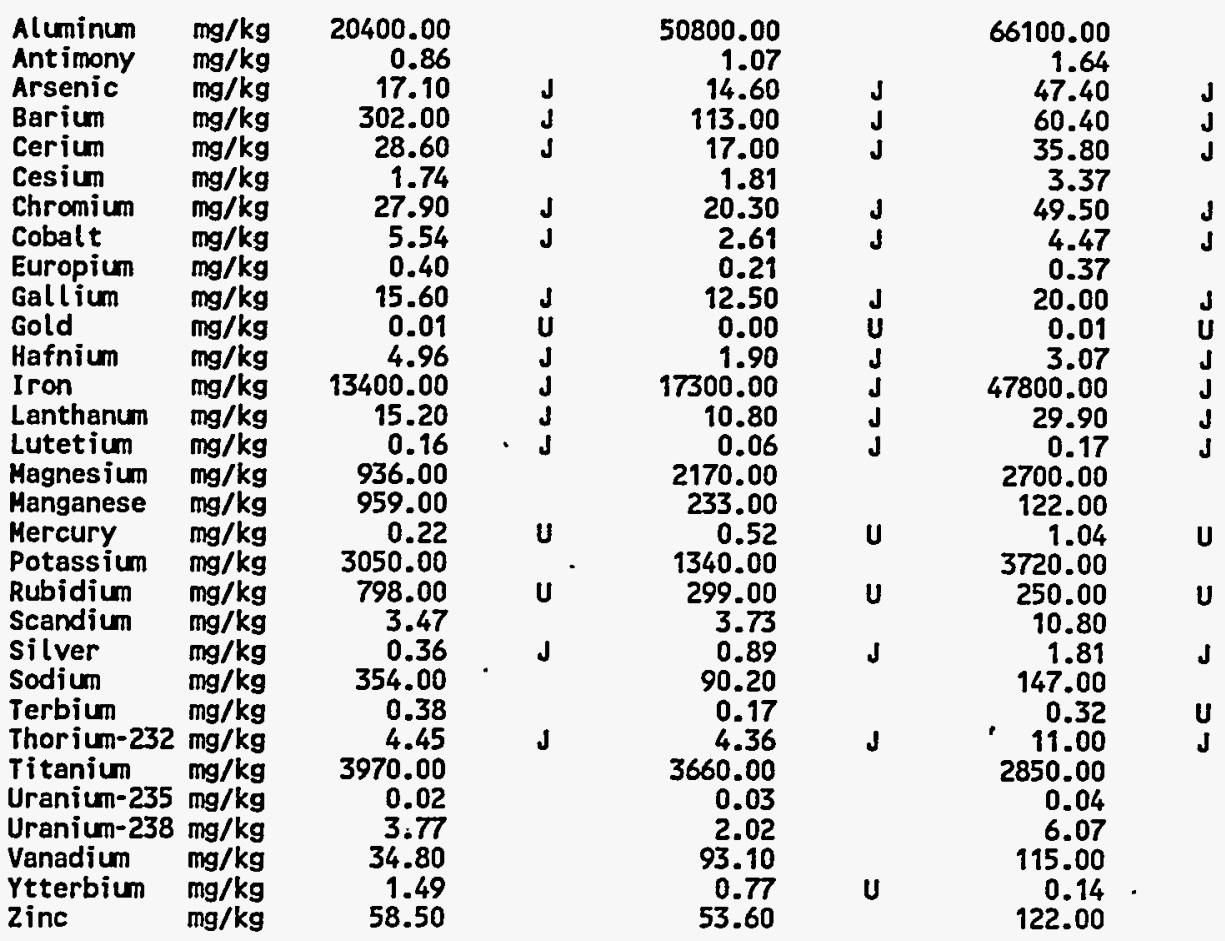


Table H.1 (continued)

\begin{tabular}{|c|c|c|c|c|c|c|c|c|c|}
\hline Analysis & Units & $\begin{array}{l}\text { A horizon } \\
\text { result }\end{array}$ & Qualifier & $\begin{array}{l}\text { B horizon } \\
\text { result }\end{array}$ & Qualifier & $\begin{array}{l}\text { C horizon } \\
\text { result }\end{array}$ & Qualifier & $\begin{array}{l}\text { A lab split } \\
\text { result }\end{array}$ & Qualifier \\
\hline
\end{tabular}

Location $=R O A ;$ Formation $=C O P P E R$ RIDGE; Site $=40,42,43$; Sample type $=$ Regular; $A=6057 ; B=6060 ; C=6063 ; A$ lab split=' '

\begin{tabular}{|c|c|c|c|c|c|c|}
\hline $\begin{array}{l}\text { Aluminum } \\
\text { Ant imony } \\
\text { Arsenic } \\
\text { Barium } \\
\text { Cerium } \\
\text { Cesium } \\
\text { Chromium } \\
\text { Cobalt } \\
\text { Europium } \\
\text { Gallium } \\
\text { Gold } \\
\text { Hafnium } \\
\text { Iron } \\
\text { Lanthanum } \\
\text { Lutetium } \\
\text { Magnesium } \\
\text { Manganese } \\
\text { Mercury } \\
\text { Potassium } \\
\text { Rubidium } \\
\text { Scandium } \\
\text { Silver } \\
\text { Sodium } \\
\text { Terbium } \\
\text { Thorium-23 } \\
\text { Titanium } \\
\text { Uranium-23 } \\
\text { Uranium-23 } \\
\text { Vanadium } \\
\text { Ytterbium } \\
\text { Zinc }\end{array}$ & $\begin{array}{l}\mathrm{mg} / \mathrm{kg} \\
\mathrm{mg} / \mathrm{kg} \\
\mathrm{mg} / \mathrm{kg} \\
\mathrm{mg} / \mathrm{kg} \\
\mathrm{mg} / \mathrm{kg} \\
\mathrm{mg} / \mathrm{kg} \\
\mathrm{mg} / \mathrm{kg} \\
\mathrm{mg} / \mathrm{kg} \\
\mathrm{mg} / \mathrm{kg} \\
\mathrm{mg} / \mathrm{kg} \\
\mathrm{mg} / \mathrm{kg} \\
\mathrm{mg} / \mathrm{kg} \\
\mathrm{mg} / \mathrm{kg} \\
\mathrm{mg} / \mathrm{kg} \\
\mathrm{mg} / \mathrm{kg} \\
\mathrm{mg} / \mathrm{kg} \\
\mathrm{mg} / \mathrm{kg} \\
\mathrm{mg} / \mathrm{kg} \\
\mathrm{mg} / \mathrm{kg} \\
\mathrm{mg} / \mathrm{kg} \\
\mathrm{mg} / \mathrm{kg} \\
\mathrm{mg} / \mathrm{kg} \\
\mathrm{mg} / \mathrm{kg} \\
\mathrm{mg} / \mathrm{kg} \\
\mathrm{mg} / \mathrm{kg} \\
\mathrm{mg} / \mathrm{kg} \\
\mathrm{mg} / \mathrm{kg} \\
\mathrm{mg} / \mathrm{kg} \\
\mathrm{mg} / \mathrm{kg} \\
\mathrm{mg} / \mathrm{kg} \\
\mathrm{mg} / \mathrm{kg}\end{array}$ & $\begin{array}{r}16300.00 \\
0.58 \\
4.50 \\
132.00 \\
27.90 \\
1.65 \\
23.80 \\
2.59 \\
0.39 \\
15.20 \\
0.01 \\
6.63 \\
8370.00 \\
14.80 \\
0.17 \\
818.00 \\
232.00 \\
0.61 \\
2960.00 \\
253.00 \\
2.52 \\
0.94 \\
431.00 \\
0.42 \\
4.28 \\
3920.00 \\
0.02 \\
2.80 \\
30.00 \\
1.35 \\
34.20\end{array}$ & $\begin{array}{l}\mathbf{J} \\
\mathbf{U} \\
\mathbf{J} \\
\mathbf{J} \\
\mathbf{J} \\
\mathbf{J}\end{array}$ & $\begin{array}{r}42000.00 \\
0.85 \\
12.40 \\
86.50 \\
19.80 \\
3.25 \\
37.30 \\
3.95 \\
0.35 \\
17.50 \\
0.01 \\
5.06 \\
20600.00 \\
12.70 \\
0.16 \\
2170.00 \\
78.50 \\
0.79 \\
3150.00 \\
263.00 \\
4.33 \\
1.33 \\
224.00 \\
0.42 \\
6.88 \\
4510.00 \\
0.02 \\
3.88 \\
76.70 \\
1.34 \\
51.10\end{array}$ & $\begin{array}{l}\mathbf{J} \\
\mathbf{U} \\
\mathbf{J} \\
\mathbf{J} \\
\mathbf{J} \\
\mathbf{J}\end{array}$ & $\begin{array}{r}60300.00 \\
1.18 \\
22.80 \\
92.50 \\
17.20 \\
3.55 \\
48.00 \\
4.27 \\
0.21 \\
15.00 \\
0.00 \\
3.14 \\
35000.00 \\
11.80 \\
0.08 \\
3200.00 \\
53.50 \\
0.78 \\
3160.00 \\
413.00 \\
5.99 \\
1.32 \\
148.00 \\
0.24 \\
9.76 \\
3550.00 \\
0.03 \\
3.79 \\
113.00 \\
1.42 \\
42.10\end{array}$ \\
\hline
\end{tabular}

Location $=R O A ;$ Formation $=C O P P E R$ RIDGE; Site $=45,46,47$; Sample type $=$ Regular; $A=6075 ; B=6072 ; C=6066 ; A$ lab split="

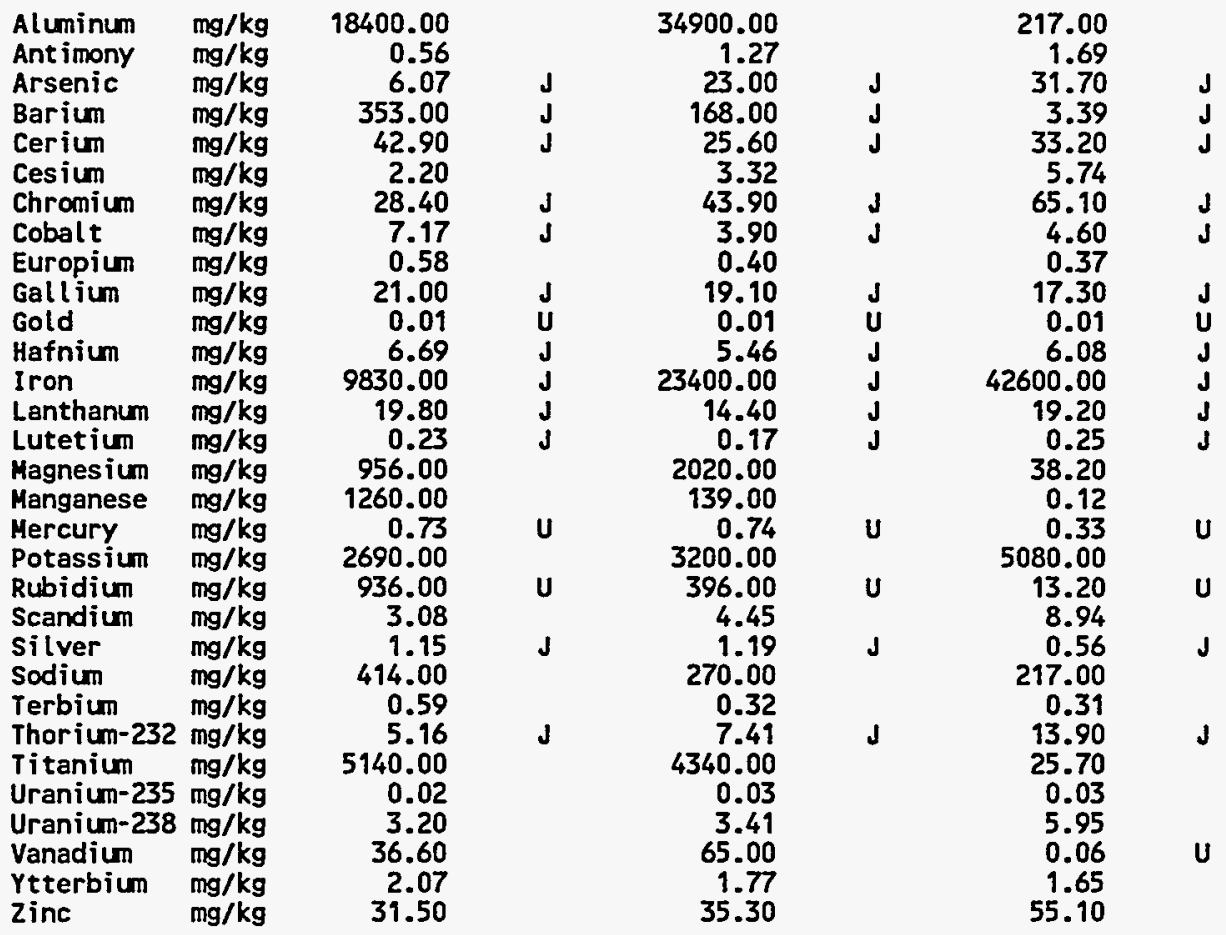


$\mathrm{H}-23$

Table H.1 (continued)

\begin{tabular}{|c|c|c|c|c|c|c|c|c|c|}
\hline Analysis & Units & $\begin{array}{l}\text { A horizon } \\
\text { result }\end{array}$ & Qualifier & $\begin{array}{l}\text { B horizon } \\
\text { result }\end{array}$ & Qualifier & $\begin{array}{l}\text { C horizon } \\
\text { result }\end{array}$ & Qualifier & $\begin{array}{l}\text { A lab split } \\
\text { result }\end{array}$ & Qualifier \\
\hline
\end{tabular}

Location=ROA; Formation $=C O P P E R$ RIDGE; Site $=45,46,47$; Sample type $=$ Field Duplicate; $A=6078 ; B=6081 ; C=6069$ A lab split=' '

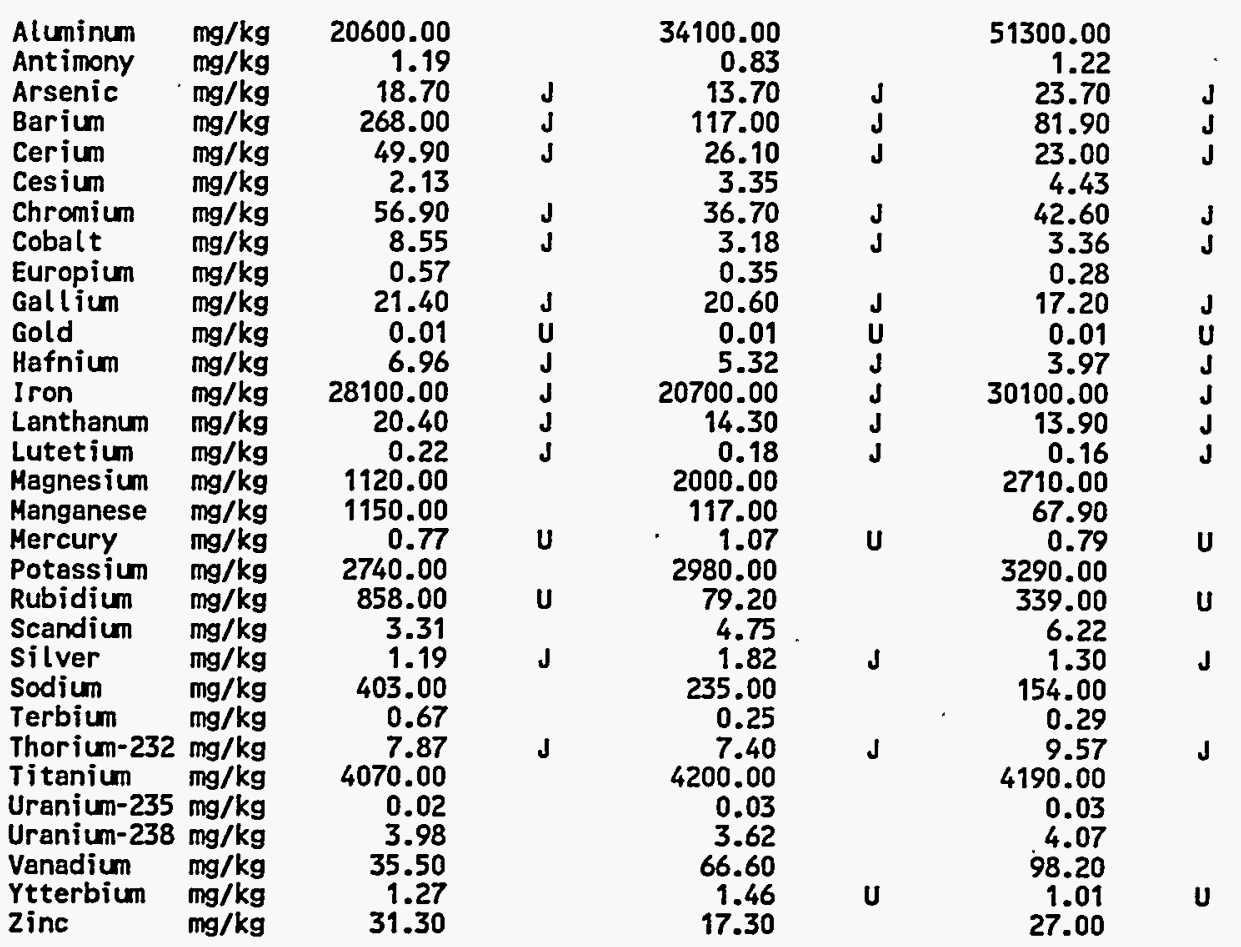

Location $=R O A ;$ Formation $=D I S M A L$ GAP; Site $=10,13,14 ;$ Sample type $=$ Regular; $A=6030 ; B=6033 ; C=6036 ; A$ lab split=' '

$\begin{array}{ll}\text { Aluminum } & \mathrm{mg} / \mathrm{kg} \\ \text { Antimony } & \mathrm{mg} / \mathrm{kg} \\ \text { Arsenic } & \mathrm{mg} / \mathrm{kg} \\ \text { Barium } & \mathrm{mg} / \mathrm{kg} \\ \text { Cerium } & \mathrm{mg} / \mathrm{kg} \\ \text { Cesium } & \mathrm{mg} / \mathrm{kg} \\ \text { Chromium } & \mathrm{mg} / \mathrm{kg} \\ \text { Cobalt } & \mathrm{mg} / \mathrm{kg} \\ \text { Europium } & \mathrm{mg} / \mathrm{kg} \\ \text { Gallium } & \mathrm{mg} / \mathrm{kg} \\ \text { Gold } & \mathrm{mg} / \mathrm{kg} \\ \text { Hafnium } & \mathrm{mg} / \mathrm{kg} \\ \text { Iron } & \mathrm{mg} / \mathrm{kg} \\ \text { Lanthanum } & \mathrm{mg} / \mathrm{kg} \\ \text { Lutetium } & \mathrm{mg} / \mathrm{kg} \\ \text { Magnesium } & \mathrm{mg} / \mathrm{kg} \\ \text { Manganese } & \mathrm{mg} / \mathrm{kg} \\ \text { Mercury } & \mathrm{mg} / \mathrm{kg} \\ \text { Potassium } & \mathrm{mg} / \mathrm{kg} \\ \text { Rubidium } & \mathrm{mg} / \mathrm{kg} \\ \text { Scandium } & \mathrm{mg} / \mathrm{kg} \\ \text { Silver } & \mathrm{mg} / \mathrm{kg} \\ \text { Sodium } & \mathrm{mg} / \mathrm{kg} \\ \text { Terbium } & \mathrm{mg} / \mathrm{kg} \\ \text { Thorium-232 } & \mathrm{mg} / \mathrm{kg} \\ \text { Titanium } & \mathrm{mg} / \mathrm{kg} \\ \text { Uranium-235 } & \mathrm{mg} / \mathrm{kg} \\ \text { Uranium-238 } & \mathrm{mg} / \mathrm{kg} \\ \text { Vanadium } & \mathrm{mg} / \mathrm{kg} \\ \text { Ytterbium } & \mathrm{mg} / \mathrm{kg} \\ \text { Zinc } & \mathrm{mg} / \mathrm{kg}\end{array}$
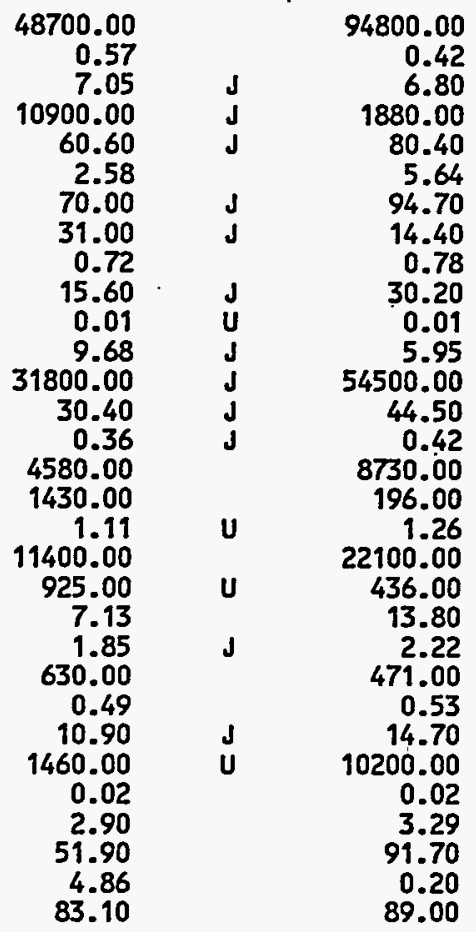

97000.00

$\begin{array}{rr}0.27 & \\ 5.53 & \mathrm{~J} \\ 5200.00 & \mathrm{~J} \\ 87.40 & \mathrm{~J} \\ 5.51 & \\ 96.70 & \mathrm{~J} \\ 17.10 & \mathrm{~J} \\ 0.97 & \\ 31.30 & \mathrm{~J} \\ 0.01 & \mathrm{U} \\ 5.09 & \mathrm{~J} \\ 52300.00 & \mathrm{~J} \\ 45.80 & \mathrm{~J} \\ 0.33 & \mathrm{~J}\end{array}$

8890.00

326.00 $1.40 \quad u$

25.00

7.13

1.85
30.00

0.49

10.90
1460.00

0.02

2.90
51.90

4.86

89.00

$\begin{array}{rr}1.40 & \text { U } \\ 27800.00 & \\ 441.00 & \mathrm{U} \\ 15.70 & \\ 2.50 & \mathrm{~J} \\ 836.00 & \\ 0.67 & \\ 15.30 & \mathrm{~J} \\ 637.00 & \mathrm{U} \\ 0.02 & \\ 2.86 & \\ 86.70 & \\ 0.41 & \\ 97.80 & \end{array}$


$\mathrm{H}-24$

Table H.1 (continued)

\begin{tabular}{|c|c|c|c|c|c|c|c|c|c|}
\hline Analysis & Units & $\begin{array}{l}\text { A horizon } \\
\text { result }\end{array}$ & Qualifier & $\begin{array}{l}\text { B horizon } \\
\text { result }\end{array}$ & Qualifier & $\begin{array}{l}\text { C horizon } \\
\text { result }\end{array}$ & Qualifier & $\begin{array}{l}\text { A lab split } \\
\text { result }\end{array}$ & Qualifier \\
\hline
\end{tabular}

Location=ROA; Formation $=$ DISMAL GAP; Site $=10,13,14 ;$ Sample type $=$ Field Duplicate; $A=6039 ; B=6042 ; C=6045 ; A$ lab split $=$ '

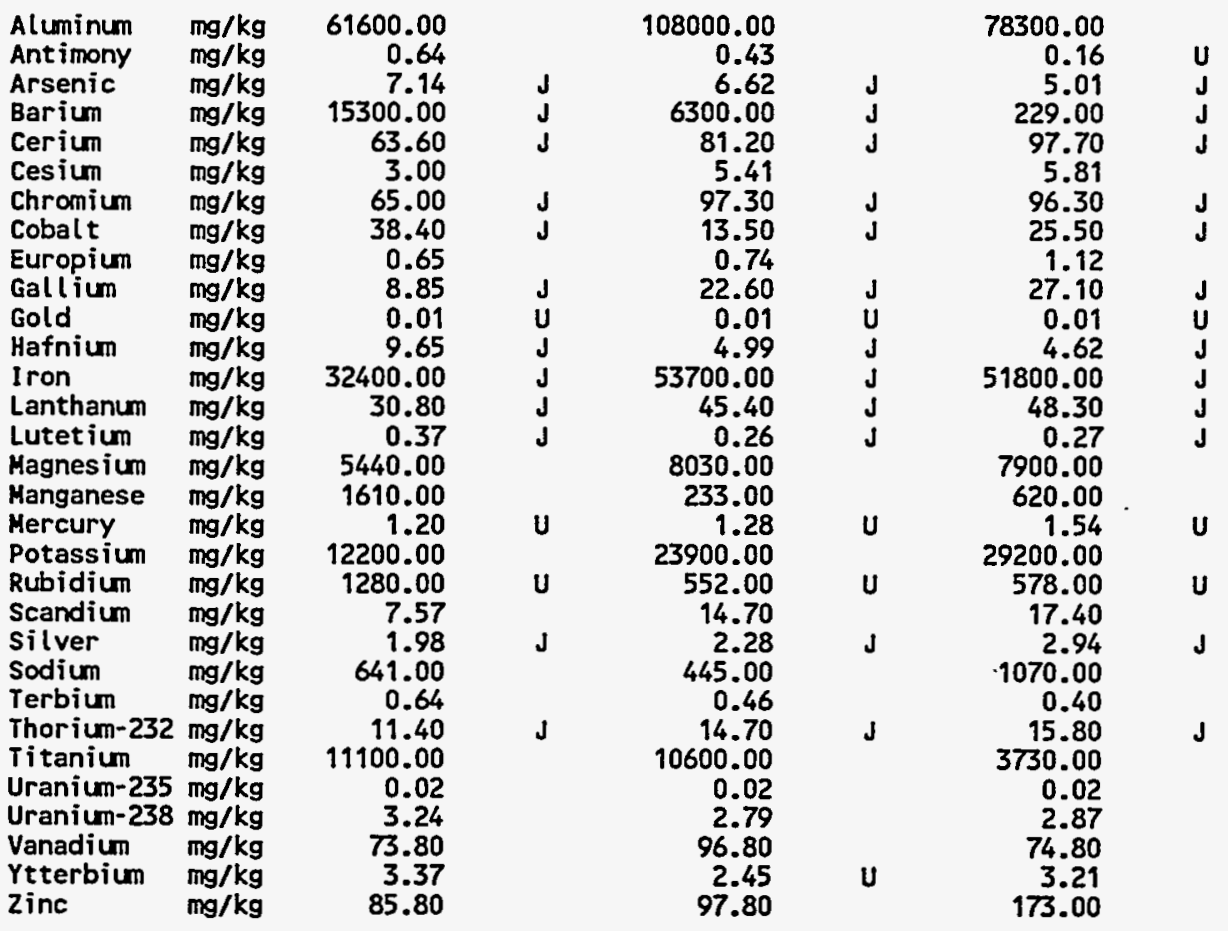

Location=ROA; Formation=DISMAL GAP; Site=3,7,21; Sample type=Regular; $A=6021 ; B=6024 ; C=6027 ; A$ lab split='

$\begin{array}{ll}\text { Aluminum } & \mathrm{mg} / \mathrm{kg} \\ \text { Antimony } & \mathrm{mg} / \mathrm{kg} \\ \text { Arsenic } & \mathrm{mg} / \mathrm{kg} \\ \text { Barium } & \mathrm{mg} / \mathrm{kg} \\ \text { Cerium } & \mathrm{mg} / \mathrm{kg} \\ \text { Cesium } & \mathrm{mg} / \mathrm{kg} \\ \text { Chromium } & \mathrm{mg} / \mathrm{kg} \\ \text { Cobalt } & \mathrm{mg} / \mathrm{kg} \\ \text { Europium } & \mathrm{mg} / \mathrm{kg} \\ \text { Gallium } & \mathrm{mg} / \mathrm{kg} \\ \text { Gold } & \mathrm{mg} / \mathrm{kg} \\ \text { Hafnium } & \mathrm{mg} / \mathrm{kg} \\ \text { Iron } & \mathrm{mg} / \mathrm{kg} \\ \text { Lanthanum } & \mathrm{mg} / \mathrm{kg} \\ \text { Lutetium } & \mathrm{mg} / \mathrm{kg} \\ \text { Magnesium } & \mathrm{mg} / \mathrm{kg} \\ \text { Manganese } & \mathrm{mg} / \mathrm{kg} \\ \text { Mercury } & \mathrm{mg} / \mathrm{kg} \\ \text { Potassium } & \mathrm{mg} / \mathrm{kg} \\ \text { Rubidium } & \mathrm{mg} / \mathrm{kg} \\ \text { Scandium } & \mathrm{mg} / \mathrm{kg} \\ \text { Silver } & \mathrm{mg} / \mathrm{kg} \\ \text { Sodium } & \mathrm{mg} / \mathrm{kg} \\ \text { Terbium } & \mathrm{mg} / \mathrm{kg} \\ \text { Thorium-232 } & \mathrm{mg} / \mathrm{kg} \\ \text { Titanium } & \mathrm{mg} / \mathrm{kg} \\ \text { Uranium-235 } \mathrm{mg} / \mathrm{kg} \\ \text { Uranium-238 } \mathrm{mg} / \mathrm{kg} \\ \text { Vanadium } & \mathrm{mg} / \mathrm{kg} \\ \text { Ytterbium } & \mathrm{mg} / \mathrm{kg} \\ \text { Zine } & \mathrm{mg} / \mathrm{kg} \\ & \end{array}$

61900.00

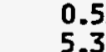

12400.01000

62.60

3.14

73.00

19.50

0.65

16.90

0.01

10.30

31900.00

31.70

0.36

6060.00

1150.00

1.13

14300.00

997.00

8.94

1.91

536.00

0.45

11.60

9810.00

0.02

3.37

72.60

3.14

72.40

$\begin{array}{rr}64100.00 & \\ 0.54 & \\ 5.81 & \mathrm{~J} \\ 6510.00 & \mathrm{~J} \\ 63.00 & \mathrm{~J} \\ 3.82 & \\ 63.70 & \mathrm{~J} \\ 22.50 & \mathrm{~J} \\ 0.64 & \\ 13.80 & \mathrm{~J} \\ 0.01 & \mathrm{U} \\ 11.10 & \mathrm{~J} \\ 31700.00 & \mathrm{~J} \\ 31.80 & \mathrm{~J} \\ 0.47 & \mathrm{~J} \\ 4500.00 & \\ 718.00 & \\ 1.08 & \mathrm{U} \\ 12200.00 & \\ 561.00 & \mathrm{U} \\ 8.87 & \\ 1.82 & \mathrm{~J} \\ 543.00 & \\ 0.62 & \\ 12.10 & \mathrm{~J} \\ 980.00 & \mathrm{U} \\ 0.03 & \\ 3.15 & \\ 71.60 & \\ 2.40 & \\ 62.40 & \end{array}$

93300.00

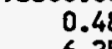

82.50 
Table H.1 (continued)

\begin{tabular}{|c|c|c|c|c|c|c|c|c|c|}
\hline Analysis & Units & $\begin{array}{l}\text { A horizon } \\
\text { result }\end{array}$ & Qualifier & $\begin{array}{l}\text { B horizon } \\
\text { result }\end{array}$ & Qualifier & $\begin{array}{l}C \text { horizon } \\
\text { result }\end{array}$ & Qualifier & $\begin{array}{l}\text { A lab split } \\
\text { result }\end{array}$ & Qualifier \\
\hline
\end{tabular}

Location=ROA; Formation=DISMAL GAP; Site=8,20,22; Sample type=Regular; $A=6012 ; B=6015 ; C=6018 ; A$ lab split=' '

\begin{tabular}{|c|c|c|c|c|c|c|c|}
\hline $\begin{array}{l}\text { Aluminum } \\
\text { Antimony } \\
\text { Arsenic } \\
\text { Barium } \\
\text { Cerium } \\
\text { Cesium } \\
\text { Chromium } \\
\text { Cobalt } \\
\text { Europium } \\
\text { Gallium } \\
\text { Gold } \\
\text { Hafnium } \\
\text { Iron } \\
\text { Lanthanum } \\
\text { Lutetium } \\
\text { Magnesium } \\
\text { Manganese } \\
\text { Mercury } \\
\text { Potassium } \\
\text { Rubidium } \\
\text { Scandium } \\
\text { Silver } \\
\text { Sodium } \\
\text { Terbium } \\
\text { Thorium-232 } \\
\text { Titanium } \\
\text { Uranium-235 } \\
\text { Uranium-238 } \\
\text { Vanadium } \\
\text { Ytterbium } \\
\text { Zinc }\end{array}$ & $\begin{array}{l}\mathrm{mg} / \mathrm{kg} \\
\mathrm{mg} / \mathrm{kg} \\
\mathrm{mg} / \mathrm{kg} \\
\mathrm{mg} / \mathrm{kg} \\
\mathrm{mg} / \mathrm{kg} \\
\mathrm{mg} / \mathrm{kg} \\
\mathrm{mg} / \mathrm{kg} \\
\mathrm{mg} / \mathrm{kg} \\
\mathrm{mg} / \mathrm{kg} \\
\mathrm{mg} / \mathrm{kg} \\
\mathrm{mg} / \mathrm{kg} \\
\mathrm{mg} / \mathrm{kg} \\
\mathrm{mg} / \mathrm{kg} \\
\mathrm{mg} / \mathrm{kg} \\
\mathrm{mg} / \mathrm{kg} \\
\mathrm{mg} / \mathrm{kg} \\
\mathrm{mg} / \mathrm{kg} \\
\mathrm{mg} / \mathrm{kg} \\
\mathrm{mg} / \mathrm{kg} \\
\mathrm{mg} / \mathrm{kg} \\
\mathrm{mg} / \mathrm{kg} \\
\mathrm{mg} / \mathrm{kg} \\
\mathrm{mg} / \mathrm{kg} \\
\mathrm{mg} / \mathrm{kg} \\
\mathrm{mg} / \mathrm{kg} \\
\mathrm{mg} / \mathrm{kg} \\
\mathrm{mg} / \mathrm{kg} \\
\mathrm{mg} / \mathrm{kg} \\
\mathrm{mg} / \mathrm{kg} \\
\mathrm{mg} / \mathrm{kg} \\
\mathrm{mg} / \mathrm{kg}\end{array}$ & $\begin{array}{r}42900.00 \\
0.46 \\
4.41 \\
5640.00 \\
48.40 \\
2.23 \\
56.80 \\
10.70 \\
0.51 \\
9.16 \\
0.01 \\
12.90 \\
23600.00 \\
25.40 \\
0.37 \\
2760.00 \\
589.00 \\
0.94 \\
8230.00 \\
462.00 \\
6.45 \\
1.57 \\
437.00 \\
0.50 \\
9.57 \\
10300.00 \\
0.02 \\
3.11 \\
48.20 \\
4.85 \\
55.10\end{array}$ & $\begin{array}{l}\mathrm{J} \\
\mathbf{U} \\
\mathrm{J} \\
\mathrm{J} \\
\mathrm{J} \\
\mathrm{J}\end{array}$ & $\begin{array}{r}106000.00 \\
0.56 \\
6.05 \\
8490.00 \\
68.20 \\
5.44 \\
84.10 \\
8.95 \\
0.66 \\
21.20 \\
0.01 \\
9.30 \\
43000.00 \\
37.60 \\
0.32 \\
6630.00 \\
88.40 \\
1.34 \\
16100.00 \\
938.00 \\
11.60 \\
2.37 \\
356.00 \\
0.42 \\
13.70 \\
383.00 \\
0.02 \\
3.90 \\
102.00 \\
3.35 \\
82.20\end{array}$ & $\begin{array}{l}\mathbf{J} \\
\mathrm{J} \\
\mathrm{J} \\
\mathbf{J}\end{array}$ & $\begin{array}{r}124000.00 \\
0.27 \\
4.30 \\
5960.00 \\
78.40 \\
5.63 \\
92.40 \\
9.43 \\
0.80 \\
30.70 \\
0.01 \\
5.94 \\
49900.00 \\
43.30 \\
0.29 \\
9560.00 \\
116.00 \\
1.52 \\
26200.00 \\
554.00 \\
15.20 \\
2.72 \\
399.00 \\
0.64 \\
14.50 \\
10600.00 \\
0.02 \\
2.65 \\
104.00 \\
1.84 \\
108.00\end{array}$ & $\begin{array}{l}\mathbf{J} \\
\mathbf{U} \\
\mathbf{J} \\
\mathbf{J} \\
\mathbf{J}\end{array}$ \\
\hline
\end{tabular}

Location=ROA; Formation=DISMAL GAP; Site=9,17,19; Sample type=Regular; $A=6006 ; B=6003 ; C=6009 ; A$ lab split=' '

\begin{tabular}{|c|c|c|c|c|c|c|}
\hline $\begin{array}{l}\text { Aluminum } \\
\text { Ant imony } \\
\text { Arsenic } \\
\text { Barium } \\
\text { Cerium } \\
\text { Cesium } \\
\text { Chromium } \\
\text { Cobalt } \\
\text { Europium } \\
\text { Gallium } \\
\text { Gold } \\
\text { Hafnium } \\
\text { Iron } \\
\text { Lanthanum } \\
\text { Lutetium } \\
\text { Magnesium } \\
\text { Manganese } \\
\text { Mercury } \\
\text { Potassium } \\
\text { Rubidium } \\
\text { Scandium } \\
\text { Silver } \\
\text { Sodium } \\
\text { Terbium } \\
\text { Thorium-232 } \\
\text { Titanium } \\
\text { Uranium-235 } \\
\text { Uranium-238 } \\
\text { Vanadium } \\
\text { Ytterbium } \\
\text { Zinc }\end{array}$ & $\begin{array}{l}\mathrm{mg} / \mathrm{kg} \\
\mathrm{mg} / \mathrm{kg} \\
\mathrm{mg} / \mathrm{kg} \\
\mathrm{mg} / \mathrm{kg} \\
\mathrm{mg} / \mathrm{kg} \\
\mathrm{mg} / \mathrm{kg} \\
\mathrm{mg} / \mathrm{kg} \\
\mathrm{mg} / \mathrm{kg} \\
\mathrm{mg} / \mathrm{kg} \\
\mathrm{mg} / \mathrm{kg} \\
\mathrm{mg} / \mathrm{kg} \\
\mathrm{mg} / \mathrm{kg} \\
\mathrm{mg} / \mathrm{kg} \\
\mathrm{mg} / \mathrm{kg} \\
\mathrm{mg} / \mathrm{kg} \\
\mathrm{mg} / \mathrm{kg} \\
\mathrm{mg} / \mathrm{kg} \\
\mathrm{mg} / \mathrm{kg} \\
\mathrm{mg} / \mathrm{kg} \\
\mathrm{mg} / \mathrm{kg} \\
\mathrm{mg} / \mathrm{kg} \\
\mathrm{mg} / \mathrm{kg} \\
\mathrm{mg} / \mathrm{kg} \\
\mathrm{mg} / \mathrm{kg} \\
\mathrm{mg} / \mathrm{kg} \\
\mathrm{mg} / \mathrm{kg} \\
\mathrm{mg} / \mathrm{kg} \\
\mathrm{mg} / \mathrm{kg} \\
\mathrm{mg} / \mathrm{kg} \\
\mathrm{mg} / \mathrm{kg} \\
\mathrm{mg} / \mathrm{kg}\end{array}$ & $\begin{array}{r}56400.00 \\
0.53 \\
6.20 \\
155.00 \\
59.40 \\
3.57 \\
66.80 \\
17.60 \\
0.63 \\
18.00 \\
0.01 \\
9.72 \\
27400.00 \\
32.50 \\
0.36 \\
3730.00 \\
826.00 \\
1.09 \\
11300.00 \\
1300.00 \\
8.71 \\
2.14 \\
571.00 \\
0.46 \\
11.50 \\
7130.00 \\
0.03 \\
3.19 \\
78.00 \\
3.34 \\
105.00\end{array}$ & $\begin{array}{l}\mathbf{J} \\
\mathbf{J} \\
\mathbf{J} \\
\mathbf{U} \\
\mathbf{J} \\
\mathbf{J} \\
\mathbf{J} \\
\mathbf{J}\end{array}$ & $\begin{array}{r}56300.00 \\
0.61 \\
7.72 \\
10700.00 \\
66.10 \\
3.39 \\
75.90 \\
21.60 \\
0.72 \\
14.30 \\
0.01 \\
10.30 \\
33100.00 \\
33.00 \\
0.37 \\
6780.00 \\
668.00 \\
1.25 \\
11900.00 \\
897.00 \\
8.95 \\
2.19 \\
562.00 \\
0.62 \\
12.90 \\
11600.00 \\
0.03 \\
3.97 \\
77.40 \\
3.38 \\
70.10\end{array}$ & $\begin{array}{l}\mathbf{J} \\
\mathbf{U} \\
\mathbf{J} \\
\mathbf{J} \\
\mathbf{J} \\
\mathbf{J}\end{array}$ & $\begin{array}{r}94200.00 \\
0.51 \\
6.98 \\
5090.00 \\
77.90 \\
5.20 \\
90.10 \\
27.60 \\
0.66 \\
25.50 \\
0.01 \\
6.46 \\
49600.00 \\
41.20 \\
0.32 \\
8630.00 \\
631.00 \\
1.47 \\
23200.00 \\
630.00 \\
14.10 \\
2.60 \\
621.00 \\
0.29 \\
14.80 \\
10300.00 \\
0.02 \\
3.34 \\
91.90 \\
2.20 \\
90.60\end{array}$ \\
\hline
\end{tabular}




\section{$\mathrm{H}-26$}

Table H.1 (continued)

\begin{tabular}{|c|c|c|c|c|c|c|c|c|c|}
\hline Analysis & Units & $\begin{array}{l}\text { A horizon } \\
\text { result }\end{array}$ & Qualifier & $\begin{array}{l}\text { B horizon } \\
\text { result }\end{array}$ & Qualifier & $\begin{array}{c}\mathrm{C} \text { horizon } \\
\text { result }\end{array}$ & Qualifier & $\begin{array}{l}\text { A lab split } \\
\text { result }\end{array}$ & Qualifier \\
\hline
\end{tabular}

Location=AND; Formation=COPPER RIDGE; Site=31,32,36; Sample type=Regular; $A=7059 ; B=7062 ; C=7065 ;$ A lab split=' '

$\begin{array}{lllllll}\text { Potassium-40 } & \mathrm{PCi} / \mathrm{g} & & & 5.2761 & & 6.1929 \\ \text { Thorium-232 } & \mathrm{PCi} / \mathrm{g} & 0.5761 & \mathrm{~J} & 0.8569 & \mathrm{~J} & 0.9413 \\ \text { Uranium-235 } & \mathrm{PCi} / \mathrm{g} & 0.0527 & & 0.0716 & & 0.0705 \\ \text { Uranium-238 } & \mathrm{PCi} / \mathrm{g} & & 1.6132 & & 1.5965\end{array}$

Location $=$ AND; Formation $=C O P P E R$ RIDGE; Site=33,34,35; Sample type=Regular; $A=7050 ; B=7053 ; C=7056 ; A$ lab split=' '

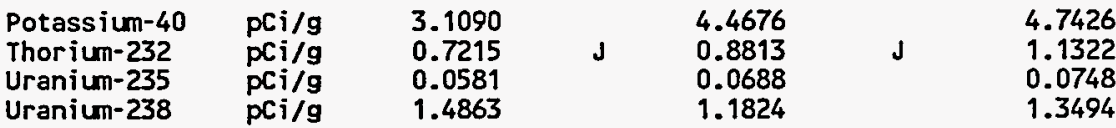

Location =AND; Formation =COPPER RIDGE; Site=37,38,41; Sample type=Regular; $A=7077 ; B=7080 ; C=7083 ; A$ lab split='

\begin{tabular}{|c|c|c|c|c|c|}
\hline $\begin{array}{l}\text { Potassium-40 } \\
\text { Thorium-232 } \\
\text { Uranium-235 } \\
\text { Uranium- } 238\end{array}$ & $\begin{array}{l}\mathrm{pCi} / \mathrm{g} \\
\mathrm{pCi} / \mathrm{g} \\
\mathrm{pCi} / \mathrm{g} \\
\mathrm{pCi} / \mathrm{g}\end{array}$ & $\begin{array}{l}4.1258 \\
0.7526 \\
0.0656 \\
1.6566\end{array}$ & J & $\begin{array}{l}0.1575 \\
1.2210 \\
0.1047 \\
5.7448\end{array}$ & $\begin{array}{l}\mathbf{U} \\
\mathbf{J}\end{array}$ \\
\hline
\end{tabular}

Location $=A N D ;$ Formation $=$ COPPER RIDGE; Site $=39,40,42 ;$ Sample type=Regular; $A=7068 ; B=7071 ; C=7074 ; A$ lab split=' '

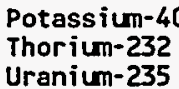

Uranium-235

Uranium-238

Potassium-40
Thorium- 232
Uranium- 235
Uranium- 238

$\mathrm{pCi} / \mathrm{g}$

0.8802

0.0716

1.6934
4.5926
1.1544
0.0884

2.0574
4.6426

1.2432

0.0821

1.8871

Location $=A N D ;$ Formation $=D I S M A L$ GAP; Site $=1,20,22 ;$ Sample type $=$ Regular; $A=7030 ; B=7033 ; C=7036 ; A$ lab split=' '

$\begin{array}{lrrrrr}\mathrm{pCi} / \mathrm{g} & 18.5870 & 20.8375 & & 24.8383 \\ \mathrm{pCi} / \mathrm{g} & 1.1544 & \mathrm{~J} & 1.6428 & \mathrm{~J} & 1.3875 \\ \mathrm{pCi} / \mathrm{g} & 0.0510 & & 0.0516 & & 0.0456 \\ \mathrm{pCi} / \mathrm{g} & 1.1356 & & 0.9452 & & 0.7381\end{array}$

Location $=A N D ;$ Formation $=D I S M A L$ GAP; Site=3,5,11; Sample type=Regular; $A=7012 ; B=7015 ; C=7018 ; A$ lab split=' '

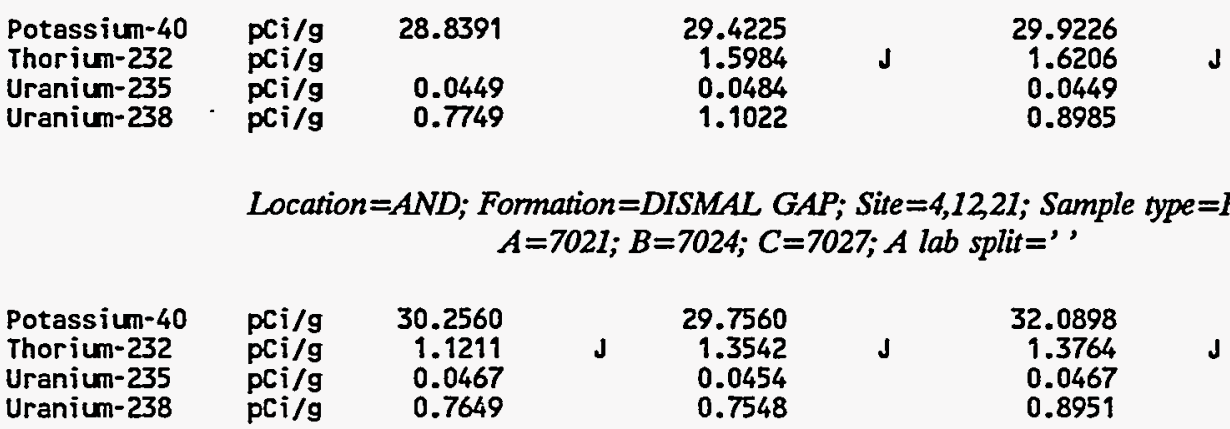


$\mathrm{H}-27$

Table H.1 (continued)

\begin{tabular}{|c|c|c|c|c|c|c|c|c|}
\hline Analysis & Units & $\begin{array}{l}\text { A horizon } \\
\text { result }\end{array}$ & Qualifier & $\begin{array}{l}\text { B horizon } \\
\text { result }\end{array}$ & Qualifier & $\begin{array}{l}C \text { horizon } \\
\text { result }\end{array}$ & $\begin{array}{cc}\text { A lab split } \\
\text { Qualifier } \quad \text { result }\end{array}$ & Qualifier \\
\hline & \multicolumn{7}{|c|}{$\begin{array}{l}\text { Location }=A N D ; \text { Formation }=D I S M A L \text { GAP; Site }=9,10,19 ; \text { Sample type }=\text { Regular; } \\
\qquad A=7003 ; B=7006 ; C=7009 ; A \text { lab split=' }\end{array}$} & $4 \cdots: \cdots$ \\
\hline $\begin{array}{l}\text { Potassium-40 } \\
\text { Thorium-232 } \\
\text { Uranium-235 } \\
\text { Uranium-238 }\end{array}$ & $\begin{array}{l}\mathrm{pCi} / \mathrm{g} \\
\mathrm{pCi} \mathrm{g} \\
\mathrm{pCi} / \mathrm{g} \\
\mathrm{pCi} \mathrm{g}\end{array}$ & $\begin{array}{r}13.9195 \\
1.0745 \\
0.0522 \\
1.0955\end{array}$ & J & $\begin{array}{r}16.8367 \\
1.3431 \\
0.0548 \\
1.0989\end{array}$ & J & $\begin{array}{r}20.6708 \\
1.4763 \\
0.0518 \\
1.0788\end{array}$ & $\mathrm{~J}$ & \\
\hline
\end{tabular}

Location=AND; Formation=DISMAL GAP; Site=9,10,19; Sample type=Field Duplicate; $A=7039 ; B=7042 ; C=7045 ; A$ lab split="

\begin{tabular}{|c|c|c|c|c|c|c|}
\hline $\begin{array}{l}\text { Potassium-40 } \\
\text { Thorium-232 } \\
\text { Uranium-235 } \\
\text { Uranium-238 }\end{array}$ & $\begin{array}{l}\mathrm{pCi} / \mathrm{g} \\
\mathrm{pCi} / \mathrm{g} \\
\mathrm{pCi} / \mathrm{g} \\
\mathrm{pCi} / \mathrm{g}\end{array}$ & $\begin{array}{r}15.4198 \\
1.1022 \\
0.0507 \\
1.1122\end{array}$ & $\mathbf{J}$ & $\begin{array}{r}17.0034 \\
1.3653 \\
0.0555 \\
1.2525\end{array}$ & $\mathbf{J}$ & $\begin{array}{r}19.7539 \\
1.5207 \\
0.0557 \\
1.0454\end{array}$ \\
\hline
\end{tabular}

Location=ORR; Formation $=$ CHEPULTEPEC; Site $=50,66,73$; Sample type $=$ Regular; $A=5169 ; B=5172 ; C=5183 ; A$ lab split $=5215$

\begin{tabular}{|c|c|c|c|c|c|c|c|c|}
\hline $\begin{array}{l}\text { Potassium-40 } \\
\text { Thorium-232 } \\
\text { Uranium-235 } \\
\text { Uranium-238 }\end{array}$ & $\begin{array}{l}\mathrm{pCi} / \mathrm{g} \\
\mathrm{pCi} / \mathrm{g} \\
\mathrm{pCi} / \mathrm{g} \\
\mathrm{pCi} / \mathrm{g}\end{array}$ & $\begin{array}{l}2.6172 \\
0.6138 \\
0.0563 \\
1.1824\end{array}$ & $\mathbf{J}$ & $\begin{array}{l}6.6430 \\
1.2543 \\
0.0727 \\
1.0454\end{array}$ & $\mathbf{J}$ & $\begin{array}{r}10.6688 \\
1.2876 \\
0.0813 \\
1.3427\end{array}$ & $\mathbf{J}$ & $\begin{array}{l}0.51393 \\
0.04730\end{array}$ \\
\hline
\end{tabular}

Location=ORR; Formation=CHEPULTEPEC; Site =50,66,73; Sample type=Field Duplicate; $A=5177 ; B=5180 ; C=5186 ; A$ lab split $=5214$

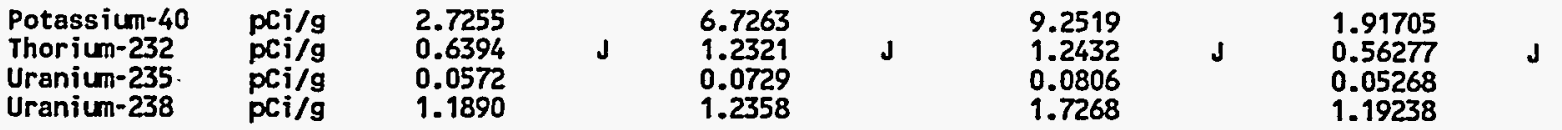

Location=ORR; Formation =CHEPULTEPEC; Site=52,53,78; Sample type=Regular; $A=5129 ; B=5132 ; C=5135 ; A$ lab split=',

$\begin{array}{llllllll}\text { Potassium-40 } & p C j / g & 2.4005 & & 5.6011 & & 10.3354 & \\ \text { Thorium-232 } & p C j / g & 0.5483 & \mathrm{~J} & 1.0001 & \mathrm{~J} & 1.1655 & \mathrm{~J} \\ \text { Uranium-235 } & \mathrm{PCj} / \mathrm{g} & 0.0563 & & 0.0705 & & 0.0875 & \\ \text { Uranium-238 } & \mathrm{pCi} / \mathrm{g} & 1.3393 & & 1.6967 & & 1.9005\end{array}$

Location=ORR; Formation=CHEPULTEPEC; Site=68,74,90; Sample type=Regular; $A=5158 ; B=5161 ; C=5164 ; A$ lab split $=$ ',

\begin{tabular}{|c|c|c|c|c|c|c|}
\hline $\begin{array}{l}\text { Potassium-40 } \\
\text { Thorium-232 } \\
\text { Uranium-235 } \\
\text { Uranium-238 }\end{array}$ & $\begin{array}{l}\mathrm{pCi} / \mathrm{g} \\
\mathrm{pCi} / \mathrm{g} \\
\mathrm{pCi} / \mathrm{g} \\
\mathrm{pCi} / \mathrm{g}\end{array}$ & $\begin{array}{l}2.2171 \\
0.5872 \\
0.0531 \\
1.4997\end{array}$ & $\mathbf{J}$ & $\begin{array}{l}7.7099 \\
1.1877 \\
0.0643 \\
1.5631\end{array}$ & $\mathbf{J}$ & $\begin{array}{l}9.6686 \\
1.4097 \\
0.0688 \\
1.5230\end{array}$ \\
\hline
\end{tabular}

Location=ORR; Formation=CHEPULTEPEC; Site=77,85,86; Sample type=Regular; $A=5138 ; B=5141 ; C=5144 ; A$ lab split="

Potassium-40 Thorium-232 Uranium-235 Uranium-238

$$
\begin{aligned}
& \text { pci/g } \\
& \text { pci/g } \\
& \text { pci/g } \\
& \text { pci/g }
\end{aligned}
$$

$\begin{array}{lll}6.7597 & 7.0431 \\ 0.9890 & & 1.0223 \\ 0.0557 & 0.0542 \\ 1.2993 & & 1.3260\end{array}$




\section{$\mathrm{H}-28$}

Table H.1 (continued)

\begin{tabular}{|c|c|c|c|c|c|c|c|c|c|}
\hline Analysis & Units & $\begin{array}{l}\text { A horizon } \\
\text { result }\end{array}$ & Qualifier & $\begin{array}{l}\text { B horizon } \\
\text { result }\end{array}$ & Qualifier & $\begin{array}{c}\mathrm{C} \text { horizon } \\
\text { result }\end{array}$ & Qualifier & $\begin{array}{l}\text { A lab split } \\
\text { result }\end{array}$ & Qualifier \\
\hline
\end{tabular}

Location=ORR; Formation $=$ CHEPULTEPEC; Site $=77,85,86 ;$ Sample type =Field Duplicate; $A=5147 ; B=5150 ; C=5153 ; A$ lab split=' '

\begin{tabular}{|c|c|c|c|c|c|c|}
\hline $\begin{array}{l}\text { Potassium-40 } \\
\text { Thorium-232 } \\
\text { Uranium-235 } \\
\text { Uranium- } 238\end{array}$ & $\begin{array}{l}\mathrm{pCi} / \mathrm{g} \\
\mathrm{pCi} i / g \\
\mathrm{pCi} / \mathrm{g} \\
\mathrm{pCi} / \mathrm{g}\end{array}$ & $\begin{array}{l}3.1256 \\
0.6227 \\
0.0585 \\
1.4896\end{array}$ & $\mathbf{J}$ & $\begin{array}{l}6.5930 \\
0.9901 \\
0.0574 \\
1.1289\end{array}$ & $\mathrm{~J}$ & $\begin{array}{l}4.0175 \\
0.5683 \\
0.0630 \\
0.6814\end{array}$ \\
\hline
\end{tabular}

Location=ORR; Formation=CHICKAMAUGA Bethel V; Site=101,102,103; Sample type=Regular; $A=5198 ; B=5201 ; C=5204 ; A$ lab split=' '

\begin{tabular}{|c|c|c|c|c|c|c|}
\hline $\begin{array}{l}\text { Potassium-40 } \\
\text { Thorium-232 } \\
\text { Uranium-235 } \\
\text { Uranium-238 }\end{array}$ & $\begin{array}{l}\mathrm{pCi} i / g \\
\mathrm{pCi} / \mathrm{g} \\
\mathrm{pCi} \mathrm{g} \\
\mathrm{pCi} / \mathrm{g}\end{array}$ & $\begin{array}{r}21.5043 \\
1.2210 \\
0.0527 \\
1.2892\end{array}$ & $\mathbf{J}$ & $\begin{array}{r}21.6710 \\
1.4541 \\
0.0462 \\
0.9085\end{array}$ & $\mathrm{~J}$ & $\begin{array}{r}21.6710 \\
1.5429 \\
0.0441 \\
0.9419\end{array}$ \\
\hline
\end{tabular}

Location=ORR; Formation $=$ CHICKAMAUGA Bethel V; Site $=104,108,110 ;$ Sample type $=$ Regular; $A=5207 ; B=5210 ; C=5213 ; A$ lab split $=$ "

\begin{tabular}{|c|c|c|c|c|}
\hline $\begin{array}{l}\text { Potassium-40 } \\
\text { Thor ium-232 } \\
\text { Uranium-235 } \\
\text { Uranium-238 }\end{array}$ & $\begin{array}{l}\mathrm{pCi} / \mathrm{g} \\
\mathrm{pCi} / \mathrm{g} \\
\mathrm{pCi} / \mathrm{g} \\
\mathrm{pCi} / \mathrm{g}\end{array}$ & $\begin{array}{l}8.5851 \\
0.9546 \\
0.0565 \\
0.9719\end{array}$ & $\mathbf{J}$ & $\begin{array}{r}15.0864 \\
1.5762 \\
0.0490 \\
1.2725\end{array}$ \\
\hline
\end{tabular}

Location=ORR; Formation=CHICKAMAUGA Bethel V; Site=115,116,117; Sample type=Regular; $A=5220 ; B=5224 ; C=5227 ; A$ lab split $=5221$

\begin{tabular}{|c|c|c|c|c|c|c|}
\hline $\begin{array}{l}\text { Potassium-40 } \\
\text { Thorium-232 } \\
\text { Uranium-235 } \\
\text { Uranium-238 }\end{array}$ & $\begin{array}{l}\mathrm{pCi} / \mathrm{g} \\
\mathrm{pCi} / \mathrm{g} \\
\mathrm{pCi} / \mathrm{g} \\
\mathrm{pCi} / \mathrm{g}\end{array}$ & $\begin{array}{l}6.4680 \\
1.0589 \\
0.0490 \\
1.0621\end{array}$ & $\mathrm{~J}$ & $\begin{array}{r}16.8367 \\
1.7205 \\
0.0585 \\
1.1289\end{array}$ & $J$ & $\begin{array}{r}19.0038 \\
1.4541 \\
0.0535 \\
1.5164\end{array}$ \\
\hline
\end{tabular}

Location=ORR; Formation=CHICKAMAUGA Bethel V; Site=93,99,100; Sample type=Regular; $A=5189 ; B=5192 ; C=5195 ; A$ lab split=',

\begin{tabular}{|c|c|c|c|c|c|c|}
\hline $\begin{array}{l}\text { Potassium-40 } \\
\text { Thorium-232 } \\
\text { Uranium-235 } \\
\text { Uranium- } 238\end{array}$ & $\begin{array}{l}\mathrm{pCi} / \mathrm{g} \\
\mathrm{pCi} / \mathrm{g} \\
\mathrm{pCi} / \mathrm{g} \\
\mathrm{pCi} / \mathrm{g}\end{array}$ & $\begin{array}{r}13.5861 \\
1.2765 \\
0.0559 \\
1.5197\end{array}$ & $\mathrm{~J}$ & $\begin{array}{r}20.5041 \\
1.5207 \\
0.0499 \\
1.0354\end{array}$ & $\mathbf{J}$ & $\begin{array}{r}23.0880 \\
1.5540 \\
0.0467 \\
1.0053\end{array}$ \\
\hline
\end{tabular}

Location=ORR; Formation $=$ CHICKAMAUGA K-25; Site=118,122,124; Sample type=Regular; $A=5244 ; B=5248 ; C=5251 ; A$ lab split $=5245$

Potassium-40 Thorium-232 Uranium-235

$\mathrm{pCi} / \mathrm{g} \quad 10.5021$

pci/g $\quad 1.2321$

pCi/g $\quad 0.0690$

$\mathrm{pCi} / \mathrm{g} \quad 1.6700$

$\begin{array}{rrr}22.3378 \\ \text { J } \quad 1.6206 \quad \mathrm{~J} & 1.5651 \\ 0.0587 & & 0.0499 \\ & 0.9653\end{array}$

22.3378

1.33934

Location=ORR; Formation=CHICKAMAUGA $K-25 ;$ Site $=119,123,127 ;$ Sample type $=$ Regular; $A=5256 ; B=5259 ; C=5262 ; A$ lab split=' '

\begin{tabular}{|c|c|c|c|c|c|c|}
\hline $\begin{array}{l}\text { Potassium-40 } \\
\text { Thorium-232 } \\
\text { Uranium-235 } \\
\text { Uranium-238 }\end{array}$ & $\begin{array}{l}\mathrm{pCi} / \mathrm{g} \\
\mathrm{pCi} / \mathrm{g} \\
\mathrm{pCi} / \mathrm{g} \\
\mathrm{pCi} i \mathrm{~g}\end{array}$ & $\begin{array}{r}10.8355 \\
1.1433 \\
0.0725 \\
1.8270\end{array}$ & $\mathbf{J}$ & $\begin{array}{r}22.0877 \\
1.6761 \\
0.0589 \\
1.3059\end{array}$ & $\mathbf{J}$ & $\begin{array}{r}22.1711 \\
1.6872 \\
0.0602 \\
1.3093\end{array}$ \\
\hline
\end{tabular}


H-29

Table H1 (continued)

\begin{tabular}{|c|c|c|c|c|c|c|c|c|c|}
\hline Analysis & Units & $\begin{array}{l}\text { A horizon } \\
\text { result }\end{array}$ & Qualifier & $\begin{array}{l}\text { B horizon } \\
\text { result }\end{array}$ & Qualifier & $\begin{array}{c}\mathrm{C} \text { horizon } \\
\text { result }\end{array}$ & Qualifier & $\begin{array}{l}\text { A lab split } \\
\text { result }\end{array}$ & Qualifier \\
\hline
\end{tabular}

Location=ORR; Formation=CHICKAMAUGA K-25; Site=120,126,129; Sample type=Regular $A=5232 ; B=5236 ; C=5239 ; A$ lab split $=5233$

\begin{tabular}{|c|c|c|c|c|c|c|c|c|}
\hline $\begin{array}{l}\text { Potassium-40 } \\
\text { Thorium-232 } \\
\text { Uranium-235 } \\
\text { Uranium- } 238\end{array}$ & $\begin{array}{l}\mathrm{pCi} / \mathrm{g} \\
\mathrm{pCi} / \mathrm{g} \\
\mathrm{pCi} / \mathrm{g} \\
\mathrm{pCi} / \mathrm{g}\end{array}$ & $\begin{array}{l}6.3179 \\
1.0145 \\
0.0742 \\
1.6099\end{array}$ & $\mathbf{J}$ & $\begin{array}{r}16.5033 \\
1.6428 \\
0.0641 \\
1.6900\end{array}$ & $\mathrm{~J}$ & $\begin{array}{r}24.8383 \\
1.6428 \\
0.0578 \\
1.2993\end{array}$ & $\mathbf{J}$ & $\begin{array}{l}5.87618 \\
1.05672 \\
0.07546 \\
1.63326\end{array}$ \\
\hline
\end{tabular}

Location=ORR; Formation=CHICKAMAUGA K-25; Site=121,125,128; Sample type=Regular; $A=5267 ; B=5270 ; C=5273 ; A$ lab split="

\begin{tabular}{|c|c|c|c|c|c|c|}
\hline $\begin{array}{l}\text { Potassium-40 } \\
\text { Thorium-232 } \\
\text { Uranium-235 } \\
\text { Uranium-238 }\end{array}$ & $\begin{array}{l}\mathrm{pCi} / \mathrm{g} \\
\mathrm{pCi} / \mathrm{g} \\
\mathrm{pCi} / \mathrm{g} \\
\mathrm{pCi} / \mathrm{g}\end{array}$ & $\begin{array}{l}7.3515 \\
0.9957 \\
0.0559 \\
1.3226\end{array}$ & $\mathbf{J}$ & $\begin{array}{r}21.5876 \\
1.5651 \\
0.0538 \\
1.2158\end{array}$ & $\mathbf{J}$ & $\begin{array}{r}23.3380 \\
1.5096 \\
0.0471 \\
1.2592 .\end{array}$ \\
\hline
\end{tabular}

Location=ORR; Formation $=$ COPPER RIDGE; Site $=45,60,75 ;$ Sample type =Regular; $A=5111 ; B=5114 ; C=5117 ; A$ lab split=' '

$\begin{array}{llllllll}\text { Potassium-40 } & \mathrm{pCi} / \mathrm{g} & 2.5922 & & 3.0923 & & 5.2260 & \\ \text { Thorium-232 } & \mathrm{PCi} / \mathrm{g} & 0.6682 & \mathrm{~J} & 1.0745 & \mathrm{~J} & 1.3431 & \mathrm{~J} \\ \text { Uranium-235 } & \mathrm{PCi} / \mathrm{g} & 0.0677 & & 0.0675 & & 0.0813 \\ \text { Uranium-238 } & \mathrm{pCi} / \mathrm{g} & 1.5297 & & 1.6032 & & 0.9886\end{array}$

Location=ORR; Formation=COPPER RIDGE; Site=51,55,62; Sample type=Regular; $A=5102 ; B=5105 ; C=5108 ; A$ lab split=' '

\begin{tabular}{|c|c|c|c|c|c|c|}
\hline $\begin{array}{l}\text { Potassium-40 } \\
\text { Thorium-232 } \\
\text { Uranium-235 } \\
\text { Uranium-238 }\end{array}$ & $\begin{array}{l}\mathrm{pCi} i / g \\
\mathrm{pCi} i / g \\
\mathrm{pCi} / \mathrm{g} \\
\mathrm{pCi} / \mathrm{g}\end{array}$ & $\begin{array}{l}2.7756 \\
0.6538 \\
0.0578 \\
1.1590\end{array}$ & $\mathbf{J}$ & $\begin{array}{l}4.0258 \\
1.0645 \\
0.0763 \\
2.0207\end{array}$ & J & $\begin{array}{l}5.8178 \\
1.4985 \\
0.0931 \\
1.6600\end{array}$ \\
\hline
\end{tabular}

Location=ORR; Formation =COPPER RIDGE; Site=54,64,83; Sample type $=$ Regular; $A=5120 ; B=5123 ; C=5126 ; A$ lab split=" '

Potassium-40 Thorium-232 Uranium-238

$\begin{array}{ll}\mathrm{pCi} / \mathrm{g} & 3.592 \\ \mathrm{pCi} / \mathrm{g} & 0.718 \\ \mathrm{pCi} / \mathrm{g} & 0.074 \\ \mathrm{pCi} / \mathrm{g} & 1.880\end{array}$

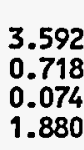

7.8432

1.3542

0.1056

2.1242

Location=ORR; Formation=COPPER RIDGE; Site=58,59,91; Sample type=Regular; $A=5093 ; B=5096 ; C=5099 ; A$ lab split="

$\begin{array}{llllllll}\text { Potassium-40 } & \mathrm{pCi} / \mathrm{g} & 3.2423 & & 5.0927 & & 7.2431 & \\ \text { Thorium-232 } & \mathrm{pCi} / \mathrm{g} & 0.8669 & \mathrm{~J} & 1.3320 & \mathrm{~J} & 1.7538 & \mathrm{~J} \\ \text { Uranium-235 } & \mathrm{pCi} / \mathrm{g} & 0.0709 & & 0.0890 & & 0.1086 & \\ \text { Uranium-238 } & \mathrm{pCi} / \mathrm{g} & 1.5564 & & 1.8871 & & 2.1443\end{array}$

Location=ORR; Formation =DISMAL GAP; Site=10,33,35; Sample type $=$ Regular; $A=5021 ; B=5024 ; C=5027 ; A$ lab split $=$ ' '

\begin{tabular}{|c|c|c|c|c|c|c|}
\hline $\begin{array}{l}\text { Potassium-40 } \\
\text { Thorium-232 } \\
\text { Uranium-235 } \\
\text { Uranium-238 }\end{array}$ & $\begin{array}{l}\mathrm{pCi} / \mathrm{g} \\
\mathrm{pCi} / \mathrm{g} \\
\mathrm{pCi} / \mathrm{g} \\
\mathrm{pCi} / \mathrm{g}\end{array}$ & $\begin{array}{r}12.1691 \\
1.0434 \\
0.0568 \\
1.3828\end{array}$ & $\mathrm{~J}$ & $\begin{array}{r}16.3366 \\
1.3209 \\
0.0456 \\
1.0387\end{array}$ & $\mathbf{J}$ & $\begin{array}{r}17.8369 \\
1.3320 \\
0.0393 \\
0.4943\end{array}$ \\
\hline
\end{tabular}


$\mathrm{H}-30$

Table H.1 (continued)

\begin{tabular}{|c|c|c|c|c|c|c|c|c|c|}
\hline Analysis & Units & $\begin{array}{l}\text { A horizon } \\
\text { result }\end{array}$ & Qualifier & $\begin{array}{l}\text { B horizon } \\
\text { result }\end{array}$ & Qualifier & $\begin{array}{c}C \text { horizon } \\
\text { result }\end{array}$ & Qualifier & $\begin{array}{l}\text { A lab split } \\
\text { result }\end{array}$ & Qualifier \\
\hline
\end{tabular}

Location $=O R R ;$ Formation $=D I S M A L$ GAP; Site $=11,27,41 ;$ Sample type $=$ Regular; $A=5003 ; B=5006 ; C=5009 ; A$ lab split=' ,

\begin{tabular}{|c|c|c|c|c|c|c|}
\hline $\begin{array}{l}\text { Potassium-40 } \\
\text { Thorium-232 } \\
\text { Uranium-235 } \\
\text { Uranium-238 }\end{array}$ & $\begin{array}{l}\mathrm{pCi} / \mathrm{g} \\
\mathrm{pCi} / \mathrm{g} \\
\mathrm{pCi} / \mathrm{g} \\
\mathrm{pCi} / \mathrm{g}\end{array}$ & $\begin{array}{r}10.8355 \\
1.0445 \\
0.0581 \\
1.2926\end{array}$ & J & $\begin{array}{r}16.4199 \\
1.2654 \\
0.0439 \\
1.3226\end{array}$ & $\mathrm{~J}$ & $\begin{array}{r}22.0877 \\
1.4319 \\
0.0419 \\
1.1323\end{array}$ \\
\hline
\end{tabular}

Location =ORR; Formation =DISMAL GAP; Site=19,22,32; Sample type $=$ Regular; $A=5012 ; B=5015 ; C=5018 ; A$ lab split=' '

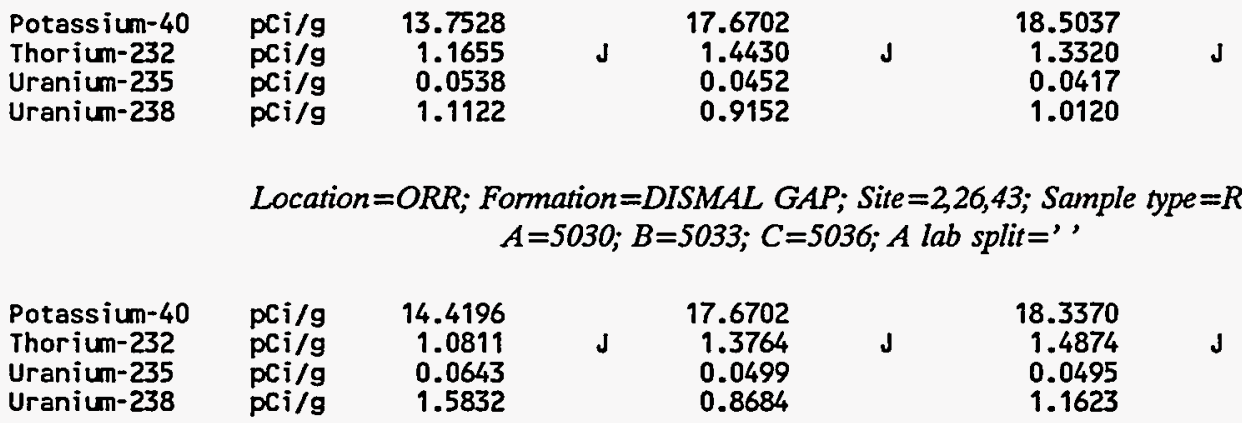

Location=ORR; Formation=DISMAL GAP; Site=2,26,43; Sample type=Field Duplicate; $A=5039 ; B=5042 ; C=5045 ; A$ lab split $=$ ',

\begin{tabular}{|c|c|c|c|c|c|c|}
\hline $\begin{array}{l}\text { Potassium-40 } \\
\text { Thorium-232 } \\
\text { Uranium-235 } \\
\text { Uranium-238 }\end{array}$ & $\begin{array}{l}\mathrm{pCi} / \mathrm{g} \\
\mathrm{pCi} / \mathrm{g} \\
\mathrm{pCi} / \mathrm{g} \\
\mathrm{pCi} i \mathrm{~g}\end{array}$ & $\begin{array}{r}13.3360 \\
1.0711 \\
0.0624 \\
1.5798\end{array}$ & $\boldsymbol{J}$ & $\begin{array}{r}22.7545 \\
1.4652 \\
0.0499 \\
1.4262\end{array}$ & $\mathrm{~J}$ & $\begin{array}{r}16.5033 \\
1.2654 \\
0.0310 \\
1.1490\end{array}$ \\
\hline
\end{tabular}

Location $=O R R ;$ Formation $=$ NOLICHUCKY; Site $=15,23,25 ;$ Sample type $=$ Regular; $A=5057 ; B=5060 ; C=5063 ; A$ lab split=' '

$\begin{array}{lllllll}\text { Potassium-40 } & \mathrm{pCi} / \mathrm{g} & 13.4194 & & 17.4202 & & 23.6714 \\ \text { Thorium-232 } & \mathrm{pCi} / \mathrm{g} & 1.2987 & \mathrm{~J} & 1.6761 & \mathrm{~J} & 1.7538 \\ \text { Uranium-235 } & \mathrm{PCi} / \mathrm{g} & 0.0542 & & 0.0439 & & 0.0417 \\ \text { Uraniun-238 } & \mathrm{pCi} / \mathrm{g} & 1.5063 & & 0.8985 & & 1.0688\end{array}$

Location=ORR; Formation $=$ NOLICHUCKY; Site $=16,28,42 ;$ Sample type $=$ Regular; $A=5066 ; B=5069 ; C=5072 ; A$ lab split $=$ '

\begin{tabular}{|c|c|c|c|c|c|c|}
\hline $\begin{array}{l}\text { Potassium-40 } \\
\text { Thorium- } 232 \\
\text { Uranium-235 } \\
\text { Uranium-238 }\end{array}$ & $\begin{array}{l}\mathrm{pCi} i / g \\
\mathrm{pCi} / \mathrm{g} \\
\mathrm{pCi} / \mathrm{g} \\
\mathrm{pCi} / \mathrm{g}\end{array}$ & $\begin{array}{r}13.3360 \\
1.3209 \\
0.0572 \\
1.0387\end{array}$ & $\mathrm{~J}$ & $\begin{array}{r}16.7533 \\
1.7538 \\
0.0520 \\
1.0154\end{array}$ & $\mathbf{J}$ & $\begin{array}{r}23.4213 \\
1.5984 \\
0.0434 \\
0.9486\end{array}$ \\
\hline
\end{tabular}

Location=ORR; Formation=NOLICHUCKY; Site=3,13,24; Sample type=Regular; $A=5084 ; B=5087 ; C=5090 ; A$ lab split=" '

$\begin{array}{llrrrrr}\text { Potassium-40 } & \mathrm{pCi} / \mathrm{g} & 13.8361 & & 19.8373 & & 35.6738 \\ \text { Thorium-232 } & \mathrm{pCi} / \mathrm{g} & 1.2654 & \mathrm{~J} & 1.6650 & \mathrm{~J} & 2.6640 \\ \text { Uranium-235 } & \mathrm{pCi} / \mathrm{g} & 0.0533 & & 0.0488 & & 0.0460 \\ \text { Uranium-238 } & \mathrm{pCi} / \mathrm{g} & 1.3794 & & 1.1957 & & .1 .7234\end{array}$


$\mathrm{H}-31$

Table H.1 (continued)

\begin{tabular}{|c|c|c|c|c|c|c|c|c|}
\hline Analysis & Units & $\begin{array}{l}\text { A horizon } \\
\text { result }\end{array}$ & Qualifier & $\begin{array}{l}\text { B horizon } \\
\text { result }\end{array}$ & Qualifier & $\begin{array}{l}\text { C horizon } \\
\text { result }\end{array}$ & $\begin{array}{cc}\text { A lab split } \\
\text { Qualifier } \quad \text { result }\end{array}$ & Qualifier \\
\hline & \multicolumn{7}{|c|}{$\begin{array}{l}\text { Location }=\text { ORR; Formation }=\text { NOLICHUCKY; Site =5,21,31; Sample type =Regular; } \\
\qquad A=5075 ; B=5078 ; C=5081 ; A \text { lab split=', }\end{array}$} & $: \pi$ \\
\hline $\begin{array}{l}\text { Potassium-40 } \\
\text { Thorium-232 } \\
\text { Uranium-235 } \\
\text { Uranium-238 }\end{array}$ & $\begin{array}{l}\mathrm{pCi} i / g \\
\mathrm{pCi} i / g \\
\mathrm{pCi} i \mathrm{~g} \\
\mathrm{pCi} i / g\end{array}$ & $\begin{array}{r}12.9193 \\
1.2987 \\
0.0544 \\
1.2558\end{array}$ & J & $\begin{array}{r}17.0034 \\
1.5540 \\
0.0464 \\
1.1289\end{array}$ & J & $\begin{array}{l}21.5043 \\
0.0452 \\
0.9051\end{array}$ & & ' \\
\hline
\end{tabular}

Location=ROA; Formation=COPPER RIDGE; Site=33,35,44; Sample type=Regular; $A=6048 ; B=6051 ; C=6054 ; A$ lab split=' '

$\begin{array}{llllllll}\text { Potassium-40 } & \text { pCi/g } & 3.6174 & & 3.9841 & & 3.8424 & \\ \text { Thorium-232 } & \text { pCi/g } & 0.6083 & \mathrm{~J} & 0.9735 & \mathrm{~J} & 1.4652 & \mathrm{~J} \\ \text { Uranium-235 } & \text { pCi/g } & 0.0497 & & 0.0658 & & 0.0830 \\ \text { Uranium-238 } & \text { pCi/g } & 0.9552 & & 1.4963 & & 1.8637\end{array}$

Location=ROA; Formation=COPPER RIDGE; Site=34,39,41; Sample type=Regular; $A=6086 ; B=6089 ; C=6092 ; A$ lab split $=$ ' '

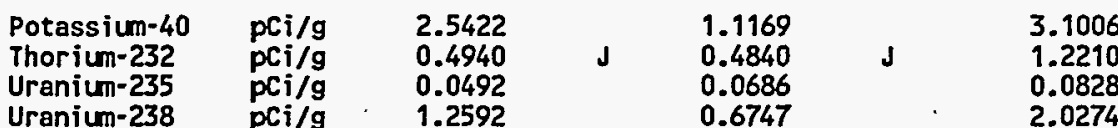

Location=ROA; Formation=COPPER RIDGE; Site=40,42,43; Sample type=Regular; $A=6057 ; B=6060 ; C=6063 ; A$ lab split="

Potassium-40 Thorium-232 Uranium-235

Uranium-238

$\begin{array}{llllll}\mathrm{PCi} / \mathrm{g} & 2.4672 & & 2.6255 & & 2.6339 \\ \mathrm{PCi} / \mathrm{g} & 0.4751 & \mathrm{~J} & 0.7637 & \mathrm{~J} & 1.0834 \\ \mathrm{PCi} / \mathrm{g} & 0.0402 & & 0.0495 & & 0.0565 \\ \mathrm{PCi} / \mathrm{g} & 0.9352 & & 1.2959 & & 1.2659\end{array}$

Location=ROA; Formation=COPPER RIDGE; Site=45,46,47; Sample type=Regular; $A=6075 ; B=6072 ; C=6066 ; A$ lab split=' '

Potassium-40 Thoriun-232

Uranium-235

$\mathrm{pCi} / \mathrm{g}$

$\mathrm{pCi} / \mathrm{g}$

$\mathrm{pCi} / \mathrm{g}$

2.2421
0.5728

0.0469

$\mathrm{pCi} / \mathrm{g}$

1.0688

2.6672
0.8225
0.0544
1.1389

4.2342

1.5429

0.0608

1.9873

Location=ROA; Formation=COPPER RIDGE; Site $=45,46,47 ;$ Sample type $=$ Field Duplicate; $A=6078 ; B=6081 ; C=6069 ; A$ lab split="

Potassium- 40

Thorium-232

Uranium-235

Uranium-238

$\mathrm{PCi} / \mathrm{g}$

$\mathrm{pCi} / \mathrm{g}$

2.2838

$\mathrm{pCi} / \mathrm{g}$

0.8736

2.4838

0.8214

2.7422

$\mathrm{pCi} / \mathrm{g}$

1.3293

0.0553

1.0623

0.0602

Location=ROA; Formation=DISMAL GAP; Site=10,13,14; Sample type=Regular; $A=6030 ; B=6033 ; C=6036 ; A$ lab split=' '

Potassium-40
Thorium-232
Urenium-235
Uranium-238

$\mathrm{pCi} / \mathrm{g}$

$\mathrm{pCi} / \mathrm{g}$

$\mathrm{pCi} / \mathrm{g}$

9.5019

18.4204

$\mathrm{pCi} / \mathrm{g}$

1.2099
0.0452

1.6317

0.0471

1.0989

23.1713

$1.6983 \mathrm{~J}$

0.0419

0.9552 


\section{H-32}

Table H.1 (continued)

\begin{tabular}{|c|c|c|c|c|c|c|c|c|c|}
\hline Analysis & Units & $\begin{array}{l}\text { A horizon } \\
\text { result }\end{array}$ & Qualifier & $\begin{array}{l}\text { B horizon } \\
\text { result }\end{array}$ & Qualifier & $\begin{array}{c}C \text { horizon } \\
\text { result }\end{array}$ & Qualifier & $\begin{array}{l}\text { A lab split } \\
\text { result }\end{array}$ & Qualifier \\
\hline
\end{tabular}

Location $=R O A ;$ Formation $=D I S M A L$ GAP; Site $=10,13,14 ;$ Sample type $=$ Field Duplicate; $A=6039 ; B=6042 ; C=6045 ;$ A lab split=' ,

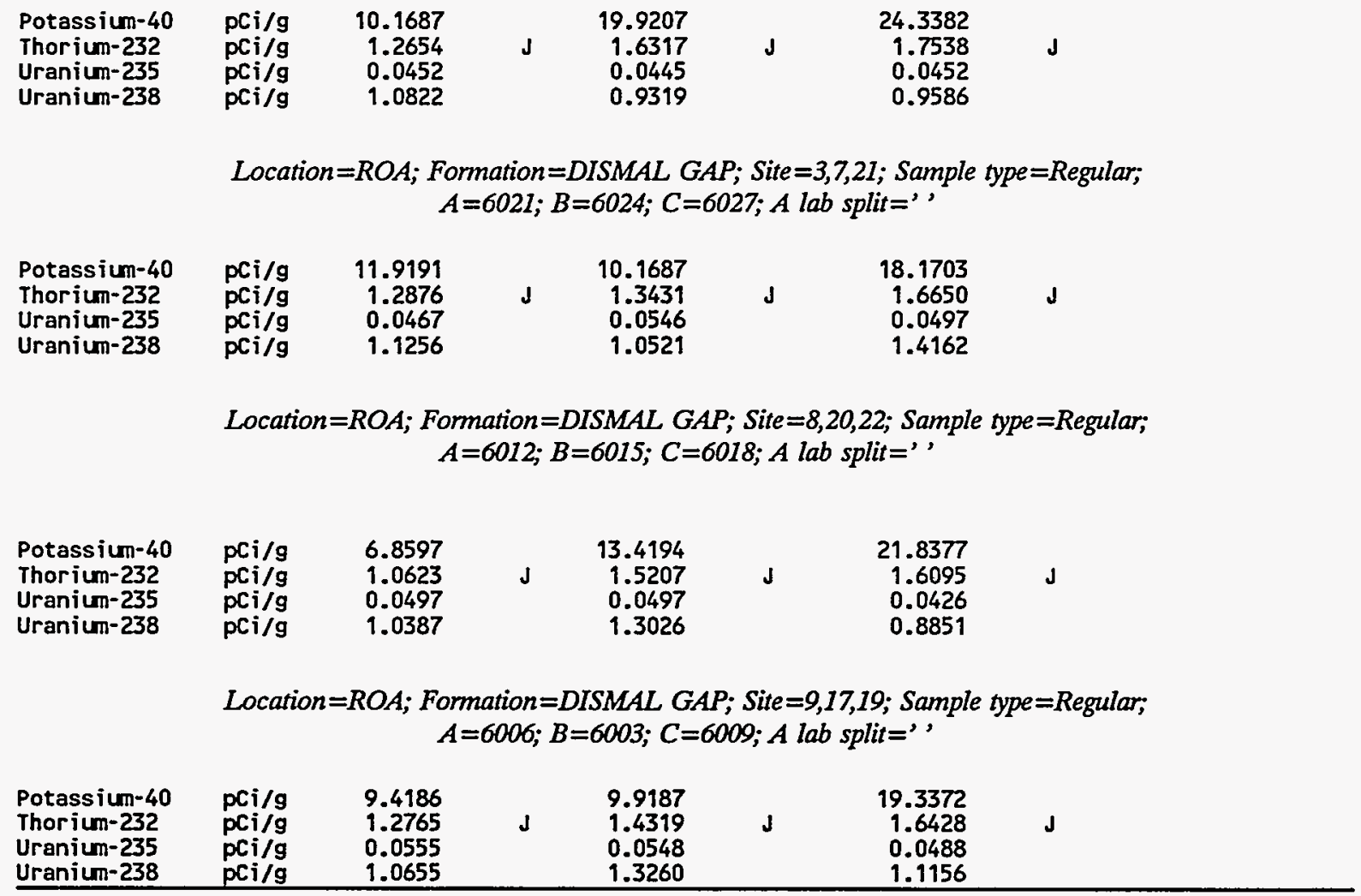


Appendix I

ICP/MS ANALYSIS DATA 

Table L1. ICP/MS metals results for composite soil samples

\begin{tabular}{|c|c|c|c|c|c|c|c|c|c|}
\hline Analysis & Units & $\begin{array}{l}\text { A horizon } \\
\text { result }\end{array}$ & Qualifier & $\begin{array}{l}\text { B horizon } \\
\text { result }\end{array}$ & Qualifier & $\begin{array}{l}C \text { horizon } \\
\text { result }\end{array}$ & Qualifier & $\begin{array}{l}\text { A lab split } \\
\text { result }\end{array}$ & Qualifier \\
\hline
\end{tabular}

Location=AND; Formation=COPPER RIDGE; Phase=2; Site=31,32,36; Sample type=Regular; $A=7057 ; B=7060 ; C=7063 ; A$ lab split $=$ "'

$\begin{array}{lrrrrrrr}\text { Aluminum } & \mathrm{mg} / \mathrm{kg} & 9200.00 & & 12100.00 & & 10000.00 & \\ \text { Antimony } & \mathrm{mg} / \mathrm{kg} & 0.24 & \mathrm{U} & 0.22 & \mathrm{U} & 0.21 & \mathrm{U} \\ \text { Arsenic } & \mathrm{mg} / \mathrm{kg} & 13.70 & & 15.40 & & 18.30 & \\ \text { Barium } & \mathrm{mg} / \mathrm{kg} & 47.10 & \mathrm{~B} & 22.40 & \mathrm{~B} & 11.00 & \mathrm{~B} \\ \text { Beryllium } & \mathrm{mg} / \mathrm{kg} & 0.40 & \mathrm{~B} & 0.29 & \mathrm{~B} & 0.32 & \mathrm{~B} \\ \text { Cadmium } & \mathrm{mg} / \mathrm{kg} & 0.24 & \mathrm{U} & 0.22 & \mathrm{U} & 0.21 & \mathrm{U} \\ \text { Chromium } & \mathrm{mg} / \mathrm{kg} & 28.60 & & 21.90 & & 22.90 & \\ \text { Cobalt } & \mathrm{mg} / \mathrm{kg} & 10.60 & \mathrm{~B} & 3 . .40 & \mathrm{~B} & 2.40 & \mathrm{~B} \\ \text { Copper } & \mathrm{mg} / \mathrm{kg} & 6.40 & & 17.70 & & 23.60 & \\ \text { Lead } & \mathrm{mg} / \mathrm{kg} & 26.70 & & 9.00 & & 10.10 \\ \text { Manganese } & \mathrm{mg} / \mathrm{kg} & 1600.00 & & 72.30 & & 55.50 & \\ \text { Nickel } & \mathrm{mg} / \mathrm{kg} & 5.30 & \mathrm{~B} & 8.30 & \mathrm{~B} & 9.60 & \\ \text { Silver } & \mathrm{mg} / \mathrm{kg} & 0.24 & \mathrm{U} & 0.22 & \mathrm{U} & 0.21 & \mathrm{U} \\ \text { Thallium } & \mathrm{mg} / \mathrm{kg} & 0.24 & \mathrm{U} & 0.24 & \mathrm{~B} & 0.21 & \mathrm{~B} \\ \text { Zinc } & \mathrm{mg} / \mathrm{kg} & 29.10 & & 54.70 & & 76.70 & \end{array}$

Location=AND; Formation=COPPER RIDGE; Phase=2; Site=33,34,35; Sample type=Regular; $A=7046 ; B=7051 ; C=7054 ;$ A lab split $=7047$

\begin{tabular}{|c|c|c|c|c|c|c|c|c|}
\hline $\begin{array}{l}\text { Aluminum } \\
\text { Ant imony } \\
\text { Arsenic } \\
\text { Barium } \\
\text { Beryllium } \\
\text { Cadmium } \\
\text { Chromium } \\
\text { Cobalt } \\
\text { Copper } \\
\text { Lead } \\
\text { Hanganese } \\
\text { Nickel } \\
\text { Silver } \\
\text { Thallium } \\
\text { Zinc }\end{array}$ & $\begin{array}{l}\mathrm{mg} / \mathrm{kg} \\
\mathrm{mg} / \mathrm{kg} \\
\mathrm{mg} / \mathrm{kg} \\
\mathrm{mg} / \mathrm{kg} \\
\mathrm{mg} / \mathrm{kg} \\
\mathrm{mg} / \mathrm{kg} \\
\mathrm{mg} / \mathrm{kg} \\
\mathrm{mg} / \mathrm{kg} \\
\mathrm{mg} / \mathrm{kg} \\
\mathrm{mg} / \mathrm{kg} \\
\mathrm{mg} / \mathrm{kg} \\
\mathrm{mg} / \mathrm{kg} \\
\mathrm{mg} / \mathrm{kg} \\
\mathrm{mg} / \mathrm{kg} \\
\mathrm{mg} / \mathrm{kg}\end{array}$ & $\begin{array}{r}11200.00 \\
0.23 \\
9.40 \\
88.50 \\
0.44 \\
0.23 \\
20.80 \\
9.70 \\
7.20 \\
31.20 \\
1620.00 \\
6.80 \\
0.23 \\
0.23 \\
30.50\end{array}$ & $\begin{array}{l}\mathbf{B} \\
\mathbf{U} \\
\mathbf{U}\end{array}$ & $\begin{array}{r}15100.00 \\
0.20 \\
14.30 \\
32.90 \\
0.28 \\
0.20 \\
24.30 \\
4.40 \\
19.10 \\
9.60 \\
216.00 \\
8.50 \\
0.20 \\
0.25 \\
44.30\end{array}$ & $\begin{array}{l}B \\
B \\
U\end{array}$ & $\begin{array}{r}15300.00 \\
0.22 \\
21.60 \\
18.90 \\
0.35 \\
0.22 \\
30.70 \\
2.70 \\
28.50 \\
10.40 \\
85.90 \\
11.10 \\
0.22 \\
0.27 \\
64.10\end{array}$ & $\begin{array}{l}\mathbf{B} \\
\mathbf{B} \\
\mathbf{U}\end{array}$ & $\begin{array}{r}11700.00 \\
0.23 \\
10.60 \\
98.00 \\
0.50 \\
0.23 \\
21.30 \\
14.40 \\
7.20 \\
26.90 \\
2630.00 \\
6.90 \\
0.23 \\
0.25 \\
34.60\end{array}$ \\
\hline
\end{tabular}

Location $=A N D ;$ Formation $=$ COPPER RIDGE; Phase $=2 ;$ Site $=37,38,41$; Sample type=Regular; $A=7075 ; B=7078 ; C=7081 ; A$ lab split=' '

$\begin{array}{lrrrrrrr}\text { Aluminum } & \mathrm{mg} / \mathrm{kg} & 16500.00 & & 24200.00 & & 36200.00 & \\ \text { Antimony } & \mathrm{mg} / \mathrm{kg} & 0.25 & \mathrm{U} & 0.21 & \mathrm{U} & 0.22 & \mathrm{U} \\ \text { Arsenic } & \mathrm{mg} / \mathrm{kg} & 12.80 & & 20.00 & & 27.00 & \\ \text { Barium } & \mathrm{mg} / \mathrm{kg} & 144.00 & & 55.60 & & 46.30 & \\ \text { Beryllium } & \mathrm{mg} / \mathrm{kg} & 0.88 & \mathrm{~B} & 0.74 & \mathrm{~B} & 1.00 & \mathrm{~B} \\ \text { Cacmium } & \mathrm{mg} / \mathrm{kg} & 0.25 & \mathrm{U} & 0.21 & \mathrm{U} & 0.22 & \mathrm{U} \\ \text { Chromium } & \mathrm{mg} / \mathrm{kg} & 21.90 & & 30.30 & & 35.20 & \\ \text { Cobalt } & \mathrm{mg} / \mathrm{kg} & 38.70 & & 64.50 & & 44.70 \\ \text { Copper } & \mathrm{mg} / \mathrm{kg} & 14.70 & & 39.50 & & 65.50 \\ \text { Lead } & \mathrm{mg} / \mathrm{kg} & 53.80 & & 48.30 & & 78.20 \\ \text { Manganese } & \mathrm{mg} / \mathrm{kg} & 2150.00 & & 1420.00 & & 1630.00 \\ \text { Nickel } & \mathrm{mg} / \mathrm{kg} & 15.00 & & 25.20 & & 43.40 & \\ \text { Silver } & \mathrm{mg} / \mathrm{kg} & 0.25 & \mathrm{U} & 0.21 & \mathrm{U} & 0.22 & \mathrm{U} \\ \text { Thallium } & \mathrm{mg} / \mathrm{kg} & 0.35 & \mathrm{~B} & 0.46 & \mathrm{~B} & 0.65 & \mathrm{~B} \\ \text { Zinc } & \mathrm{mg} / \mathrm{kg} & 69.20 & & 110.00 & & 160.00 & \end{array}$

Location=AND; Formation=COPPER RIDGE; Phase =2; Site=39,40,42; Sample type=Regular; $A=706 \dot{6} ; B=7069 ; C=7072 ; A$ lab split =' '

$\begin{array}{lrrrrrrr}\text { Aluminum } & \mathrm{mg} / \mathrm{kg} & 20400.00 & & 27500.00 & & 29700.00 & \\ \text { Antimony } & \mathrm{mg} / \mathrm{kg} & 0.23 & \mathrm{U} & 0.22 & \mathrm{U} & 0.22 & \mathrm{U} \\ \text { Arsenic } & \mathrm{mg} / \mathrm{kg} & 14.10 & & 18.60 & & 17.10 & \\ \text { Barium } & \mathrm{mg} / \mathrm{kg} & 204.00 & & 56.10 & & 46.00 & \\ \text { Beryll lium } & \mathrm{mg} / \mathrm{kg} & 0.94 & \mathrm{~B} & 0.42 & \mathrm{~B} & 0.42 & \mathrm{~B} \\ \text { Cadmium } & \mathrm{mg} / \mathrm{kg} & 0.23 & \mathrm{U} & 0.22 & \mathrm{U} & 0.22 & \mathrm{U}\end{array}$


$\mathrm{I}-4$

Table I1 (continued)

\begin{tabular}{|c|c|c|c|c|c|c|c|c|c|}
\hline Analysis & Units & $\begin{array}{l}\text { A horizon } \\
\text { result }\end{array}$ & Qualifier & $\begin{array}{l}\text { B horizon } \\
\text { result }\end{array}$ & Qualifier & $\begin{array}{l}C \text { horizon } \\
\text { result }\end{array}$ & Qualifier & $\begin{array}{l}\text { A lab split } \\
\text { result }\end{array}$ & Qualifier \\
\hline $\begin{array}{l}\text { Chromium } \\
\text { Cobalt } \\
\text { Copper } \\
\text { Lead } \\
\text { Manganese } \\
\text { Nickel } \\
\text { Silver } \\
\text { Thallium } \\
\text { Zinc }\end{array}$ & $\begin{array}{l}\mathrm{mg} / \mathrm{kg} \\
\mathrm{mg} / \mathrm{kg} \\
\mathrm{mg} / \mathrm{kg} \\
\mathrm{mg} / \mathrm{kg} \\
\mathrm{mg} / \mathrm{kg} \\
\mathrm{mg} / \mathrm{kg} \\
\mathrm{mg} / \mathrm{kg} \\
\mathrm{mg} / \mathrm{kg} \\
\mathrm{mg} / \mathrm{kg}\end{array}$ & $\begin{array}{r}26.00 \\
17.30 \\
10.90 \\
40.50 \\
4250.00 \\
14.30 \\
0.23 \\
0.37 \\
38.70\end{array}$ & $\begin{array}{l}\text { U } \\
\text { B }\end{array}$ & $\begin{array}{r}38.40 \\
8.80 \\
23.00 \\
17.80 \\
558.00 \\
17.10 \\
0.22 \\
0.36 \\
45.20\end{array}$ & $\begin{array}{l}\text { U } \\
\text { B }\end{array}$ & $\begin{array}{r}32.20 \\
8.90 \\
20.30 \\
27.30 \\
558.00 \\
17.90 \\
0.22 \\
0.33 \\
41.60\end{array}$ & $\begin{array}{l}\text { U } \\
\text { B }\end{array}$ & & \\
\hline
\end{tabular}

Location=AND; Formation=DISMAL GAP; Phase =1; Site=1,20,22; Sample type=Regular; $A=7028 ; B=7031 ; C=7034 ; A$ lab split='

$\begin{array}{lrrrrrrr}\text { Aluminum } & \mathrm{mg} / \mathrm{kg} & 21100.00 & & 30900.00 & & 32300.00 & \\ \text { Antimony } & \mathrm{mg} / \mathrm{kg} & 0.25 & \mathrm{U} & 0.24 & \mathrm{U} & 0.25 & \mathrm{U} \\ \text { Arsenic } & \mathrm{mg} / \mathrm{kg} & 3.50 & & 3.60 & & 2.90 & \\ \text { Barium } & \mathrm{mg} / \mathrm{kg} & 88.80 & & 67.20 & & 84.80 & \\ \text { Beryllium } & \mathrm{mg} / \mathrm{kg} & 0.82 & \mathrm{~B} & 1.10 & \mathrm{~B} & 1.10 & \mathrm{~B} \\ \text { Cadmium } & \mathrm{mg} / \mathrm{kg} & 0.25 & \mathrm{U} & 0.24 & \mathrm{U} & 0.25 & \mathrm{U} \\ \text { Chromium } & \mathrm{mg} / \mathrm{kg} & 24.90 & & 33.70 & & 29.00 & \\ \text { Cobalt } & \mathrm{mg} / \mathrm{kg} & 13.00 & & 10.30 & \mathrm{~B} & 17.50 \\ \text { Copper } & \mathrm{mg} / \mathrm{kg} & 19.60 & & 20.60 & & 19.10 \\ \text { Lead } & \mathrm{mg} / \mathrm{kg} & 31.40 & & 17.30 & & 13.90 \\ \text { Manganese } & \mathrm{mg} / \mathrm{kg} & 927.00 & & 462.00 & & 744.00 & \\ \text { Nickel } & \mathrm{mg} / \mathrm{kg} & 17.40 & & 21.70 & & 24.60 & \\ \text { Selenium } & \mathrm{mg} / \mathrm{kg} & 0.48 & \mathrm{~B} & 0.57 & \mathrm{~B} & 0.25 & \mathrm{~B} \\ \text { Silver } & \mathrm{mg} / \mathrm{kg} & 0.25 & \mathrm{U} & 0.24 & \mathrm{U} & 0.25 & \mathrm{U} \\ \text { Thallium } & \mathrm{mg} / \mathrm{kg} & 0.25 & \mathrm{U} & 0.24 & \mathrm{U} & 0.25 & \mathrm{U} \\ \text { Zinc } & \mathrm{mg} / \mathrm{kg} & 44.40 & & 45.40 & & 43.80 & \end{array}$

Location=AND; Formation=DISMAL GAP; Phase=1; Site=3,5,11; Sample type=Regular; $A=7010 ; B=7013 ; C=7016 ; A$ lab split='

$\begin{array}{lrrrrrrr}\text { Aluminum } & \mathrm{mg} / \mathrm{kg} & 21300.00 & & 32400.00 & & 34300.00 & \\ \text { Antimony } & \mathrm{mg} / \mathrm{kg} & 0.23 & \mathrm{U} & 0.24 & \mathrm{U} & 0.23 . & \mathrm{U} \\ \text { Arsenic } & \mathrm{mg} / \mathrm{kg} & 3.20 & & 3.90 & & 2.80 & \\ \text { Barium } & \mathrm{mg} / \mathrm{kg} & 71.30 & & 73.00 & & 58.20 & \\ \text { Beryllium } & \mathrm{mg} / \mathrm{kg} & 0.91 & \mathrm{~B} & 1.00 & \mathrm{~B} & 1.00 & \mathrm{~B} \\ \text { Cadmium } & \mathrm{mg} / \mathrm{kg} & 0.23 & \mathrm{U} & 0.24 & \mathrm{U} & 0.23 & \mathrm{U} \\ \text { Chromium } & \mathrm{mg} / \mathrm{kg} & 28.90 & & 38.40 & & 35.80 & \\ \text { Cobalt } & \mathrm{mg} / \mathrm{kg} & 14.00 & & 11.30 & \mathrm{~B} & 9.70 & \mathrm{~B} \\ \text { Copper } & \mathrm{mg} / \mathrm{kg} & 22.10 & & 25.90 & & 26.70 & \\ \text { Lead } & \mathrm{mg} / \mathrm{kg} & 25.30 & & 22.80 & & 24.50 & \\ \text { Manganese } & \mathrm{mg} / \mathrm{kg} & 535.00 & & 251.00 & & 330.00 & \\ \text { Nickel } & \mathrm{mg} / \mathrm{kg} & 20.90 & & 24.70 & & 21.60 & \\ \text { Selenium } & \mathrm{mg} / \mathrm{kg} & 0.33 & \mathrm{~B} & 0.24 & \mathrm{~B} & 0.23 & \mathrm{U} \\ \text { Silver } & \mathrm{mg} / \mathrm{kg} & 0.23 & \mathrm{U} & 0.24 & \mathrm{U} & 0.23 & \mathrm{U} \\ \text { Thallium } & \mathrm{mg} / \mathrm{kg} & 0.23 & \mathrm{U} & 0.26 & \mathrm{~B} & 0.25 & \mathrm{~B} \\ \text { Zinc } & \mathrm{mg} / \mathrm{kg} & 53.80 & & 62.50 & & 51.40 & \end{array}$

Location=AND; Formation=DISMAL GAP; Phase=1; Site=4,12,21; Sample type=Regular; $A=7019 ; B=7022 ; C=7025 ;$ lab split=" '

$\begin{array}{llrlrlrl}\text { Aluminum } & \mathrm{mg} / \mathrm{kg} & 18600.00 & & 33300.00 & & 33700.00 & \\ \text { Antimony } & \mathrm{mg} / \mathrm{kg} & 0.24 & \mathrm{U} & 0.23 & \mathrm{U} & 0.24 & \mathrm{U} \\ \text { Arsenic } & \mathrm{mg} / \mathrm{kg} & 2.30 & \mathrm{~B} & 2.30 & & 2.20 & \mathrm{~B} \\ \text { Barium } & \mathrm{mg} / \mathrm{kg} & 72.50 & & 60.90 & & 54.40 & \\ \text { Beryllium } & \mathrm{mg} / \mathrm{kg} & 0.85 & \mathrm{~B} & 1.30 & & 1.40 & \mathrm{U} \\ \text { Cactnium } & \mathrm{mg} / \mathrm{kg} & 0.24 & \mathrm{U} & 0.23 & \mathrm{U} & 0.24 & \mathrm{U} \\ \text { Chromium } & \mathrm{mg} / \mathrm{kg} & 23.20 & & 31.90 & & 25.60 & \\ \text { Cobalt } & \mathrm{mg} / \mathrm{kg} & 12.80 & & 6.70 & \mathrm{~B} & 15.90 \\ \text { Copper } & \mathrm{mg} / \mathrm{kg} & 20.20 & & 22.80 & & 29.60 \\ \text { Lead } & \mathrm{mg} / \mathrm{kg} & 27.80 & & 18.10 & & 43.80 \\ \text { Manganese } & \mathrm{mg} / \mathrm{kg} & 559.00 & & 95.80 & & 595.00 & \\ \text { Nickel } & \mathrm{mg} / \mathrm{kg} & 18.00 & & 20.50 & & 21.30 & \\ \text { Selenium } & \mathrm{mg} / \mathrm{kg} & 0.24 & \mathrm{U} & 0.24 & \mathrm{~B} & 0.24 & \mathrm{U}\end{array}$


Table I.1 (continued)

\begin{tabular}{|c|c|c|c|c|c|c|c|c|c|}
\hline Analysis & Units & $\begin{array}{l}\text { A horizon } \\
\text { result }\end{array}$ & Qualifier & $\begin{array}{l}\text { B horizon } \\
\text { result }\end{array}$ & Qualifier & $\begin{array}{l}\mathrm{C} \text { horizon } \\
\text { result }\end{array}$ & Qualifier & $\begin{array}{l}\text { A lab split } \\
\text { result }\end{array}$ & Qualifier \\
\hline $\begin{array}{l}\text { Silver } \\
\text { Thallium } \\
\text { Zinc }\end{array}$ & $\begin{array}{l}\mathrm{mg} / \mathrm{kg}^{\prime} \\
\mathrm{mg} / \mathrm{kg} \\
\mathrm{mg} / \mathrm{kg}\end{array}$ & $\begin{array}{r}0.24 \\
0.24 \\
44.40\end{array}$ & $\begin{array}{l}\mathbf{U} \\
\mathbf{U}\end{array}$ & $\begin{array}{r}0.23 \\
0.23 \\
38.50\end{array}$ & $\begin{array}{l}\text { u } \\
\mathbf{u}\end{array}$ & $\begin{array}{r}0.24 \\
0.24 \\
41.70\end{array}$ & $\begin{array}{l}\mathbf{U} \\
\mathbf{U}\end{array}$ & & ... is \\
\hline
\end{tabular}

Location=AND; Formation=DISMAL GAP; Phase=1; Site=9,10,19; Sample type=Field duplicate; $A=7037 ; B=7040 ; C=7043 ;$ A lab split $=$ ',

$\begin{array}{lrrrrrrr}\text { Aluminum } & \mathrm{mg} / \mathrm{kg} & 15900.00 & & 23600.00 & & 30500.00 & \\ \text { Antimony } & \mathrm{mg} / \mathrm{kg} & 0.24 & \mathrm{U} & 0.23 & \mathrm{U} & 0.23 & \mathrm{U} \\ \text { Arsenic } & \mathrm{mg} / \mathrm{kg} & 3.70 & & 4.40 & & 3.40 & \\ \text { Barium } & \mathrm{mg} / \mathrm{kg} & 71.80 & & 75.80 & & 108.00 & \\ \text { Beryllium } & \mathrm{mg} / \mathrm{kg} & 0.68 & \mathrm{~B} & 0.83 & \mathrm{~B} & 1.20 & \mathrm{U} \\ \text { Cadmium } & \mathrm{mg} / \mathrm{kg} & 0.24 & \mathrm{U} & 0.23 & \mathrm{U} & 0.23 & \mathrm{U} \\ \text { Chromium } & \mathrm{mg} / \mathrm{kg} & 24.00 & & 32.50 & & 32.50 & \\ \text { Cobalt } & \mathrm{mg} / \mathrm{kg} & 12.90 & & 17.40 & & 26.60 \\ \text { Copper } & \mathrm{mg} / \mathrm{kg} & 14.80 & & 21.50 & & 52.40 \\ \text { Lead } & \mathrm{mg} / \mathrm{kg} & 32.50 & & 26.30 & & 28.80 \\ \text { Manganese } & \mathrm{mg} / \mathrm{kg} & 947.00 & & 296.00 & & 1510.00 & \\ \text { Nickel } & \mathrm{mg} / \mathrm{kg} & 16.70 & & 17.20 & & 27.20 & \\ \text { Selenium } & \mathrm{mg} / \mathrm{kg} & 0.55 & \mathrm{~B} & 0.29 & \mathrm{~B} & 0.40 & \mathrm{~B} \\ \text { Silver } & \mathrm{mg} / \mathrm{kg} & 0.24 & \mathrm{U} & 0.23 & \mathrm{U} & 0.23 & \mathrm{U} \\ \text { Thallium } & \mathrm{mg} / \mathrm{kg} & 0.24 & \mathrm{U} & 0.23 & \mathrm{U} & 0.23 & \mathrm{U} \\ \text { Zinc } & \mathrm{mg} / \mathrm{kg} & 38.30 & & 36.60 & & 50.50 & \end{array}$

Location=AND; Formation=DISMAL GAP; Phase=1; Site=9,10,19; Sample type=Regular; $A=7001 ; B=7004 ; C=7007 ;$ A lab split=" '

\begin{tabular}{|c|c|c|c|c|c|c|}
\hline $\begin{array}{l}\text { Aluminum } \\
\text { Antimony } \\
\text { Arsenic } \\
\text { Barium } \\
\text { Beryllium } \\
\text { Cadmium } \\
\text { Chromium } \\
\text { Cobalt } \\
\text { Copper } \\
\text { Lead } \\
\text { Manganese } \\
\text { Hickel } \\
\text { Selenium } \\
\text { Silver } \\
\text { Thallium } \\
\text { Zinc }\end{array}$ & $\begin{array}{l}\mathrm{mg} / \mathrm{kg} \\
\mathrm{mg} / \mathrm{kg} \\
\mathrm{mg} / \mathrm{kg} \\
\mathrm{mg} / \mathrm{kg} \\
\mathrm{mg} / \mathbf{k g} \\
\mathrm{mg} / \mathbf{k g} \\
\mathrm{mg} / \mathbf{k g} \\
\mathrm{mg} / \mathbf{k g} \\
\mathrm{mg} / \mathbf{k g} \\
\mathrm{mg} / \mathbf{k g} \\
\mathrm{mg} / \mathbf{k g} \\
\mathrm{mg} / \mathrm{kg} \\
\mathrm{mg} / \mathbf{k g} \\
\mathrm{mg} / \mathbf{k g} \\
\mathrm{mg} / \mathbf{k g} \\
\mathrm{mg} / \mathrm{kg}\end{array}$ & $\begin{array}{r}22200.00 \\
0.25 \\
3.90 \\
79.40 \\
0.83 \\
0.25 \\
30.30 \\
14.50 \\
15.30 \\
27.70 \\
963.00 \\
22.30 \\
0.53 \\
0.25 \\
0.25 \\
47.60\end{array}$ & $\begin{array}{l}B \\
\mathbf{U} \\
\mathbf{U}\end{array}$ & $\begin{array}{r}30100.00 \\
0.23 \\
4.20 \\
83.90 \\
0.84 \\
0.23 \\
34.10 \\
15.80 \\
22.70 \\
25.00 \\
311.00 \\
21.00 \\
0.58 \\
0.23 \\
0.25 \\
46.50\end{array}$ & $\begin{array}{l}B \\
U \\
B\end{array}$ & $\begin{array}{r}36100.00 \\
0.25 \\
3.30 \\
102.00 \\
1.20 \\
0.25 \\
38.60 \\
17.70 \\
42.30 \\
27.80 \\
893.00 \\
27.80 \\
0.52 \\
0.25 \\
0.25 \\
53.90\end{array}$ \\
\hline
\end{tabular}

Location=ORR; Fomation=CHEPULTEPEC; Phase $=2 ;$ Site $=50,66,73 ;$ Sample type $=$ Field duplicate; $A=5173 ; B=5178 ; C=5184 ; A$ lab split $=5174$

\begin{tabular}{|c|c|c|c|c|c|c|c|c|}
\hline $\begin{array}{l}\text { Aluminum } \\
\text { Antimony } \\
\text { Arsenic } \\
\text { Barium } \\
\text { Beryl lium } \\
\text { Cacmium } \\
\text { Chromium } \\
\text { Cobalt } \\
\text { Copper } \\
\text { Lead } \\
\text { Manganese } \\
\text { Nickel } \\
\text { Silver } \\
\text { Thallium } \\
\text { Zinc }\end{array}$ & $\begin{array}{l}\mathrm{mg} / \mathrm{kg} \\
\mathrm{mg} / \mathrm{kg} \\
\mathrm{mg} / \mathrm{kg} \\
\mathrm{mg} / \mathrm{kg} \\
\mathrm{mg} / \mathrm{kg} \\
\mathrm{mg} / \mathrm{kg} \\
\mathrm{mg} / \mathrm{kg} \\
\mathrm{mg} / \mathrm{kg} \\
\mathrm{mg} / \mathrm{kg} \\
\mathrm{mg} / \mathrm{kg} \\
\mathrm{mg} / \mathrm{kg} \\
\mathrm{mg} / \mathrm{kg} \\
\mathrm{mg} / \mathrm{kg} \\
\mathrm{mg} / \mathrm{kg} \\
\mathrm{mg} / \mathrm{kg}\end{array}$ & $\begin{array}{r}8010.00 \\
0.21 \\
15.50 \\
29.20 \\
0.25 \\
0.21 \\
27.30 \\
10.50 \\
4.90 \\
16.50 \\
734.00 \\
5.50 \\
0.21 \\
0.21 \\
27.30\end{array}$ & $\begin{array}{l}\mathbf{B} \\
\mathbf{U} \\
\mathbf{U}\end{array}$ & $\begin{array}{r}20900.00 \\
0.22 \\
16.00 \\
38.30 \\
0.32 \\
0.22 \\
36.50 \\
2.70 \\
21.30 \\
9.90 \\
62.80 \\
10.90 \\
0.22 \\
0.30 \\
97.20\end{array}$ & $\begin{array}{l}B \\
B \\
U\end{array}$ & $\begin{array}{r}17900.00 \\
0.21 \\
17.90 \\
19.90 \\
0.42 \\
0.21 \\
32.30 \\
22.40 \\
24.40 \\
30.50 \\
378.00 \\
14.10 \\
0.21 \\
0.26 \\
163.00\end{array}$ & $\begin{array}{l}B \\
B \\
U\end{array}$ & $\begin{array}{r}7650.00 \\
0.22 \\
7.50 \\
34.30 \\
0.23 \\
0.22 \\
13.30 \\
11.40 \\
5.30 \\
14.90 \\
510.00 \\
5.80 \\
0.22 \\
0.22 \\
29.10\end{array}$ \\
\hline
\end{tabular}


Table I.1 (continued)

\begin{tabular}{lcccccccc}
\hline & & A horizon & & B horizon & & C horizon & & A lab split \\
Analysis & Units & result & Qualifier & $\begin{array}{c}\text { result } \\
\text { Q Qualifier }\end{array}$ & result & Qualifier & result & Qualifier \\
\hline
\end{tabular}

Location =ORR; Formation =CHEPULTEPEC; Phase=2; Site=50,66,73; Sample type=Regular; $A=5165 ; B=5170 ; C=5181 ; A$ lab split $=5166$

\begin{tabular}{|c|c|c|c|c|c|c|c|c|}
\hline $\begin{array}{l}\text { Aluminum } \\
\text { Antimony } \\
\text { Arsenic } \\
\text { Barium } \\
\text { Beryllium } \\
\text { Cadmium } \\
\text { Chromium } \\
\text { Cobalt } \\
\text { Copper } \\
\text { Lead } \\
\text { Manganese } \\
\text { Nickel } \\
\text { Silver } \\
\text { Thallium } \\
\text { Zinc }\end{array}$ & $\begin{array}{l}\mathrm{mg} / \mathrm{kg} \\
\mathrm{mg} / \mathrm{kg} \\
\mathrm{mg} / \mathrm{kg} \\
\mathrm{mg} / \mathrm{kg} \\
\mathrm{mg} / \mathrm{kg} \\
\mathrm{mg} / \mathrm{kg} \\
\mathrm{mg} / \mathrm{kg} \\
\mathrm{mg} / \mathrm{kg} \\
\mathrm{mg} / \mathrm{kg} \\
\mathrm{mg} / \mathrm{kg} \\
\mathrm{mg} / \mathrm{kg} \\
\mathrm{mg} / \mathrm{kg} \\
\mathrm{mg} / \mathrm{kg} \\
\mathrm{mg} / \mathrm{kg} \\
\mathrm{mg} / \mathrm{kg}\end{array}$ & $\begin{array}{r}8550.00 \\
0.23 \\
10.70 \\
37.20 \\
0.27 \\
0.23 \\
25.80 \\
9.80 \\
8.50 \\
15.50 \\
615.00 \\
6.10 \\
0.23 \\
0.23 \\
34.80\end{array}$ & $\begin{array}{l}8 \\
U \\
U\end{array}$ & $\begin{array}{r}18300.00 \\
0.20 \\
15.70 \\
56.50 \\
0.38 \\
0.20 \\
31.90 \\
2.10 \\
17.50 \\
8.40 \\
55.60 \\
10.60 \\
0.20 \\
0.27 \\
118.00\end{array}$ & $\begin{array}{l}B \\
U\end{array}$ & $\begin{array}{r}20700.00 \\
0.20 \\
25.80 \\
27.50 \\
0.54 \\
0.20 \\
29.90 \\
5.00 \\
27.60 \\
19.40 \\
105.00 \\
19.80 \\
0.20 \\
0.26 \\
178.00\end{array}$ & $\begin{array}{l}B \\
B \\
U\end{array}$ & $\begin{array}{r}9000.00 \\
0.22 \\
17.30 \\
33.40 \\
0.32 \\
0.22 \\
28.10 \\
9.20 \\
6.70 \\
16.90 \\
717.00 \\
5.40 \\
0.22 \\
0.22 \\
32.80\end{array}$ \\
\hline
\end{tabular}

Location $=$ ORR; Formation $=$ CHEPULTEPEC; Phase $=2 ;$ Site $=52,53,78 ;$ Sample type $=$ Regular; $A=5127 ; B=5130 ; C=5133 ; A$ lab split $=$ ' '

$\begin{array}{lrrrrrrr}\text { Aluminum } & \mathrm{mg} / \mathrm{kg} & 8410.00 & & 13600.00 & & 12800.00 & \\ \text { Antimony } & \mathrm{mg} / \mathrm{kg} & 0.21 & \mathrm{U} & 0.22 & \mathrm{U} & 0.22 & \mathrm{U} \\ \text { Arsenic } & \mathrm{mg} / \mathrm{kg} & 6.80 & & 21.50 & & 36.90 & \\ \text { Barium } & \mathrm{mg} / \mathrm{kg} & 64.70 & & 28.60 & \mathrm{~B} & 10.30 & \mathrm{~B} \\ \text { Beryllium } & \mathrm{mg} / \mathrm{kg} & 0.37 & \mathrm{~B} & 0.37 & \mathrm{~B} & 0.61 & \mathrm{~B} \\ \text { Cadmium } & \mathrm{mg} / \mathrm{kg} & 0.21 & \mathrm{U} & 0.22 & \mathrm{U} & 0.22 & \mathrm{U} \\ \text { Chromium } & \mathrm{mg} / \mathrm{kg} & 12.00 & & 28.90 & & 32.20 & \\ \text { Cobalt } & \mathrm{mg} / \mathrm{kg} & 8.80 & \mathrm{~B} & 3.20 & \mathrm{~B} & 3.60 & \mathrm{~B} \\ \text { Copper } & \mathrm{mg} / \mathrm{kg} & 5.90 & & 19.20 & & 32.50 & \\ \text { Lead } & \mathrm{mg} / \mathrm{kg} & 18.50 & & 9.30 & & 15.30 & \\ \text { Manganese } & \mathrm{mg} / \mathrm{kg} & 1000.00 & & 133.00 & & 58.20 & \\ \text { Nickel } & \mathrm{mg} / \mathrm{kg} & 7.40 & \mathrm{~B} & 7.90 & \mathrm{~B} & 18.20 & \\ \text { Selenium } & \mathrm{mg} / \mathrm{kg} & 0.63 & \mathrm{~B} & 0.80 & \mathrm{~B} & 0.60 & \mathrm{~B} \\ \text { Silver } & \mathrm{mg} / \mathrm{kg} & 0.21 & \mathrm{U} & 0.22 & \mathrm{U} & 0.22 & \mathrm{U} \\ \text { Thallium } & \mathrm{mg} / \mathrm{kg} & 0.21 & \mathrm{U} & 0.23 & \mathrm{~B} & 0.22 & \mathrm{U} \\ \text { Zinc } & \mathrm{mg} / \mathrm{kg} & 27.60 & & 66.60 & & 163.00 & \end{array}$

Location=ORR; Formation $=$ CHEPULTEPEC; Phase $=2 ;$ Site $=68,74,90 ;$ Sample type $=$ Regular; $A=5154 ; B=5159 ; C=5162 ; A$ lab split $=5155$

\begin{tabular}{|c|c|c|c|c|c|c|c|c|}
\hline $\begin{array}{l}\text { Aluminum } \\
\text { Antimony } \\
\text { Arsenic } \\
\text { Barium } \\
\text { Beryll ium } \\
\text { Cadmium } \\
\text { Chromium } \\
\text { Cobalt } \\
\text { Copper } \\
\text { Lead } \\
\text { Manganese } \\
\text { Nickel } \\
\text { Silver } \\
\text { Thal lium } \\
\text { Zinc }\end{array}$ & $\begin{array}{l}\mathrm{mg} / \mathrm{kg} \\
\mathrm{mg} / \mathrm{kg} \\
\mathrm{mg} / \mathrm{kg} \\
\mathrm{mg} / \mathrm{kg} \\
\mathrm{mg} / \mathrm{kg} \\
\mathrm{mg} / \mathrm{kg} \\
\mathrm{mg} / \mathrm{kg} \\
\mathrm{mg} / \mathrm{kg} \\
\mathrm{mg} / \mathrm{kg} \\
\mathrm{mg} / \mathrm{kg} \\
\mathrm{mg} / \mathrm{kg} \\
\mathrm{mg} / \mathrm{kg} \\
\mathrm{mg} / \mathrm{kg} \\
\mathrm{mg} / \mathrm{kg} \\
\mathrm{mg} / \mathrm{kg}\end{array}$ & $\begin{array}{r}7600.00 \\
0.20 \\
4.60 \\
30.50 \\
0.25 \\
0.20 \\
8.70 \\
12.00 \\
4.20 \\
15.20 \\
597.00 \\
5.00 \\
0.20 \\
0.20 \\
18.10\end{array}$ & $\begin{array}{l}\mathbf{B} \\
\mathbf{B} \\
\mathbf{U}\end{array}$ & $\begin{array}{r}3800.00 \\
0.21 \\
18.70 \\
27.90 \\
0.31 \\
0.21 \\
33.90 \\
2.30 \\
14.90 \\
9.70 \\
46.00 \\
9.90 \\
0.21 \\
0.25 \\
54.20\end{array}$ & $\begin{array}{l}\text { B } \\
\text { B } \\
U\end{array}$ & $\begin{array}{r}20900.00 \\
0.23 \\
16.70 \\
12.00 \\
0.39 \\
0.23 \\
30.40 \\
1.90 \\
17.60 \\
11.80 \\
76.90 \\
12.10 \\
0.23 \\
0.24 \\
77.30\end{array}$ & $\begin{array}{l}\mathbf{B} \\
\mathbf{B} \\
\mathbf{U}\end{array}$ & $\begin{array}{r}8480.00 \\
0.18 \\
7.00 \\
31.60 \\
0.33 \\
0.18 \\
12.80 \\
10.10 \\
4.80 \\
15.80 \\
475.00 \\
5.10 \\
0.18 \\
0.18 \\
21.60\end{array}$ \\
\hline
\end{tabular}

Location=ORR; Formation=CHEPULTEPEC; Phase=2; Site=77,85,86; Sample type=Field duplicate; $A=5145 ; B=5148 ; C=5151 ; A$ lab split $=$ ',

\begin{tabular}{|c|c|c|c|c|c|c|}
\hline $\begin{array}{l}\text { Aluminum } \\
\text { Antimony } \\
\text { Arsenic } \\
\text { Barium } \\
\text { Beryllium }\end{array}$ & $\begin{array}{l}\mathrm{mg} / \mathrm{kg} \\
\mathrm{mg} / \mathrm{kg} \\
\mathrm{mg} / \mathrm{kg} \\
\mathrm{mg} / \mathrm{kg} \\
\mathrm{mg} / \mathrm{kg}\end{array}$ & $\begin{array}{r}9290.00 \\
0.21 \\
4.50 \\
141.00 \\
0.95\end{array}$ & $\mathbf{U}$ & $\begin{array}{r}15900.00 \\
0.41 \\
18.40 \\
68.60 \\
1.10\end{array}$ & B & $\begin{array}{r}17200.00 \\
0.21 \\
13.10 \\
57.30 \\
1.60\end{array}$ \\
\hline
\end{tabular}


Table I.1 (continued)

\begin{tabular}{|c|c|c|c|c|c|c|c|c|c|}
\hline Analysis & Units & $\begin{array}{l}\text { A horizon } \\
\text { result }\end{array}$ & Qualifier & $\begin{array}{c}\text { B horizon } \\
\text { result }\end{array}$ & Qualifier & $\begin{array}{l}C \text { horizon } \\
\text { result }\end{array}$ & Qualifier & $\begin{array}{l}\text { A lab split } \\
\text { result }\end{array}$ & Qualifier \\
\hline $\begin{array}{l}\text { Cadmium } \\
\text { Chromium } \\
\text { Cobalt } \\
\text { Copper } \\
\text { Lead } \\
\text { Manganese } \\
\text { Nickel } \\
\text { Selenium } \\
\text { silver } \\
\text { Thallium } \\
\text { Zinc }\end{array}$ & $\begin{array}{l}\mathrm{mg} / \mathrm{kg} \\
\mathrm{mg} / \mathrm{kg} \\
\mathrm{mg} / \mathrm{kg} \\
\mathrm{mg} / \mathrm{kg} \\
\mathrm{mg} / \mathrm{kg} \\
\mathrm{mg} / \mathrm{kg} \\
\mathrm{mg} / \mathrm{kg} \\
\mathrm{mg} / \mathrm{kg} \\
\mathrm{mg} / \mathrm{kg} \\
\mathrm{mg} / \mathrm{kg} \\
\mathrm{mg} / \mathrm{kg}\end{array}$ & $\begin{array}{r}0.21 \\
9.60 \\
17.20 \\
7.20 \\
20.90 \\
2250.00 \\
13.60 \\
0.77 \\
0.21 \\
0.21 \\
82.00\end{array}$ & $\begin{array}{l}B \\
U \\
U\end{array}$ & $\begin{array}{r}0.21 \\
37.80 \\
17.70 \\
19.80 \\
35.60 \\
669.00 \\
18.70 \\
0.72 \\
0.21 \\
0.40 \\
223.00\end{array}$ & $\begin{array}{l}\text { B } \\
\text { U } \\
B\end{array}$ & $\begin{array}{r}0.21 \\
24.70 \\
31.70 \\
34.00 \\
54.30 \\
1360.00 \\
37.80 \\
0.73 \\
0.21 \\
0.42 \\
371.00\end{array}$ & $\begin{array}{l}\text { B } \\
\text { U } \\
\text { B }\end{array}$ & & $\therefore$ \\
\hline
\end{tabular}

Location=ORR; Formation $=$ CHEPULTEPEC; Phase $=2 ;$ Site $=77,85,86 ;$ Sample type $=$ Regular; $A=5136 ; B=5139 ; C=5142 ; A$ lab split=",

$\begin{array}{lrrrrrrr}\text { Aluminum } & \mathrm{mg} / \mathrm{kg} & 9690.00 & & 16700.00 & & 15900.00 & \\ \text { Antimony } & \mathrm{mg} / \mathrm{kg} & 0.22 & \mathrm{U} & 0.21 & \mathrm{U} & 0.23 & \mathrm{U} \\ \text { Arsenic } & \mathrm{mg} / \mathrm{kg} & 4.40 & , & 10.20 & & 12.60 & \\ \text { Barium } & \mathrm{mg} / \mathrm{kg} & 145.00 & & 44.20 & & 26.00 & \mathrm{~B} \\ \text { Beryllium } & \mathrm{mg} / \mathrm{kg} & 0.94 & \mathrm{~B} & 1.40 & & 1.40 & \\ \text { Cadmium } & \mathrm{mg} / \mathrm{kg} & 0.22 & \mathrm{U} & 0.21 & \mathrm{U} & 0.23 & \mathrm{U} \\ \text { Chromium } & \mathrm{mg} / \mathrm{kg} & 10.60 & & 24.10 & & 24.90 & \\ \text { Cobalt } & \mathrm{mg} / \mathrm{kg} & 15.60 & & 8.30 & \mathrm{~B} & 13.30 \\ \text { Copper } & \mathrm{mg} / \mathrm{kg} & 7.60 & & 20.30 & & 23.70 \\ \text { Lead } & \mathrm{mg} / \mathrm{kg} & 21.00 & & 16.50 & & 20.70 \\ \text { Manganese } & \mathrm{mg} / \mathrm{kg} & 2190.00 & & 228.00 & & 447.00 & \\ \text { Nickel } & \mathrm{mg} / \mathrm{kg} & 12.70 & & 20.10 & & 21.60 & \\ \text { Selenium } & \mathrm{mg} / \mathrm{kg} & 0.67 & \mathrm{~B} & 0.58 & \mathrm{~B} & 0.59 & \mathrm{~B} \\ \text { Silver } & \mathrm{mg} / \mathrm{kg} & 0.22 & \mathrm{U} & 0.21 & \mathrm{U} & 0.23 & \mathrm{U} \\ \text { Thallium } & \mathrm{mg} / \mathrm{kg} & 0.22 & \mathrm{U} & 0.21 & \mathrm{U} & 0.25 & \mathrm{~B} \\ \text { Zinc } & \mathrm{mg} / \mathrm{kg} & 91.10 & & 304.00 & & 295.00 & \end{array}$

Location=ORR; Formation=CHICKAMAUGA Bethel V; Phase=2; Site=101,102,103; Sample type=Regular; $A=5196 ; B=5199 ; C=5202 ; A$ lab split=" '

$\begin{array}{lrrrrrrr}\text { Aluminum } & \mathrm{mg} / \mathrm{kg} & 22400.00 & & 40000.00 & & 41700.00 & \\ \text { Antimony } & \mathrm{mg} / \mathrm{kg} & 0.22 & \mathrm{U} & 0.22 & \mathrm{U} & \mathbf{0 . 2 2} & \mathrm{U} \\ \text { Arsenic } & \mathrm{mg} / \mathrm{kg} & 4.50 & & 5.90 & & 12.80 & \\ \text { Barium } & \mathrm{mg} / \mathrm{kg} & 113.00 & & 189.00 & & 145.00 & \\ \text { Beryllium } & \mathrm{mg} / \mathrm{kg} & 1.30 & & 2.40 & & 2.10 & \\ \text { Cacmium } & \mathrm{mg} / \mathrm{kg} & 0.22 & \mathrm{U} & 0.22 & \mathrm{U} & 0.22 & \mathrm{U} \\ \text { Chromium } & \mathrm{mg} / \mathrm{kg} & 25.40 & & 37.70 & & 37.40 & \\ \text { Cobalt } & \mathrm{mg} / \mathrm{kg} & 17.90 & & 16.50 & & 45.90 \\ \text { Copper } & \mathrm{mg} / \mathrm{kg} & 19.70 & & 28.90 & & 37.10 \\ \text { Lead } & \mathrm{mg} / \mathrm{kg} & 36.90 & & 25.60 & & 50.00 \\ \text { Manganese } & \mathrm{mg} / \mathrm{kg} & 1760.00 & & 762.00 & & 818.00 \\ \text { Nickel } & \mathrm{mg} / \mathrm{kg} & 19.60 & & 35.20 & & 43.10 & \\ \text { Silver } & \mathrm{mg} / \mathrm{kg} & 0.22 & \mathrm{U} & 0.22 & \mathrm{U} & 0.22 & \mathrm{U} \\ \text { Thallium } & \mathrm{mg} / \mathrm{kg} & 0.22 & \mathrm{U} & 0.28 & \mathrm{~B} & 0.28 & \mathrm{~B} \\ \text { Zinc } & \mathrm{mg} / \mathrm{kg} & 46.90 & & 61.70 & & 95.00 & \end{array}$

Location=ORR; Formation $=$ CHICKAMAUGA Bethel V; Phase =2; Site =104,108,110; Sample type=Regular; $A=5205 ; B=5208 ; C=5211 ; A$ lab split $=$ ' '

$\begin{array}{lrrrrrrr}\text { Aluminum } & \mathrm{mg} / \mathrm{kg} & 14500.00 & & 29700.00 & & 32200.00 & \\ \text { Antimony } & \mathrm{mg} / \mathrm{kg} & 0.22 & \mathrm{U} & 0.21 & \mathrm{U} & 0.23 & \mathrm{U} \\ \text { Arsenic } & \mathrm{mg} / \mathrm{kg} & 5.80 & & 5.00 & & 4.50 & \\ \text { Barium } & \mathrm{mg} / \mathrm{kg} & 68.50 & & 102.00 & & 176.00 & \\ \text { Beryllium } & \mathrm{mg} / \mathrm{kg} & 0.98 & \mathrm{~B} & 1.40 & & 1.90 & \\ \text { Cadmium } & \mathrm{mg} / \mathrm{kg} & 0.22 & \mathrm{U} & 0.21 & \mathrm{U} & 0.23 & \mathrm{U} \\ \text { Chromium } & \mathrm{mg} / \mathrm{kg} & 35.10 & & 41.30 & & 37.50 & \\ \text { Cobalt } & \mathrm{mg} / \mathrm{kg} & 18.30 & & 15.00 & & 20.30 & \\ \text { Copper } & \mathrm{mg} / \mathrm{kg} & 8.30 & & 21.80 & & 26.40 \\ \text { Lead } & \mathrm{mg} / \mathrm{kg} & 37.00 & & 31.70 & & 41.50 \\ \text { Manganese } & \mathrm{mg} / \mathrm{kg} & 672.00 & & 368.00 & & 845.00 \\ \text { Hickel } & \mathrm{mg} / \mathrm{kg} & 8.60 & \mathrm{~B} & 17.20 & & 25.90\end{array}$


I-8

Table I.1 (continued)

\begin{tabular}{|c|c|c|c|c|c|c|c|c|c|}
\hline Analysis & Units & $\begin{array}{l}\text { A horizon } \\
\text { result }\end{array}$ & Qualifier & $\begin{array}{c}\text { B horizon } \\
\text { result }\end{array}$ & Qualifier & $\begin{array}{l}\mathrm{C} \text { horizon } \\
\text { result }\end{array}$ & Qualifier & $\begin{array}{l}\text { A lab split } \\
\text { result }\end{array}$ & Qualifier \\
\hline $\begin{array}{l}\text { Silver } \\
\text { Thallium } \\
\text { Zinc }\end{array}$ & $\begin{array}{l}\mathrm{mg} / \mathrm{kg} \\
\mathrm{mg} / \mathrm{kg} \\
\mathrm{mg} / \mathrm{kg}\end{array}$ & $\begin{array}{r}0.22 \\
0.22 \\
24.70\end{array}$ & $\underset{U}{U}$ & $\begin{array}{r}0.21 \\
0.26 \\
39.50\end{array}$ & $\begin{array}{l}\text { U } \\
\text { B }\end{array}$ & $\begin{array}{r}0.23 \\
0.23 \\
58.30\end{array}$ & $\begin{array}{l}U \\
U\end{array}$ & & \\
\hline
\end{tabular}

Location=ORR; Formation=CHICKAMAUGA Bethel V; Phase=2; Site=115,116,117; Sample type=Regular; $A=5216 ; B=5222 ; C=5225 ; A$ lab split $=5217$

\begin{tabular}{|c|c|c|c|c|c|c|c|c|}
\hline $\begin{array}{l}\text { Aluminum } \\
\text { Antimony } \\
\text { Arsenic } \\
\text { Barium } \\
\text { Beryllium } \\
\text { Cadmium } \\
\text { Chromiun } \\
\text { Cobalt } \\
\text { Copper } \\
\text { Lead } \\
\text { Manganese } \\
\text { Nickel } \\
\text { Silver } \\
\text { Thallium } \\
\text { Zinc }\end{array}$ & $\begin{array}{l}\mathrm{mg} / \mathrm{kg} \\
\mathrm{mg} / \mathrm{kg} \\
\mathrm{mg} / \mathrm{kg} \\
\mathrm{mg} / \mathrm{kg} \\
\mathrm{mg} / \mathrm{kg} \\
\mathrm{mg} / \mathrm{kg} \\
\mathrm{mg} / \mathrm{kg} \\
\mathrm{mg} / \mathrm{kg} \\
\mathrm{mg} / \mathrm{kg} \\
\mathrm{mg} / \mathrm{kg} \\
\mathrm{mg} / \mathrm{kg} \\
\mathrm{mg} / \mathrm{kg} \\
\mathrm{mg} / \mathrm{kg} \\
\mathrm{mg} / \mathrm{kg} \\
\mathrm{mg} / \mathrm{kg}\end{array}$ & $\begin{array}{r}14700.00 \\
0.23 \\
4.20 \\
63.10 \\
0.68 \\
0.23 \\
35.30 \\
15.10 \\
11.40 \\
34.50 \\
852.00 \\
9.70 \\
0.23 \\
0.23 \\
32.80\end{array}$ & $\begin{array}{l}\mathbf{U} \\
\mathbf{U}\end{array}$ & $\begin{array}{r}29200.00 \\
0.22 \\
5.80 \\
80.00 \\
0.93 \\
0.22 \\
36.10 \\
15.10 \\
19.90 \\
28.10 \\
307.00 \\
14.00 \\
0.22 \\
0.22 \\
36.00\end{array}$ & $\begin{array}{l}\text { B } \\
\text { U }\end{array}$ & $\begin{array}{r}34000.00 \\
0.23 \\
5.50 \\
150.00 \\
2.70 \\
0.23 \\
33.50 \\
38.80 \\
25.80 \\
52.30 \\
823.00 \\
30.90 \\
0.23 \\
0.23 \\
65.80\end{array}$ & $\begin{array}{l}\mathbf{U} \\
\mathbf{U}\end{array}$ & $\begin{array}{r}14400.00 \\
0.23 \\
5.30 \\
70.50 \\
0.77 \\
0.23 \\
46.20 \\
16.60 \\
10.90 \\
42.60 \\
1180.00 \\
9.30 \\
0.23 \\
0.23 \\
31.40\end{array}$ \\
\hline
\end{tabular}

Location=ORR; Formation=CHICKAMAUGA Bethel V; Phase=2; Site=93,99,100; Sample type=Regular; $A=5187 ; B=5190 ; C=5193 ; A$ lab split=' '

$\begin{array}{lrrrrrrr}\text { Aluminum } & \mathrm{mg} / \mathrm{kg} & 21200.00 & & 40500.00 & & 42700.00 & \\ \text { Antimony } & \mathrm{mg} / \mathrm{kg} & 0.22 & \mathrm{U} & 0.22 & \mathrm{U} & 0.23 & \mathrm{U} \\ \text { Arsenic } & \mathrm{mg} / \mathrm{kg} & 6.00 & & 4.00 & & 3.20 & \\ \text { Barium } & \mathrm{mg} / \mathrm{kg} & 79.60 & & 156.00 & & 191.00 & \\ \text { Beryllium } & \mathrm{mg} / \mathrm{kg} & 0.97 & \mathrm{~B} & 1.60 & & 1.70 & \\ \text { Cadmium } & \mathrm{mg} / \mathrm{kg} & 0.22 & \mathrm{U} & 0.22 & \mathrm{U} & 0.23 & \mathrm{U} \\ \text { Chromium } & \mathrm{mg} / \mathrm{kg} & 29.50 & & 38.50 & & 39.60 & \\ \text { Cobalt } & \mathrm{mg} / \mathrm{kg} & 27.70 & & 17.30 & & 13.60 \\ \text { Copper } & \mathrm{mg} / \mathrm{kg} & 18.20 & & 30.10 & & 35.00 \\ \text { Lead } & \mathrm{mg} / \mathrm{kg} & 47.60 & & 30.10 & & 33.20 \\ \text { Manganese } & \mathrm{mg} / \mathrm{kg} & 1150.00 & & 463.00 & & 586.00 & \\ \text { Mickel } & \mathrm{mg} / \mathrm{kg} & 15.60 & & 31.70 & & 37.80 & \\ \text { Silver } & \mathrm{mg} / \mathrm{kg} & 0.22 & \mathrm{U} & 0.22 & \mathrm{U} & 0.23 & \mathrm{U} \\ \text { Thallium } & \mathrm{mg} / \mathrm{kg} & 0.32 & \mathrm{~B} & 0.27 & \mathrm{~B} & 0.27 & \mathrm{~B} \\ \text { Zinc } & \mathrm{mg} / \mathrm{kg} & 46.00 & & 73.10 & & 88.20 & \end{array}$

Location=ORR; Formation $=C H I C K A M A U G A$ K-25; Phase $=2 ;$ Site=118,122,124; Sample type=Regular; $A=5240 ; B=5246 ; C=5249 ; A$ lab split $=5241$

\begin{tabular}{|c|c|c|c|c|c|c|c|c|}
\hline $\begin{array}{l}\text { Aluminum } \\
\text { Antimony } \\
\text { Arsenic } \\
\text { Barium } \\
\text { Beryllium } \\
\text { Cadmium } \\
\text { Chromium } \\
\text { Cobalt } \\
\text { Copper } \\
\text { Lead } \\
\text { Manganese } \\
\text { Hickel } \\
\text { Silver } \\
\text { Thallium } \\
\text { Zinc }\end{array}$ & $\begin{array}{l}\mathrm{mg} / \mathrm{kg} \\
\mathrm{mg} / \mathrm{kg} \\
\mathrm{mg} / \mathrm{kg} \\
\mathrm{mg} / \mathrm{kg} \\
\mathrm{mg} / \mathrm{kg} \\
\mathrm{mg} / \mathrm{kg} \\
\mathrm{mg} / \mathrm{kg} \\
\mathrm{mg} / \mathrm{kg} \\
\mathrm{mg} / \mathrm{kg} \\
\mathrm{mg} / \mathrm{kg} \\
\mathrm{mg} / \mathrm{kg} \\
\mathrm{mg} / \mathrm{kg} \\
\mathrm{mg} / \mathrm{kg} \\
\mathrm{mg} / \mathrm{kg} \\
\mathrm{mg} / \mathrm{kg}\end{array}$ & $\begin{array}{r}20000.00 \\
0.26 \\
6.90 \\
82.00 \\
0.96 \\
0.26 \\
28.80 \\
15.00 \\
20.50 \\
39.90 \\
1070.00 \\
27.30 \\
0.26 \\
0.26 \\
55.90\end{array}$ & $\begin{array}{l}\mathbf{B} \\
\mathbf{U}\end{array}$ & $\begin{array}{r}36900.00 \\
0.22 \\
5.60 \\
77.50 \\
2.00 \\
0.22 \\
34.30 \\
26.70 \\
25.50 \\
24.70 \\
255.00 \\
30.40 \\
0.22 \\
0.26 \\
67.40\end{array}$ & $\begin{array}{l}\mathbf{U} \\
\mathbf{B}\end{array}$ & $\begin{array}{r}40900.00 \\
0.22 \\
3.70 \\
86.30 \\
2.00 \\
0.22 \\
34.10 \\
31.60 \\
25.10 \\
28.40 \\
384.00 \\
33.80 \\
0.22 \\
0.34 \\
68.10\end{array}$ & $\begin{array}{l}\mathbf{U} \\
\mathbf{B}\end{array}$ & $\begin{array}{r}18600.00 \\
0.26 \\
6.90 \\
74.00 \\
0.91 \\
0.26 \\
28.00 \\
15.10 \\
19.50 \\
35.80 \\
997.00 \\
25.00 \\
0.26 \\
0.26 \\
54.20\end{array}$ \\
\hline
\end{tabular}


Table L1 (continued)

\begin{tabular}{|c|c|c|c|c|c|c|c|c|c|}
\hline Analysis & Units & $\begin{array}{l}\text { A horizon } \\
\text { result }\end{array}$ & Qualifier & $\begin{array}{l}\text { B horizon } \\
\text { result }\end{array}$ & Qualifier & $\begin{array}{l}\text { C horizon } \\
\text { result }\end{array}$ & Qualifier & $\begin{array}{l}\text { A lab split } \\
\text { result }\end{array}$ & Qualifier \\
\hline \multicolumn{10}{|c|}{$\begin{array}{c}\text { Location }=\text { ORR; Formation }=\text { CHICKAMAUGA } K-25 ; \text { Phase }=2 ; \text { Site }=119,123,127 ; \text { Sample type }=\text { Regular; } \\
\qquad A=5252 ; B=5257 ; C=5260 ; A \text { lab split }=5253\end{array}$} \\
\hline $\begin{array}{l}\text { Aluminum } \\
\text { Antimony } \\
\text { Arsenic } \\
\text { Barium } \\
\text { Beryllium } \\
\text { Cactium } \\
\text { Chromium } \\
\text { Cobalt } \\
\text { Copper } \\
\text { Lead } \\
\text { Manganese } \\
\text { Nickel } \\
\text { Silver } \\
\text { Thallium } \\
\text { Zinc }\end{array}$ & $\begin{array}{l}\mathrm{mg} / \mathrm{kg} \\
\mathrm{mg} / \mathrm{kg} \\
\mathrm{mg} / \mathrm{kg} \\
\mathrm{mg} / \mathrm{kg} \\
\mathrm{mg} / \mathrm{kg} \\
\mathrm{mg} / \mathrm{kg} \\
\mathrm{mg} / \mathrm{kg} \\
\mathrm{mg} / \mathrm{kg} \\
\mathrm{mg} / \mathrm{kg} \\
\mathrm{mg} / \mathrm{kg} \\
\mathrm{mg} / \mathrm{kg} \\
\mathrm{mg} / \mathrm{kg} \\
\mathrm{mg} / \mathrm{kg} \\
\mathrm{mg} / \mathrm{kg} \\
\mathrm{mg} / \mathrm{kg}\end{array}$ & $\begin{array}{r}27900.00 \\
0.24 \\
6.30 \\
107.00 \\
1.30 \\
0.24 \\
32.90 \\
15.70 \\
12.60 \\
32.60 \\
1820.00 \\
22.30 \\
0.24 \\
0.33 \\
51.70\end{array}$ & $\begin{array}{l}\text { U } \\
\text { B }\end{array}$ & $\begin{array}{r}40100.00 \\
0.22 \\
5.30 \\
130.00 \\
1.70 \\
0.22 \\
39.10 \\
13.20 \\
16.50 \\
20.00 \\
535.00 \\
24.30 \\
0.22 \\
0.33 \\
74.90\end{array}$ & $\begin{array}{l}\mathbf{U} \\
\mathbf{B}\end{array}$ & $\begin{array}{r}42200.00 \\
0.22 \\
5.20 \\
193.00 \\
2.00 \\
0.22 \\
40.30 \\
17.70 \\
18.60 \\
18.20 \\
1830.00 \\
29.60 \\
0.22 \\
0.37 \\
66.70\end{array}$ & $\begin{array}{l}\mathbf{U} \\
\mathbf{B}\end{array}$ & $\begin{array}{r}23200.00 \\
0.24 \\
6.40 \\
93.70 \\
1.20 \\
0.24 \\
36.30 \\
21.40 \\
12.20 \\
39.30 \\
1980.00 \\
23.00 \\
0.24 \\
0.26 \\
50.60\end{array}$ & B \\
\hline
\end{tabular}

Location=ORR; Formation =CHICKAMAUGA K-25; Phase=2; Site=120,126,129; Sample type=Regular; $A=5228 ; B=5234 ; C=5237 ; A$ lab split $=5229$

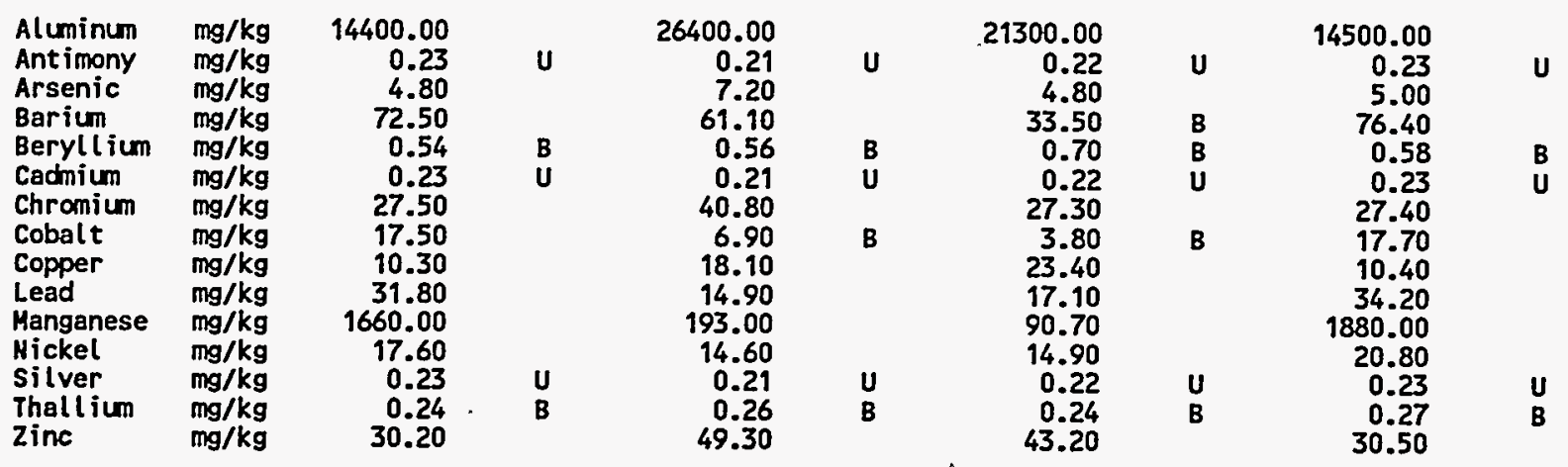

Location=ORR; Formation=CHICKAMAUGA K-25; Phase=2; Site=121,125,128; Sample type=Regular; $A=5263 ; B=5268 ; C=5271 ; A$ lab split $=5264$

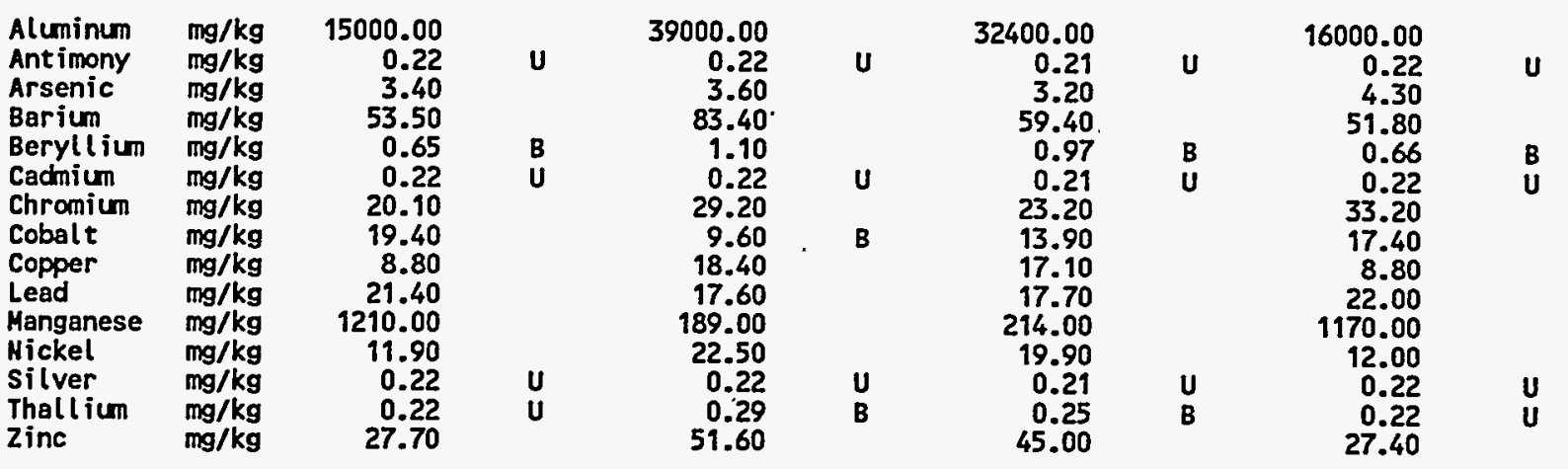

Location=ORR; Formation $=C O P P E R$ RIDGE; Phase $=2 ;$ Site $=45,60,75 ;$ Sample type=Regular; $A=5109 ; B=5112 ; C=5115 ; A$ lab split=" '

$\begin{array}{lrrrrrrr}\text { Aluminum } & \mathrm{mg} / \mathrm{kg} & 10200.00 & & 13900.00 & & 8490.00 & \\ \text { Antimony } & \mathrm{mg} / \mathrm{kg} & 0.21 & \mathrm{U} & 0.21 & \mathrm{U} & 0.21 & \mathrm{U} \\ \text { Arsenic } & \mathrm{mg} / \mathrm{kg} & 13.20 & & 20.20 & & 34.50 & \\ \text { Barium } & \mathrm{mg} / \mathrm{kg} & 89.30 & & 33.30 & \mathrm{~B} & 5.90 & \mathrm{~B} \\ \text { Beryllium } & \mathrm{mg} / \mathrm{kg} & 0.52 & \mathrm{~B} & 0.21 & \mathrm{~B} & 0.26 & \mathrm{~B} \\ \text { Cadmium } & \mathrm{mg} / \mathrm{kg} & 0.21 & \mathrm{U} & 0.21 & \mathrm{U} & 0.21 & \mathrm{U} \\ \text { Chromium } & \mathrm{mg} / \mathrm{kg} & 19.00 & & 35.40 & & 18.70 & \end{array}$


Table I.1 (continued)

\begin{tabular}{|c|c|c|c|c|c|c|c|c|c|}
\hline Analysis & Units & $\begin{array}{l}\text { A horizon } \\
\text { result }\end{array}$ & Qualifier & $\begin{array}{l}\text { B horizon } \\
\text { result }\end{array}$ & Qualifier & $\begin{array}{l}\text { C horizon } \\
\text { result }\end{array}$ & Qualifier & $\begin{array}{l}\text { A lab split } \\
\text { result }\end{array}$ & Qualifier \\
\hline $\begin{array}{l}\text { Cobalt } \\
\text { Copper } \\
\text { Lead } \\
\text { Manganese } \\
\text { Hickel } \\
\text { Silver } \\
\text { Thallium } \\
\text { Zinc }\end{array}$ & $\begin{array}{l}\mathrm{mg} / \mathrm{kg} \\
\mathrm{mg} / \mathrm{kg} \\
\mathrm{mg} / \mathrm{kg} \\
\mathrm{mg} / \mathrm{kg} \\
\mathrm{mg} / \mathrm{kg} \\
\mathrm{mg} / \mathrm{kg} \\
\mathrm{mg} / \mathrm{kg} \\
\mathrm{mg} / \mathrm{kg}\end{array}$ & $\begin{array}{r}7.50 \\
6.20 \\
24.00 \\
1520.00 \\
6.80 \\
0.21 \\
0.21 \\
22.20\end{array}$ & $\begin{array}{l}\mathbf{B} \\
\mathbf{U} \\
\mathbf{U}\end{array}$ & $\begin{array}{r}3.00 \\
10.60 \\
10.90 \\
119.00 \\
5.60 \\
0.21 \\
0.21 \\
32.00\end{array}$ & $\begin{array}{l}\mathbf{B} \\
\mathbf{U} \\
\mathbf{B}\end{array}$ & $\begin{array}{r}1.80 \\
18.90 \\
31.80 \\
86.50 \\
8.30 \\
0.21 \\
0.21 \\
81.20\end{array}$ & $\begin{array}{l}\mathbf{B} \\
\mathbf{U} \\
\mathbf{U}\end{array}$ & & \\
\hline
\end{tabular}

Location=ORR; Formation=COPPER RIDGE; Phase =2; Site=51,55,62; Sample type=Regular; $A=5100 ; B=5104 ; C=5106 ; A$ lab split =' '

$\begin{array}{lrrrrrrr}\text { Aluminum } & \mathrm{mg} / \mathrm{kg} & 8920.00 & & 9260.00 & & 10100.00 & \\ \text { Antimony } & \mathrm{mg} / \mathrm{kg} & 0.21 & \mathrm{U} & 0.20 & \mathrm{U} & 0.23 & \mathrm{U} \\ \text { Arsenic } & \mathrm{mg} / \mathrm{kg} & 12.10 & & 22.40 & & 32.10 & \\ \text { Barium } & \mathrm{mg} / \mathrm{kg} & 57.60 & & 28.70 & \mathrm{~B} & 7.30 & \mathrm{~B} \\ \text { Beryllium } & \mathrm{mg} / \mathrm{kg} & 0.52 & \mathrm{~B} & 0.23 & \mathrm{~B} & 0.32 & \mathrm{~B} \\ \text { Cadmium } & \mathrm{mg} / \mathrm{kg} & 0.21 & \mathrm{U} & 0.20 & \mathrm{U} & 0.23 & \mathrm{U} \\ \text { Chromium } & \mathrm{mg} / \mathrm{kg} & 15.80 & & 22.30 & & 28.50 & \\ \text { Cobalt } & \mathrm{mg} / \mathrm{kg} & 8.80 & \mathrm{~B} & 1.80 & \mathrm{~B} & 2.60 & \mathrm{~B} \\ \text { Copper } & \mathrm{mg} / \mathrm{kg} & 5.00 & \mathrm{~B} & 13.60 & & 24.90 & \\ \text { Lead } & \mathrm{mg} / \mathrm{kg} & 27.00 & & 14.50 & & 22.20 & \\ \text { Manganese } & \mathrm{mg} / \mathrm{kg} & 1530.00 & & 63.50 & & 85.40 & \\ \text { Nickel } & \mathrm{mg} / \mathrm{kg} & 6.90 & \mathrm{~B} & 5.40 & \mathrm{~B} & 9.30 & \\ \text { Silver } & \mathrm{mg} / \mathrm{kg} & 0.21 & \mathrm{U} & 0.20 & \mathrm{U} & 0.23 & \mathrm{U} \\ \text { Thallium } & \mathrm{mg} / \mathrm{kg} & 0.21 & \mathrm{U} & 0.20 & \mathrm{U} & 0.23 & \mathrm{U} \\ \text { Zinc } & \mathrm{mg} / \mathrm{kg} & 20.50 & & 44.40 & & 87.10 & \end{array}$

Location $=O R R ;$ Formation $=C O P P E R$ RIDGE; Phase $=2$; Site=54,64,83; Sample type=Regular; $A=5118 ; B=5121 ; C=5124 ; A$ lab split $=$ '

$\begin{array}{lrrrrrrr}\text { Aluminum } & \mathrm{mg} / \mathrm{kg} & 9660.00 & & 19500.00 & & 17500.00 & \\ \text { Antimony } & \mathrm{mg} / \mathrm{kg} & 0.26 & \mathrm{~B} & 0.22 & \mathrm{U} & 0.22 & \text { U } \\ \text { Arsenic } & \mathrm{mg} / \mathrm{kg} & 24.20 & & 52.40 & & 54.00 & \\ \text { Barium } & \mathrm{mg} / \mathrm{kg} & 68.90 & & 42.20 & \mathrm{~B} & 11.40 & \mathrm{~B} \\ \text { Beryllium } & \mathrm{mg} / \mathrm{kg} & 0.48 & \mathrm{~B} & 0.50 & \mathrm{~B} & 0.49 & \mathrm{~B} \\ \text { Cactiium } & \mathrm{mg} / \mathrm{kg} & 0.22 & \mathrm{U} & 0.22 & \mathrm{U} & 0.22 & \text { U } \\ \text { Chromium } & \mathrm{mg} / \mathrm{kg} & 17.40 & & 25.60 & & 34.00 & \\ \text { Cobalt } & \mathrm{mg} / \mathrm{kg} & 20.60 & & 4.90 & \mathrm{~B} & 3.50 & \mathrm{~B} \\ \text { Copper } & \mathrm{mg} / \mathrm{kg} & 12.00 & & 31.40 & & 29.80 & \\ \text { Lead } & \mathrm{mg} / \mathrm{kg} & 38.80 & & 24.10 & & 33.30 & \\ \text { Manganese } & \mathrm{mg} / \mathrm{kg} & 1570.00 & & 89.30 & & 104.00 & \\ \text { Nickel } & \mathrm{mg} / \mathrm{kg} & 9.90 & & 18.00 & & 20.00 & \\ \text { Selenium } & \mathrm{mg} / \mathrm{kg} & 0.68 & \mathrm{~B} & 0.93 & \mathrm{~B} & 0.50 & \mathrm{~B} \\ \text { Silver } & \mathrm{mg} / \mathrm{kg} & 0.22 & \mathrm{U} & 0.22 & \mathrm{U} & 0.22 & \mathrm{U} \\ \text { Thallium } & \mathrm{mg} / \mathrm{kg} & 0.23 & \mathrm{~B} & 0.34 & \mathrm{~B} & 0.34 & \mathrm{~B} \\ \text { Zinc } & \mathrm{mg} / \mathrm{kg} & 38.10 & & 83.20 & & 97.10 & \end{array}$

Location $=O R R ;$ Formation $=$ COPPER RIDGE; Phase $=2 ;$ Site $=58,59,91 ;$ Sample type $=$ Regular; $A=5091 ; B=5094 ; C=5097 ; A$ lab split="

$\begin{array}{lrrrrrrr}\text { Aluminum } & \mathrm{mg} / \mathrm{kg} & 8630.00 & & 10800.00 & & 13100.00 & \\ \text { Antimony } & \mathrm{mg} / \mathrm{kg} & 0.23 & \mathrm{U} & 0.23 & \mathrm{U} & 0.24 & \mathrm{U} \\ \text { Arsenic } & \mathrm{mg} / \mathrm{kg} & 11.80 & & 37.60 & & 60.50 & \mathrm{~B} \\ \text { Barium } & \mathrm{mg} / \mathrm{kg} & 68.00 & & 23.40 & \mathrm{~B} & 9.40 & \mathrm{~B} \\ \text { Beryllium } & \mathrm{mg} / \mathrm{kg} & 0.39 & \mathrm{~B} & 0.29 & \mathrm{~B} & 0.35 & \mathrm{~B} \\ \text { Cadmium } & \mathrm{mg} / \mathrm{kg} & 0.23 & \mathrm{U} & 0.23 & \mathrm{U} & 0.24 & \mathrm{U} \\ \text { Chromium } & \mathrm{mg} / \mathrm{kg} & 14.00 & & 32.30 & & 30.00 & \\ \text { Cobalt } & \mathrm{mg} / \mathrm{kg} & 4.90 & \mathrm{~B} & 2.60 & \mathrm{~B} & 1.60 & \mathrm{~B} \\ \text { Copper } & \mathrm{mg} / \mathrm{kg} & 6.40 & & 17.10 & & 27.50 & \\ \text { Lead } & \mathrm{mg} / \mathrm{kg} & 20.80 & & 15.80 & & 25.80 & \\ \text { Manganese } & \mathrm{mg} / \mathrm{kg} & 1000.00 & \mathrm{Hg} & 115.00 & & 77.40 & \\ \text { Nickel } & \mathrm{mg} / \mathrm{kg} & 6.20 & \mathrm{~B} & 7.30 & \mathrm{~B} & 11.10 & \\ \text { Silver } & \mathrm{mg} / \mathrm{kg} & 0.23 & \mathrm{U} & 0.23 & U & 0.24 & \mathrm{U} \\ \text { Thallium } & \mathrm{mg} / \mathrm{kg} & 0.23 & \mathrm{U} & 0.23 & \mathrm{U} & 0.24 & \mathrm{~B} \\ \text { Zinc } & \mathrm{mg} / \mathrm{kg} & 20.50 & & 52.40 & & 89.00 & \end{array}$


Table I.1 (continued)

\begin{tabular}{|c|c|c|c|c|c|c|c|c|c|}
\hline Analysis & Units & $\begin{array}{l}\text { A horizon } \\
\text { result }\end{array}$ & Qualifier & $\begin{array}{c}\text { B horizon } \\
\text { result }\end{array}$ & Qualifier & $\begin{array}{l}\mathrm{C} \text { horizon } \\
\text { result }\end{array}$ & Qualifier & $\begin{array}{l}\text { A lab split } \\
\text { result }\end{array}$ & Qualifiet \\
\hline
\end{tabular}

Location=ORR; Formation=DISMAL GAP; Phase =1; Site=10,33,35; Sample type=Regular; $A=5019 ; B=5022 ; C=5025 ; A$ lab split=' '

$\begin{array}{lrrrrrrr}\text { Aluminum } & \mathrm{mg} / \mathrm{kg} & 18800.00 & & 32700.00 & & 36900.00 & \\ \text { Antimony } & \mathrm{mg} / \mathrm{kg} & 0.22 & \mathrm{U} & 0.23 & \mathrm{U} & 0.23 & \mathrm{U} \\ \text { Arsenic } & \mathrm{mg} / \mathrm{kg} & 5.00 & & 3.10 & & 3.20 & \\ \text { Barium } & \mathrm{mg} / \mathrm{kg} & 87.30 & & 94.30 & & 104.00 & \\ \text { Beryllium } & \mathrm{mg} / \mathrm{kg} & 0.73 & \mathrm{~B} & 0.77 & \mathrm{~B} & 1.00 & \mathrm{~B} \\ \text { Cadmium } & \mathrm{mg} / \mathrm{kg} & 0.22 & \mathrm{U} & 0.23 & \mathrm{U} & 0.23 & \mathrm{U} \\ \text { Chromium } & \mathrm{mg} / \mathrm{kg} & 36.40 & & 39.90 & & 45.30 & \\ \text { Cobalt } & \mathrm{mg} / \mathrm{kg} & 14.60 & & 9.30 & \mathrm{~B} & 10.20 & \mathrm{~B} \\ \text { Copper } & \mathrm{mg} / \mathrm{kg} & 20.00 & & 20.70 & & 23.30 & \\ \text { Lead } & \mathrm{mg} / \mathrm{gg} & 21.40 & & 17.80 & & 25.30 & \\ \text { Manganese } & \mathrm{mg} / \mathrm{kg} & 919.00 & & 207.00 & & 213.00 & \\ \text { Nickel } & \mathrm{mg} / \mathrm{kg} & 19.80 & & 20.00 & & 23.20 & \\ \text { Selenium } & \mathrm{mg} / \mathrm{gg} & 0.22 & \mathrm{U} & 0.23 & \mathrm{U} & 0.23 & \mathrm{U} \\ \text { Silver } & \mathrm{mg} / \mathrm{kg} & 0.22 & \mathrm{U} & 0.23 & \mathrm{U} & 0.23 & \mathrm{U} \\ \text { Thallium } & \mathrm{mg} / \mathrm{kg} & 0.22 & \mathrm{U} & 0.23 & \mathrm{~B} & 0.26 & \mathrm{~B} \\ \text { Zinc } & \mathrm{mg} / \mathrm{kg} & 42.30 & & 46.30 & & 50.40 & \end{array}$

Location=ORR; Formation=DISMAL GAP; Phase=1; Site=19,22,32; Sample type=Regular; $A=5010 ; B=5013 ; C=5016 ; A$ lab split $=$ ',

$\begin{array}{lrrrrrrr}\text { Aluminum } & \mathrm{mg} / \mathrm{kg} & 19700.00 & & 36700.00 & & 39600.00 & \\ \text { Antimony } & \mathrm{mg} / \mathrm{kg} & 0.21 & \mathrm{Ug} & 0.22 & \mathrm{U} & 0.22 & \mathrm{U} \\ \text { Arsenic } & \mathrm{mg} / \mathrm{kg} & 2.80 & & 3.40 & & 2.80 & \\ \text { Barium } & \mathrm{mg} / \mathrm{kg} & 92.60 & & 127.00 & & 147.00 & \\ \text { Beryllium } & \mathrm{mg} / \mathrm{kg} & 0.76 & \mathrm{~B} & 0.99 & \mathrm{~B} & 1.10 & \\ \text { Cadmium } & \mathrm{mg} / \mathrm{kg} & 0.21 & \mathrm{U} & 0.22 & \mathrm{U} & 0.22 & \mathrm{U} \\ \text { Chromium } & \mathrm{mg} / \mathrm{kg} & 26.40 & & 39.40 & & 40.80 & \\ \text { Cobalt } & \mathrm{mg} / \mathrm{kg} & 14.10 & & 16.90 & & 12.00 & \\ \text { Copper } & \mathrm{mg} / \mathrm{kg} & 15.10 & & 21.00 & & 22.90 & \\ \text { Lead } & \mathrm{mg} / \mathrm{kg} & 13.20 & & 14.00 & & 13.90 & \\ \text { Manganese } & \mathrm{mg} / \mathrm{kg} & 772.00 & & 477.00 & & 554.00 & \\ \text { Hickel } & \mathrm{mg} / \mathrm{kg} & 19.20 & & 22.20 & & 23.60 & \\ \text { Selenium } & \mathrm{mg} / \mathrm{kg} & 0.60 & \mathrm{~B} & 0.22 & U & 0.22 & U \\ \text { Silver } & \mathrm{mg} / \mathrm{kg} & 0.21 & U & 0.22 & U & 0.22 & U \\ \text { Thallium } & \mathrm{mg} / \mathrm{kg} & 0.21 & U & 0.28 & \mathrm{U} & 0.28 & \mathrm{~B} \\ \text { Zinc } & \mathrm{mg} / \mathrm{kg} & 36.10 & & 58.10 & & 48.50 & \end{array}$

Location=ORR; Formation=DISMAL GAP; Phase=1; Site=2,26,43; Sample type=Field duplicate; $A=5037 ; B=5040 ; C=5043 ; A$ lab split $=$ ' '

\begin{tabular}{|c|c|c|c|c|c|c|}
\hline $\begin{array}{l}\text { Aluminum } \\
\text { Antimony } \\
\text { Arsenic } \\
\text { Barium } \\
\text { Beryllium } \\
\text { Cadmium } \\
\text { chromium } \\
\text { Cobalt } \\
\text { Copper } \\
\text { Lead } \\
\text { Manganese } \\
\text { Nickel } \\
\text { Selenium } \\
\text { silver } \\
\text { Thallium } \\
\text { Zinc }\end{array}$ & $\begin{array}{l}\mathrm{mg} / \mathrm{kg} \\
\mathrm{mg} / \mathrm{kg} \\
\mathrm{mg} / \mathrm{kg} \\
\mathrm{mg} / \mathrm{kg} \\
\mathrm{mg} / \mathrm{kg} \\
\mathrm{mg} / \mathrm{kg} \\
\mathrm{mg} / \mathrm{kg} \\
\mathrm{mg} / \mathrm{kg} \\
\mathrm{mg} / \mathrm{kg} \\
\mathrm{mg} / \mathrm{kg} \\
\mathrm{mg} / \mathrm{kg} \\
\mathrm{mg} / \mathrm{kg} \\
\mathrm{mg} / \mathrm{kg} \\
\mathrm{mg} / \mathrm{kg} \\
\mathrm{mg} / \mathrm{kg} \\
\mathrm{mg} / \mathrm{kg}\end{array}$ & $\begin{array}{r}20400.00 \\
0.23 \\
2.80 \\
95.50 \\
0.77 \\
0.23 \\
29.00 \\
21.50 \\
17.50 \\
16.20 \\
1220.00 \\
22.60 \\
0.23 \\
0.23 \\
0.23 \\
43.40\end{array}$ & $\underset{U}{u}$ & $\begin{array}{r}29000.00 \\
0.23 \\
3.50 \\
102.00 \\
0.91 \\
0.23 \\
38.10 \\
17.90 \\
26.10 \\
15.10 \\
581.00 \\
26.00 \\
0.23 \\
0.23 \\
0.23 \\
49.50\end{array}$ & $\begin{array}{l}u \\
\mathbf{u} \\
\mathbf{u}\end{array}$ & $\begin{array}{r}38300.00 \\
0.24 \\
3.00 \\
136.00 \\
1.20 \\
0.24 \\
46.00 \\
14.20 \\
30.10 \\
15.00 \\
262.00 \\
34.60 \\
0.81 \\
0.24 \\
0.27 \\
59.80\end{array}$ \\
\hline
\end{tabular}

Location=ORR; Formation=DISMAL GAP; Phase $=1 ;$ Site $=2,26,43 ;$ Sample type=Regular; $A=5028 ; B=5031 ; C=5034 ; A$ lab split=' '
Aluminum
$\mathrm{mg} / \mathrm{kg}$
20700.00
0.23
$\mathbf{u}$
34300.00
0.24
u
40000.00
$0.24 \quad U$ 
Table L1 (continued)

\begin{tabular}{|c|c|c|c|c|c|c|c|c|c|}
\hline Analysis & Units & $\begin{array}{l}\text { A horizon } \\
\text { result }\end{array}$ & Qualifier & $\begin{array}{l}\text { B horizon } \\
\text { result }\end{array}$ & Qualifier & $\begin{array}{c}\mathrm{C} \text { horizon } \\
\text { result }\end{array}$ & Qualifier & $\begin{array}{l}\text { A lab split } \\
\text { result }\end{array}$ & Qualifier \\
\hline $\begin{array}{l}\text { Arsenic } \\
\text { Barium } \\
\text { Beryllium } \\
\text { Cadmium } \\
\text { Chromium } \\
\text { Cobalt } \\
\text { Copper } \\
\text { Lead } \\
\text { Manganese } \\
\text { Nickel } \\
\text { Selenium } \\
\text { Silver } \\
\text { Thallium } \\
\text { Zinc }\end{array}$ & $\begin{array}{l}\mathrm{mg} / \mathrm{kg} \\
\mathrm{mg} / \mathrm{kg} \\
\mathrm{mg} / \mathrm{kg} \\
\mathrm{mg} / \mathrm{kg} \\
\mathrm{mg} / \mathrm{kg} \\
\mathrm{mg} / \mathrm{kg} \\
\mathrm{mg} / \mathrm{kg} \\
\mathrm{mg} / \mathrm{kg} \\
\mathrm{mg} / \mathrm{kg} \\
\mathrm{mg} / \mathrm{kg} \\
\mathrm{mg} / \mathrm{kg} \\
\mathrm{mg} / \mathrm{kg} \\
\mathrm{mg} / \mathrm{kg} \\
\mathrm{mg} / \mathrm{kg}\end{array}$ & $\begin{array}{r}3.30 \\
111.00 \\
0.76 \\
0.23 \\
28.20 \\
19.50 \\
19.40 \\
18.80 \\
1230.00 \\
24.90 \\
0.61 \\
0.23 \\
0.23 \\
48.80\end{array}$ & $\begin{array}{l}B \\
U \\
U\end{array}$ & $\begin{array}{r}5.10 \\
86.90 \\
0.82 \\
0.24 \\
40.80 \\
17.00 \\
31.50 \\
18.90 \\
309.00 \\
24.50 \\
0.57 \\
0.24 \\
0.30 \\
46.20\end{array}$ & $\begin{array}{l}B \\
U \\
B\end{array}$ & $\begin{array}{r}3.40 \\
118.00 \\
1.00 \\
0.24 \\
40.10 \\
36.20 \\
39.60 \\
20.50 \\
974.00 \\
33.90 \\
0.35 \\
0.24 \\
0.27 \\
57.90\end{array}$ & $\begin{array}{l}B \\
U \\
B\end{array}$ & & \\
\hline
\end{tabular}

Location=ORR; Formation $=$ NOLICHUCKY; Phase $=1 ;$ Site=15,23,25; Sample type=Regular; $A=5055 ; B=5058 ; C=5061 ; A$ lab split=' '

$\begin{array}{lrrrrrrr}\text { Aluminum } & \mathrm{mg} / \mathrm{kg} & 16500.00 & & 26400.00 & & 23300.00 & \\ \text { Antimony } & \mathrm{mg} / \mathrm{kg} & 0.22 & \mathrm{U} & 0.23 & \mathrm{U} & 0.23 & \mathrm{U} \\ \text { Arsenic } & \mathrm{mg} / \mathrm{kg} & 4.00 & & 4.00 & & 2.80 & \\ \text { Barium } & \mathrm{mg} / \mathrm{kg} & 47.70 & & 59.40 & & 57.50 & \\ \text { Beryllium } & \mathrm{mg} / \mathrm{kg} & 0.53 & \mathrm{~B} & 0.61 & \mathrm{~B} & 0.80 & \mathrm{~B} \\ \text { Cadmium } & \mathrm{mg} / \mathrm{kg} & 0.22 & \mathrm{U} & 0.23 & \mathrm{U} & 0.23 & \mathrm{U} \\ \text { Chromium } & \mathrm{mg} / \mathrm{kg} & 36.10 & & 39.20 & & 39.60 & \\ \text { Cobalt } & \mathrm{mg} / \mathrm{kg} & 11.90 & & 6.80 & \mathrm{~B} & 9.00 & \mathrm{~B} \\ \text { Copper } & \mathrm{mg} / \mathrm{kg} & 13.90 & & 19.80 & & 24.10 & \\ \text { Lead } & \mathrm{mg} / \mathrm{kg} & 18.90 & & 11.80 & & 13.50 & \\ \text { Manganese } & \mathrm{mg} / \mathrm{kg} & 558.00 & & 124.00 & & 208.00 & \\ \text { Mickel } & \mathrm{mg} / \mathrm{kg} & 10.90 & & 13.40 & & 15.20 & \\ \text { Selenium } & \mathrm{mg} / \mathrm{kg} & 0.31 & \mathrm{~B} & 0.50 & \mathrm{~B} & 0.23 & \mathrm{U} \\ \text { Silver } & \mathrm{mg} / \mathrm{kg} & 0.22 & \mathrm{U} & 0.23 & \mathrm{U} & 0.23 & \mathrm{U} \\ \text { Thallium } & \mathrm{mg} / \mathrm{kg} & 0.22 & \mathrm{U} & 0.25 & \mathrm{~B} & 0.24 & \mathrm{~B} \\ \text { Zinc } & \mathrm{mg} / \mathrm{kg} & 24.50 & & 29.20 & & 28.00 & \end{array}$

Location=ORR; Formation=NOLICHUCKY; Phase =1; Site=16,28,42; Sample type=Regular; $A=5064 ; B=5067 ; C=5070 ; A$ lab split=' '

$\begin{array}{lrrrrrrr}\text { Aluminum } & \mathrm{mg} / \mathrm{kg} & 12800.00 & & 21000.00 & & 22600.00 & \\ \text { Antimony } & \mathrm{mg} / \mathrm{kg} & 0.22 & \mathrm{U} & 0.23 & \mathrm{U} & 0.24 & \mathrm{U} \\ \text { Arsenic } & \mathrm{mg} / \mathrm{kg} & 3.40 & & 5.00 & & 2.90 & \\ \text { Barium } & \mathrm{mg} / \mathrm{kg} & 54.30 & & 64.50 & & 62.50 & \\ \text { Beryllium } & \mathrm{mg} / \mathrm{kg} & 0.57 & \mathrm{~B} & 0.64 & \mathrm{~B} & 0.79 & \mathrm{~B} \\ \text { Cadmium } & \mathrm{mg} / \mathrm{kg} & 0.22 & \mathrm{U} & 0.23 & \mathrm{U} & 0.24 & \mathrm{U} \\ \text { Chromium } & \mathrm{mg} / \mathrm{kg} & 28.40 & & 37.50 & & 35.20 & \\ \text { Cobalt } & \mathrm{mg} / \mathrm{kg} & 12.80 & & 10.50 & \mathrm{~B} & 15.40 \\ \text { Copper } & \mathrm{mg} / \mathrm{kg} & 10.70 & & 18.80 & & 19.40 \\ \text { Lead } & \mathrm{mg} / \mathrm{kg} & 13.60 & & 12.20 & & 10.90 \\ \text { Hanganese } & \mathrm{mg} / \mathrm{kg} & 668.00 & & 284.00 & & 328.00 & \\ \text { Nickel } & \mathrm{mg} / \mathrm{kg} & 10.70 & & 13.40 & & 16.40 & \\ \text { Selenium } & \mathrm{mg} / \mathrm{kg} & 0.29 & \mathrm{~B} & 0.45 & \mathrm{~B} & 0.36 & \mathrm{~B} \\ \text { Silver } & \mathrm{mg} / \mathrm{kg} & 0.22 & \mathrm{U} & 0.23 & \mathrm{U} & 0.24 & \mathrm{U} \\ \text { Thallium } & \mathrm{mg} / \mathrm{kg} & 0.22 & \mathrm{U} & 0 . .23 & \mathrm{U} & 0.24 & \mathrm{U} \\ \text { Zinc } & \mathrm{mg} / \mathrm{kg} & 20.00 & & 25.70 & & 26.90 & \end{array}$

Location=ORR; Formation=NOLICHUCKY; Phase=1; Site=3,13,24; Sample type=Regular; $A=5082 ; B=5085 ; C=5088 ; A$ lab split=' '

$\begin{array}{lrrrrrrr}\text { Aluminum } & \mathrm{mg} / \mathrm{kg} & 12300.00 & & 25600.00 & & 27900.00 & \\ \text { Antimony } & \mathrm{mg} / \mathrm{kg} & 0.23 & \mathrm{U} & 0.21 & \mathrm{U} & 0.24 & \mathrm{U} \\ \text { Arsenic } & \mathrm{mg} / \mathrm{kg} & 4.10 & & 3.10 & & 2.90 & \\ \text { Barium } & \mathrm{mg} / \mathrm{kg} & 101.00 & & 91.00 & & 69.00 & \\ \text { Beryllium } & \mathrm{mg} / \mathrm{kg} & 0.51 & \mathrm{~B} & 0.83 & \mathrm{~B} & 0.97 & \mathrm{~B} \\ \text { Cactmium } & \mathrm{mg} / \mathrm{kg} & 0.23 & \mathrm{U} & 0.21 & \mathrm{U} & 0.24 & \mathrm{U} \\ \text { Chromium } & \mathrm{mg} / \mathrm{kg} & 24.30 & & 32.10 & & 31.30 & \\ \text { Cobalt } & \mathrm{mg} / \mathrm{kg} & 18.10 & & 14.40 & & 13.20 & \end{array}$


Table I.1 (continued)

\begin{tabular}{|c|c|c|c|c|c|c|c|c|c|}
\hline Analysis & Units & $\begin{array}{l}\text { A horizon } \\
\text { result }\end{array}$ & Qualifier & $\begin{array}{l}\text { B horizon } \\
\text { result }\end{array}$ & Qualifier & $\begin{array}{l}\mathrm{C} \text { horizon } \\
\text { result }\end{array}$ & Qualifier & $\begin{array}{l}\text { A' lab split } \\
\text { result }\end{array}$ & Qualifier \\
\hline $\begin{array}{l}\text { Copper } \\
\text { Lead } \\
\text { Manganese } \\
\text { Nickel } \\
\text { Selenium } \\
\text { silver } \\
\text { Thall ium } \\
\text { Zinc }\end{array}$ & $\begin{array}{l}\mathrm{mg} / \mathrm{kg} \\
\mathrm{mg} / \mathrm{kg} \\
\mathrm{mg} / \mathrm{kg} \\
\mathrm{mg} / \mathrm{kg} \\
\mathrm{mg} / \mathrm{kg} \\
\mathrm{mg} / \mathrm{kg} \\
\mathrm{mg} / \mathrm{kg} \\
\mathrm{mg} / \mathrm{kg}\end{array}$ & $\begin{array}{r}11.90 \\
21.30 \\
1020.00 \\
13.70 \\
0.39 \\
0.23 \\
0.23 \\
30.00\end{array}$ & $\begin{array}{l}B \\
U \\
U\end{array}$ & $\begin{array}{r}23.20 \\
12.60 \\
307.00 \\
22.00 \\
0.48 \\
0.21 \\
0.22 \\
38.30\end{array}$ & $\begin{array}{l}B \\
\text { U } \\
B\end{array}$ & $\begin{array}{r}28.80 \\
18.00 \\
313.00 \\
21.90 \\
0.33 \\
0.24 \\
0.24 \\
36.80\end{array}$ & $\begin{array}{l}8 \\
U \\
U\end{array}$ & & $\cdots$ \\
\hline
\end{tabular}

Location=ORR; Formation=NOLICHUCKY; Phase=1; Site=5,21,31; Sample type=Regular; $A=5073 ; B=5076 ; C=5079 ; A$ lab split=" '

$\begin{array}{lrrrrrrr}\text { Aluminum } & \mathrm{mg} / \mathrm{kg} & 13700.00 & & 19600.00 & & 23800.00 & \\ \text { Antimony } & \mathrm{mg} / \mathrm{kg} & 0.23 & \mathrm{U} & 0.23 & \mathrm{U} & 0.24 & \mathrm{U} \\ \text { Arsenic } & \mathrm{mg} / \mathrm{kg} & 4.30 & & 3.50 & & 3.60 & \\ \text { Barium } & \mathrm{mg} / \mathrm{kg} & 59.20 & & 64.10 & & 66.30 & \\ \text { Beryllium } & \mathrm{mg} / \mathrm{kg} & 0.58 & \mathrm{~B} & 0.52 & \mathrm{~B} & 0.64 & \mathrm{~B} \\ \text { Cadmium } & \mathrm{mg} / \mathrm{kg} & 0.23 & \mathrm{U} & 0.23 & \mathrm{U} & 0.24 & \mathrm{U} \\ \text { Chromium } & \mathrm{mg} / \mathrm{kg} & 29.60 & & 30.00 & & 37.20 & \\ \text { Cobalt } & \mathrm{mg} / \mathrm{kg} & 16.50 & & 7.80 & \mathrm{~B} & 39.20 \\ \text { Copper } & \mathrm{mg} / \mathrm{kg} & 11.20 & & 13.70 & & 26.60 \\ \text { Lead } & \mathrm{mg} / \mathrm{kg} & 20.00 & & 11.90 & & 23.50 \\ \text { Manganese } & \mathrm{mg} / \mathrm{kg} & 828.00 & & 166.00 & & 919.00 & \\ \text { Nickel } & \mathrm{mg} / \mathrm{kg} & 14.00 & & 17.30 & & 24.80 & \\ \text { Selenium } & \mathrm{mg} / \mathrm{kg} & 0.28 & \mathrm{~B} & 0.28 & \mathrm{~B} & 0.39 & \mathrm{~B} \\ \text { Silver } & \mathrm{mg} / \mathrm{kg} & 0.23 & \mathrm{U} & 0.23 & \mathrm{U} & 0.24 & \mathrm{U} \\ \text { Thallium } & \mathrm{mg} / \mathrm{kg} & \mathbf{0 . 2 3} & \mathrm{U} & 0.23 & \mathrm{U} & 0.25 & \mathrm{~B} \\ \text { Zinc } & \mathrm{mg} / \mathrm{kg} & 28.60 & & 33.90 & & 40.80 & \end{array}$

Location=ROA; Formation=COPPER RIDGE; Phase=2; Site =33,35,44; Sample type=Regular; $A=6046 ; B=6049 ; C=6052 ; A$ lab split=' '

$\begin{array}{lrrrrrrr}\text { Aluminum } & \mathrm{mg} / \mathrm{kg} & 7180.00 & & 12800.00 & & 13900.00 & \\ \text { Antimony } & \mathrm{mg} / \mathrm{kg} & 0.22 & \mathrm{U} & 0.22 & \mathrm{U} & 0.23 & \mathrm{U} \\ \text { Arsenic } & \mathrm{mg} / \mathrm{kg} & 5.60 & & 13.00 & & 20.30 & \\ \text { Barium } & \mathrm{mg} / \mathrm{kg} & 58.10 & & 27.80 & \mathrm{~B} & 11.00 & \mathrm{~B} \\ \text { Beryllium } & \mathrm{mg} / \mathrm{kg} & 0.36 & \mathrm{~B} & 0.29 & \mathrm{~B} & 0.24 & \mathrm{~B} \\ \text { Cachium } & \mathrm{mg} / \mathrm{kg} & 0.22 & \mathrm{U} & 0.22 & \mathrm{U} & 0.23 & \mathrm{U} \\ \text { Chromium } & \mathrm{mg} / \mathrm{kg} & 11.00 & & 25.90 & & 40.20 & \\ \text { Cobalt } & \mathrm{mg} / \mathrm{kg} & 7.50 & \mathrm{~B} & 5.10 & \mathrm{~B} & 3.30 & \mathrm{~B} \\ \text { Copper } & \mathrm{mg} / \mathrm{kg} & 5.80 & & 18.00 & & 19.70 & \\ \text { Lead } & \mathrm{mg} / \mathrm{kg} & 22.70 & & 20.30 & & 30.40 & \\ \text { Manganese } & \mathrm{mg} / \mathrm{kg} & 1100.00 & & 173.00 & & 79.30 & \\ \text { Nickel } & \mathrm{mg} / \mathrm{kg} & 4.50 & \mathrm{~B} & 10.10 & & 10.50 & \\ \text { Silver } & \mathrm{mg} / \mathrm{kg} & 0.22 & \mathrm{U} & 0.22 & \mathrm{U} & 0.23 & \mathrm{U} \\ \text { Thallium } & \mathrm{mg} / \mathrm{kg} & 0.22 & \mathrm{U} & 0.25 & \mathrm{~B} & 0.25 & \mathrm{~B} \\ \text { Zinc } & \mathrm{mg} / \mathrm{kg} & 24.00 & & 42.20 & & 59.90 & \end{array}$

Location=ROA; Formation=COPPER RIDGE; Phase =2; Site=34,39,41; Sample type=Regular; $A=6082 ; B=6087 ; C=6090 ; A$ lab split $=6084$

\begin{tabular}{|c|c|c|c|c|c|c|c|c|}
\hline $\begin{array}{l}\text { Aluminum } \\
\text { Ant imony } \\
\text { Arsenic } \\
\text { Barium } \\
\text { Beryllium } \\
\text { Cacmium } \\
\text { Chromium } \\
\text { Cobalt } \\
\text { Copper } \\
\text { Lead } \\
\text { Manganese } \\
\text { Nickel } \\
\text { Silver } \\
\text { Thallium } \\
\text { Zinc }\end{array}$ & $\begin{array}{l}\mathrm{mg} / \mathrm{kg} \\
\mathrm{mg} / \mathrm{kg} \\
\mathrm{mg} / \mathrm{kg} \\
\mathrm{mg} / \mathrm{kg} \\
\mathrm{mg} / \mathrm{kg} \\
\mathrm{mg} / \mathrm{kg} \\
\mathrm{mg} / \mathrm{kg} \\
\mathrm{mg} / \mathrm{kg} \\
\mathrm{mg} / \mathrm{kg} \\
\mathrm{mg} / \mathrm{kg} \\
\mathrm{mg} / \mathrm{kg} \\
\mathrm{mg} / \mathrm{kg} \\
\mathrm{mg} / \mathrm{kg} \\
\mathrm{mg} / \mathrm{kg} \\
\mathrm{mg} / \mathrm{kg}\end{array}$ & $\begin{array}{r}9820.00 \\
0.23 \\
13.30 \\
56.60 \\
0.41 \\
0.23 \\
12.80 \\
5.50 \\
10.50 \\
28.80 \\
887.00 \\
6.60 \\
0.23 \\
0.23 \\
44.50\end{array}$ & $\begin{array}{l}\mathrm{B} \\
\mathrm{U} \\
\mathrm{B}\end{array}$ & $\begin{array}{r}24300.00 \\
0.20 \\
31.60 \\
31.70 \\
0.44 \\
0.20 \\
28.40 \\
3.80 \\
26.00 \\
17.60 \\
121.00 \\
13.80 \\
0.20 \\
0.33 \\
92.10\end{array}$ & $\begin{array}{l}\mathbf{B} \\
\mathbf{B} \\
\mathbf{U}\end{array}$ & $\begin{array}{r}21200.00 \\
0.21 \\
36.00 \\
12.20 \\
0.45 \\
0.21 \\
30.50 \\
3.40 \\
30.00 \\
23.90 \\
109.00 \\
14.20 \\
0.21 \\
0.24 \\
100.00\end{array}$ & $\begin{array}{l}\mathbf{B} \\
\mathbf{B} \\
\mathbf{U}\end{array}$ & $\begin{array}{r}10600.00 \\
0.23 \\
12.40 \\
56.50 \\
0.42 \\
0.23 \\
13.90 \\
5.40 \\
15.20 \\
28.50 \\
895.00 \\
7.10 \\
0.23 \\
0.23 \\
50.70\end{array}$ \\
\hline
\end{tabular}


Table I.1 (continued)

\begin{tabular}{|c|c|c|c|c|c|c|c|c|c|}
\hline Analysis & Units & $\begin{array}{l}\text { A horizon } \\
\text { result }\end{array}$ & Qualifier & $\begin{array}{c}\text { B horizon } \\
\text { result }\end{array}$ & Qualifier & $\begin{array}{l}\mathrm{C} \text { horizon } \\
\text { result }\end{array}$ & Qualifier & $\begin{array}{l}\text { A lab split } \\
\text { result }\end{array}$ & Qualifier \\
\hline
\end{tabular}

Location=ROA; Formation=COPPER RIDGE; Phase=2; Site=40,42,43; Sample type=Regular; $A=6055 ; B=6058 ; C=6061 ; A$ lab split $=$ ',

$\begin{array}{lrrrrrrr}\text { Aluminum } & \mathrm{mg} / \mathrm{kg} & 8970.00 & & 12300.00 & & 9930.00 & \\ \text { Antimony } & \mathrm{mg} / \mathrm{kg} & 0.21 & \mathrm{U} & 0.22 & \mathrm{U} & 0.22 & \mathrm{U} \\ \text { Arsenic } & \mathrm{mg} / \mathrm{kg} & 8.10 & & 7.70 & & 9.70 & \mathrm{~B} \\ \text { Barium } & \mathrm{mg} / \mathrm{kg} & 27.90 & \mathrm{~B} & 24.50 & \mathrm{~B} & 9.70 & \mathrm{U} \\ \text { Beryllium } & \mathrm{mg} / \mathrm{kg} & 0.51 & \mathrm{~B} & 0.22 & \mathrm{U} & 0.22 & \mathrm{U} \\ \text { Cadmium } & \mathrm{mg} / \mathrm{kg} & 0.21 & \mathrm{U} & 0.22 & \mathrm{U} & 0.22 & \\ \text { Chromium } & \mathrm{mg} / \mathrm{kg} & 20.30 & & 17.40 & & 23.30 & \\ \text { Cobalt } & \mathrm{mg} / \mathrm{kg} & 2.70 & \mathrm{~B} & 1.80 & \mathrm{~B} & 1.10 & \mathrm{~B} \\ \text { Copper } & \mathrm{mg} / \mathrm{kg} & 2.90 & \mathrm{~B} & 5.60 & & 7.70 & \\ \text { Lead } & \mathrm{mg} / \mathrm{kg} & 17.10 & & 6.20 & & 6.90 & \\ \text { Manganese } & \mathrm{mg} / \mathrm{kg} & 379.00 & & 66.80 & & 24.40 & \mathrm{~B} \\ \text { Nickel } & \mathrm{mg} / \mathrm{kg} & 2.80 & \mathrm{~B} & 3.70 & \mathrm{~B} & 3.30 & \mathrm{~B} \\ \text { Silver } & \mathrm{mg} / \mathrm{kg} & 0.21 & \mathrm{U} & 0.22 & \mathrm{U} & 0.22 & \mathrm{U} \\ \text { Thallium } & \mathrm{mg} / \mathrm{kg} & 0.21 & \mathrm{U} & 0.22 & \mathrm{U} & 0.22 & \mathrm{U} \\ \text { Zinc } & \mathrm{mg} / \mathrm{kg} & 13.60 & & 18.00 & & 19.00 & \end{array}$

Location=ROA; Formation=COPPER RIDGE; Phase =2; Site=45,46,47; Sample type =Field duplicate; $A=6076 ; B=6079 ; C=6067 ; A$ lab split=' '

$\begin{array}{lrrrrrrr}\text { Aluminum } & \mathrm{mg} / \mathrm{kg} & 11100.00 & & 14100.00 & & 13300.00 & \\ \text { Antimony } & \mathrm{mg} / \mathrm{kg} & 0.21 & \mathrm{U} & 0.21 & \mathrm{U} & 0.35 & \mathrm{~B} \\ \text { Arsenic } & \mathrm{mg} / \mathrm{kg} & 6.10 & & 11.60 & & 19.20 & \\ \text { Barium } & \mathrm{mg} / \mathrm{kg} & 88.30 & & 46.60 & & 20.20 & \mathrm{~B} \\ \text { Beryllium } & \mathrm{mg} / \mathrm{kg} & 0.51 & \mathrm{~B} & 0.21 & \mathrm{U} & 0.22 & \mathrm{U} \\ \text { Cadmium } & \mathrm{mg} / \mathrm{kg} & 0.21 & \mathrm{U} & 0.21 & \mathrm{U} & 0.22 & \mathrm{U} \\ \text { Chromium } & \mathrm{mg} / \mathrm{kg} & 15.00 & & 25.40 & & 35.90 & \\ \text { Cobalt } & \mathrm{mg} / \mathrm{kg} & 7.60 & \mathrm{~B} & 2.60 & \mathrm{~B} & 1.50 & \mathrm{~B} \\ \text { Copper } & \mathrm{mg} / \mathrm{kg} & 5.00 & \mathrm{~B} & 10.60 & & 13.70 & \\ \text { Lead } & \mathrm{mg} / \mathrm{kg} & 16.30 & & 8.10 & & 10.60 & \\ \text { Manganese } & \mathrm{mg} / \mathrm{kg} & 1310.00 & & 74.80 & & 51.50 & \\ \text { Nickel } & \mathrm{mg} / \mathrm{kg} & 6.20 & \mathrm{~B} & 7.00 & \mathrm{~B} & 6.80 & \mathrm{~B} \\ \text { Selenium } & \mathrm{mg} / \mathrm{kg} & 0.47 & \mathrm{~B} & 0.56 & \mathrm{~B} & 0.71 & \mathrm{~B} \\ \text { Silver } & \mathrm{mg} / \mathrm{kg} & 0.21 & \mathrm{U} & 0.21 & \mathrm{U} & 0.22 & \mathrm{U} \\ \text { Thallium } & \mathrm{mg} / \mathrm{kg} & 0.21 & \mathrm{U} & 0.21 & \mathrm{U} & 0.22 & \mathrm{U} \\ \text { Zinc } & \mathrm{mg} / \mathrm{kg} & 23.00 & & 21.20 & & 20.20 & \end{array}$

Location=ROA; Formation=COPPER RIDGE; Phase =2; Site=45,46,47; Sample type=Regular; $A=6073 ; B=6070 ; C=6064 ; A$ lab split="'

$\begin{array}{lrrrrrrr}\text { Aluminum } & \mathrm{mg} / \mathrm{kg} & 10600.00 & & 15200.00 & & 12600.00 & \\ \text { Antimony } & \mathrm{mg} / \mathrm{kg} & 0.21 & \mathrm{U} & 0.22 & \mathrm{U} & 0.22 & \mathrm{U} \\ \text { Arsenic } & \mathrm{mg} / \mathrm{kg} & 5.50 & & 12.20 & & 17.10 & \\ \text { Barium } & \mathrm{mg} / \mathrm{kg} & 80.20 & & 60.30 & & 18.90 & \mathrm{~B} \\ \text { Beryllium } & \mathrm{mg} / \mathrm{kg} & 0.47 & \mathrm{~B} & 0.27 & \mathrm{~B} & 0.22 & \mathrm{U} \\ \text { Cactmium } & \mathrm{mg} / \mathrm{kg} & 0.21 & \mathrm{U} & 0.22 & \mathrm{U} & 0.22 & \mathrm{U} \\ \text { Chromium } & \mathrm{mg} / \mathrm{kg} & 14.50 & & 40.20 & & 30.20 & \\ \text { Cobalt } & \mathrm{mg} / \mathrm{kg} & 6.40 & \mathrm{~B} & 2.90 & \mathrm{~B} & 1.30 & \mathrm{~B} \\ \text { Copper } & \mathrm{mg} / \mathrm{kg} & 5.20 & & 10.50 & & 13.60 & \\ \text { Lead } & \mathrm{mg} / \mathrm{kg} & 16.10 & & 9.30 & & 10.10 & \\ \text { Manganese } & \mathrm{mg} / \mathrm{kg} & 1180.00 & & 120.00 & & 41.50 & \\ \text { Hickel } & \mathrm{mg} / \mathrm{kg} & 6.10 & \mathrm{~B} & 7.90 & \mathrm{~B} & 6.80 & \mathrm{~B} \\ \text { Selenium } & \mathrm{mg} / \mathrm{kg} & 0.48 & \mathrm{~B} & 0.41 & \mathrm{~B} & 0.44 & \mathrm{~B} \\ \text { Silver } & \mathrm{mg} / \mathrm{kg} & 0.21 & \mathrm{U} & 0.22 & \mathrm{U} & 0.22 & \mathrm{U} \\ \text { Thallium } & \mathrm{mg} / \mathrm{kg} & 0.21 & \mathrm{U} & 0.23 & \mathrm{~B} & 0.22 & \mathrm{U} \\ \text { Zinc } & \mathrm{mg} / \mathrm{kg} & 24.10 & & 22.80 & & 20.40 & \end{array}$

Location $=R O A ;$ Formation $=D I S M A L$ GAP; Phase $=1 ;$ Site $=10,13,14 ;$ Sample type $=$ Field duplicate; $A=6037 ; B=6040 ; C=6043 ; A$ lab split=' '

$\begin{array}{lrrrrrrr}\text { Aluminum } & \mathrm{mg} / \mathrm{kg} & 16300.00 & & 37500.00 & & 31000.00 & \\ \text { Antimony } & \mathrm{mg} / \mathrm{kg} & 0.25 & \mathrm{U} & 0.24 & \mathrm{U} & 0.23 & \mathrm{U} \\ \text { Arsenic } & \mathrm{mg} / \mathrm{kg} & 4.40 & & 6.20 & & 3.80 & \end{array}$


I-15

Table I.1 (continued)

\begin{tabular}{|c|c|c|c|c|c|c|c|c|c|}
\hline Analysis & Units & $\begin{array}{l}\text { A horizon } \\
\text { result }\end{array}$ & Qualifier & $\begin{array}{l}\text { B horizon } \\
\text { result }\end{array}$ & Qualifier & $\begin{array}{l}C \text { horizon } \\
\text { result }\end{array}$ & Qualifier & $\begin{array}{l}\text { A lab split } \\
\text { result }\end{array}$ & Qualifier \\
\hline $\begin{array}{l}\text { Barium } \\
\text { Beryllium } \\
\text { Cacmium } \\
\text { Chromium } \\
\text { Cobalt } \\
\text { Copper } \\
\text { Lead } \\
\text { Manganese } \\
\text { Nickel } \\
\text { Selenium } \\
\text { Silver } \\
\text { Thallium } \\
\text { Zinc }\end{array}$ & $\begin{array}{l}\mathrm{mg} / \mathbf{k g} \\
\mathrm{mg} / \mathrm{kg} \\
\mathrm{mg} / \mathbf{k g} \\
\mathrm{mg} / \mathbf{k g} \\
\mathrm{mg} / \mathbf{k g} \\
\mathrm{mg} / \mathbf{k g} \\
\mathrm{mg} / \mathbf{k g} \\
\mathrm{mg} / \mathbf{k g} \\
\mathrm{mg} / \mathbf{k g} \\
\mathrm{mg} / \mathbf{k g} \\
\mathrm{mg} / \mathbf{k g} \\
\mathrm{mg} / \mathbf{k g} \\
\mathrm{mg} / \mathrm{kg}\end{array}$ & $\begin{array}{r}115.00 \\
0.84 \\
0.25 \\
33.70 \\
26.40 \\
14.30 \\
25.70 \\
1730.00 \\
19.40 \\
0.25 \\
0.25 \\
0.25 \\
43.90\end{array}$ & $\begin{array}{l}U \\
U \\
U\end{array}$ & $\begin{array}{r}222.00 \\
1.10 \\
0.24 \\
71.90 \\
16.90 \\
25.30 \\
32.40 \\
869.00 \\
26.20 \\
0.81 \\
0.24 \\
0.33 \\
49.80\end{array}$ & $\begin{array}{l}\text { B } \\
\mathrm{B}\end{array}$ & $\begin{array}{r}111.00 \\
1.20 \\
0.23 \\
43.40 \\
26.60 \\
23.00 \\
17.50 \\
823.00 \\
35.90 \\
0.23 \\
0.23 \\
0.25 \\
57.00\end{array}$ & $\begin{array}{l}\text { U } \\
U \\
B\end{array}$ & + & $*$ \\
\hline
\end{tabular}

Location=ROA; Formation=DISMAL GAP; Phase=1; Site=10,13,14; Sample type=Regular; $A=6028 ; B=6031 ; C=6034 ; A$ lab split=' '

\begin{tabular}{|c|c|c|c|c|c|c|}
\hline $\begin{array}{l}\text { Aluminum } \\
\text { Antimony } \\
\text { Arsenic } \\
\text { Barium } \\
\text { Beryl l ium } \\
\text { Cadmium } \\
\text { Chromium } \\
\text { Cobalt } \\
\text { Copper } \\
\text { Lead } \\
\text { Hanganese } \\
\text { Hickel } \\
\text { Selenium } \\
\text { Silver } \\
\text { Thallium } \\
\text { Zinc }\end{array}$ & $\begin{array}{l}\mathrm{mg} / \mathbf{k g} \\
\mathrm{mg} / \mathbf{k g} \\
\mathrm{mg} / \mathbf{k g} \\
\mathrm{mg} / \mathbf{k g} \\
\mathrm{mg} / \mathbf{k g} \\
\mathrm{mg} / \mathbf{k g} \\
\mathrm{mg} / \mathbf{k g} \\
\mathrm{mg} / \mathbf{k g} \\
\mathrm{mg} / \mathbf{k g} \\
\mathrm{mg} / \mathbf{k g} \\
\mathrm{mg} / \mathbf{k g} \\
\mathrm{mg} / \mathbf{k g} \\
\mathrm{mg} / \mathbf{k g} \\
\mathrm{mg} / \mathbf{k g} \\
\mathrm{mg} / \mathbf{k g} \\
\mathrm{mg} / \mathbf{k g}\end{array}$ & $\begin{array}{r}17300.00 \\
0.25 \\
4.50 \\
117.00 \\
0.80 \\
0.25 \\
30.20 \\
26.90 \\
15.40 \\
26.70 \\
1860.00 \\
20.00 \\
0.36 \\
0.25 \\
0.25 \\
46.70\end{array}$ & $\begin{array}{l}\mathbf{B} \\
\mathbf{U} \\
\mathbf{U}\end{array}$ & $\begin{array}{r}31900.00 \\
0.23 \\
4.20 \\
94.20 \\
0.93 \\
0.23 \\
44.40 \\
13.50 \\
21.50 \\
16.50 \\
225.00 \\
25.30 \\
0.58 \\
0.23 \\
0.25 \\
47.40\end{array}$ & $\begin{array}{l}\mathbf{B} \\
\mathbf{U} \\
\mathbf{B}\end{array}$ & $\begin{array}{r}30100.00 \\
0.22 \\
3.30 \\
103.00 \\
1.10 \\
0.22 \\
45.70 \\
14.60 \\
24.90 \\
14.50 \\
300.00 \\
36.50 \\
0.22 \\
0.22 \\
0.24 \\
58.60\end{array}$ \\
\hline
\end{tabular}

Location=ROA; Formation=DISMAL GAP; Phase=1; Site=3,7,21; Sample type=Regular; $A=6019 ; B=6022 ; C=6025 ; A$ lab split="'

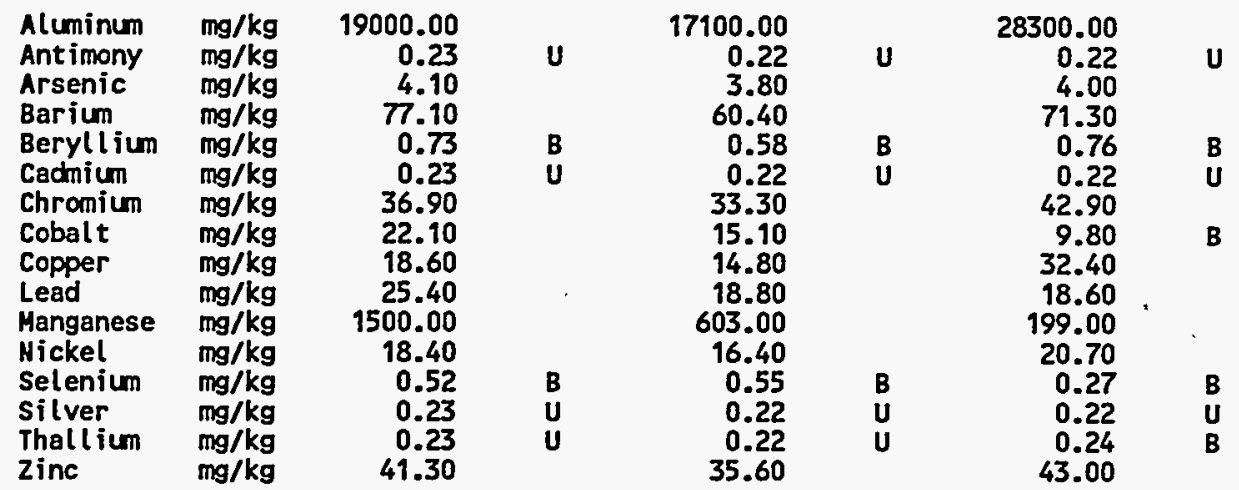

Location=ROA; Formation=DISMAL GAP; Phase =1; Site=8,20,22; Sample type=Regular; $A=6010 ; B=6013 ; C=6016 ; A$ lab split='

\begin{tabular}{|c|c|c|c|c|c|c|}
\hline $\begin{array}{l}\text { Aluminum } \\
\text { Antimony } \\
\text { Arsenic } \\
\text { Barium } \\
\text { Beryllium } \\
\text { Cadmium } \\
\text { Chromium } \\
\text { Cobalt } \\
\text { Copper }\end{array}$ & $\begin{array}{l}\mathrm{mg} / \mathrm{kg} \\
\mathrm{mg} / \mathrm{kg} \\
\mathrm{mg} / \mathrm{kg} \\
\mathrm{mg} / \mathrm{kg} \\
\mathrm{mg} / \mathrm{kg} \\
\mathrm{mg} / \mathrm{kg} \\
\mathrm{mg} / \mathrm{kg} \\
\mathrm{mg} / \mathrm{kg} \\
\mathrm{mg} / \mathrm{kg}\end{array}$ & $\begin{array}{r}15700.00 \\
0.22 \\
3.80 \\
60.90 \\
0.50 \\
0.22 \\
30.40 \\
11.30 \\
13.30\end{array}$ & $\begin{array}{l}\mathbf{B} \\
\mathbf{U}\end{array}$ & $\begin{array}{r}30000.00 \\
0.22 \\
4.60 \\
71.60 \\
0.69 \\
0.22 \\
54.00 \\
7.20 \\
19.50\end{array}$ & $\begin{array}{l}B \\
U\end{array}$ & $\begin{array}{r}31500.00 \\
0.22 \\
2.20 \\
72.50 \\
0.86 \\
0.22 \\
48.50 \\
8.20 \\
24.40\end{array}$ \\
\hline
\end{tabular}




\section{I-16}

Table I.1 (continued)

\begin{tabular}{|c|c|c|c|c|c|c|c|c|c|}
\hline Analysis & Units & $\begin{array}{l}\text { A horizon } \\
\text { result }\end{array}$ & Qualifier & $\begin{array}{l}\text { B horizon } \\
\text { result }\end{array}$ & Qualifier & $\begin{array}{l}C \text { horizon } \\
\text { result }\end{array}$ & Qualifier & $\begin{array}{l}\text { A lab split } \\
\text { result }\end{array}$ & Qualifier \\
\hline $\begin{array}{l}\text { Lead } \\
\text { Manganese } \\
\text { Nickel } \\
\text { Selenium } \\
\text { Silver } \\
\text { Thallium } \\
\text { Zinc }\end{array}$ & $\begin{array}{l}\mathrm{mg} / \mathrm{kg} \\
\mathrm{mg} / \mathrm{kg} \\
\mathrm{mg} / \mathrm{kg} \\
\mathrm{mg} / \mathbf{k g} \\
\mathrm{mg} / \mathrm{kg} \\
\mathrm{mg} / \mathbf{k g} \\
\mathrm{mg} / \mathrm{kg}\end{array}$ & $\begin{array}{r}21.00 \\
949.00 \\
11.50 \\
0.44 \\
0.22 \\
0.22 \\
30.60\end{array}$ & $\begin{array}{l}\mathbf{B} \\
\mathbf{U} \\
\mathbf{U}\end{array}$ & $\begin{array}{r}12.30 \\
120.00 \\
18.00 \\
0.52 \\
0.22 \\
0.24 \\
41.60\end{array}$ & $\begin{array}{l}\text { B } \\
\text { U } \\
B\end{array}$ & $\begin{array}{r}11.40 \\
99.40 \\
29.20 \\
0.29 \\
0.22 \\
0.25 \\
48.70\end{array}$ & $\begin{array}{l}\text { B } \\
\text { U } \\
\text { B }\end{array}$ & & \\
\hline
\end{tabular}


Appendix J

OCCURRENCES OF REJECTED DATA 
Table J.1. Occurrences of rejected data

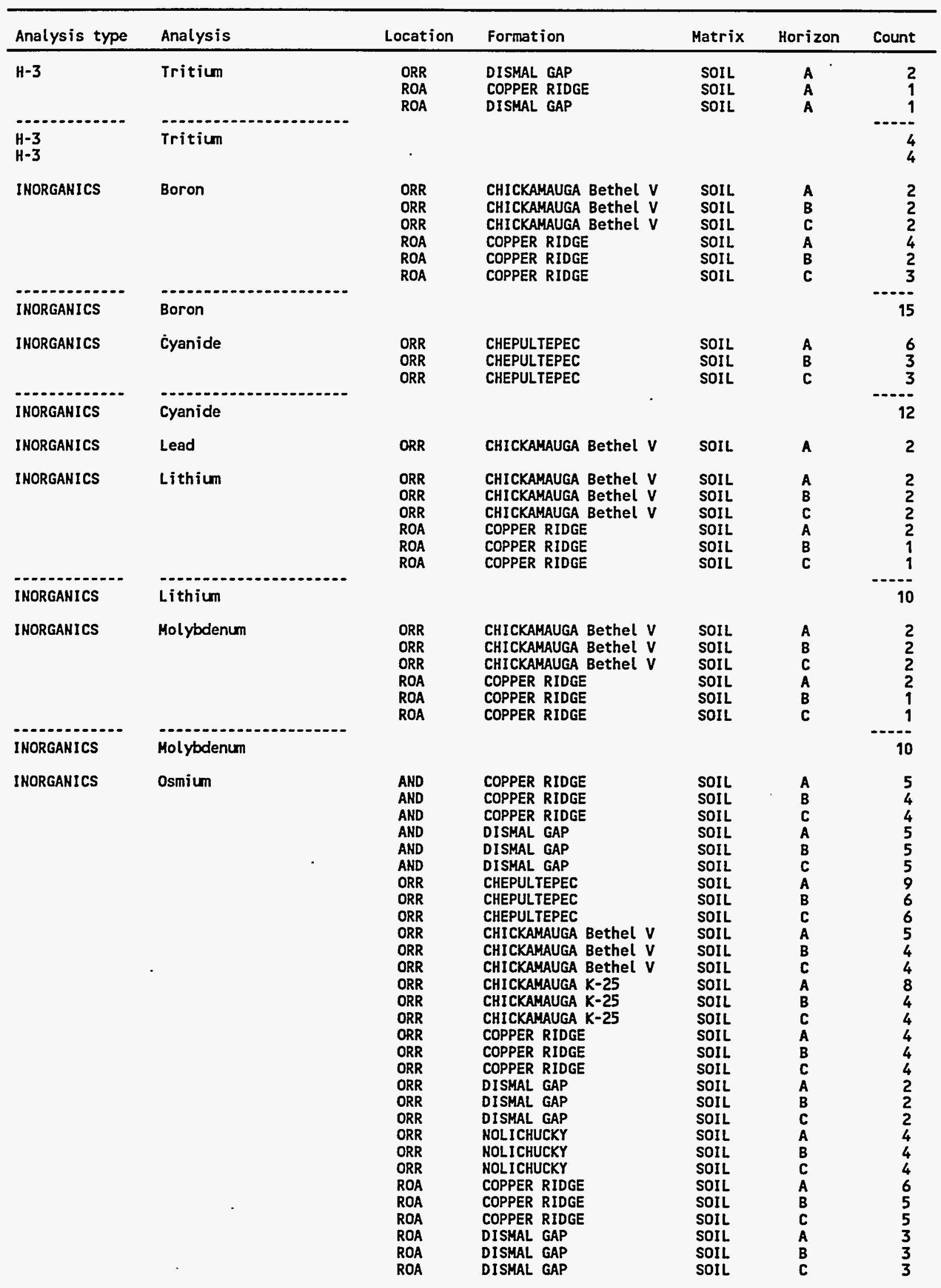


Table J.1 (continued)

\begin{tabular}{|c|c|c|c|c|c|c|}
\hline Analys is type & Analysis & Location & Formation & Matrix & Horizon & Count \\
\hline INORGANICS & Osmium & & & & & 133 \\
\hline IHORGANICS & Potassium & $\begin{array}{l}\text { ORR } \\
\text { ROA } \\
\text { ROA } \\
\text { ROA }\end{array}$ & $\begin{array}{l}\text { CHEPULTEPEC } \\
\text { COPPER RIDGE } \\
\text { COPPER RIDGE } \\
\text { COPPER RIDGE }\end{array}$ & $\begin{array}{l}\text { SOIL } \\
\text { SOIL } \\
\text { SOIL } \\
\text { SOIL }\end{array}$ & $\begin{array}{l}B \\
A \\
B \\
C\end{array}$ & $\begin{array}{l}1 \\
2 \\
1 \\
1\end{array}$ \\
\hline IHORGANICS & Potassium & & & & & $\cdots$ \\
\hline ב. & - & $\begin{array}{l}\text { ORR } \\
\text { ORR } \\
\text { ORR }\end{array}$ & $\begin{array}{l}\text { CHEPULTEPEC } \\
\text { CHEPULTEPEC } \\
\text { CHEPULTEPEC }\end{array}$ & $\begin{array}{l}\text { SOIL } \\
\text { SOIL } \\
\text { SOIL }\end{array}$ & $\begin{array}{l}\text { A } \\
\mathbf{B} \\
\mathbf{C}\end{array}$ & $\begin{array}{r}6 \\
2 \\
2 \\
--\end{array}$ \\
\hline IHORGANICS & Selenium & & & & & 10 \\
\hline IMORGANICS & silicon & $\begin{array}{l}\text { ORR } \\
\text { ORR } \\
\text { ORR } \\
\text { ORR } \\
\text { ORR } \\
\text { ORR } \\
\text { ROA } \\
\text { ROA } \\
\text { ROA }\end{array}$ & $\begin{array}{l}\text { CHICKAMAUGA Bethel } v \\
\text { CHICKAMAUGA Bethel } v \\
\text { CHICKAMAUGA Bethel } v \\
\text { COPPER RIDGE } \\
\text { COPPER RIDGE } \\
\text { COPPER RIDGE } \\
\text { COPPER RIDGE } \\
\text { COPPER RIDGE } \\
\text { COPPER RIDGE }\end{array}$ & $\begin{array}{l}\text { SOIL } \\
\text { SOIL } \\
\text { SOIL } \\
\text { SOIL } \\
\text { SOIL } \\
\text { SOIL } \\
\text { SOIL } \\
\text { SOIL } \\
\text { SOIL }\end{array}$ & $\begin{array}{l}\text { A } \\
B \\
C \\
A \\
B \\
C \\
A \\
B \\
C\end{array}$ & $\begin{array}{l}2 \\
2 \\
2 \\
3 \\
3 \\
3 \\
4 \\
3 \\
3 \\
-.\end{array}$ \\
\hline INORGANICS & Silicon & & & & & 25 \\
\hline INORGANICS & Strontium & $\begin{array}{l}\text { ORR } \\
\text { ORR } \\
\text { ORR } \\
\text { ROA } \\
\text { ROA } \\
\text { ROA }\end{array}$ & $\begin{array}{l}\text { CHICKAMAUGA Bethel } v \\
\text { CHICKAMAUGA Bethel } v \\
\text { CHICKAMAUGA Bethel } v \\
\text { COPPER RIDGE } \\
\text { COPPER RIDGE } \\
\text { COPPER RIDGE }\end{array}$ & $\begin{array}{l}\text { SOIL } \\
\text { SOIL } \\
\text { SOIL } \\
\text { SOIL } \\
\text { SOIL } \\
\text { SOIL }\end{array}$ & $\begin{array}{l}\text { A } \\
\text { B } \\
\text { C } \\
\text { A } \\
\text { B } \\
\text { C }\end{array}$ & $\begin{array}{l}2 \\
2 \\
2 \\
2 \\
1 \\
1\end{array}$ \\
\hline INORGANICS & Strontium & & & & & 10 \\
\hline - & n.mon & $\begin{array}{l}\text { ORR } \\
\text { ORR } \\
\text { ORR }\end{array}$ & $\begin{array}{l}\text { CHEPULTEPEC } \\
\text { CHEPULTEPEC } \\
\text { CHEPULTEPEC }\end{array}$ & $\begin{array}{l}\text { SOIL } \\
\text { SOIL } \\
\text { SOIL }\end{array}$ & $\begin{array}{l}A \\
B \\
C\end{array}$ & $\begin{array}{r}6 \\
3 \\
3 \\
\end{array}$ \\
\hline $\begin{array}{l}\text { INORGANICS } \\
\text { INORGANICS }\end{array}$ & Thallium & & & & & $\begin{array}{r}12 \\
244\end{array}$ \\
\hline ORGANICS & alpha-BHC & ORR & CHICKAMAUGA K-25 & SOIL & A & 1 \\
\hline ORGANICS & alpha-Chlordane & ORR & CHICKAMAUGA K-25 & SOIL & A & 1 \\
\hline ORGAHICS & beta-BHC & ORR & CHICKAMAUGA K-25 & SOIL & A & 1 \\
\hline ORGANICS & del ta-BHC & ORR & CHICKAMAUGA K-25 & SOIL & A & 1 \\
\hline ORGANICS & gamma-Chlordane & ORR & CHICKAMAUGA $\mathrm{K}-25$ & SOIL & A & 1 \\
\hline ORGANICS & Acenaph thene & $\begin{array}{l}\text { AND } \\
\text { AND } \\
\text { ORR } \\
\text { ORR } \\
\text { ORR } \\
\text { ORR } \\
\text { ORR } \\
\text { ROA } \\
\text { ROA }\end{array}$ & $\begin{array}{l}\text { COPPER RIDGE } \\
\text { DISMAL GAP } \\
\text { CHEPULTEPEC } \\
\text { CHICKAMAUGA Bethel } v \\
\text { CHICKAMAUGA } \mathrm{V}-25 \\
\text { COPPER RIDGE } \\
\text { DISHAL GAP } \\
\text { COPPER RIDGE } \\
\text { DISMAL GAP }\end{array}$ & $\begin{array}{l}\text { SOIL } \\
\text { SOIL } \\
\text { SOIL } \\
\text { SOIL } \\
\text { SOIL } \\
\text { SOIL } \\
\text { SOIL } \\
\text { SOIL } \\
\text { SOIL }\end{array}$ & $\begin{array}{l}A \\
A \\
A \\
A \\
A \\
A \\
A \\
A \\
A\end{array}$ & $\begin{array}{r}10 \\
3 \\
3 \\
11 \\
9 \\
6 \\
1 \\
4 \\
1\end{array}$ \\
\hline ORGANICS & Acenaphthene & & & & & 48 \\
\hline ORGANICS & Acenaphthylene & $\begin{array}{l}\text { AND } \\
\text { ORR } \\
\text { ORR } \\
\text { ROA }\end{array}$ & $\begin{array}{l}\text { DISMAL GAP } \\
\text { COPPER RIDGE } \\
\text { DISMAL GAP } \\
\text { COPPER RIDGE }\end{array}$ & $\begin{array}{l}\text { SOIL } \\
\text { SOIL } \\
\text { SOIL } \\
\text { SOIL }\end{array}$ & $\begin{array}{l}A \\
A \\
A \\
A\end{array}$ & $\begin{array}{r}13 \\
2 \\
1 \\
4\end{array}$ \\
\hline
\end{tabular}


Table J.1 (continued)

\begin{tabular}{|c|c|c|c|c|c|c|}
\hline Analysis type & Analysis & Location & Formation & Matrix & Horizon & Count \\
\hline ORGAHICS & Acenaphthylene & ROA & DISHAL GAP & SOIL & A & $\begin{array}{r}1 \\
21\end{array}$ \\
\hline ORGAHICS & Aldrin & ORR & CHICKAMAUGA K-25 & SOIL & A & 1 \\
\hline ORGANICS & Anthracene & $\begin{array}{l}\text { AND } \\
\text { AND } \\
\text { ORR } \\
\text { ORR } \\
\text { ORR } \\
\text { ORR } \\
\text { ORR } \\
\text { ROA } \\
\text { ROA }\end{array}$ & $\begin{array}{l}\text { COPPER RIDGE } \\
\text { DISMAL GAP } \\
\text { CHEPULTEPEC } \\
\text { CHICKAMAUGA Bethel } v \\
\text { CHICKAMAUGA K-25 } \\
\text { COPPER RIDGE } \\
\text { DISMAL GAP } \\
\text { COPPER RIDGE } \\
\text { DISHAL GAP }\end{array}$ & $\begin{array}{l}\text { SOIL } \\
\text { SOIL } \\
\text { SOIL } \\
\text { SOIL } \\
\text { SOIL } \\
\text { SOIL } \\
\text { SOIL } \\
\text { SOIL } \\
\text { SOIL }\end{array}$ & $\begin{array}{l}\mathbf{A} \\
\mathbf{A} \\
\mathbf{A} \\
\mathbf{A} \\
\mathbf{A} \\
\mathbf{A} \\
\mathbf{A} \\
\mathbf{A} \\
\mathbf{A}\end{array}$ & $\begin{array}{r}5 \\
11 \\
3 \\
7 \\
2 \\
4 \\
1 \\
2 \\
1\end{array}$ \\
\hline ORGANICS & Anthracene & & & & & 36 \\
\hline$\ldots \ldots$ & (1) & $\begin{array}{l}\text { AND } \\
\text { AND } \\
\text { ORR } \\
\text { ORR } \\
\text { ORR } \\
\text { ROA }\end{array}$ & $\begin{array}{l}\text { COPPER RIDGE } \\
\text { DISMAL GAP } \\
\text { CHICKAMAUGA Bethel } v \\
\text { DISMAL GAP } \\
\text { HOLICHUCKY } \\
\text { DISMAL GAP }\end{array}$ & $\begin{array}{l}\text { SOIL } \\
\text { SOIL } \\
\text { SOIL } \\
\text { SOIL } \\
\text { SOIL } \\
\text { SOIL }\end{array}$ & $\begin{array}{l}A \\
A \\
A \\
A \\
A \\
A\end{array}$ & $\begin{array}{r}1 \\
13 \\
6 \\
1 \\
10 \\
3\end{array}$ \\
\hline ORGANICS & Benzo(a)anthracene & & & & & 34 \\
\hline ORGAHICS & Benzo(a)pyrene & $\begin{array}{l}\text { AND } \\
\text { AND } \\
\text { ORR } \\
\text { ORR } \\
\text { ORR } \\
\text { ROA }\end{array}$ & $\begin{array}{l}\text { COPPER RIDGE } \\
\text { DISMAL GAP } \\
\text { CHEPULTEPEC } \\
\text { COPPER RIDGE } \\
\text { DISMAL GAP } \\
\text { DISMAL GAP }\end{array}$ & $\begin{array}{l}\text { SOIL } \\
\text { SOIL } \\
\text { SOIL } \\
\text { SOIL } \\
\text { SOIL } \\
\text { SOIL }\end{array}$ & $\begin{array}{l}\mathbf{A} \\
\mathbf{A} \\
\mathbf{A} \\
\mathbf{A} \\
\mathbf{A} \\
\mathbf{A}\end{array}$ & $\begin{array}{l}2 \\
2 \\
2 \\
2 \\
1 \\
1\end{array}$ \\
\hline ORGANICS & Benzo(a)pyrene & & & & $\cdot$ & 10 \\
\hline ORGANICS & Benzo(b)fluoranthene & $\begin{array}{l}\text { AND } \\
\text { AND } \\
\text { ORR } \\
\text { ORR } \\
\text { ORR } \\
\text { ORR } \\
\text { ROA }\end{array}$ & $\begin{array}{l}\text { COPPER RIDGE } \\
\text { DISHAL GAP } \\
\text { CHEPULTEPEC } \\
\text { CHICKAMAUGA Bethel } v \\
\text { COPPER RIDGE } \\
\text { DISMAL GAP } \\
\text { DISHAL GAP }\end{array}$ & $\begin{array}{l}\text { SOIL } \\
\text { SOIL } \\
\text { SOIL } \\
\text { SOIL } \\
\text { SOIL } \\
\text { SOIL } \\
\text { SOIL }\end{array}$ & $\begin{array}{l}A \\
A \\
A \\
A \\
A \\
A \\
A\end{array}$ & $\begin{array}{r}4 \\
4 \\
3 \\
4 \\
4 \\
1 \\
1\end{array}$ \\
\hline ORGANICS & Benzo(b)fluoranthene & & & & & 21 \\
\hline ORGANICS & Benzo(ghi)perylene & $\begin{array}{l}\text { AND } \\
\text { AND } \\
\text { ORR } \\
\text { ORR } \\
\text { ORR } \\
\text { ORR } \\
\text { ROA } \\
\text { ROA }\end{array}$ & $\begin{array}{l}\text { COPPER RIDGE } \\
\text { DISMAL GAP } \\
\text { CHEPULTEPEC } \\
\text { CHICKAMAUGA Bethel } v \\
\text { COPPER RIDGE } \\
\text { DISHAL GAP } \\
\text { COPPER RIDGE } \\
\text { DISHAL GAP }\end{array}$ & $\begin{array}{l}\text { SOIL } \\
\text { SOIL } \\
\text { SOIL } \\
\text { SOIL } \\
\text { SOIL } \\
\text { SOIL } \\
\text { SOIL } \\
\text { SOIL }\end{array}$ & $\begin{array}{l}A \\
A \\
A \\
A \\
A \\
A \\
A \\
A\end{array}$ & $\begin{array}{r}2 \\
23 \\
1 \\
7 \\
3 \\
1 \\
1 \\
1\end{array}$ \\
\hline ORGANICS & Benzo(ghi)perylene & & & & & 39 \\
\hline ORGAHICS & Benzo(k)fluoranthene & $\begin{array}{l}\text { AHD } \\
\text { AND } \\
\text { ORR } \\
\text { ORR } \\
\text { ORR } \\
\text { ROA }\end{array}$ & $\begin{array}{l}\text { COPPER RIDGE } \\
\text { DISHAL GAP } \\
\text { CHEPULTEPEC } \\
\text { COPPER RIDGE } \\
\text { DISHAL GAP } \\
\text { DISHAL GAP }\end{array}$ & $\begin{array}{l}\text { SOIL } \\
\text { SOIL } \\
\text { SOIL } \\
\text { SOIL } \\
\text { SOIL } \\
\text { SOIL }\end{array}$ & $\begin{array}{l}\mathbf{A} \\
\mathbf{A} \\
\mathbf{A} \\
\mathbf{A} \\
\mathbf{A} \\
\mathbf{A}\end{array}$ & $\begin{array}{r}3 \\
12 \\
2 \\
1 \\
1 \\
1\end{array}$ \\
\hline ORGANICS & Benzo(k)fluoranthene & & & & & 20 \\
\hline ORGAMICS & Chrysene & $\begin{array}{l}\text { AND } \\
\text { AND } \\
\text { ORR } \\
\text { ORR }\end{array}$ & $\begin{array}{l}\text { COPPER RIDGE } \\
\text { DISMAL GAP } \\
\text { CHEPULTEPEC } \\
\text { CHICKAMAUGA Bethel } v\end{array}$ & $\begin{array}{l}\text { SOIL } \\
\text { SOIL } \\
\text { SOIL } \\
\text { SOIL }\end{array}$ & $\begin{array}{l}A \\
A \\
A \\
A\end{array}$ & $\begin{array}{r}11 \\
16 \\
4 \\
7\end{array}$ \\
\hline
\end{tabular}


Table J.1 (continued)

\begin{tabular}{|c|c|c|c|c|c|c|}
\hline Analysis type & Analysis & Location & Formation & Matrix & Horizon & Count \\
\hline & & $\begin{array}{l}\text { ORR } \\
\text { ORR } \\
\text { ORR } \\
\text { ORR } \\
\text { ROA } \\
\text { ROA }\end{array}$ & $\begin{array}{l}\text { CHICKAMAUGA K-25 } \\
\text { COPPER RIDGE } \\
\text { DISHAL GAP } \\
\text { NOLICHUCKY } \\
\text { COPPER RIDGE } \\
\text { DISHAL GAP }\end{array}$ & $\begin{array}{l}\text { SOIL } \\
\text { SOIL } \\
\text { SOIL } \\
\text { SOIL } \\
\text { SOIL } \\
\text { SOIL }\end{array}$ & $\begin{array}{l}A \\
A \\
A \\
A \\
A \\
A\end{array}$ & $\begin{array}{r}6 \\
3 \\
1 \\
10 \\
1 \\
3\end{array}$ \\
\hline ORGANICS & Chrysene & & & & & 62 \\
\hline . & Dalapon & $\begin{array}{l}\text { AND } \\
\text { ORR } \\
\text { ORR } \\
\text { ROA }\end{array}$ & $\begin{array}{l}\text { COPPER RIDGE } \\
\text { CHICKAMAUGA Bethel } v \\
\text { DISMAL GAP } \\
\text { DISMAL GAP }\end{array}$ & $\begin{array}{l}\text { SOIL } \\
\text { SOIL } \\
\text { SOIL } \\
\text { SOIL }\end{array}$ & $\begin{array}{l}A \\
A \\
A \\
A\end{array}$ & $\begin{array}{l}3 \\
3 \\
9 \\
3\end{array}$ \\
\hline ORGANICS & Dalapon & & & & & 18 \\
\hline ORGANICS & Dibenzo $(a, h)$ anthracene & $\begin{array}{l}\text { AND } \\
\text { AND } \\
\text { ORR } \\
\text { ORR } \\
\text { ORR } \\
\text { ORR } \\
\text { ORR } \\
\text { ROA }\end{array}$ & $\begin{array}{l}\text { COPPER RIDGE } \\
\text { DISHAL GAP } \\
\text { CHEPULTEPEC } \\
\text { CHICKAMAUGA Bethel } v \\
\text { CHICKAMAUGA K-25 } \\
\text { COPPER RIDGE } \\
\text { DISMAL GAP } \\
\text { DISMAL GAP }\end{array}$ & $\begin{array}{l}\text { SOIL } \\
\text { SOIL } \\
\text { SOIL } \\
\text { SOIL } \\
\text { SOIL } \\
\text { SOIL } \\
\text { SOIL } \\
\text { SOIL }\end{array}$ & $\begin{array}{l}A \\
A \\
A \\
A \\
A \\
A \\
A \\
A\end{array}$ & $\begin{array}{r}10 \\
24 \\
2 \\
9 \\
9 \\
4 \\
1 \\
1\end{array}$ \\
\hline ORGANICS & Dibenzo $(a, h)$ anthracene & & & & & 60 \\
\hline ORGANICS & Dicamba & $\begin{array}{l}\text { AND } \\
\text { ORR }\end{array}$ & $\begin{array}{l}\text { COPPER RIDGE } \\
\text { CHICKAMAUGA Bethel } v\end{array}$ & $\begin{array}{l}\text { SOIL } \\
\text { SOIL }\end{array}$ & $\begin{array}{l}A \\
A\end{array}$ & $\begin{array}{l}3 \\
3\end{array}$ \\
\hline ORGANICS & Dicamba & & 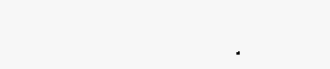 & & & 6 \\
\hline ORGANICS & Dichlorprop & $\begin{array}{l}\text { AND } \\
\text { ORR }\end{array}$ & $\begin{array}{l}\text { COPPER RIDGE } \\
\text { CHICKAMAUGA Bethel } v\end{array}$ & $\begin{array}{l}\text { SOIL } \\
\text { SOIL }\end{array}$ & $\begin{array}{l}A \\
A\end{array}$ & $\begin{array}{l}3 \\
3\end{array}$ \\
\hline ORGANICS & Dichlorprop & & & & & $-\overline{6}$ \\
\hline ORGAHICS & Dieldrin & ORR & CHICKAMAUGA K-25 & SOIL & A & 1 \\
\hline ORGANICS & Dinoseb & $\begin{array}{l}\text { AND } \\
\text { ORR }\end{array}$ & $\begin{array}{l}\text { COPPER RIDGE } \\
\text { CHICKAMAUGA Bethel } v\end{array}$ & $\begin{array}{l}\text { SOIL } \\
\text { SOIL }\end{array}$ & A & $\begin{array}{r}3 \\
3 \\
-\end{array}$ \\
\hline ORGANICS & Dinoseb & & & & & 6 \\
\hline ORGAHICS & Endosulfan sulfate & ORR & CHICKAMAUGA K-25 & SOIL & A & 1 \\
\hline ORGANICS & Endosulfan I & $\begin{array}{l}\text { ORR } \\
\text { ORR }\end{array}$ & $\begin{array}{l}\text { CHICKAMAUGA } \mathrm{K}-25 \\
\text { COPPER RIDGE }\end{array}$ & $\begin{array}{l}\text { SOIL } \\
\text { SOIL }\end{array}$ & A & 1 \\
\hline ORGANICS & Endosulfan I & & & & & 2 \\
\hline ORGANICS & Endosulfan II & ORR & CHICKAMAUGA K-25 & SOIL & A & 1 \\
\hline ORGANICS & Endrin & ORR & CHICKAMAUGA K-25 & SOIL & A & 1 \\
\hline ORGANICS & Endrin aldehyde & ORR & CHICKAMAUGA K-25 & SOIL & $A$ & 1 \\
\hline ORGAHICS & Endrin ketone & ORR & CHICKAMAUGA K-25 & SOIL & A & 1 \\
\hline ORGAHICS & Fluoranthene & $\begin{array}{l}\text { AND } \\
\text { AND } \\
\text { ORR } \\
\text { ORR } \\
\text { ORR } \\
\text { ORR } \\
\text { ROA }\end{array}$ & $\begin{array}{l}\text { COPPER RIDGE } \\
\text { DISHAL GAP } \\
\text { CHICKAMAUGA Bethel } v \\
\text { CHICKAMAUGA } \mathrm{K}-25 \\
\text { DISHAL GAP } \\
\text { NOLICHUCKY } \\
\text { DISHAL GAP }\end{array}$ & $\begin{array}{l}\text { SOIL } \\
\text { SOIL } \\
\text { SOIL } \\
\text { SOIL } \\
\text { SOIL } \\
\text { SOIL } \\
\text { SOIL }\end{array}$ & $\begin{array}{l}A \\
A \\
A \\
A \\
A \\
A \\
A\end{array}$ & $\begin{array}{r}6 \\
13 \\
4 \\
1 \\
10 \\
12 \\
12\end{array}$ \\
\hline ORGANICS & Fluoranthene & & & & & 58 \\
\hline ORGANICS & Fluorene & AND & COPPER RIDGE & SOIL & A & 9 \\
\hline
\end{tabular}


Table J.1 (continued)

\begin{tabular}{|c|c|c|c|c|c|c|}
\hline Analysis type & Analysis & Location & Formation & Matrix & Horizon & Count \\
\hline & . & $\begin{array}{l}\text { AND } \\
\text { ORR } \\
\text { ORR } \\
\text { ORR } \\
\text { ORR } \\
\text { ORR } \\
\text { ROA } \\
\text { ROA }\end{array}$ & $\begin{array}{l}\text { DISHAL GAP } \\
\text { CHEPULTEPEC } \\
\text { CHICKAMAUGA Bethel v } \\
\text { CHICKAMAUGA K-25 } \\
\text { COPPER RIDGE } \\
\text { DISMAL GAP } \\
\text { COPPER RIDGE } \\
\text { DISHAL GAP }\end{array}$ & $\begin{array}{l}\text { SOIL } \\
\text { SOIL } \\
\text { SOIL } \\
\text { SOIL } \\
\text { SOIL } \\
\text { SOIL } \\
\text { SOIL } \\
\text { SOIL }\end{array}$ & $\begin{array}{l}A \\
A \\
A \\
A \\
A \\
A \\
A \\
A\end{array}$ & $\begin{array}{r}14 \\
1 \\
10 \\
5 \\
6 \\
1 \\
3 \\
1\end{array}$ \\
\hline ORGANICS & Fluorene & & & & & 50 \\
\hline ORGANICS & Heptachlor & ORR & CHICKAMAUGA K-25 & SOIL & A - & 1 \\
\hline ORGANICS & Heptachlor epoxide & ORR & CHICKAMAUGA K-25 & SOIL & A & 1 \\
\hline ORGANICS & Indeno $(1,2,3-c d)$ pyrene & $\begin{array}{l}\text { AND } \\
\text { ORR } \\
\text { ORR } \\
\text { ROA } \\
\text { ROA }\end{array}$ & $\begin{array}{l}\text { DISMAL GAP } \\
\text { CHICKAMAUGA Bethel } v \\
\text { DISMAL GAP } \\
\text { COPPER RIDGE } \\
\text { DISMAL GAP }\end{array}$ & $\begin{array}{l}\text { SOIL } \\
\text { SOIL } \\
\text { SOIL } \\
\text { SOIL } \\
\text { SOIL }\end{array}$ & $\begin{array}{l}A \\
A \\
A \\
A \\
A\end{array}$ & $\begin{array}{r}15 \\
1 \\
1 \\
2 \\
1\end{array}$ \\
\hline ORGANICS & Indeno $(1,2,3-\mathrm{cd})$ pyrene & & & & & 20 \\
\hline ORGANICS & Lindane & ORR & CHICKAMAUGA K-25 & SOIL & A & 1 \\
\hline ORGAHICS & Methoxychlor & ORR & CHICKAMAUGA K-25 & SOIL & A & 1 \\
\hline ORGANICS & MCPA & $\begin{array}{l}\text { AND } \\
\text { ORR }\end{array}$ & $\begin{array}{l}\text { COPPER RIDGE } \\
\text { CHICKAMAUGA Bethel } v\end{array}$ & $\begin{array}{l}\text { SOIL } \\
\text { SOIL }\end{array}$ & $\begin{array}{l}A \\
A\end{array}$ & $\begin{array}{l}3 \\
3\end{array}$ \\
\hline ORGANICS & MCPA & & & & & 6 \\
\hline ORGANICS & МСРP & $\begin{array}{l}\text { AND } \\
\text { ORR }\end{array}$ & $\begin{array}{l}\text { COPPER RIDGE } \\
\text { CHICKAMAUGA Bethel } v\end{array}$ & $\begin{array}{l}\text { SOIL } \\
\text { SOIL }\end{array}$ & $\begin{array}{l}A \\
A\end{array}$ & $\begin{array}{l}3 \\
3\end{array}$ \\
\hline ORGANICS & MCPP & & & & & 6 \\
\hline ORGANICS & Naphthalene & $\begin{array}{l}\text { AND } \\
\text { AND } \\
\text { ORR } \\
\text { ORR } \\
\text { ORR } \\
\text { ORR } \\
\text { ORR } \\
\text { ROA }\end{array}$ & $\begin{array}{l}\text { COPPER RIDGE } \\
\text { DISMAL GAP } \\
\text { CHEPULTEPEC } \\
\text { CHICKAMAUGA Bethel } v \\
\text { CHICKAMAUGA K-25 } \\
\text { COPPER RIDGE } \\
\text { DISHAL GAP } \\
\text { COPPER RIDGE }\end{array}$ & $\begin{array}{l}\text { SOIL } \\
\text { SOIL } \\
\text { SOIL } \\
\text { SOIL } \\
\text { SOIL } \\
\text { SOIL } \\
\text { SOIL. } \\
\text { SOIL }\end{array}$ & $\begin{array}{l}\hat{A} \\
\hat{A} \\
\hat{A} \\
\hat{A} \\
\hat{A} \\
\hat{A} \\
\hat{A} \\
\hat{A}\end{array}$ & $\begin{array}{r}12 \\
10 \\
3 \\
5 \\
6 \\
5 \\
1 \\
5\end{array}$ \\
\hline ORGAHICS & Naphthalene & & & & & 47 \\
\hline ORGANICS & Phenanthrene & $\begin{array}{l}\text { AND } \\
\text { ORR } \\
\text { ROA }\end{array}$ & $\begin{array}{l}\text { DISHAL GAP } \\
\text { DISMAL GAP } \\
\text { DISMAL GAP }\end{array}$ & $\begin{array}{l}\text { SOIL } \\
\text { SOIL } \\
\text { SOIL }\end{array}$ & $\begin{array}{l}\mathbf{A} \\
\mathbf{A} \\
\mathbf{A}\end{array}$ & $\begin{array}{l}2 \\
1 \\
1\end{array}$ \\
\hline ORGANICS & Phenanthrene & & & & & 4 \\
\hline ORGANICS & Pyrene & $\begin{array}{l}\text { AND } \\
\text { AND } \\
\text { ORR } \\
\text { ORR } \\
\text { ROA }\end{array}$ & $\begin{array}{l}\text { COPPER RIDGE } \\
\text { DISHAL GAP } \\
\text { CHICKAMAUGA Bethel } v \\
\text { DISHAL GAP } \\
\text { DISHAL GAP }\end{array}$ & $\begin{array}{l}\text { SOIL } \\
\text { SOIL } \\
\text { SOIL } \\
\text { SOIL } \\
\text { SOIL }\end{array}$ & $\begin{array}{l}\mathbf{A} \\
\hat{A} \\
\hat{A} \\
\hat{A} \\
\mathbf{A}\end{array}$ & $\begin{array}{l}2 \\
6 \\
6 \\
1 \\
1\end{array}$ \\
\hline ORGANICS & Pyrene & & & & & 16 \\
\hline ORGAHICS & РСB-1016 & ORR & CHICKAMAUGA K-25 & SOIL & A & 1 \\
\hline ORGAHICS & РCB-1221 & ORR & CHICKAMAUGA K-25 & SOIL & A & 1 \\
\hline ORGAMICS & РСB-1232 & ORR & CHICKAMAUGA K-25 & SOIL & A & 1 \\
\hline ORGANICS & PCB-1242 & ORR & CHICKAMAUGA K-25 & SOIL & A & 1 \\
\hline
\end{tabular}


Table J.1 (continued)

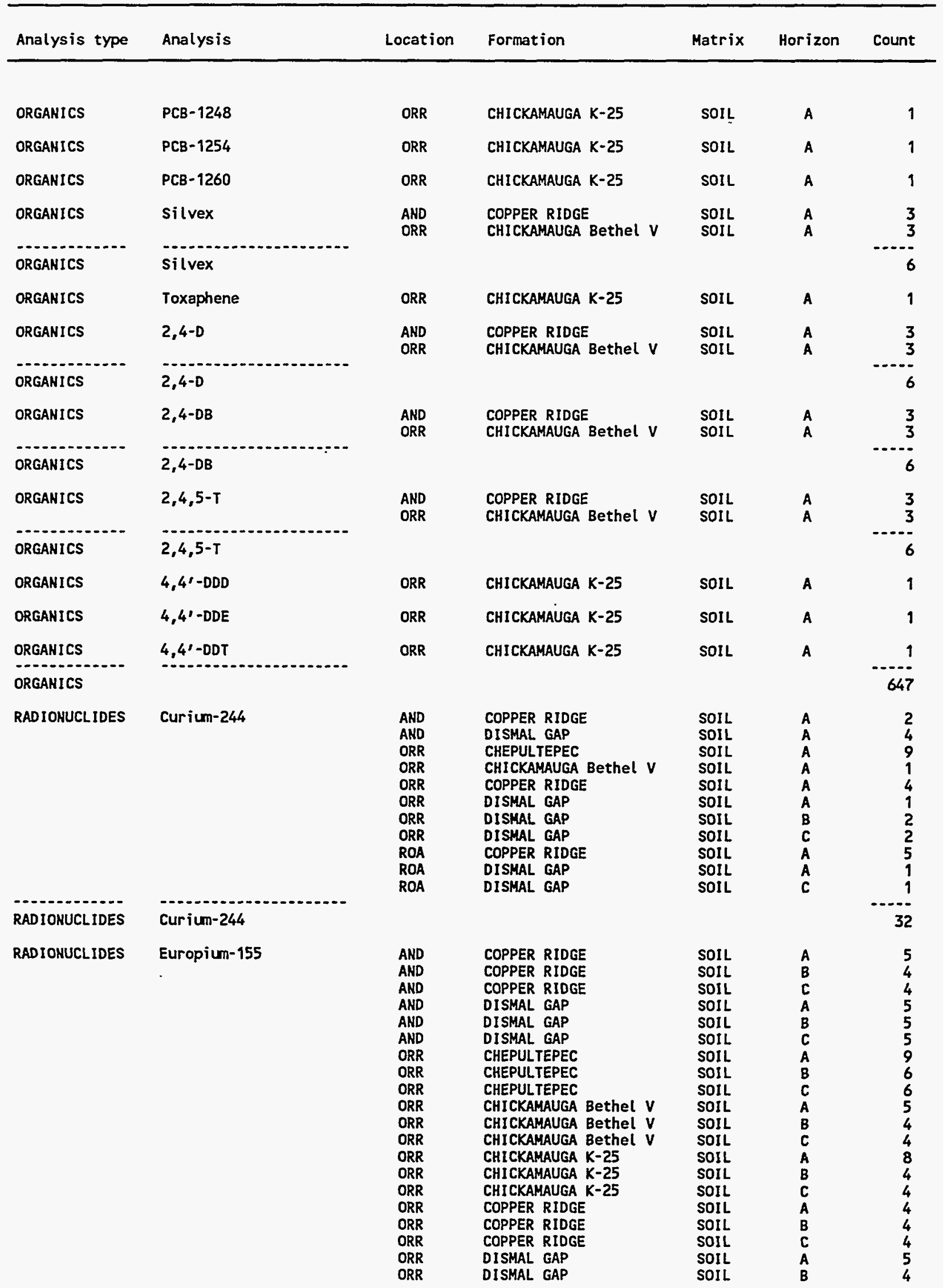


Table J.1 (continued)

\begin{tabular}{|c|c|c|c|c|c|c|}
\hline Analysis type & Analysis & Location & Formation & Matrix & Horizon & Count \\
\hline & & $\begin{array}{l}\text { ORR } \\
\text { ORR } \\
\text { ORR } \\
\text { ORR } \\
\text { ROA } \\
\text { ROA } \\
\text { ROA } \\
\text { ROA } \\
\text { ROA } \\
\text { ROA }\end{array}$ & $\begin{array}{l}\text { DISMAL GAP } \\
\text { HOLICHUCKY } \\
\text { HOLICHUCKY } \\
\text { HOLICHUCKY } \\
\text { COPPER RIDGE } \\
\text { COPPER RIDGE } \\
\text { COPPER RIDGE } \\
\text { DISMAL GAP } \\
\text { DISMAL GAP } \\
\text { DISMAL GAP }\end{array}$ & $\begin{array}{l}\text { SOIL } \\
\text { SOIL } \\
\text { SOIL } \\
\text { SOIL } \\
\text { SOIL } \\
\text { SOIL } \\
\text { SOIL } \\
\text { SOIL } \\
\text { SOIL } \\
\text { SOIL }\end{array}$ & $\begin{array}{l}\text { C } \\
\text { A } \\
B \\
C \\
A \\
B \\
C \\
A \\
B \\
C\end{array}$ & $\begin{array}{l}4 \\
4 \\
4 \\
4 \\
6 \\
5 \\
5 \\
5 \\
5 \\
5\end{array}$ \\
\hline RADIONUCLIDES & Europium-155 & & & & & 146 \\
\hline RADIOHUCLIDES & Neptunium-237 & $\begin{array}{l}\text { AHD } \\
\text { ORR } \\
\text { ORR } \\
\text { ORR } \\
\text { ORR } \\
\text { ROA } \\
\text { ROA } \\
\text { ROA }\end{array}$ & $\begin{array}{l}\text { DISMAL GAP } \\
\text { DISMAL GAP } \\
\text { DISMAL GAP } \\
\text { DISMAL GAP } \\
\text { NOLICHUCKY } \\
\text { DISMAL GAP } \\
\text { DISMAL GAP } \\
\text { DISMAL GAP }\end{array}$ & $\begin{array}{l}\text { SOIL } \\
\text { SOIL } \\
\text { SOIL } \\
\text { SOIL } \\
\text { SOIL } \\
\text { SOIL } \\
\text { SOIL } \\
\text { SOIL }\end{array}$ & $\begin{array}{l}A \\
A \\
B \\
C \\
A \\
A \\
B \\
C\end{array}$ & $\begin{array}{l}1 \\
5 \\
2 \\
2 \\
2 \\
5 \\
1 \\
1\end{array}$ \\
\hline RADIONUCLIDES & Keptunium-237 & & & & & 19 \\
\hline RADIONUCLIDES & Plutonium-238 & ROA & COPPER RIDGE & SOIL & A & 1 \\
\hline RADIONUCLIDES & Plutonium-239/240 & ROA & COPPER RIDGE & SOIL & A & 1 \\
\hline RADIONUCLIDES & Technetium-99 & $\begin{array}{l}\text { AND } \\
\text { AND } \\
\text { ORR } \\
\text { ORR } \\
\text { ORR } \\
\text { ORR } \\
\text { ORR } \\
\text { ORR } \\
\text { ORR } \\
\text { ORR } \\
\text { ROA } \\
\text { ROA } \\
\text { ROA } \\
\text { ROA }\end{array}$ & $\begin{array}{l}\text { COPPER RIDGE } \\
\text { DISMAL GAP } \\
\text { CHEPULTEPEC } \\
\text { CHICKAMAUGA Bethel } v \\
\text { CHICKAMAUGA K-25 } \\
\text { COPPER RIDGE } \\
\text { DISMAL GAP } \\
\text { DISHAL GAP } \\
\text { DISHAL GAP } \\
\text { NOLICHUCKY } \\
\text { COPPER RIDGE } \\
\text { DISHAL GAP } \\
\text { DISHAL GAP } \\
\text { DISHAL GAP }\end{array}$ & $\begin{array}{l}\text { SOIL } \\
\text { SOIL } \\
\text { SOIL } \\
\text { SOIL } \\
\text { SOIL } \\
\text { SOIL } \\
\text { SOIL } \\
\text { SOIL } \\
\text { SOIL } \\
\text { SOIL } \\
\text { SOIL } \\
\text { SOIL } \\
\text { SOIL } \\
\text { SOIL }\end{array}$ & $\begin{array}{l}\text { A } \\
\text { A } \\
\text { A } \\
\text { A } \\
\text { A } \\
\text { A } \\
\text { A } \\
\text { B } \\
\text { C } \\
\text { A } \\
\text { A } \\
\text { A } \\
\text { B } \\
\text { C }\end{array}$ & $\begin{array}{l}5 \\
5 \\
9 \\
4 \\
8 \\
4 \\
5 \\
2 \\
2 \\
4 \\
6 \\
5 \\
1 \\
1\end{array}$ \\
\hline $\begin{array}{l}\text { RADIONUCLIDES } \\
\text { RADIONUCLIDES }\end{array}$ & Technetium-99 & & & & & $\begin{array}{r}61 \\
260\end{array}$ \\
\hline MAA & Cactnium & $\begin{array}{l}\text { AND } \\
\text { AND } \\
\text { AHD } \\
\text { AND } \\
\text { AHD } \\
\text { AND } \\
\text { ORR } \\
\text { ORR } \\
\text { ORR } \\
\text { ORR } \\
\text { ORR } \\
\text { ORR } \\
\text { ORR } \\
\text { ORR } \\
\text { ORR } \\
\text { ORR } \\
\text { ORR } \\
\text { ORR } \\
\text { ORR } \\
\text { ORR } \\
\text { ORR } \\
\text { ORR } \\
\text { ORR } \\
\text { ORR }\end{array}$ & $\begin{array}{l}\text { COPPER RIDGE } \\
\text { COPPER RIDGE } \\
\text { COPPER RIDGE } \\
\text { DISHAL GAP } \\
\text { DISHAL GAP } \\
\text { DISMAL GAP } \\
\text { CHEPULTEPEC } \\
\text { CHEPULTEPEC } \\
\text { CHEPULTEPEC } \\
\text { CHICKAMAUGA Bethel } v \\
\text { CHICKAMAUGA Bethel V } \\
\text { CHICKAMAUGA Bethel V } \\
\text { CHICKAMAUGA K-25 } \\
\text { CHICKAMAUGA K-25 } \\
\text { CHICKAMAUGA K-25 } \\
\text { COPPER RIDGE } \\
\text { COPPER RIDGE } \\
\text { COPPER RIDGE } \\
\text { DISMAL GAP } \\
\text { DISMAL GAP } \\
\text { DISMAL GAP } \\
\text { NOLICHUCKY } \\
\text { NOLICHUCKY } \\
\text { NOLICHUCKY }\end{array}$ & $\begin{array}{l}\text { SOIL } \\
\text { SOIL } \\
\text { SOIL } \\
\text { SOIL } \\
\text { SOIL } \\
\text { SOIL } \\
\text { SOIL } \\
\text { SOIL } \\
\text { SOIL } \\
\text { SOIL } \\
\text { SOIL } \\
\text { SOIL } \\
\text { SOIL } \\
\text { SOIL } \\
\text { SOIL } \\
\text { SOIL } \\
\text { SOIL } \\
\text { SOIL } \\
\text { SOIL } \\
\text { SOIL } \\
\text { SOIL } \\
\text { SOIL } \\
\text { SOIL } \\
\text { SOIL }\end{array}$ & $\begin{array}{l}A \\
B \\
C \\
A \\
B \\
C \\
A \\
B \\
C \\
A \\
B \\
C \\
A \\
B \\
C \\
A \\
B \\
C \\
A \\
B \\
C \\
A \\
B \\
C\end{array}$ & $\begin{array}{l}3 \\
4 \\
4 \\
5 \\
5 \\
5 \\
6 \\
6 \\
6 \\
5 \\
4 \\
4 \\
6 \\
3 \\
4 \\
4 \\
4 \\
4 \\
5 \\
5 \\
5 \\
4 \\
4 \\
4\end{array}$ \\
\hline
\end{tabular}


Table J.1 (continued)

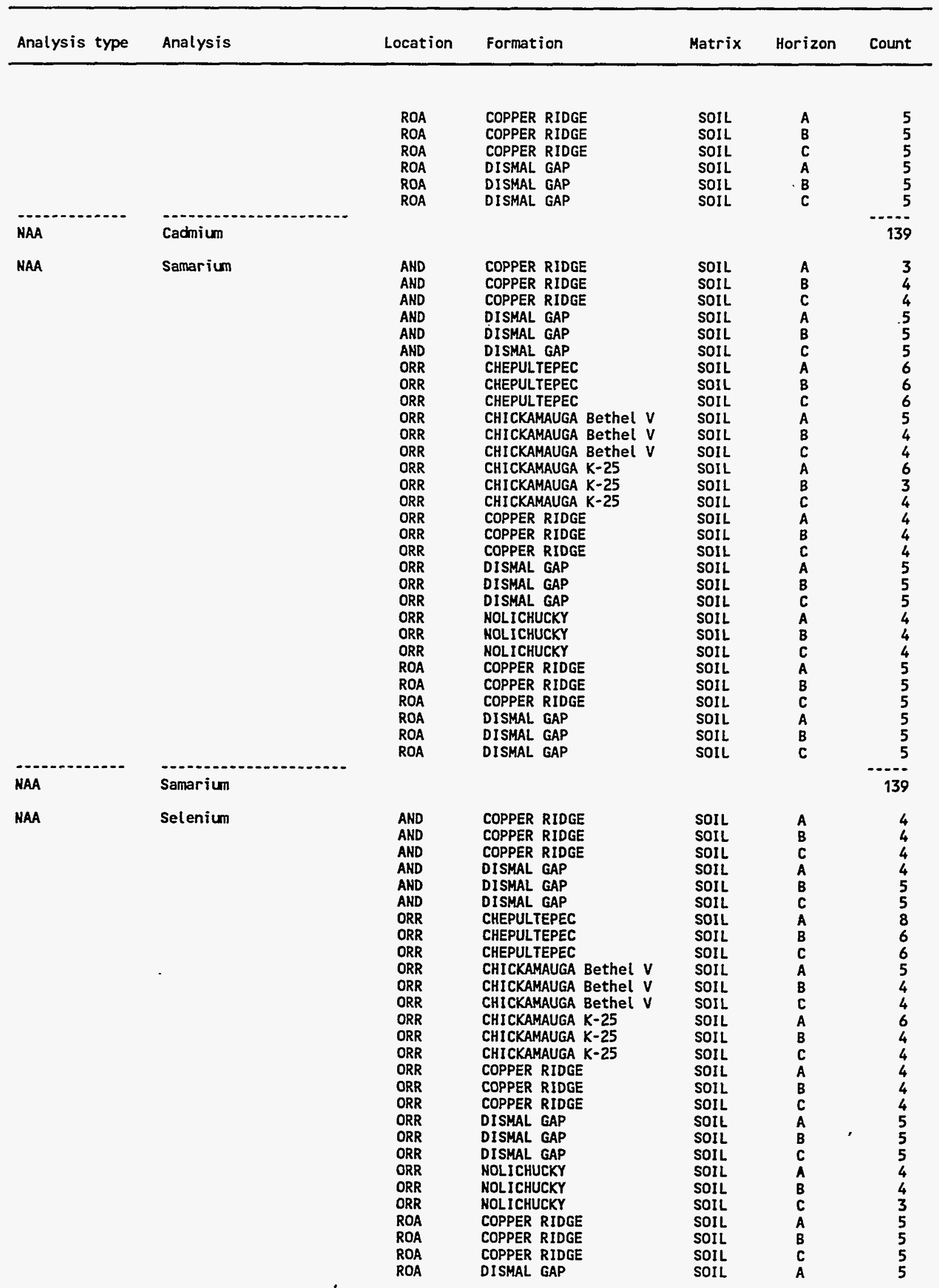


J-11

Table J.1 (continued)

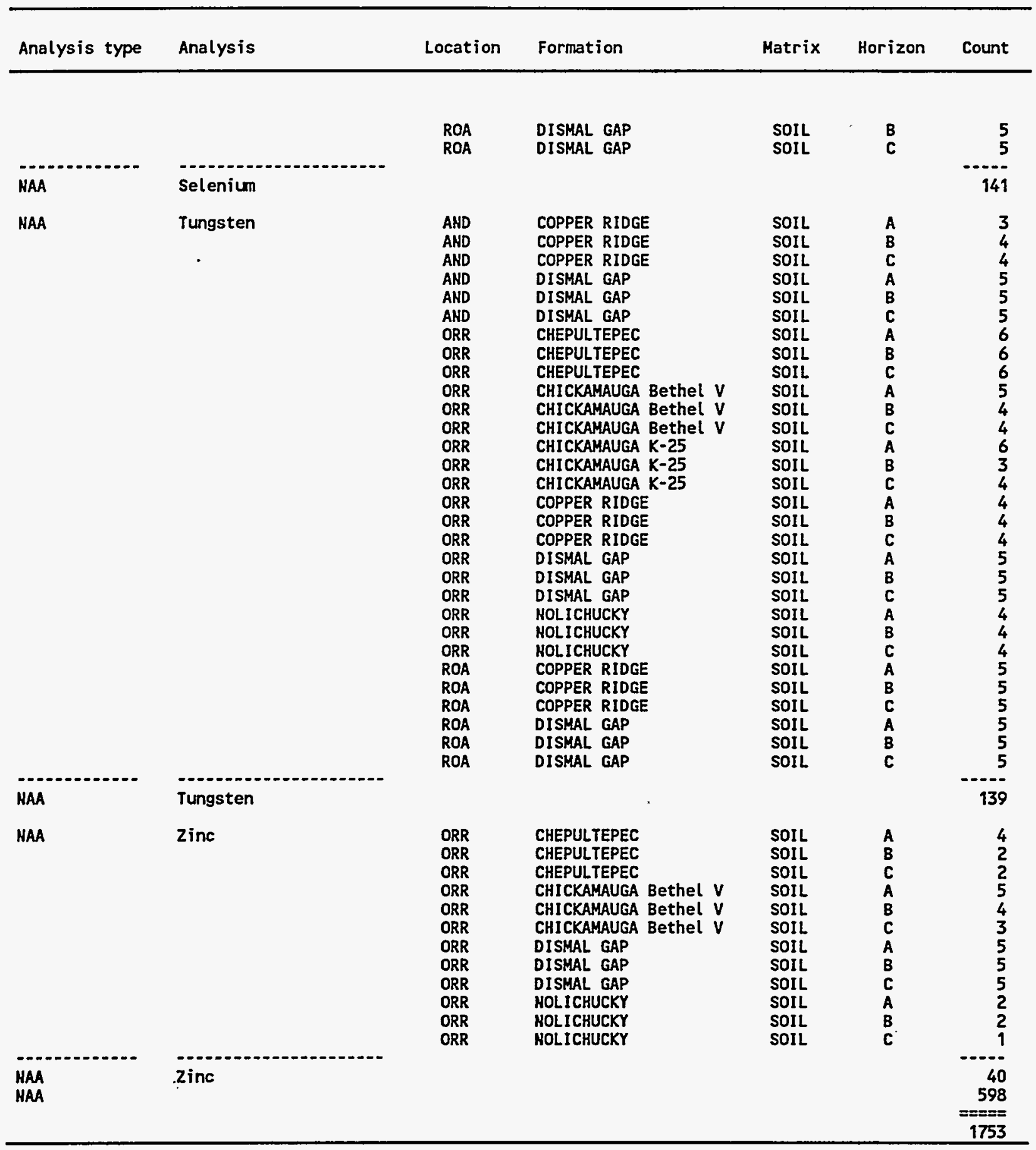




\section{DISTRIBUTION}

1. R. O. Barnett (PORTS)

2. F. P. Baxter

3. D. T. Bell

4. H. L. Boston

5. V. J. Brumback

6. R. B. Cook

7. T. O. Early

8. C. W. Francis

9. P. L. Goddard

10. P. A. Gourieux (PGDP)

11. T. L. Hatmaker

12. L. A. Hook

13. R. H. Ketelle

14. C. W. Kimbrough

15. B. L. Kimmel

16. A. J. Kuhaida

17. S. Y. Lee
18-20. D. M. Matteo

21. C. W. McGinn

22. S. A. Meacham

23. M. J. Norris

24. B. D. Nourse

25-26. P. T. Owen

27. R. L. Schmoyer

28. S. H. Stow

29. R. R. Turner

30-32. D. R. Watkins

33. P. S. Wood

34. Central Research Library

35-37. ESD Library

38. Laboratory Records Department

39. ORNL Patent Section

40-42. Central ER Doc. Mgmt. Center

43-44. ORNL ER Doc. Mgmt. Center

45. Office of Assistant Manager for Energy Research and Development, DOE Oak Ridge Operations Office, P.O. Box 2001, Oak Ridge, TN 37831-8600

46. J. T. Ammons, Department of Plant and Soil Science, University of Tennessee, Knoxville, TN 37916

47. G. W. Bodenstein, DOE Oak Ridge Operations Office, P.O. Box 2001, Oak Ridge, TN 37831-8541

48. D. M. Carden, DOE Oak Ridge Operations Office, P.O. Box 2001, Oak Ridge, TN 37831-8541

49. R. N. Farvolden, Department of Earth Sciences, University of Waterloo, Waterloo, Ontario N21 3G1 Canada

50. M. S. Ferre, DOE Oak Ridge Operations Office, P.O. Box 2001, Oak Ridge, TN 37831-8541

51. C. S. Gist, DOE Oak Ridge Operations Office, P.O. Box 2001, Oak Ridge, TN 37831-8541

52. D. A. Lietzke, Lietzke Soil Services, Route No. 3, Box 607, Rutledge, TN 37861

53. W. N. Lingle, DOE Oak Ridge Operations Office, P.O. Box 2001, Oak Ridge, TN 37831-8541

54. S. P. Riddle, DOE Oak Ridge Operations Office, P.O. Box 2001, Oak Ridge, TN 37831-8541

55. R. C. Sleeman, DOE Oak Ridge Operations Office, P.O. Box 2001, Oak Ridge, TN 37831-8541

56. J. T. Sweeney, DOE Oak Ridge Operations Office, P.O. Box 2001, Oak Ridge, TN 37831-8541

57-58. Office of Scientific and Technical Information, P.O. Box 62, Oak Ridge, TN 37831 